


\title{
GÖYTEPE \\ NEOLITHIC EXCAVATIONS \\ IN THE MIDDLE KURA VALLEY, AZERBAIJAN
}

\author{
Edited by
}

Yoshihiro Nishiaki and Farhad Guliyev 


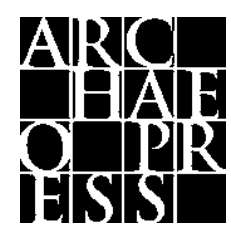

ARCHAEOPRESS PUBLISHING LTD

Summertown Pavilion

18-24 Middle Way

Summertown

Oxford OX2 7LG

www.archaeopress.com

ISBN 978-1-78969-878-7

ISBN 978-1-78969-879-4 (e-Pdf)

(c) the individual authors and Archaeopress 2020

\section{(c) $(1) \Theta \Theta$}

This work is licensed under a Creative Commons Attribution-NonCommercial-NoDerivatives 4.0 International (CC BY-NC-ND 4.0) Licence. https://creativecommons.org/licenses/by/4.0/ 


\section{PREFACE}

This volume presents the results of the archaeological fieldwork conducted by the Azerbaijani-Japanese Archaeological Mission at Göytepe from 2008-2013. The fieldwork was carried out under the collaboration agreement made between the Institute of Archaeology and Ethnography, the National Academy of Science, Azerbaijan, and the University Museum, the University of Tokyo, Japan.

The investigation at Göytepe is led by issues on the emergence and development of food-producing economies and communities in the South Caucasus. Although such issues have been a major focus of archaeological studies for decades in the South Caucasus, similar to Southwest Asia where the large number of investigations have been in progress, at the time of our research planning, our archaeological knowledge on the timing and processes of the transition from hunter-gatherers to farmers was limited. in the latter. However, a number of archaeological studies in the 1980s and 1990s on the Neolithic mound sites in the South Caucasus, including Göytepe, indicated their potential significance in contributing to the clarification of the socio-economy at the dawn of agriculture. In light of this research background, the Azerbaijani-Japanese joint project conducted archaeological investigations at Göytepe aiming to provide new archaeological evidence about the early agricultural societies in the South Caucasus by employing contemporary field and analytical methods.

Many of the chapters of the present volume were completed in early 2012 and, on the occasion of this publication, updated to include subsequent seasons' results and more recent references. However, some chapters may not have been fully updated for a variety of reasons. Nevertheless, we believe that the present volume provides essential information for the research of the South Caucasian Neolithic because its archaeological records have never before been published in a single work in this degree of detail using a high-resolution chronology based on dozens of radiocarbon dates.

The research was made possible with support from a number of sources. Most important was the understanding of the significance of the project and kind permission rendered by Dr. Maisa N. Ragimova of the Institute of Archaeology and Ethnography, the National Academy of Science of Azerbaijan. Dr. Bertille Lyonnet, Laboratoire ProCauLAC, CNRS, France, made a significant contribution to the collaboration arrangement between the two institutions in Azerbaijan and Japan. Financial support was obtained from grants by the Institute of Archaeology and Ethnography, the National Academy of Science of Azerbaijan, the Science Development Foundation under the president of the Republic of Azerbaijan, the Japanese Ministry of Education, Culture, Science, Sports and Technology (17063003 and 22101002), Japan Society for Promotion of Sciences (20401030 and 24251014), the Heiwa Nakajima Foundation (2009), and the Japanese Ministry of Education, Culture, Science, Sports, and Technology (16H06408).

The fieldwork was conducted by numerous colleagues. The major participants of the Azerbaijani team are Farhad Guliyev, Fuad Huseynov, Narqis Hazizade, Tarana Babayeva, Orkhan Zamanov, Mir Jafar Gedirov, Elena Muradova, Jaqob Mammadov, Aygun Alieva, Valeh Alakbarov, Ajhdal Babazadeh, and Shahin Salimbayov, and the Japanese team are Yoshihiro Nishiaki, Seiji Kadowaki, Yui Arimatsu, Shogo Kume, Kazuya Shimogama, Ken-ichi Tanno, Chie Akashi, Yuichi Hayakawa, Takahiro Odaka, Hiroto Nakata, Saiji Arai, Takehiro Miki, and Keiko Ohnishi.

We appreciate the various logistical assistance provided by local colleagues, their warm support and cooperation in the field and camp, notably Namiq Huseynli. We also appreciate the diligent work by the workers at the site, led by Zeki Jeferov, who helped us realize fruitful excavation seasons.

Yoshihiro Nishiaki

The University Museum, The University of Tokyo, Japan

Farhad Guliyev

Institute of Archaeology and Ethnography, The National Academy of Science, Azerbaijan 


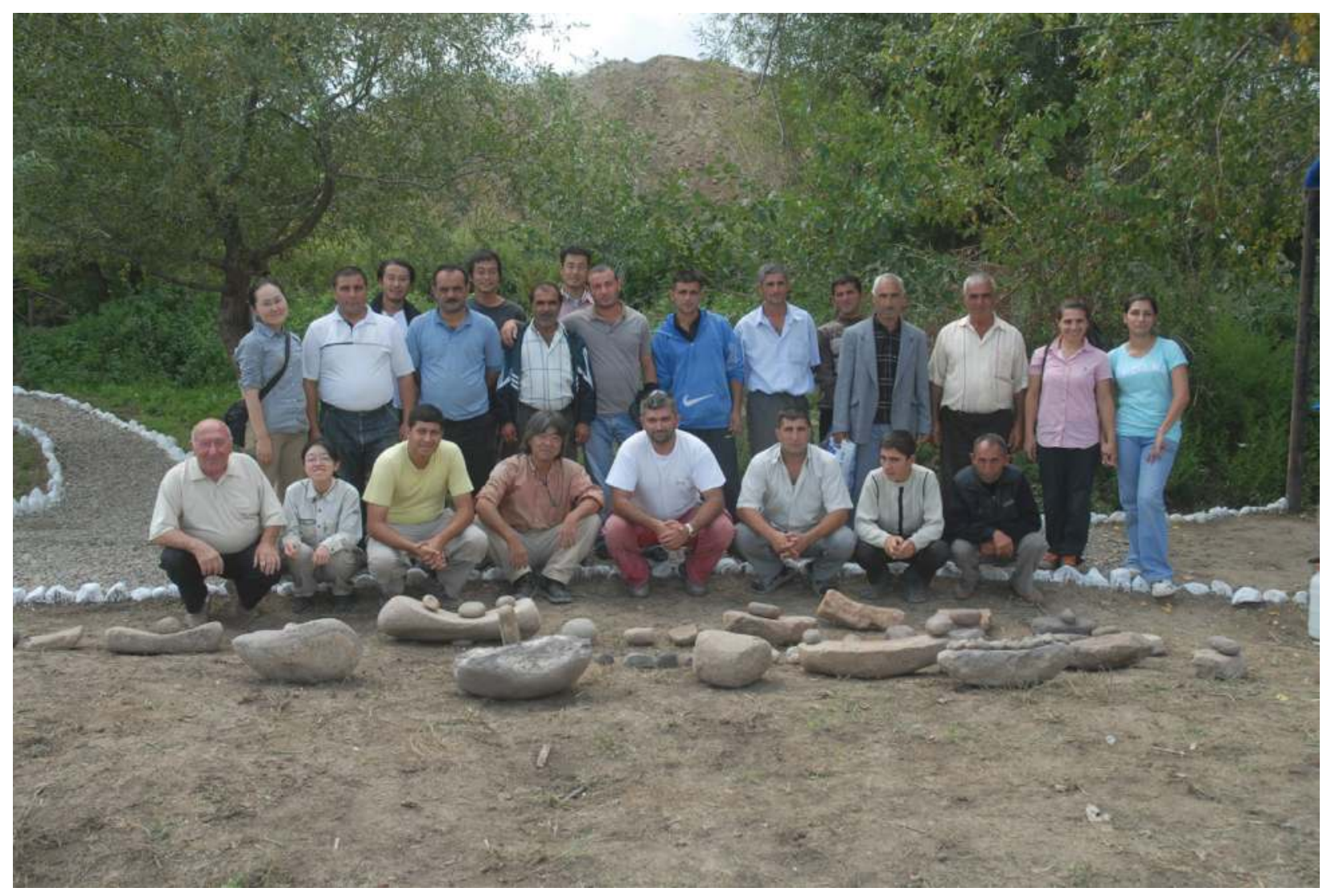

Research crew for the 2009 season's excavations at Göytepe. 


\section{TABLE OF CONTENTS}

Preface

Yoshihiro Nishiaki and Farhad Guliyev

iii

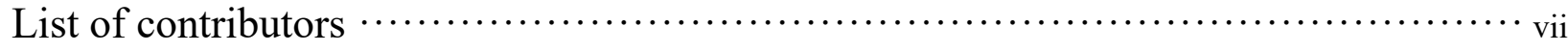

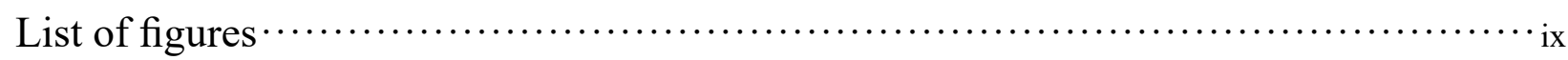

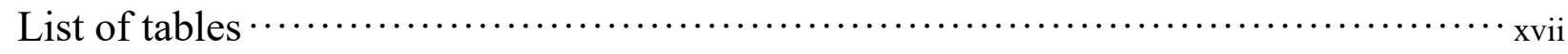

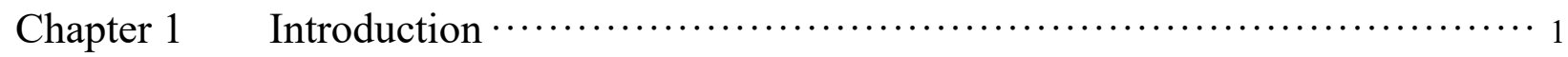

Yoshihiro Nishiaki and Farhad Guliyev

\section{Part I: Field Investigations}

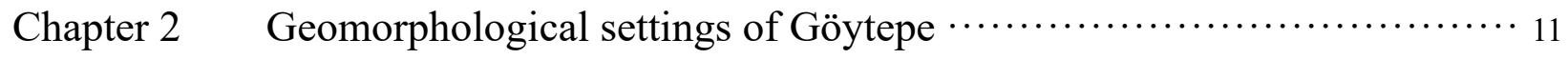
Yuichi Hayakawa

Chapter 3 Stratigraphy and architecture in the main excavation area

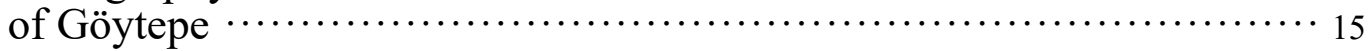
Yoshihiro Nishiaki, Farhad Guliyev, Fuad Hoseynov, and Kazuya Shimogama

Chapter 4 Excavation, stratigraphy, and architecture of Square 4B

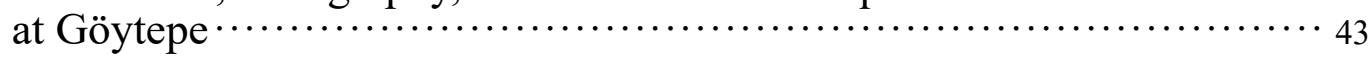
Seiji Kadowaki, Yui Arimatsu, and Yoshihiro Nishiaki

Chapter 5 Soundings at the edges of Göytepe

Yoshihiro Nishiaki, Yui Arimatsu, and Saiji Arai

Chapter 6 Mud-bricks in Neolithic architecture at Göytepe

Yoshihiro Nishiaki, Farhad Guliyev, and Emmanuel Baudouin

Chapter 7 Geoarchaeological investigation of storage space at Göytepe: Phytolith, dung spherulite, and micromorphological analyses …................ 109 Seiji Kadowaki, Lisa Maher, Marta Portillo, and Rosa M. Albert

Chapter 8 Archaeological reconnaissance survey around Göytepe,

Tovuz-Qovlar region

Kazuya Shimogama and Valeh Alakbarov

\section{Part II: Technology and Subsistence}

Chapter 9 Neolithic flaked stone assemblages from Göytepe

Yoshihiro Nishiaki

Chapter 10 Use-wear analysis of chipped stone artifacts from Göytepe 191

Katsunori Takase

Chapter 11 Fracture wing analysis for identification of obsidian blank production techniques at Göytepe 209 Jun Takakura and Yoshihiro Nishiaki 
Chapter 12 Neolithic ground stone typology and technology at Göytepe

Seiji Kadowaki

Chapter 13 Neolithic pottery from Göytepe

Yui Arimatsu

With an Appendix by Takahiro Odaka

Chapter 14 Neolithic figurines from Göytepe

Kazuya Shimogama

Chapter 15 The Neolithic bone and antler industry from Göytepe $\cdots \cdots \cdots \cdots \cdots \cdots \cdots \cdots \cdots \cdots \cdots$ Saiji Arai

Chapter 16 Plant remains from Göytepe

Chie Akashi and Ken-ichi Tanno

Chapter 17 Faunal remains from Göytepe

Saiji Arai

Rezume 365

Fərhad Quliyev, Yoşihiro Nişiyaki 


\section{LIST OF CONTRIBUTORS}

Chie Akashi

The University Museum, The University of Tokyo, Tokyo, JAPAN

Valeh Alakbarov

Institute of Archaeology and Ethnography, The National Academy of Science, Baku, AZERBAIJAN

Rosa M. Albert

ICREA / Department of Prehistory, Ancient History and Archaeology, University of Barcelona, Barcelona, SPAIN

Saiji Arai

Department of Evolutionary Studies of Biosystems, School of Advanced Sciences, The Graduate University for Advanced Studies, Miura, JAPAN

Yui Arimatsu

Graduate School of Humanities and Social Sciences, Hiroshima University, Hiroshima, JAPAN

Emmanuel Baudouin

Université Toulouse Jean Jaurès, Toulouse, FRANCE

Farhad Guliyev (editor)

Institute of Archaeology and Ethnography, The National Academy of Science, Baku, AZERBAIJAN

Yuichi Hayakawa

Faculty of Environmental Earth Science, Hokkaido University, Sapporo, JAPAN

Fuad Hoseynov

Institute of Archaeology and Ethnography, The National Academy of Science, Baku, AZERBAIJAN

Seiji Kadowaki

Nagoya University Museum, Nagoya University, Nagoya, JAPAN

Lisa Maher

Department of Anthropology, Berkeley, University of California, Berkely, USA

Yoshihiro Nishiaki (editor)

The University Museum, The University of Tokyo, Tokyo, JAPAN

Takahiro Odaka

Center for Cultural Resource Studies, Kanazawa University, Kanazawa, JAPAN

Marta Portillo

Department of Archaeology and Anthropology, Institució Milà i Fontanals de Recerca en Humanitats, Spanish National Research Council (CSIC), Barcelona, SPAIN

Kazuya Shimogama

Ancient Orient Museum, Tokyo, Tokyo, JAPAN 
Jun Takakura

Archaeological Research Center, Hokkaido University, Sapporo, JAPAN

Katsunori Takase

Graduate School of Humanities and Human Sciences, Hokkaido University, Sapporo, JAPAN

Ken-ichi Tanno

Faculty of Letters, Ryukoku University, Kyoto, JAPAN 


\section{LIST OF FIGURES}

Fig. 1.1 Map showing the location of Göytepe and related Neolithic sites in the South Caucasus. ……………........ 2

Fig. 1.2 Probability distributions of dates estimated with the sequence and phase models of the OxCal program. $\cdots 3$

Fig. 1.3 Summed probability distribution of radiocarbon dates from Göytepe. ................................................... 7

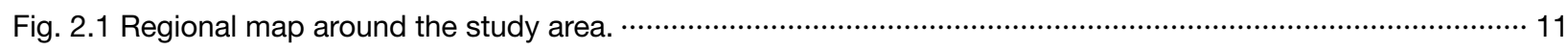

Fig. 2.2 Topographic map around Göytepe, measured in the field with a laser range finder and a differential GNSS

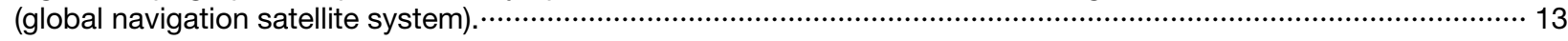

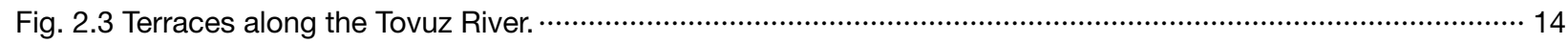

Fig. 3.1 Plan of Göytepe showing the location of excavation squares. ……................................................... 15

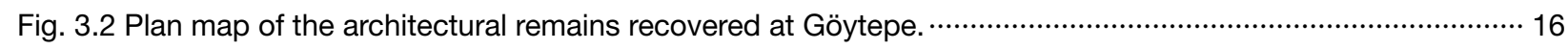

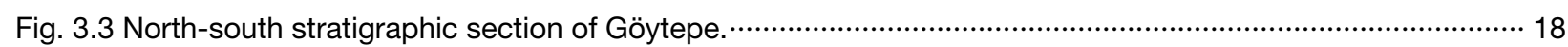

Fig. 3.4 Mud-brick wall of Building 101 in Square 2BI looking northwest. ….................................................. 19

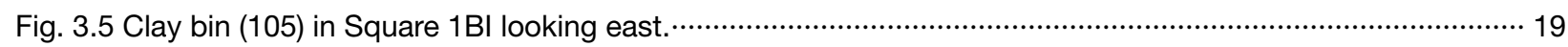

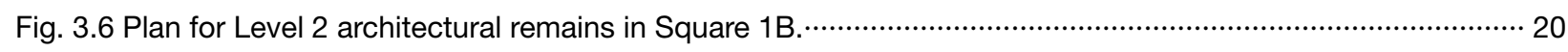

Fig. 3.7 Level 2 architectural remains in Square 1B looking southwest...............................................................20

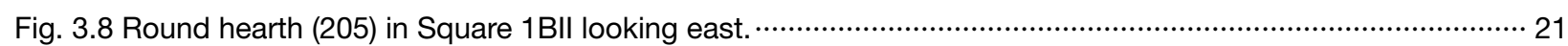

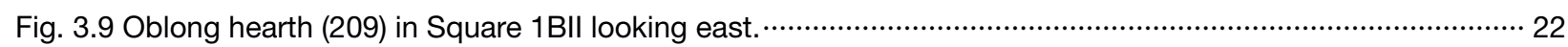

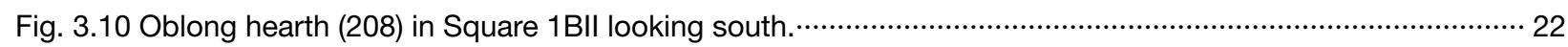

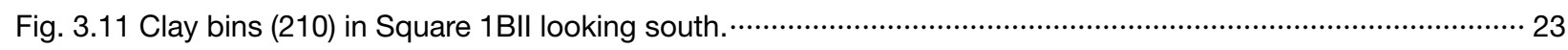

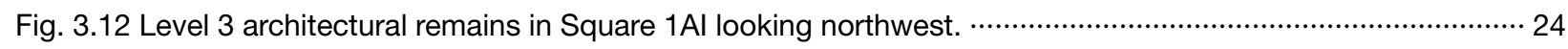

Fig. 3.13 Concentration of sling stones near clay bins (305) in Square 1Al. ...................................................24

Fig. 3.14 General view of the courtyard of the Square 3A compound looking southeast. …................................25

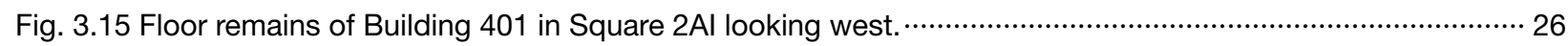

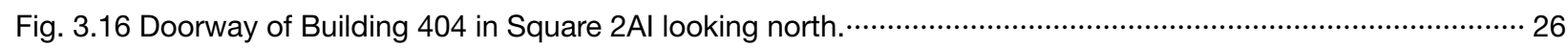

Fig. 3.17 Floor remains of the courtyard of the compound of Square 3A looking east. …....................................27

Fig. 3.18 General view of the compound of Square 2A looking southeast. ....................................................28

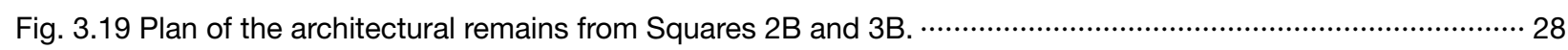

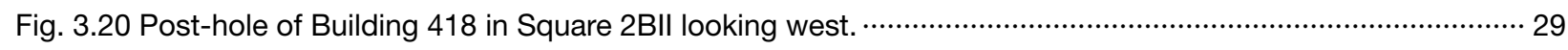

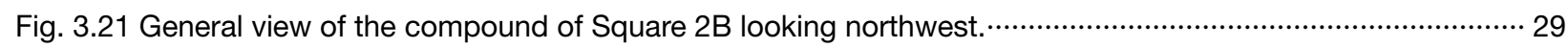

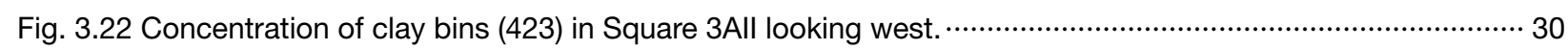

Fig. 3.23 Distribution of obsidian artifacts near clay bins (423) in Square 3All looking west................................. 31

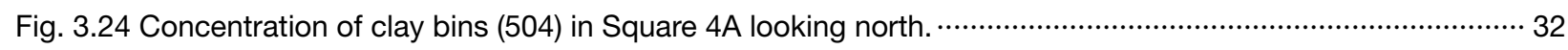

Fig. 3.25 Hearth (501) and a ground stone (502) from Square 4A1 looking east. ……......................................... 32

Fig. 3.26 Concentration of cattle scapulae on the room floor $(510)$ in Square 3BII looking south.............................3 33

Fig. 3.27 Concentration of stone and bone objects in Square 3BI (507) looking south.........................................33

Fig. 3.28 Wall foundation made of clay cob for Level 2 buildings in $1 \mathrm{BI}$ looking east. .........................................35

Fig. 3.29 Wall of Building 511 of Level 5 showing the use of mud-bricks with different colors. …........................... 36

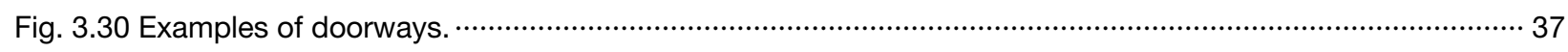

Fig. 3.31 Doorway closed with mud-bricks, Building 419 of Level 4, looking northwest. …….............................. 38

Fig. 3.32 Doorway closed with mud-bricks and clay, Building 512 of Level 5, looking west. ….............................39

Fig. 3.33 Schematic reconstruction of a Level 4 compound. …...................................................................... 40

Fig. 3.34 Inner diameters of the circular buildings from Levels 1-6 of Göytepe. …...........................................40

Fig. 3.35 Schematic reconstruction showing repeated construction of the household compounds. ……………...... 41

Fig. 4.1 Topographic map of Göytepe showing the excavation squares and disturbed area to the northeast. $\cdots \cdots \cdots . . . .43$ 
Fig. 4.2 Square 4B seen from the east.

Fig. 4.3 Square 4B seen from the north.

Fig. 4.4 Topography of Square 4B after the 2009-2013 seasons showing the building levels excavated in different parts of the square and locations of stratigraphic sections presented in this chapter. ……........................................45

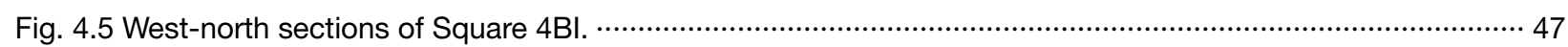

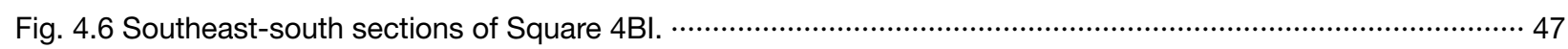

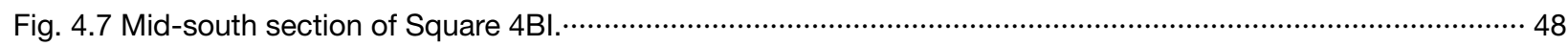

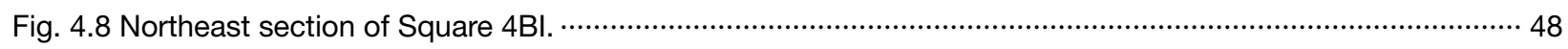

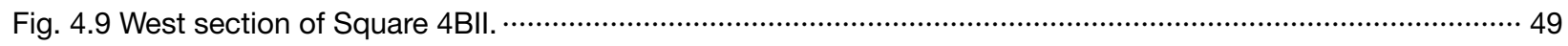

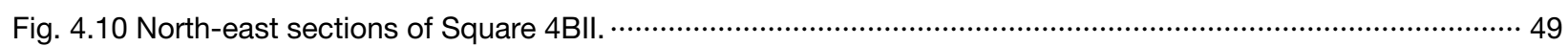

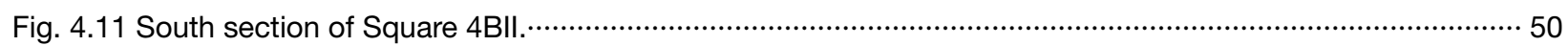

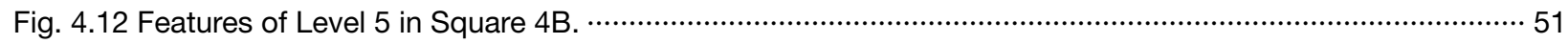

Fig. 4.13 Building remains of the upper phase of Level 6 in Square 4B. ……..................................................5

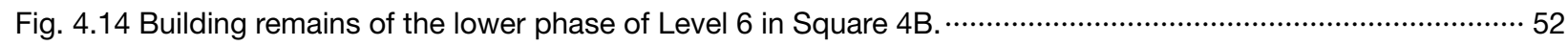

Fig. 4.15 Building remains of the upper phase of Level 7 in Square 4B. …...................................................53

Fig. 4.16 Building remains of the lower phase of Level 7 in Square 4B. …......................................................53

Fig. 4.17 Mud-brick walls of Levels 6 and 7 in Square 4B, seen from the south. …...........................................5 54

Fig. 4.18 Architectural features of Levels 6 and 7 in Square 4BI, seen from the southwest. ………………...........5 54

Fig. 4.19 Mud-brick walls and other features of Levels 7 and 8 in Square 4BII, seen from the northwest.………....55

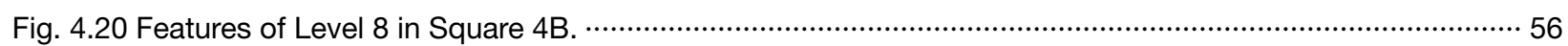

Fig. 4.21 Features recovered in Level 8 in Square 4BI, seen from the north. ….................................................56

Fig. 4.22 Building remains of the upper phase of Level 9 in Square 4B. …......................................................

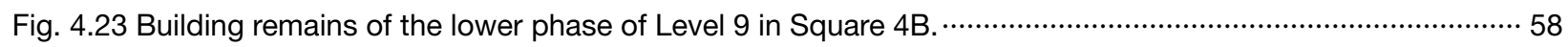

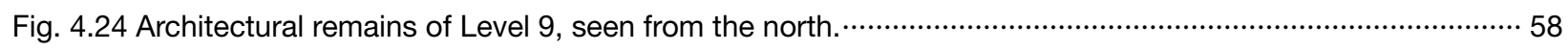

Fig. 4.25 Hearth with burnt cobbles (4BII-56) from Level 9 in Square 4BII, seen from the southwest. ……............59

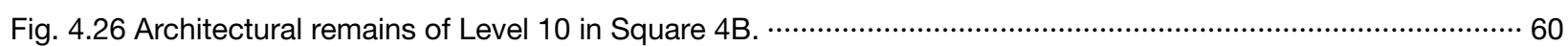

Fig. 4.27 Architectural remains of Levels 9, 10, and 11 in Square 4BII, seen from the northeast. ……..................60

Fig. 4.28 Stratigraphic section of room fill and a floor inside the round structure (4BIIX-12), seen from the east. ......61

Fig. 4.29 The upper phase of the round building (4BIIX-16), corresponding to Level 10. ......................................61

Fig. 4.30 Indoor clay bins (4BI-118 and 121) showing sections of fill, seen from the south. ...............................6.

Fig. 4.31 Front view of the round building's (4BIIX-16) entrance, seen from the southwest.................................63

Fig. 4.32 Concentration of chipped obsidian artifacts inside the building (4BIIX-16), seen from the north...............6 64

Fig. 4.33 Sampling grids within the round building (4BIIX-16) of Level 10 in Square 4B.......................................6

Fig. 4.34 The building's interior (4BIIX-16) was excavated using $50 \times 50 \mathrm{~cm}$ grids with a combination of arbitrary and

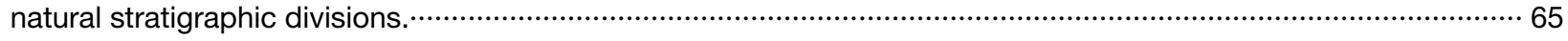

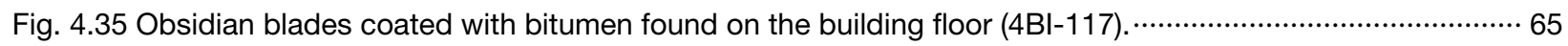

Fig. 4.36 Plans and profiles of clay bins in Level 10 in Square 4BII. ..................................................................6.

Fig. 4.37 A clay bin (4BIIX-94) in Level 10, adjacent to an area scattered with various domestic refuse, including a complete grinder, bone tools, angular cobbles, animal bones, and charcoal fragments in ashy sediments (4BIIX-92), seen from the west.

Fig. 4.38 White fibrous remains attached to the surface of a handstone (4BIIX-94a) found near the bottom of the clay

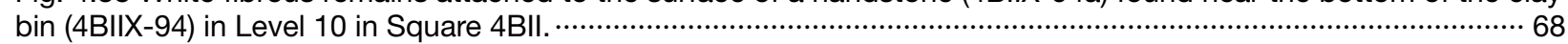

Fig. 4.39 Outdoor features south of Square 4BII, seen from the southwest. …................................................68

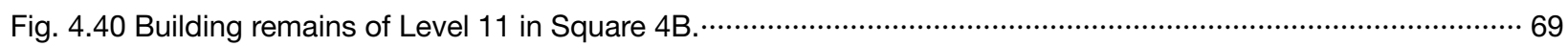


Fig. 4.41 Lower phase of the round building (4BIIX-16) in Level 11, seen from the southwest.

Fig. 4.42 Superposition of the round building (4BIIX-12) of Level 10 over that of Level 11 (4BIIX-34, 52, and 56), seen from the northeast.

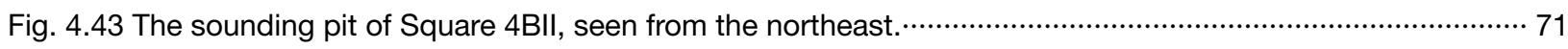

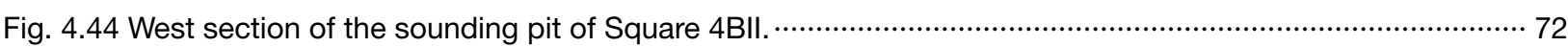

Fig. 4.45 Building remains of Level 12 in Square 4B, seen from the west. ….................................................... 73

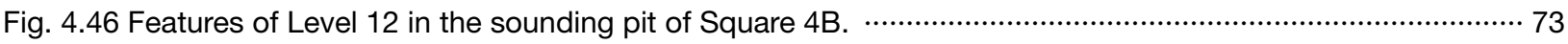

Fig. 4.47 Side view of the mud-brick wall of Level 12 in the sounding pit of Square 4B, seen from the east. ……... 74

Fig. 4.48 Top view of the mud-brick wall of Level 12 in the sounding pit of Square 4B. ……………………….... 74

Fig. 4.49 Features of Level 13 in the sounding pit of Square 4B. ….........................................................

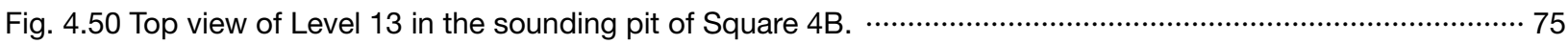

Fig. 4.51 Side view of the structure of Level 13 in the sounding pit of Square 4B, seen from the northwest. ……... 76

Fig. 4.52 Excavation in the northwest corner of the sounding pit in Square 4B, seen from the southeast. ………...76

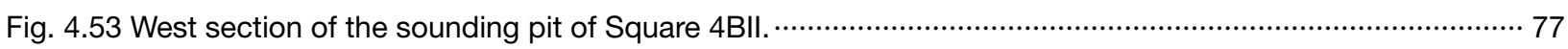

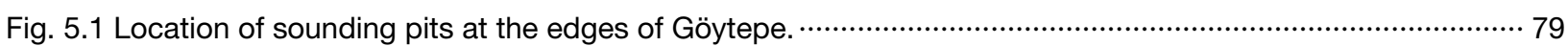

Fig. 5.2 Upper portion of the sounding pit in Square 7B, seen from the east................................................. 80

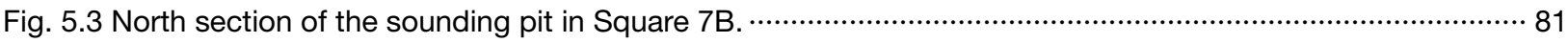

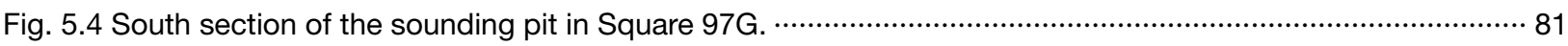

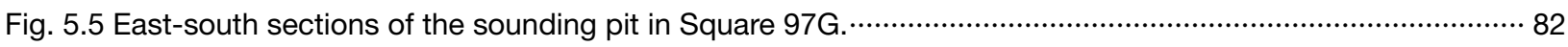

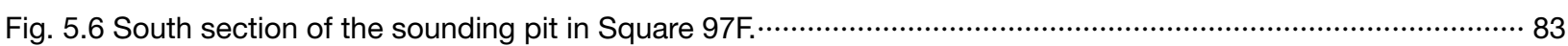

Fig. 5.7 East-south-west sections of the sounding pit in Square 97F. ............................................................ 83

Fig. 5.8 A massive wall revealed by a small sounding in Square 96F, looking south. ............................................ 84

Fig. 5.9 A massive wall revealed by a small sounding in Square 96F, looking southwest. .................................... 84

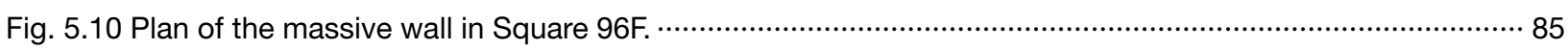

Fig. 5.11 South-west-north sections of the massive wall in Square 96F...................................................... 85

Fig. 5.12 Mud-bricks at the base of the wall in Square 96F, looking west. ........................................................ 86

Fig. 5.13 Inner surface of the massive wall in Square 96F, seen from the west................................................. 86

Fig. 5.14 Base of the massive wall in Square 96F, seen from the east. …….................................................... 87

Fig. 5.15 West section of the massive wall's inner part in Square 96F, seen from the south. …............................ 87

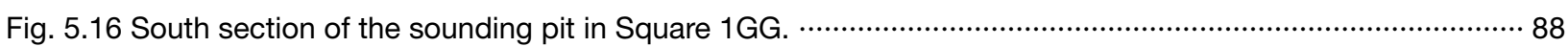

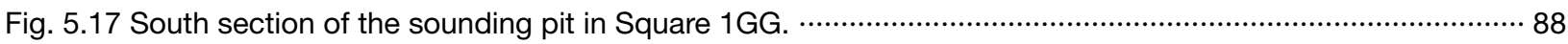

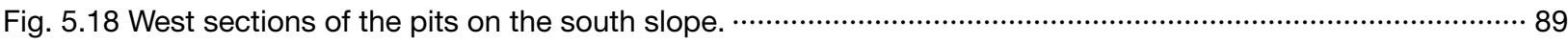

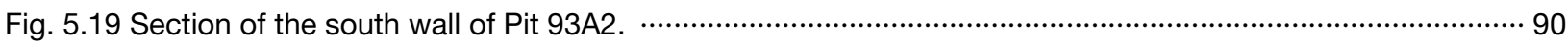

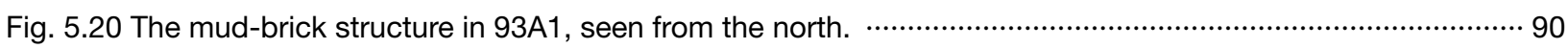

Fig. 5.21 The base of the curvilinear wall in 93A1, seen from the east. .......................................................... 91

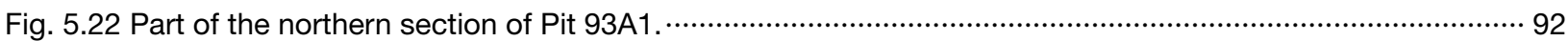

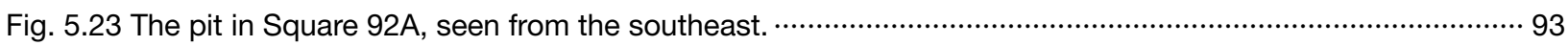

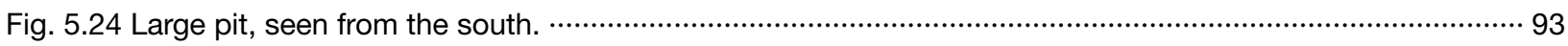

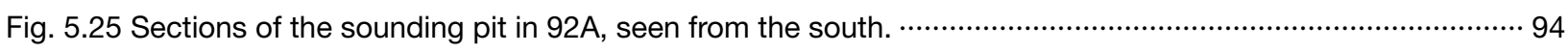

Fig. 5.26 Excavations of the sounding pit in $92 \mathrm{~A}$, seen from the northeast. .................................................. 94

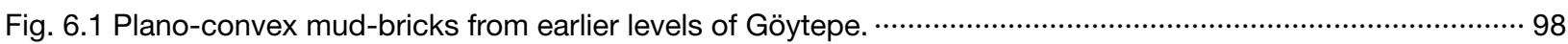

Fig. 6.2 Larger and less-convexed mud-bricks from later levels of Göytepe. ……............................................. 99

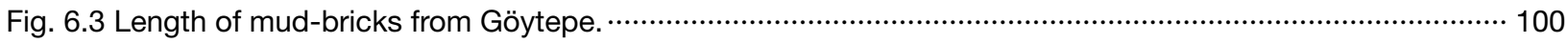




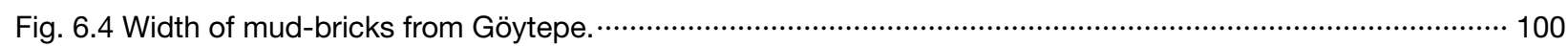

Fig. 6.5 Modern stone block wall in Qovlar Village, Tovuz, Azerbaijan. …....................................................... 101

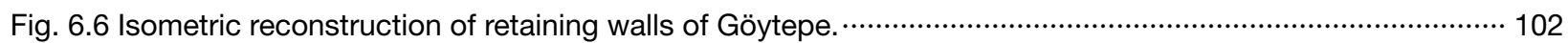

Fig. 6.7 Stratigraphic changes in mud-brick size at Göytepe. ………................................................................. 103

Fig. 6.8 Map showing regional variability of Neolithic mud-brick types in the southern Caucasus. ........................... 104

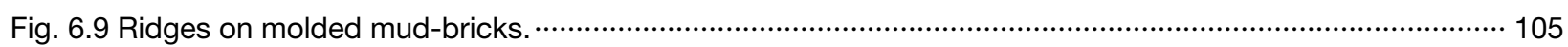

Fig. 7.1 Architectural plan revealed during the 2008-2011 seasons at Göytepe. …….......................................... 110

Fig. 7.2 A cluster of bin features in an outdoor area (Level 4 in Square 3All), indicating the contexts sampled for

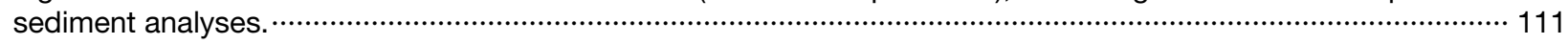

Fig. 7.3 Sections of fills in two bins (3All-1 and 2), indicating sampling locations.

Fig. 7.4 Architectural plan of Level 10 in Square 4B, indicating the contexts sampled for sediment analyses. ........114

Fig. 7.5 A clay bin (4BIIX-94) and its adjacent area (4BIIX-92) distributed with domestic refuse. ………………...... 114

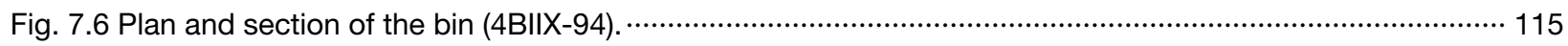

Fig. 7.7 Grinder and handstone placed together near the bottom of the bin (4BIIX-94). ………............................ 116

Fig. 7.8 Histogram showing the relative abundances (\%) of phytoliths from grasses, dicotyledonous leaves,

dicotyledonous wood/bark and weathered morphotypes (WM) obtained from Square 3All samples. …………….. 118

Fig. 7.9 Anatomical origin of grass phytoliths in Square 3All samples. …….......................................................... 119

Fig. 7.10 Photomicrographs of phytoliths and other microremains identified in the samples. …............................ 119

Fig. 7.11 Histogram showing the relative abundances (\%) of phytoliths from grasses, dicotyledonous leaves, dicotyledonous wood/bark and weathered morphotypes (WM) obtained from Square 4BIIX samples. ………….... 120

Fig. 7.12 Anatomical origin of grass phytoliths in Square 4BIIX samples. ……..................................................... 121

Fig. 7.13 Excavated sediment column from S25 removed en bloc with five thin section slides cut from the block. 122

Fig. 7.14 Photomicrograph images of features from the bottom fill and clay wall of bin S25. ……....................... 123

Fig. 7.15 Excavated sediment column from S36 removed en bloc with five thin section slides cut from the block. 124

Fig. 7.16 Photomicrograph images of features from the bottom fill and clay wall of bin S36. ……....................... 131

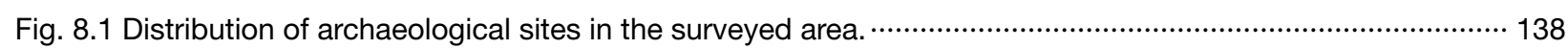

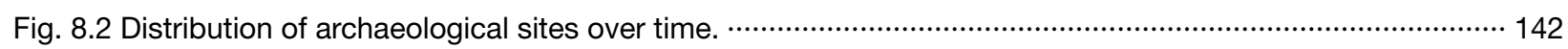

Fig. 8.3 Diagram of the numbers of sites from each time period. ……………............................................ 143

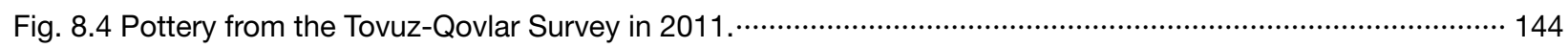

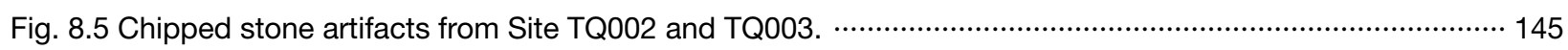

Fig. 8.6 Site TQ009 (Hacı Elamxanli), seen from the southwest. ……................................................................. 146

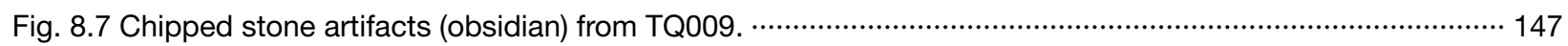

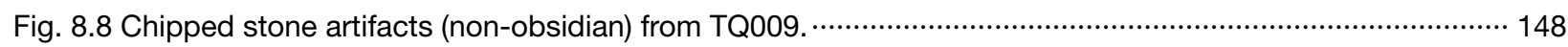

Fig. 8.9 Site TQ024 (Toretepe), seen from the southeast. ………………………………………………………... 149

Fig. 8.10 Chipped stone artifacts (obsidian) from TQ024, TQ026, and TQ027. ……………………………........ 149

Fig. 8.11 Site TQ011 (Aytepe) cut by modern road in the foreground and TQ012 (Ibrahim Hacili Tepe) in the

background behind village houses, seen from the north. ……....................................................................... 151

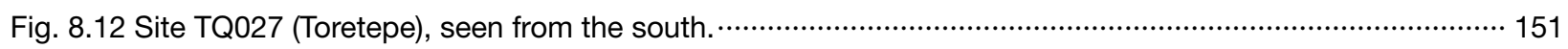

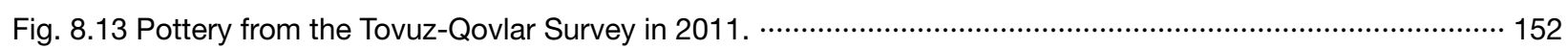

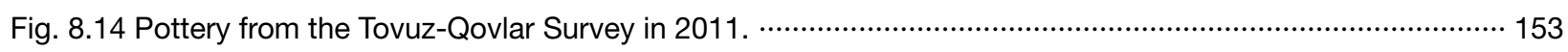

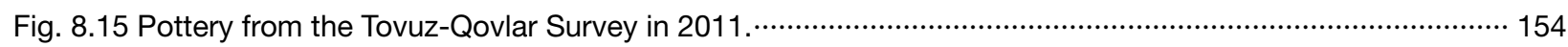

Fig. 8.16 Pottery from the Tovuz-Qovlar Survey in 2011.…................................................................. 155

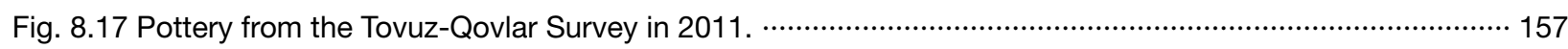

Fig. 8.18 Pottery and small finds from the Tovuz-Qovlar Survey in 2011. .......................................................... 158 


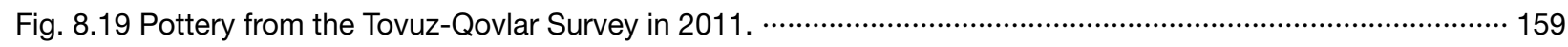

Fig. 8.20 Pottery from the Tovuz-Qovlar Survey in 2011. ........................................................................ 160

Fig. 8.21 Pottery from the Tovuz-Qovlar Survey in 2011.......................................................................... 161

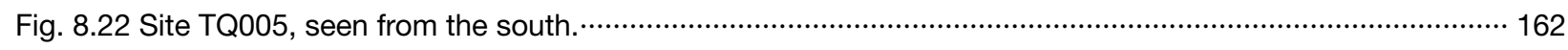

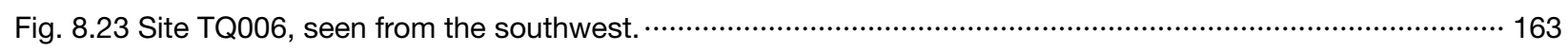

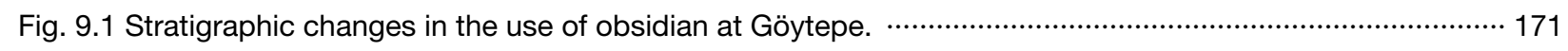

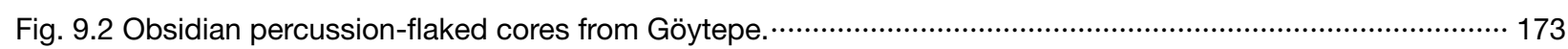

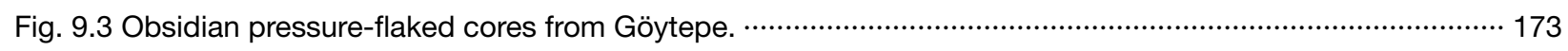

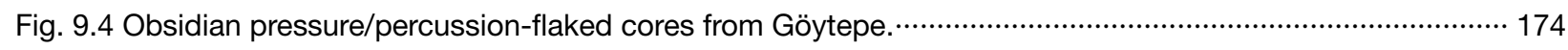

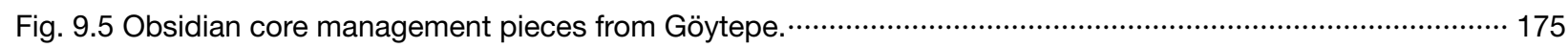

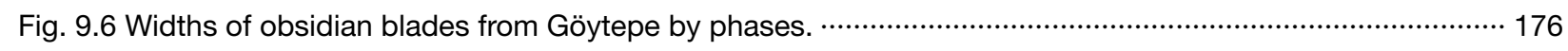

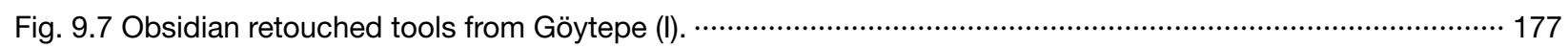

Fig. 9.8 Complete sickle with obsidian blades inserted in a jagged way (Upper Area).....................................179

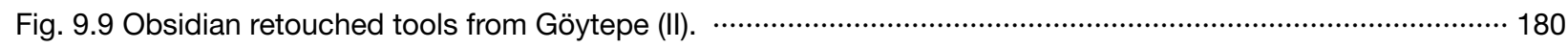

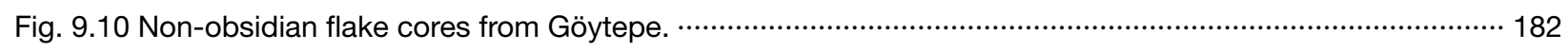

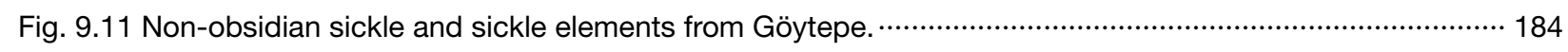

Fig. 9.12 Non-obsidian retouched tools from Göytepe (I). ….................................................................. 185

Fig. 9.13 Non-obsidian retouched tools from Göytepe (II). …...................................................................... 186

Fig. 9.14 Non-obsidian retouched tools from Göytepe (III). …….................................................................. 187

Fig. 10.1 Polish distribution on obsidian artifacts from Göytepe (I). …........................................................... 197

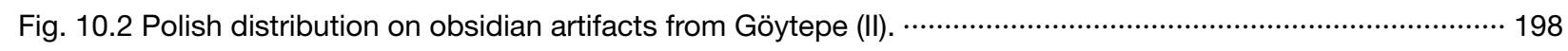

Fig. 10.3 Polish distribution on obsidian artifacts from Göytepe (III). …........................................................ 199

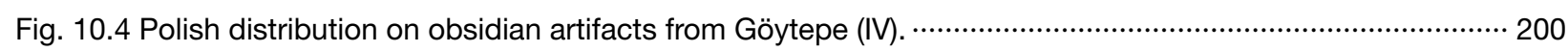

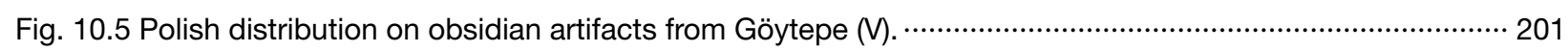

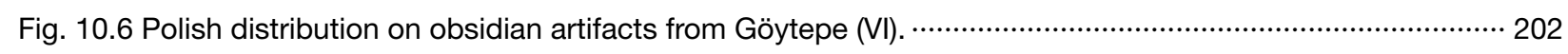

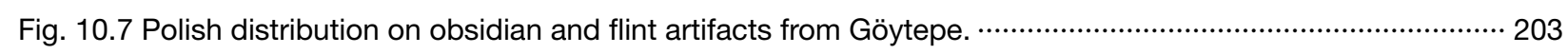

Fig. 11.1 Obsidian artifacts from Göytepe showing the location of fracture wing measurement points. …….......210

Fig. 11.2 Microphotographs of fracture wings from Göytepe (I). …............................................................214

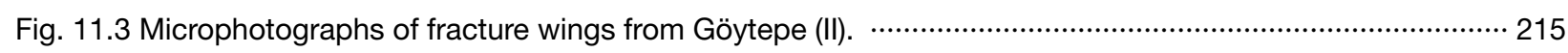

Fig. 11.4 Microphotographs of fracture wings from Göytepe (III). ...................................................................216

Fig. 11.5 Measurement values of the crack velocity of 19 samples from Göytepe. …….................................220

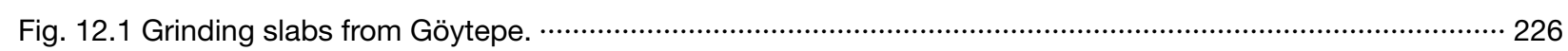

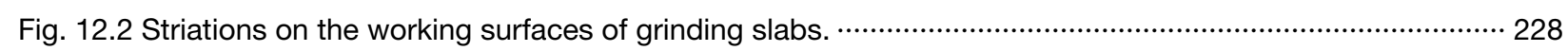

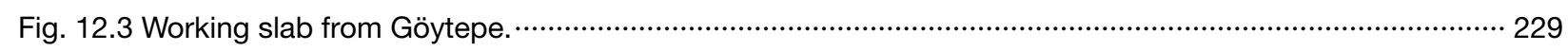

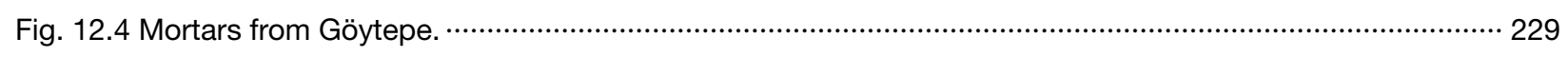

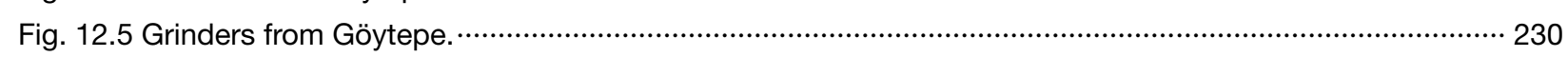

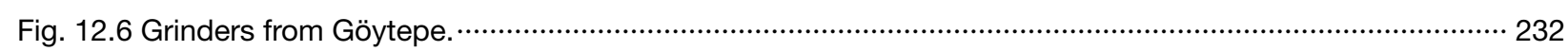

Fig. 12.7 Size-frequency distributions of complete grinders and handstones in comparison with grinding slabs. $\cdots 233$

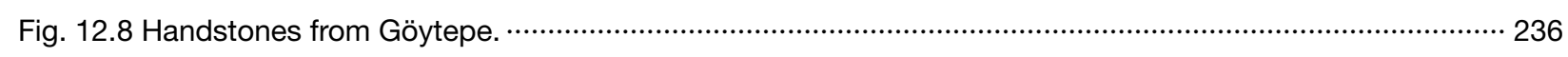

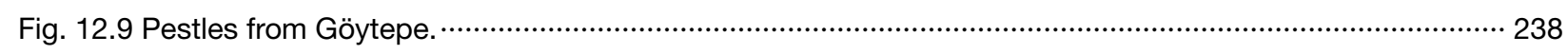

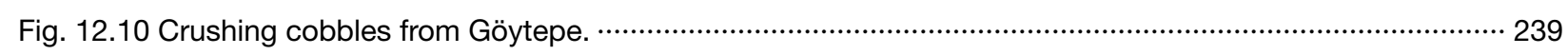

Fig. 12.11 Pounders and flaked cobble from Göytepe. ….....................................................................240

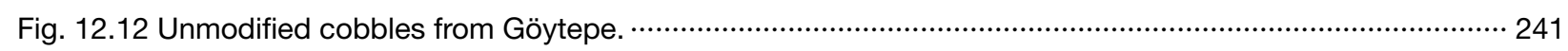


Fig. 12.13 Pebbles from Göytepe.

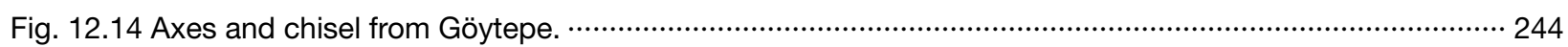

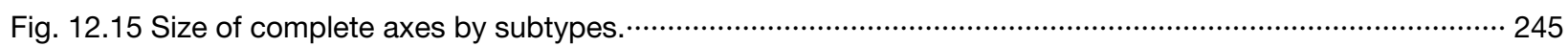

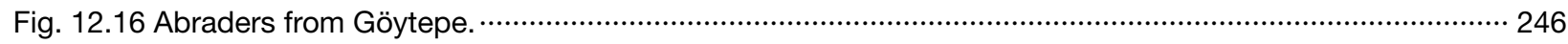

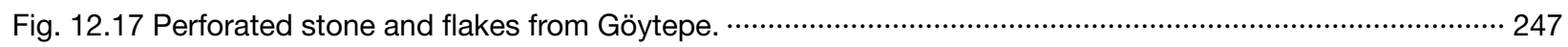

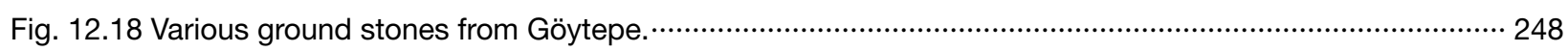

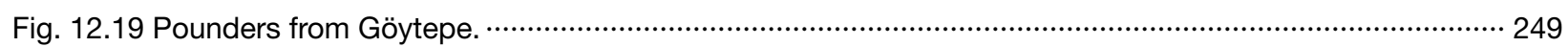

Fig. 12.20 Schematic chart of technological behaviors in the raw material selection, production, use, re-use, and

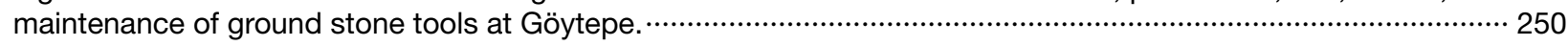

Fig. 12.21 Maximum length of complete ground stones, excluding flakes and pebbles, by raw material types. …251

Fig. 12.22 Maximum length of complete ground stones, excluding flakes and pebbles, by tool types. …………2 252

Fig. 12.23 Ground stones and other tools recovered in the clay bins. …..........................................................253

Fig. 12.24 Symmetric display of sites and their ground stone tool types in the space defined by Axes 1 and 2 that account for $54.0 \%$ and $35.4 \%$ of inertia of tool types respectively.

Fig. 12.25 Length of complete querns/slabs and mortars at Göytepe and select Neolithic sites in the Kwemo-Kartli

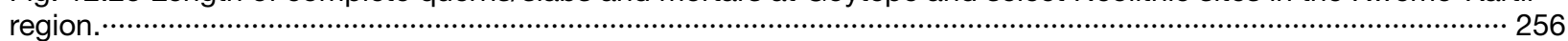

Fig. 12.26 Size-frequency distributions of the maximum length of complete grinders and handstones at Göytepe and

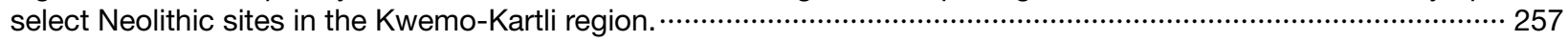

Fig. 13.1 Proportion of pottery types in the study collection of Göytepe. …................................................264

Fig. 13.2 Diachronic changes in the pottery types by level at Göytepe. ….....................................................265

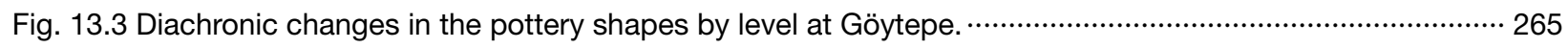

Fig. 13.4 Proportion of pottery types in open-shape pottery assemblages by level at Göytepe. ………………....2.266

Fig. 13.5 Proportion of pottery types in closed-shape pottery assemblages by level at Göytepe. ……….............267

Fig. 13.6 Proportion of pottery types in decorated pottery assemblages by level at Göytepe.............................268

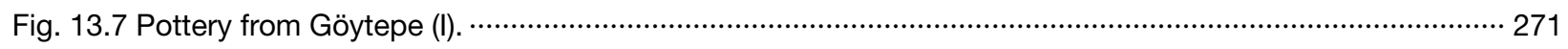

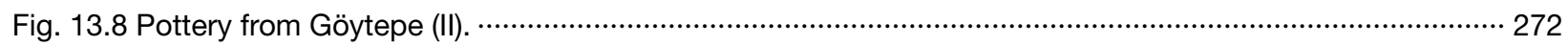

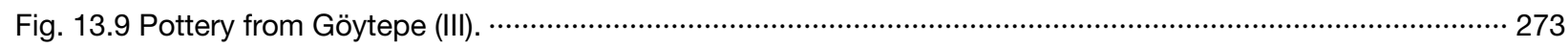

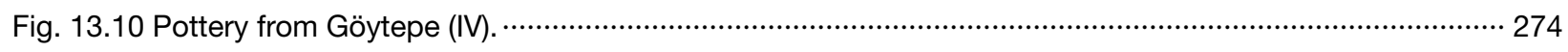

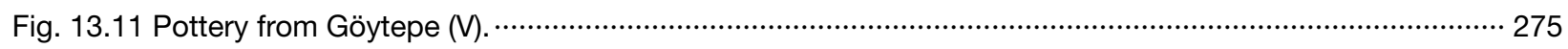

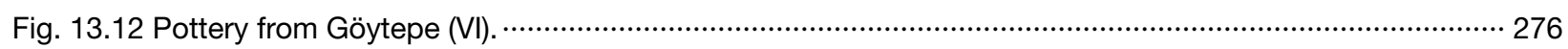

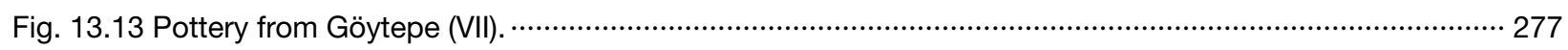

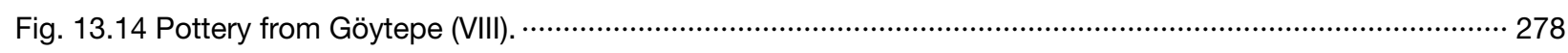

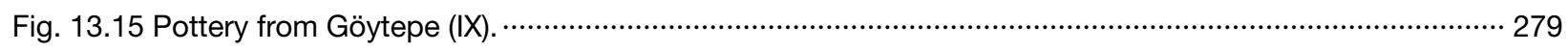

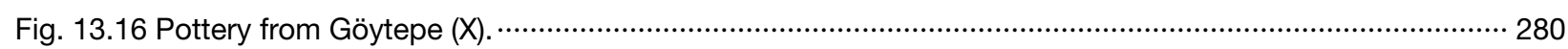

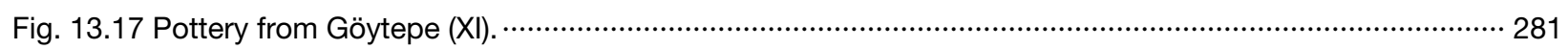

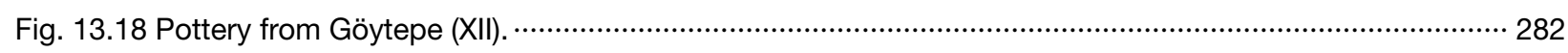

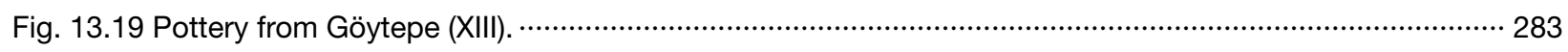

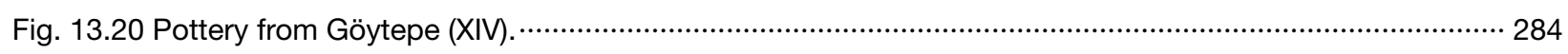

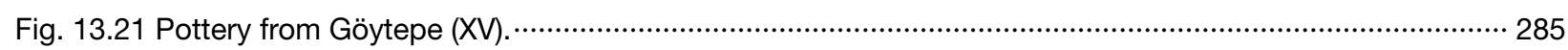

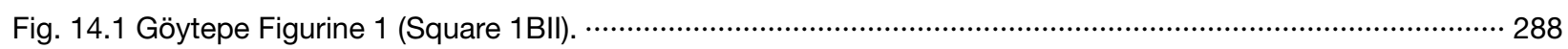

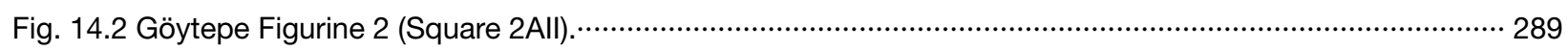

Fig. 15.1 Chronological changes in the use of skeletal parts used to manufacture awls at Göytepe. ……………2297

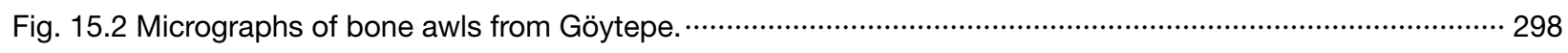

Fig. 15.3 Manufacturing scheme of worked antler implements at Göytepe. …............................................299 
Fig. 15.4 Bone tools and non-worked boned from the locus 4BI-131 KK at Göytepe. …………………...............300

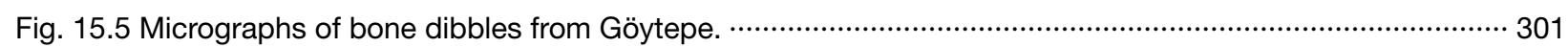

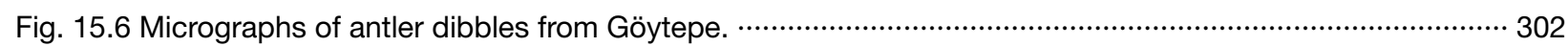

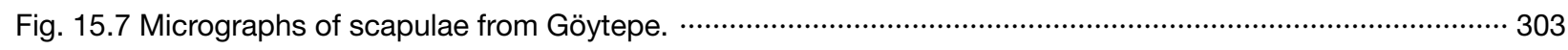

Fig. 15.8 Manufacturing scheme of worked scapula implements. ……........................................................... 304

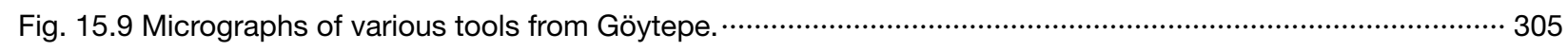

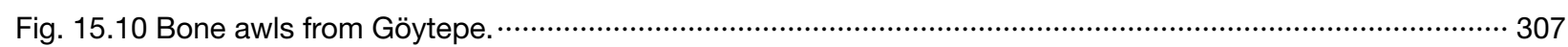

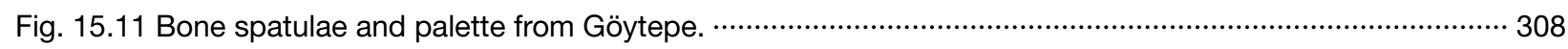

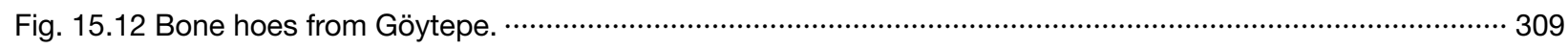

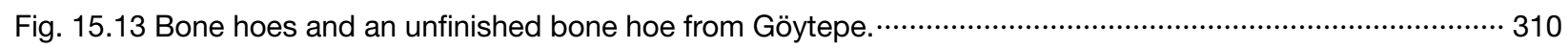

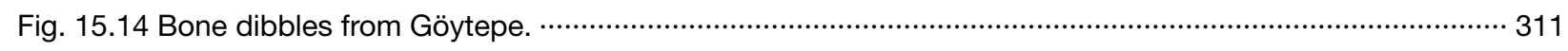

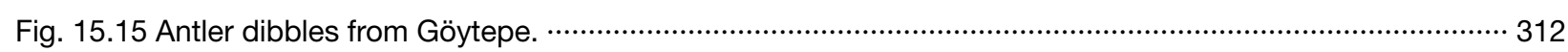

Fig. 15.16 Antler hammers and a possible punch from Göytepe. …............................................................... 313

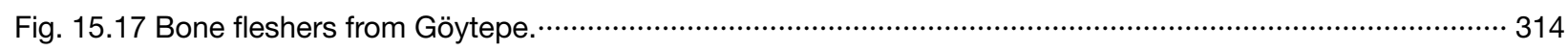

Fig. 15.18 Arrowhead, buttonette, knife, counter, ornament, and sickle? from Göytepe. ………………………... 315

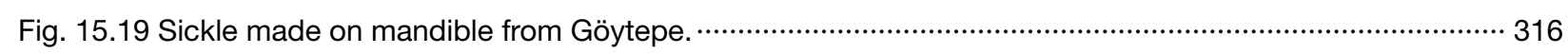

Fig. 15.20 Representative bone awls from Göytepe. …........................................................................... 317

Fig. 15.21 Representative bone palettes from Göytepe. ….......................................................................... 317

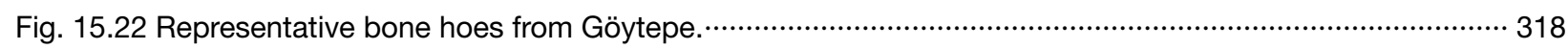

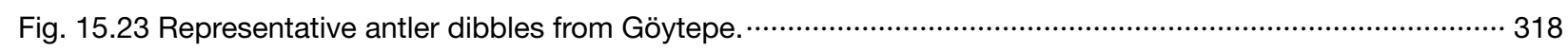

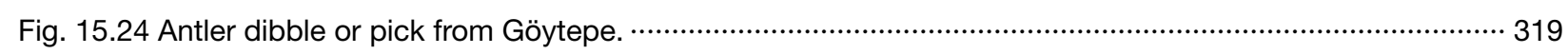

Fig. 15.25 Representative antler hammers from Göytepe.......................................................................... 319

Fig. 15.26 Fleshers made on various elements from Göytepe. ….................................................................. 320

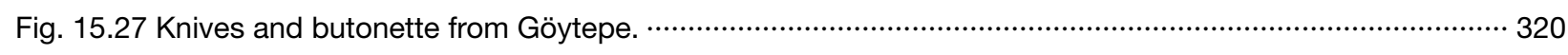

Fig. 15.28 Unfinished sickle handle made on cattle mandible from Göytepe. ……............................................. 321

Fig. 15.29 Debitage splintered by grooving technique from Göytepe. ........................................................... 321

Fig. 15.30 Worked dog mandibles (sickle?) from Göytepe. …...................................................................... 322

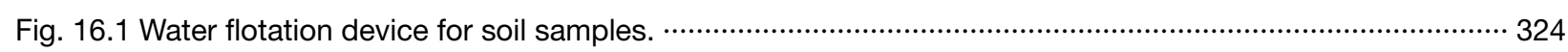

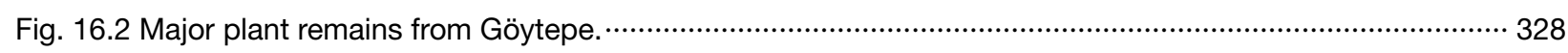

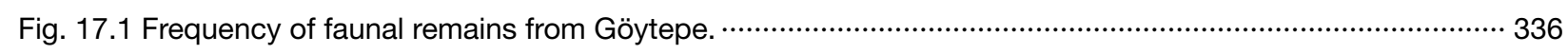

Fig. 17.2 Histogram showing the distribution of LSI values of sheep and goat. .......................................... 337

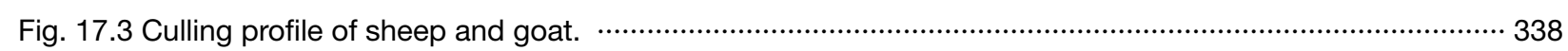

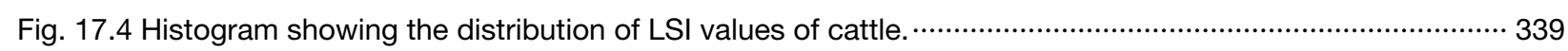

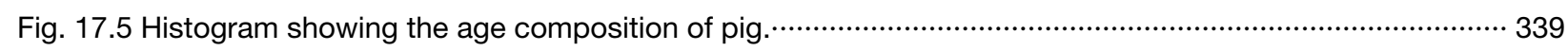

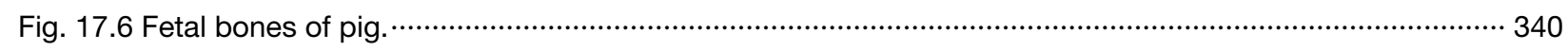

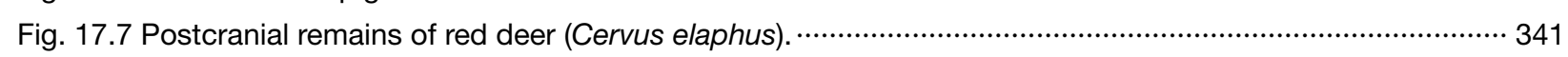

Fig. 17.8 Sequential change in the frequency of game species. ….............................................................. 342

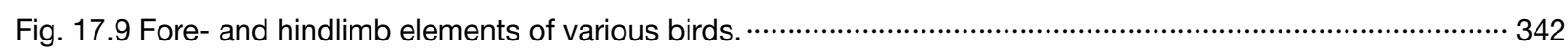

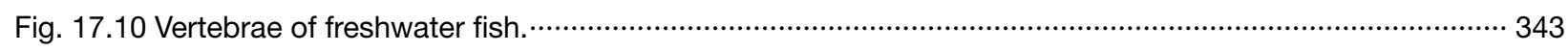





\section{LIST OF TABLES}

Table 1.1 Results of the Bayesian analysis of radiocarbon dates from Göytepe. …………………………………... 4

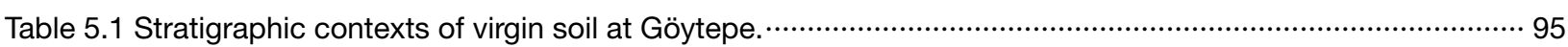

Table 7.1 List of sediment samples examined for phytoliths, faecal spherulite, and micromorphology. …….......... 113

Table 7.2 Main phytolith and spherulite results obtained from Squares 3All and 4BII. ........................................... 117

Table 7.3 Summary descriptions of thin section samples from two clay bins. …………………………………..... 125

Table 8.1 List of archaeological sites visited during the 2011 survey. ……........................................................ 140

Table 9.1 General categories of lithic assemblages from Göytepe (2008-2013). ….............................................. 170

Table 9.2 Cores and debitage types for obsidian assemblages from Göytepe (2008-2013). …………………....... 172

Table 9.3 Mean widths of obsidian blades from Göytepe (2008-2013). …….................................................... 176

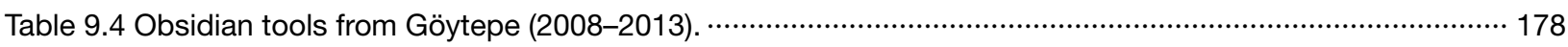

Table 9.5 Flint cores, core management pieces, and debitage from Göytepe (2008-2013). ………….................. 181

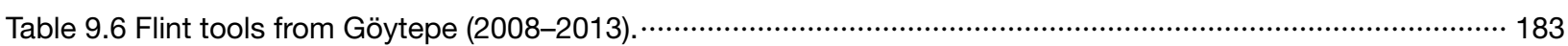

Table 10.1 Results of use-wear analysis of stone artifacts from Göytepe. ……………………………………..... 192

Table 10.2 Estimated use of each part (edge) of stone artifacts from Göytepe. ……………................................. 204

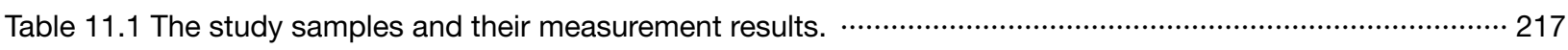

Table 12.1 Inventory of ground stone artifacts from Göytepe (2008-2011 seasons). ……………………………... 224

Table 12.2 Frequencies of raw materials by ground stone tool types at Göytepe. ………………………………... 225

Table 12.3 Frequencies of plan forms of grinders and handstones. ……....................................................... 234

Table 12.4 List of ground stone artifacts recovered in clay bins. ……............................................................. 252

Table 12.5 Occurrences of ground stone artifact types, excluding flakes, pebbles, and unidentifiable pieces, by

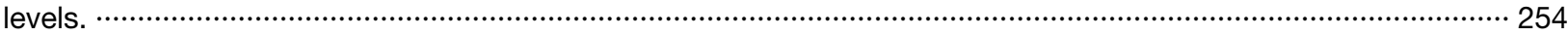

Table 12.6 Frequencies of ground stone tool types at Göytepe and select Neolithic sites in the Kwemo-Kartli

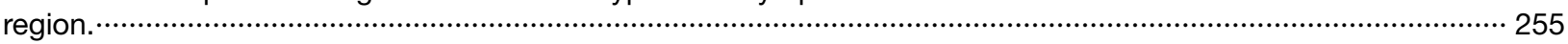

Table 13.1 The pottery assemblages described in this chapter by squares and levels. ……................................ 261

Table 13.2 Classification of pottery types for Göytepe. ……………......................................................... 263

Table 15.1 Worked bone materials from Square 4B at Göytepe. ……………………………………………... 293

Table 15.2 Animal species used as raw material for bone tools. ………............................................................ 296

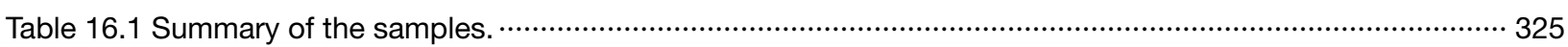

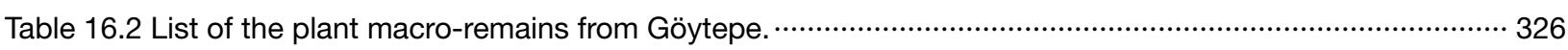

Table 17.1 Animal remains from Square 4B of Göytepe by levels. ……………………………………….... 334

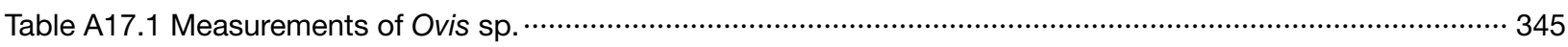

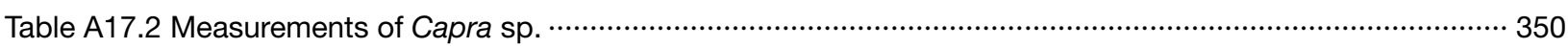

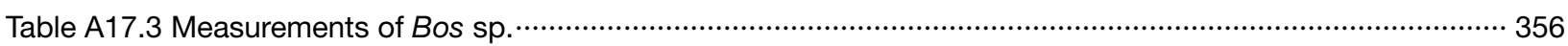

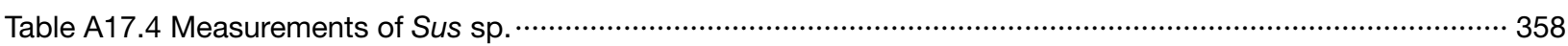

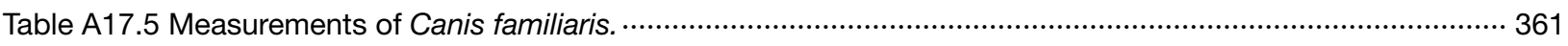

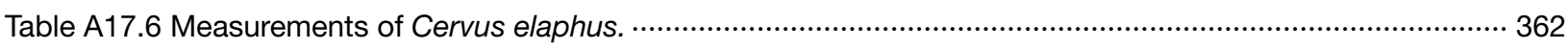

Table A17.7 Measurements of Gazella subgutturosa. ……………………………………………………..... 363

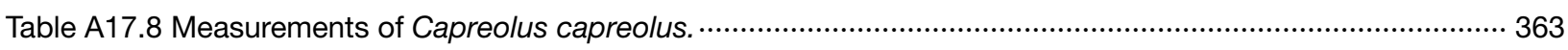

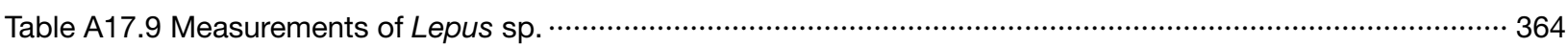





\title{
Chapter 1
}

\section{Introduction}

\author{
Yoshihiro Nishiaki and Farhad Guliyev
}

The advent of farming in human history has attracted global attention since the early history of archaeology and anthropology because of its significant impact on the subsequent development of society in a given region. The transition from hunting-gathering to farming economies was even termed the "Neolithic revolution" or "Agricultural revolution" in some studies of the 20th century. The intensive ongoing archaeological research has now demonstrated that one of the earliest farming economies emerged in the Fertile Crescent of Southwest Asia, a region that reaches from the Levant to the hilly flanks of the Anatolian and Zagros Mountains. Research has also shown that the beginning of farming was a long-term process, and thereby better termed a "Neolithization," rather than a revolution. Consequently, the timing of its advent depends on the interpretation of how the earliest farming should be defined. Most researchers today agree that farming came to be practiced in the Fertile Crescent at the beginning of the Holocene period, approximately eleven thousand years ago, when the first Neolithic cultures appeared (Zeder 2011; Willcox 2013; Ibáñez et al. 2018 and references therein).

In the case of the Neolithization of the South Caucasus, our knowledge has also greatly increased recently, particularly in the past two decades, owing to intensive international field campaigns. Archaeologists have excavated important sites such as Mentesh (Lyonnet and Guliyev 2017), Hacı Elamxanlı (Nishiaki et al. 2015a), and Mil Plain (Helwing and Aliyev 2017) in Azerbaijan; Aratashen (Petrosyan et al. 2014), Aknashen (Badalyan et al 2010), and Masis Blur (Martirosyan-Olshansky et al. 2013) in Armenia; and Aruchlo (Hansen and Mirtskhulava 2017) and Gadachrili Gora (Hamon et al. 2016) in Georgia, to mention only a few (Fig. 1.1). Consequently, while some studies in the mid 20th century may have suggested that farming originated independently in various regions, the current consensus argues that the Neolithization of the South Caucasus was a result of dispersals from the Fertile Crescent of Southwest Asia. However, scholars have not yet clarified the details of the dispersal processes: for example, the chronological patterns and geographic contexts of the dispersals, the cultural and population interaction of the incoming farmers and the local hunter-gatherers, if any were present, and the cultural development of the first farming societies after their acceptance of this novel economy. All of these represent important avenues of research for future studies.

Our research in the Middle Kura Valley of the Ganja-Kazakh Plain, Azerbaijan, also aims to contribute to a better understanding of the Neolithization in the South Caucasus. Substantial investigations of the Neolithic sites of this valley began with the pioneering fieldwork of Ideal Narimanov in the 1960s and 1970s. The most remarkable achievements of his study were the excavations of Shomutepe, Gargalartepe, and Toyretepe in this plain. From these excavations, Narimanov (1987: 17) proposed that the Shomutepe culture was the oldest Neolithic culture in the region. His description of the architectural and artifactual remains provided sufficient evidence that this site exhibited all aspects of Neolithic culture, such as plant cultivation, stock-breeding, mud-brick architecture, and the use of early pottery. Narimanov argued that this culture represented the first full-fledged Neolithic entity in Azerbaijan at that time, and scholars still agree with this today. However, despite this valuable 


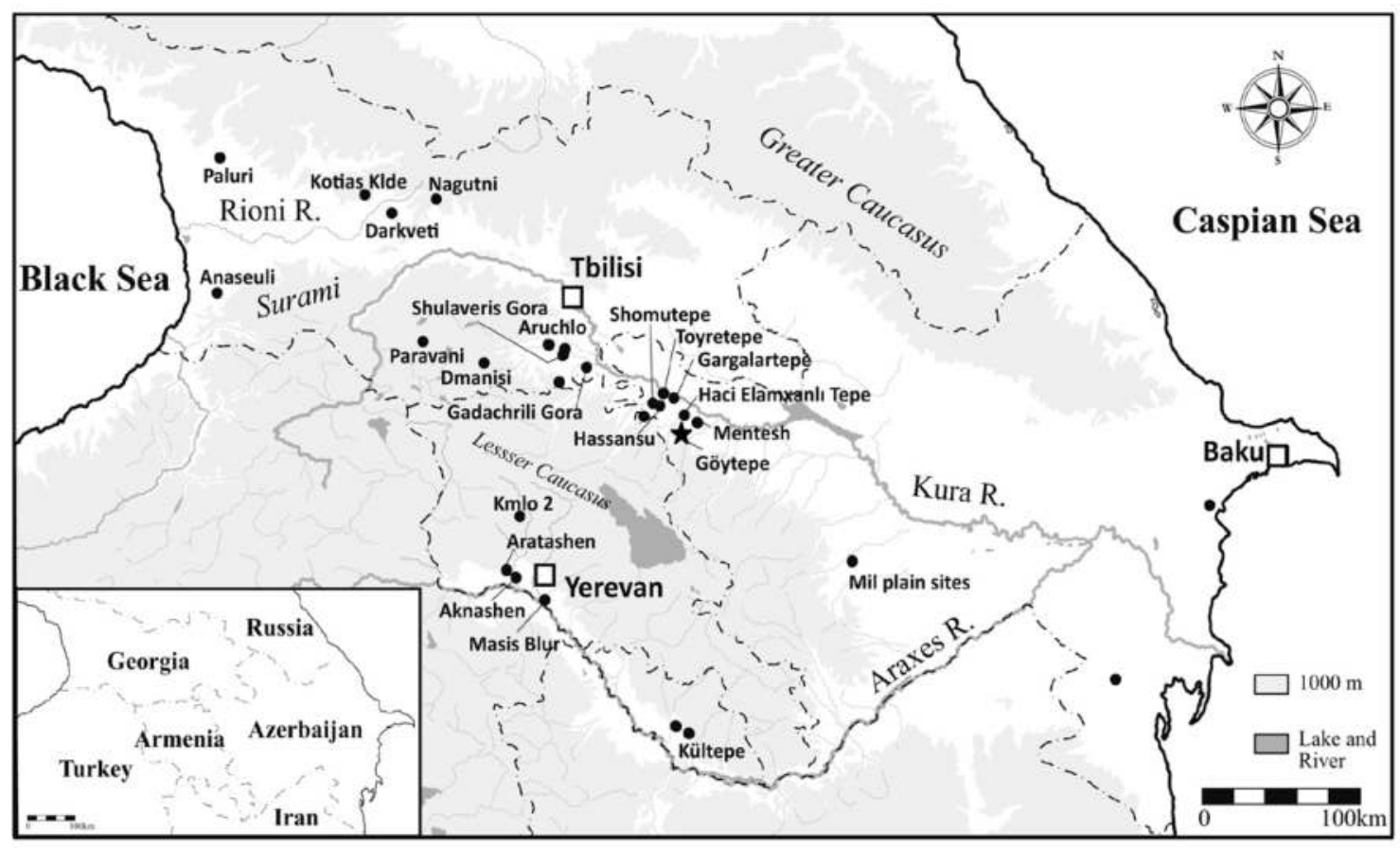

Fig. 1.1 Map showing the location of Göytepe and related Neolithic sites in the South Caucasus.

contribution, in light of the current standards, even the proposed cultural assemblage and chronology needs refinement. Narimanov developed the definition of the Shomutepe culture before adequate availability of modern excavation techniques such as stratigraphic sampling, recovering strategies for botanical and faunal remains, and radiocarbon dating. Nevertheless, because of a number of reasons, the next two decades did not witness any follow-up field research in Azerbaijan with regard to the Shomutepe culture.

Accordingly, the major objective of our research is to redefine the archaeological elements of the Shomutepe culture in detail and establish its chronological framework by means of scientific excavations that employ up-to-date field techniques. Through these, we intend to shed new light on the origin and development of the early farming communities in the Middle Kura Valley. The site we chose for intensive field investigations is the mound of Göytepe, situated on the right bank of the Middle Kura Valley at an altitude of about $400 \mathrm{~m}$, approximately $10 \mathrm{~km}$ east of Tovus city (Fig. 1.1). It is, to date, one of the largest Neolithic sites known in the region. This mound was first identified as a Neolithic site of the Shomutepe type during the survey by Narimanov (1987: 31). Later in 2007, his interpretation was confirmed by an Azerbaijan-
French survey, which made analyses of the surface archaeological materials and charcoal specimens for radiocarbon dating from the stratigraphic section exposed at the northern edge of this mound (Guliyev et al. 2009; see also Chapter 4). These investigations guided our research from the 2008 season. It revealed that, despite Narimanov's estimate of about $5 \mathrm{~m}$ high and covered an area of one hectare at the base, our excavations revealed that this mound is much larger, about $9 \mathrm{~m}$ high and nearly 1.5 ha in area. Moreover, cultural deposits were found to continue for $2 \mathrm{~m}$ below the present ground surface. Therefore, the total cultural deposits, all from the Neolithic period, are a total of $11 \mathrm{~m}$ deep.

The present volume addresses the results of the excavations conducted by the Azerbaijan-Japan Archaeological Mission from 2008 to 2013, under the direction of the editors of this volume. Although the fieldwork at this important site continues today, the first six seasons' excavations were more substantial than the later ones (Guliyev and Nishiaki 2012, 2014). The results of the first seasons' work are considered worth being published as a separate monograph. The present volume consists of two parts: Part I presents results from fieldwork that deal with our observations as to the geomorphological setting (Chapter 2), stratigraphy and architecture (Chapters 3-6), geoarchaeological aspects of selected features (Chapter 
7), and the distribution of related archaeological sites in the surrounding region (Chapter 8). Part II then refers to laboratory analyses of the excavated materials such as flaked stone artifacts (Chapters 9-11), ground stone artifacts (Chapter 12), pottery (Chapter 13), clay figurines (Chapter 14), bone objects (Chapter 15), plant remains (Chapter 16), and animal remains (Chapter 17).

Before we begin the descriptions, we shall address the chronological framework of the Göytepe site. The statistical analyses of nearly 50 radiocarbon dates indicates that the Neolithic occupations at Göytepe started in approximately $5650 \mathrm{cal}$. BC and ended about $5460 \mathrm{cal}$. BC (Table 1.1). These results imply that the site was occupied for a relatively short period of about 200 years in the middle of the 6th millennium BC. According to the current chronological framework of the Shomutepe culture, the site at Göytepe was occupied in the late phase (Fig. 1.2).

This radiocarbon chronology has raised two important issues related to how we understand the Neolithic lifestyles at Göytepe. One is the very rapid cycle of rebuilding the architecture. As detailed in Chapters 3 and 4, the sequence of $11 \mathrm{~m}$ cultural deposits of Göytepe is divided into 14 architectural levels. Our Bayesian analyses of the radiocarbon dates estimate a duration for each level, 5 to 15 years at average (Table 1.1; Fig. 1.3). The lack of the comparable data from other sites in the South Caucasus prevents an evaluation of this pattern in the regional context. However, the available literature on the life history of mud-brick architecture of the archaeological and ethnographic examples in Southwest Asia point to a much longer cycle of 20 to 50 years (Nishiaki et al. 2018). Therefore, the unexpectedly short rebuilding cycle for the Göytepe architecture requires adequate explanation. The data from Göytepe, equipped with numerous radiocarbon dates on a hitherto unparalleled scale, provide us with an important insight into the residential/settlement patterns of the early 6 th millennium BC in this part

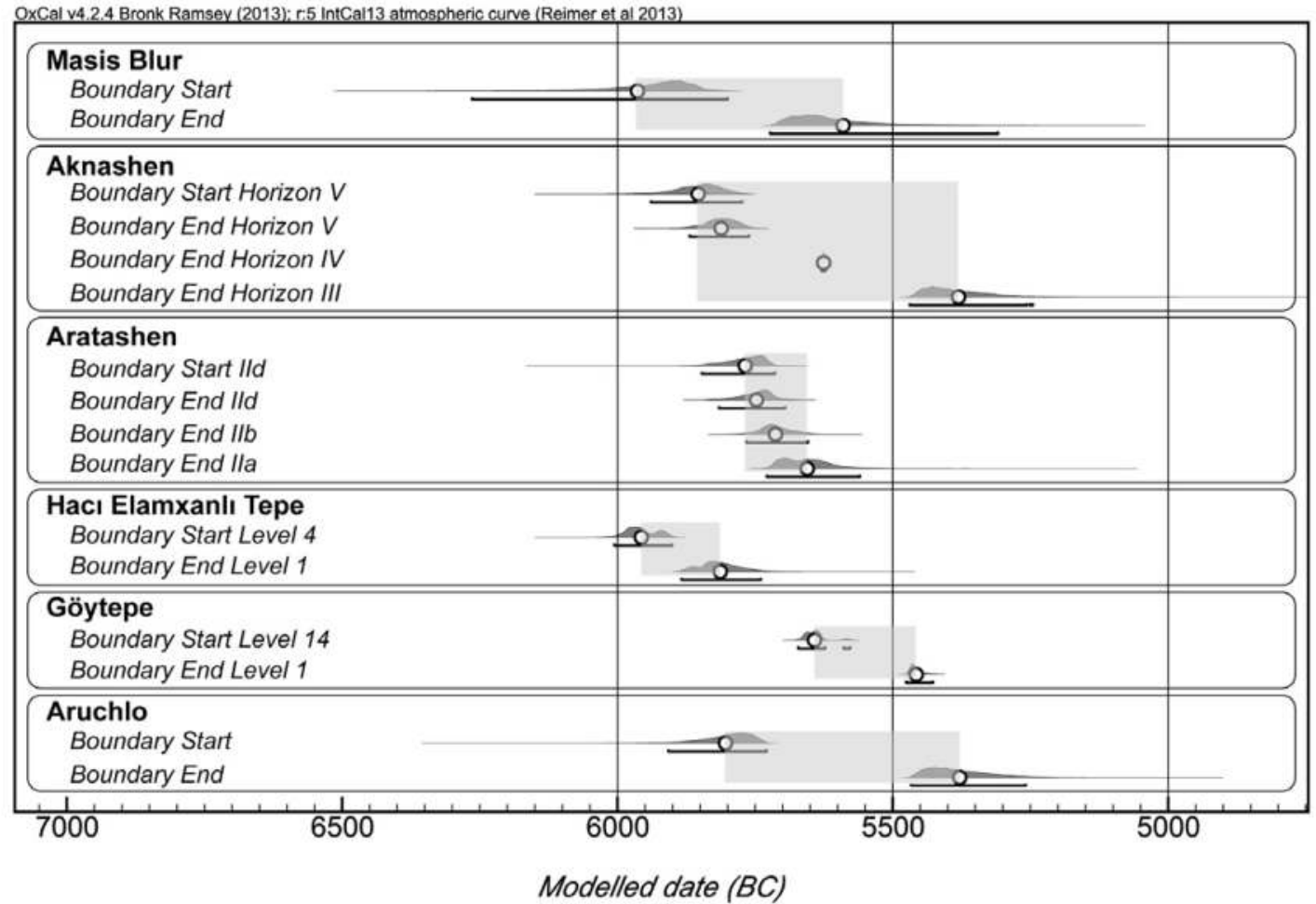

Fig. 1.2 Probability distributions of dates estimated with the sequence and phase models of the OxCal program (Nishiaki et al. 2015b). Gray areas mark the range between the mean values estimated for the start and end of the occupations at each site. 


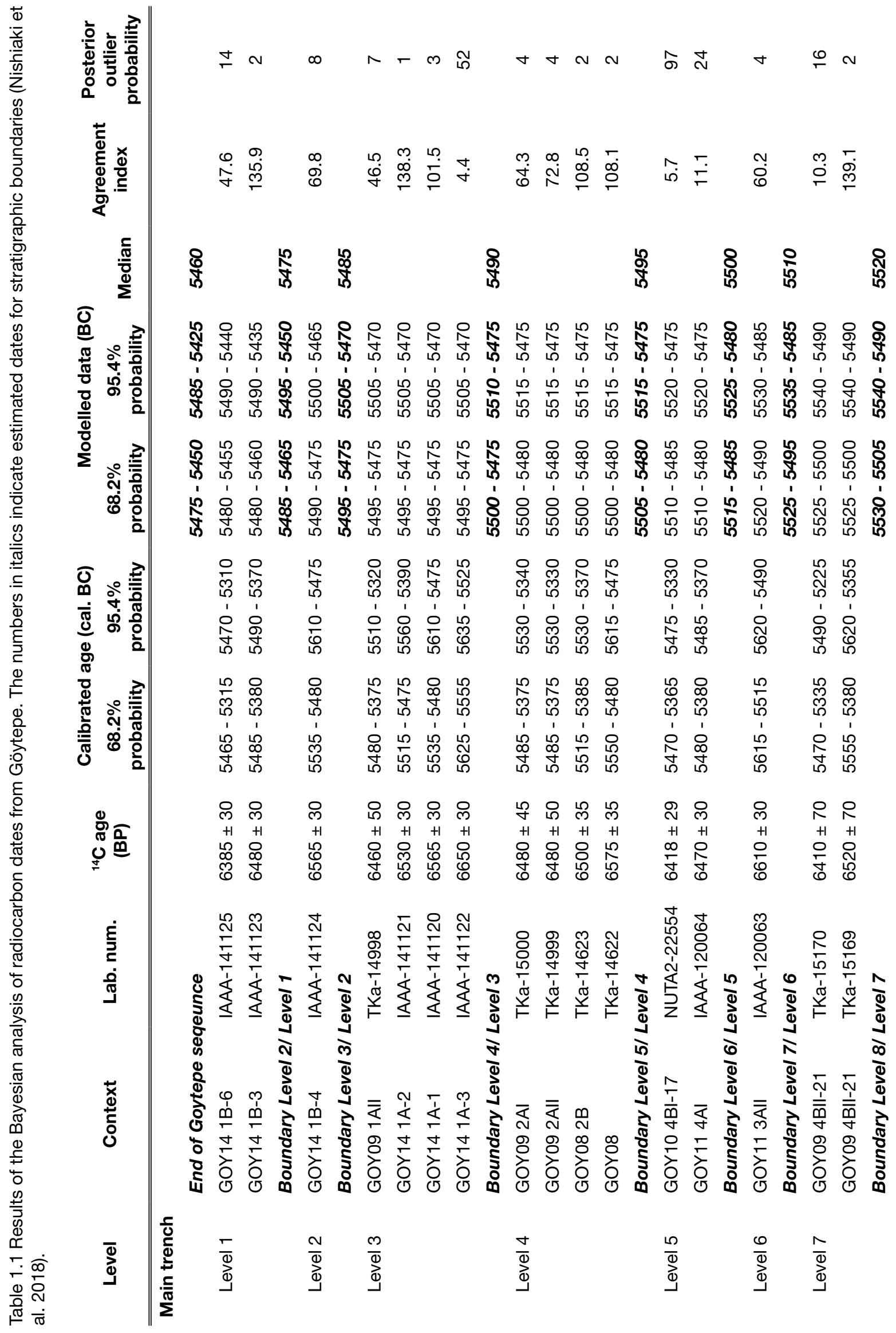




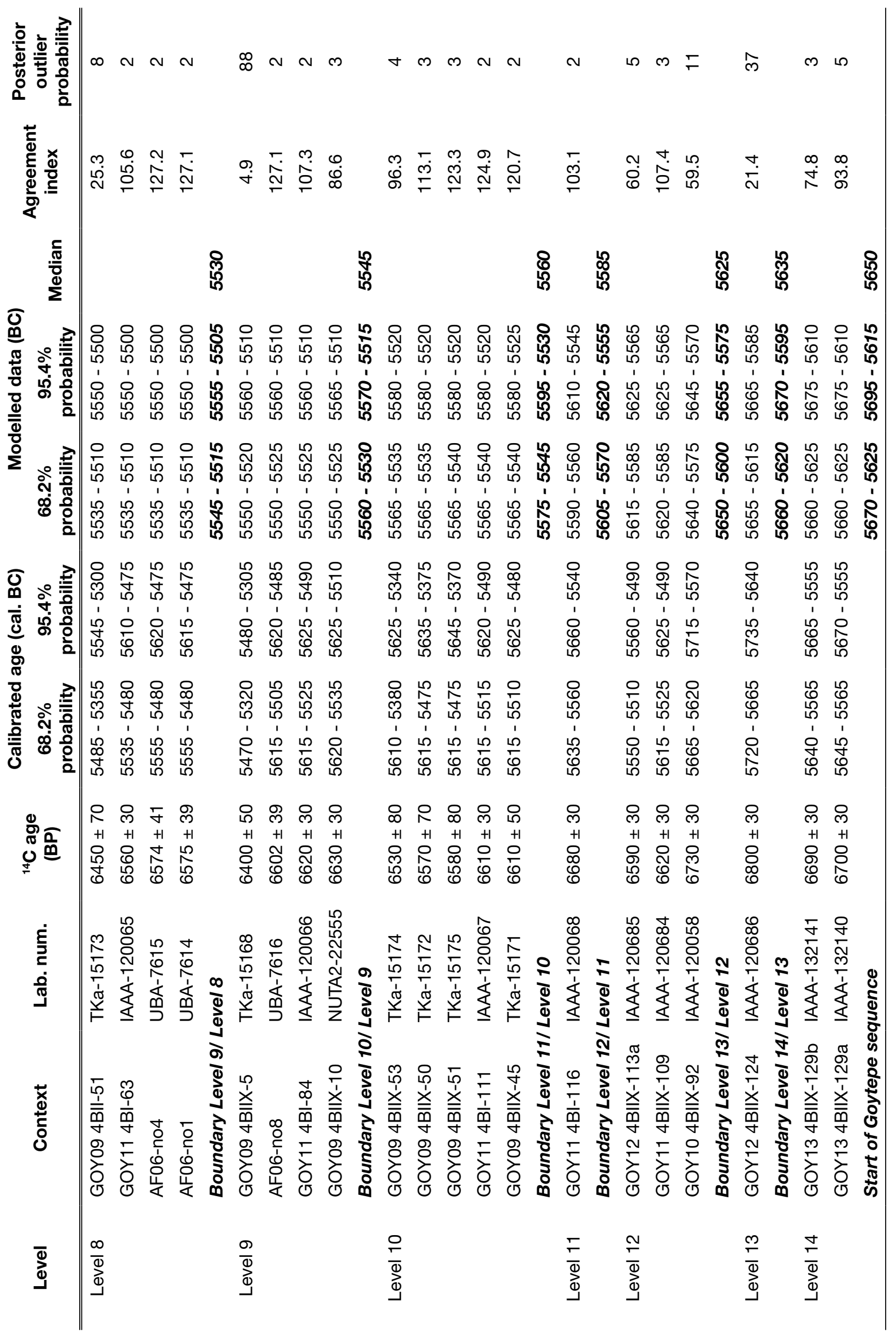




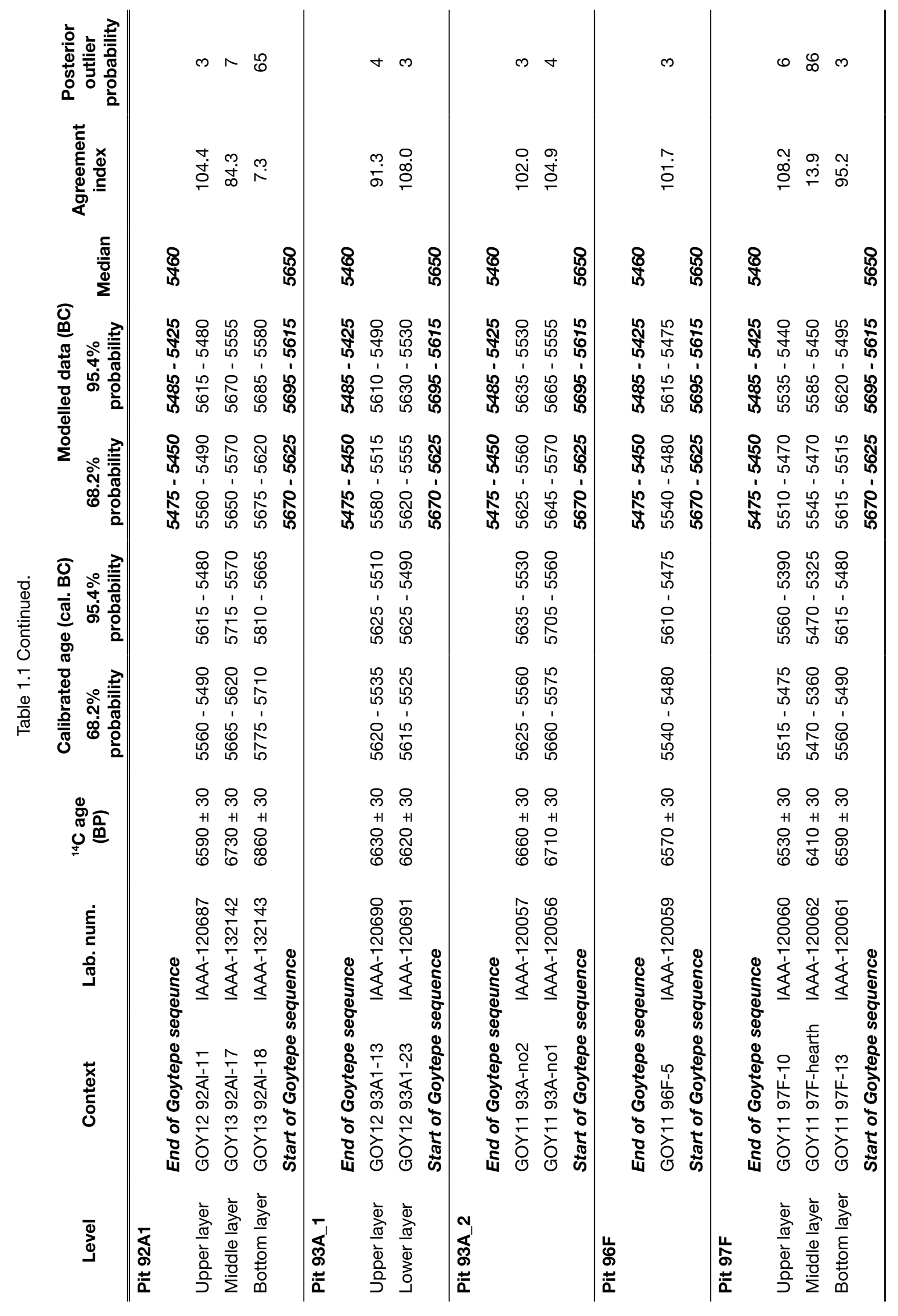




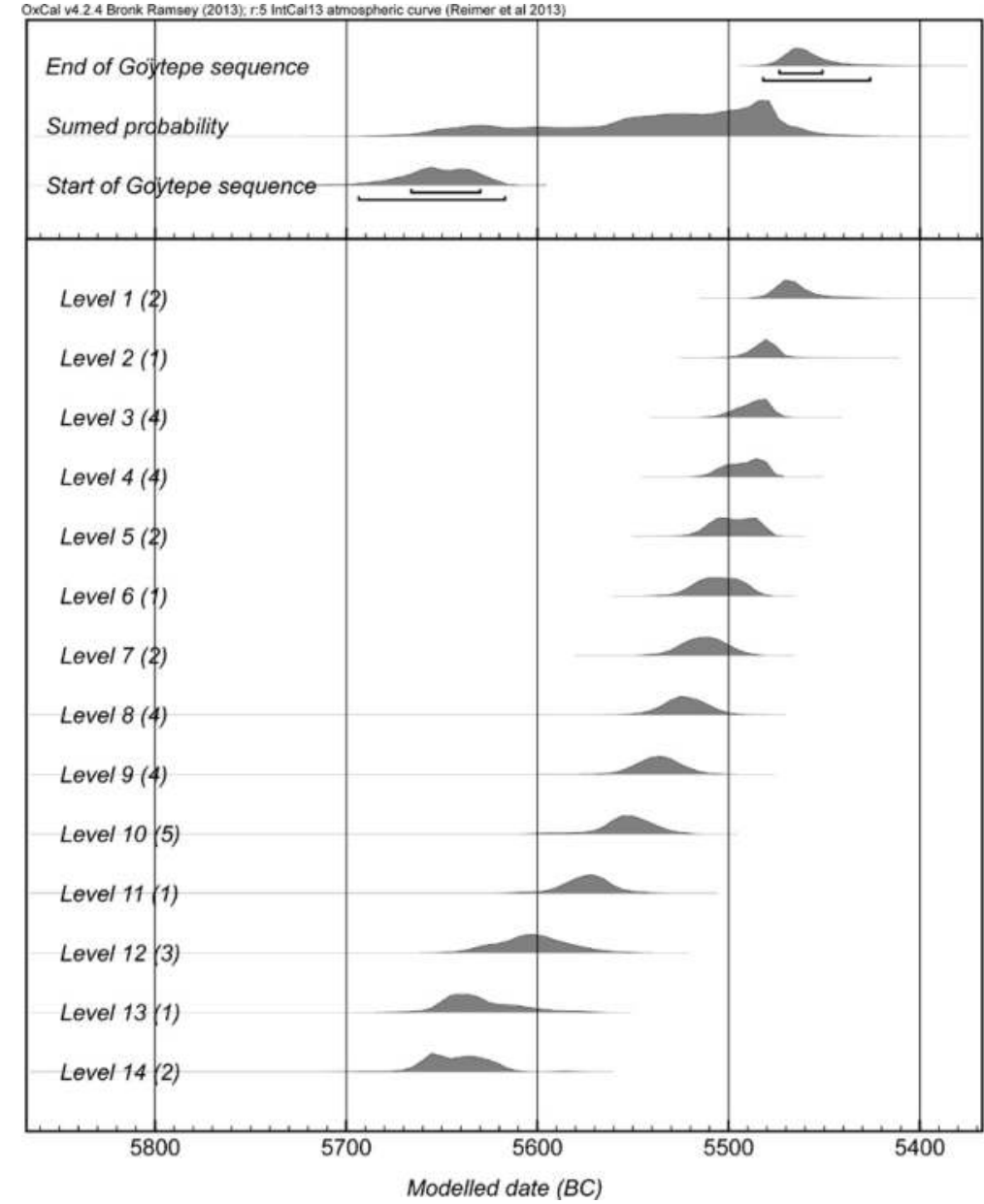

Fig. 1.3 Summed probability distribution of radiocarbon dates from Göytepe. Top: combined model using all the dates. Bottom: dates from the main excavation trench by level. Number of dates used for modeling in parentheses (Nishiaki et al. 2018). of Eurasia. Our preliminary interpretation is that at least the late Shomutepe communities were not as sedentary as we envisage for the Neolithic period in general and these findings deserve verification with further evidence.

Second, our stratigraphic analysis of the archaeological record indicates a major break during the Göytepe sequence, between Levels 8 and 7, around 5530 cal. BC (Nishiaki et al. 2015b). At least four changes have been identified; 1) the use of pottery became common (Chapter 13), 2) the sources of obsidian procurement shifted from those of Southeast Anatolia to the Lessor Caucasus (Chapter 9) after Level 8, 3) the mud-brick size changed between Levels 8 and 7 (Chapter 6), and 4 ) the acceleration of rebuilding cycles of the mudbrick architecture. We estimate that the duration of each level from Level 7 and later is as short as five years or so (Nishiaki et al. 2018). These changes are best interpreted to reflect a substantial event in the Neolithization processes of the Göytepe communities and likely the neighboring communities during this time period. The detailed dataset for a range of archaeological findings presented in this volume will help interpret the socio-economic implications of those changes.

\section{References}

Badalyan, R. S., A. A. Harutyunyan, C. Chataigner, F. Le Mort, J. Chabot, J. -E. Brochier, A. Balalescu, V. Radu, and R. Hovsepyan (2010) The settlement of Aknashen-Khatunarkh. A Neolithic site in the Ararat plain (Armenia): excavation results (2004-2009). Turkiye Bilimler Akademisi Arkeoloji Dergisi (TÜBA$A R), 13: 187-220$.

Bronk Ramsey, C. (2009) Bayesian analysis of radiocarbon dates. Radiocarbon, 51(1): 337-360.

Guliyev, F., F. Husejnov, and H. Almamedov (2009) 2009 Excavations of a Neolithic settlement at Göytepe (Azerbaijan). In: Proceedings of the International Symposium - Land between East and West, April 1-3, 2009, edited by the German Archaeological Institute, pp. 29-30. Baku: Azerbaijan.

Guliyev, F. and Y. Nishiaki (2012) Excavations at the Neolithic settlement of Göytepe, the middle Kura 
Valley, Azerbaijan, 2008-2009. In: Proceedings of the 7th International Congress of the Archaeology of the Ancient Near East, Vol. 3, edited by R. Matthews and J. Curtis, pp. 71-84. Wiesbaden: Harrassowitz Verlag.

Guliyev, F. and Y. Nishiaki (2014) Excavations at the Neolithic settlement of Göytepe, West Azerbaijan, 2010-2011. In: Proceedings of the 8th International Congress of the Archaeology of the Ancient Near East, Vol. 2, edited by P. Bieliński, M. Gawlikowski, R. Koliński, D. Ławecka, A. Sołtysiak, and Z. Wygnańska, pp. 3-16. Wiesbaden: Harrassowitz Verlag.

Hamon, C., M. Jalabadze, T. Agapishvili, E. Baudouin, I. Koridze, and E. Messager (2016) Gadachrili Gora: architecture and organisation of a Neolithic settlement in the middle Kura Valley (6th millennium BC, Georgia). Quaternary International, 395: 154169.

Hansen, S. and C. Mirtskhulava (2017) Excavations in Aruchlo 2005-2014. In: The Kura Projects: New Research on the Later Prehistory of the Southern Caucasus, edited by B. Helwing, T. Aliyev, B. Lyonnet, F. Guliyev, S. Hansen, and G. Mirtskhulava, pp. 195-297. Berlin: German Institute of Archaeology, Eurasian Department.

Helwing, B. and T. Aliyev (2017) Excavations in the Mil Plain sites 2012-2014. In: The Kura Projects: New Research on the Later Prehistory of the Southern Caucasus, edited by B. Helwing, T. Aliyev, B. Lyonnet, F. Guliyev, S. Hansen, and G. Mirtskhulava, pp. 11-42. Berlin: German Institute of Archaeology, Eurasian Department.

Ibáñez, J. J., J. González-Urquijo, L.C. Teira-Mayolini, and T. Lazuén (2018) The emergence of the Neolithic in the near east: a protracted and multi-regional model. Quaternary International, 470: 226-252.

Lyonnet, B. and F. Guliyev (2017) Mentesh Tepe (Azerbaijan), a preliminary report on the 2012-2014 excavations. In: The Kura Projects: New Research on the Later Prehistory of the Southern Caucasus, edited by B. Helwing, T. Aliyev, B. Lyonnet, F. Guliyev, S. Hansen, and G. Mirtskhulava, pp. 125-140. Berlin: German Institute of Archaeology, Eurasian Department.

Martirosyan-Olshansky, K., G. E. Areshian, P. S. Avestiyan, and A. Hayrapetyan (2013) Masis Blur: a late Neolithic settlement in the plain of Ararat, Armenia. Backdirt, 2013: 142-146.

Narimanov, I. (1987) The Culture of the Most Ancient Farming and Stock-Breeding Population of Azerbaijan. Baku: National Academy of Sciences (in Russian with an English summary).

Nishiaki, Y., F. Guliyev, S. Kadowaki, V. Alakbarov, T. Miki, S. Salimbeyov, C. Akashi, and S. Arai (2015a) Investigating cultural and socioeconomic change at the beginning of the Pottery Neolithic in the Southern Caucasus - The 2013 Excavations at $\mathrm{Hacl}$ Elamxanlı Tepe, Azerbaijan. Bulletin of the American School of Oriental Research, 374: 1-28.

Nishiaki, Y., F. Guliyev and S. Kadowaki (2015b) Chronological contexts of the earliest Pottery Neolithic in the Southern Caucasus: Radiocarbon dates for Göytepe and Hacı Elamxanlı Tepe, West Azerbaijan. American Journal of Archaeology, 119(3): 279-294.

Nishiaki, Y., F. Guliyev, S. Kadowaki, and T. Omori (2018) Neolithic residential patterns in the southern Caucasus: Radiocarbon analysis of rebuilding cycles of mudbrick architecture at Göytepe, west Azerbaijan. Quaternary International, 474: 119-130.

Petrosyan, A., M. Arimura, B. Gasparyan, S. Nahapetyan, and C. Chataigner (2014) Early Holocene sites of the Republic of Armenia: Questions of cultural distribution and chronology. In: Stone Age of Armenia, edited by B. Gasparyan and M. Arimura, pp. 138-158. Kanazawa: Kanazawa University.

Reimer, P. J. et al. (2012) IntCal13 and Marine13 radiocarbon age calibration curves $0-50,000$ years. Radiocarbon, 55(4): 1869-1887.

Willcox, G. (2013) The roots of cultivation in Southwestern Asia. Science, 341 (39): 39-40.

Zeder, M. (2011) The origins of agriculture: new data, new ideas. Current Anthropology, 52 (S4): S221S235. 
Part I: Field Investigations 

Chapter 2

\title{
Geomorphological settings of Göytepe
}

\author{
Yuichi Hayakawa
}

\subsection{Location}

Göytepe is located in a foothill area of the Lesser Caucasus Mountains. Its landscape is locally gentle on an alluvial fan, but more rugged and steeper terrains are present in surrounding areas. On the northern side of the Lesser Caucasus, rivers draining northward from the mountains form many alluvial fans along mountain foothills (Fig. 2.1). The toes of the alluvial fans are bounded by the Kura River, which drains eastward into the Caspian Sea. The climate of the area is warm and humid, with an annual mean precipitation of $300 \mathrm{~mm}$, a winter monthly mean temperature of $-2.3-6.5^{\circ} \mathrm{C}$, and a summer monthly mean of 19.5-31.7 (data at Ganja; World Meteorological Information, 2013). The modern climatic type ranges from BSk (arid steppe and cold) to $\mathrm{Cfa}$ (warm temperature, fully humid and hot summer) in Köppen's classification (Kottek et al. 2006). Vegetation cover in the area is characterized

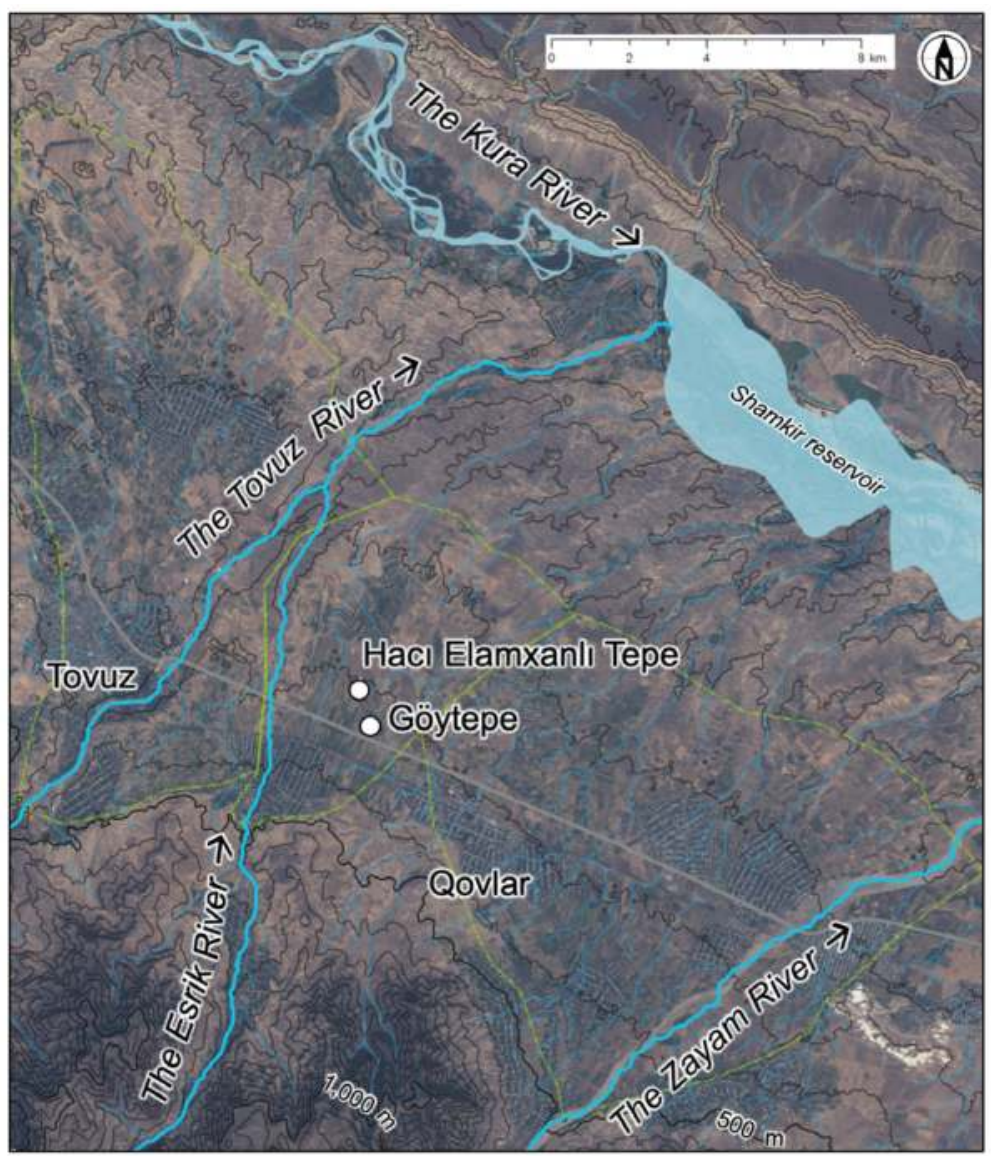

Fig. 2.1 Regional map around the study area. The background is a satellite image by ALOS AVNIR-2, and the topographic contour lines with an interval of $50 \mathrm{~m}$ are derived from ASTER GDEM (a 30-m resolution DEM). The approximate extent of the alluvial fans formed by the Tovuz, Esrik, and Zayam rivers are shown with green dashed lines. 
by steppe, and the dominant land use, given the abundant groundwater and the surface river water of the alluvial fans, is agriculture and meadow.

The mound of Göytepe, near Qovlar town, is located on an alluvial fan of the Esrik River, which is affected by the two major adjacent rivers, the Zayam and Tovuz (Fig. 2.1). Although the modern channel of the Esrik River has less discharge and an upstream catchment area of only $146 \mathrm{~km}^{2}$ at its fan apex, the discharge could have been much larger in the Pleistocene, judging by the particle sizes and rock types of sediments in the modern channel and the terrace cover along the river. The long axes of the gravels in the Esrik River are predominantly 10-20 $\mathrm{cm}$ (up to $55 \mathrm{~cm}$ at a sampling point with a similar elevation to Göytepe), and the rock types of these gravels vary and include andesite, carbonate rocks, and pyroclastic flow deposits. These are comparable to those in the larger Tovuz River, located farther west. The discharge of the Esrik River has thus likely been affected by the flow income from the Tovuz River catchment and the Zayam River to the east. The abandonment by the Esrik River of the Tovuz River catchment is supposed to have occurred before the Last Glacial Maximum (LGM), at probably Marine Isotope Stage (MIS) 3-4 (Mousterian) or earlier. At this stage, coarse gravels with lengths of up to 15 $\mathrm{cm}$, found in surficial sediments around Göytepe, have likely been transported from either the Tovuz or Zayam Rivers.

\subsection{Geomorphological setting}

With a large-scale topographic map, derived from field surveys with a laser range finder and global navigation satellite system measurements in 2009 and 2012, it is indicated that Göytepe is located on a fewmeter-high ridge, running in a southwest-northeast direction on the alluvial fan (Fig. 2.2). The slope of the ridge around the mound is approximately $1.5-2 \%$ toward the northeast. As shown in the survey of the sounding pits surrounding the mound (Chapter 5), subsurface loess deposits at the south of the mound are well developed, while thin loess layers over thick gravel sediments are more frequently found in the other portions of the mound. Moreover, it is suggested that the altitude of the basement of the mound is $1-2 \mathrm{~m}$ higher at its southwest edge than at the northeast and that the gap in basement height seems to correspond to the slope of surrounding areas. Because the general trend of streams on the alluvial fan is from southwest to northeast, the location of the mound on the ridge likely affects the preferable preservation of loess deposits on its southern or southwestern side, which could be blocked by the settlement. Such deposits on the northern or northeastern side could have been eroded by overland flows around the settlement or by the headward erosion of gullies downstream (north to east) of the mound.

On the surface of the alluvial fans, several fluvial terraces are found along the Esrik and Tovuz Rivers (Fig. 2.1). The relative height of the uppermost terrace from the modern riverbed along the Esrik River is approximately $20 \mathrm{~m}$ at an elevation of ca. $400 \mathrm{~m}$ a.s.1., whereas that along the Tovuz River is 60 $\mathrm{m}$, with a maximum valley width (i.e., edge to edge length of the uppermost terraces on the left and right sides of the river) of $1,000 \mathrm{~m}$. Such deep incision along the Tovuz River may have occurred through the Pleistocene, following abundant alluvial filling events in the glacial periods. Several terraces are present at about $4 \mathrm{~m}$ and $20 \mathrm{~m}$ higher than the modern riverbed, which comprises several alternating layers of gravel and sandy to muddy sediments. The modern riverbed is suffering from gravel quarrying, probably causing recent incisions of a few meters.

In the late Pleistocene, discharges in the rivers on the alluvial fans around Qovlar were large enough to transport coarse sediments from their mountain catchments, where the sediment supply from slopes, under a glacial or periglacial environment, could have been greater than that of the present. After the LGM about $20 \mathrm{ka}$ (MIS 2), several incision events have been identified (Ollivier et al. 2011). The incision events are supposed to have coincided, in part, with the lowering of the Caspian Sea level, but those not related to sea level change have also been found. Alluvial infilling could also have occurred in colder periods, and the $20-\mathrm{m}$ high terraces in the Tovuz River are supposed to have filled in the Younger Dryas at about 12-13 ka (Ollivier et al. 2011). Such terraces are also observed in the Zayam River, although their formative age may be different from those in the Tovuz River. Terraces with 4-m 


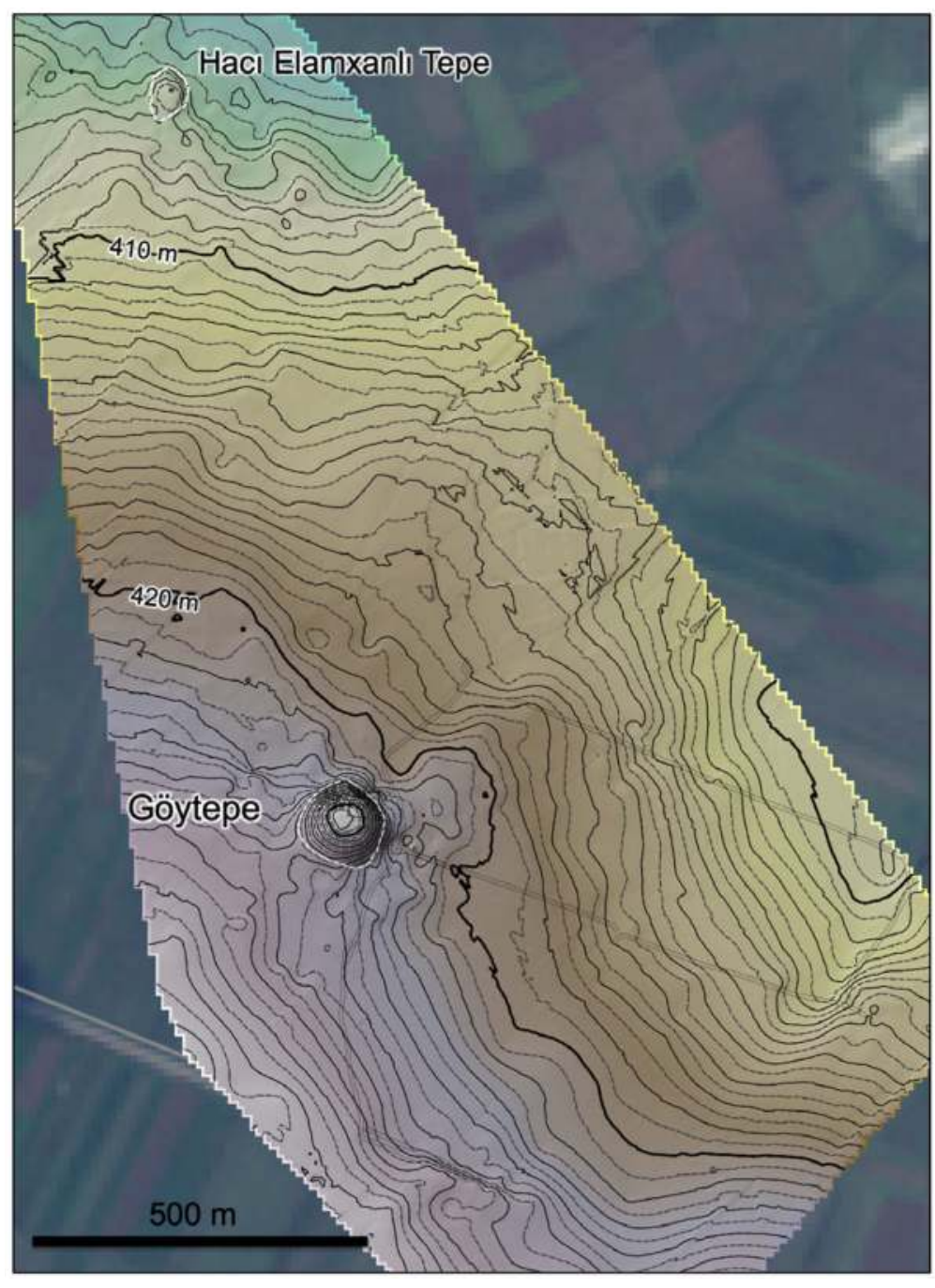

Fig. 2.2 Topographic map around Göytepe, measured in the field with a laser range finder and a differential GNSS (global navigation satellite system). The field survey was carried out in the 2009 and 2012 seasons. Contour lines are shown at a $0.5 \mathrm{~m}$ interval. The background image is from ALOS AVNIR-2. height in the Tovuz River are supposed to have been formed in the Holocene, whose timing is possibly 2-6 ka (Ollivier et al. 2011).

The 60-m deep valley of the Tovuz River (Fig. 2.3) suggests that the region around Qovlar is subject to an incision-dominated trend, at least after the late Pleistocene. The incision is caused by Caspian Sea level fluctuations, tectonic displacement by faults running along the Kura River, or climatic changes. The longitudinal profile of the Tovuz River shows a prominent knickzone, i.e., a locally steep segment, at ca. 700-1,300 $\mathrm{m}$ a.s.1., and an incision in the downstream alluvial reach with a straight profile is likely associated with the propagation of the knickzone or knickpoints.

Along with the long-term incision trend of the surrounding rivers in the Holocene, the availability of water for humans on the alluvial fans could have changed (Ollivier and Fontugne 2012). The rapid incision that formed the 20-m high terraces along the Tovuz and Zayam Rivers may have affected the water tables of the alluvial fans, and the base-level lowering could have been linked to locational shifts of surficial springs on the alluvial fans. Further, associated decreases in surface water discharges from the upstream mountains could have occurred. Although detailed behaviors of water availability on the alluvial fans, which can be associated with eustatic variations of the Caspian Sea level downstream of the mainstream of the Kura, remains to be further investigated (Ollivier and Fontugne 2012), a systematic shift of the archaeological site distribution from alluvial fan surface to valley-side domains, observed in Neolithic to Early Iron Age (Chapter 8), likely suggests that the decrease in groundwater availability is crucial for the development of those settlements along the timeline. 

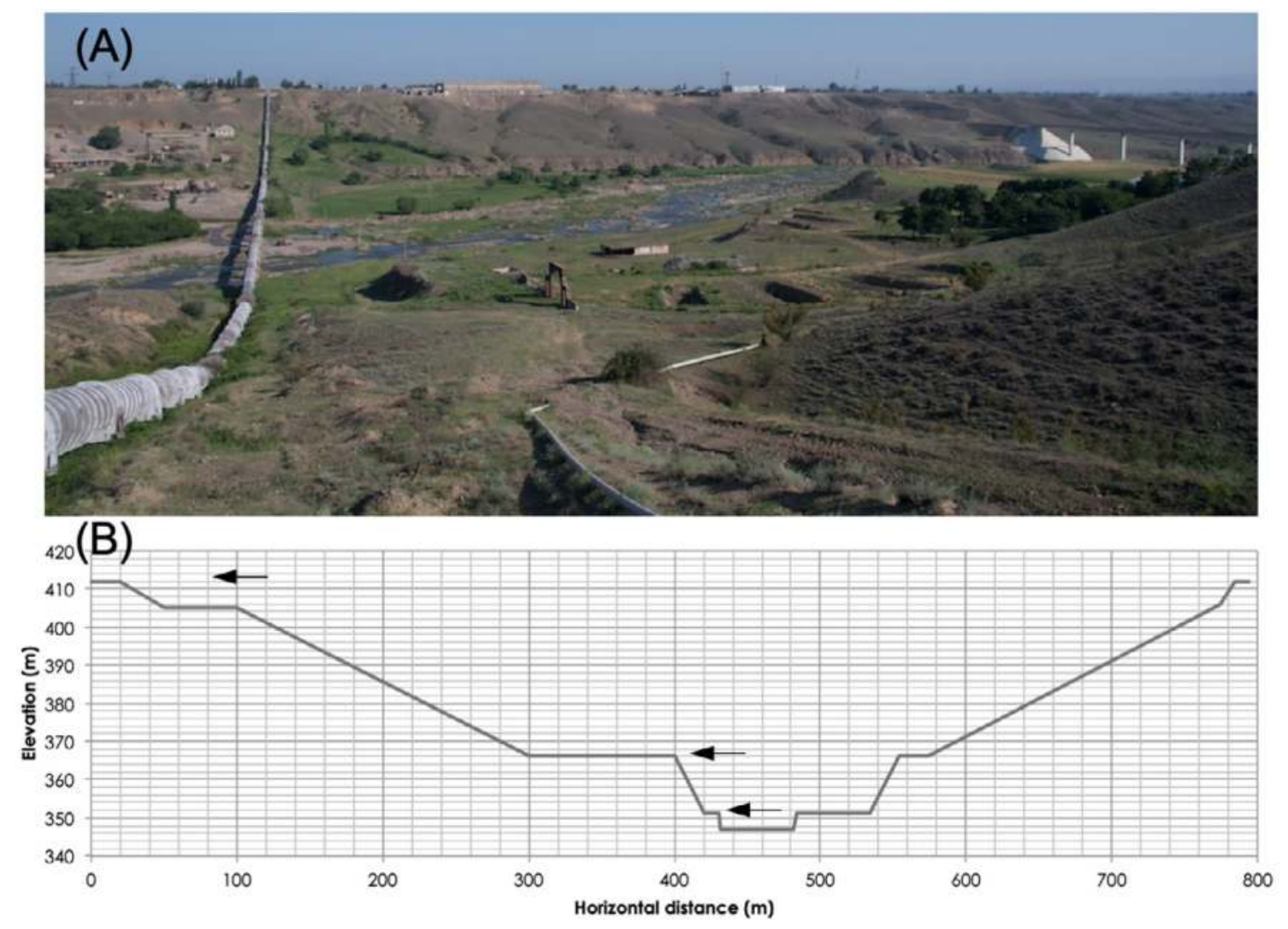

Fig. 2.3 Terraces along the Tovuz River. (A) A picture looking downstream (northwest). The big pipe on the left is for irrigation, which delivers water from the west to east over the Tovuz (and terminates at the Esrik). Next to the pipe, a quarry plant is shown on the opposite (left) side of the river. (B) A cross profile of the Tovuz River generated with a laser range finder (view toward upstream). Terraces at $60 \mathrm{~m}, 20 \mathrm{~m}$, and $4 \mathrm{~m}$ high are shown as indicated by arrows.

\section{References}

Kottek, M., J. Grieser, C. Beck, B. Rudolf, and F. Rubel (2006) World map of the Köppen-Geiger climate classification updated. Meteorologische Zeitschrift, 15: 259-263.

Ollivier, V., M. Fontugne, B. Lyonnet, and B. Helwing (2011) Geomorphological studies in the Middle Valley of the Kura River (Azerbaijan): First key to understand the postglacial landscape mutations linked to climate changes and human occupation. European Geosciences Uniron 2011.

Ollivier, V. and M. Fontugne (2012) Holocene landscape and human modes of occupation in the Kura Valley (Azerbaijan). Archäologische Mitteilungen aus Iran und Turan, 44: 121-127.

World Meteorological Information (2013) World Weather Information Service, http://worldweather.wmo. int/007/c00019.htm (Last accessed 17 March 2013) 


\title{
Chapter 3
}

\section{Stratigraphy and architecture in the main excavation area of Göytepe}

\author{
Yoshihiro Nishiaki, Farhad Guliyev, Fuad Hoseynov, and Kazuya Shimogama
}

\subsection{Introduction}

The excavations of Göytepe in 2008-2013 were conducted in three different areas to ascertain (1) the large-scale exposure of the squares across the highest part of the mound ("Upper Area") to understand the settlement layout and structure; (2) the deep soundings at one square along the northern slope (Square 4B) to determine the principle stratigraphic sequence; and (3) the small-scale pit soundings at the mound peripheries to investigate the extension of the Neolithic settlement (Fig. 3.1). This chapter is devoted to descriptions of the primary results of the large-scale exposure. The deep and small-scale pit soundings are presented in Chapters 4 and 5 .

One of the most important characteristics of the Göytepe mound is its large size for the Neolithic sites in the Southern Caucasus. Indeed, it is the largest known to date in the Middle Kura Valley of Azerbaijan (see Narimanov 1987; Chataigner 1995; Helwing et al. 2017). Therefore, this site provides a privileged case to investigate the settlement structure through extensive excavations. The excavation squares we set up for this purpose consist of ten $10 \mathrm{~m}$ by $10 \mathrm{~m}$ squares $(99 \mathrm{~A} / \mathrm{B}$ to $4 \mathrm{~A} / \mathrm{B})$, encompassing the sounding square of $4 \mathrm{~B}$. Each square was subdivided into two rectangular areas of $5 \mathrm{~m}$ by $10 \mathrm{~m}$ (I and II; Fig. 3.2). The excavations proceeded with preserving

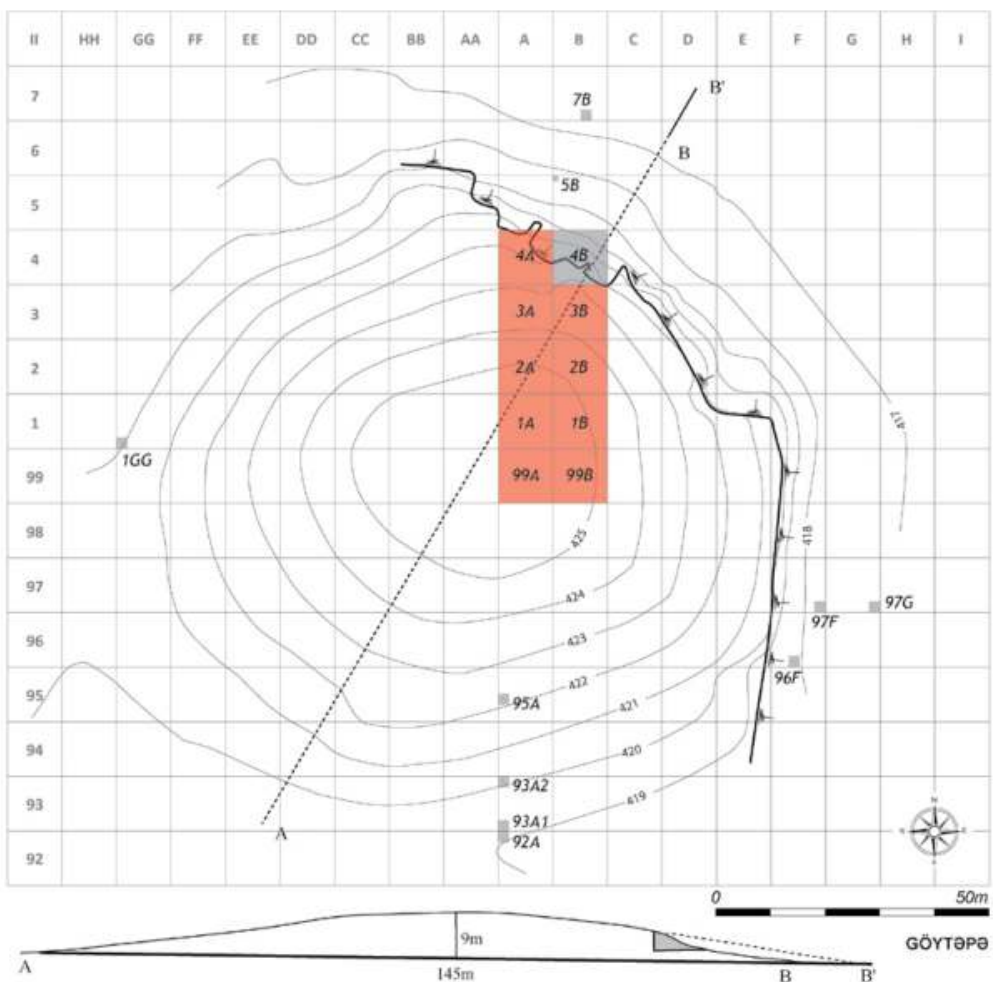

Fig. 3.1 Plan of Göytepe showing the location of excavation squares. 


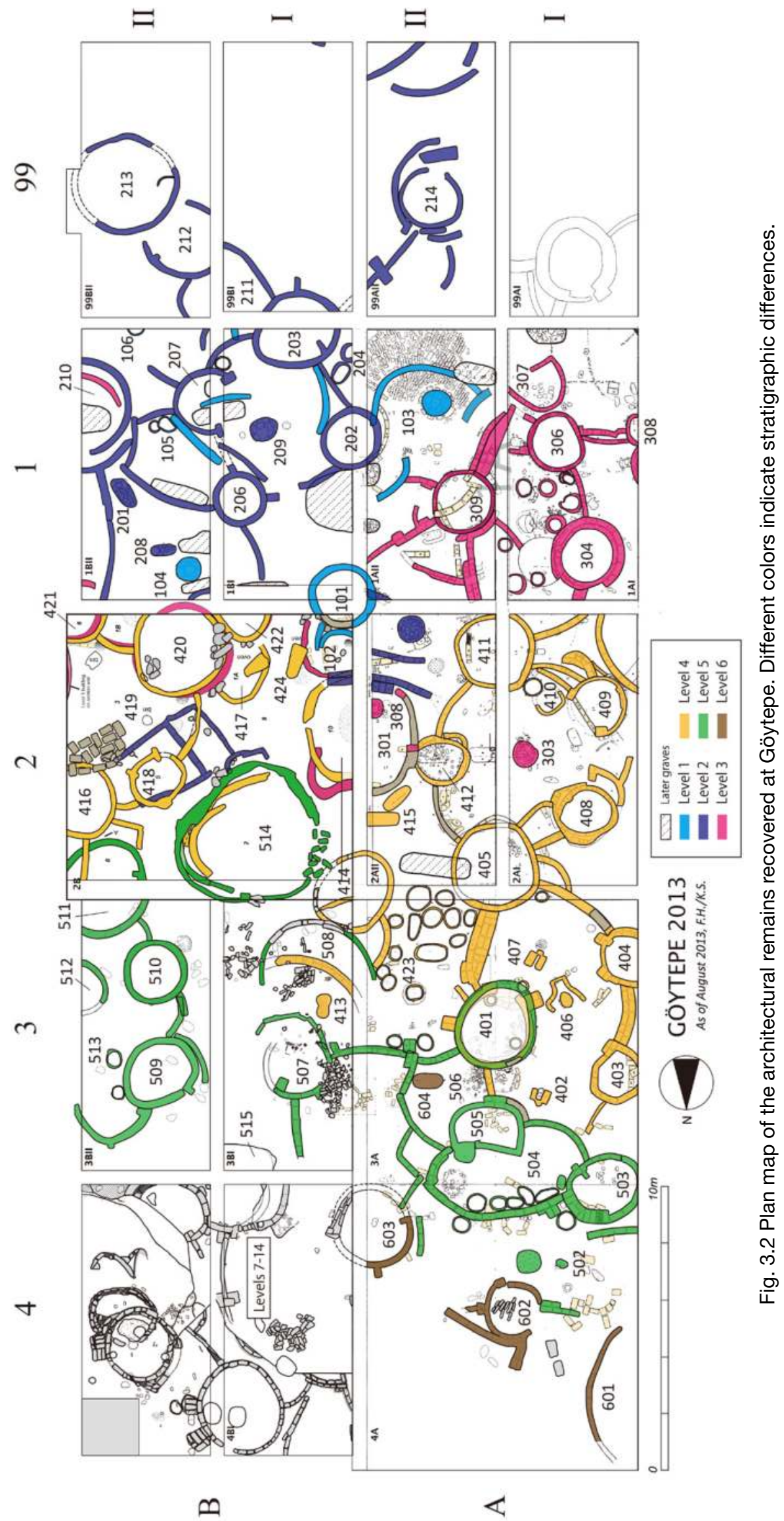


the architectural remains after their exposure and examination. Accordingly, lower levels were only excavated in an area where substantial buildings or features of the upper levels were not distributed. This excavation strategy was employed to install a field museum at Göytepe in the near future, which would allow visitors to look at the standing Neolithic buildings and domestic structures in the primary positions. It has a distinct disadvantage in the significant reduction of an excavation area when attempting to deepen the same square. However, this drawback is recovered by moving the excavation squares from the central to the marginal parts of this sufficiently large mound site. Moreover, this strategy has a notable advantage in leaving an opportunity for future archaeologists to re-examine the architecture and stratigraphy whenever necessary. In the following, we present preliminary results of our examination of the stratigraphy and architectural remains, which are derived from the latest six architectural levels. For the earlier levels, see Chapters 4 and 5.

\subsection{Stratigraphy}

The "level" in this research project denotes an identifiable major ground surface on which any substantial structure was originally built. It also denotes the deposits between the given level and its upper level (Lloyd 1963). To define building levels, we focused on the stratigraphic positions of the construction: the base in the case of standing architecture, and the top in the case of pits and ditches. Using the architecture and features as a stratigraphic key point, we include in the same building level the deposits and features related to the construction, use, and abandonment of the major building remains.

Two difficulties in assigning architectural remains to building levels should be mentioned. First, a difficulty specific to the Göytepe campaign exists: most of the structures have not been fully excavated. The excavation strategy to preserve the buildings does not allow it to fully reveal the base of any standing architecture. For example, excavations of a building that might have been used for more than one level by re-flooring were often halted when the first (upper) floor was recovered. The lowest part of those structures might have continued downward.
Consequently, the assigned level is considered to represent the latest stage of the building's life history. This is particularly the case in the large excavation area where the architecture of Levels 1-6 was identified. This difficulty was less significant in the excavations of Square 4B because this square was situated in an eroded slope that exposed sections of buildings on the surface. Therefore, the identification of building levels in Square 4B is much more reliable, although the smaller excavation area often prevents us from characterizing the architectural plan.

The second issue relates to frequent additions to or reforms of the extant buildings. Although this is a well-known phenomenon at Neolithic settlements with mud-brick walled buildings in general, it was also commonly recognized at Göytepe. In particular, additions of curvilinear partition walls were repeatedly observed. Moreover, it was also common that structures shared sidewalls and, even, some part of a building. The structures of this settlement, often connected to each other in this way, seemed to have comprised much larger complexes. A key feature in comprehending a group of structures used in a certain period must be the entrances to structures. Our analysis shows that the entrances of circular buildings were exclusively open toward their courtyard. This finding helped us distinguish one dwelling group from others, and also reconstruct the past social organization at this site (see below). Accordingly, major groups of buildings were identified as comprising the same levels. Nonsubstantial additions or reforms were not considered to represent a separate building level but identified as different construction phases of a building level. They were designated as a sub-level with a, b, c, and so on when necessary.

We defined 14 occupational levels in the 11 m-thick Neolithic deposits at Göytepe (Fig. 3.3). According to radiocarbon dating, this "massive" sequence belongs to a relatively short period of ca. $5650-5460$ cal. BC, or approximately 200 years (Nishiaki et al. 2018).

\subsection{Architectural remains by levels}

\subsubsection{Level 1}

The highest part of this mound, especially the 


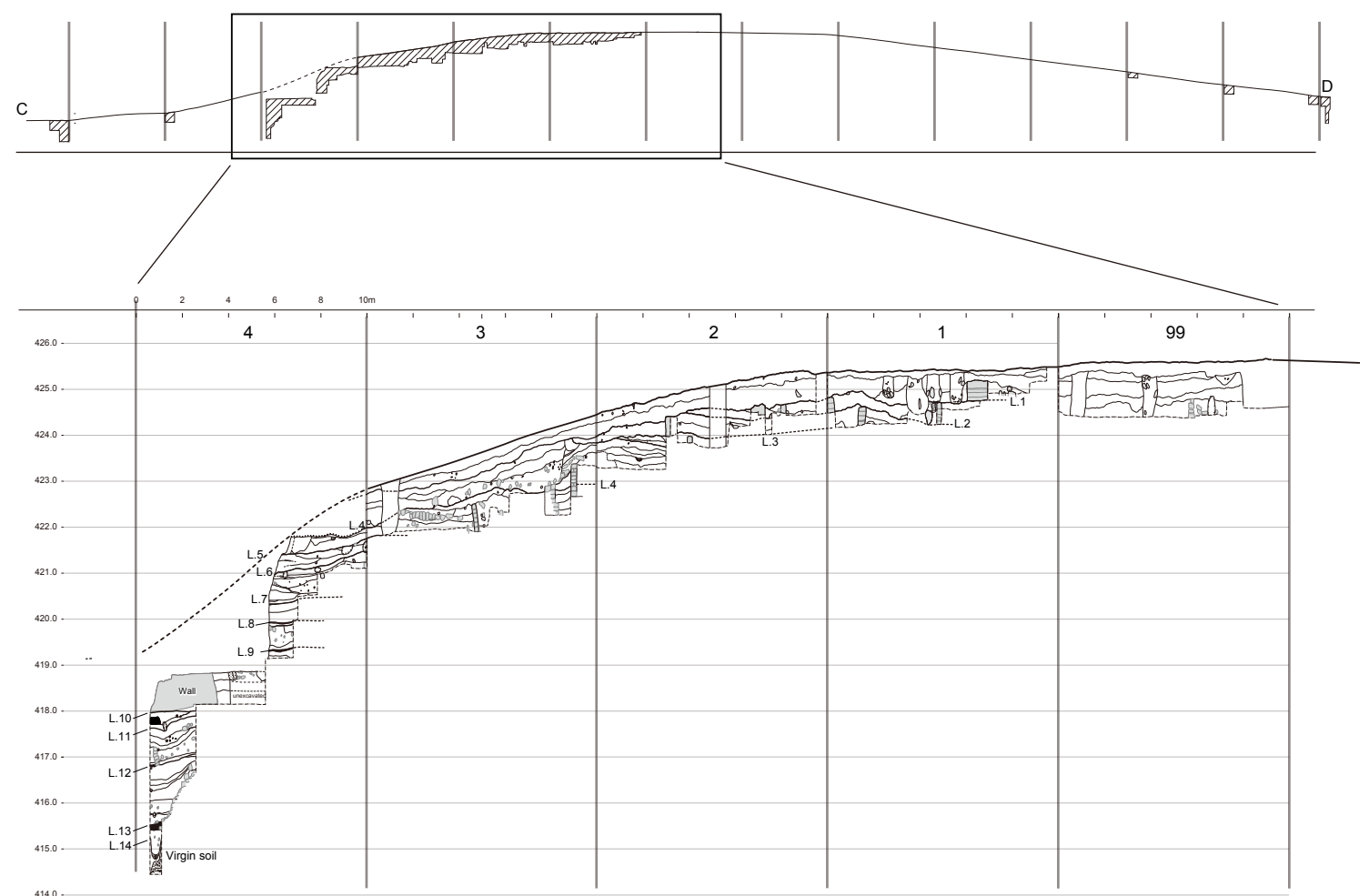

Fig. 3.3 North-south stratigraphic section of Göytepe. The vertical scale is twice the size of the horizontal scale.

areas including Squares 99A and 99B, was used for cemeteries in the Bronze Age and later periods. Consequently, the latest Neolithic occupation phase of this mound is not well preserved. However, we identified one circular mud-brick walled structure, isolated remnants of mud-brick walls, and two hearths in Squares 1AII and 2B.

The circular building (101) in Square 2BI, made of sun-dried mud-bricks, measures about 2 $\mathrm{m}$ in diameter (Figs. 3.2 and 3.4) and has a short sidewall running to the north, which is truncated by later disturbance. There seems to have been another circular building of similar size nearby (102), although it is heavily damaged. The fragmented walls in Square 1AII is likely a portion of the sidewalls encircling a courtyard. A circular building of this possible compound (202) was built in the underlying level (Level 2) and continued to have functioned in Level 1. The courtyard encompasses a hearth in the center (103), which is made of round cobbles paved in a round form of approximately $90 \mathrm{~cm}$ in diameter. It has larger stones in the middle and smaller stones at the edges. The cobbles retain reddish-brown surfaces with black soot, demonstrating their exposure to fire.

There were fragments of mud-brick walls in
Square 1B. They may have constituted another compound using earlier Level 2 buildings (203 and 207), but the poor preservation prevents it from reconstructing the original architecture. The remaining features in this level are one hearth (104) and two clay bins (105 and 106) in Square 1BII. The hearth (104) shows a similar size and structure to those of the one described earlier (103). The bins are made of clay walls in a round shape with a diameter of approximately $50 \mathrm{~cm}$. Its interior side was clayplastered. One of the bins (105), standing about 40 $\mathrm{cm}$ high and attached to a sidewall, was found to contain an unmodified cattle scapula (Fig. 3.5). The excavation boundary cuts the other bin (106), and its spatial context is unknown.

\subsubsection{Level 2}

The architecture of this level is better preserved. Several building remains were recovered in Squares 1B, 99AII, 99B, and in an area between 2AII and 2BI (Fig. 3.2). The best-preserved structures were recovered in Square 1B (Figs. 3.6 and 3.7). A group of five circular buildings $(202,203,206,207$, and 210) connected with curvilinear sidewalls was identified. Together with its courtyard, this can be 


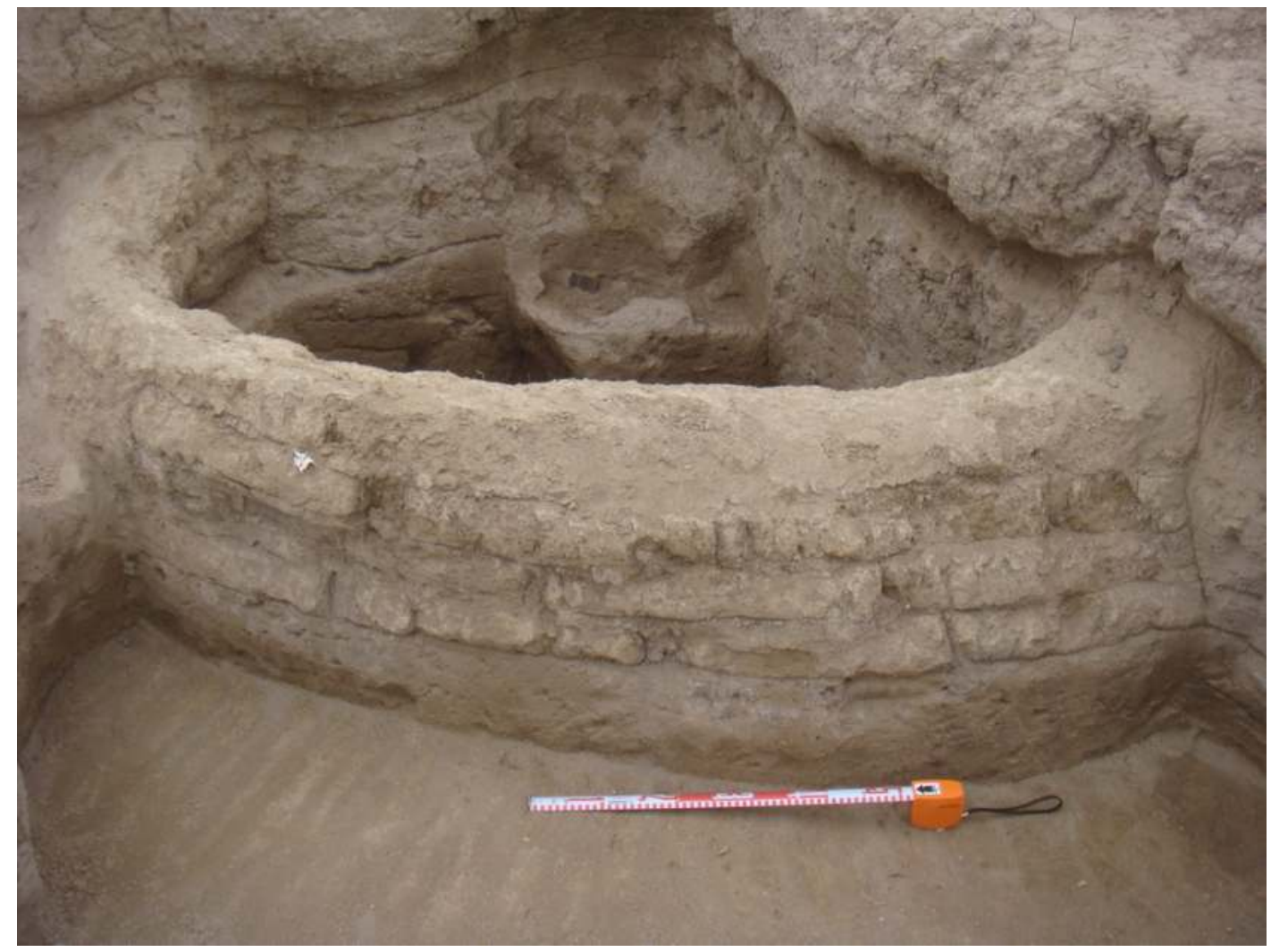

Fig. 3.4 Mud-brick wall of Building 101 in Square 2BI looking northwest. Four rows of mud-brick are visible.

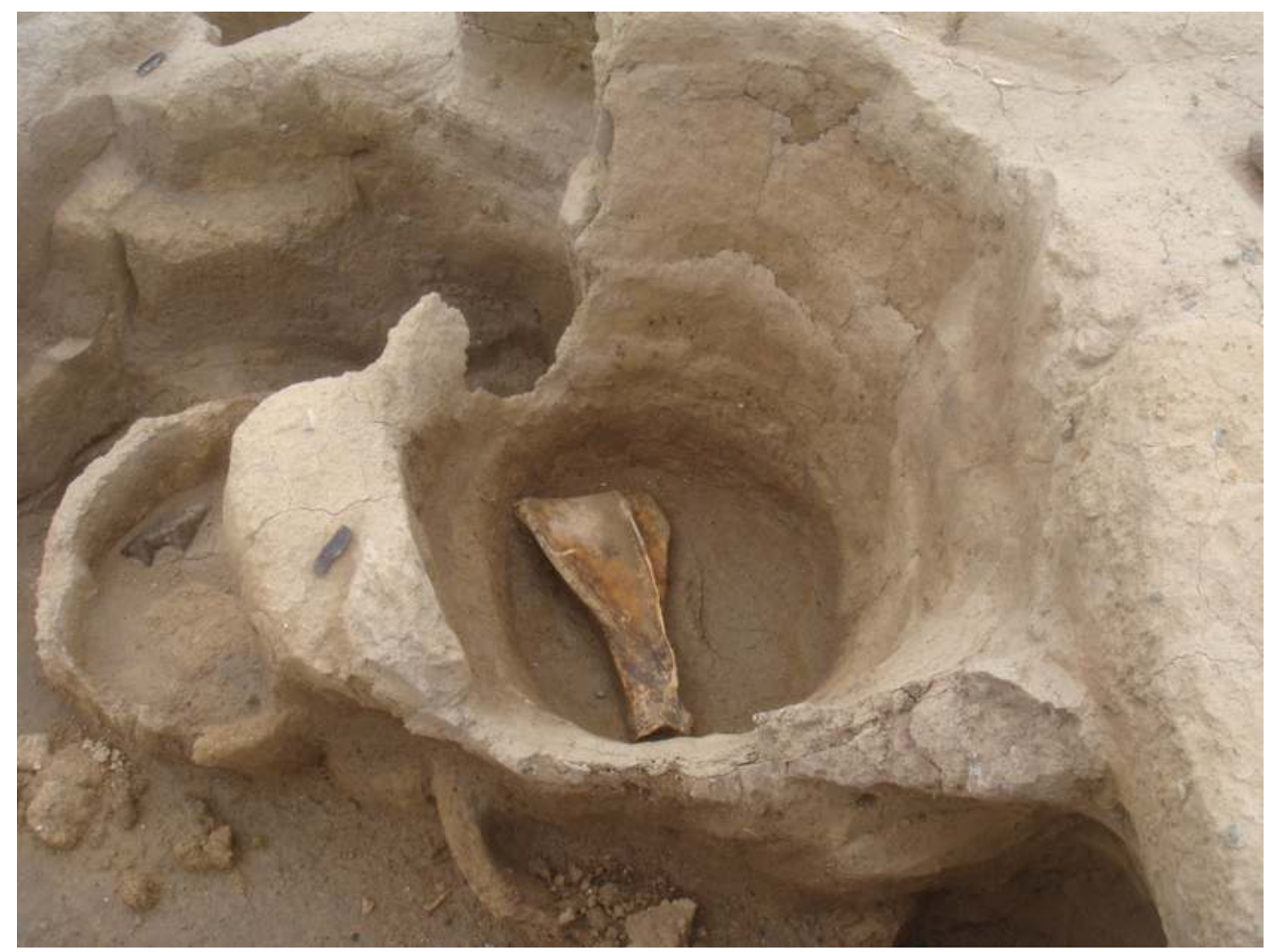

Fig. 3.5 Clay bin (105) in Square 1BI looking east. Note a cattle scapula inside. 


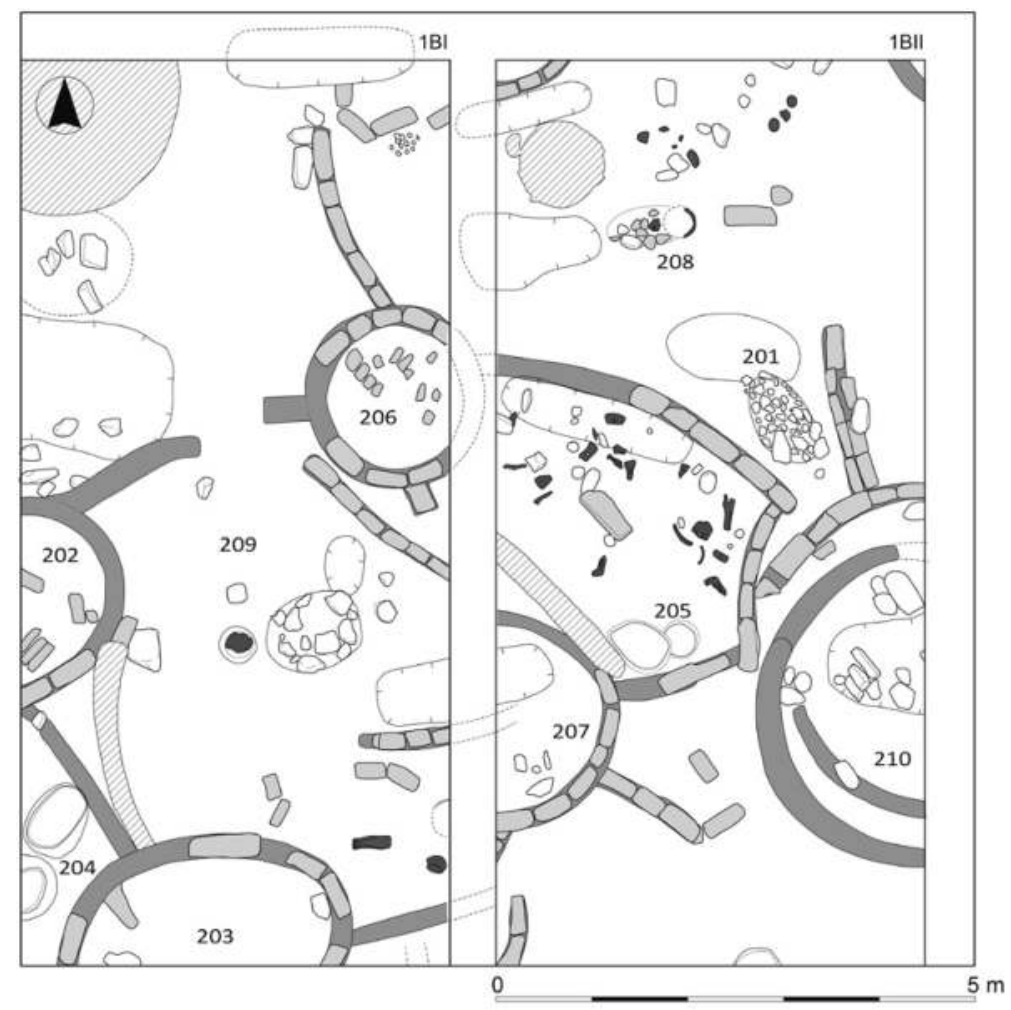

Fig. 3.6 Plan for Level 2 architectural remains in Square 1B.

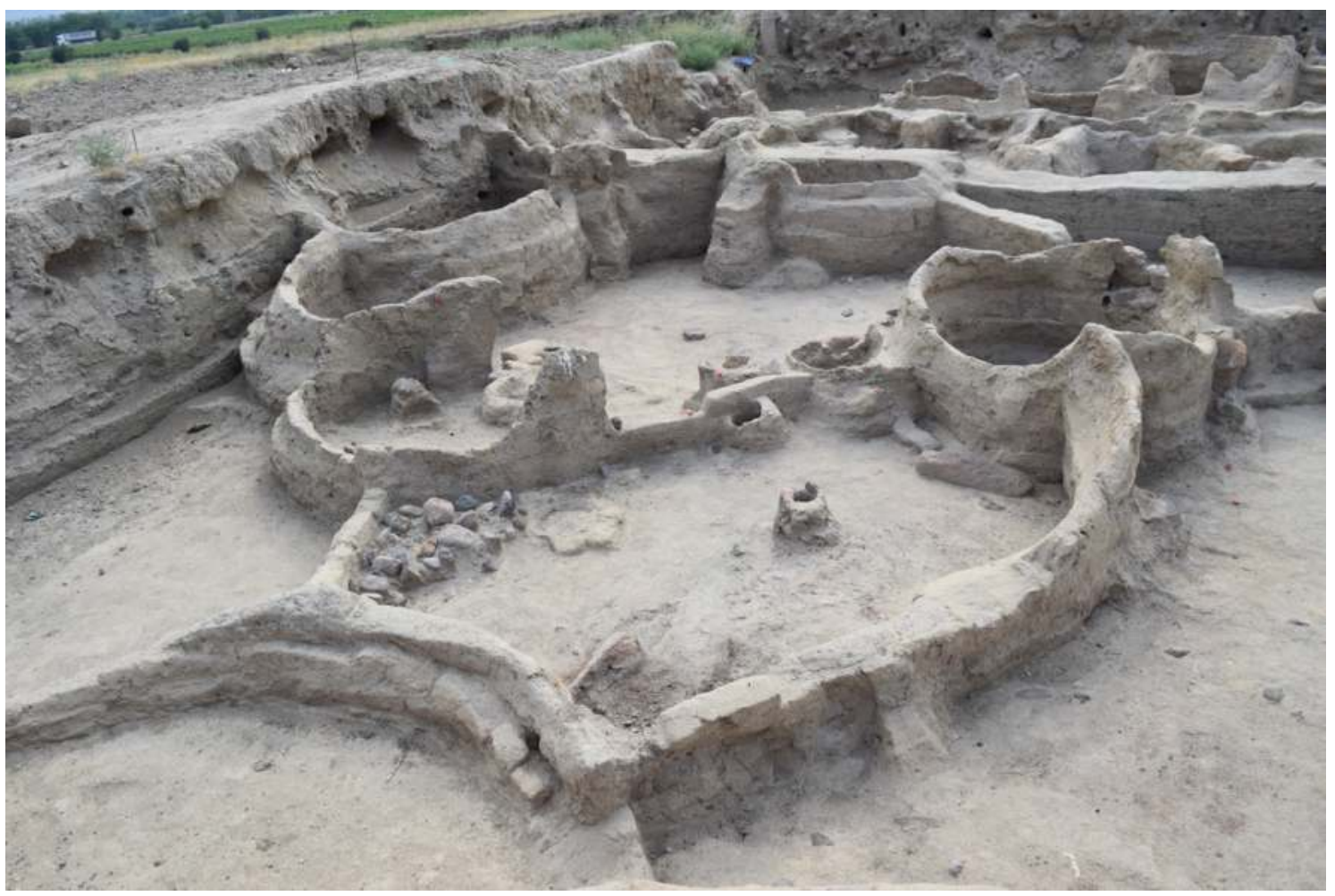

Fig. 3.7 Level 2 architectural remains in Square 1B looking southwest. 
interpreted as a household compound. The largest building structure measures $3.3 \mathrm{~m}$ in its inner diameter (210). However, this building does not have an entrance from the courtyard and has curvilinear sidewall extending to the north. Accordingly, the role of Building 210, originally built for another compound, was not to provide residential space for this compound but to enclose its courtyard with the outer wall. The diameters of the other buildings range from 1.5 to $2.4 \mathrm{~m}$. They enclose an oblong courtyard with sidewalls, whose entrance is situated in Square 1AI. At least one structure (207) showed a well-made doorway facing the courtyard. As in Level 1, a stonepaved hearth (205) was recovered at the center of the courtyard (Fig. 3.8). Further, it is remarkable that the small area situated to the east of the courtyard yielded numerous stone artifacts and bone tools in situ (Fig. 3.6), and it may have been a kind of annex of the main courtyard used as a workspace.

The structures to the north and east of this compound are less defined because of the cutting by the excavation limits. It is equipped with at least two hearths, of which both are stone-paved like the hearths mentioned earlier, but show an oblong plan, measuring $100 \times 55 \mathrm{~cm}(209$; Fig. 3.9$)$ and $80 \times 30$ $\mathrm{cm}$ (208; Fig. 3.10). The latter preserved a standing pottery vessel on the paved stones.

Even fewer clear structures are distributed in Squares of 99AII to 99BII. In addition to the later disturbance, the suspension of deeper excavations for the future archaeological park prevented defining the architectural details. Therefore, the stratigraphic assignment in 99A and 99B should be treated as a preliminary one. Also, structures in 2AII and 2BI were hardly identifiable due to the heavy disturbance in the later periods. The notable features were only a couple of isolated mud-brick walls and a stonepaved hearth (201), as noted in Level 1. The clay bin located in Square 1BII (210) was remarkable in containing complete ground stone artifacts and

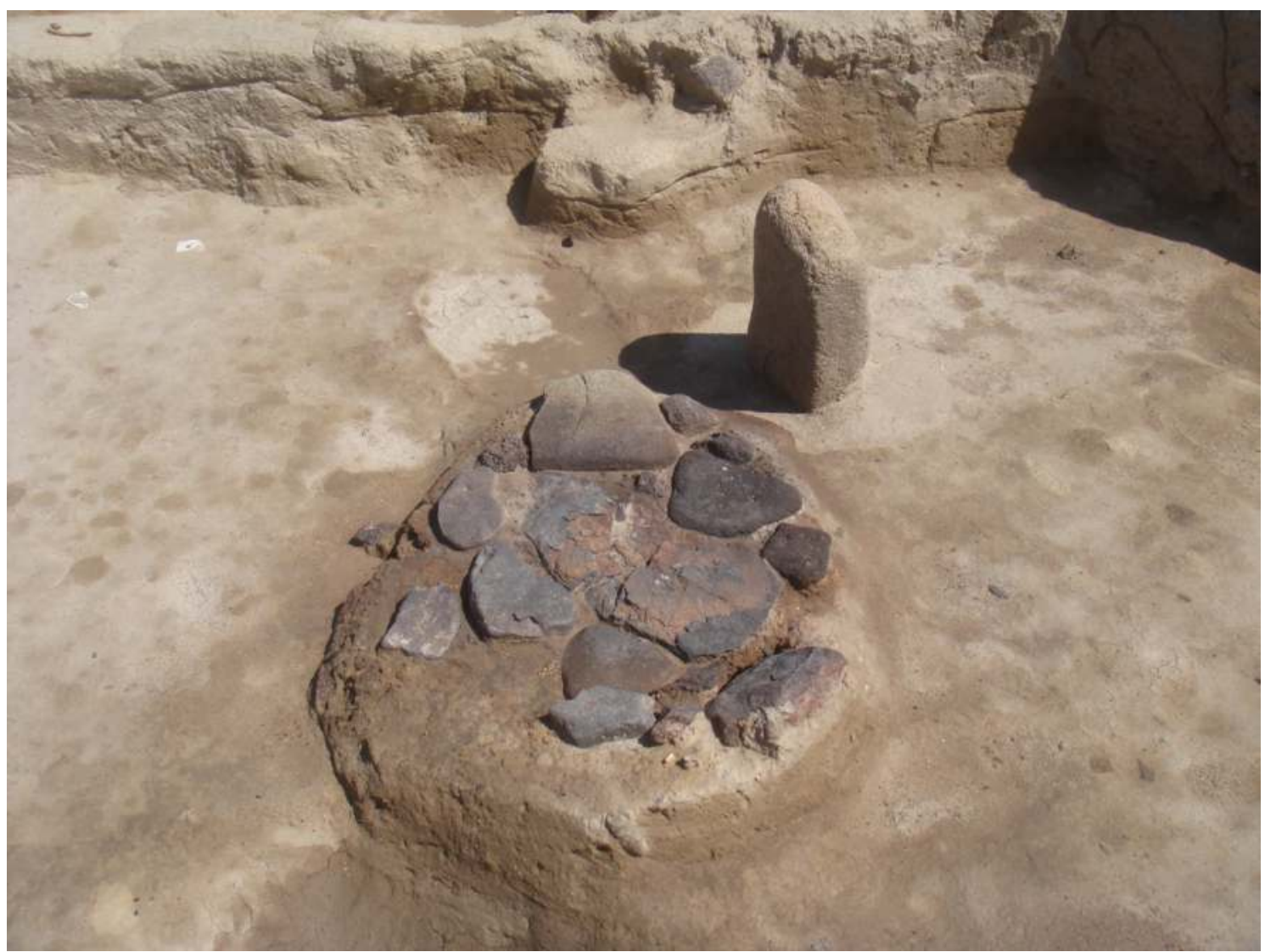

Fig. 3.8 Round hearth (205) in Square 1Bll looking east. A large ground stone slab was found standing nearby. 


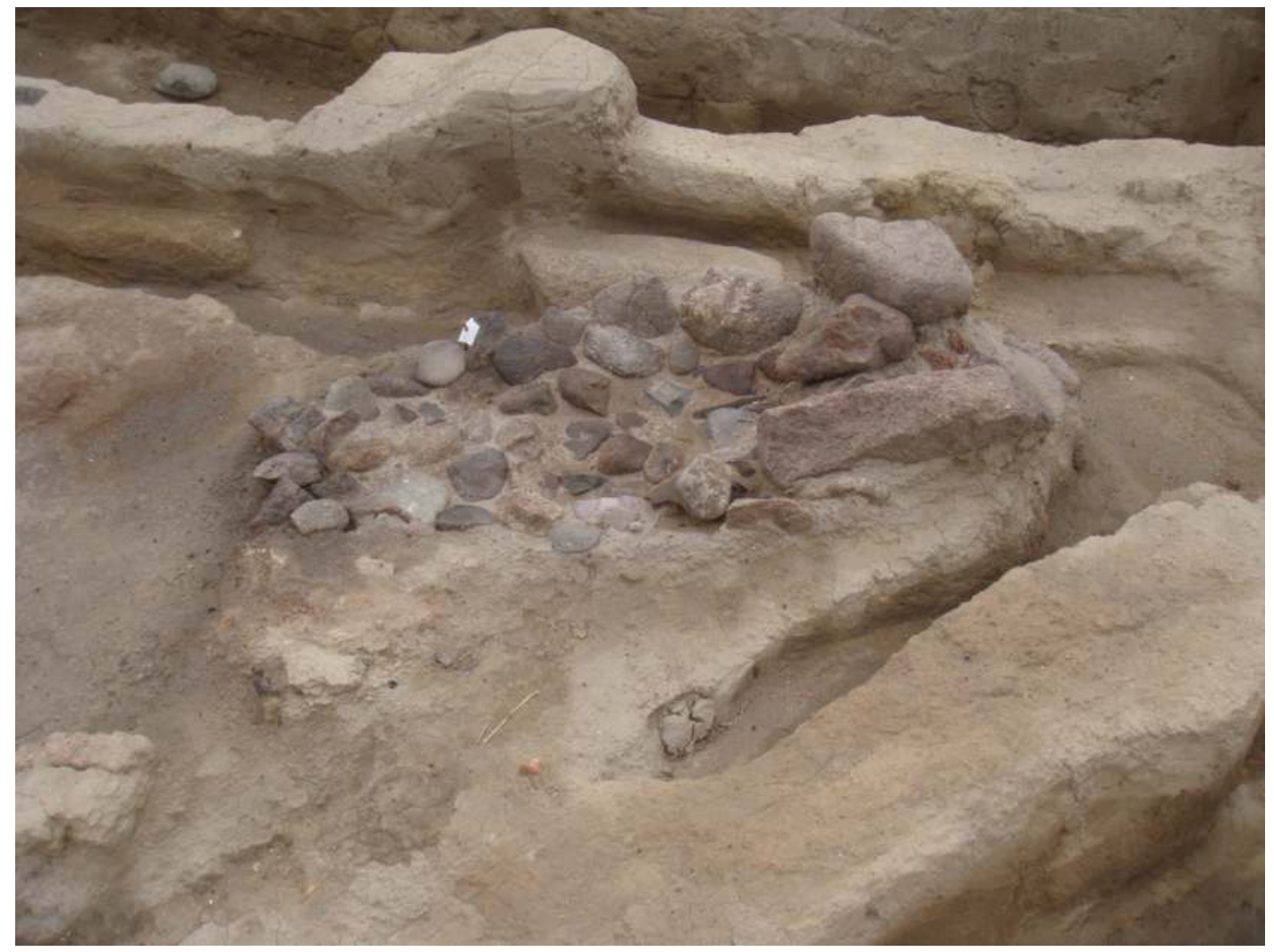

Fig. 3.9 Oblong hearth (209) in Square 1Bll looking east.

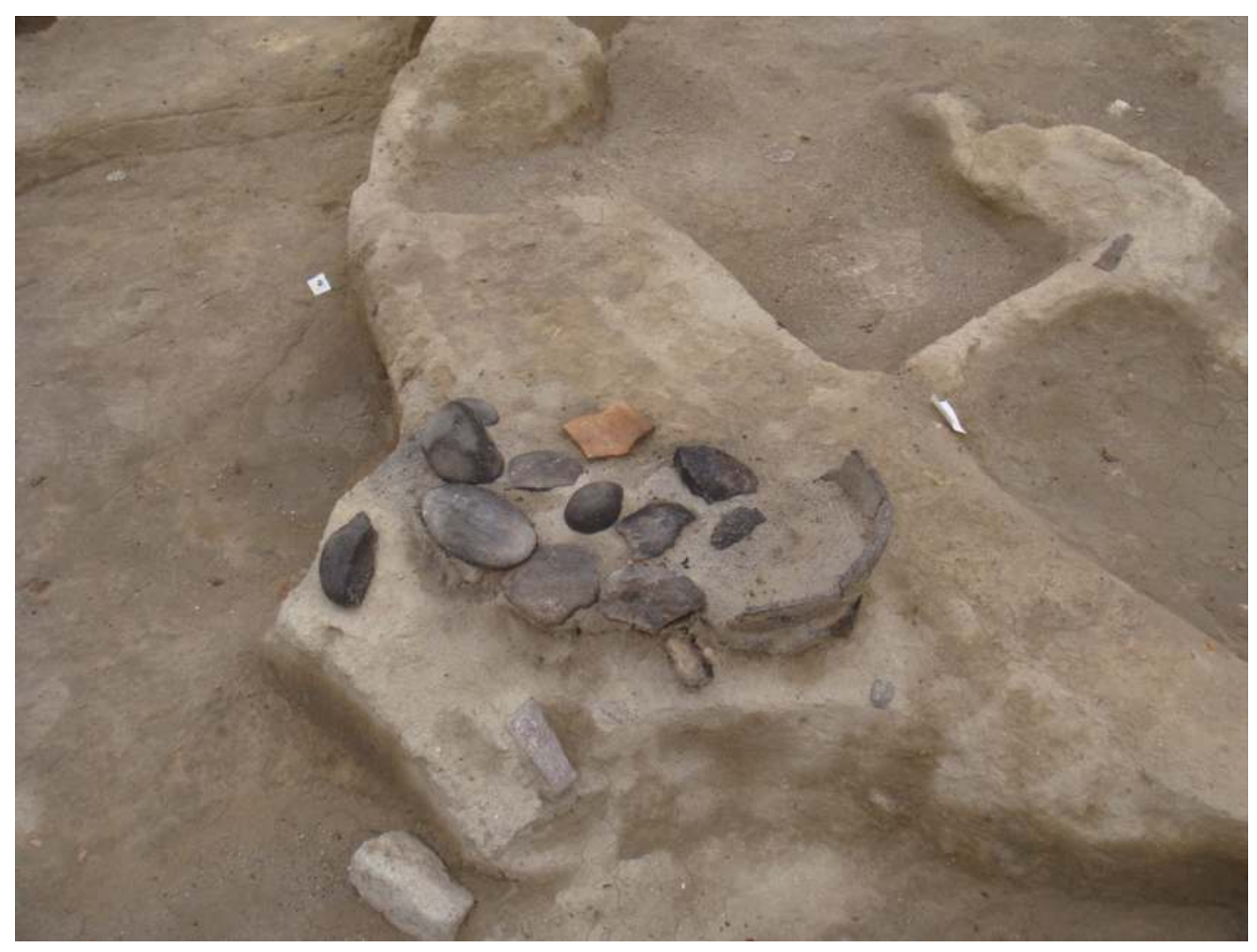

Fig. 3.10 Oblong hearth (208) in Square 1Bll looking south. A standing pottery vessel is visible on its right side. 
unworked cobbles inside (Fig. 3.11), which indicates, as the bins of Level 1, that some of the clay bins were used as storage of tools for future use.

\subsubsection{Level 3}

Architectural remains belonging to this level have been recovered mainly in Square 1A (Fig. 3.2). A few structures are also distributed in Squares $2 \mathrm{~A}$ and $2 \mathrm{~B}$ but fade away due to slope erosion. The structures in Square 2A comprise a compound of four circular buildings $(304,306,309$, and 410) connected by sidewalls (Fig. 3.12). They include a building originally built in the earlier Level 4 (410) in Square 2A. The location of its doorway, opening to the north, indicates that this building was inaccessible to the inhabitants of this compound. In other words, the southern wall of Building 410 was used to enclose the compound of this level. The remaining three structures show a similar shape and size. Remarkably, the courtyard yielded at least seven clay bins, each of which had a 60 to $70 \mathrm{~cm}$ diameter and a depth of some 40 to $60 \mathrm{~cm}$. Our observations show that their rim was above the ground, while the lower part was buried in the ground. Another interesting feature of this compound was the distribution of abundant objects such as pottery vessels, obsidian blades, large bone tools, and a variety of ground stones, including sling stones in its courtyard (Fig. 3.13). They are obviously still usable. It raises the question of whether they were left for future use because of an unexpected disaster or other reasons (Nishiaki et al. 2018). The in situ discovery of usable objects is repeatedly encountered in other building levels, a pattern characterizing the discovery context of the archaeological remains at Göytepe.

This compound was likely connected to another to the south in Square 1AI, involving structures of 307 and 308, and one in the north in Square 2BI, linking with the building of 301 . However, details of those structures are unknown because of the distribution of later structures. Two hearths found in 2AI (302) and 2AII (303) are the rare recognizable features in those squares. As in the later levels, they

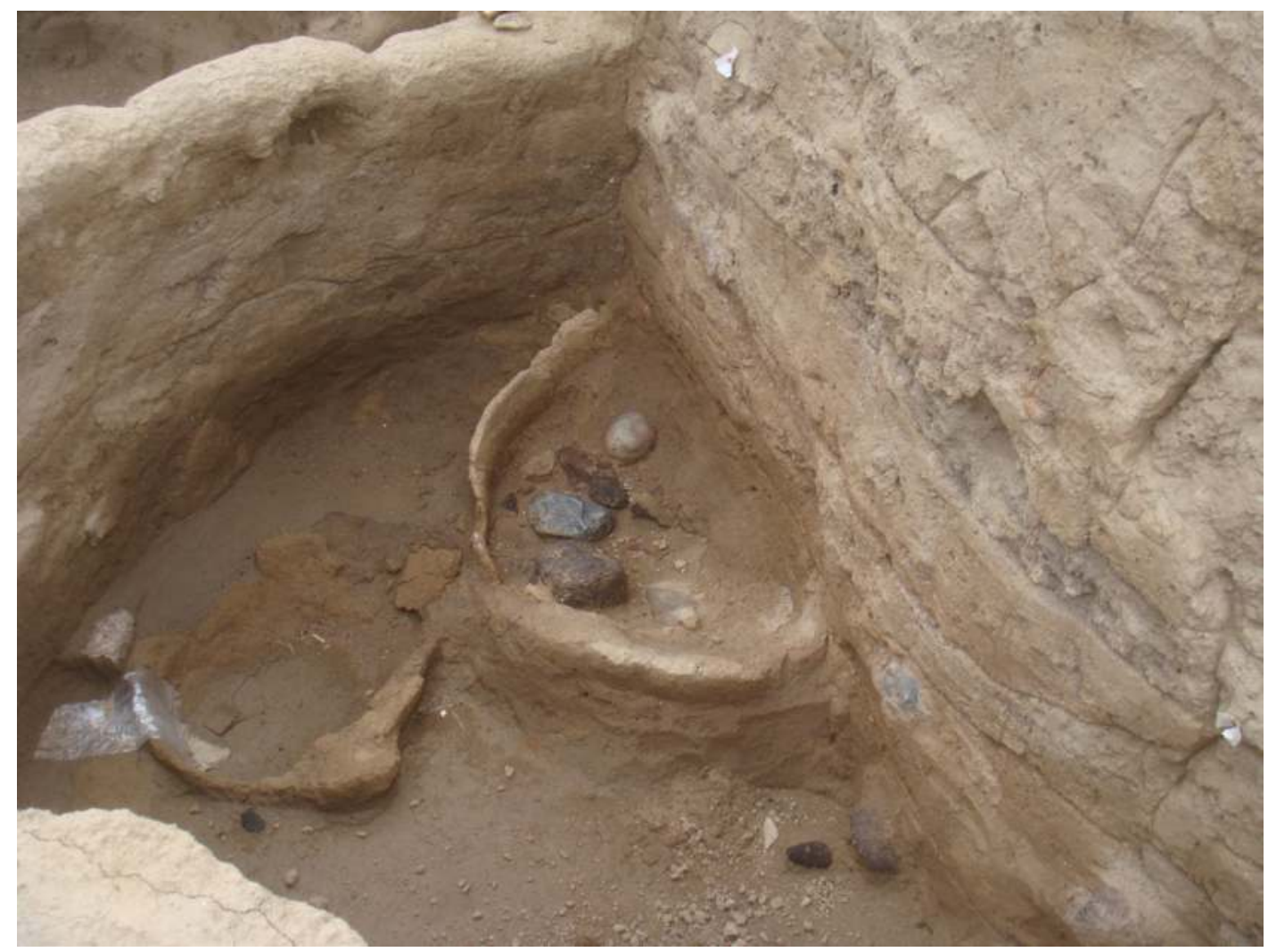

Fig. 3.11 Clay bins (210) in Square 1BII looking south. Note lithic materials inside. 


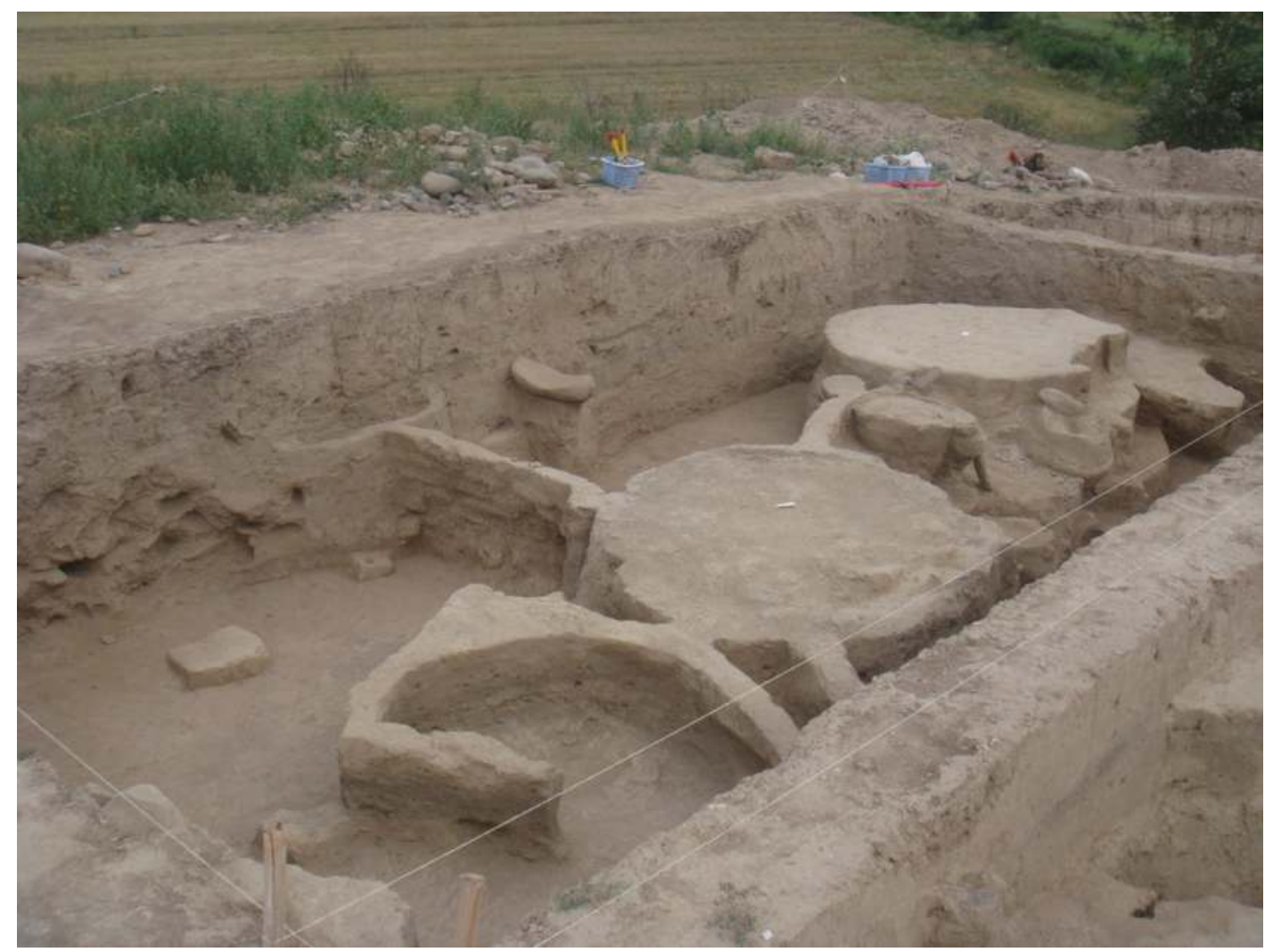

Fig. 3.12 Level 3 architectural remains in Square 1Al looking northwest.

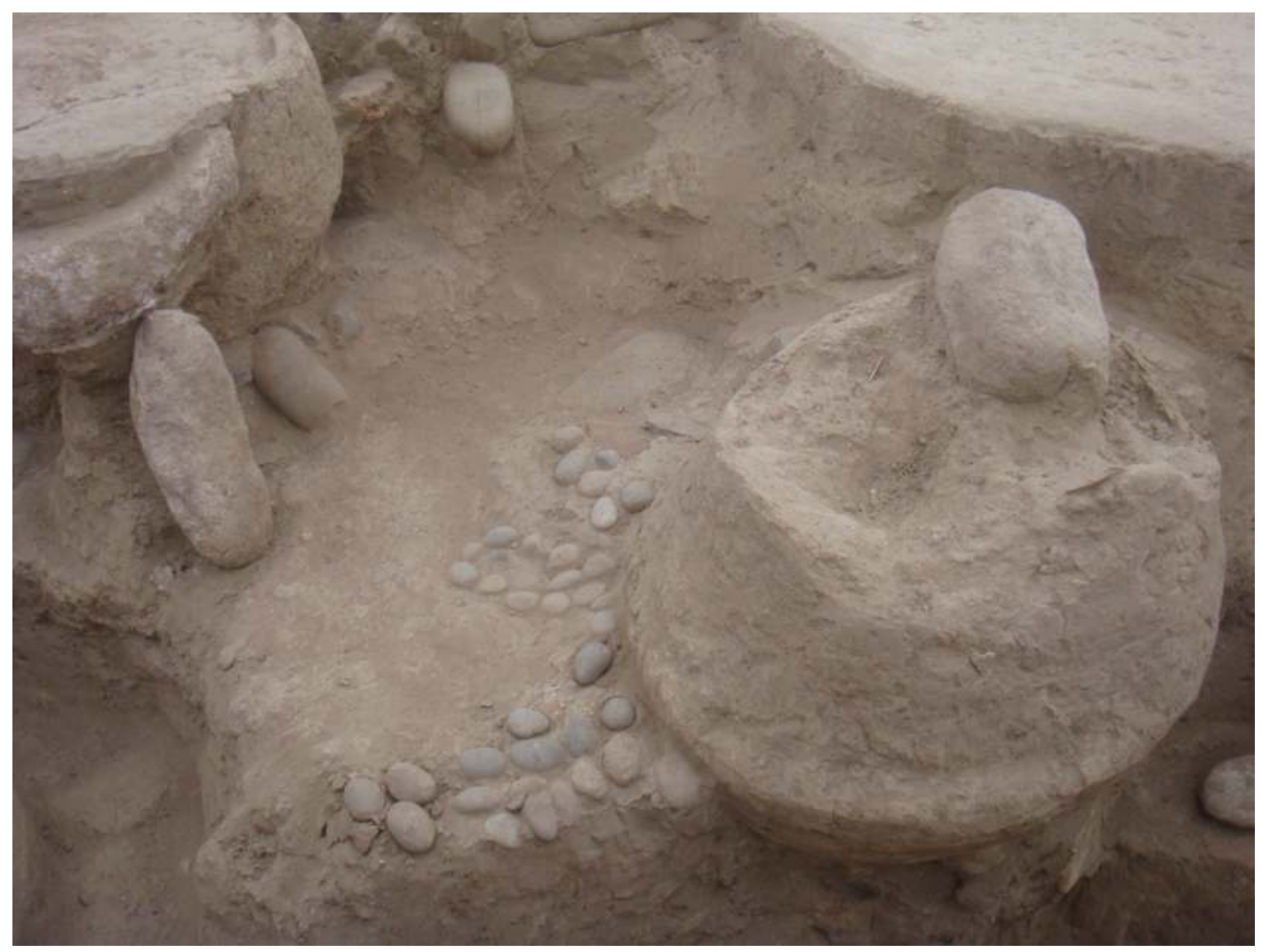

Fig. 3.13 Concentration of sling stones near clay bins (305) in Square 1Al. 
were made of river cobbles arranged in a round shape of 60 to $75 \mathrm{~cm}$ in diameter. Besides these remains, we have provisionally assigned two more isolated mudbrick walls of 1BII to Level 3 (Fig. 3.2), because they were discovered directly below the Level 2 structures. Their precise relationship to the other Level 3 structures will not be determined before the entire structures of Levels 1 and 2 are removed.

\subsubsection{Level 4}

This level exposed its architectural remains more extensively than the later ones. Numerous buildings and features were recovered in Squares 2A, 2B, and $3 \mathrm{~A}$ (Fig. 3.2). Their extension to the south is also indicated by a curvilinear wall below a circular building of Level 3 in Square 1AII (309), but are mostly hidden by later structures. Conversely, architectural remains on the northern side are missing due to the slope erosion. In the better-preserved squares, at least three circular compounds were recognized. Those of Squares 2A and 3A are linked by sharing one circular building in the north (405). The compound of $2 \mathrm{~A}$ is connected with the one in $1 \mathrm{~A}$ through its southernmost building in (411).

The compound in $3 \mathrm{~A}$ consists of at least four circular buildings $(401,403,404$, and 405) that are joined with curvilinear walls (Fig. 3.14), and attached to a wall originally built in Level 5 (Fig. 3.2). Some of the Level 5 buildings were still standing when the Level 4 structures were built. For example, the circular building of 401 had two floors, corresponding to different levels, respectively. That is, walls of an older building were used as foundations for the later building. A possible post-hole of about $15 \mathrm{~cm}$ in diameter, associated with a group of stones, was identified on the floor of Building 401. Further, the floor was characterized by a distribution of numerous in situ objects like stone tools, sling stones, bone objects, and pottery sherds (Fig. 3.15). Doorways are preserved at three buildings (401, 403, and 404; Fig. 3.16). The construction method of these doorways, all opening toward the courtyard, is as follows. Two short buttress walls, made of mud-bricks in British bond, were laid on both sides of the entrance, and a mud-brick threshold was laid in-between. The width of the entrance is between 50 and $80 \mathrm{~cm}$. In the

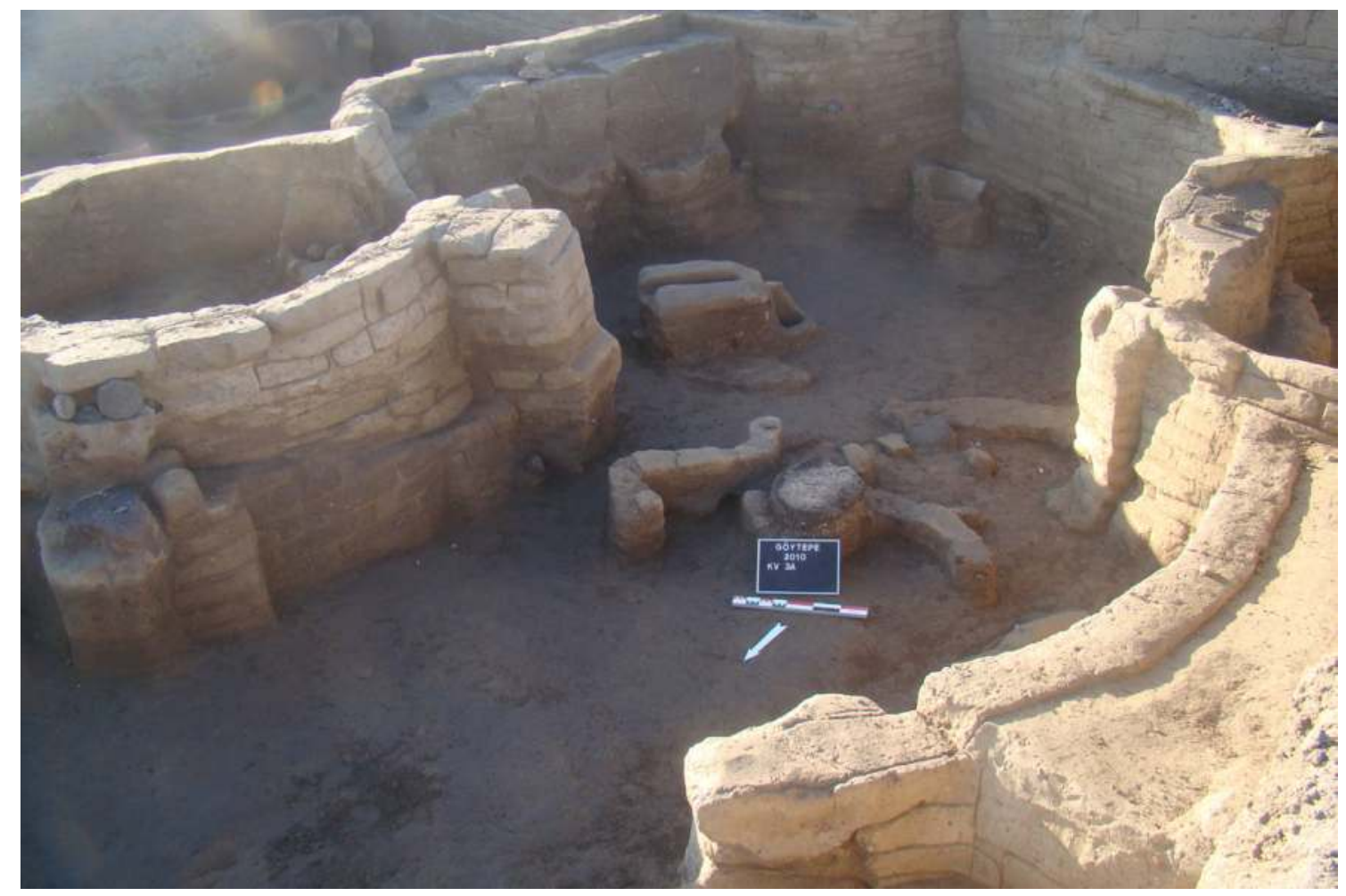

Fig. 3.14 General view of the courtyard of the Square 3A compound looking southeast. 


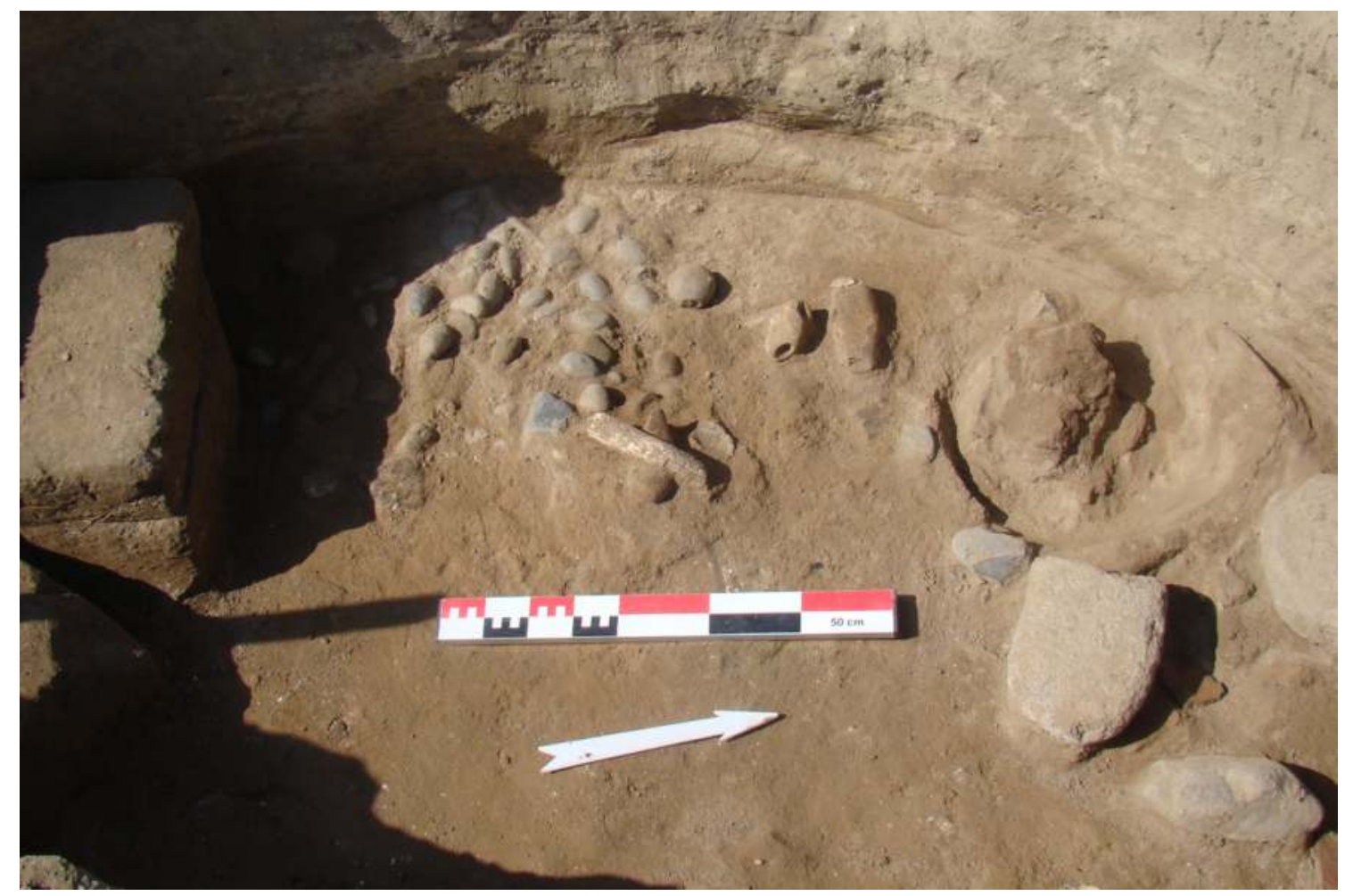

Fig. 3.15 Floor remains of Building 401 in Square 2Al looking west.

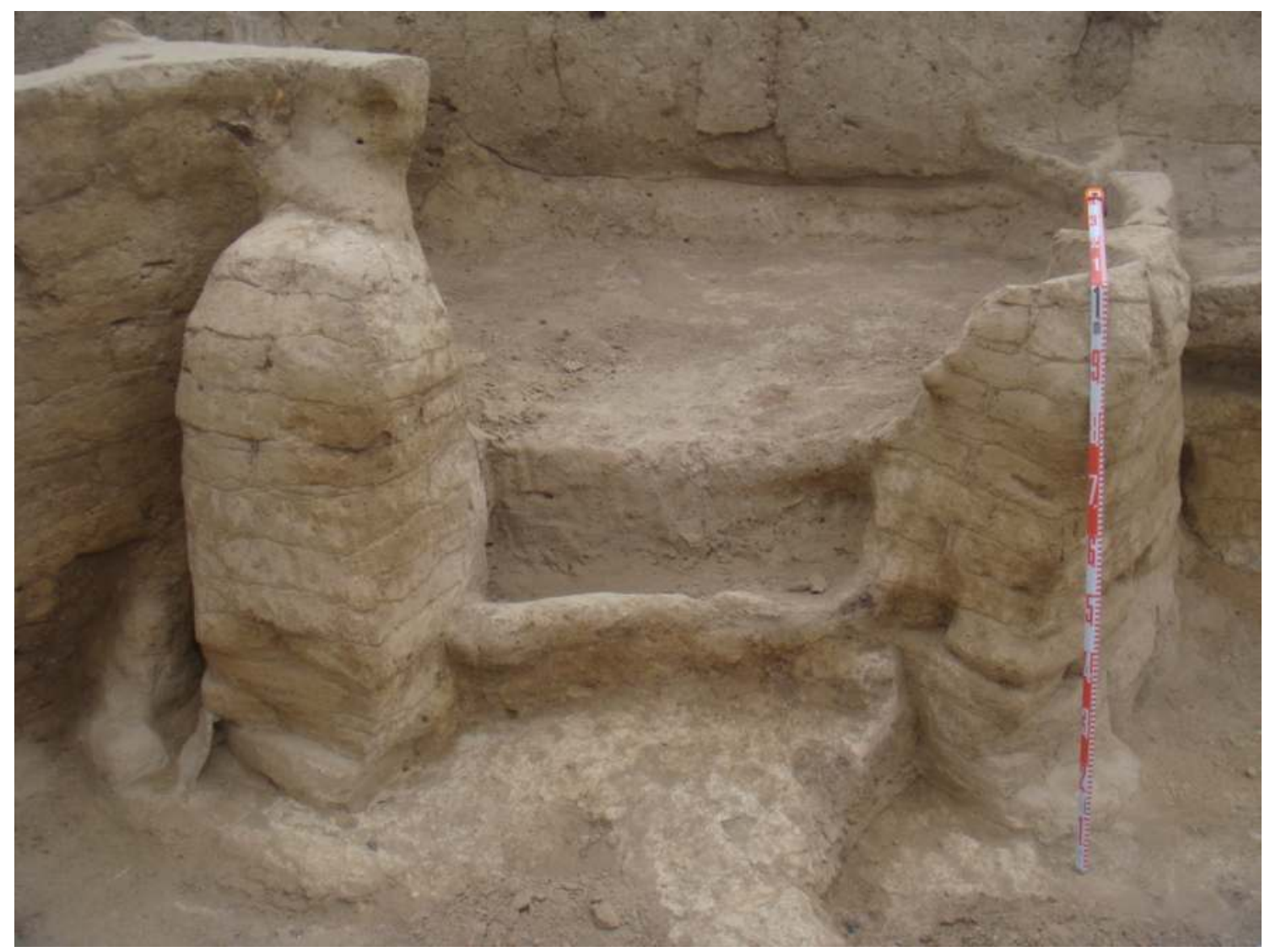

Fig. 3.16 Doorway of Building 404 in Square 2Al looking north. 
courtyard of this compound, two mud-brick features (402 and 407) and a hearth (406) were identified. The hearth, situated in the center of the courtyard, is a round stone-paved one with mud-brick walls laid on both sides in a V shape (Fig. 3.14). Several stone and bone tools were discovered in this compound, especially in its southeast area (Fig. 3.17).

The compound in Square 2A, consisting of five circular buildings (405, 408, 409, 411, and 412; Fig. 3.18 ) and sidewalls, is also well preserved. It shared a circular building in Square 3A (405). However, this building has a doorway open to the courtyard in $2 \mathrm{~A}$; therefore, it was accessible for the inhabitants of this compound alone. The other four buildings also had a doorway respectively facing the courtyard. A few clay bins are located in the south-western part of the courtyard (410). Hearths or fireplaces often recovered at the center of a courtyard in other compounds, have not been identified. This may be because of the incomplete excavations: the central part of the courtyard deposits has not been excavated to preserve a hearth of Level 3 (303).

The third compound of this level was revealed in
Square 2B (Fig. 3.19). It is located in the southwest corner of this square, whose excavation limits hide the southern and the eastern extensions. Curvilinear sidewalls join at least four circular buildings. The room at the northern end (418) showed a possible post-hole in the center of the floor (Fig. 3.20). The entrance to the courtyard of this compound should probably be located in the unexcavated square. This compound stands out in yielding a series of in situ discoveries from the courtyard. An oval hearth with a stone pavement is situated in the central part nearby, in which a complete pottery vessel standing on the stones was found (Fig. 3.21). The extensive black soot on the outer surface suggests heavy firing at this hearth, and two clay bins were among notable features. One is located near the west wall and is accompanied by a group of ground stones. The other is situated close to the building at the southeast end (421), where several clay sling missiles were discovered together. Other interesting artifacts found in situ include a large obsidian core (Chapter 9), recovered from an area near the northern building (418). An unfired clay figurine with elaborate

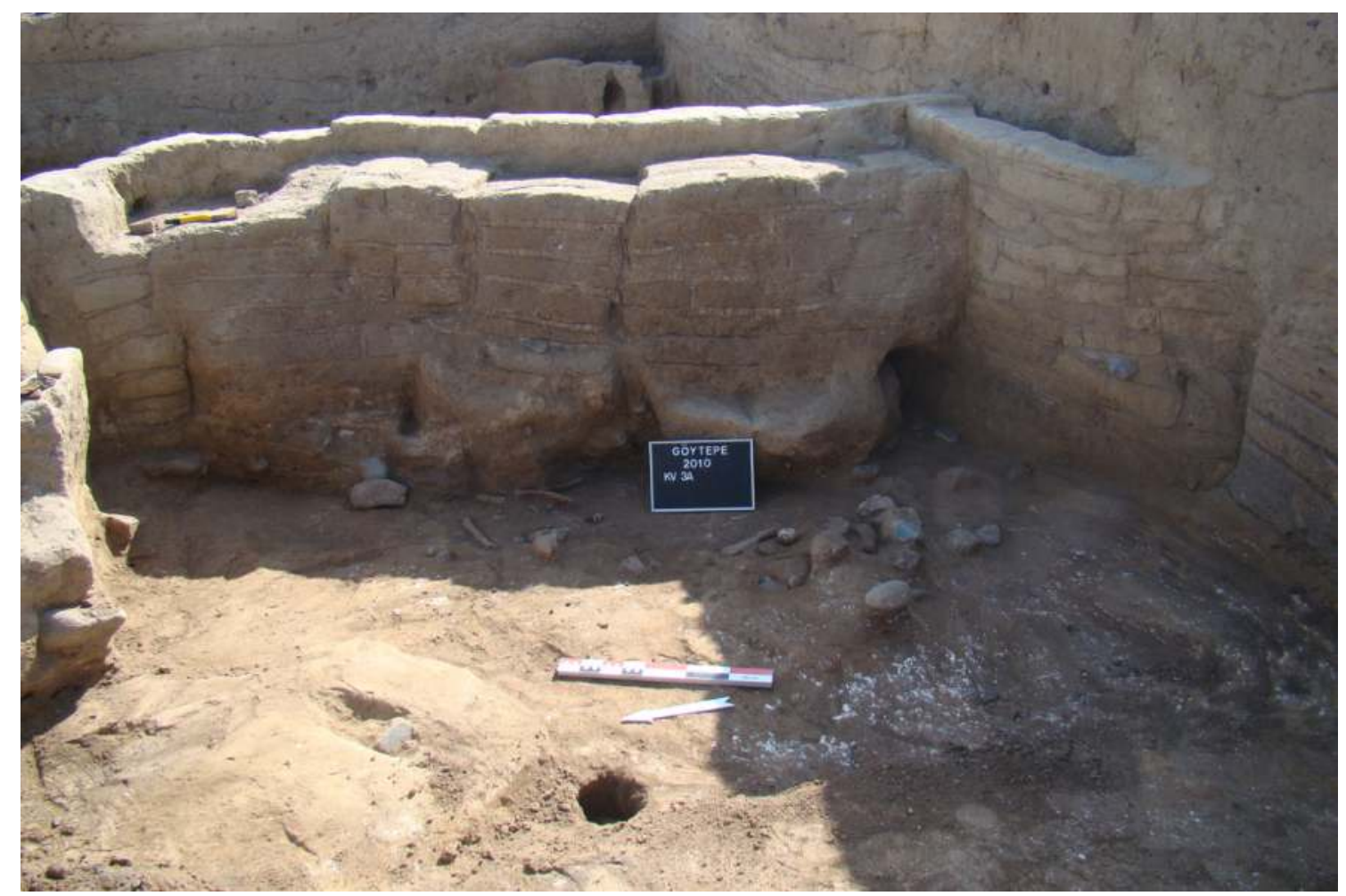

Fig. 3.17 Floor remains of the courtyard of the compound of Square 3A looking east. 


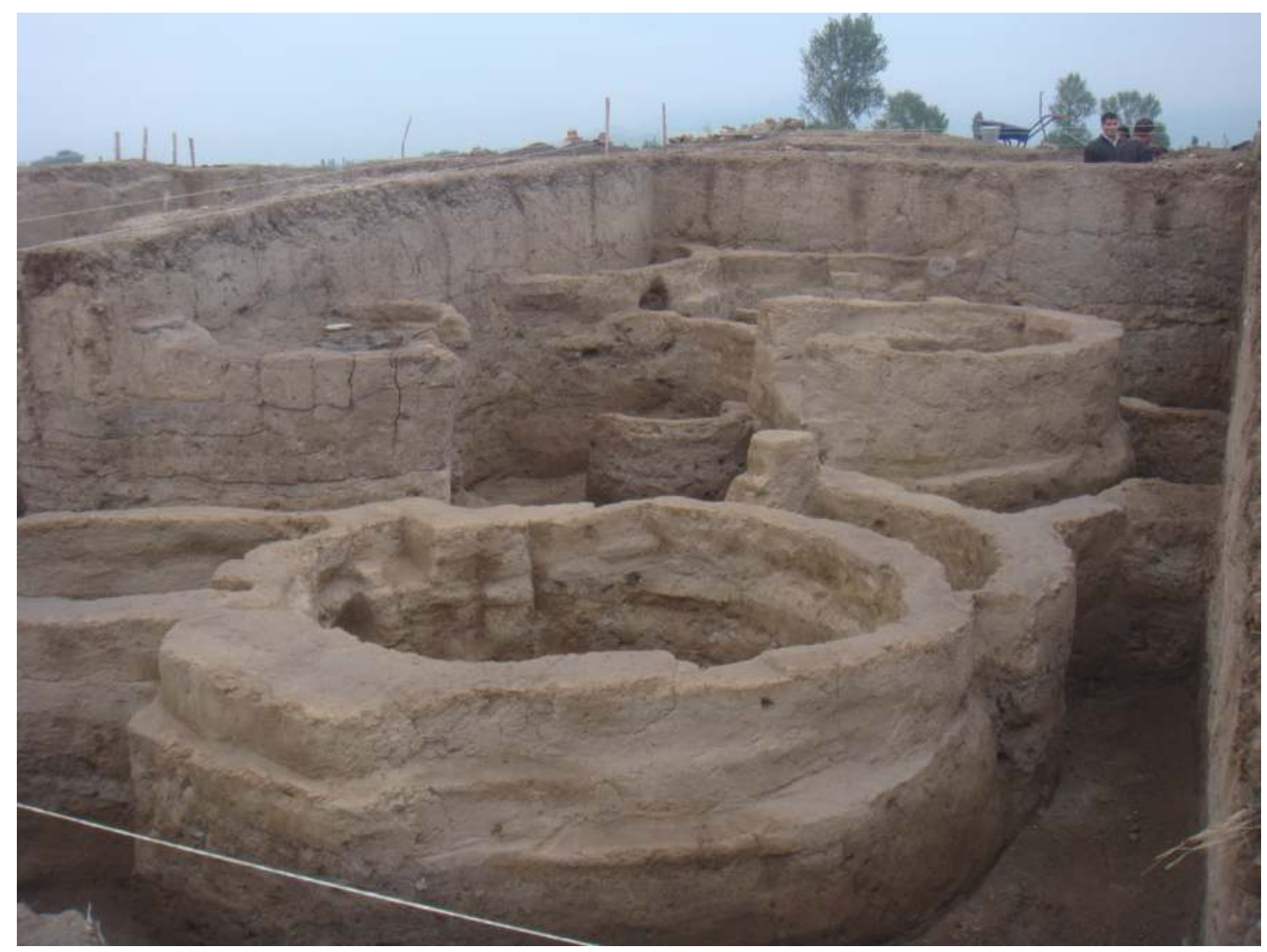

Fig. 3.18 General view of the compound of Square 2A looking southeast.

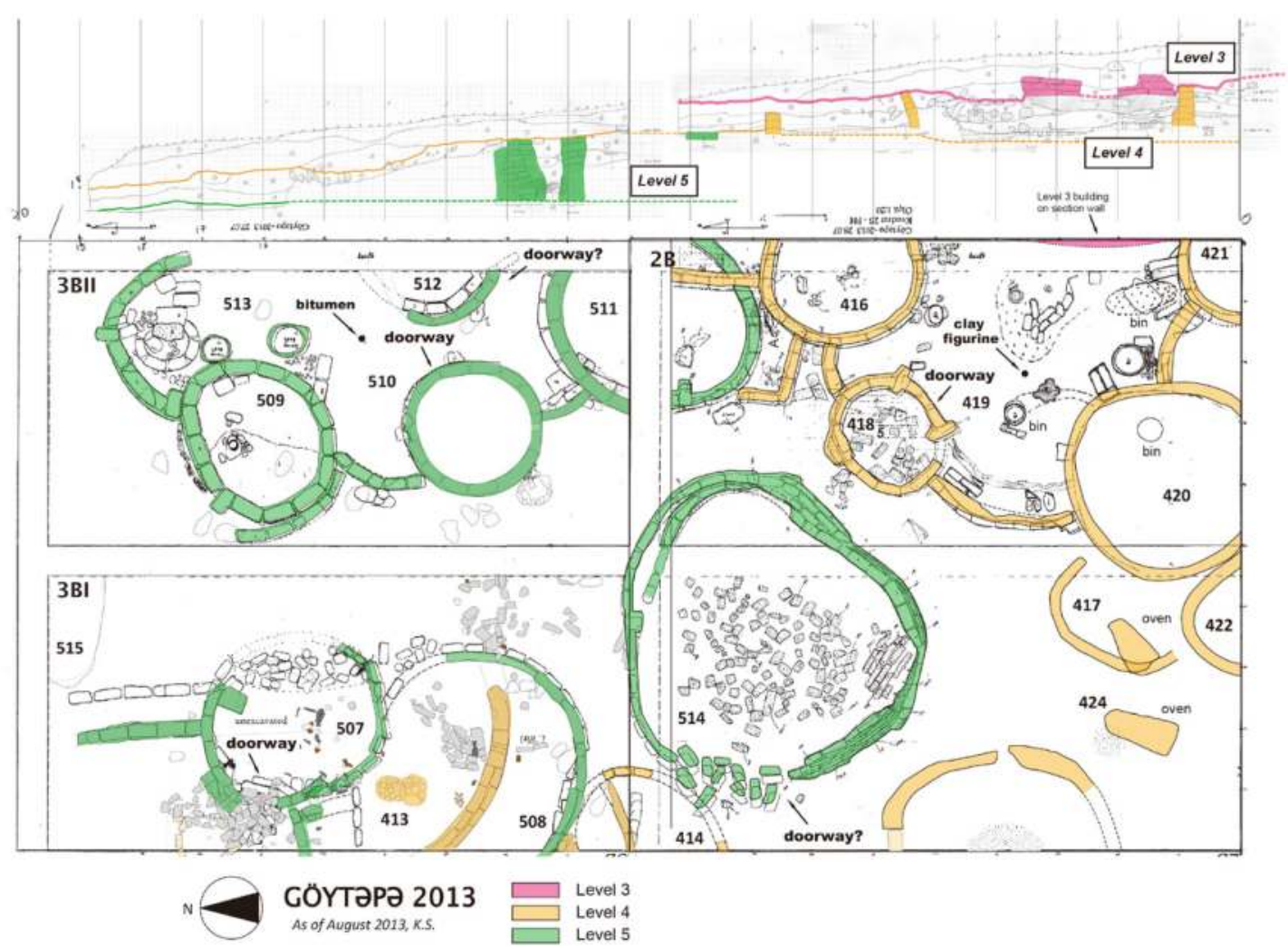

Fig. 3.19 Plan of the architectural remains from Squares 2B and 3B. 


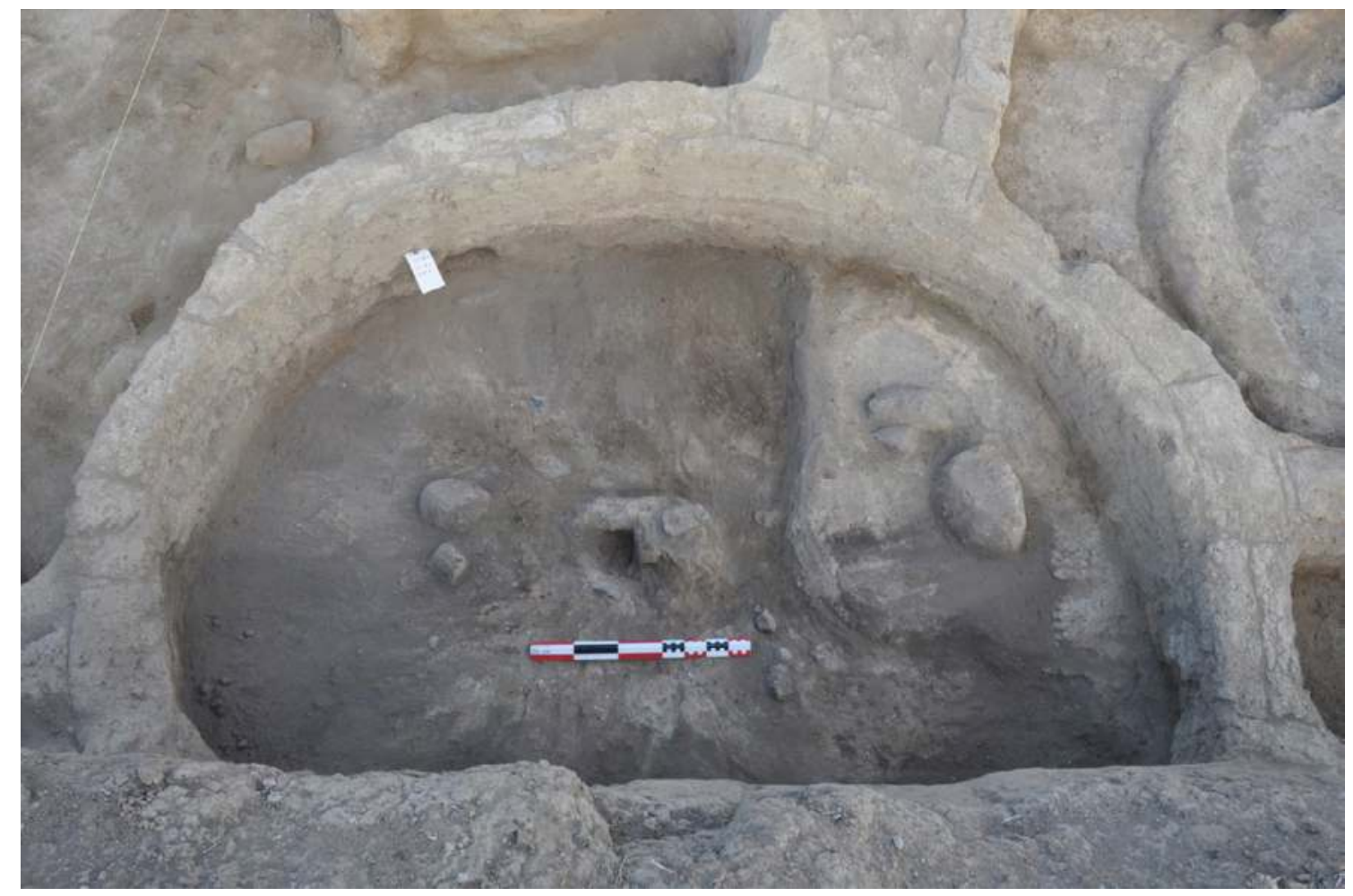

Fig. 3.20 Post-hole of Building 418 in Square 2BII looking west.

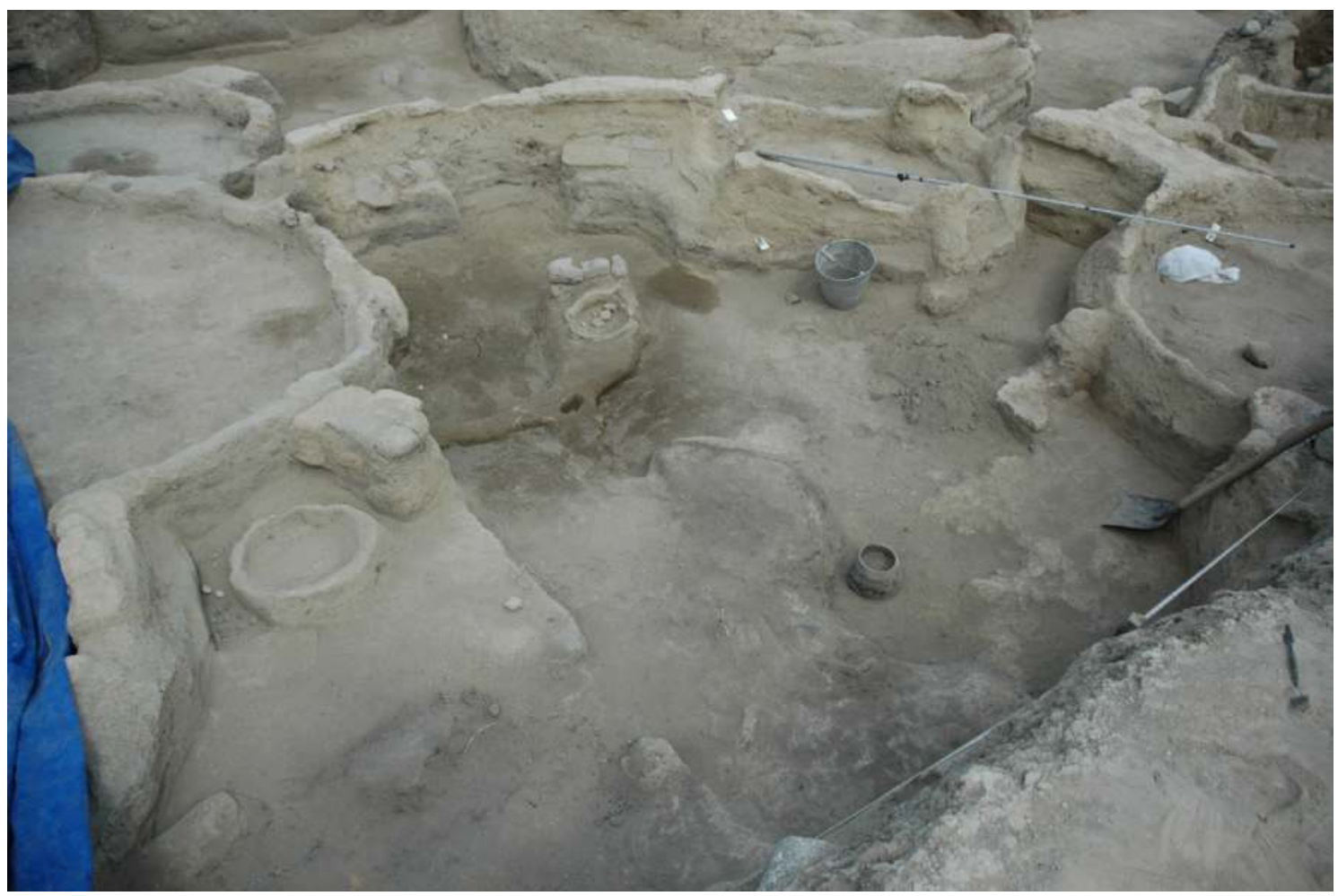

Fig. 3.21 General view of the compound of Square 2B looking northwest. 
punctate designs, $3 \mathrm{~cm}$ tall (Chapter 14), was found near the clay bin in the west.

Between the 2A and 2B compounds, several isolated mud-brick walls and structures were discovered. The remarkable discovery is a large circular building with a slightly deformed shape in Square 2B (514; Fig. 3.19). This building was originally constructed in Level 5 but was also used in Level 4. It is the largest building discovered at Göytepe to date, measuring about $4.5 \mathrm{~m}$ in diameter. The entrance to this building was located at the western wall, apparently closed with mud-bricks for an unknown purpose. Unconnected to any other buildings, this large building could have served for a communal use like an assembly house. However, the floor was covered with many collapsed mudbricks and has not been fully exposed to study the distribution of specific remains suggesting its use or

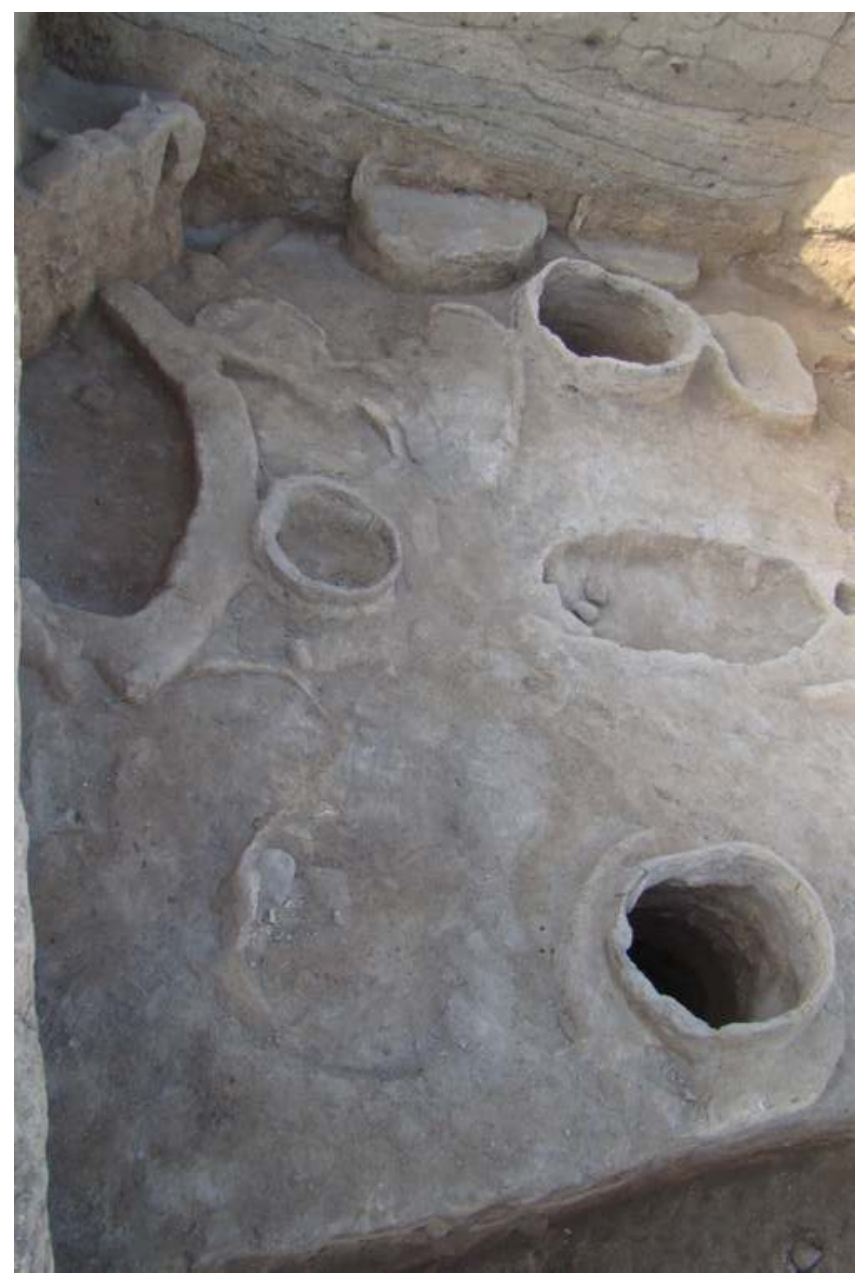

function.

Other notable finds from Level 4 included a concentration of clay bins in an area between the $3 \mathrm{~A}$ compound and fragmentary mud-brick walls in Square 3B (413 and 414). As in the courtyard of the 1A compound of Level 3 (305), the clay bins amounted to a dozen (Fig. 3.22). This area yielded another interesting find from the north part, which is a large obsidian core. Interestingly, it was found in an upright position on the ground (Fig. 3.23). Additionally, numerous obsidian flakes were recovered nearby, suggesting that obsidian tool production was conducted in this area.

\subsubsection{Level 5}

This level has been exposed mainly in Squares $3 \mathrm{~A}$ and $3 \mathrm{~B}$, and partly in $4 \mathrm{~A}$ and $2 \mathrm{~B}$, close to the northern edge of the mound (Fig. 3.2). However, due 


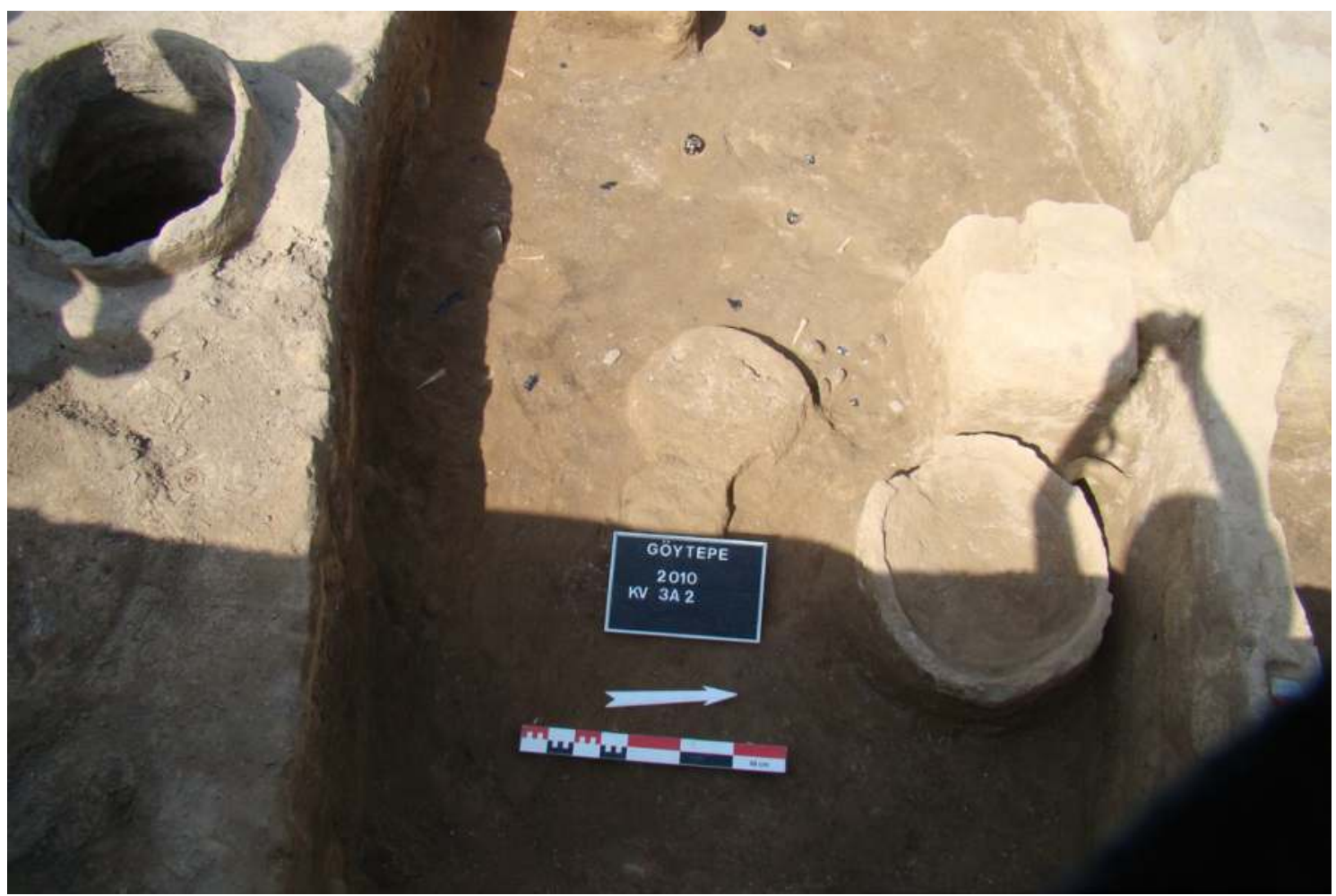

Fig. 3.23 Distribution of obsidian artifacts near clay bins (423) in Square 3All looking west. Note that the large core was recovered standing with its platform top (top center).

to the incomplete excavations, much more work is needed to obtain more precise data on the structures and their spatial organization. Structures particularly challenging to interpret are those in $4 \mathrm{~A}, 3 \mathrm{~A}$, and 3BI. Several circular buildings, some of which were connected with mud-brick walls, have been noted, and a series of clay bins were discovered along a curvilinear mud-brick sidewall (504; Fig. 3.24). We also note a hearth made with mud-bricks (501) in an open space of Square 4A. A large ground stone (502) is situated nearby (Fig. 3.25).

The relatively well-defined architectural complex was found in Square 3BII, where a household compound consisting of at least four circular buildings encloses an oval courtyard (Fig. 3.19). The building to the south (511) is slightly larger than the others and is approximately $3 \mathrm{~m}$ in diameter. Important finds from this compound include two ground stones from the floor of the northwestern circular building (509) and a group of unworked scapulae from the room in its south (510; Fig. 3.26). The open area in their east was associated with two clay bins and a hearth encircled with mud-bricks (513). Numerous ground stones were found nearby. These features suggest that the northern part of this compound was used for cooking. A lump of bitumen was found in the middle of this space.

Other remarkable structures include a large circular building in Square 2B (514; Figs. 3.2 and 3.19). It was a long-lived construction that has been continuously used in Level 4, as mentioned earlier. A similar structure was partially excavated in 3BI (508). Although it is slightly smaller than Building 514, about $4 \mathrm{~m}$ in diameter, and the intramural deposits are to be excavated, the 508 circular building might also represent some kind of a public character. The building of 507, though also only partially excavated, represents another remarkable construction because of the rich objects discovered on the floor (Fig. 3.27). Abundant stone and bone artifacts were recovered near a stone-paved hearth.

From a stratigraphic perspective, the presence of a pit at the northern end of Square 3BI (515) is worth mentioning. This pit continues to distribute toward the north in Square 4B, bridging the stratigraphies of the Upper and Sounding Areas. Thanks to this pit, the latest level of the latter area, to be described in Chapter 4, was determined as Level 5. 


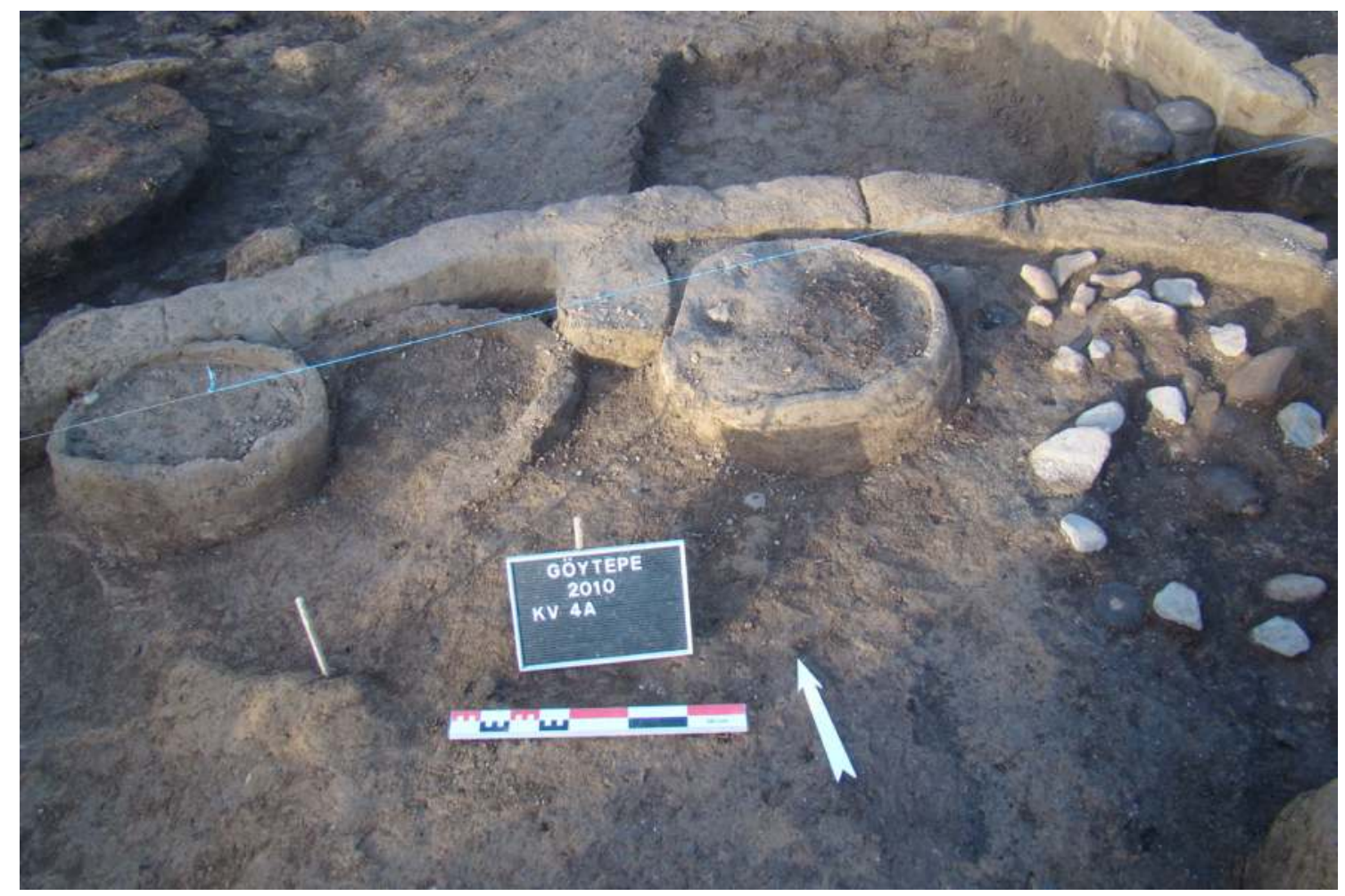

Fig. 3.24 Concentration of clay bins (504) in Square 4A looking north.

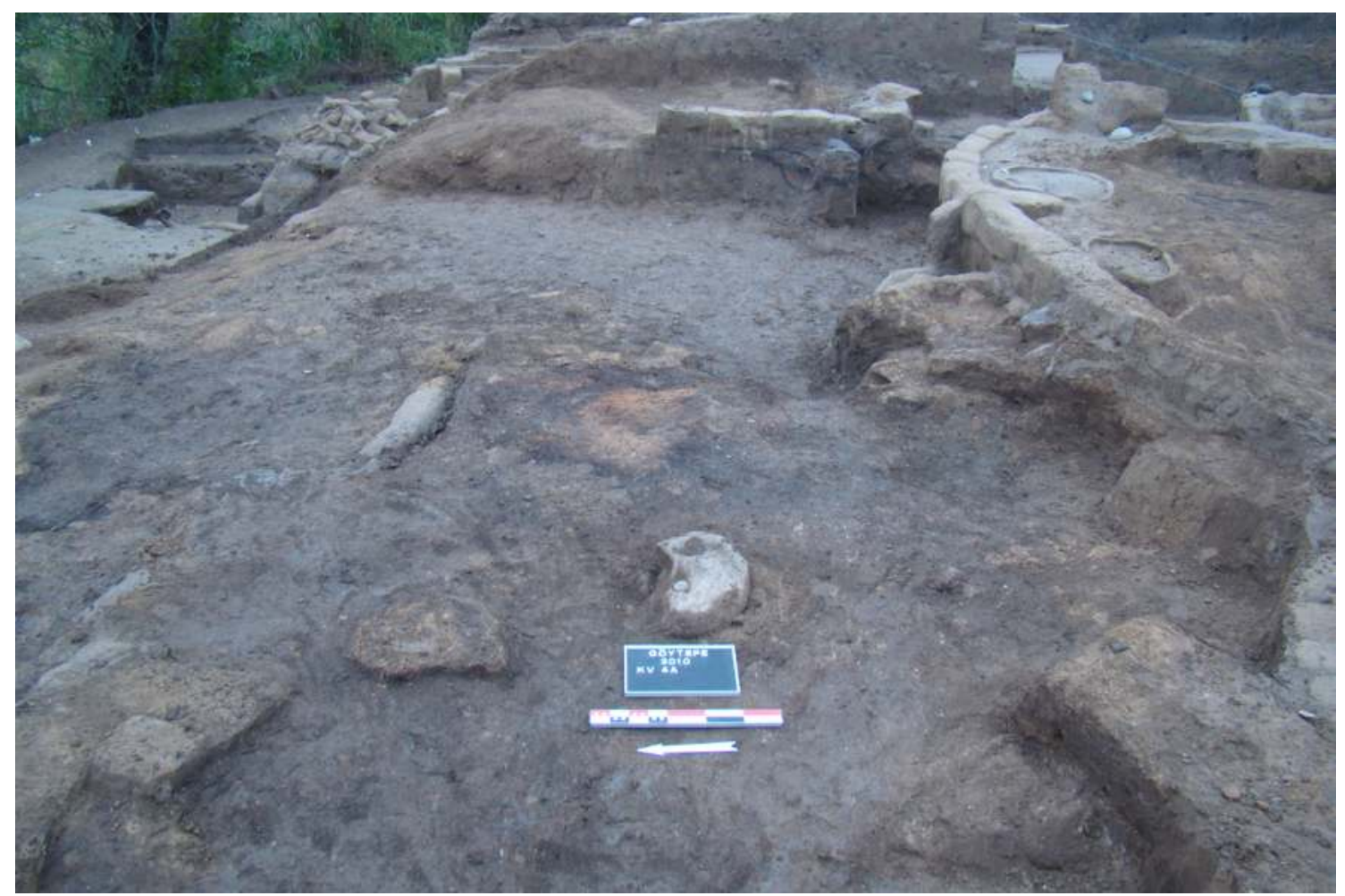

Fig. 3.25 Hearth (501) and a ground stone (502) from Square 4A1 looking east. 


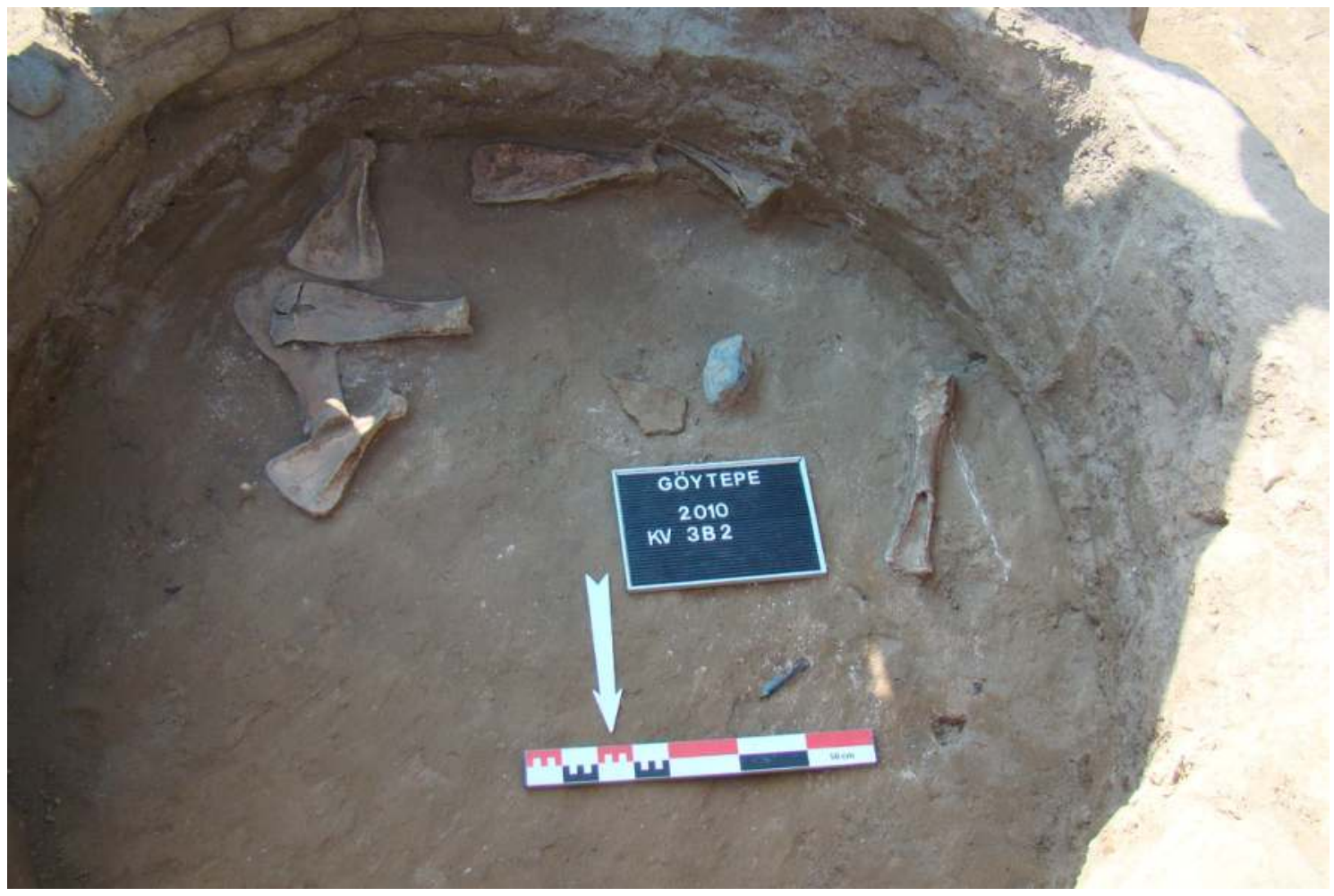

Fig. 3.26 Concentration of cattle scapulae on the room floor (510) in Square 3BII looking south.

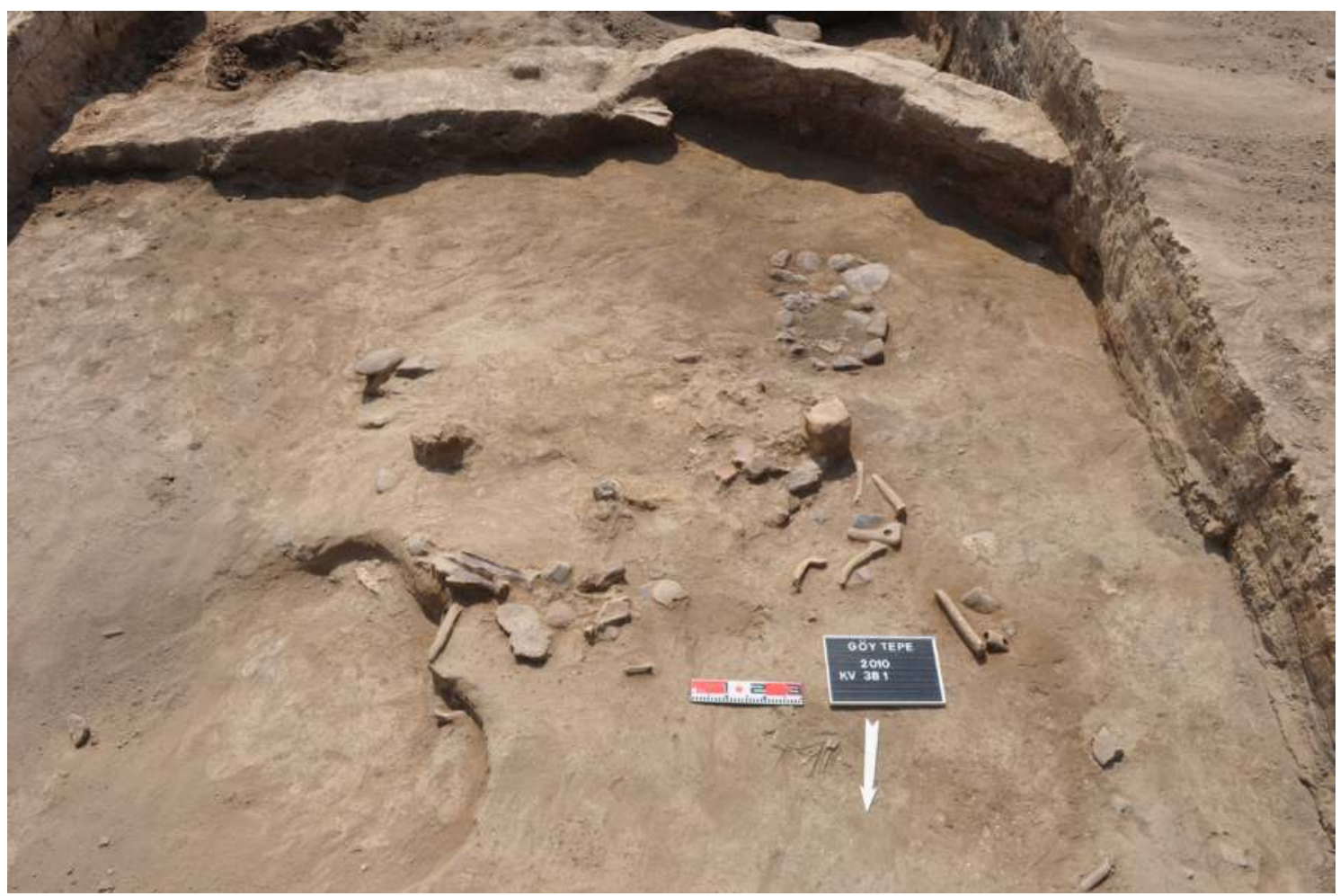

Fig. 3.27 Concentration of stone and bone objects in Square 3BI (507) looking south. 


\subsubsection{Level 6}

Regarding this level, only the small portions of the architectural remains have been exposed. They are located mainly in Square 4A (Fig. 3.2). Due to the slope erosion along the northern edge of the mound, this level had been exposed close to the surface. Two circular buildings with fragmented sidewalls and one isolated curvilinear wall, which may have comprised part of a large building or a wing-wall, have been identified. One of the buildings in Square 4AII represents the western half of a Level 6 building in 4B (Chapter 4). It served as another key to connect the stratigraphies of the Upper and Sounding areas.

\subsection{Discussion}

\subsubsection{Architectural techniques}

Similar to other Shomutepe settlements, the most characteristic architectural form of Göytepe was a circular mud-brick walled construction (Narimanov 1987; Azimov 2006). Semi-subterranean buildings, known at several sites including Shomutepe and Hacı Elamxanlitepe (Baudouin 2019), have not been recovered in Levels 1 to 6 of Göytepe.

The walls were made of sun-dried mud-bricks with plant temper. The literature indicates the use of cob (packed mud) at related settlements in the Araxes Valley (Badalyan et al. 2010; Baudouin 2019). However, cob walls were never popular at Göytepe, and their use was principally limited to constructing foundations. While most of the mud-brick walls were laid directly on the ground for architecture, some occasionally show clay cob foundations (Fig. 3.28). Stone foundations may also have been employed, but not commonly (see Baudouin 2019).

Mud-bricks of the Shomutepe culture are known to have a rectangular shape with a planoconvex section: the bottom is flat, whereas the top is convexed. Examples of this type, like those described at Aruchlo (Hansen and Ullrich 2017), have also been identified at Göytepe. The precise size and shape of mud-bricks can only be determined by removing them from walls. This has not been accomplished due to our particular excavation technique. However, some isolated mud-bricks were found from collapsed walls, like those found in a large circular building of Level 5 (Fig. 3.19). According to their measurements, the most popular ones in Levels 1-6 of Göytepe are approximately $40 \times 20 \mathrm{~cm}$, followed by those of $48 \times$ $20 \mathrm{~cm}$. Their size match well with the data reported from Shomutepe (Narimanov 1987). Nevertheless, the size of mud-bricks might have varied by period, architectural type, and others (see Chapter 6).

The colors of mud-bricks at Göytepe consist of two-colored ones: gray and yellow. As far as the data from Levels 1-6 are concerned, there is no indication for the patterned use for these two different colored mud-bricks, either in a diachronic or functional way. There are examples using both types of mudbricks, even for the same buildings (Fig. 3.29). Our observations at trenches at the periphery (Chapter 5), which penetrated the virgin soil, suggest that the color of mud-bricks depends on the source of clay in the building areas rather than a cultural choice. The virgin soil of Göytepe consists of yellowish loess; therefore, mud-bricks made of clay taken from the loess deposits naturally become yellow. Conversely, mud-bricks manufactured with clay or soil taken from the secondary deposits of the mound, which constituted greyish brown sediments containing ash and old mud-brick rubble, show a greyish brown color. Indeed, all of the walls recovered at the southern edge of the mound, which belong to the earliest occupational levels on virgin soil with a deep pit dug into the loess deposits, showed a yellow color (Chapter 5).

Mud-bricks were bonded with fine clay as a binding material. They usually show a stretcher bonding longitudinally, except for a couple of special constructions as discovered at the peripheries of the settlement (Chapters 4 and 6). While the bottom parts of the walls were occasionally constructed in two or three rows, the row is one in principle. Accordingly, the walls of the Göytepe architecture are quite narrow in comparison with those of the Upper Mesopotamian settlements in the same period (Nishiaki et al. 2018). The exterior and interior surfaces were plastered with fine clay. The thickness varies from a few millimeters to more than $2 \mathrm{~cm}$.

The circular buildings of the Shomutepe culture may be interpreted to have a domed roof made of mud-bricks, similar to those of the Halaf culture in Upper Mesopotamia. This possibility cannot be entirely ruled out, but the discovery of a few post- 


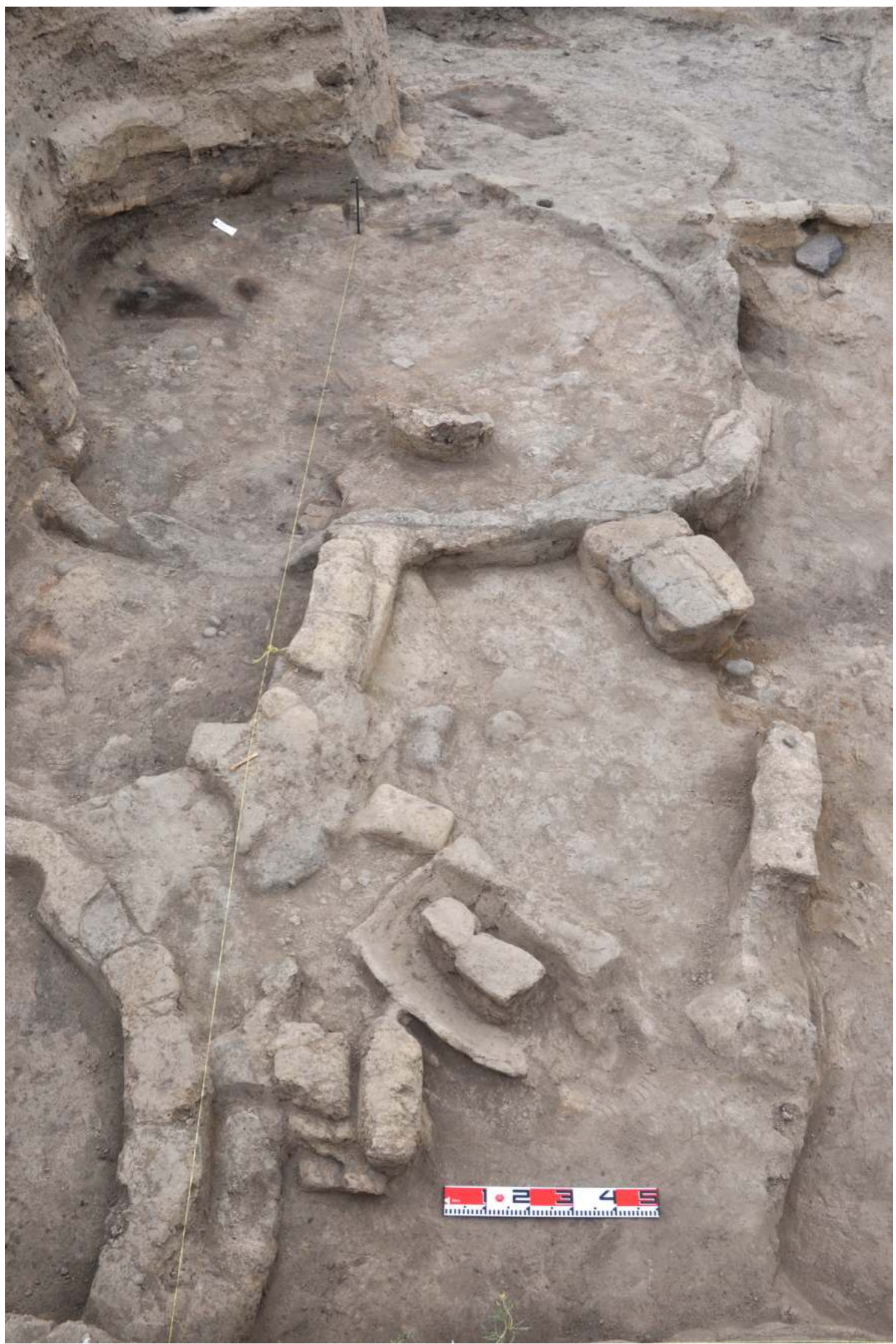

Fig. 3.28 Wall foundation made of clay cob for Level 2 buildings in 1BI looking east. 


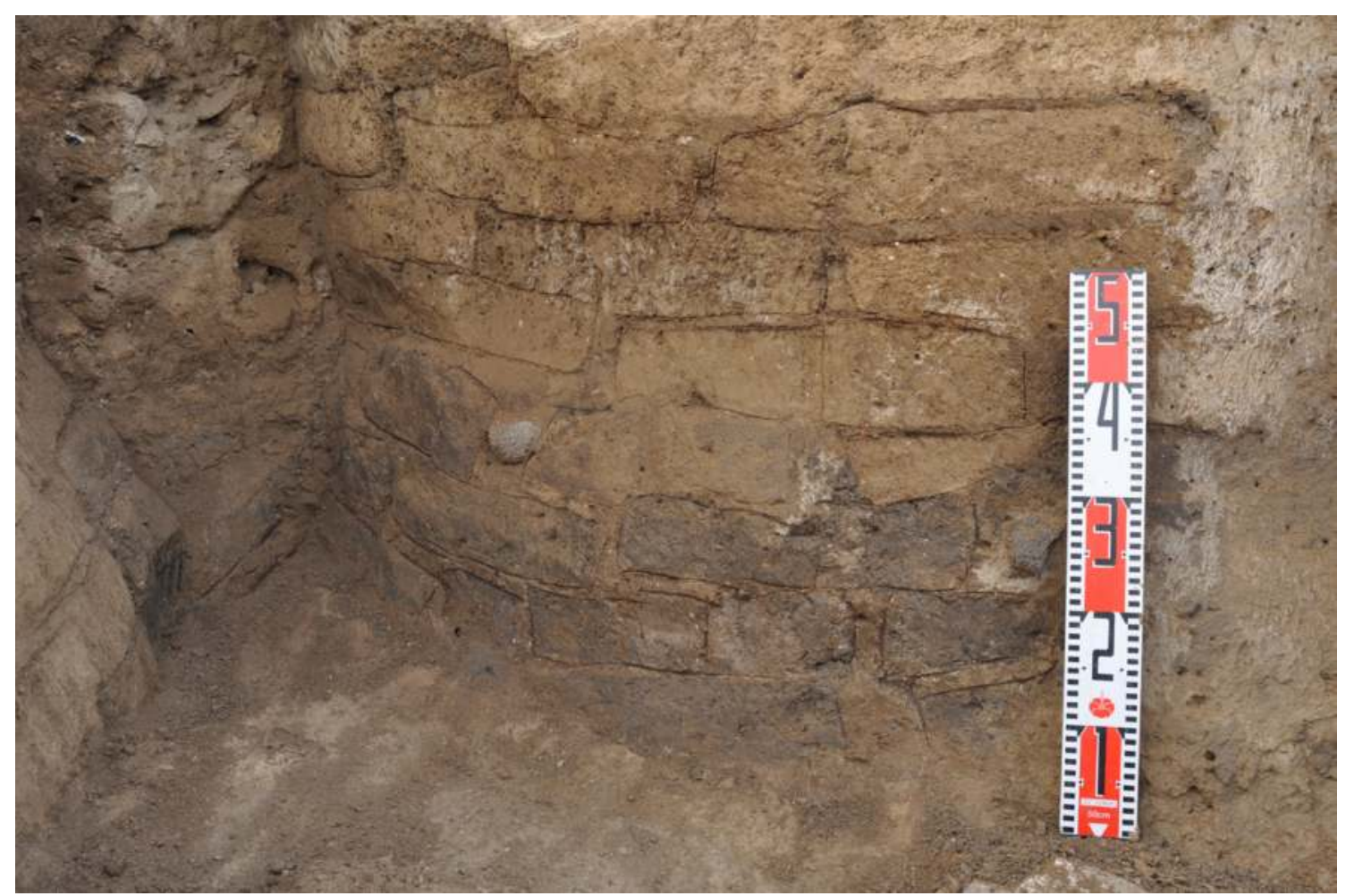

Fig. 3.29 Wall of Building 511 of Level 5 showing the use of mud-bricks with different colors. Yellowishbrown mud-bricks were laid above dark grey ones. Looking southeast.

holes at Göytepe, resembling those reported from Shomutepe (Narimanov 1987: 15), demonstrates the existence of buildings with a roof made by other techniques like thatching supported by a central post (Munchaev 1982: 141). A few stone cobbles, which may have supported the wooden post, were found near the hole. The relatively thin walls, made of only one course of narrow mud-bricks for all the structures of the Shomutepe culture, would not have been robust enough to support a domed roof made of mud-bricks as in the Halafian culture. It is particularly the case for larger buildings with a diameter of more than 3 $\mathrm{m}$. The Halafian architecture had much thicker walls, often with a stone foundation at the base (Mallowan and Rose 1935), features that are never seen in Shomutepe architecture. Simultaneously, we should note that not every circular building necessarily retains traces of a post-hole. This may indicate the use of a variety of construction methods to support the roof of the Neolithic buildings of Göytepe and a difficulty in fieldwork. Smaller buildings may have been constructed in a dome shape. For example, it may also have been possible to place a post without leaving archaeological records on the floor.

The doorways of circular buildings are relatively small, approximately 60 to $80 \mathrm{~cm}$ wide (Figs. 3.16 and 3.30). The typical type is characterized by a pair of buttresses at both sides of the entrance, accompanied by a threshold also made of mud-bricks. Some of these doorways probably had an actual door. Although such evidence has not been identified in the Upper Area, one building in the Sounding Area revealed a post-hole for the door (see Chapter 4; Figs. 4.17 and 4.18). Another type of doorways was also present. It was like a window, open on the wall higher than the ground surface. Some of those doorways were open about $40 \mathrm{~cm}$ or higher from the ground (Fig. 3.30: 4). A comparable doorway constructed $25 \mathrm{~cm}$ above the ground has been reported from Shomutepe (Narimanov 1987: 15). The use of such techniques may help us interpret why doorways have not always been identified at all buildings, particularly at those only remaining at the base. It is also worth mentioning that some of the doorways were found to be entirely closed with either mud-bricks (Fig. 3.31) or a combination of mud-bricks and cob (Fig. 3.32). These doorways were regarded intentionally closed at the time of abandonment. The common occurrences of such examples suggest abandonment of the buildings in anticipation of returning (Nishiaki 


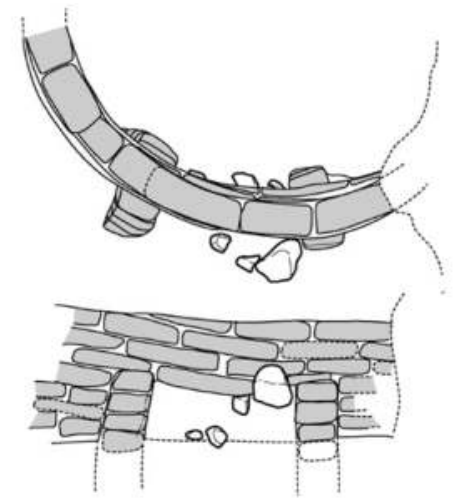

1

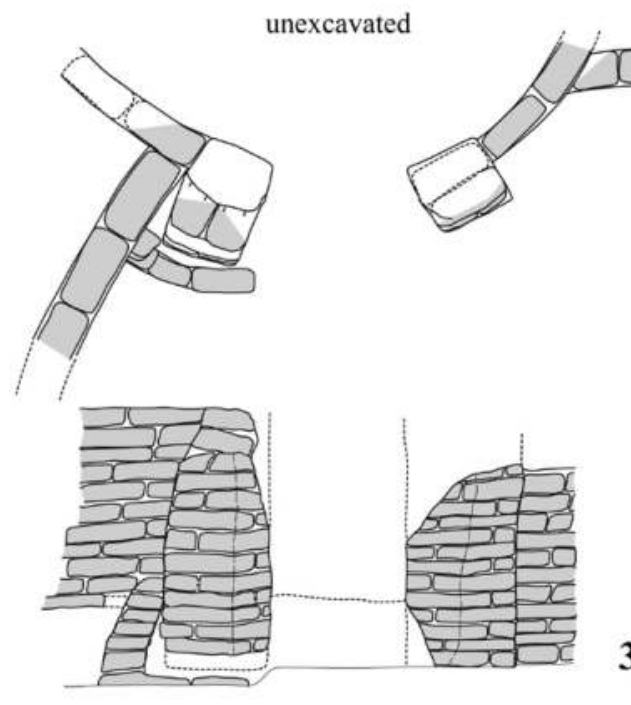

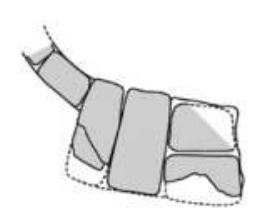
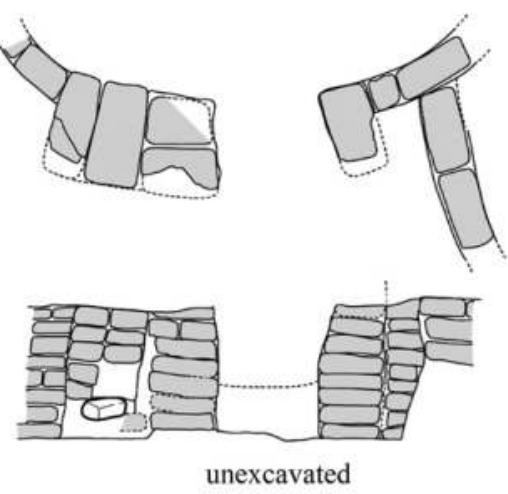

2
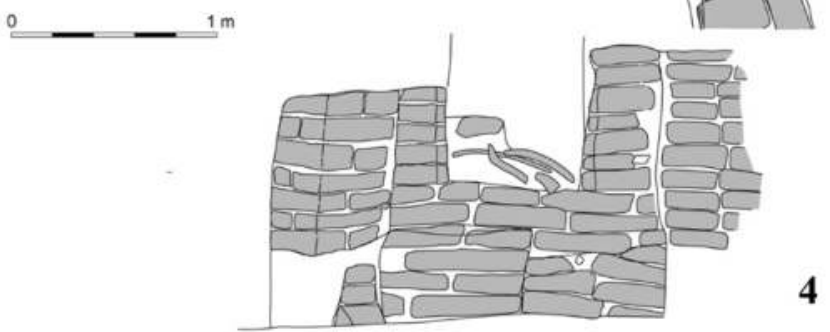

Fig. 3.30 Examples of doorways. 1: Upper part, Building 408 of Level 4, looking northwest; 2: Doorway, upper part, Building 415 of Level 4 looking west; 3: Doorway with a low threshold, Building 404 of Level 4; 4: Lower part, window type, Building 401 of Level 4.

et al. 2018). This interpretation agrees with the frequent abandonment of still-usable objects; hence de facto refuses (Schiffer 2010).

\subsubsection{Small structures}

In addition to the larger buildings, the construction of smaller structures was also common at this settlement, such as clay bins, which had a round or oblong form, with a diameter of 50-60 $\mathrm{cm}$ and a height of about $50 \mathrm{~cm}$. The bottom of these bins is often buried approximately $10-15 \mathrm{~cm}$ below the ground; therefore, they are better described as semisubterranean bins. Some were found to contain complete bone and stone tools, suggesting their function as a storage of precious tools for future use, and some were closed with mud-bricks. Our geochemical analysis has demonstrated that at least some of the bins stored cereals (Kadowaki et al. 2015; Chapter 7).
Further, fireplaces comprise a major feature of the Göytepe records. They can be divided into two types. The more conspicuous are hearths with a rivercobble pavement. The cobbles are neatly arranged in either a round (Fig. 3.8) or an oblong shape (Figs. 3.9 and 3.10). Typically, larger stones are laid in the center, and smaller ones at the edges. Their size varied, and the largest hearth of a round shape is about $80 \mathrm{~cm}$ in diameter, and that of an oblong one was $40 \mathrm{~cm}$ by $80 \mathrm{~cm}$. There were fireplaces made without using stones. Some seem to have been constructed with mud-bricks (Fig. 3.14), as reported at Shomutepe (Narimanov 1987: 16). The burnt stones with reddish colors, charred black soot, and burnt soil indicate the heavy use of firing. Given the discovery of pottery vessels in or nearby (Figs. 3.10 and 3.21), it is likely that at least some of those hearths were used for cooking. The function of the variety of firing facilities would constitute a good 


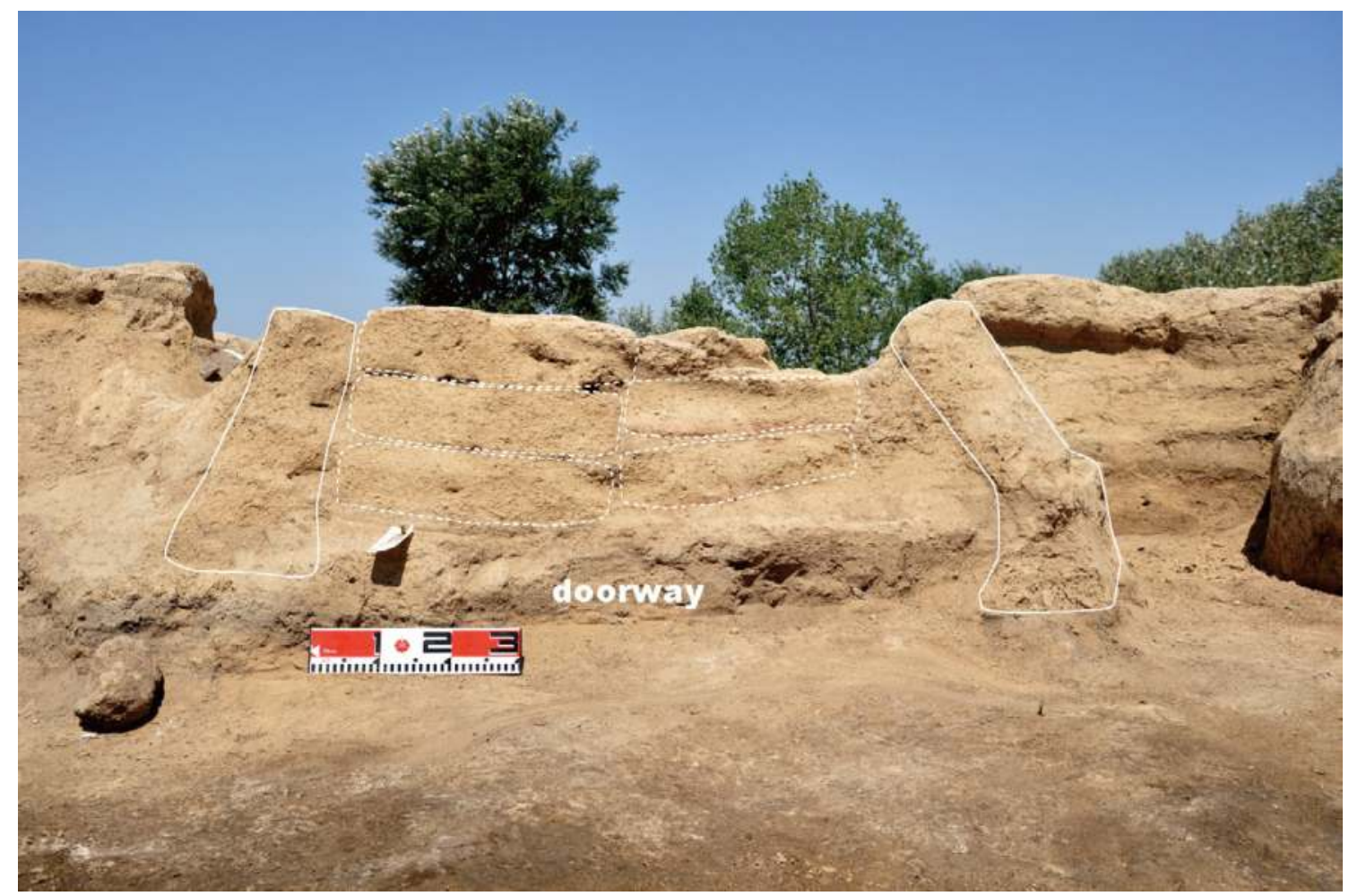

Fig. 3.31 Doorway closed with mud-bricks, Building 419 of Level 4, looking northwest.

subject for future archaeometric studies. Meanwhile, it is worthwhile stressing that the clay structures so far discovered at Göytepe do not include any oven of the tannor type, widely known at contemporaneous settlements of the 6th millennium BC in the Fertile Crescent of Southwest Asia.

\subsubsection{Settlement organization}

The large-scale excavations in the Upper Area revealed numerous mud-brick buildings and walls, whose distribution patterns help us reconstruct the settlement organizations at the Shomutepe culture settlement of Göytepe.

The settlement layout appears to be complicated, consisting of numerous small and large circular buildings, sidewalls, and other structures (Fig. 3.2). This is likely a result of repeated constructions and additions during the occupations. Careful examination revealed that the basic architectural unit is what we termed a "household compound," defined as a spatial unit delineated by four to six circular buildings connected with sidewalls, which functioned as enclosure walls, in a round to oblong shape of 6 to $8 \mathrm{~m}$ by 4 to $5 \mathrm{~m}$. Each compound had one break on the enclosure wall, serving the entrance to the courtyard. A schematic reconstruction of the compound discovered in Square 2A (Level 4) is shown in Fig. 3.33. The compound comprises a combination and larger and smaller circular buildings, with doorways facing the courtyard, which accommodated clay bins and hearths.

The size of the circular buildings may reflect their functions. In the Upper Area of Göytepe, they varied from $1.5 \mathrm{~m}$ to more than $4 \mathrm{~m}$ in diameter. According to Narimanov (1987), the excavator of the settlement of Shomutepe, the buildings of the Shomutepe culture, were of two types: small and large, and the smaller ones might have been used for non-dwelling purposes such as storage, while the larger ones were for dwelling. To test this empirical interpretation, we compared the diameters of the circular buildings in the Upper Area. The results are shown in Fig. 3.34, which indicates a unimodal distribution without showing large and small clusters. Most common buildings have a diameter of 1.5-2.0 m. Larger buildings were present, but do not show a particular cluster in size. This result suggests that the differentiated function of each circular building, which must have existed, cannot be determined by their size only. Instead, a wide range of evidence, especially the information of in situ floor remains, needs to be analyzed for this purpose. Our preliminary observations show 


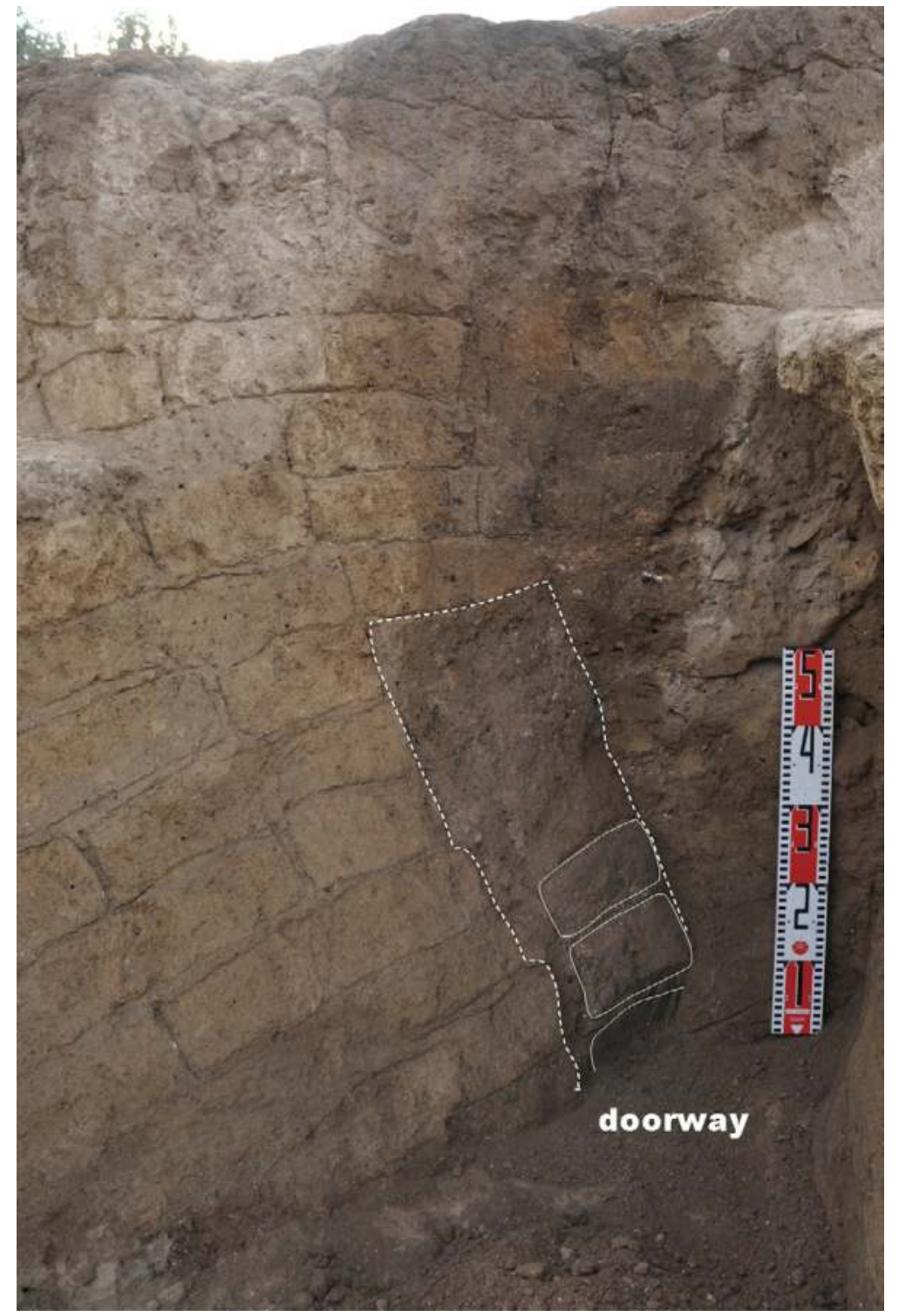

Fig. 3.32 Doorway closed with mud-bricks and clay, Building 512 of Level 5, looking west. that tools and other objects were rarely found in the interiors of the smaller circular constructions, while many are from the larger circular constructions. Such patterns certainly deserve intensive analysis in the future. Meanwhile, the courtyard of each compound has produced numerous findings in situ on the floors, which point to its use for the major daily activities of the inhabitants. Domestic features, such as bins and ovens, and utensils such as ground stone tools and pottery vessels, were discovered in the courtyard, emphasizing the importance of the courtyard for domestic activities.

The Neolithic settlement of Göytepe is formed with numerous such compounds. They were sequentially built one by one, often using buildings constructed in the previous level (Fig. 3.35). This fact indicates that a new building was built while the previous ones were still standing. A remarkable occupational continuity and rapid house construction cycles at this mound are evident. By extension, this suggests strong social ties maintained by the inhabitants of these compounds over generations.

In our view, each compound represents a residential unit for a household. Indeed, the artifacts and other remains recovered indicate a domestic character in nature, rather than religious, cultic, and/ or communal. However, exceptional buildings have been recovered. Two buildings are noted: 514 of Levels 4/5 and 508 of level 5, located in Squares 2B to 3B (Figs. 3.2 and 3.19). While the latter is still under excavation, the former (514) shows an exceptionally large size, measuring up to $4.5 \mathrm{~m}$ in diameter (Fig. 


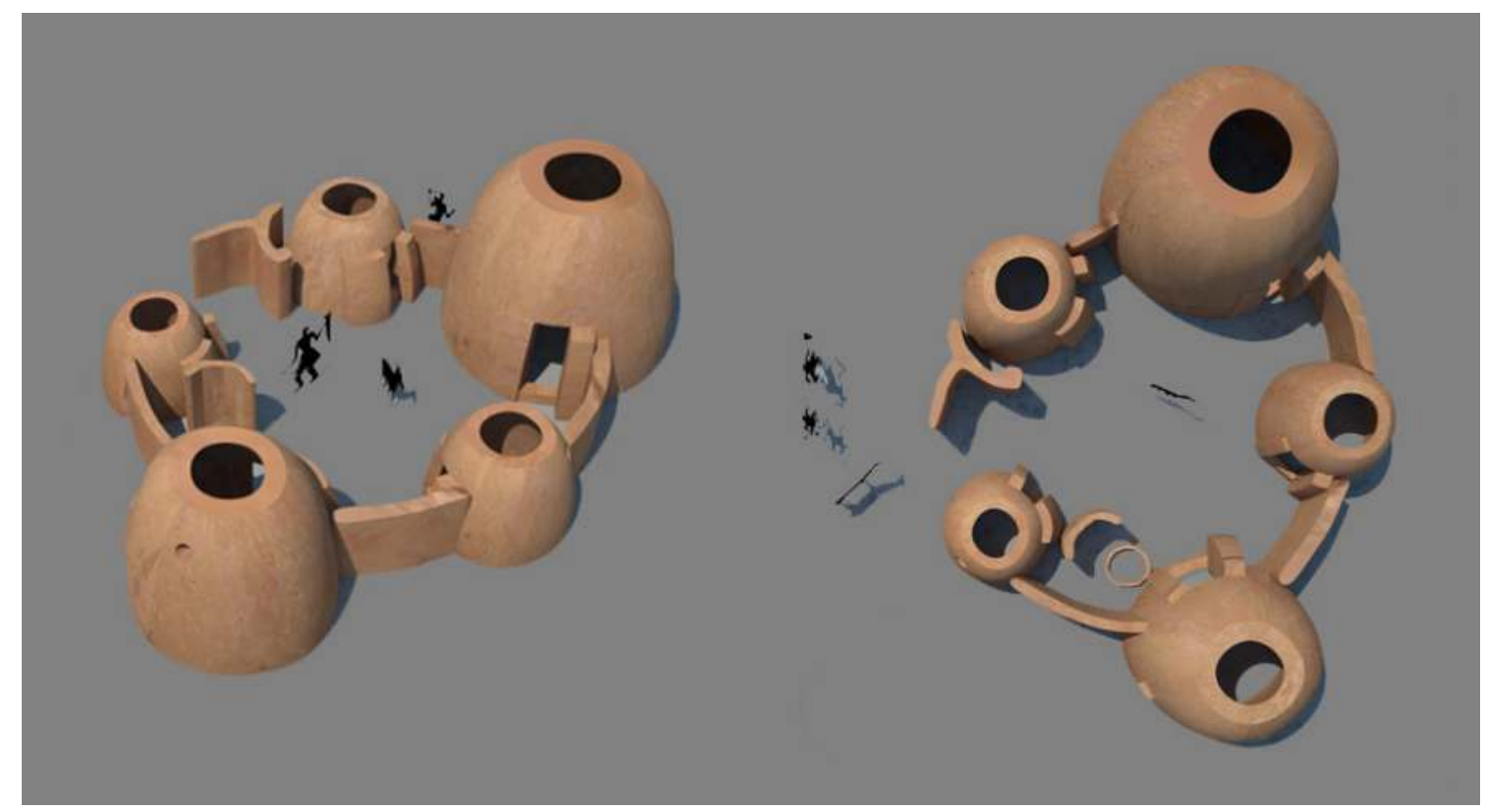

Fig. 3.33 Schematic reconstruction of a Level 4 compound.

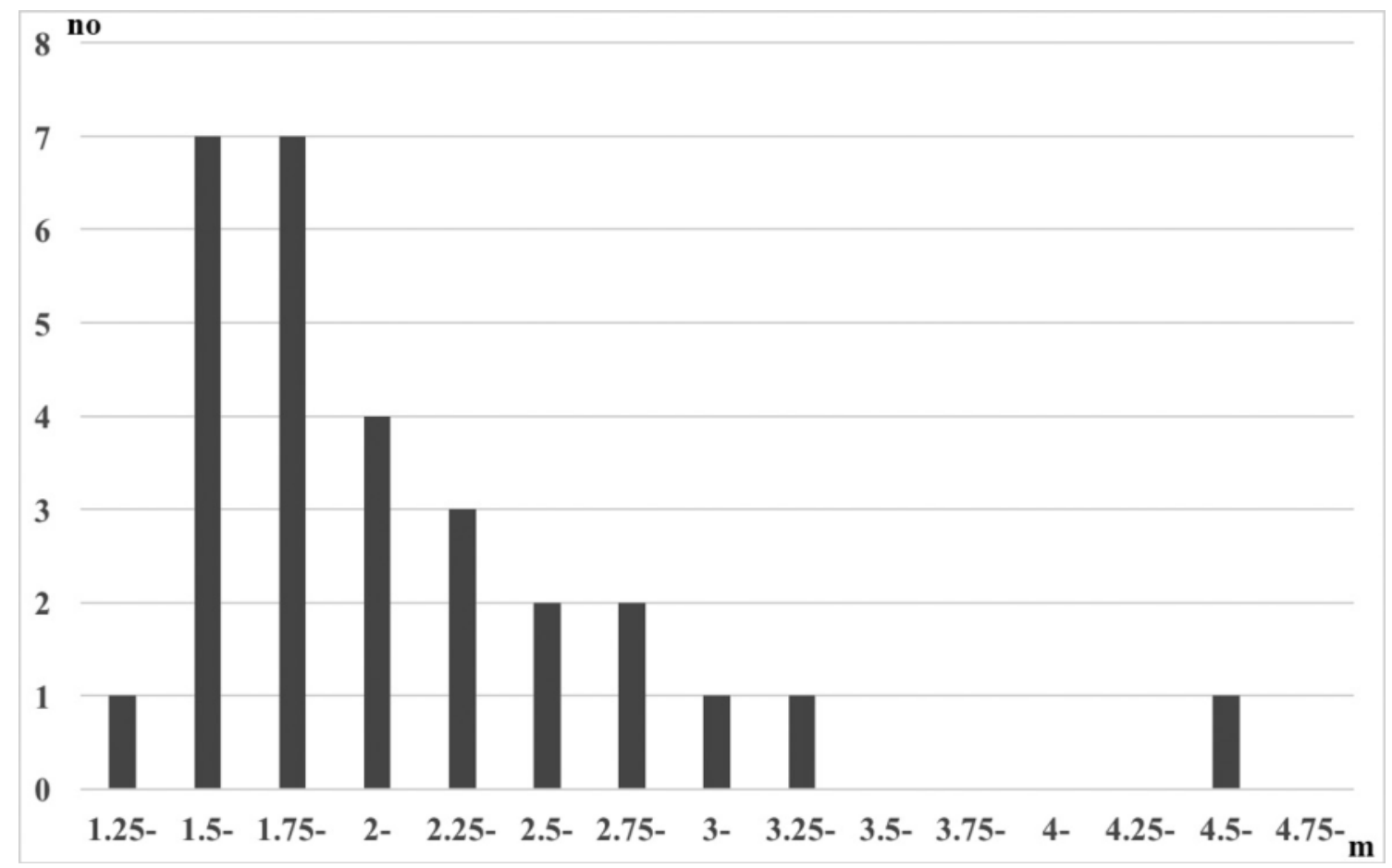

Fig. 3.34 Inner diameters of the circular buildings from Levels 1-6 of Göytepe.

3.34). Moreover, it is not connected to other circular buildings with sidewalls. Furthermore, it seems to be encircled with plenty of household compounds that consist of much smaller buildings (Fig. 3.2). These observations suggest a distinct function of this large building, and our current interpretation is that this had a communal use. At the present stage of research, its floor remains have not been recovered. Future excavations will elucidate this suggestion. 


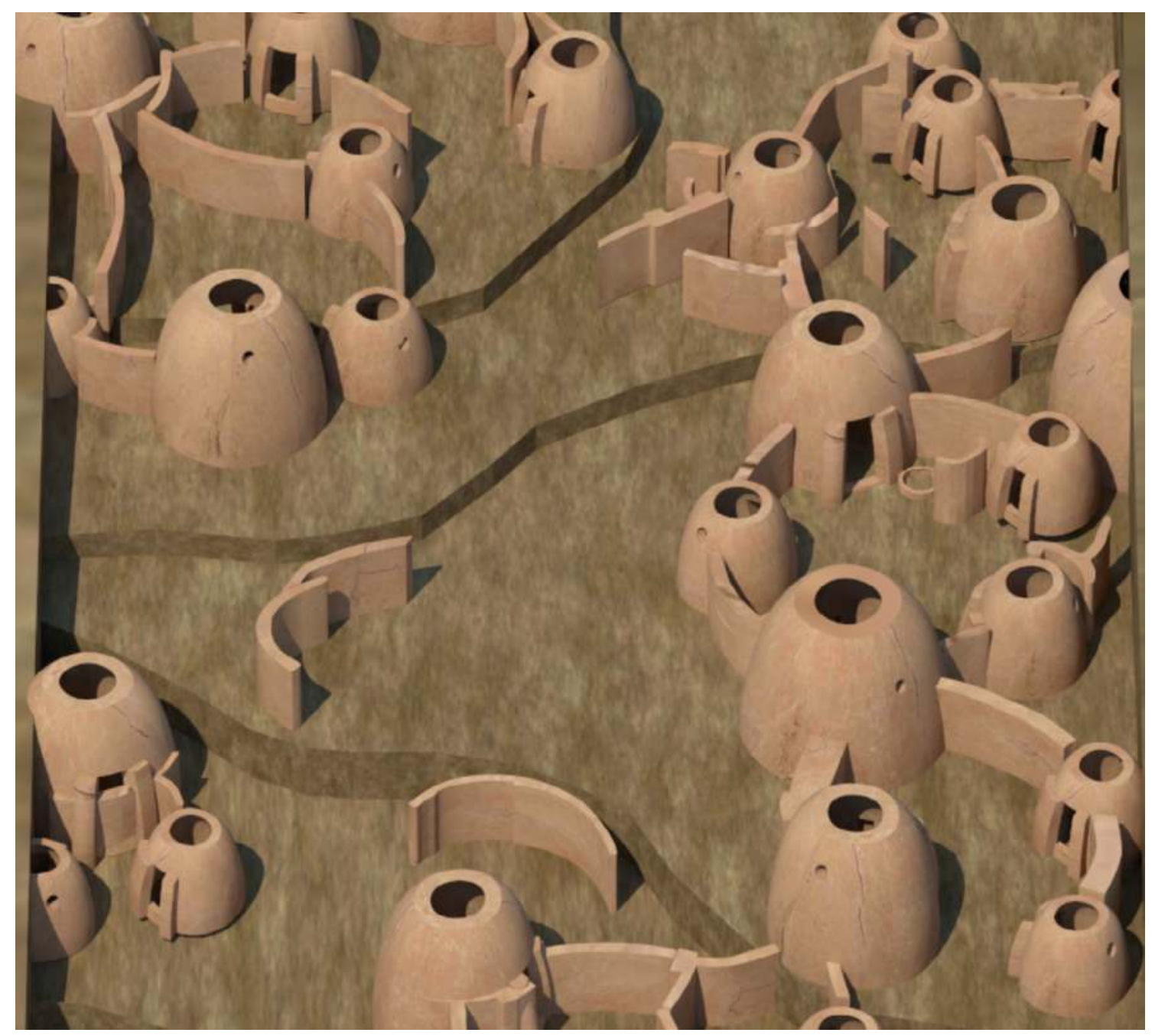

Fig. 3.35 Schematic reconstruction showing repeated construction of the household compounds.

\subsection{Conclusions}

The excavations in the Upper Area of Göytepe produced rich architectural remains of the mid 6th millennium BC in stratified contexts, best compared to those of a late phase of the Shomutepe culture. Although a good number of Neolithic sites have been assigned to this culture in the Southern Caucasus, few have received systematic excavation with controlled field strategies. The excavations of Göytepe stand out in this regard, representing the largest of those ever conducted, at least in Azerbaijan. As illustrated here, the results showed us the first picture of important facets of the Neolithic settlement in the region.

First, the well-preserved architecture of Göytepe is a valuable source of information to define architectural techniques that help place the cultural tradition of the communities in the Middle Kura Valley in the larger cultural contexts of the South
Caucasian Neolithic. Second, the distinct discovery pattern of the floor remains deserves further research concerning the residential and settlement patterns of the communities. Most of the household compounds of Göytepe were found abandoned with plenty of still usable tools and materials on the floor, indicating de facto refuse. This pattern, not always common in the Neolithic of the Fertile Crescent, characterizes the communities of the study region, raising an important question to be further pursued. Third, the extensive excavations with a stratigraphic control have produced an exceptional dataset to study the settlement organization at this large Neolithic settlement. While this chapter addressed our preliminary idea as to the basic architectural unit and its general distribution pattern, further research is deserved. When the analysis proceeds further, as the excavations do, the research at Göytepe will make 
an important contribution to define the nature of the Shomutepe Neolithic society.

\section{References}

Azimov, M. S. (2006) Architectural Development in the Earliest Settled Agricultural Phases of Azerbaijan. Oxford: Archaeopress.

Badalyan, R. S., A. A. Harutyunyan, C. Chataigner, F. Le Mort, J. Chabot, J. -E. Brochier, A. Balalescu, V. Radu, and R. Hovsepyan (2010) The settlement of Aknashen-Khatunarkh. A Neolithic site in the Ararat plain (Armenia): excavation results (2004-2009). Turkiye Bilimler Akademisi Arkeoloji Dergisi (TÜBA$A R), 13: 187-220$.

Baudouin, E. (2019) Rethinking architectural techniques of southern Caucasus in the $6^{\text {th }}$ Millennium BC: A re-examination of former data and new insights. Paleorient, 45(1): 115-40.

Chataigner, C. (1995) La Transcaucasie au Néolithique et au Chalcolithique. Oxford: Tempus Reparatum.

Hansen, S. and M. Ullrich (2017) Excavations at Aruchlo. Preliminary report on the 2012-2014 campaigns. I In: The Kura Projects: New Research on the Later Prehistory of the Southern Caucasus, edited by B. Helwing, T. Aliyev, B. Lyonnet, F. Guliyev, S. Hansen, and G. Mirtskhulava, pp. 201-222. Berlin: German Institute of Archaeology, Eurasian Department.

Helwing, B., T. Aliyev, B. Lyonnet, F. Guliyev, S. Hansen, and G. Mirtshulava (eds.) (2017) The Kura Projects: New Research on the Late Prehistory of the Southern Caucasus. Berlin: German Institute of Archaeology, Eurasian Department.

Kadowaki, S., L. Maher, M. Portillo, R. M Albert, C. Akashi, F. Guliyev, and Y. Nishiaki (2015) Geoarchaeological and palaeobotanical evidence for prehistoric cereal storage at the Neolithic settlement of Göytepe (mid 8th millennium BP) in the southern Caucasus. Journal of Archaeological Science, 53: 408-425.

Lloyd, S. (1963) Mounds of the Near East. Edinburgh: Edinburgh University Press.

Mallowan, M. E. L. and J. C. Rose (1935) Excavations at Tall Arpachiyah, 1933. Iraq, 2: 1-178.

Munchaev, R. M. (1982) Eneolit Kavkaza. In: Eneolit SSSR, edited by V. M. Masson and N. Merpert, pp. 100-164. Moscow: Nauk.

Narimanov, I. (1987) The Culture of the Most Ancient Farming and Stock-Breeding Population of Azerbaijan. Baku: National Academy of Sciences (in Russian with an English summary).

Nishiaki, Y., F. Guliyev, S. Kadowaki, and T. Omori (2018) Neolithic residential patterns in the southern Caucasus: Radiocarbon analysis of rebuilding cycles of mudbrick architecture at Göytepe, West
Azerbaijan. Quaternary International, 474: 119-130. Schiffer, M. (2010) Behavioral Archaeology: Principles and Practice. London: Routledge. 
Chapter 4

\title{
Excavation, stratigraphy, and architecture of Square 4B at Göytepe
}

\author{
Seiji Kadowaki, Yui Arimatsu, and Yoshihiro Nishiaki
}

\subsection{Purposes of the excavation of Square 4B}

One of the main purposes of the investigations at Göytepe by the Japanese mission was to obtain an overall picture of occupation history at the site by establishing building levels or phases correlated with radiocarbon dates. We aimed to uncover lower occupation levels at Göytepe to collaborate with the Azerbaijani mission that had already started excavating upper portions of the mound (Chapter 3 ). Through this collaboration, we wanted to efficiently obtain archaeological data for studying diachronic changes in material culture and the socioeconomic strategies of Neolithic inhabitants at Göytepe.
For these reasons, Square 4B (10 x $10 \mathrm{~m})$ was chosen as the focus of our excavation (Fig. 4.1). The square is located in the northeastern part of Göytepe and at least $5 \mathrm{~m}$ below the top of the mound. The area at Square $4 \mathrm{~B}$ had been disturbed by modern building construction that created a steep slope descending from south to north. Consequently, the disturbed area revealed stratigraphic sections of archaeological deposits. These sections had been cleaned and examined in 2007 by an Azerbaijani-French mission (Lyonnet 2009). Before starting our excavations of Square 4B, we reexamined the exposed stratigraphic sections and confirmed that intact Neolithic deposits were preserved.

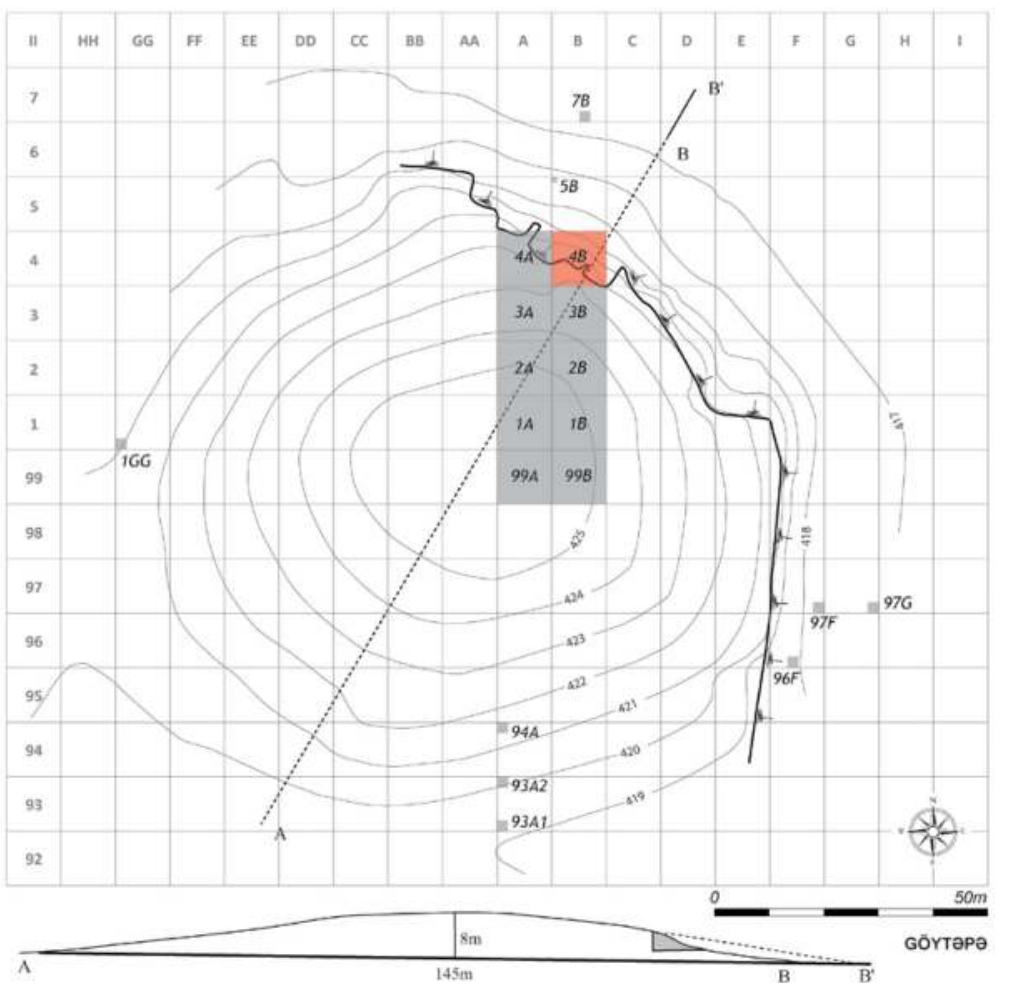

Fig. 4.1 Topographic map of Göytepe showing the excavation squares and disturbed area to the northeast. 


\subsection{Excavation methods}

We subdivided Square 4B into two rectangular areas, $4 \mathrm{BI}$ and 4BII, each of which measures $5 \times 10 \mathrm{~m}$. We left $50 \mathrm{~cm}$-wide baulks on their northern and eastern sides for stratigraphic observations. In addition, because of the steep slope, we divided the square into upper and lower excavation areas, somewhat like a stepped trench but with an irregular boundary due to the modern disturbance (Fig. 4.2). The upper excavation area is located in the southern section, while the lower one is in the northern section. This allowed us to conduct excavations at different levels concurrently and, in turn, quickly uncover a sequence of occupations by identifying building levels.

For the investigation of multilayered sites like tepes, it is usually necessary to remove architectural features before uncovering lower archaeological layers in the excavation areas. However, in the case of Göytepe, we left well-preserved architectural remains intact and restricted the excavation areas when we continued from upper to lower building levels (Fig. 4.3). This decision was based on plans to preserve Göytepe as an open-air museum where some of the in-situ archaeological remains will be presented to visitors. Due to these preservation procedures and the modern surface disturbance, the forms of excavated areas were somewhat irregular (Fig. 4.4). Nonetheless, the preserved deposits contained architectural remains that were dense enough for us to recognize the construction levels.

To systematically record various architectural remains and archaeological deposits, we employed spatial units, "context," which corresponds to a depositional unit (e.g., mud-brick wall, building floor, pit fill, or a cluster of artifacts; see Nishiaki et al. 2001: 49). We assigned a numerical name to each context and used a series of context numbers for Squares 4BI and 4BII. For the latter, we used another series of context numbers for lower levels (Levels 9-11) in the northern area, which was named "4BIIX" to distinguish between the context numbers

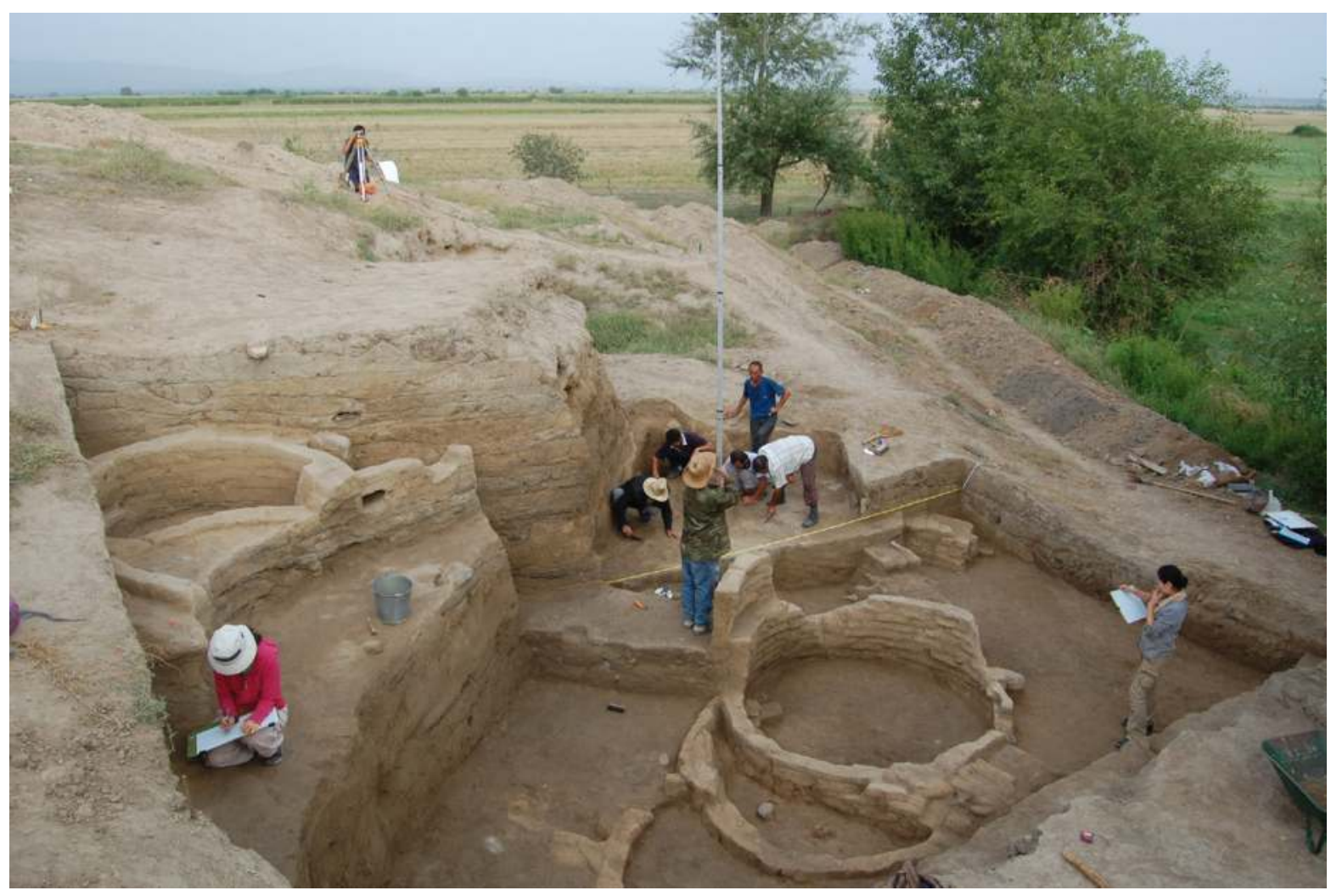

Fig. 4.2 Square 4B seen from the east. Note that a modern disturbance created a stratigraphic section diagonal to the square. Architectural remains of two different levels are exposed in Square 4BII. 


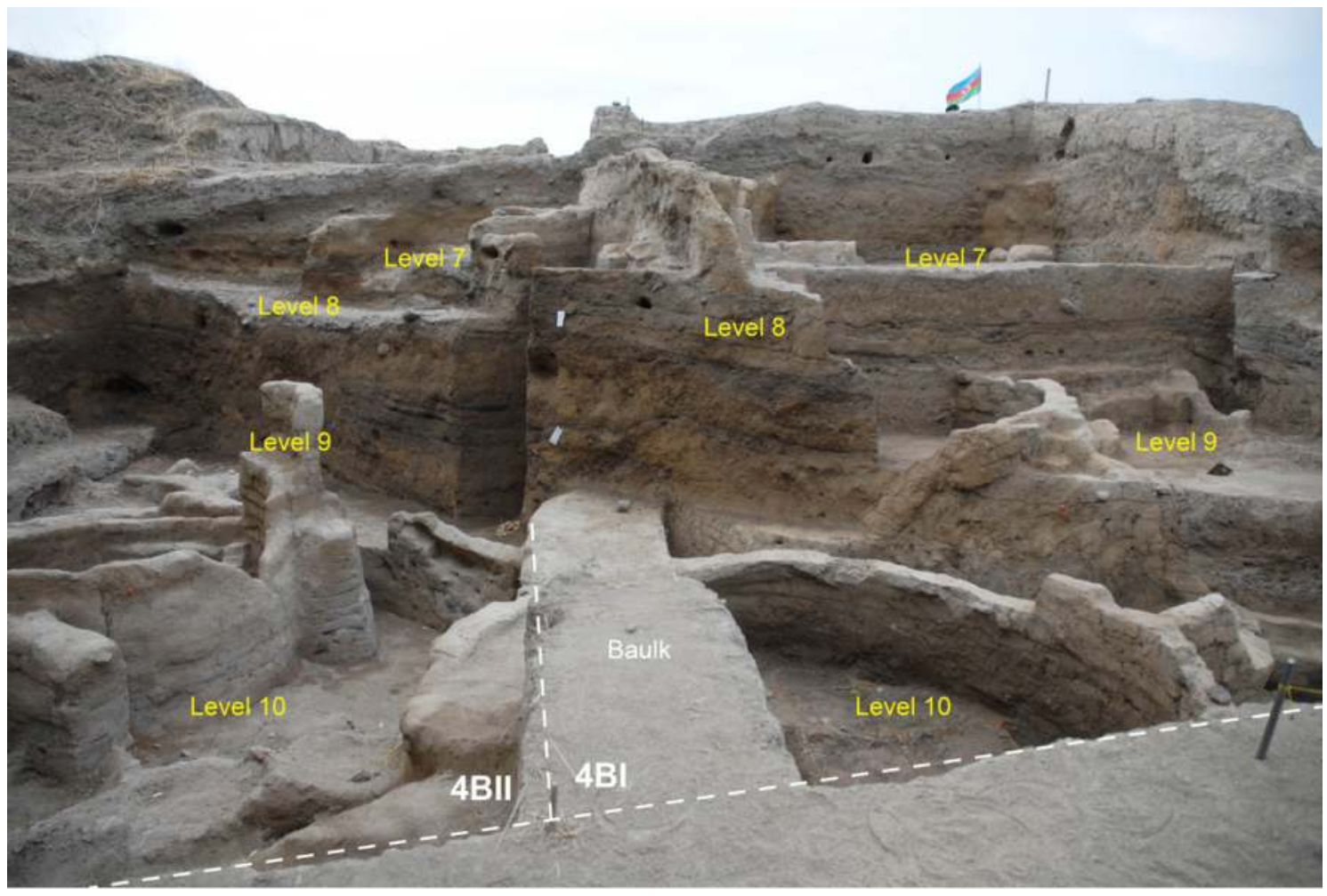

Fig. 4.3 Square 4B seen from the north. Note that a modern disturbance created a stratigraphic section diagonal to the square. Numbers indicate the building levels with architectural remains preserved for a future open-air museum at Göytepe.

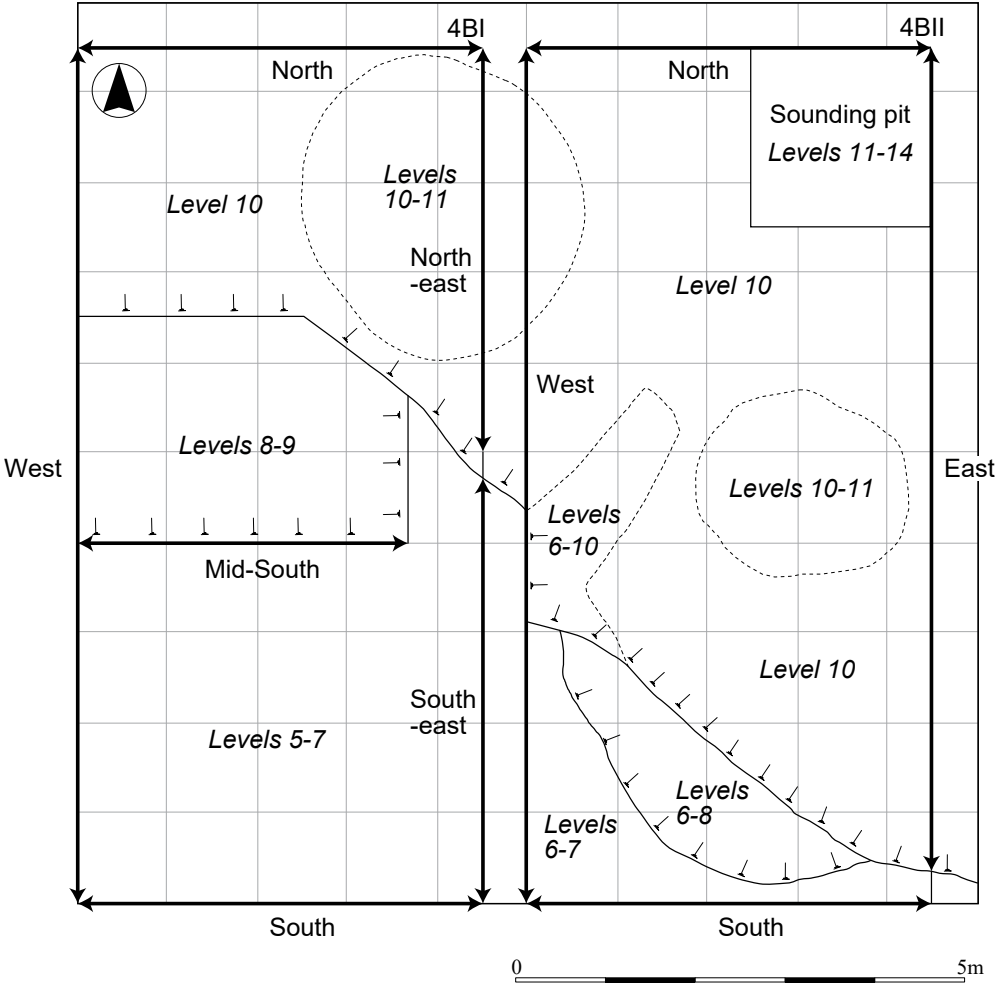

Fig. 4.4 Topography of Square 4B after the 2009-2013 seasons showing the building levels excavated in different parts of the square and locations of stratigraphic sections presented in this chapter. Note that two round areas (dashed line) of Level 11 correspond to two round buildings excavated in this level, while the tilted rectangular area (dashed line) of Levels 6-10 in Square 4BII had been created by a modern disturbance before our excavation. 
of lower and upper levels of the same square. In this report, we refer to the context numbers by their square names in order to specify architectural features or archaeological deposits, such as Wall (4BI-129) or Clay bin fill (4BIIX-94).

\subsection{Sampling methods}

We collected artifacts, ecofacts, and other samples primarily by context. In the excavation of each one, we used "context sheets" to record the context definition, spatial extent, stratigraphic position, lithological characteristics of sediments, and excavation methods. Sediment samples were also taken for water flotation and subsequent analysis of phytoliths, fecal spherulites, and micromorphology, particularly from contexts related to activity areas or storage, such as building floors, hearths, and clay bins (see Chapter 7 for the geoarchaeological study of clay bins). Relatively good preservation of charred botanical remains at Göytepe facilitated sampling for both anthracological studies and radiocarbon dating. For the latter purpose, we selected charcoal samples primarily from hearths.

A round mud-brick building (4BIIX-16) in Level 10 was the best preserved structure in Square 4B (see below). We recorded the spatial distribution of refuse inside the building by collecting artifacts, ecofacts, and sediment samples by $50 \times 50 \mathrm{~cm}$ grids. Using this grid system, we separated five stratigraphic units in the deposits starting with the house fill, moving through a floor surface, and ending at the bottom level of the building wall. More details of this building are described in section 4.5 below.

\subsection{Building levels}

We organized the occupational history of Göytepe by defining building levels. A building level is defined as a stratigraphic unit of archaeological deposits and features that we associate with the construction, use, and abandonment of major architectural remains. In the case of Göytepe, these are round mud-brick structures. Using contexts as units of stratigraphic analysis, we grouped them by building level on the basis of their stratigraphic relationship with round mud-brick buildings.
During the 2009-2013 seasons, we identified Neolithic Levels 1-14, which cover ca. 11 m-thick deposits from the top of the site. These building levels were established by connecting the stratigraphy between excavation areas of both the Azerbaijani and Japanese missions, which include Squares 1A, 1B, 2A, 2B, 3A, 3B, 4A, and 4B in total (Chapter 3 ). Among these areas, Square $4 B$ contained Levels 5-14 (Fig. 4.4). Figs. 4.5 to 4.9 and 4.44 show major stratigraphic sections indicating the extent of building levels.

\subsection{Architectural remains in Square 4B}

\subsubsection{Level 5}

The deposits of Level 5 were limited to Square 4BI (Fig. 4.12), since the surface descends from west to east, i.e., from 4BI to 4BII (Figs. 4.6 and 4.11). In Square 4BI, we recovered two large ash pits (4BI-12 and $13 / 15 / 17 / 32$ ) that disturb underlying buildings in Level 6. The larger pit (13/15/17/32) measures $4.1 \times$ $3.5 \mathrm{~m}$ in plan and $0.8 \mathrm{~m}$ in depth, while the smaller one (12) is $1.7 \mathrm{~m} \times 1.7 \mathrm{~m}$ in plan and $0.5 \mathrm{~m}$ in depth. The latter pit was found to extend further south into Square 3BI, where its continuation was recorded as pit 3BI-42, allowing us to stratigraphically connect the two squares.

\subsubsection{Level 6}

This level includes a round mud-brick structure (1.8 $\mathrm{m}$ in diameter) in the southeastern corner of Square 4BI and a curvilinear wall attached to its northern exterior (Figs. 4.13 and 4.14). This massive northsouth wall appears to have functioned as an outdoor boundary instead of a portion of a roofed building.

Level 6 was subdivided into two subphases based on the identification of two floor levels in the round building. The upper floor (4BI-22) is made of yellowish-brown mud plaster, on which a large rectangular stone was found (Fig. 4.13). A cluster of mud-brick fragments and stones (4BI-20) was discovered in the upper level beside the massive north-south wall. The lower floor of the round building (4BI-31) was not as clear as the upper one, but two large burnt cobbles (4BI-30) were set against the northern wall (Fig. 4.14).

A large ash pit was found in the southern part 

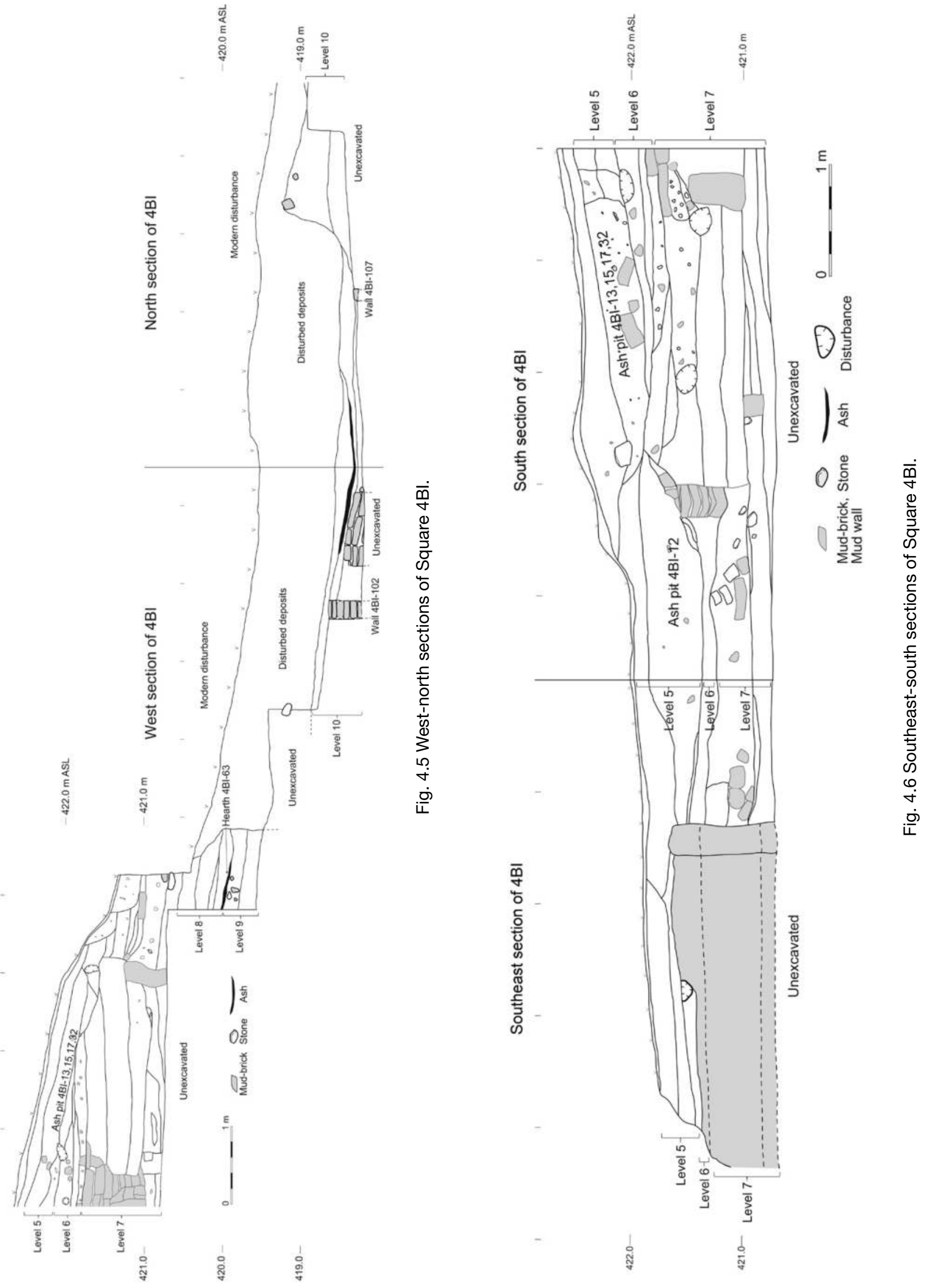

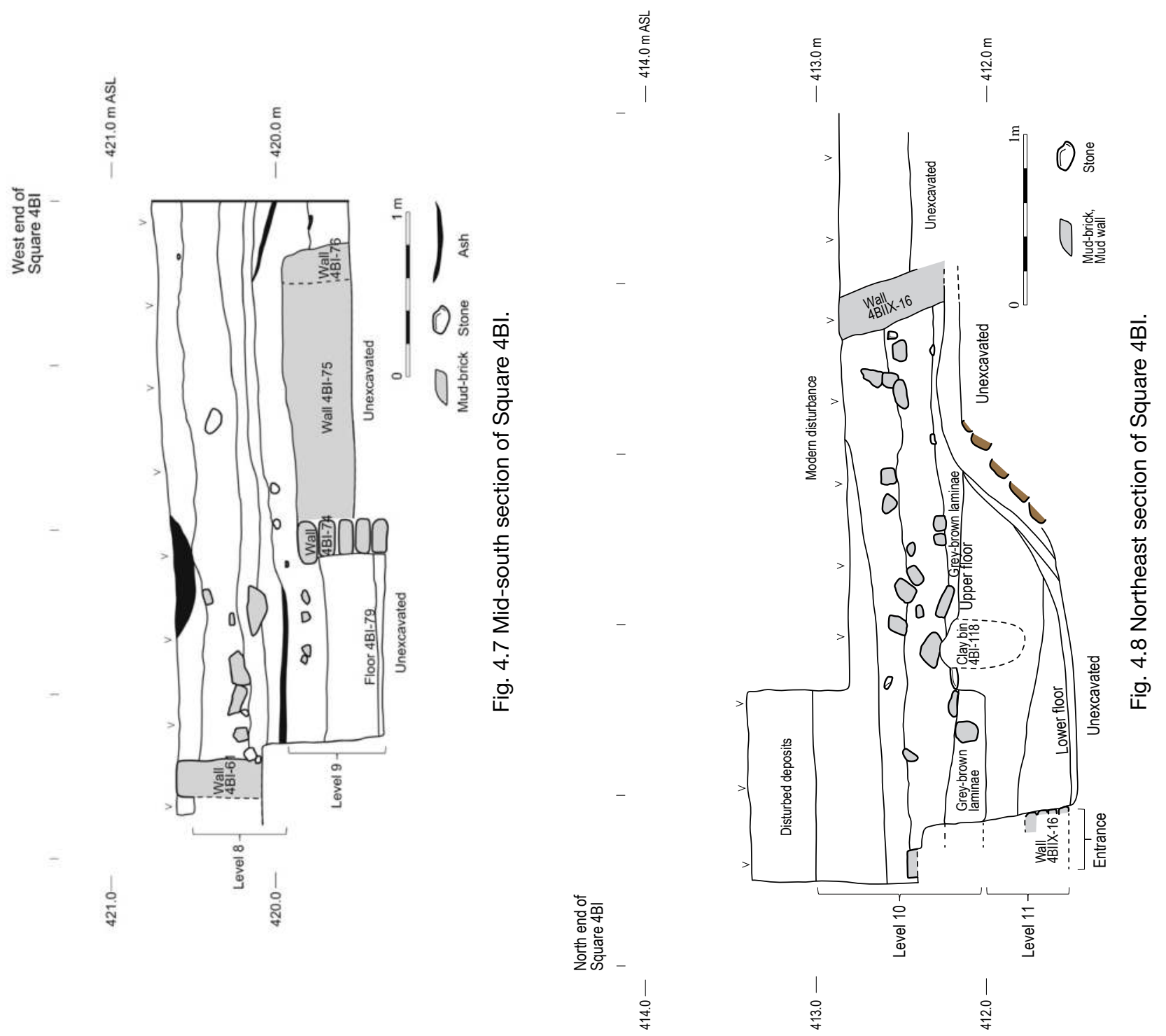

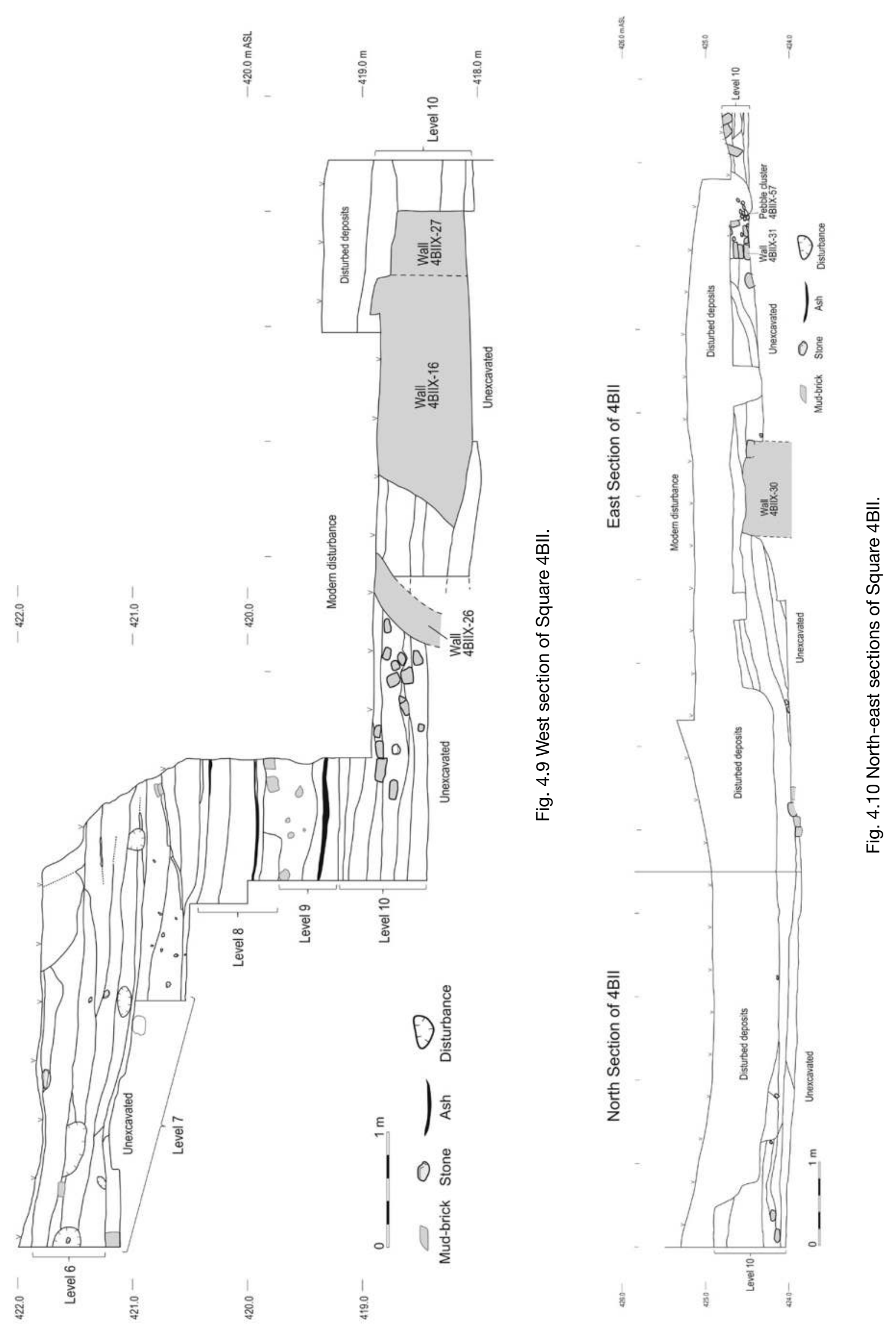


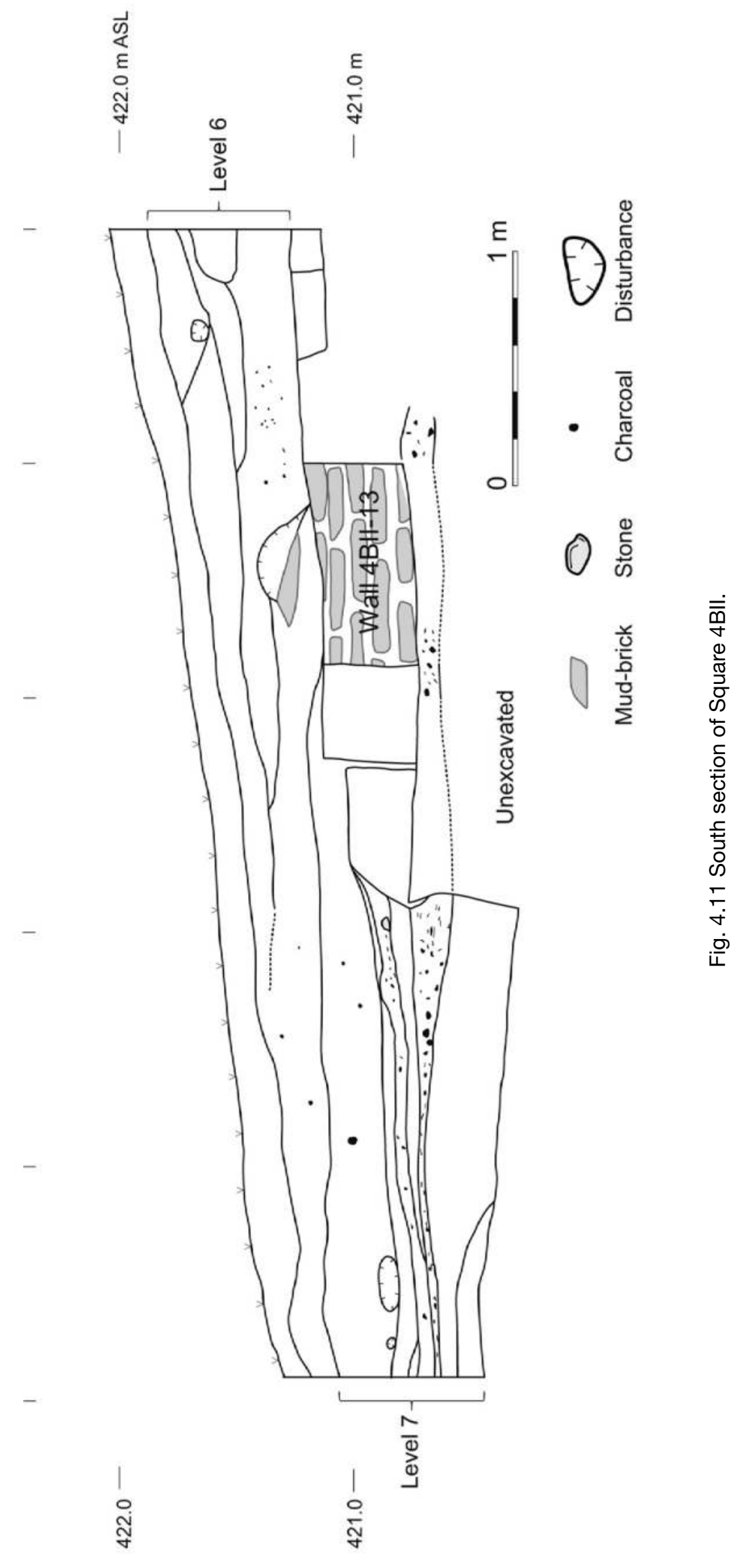



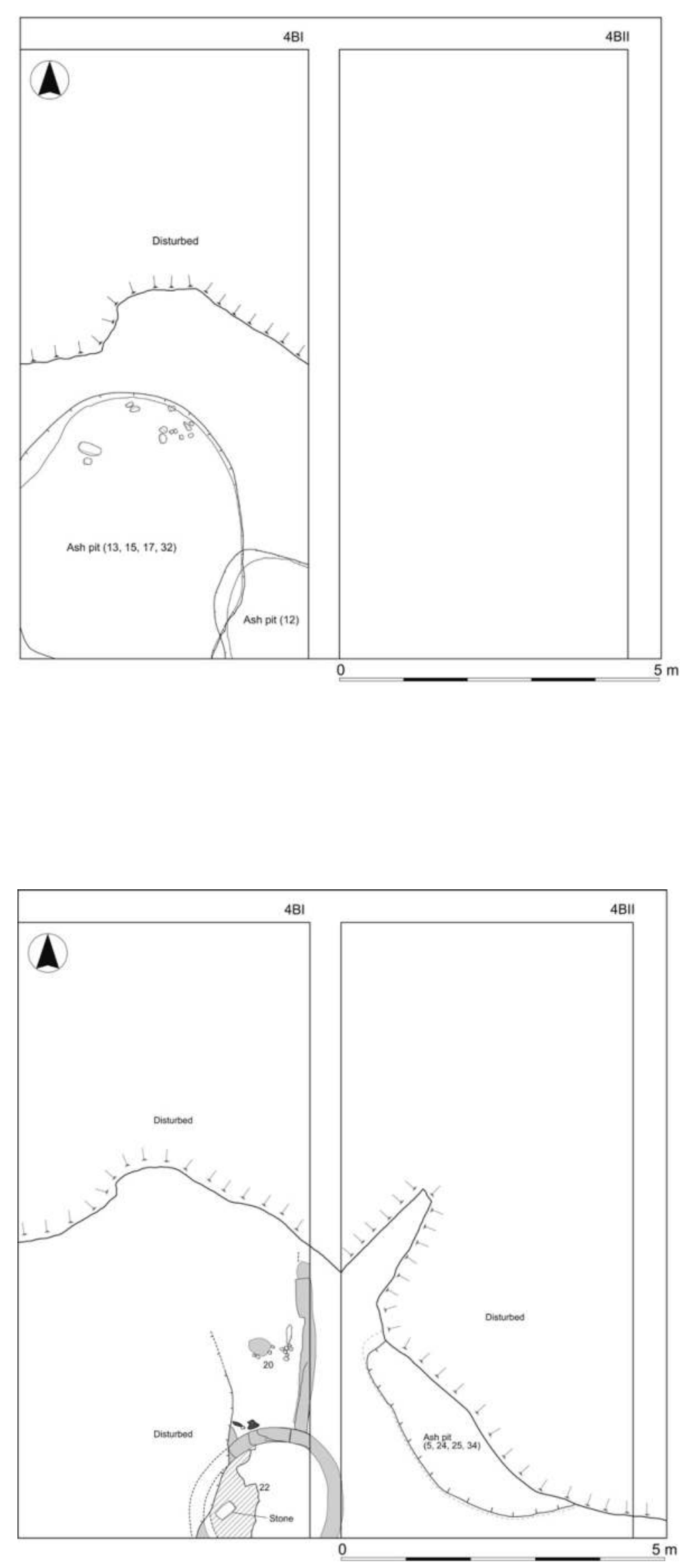

Fig. 4.12 Features of Level 5 in Square 4B. Deposits in this level were not preserved in Square 4BII since the surface descends from west to east.
Fig. 4.13 Building remains of the upper phase of Level 6 in Square 4B. 


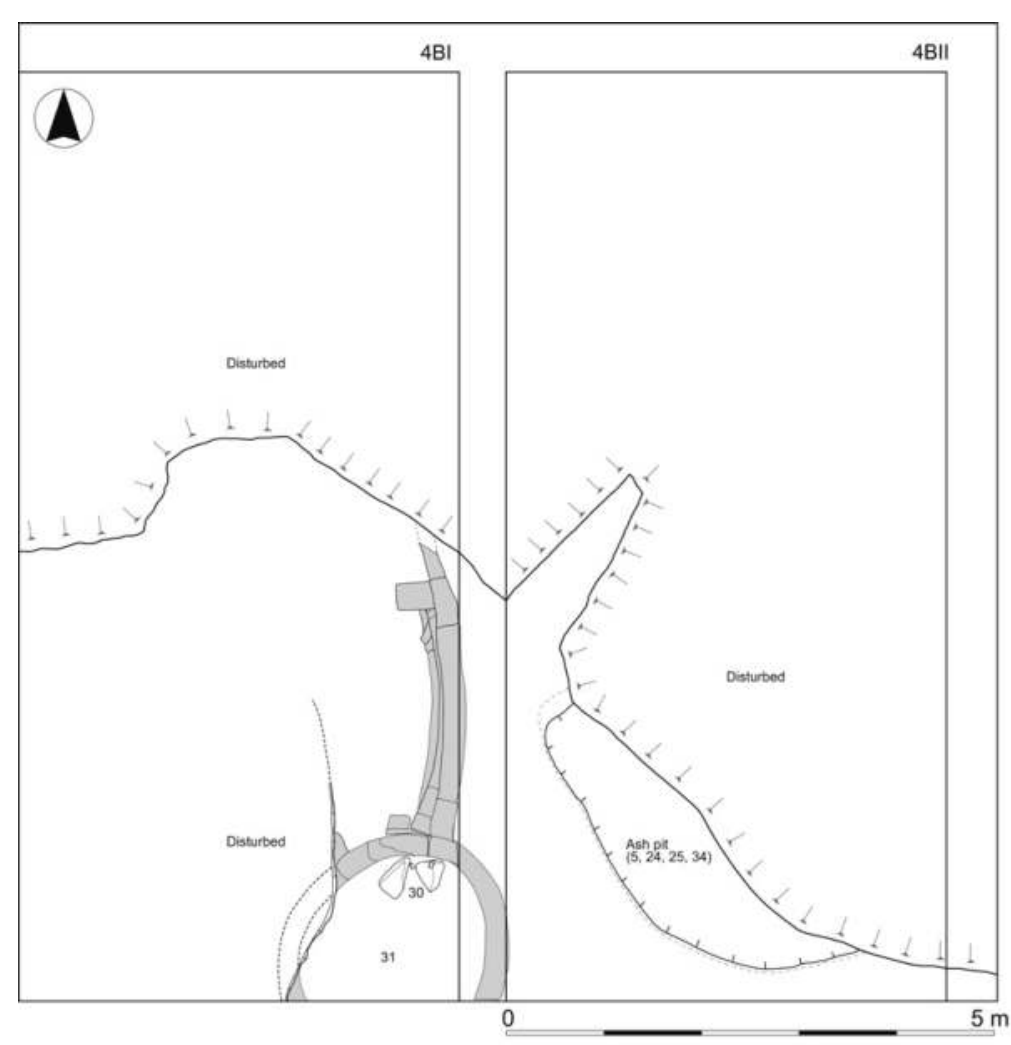

Fig. 4.14 Building remains of the lower phase of Level 6 in Square 4B. of Square 4BII. The pit measures $3.5 \mathrm{~m}$ in diameter and $1 \mathrm{~m}$ in depth. Its construction partially destroyed a building structure in Level 7. A large volume of refuse recovered from the pit fill indicates that it was used for disposing domestic waste.

\subsubsection{Level 7}

In Square 4BI, this level contains a round mudbrick building in the southeastern corner and by its appendicular wall extending to north (Figs. 4.154.18). These walls were constructed with grayishbrown mud-bricks, in contrast to the yellowishbrown mud-brick walls at the same location in Level 6. Other features in Square 4BI include a mud-brick wall in the southwestern corner and a pile of mudbricks (4BI-37) in the northern section. Not disturbed by the ash pits in Level 5, deposits in this level yielded some features. One is a concentration of clay sling balls (ca. 20 pieces) and small pebbles (4BI-40) that were located on the exterior side of the building.

In Square 4BII, a round mud-brick structure (4BII-13) was uncovered in the southwestern corner of the square (Fig. 4.19). The structure measures ca. $2.5 \mathrm{~m}$ in diameter and has a mud-plastered floor (4BII-26). Its northeastern quarter has been obliterated by the pit construction in Level 6 . We detected a prepared surface in what looked to be an external space immediately east of this round structure. The surface was created using lime or gypsum white plaster. Two hearths (4BII-17 and 19) were also discovered in the outdoor area north of the building.

A lower phase of this level was detected in Square 4BII (Figs. 4.16 and 4.19). The lower phase is defined by a round mud-brick structure (4BII-39) measuring $2 \mathrm{~m}$ in diameter. This structure is thought to represent an earlier stage of the round structure in the upper phase (4BI-13) because the eastern part of the wall continues from the lower to upper phase. However, the wall's western part was rebuilt in the upper phase to widen the interior space. Thus, the lower phase building is smaller and has a separate mud-plastered floor (4BII-40). Like the upper phase, two hearths (4BII-41 and 42) were found in the external space north of the structure. Besides these hearths and the north-south wall in the middle of the square, we found a great volume of artifacts, which indicate the use of this space for various activities.

\subsubsection{Level 8}

The excavation of this level was restricted to narrow and irregular areas due to the modern disturbance 


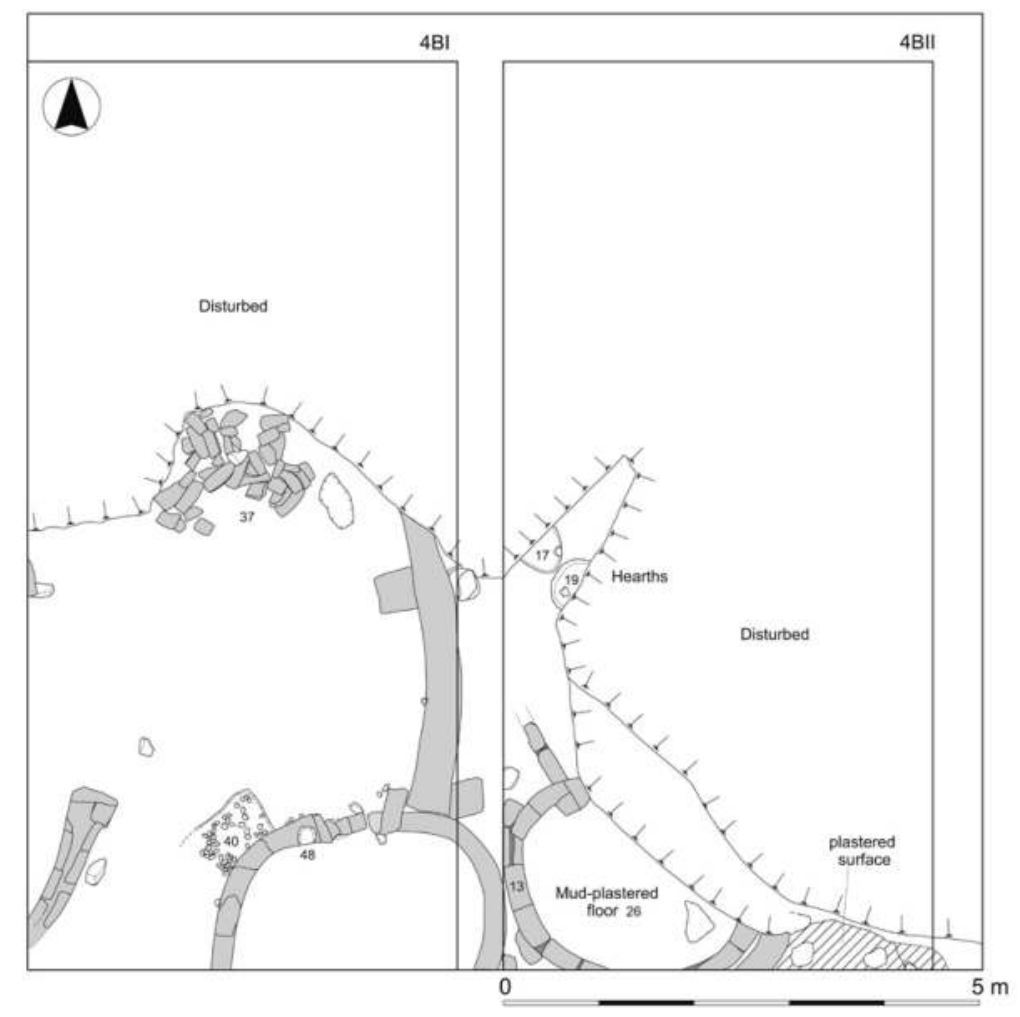

Fig. 4.15 Building remains of the upper phase of Level 7 in Square 4B.

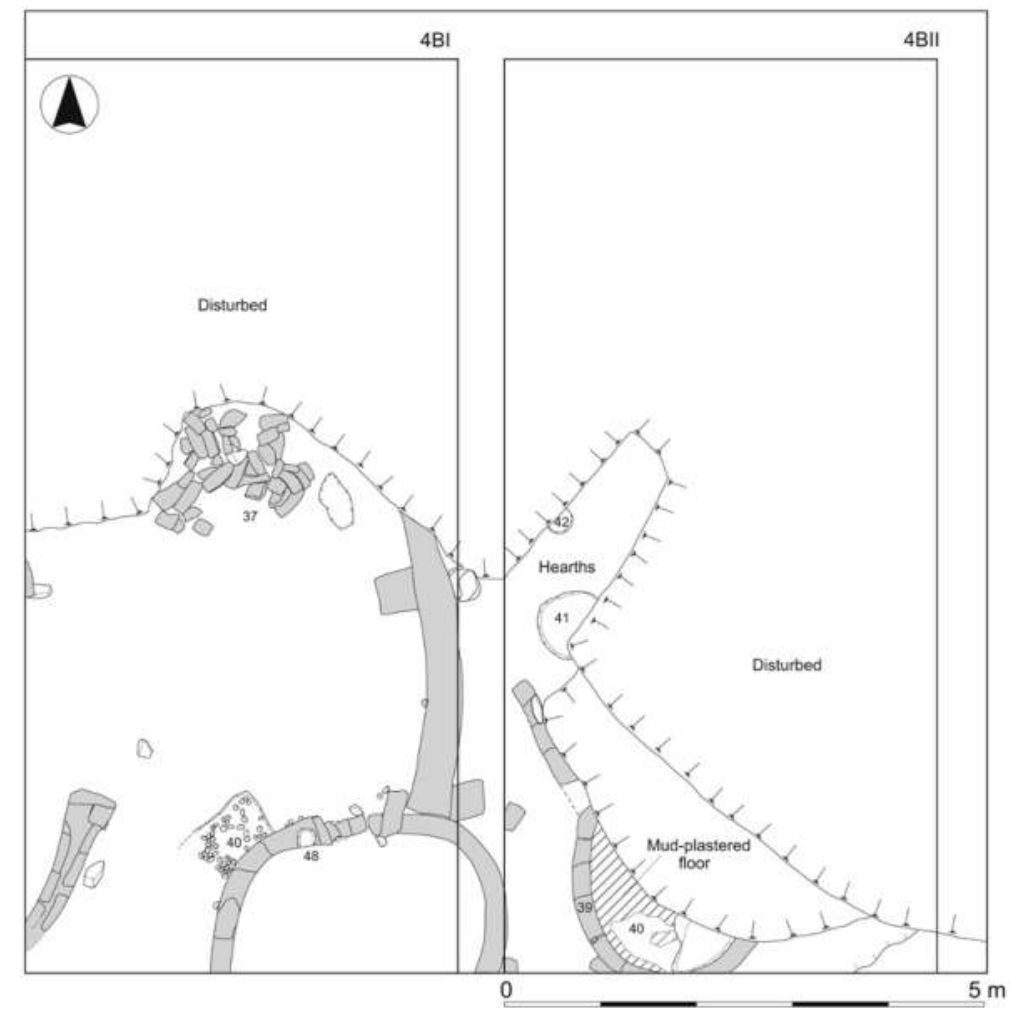

Fig. 4.16 Building remains of the lower phase of Level 7 in Square 4B. 


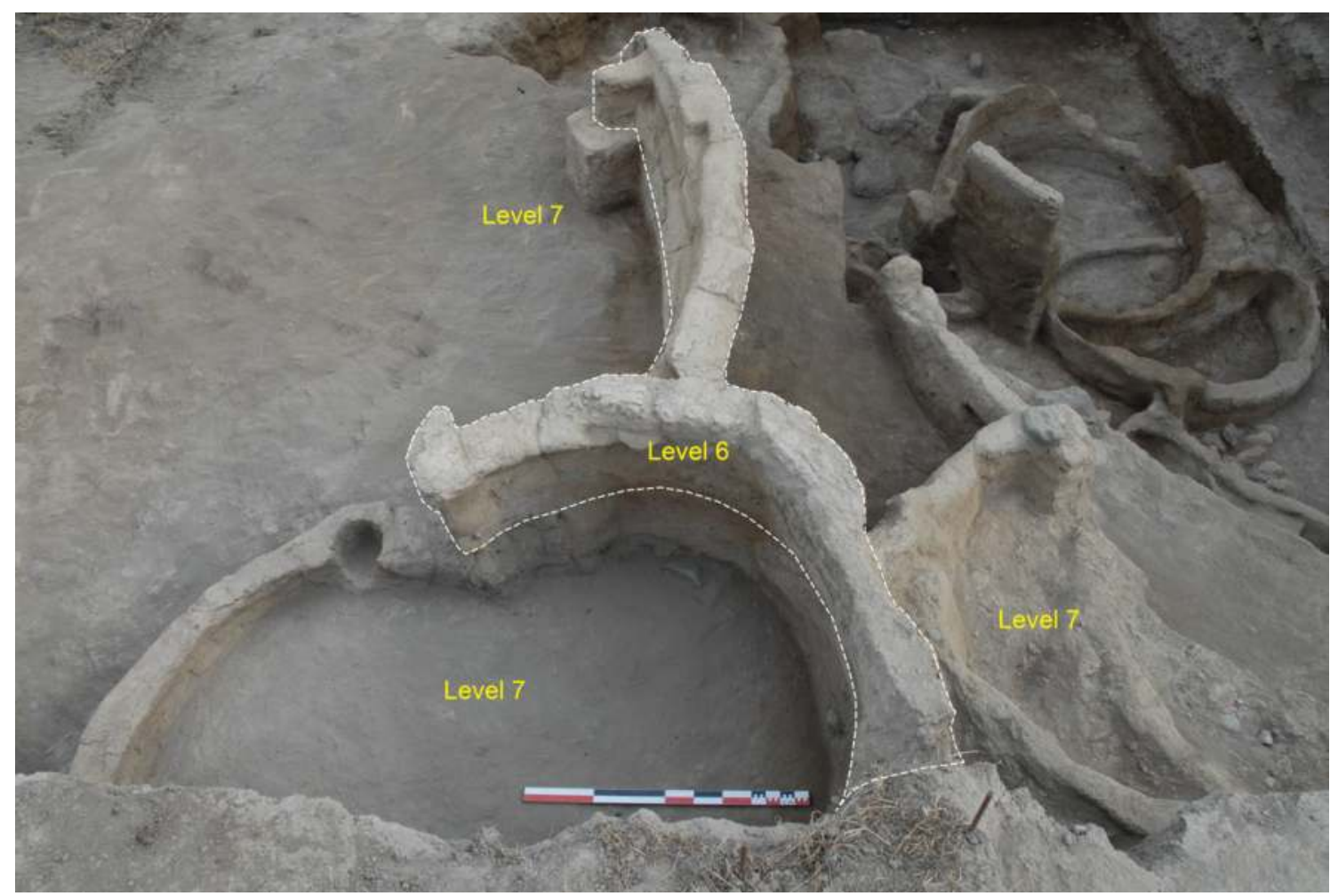

Fig. 4.17 Mud-brick walls of Levels 6 and 7 in Square 4B, seen from the south. The walls of Level 6 (dashed line) and Level 7 were constructed at almost the same location.

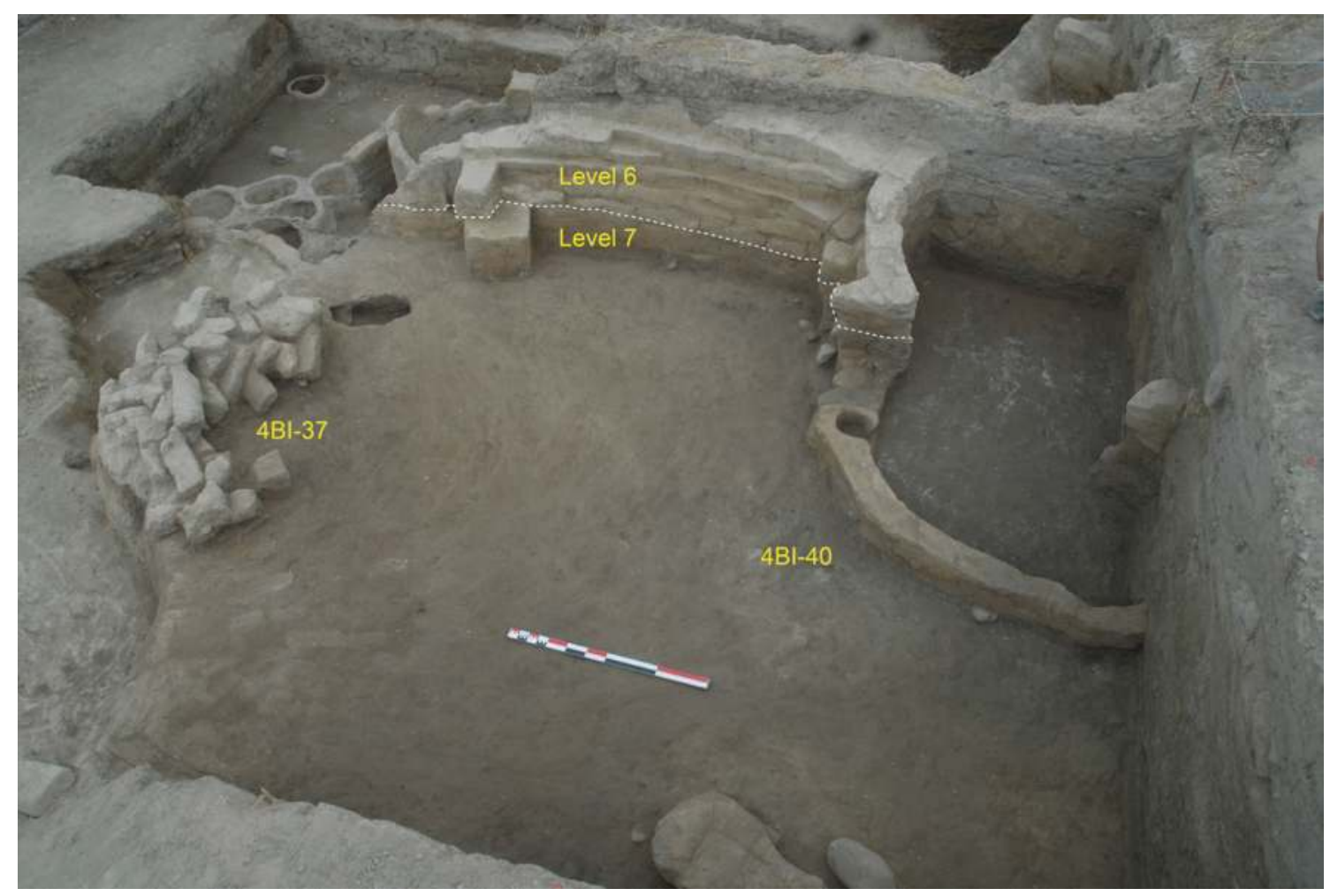

Fig. 4.18 Architectural features of Levels 6 and 7 in Square 4BI, seen from the southwest. The bottom walls of Level 6 (dashed line) and Level 7 were constructed at almost the same location. 


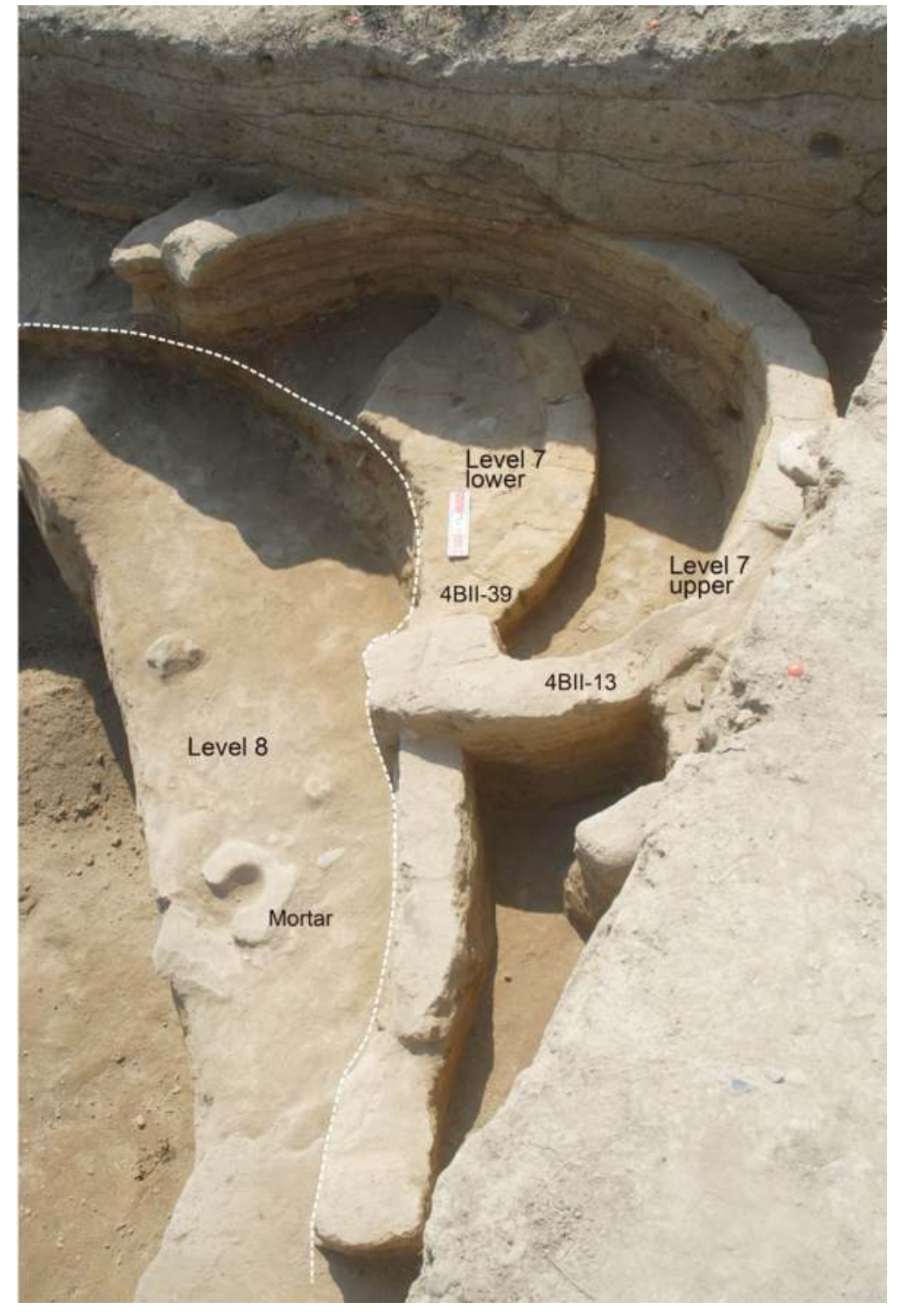

Fig. 4.19 Mud-brick walls and other features of Levels 7 and 8 in Square 4BII, seen from the northwest. An irregular step between these levels (dashed line) was created by a large ash pit intrusive from Level 5. and for the purpose of preserving the architectural remains in Level 7 (Fig. 4.20).

In Square 4BI, we recovered a slightly curvilinear wall (4BI-61) extending in a north-south direction, somewhat similar to the outdoor walls in Levels 6 and 7. Immediately to the west of this wall was an apparent outdoor space associated with a hearth (4BI-63) and cluster of cobbles (4BI-64) (Figs. 4.20 and 4.21). The latter feature was also associated with a round, shallow clay basin, which may represent the bottom of a clay bin. Clay bins are prevalent architectural features at Göytepe and may have been used for storage (see Chapter 7 for the geoarchaeological study of clay bins). Around these features, we recovered pottery sherds, chipped stones, and ground stones.
In Square 4BII, a broken mortar was found lying beside an ash pit (4BII-50) (Fig. 4.20). A small portion of a curvilinear wall (4BII-47) was preserved north of these finds. This may be a remnant of a round mud-brick structure standing to the north, considering that a round building was recovered in the same (but slightly shifted) location in underlying Levels 9 and 10.

\subsubsection{Level 9}

Although the excavation of this Level was also restricted to narrow and irregular areas due to the modern disturbance, we recovered a number of mud-brick walls substantial enough for identifying a building level.

In Square 4BI, we uncovered three round 


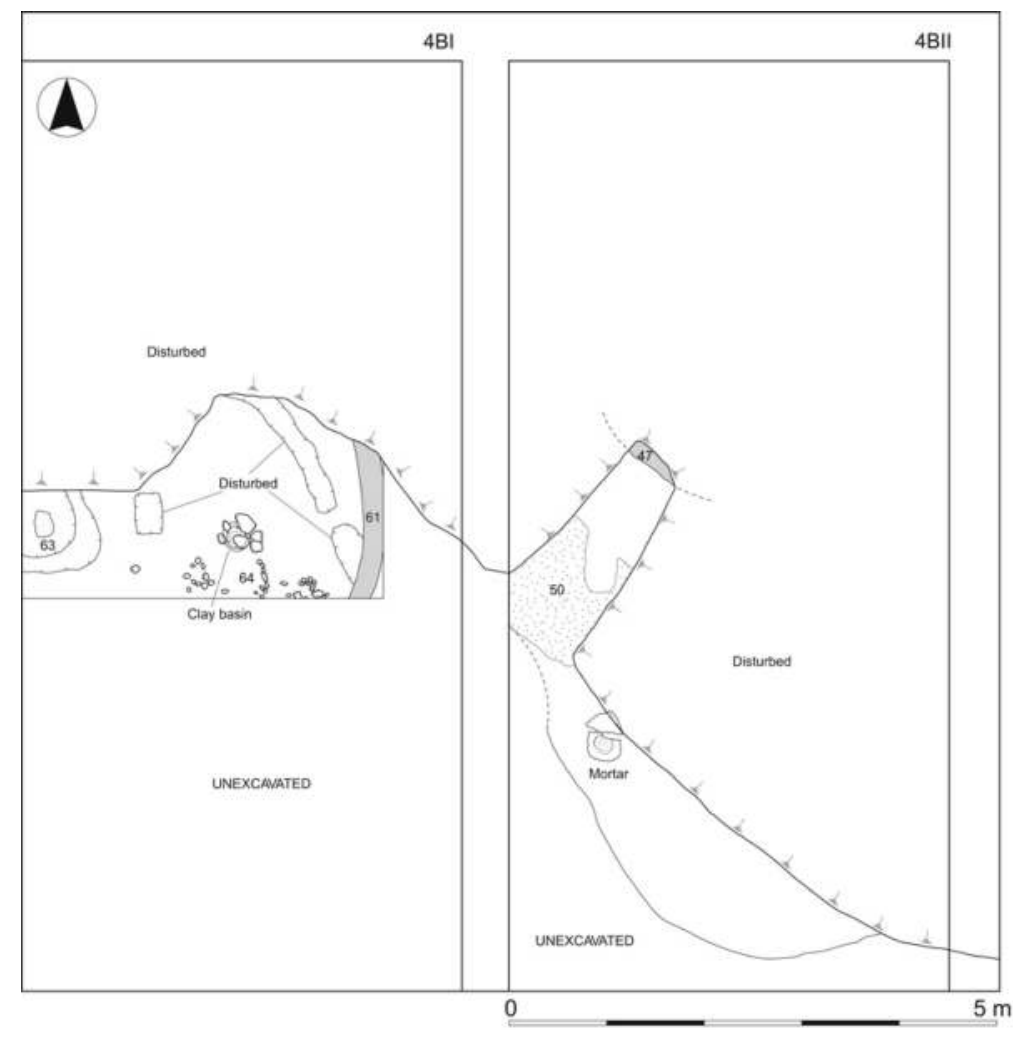

Fig. 4.20 Features of Level 8 in Square 4B.

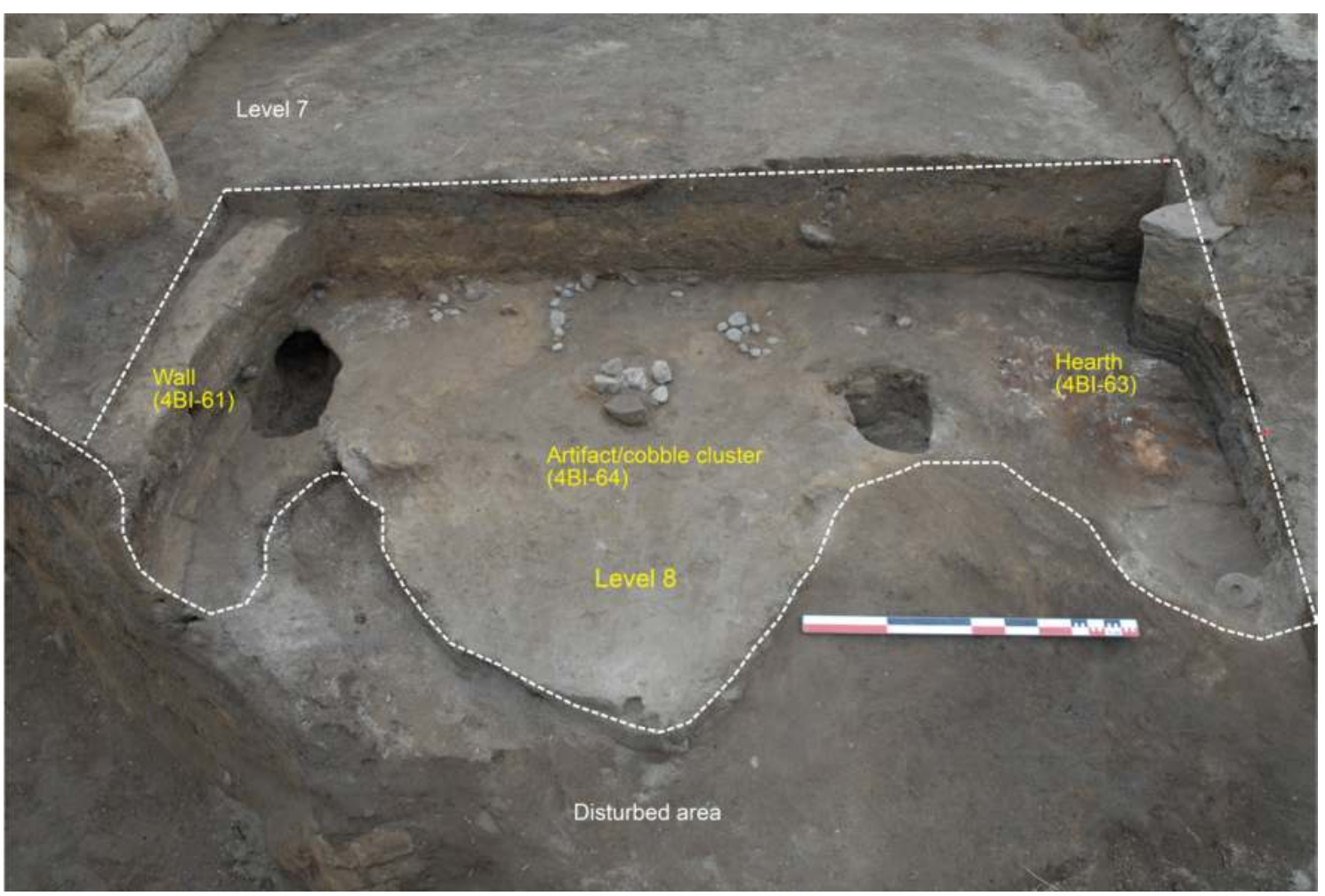

Fig. 4.21 Features recovered in Level 8 in Square 4BI, seen from the north. Although the excavated area was narrow, a building wall was found beside a probable activity area with a hearth. 
structures (4BI-74, 76, and 81) and their appendicular walls (4BI-75, 83, and 90) (Figs. 4.22-4.24). Among these, two round structures (4BI-74 and 76) are connected to each other by a straight thick wall (4BI$75)$, while another wall (4BI-74) is also connected to the northern round house (4BI-81) by a third wall (4BI-83). The northern round house (4BI-81) may have been further linked to another structure by a slightly curved wall (4BI-90), indicating that the three round buildings were part of a courtyard structure extending from Square 4BI to the northwest.

Inside the round wall (4BI-74) in Square 4BI, a building floor was detected in the form of ca. 10 cm-thick deposits (4BI-79), consisting of laminae of ashy, black, and brown sediments without features (Fig. 4.7). This floor deposit formed a shallow basin. The center of the room was slightly lower than the periphery near the wall, although this may have been the result of pressure from overlying deposits. To the north, the round building (4BI-81) was excavated with particular attention to floor deposits (4BI-84), although they were not significantly distinct from the room fill and were not associated with features or artifacts. With regard to outdoor features, a hearth (4BI-78) and ash deposits (4BI-73) were found between the two round buildings (4BI-74 and 76). The density of materials from these areas was higher than in room fills.

Near the center of Square 4BII, we uncovered portions of a round mud-brick structure (4BII-66 and 4BIIX-3). The wall (4BIIX-3) retained six courses (rows) of bricks. It is unclear whether this building is structurally related to those in Square 4BI since a disturbed area separates them. We identified two construction phases in what look to be outdoor deposits of Square 4BII. The upper phase is associated with a hearth (4BII-55), a stone feature (4BIIX-6), and a mud-plastered bin (4BIIX-11) (Fig. 4.22). The lower phase (Fig. 4.23) includes a flay-lying grinding slab (4BIIX-13) hearths with burnt cobbles (4BII-56 and 65; Fig. 4.25), and an ash-filled pit (4BIIX-65) measuring at least $1 \mathrm{~m}$ in diameter.

\subsubsection{Level 10}

\subsubsection{Round mud-brick building (4BIIX-12)}

In Square 4BII, we uncovered a round mud-brick structure (4BIIX-12; Fig. 4.26). The top of its wall

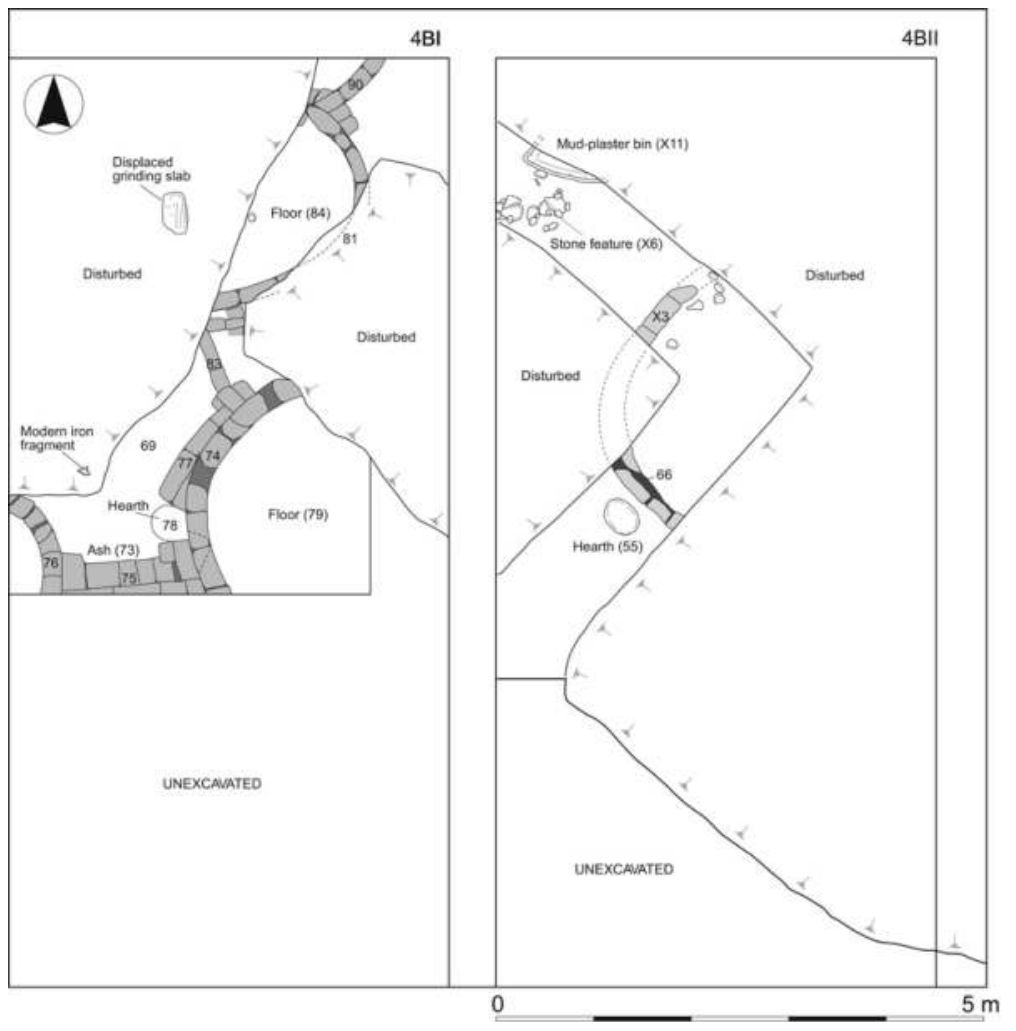

Fig. 4.22 Building remains of the upper phase of Level 9 in Square 4B. 


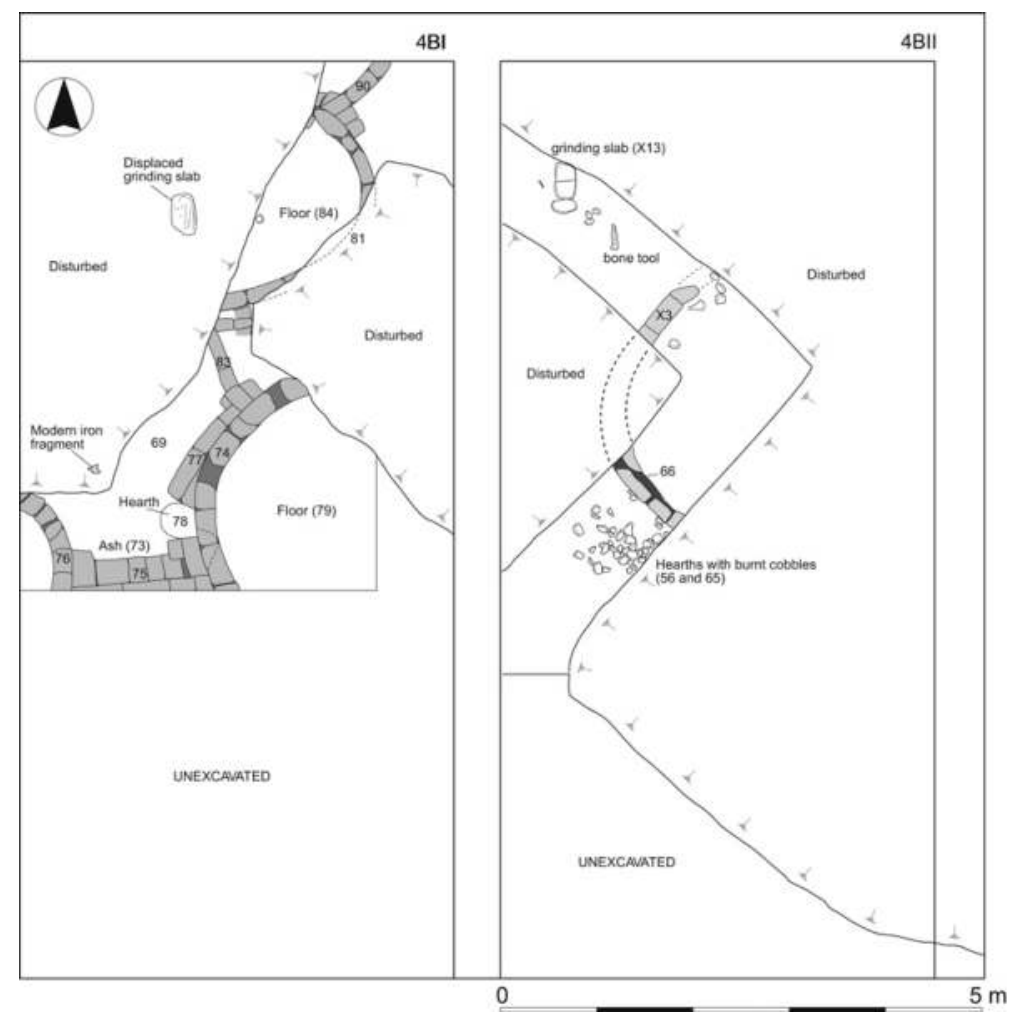

Fig. 4.23 Building remains of the lower phase of Level 9 in Square 4B.

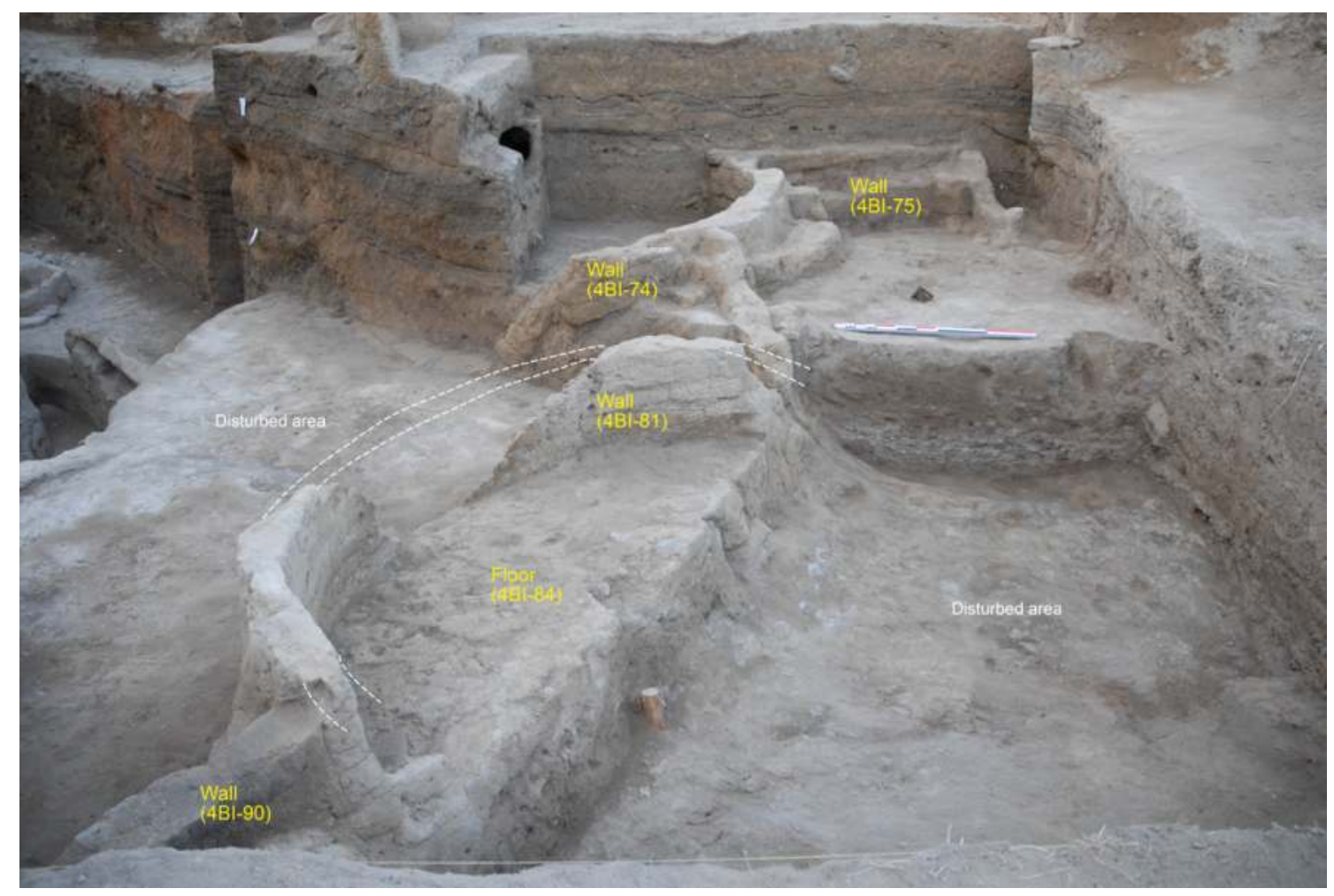

Fig. 4.24 Architectural remains of Level 9, seen from the north. Note that a curvilinear wall (4BI-81) in front was heavily disturbed from both sides. 


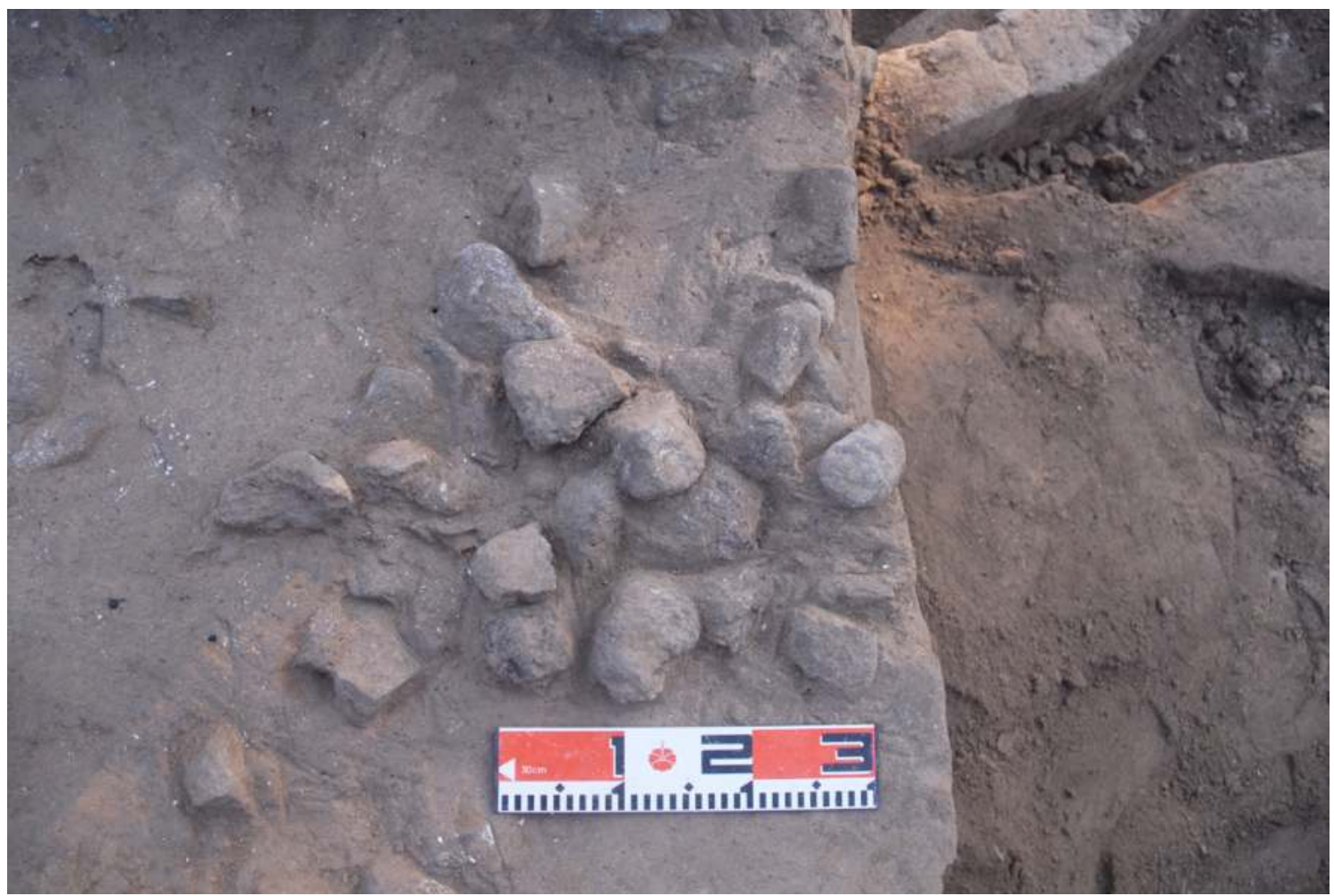

Fig. 4.25 Hearth with burnt cobbles (4BII-56) from Level 9 in Square 4BII, seen from the southwest. Note that the feature's right side was removed by a modern disturbance.

was located immediately below the building walls at almost the same location of Level 9 (4BII-66 and 4BIIX-3). The western half of the wall (4BIIX12) was better preserved, measuring ca. $80 \mathrm{~cm}$ in height (Fig. 4.27). The building had two floor levels consisting of thin, alternating deposits of ashy, white, black, and brown sediments (Fig. 4.28). Neither floor had been mud-plastered in contrast to the building floors in Level 7 (4BII-26 and 40). Although we found some cobbles, an antler, and some small obsidian fragments in the floor deposits, the overall density of materials inside the building was clearly lower than in outdoor deposits, indicating that the inhabitants maintained their indoor space and kept it free of refuse. To further investigate the nature of building floors and obtain evidence of indoor activities, we collected sediment samples from the floor deposits for microrefuse and micromorphological analyses. The studies of these samples are in progress.

Immediately east of this building, we found a half-circular wall (4BIIX-34) that creates a narrow internal space between another wall (4BIIX-12). The former is a remnant of a round building in Level 11 after it was partly destroyed by the construction of the latter in Level 10. As described below, the lower courses of one wall (4BIIX-34) continue below another (4BIIX-12) (Fig. 4.42). In addition, a rectangular mud-brick structure (4BIIX-30) was found attached to the exterior surface of the round building (4BIIX-12) on its eastern side. The rectangular structure was preserved to the height of only a few courses of mud-bricks but its function is unclear. To the west of the round structure (4BIIX12), an appendicular wall (4BIIX-26) connects to another round building (4BIIX-16).

\subsubsection{Upper phase of a round mud-brick building (4BIIX-16)}

A round mud-brick structure (4BIIX-16) was fully uncovered in Squares 4BI and 4BII (Figs. 4.26 and 4.29). The eastern side of this building is connected by an appendicular wall (4BIIX-26) to the round structure (4BIIX-12) in Square 4BII, while the western side is linked by the wall (4BI-94) to a double-leaf mud-brick wall (4BI-95).

The structure (4BIIX-16) measures $3.5 \times 3 \mathrm{~m}$ in diameter and represents one of the largest round buildings uncovered thus far at Göytepe. In addition to its large size, this building is distinguished from 


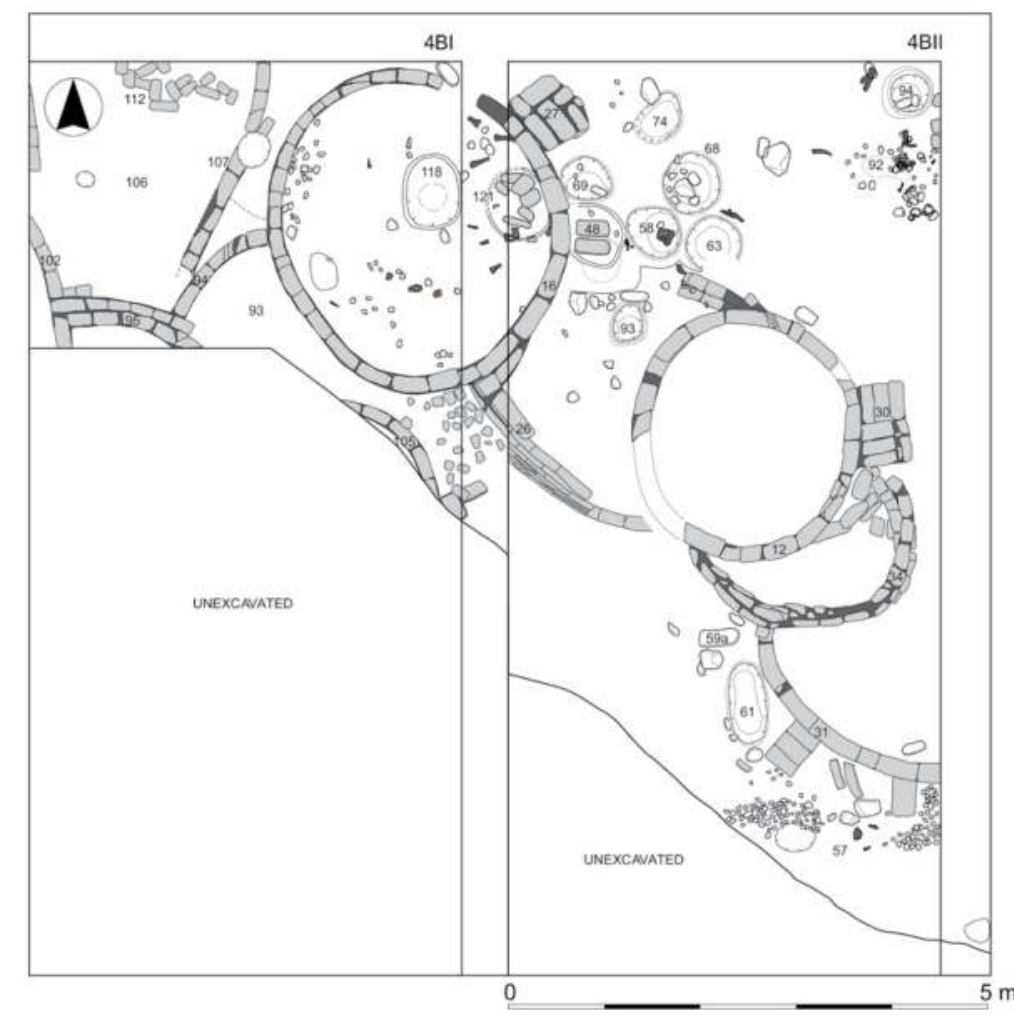

Fig. 4.26 Architectural remains of Level 10 in Square 4B.

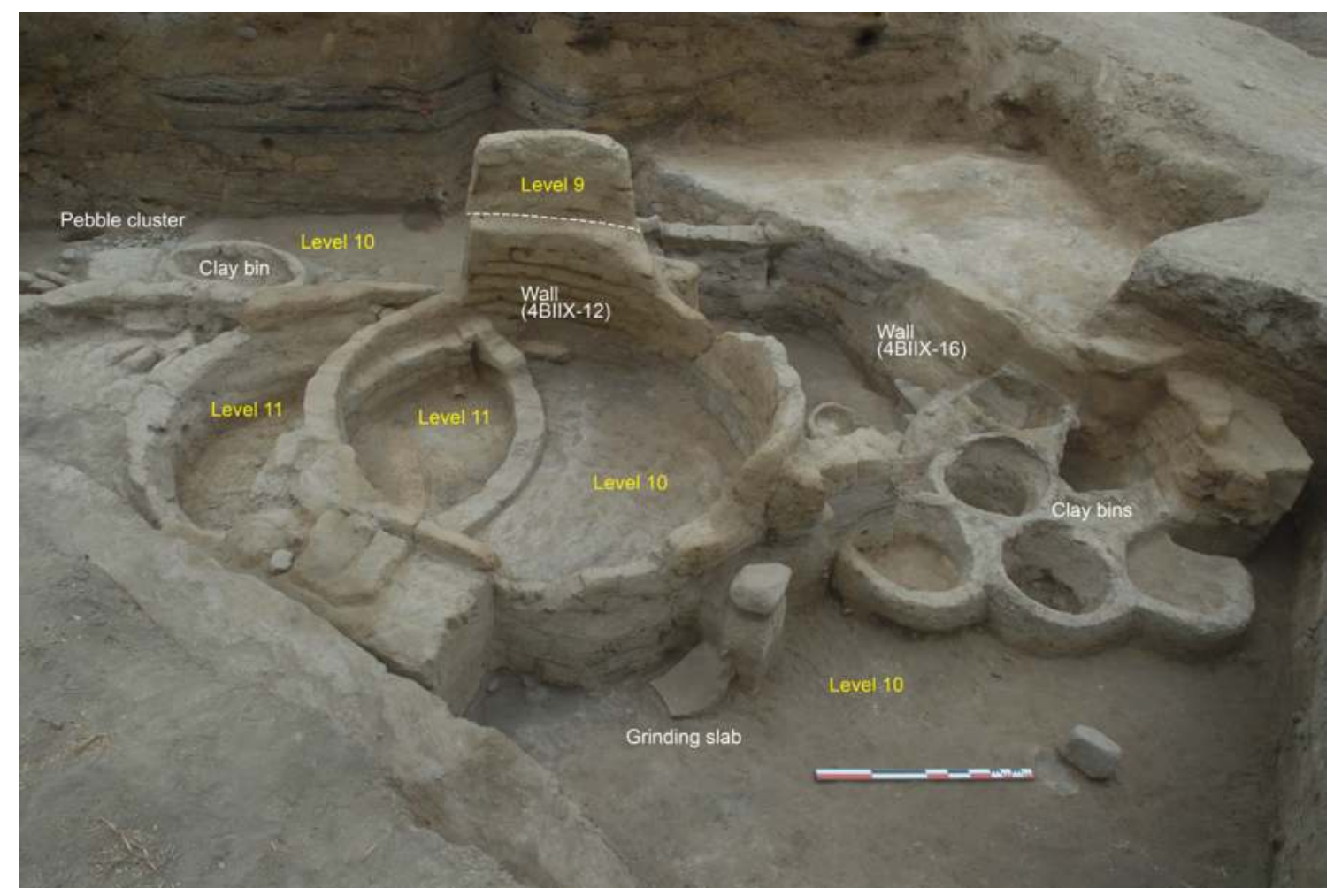

Fig. 4.27 Architectural remains of Levels 9, 10, and 11 in Square 4BII, seen from the northeast. Note that the round building (4BIIX-16) remains unexcavated. 


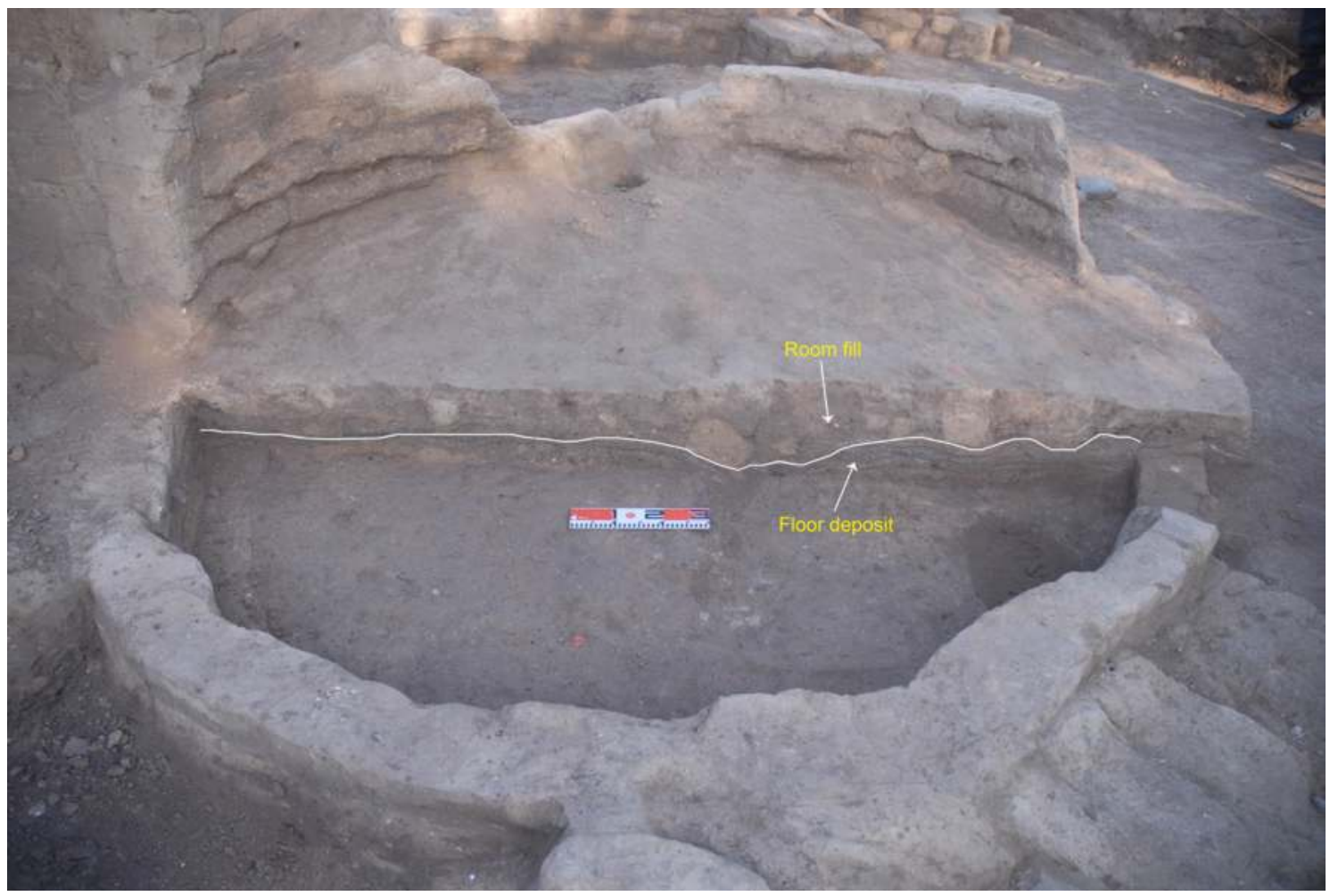

Fig. 4.28 Stratigraphic section of room fill and a floor inside the round structure (4BIIX-12), seen from the east. Note that the lamina of ashy, white, black, and brown sediments underlies the light brown sediment including tumbled mud-bricks.

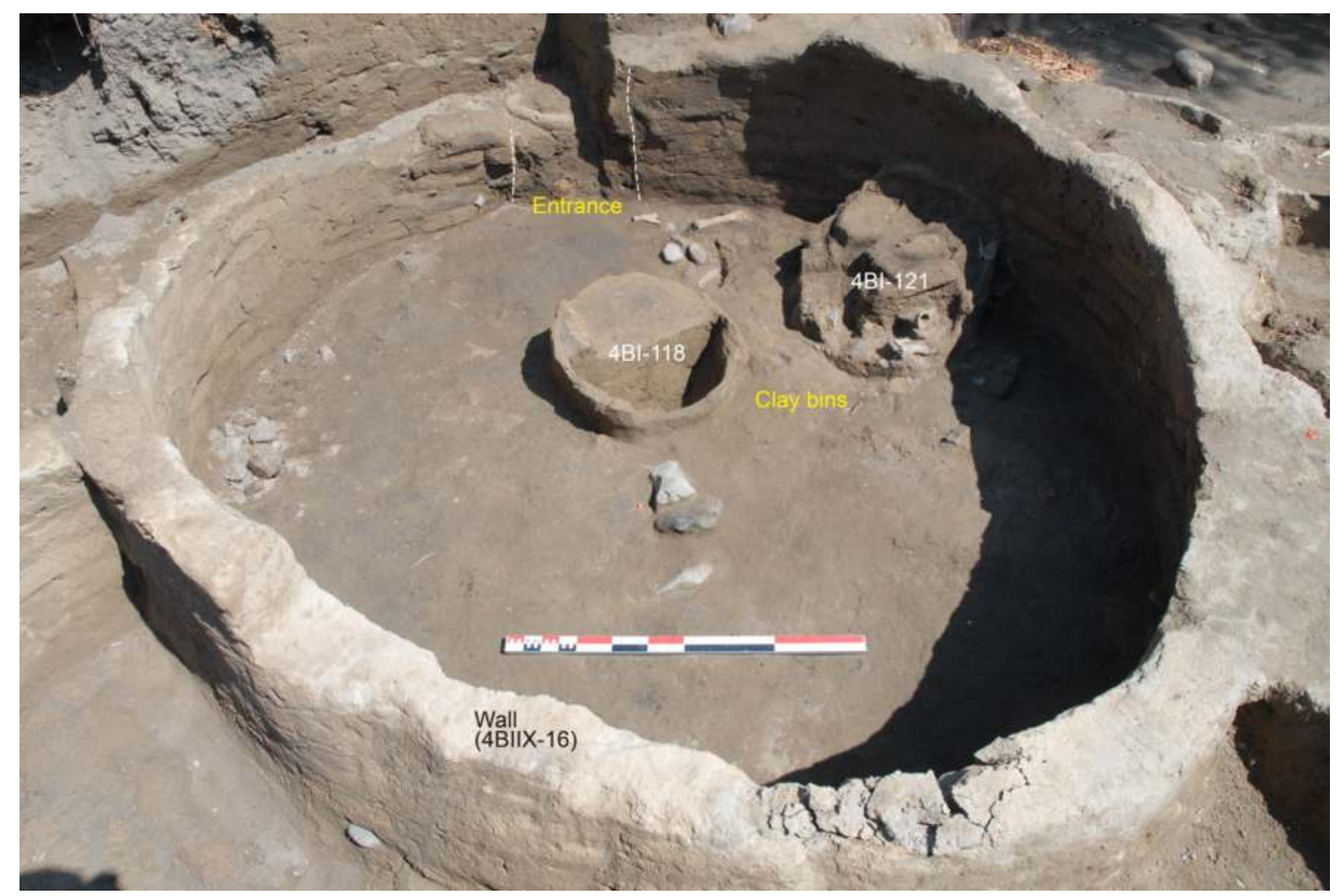

Fig. 4.29 The upper phase of the round building (4BIIX-16), corresponding to Level 10. Shown are two oval clay bins (4BI-118 and 121), an entrance, and the floor associated with cultural materials, seen from the south. 
other round structures by several architectural features (e.g., clay bins and an entrance) and finds (e.g., obsidian concentration and floor surface materials). We identified two occupational phases of this building (Fig. 4.8): the upper phase corresponds to Level 10, while the lower one corresponds to Level 11. Here, we describe several significant features and finds of the upper phase.

\section{Clay bins}

Two oval clay bins (4BI-118 and 121) were found inside the building (Figs. 4.29 and 4.30) at the northeastern area near the entrance. Although clay bins are prevalent features at Göytepe, they are usually located in external spaces. In fact, this building is also associated with a cluster of clay bins in the outdoor area to the east (Fig. 4.36). These outdoor bins are described later.

One indoor bin (4BI-118) measures ca. $50 \times 65$ $\mathrm{cm}$ and is a semi-subterranean feature, although its original height is unknown due to the destruction of the upper wall. The bin contained minimal refuse. The other indoor bin (4BI-121) is slightly larger (ca.
$65 \times 65 \mathrm{~cm}$ ) and at least $50-60 \mathrm{~cm}$ higher than the floor. Built against the house wall, its fill contained one bone artifact and a sheep skull with horncores. Although the fill was capped with many mud-bricks, these were randomly arranged and may have actually fallen from the wall during its collapse.

\section{Entrance}

Another feature characterizing this building is its entrance (Figs. 4.29 and 4.31). At the northern section of the building wall, we detected a ca. $60 \mathrm{~cm}$-wide gap filled with loose sediments and mud-brick fragments, some of which are burnt. A large handstone was also found. Although the upper portion of the entrance is missing, the opening was preserved no higher than $80 \mathrm{~cm}$. The bottom of the opening is higher than the base of the building wall by $30 \mathrm{~cm}$ (i.e., four courses of mud-bricks). People probably used the entrance continuously throughout both the lower and upper occupational phases.

\section{Obsidian concentration}

A concentration of chipped obsidian (4BI-111) was

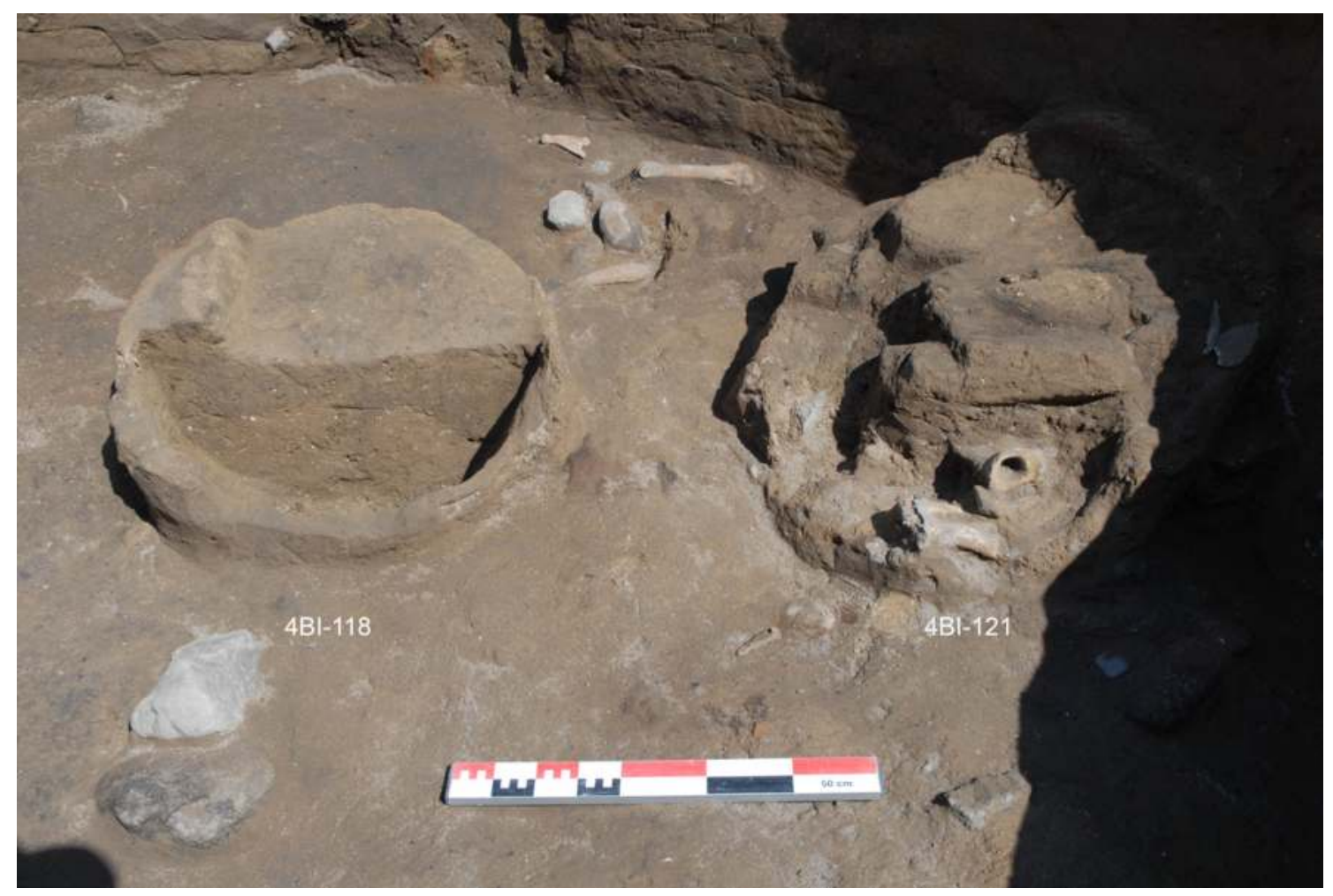

Fig. 4.30 Indoor clay bins (4BI-118 and 121) showing sections of fill, seen from the south. 

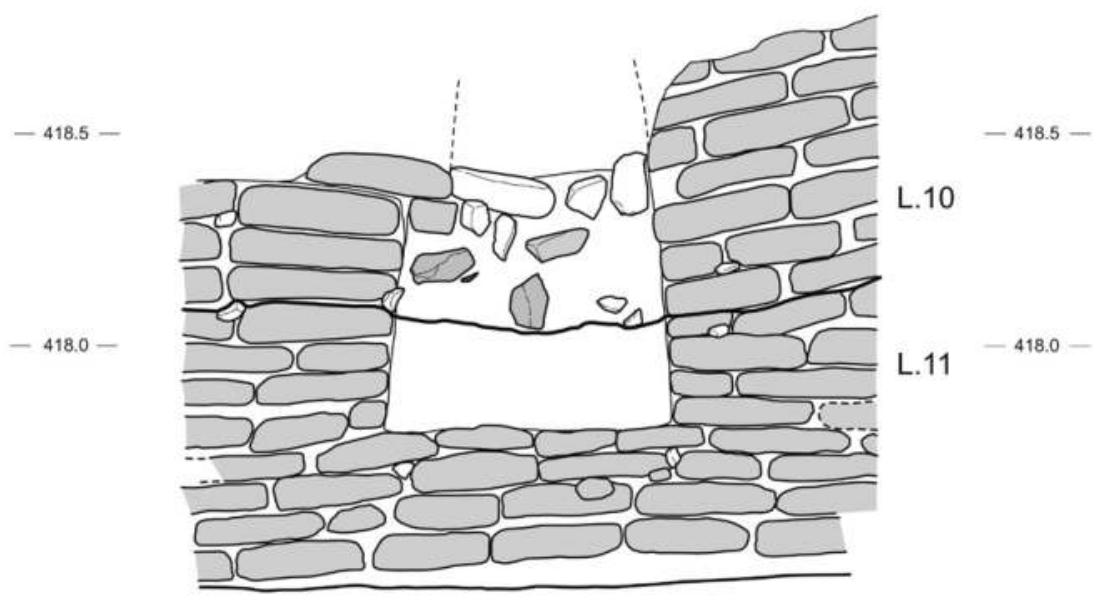

0 $1 \mathrm{~m}$

Fig. 4.31 Front view of the round building's (4BIIX-16) entrance, seen from the southwest. Note that the upper floor corresponds to the bottom of the upper fill.

recovered in the building's room fill (Fig. 4.32). Located in the southern part of the building's interior, the main cluster includes ca. 230 pieces, mostly consisting of flakes. These typically include cortical flakes and core-trimming elements, particularly platform rejuvenation flakes from blade cores. Although several different types of obsidian are observable, a translucent dark brown type is dominant. Because most obsidian pieces were deposited in the room fill containing mud-brick fragments from collapsed walls, they likely represent secondary deposition instead of the primary residue of stone tool production. To test this idea, sediment samples were collected from the concentration and immediately underlying deposits (4BI-113) for water sieving. This process should allow recovery of small chips, whose presence or absence would provide evidence of the depositional processes of the obsidian pieces.

\section{Grid sampling of room fill and floor deposits}

From immediately below the level of the obsidian cluster (4BI-111) to the floor level, deposits inside the building were excavated by $50 \times 50 \mathrm{~cm}$ grids to record spatial distributions of artifacts and ecofacts (Figs. 4.33 and 4.34). Deposits were vertically separated into five units (4BI-113, 114, 115, 116, and 117) for the upper phase. The former three units consist of room fill above the floor. The deposit of
4BI-113 represents the top unit and was $10-15 \mathrm{~cm}$ above the floor, while 4BI-115 is ca. $5-10 \mathrm{~cm}$ thick and was directly on the floor. In addition to these finds, the 4BI-116 deposit was detectable in the field as a surface marked by white fibrous remains (probably phytoliths as indicated by the geoarchaeological analyses presented in Chapter 7). The floor deposits (4BI-117) are 5-20 cm thick and consist of ashy grey sediments in the southern area (Grids A-N), which were gradually replaced by dark brown sediments in the northern area (Grids $\mathrm{O}-\mathrm{Y}$ ). The white fibrous remains were distributed across both grid areas. The floor sediments (4BI-116 and 117) were sampled for water-flotation to recover botanical and microrefuse remains.

\section{On-floor finds}

We recovered a high density of artifacts in the lower room fill and floor deposits inside the round building (4BIIX-16). This is in contrast to the other round structures in Square 4B, which usually contain very few remains. The finds include animal bones, bone artifacts (e.g., awls, hammers, and spatulas), chipped obsidian, and ground stones (e.g., axes, pounders, and handstones). Among the notable finds are obsidian blades coated with bitumen (Fig. 4.35). Three blade segments were fixed with bitumen in a slightly oblique position apparently without other haft material. 


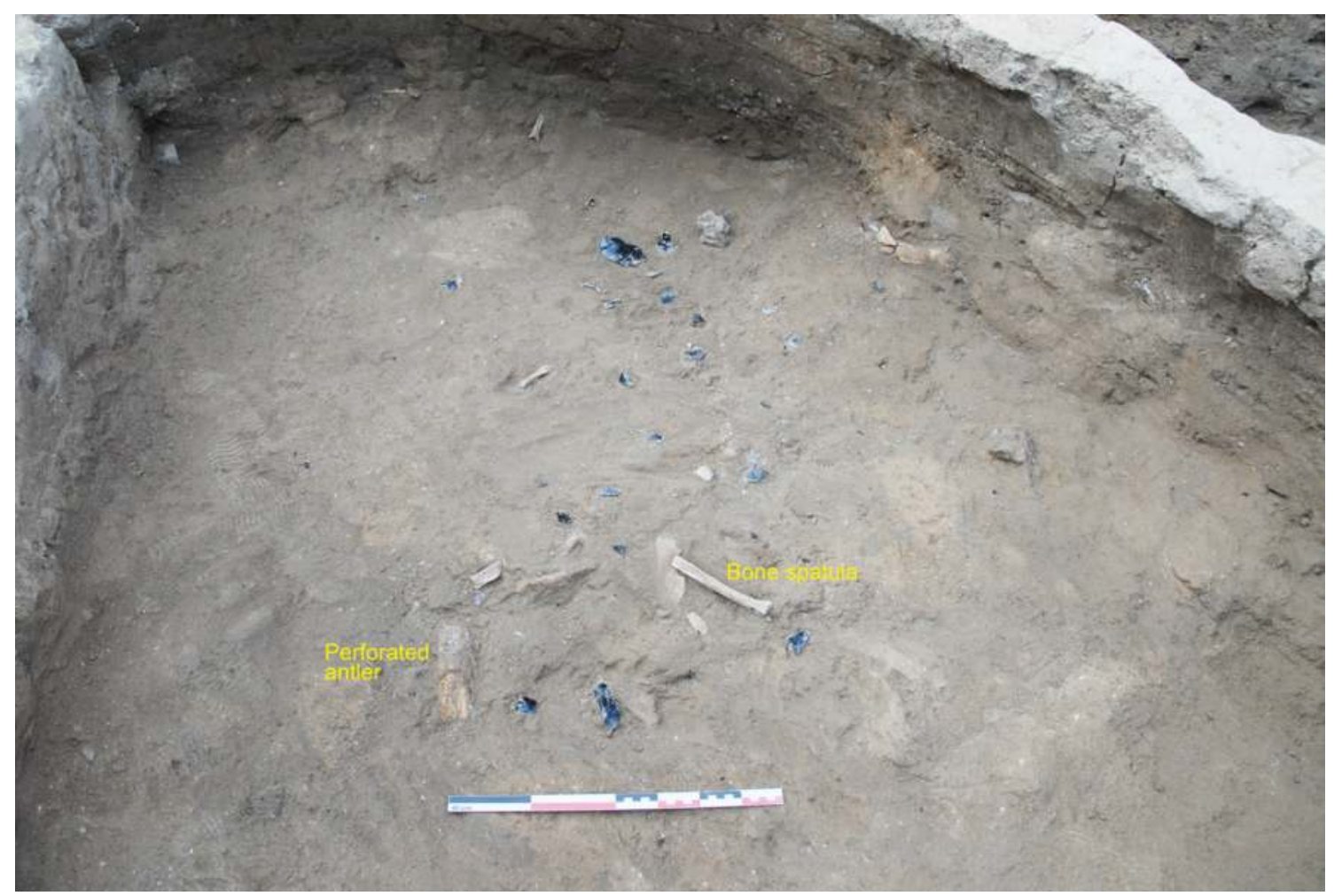

Fig. 4.32 Concentration of chipped obsidian artifacts inside the building (4BIIX-16), seen from the north. Note that obsidian pieces were associated with a perforated antler (hammer?) and a bone spatula.

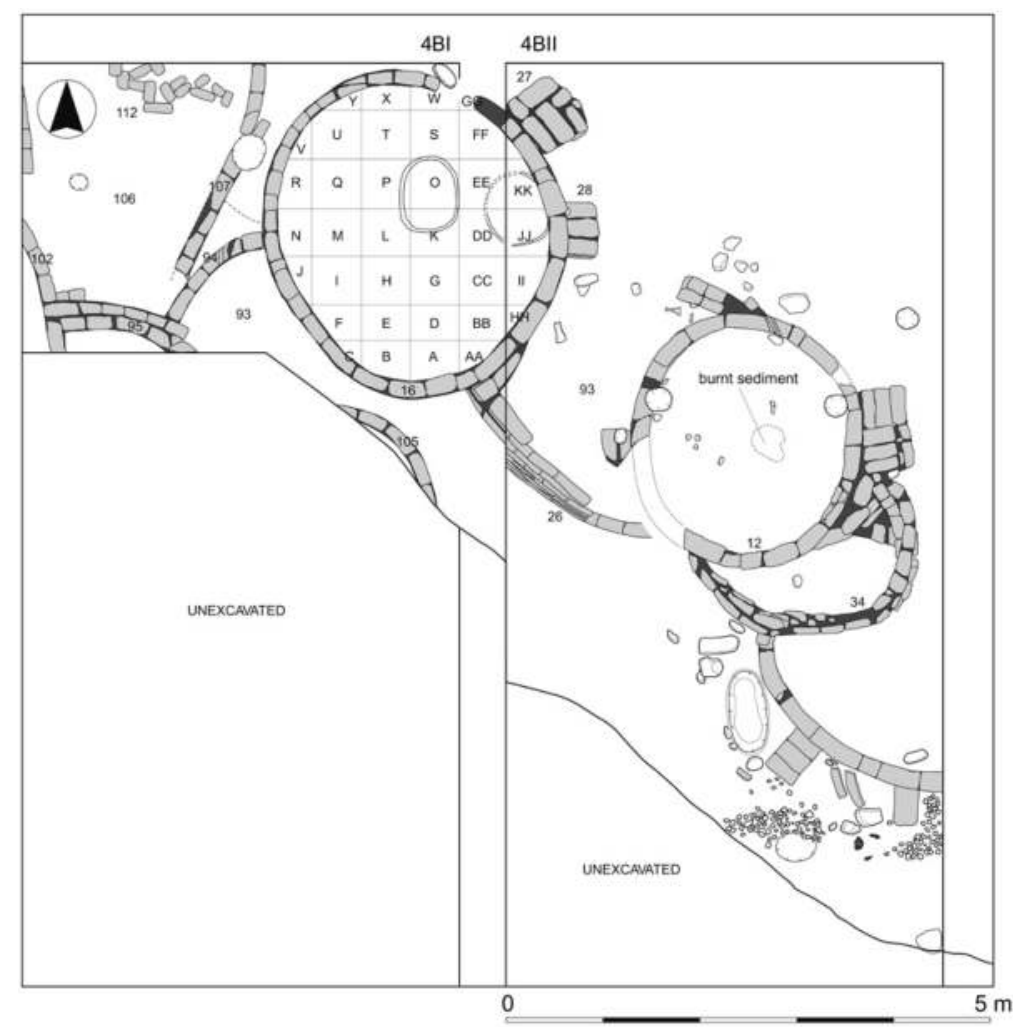

Fig. 4.33 Sampling grids within the round building (4BIIX-16) of Level 10 in Square 4B. 


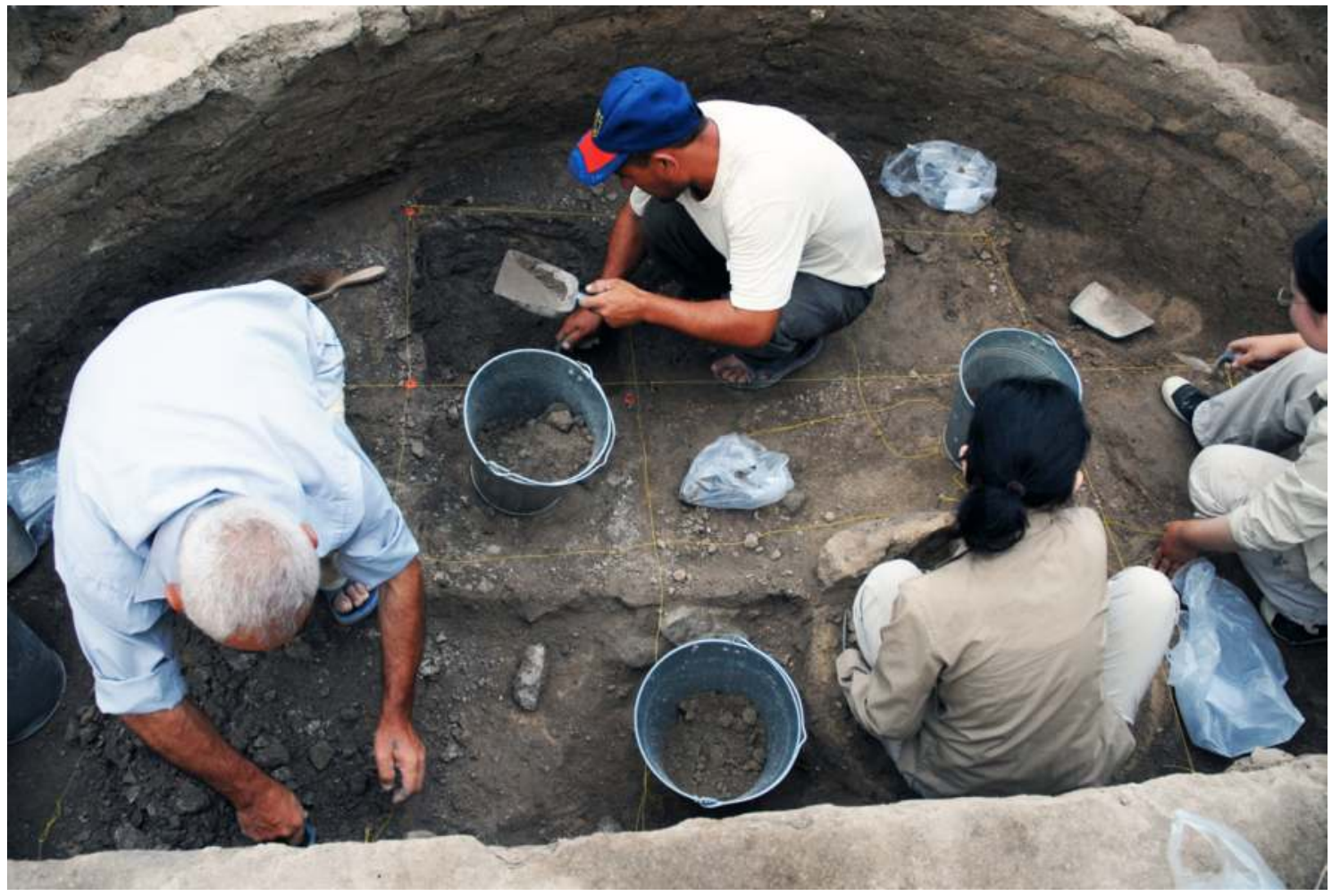

Fig. 4.34 The building's interior (4BIIX-16) was excavated using 50 x $50 \mathrm{~cm}$ grids with a combination of arbitrary and natural stratigraphic divisions.

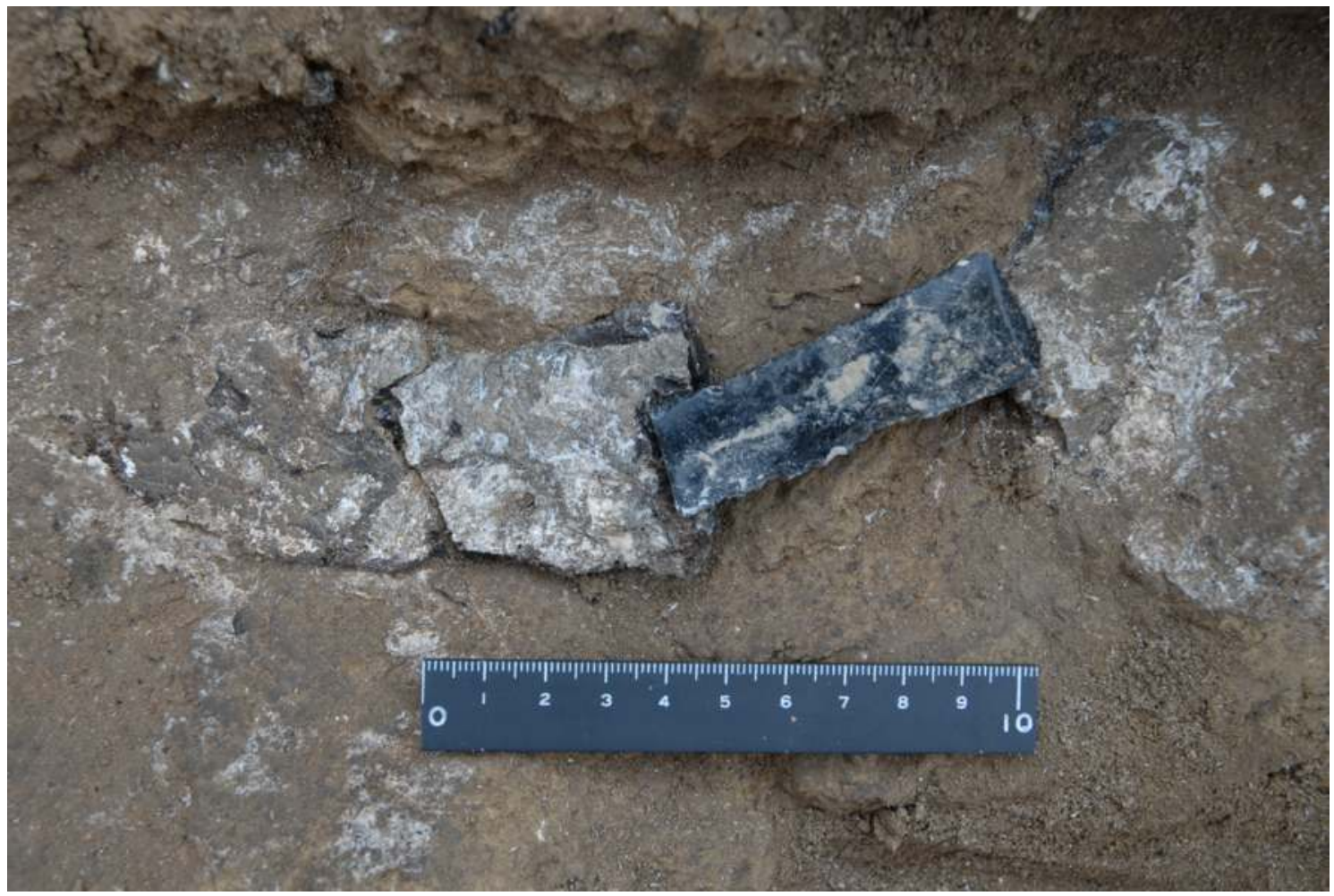

Fig. 4.35 Obsidian blades coated with bitumen found on the building floor (4BI-117). The floor deposits contained white fibrous remains (probably phytoliths). 


\subsubsection{Outdoor features: clay bins and activity areas}

In addition to the round buildings, we uncovered a number of other architectural features in what appear to have been outdoor activity areas.

\section{Clay bin cluster}

A concentration of seven mud-plastered oval bins was found between the two round structures (Figs. 4.26 and 4.27). Five are located in the middle of the cluster and are 50-60 cm in diameter (Fig. 4.36). The bin of 4BIIX-48 has a preserved rim and stands 50 $\mathrm{cm}$ tall, whereas the others lack upper portions. The two bins at 4BIIX-74 and 93 are smaller, measuring ca. $40 \mathrm{~cm}$ in diameter, and much shallower (ca. 10 $\mathrm{cm}$ ), although their true depth is unknown because the upper portions were largely destroyed.

These clay bins were likely associated with the round house of 4BIIX-16 because two of them (4BIIX-48 and 69) were built against the building's walls (4BIIX-16 and 27; Fig. 4.36). The bins' profiles suggest that those situated against the walls were built first, followed by the construction of additional bins leaning on the previous ones.

The bins were filled with dark brown to yellowish sediments, sometimes containing large bone tools and handstones in the middle of the fill. One of the bins (4BIIX-48) contained four courses of mud-bricks standing over the rim to make a short wall (4BIIX-28) like that of 4BIIX-27. To identify the bins' primary contents, we collected sediment samples of the fill for macro- and microbotanical studies.

\section{Clay bin with white fibrous deposits}

Another mud-plastered bin (4BIIX-94) was found in the northeastern corner of Square 4BII (Figs. 4.26 and 4.37). Although this bin is structurally similar to others in this level, it is somewhat distinct from the others in two ways. First, two complete upper grinding stones (4BIIX-94a and 94b) were placed near its base. Second, the sediments at the very

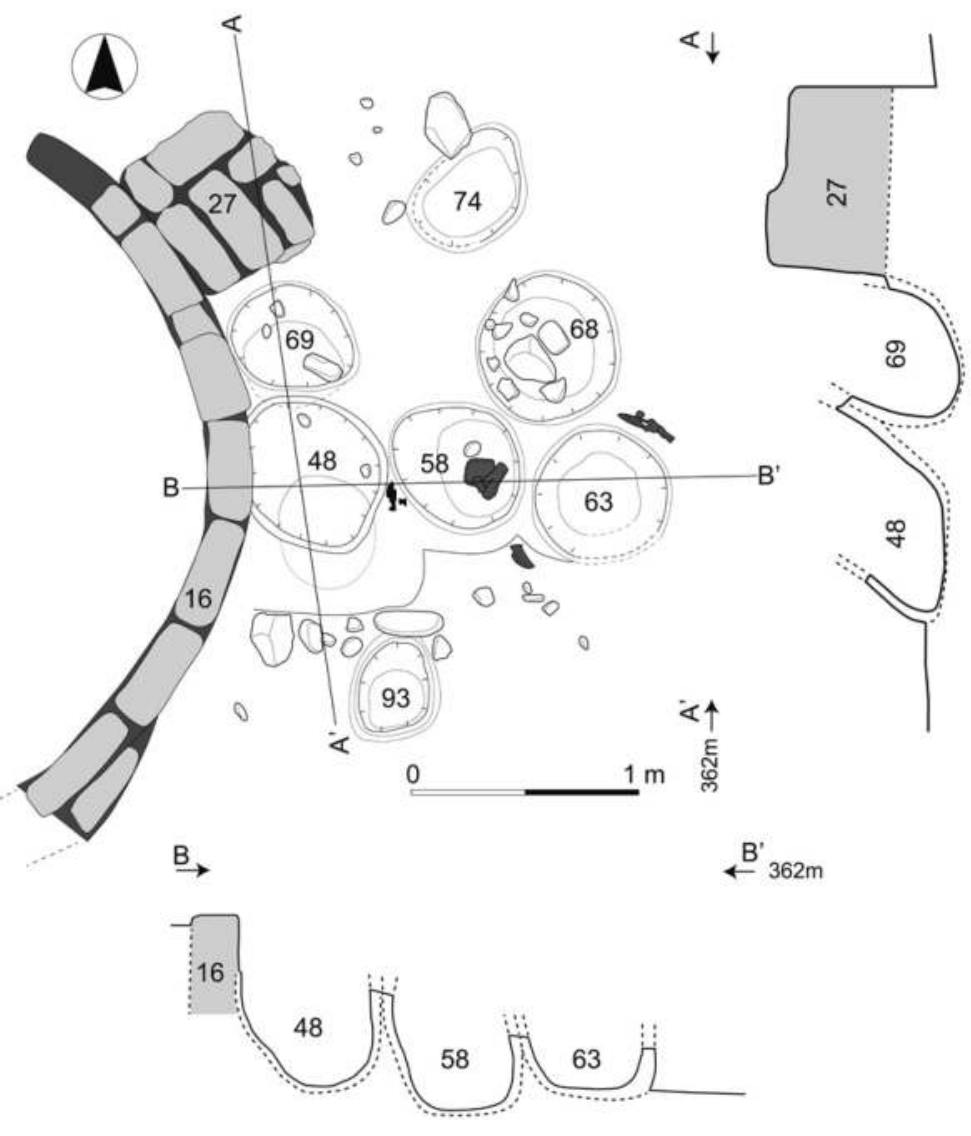

Fig. 4.36 Plans and profiles of clay bins in Level 10 in Square 4BII. 


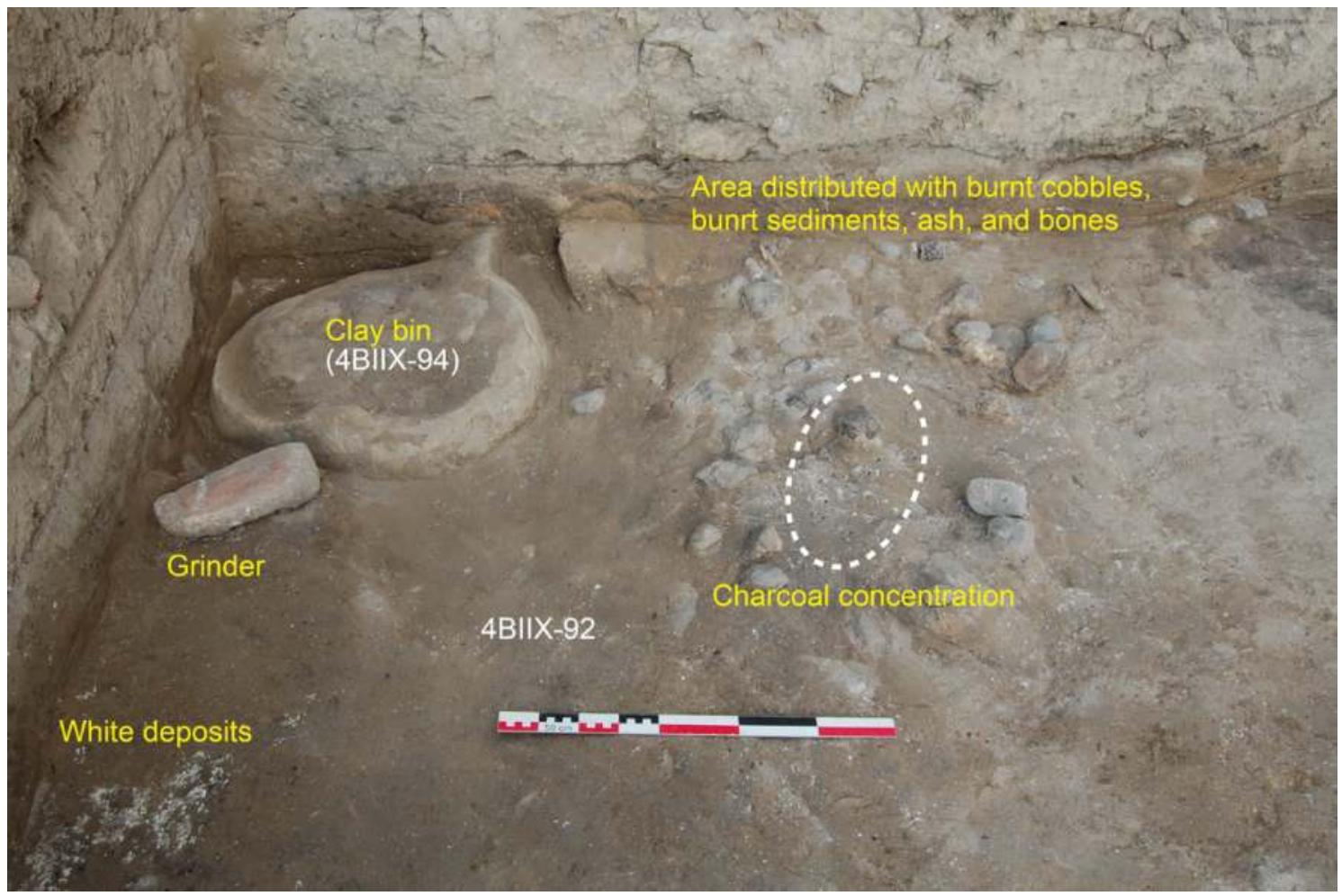

Fig. 4.37 A clay bin (4BIIX-94) in Level 10, adjacent to an area scattered with various domestic refuse, including a complete grinder, bone tools, angular cobbles, animal bones, and charcoal fragments in ashy sediments (4BIIX-92), seen from the west.

base, which were approximately $4 \mathrm{~cm}$ thick and found between the bin walls and overlying grinding stones, exhibited a white, fibrous appearance, likely reflecting rich phytolith concentrations (Fig. 4.38).

To examine the depositional processes of these distinct finds, we took sediment samples for phytolith and fecal spherulite analyses from several locations, including the middle and bottom fills, bottom wall of the bin, working surfaces of grinding stones left in the bin, and an adjacent activity area. The feature was also sampled for micromorphological analysis; a column of sediment approximately $14 \times 11 \times 12 \mathrm{~cm}$ in size was left unexcavated at the base of the bin and then taken en bloc (see Chapter 7 and Kadowaki et al. 2015 for results of the analyses).

\section{Outdoor activity areas}

We often recovered various domestic refuse near the clay bins. For example, the area adjacent to the bin at 4BIIX-94 was scattered with angular cobbles, animal bones, bone tools, and charcoal fragments in ashy sediments (4BIIX-92; Fig. 4.37). Beside this area, there was another concentration of bones and bone tools (4BIIX-97). A grinding slab was found in a tilted position south of the bone concentration (Fig. 4.27).

Another example of a probable activity area is located south of the wall of 4BIIX-31 (Figs. 4.26 and 4.39). This wall is attached to another one (4BIIX34) that was originally built in Level 11 and partly obliterated by construction of the round building (4BIIX-12) in Level 10. The area contained several different features, including the concentration of pebbles, burnt bones, and burnt bricks (4BIIX-57). Pebble sizes are homogeneous, measuring ca. $5 \mathrm{~cm}$ in diameter. They make up two concentrations, between which a cluster of burnt bones, burnt mud-bricks, and large stones were distributed. To the west of these, we found an oval clay bin (4BIIX-61) and a complete grinder (4BIIX-59a) lying horizontally. Although the upper part of the bin had been destroyed, a profile suggests that it was built against the wall (4BIIX-31).

\subsubsection{Level 11}

\subsubsection{Lower phase of a round mud-brick building (4BIIX-16)}

The lower phase of this building is defined by a floor close to the bottom of its wall (Figs. 4.8, 4.40, and 


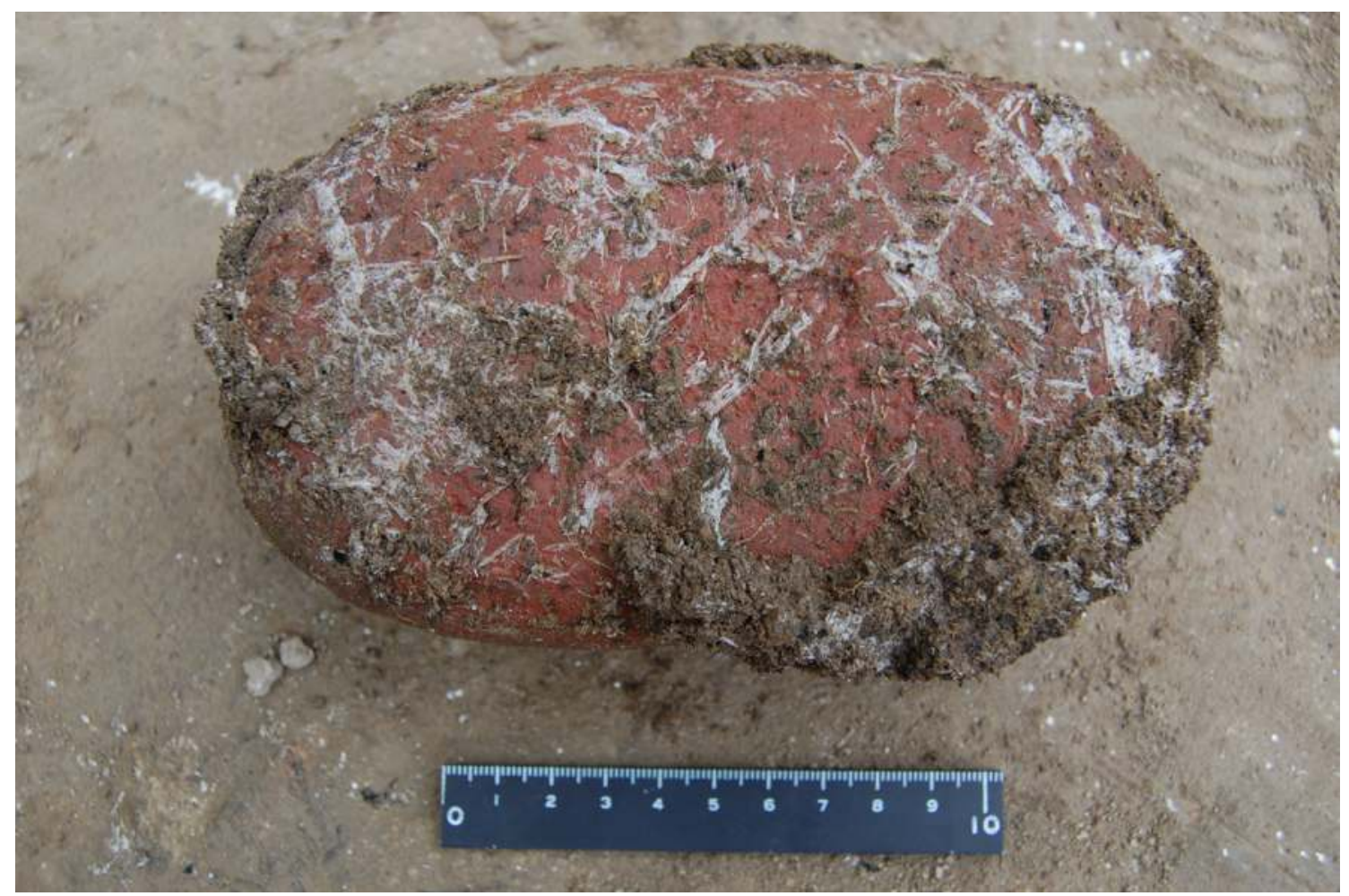

Fig. 4.38 White fibrous remains attached to the surface of a handstone (ca. $20 \mathrm{~cm}, 4 \mathrm{BIIX}-94 \mathrm{a}$ ) found near the bottom of the clay bin (4BIIX-94) in Level 10 in Square 4BII.

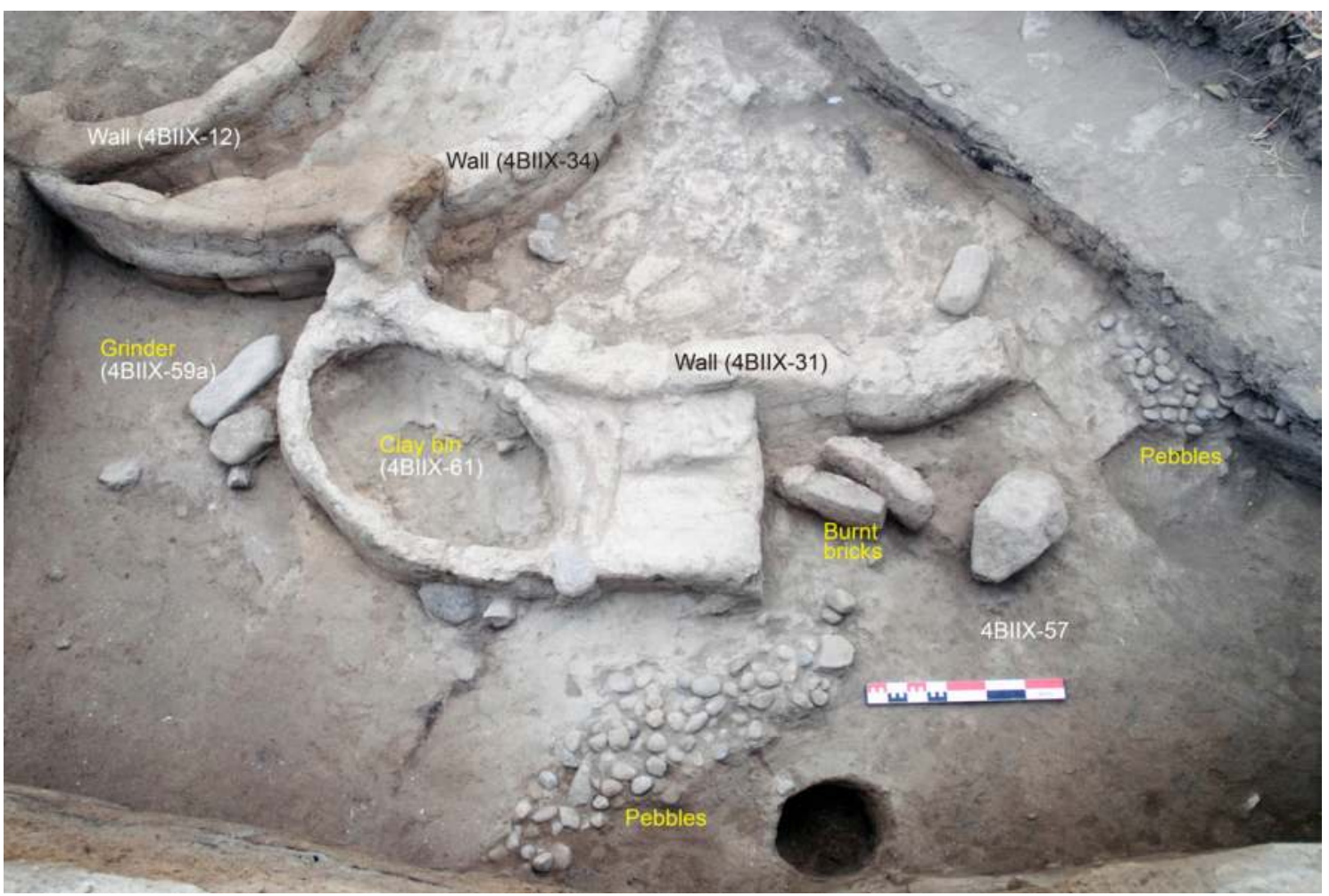

Fig. 4.39 Outdoor features south of Square 4BII, seen from the southwest. Note pebble clusters, clay oval bin, and a large grinder beside the bin. 


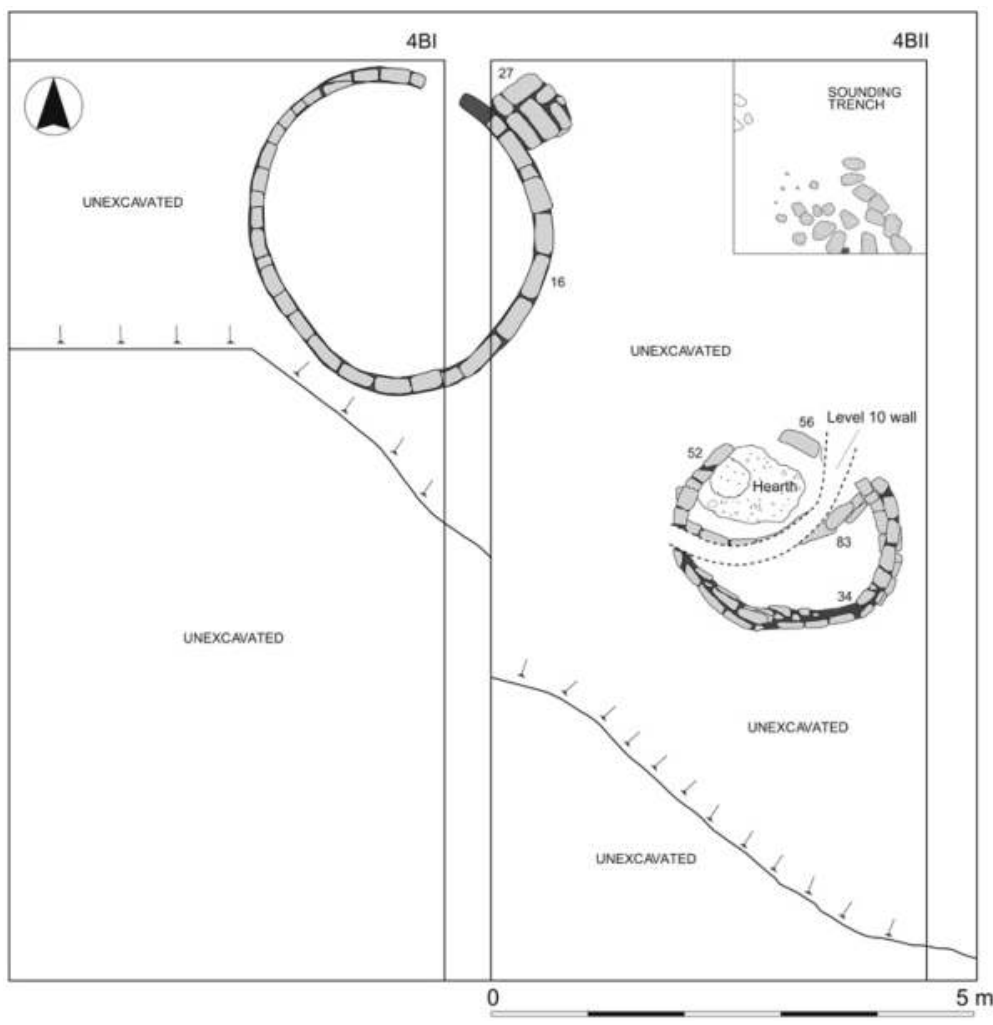

Fig. 4.40 Building remains of Level 11 in Square 4B.
4.41) and is thus considered to be part of the original occupation of the building after its construction. It was constructed on the surfaces of two different levels that were connected by a slope descending to the north. The higher surface to the south overlies an abandoned mud-brick structure of a lower building level. Thus, we believe that this building originally had a raised floor (or bench) in the southern part, where the surface is ca. $80 \mathrm{~cm}$ higher than the northern part near the entrance.

The stepped floor was subsequently leveled off by filling the northern area with mud-bricks, cobbles, ash, burnt sediments, and items such as ground stones and bone tools (4BI-131 and 132). As a result, the building's upper phase (Level 10) had a flat floor, on which the two clay bins were constructed (4BI118 and 121; see descriptions above). The entrance was partially filled at the bottom but remained open during the upper phase (Fig. 4.31).

\subsubsection{Round mud-brick building (4BIIX-34, 52, and 56)}

In the middle of Square 4BII, we detected several curvilinear walls (4BIIX-34, 52, and 56) that probably formed a round building ca. $2 \mathrm{~m}$ in diameter (Figs. 4.40 and 4.42). A hearth was recovered inside near the northern wall. We also found a curvilinear wall (4BIIX-83) that partitions the building's interior. However, this wall may represent a foundation for the upper level wall (4BIIX-12 in Level 10) that partially destroyed the round structure of Level 11 . In fact, the northwestern quarter of the building in Level 11 (walls 4BIIX-52 and 56) underlies the round structure (4BIIX-12) in Level 10, whereas the rest of the other (wall 4BIIX-34) was still exposed on the surface during Level 10.

\subsubsection{Level 12}

From this level downward, we limited excavations to a small pit of $2 \times 2 \mathrm{~m}$ located in the northeast corner of 4BII in order to preserve the architectural remains of Levels 10 and 11 (Fig. 4.43). The stratigraphic section of this pit is shown in Fig. 4.44. Similar to the upper levels, the levels on which each structure was built are distinguished by alternating thin layers of blackish-gray ash containing plenty of charcoal and small clay rubble, most likely representing occupational debris.

Architectural remains in Level 12 were discovered immediately below the ground surface of Level 11. A mud-brick curvilinear wall (4BIIX-107) was located in the southwest corner of this pit, presumably 


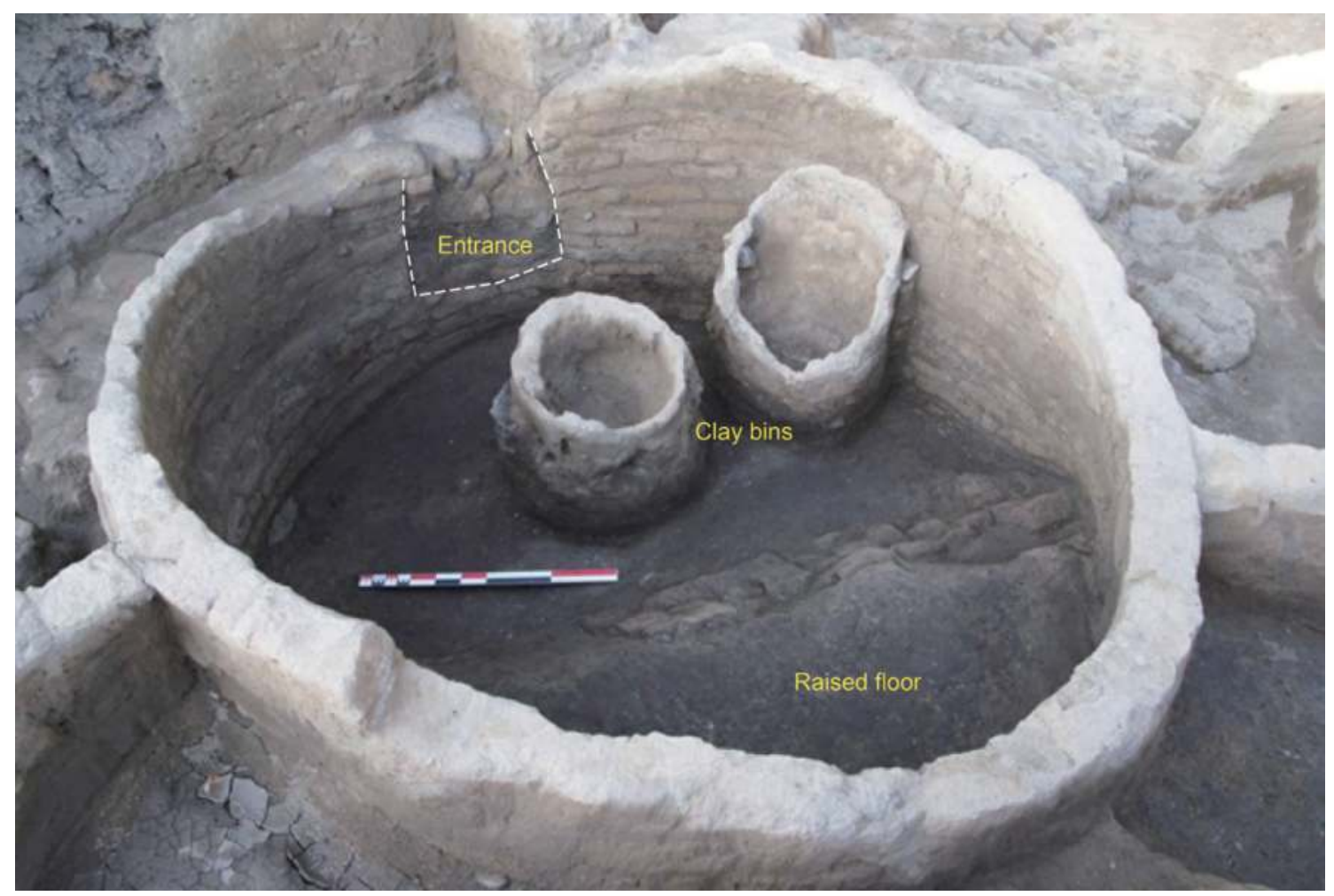

Fig. 4.41 Lower phase of the round building (4BIIX-16) in Level 11, seen from the southwest. Note the raised floor in the southern interior. Two clay bins were constructed on the upper floor (Level 10) after the northern interior was filled to level off the floor surface.

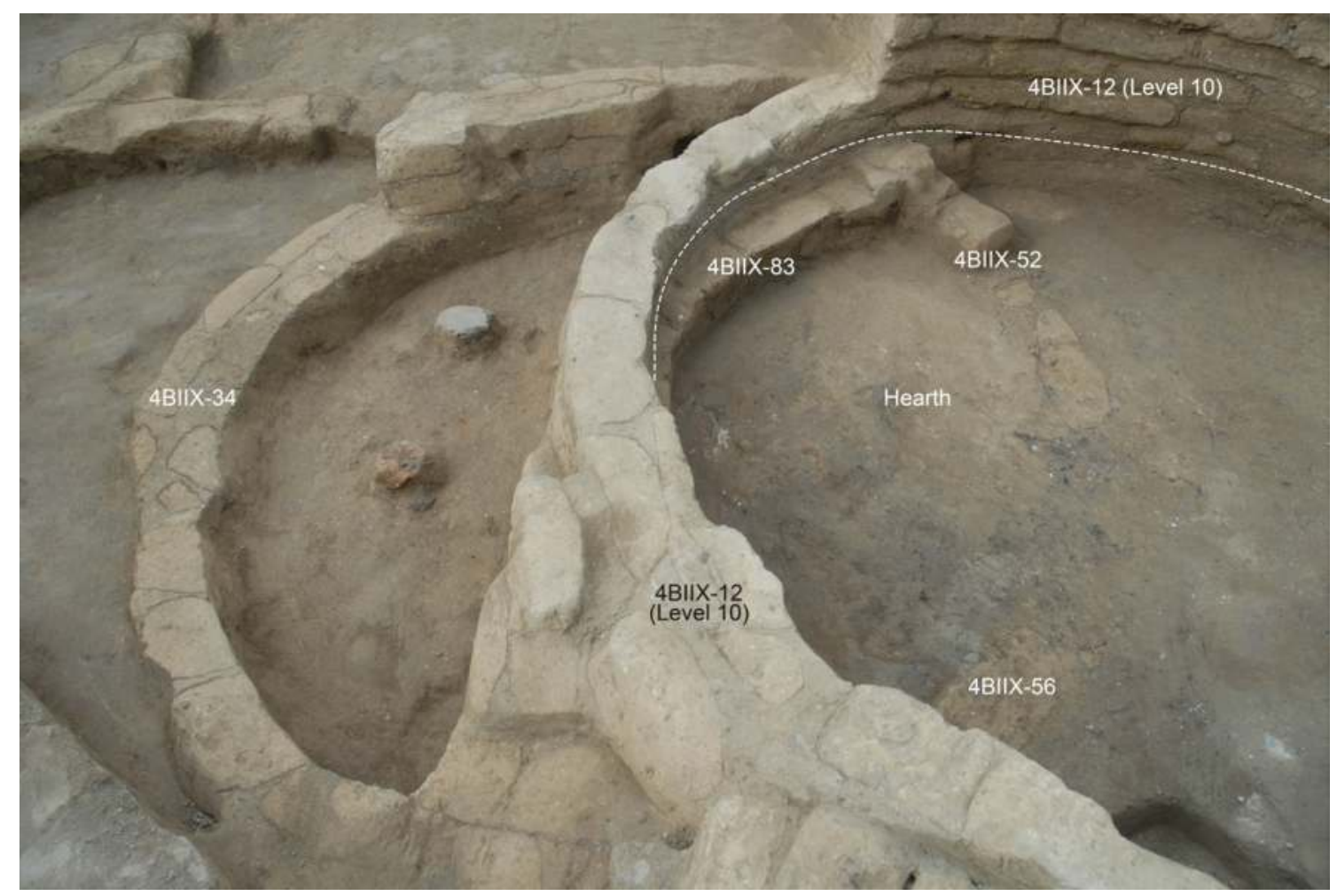

Fig. 4.42 Superposition of the round building (4BIIX-12) of Level 10 over that of Level 11 (4BIIX-34, 52, and $56)$, seen from the northeast. A dashed line marks the bottom of the wall (4BIIX-12). The structure of Level 11 contains a hearth and wall (4BIIX-83). The wall may have served as a foundation for the $4 \mathrm{BIIX}-12$ wall. 


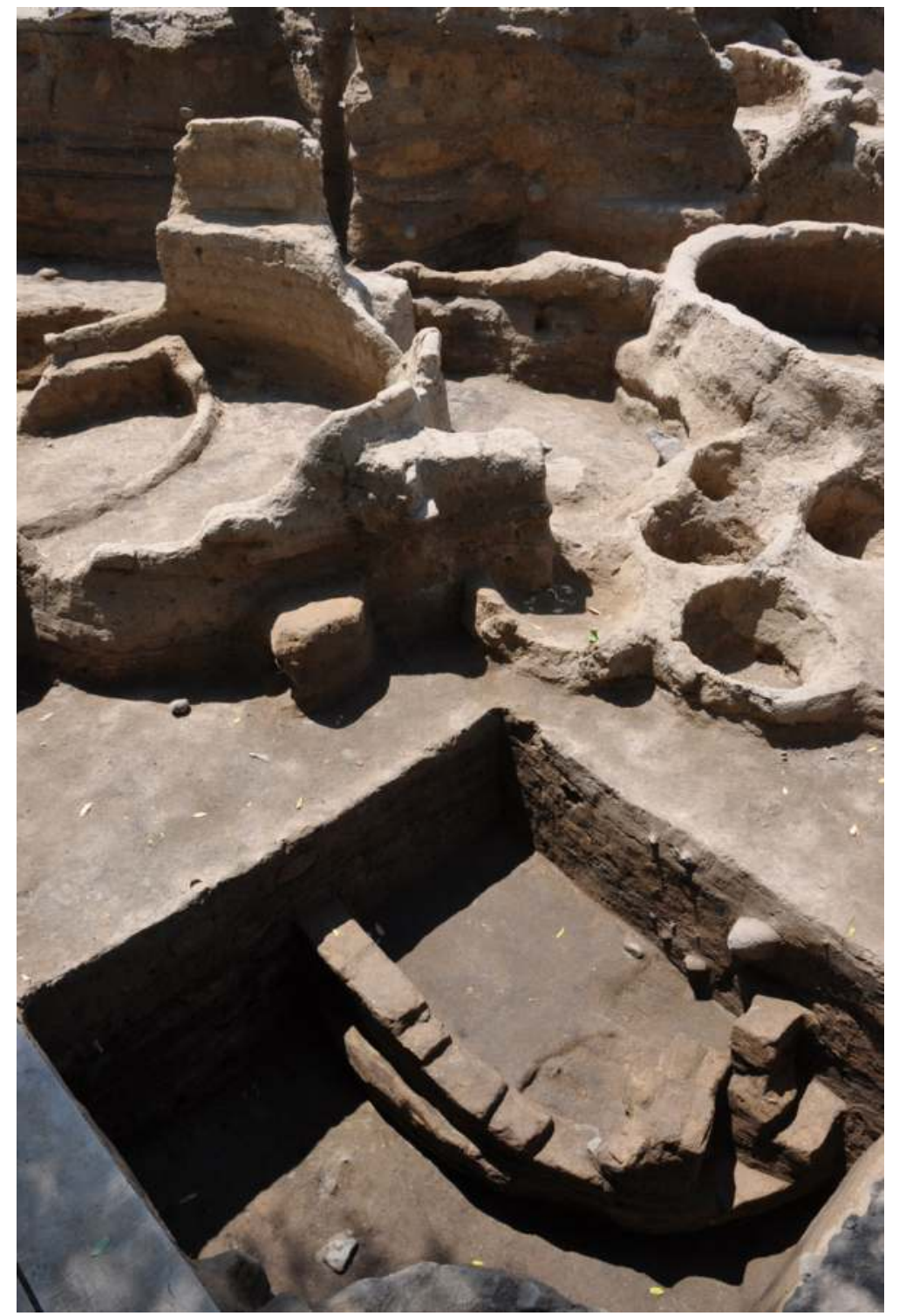

Fig. 4.43 The sounding pit of Square 4BII, seen from the northeast. forming a round structure similar to those found in other excavation areas of this mound. Although the upper part had been eroded following the slope of the mound, preservation was quite good (Figs. 4.45 and 4.46). The wall was made of yellowish-brown mud-bricks (Fig. 4.47) and stood nearly $70 \mathrm{~cm}$ high. Bricks were arranged in one row in the upper parts while the lower and bottom parts were constructed in triple or double rows, respectively (Fig. 4.48). Both regular mud-bricks and halved bricks were used to construct the wall. Ashy gray mortar with charcoal and gypsum inclusions was used to cement each brick; sometimes small cobbles were embedded in the mortar.

This structure's fill consisted of plenty of mudbrick rubble, under which a living floor (4BIIX-
126) was recovered. This was characterized by blackish-brown organic sediments containing animal bones and charcoal. However, the floor deposits did not yield white fibrous remains, which had been occasionally recovered from upper level building floors and thought to represent some sort of a floor structure.

\subsubsection{Level 13}

The underlying Level 13 yielded a unique structure (Figs. 4.49 and 4.50) consisting of a bank neatly covered with yellowish-brown mud-bricks (4BIIX122 and 123). Excavations revealed fairly good preservation. The bank faces north and stands at least $150 \mathrm{~cm}$ high. It was made of at least ten layers of mud-bricks laid in short rows, with longer rows seen 


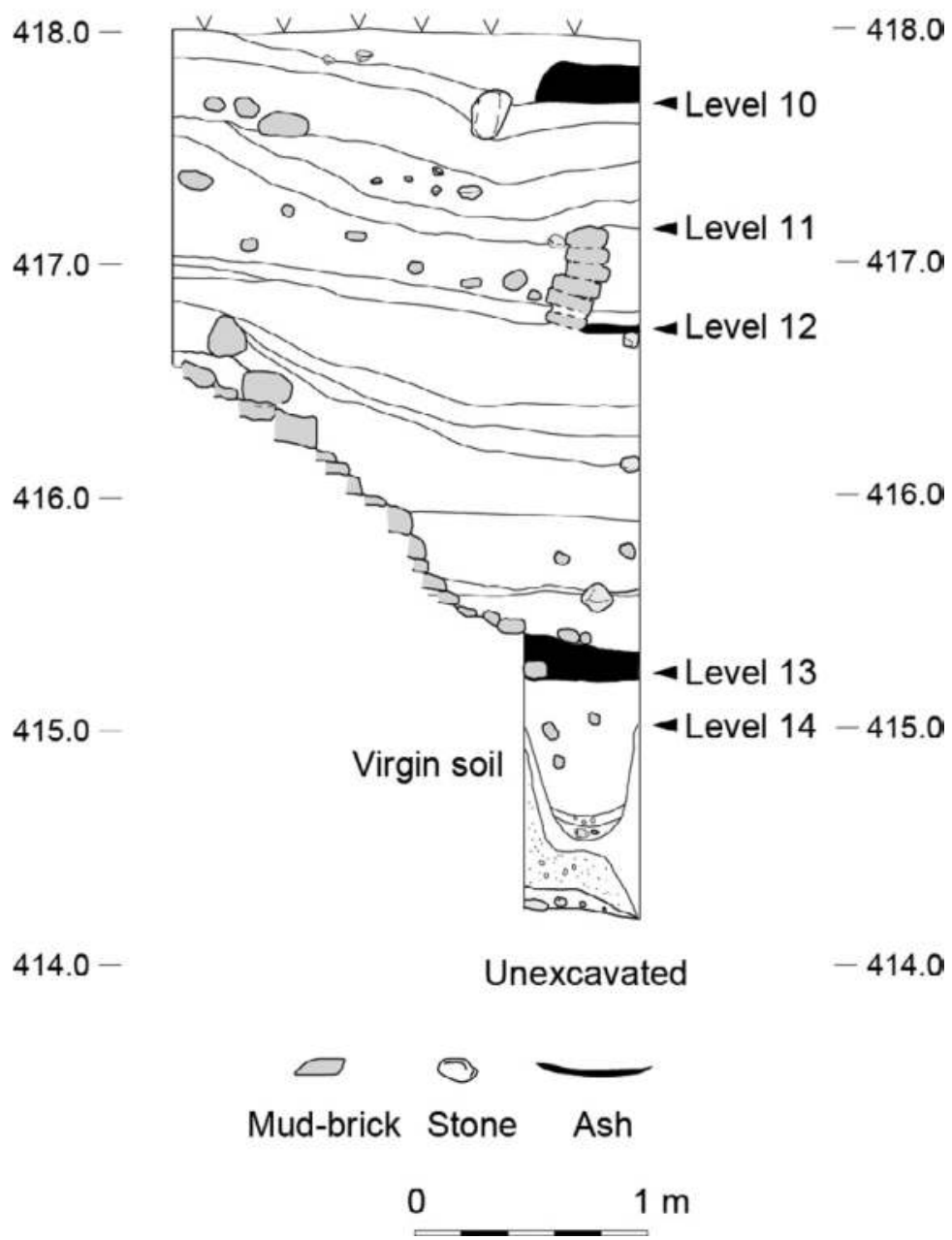

Fig. 4.44 West section of the sounding pit of Square 4BII. at the top (Fig. 4.51). Since the interior part was not excavated, it remains unknown whether it was filled with mud-bricks. Artifacts unambiguously associated with this construction were extremely rare; only a small number of pottery sherds were recovered from the fill.

The function of this bank itself remains unknown. Possible functions include a wall protecting against water; a settlement boundary wall; the foundation for a large structure; or protection against a landslide of the mound slope. Regardless, its function would have been comparable to another massive wall or bank discovered on the mound's eastern edge (Square 96F; see Chapter 5). This structure is also reminiscent of the Level 11 structure (4BIIX-16) mentioned earlier that had a floor with two steps connected by a slope descending north. The slope actually represents an abandoned mud-brick structure from a lower building level, which might have actually been a bank comparable to the one in Level 13. In sum, similar banks or terrace structures have always been recovered close to the edge of the mound.

\subsubsection{Level 14}

We again limited our excavation area from this level downward to preserve the unique structure of Level 13 (Fig. 4.52). A tiny area of $100 \times 80 \mathrm{~cm}$ in the northwestern corner of 4BIIX, where the Level 13 terrace wall was absent, was chosen for further excavation. As a result, an interesting feature was recovered: a ditch running in an east-west direction with a V-shaped profile (4BIIX-129; Fig. 4.53). Its current depth was at least $60 \mathrm{~cm}$, but its original depth or width could not be determined because both upper side portions were beyond the excavation boundary. The ditch contained distinct fill; the upper part included brown soil with patches of gypsum, while the lower part was filled with pebbles. This 


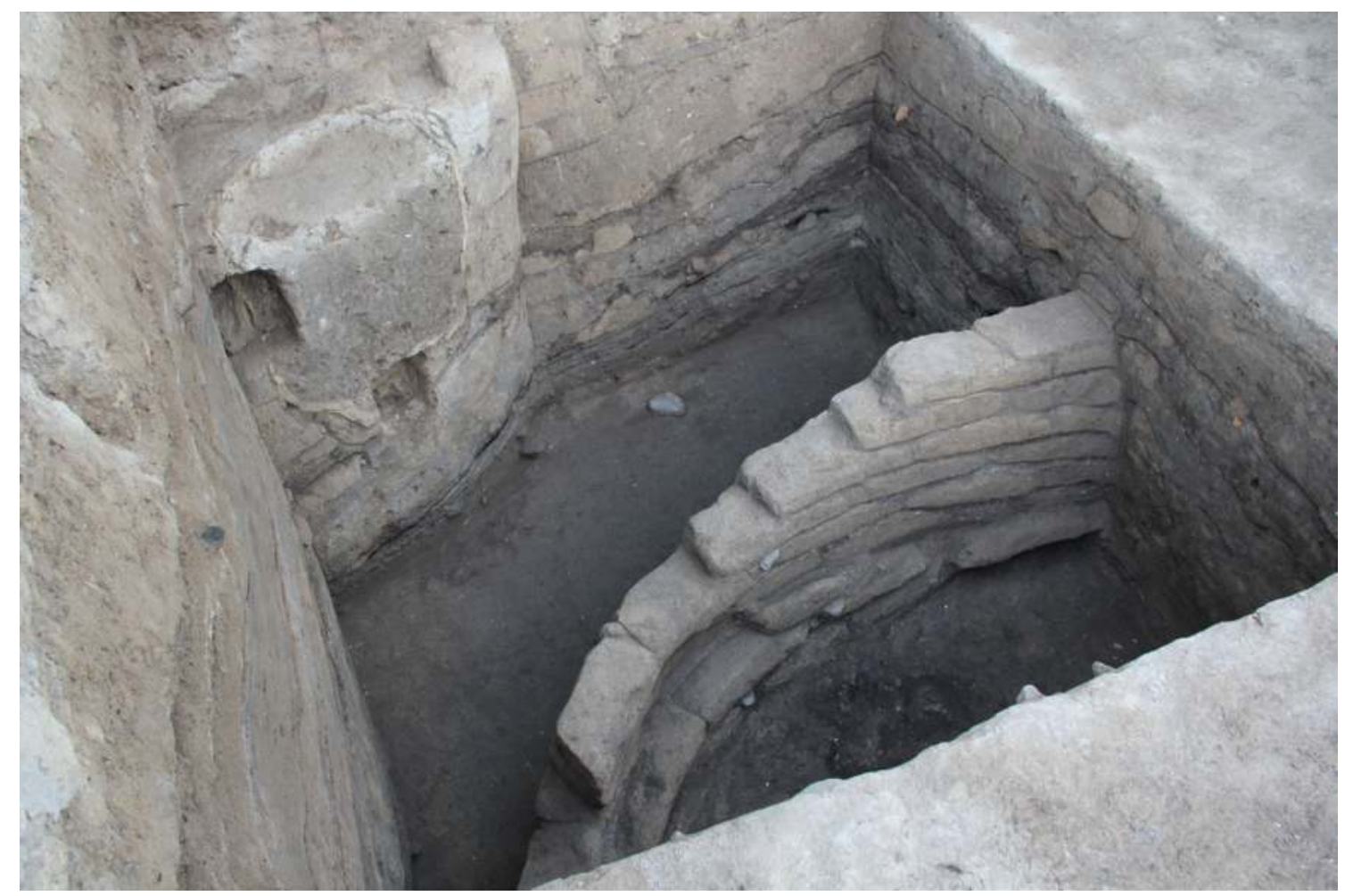

Fig. 4.45 Building remains of Level 12 in Square 4B, seen from the west. Note that the bin to the left belongs to Level 10.
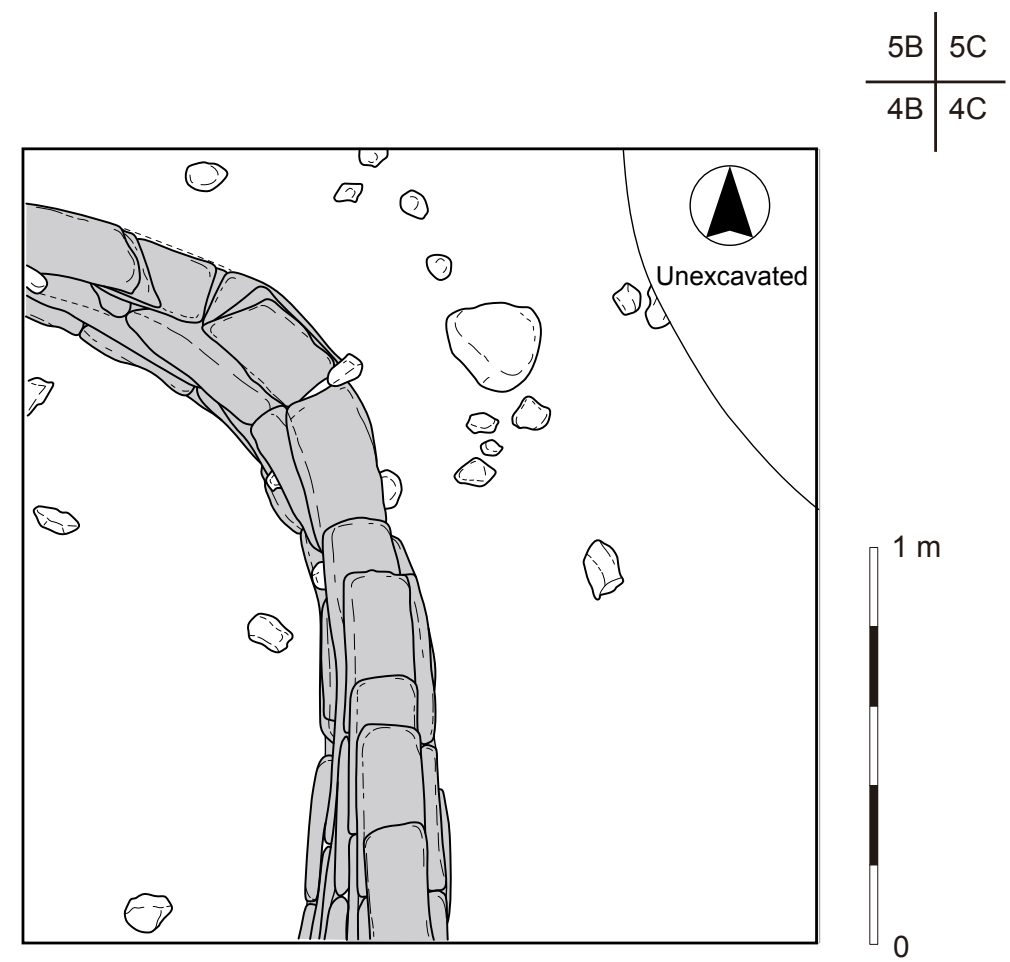

Fig. 4.46 Features of Level 12 in the sounding pit of Square 4B. 


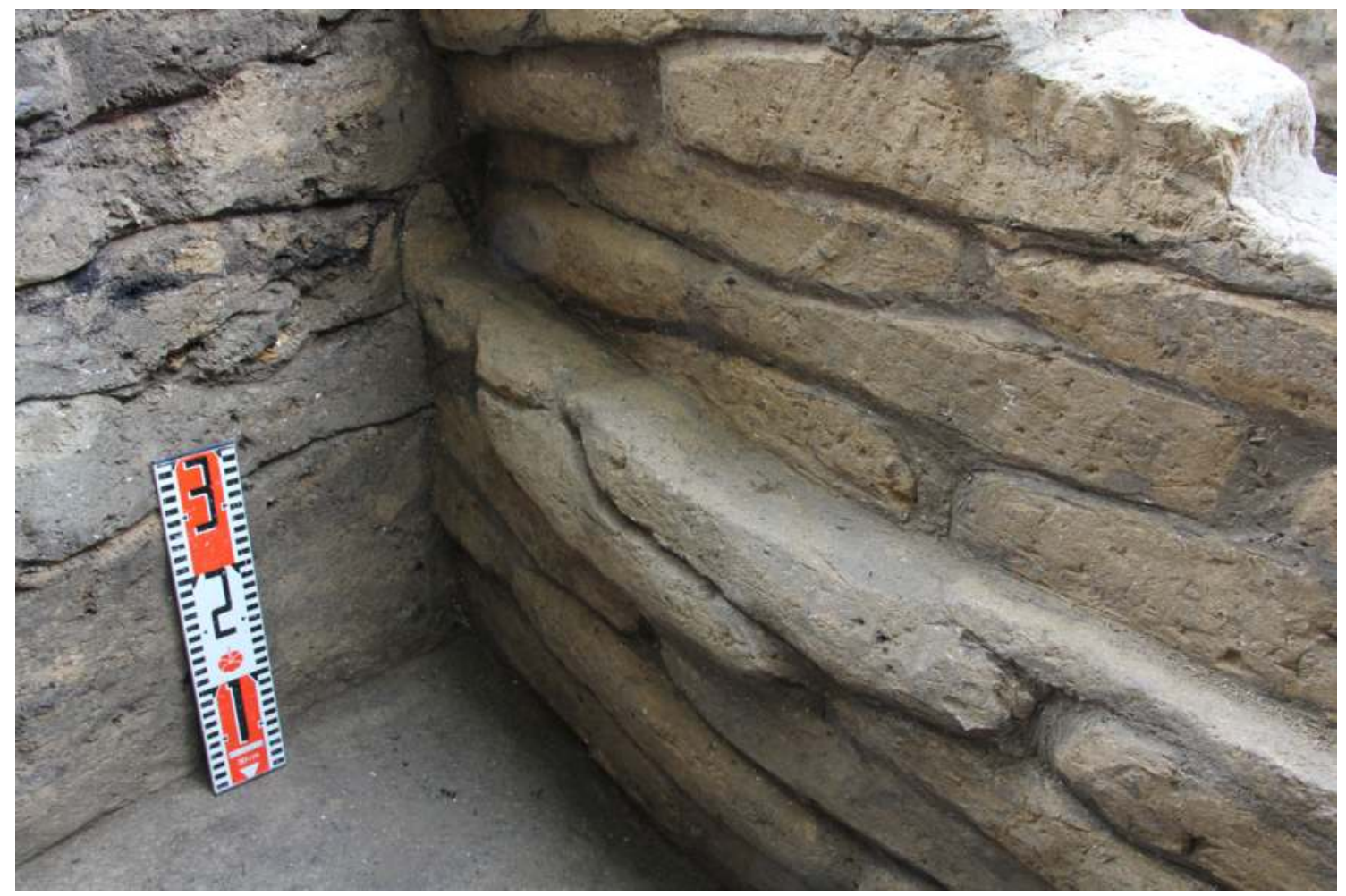

Fig. 4.47 Side view of the mud-brick wall of Level 12 in the sounding pit of Square 4B, seen from the east.

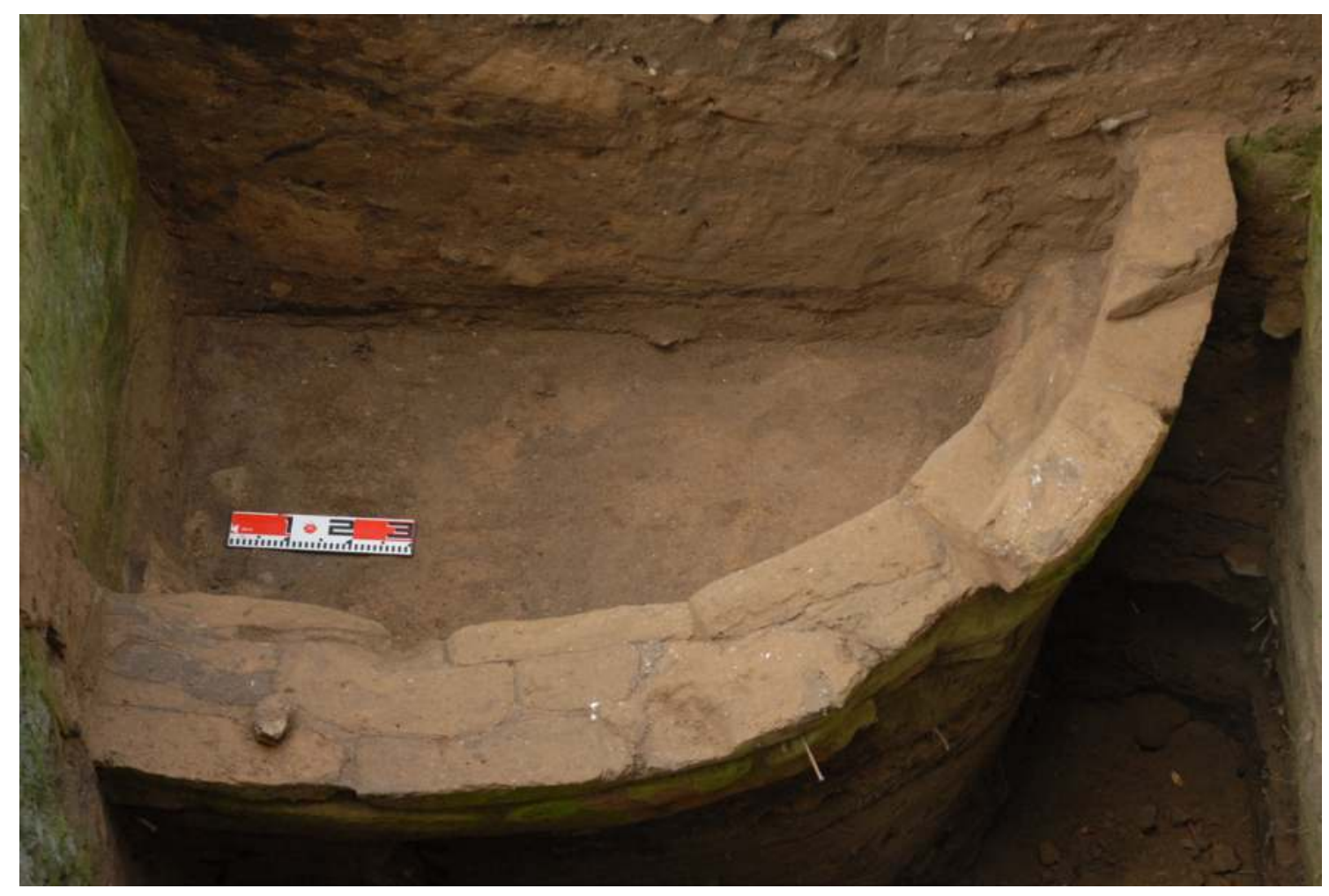

Fig. 4.48 Top view of the mud-brick wall of Level 12 in the sounding pit of Square 4B. 


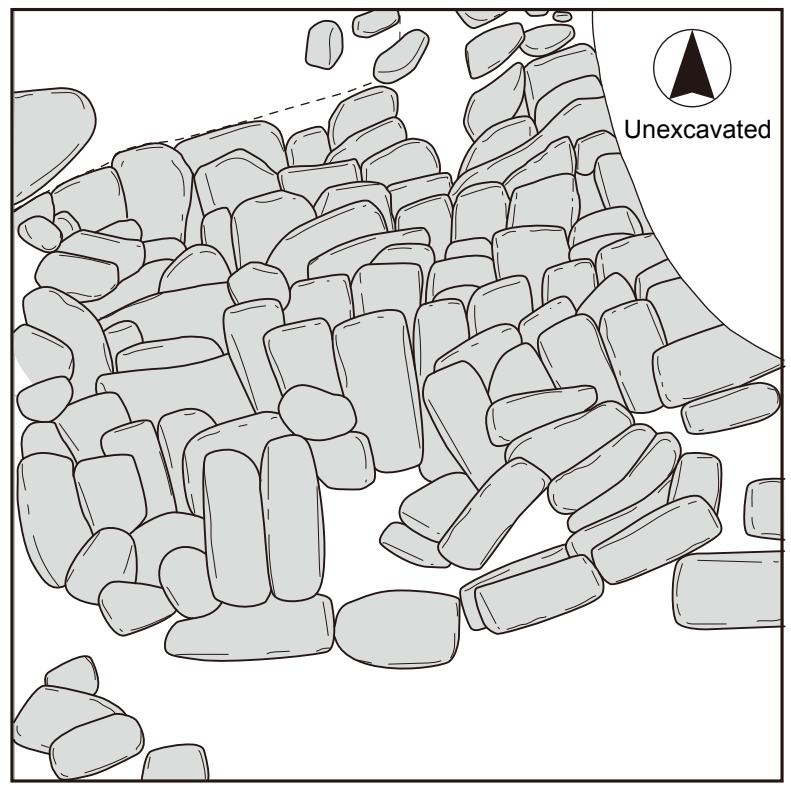

$$
\begin{array}{l|l}
5 \mathrm{~B} & 5 \mathrm{C} \\
\hline 4 \mathrm{~B} & 4 \mathrm{C}
\end{array}
$$

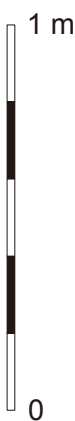

Fig. 4.49 Features of Level 13 in the sounding pit of Square 4B.

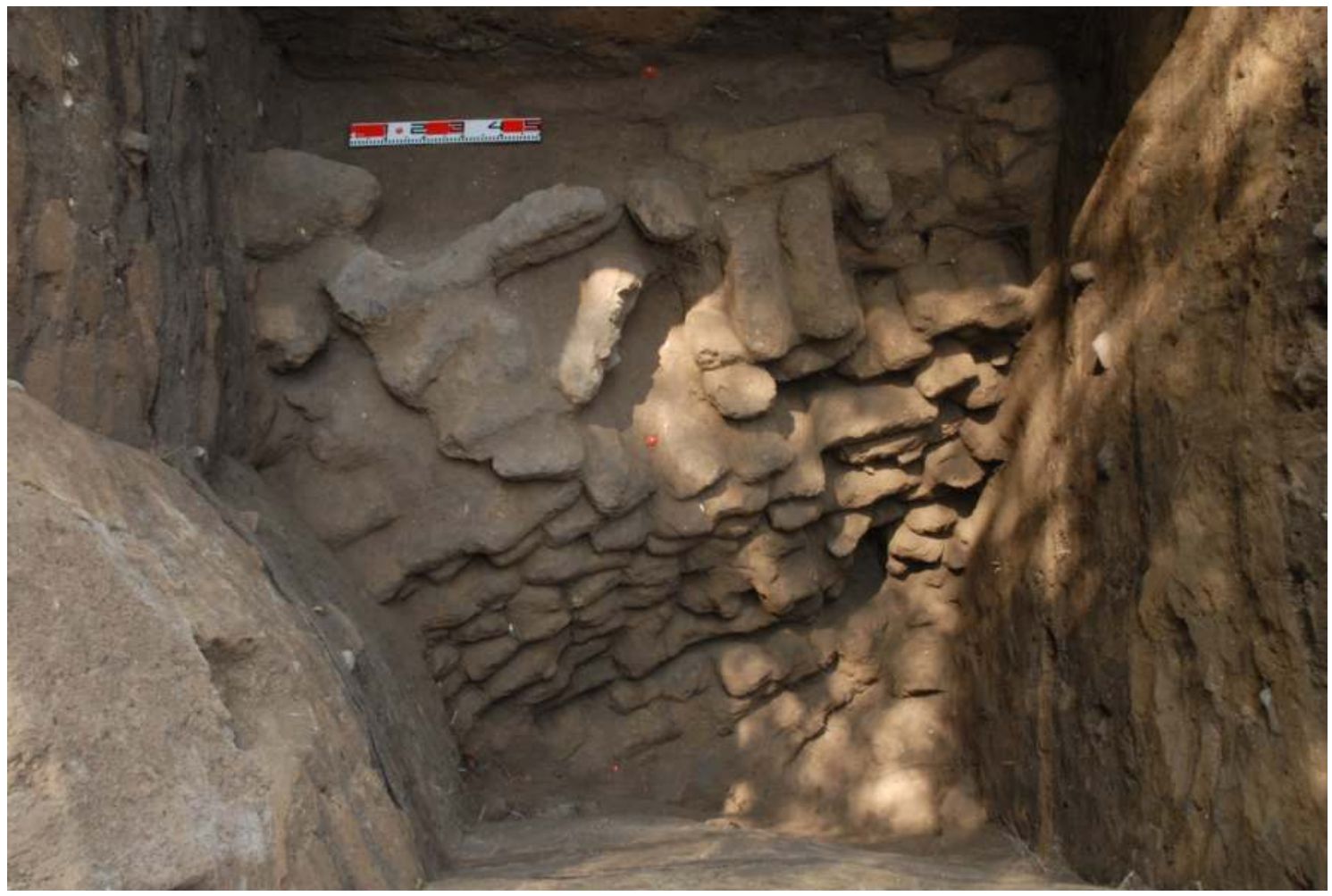

Fig. 4.50 Top view of Level 13 in the sounding pit of Square 4B. 


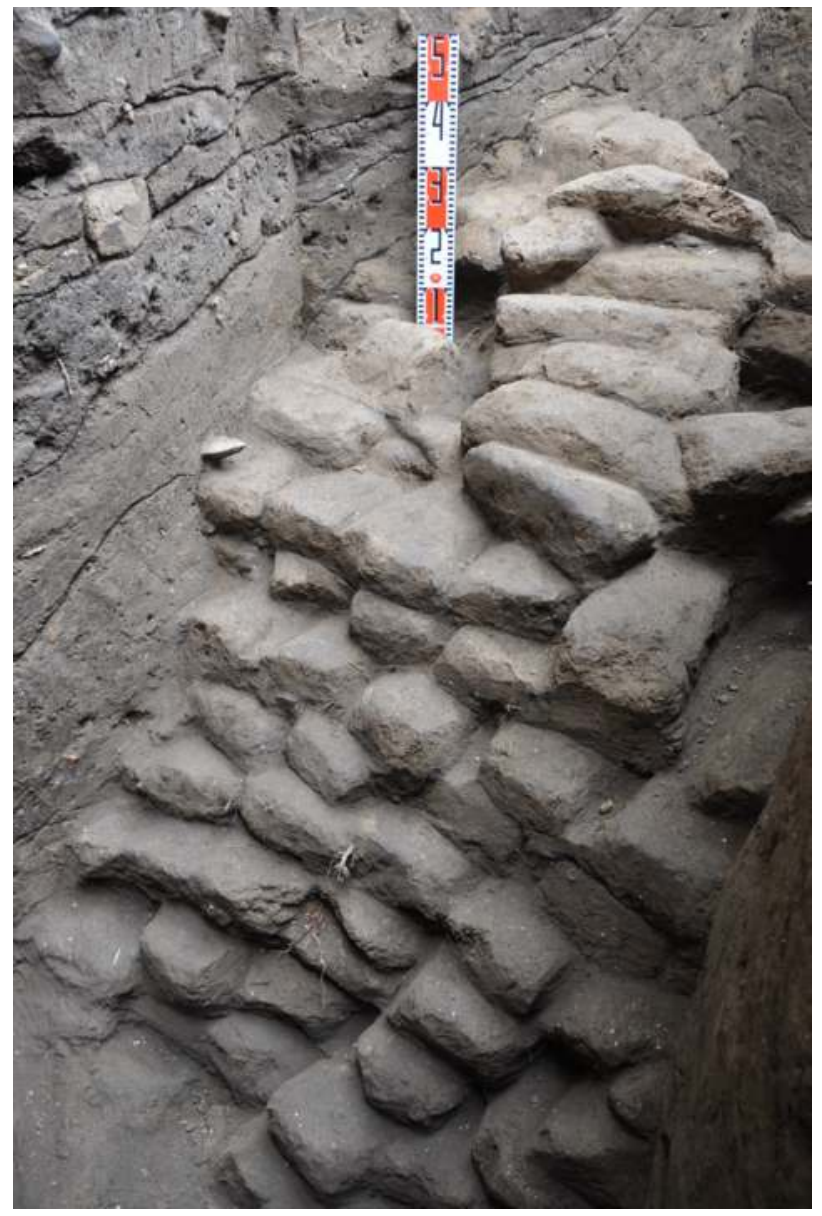

Fig. 4.51 Side view of the structure of Level 13 in the sounding pit of Square $4 \mathrm{~B}$, seen from the northwest.

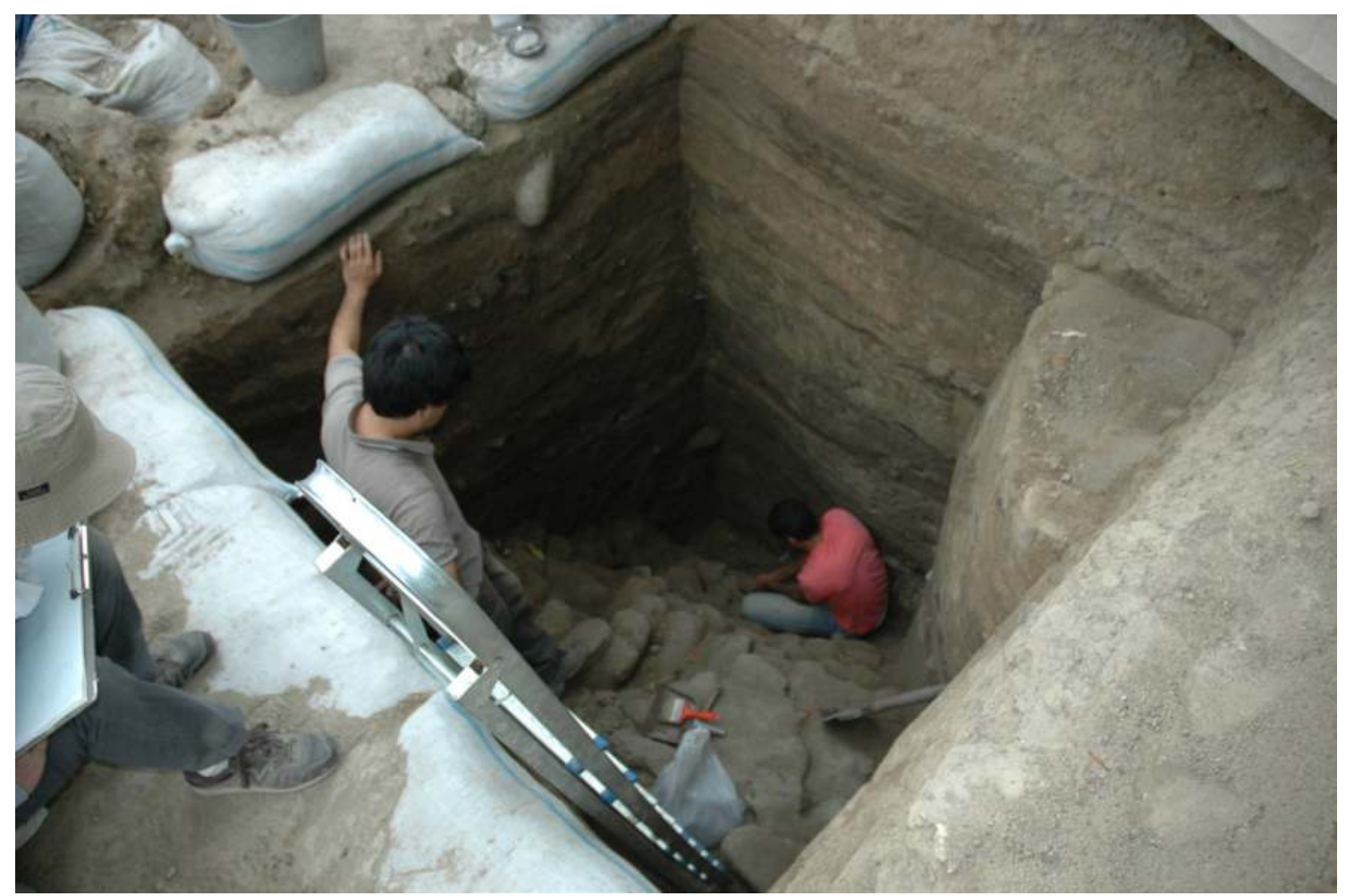

Fig. 4.52 Excavation in the northwest corner of the sounding pit in Square 4B, seen from the southeast. 


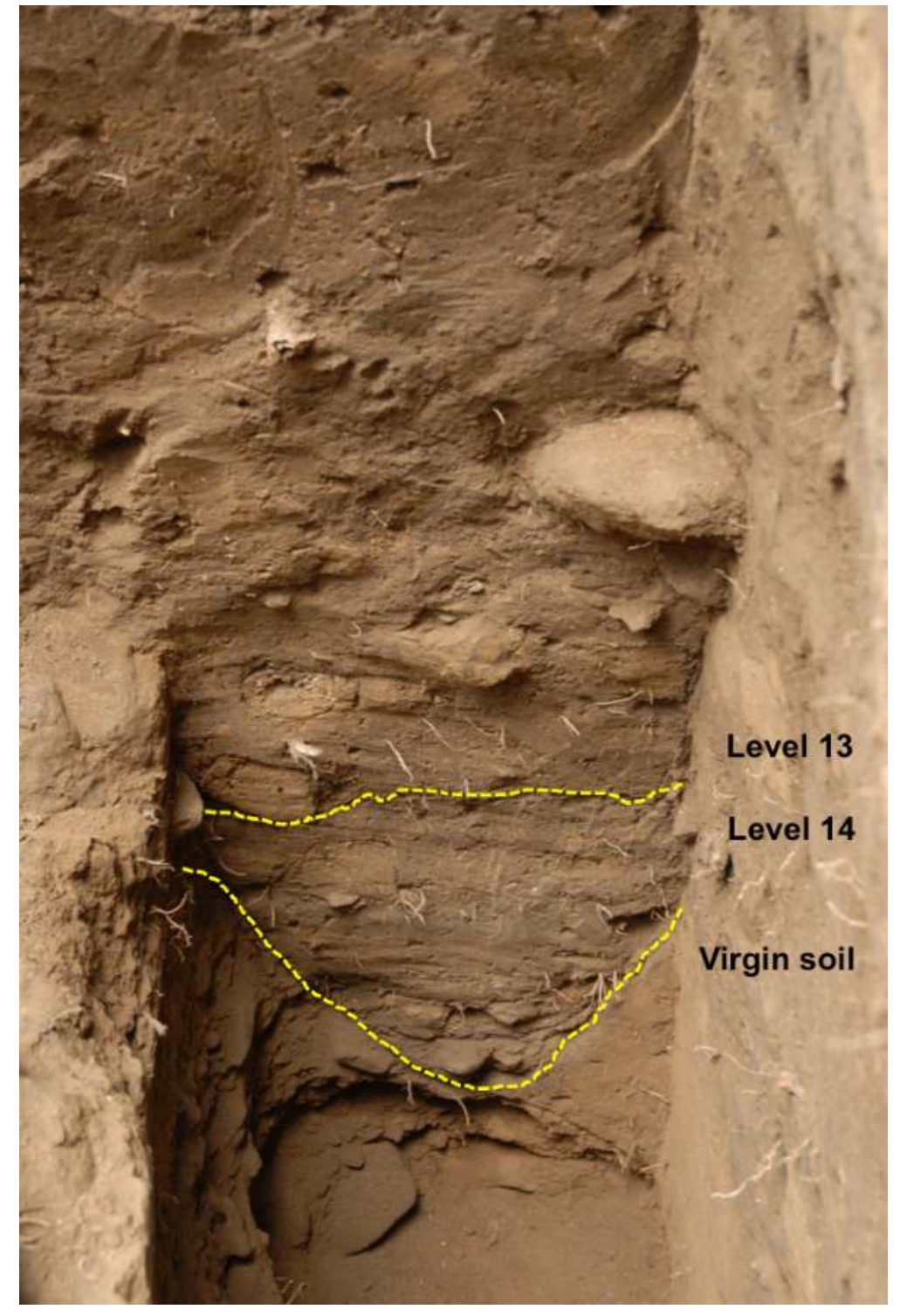

Fig. 4.53 West section of the sounding pit of Square 4BII. The V-shaped profile of the Level 14 ditch is visible at the lowest part. ditch was apparently used for water drainage. It was originally cut into a homogeneous grayishyellow soil consisting of very fine sediments lacking artifacts or other organic remains. Below the grayishyellow soil was a thin layer of sands overlying a thick gravel layer continuing downward. This stratigraphic succession indicates that the $80 \mathrm{~cm}$-thick layer of grayish-yellow soil represents the top of virgin soil in this part of the mound. Thus, excavation of 4BII reached virgin soil, which allowed us to identify the mound's first occupation level as Level 14.

\subsection{Summary}

The main aim of excavating Square 4B was to reveal an earlier part of Göytepe's occupational sequence.
This work would supplement excavations by the Azerbaijani team that focused on the later sequence in the larger excavation area on the mound's upper part. Our excavations indeed resulted in the exposure of stratified occupation levels (5 to 14). Since the Azerbaijanis' excavation uncovered Levels 1 to 5 (Chapter 3), the sequences from both campaigns can thus be connected in order to reveal the entire Neolithic sequence at Göytepe. This remarkable stratigraphy, consisting of 14 levels in $11 \mathrm{~m}$ of deposits, is unparalleled by any known Neolithic mound in western Azerbaijan. This unique sequence allows us to study diachronic changes in the material culture and socioeconomy of Neolithic societies with solid stratigraphic evidence.

Another important achievement of the excavations 
of Square 4B involves the stratigraphic sampling of a variety of geoarchaeological and environmental materials spanning a long chronological range. For example, this project collected detailed contextual information on charcoal for radiocarbon dating and sediments for analyses of phytoliths, fecal spherulites, micromorphology (Chapter 7 and Kadowaki et al. 2015), macro- and micro-organic remains, and other artifactual and non-artifactual remains. The horizontal distribution of objects was also carefully recorded in relation to the architecture. Archaeological data combined from both the highresolution excavations of Square 4B and the largescale excavations on top of the mound comprise an exceptional cultural record that enhances the research potential of Göytepe for extensive studies in the future.

\section{References}

Kadowaki, S., L. Maher, M. Portillo, R. M Albert, C. Akashi, F. Guliyev, and Y. Nishiaki (2015) Geoarchaeological and palaeobotanical evidence for prehistoric cereal storage at the Neolithic settlement of Göytepe (mid 8th millennium BP) in the southern Caucasus. Journal of Archaeological Science, 53: 408-425.

Lyonnet, B. (2009) Surveys and excavations in Western Azerbaijan: Settlement changes and relations with surrounding areas, from the Neolithic to the Bronze Age. In: Proceedings of the International Symposium - Land between East and West, April 1-3, 2009, edited by the German Archaeological Institute, pp. 41-48. Baku: Azerbaijan.

Nishiaki, Y., M. Tao, S. Kadowaki, M. Abe, and H. Tano (2001) Excavations in Sector A of Tell Kosak Shamali: the stratigraphy and architectures. In: Tell Kosak Shamali - The Archaeological Investigations on the Upper Euphrates, Syria. Volume 1: Chalcolithic Architecture and the Earlier Prehistoric Remains, edited by Y. Nishiaki and T. Matsutani, pp. 49-113. UMUT Monograph 1. Oxford: Oxbow Books. 


\title{
Chapter 5
}

\section{Soundings at the edges of Göytepe}

\author{
Yoshihiro Nishiaki, Yui Arimatsu, and Saiji Arai
}

\subsection{Introduction}

Göytepe represents one of the largest Neolithic mounds thus far known in Azerbaijan. Yet, its precise horizontal extension in this cultural period remains unconfirmed. What blurs any estimate of the settlement size at Göytepe is the extensive disturbance at its northern and eastern edges, where modern farmers and shepherds have carried out repeated slope cuttings for the construction of buildings, an irrigation canal, and an animal shelter (Chapter 3). Acknowledging this limitation, we opened a series of small sounding pits at the edges of the mound to investigate the horizontal distribution of Neolithic cultural deposits (Fig. 5.1). These sounding pits should give a minimum size estimate for the Neolithic settlement of Göytepe. At the same time, they can also help reveal other important aspects of the settlement. For example, they could indicate whether particular differences existed in the use of space between the core and marginal portions of the settlement. Moreover, the soundings may also provide us with insights on the Neolithic landscape in which the first inhabitants settled, since these deep test pits at varied localities help us identify the stratigraphic contexts of virgin soil over a wide area.

A total of nine $2 \times 2 \mathrm{~m}$ and one $1 \times 1 \mathrm{~m}$ pits

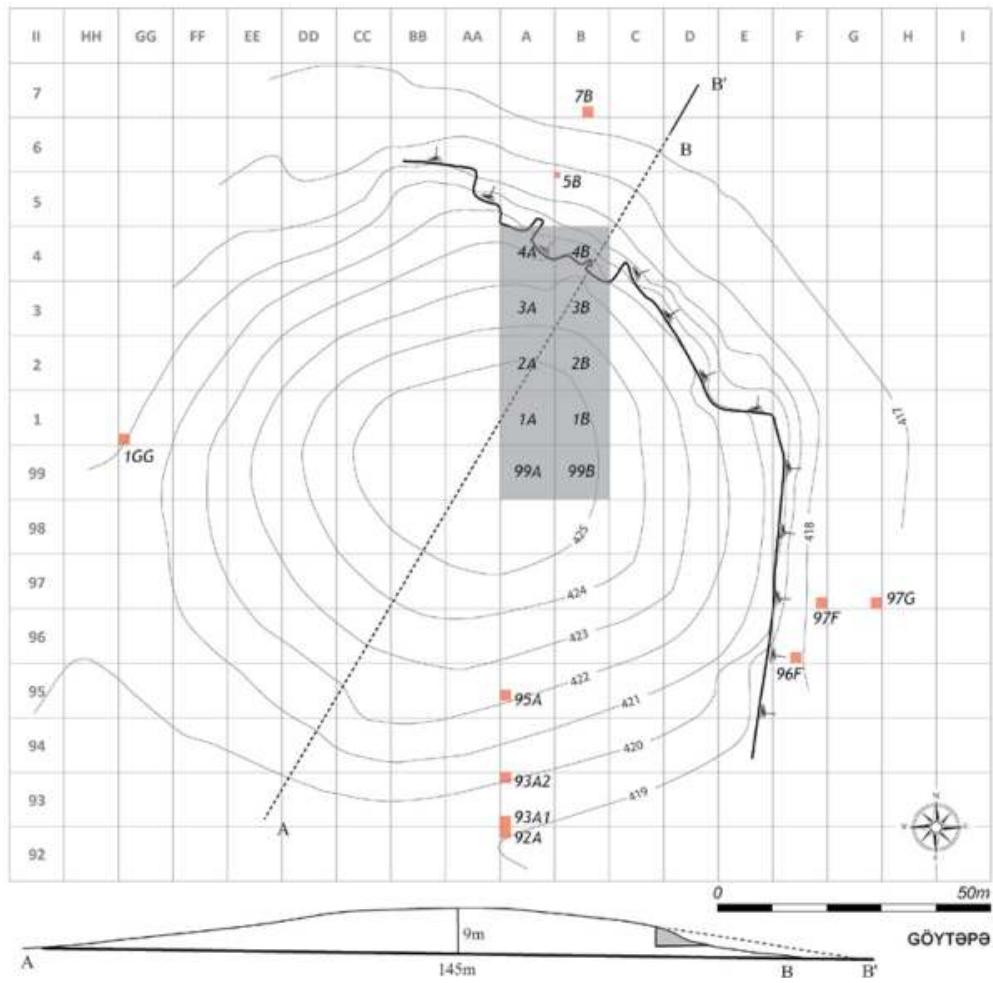

Fig. 5.1 Location of sounding pits at the edges of Göytepe. 
were opened at the northern, eastern, western, and southern edges of the mound. Results of these test pit excavations will be reported in this sequence.

\subsection{Northern edge}

\subsubsection{Pit 7B}

A modern irrigation canal is situated along the eastern and northern edges of the mound, running from the south to the northwest. At the northern edge, it runs through Square 6B from east to west. The pit in Square 7B was set up away from this canal (Fig. 5.1). Excavations reached to the depth of $2.2 \mathrm{~m}$ from the present surface. The top layers, $50-60 \mathrm{~cm}$ in thickness, consisted of grayish-brown sediments (Fig. 5.2) containing fragments of modern construction materials and a small amount of Neolithic pottery sherds and flaked stone artifacts. These layers evidently represent secondary deposits, which might have included those derived from digging the canal and the construction of associated buildings. Underneath is virgin soil, comprising thick gravel deposits, in which thin silt layers are occasionally embedded in some parts (Fig. 5.3). No Neolithic cultural deposits remain in this area. If present, they would have been removed through erosional processes of natural and/or human factors.

\subsubsection{Pit 5B}

The small $1 \times 1 \mathrm{~m}$ pit is located in the northwestern corner of Square 5B (Fig. 5.1), immediately south of the canal. Excavation proceeded to a depth of just one meter because of its close proximity to the canal used today by local farmers. The excavated deposits contained abundant Neolithic stone artifacts, pottery sherds, animal bones, and mud-brick fragments, all considered to have been materials that eroded out of the mound. Modern items such as metal wire and glass fragments were also uncovered. No architectural remains were recovered. Unfortunately, the virgin soil was not reached.

\subsection{Eastern edge}

\subsubsection{Pit 97G}

The sounding pit in Square $97 \mathrm{G}$ is situated about 20 $\mathrm{m}$ east of the modern disturbance (Fig. 5.1). Above the virgin soil, we found approximately $1.5 \mathrm{~m}$-thick deposits bearing Neolithic artifacts (Fig. 5.4). These deposits contained large fragments of mud-bricks, a small number of Neolithic sherds and chipped stones, as well as modern metal and plastic objects. The latter group shows the secondary nature of these deposits. However, the grayish-brown clay layer, which was found below a brown soil layer with plenty of gravel (Figs. 5.4 and 5.5), did not yield

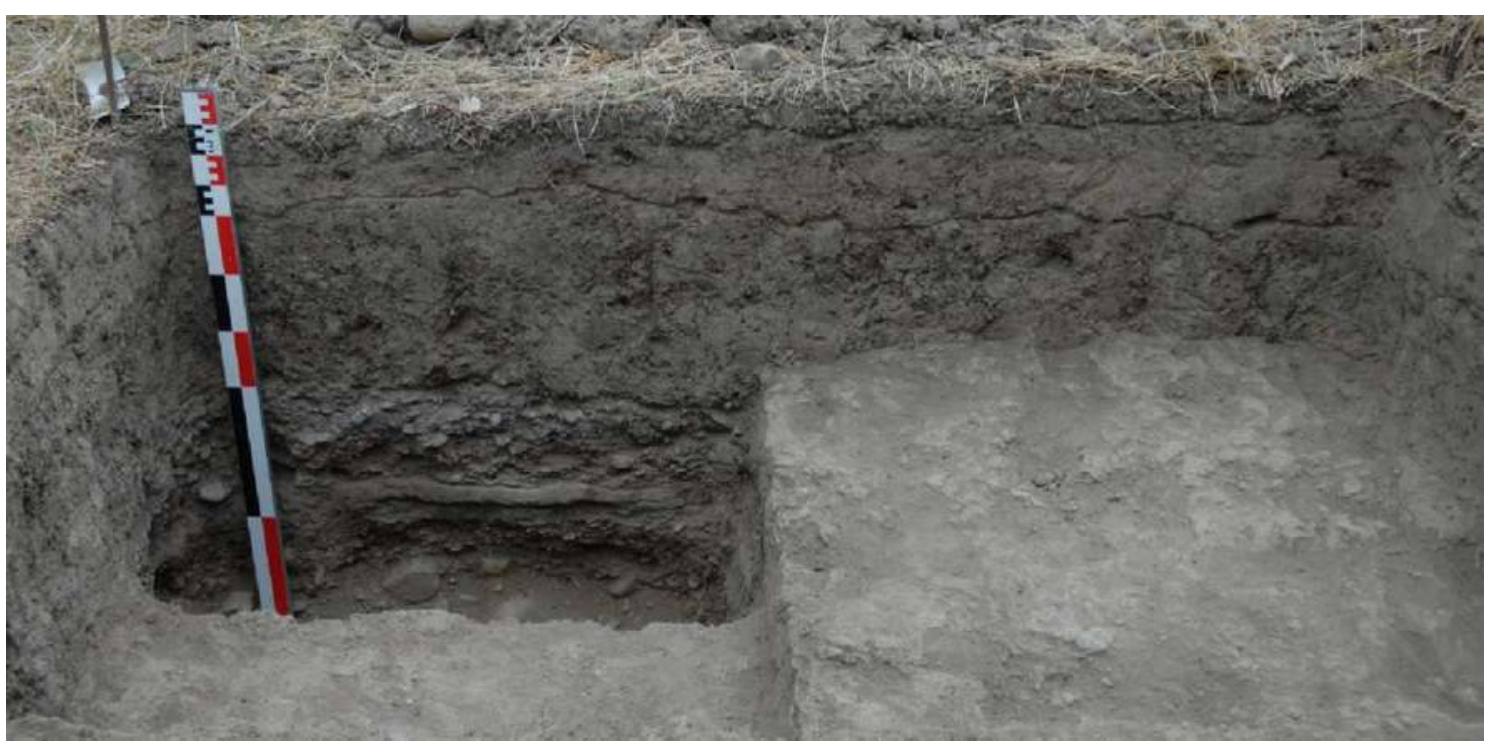

Fig. 5.2 Upper portion of the sounding pit in Square 7B, seen from the east. 

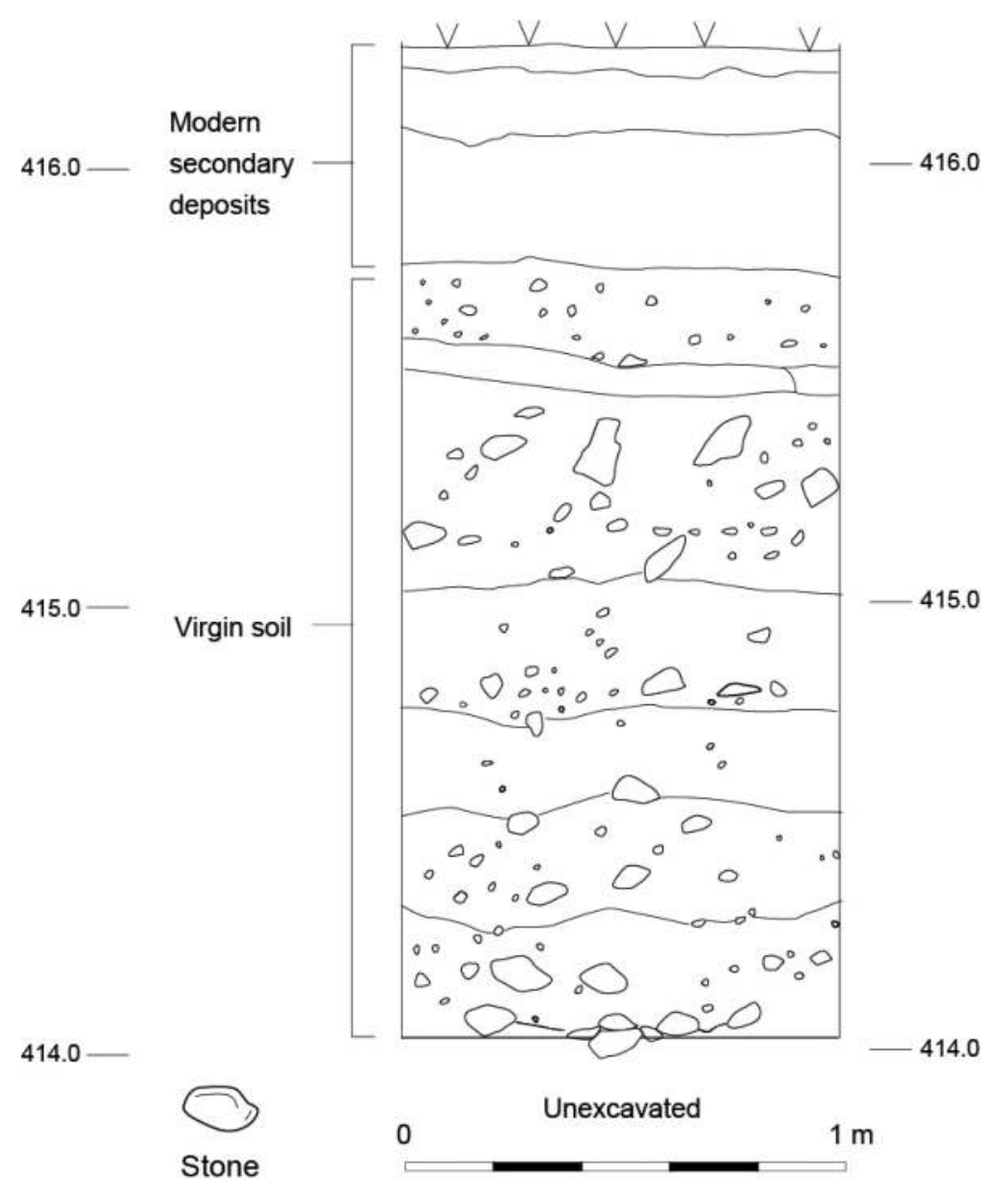

Fig. 5.3 North section of the sounding pit in Square $7 \mathrm{~B}$.

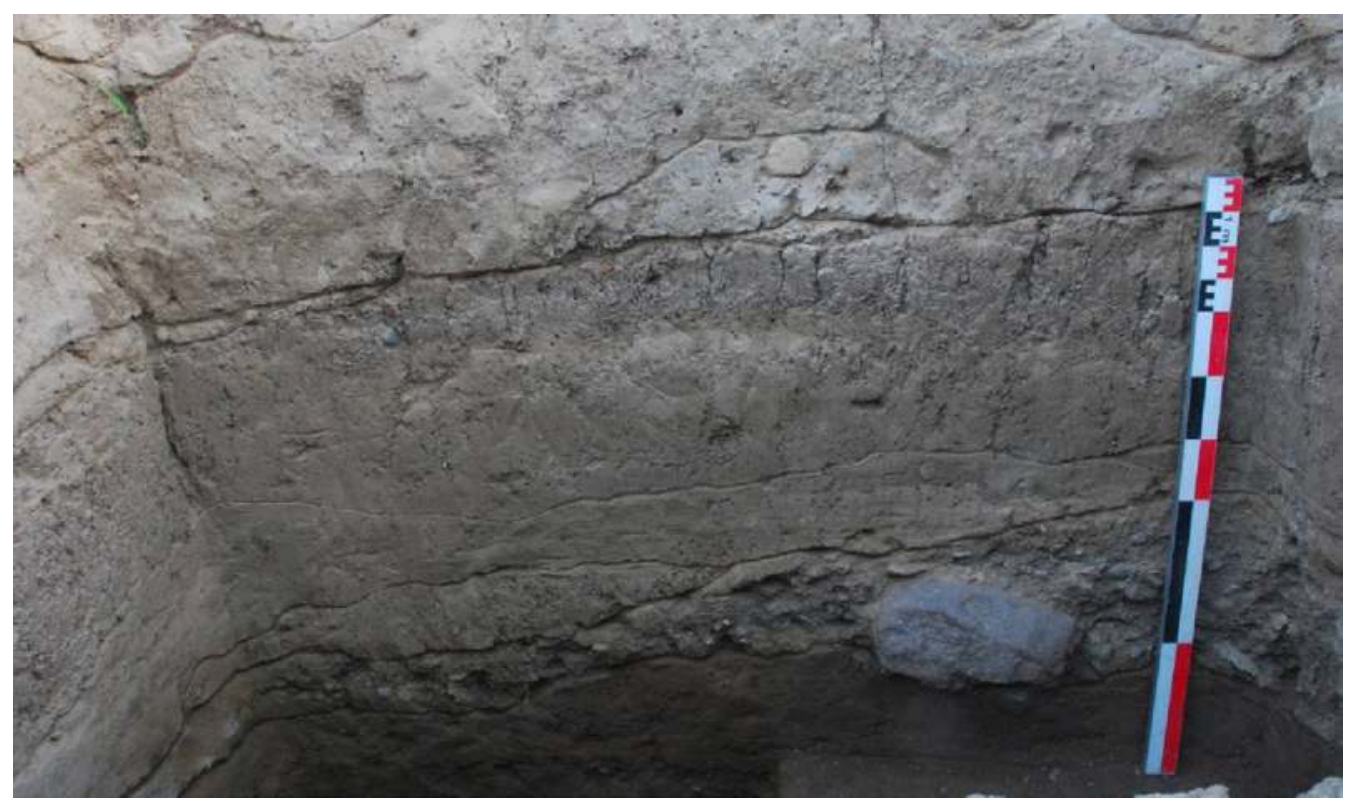

Fig. 5.4 South section of the sounding pit in Square 97G. 


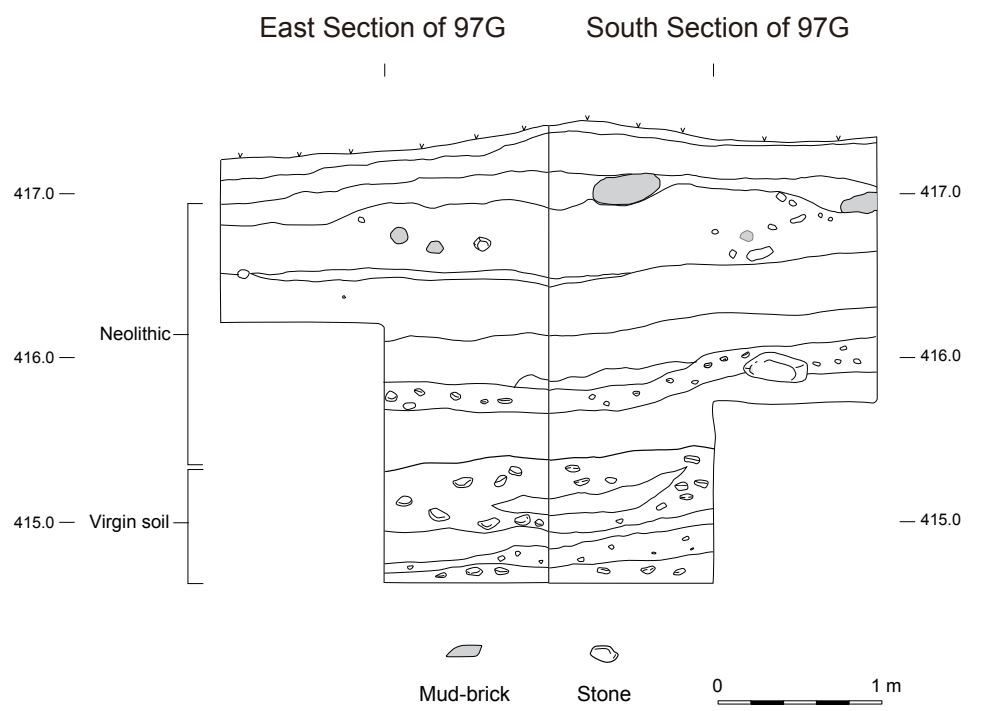

Fig. 5.5 East-south sections of the sounding pit in Square 97G. modern objects but did contain a few stone artifacts. This layer may represent Neolithic sediments, although they unlikely represent primary occupation floors. The virgin soil in this pit consisted of gravel layers, occasionally containing thin silt layers (Fig. 5.5). Both the altitude of the top of the virgin soil and the stratigraphic succession are quite comparable to those of the pit in Square 7B.

\subsubsection{Pit 97F}

In this square, the test pit $(2 \times 2 \mathrm{~m})$ was opened in its southeast corner (Fig. 5.1), where we discovered relatively thick primary Neolithic deposits. No standing architecture was recovered, but a simple fireplace was identified within Neolithic deposits more than $2 \mathrm{~m}$ thick (Figs. 5.6 and 5.7). This indicates that some open-air activities took place during the Neolithic occupations. The fireplace yielded charcoal remains for radiocarbon dating (see Nishiaki et al. 2018). Below this feature were deposits principally consisting of clayish sediments, including a smaller amount of Neolithic cultural remains. The sediments are gradually replaced by sandy ones in the lower part, suggesting that fluvial depositional processes might have taken place during the Neolithic period. The virgin soil was a thin layer of yellowish-gray silt, below which river gravel continued downward (Fig. 5.7).

\subsubsection{Pit 96F}

The $2 \times 2 \mathrm{~m}$ pit in this square yielded a substantial Neolithic structure immediately below the surface. It consisted of a massive double-framed mud-brick wall at least one meter in width at the base, and partially destroyed by the modern canal construction (Figs. 5.8 and 5.9). The excavation was suspended at the bottom of the wall's base due to water from the nearby irrigation canal flowing to the north. Therefore, the virgin soil of this square has not been exposed.

The wall is slightly curvilinear in plan, more or less following the mound's round edge (Fig. 5.10). The preserved portion of the wall stands one meter high and consists of seven mud-brick courses that are laid alternately in different orientations (Fig. 5.11). The mud-bricks are generally yellowish-brown in color, but grayish ones were also used for some parts (Figs. 5.12 and 5.13). This wall was constructed on a layer of brown soil with river gravel that was at least $20 \mathrm{~cm}$ thick (Figs. 5.11 and 5.14). This layer is not considered virgin soil, but was likely deposited intentionally as a foundation. Interestingly, a similar gravel-rich layer was also recovered at the height of about $50 \mathrm{~cm}$ from its base. This upper gravel layer, dispersed west of the wall, was covered with a layer of mud-brick rubble (Figs. 5.11 and 5.15). This might have served as another foundation floor or a ground surface for various open-air activities, although further excavation inside the mound is needed to verify this theory.

This structure reminds us of the bank wall made of mud-bricks recovered in Square 4BII at the northern edge of the mound (Chapter 4). The repeated occurrence of such massive structures indicates that Neolithic architecture on the mound's periphery consisted of structures different from 


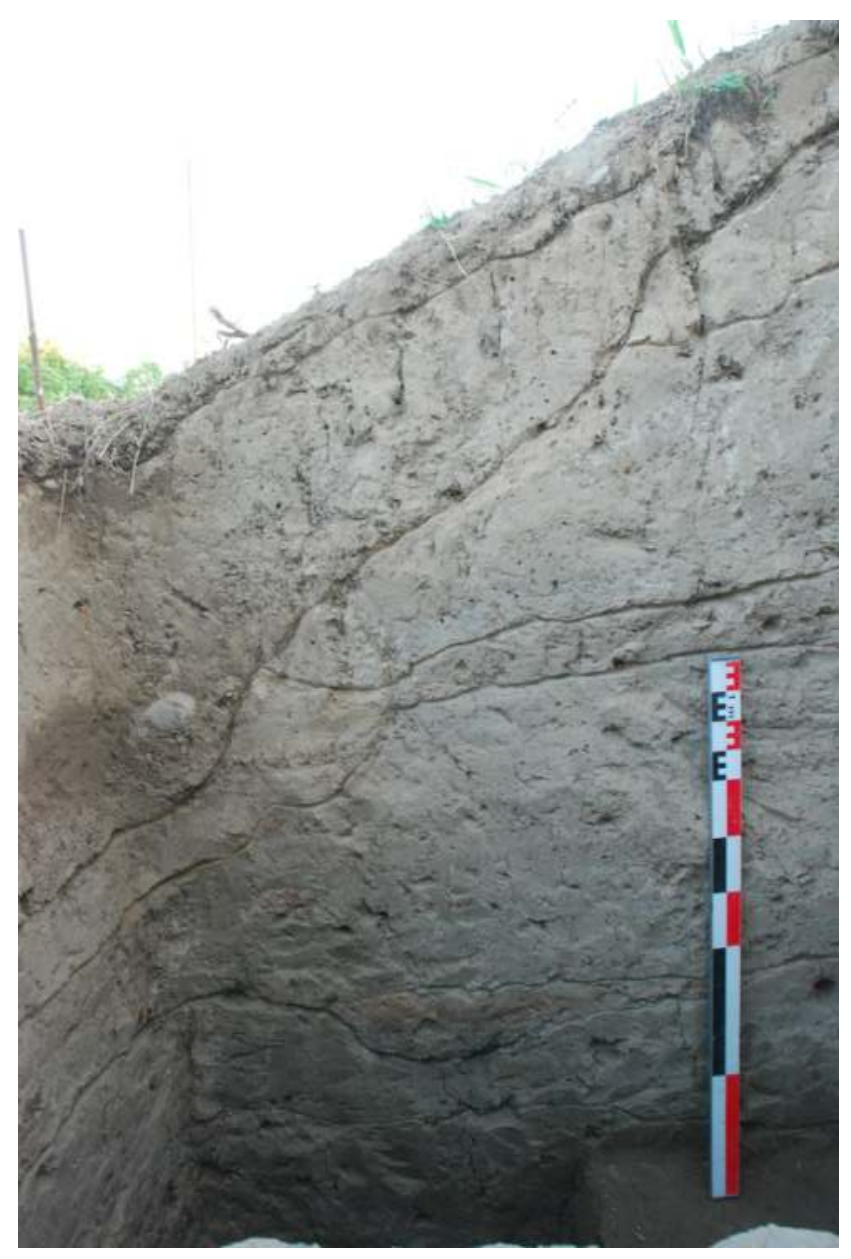

Fig. 5.6 South section of the sounding pit in Square 97F.

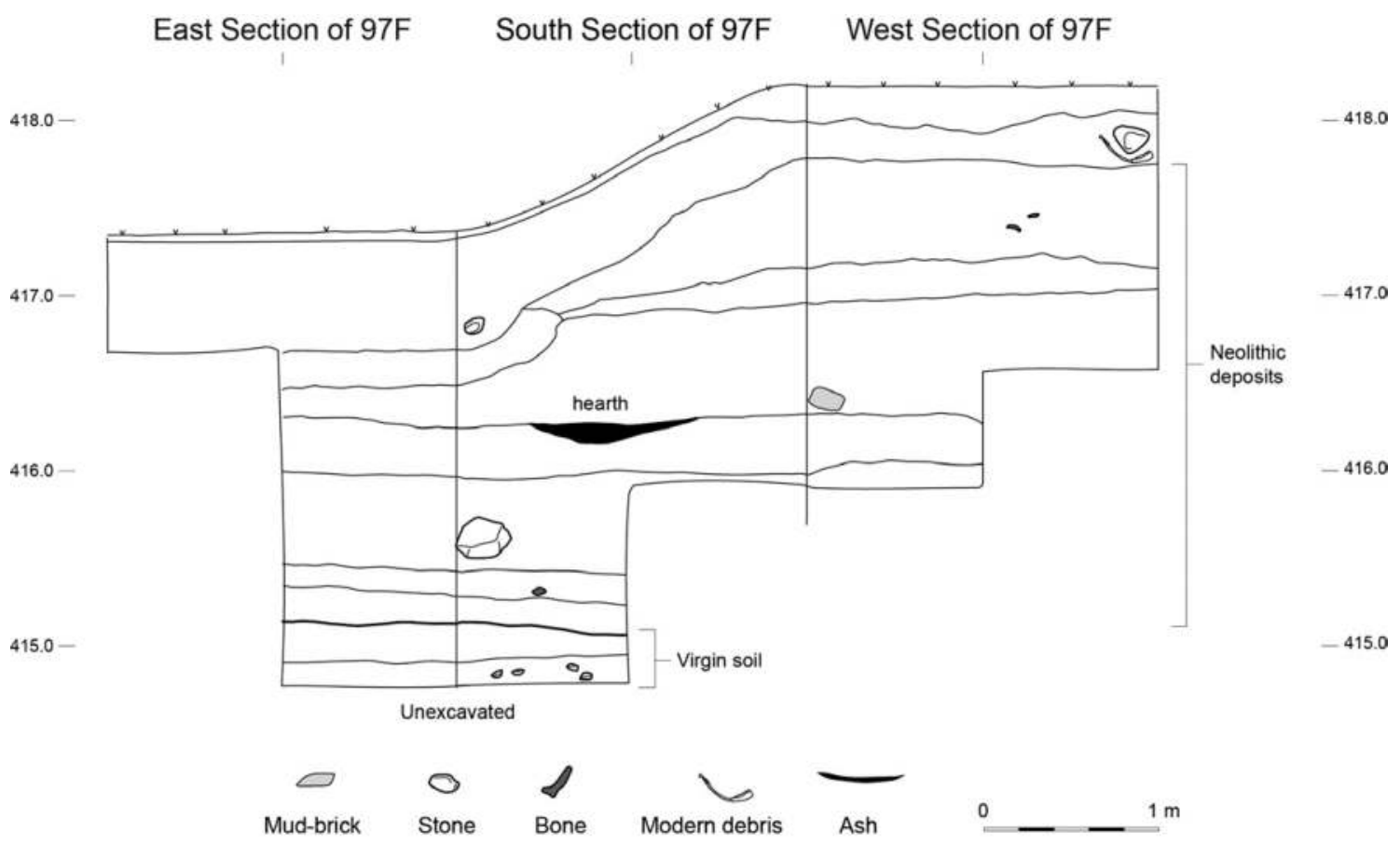

Fig. 5.7 East-south-west sections of the sounding pit in Square 97F. 


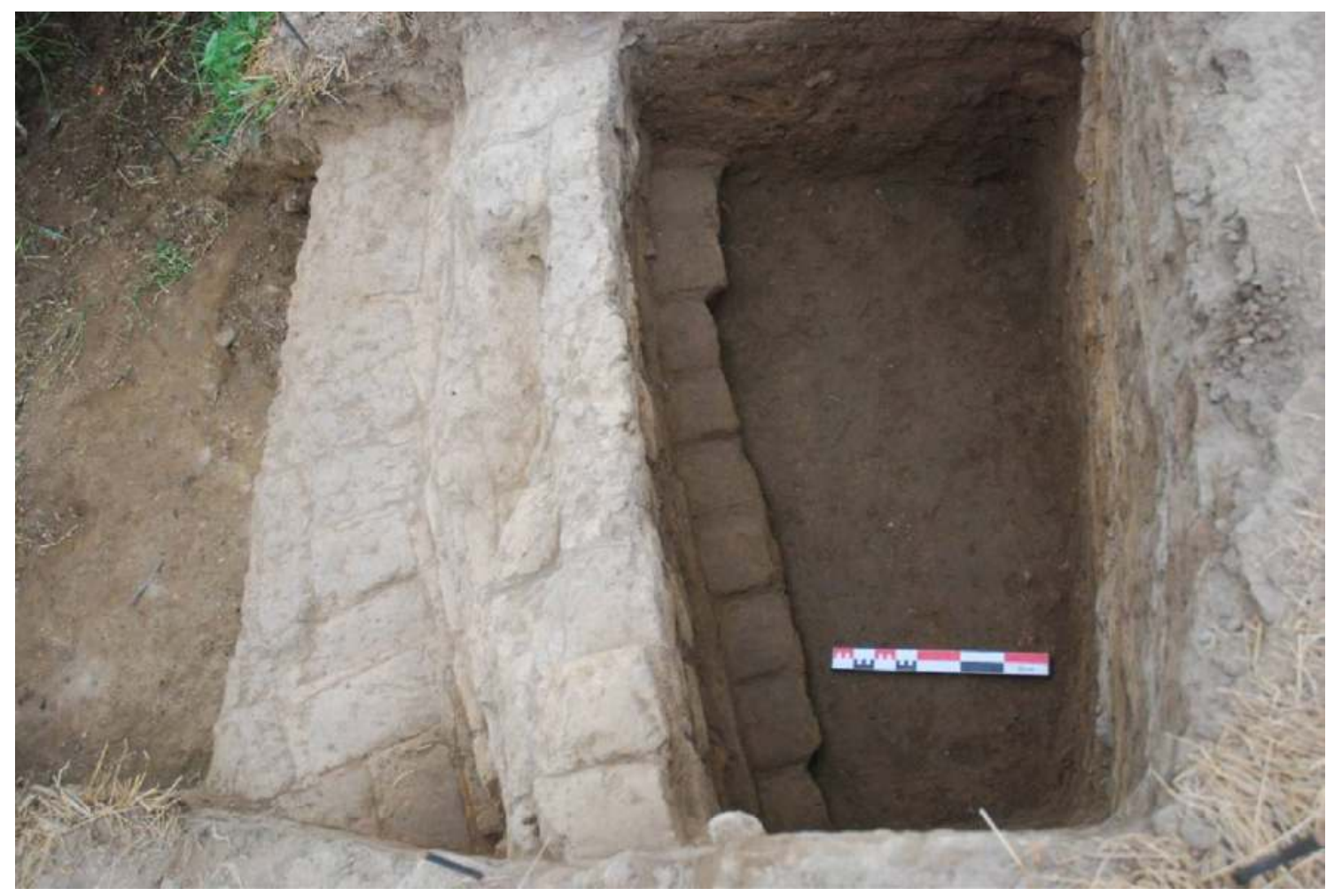

Fig. 5.8 A massive wall revealed by a small sounding in Square 96F, looking south.

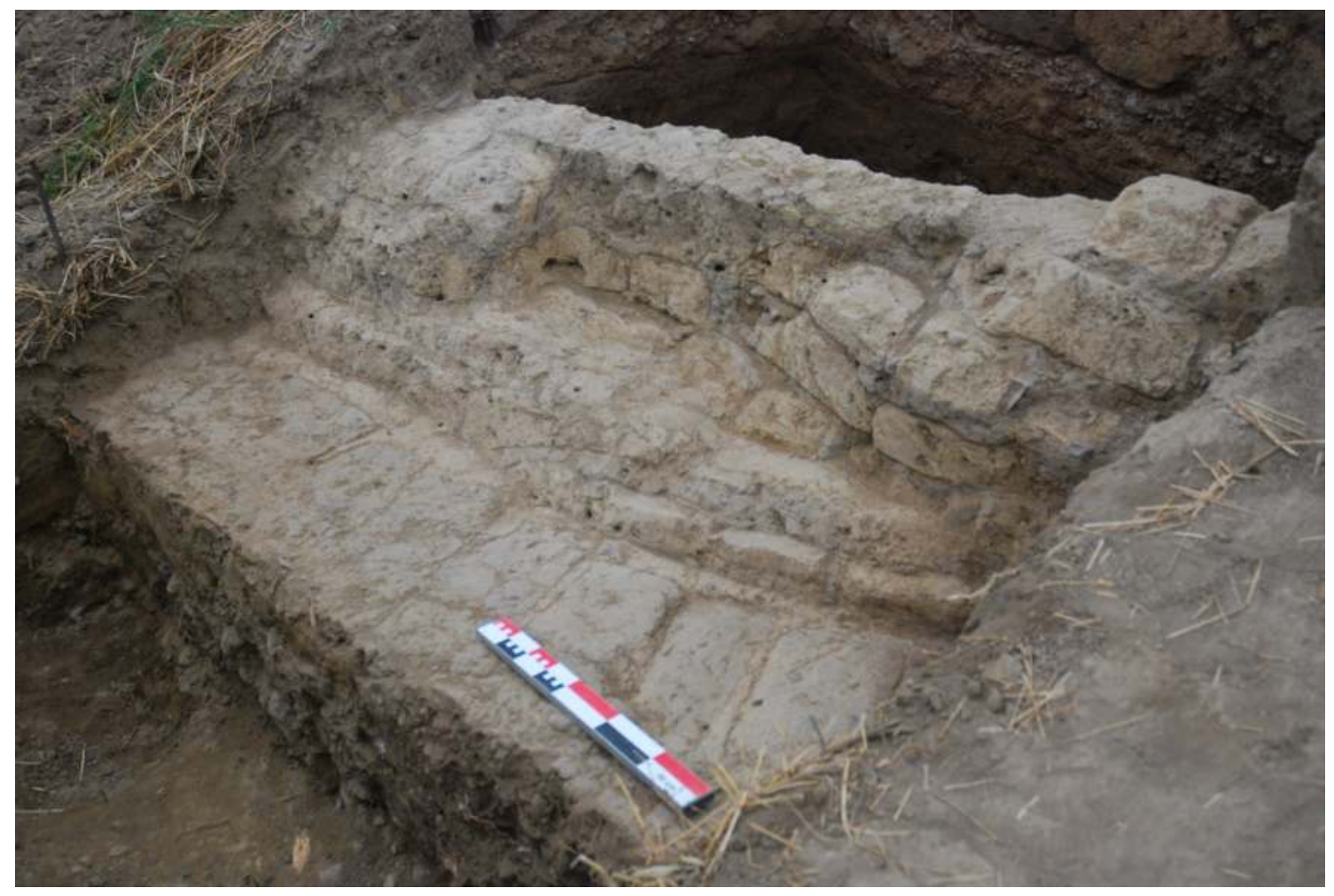

Fig. 5.9 A massive wall revealed by a small sounding in Square 96F, looking southwest. 


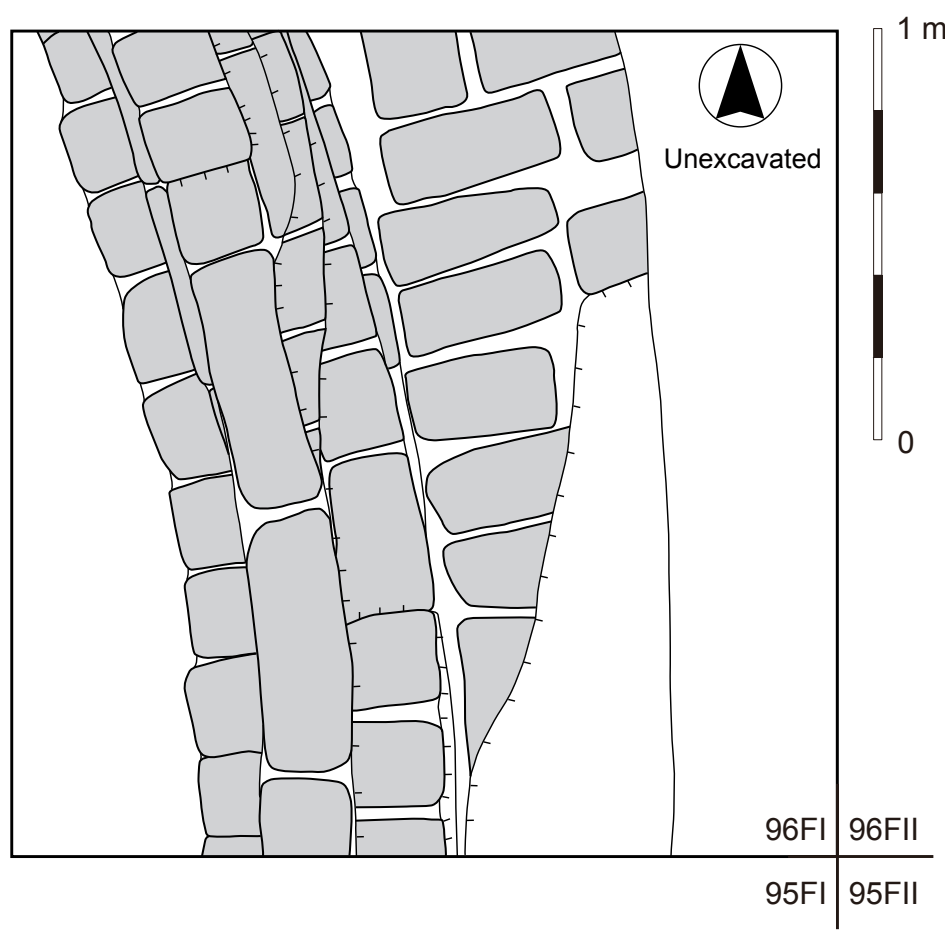

Fig. 5.10 Plan of the massive wall in Square 96F.

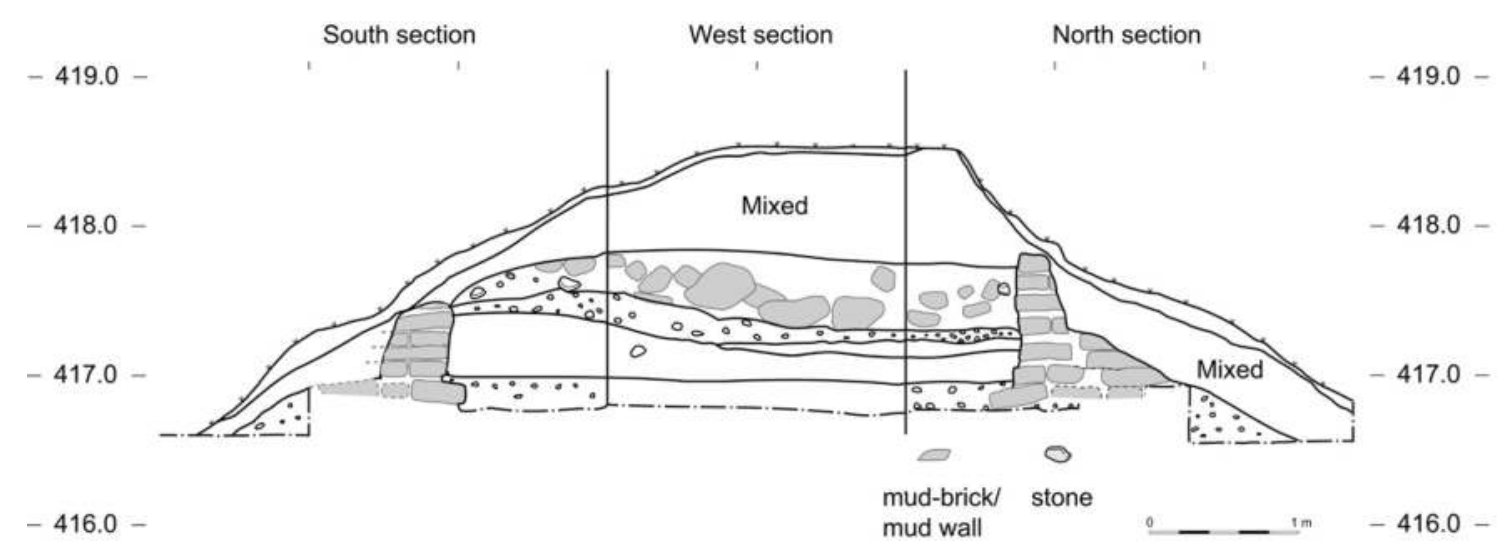

Fig. 5.11 South-west-north sections of the massive wall in Square 96F.

domestic houses that are densely distributed in the center of the settlement.

\subsection{Western edge}

\section{Pit 1GG}

A $2 \times 2 \mathrm{~m}$ test pit was opened at the western periphery in the southwest corner of Square $1 \mathrm{GG}$ (Fig. 5.1). The virgin soil was reached about $2 \mathrm{~m}$ below the surrounding surface (Fig. 5.16). The excavated deposits contained no architecture, but a large amount of Neolithic remains such as pottery sherds, flaked stones, and mud-brick fragments were found. Most of them were obtained from the thick grayish-brown soil layer, which most likely derived from either garbage disposal during the Neolithic period or slope erosion in later periods. However, the primary Neolithic layers could also be identified in the lower section. One such layer contains patches of ash, which were associated with abundant cultural remains (Fig. 5.17). The underlying layer, found directly on virgin soil, also revealed a horizontal distribution of Neolithic cultural remains. Although this layer contained plenty of gravel that probably derived from the gravel layer beneath, it likely represents part of the primary Neolithic settlement. 


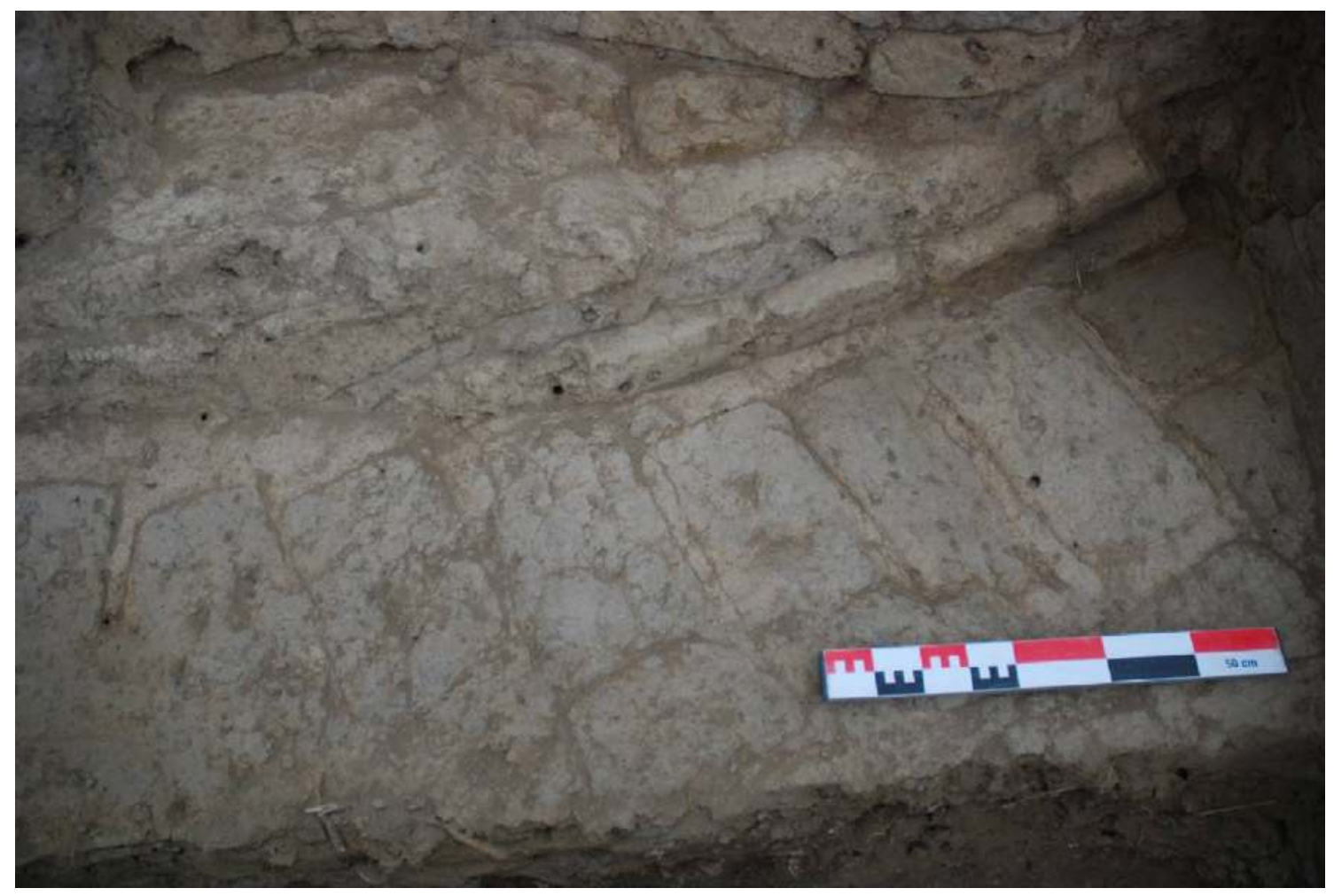

Fig. 5.12 Mud-bricks at the base of the wall in Square 96F, looking west.

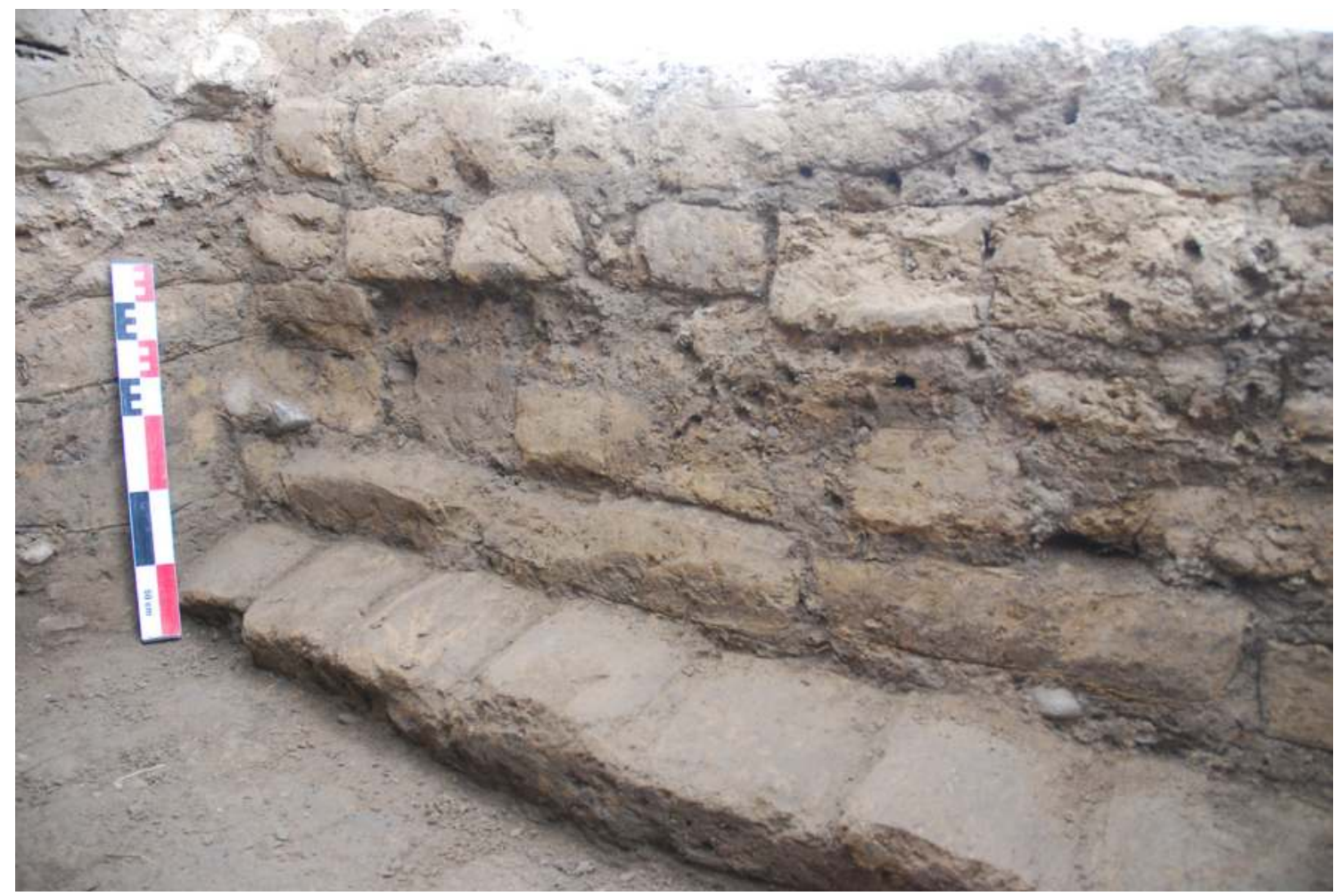

Fig. 5.13 Inner surface of the massive wall in Square 96F, seen from the west. 


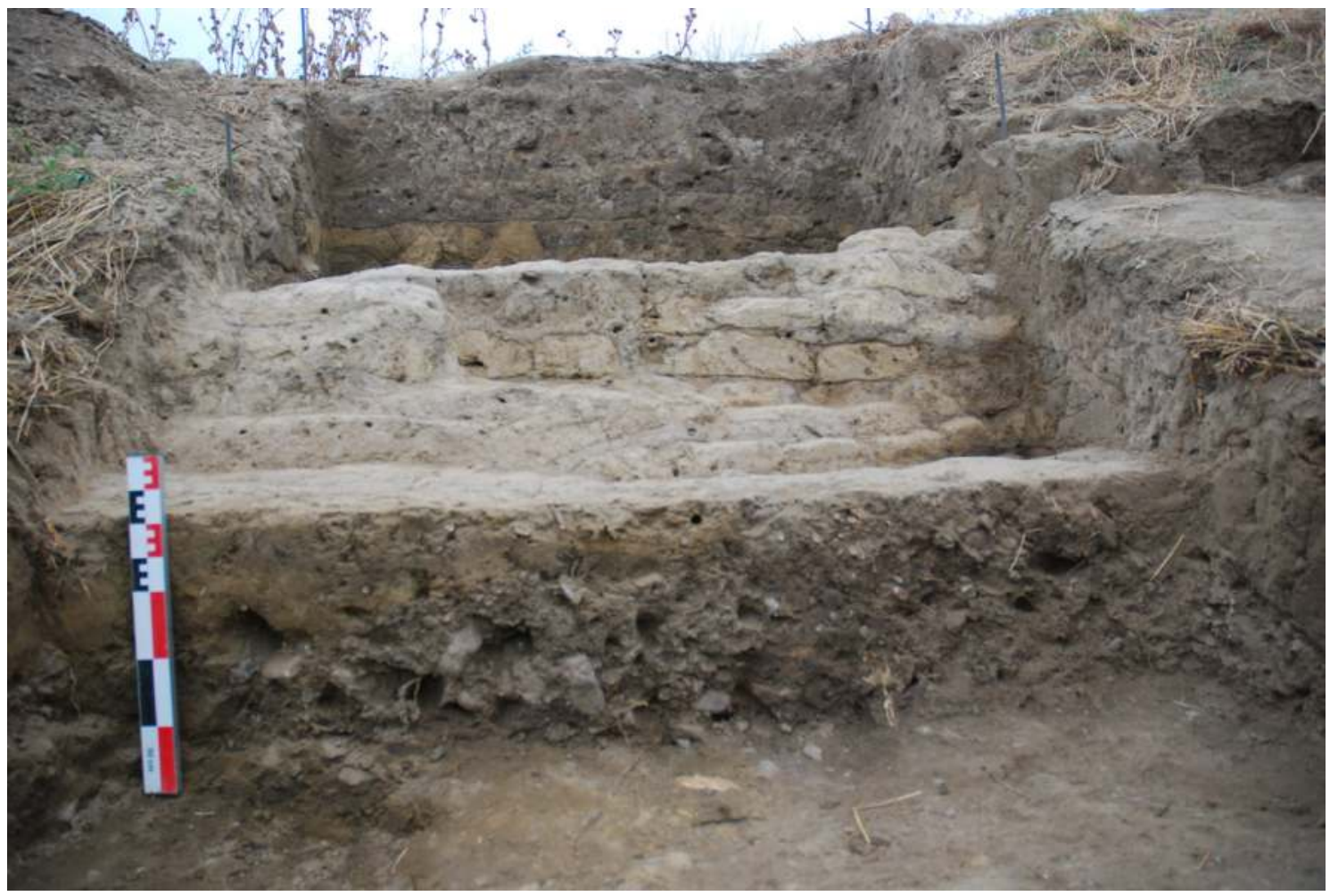

Fig. 5.14 Base of the massive wall in Square 96F, seen from the east.

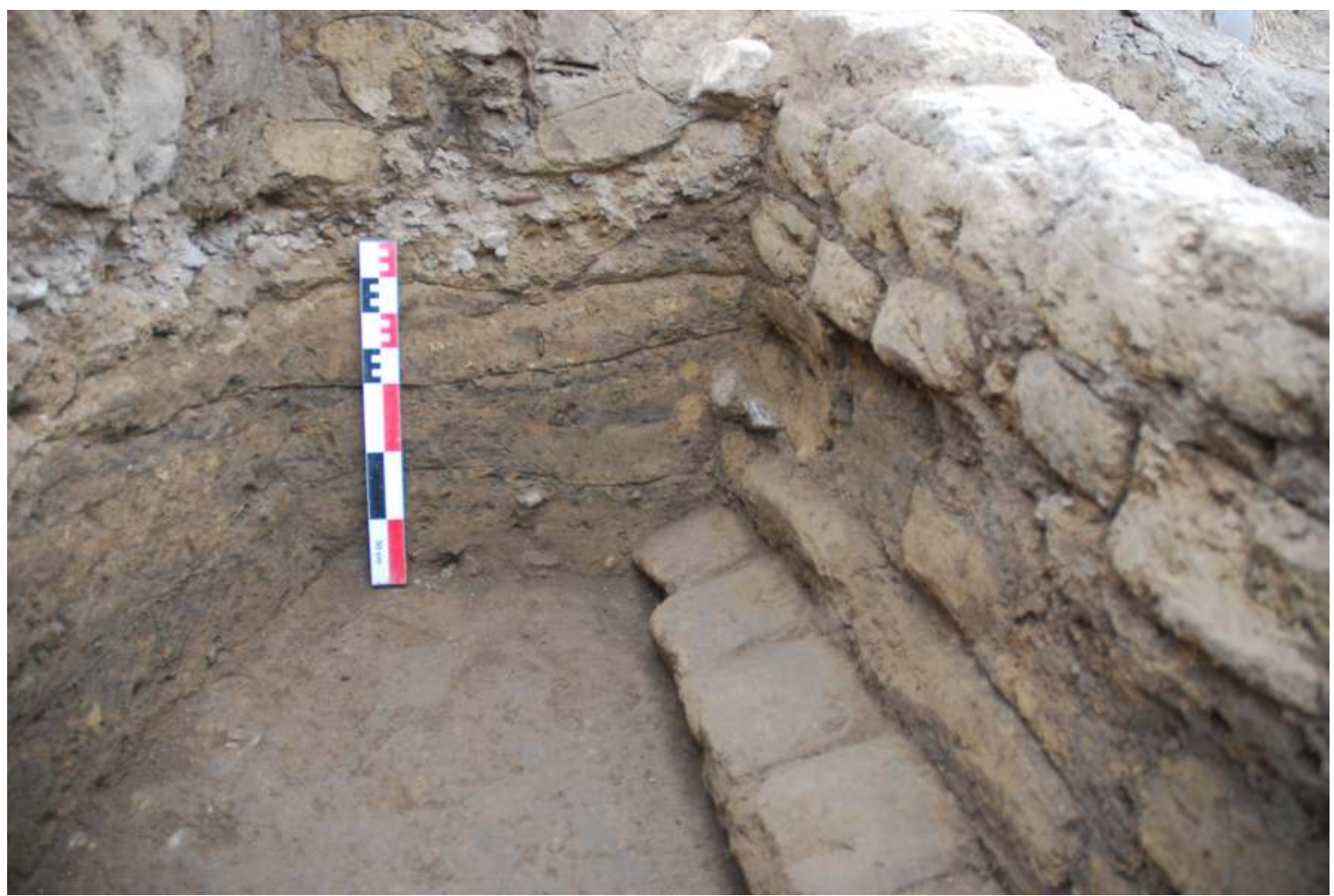

Fig. 5.15 West section of the massive wall's inner part in Square 96F, seen from the south. 


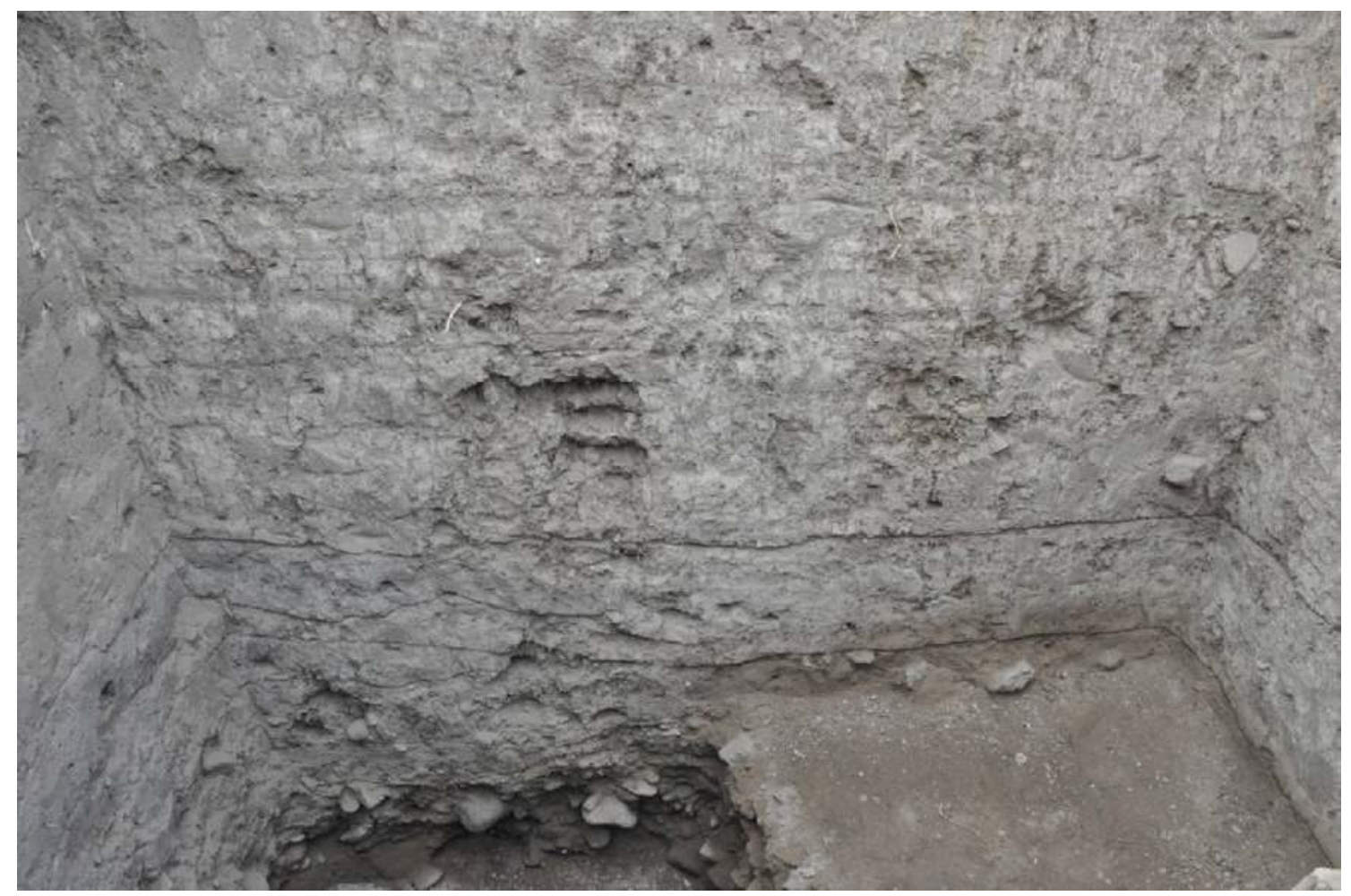

Fig. 5.16 South section of the sounding pit in Square 1GG.

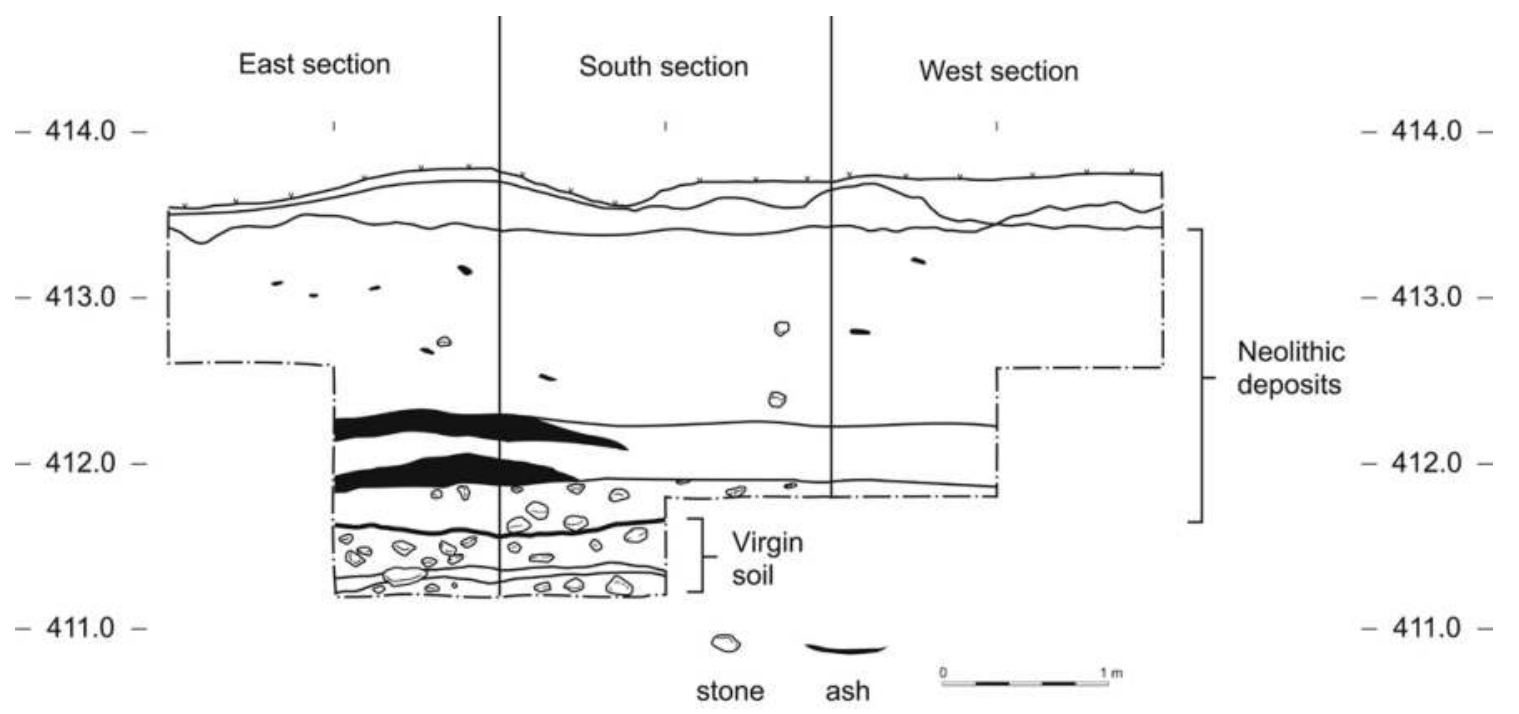

Fig. 5.17 South section of the sounding pit in Square 1GG.

Based on these finds, it is surmised that the Neolithic settlement expanded to at least this part of the mound. At the same time, the thick accumulation of possible slope erosion deposits - but an absence of standing architecture-suggest that this area was situated close to the mound's edge.

\subsection{Southern edge}

\subsubsection{Pit 95A}

Four $2 \times 2 \mathrm{~m}$ pits were opened along the AA/A line on the southern slope (Figs. 5.1 and 5.18). The northernmost sounding is situated in Square 95A. The excavation revealed the top of a circular mudbrick structure about $20 \mathrm{~cm}$ below the ground surface. 


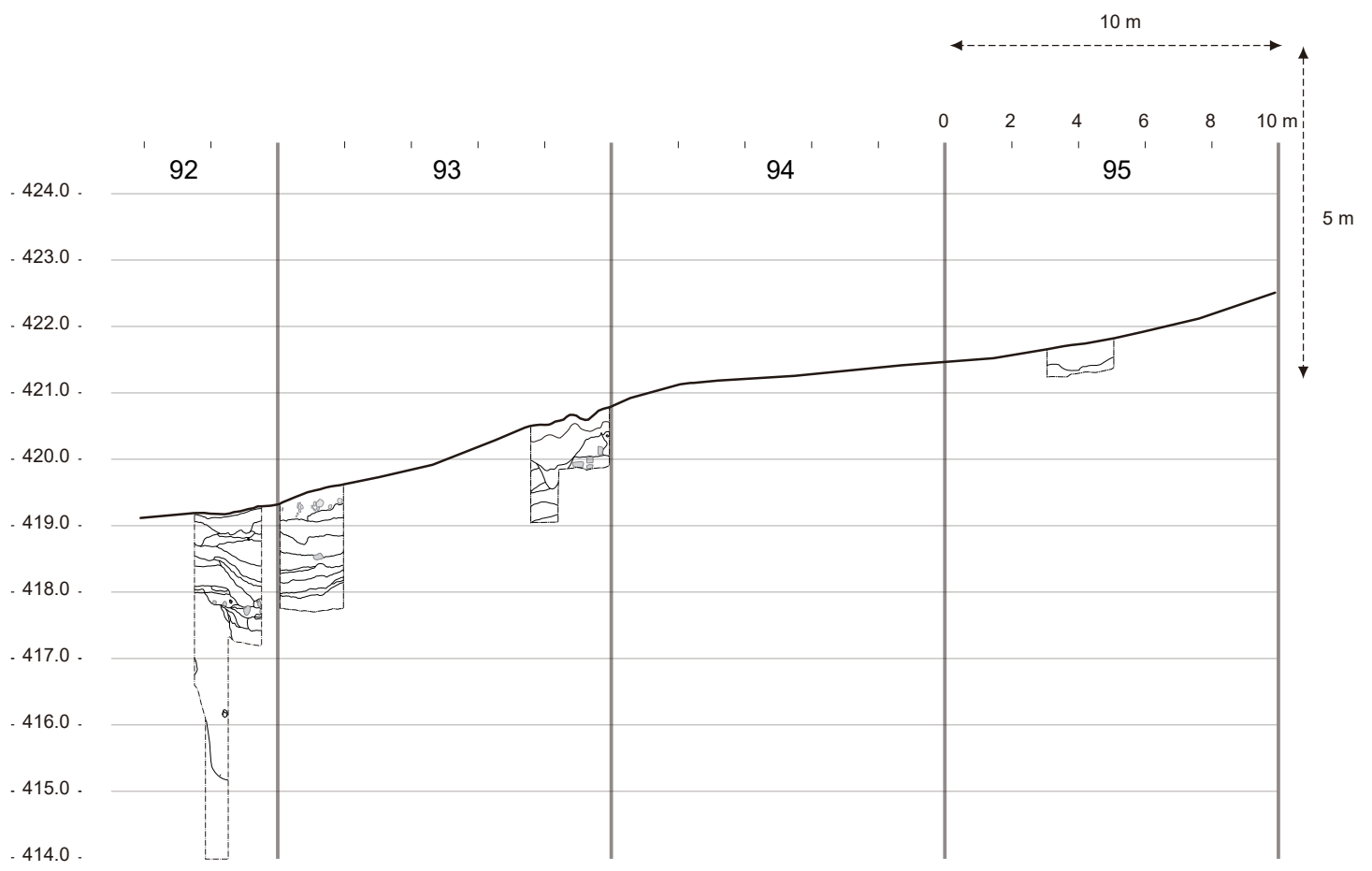

Fig. 5.18 West sections of the pits on the south slope.

After confirming the presence of a well-preserved Neolithic structure in this area, the excavation was suspended at the depth of $40 \mathrm{~cm}$.

\subsubsection{Pit 93A2}

Two pits were opened in Square 93A-one in the northwest corner was designated as 93A2 and another in the southwest as 93A1. Pit 93A2 was excavated down to the depth of $140 \mathrm{~cm}$ from the surface. As a result, three Neolithic architectural levels were identified. The uppermost is a level on which a ditch, running from the northwest to southeast, was discovered. It was at least $80 \mathrm{~cm}$ in width and $60 \mathrm{~cm}$ in depth, measurements that are minimum estimates due to erosion of the upper part of the ditch. Interestingly, directly below the bottom, the profile of another possible ditch was visible (Fig. 5.19). While the available evidence did not reveal the construction level of these ditches, the accompanying artifacts identify them as Neolithic. The second and third architectural levels yielded parts of round mud-brick structures that were found to be cut by the ditches. Neolithic deposits evidently continue underneath, but the excavations are currently suspended at this level.

\subsubsection{Pit 93A1}

The pit of $93 \mathrm{~A} 1$ revealed the top of a Neolithic mud-brick structure around $50 \mathrm{~cm}$ below the present surface (Fig. 5.20). The preservation of this structure was excellent. The base of the wall was identified at a depth of approximately $2 \mathrm{~m}$. In other words, the wall stood up to $150 \mathrm{~cm}$ high. The wall's surface was covered with a layer of thick mud plaster (Fig. 5.21). Careful examination showed that this structure had two floors, suggesting its prolonged use (Fig. 5.22). The upper floor is situated about $70 \mathrm{~cm}$ above the lower one. Although this structure was constructed on a slope descending to the southeast, these occupation floors were constructed rather flat. Virtually no Neolithic remains except mud-brick rubble were recovered from the floors. The living floors seem to have been kept quite tidy during the Neolithic.

\subsubsection{Pit 92A}

Another periphery sounding, Pit 92A, was excavated to the south of Pit 93A1 (Fig. 5.1), leaving a baulk $50 \mathrm{~cm}$ wide for stratigraphic examination. Pit $92 \mathrm{~A}$ was excavated more than $5 \mathrm{~m}$ below the surface, reducing the excavation area as it went deeper. Four building levels were identified. The uppermost level is represented by a post-Neolithic pit, whereas the second is defined by thin, dark brown ash layers (Fig. 


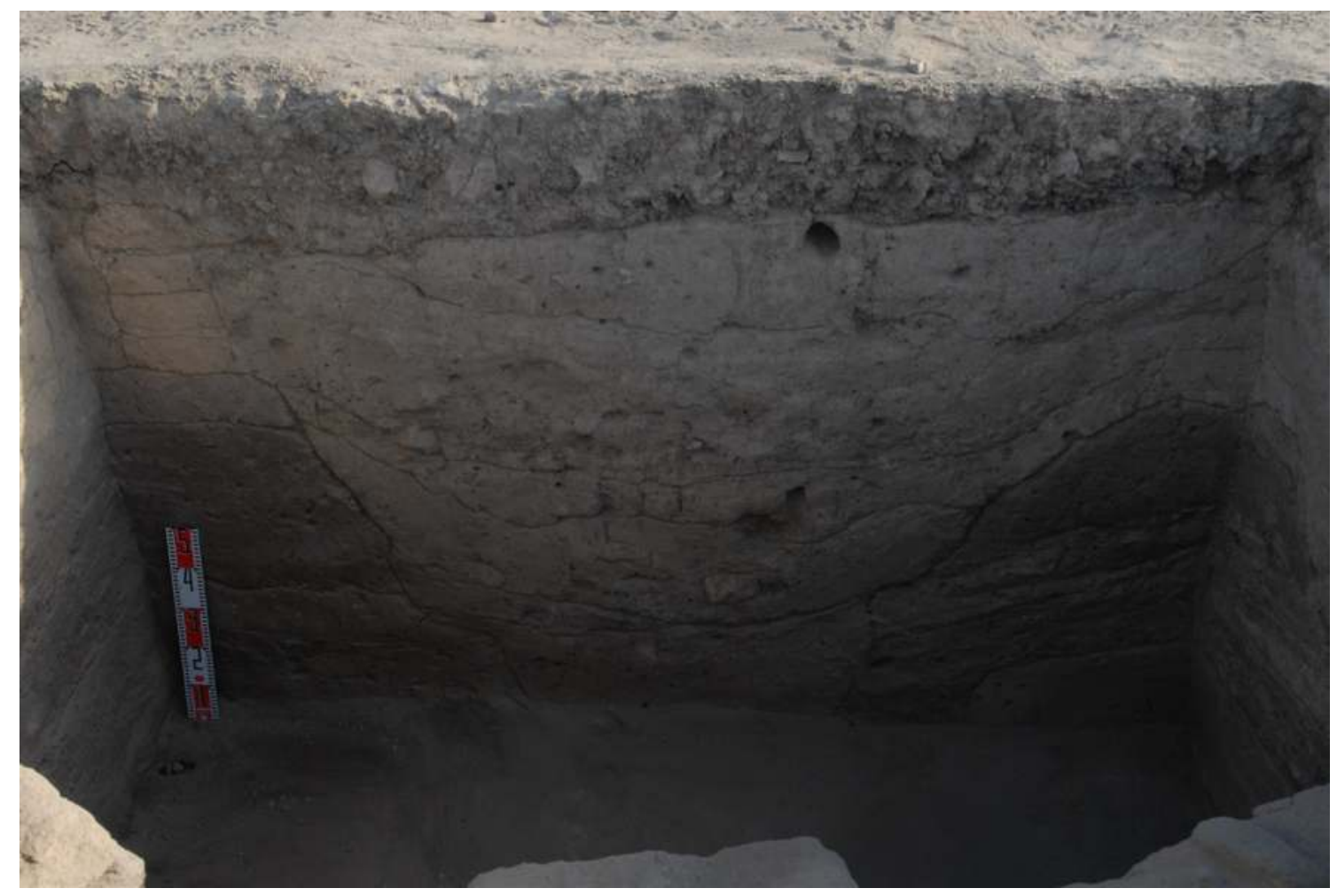

Fig. 5.19 Section of the south wall of Pit 93A2. Note that two ditches are overlapped.

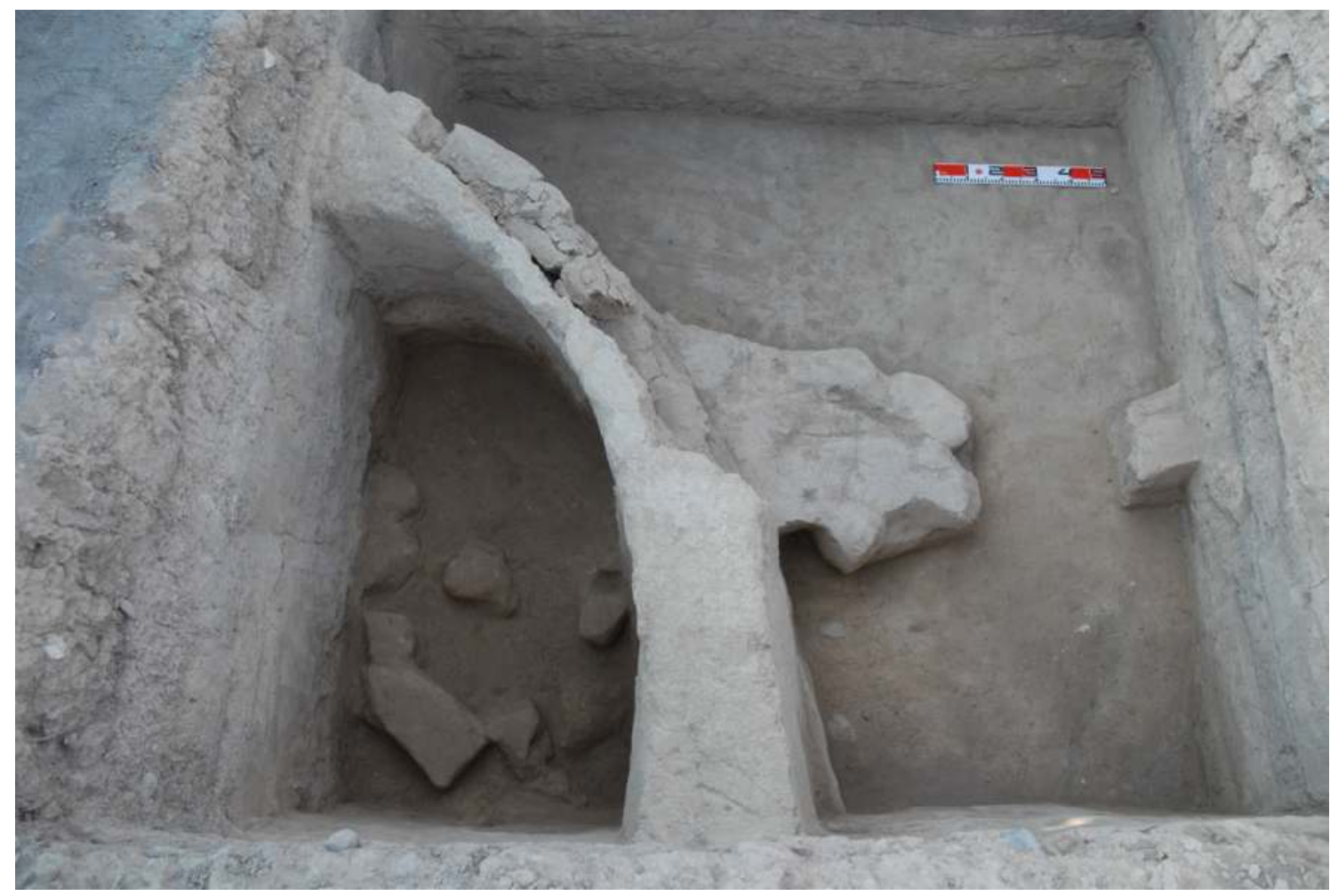

Fig. 5.20 The mud-brick structure in 93A1, seen from the north. Note that mud-brick rubble on both sides of the wall was discovered on the first floor. This structure had another floor underneath. 


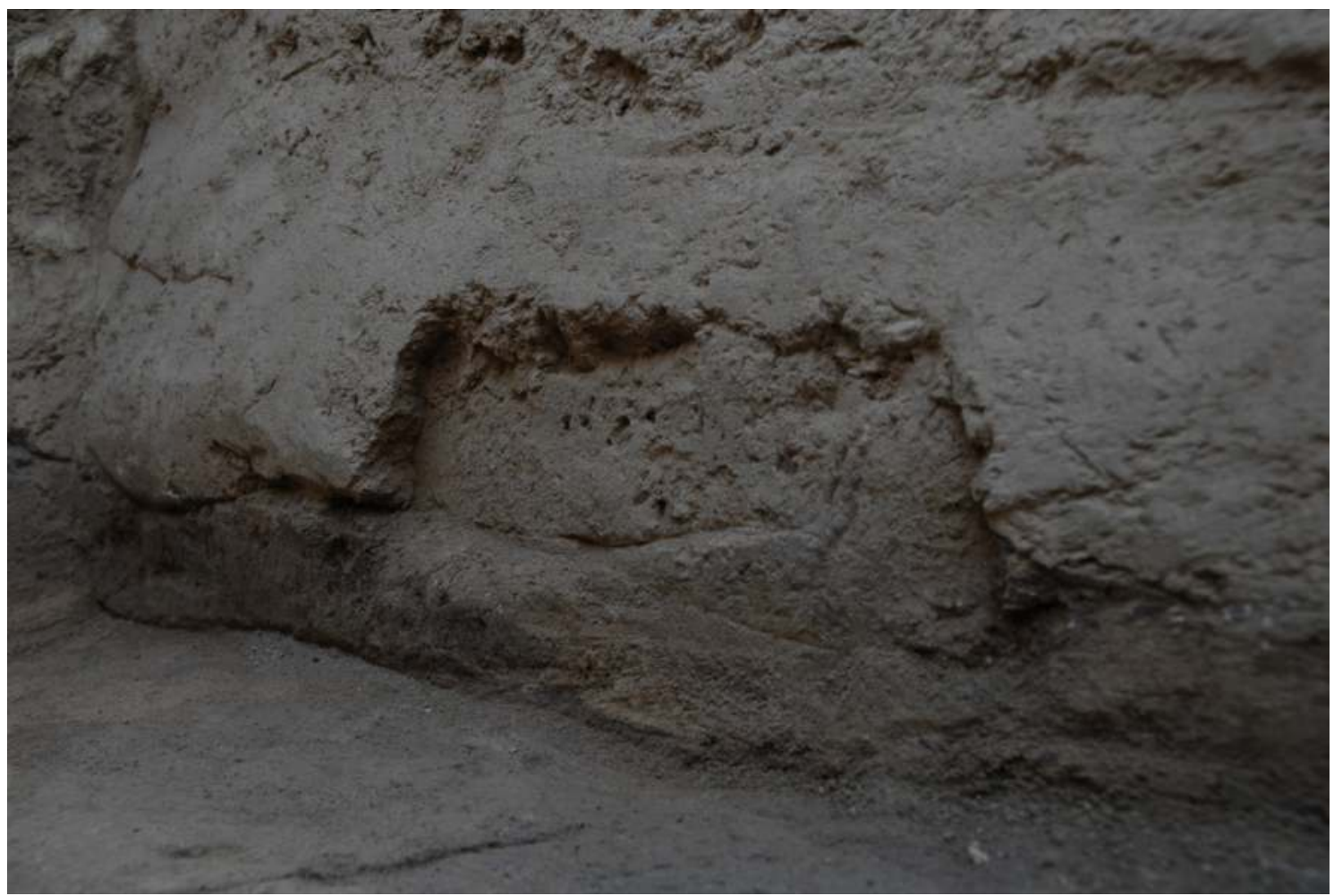

Fig. 5.21 The base of the curvilinear wall in 93A1, seen from the east

5.23). This level corresponds to the one on which the above-mentioned Neolithic architecture of Pit 93A1 was constructed. It inclines slightly downward to the north. The third level is situated $70 \mathrm{~cm}$ below the second one. It is also characterized by ash layers (93A1-13), probably denoting living floors (Fig. 5.23 ), on which plenty of mud-brick rubble had accumulated.

An exceptionally deep pit was dug into the virgin soil from the fourth or earliest level (Fig. 5.24). One "step" was found on the north wall, possibly used as a platform for digging (Fig. 5.25). The fill of the pit consisted of homogeneous grayish-brown sediments including ash, charcoal, lithics, bones, and cobbles; the loose matrix suggests that the pit was filled relatively quickly. Some burnt soil was also recovered. The pit was at least $2.5 \mathrm{~m}$ deep, but our excavation did not reach its bottom. Furthermore, the precise dimensions were not determined. Accordingly, it cannot be ruled out that the "pit" might in fact have been a ditch or water well. Future enlargement of the excavation area would verify the interpretation of this feature.

The virgin soil is grayish-yellow loess, consisting of very fine homogeneous sediments containing no artifacts or other organic remains. Although this layer continued downward, our excavations were suspended at the depth of $5 \mathrm{~m}$ from the present ground surface due to the practical difficulty in digging such a deep pit (Fig. 5.26). We have not been able to reach the gravel layer, whose presence is suggested from the soundings in other squares. Whatever the function of the pit (ditch), the exploited grayish-yellow loess must have served as an ideal raw material for mud-brick architecture. In fact, all of the mud-bricks recovered in the pits of Squares 92A and 93A exhibit a homogeneous grayish-yellow color. These mud-bricks have also been discovered in the central part of the mound, but they are usually used together with gray mud-bricks, which evidently contain ash rather than virgin soil. The use of mudbricks of two different colors has been known at Neolithic mounds of the Shomutepe culture since the early stage of research (Narimanov 1987). The case at Göytepe suggests that the colors of mud-bricks related to the location or depth of the quarries for obtaining building materials.

\subsection{Conclusions}

The ten sounding pits discussed above provided significant insight on the extension of the remaining 


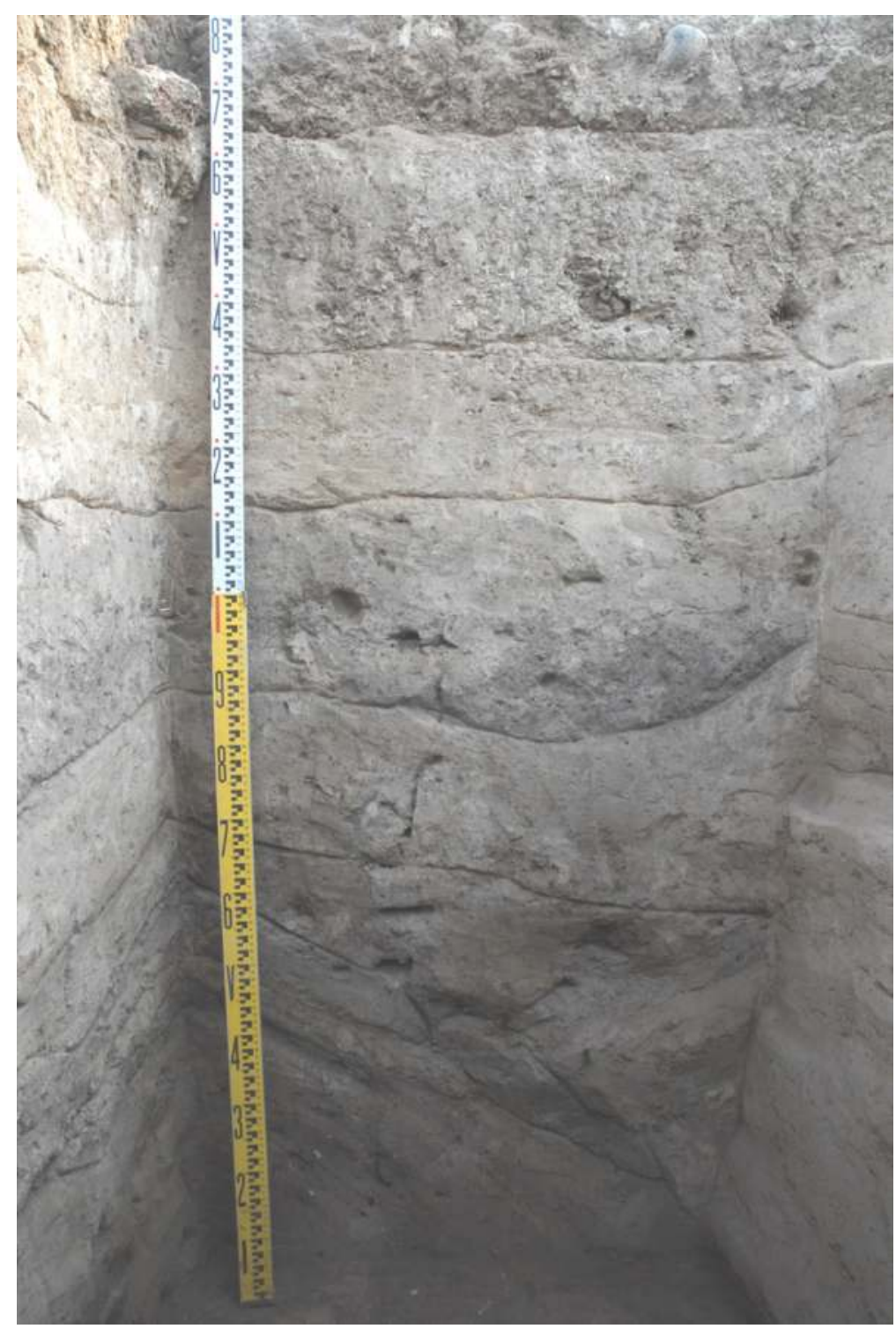

Fig. 5.22 Part of the northern section of Pit 93A1.
Neolithic settlement at Göytepe. It covers an area of at least $120 \mathrm{~m}$ north to south and $135 \mathrm{~m}$ east to west. While the northern and eastern boundaries of the mound have been delineated by natural erosion and human disturbances, the Neolithic deposits in the south, and partly in the west as well, are fairly well preserved. The intact Neolithic deposits at the mound's south edge accumulated as much as $2 \mathrm{~m}$ below the flat surface of the modern agricultural field, and evidently continue further to the south of Square 92A. Therefore, the original size of the mound must have been larger than it is today. Supposing that the mound was round, 140-145 $\mathrm{m}$ would be a reasonable estimate for its diameter. According to our site reconnaissance survey, comparably large Neolithic mounds have not been discovered in the vicinity (Chapter 8). Thus, Göytepe can be regarded as a major settlement that may have played a significant social role in the regional Neolithic community.

Another research question that the periphery soundings aimed to address was whether structures unique to the marginal area of the settlement existed. In fact, the soundings revealed two unique types of Neolithic architecture: the massive double wall recovered at the eastern edge $(96 \mathrm{~F})$ and the ditches or deep pits at the southern edge (93A2 and 92A). Comparable structures have been recovered at the northern end in the main excavation sector of Göytepe (Square 4BII; Chapter 4), but not from the central part of the main sector. Ditches and bank walls might have had more specific functions at the edge of the settlement. Ditches have also been known from marginal areas of the Neolithic settlements of Kamiltepe (Helwing and Aliyev 2017) 


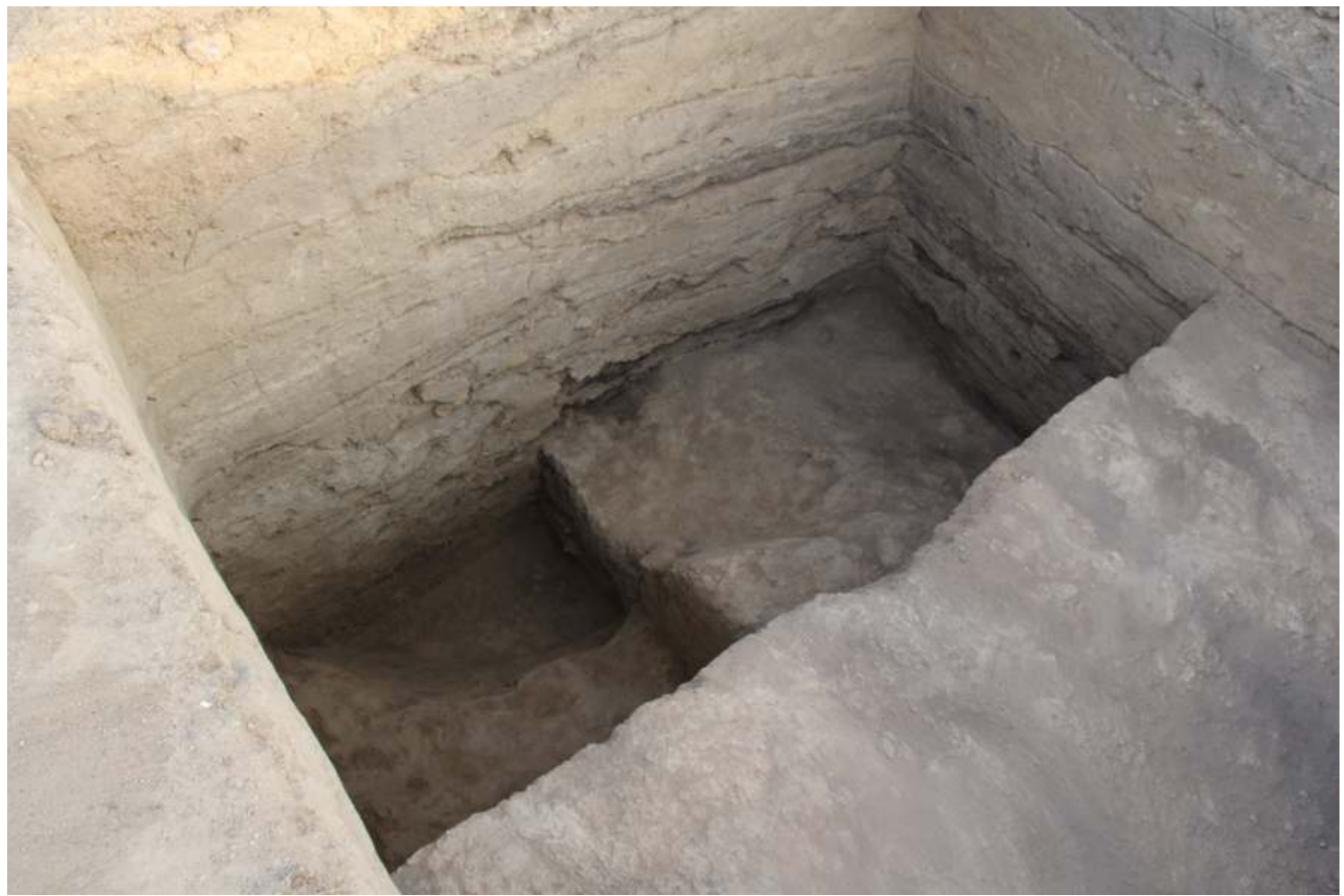

Fig. 5.23 The pit in Square 92A, seen from the southeast. The second and third levels are visible as blackish bands on the section.

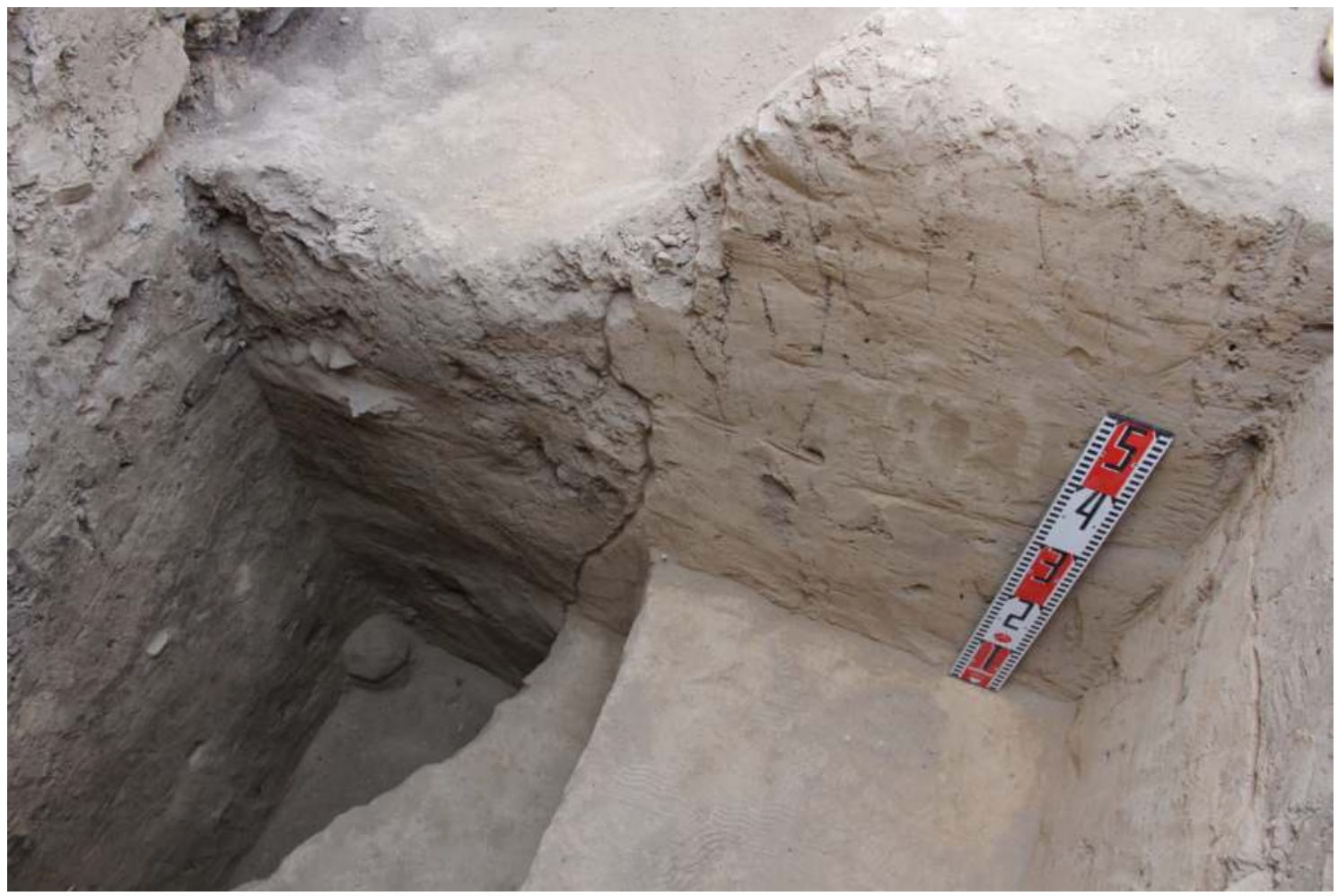

Fig. 5.24 Large pit, seen from the south. 


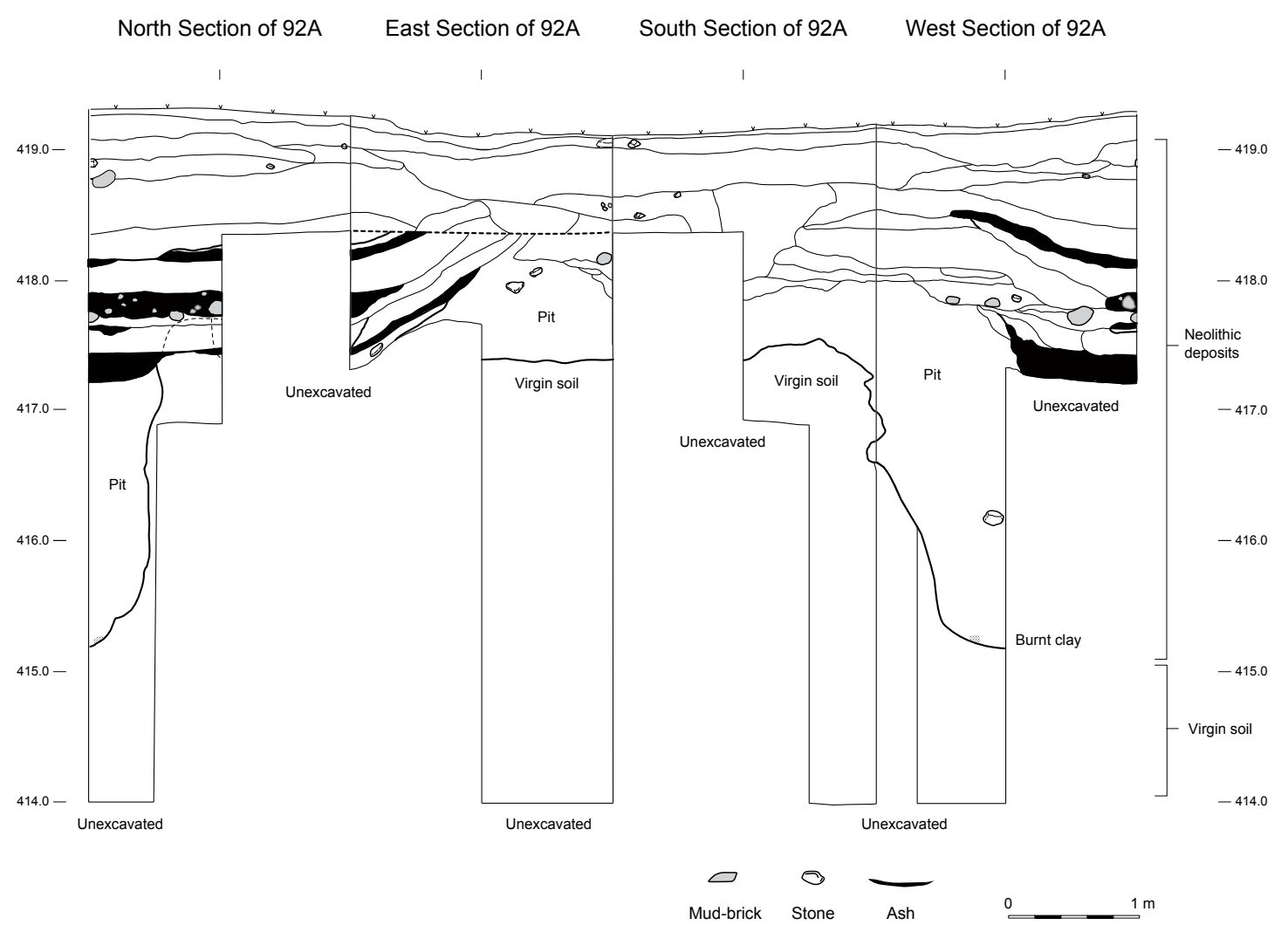

Fig. 5.25 Sections of the sounding pit in 92A, seen from the south.

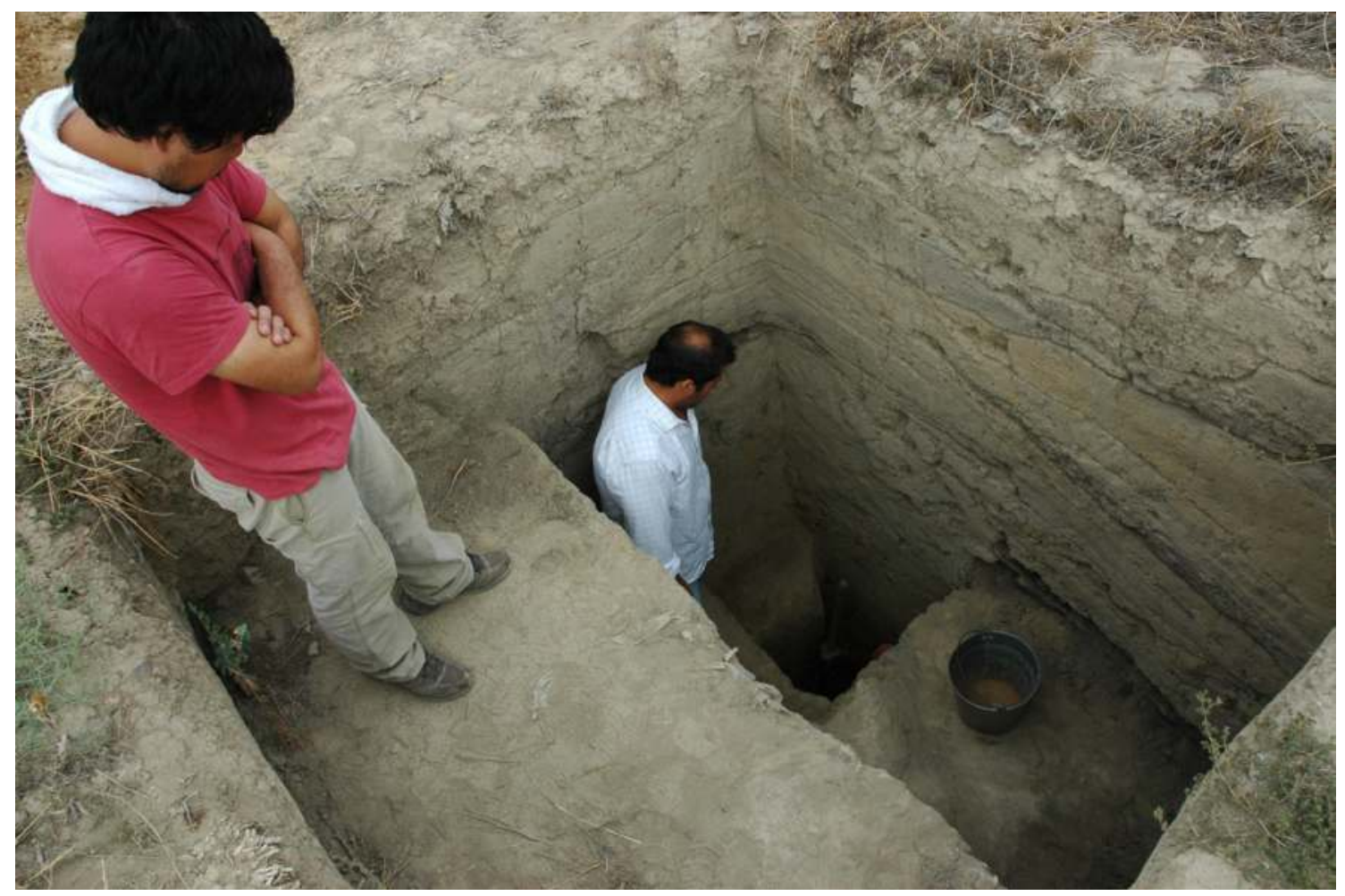

Fig. 5.26 Excavations of the sounding pit in 92A, seen from the northeast. 
Table 5.1 Stratigraphic contexts of virgin soil at Göytepe.

\begin{tabular}{|l|l|l|l|}
\hline \multicolumn{1}{|c|}{ Square } & \multicolumn{1}{c|}{ Overlying deposits } & \multicolumn{1}{c|}{ Virgin soil } & \multicolumn{1}{c|}{ a.s.I } \\
\hline 7B (north) & modern & gravel & $415.7 \mathrm{~m}$ \\
\hline 4BII (north) & Neolithic & yellow loess (thin) & $415.2 \mathrm{~m}$ \\
\hline 97G (east) & modern & gravel & $415.4 \mathrm{~m}$ \\
\hline 97F (east) & Neolithic & yellow loess (thin) & $415.1 \mathrm{~m}$ \\
\hline 1GG (west) & Neolithic & gravel & $415.5 \mathrm{~m}$ \\
\hline 92A (south) & Neolithic & yellow loess (thick) & $415.4 \mathrm{~m}$ \\
\hline
\end{tabular}

and Aruchlo (Hansen and Ullrich 2017). Examples of terrace/bank walls are unknown from the available literature, although they may be recovered when the excavations of other settlements are also extended to the edges.

In terms of the Neolithic landscape, the nature of virgin soil provides useful information for site interpretation. The stratigraphic contexts of virgin soil in the sounding pits are summarized in Table 5.1. Data from the excavations of Square 4B is also listed (Chapter 4). The virgin soil at the northern and western ends consists of river gravel, on which secondary Neolithic and later deposits were accumulated. On the other hand, the virgin soil in the main part of the mound is represented by a layer of grayish-yellow loess, whose thickness varies from its maximum in the south (more than $3 \mathrm{~m}$ ) and minimum in the north and east (less than $50 \mathrm{~cm}$ ). The altitude at the top of the virgin soil also varies, being higher to the south and lower to the north and east. The uneven accumulation of loess by locality is remarkable. The lack or thinness of loess deposits at the north, west, and east edges of the mound suggest the occurrence of complicated local erosional processes in the past. It is also notable that we never reached any gravel layer in Square 92A at the low altitude of $408.0 \mathrm{~m}$ a.s.l. If present, the top of the gravel should be lowest in this area, a fact to be considered when attempting to reconstruct the geomorphology of the surrounding area over a longer time span.

Whatever the case, the relatively similar altitudes (ca. $409.5 \mathrm{~m}$ ) at the top of virgin soil at the northern and eastern edges are remarkable, while those at the south and west are 1 to $2 \mathrm{~m}$ higher. This configuration suggests that the first Neolithic society lived on the northern edge of a terrace of yellowish-brown loess, which was 1 to $2 \mathrm{~m}$ higher than the lower terrace and gently sloped to the north. After the mound was abandoned, erosion also occurred that completely removed Neolithic sediments in the surrounding area. This reconstruction is worthy of testing and further elaboration using geoarchaeological and geomorphological data.

\section{References}

Hansen S. and M. Ullrich (2017) Excavations at Aruchlo. Preliminary report on the 2012-2014 campaigns. In: The Kura Projects: New Research on the Later Prehistory of the Southern Caucasus, edited by B. Helwing, T. Aliyev, B. Lyonnet, F. Guliyev, S. Hansen, and G. Mirtskhulava, pp. 201-222. Berlin: German Institute of Archaeology, Eurasian Department.

Helwing, B. and T. Aliyev (2017) Excavations at Aruchlo. Preliminary report on the 2012-2014 campaigns. In: The Kura Projects: New Research on the Later Prehistory of the Southern Caucasus, edited by B. Helwing, T. Aliyev, B. Lyonnet, F. Guliyev, S. Hansen, and G. Mirtskhulava, pp. 11-42. Berlin: German Institute of Archaeology, Eurasian Department.

Narimanov, I. (1987) The Culture of the Most Ancient Farming and Stock-Breeding Population of Azerbaijan. Baku: National Academy of Sciences (in Russian with an English summary).

Nishiaki, Y., F. Guliyev, S. Kadowaki, and T. Omori (2018) Neolithic residential patterns in the southern Caucasus: Radiocarbon analysis of rebuilding cycles of mudbrick architecture at Göytepe, west Azerbaijan. Quaternary International, 474: 119-130. 



\title{
Chapter 6
}

\section{Mud-bricks in Neolithic architecture at Göytepe}

\author{
Yoshihiro Nishiaki, Farhad Guliyev, and Emmanuel Baudouin
}

\subsection{Introduction}

An increasing amount of evidence indicates that the Neolithic cultures of the South Caucasus underwent rapid cultural evolution in the early 6th millennium $\mathrm{BC}$ soon after the emergence of Neolithic society. Such evidence attests to autonomous development, regardless of whether Neolithic culture was introduced from southwest Asia (Nishiaki et al. 2019). One of the best examples of this cultural evolution is the production and use of pottery. Although the material culture of Fertile Crescent communities in southwest Asia around $6000 \mathrm{BC}$ was characterized by the proliferation of pottery, the earliest southern Caucasus Neolithic sites do not always yield a comparably large amount of pottery. In the Middle Kura Valley of West Azerbaijan, the substantial production of pottery began only a few centuries after the introduction of the Neolithic economy (Nishiaki et al. 2015a; cf. Marro et al. 2019).

A local development can also be seen in architecture. Circular buildings were prevalent in the Neolithic cultures of the South Caucasus, although they have also been found in the Halafian culture of the Fertile Crescent. The classic architectural style is characterized by ring-shaped compounds consisting of a series of circular buildings $2-3 \mathrm{~m}$ in diameter connected with wing walls surrounding a courtyard (see Chapter 3). Recent field investigations in the Middle Kura Valley have revealed that this architectural style was a local development. In its early stages, a different style with snowman-shaped buildings was popular with a larger, roughly 5-6 $\mathrm{m}$ circular structure abutted by a smaller structure of 1.5-2 $\mathrm{m}$ and connected via a narrow passage (Nishiaki et al. 2015a). Furthermore, in the early stages, this building type was accompanied by circular semi-subterranean buildings reminiscent of the domestic structures used by incipient farmers of the Fertile Crescent (Baudouin 2019).

These changes in architectural style are likely related to other socio-economic changes that occurred during the formative period of the Neolithic group in the region. The aim of this chapter is to provide new data on the architectural development of the Shomutepe culture of the Middle Kura Valley, namely regarding the production of mud-bricks at Göytepe. Based on stratigraphic data, we examine how mud-brick manufacturing technology might have developed during the Neolithic period in Göytepe.

\subsection{Mud-bricks in Göytepe architecture}

Mud-bricks can be examined from a variety of perspectives including those based on raw material and techno-morphology (Love 2012). The present study focuses on their morphology. The shape and size of mud-bricks excavated at Göytepe were examined in the field. Mud-bricks observable on the wall's surface were chosen for examination to avoid dismantling the walls. This study method was employed because Göytepe architecture was preserved intact for a planned archeological park. This method can admittedly result in uncertain identification and/or measurement of mud-bricks. For example, the precise thickness of mud-bricks cannot be determined unless they form a precise rectangular shape. Furthermore, the bonding mortar makes the edges and shape of the mud-bricks unidentifiable on the wall surfaces. Measuring the width of the mud-bricks is also difficult because of the clay plaster covering them; therefore, the measured width 
depends on the amount of clay plaster removed during excavations. Given these constraints, the data presented below, which does not include thickness measurements, should be treated as preliminary. Nevertheless, our method allowed it to study a large number of mud-bricks. We believe that this pilot study of the data is worthy of analysis.

\subsubsection{Mud-brick shape}

The literature indicates that South Caucasian Neolithic communities manufactured at least two types of mud-bricks. Both are rectangular, but differ in their upper and/or lower surface shapes: there is a flat type and a plano-convex face type (Chataigner
1995). The latter displays a distinct morphology with a flat base and domed top with rectangular sides (Hansen and Ullrich 2017).

The representative shapes of the mud-bricks were recorded for each building level at Göytepe. Despite the constraints in determining the precise shape of the mud-bricks, our preliminary conclusion was that the mud-bricks of Göytepe were made principally in a plano-convex shape. The plano-convex mud-bricks were laid with a convex surface facing upward. Although plano-convex mud-bricks were identified throughout the sequence, the convexity seems more conspicuous in the earlier phase (Levels 14-8; Fig. 6.1) than in the later phase (Levels 7-1; Fig. 6.2). For
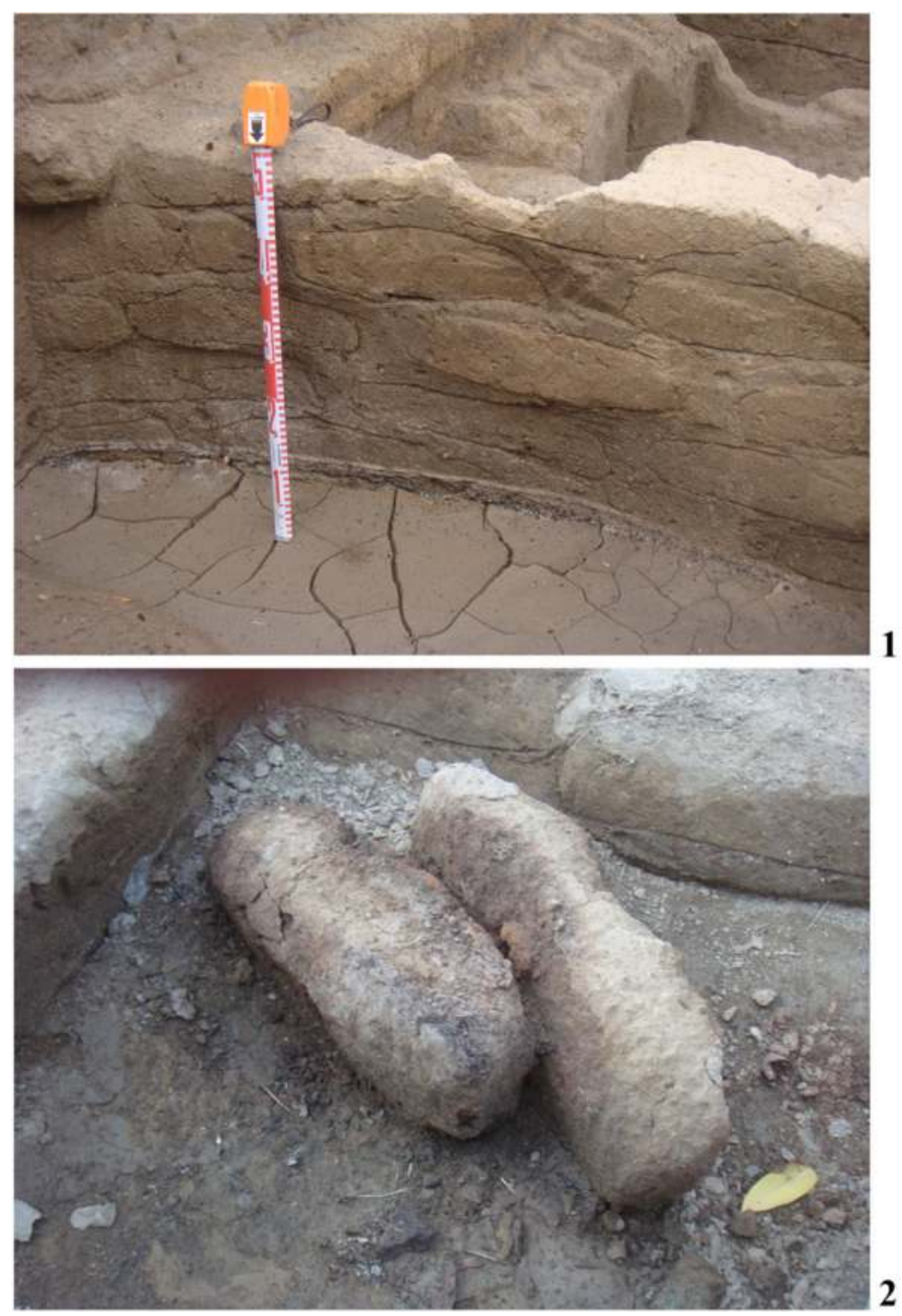

Fig. 6.1 Plano-convex mud-bricks from earlier levels of Göytepe. 1: Level 10, ca. 32 cm long; 2: Level 10, ca. 32 cm long. 

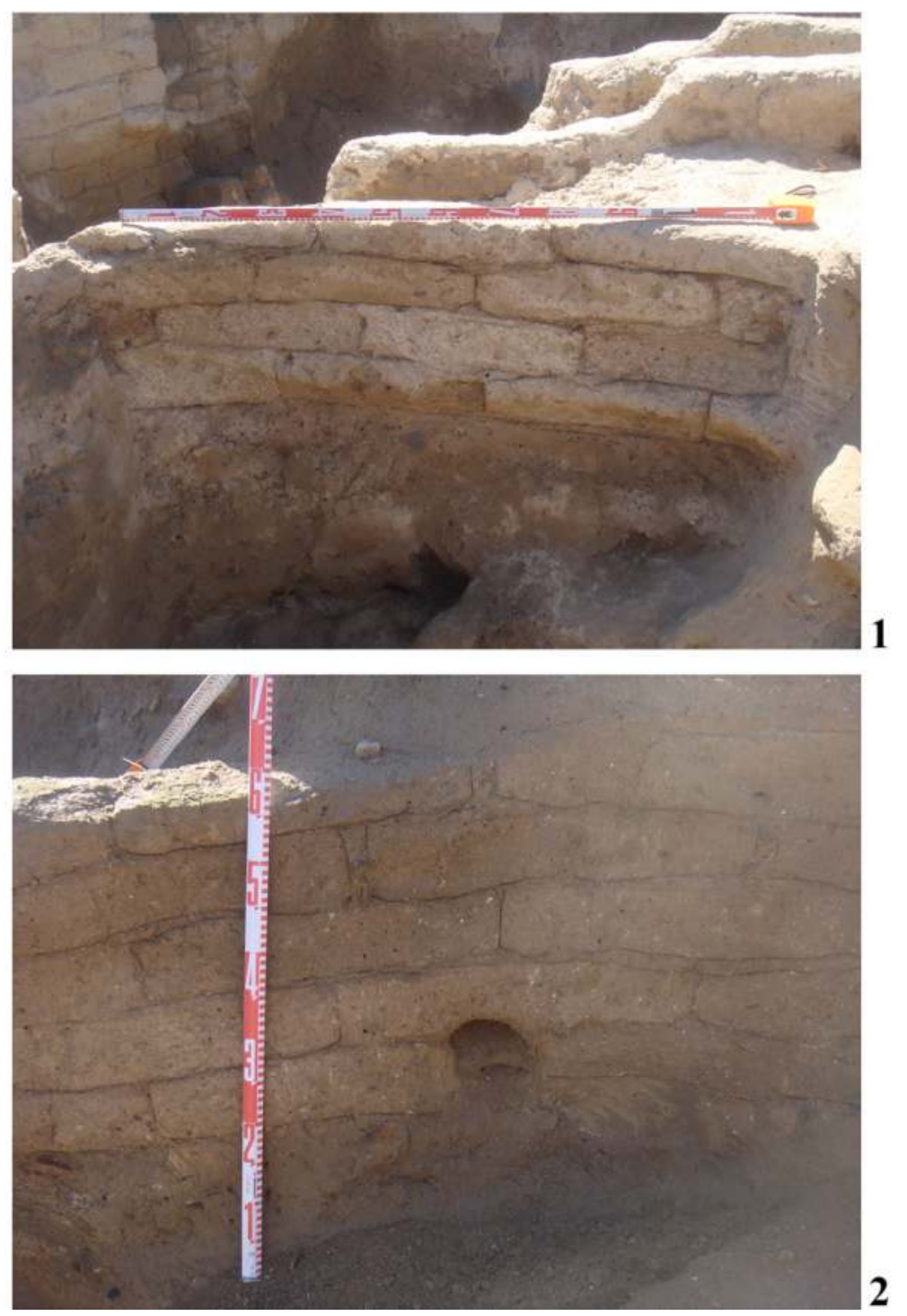

Fig. 6.2 Larger and less-convexed mudbricks from later levels of Göytepe. 1: Level 4, ca. $40 \mathrm{~cm}$ long; 2: Level 7, ca. $40 \mathrm{~cm}$ long.

the longer mud-bricks, which were more common in the later phase, the convexity seemed less clear. These samples led us to report the dual use of planoconvex and flat mud-bricks at Göytepe (Guliyev and Nishiaki 2012). However, it is still unclear whether any solid modality for flat mud-brick production exists. It may simply reflect the difficulty of identifying the convexity of longer mud-bricks. It is currently clear, however, that plano-convex mudbricks were maintained throughout Göytepe.

\subsubsection{Mud-brick size}

A total of 1,080 mud-bricks from 78 structures at Göytepe were measured. Their lengths and widths are shown in Figs. 6.3 and 6.4, according to the cultural phases. First, the large variations in the sizes of the mud-bricks should be noted. The lengths ranged between 16 and $52 \mathrm{~cm}$ (Fig. 6.3) and widths ranged between 11 and $24 \mathrm{~cm}$ (Fig. 6.4). Assuming that Neolithic builders employed molding frames for mud-brick production, this large variability is impressive. In this regard, however, we should acknowledge the possibility that mud-brick sizes could be misidentified in the field rather than presume the use of many types of molding frames during the same period or the shaping of mud-bricks by hand. Breakage in a single mud-brick may have been identified as a joint consisting of two bricks, and some joints may have been overlooked, leading to situations in which multiple bricks were measured as one large brick.

On the other hand, if our measurements more or less represent the archaeological reality, other noises in measurement can also be postulated. Short 


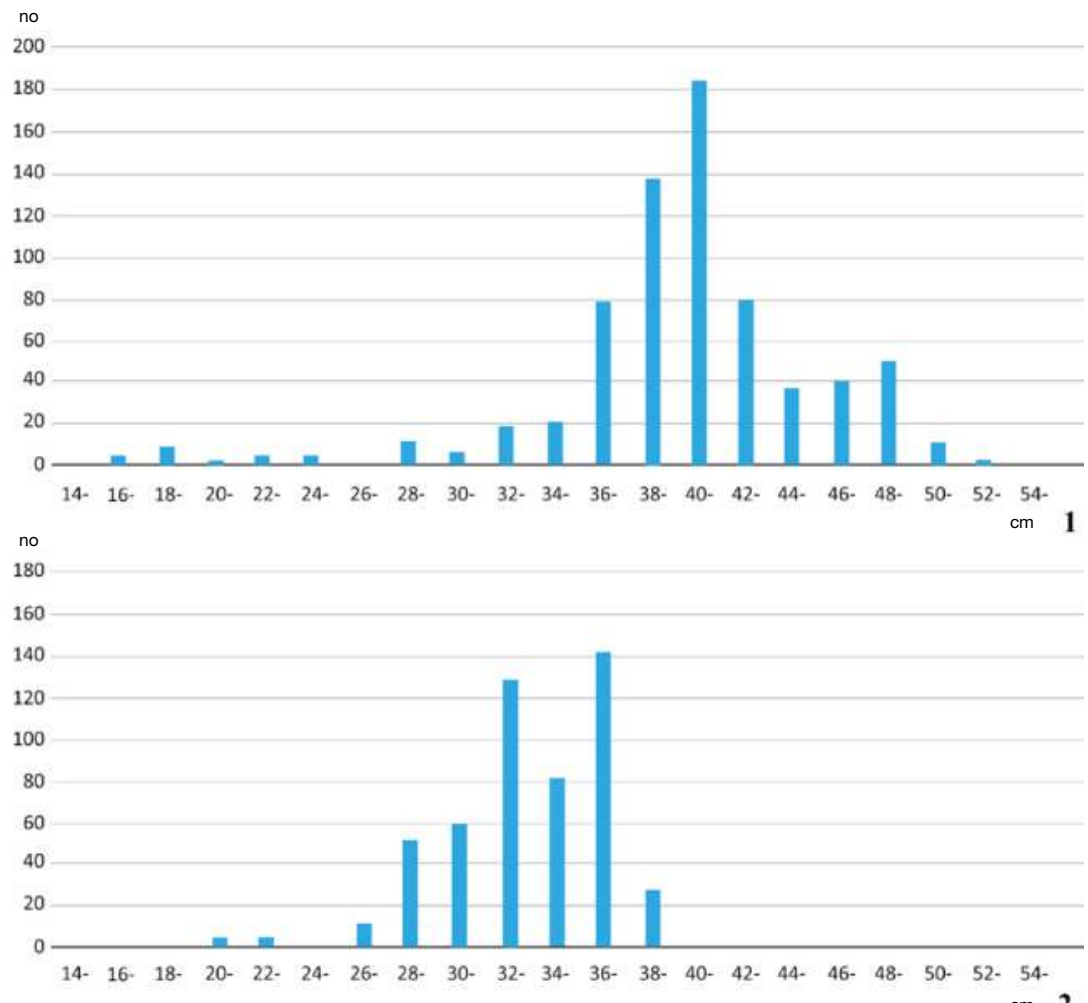

Fig. 6.3 Length of mud-bricks from Göytepe. 1: Levels 7-1 ( $n=924) ; 2$ : Levels 14-8 $(n=156)$.
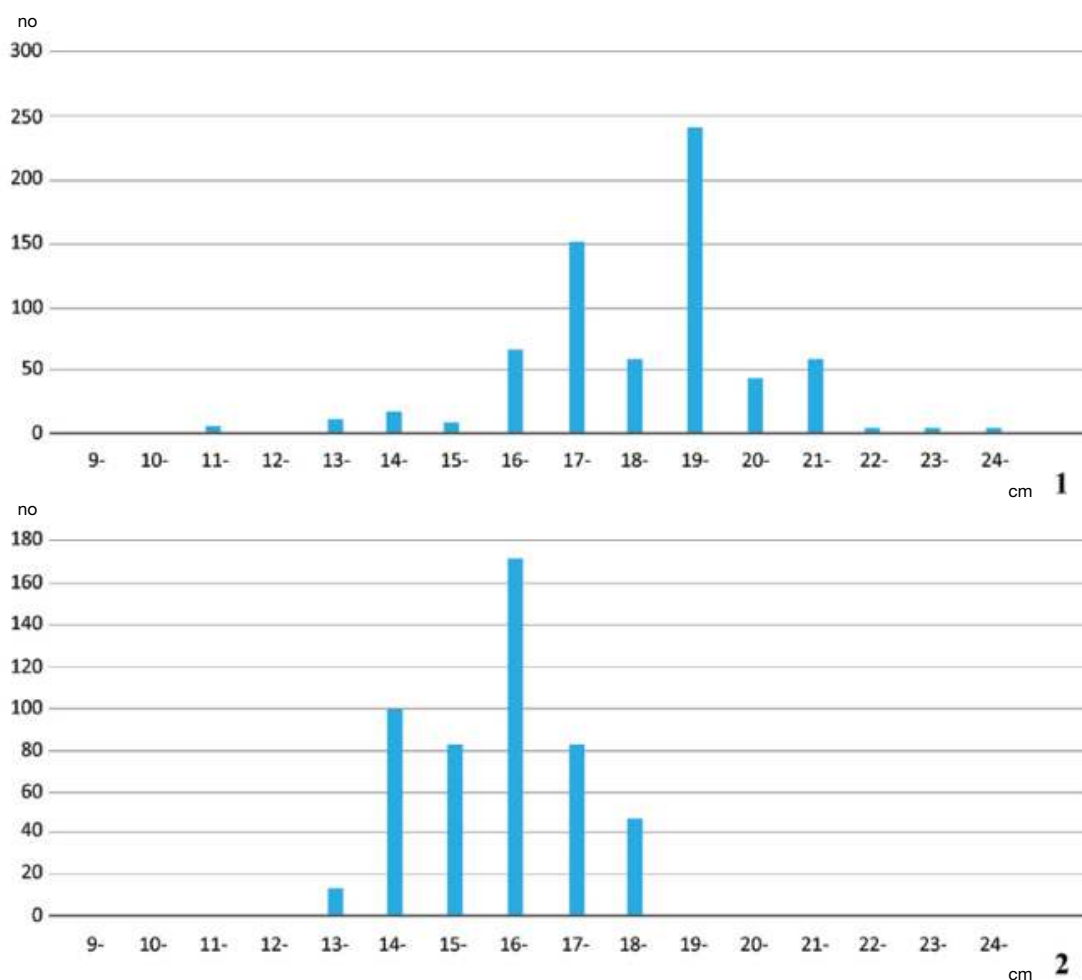

Fig. 6.4 Width of mud-bricks from Göytepe. 1: Levels 7-1 ( $n=153) ; 2$ : 2 Levels 14-8 $(n=131)$. 
mud-bricks may indicate that Neolithic artisans deliberately broke longer mud-bricks to fit them into the length of a wall. This practice is often noted in ethnographic records as it is even used in modern stone block building construction in this region (Fig. 6.5). Thus, it would not be surprising if this practice was present in prehistoric times. Examples can be found in a number of photos of 6th millennium-BC sites in the Fertile Crescent such as those of Telul eth-Thalathat II Level XIV (Fukai and Matsutani 1981) and Tepe Gawra Level XIII (Tobler 1950).

Under such circumstances, it may be advisable to overlook detailed variations and instead emphasize the general patterns in size measurement (see Nishiaki et al. 2001). The histograms of the length and width measurements at Göytepe sites suggest at least two types (Figs. 6.3 and 6.4): a small type, approximately $32-36 \times 16-17 \mathrm{~cm}$, and a larger (middle) type, 38-42 $\times 18-20 \mathrm{~cm}$. In addition, an even larger mud-brick might have existed, measuring 48-50 × 20-22 cm (Fig. 6.3: 1). As expected, the longer bricks had a larger width. The proportional relationship was more securely maintained for some mud-bricks. Certain mud-bricks popular in the earlier phase were regularly manufactured with a size of ca. $32 \times$ $16 \mathrm{~cm}$. This 2-1 length-width ratio allowed several standardized brick-laying methods (Fig. 6.6).

Fig. 6.7 shows the results of a seriation analysis in which the horizontal bars indicate the occurrence frequency (\%) of mud-bricks with a specific length and width at each level. We can define a marked size change through time. The mud-brick size showed a dramatic increase between the early and late phases of Göytepe (Fig. 6.7). The late phase apparently even includes very large mud-bricks, the largest type (ca. $48-50 \mathrm{~cm}$ long), though in a smaller number. On the contrary, in the early phase, the mud-brick size seems become smaller through time. These minor changes within each of the phases need to be verified in the future studies. In the meantime, the marked size change of mud-bricks in a single occupational sequence at Göytepe, an phenomenon previously unknown at any other South Caucasian Neolithic sites calls for much attention.

\subsection{Discussion}

\subsubsection{Mud-bricks of the South Caucasian Neolithic}

The dataset presented above is the first systematically collected from mud-bricks at a Neolithic settlement in the South Caucasus. Though excavation reports mentioning the shapes, sizes, and other features of mud-bricks from related sites do exist (Baudouin 2019), the accuracy with which each reference represents the archeological reality is unknown. As discussed in the previous section, it is not always easy to determine the morphological characteristics and size of sun-dried mud-bricks. Nevertheless, the patterns discovered in this study can be significant
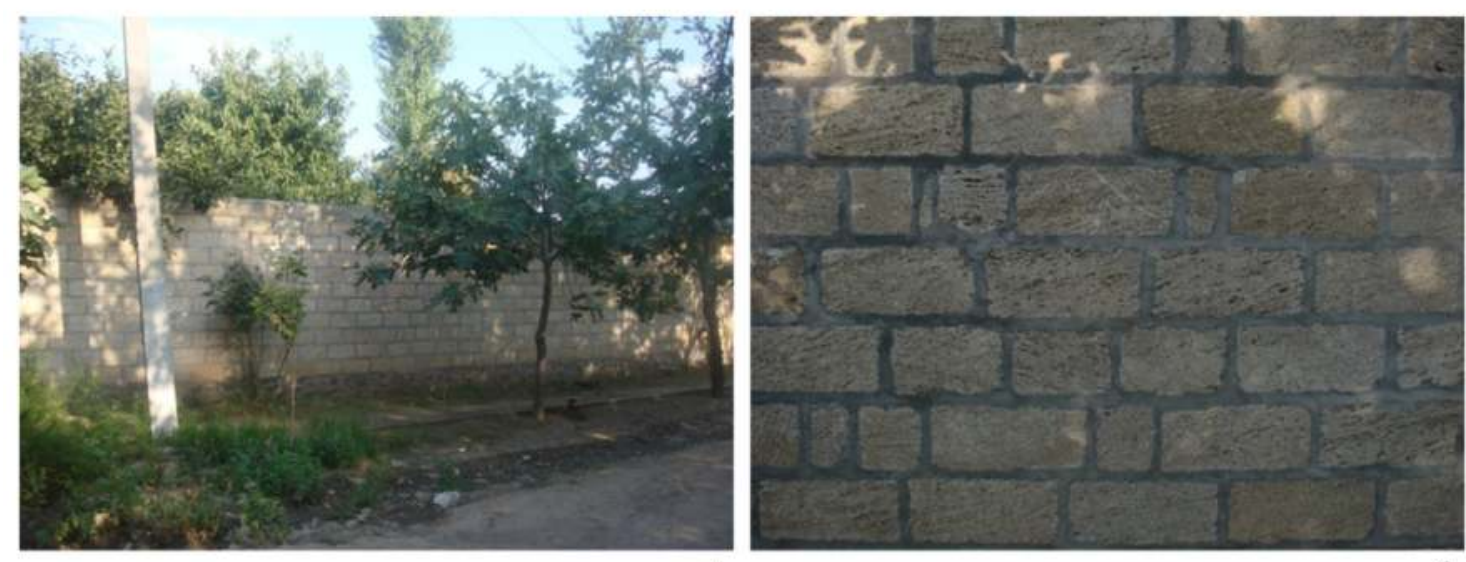

Fig. 6.5 Modern stone block wall in Qovlar Village, Tovuz, Azerbaijan. 1: General view; 2: Close-up view. Note the large variability in block length visible on the wall surface. 

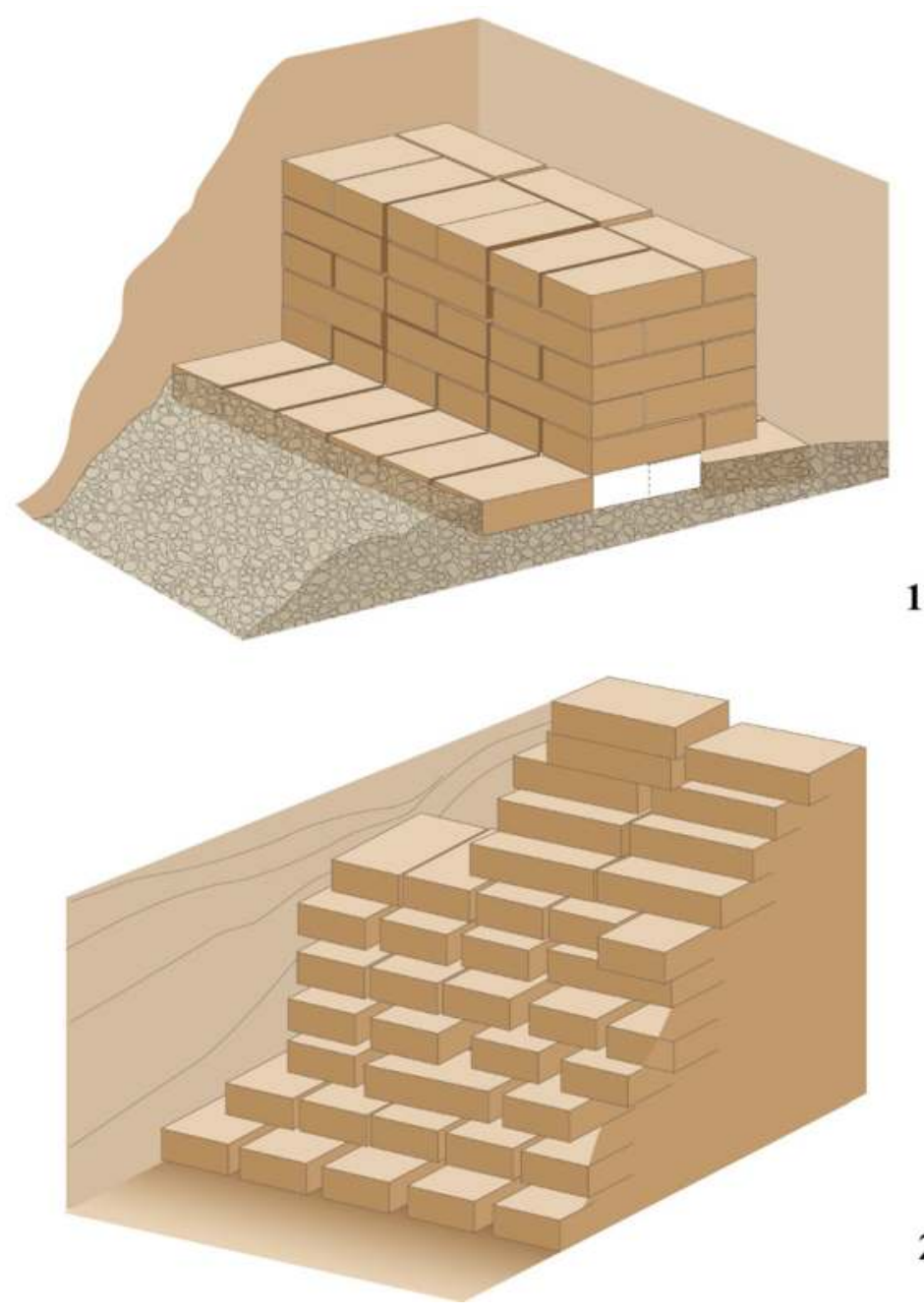

Fig. 6.6 Isometric reconstruction of retaining walls of Göytepe. 1: Square 96F built on virgin soil (river gravel). See Fig. 5.10 of Chapter 5; 2: Level 13 of Square 4BII. See Fig. 4.50 of Chapter 4. because they include previously unknown findings.

Evidence has been found for the use of mudbricks from the beginning of the Neolithic Period in the Middle Kura Valley. One of the oldest Neolithic sites, Hac1 Elamxanlı Tepe, already showed evidence of the mastery of mud-brick production technology (Nishiaki et al. 2015b). The early use of mud-bricks has also been recognized at other sites in the Middle Kura Valley, but not so evidently in other regions of the southern Caucasus. In the Ararat Plain of Armenia, the literature mentions the use of mudbricks at sites such as Akhnashen and Aratashen, but the use of cob walls has also been reported as at Masis Blur (Martirosyan-Olshansky et al. 2013). In the Mil Plain in southeast Azerbaijan, the use of mud-bricks was confirmed only after $5600 \mathrm{BC}$, a few centuries later than the start of the Neolithic Period (Helwing 2017). Similarly, recent excavations confirmed the use of cob walls in the earliest Neolithic architecture at Kültepe, the Middle Araxes Valley (Marro et al.
2019).

Region-specific processes in the earliest mudbrick production history of the South Caucasus are also illustrated by the morphology of mudbricks. Chataigner (1995: 58) has argued that the production of plano-convex mud-bricks is an important "technocultural" marker for Neolithic communities in the Middle Kura Valley, as opposed to the prevalent use of flat mud-bricks in the Ararat Plain. Our research at Hac1 Elamxanlı Tepe demonstrated the use of plano-convex mud-bricks from their earliest occupational stage (Nishiaki et al. 2015b). Here, the use of such mud-bricks at Göytepe has been confirmed, showing that the communities of this mound followed the local architectural tradition. At the same time, it should be noted that most recent information from related sites suggests a need to revise this regional distinction (Fig. 6.8). For instance, plano-convex and flat mud-bricks are said to have been used in two different buildings 

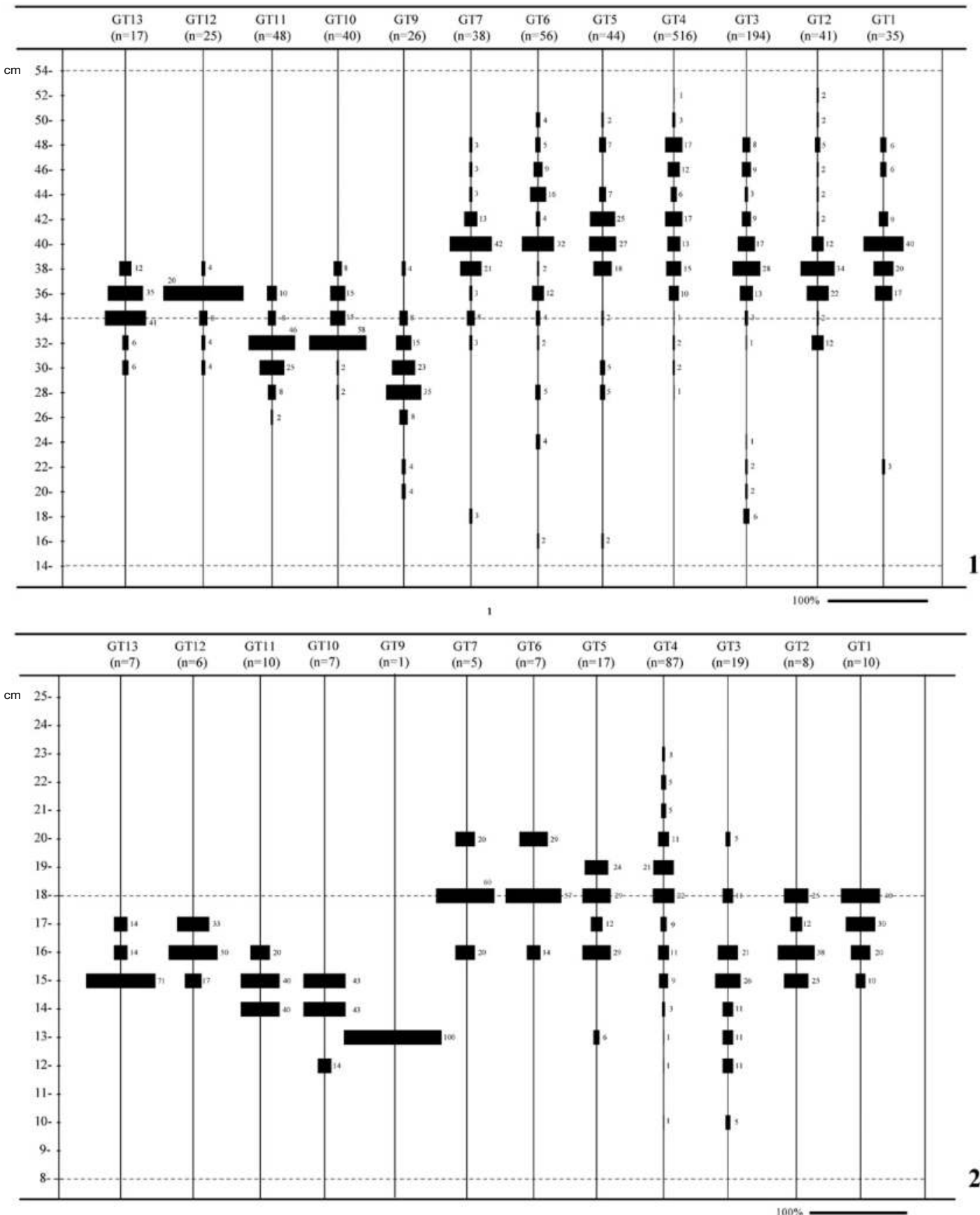

Fig. 6.7 Stratigraphic changes in mud-brick size at Göytepe. Later levels to the right. 1: Length $(n=1,080)$; 2: Width $(n=284)$.

on the same level as Gadachrili Gora (Hamon et al. 2016: 159-160). Additionally, the region in which flat mud-bricks were produced may have extended to the Lower Kura Valley and the Mil Plain. Further, the region with plano-convex mud-bricks seems to have extended beyond the Middle Kura Valley to the Ismail Tepe in the Karabagh Plain, which is situated on a tributary of the Kura River (Baudouin
2019). Nevertheless, it is important to note that there is no evidence of plano-convex mud-bricks in the Ararat Plain, the Araxes Valley, or the Mil Plain communities. Their absence in the Ararat Plain is particularly interesting when considering the current claim that the coeval Neolithic culture of the region should be grouped with that of the Middle Kura Valley as part of the Aknashen-Shulaveri-Shomutepe 


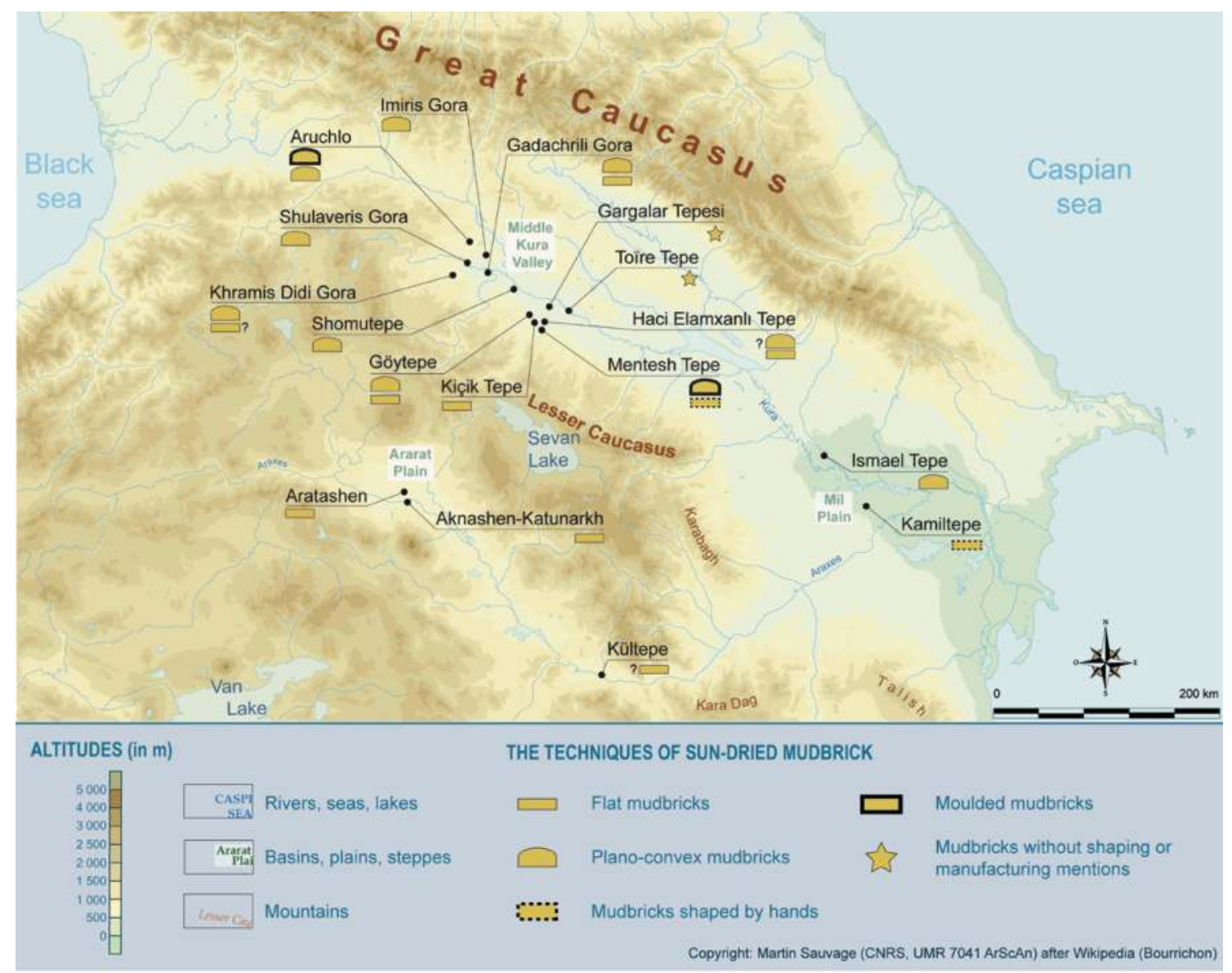

Fig. 6.8 Map showing regional variability of Neolithic mud-brick types in the southern Caucasus.

culture (Badalyan et al. 2010).

This regional variability can also be identified in the use of molding frames. Although the shape and size of the mud-bricks recovered at each site are regularly discussed, considerations of their manufacturing techniques are limited. So far, Chataigner (1995: 57-58) seems to be the first to have mentioned such details. She surmised from the then-available data that mud-bricks at the Kura Valley Neolithic sites were shaped by hand as in the Pre-Pottery Neolithic mud-bricks of the Levant. However, recent discoveries at Aruchlo (Ioseliani 2017) and Mentesh Tepe (Baudouin et al. 2018) confirm the production of molded plano-convex mud-bricks in the Kura Valley. At these sites, careful examination revealed two sets of data. The first is related to the rim at the top of the bricks, which is considered to be a consequence of the removal of the frame by the top (Fig. 6.9: 1 and 2 ). The potential rim on the bottom could indicate a depression of the frame, indicating the use of a specific technique called moule enfoncé (pastry cutter) (Aurenche 1981: 65; Sauvage 1998: 22).
Second, slight longitudinal ridges on the convex face of several mud-bricks could indicate an equalization of the surface before the removal of the frame (Fig. 6.9: 3), unless these ridges were intended for easier bonding with mortar during construction (Aurenche 1981: 62; Sauvage 1998: 41-42). They could also indicate both. Third, the straight edges of the mudbricks indicate the use of molding frames (Fig. 6.9: $3,4)$. In the Ararat Plain, no site produced such evidence (Fig. 6.8). In fact, the mud walls at those sites were not standardized. Accordingly, based on the current knowledge available from the literature, the production of the molded plano-convex mudbrick seems to represent a regional cultural trait of the Kura Valley. Although comparable analysis has not been completed at Göytepe, the regularity of the size as well as the shape closely resembling that of Aruchlo and Mentesh suggests the use of molding at this site.

Data from recently excavated sites such as Mentesh Tepe, Aruchlo, and Gadachrili Gora provide useful perspectives for mud-brick size comparison. 

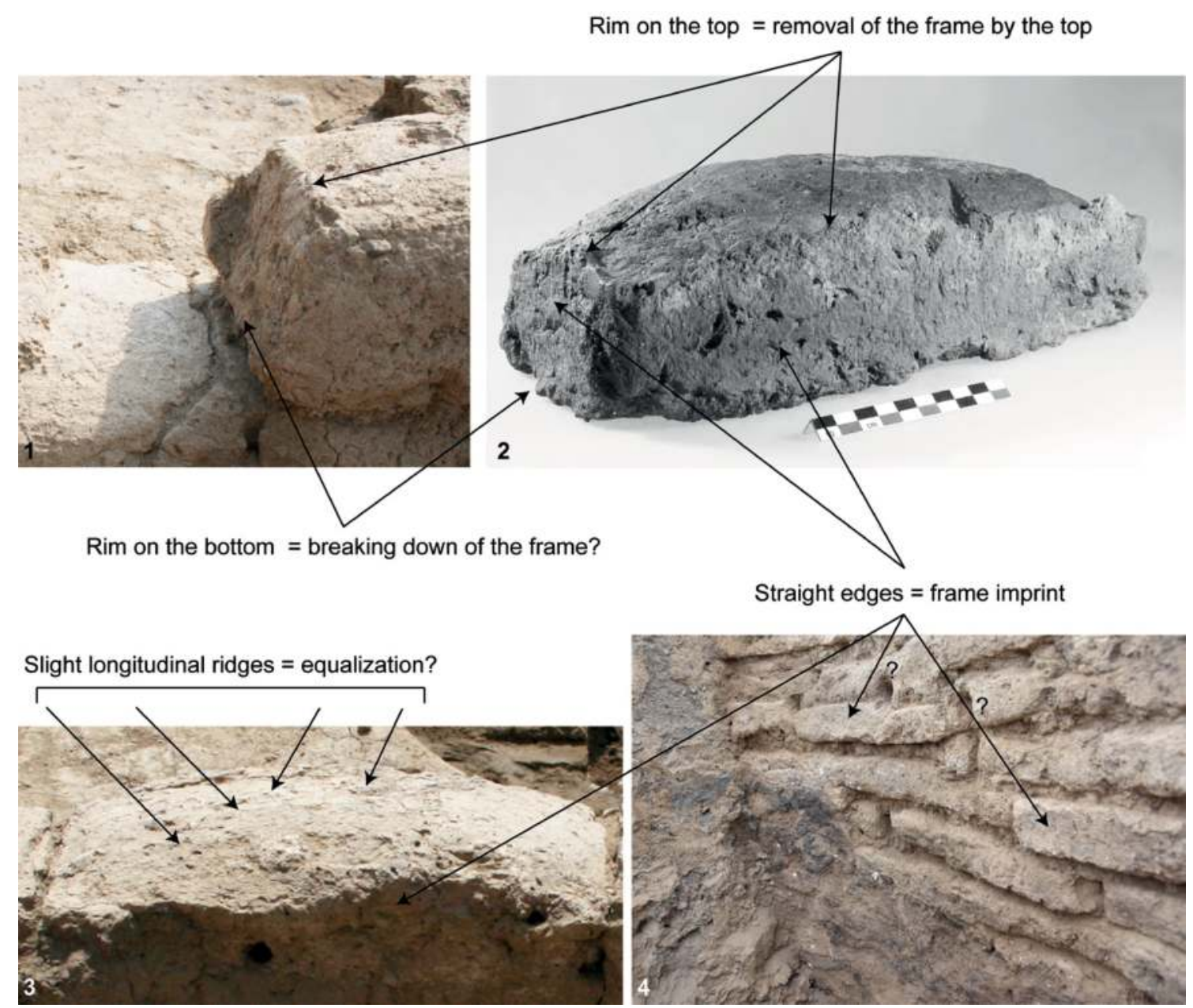

Fig. 6.9 Ridges on molded mud-bricks. 1 and 3: Mentesh Tepe, Azerbaijan; 2: Aruchlo, Georgia; 4: Kichik Tepe, Azerbaijan.

The brick size at Aruchlo has been reported to be 30 to $50 \mathrm{~cm}$ long and $20 \mathrm{~cm}$ wide (Hansen and Ullrich 2017: 202). However, more specific references are also available: a group of complete mud-bricks showed a size of 32-34 cm long and 16-18 cm wide (Ioseliani 2017: 281, tab. 1). The excavators also mentioned the existence of mud-bricks measuring 41 by $20 \mathrm{~cm}$ (Hansen and Mirtskhulava 2012). In other words, these two specific types of mud-bricks perfectly match the small and middle types noted at Göytepe. However, if the Aruchlo samples include larger ones of 50 by $20 \mathrm{~cm}$ as stated (Hansen and Ullrich 2017: 202), they can best be compared to our largest type. A similar size range was also reported for Gadachrili Gora. The excavator of this site mentioned the use of mud-bricks sized $30-40$ by $15-20 \mathrm{~cm}$ and a large type measuring 50 by $25 \mathrm{~cm}$ (Hamon et al. 2016: 164). Interestingly, the literature also refers to a far smaller mud-brick measuring $20-26$ by $12-14 \mathrm{~cm}$.
This latter type may correspond to our smallest limit of the size range identified at Göytepe (Level 9, Fig. 6.7). At Mentesh Tepe, specimens of approximately 40 by $15 \mathrm{~cm}$ were claimed to have been common, associated with a larger size measuring $44-45$ by $15-16 \mathrm{~cm}$ and a smaller one measuring 28 by $22 \mathrm{~cm}$ (Lyonnet et al. 2016: 180). These are also more or less comparable to the data from Göytepe.

These measurements of mud-brick size demonstrate that the datasets obtained at Göytepe fall in the range of mud-brick types that have been identified at other sites in the Middle Kura Valley. We should note that the measurement data from the above-mentioned sites did not exceed 20 samples per site, while the Göytepe data is based on a far larger number of measurements from stratified contexts. The stratified dataset from Göytepe can provide a more comprehensive basis for future studies. 


\subsubsection{Chronological change}

This study revealed changes in the shape and size of mud-bricks in the Neolithic sequence of Göytepe. While it was previously known that the architectural layout changed from snowman-shaped (Hac1 Elamxanlı Tepe) to ring-shaped (Göytepe) buildings, the change in mud-brick production in the later phase is a new finding. Changes occurred in both shape and size during the middle 6th millennium BC. The shape change is that from plano-convex bricks to "less-convex" forms. This change has been noted on the basis of our field notes without quantified data. Therefore, it needs to be verified when the walls are dismantled and metric data become available to characterize mud-brick shape. The timing and process of this change also require specification. On the other hand, the size change is stratigraphically defined: mud-bricks in the excavated squares of Göytepe showed an abrupt increase in the later phase starting from Level 7 (Fig. 6.7). One may wonder if the changes in mud-brick size were related to changes in the size of circular buildings themselves over this period. Indeed, the shorter mud-bricks may have facilitated the construction of smaller buildings with enhanced circular arcs. However, this proposition does not fit with the fact that the mud-brick size increased in Level 7 and later, when no radical size change in the architecture was detected (Chapter 3). Indeed, earlier buildings tend to have been larger in diameter in the Shomutepe culture (Nishiaki et al. 2015b).

The stratigraphic and radiocarbon data demonstrate that this change in mud-brick size at the middle of the occupation sequence at Göytepe was a radical phenomenon without a transitional stage, suggesting the introduction of a new tradition. It should be noted that this change occurred during a period of significant changes shown in other material records (see Chapter 1), including the rapid increase and technological changes in pottery development (Nishiaki et al. 2015a). Similarly, a sudden shift in the use of obsidian from sources in Northeast Anatolia to Central Armenia also occurred during this time period (Nishiaki et al. 2019). Our Bayesian analysis of radiocarbon dates has established a highresolution chronology for the Göytepe occupation sequence, which suggests a boundary between Levels
9 and 8 of $5530 \mathrm{BC}$, Levels 8 and 7 of $5520 \mathrm{BC}$, and Levels 7 and 6 of 5510 BC (Nishiaki et al. 2018). Assuming the mud-bricks were a product of the local communities occupying this settlement, the sudden change in mud-brick manufacturing traditions may suggest the arrival of a community with a different architectural tradition or the swift acceptance of a new tradition by the local communities. To determine this, we need more data on mud-bricks from welldated levels in the neighboring settlements. With such data, an evaluation can be made from a variety of perspectives, including the possible introduction of the cubit system developed in the Fertile Crescent in the Neolithic period (Haklay and Gopher 2019; Nishiaki et al. 2001).

\subsection{Conclusions}

This chapter presents preliminary data regarding the shape and size of mud-bricks at Göytepe. This study produced two major findings. The first is the demonstration of the common use of planoconvex mud-bricks, which confirms that the mudbrick manufacturing process at Göytepe belongs to the local tradition of the Middle Kura Valley. The second important finding is that the shape and size of mud-bricks at Göytepe changed during the mid6th millennium $\mathrm{BC}$. The most significant change occurred in size around 5520 BC. The background of these changes should be studied from multiple perspectives as it appears to have coincided with changes in other lines of evidence, including the shift of obsidian procurement sources and pottery production and use. To further develop this research, quantitative data from mud-bricks discovered at other sites should be gathered for comparison. The large variability in the shape and size of mud-bricks at Göytepe points to the need to treat metric data from a large sample. When more data from other sites become available, the preliminary data from Göytepe discussed in this chapter can be interpreted in a deeper manner.

\section{Acknowledgements}

The present study was supported by the Ministry of Education, Science, Sport, and Technology, Japan, 
under Grant \#16H06408, and the Japan Society for Promotion of Sciences under Grant \#PE18016.

\section{References}

Aurenche, O. (1981) La Maison Orientale. L'Architecture du Proche-Orient Ancien des Origines au Milieu du IVéme Millénaire. Paris: C.N.R.S.

Badalyan, R. S., A. A. Harutyunyan, C. Chataigner, F. Le Mort, J. Chabot, J. -E. Brochier, A. Balalescu, V. Radu, and R. Hovsepyan (2010) The settlement of Aknashen-Khatunarkh. A Neolithic site in the Ararat Plain (Armenia): Excavation results (2004-2009). Turkiye Bilimler Akademisi Arkeoloji Dergisi (TÜBA$A R), 13: 187-220$.

Baudouin, E. (2019) Rethinking architectural techniques of southern Caucasus in the 6 th millennium BC: A re-examination of former data and new insights. Paléorient, 45(1): 115-140.

Baudouin, E., B. Lyonnet, and C. Hamon (2018) Architectural techniques and cultural relationships between the Caucasus and Mesopotamia at the beginning of the sixth millennium B.C. In: Routes de I'Orient, Actes I, From the Caucasus to the Arabian Peninsula: Domestic Spaces in the Neolithic, edited by C. Douché and F. Pichon, 49-84. Paris: C.N.R.S.

Chataigner, C. (1995) La Transcaucasie au Néolithique et au Chalcolithique. Oxford: Tempus Reparatum.

Fukai, S. and T. Matsutani (1981) Telul eth-Thalathat II, The Fifth Season, 1976. Tokyo: The University of Tokyo.

Guliyev, F. and Y. Nishiaki (2012) Excavations at the Neolithic settlement of Göytepe, the Middle Kura Valley, Azerbaijan, 2008-2009. In: Proceedings of the 7th International Congress of the Archaeology of the Ancient Near East, Vol. 3: Fieldwork and Recent Research, edited by R. Matthews and J. Curtis, 71-84. Wiesbaden: Harrassowitz Verlag.

Haklay, G. and A. Gopher (2019) Architectural planning and measuring in the Pre-Pottery Neolithic site of Çayönü, Turkey. Paléorient, 45(1): 7-17.

Hamon, C., M. Jalabadze, T. Agapishvili, E. Baudouin, I. Koridze, and E. Messager (2016) Gadachrili Gora: Architecture and organisation of a Neolithic settlement in the Middle Kura Valley (6th millennium BC, Georgia). Quaternary International, 395: 154169.

Hansen, S. and G. Mirtskhulava (2012) The Neolithic settlement of Aruchlo. Report on the excavations in 2009-2011. Archäologische Mitteilungen aus Iran und Turan, 44: 58-70.

Hansen, S. and M. Ullrich (2017) Excavations at Aruchlo. Preliminary report on the 2012-2014 campaigns. In: The Kura Projects: New Research on the Late Prehistory of the Southern Caucasus, edited by B.
Helwing, T. Aliyev, B. Lyonnet, F. Guliyev, S. Hansen, and G. Mirtshulava, 201-222. Berlin: Deutsches Archäologisches Institut, Eurasien-Abteilung.

Helwing, B. (2017) Excavations at Mil Plain. In: The Kura Projects: New Research on the Late Prehistory of the Southern Caucasus, edited by B. Helwing, T. Aliyev, B. Lyonnet, F. Guliyev, S. Hansen, and G. Mirtshulava, 11-42. Berlin: Deutsches Archäologisches Institut, Eurasien-Abteilung.

Ioseliani, V. (2017) Architecture debris from the burnt layers. In: The Kura Projects: New Research on the Late Prehistory of the Southern Caucasus, edited by B. Helwing, T. Aliyev, B. Lyonnet, F. Guliyev, S. Hansen, and G. Mirtshulava, 281-286. Berlin: Deutsches Archäologisches Institut, EurasienAbteilung.

Love, S. (2012) The geoarchaeology of mudbricks in architecture: A methodological study from Çatalhöyük, Turkey. Geoarchaeology, 27(2): 140156.

Lyonnet, B., F. Guliyev, L. Bouquet, G. Bruley-Chabot, A. Samzun, L. Pecqueur, E. Jovenet, E. Baudouin, M. Fontugne, P. Raymond, E. Degorre, L. Astruc, D. Guilbeau, G. Le Dosseur, N. Benecke, C. Hamon, M. Poulmarc'h, A. Courcier, and E. Degorre (2016) Mentesh Tepe, an early settlement of the Shomu-Shulaveri culture in Azerbaijan. Quaternary International, 395: 170-183.

Marro, C., V. Bakhshaliyev, R. Berthon, and J. Thomalsky (2019) New light on the late prehistory of the south Caucasus: Data from the recent excavation campaigns at Kültepe I in Nakhchivan, Azerbaijan (2012-2018). Paléorient, 45(1): 81-113.

Martirosyan-Olshansky, K., G. E. Areshian, P. S. Avestiyan, and A. Hayrapetyan (2013) Masis Blur: A Late Neolithic settlement in the Plain of Ararat, Armenia. Backdirt, 2013: 142-146.

Nishiaki, Y., M. Tao, S. Kadowaki, M. Abe, and H. Tano (2001) Excavations in Sector A of Tell Kosak Shamali: The stratigraphy and architectures. In: Tell Kosak Shamali - The Archaeological Investigations on the Upper Euphrates, Syria. Volume 1: Chalcolithic Architecture and the Earlier Prehistoric Remains, edited by Y. Nishiaki and T. Matsutani, pp. 49-113. UMUT Monograph 1. Oxford: Oxbow Books.

Nishiaki, Y., F. Guliyev, and S. Kadowaki (2015a) Chronological contexts of the earliest Pottery Neolithic in the southern Caucasus: Radiocarbon dates for Göytepe and Hacı Elamxanlı Tepe, west Azerbaijan. American Journal of Archaeology, 119(3): 279-294.

Nishiaki, Y., F. Guliyev, S. Kadowaki, V. Alakbarov, T. Miki, S. Salimbeyov, C. Akashi, and S. Arai (2015b) Investigating cultural and socioeconomic change at the beginning of the Pottery Neolithic in the southern Caucasus: The 2013 Excavations at Hacl 
Elamxanlı Tepe, Azerbaijan. Bulletin of the American School of Oriental Research, 374: 1-28.

Nishiaki, Y., F. Guliyev, S. Kadowaki, and T. Omori (2018) Neolithic residential patterns in the southern Caucasus: Radiocarbon analysis of rebuilding cycles of mudbrick architecture at Göytepe, West Azerbaijan. Quaternary International, 474: 119-130.

Nishiaki, Y., O. Maeda, T. Kannari, M. Nagai, E. Healey, F. Guliyev, and S. Campbell (2019) Obsidian provenance analyses at Göytepe, Azerbaijan: Implications for understanding Neolithic socioeconomies in the southern Caucasus. Archaeometry, 61(4): 765-782.

Sauvage, M. (1998) La Brique et Sa Mise en CEuvre en Mésopotamie: Des Origines à l'Epoque Achéménide. Paris: E.R.C.

Tobler, A. J. (1950) Excavations at Tepe Gawra. Vol. II. Philadelphia: Pennsylvania University Museum. 


\title{
Geoarchaeological investigation of storage space at Göytepe: Phytolith, dung spherulite, and micromorphological analyses
}

\author{
Seiji Kadowaki, Lisa Maher, Marta Portillo, and Rosa M. Albert
}

\subsection{Introduction}

Storage is one of the main foci in archaeological research on prehistoric communities of various time periods and regions. This socio-economic practice is related to a wide range of past lifeways, such as subsistence practices, settlement patterns, and social relations, which are particularly significant aspects in the study on the transition from foragers to early farmers during the late Pleistocene and early Holocene (e.g., Kuijt 2008; Kuijt and Finlayson 2009). This chapter presents geoarchaeological analyses of clay bins discovered at Göytepe to provide evidence for storage facilities and clarify their contexts in the living space. We analyzed phytoliths (plant silica cells; Piperno 2006) and dung spherulites (microscopic calcitic particles produced in the guts of animals, especially ruminants, and later expelled in excrement; Canti 1999) deposited inside and outside the clay bins, in combination with the analyses of in situ processes of construction, deposition, use and preservation through micromorphology of the clay bins. The results have been published in Kadowaki et al. (2015) along with relevant palaeobotanical records and integrative discussion while this chapter presents detailed descriptions of the samples and observations.

Clay bins examined in this study are less than $1 \mathrm{~m}$ (often 40-50 cm) in diameter and reaching a depth of $70 \mathrm{~cm}$ at most (Figs. 7.1 and 7.2). They are probably semi-subterranean and partially dug into surrounding surfaces and fill. Similar features are frequently shown in architectural plans of the sites belonging to the Shomutepe culture, such as Shomutepe (Narimanov 1992) and Shulaveri Gora
(Chataigner 1995), although their distributional patterns are difficult to recognize in these plans because architectural features of different levels are shown together in the same map. A preliminary report of excavation at Aratashen along the Arax River shows a photo of round features, interpreted as silos or ovens, that are distributed in clusters within or outside buildings with curvilinear walls (Badalyan et al. 2007). Thus, clay bins appear to be common architectural components at Neolithic sites in the southern Caucasus (specifically those of the Shomutepe culture), including Göytepe. However, their close examination has rarely been conducted despite their potential significance as material evidence regarding socio-economic aspects of these early agro-pastoral communities.

In light of this, this study examines the context and depositional history of clay bins that have been recovered in the investigation at Göytepe, employing geoarchaeological methods including phytolith, spherulite, and micromorphological analyses.

\subsection{Contexts of clay bins at Göytepe}

A renewed investigation at Göytepe since 2008 recovered a number of clay bins that are often clustered adjacent to buildings or walls connecting the buildings (Guliyev and Nishiaki 2012). At least four such clusters of bins have been recovered in Levels 3, 4, 5, and 10 (Fig. 7.1), indicating that these features continued to have been common architectural component during most of the Neolithic occupations at Göytepe. Among the four clusters of bins, those in Levels 3,4 , and 5 were excavated by the Azerbaijani investigation, while that in Level 10 was 


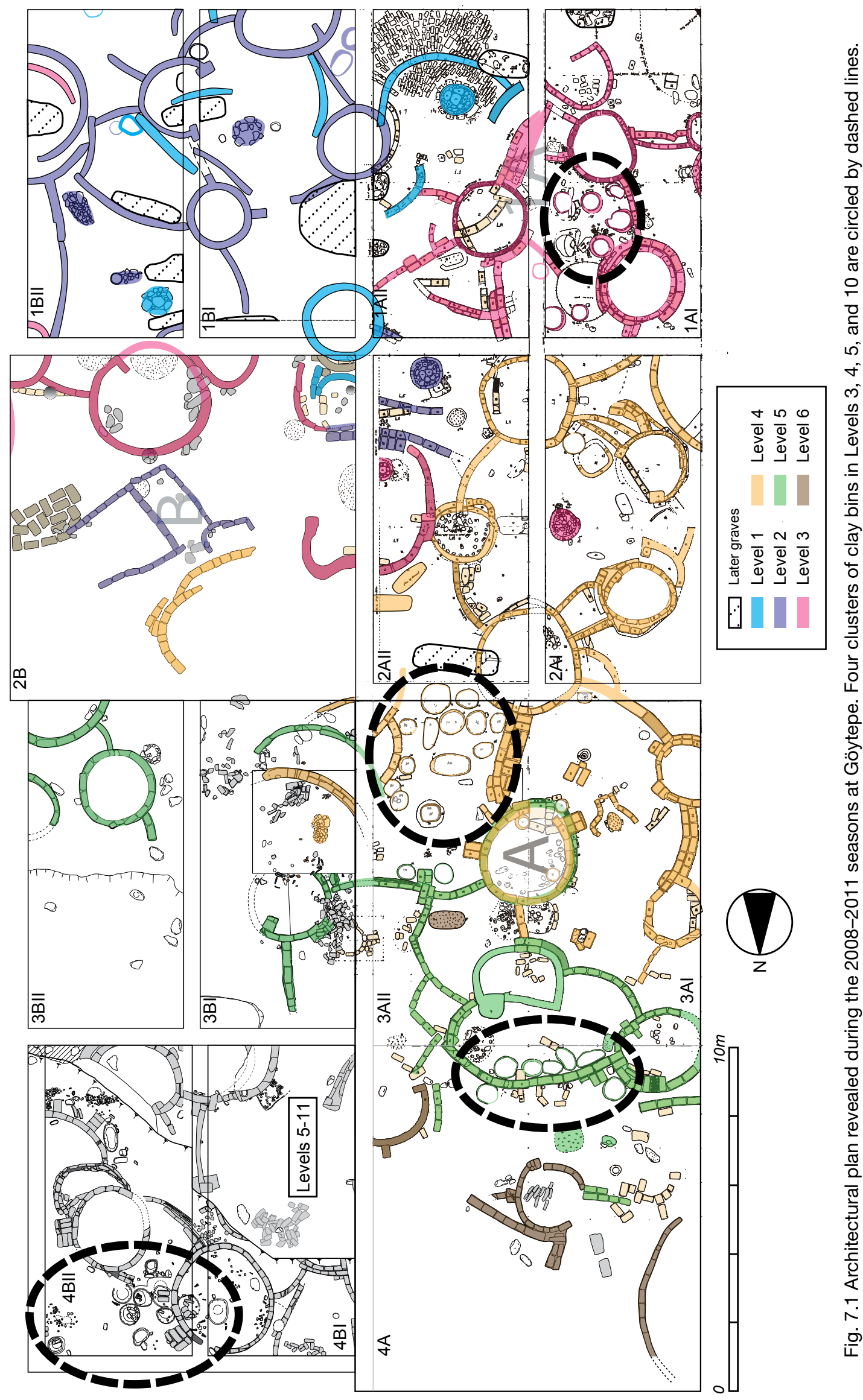




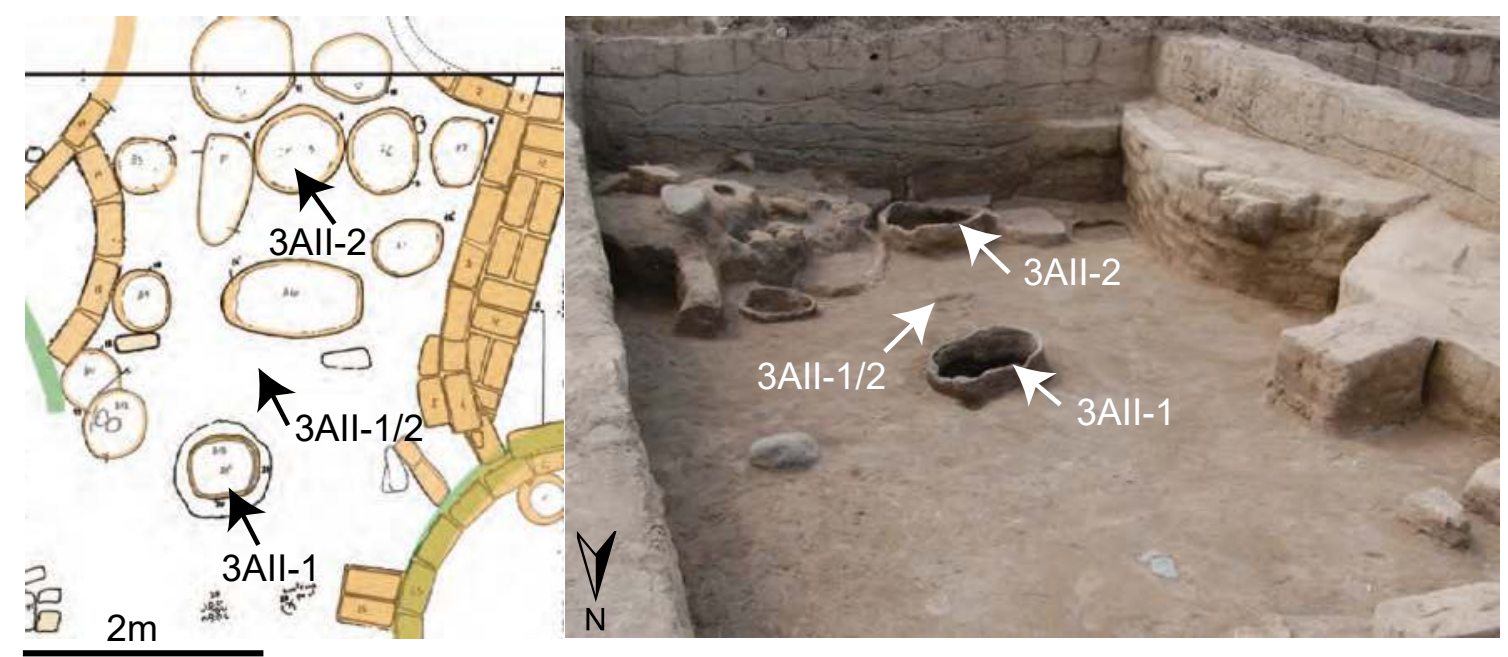

Fig. 7.2 A cluster of bin features in an outdoor area (Level 4 in Square 3AII), indicating the contexts sampled for sediment analyses.

excavated by the Japanese mission during the 20082011 seasons. A cluster of bins in Level 3 is located at Square 1A within an apparently courtyard surrounded by four round buildings connected with each other by walls. Another cluster in Level 4 is at Square 3AII, also located in outdoor space surrounded by round buildings and appendicular walls. Bins in Level 5 at Square $4 \mathrm{~A}$ are arranged in a row along a wall that is attached to a round building. Lastly, a cluster in Level 10 is located in an open space near two round buildings connected with each other by a wall.

Another significant aspect of bins at Göytepe is that they are situated adjacent to open space that often contains complete or nearly complete ground stones (particularly food processing tools: Chapter 12), other artifacts that were apparently left as de facto refuse, as well as the concentration of charcoal fragments and ash (Fig. 7.5). According to these field observations, such open space probably represents places where domestic activities took place.

With these contextual observations of the clay bins, the following analyses were conducted in order to identify the primary and secondary contents of these probably storage features and the kinds of activities in adjacent areas through the examination of depositional processes inside and outside the bins employing geoarchaeological methods, particularly phytolith, dung spherulite, and micromorphological analyses. For this purpose, we sampled sediments from various contexts, such as inside and outside the bins as well as building floors and fills in Level 10 at Square 4B and partly in Level 4 at Square 3AII. Here, we report a close examination focusing on three bins from these levels (Table 7.1). We selected samples from a clay bin (4BIIX-94 in Level 10) that is notably associated with possible primary deposits and in situ artifacts, as well as those from two bins (3AII-1 and 2 in Level 4) that appear to be filled with secondary deposits like most of the bin features recovered at the site. The three bin features reported in this chapter are similar to each other in their size, form, and context in the settlement (i.e., located in the open-air activity space), and considered fairly representative of other similar bin features common at the site.

\subsection{Excavation and sampling of clay bins}

\subsubsection{Level 4 in Square 3AII}

The excavation of Square 3AII by the Azerbaijani team recovered more than ten clay bins belonging to Level 4 (Fig. 7.2). They are clustered in an apparently outdoor area (ca. $4 \times 3 \mathrm{~m}$ ), surrounded by round buildings and walls. Adjacent to this cluster is an open space (ca. $3 \times 3 \mathrm{~m}$ ), where domestic activities could have taken place, although the excavation did not record any features, artifacts, or the nature of deposits in this area.

For the studies of phytolith and faecal spherulite, sediments were sampled from the fill (at the middle and bottom parts) and the bottom walls of two bins (Table 7.1; Figs. 7.2 and 7.3: 3AII-1 and 3AII-2) as well as outside adjacent spaces (3AII-1/2). For micromorphological analysis, a block of sediments $(15 \times 12 \times 10 \mathrm{~cm})$, including bottom fill and a bottom 


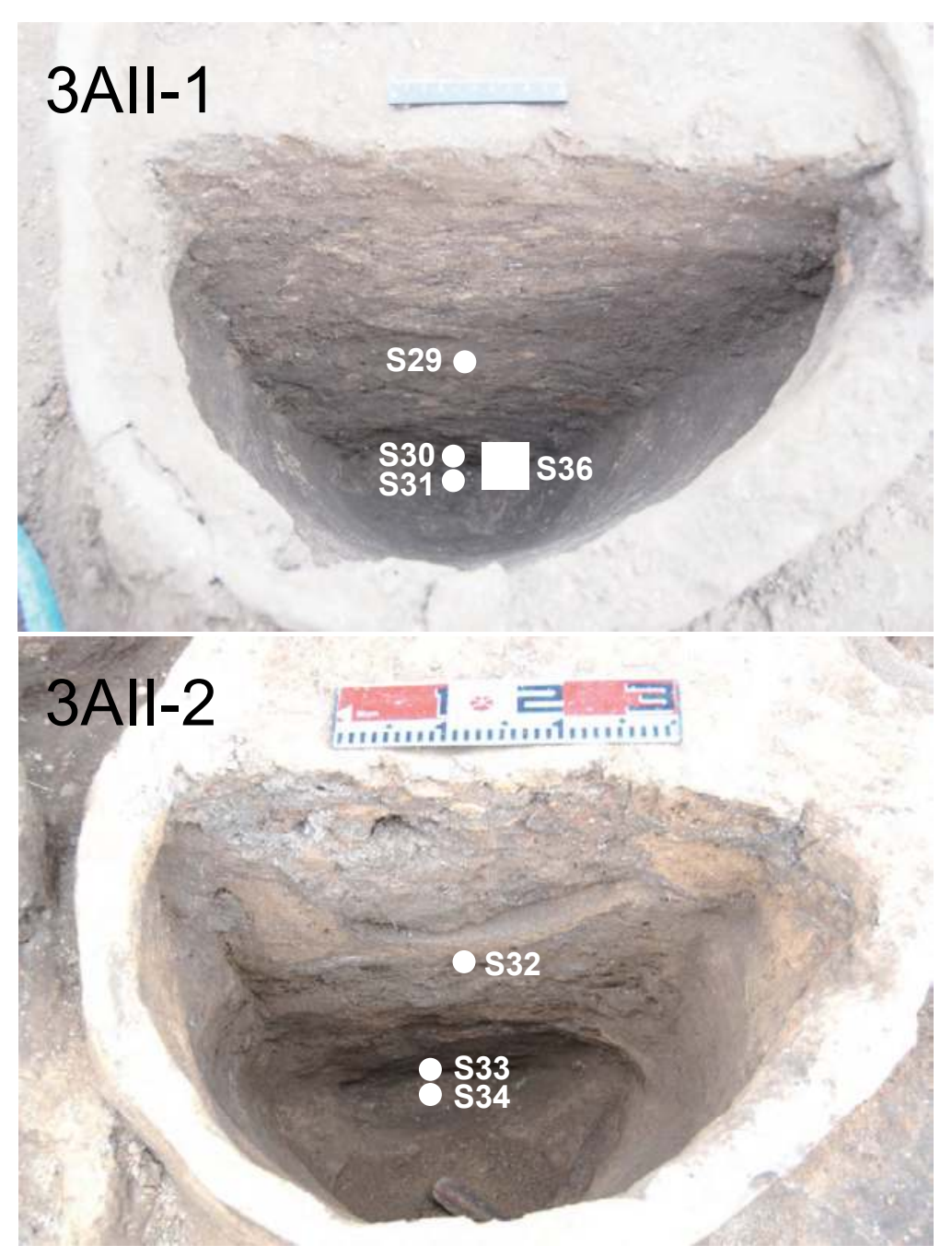

Fig. 7.3 Sections of fills in two bins (3All1 and 2), indicating sampling locations ( $O$ for phytoliths and faecal spherulites, and $\square$ for micromorphology). See Table 7.1 for the sample numbers. wall, was sampled from a bin at 3AII-1.

\subsubsection{Level 10 in Square 4BII}

The excavation of Level 10 in Square 4BII by the Japanese mission found eleven bin features, two of which are located inside a round building (4BIIX16), while the rest are distributed in outdoor areas (Fig. 7.4). Among the latter, eight bins are clustered in a space that could have been a courtyard given the layout of two round buildings (4BIIX-12 and 16), their annex walls, and a location of doorway of the building wall at 4BIIX-16. The deposits in the probable courtyard are generally ashy and associated with a concentration of charcoal fragments and burnt cobbles on reddish, hardened (burnt?) surface (4BIIX-92). This area is also characterized by the recovery of complete and nearly complete, large artifacts, such as a grinder, a grinding slab, an abrader, and bone artifacts, located near building walls or bins, indicating that they were left as de facto or provisional refuse of activities performed in this area.

We took more than 100 sediment samples from various contexts in this level, including building floors, outdoor occupational surfaces, and inside the bins. Here we report the analysis of the samples from the inside and outside a bin at 4BIIX-94 (See Table 7.1 and Figs. 7.5 and 7.6 for the list and locations of the samples). Although this feature is structurally similar to other bins in this level, it is somewhat distinct from the others for two reasons. First, two complete upper grinding stones (4BIIX-94a and 94b) were placed near the base of the bin (Figs. 7.6 and 7.7). Second, the sediments at the very base of the bin, approximately $4 \mathrm{~cm}$ in thickness, between the bin walls and the overlying grinding stones, exhibited a white, fibrous appearance, which is extremely rich in phytoliths (see the phytolith section). According to these observations, the fill deposits were subdivided 


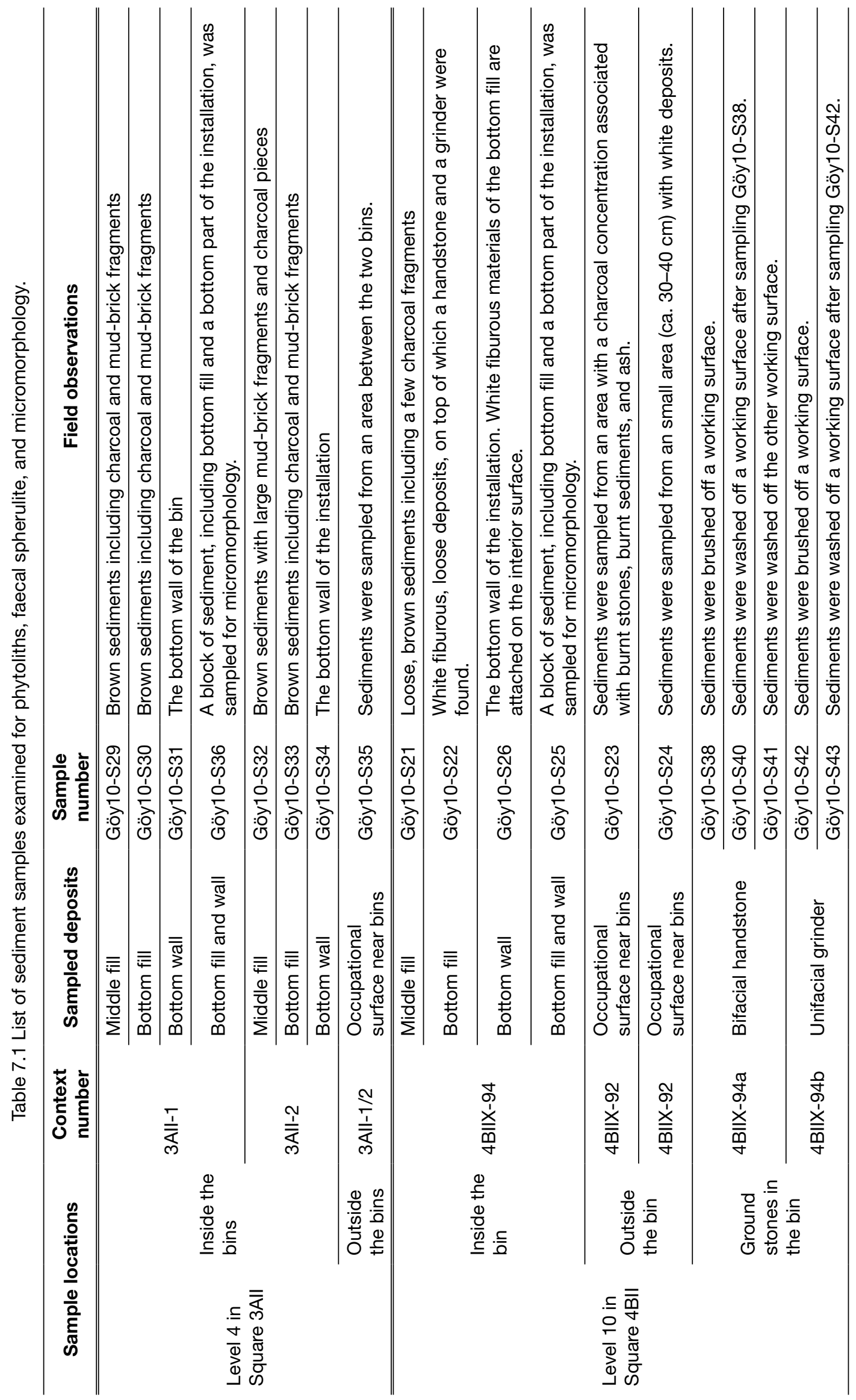




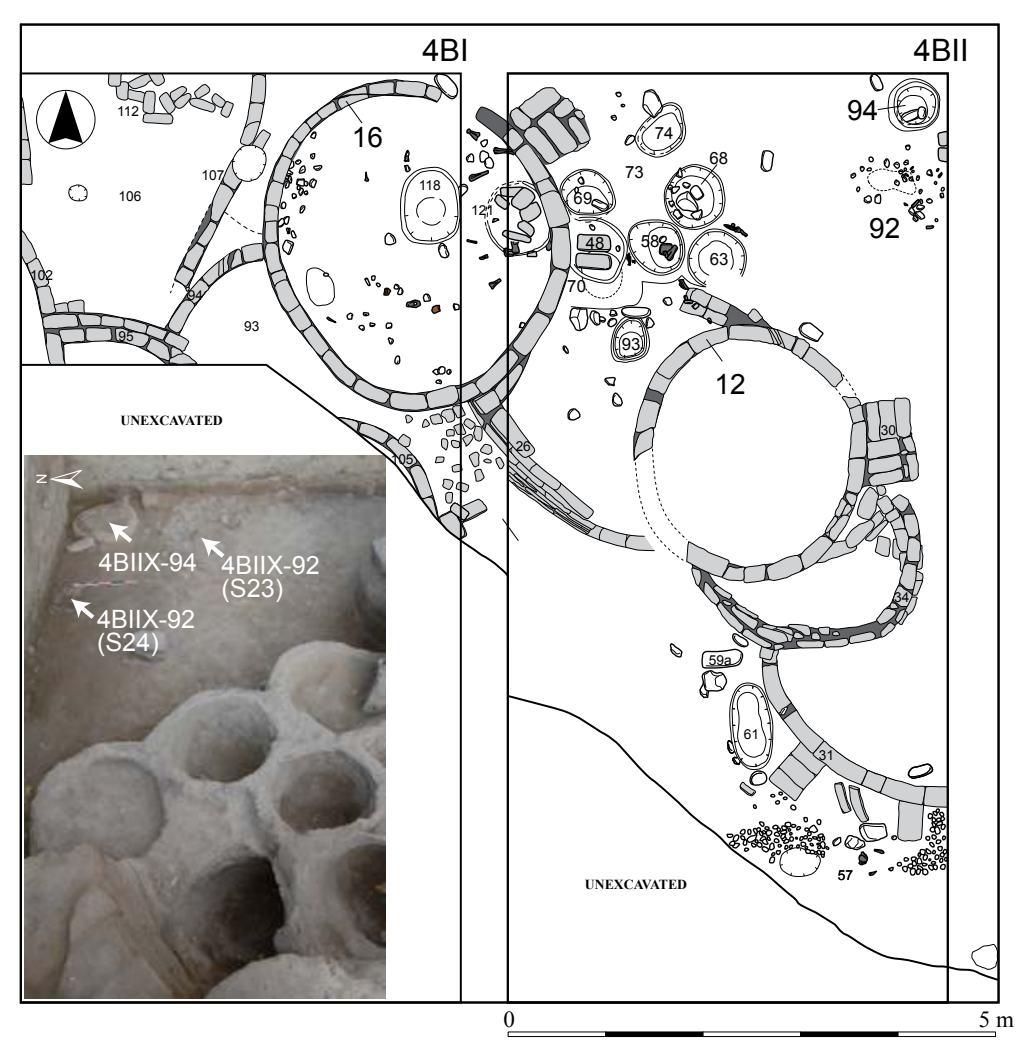

Fig. 7.4 Architectural plan of Level 10 in Square $4 \mathrm{~B}$, indicating the contexts sampled for sediment analyses.

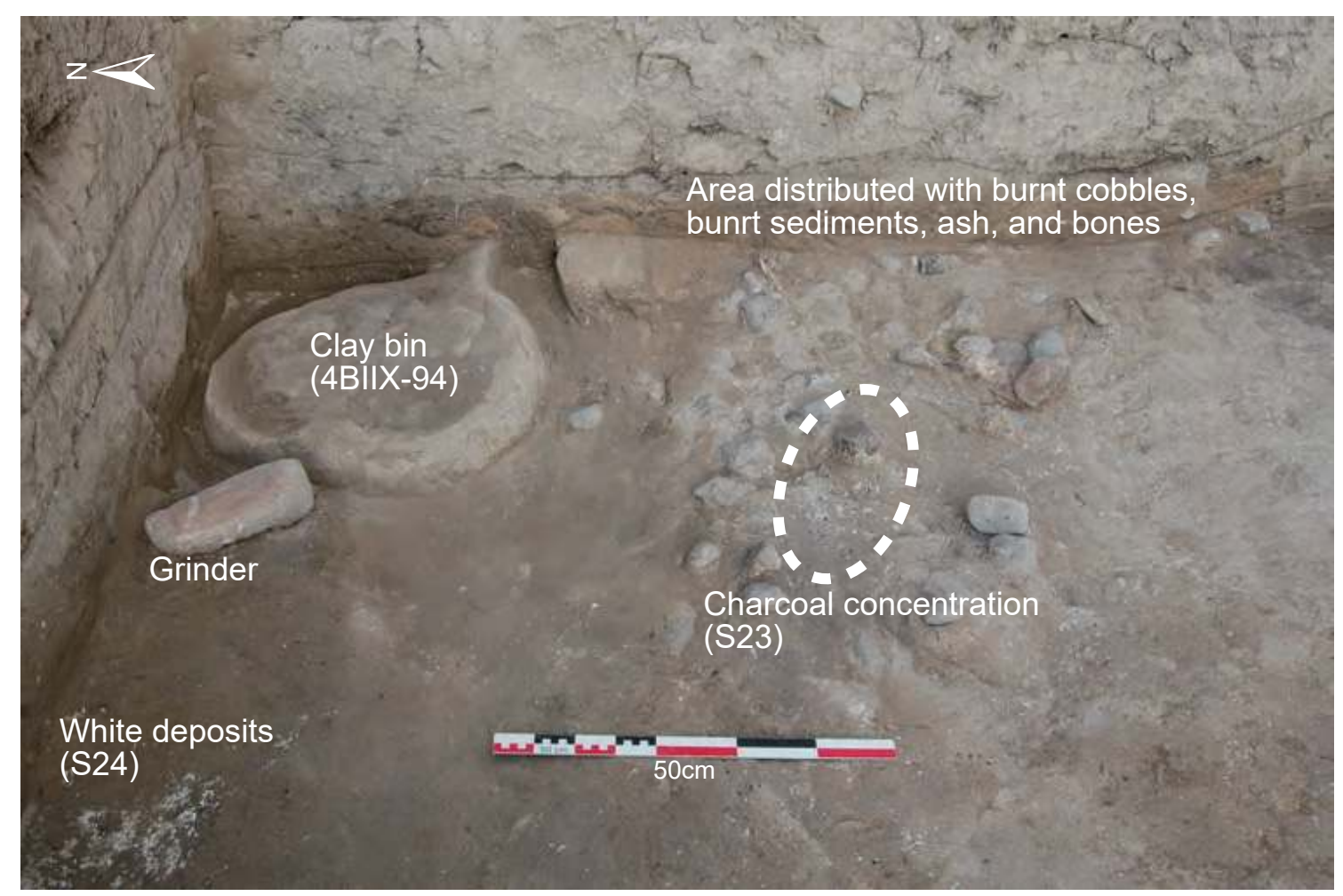

Fig. 7.5 A clay bin (4BIIX-94) and its adjacent area (4BIIX-92) distributed with domestic refuse. 


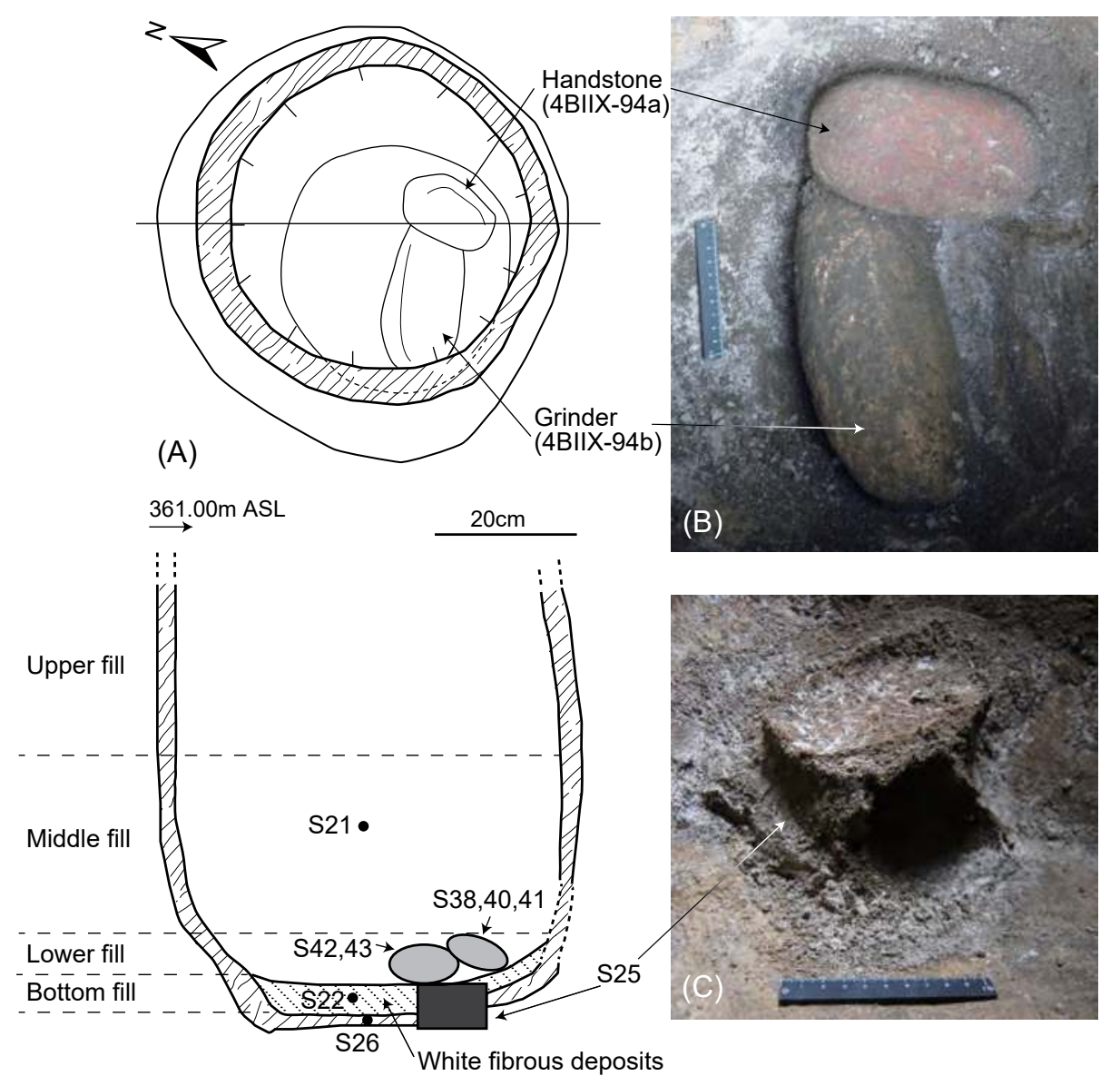

Fig. 7.6 Plan and section of the bin (4BIIX-94). (A) Plan and section of the bin (4BIIX94), showing sampling locations ( $\circ$ for phytoliths and faecal spherulites, and $\square$ for micromorphology). See Table 7.1 for the sample numbers; (B) Two in situ grinding stone found at the bottom of the bin overlying a whitish fibrous sediment at the base of the bin; (C) A column of whitish sediments sampled for micromorphology analysis at the base of the bin. into four layers (Upper, Middle, Lower, and Bottom: Fig. 7.6). The bottom fill corresponds to the white fibrous deposits at the bin base, while the lower fill is immediately above the bottom fill.

To examine the deposition processes of these distinct finds, we took sediment samples for phytolith and faecal spherulite analysis from middle and bottom fills, the bottom wall of the bin, working surfaces of the grinding stones left in the bin, as well as an adjacent activity area (Table 7.1). The feature was also sampled for micromorphological analysis; a column of sediment approximately $14 \times 11 \times 12 \mathrm{~cm}$ in size was left unexcavated at the base of the bin and then taken en bloc (Fig. 7.6).

\subsection{Phytolith and spherulite analyses}

\subsubsection{Methods}

\section{Phytolith analyses}

Phytolith analyses followed the methods of Albert et al. (1999). Samples of approximately $1 \mathrm{~g}$ of dried sediment were treated with $3 \mathrm{~N} \mathrm{HCl}, 3 \mathrm{~N} \mathrm{HNO}_{3}$ and
$\mathrm{H}_{2} \mathrm{O}_{2}$. Phytoliths were concentrated using $2.4 \mathrm{~g} /$ $\mathrm{ml}$ sodium polytungstate $\left[\mathrm{Na}_{6}\left(\mathrm{H}_{2} \mathrm{~W}_{12} \mathrm{O}_{40}\right) \cdot \mathrm{H}_{2} \mathrm{O}\right]$. Slides were prepared by weighing about $1 \mathrm{mg}$ of sample using Entellan New (Merck). A minimum of 200 phytoliths with diagnostic morphologies were counted at $400 \times$ magnification. Morphological identification was based on standard literature (Twiss et al. 1969; Brown 1984; Mulholland and Rapp 1992; Rosen 1992; Twiss 1992; Madella et al. 2005; Piperno 2006), as well as on modern plant reference collections (Albert and Weiner 2001; Tsartsidou et al. 2007; Albert et al. 2011; Portillo et al. 2014).

\section{Spherulite analyses}

Samples were prepared following Canti's (1999) methodology. Approximately $1 \mathrm{mg}$ of dried sediment was mounted on a microscope slide, as described above for phytoliths. Spherulite counting was performed using a polarized light microscope at $400 \times$ magnification. Samples were compared to modern dung reference collections (Albert et al. 2008; Portillo et al. 2012; Portillo et al. 2014). 

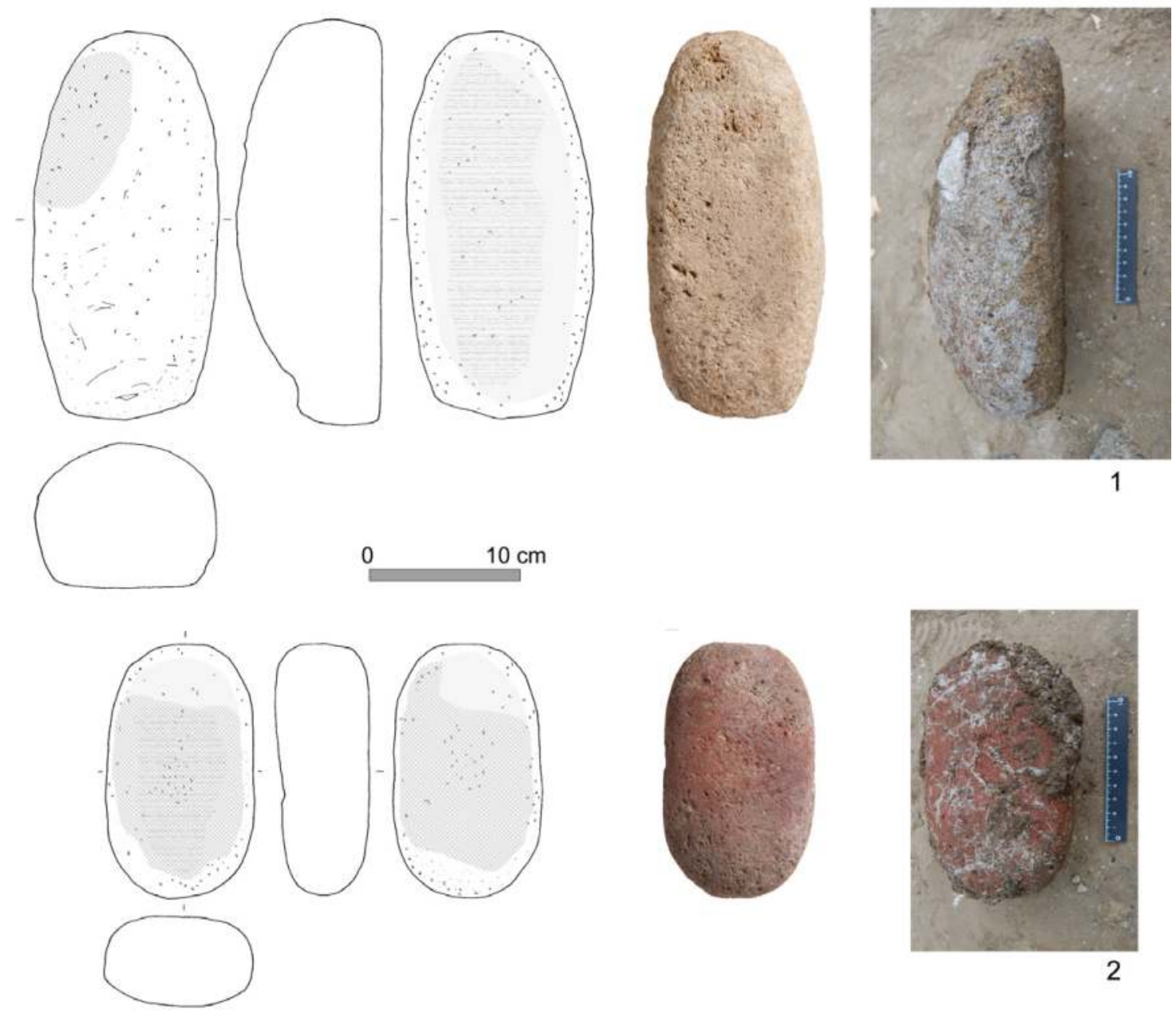

Ground or polished surface Striations $\because$ Pecking or battering scars

Fig. 7.7 Grinder (1: 4BIIX-94b) and handstone (2: 4BIIX-94a) placed together near the bottom of the bin (4BIIX-94). The rightmost photos show the two pieces covered with whitish fibrous sediments containing large amounts of phytoliths.

\subsubsection{Results and interpretation}

Table 7.2 shows the quantitative phytolith and spherulite main results, percentage of acid insoluble fraction (AIF), estimate amounts of phytoliths per gram of AIF and per gram of sediment, percentages of weathered morphotypes (WM) and multicelled phytoliths (MC) and numbers of spherulites per gram of sediment. The insoluble fraction data (\% AIF), which is the fraction that remains after the acid and peroxide treatment, indicates the percentage presence of siliceous material, which includes heavy minerals, quartz, clay and phytoliths. The AIF percentage in the samples ranged from 53 to $78 \%$ (Table 7.2). This means that siliceous minerals were major components of these sediments, although with variations among the samples. Sediments obtained from bin fills (S21, S30, and S32) showed the lowest AIF percentages (around 53-59\%), whereas in most of samples AIF raises to $65 \%$ or more.

Phytoliths and spherulites were noted in different amounts in the samples. Indications of partial dissolution of phytoliths were observed in all samples by the presence of surface pitting and etching at different degrees. Those phytoliths which were unidentifiable because of some degree of dissolution were counted and percentages of the total phytolith count were listed as weathered morphotypes (\% WM, Table 7.2). The dissolution index ranged from 1.9 to $8.9 \%$ which did not interfere in the overall morphological identification of the phytoliths. 


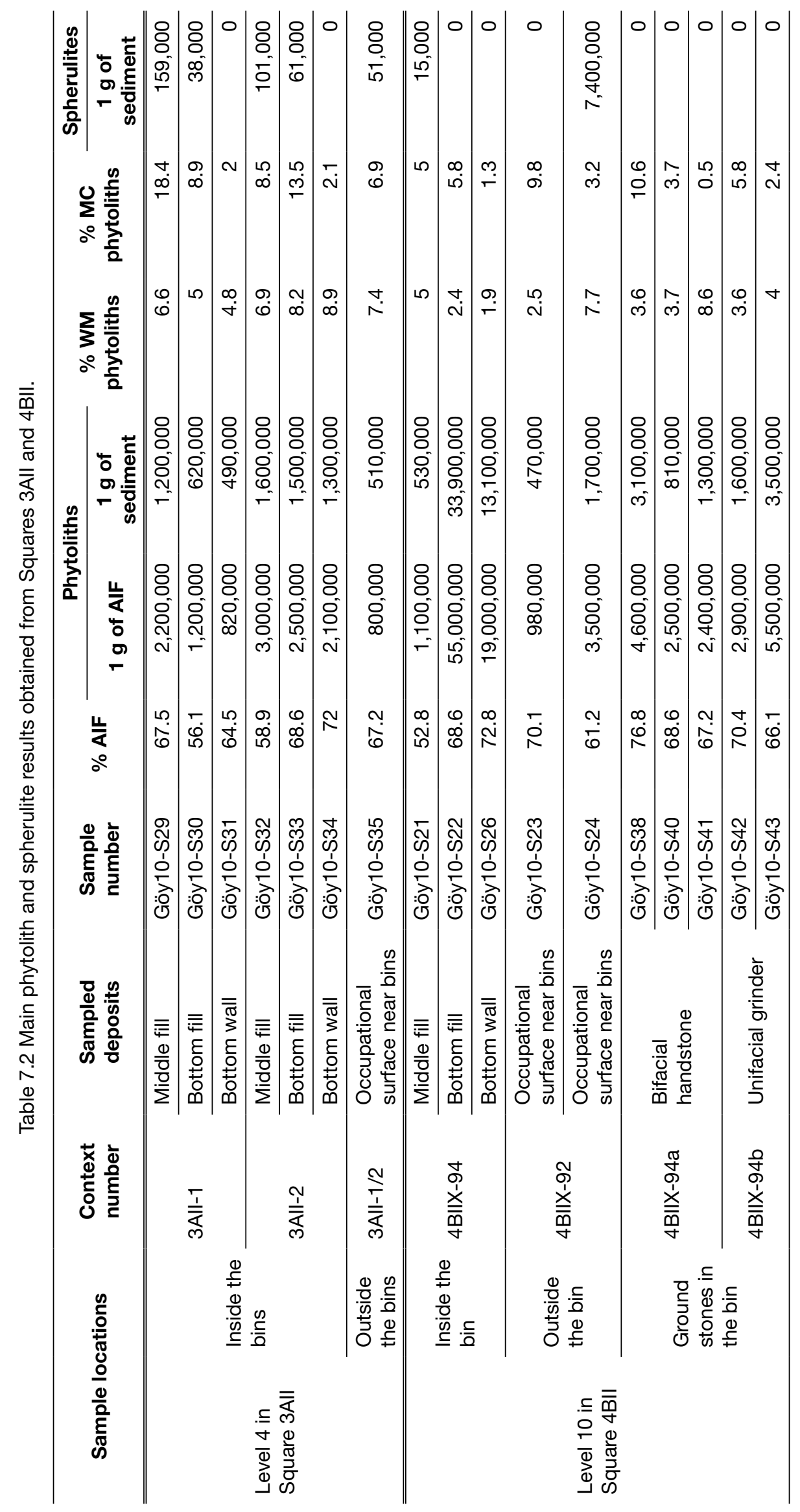


Other siliceous biogenic microremains, primarily diatoms, were also observed in most of the bin filling sediments and their bottom walls. In contrast, samples collected from outside the bins did not yield these silica microfossils. Diatoms can grow in almost any environmental condition where moisture is present (i.e., soils, deposits, mud-bricks and plasters) (Coil et al. 2003).

The results obtained from this study have been analyzed separately according to the different excavation areas (Squares 3AII and 4BII).

\subsubsection{Level 4 in Square 3AII}

Seven samples were analyzed from Level 4 in Square 3AII (Table 7.2). Most of the samples correspond to the fills of two bins (3AII-1 and 2). Two of these samples were collected in the bottom wall of each feature. An additional sample belongs to sediments from outside the bins (S35).

Phytoliths were abundantly identified in the examined assemblages with concentrations ranging from 500,000 to 1.6 million phytoliths per gram of sediment (Table 7.2). Both bins showed largest abundances in their middle fills. Grasses dominated the phytolith record, with around $85 \%$ or more of all the counted morphotypes (Fig. 7.8). Fig. 7.9 shows the grass phytolith morphological distribution according to the part plant where they were formed. Inflorescences constitute between 31 and $43 \%$ of all the morphotypes. According to the short cell morphologies, which are commonly produced both in leaves and inflorescences, most of these grasses belonged to the $\mathrm{C}_{3}$ Pooid subfamily (Fig. 7.10: a). In addition, other short cells from $\mathrm{C}_{4}$ Panicoids were also identified but in lesser amounts (Fig. 7.10: b). Inflorescences were characterized mainly by diagnostic epidermal elongate echinate long cells (Fig. 7.10: c). Other distinctive morphotypes, such as dendritic and papillae cells were also identified, although in lower proportions (Fig. 7.10: d-e). These later morphologies are considered as good indicators of preservation in phytolith assemblages (Cabanes et al. 2011). Multicellular structures - multi-celled or interconnected phytoliths, from both floral parts of cereals, and the leaves and the stems of grasses (Fig. 7.10: f) were also noted in most of the samples in different proportions (Table 7.2). The identified morphologies corresponded to the floral parts or husks of grass seeds, primarily from wheat (Triticum sp.) and barley (Hordeum sp., Fig. 7.10: g), which is consistent with direct evidence from macrobotanical remains at the site (See Chapter 16 and Kadowaki et al. 2015 for relevant macrobotanical records). Phytolith results indicate a vegetal component

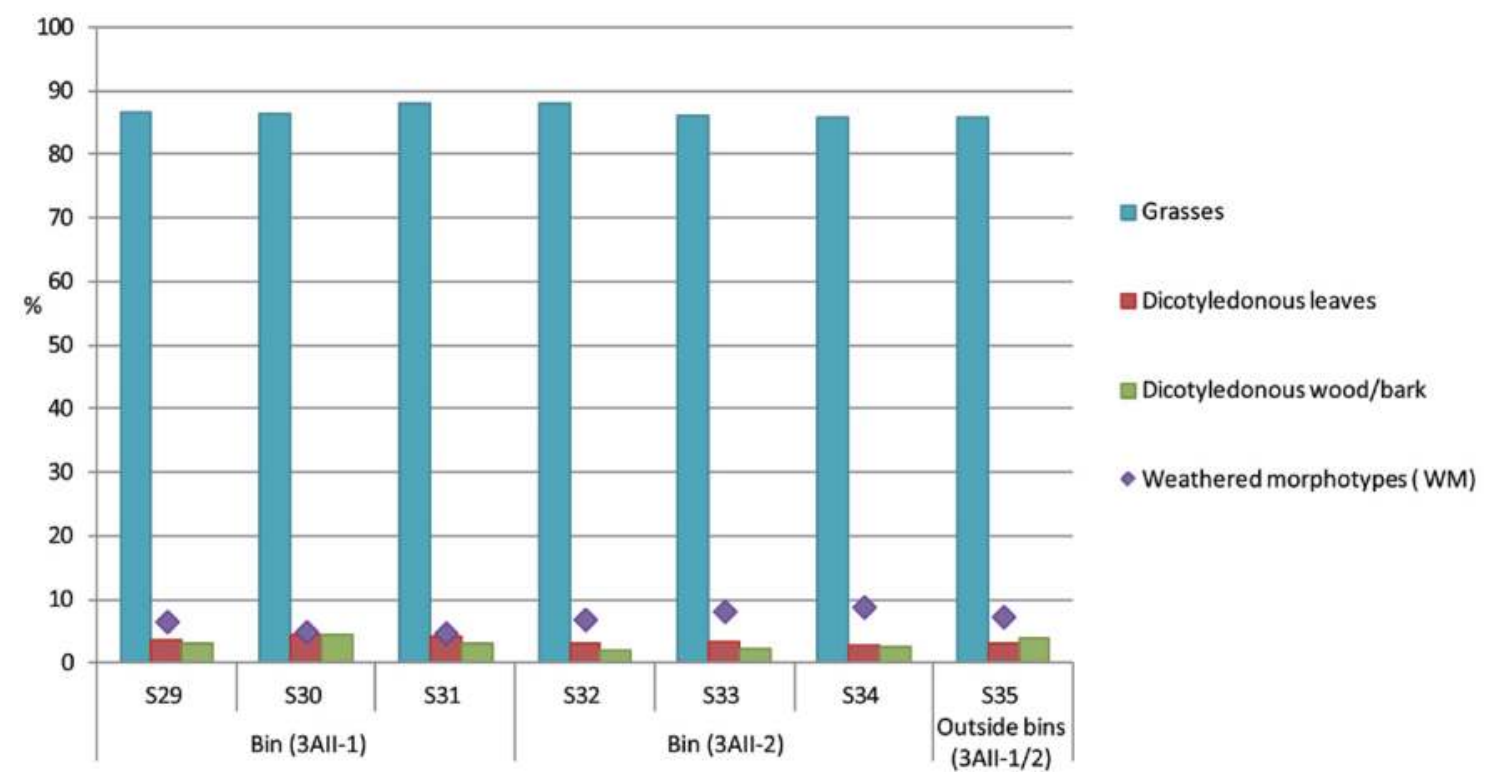

Fig. 7.8 Histogram showing the relative abundances (\%) of phytoliths from grasses, dicotyledonous leaves, dicotyledonous wood/bark and weathered morphotypes (WM) obtained from Square 3All samples. 


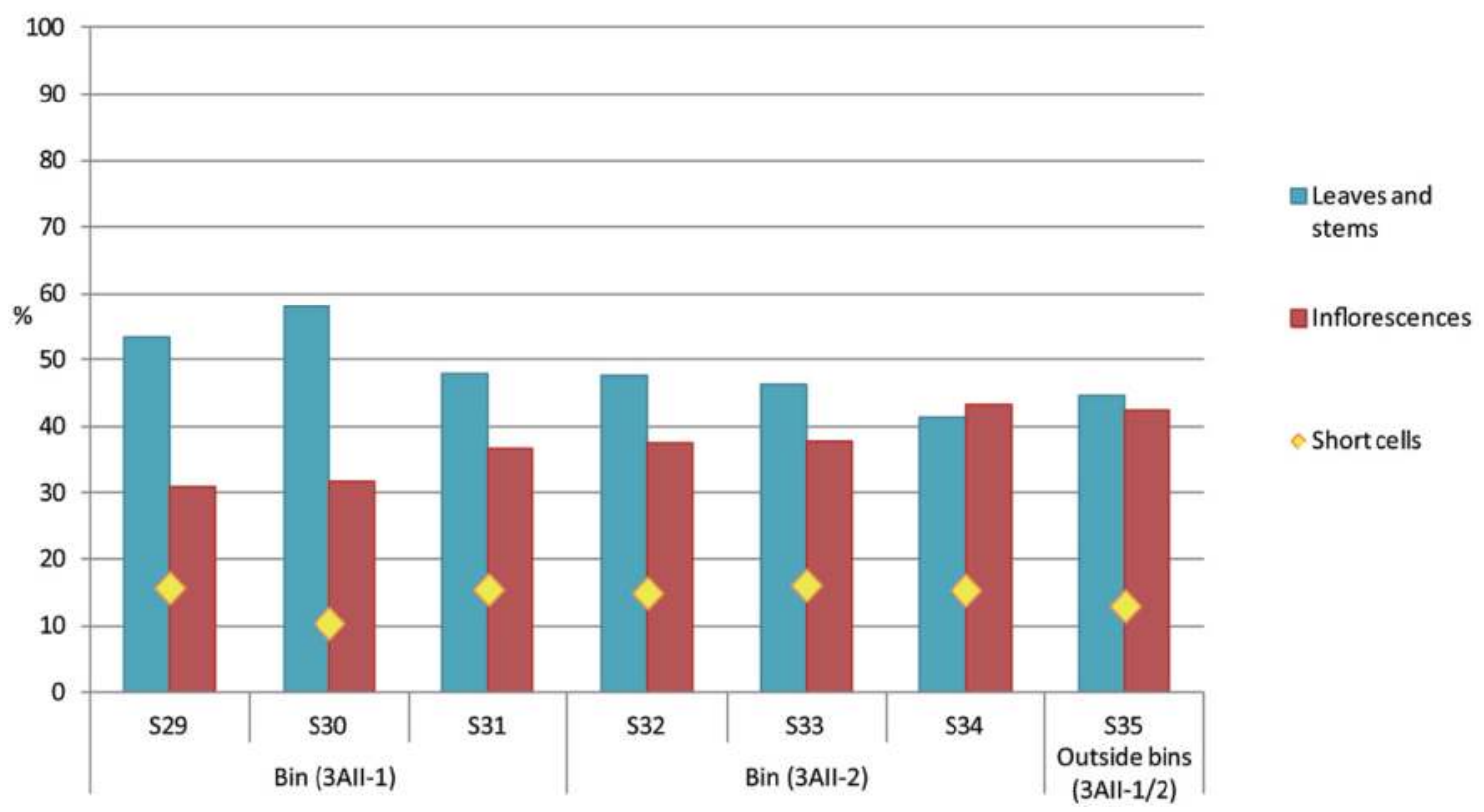

Fig. 7.9 Anatomical origin of grass phytoliths in Square 3All samples.
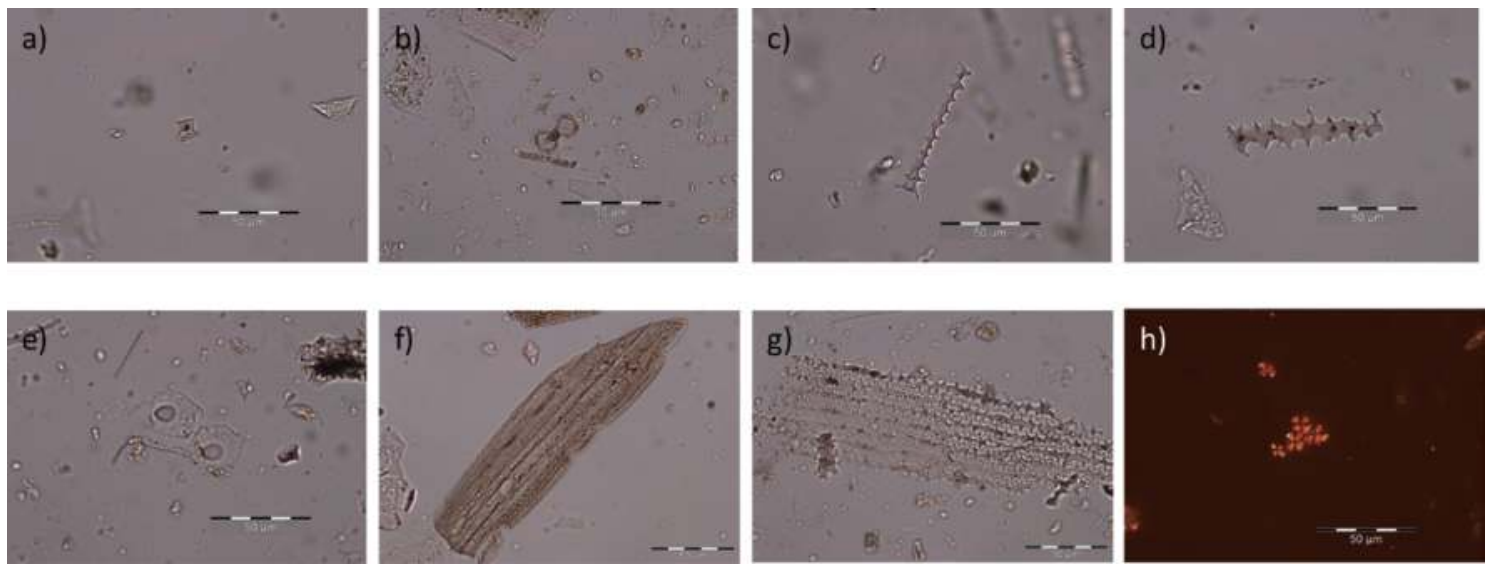

Fig. 7.10 Photomicrographs of phytoliths and other microremains identified in the samples. The photographs have been taken at 400× (a-g: PPL, h: XPL). a) Short cell rondel; b) Short cell bilobate; c) Long cell with echinate margin; d) Dendriform long cell; e) Papillae cells; f) Multicellular structure from grass leaves/stems; g) Multicellular structure from Hordeum sp. husk; h) Dung spherulites.

dominated by a mixing of inflorescence and leaves/ stems in both bin deposits, as well as in the sediments from the outside area.

Dung spherulites were noted in most of the bin samples in different amounts (Table 7.2 and Fig. 7.10: h) with the exception of those samples from the bottom walls of the bins. The middle fills yielded the largest numbers (over 100,000 spherulites/ $\mathrm{g}$ sediment) which correlate with large phytolith concentrations in these samples indicating that dung material was dumped here. Dung micro-remains were also observed in the adjacent area of the bins.
Moreover, the fact that the vegetal component is similar to the results obtained from the outside area indicates that these assemblages may represent material accumulation resulting from household debris or the remains of dumped material derived from domestic activities. These findings could represent either livestock dung remains or faecal material burnt as fuel and that its residues were dumped at the localities studied here. The latter case may be consistent with the identification of a large amount of ash and household debris in the thin sections of the bottom fill of the 3AII-1 bin (S36: see 
the following micromorphological observations). These results suggest that the bin fills are for the most part a secondary deposit and may yield little information about their primary use.

\subsubsection{Level 10 in Square 4BII}

Ten samples were selected from level 10 in Square 4BIIX (Table 7.2). Three of these samples correspond to fills of a bin (4BIIX-94), including the whitish fibrous sediments at the bottom. Additionally, five samples were obtained from the working surfaces of grinding stones which were placed near the base of the bin. Two sediment samples were obtained from outside the bins. Phytoliths were especially abundant in all the samples (from 470,000 to 34 million phytoliths per gram of sediment, Table 7.2). Contrary to Level 4 at Square 3AII, the phytolith richest sediments here were the white deposits from the bottom fill of the bin (S22 and S26, over 13 million phytoliths/g of sediment). The white sediments (S24) of the adjacent area of the bin yielded also a high amount of these micro-remains (around 1.7 m phytoliths/g sediment). Grasses constitute more than $84 \%$ of all the morphotypes in all the examined samples (Fig. 7.11). Again, $\mathrm{C}_{3}$ Pooid grasses (Fig.
7.10: a) were the most common group identified. Interestingly, grass inflorescences dominated in all the bin and grinding stones samples, whereas leaves and stems of these plants were common in sediments from the outside area (Fig. 7.12). Similar to Square 3AII samples, epidermal elongate echinate cells were abundantly identified in all the assemblages. The white sediments from the bin fills (S22 and S26) yielded the highest concentrations of inflorescent phytoliths with around $75-78 \%$, a low proportion of anatomically connected phytoliths and the presence of two complete grinding stones (4BIIX-94a and 94b). This association is consistent with previous studies carried out with grinding tools, which reveal that multicellular structures may not be well preserved as a result of the mechanical degradation of the phytoliths produced by grounding processes (Albert and Portillo 2005; Portillo 2006; Portillo et al. 2009). These findings suggest that the remains of grain processing activities, such as the grinding or dehusking of cereals may have been deposited here. Regarding the middle fill deposit (S21), this probably represents a later moment of deposition, where plants would not be so abundant. Also note that dung spherulites are not common from here especially

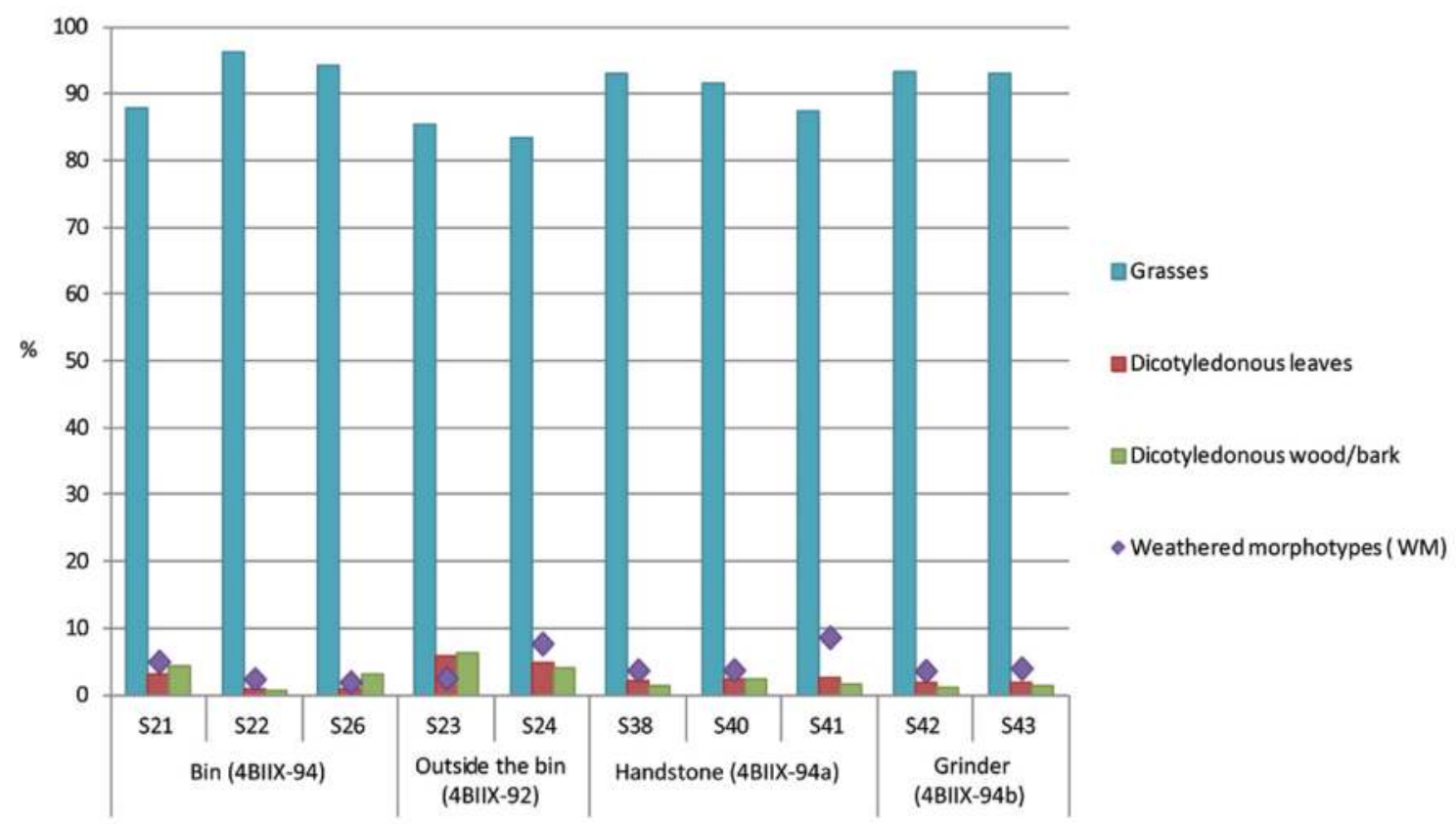

Fig. 7.11 Histogram showing the relative abundances (\%) of phytoliths from grasses, dicotyledonous leaves, dicotyledonous wood/bark and weathered morphotypes (WM) obtained from Square 4BIIX samples. 


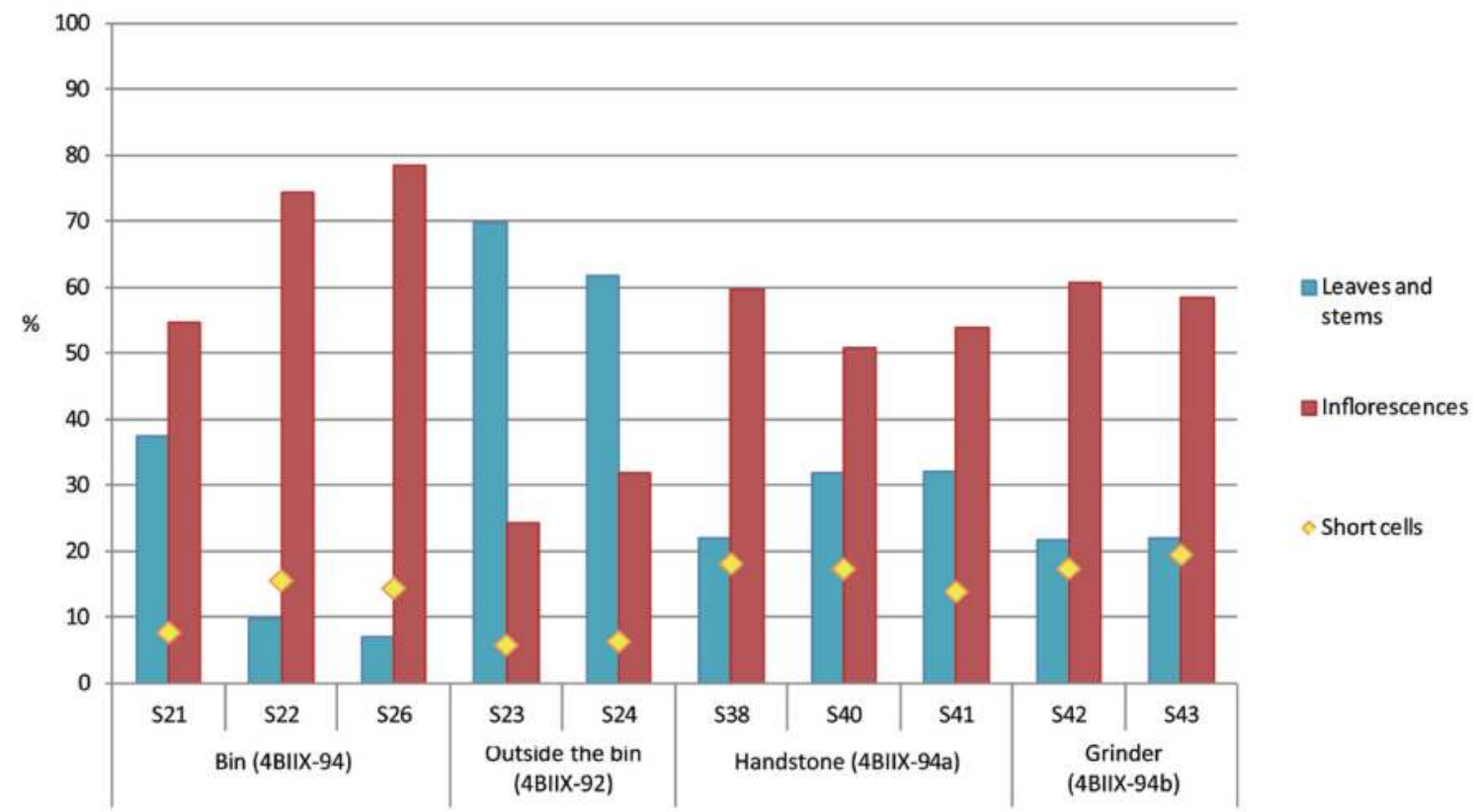

Fig. 7.12 Anatomical origin of grass phytoliths in Square 4BIIX samples.

when compared to the outside area immediately adjacent to the bin.

Also of significance were the overwhelmingly large abundances of spherulites observed in the white deposits in the adjacent area (S24 from 4BIIX-92) (over 7 million spherulites/g sediment, Table 7.2) indicative of dung accumulation. This concentration overlaps with high proportions of grass phytoliths. Inflorescences were also observed in this sample, although in much lesser proportions (32\%, Fig. 7.12). These findings indicate that these sediments were composed of animal dung derived from a grass-rich diet. This area was described in the field as white colored sediments related to cobbles, bone tools, faunal remains, charcoal and ashes (Fig. 7.5). The studied sample (S24) may represent the remains of ash dung residues.

\subsection{Micromorphological examination}

\subsubsection{Methods}

The primary aim of micromorphological examination of sediment samples from two clay bins (S36 from 3BII-1 and S25 from 4BIIX-94) was to understand the nature of the fill within these bins and compare formation processes between the two bin features that differed macroscopically during excavation. More specifically, we aim to explore the depositional processes that led to the formation of a whitish, fibrous deposit at the base of the 4BIIX-94 bin (Fig. 7.6).

During excavation of the two clay bins, an intact block of sediment (approximately $15 \times 10 \times 10 \mathrm{~cm}$ ) from the inside of each bin was left undisturbed. Each block was described, oriented, photographed and collected en bloc for micromorphological processing and analysis. They were transported to Nichika Geo-Science Materials Inc. in Kyoto, Japan. In the thin section lab, each intact sediment block was thoroughly dried and then impregnated with a clear polyester resin under a vacuum. Once consolidated, each block was prepared into five thin section slides, each $3 \times 5 \mathrm{~cm}$ in size, representing the bottom fill sediments contained within the bin, a portion of the clay bin wall and, importantly, the boundary between these two deposits (see Figs. 7.13 and 7. 15).

\subsubsection{Results: Level 10 in Square 4BII}

Our primary question related to this sample (S25) is what depositional processes are relevant to the formation of the whitish fill at the bottom of the bin (Fig. 7.6). The main objective for the examination of these slides is to determine if one can differentiate between the bin and its fill and, if so, what can we say about the use of the bin. In short, the samples of bin walls are dominated by a massive, dense clay groundmass and those of bin-fills by phytoliths and gypsum crystal intergrowths. There is a clear 


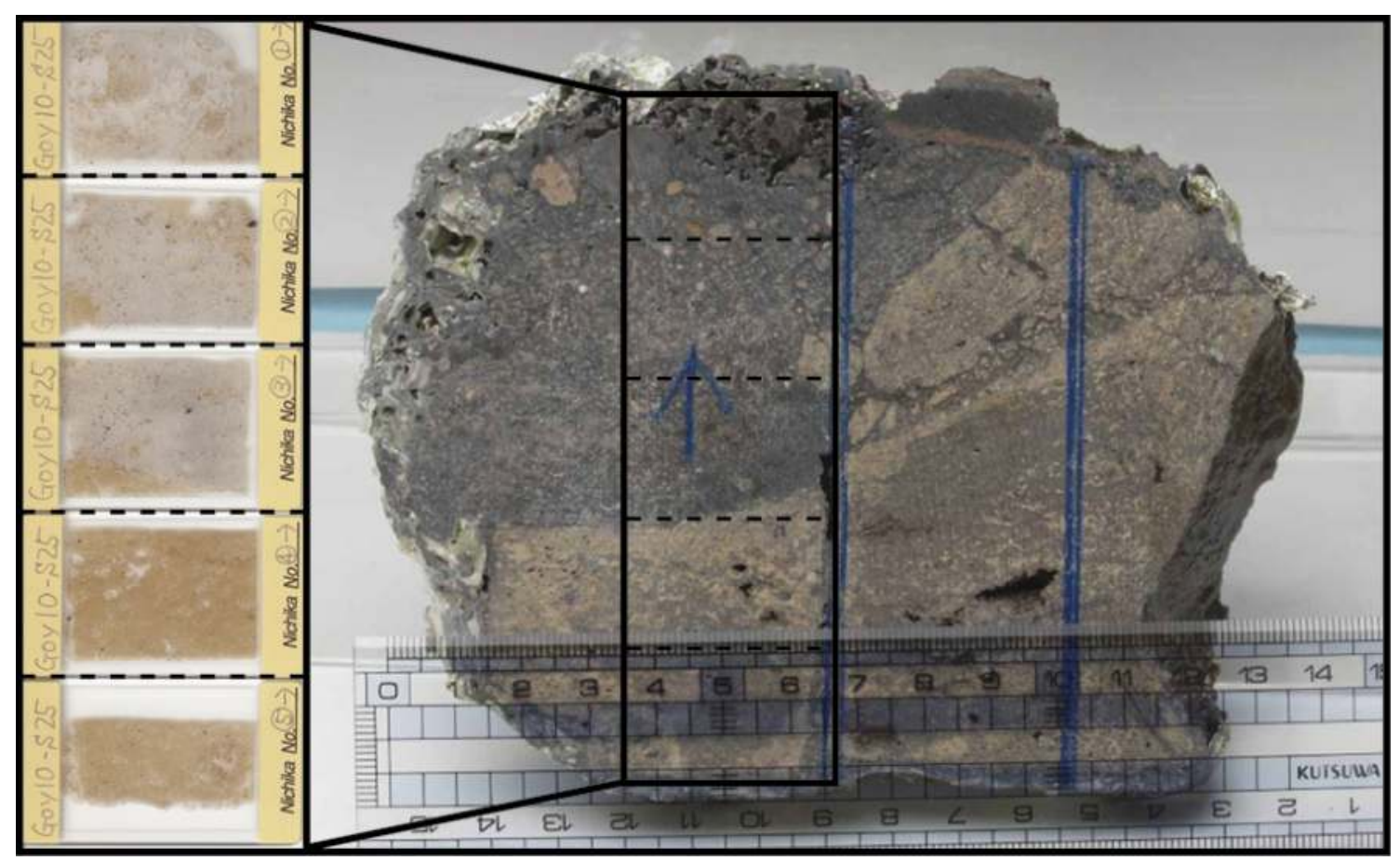

Fig. 7.13 Excavated sediment column from S25 removed en bloc (right) with five thin section slides cut from the block (left). Slides 1-3 (from top) represent the bottom fill, while Slides 4-5 consist of the bin wall material.

difference in composition and character between samples taken from the bottom fill of the 4BIIX-94 bin (Slides 1-3 of S25) versus those that represent the wall/base of the bin (Slides 4-5). The bin-fill samples exhibit a spongy, porous, yet laminated microstructure and a notably grey colour in PlanePolarised Light (PPL). They consist almost entirely of phytoliths, particularly elongate phytoliths oriented parallel to each other and to the sampled bin wall/ base creating a layered, laminated appearance, with $5-10 \%$ density of very small charcoal fragments, fine amorphous organic matter, and even higher densities of fine carbonate and gypsum crystals (Fig. 7.14: A-C; Table 7.3).

The bottom fill likely represents the remains of the last stored contents of the bin and is almost entirely dominated by grass phytoliths (predominantly inflorescences, and lesser amounts of stems and leaves, see phytolith section above for details). The fill is quite porous, a distinct grey/whitish colour, $10-15 \%$ density of very fine charcoal fragments $(<0.05 \mathrm{~mm})$, and laminated following the contours of the underlying bin shape. In particular, Slide 3 shows a very distinct, abrupt and well-marked boundary between the fill and bin wall/base, with both a colour and composition change, and a thin ( $\sim 3 \mathrm{~mm}$-thick) layer of dark grey, very porous, crumb clay aggregates and individual gypsum crystals. The whitish/grey colour seen macroscopically during excavation is also documented in thin section. It results from very fine crystals and crystal intergrowths of gypsum (and, rarely, calcite) that form a fine-textured and sparse groundmass around the phytoliths and charcoal fragments (Fig. 7.14: G).

Well-formed gypsum crystals and crystal intergrowths form in situ as the mineral precipitates out from solution in water present in a deposit (Brewer 1976; Fitzpatrick 1993; Stoops et al. 2010). They can form from infiltration of moisture through a deposit or as it pools in particular portions of a deposit, such as the undersides of stones (in this case two handstones) or when reaching a comparably impermeable layer such as the dense clay bin wall/base. In this case, the laminated phytolith layers consisting of grass parts (husks, leaves and stems) in the bottom fill, some of which suggest a possible matted texture (Fig. 7.14: C), could represent the remains of a lining on the bottom of the bin (Goldberg 2000; Goldberg et al. 2009; Wadley et al. 2012). The gypsum likely formed as moisture introduced into the plant matter contained in the bins percolated down to the lowest layers, and into the lining, where gypsum precipitated here to form small crystals and intergrowths at the bin wall boundary that was more compact and impermeable. 


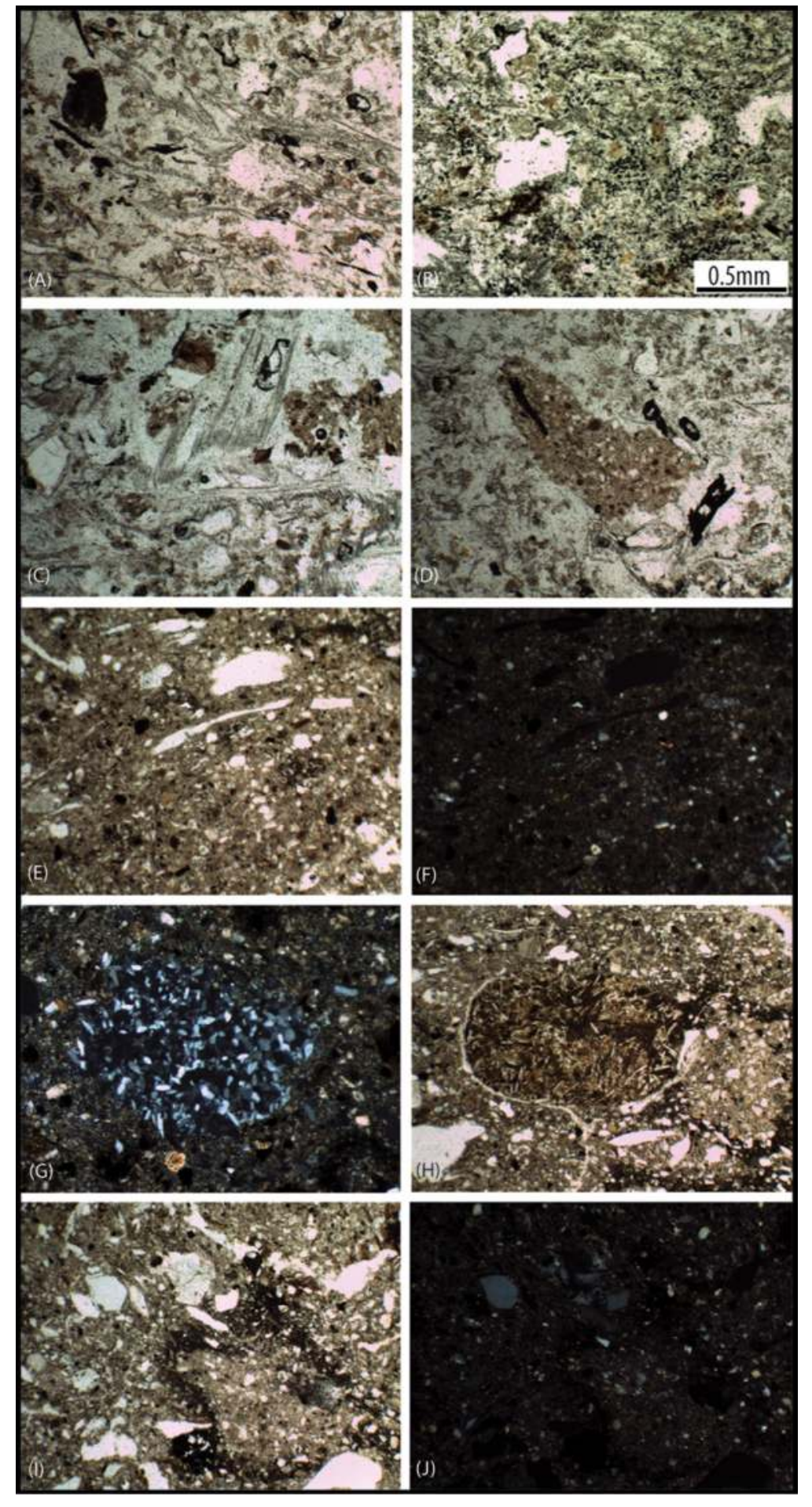

Fig. 7.14 Photomicrograph images of features from the bottom fill and clay wall of bin S25. (A) Image of the laminated phytolith layers in the bottom fill (PPL); (B) Close-up of bottom fill layer with fine groundmass of randomly oriented phytoliths, fine charcoal and gypsum crystals (clear) (PPL); (C) Image of the bottom fill showing phytolith layers perpendicular to each other (PPL); (D) Clay aggregate of bin wall incorporated into phytolith-rich bottom fill (PPL); (E-F) Image of the clay bin wall consisting of quartz and gypsum silt (clear crystals) within a dense clay fabric (E: PPL, F: XPL); (G) Gypsum crystal intergrowth within clay bin wall (XPL); (H) Spherulites from micro-mammal excrement incorporated into the clay bin wall sediment (PPL); (I-J) Secondary features in the clay bin wall deposit include localized areas of iron staining and clay translocation (darkened area) (I: PPL, J: XPL).
In essence, the clay wall formed a more impermeable boundary that the water did not infiltrate through as easily as the fill. Instead after water pooled at the base of the bin as it dried it precipitated gypsum and some carbonate from solution, forming the fine dustlike layers of gypsum and carbonate that give it a distinctive whitish, fibrous macroscopic appearance.
The clay walls of the bin form a comparatively water-resistant boundary that concentrated moisture percolating down through the bin and its contents by trapping it in the lining and lower fill, between it and the bin walls, where gypsum (mobilised from the local geology and adjacent archaeological deposits) precipitated out of solution here to form 


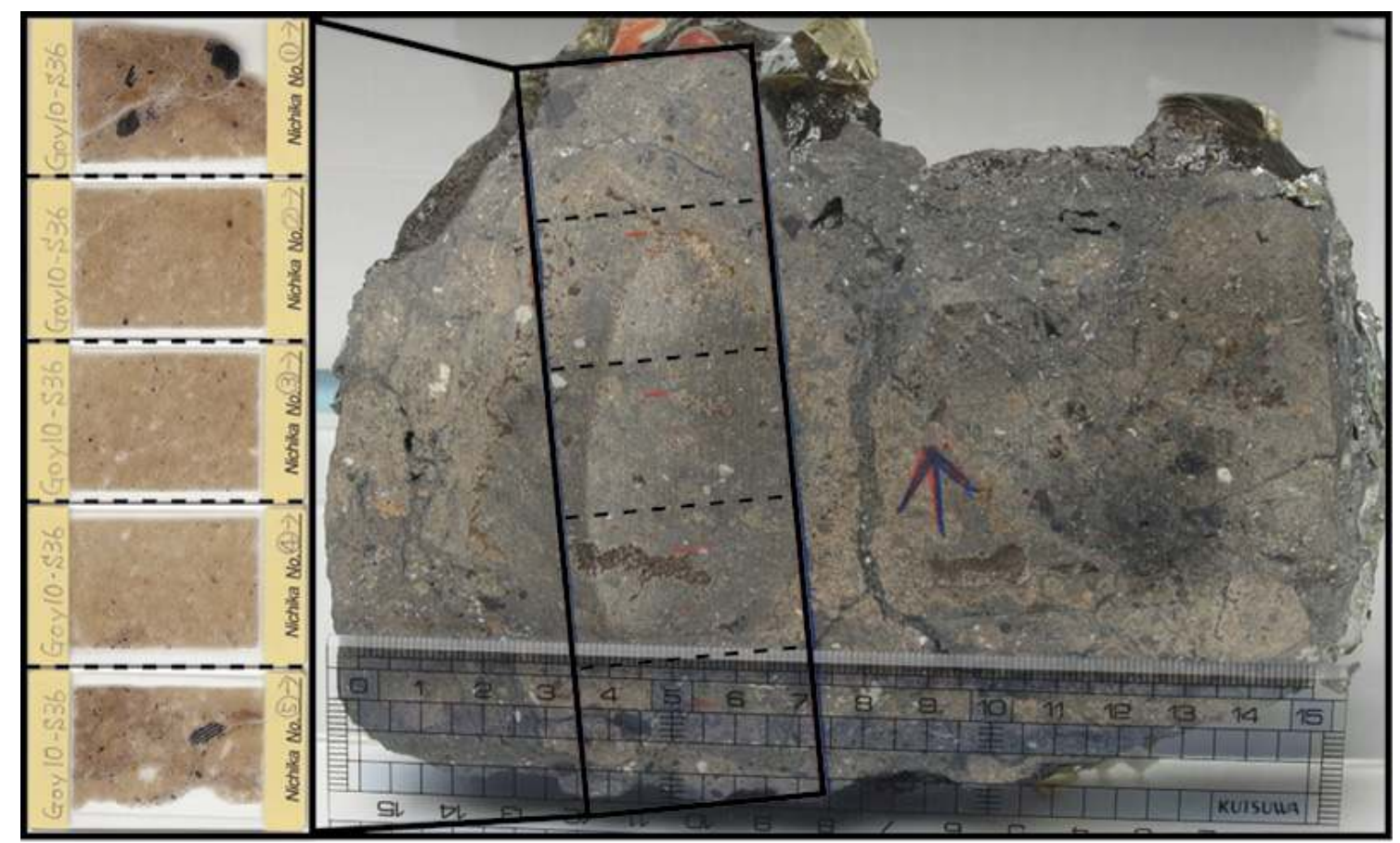

Fig. 7.15 Excavated sediment column from S36 removed en bloc (right) with five thin section slides cut from the block (left). Slide 1 (from top) represents the bottom fill, while Slides $2-5$ come from the bin wall material.

fibrous crystals and crystal intergrowths in the pores between the grass material.

The walls/base of the bin (Slides 4-5), on the other hand, are massive in appearance, with varying crumb, granular, and vughy or vesicular microstructures (termed 'complex' in Stoops et al. 2010). The groundmass is dense and clay-rich, with voids (vughs, channels and vesicles) indicative of decayed plant matter (Fig. 7.14: E-F). The samples are a reddish-brown colour in PPL and, as a clay-enriched deposit, exhibit a mosaic b-fabric in Cross-Polarised Light (XPL) caused by very fine gypsum and calcite crystals. These dense clay samples also contain $10 \%$ or more small calcitic (micritic) limestone fragments, gypsum crystals and intergrowths, charcoal, burnt bone, and rare clasts of quartz or obsidian flakes (Fig. 7.14: E-F). These samples also contain rare spherulites from animal dung (Fig. 7.14: H). Phytoliths are also present in these samples, but in much lower densities than the overlying fill deposit (5\%). Instead, a high biological content is represented by characteristic void spaces (resulting from decay of biological content in the deposit). These samples are characteristic of prepared clay construction material or mud-brick, with their groundmass density, high clay content, gypsum crystal intergrowths and evidence for some dissolution features. This is not surprising as erosion by water is the most common post-depositional alternation affecting clay construction material (Goodman-Elgar 2008; Rosen 1986). Although there are small fragments of the clay bin contained within the fill $(0.5-1 \mathrm{~mm}$ diameter; Fig. 7.14: D), the fill and bin wall samples are really quite distinct from each other in their content and structure and, unsurprisingly, represent very different depositional events and diagenesis. The samples from S36 are much less clear (see below).

Without also having micromorphology samples of the upper fill or domestic activity areas for comparison, it appears that the laminated, whitish, phytolith-rich bottom fill layer inside the bin with two large handstones sitting on top of it represents the remains of compacted plant matter that lined the bin with remnants of stored material intermixed. When put together with the phytolith data, it seems that these bins were used for the storage of grasses (wheat and barley). The three slides from the bottom fill cover about 3-4 cm depth of this deposit at its base. In this fill there are rare inclusions of clay aggregates averaging $1 \mathrm{~mm}$ in diameter (Fig. 7.14: D) that resemble in microstructure and content the samples of the bin wall (Slides 4-5). The presence of these aggregates in the bottom fill suggests re-use 


\begin{tabular}{|c|c|c|}
\hline $\begin{array}{l} \\
\check{0} \\
\stackrel{0}{0} \\
\overline{\bar{\nu}} \\
\dot{\phi} \\
\Delta\end{array}$ & 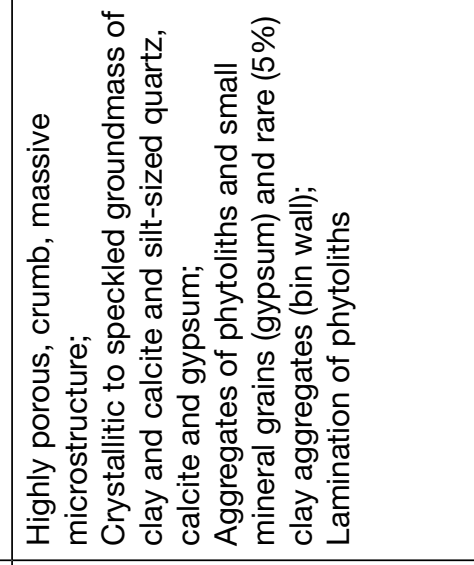 & 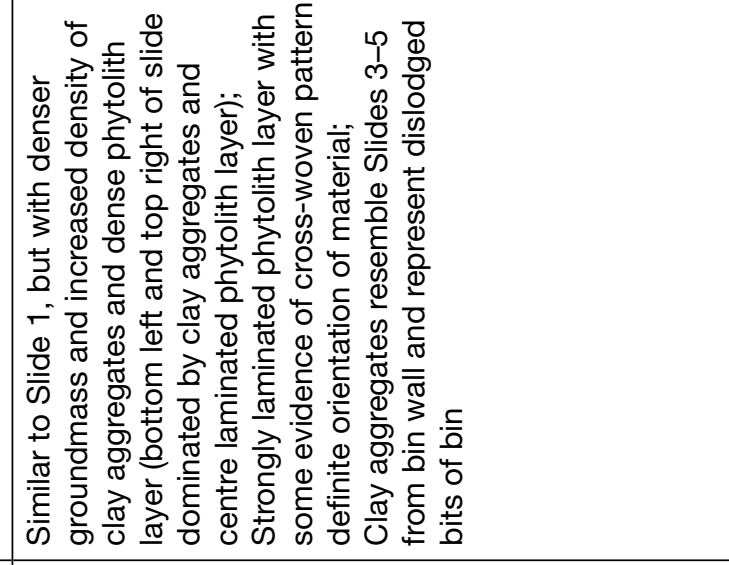 \\
\hline 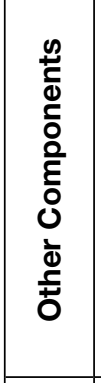 & 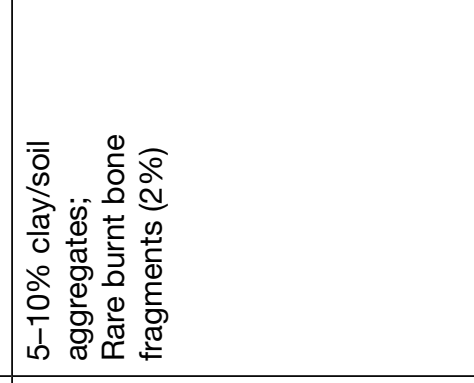 & 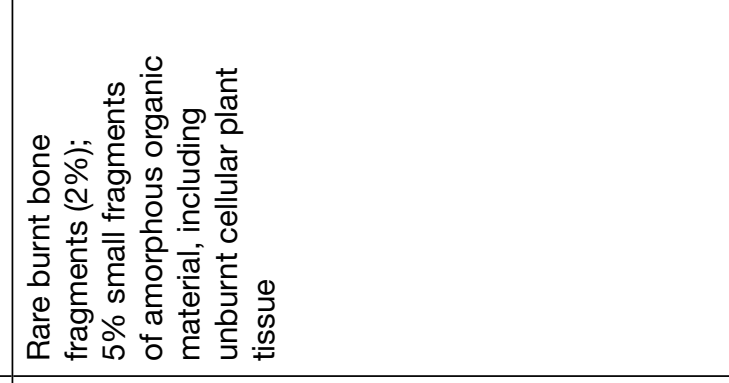 \\
\hline 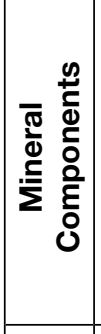 & 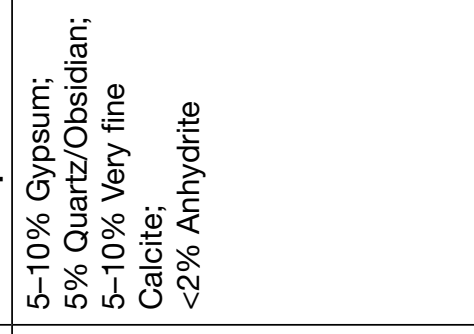 & 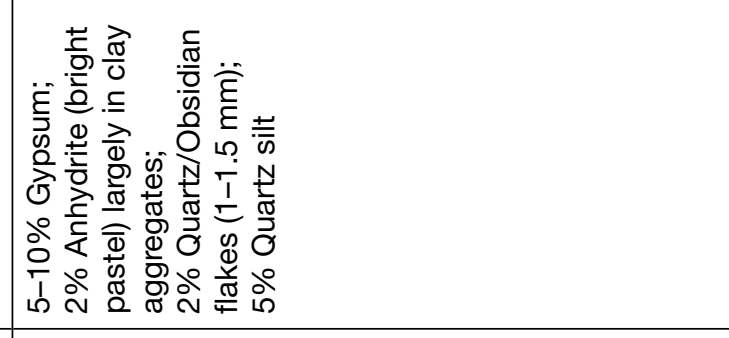 \\
\hline 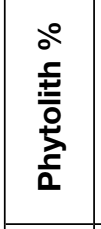 & 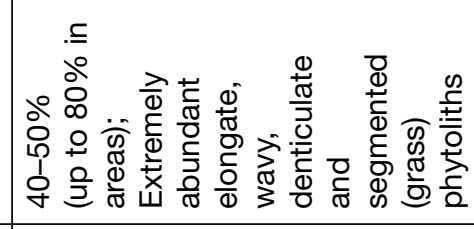 & 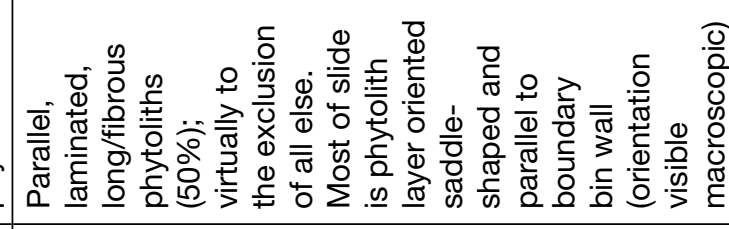 \\
\hline 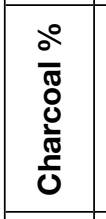 & 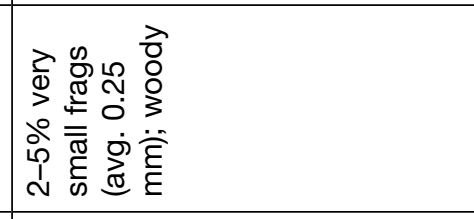 & 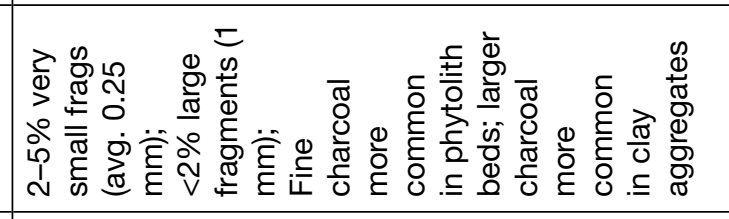 \\
\hline$\frac{\infty}{50}$ & 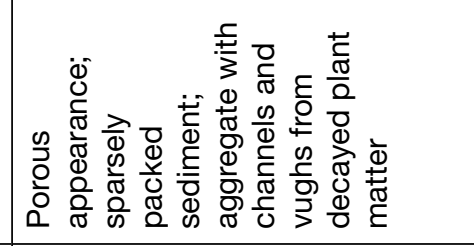 & 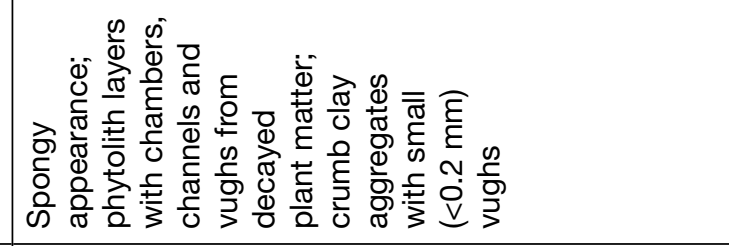 \\
\hline 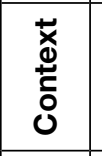 & 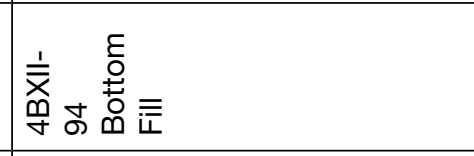 & 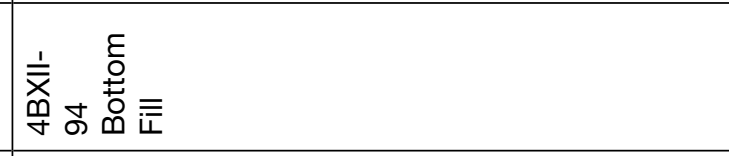 \\
\hline 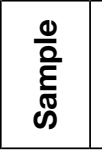 & 崖 & 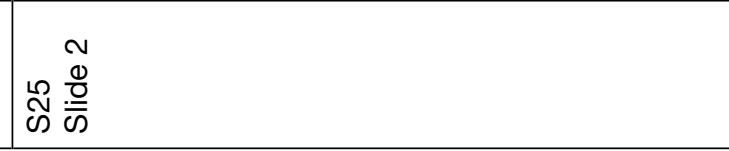 \\
\hline
\end{tabular}




\begin{tabular}{|c|c|c|}
\hline 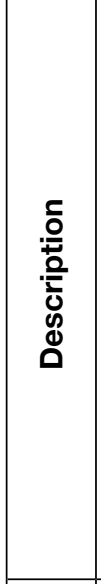 & 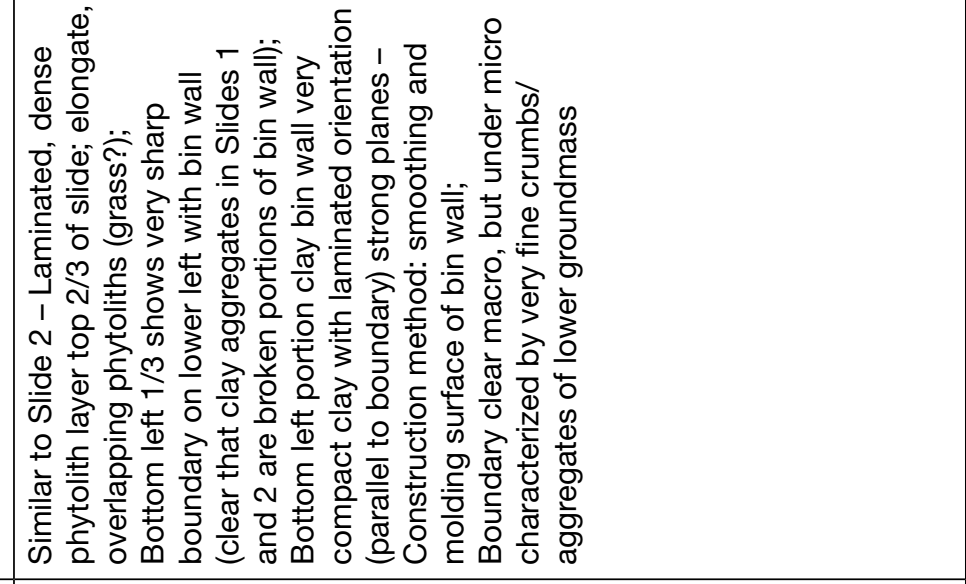 & 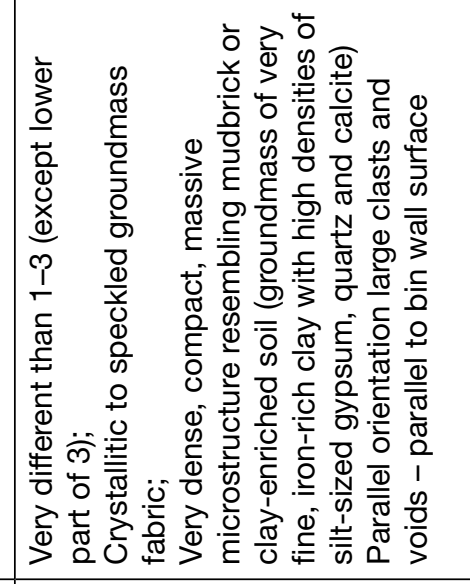 \\
\hline 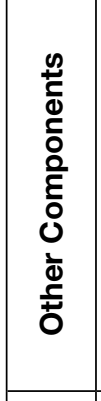 & 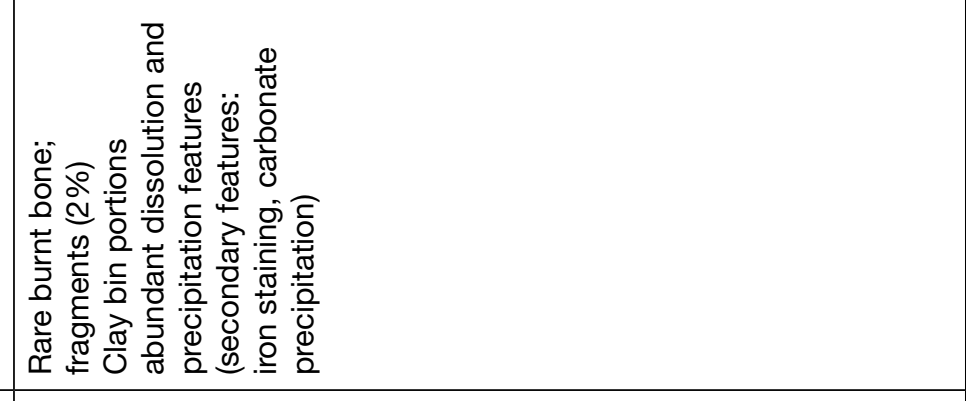 & 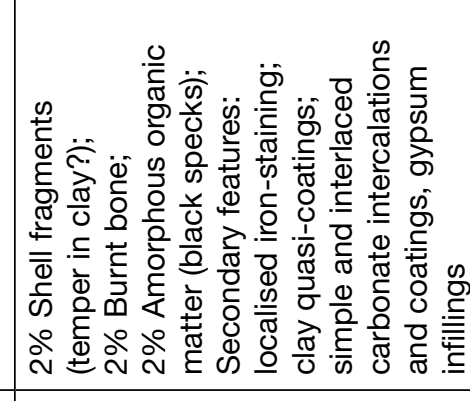 \\
\hline 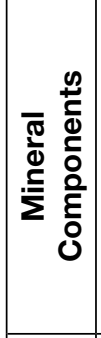 & 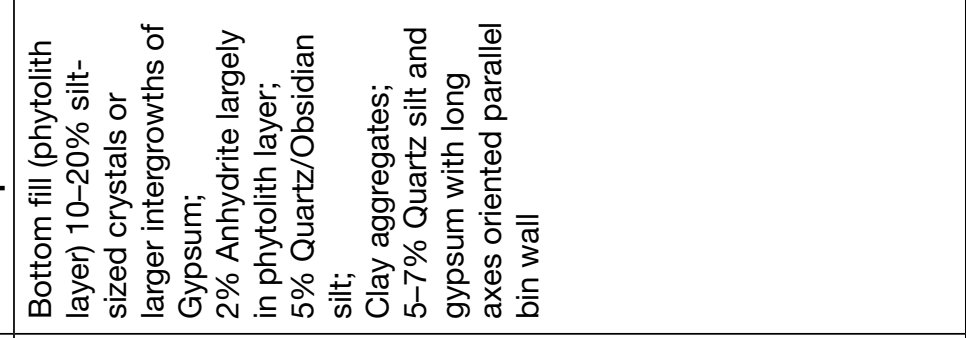 & 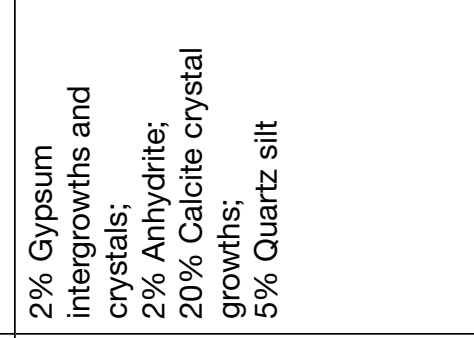 \\
\hline 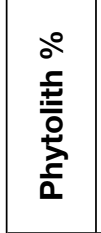 & 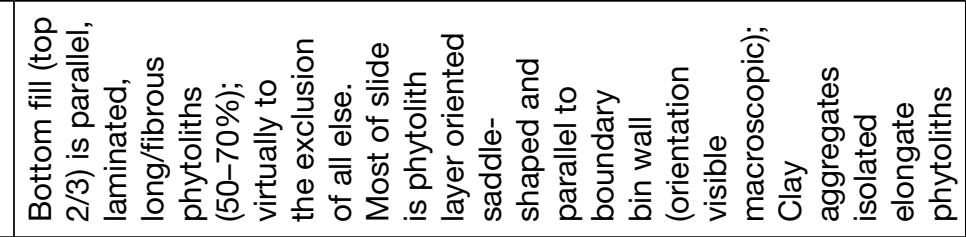 & 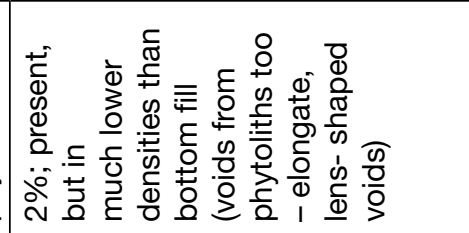 \\
\hline 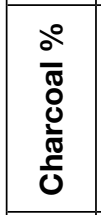 & 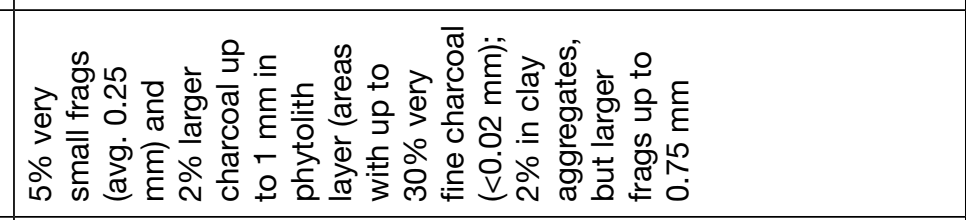 & 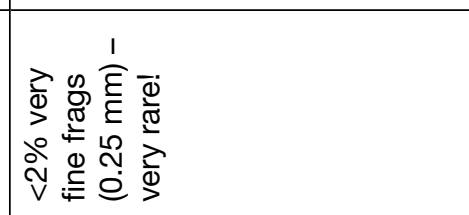 \\
\hline$\frac{\mathscr{8}}{\circ}$ & 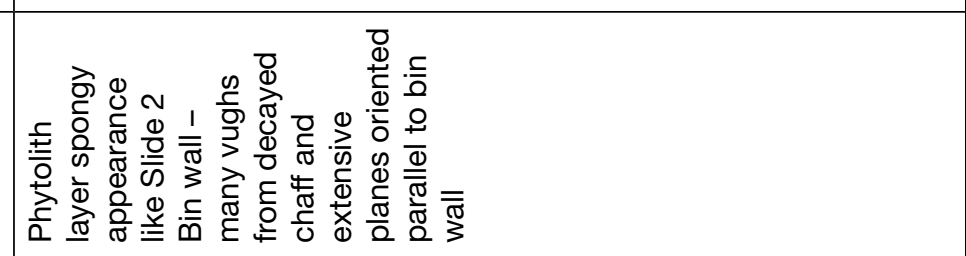 & 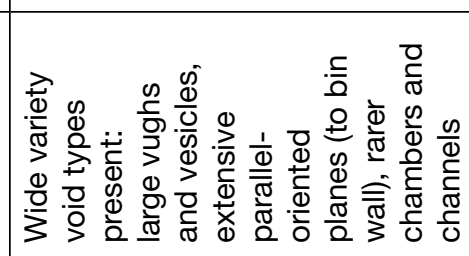 \\
\hline 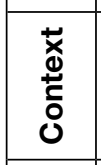 & 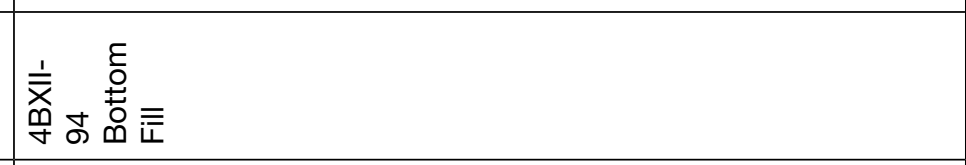 & 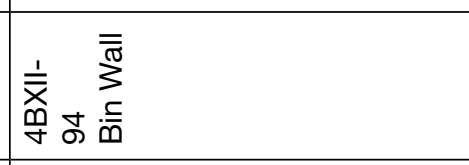 \\
\hline 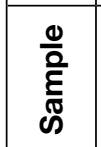 & 先 & 岕 \\
\hline
\end{tabular}




\begin{tabular}{|c|c|c|}
\hline 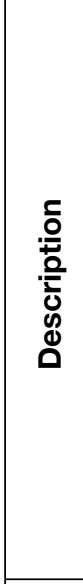 & 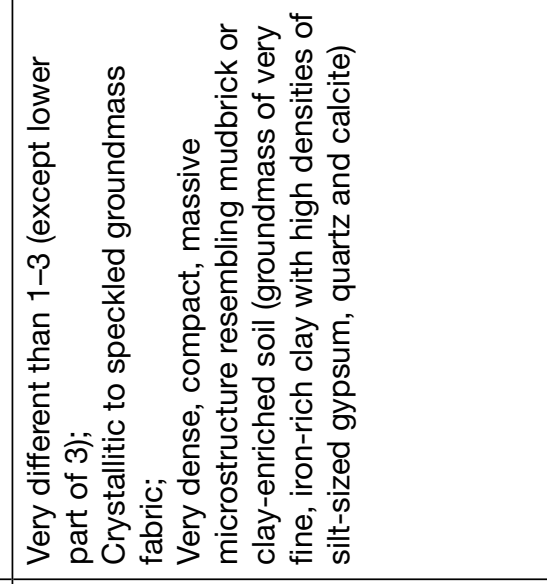 & 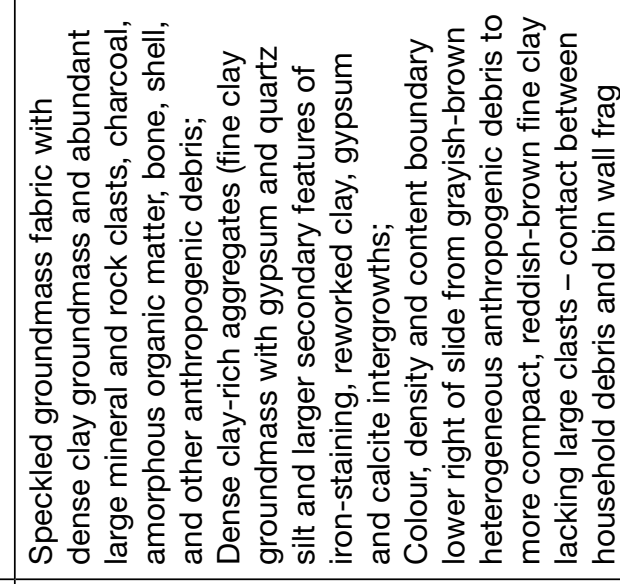 \\
\hline 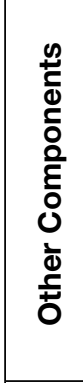 & 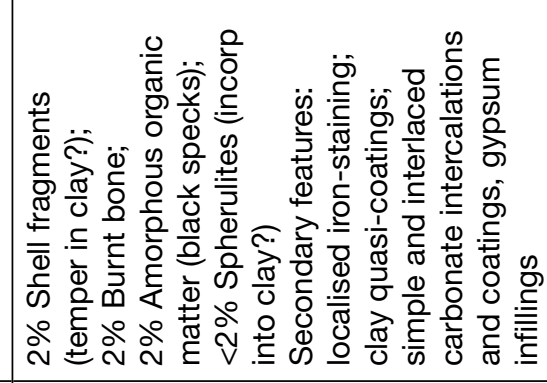 & 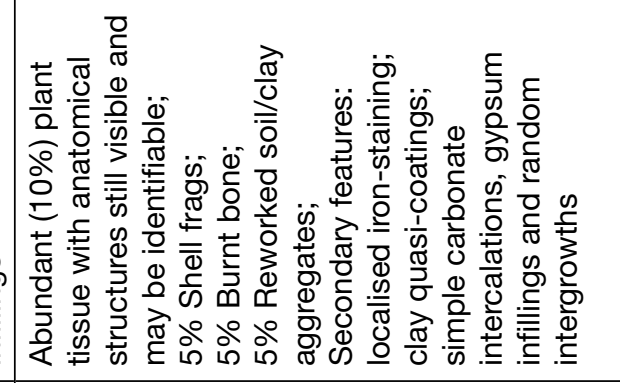 \\
\hline 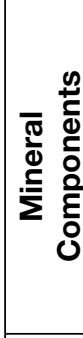 & 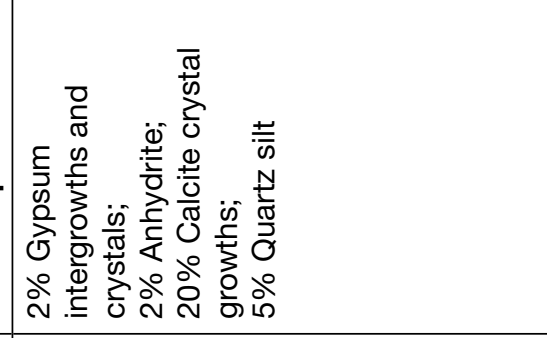 & 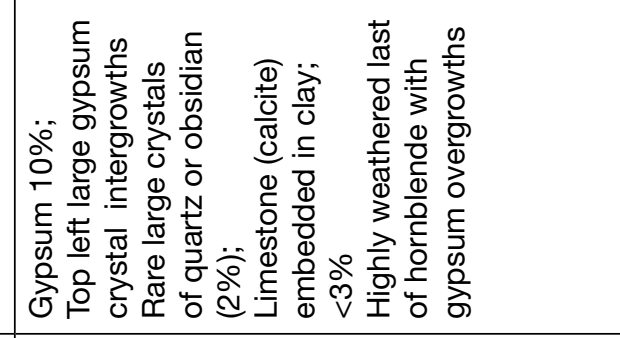 \\
\hline 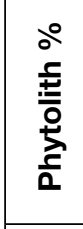 & 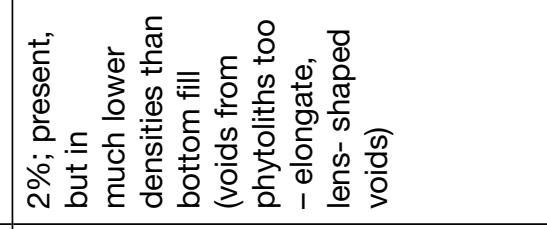 & 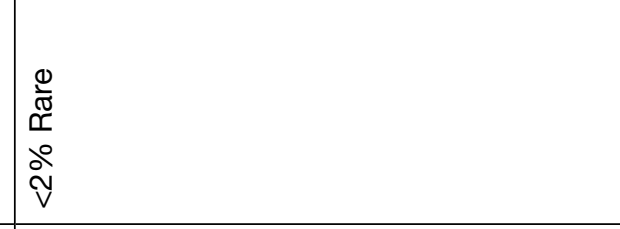 \\
\hline $\begin{array}{l}\frac{0}{0} \\
\frac{0}{0} \\
\frac{0}{0} \\
\frac{0}{0} \\
\end{array}$ & 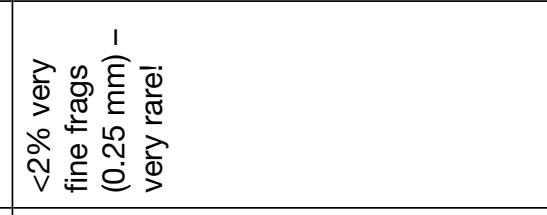 & 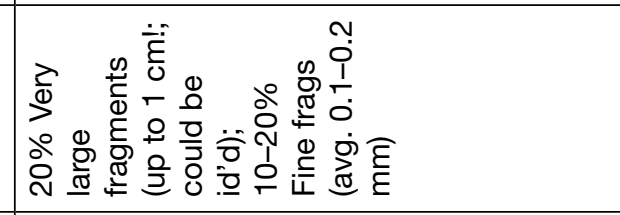 \\
\hline$\stackrel{0}{: 0}$ & 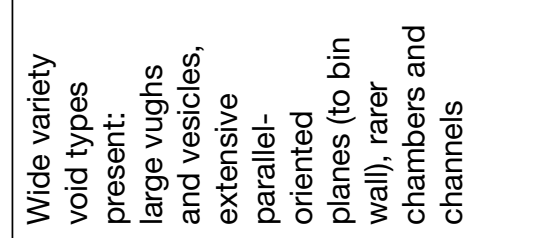 & 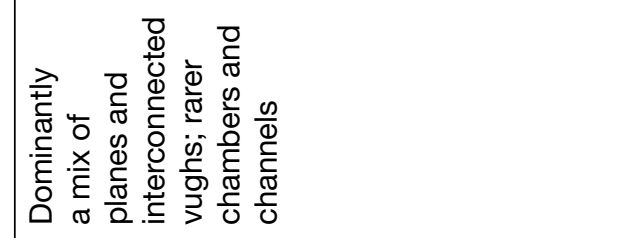 \\
\hline 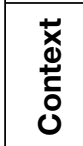 & 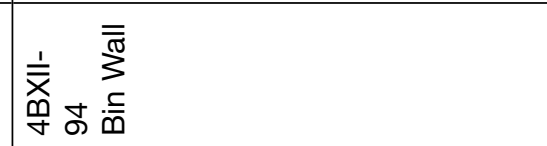 & 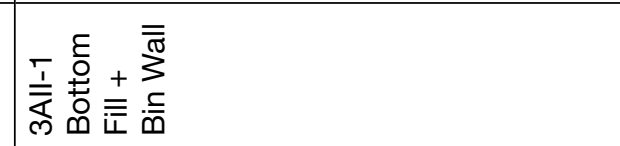 \\
\hline $\begin{array}{l}\frac{0}{0} \\
\frac{\tilde{E}}{5} \\
\stackrel{5}{\infty}\end{array}$ & 罢 & 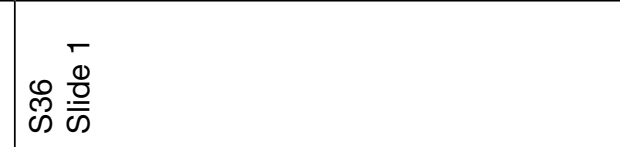 \\
\hline
\end{tabular}




\begin{tabular}{|c|c|c|}
\hline 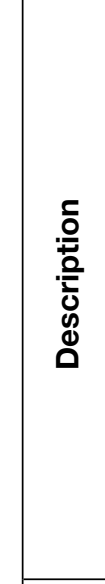 & 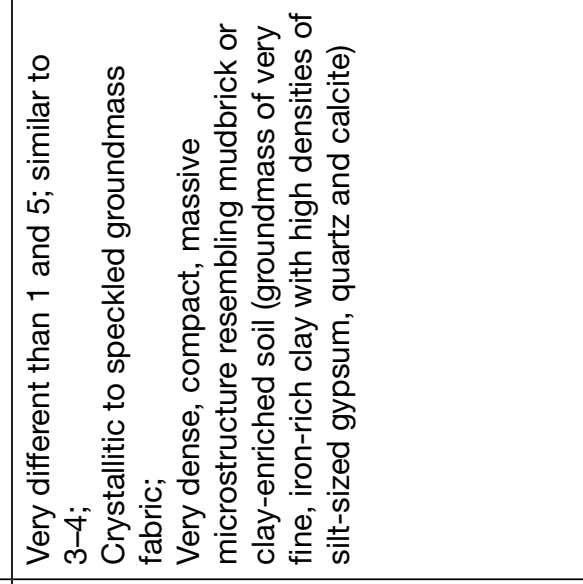 & 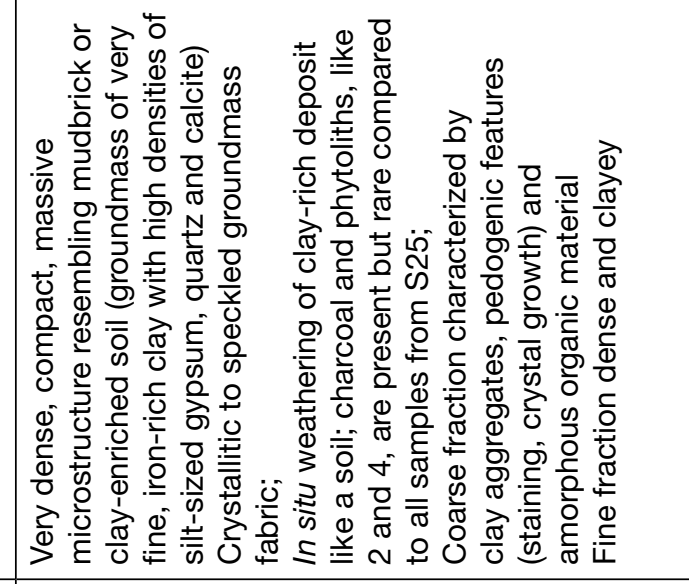 \\
\hline 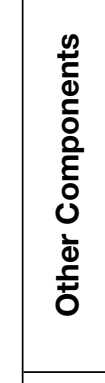 & 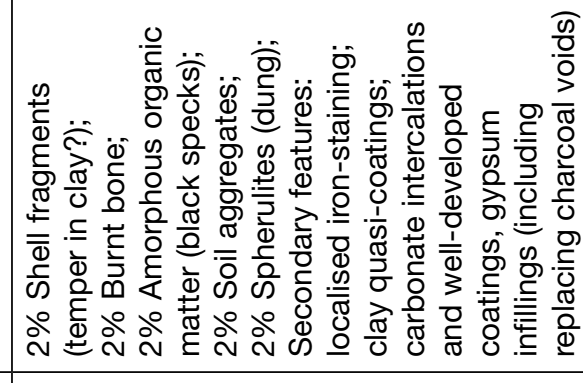 & 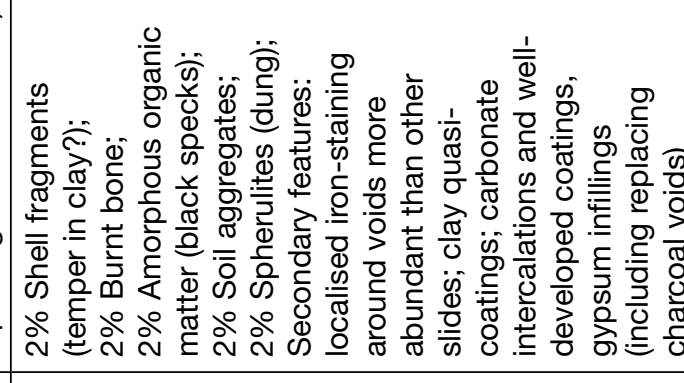 \\
\hline 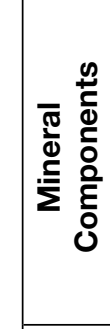 & 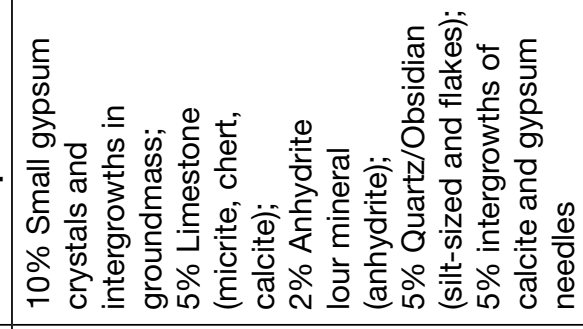 & 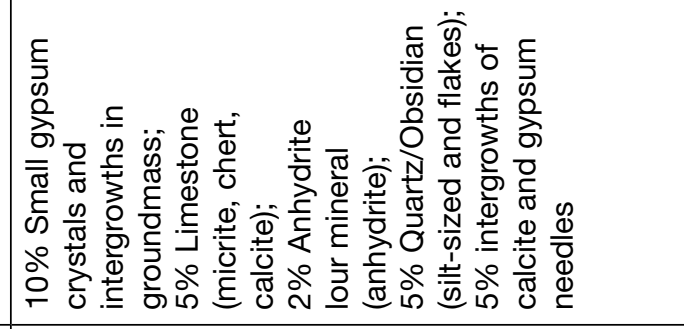 \\
\hline 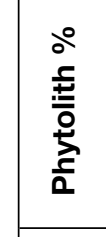 & 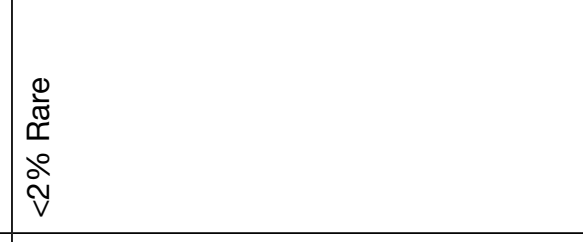 & 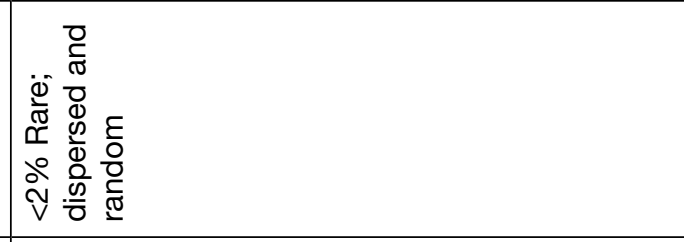 \\
\hline 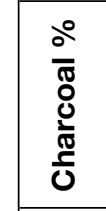 & 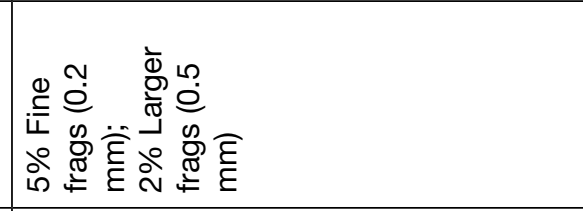 & 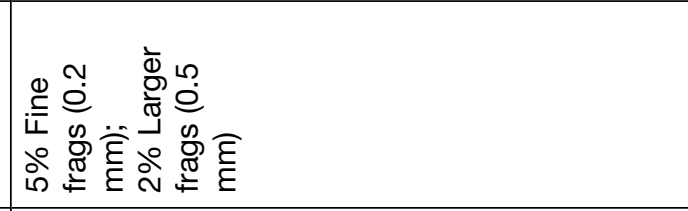 \\
\hline$\frac{0}{0}$ & 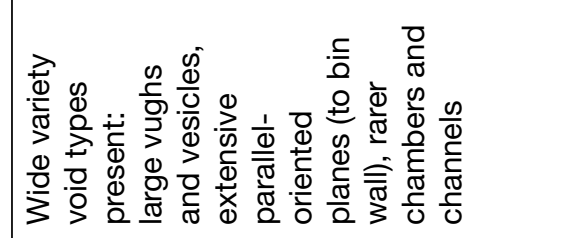 & 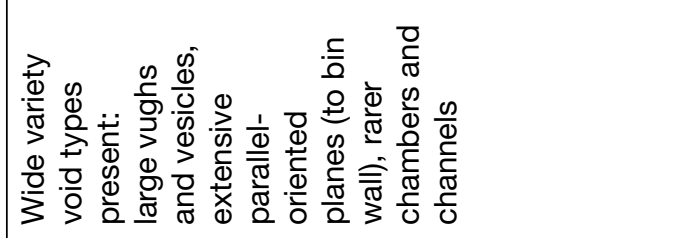 \\
\hline 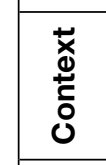 & 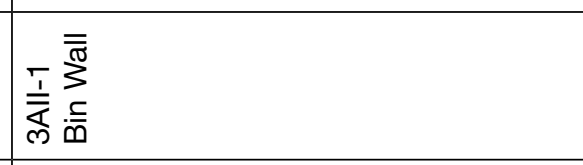 & 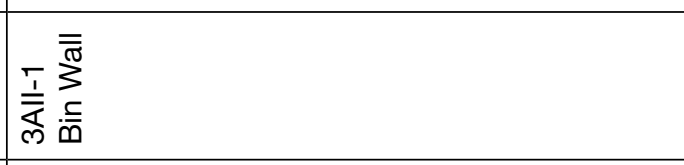 \\
\hline 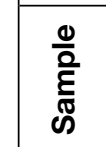 & 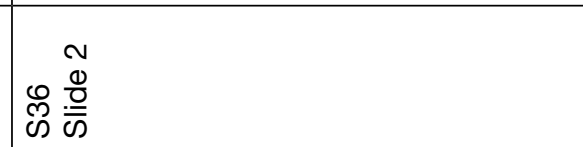 & 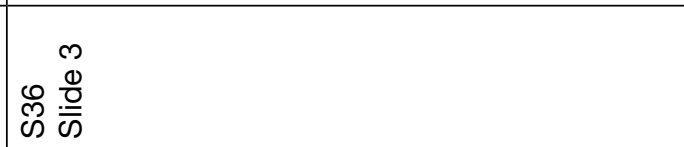 \\
\hline
\end{tabular}




\begin{tabular}{|c|c|c|}
\hline 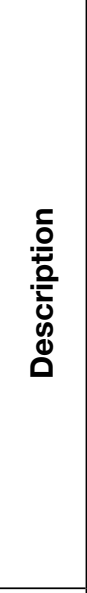 & 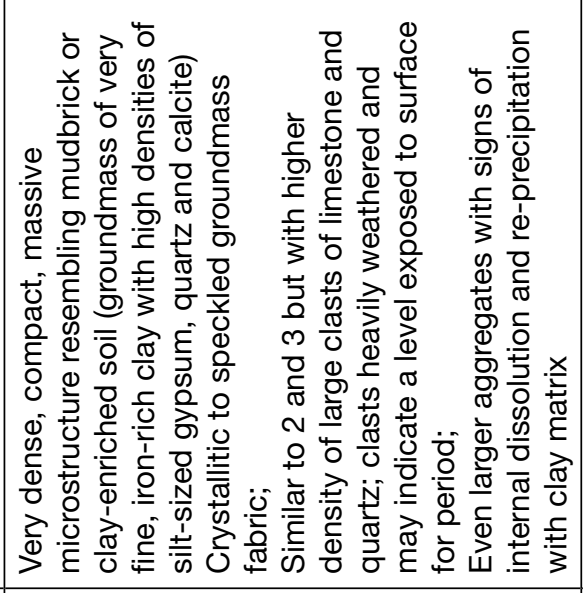 & 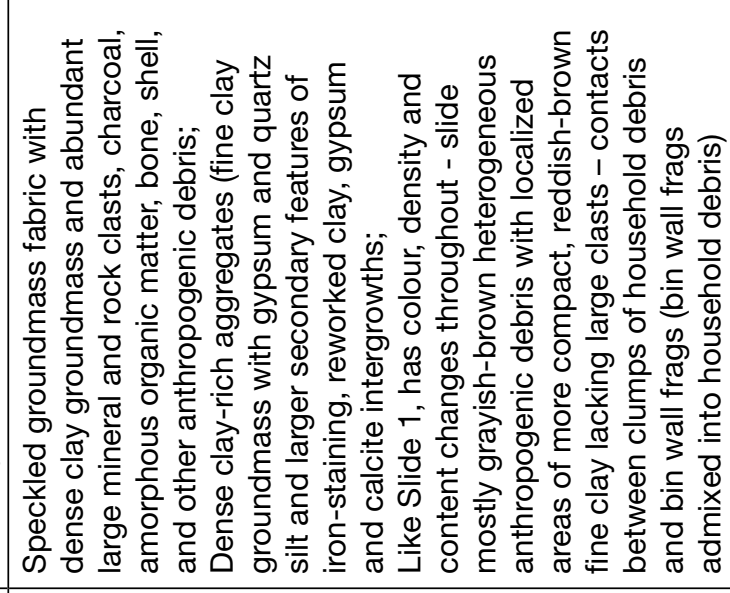 \\
\hline 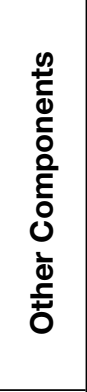 & 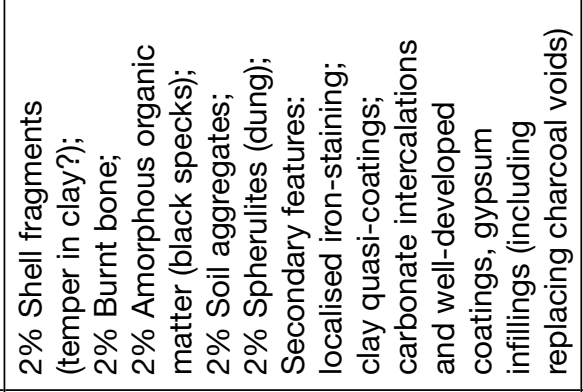 & 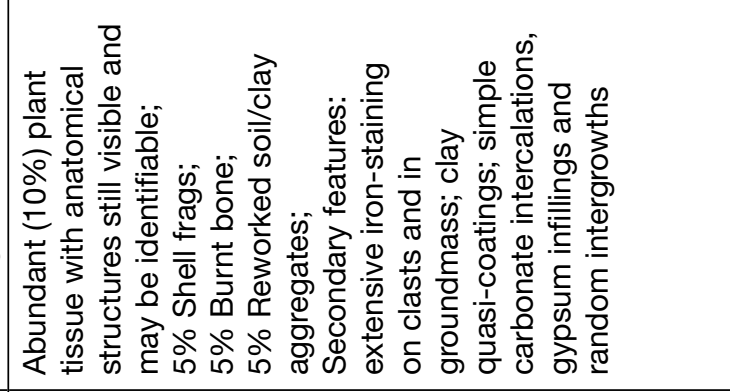 \\
\hline 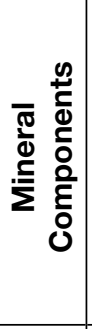 & 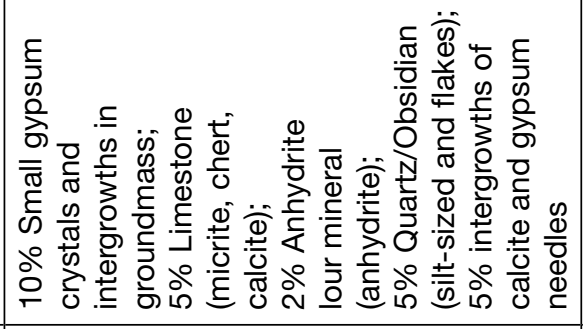 & 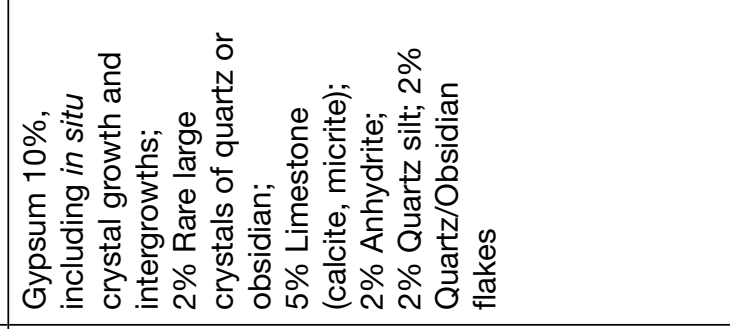 \\
\hline 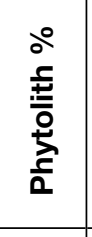 & 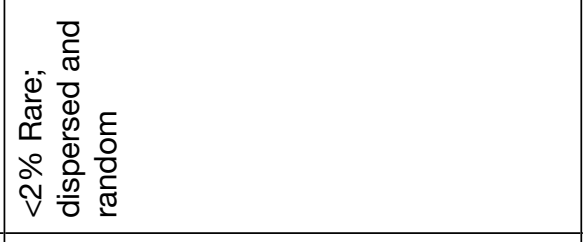 & 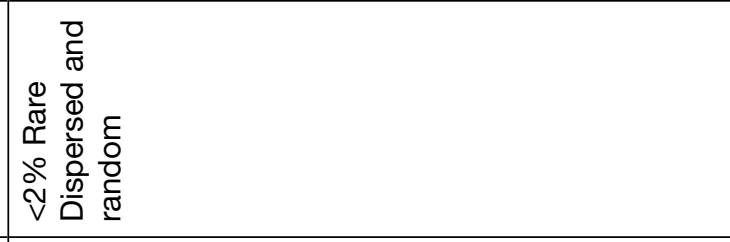 \\
\hline 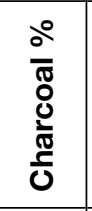 & 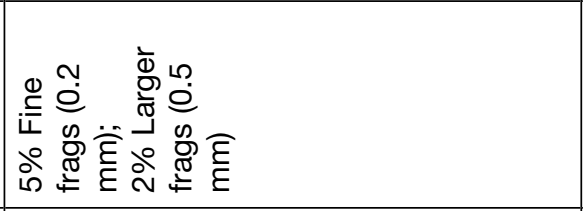 & 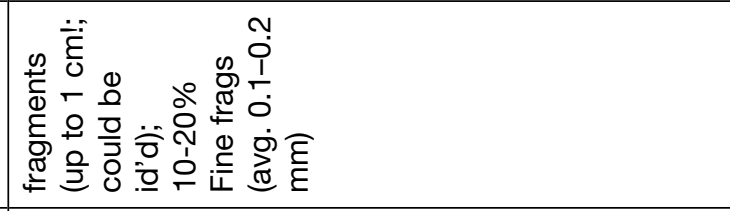 \\
\hline & 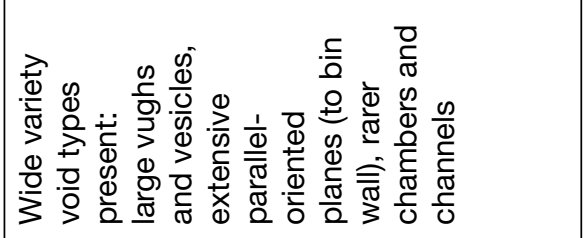 & 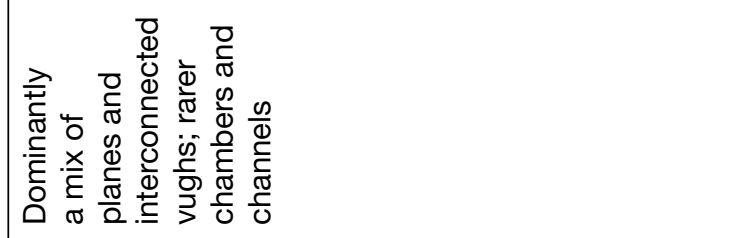 \\
\hline $\begin{array}{l}\vec{x} \\
\stackrel{x}{0} \\
\stackrel{0}{0} \\
0\end{array}$ & $\frac{\overline{1}}{\overline{\bar{\Sigma}}}$ & $\frac{\bar{T}}{\overline{\bar{\Sigma}}}$ \\
\hline $\begin{array}{l}0 \\
0 \\
\text { क्ष } \\
\text { का }\end{array}$ & 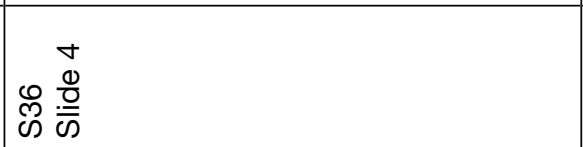 & 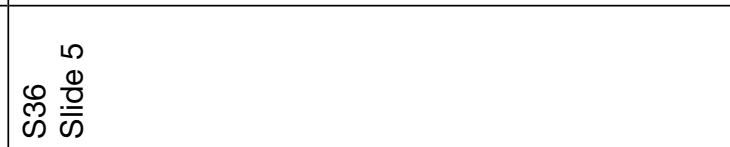 \\
\hline
\end{tabular}


of the bins where, for example, re-opening and refilling of its contents caused tiny fragments of the clay bin wall to become dislodged and amalgamated into the stored contents. Although the large planes and channels show a parallel orientation from molding and forming of the clay (and likely also from inclusions of grass stems and leaves as temper), the samples from the clay bin walls (Slides 4-5) display a dense, massive microstructure with no laminations or microstratigraphy and, thus, no evidence for regular re-surfacing of the bin walls. It appears that the bin was used repeatedly with little or no maintenance to its original structure at the base.

\subsubsection{Results: Level 4 in Square 3AII}

A sediment block (S36 in Fig. 7.3) sampled from the 3AII-1 bin covers deposits of the bottom fill and the bin wall (Fig. 7.15). However, the boundary between the bottom wall of the bin and the bottom fill is unclear as there was admixture between the fill and bin lining noted during excavation of the feature, and so the sample may contain only a few $(1-2) \mathrm{cm}$ of the bottom fill (Slide 1) and consist otherwise of either the bottom bin wall or a secondary fill that is identical to the bin walls (Slides 2-5).

The five slides from the 3AII-1 bin are quite homogeneous and, thus, different from the fill samples from S25 of the 4BIIX-94 bin (see also Table 7.3). The bottom fill and bin wall samples from S25 are distinct from each other in terms of both fine- and coarse-fraction components and structure. All of the slides from the 3AII-1 bin, representing the bottom fill (upper portion of Slides 1) and bin wall/base (Slides 2-5), are comparatively uniform in composition and suggest instead that the fill in this bin is secondary fill comprised of both remnants of the bins last contents (grass phytoliths), other debris (charcoal, burnt bone, shell, limestone/calcite clasts), and fragments of clay bin debris (Fig. 7.16). A secondary fill interpretation is also consistent with the observation that although only a portion of Slide 1 was thought to represent a sample of the bin fill (and was identified macroscopically as a greyish sediment), it, and Slide 5 (reported as bin wall material) resemble each other, while the other Slides (2-4) are homogenous, dense clay (Fig. 7.16).

Thus, depositional processes of S36 appear different from those of S25. S36 likely represents a secondary fill composed of an admixture of stored contents and debris from domestic activities and clay bin construction or repair. Although it is also high in phytolith content (in particular grass leaves and stems, versus seeds in S25), the fill (Slide 1) and wall samples (Slides 2-5) contain notably less phytoliths than the S25 fill and are more similar to the bin wall samples from S25. Both phytoliths and void spaces present in the bin wall samples here result from the use of chaff temper in the construction of mud-brick that leaves characteristic phytolith and void traces after decay (Courty et al. 1989; Goldberg 1980; Goodman-Elgar 2008; Love 2012). Other dissolution features are typical fabric changes of mud-brick construction (i.e., baking) and decay (Berna et al. 2007; Goodman-Elgar 2008).

There are no distinct differences between any of the slides in terms of lamination, other fine- and coarse-fraction content, or mineralogy. Unlike S25, S36, especially Slides 1 and 5, contain very large fragments (up to $1 \mathrm{~cm}$ ) of charcoal and ash. The presence of very small $(<0.5 \mathrm{~mm})$ fragments of charcoal like those from S25 is expected within stored processed grains and associated linings, where charcoal flying out of a hearth could easily become incorporated into foodstuffs or textiles being processed or dried nearby. Instead, these large fragments of charcoal (up to $1 \mathrm{~cm}$ ) are more commonly found in secondary fill deposits that contain the heterogeneous debris from household activities, such as hearth cleaning or floor sweeping (Matthews 2012a, b; Matthews et al. 1997; Shillito et al. 2011; Stoops et al. 2010). These large fragments of charcoal are randomly oriented and distributed. The accompanying ash reinforces this source for the charcoal.

The bin wall samples (Slides 2-4) also contain comparatively increased densities of rock fragments (limestone, obsidian or quartz flakes), soil/clay-like aggregates (silty and sandy clays with amorphous organic matter, fine charcoal, a coarse fraction of calcite, gypsum, and quartz), and more than typical of intentionally-stored foodstuffs (Finlayson et al. 2003; Kuijt and Finlayson 2009; Stoops et al. 2010). In essence, all the S36 samples, including the upper portion of Slide 1, more closely resemble the bin 


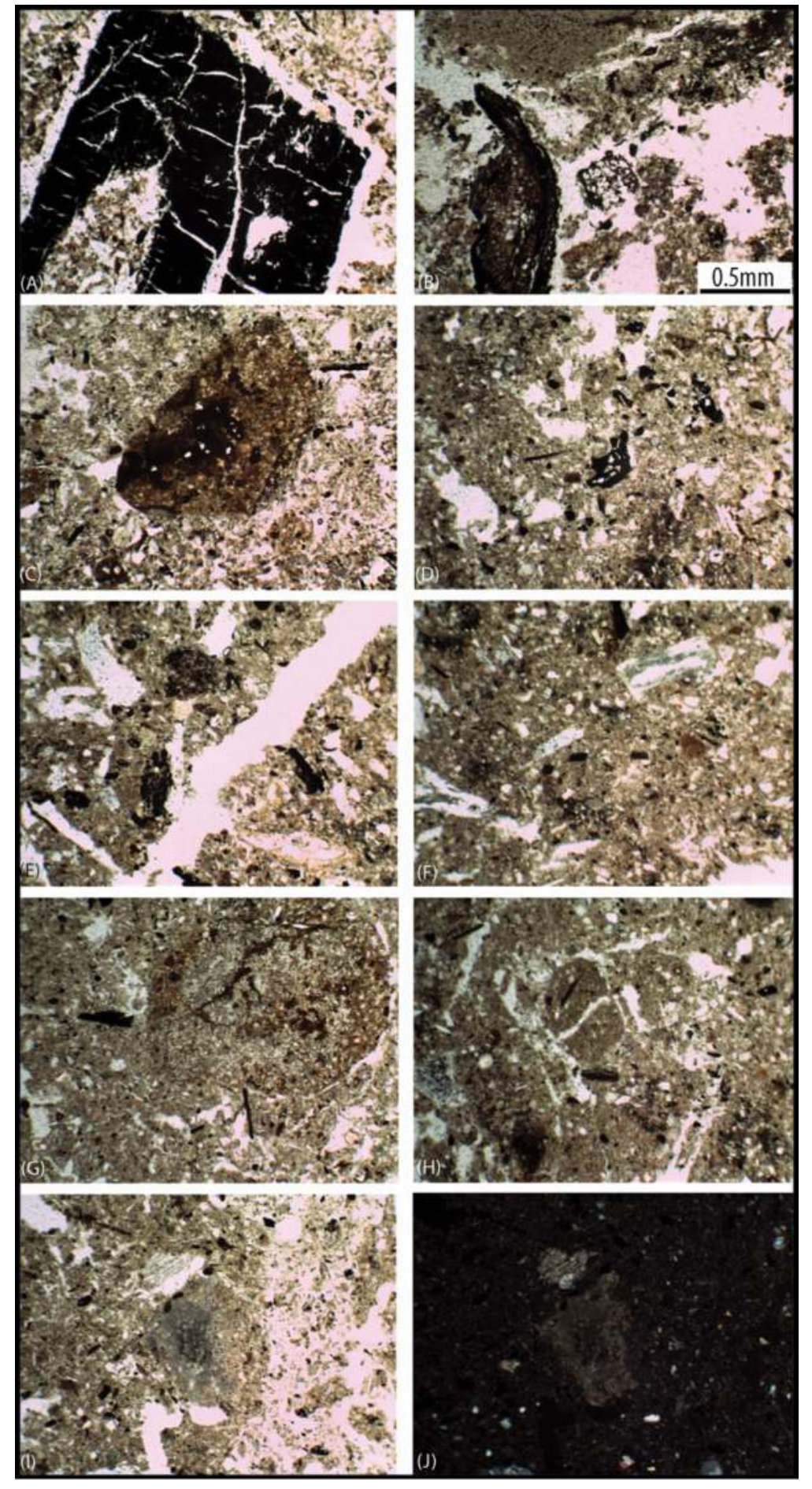

Fig. 7.16 Photomicrograph images of features from the bottom fill and clay wall of bin S36. (A) Large fragment of charcoal within a crumby, clay-rich aggregate in the bottom fill (PPL); (B) Charcoal, dense ash with fine charcoal (upper left) and amorphous organic matter (small black specks) within sparse clay groundmass of the bin wall sample (PPL); (C) Soil aggregates contained with the dense clay groundmass of the bin wall samples (PPL); (D-F, H) Clay-rich groundmass with fine charcoal, ash, amorphous organic matter, gypsum and quartz crystals, reworked clay aggregates $(\mathrm{H}$, centre) and abundant vughy and planar voids characteristic of the bin wall samples (PPL); (G) Secondary features in the bin wall samples include iron staining of clay aggregates (upper right) with clay hypocoatings (upper right, reddish ring around ashy clay aggregate) (PPL); (I-J) Secondary features also include carbonate nodule formation in the centre of the slide with in situ growth of calcite upper left. (I: PPL, J: XPL). Note the irregular and varied structure of the groundmass from a crumb structure with interconnected voids to a dense and massive groundmass. wall samples from both S25 than the distinctive bottom fill from S25 (Slides 1-2). In addition, with the exception of increased large charcoal and mineral constituents in Slides 1 and 5, the S36 slides are very consistent with each other in terms of the arrangement of components and groundmass (very fine fraction). There are no distinct boundaries between fill and bin wall (as seen in S25), the slides show no internal structure (massive, rather than laminated), and their composition (fine and coarse matter and mineralogy) is much more internally variable than the bottom fill from S25 (Table 7.3).

In summary, both macroscopically and microscopically (Figs. 7.15 and 7.16), it appears that Slides 1 and 5 might represent secondary dumping events filling in the bin, with large fragments of 
charcoal, and more ash, lending them a darker, more heterogeneous appearance, while Slides 2-4 are very homogenous, with a compact groundmass and brown colour. The latter slides are probably fill material consisting of debris of the same material as the bin wall (mud-brick) dumped into the bin to fill it. If one can assume a similar original function to that of S25 based on contextual similarities, perhaps after use as a storage bin, this clay bin feature was used for refuse or simply filled with similar material to its construction to level it off with adjacent features. While these samples are quite homogenous compared to S25 and contamination during sampling may obscure their interpretation, there is no obvious sampling reason why, from a column sample of sediment, the topmost and bottommost slides (1 and 5 ) would resemble each other while the middle ones (2-4) would be homogenous. The most parsimonious explanation is that similarities in the macroscopic appearance of the sediment column from the bin with the thin section slides cut from it suggest a secondary nature for the fill within this bin, where Slides 1 and 5 represent slightly different types of fill (household refuse dumping or hearth cleaning) than Slides 2-4 (construction material).

\subsubsection{Micromorphological discussion}

The primary purpose of micromorphological examination of sediment samples from two clay bins (S25 from 4BIIX-94 and S36 from 3AII-1) was to explore the depositional processes that led to the formation of a whitish, fibrous deposit at the base of the 4BIIX-94 bin and compare formation processes between the two bin features that differed macroscopically during excavation. The 4BIIX-94 bin contains extremely high densities of inflorescence phytoliths from grasses, with smaller densities of leaves and stems (see phytolith section) that appear to represent the storage of foodstuffs. Although evidence of anatomically unconnected phytoliths is consistent with grinding of plants, the soil thin sections from S25 show that phytoliths in the bottom fill are elongate and overlapping/layered. Within this layer, there are both articulated and individual phytoliths.

This bin was likely used more than once, as suggested by the incorporation of clay aggregate debris, charcoal, small bone fragments (all indicative of very fine domestic debris) admixed into the contents with re-opening, use, and re-filling. The whitish, fibrous material at the very base of the bin is formed by fine gypsum crystals and crystal intergrowths precipitated in situ out of water/ moisture contained in the bins as it settled below a dense accumulation of plant matter (a possible lining) at the base of the bin. The S36 samples, on the other hand, contain phytoliths, but in much lower densities and instead the slides, containing larger fragments of typical household debris (charcoal, ash, limestone, bone, shell, and clay aggregates) represent a secondary fill dumped into the bin, presumably after its use for storage.

Beyond an assessment of the fill within these bins, a few words can be said regarding the construction, use, re-use and abandonment of these bin features. However, these comments are preliminary and based on a very restricted number of sediment samples. A full discussion of other aspects of these bins requires further sampling of adjacent features and other bins.

Examination of the bin wall slides from S25 and portions of bin wall from S36 suggest from its massive, dense clay-rich structure with voids (channels and vughs) characteristics of decayed plant matter, that each bin was constructed as one event. Although the samples are limited, there is no clear evidence for re-surfacing or maintenance of the bottom of the bin wall surfaces; however, one cannot rule out repair of the sides of the bins, especially portions which appear to have sat above floor surface.

The fill from the 4BIIX-94 bin suggests its primary use was for the storage of plant products (i.e., grains or chaff of wheat and barley). In addition, examination of the abrupt transition from the fill to bin wall, and the nature of the sediment here (layers of phytolith-rich sediment) indicates that the plant material that accumulated here (both inflorescence and to a lesser degree leaves and stems are present) may have been different from the stored contents. In any case, the material was sufficiently dense to trap moisture between it and the underlying bin wall/base causing the precipitation of gypsum crystals and some carbonate here. Indeed, this may have been the purpose of an accumulation of plant matter here to drive moisture out of the stored material and to 
the base of the bins to prevent rotting or infestation. The incorporation of clay aggregates and very fine domestic debris suggests possible re-opening and reuse of the bin.

It is also possible that re-use of the bins might have inadvertently concentrated layers of elongate plant matter at the base of the bin if it was not completely cleaned out when new material was added. Over time, this might result in an accumulation of this plant matter with a layered appearance that would serve the same purpose as an intentional bin lining, and leave the same post-depositional traces. Differentiating between these two activitiesintentional bin lining or accumulated plant matter as a by-product of re-use - is not possible at this stage in their analysis.

It is difficult to assess the reasons for abandonment of the bins, although for S25 the state in which it was found gives us clues as to its last use. The 4BIIX-94 bin was used for grain or chaff storage and appears to have been left partially undisturbed (at least its bottom contents), possibly with material still stored within. The 3AII-1 bin, on the other hand, was emptied of its original contents and filled in with general debris, including material similar to the construction material of the bins themselves. Thus, it seems that the 3AII-1 bin was intentionally filled in, perhaps to level off a surface for a later floor level.

\subsection{Summary}

This chapter presented analyses of phytoliths, faecal spherulites, and micromorphology of the clay bin features to provide geoarchaeological evidence for storage practices at Göytepe (See Kadowaki et al. 2015 for further discussion in combination with macrobotanical records). Clay bins are common architectural components at Göytepe and other early agricultural settlements affiliated with the Shomutepe culture in the southern Caucasus. The excavations at Göytepe during the 2008-2011 seasons revealed four clusters of clay bins in Levels 3, 4, 5, and 10 near residential buildings and apparently open-air activity areas. Among nearly forty clay bins recovered in these clusters, one of the bins in Level 10 (4BIIX94) was distinct from others because of 1) two complete upper grinding stones (4BIIX-94a and 94b) placed near the base of the bin and 2) the whitish, fibrous (extremely rich in phytoliths) sediments at the very base of the bin, approximately $4 \mathrm{~cm}$ in thickness, between the bin walls and the overlying grinding stones. In order to examine the depositional processes that led to the whitish, fibrous deposits of the 4BIIX-94 bin, sediment samples were taken from various parts inside and outside the bin for phytoliths, faecal spherulite, and micromorphology analyses. For comparison, sediments from two other clay bins (3AII-1 and 2) were also analyzed.

The results suggest that the whitish fibrous deposits at the bottom of the 4BIIX-94 bin mainly consist of grass phytoliths with relatively high proportions of inflorescences and low percentages of anatomically connected phytoliths in comparison with deposits from upper fill or outside the bin. These finds, combined with the association of the two grinding stones, suggest that the remains of grain processing activities, such as the grinding or dehusking of cereals, may have been deposited at the base of the 4BIIX-94 bin. These interpretations are generally in accord with the micromorphological observation that the bottom fill consists almost entirely of phytoliths with $5-10 \%$ density of very small charcoal fragments, fine amorphous organic matter, and even higher densities of fine carbonate and gypsum crystals. In addition, the micromorphological observation points to particularly elongate phytoliths oriented parallel to each other and to the sampled bin wall/base creating a layered, laminated appearance.

In contrast, the middle fill of the 4BIIX-94 bin and the middle and bottom fills of the two bins at 3AII-1 and 2 are likely to represent secondary influx including domestic refuse or intentional filling of the bins with clay materials similar to bin walls to level off the abandoned bins. Although these depositional processes are not indicative of primary uses of the bins, they are consistent with the fact that the clay bins are located near open space where household activities may have taken place. In fact, the excavation of the open space near the 4BIIX-94 bin recovered complete or nearly complete ground stones and bone tools that likely represent de facto refuse, as well as the concentration of charcoal fragments and ash. These macro-remains are consistent with microremains in the sediment samples outside the bins 
(S24 and S35). These samples include a large amount of phytoliths and faecal spherulites, indicating that either livestock dung remains or faecal material burnt as fuel.

These observations on primary and secondary contents and spatial contexts of the bins collectively indicate that storage space at Göytepe were not spatially segregated from but rather closely connected to domestic areas consisting of round residential buildings and a courtyard where daily activities, such as food processing and burning, took place. Such a spatial relationship between domestic and storage areas in turn suggests that food storage was mainly performed at the level of the group who resided in round buildings adjacent to a courtyard. Furthermore, the appearance of this settlement organization through different levels (at least, Levels 3, 4, 5, and 10) suggests that a management of storage continued to be a significant component of household activities since the early occupations at Göytepe.

Thus, the studies of lower levels or sites earlier than Göytepe should help clarify the question of when and how this storage practice developed at the transition from foraging to agricultural economy in the southern Caucasus. Regarding this question, it is notable that similar clay bins have been discovered at a Neolithic settlement of Hac Elamxanlı Tepe, which predates Göytepe, along with domesticated cereal remains (Nishiaki et al. 2015a and 2015b; Akashi et al. 2018). In contrast, no such features have been found at a final Mesolithic campsite of Damjili Cave (Nishiaki et al. 2019). These current records suggest a sudden development of storage practices at the transition from the Mesolithic to the Neolithic in the southern Caucasus.

\section{References}

Akashi, C., K. Tanno, F. Guliyev, and Y. Nishiaki (2018) Neolithisation processes of the southern Caucasus: As viewed from macro-botanical analyses at $\mathrm{Hacl}$ Elamxanlı Tepe, West Azerbaijan. Paléorient, 44(2): 75-89.

Albert, R. M. and M. Portillo (2005) Plant uses in different Bronze and Iron Age settlements from the Nuoro Province (Sardinia). The results of phytolith analyses from several ceramic fragments and grinding stones. Anejos de Complutum, 10: 109-
119.

Albert, R. M. and S. Weiner (2001) Study of phytoliths in prehistoric ash layers using a quantitative approach. In: Phytoliths, Applications in Earth Sciences and Human History, edited by J. D. Meunier and F. Colin, pp. 251-266. Lisse: A. A. Balkema Publishers.

Albert, R. M., O. Lavi, L. Estroff, S. Weiner, A. Tsatskin, A. Ronen, and S. Lev-Yadun (1999) Mode of occupation of Tabun Cave, Mt Carmel, Israel during the Mousterian period: A study of the sediments and phytoliths. Journal of Archaeological Science, 26: $1249-1260$.

Albert, R. M., R. Shahack-Gross, D. Cabanes, A. Gilboa, S. Lev-Yadun, M. Portillo, I. Sharon, E. Boaretto, and S. Weiner (2008) Phytolith-rich layers from the Late Bronze and Iron Ages at Tel Dor (Israel): Mode of formation and archaeological significance. Journal of Archaeological Science, 35: 57-75.

Albert, R. M., X. Esteve, M. Portillo, A. RodríguezCintas, D. Cabanes, I. Esteban, and F. Hernández (2011) Phytolith CoRe, Phytolith Reference Collection. Retrieved May 7, 2012. http://www. gepeg.org/enter_PCORE.html.

Badalyan, R., P. Lombard, P. Avetisyan, C. Chataigner, J. Chabot, E. Vila, R. Hovsepyan, G. Willcox, and H. Pessin (2007) New data on the late prehistory of the southern Caucasus. The excavations at Aratashen (Armenia): Preliminary report. In: Les Cultures du Caucase (Vle-Ille Millénaires avant notre ère): Leur Relations avec le Proche-Orient, edited by B. Lyonnet, pp. 37-61. Paris: Éditions Recherche sur les Civilisations.

Berna, F., A. Behar, R. Shahack-Gross, J. Berg, E. Boaretto, A. Gilboa, I. Sharon, S. Shalev, S. Shilstein, N. Yahalom-Mack, J. R. Zorn, and S. Weiner (2007) Sediments exposed to high temperatures: Reconstructing pyrotechnological processes in Late Bronze and Iron Age strata at Tel Dor (Israel). Journal of Archaeological Science, 34: 358-373.

Brewer, R. (1976) Fabric and Mineral Analysis of Soils (2nd ed.). Huntington: Krieger.

Brown, D. A. (1984) Prospects and limits of a phytolith key for grasses in the central United States. Journal of Archaeological Science, 11: 345-368.

Cabanes, D., S. Weiner, and R. Shahack-Gross (2011) Stability of phytoliths in the archaeological record: a dissolution study of modern and fossil phytoliths. Journal of Archaeological Science, 38: 2480-2490.

Canti, M. G. (1999) The production and preservation of faecal spherulites: Animals, environment and taphonomy. Journal of Archaeological Science, 26: 251-258.

Chataigner, C. (1995) La Transcaucasie au Néolithique et au Chalcolithique. BAR Internatinal Series 624. Oxford: Tempus Reparatum.

Coil, J., M. A. Korstanje, S. Archer, and C. A. Hastorf 
(2003) Laboratory goals and considerations for multiple microfossil extraction in archaeology. Journal of Archaeological Science, 30: 991-1008.

Courty, M. A., P. Goldberg, and R. Macphail (1989) Soils and Micromorphology in Archaeology. Cambridge: Cambridge University Press.

Finlayson, B., I. Kuijt, T. Arpin, M. Chesson, S. Dennins, N. Goodale, S. Kadowaki, L. Maher, S. Smith, M. Schurr, and J. Mckay (2003) Dhra' Excavation Project, 2002 interim report. Levant, 25: 1-38.

Fitzpatrick, E. A. (1993) Soil Microscopy and Micromorphology. Toronto: John Wiley and Sons.

Goldberg, P. (1980) Micromorphology in archaeology and prehistory. Paléorient, 6: 159-164.

Goldberg, P. (2000) Micromorphological aspects of site formation at Keatley Creek. In: The Ancient Past of Keatley Creek, Vol. 1: Taphonomy, edited by B. Hayden, pp. 81-94. Burnaby, BC: Archaeology Press, Simon Fraser University.

Goldberg, P., C. E. Miller, S. Schiegl, B. Ligouis, F. Berna, N. J. Conard, and L. Wadley (2009) Bedding, hearths, and site maintenance in the Middle Stone Age of Sibudu Cave, KwaZulu-Natal, South Africa. Archaeological and Anthropological Science, 1(2): 95-122.

Goodman-Elgar, M. (2008) The devolution of mudbrick: Ethnoarchaeology of abandoned earthen dwellings in the Bolivian Andes. Journal of Archaeological Science, 35: 3067-3071.

Guliyev, F. and Y. Nishiaki (2012) Excavations at the Neolithic settlement of Göytepe, the middle Kura Valley, Azerbaijan, 2008-2009. In: Proceedings of the 7th International Congress of the Archaeology of the Ancient Near East, Vol. 3, edited by R. Matthews and J. Curtis, pp. 71-84. Wiesbaden: Harrassowitz Verlag.

Kadowaki, S., L. Maher, M. Portillo, R. M. Albert, C. Akashi, F. Guliyev, and Y. Nishiaki (2015) Geoarchaeological and palaeobotanical evidence for prehistoric cereal storage in the southern Caucasus: The Neolithic settlement of Göytepe (mid 8th millennium BP). Journal of Archaeological Science, 53: 408-425.

Kuijt, I. (2008) Demography and storage systems during the southern Levantine Neolithic demographic transition. In: The Neolithic Demographic Transition and Its Consequences, edited by J. -P. BocquetAppel and O. Bar Yosef, pp. 287-313. New York: Springer.

Kuijt, I. and B. Finlayson (2009) Evidence for food storage and predomestication granaries 11,000 years ago in the Jordan Valley. PNAS, 106: 1096610970.

Love, S. (2012) The geoarchaeology of mudbricks in architecture: A methodological study from Çatalhöyük, Turkey. Geoarchaeology, 27: 140-156.
Madella, M., A. Alexandre, T. B. Ball, and ICPN Working Group (2005) International code for phytolith nomenclature 1.0. Annals of Botany, 96: 253-260.

Matthews, W. (2012a) Defining households: Microcontextual analysis of early Neolithic households in the Zagros, Iran. In: New Perspectives on Household Archaeology, edited by B. Parker and C. Foster, pp. 183-218. Winona Lake: Eisenbrauns.

Matthews, W. (2012b) Household life-histories and boundaries. In: Last House on the Hill: $B A C H$ Area Reports from Çatalhöyük, Turkey, edited by R. Tringham and M. Stevanovic, pp. 205-222. Los Angeles and Ankara: Cotsen Institute of Archaeology Press and British Institute at Ankara.

Matthews, W., C. A. I. French, T. Lawrence, D. F. Cutler, and M. K. Jones (1997) Microstratigraphic traces of site formation processes and human activities. World Archaeology, 29: 281-308.

Mulholland, S. C. and G. Rapp Jr. (1992) A morphological clasification of grass silica-bodies. In: Phytolith Systematics: Emerging Issues, Advances in Archaeological and Museum Science, edited by G. Rapp Jr. and S. C. Mulholland, pp. 65-89. New York: Plenum Press.

Narimanov, I. G. (1992) The earliest agricultural settlements in the territory of Azerbaidzhan. In: Recent Discoveries in Transcaucasia, edited by P. L. Kohl, pp. 9-66. Soviet Anthropology and Archeology 30(4). M. E. Sharpe, N.Y. (English translation of Narimanov 1987: pp. 14-69).

Nishiaki, Y., F. Guliyev and S. Kadowaki (2015a) Chronological contexts of the earliest Pottery Neolithic in the southern Caucasus: Radiocarbon dates for Göytepe and $\mathrm{Hacl}$ Elamxanlı Tepe, Azerbaijan. American Journal of Archaeology, 119(3): 279-294.

Nishiaki, Y., F. Guliyev, S. Kadowaki, V. Alakbarov, T. Miki, S. Salimbayov, C. Akashi, and S. Arai (2015b) Investigating cultural and socioeconomic change at the beginning of the Pottery Neolithic in the southern Caucasus: The 2013 excavations at $\mathrm{Hacl}$ Elamxanlı Tepe, Azerbaijan. Bulletin of the American Schools of Oriental Research, 374: 1-28.

Nishiaki, Y., A. Zeynalov, M. Mansrov, C. Akashi, S. Arai, K. Shimogama and F. Guliyev (2019) The MesolithicNeolithic interface in the Southern Caucasus: 20162017 excavations at Damjili Cave, West Azerbaijan. Archaeological Research in Asia, 19: 100140.

Piperno, D.R. (2006) Phytoliths: A Comprehensive Guide for Archaeologists and Paleoecologists. Lanham: AltaMira Press.

Portillo, M. (2006) La Mòlta i Triturat d'Aliments Vegetals durant la Protohistòria a la Catalunya Oriental. Ph.D. Thesis. University of Barcelona.

Portillo, M., Albert R. M., and D. O. Henry (2009) Domestic activities and spatial distribution in Ain 
Abu Nukhayla (Wadi Rum, Southern Jordan): The use of phytoliths and spherulites studies. Quaternary International, 193: 174-183.

Portillo, M., Kadowaki, S., Nishiaki, Y., and Albert, R.M. (2014) Early Neolithic household behavior at Tell Seker al-Aheimar (Upper Khabur, Syria): A comparison to ethnoarchaeological study of phytoliths and dung spherulites. Journal of Archaeological Science, 42: 107-118.

Portillo, M., S. Valenzuela, and R. M. Albert (2012) Domestic patterns in the Numidian site of Althiburos (northern Tunisia): The results from a combined study of animal bones, dung and plant remains. Quaternary International, 275: 84-96.

Rosen, A. M. (1986) Cities of Clay: The Geoarchaeology of Tells. Chicago: University of Chicago Press.

Rosen, A. M. (1992) Preliminary identification of silica skeletons from Near Eastern archaeological sites: An anatomical approach. In: Phytolith Systematics: Emerging Issues, Advances in Archaeological and Museum Science, edited by G. Rapp Jr. and S. C. Mulholland, pp. 129-147. New York: Plenum Press.

Shillito, M., W. Matthews, M. Almond, and I. Bull (2011) The microstratigraphy of middens: Capturing daily routine in rubbish at Neolithic Catalhoyuk, Turkey. Antiquity, 85: 1024-1038.

Stoops, G., V. Marcelino, and F. Mees (eds.) (2010) Interpretation of Micromorphological Features of Soils and Regoliths. Amsterdam: Elsevier.

Tsartsidou, G., S. Lev-Yadun, R. M. Albert, A. MillerRosen, N. Efstratiou, and S. Weiner (2007) The phytolith archaeological record: Strengths and weaknesses based on a quantitative modern reference collection from Greece. Journal of Archaeological Science, 34: 1262-1275.

Twiss, P. C. (1992) Predicted world distribution of C3 and C4 grass phytoliths. In: Phytolith Systematics: Emerging Issues, Advances in Archaeological and Museum Science, edited by G. Rapp Jr. and S. C. Mulholland, pp. 113-128. New York: Plenum Press.

Twiss, P. C., E. Suess, and R. M. Smith (1969) Morphological classification of grass phytoliths. Soil Science Society of America Proceedings, 33: 109-115.

Wadley, L., C. Sievers, M. Bamford, P. Goldberg, F. Berna, and C. Miller (2012) Middle Stone Age bedding construction and settlement patterns at Sibudu, South Africa. Science, 334: 1388-1391. 


\title{
Archaeological reconnaissance survey around Göytepe, Tovuz-Qovlar region
}

\author{
Kazuya Shimogama and Valeh Alakbarov
}

\subsection{Introduction}

Archaeological surveys around Göytepe were undertaken during the 2011, 2012, and 2013 summer excavation seasons. The first preliminary results of the 2011 field season are presented in this chapter.

Prior to our investigations, Ideal Narimanov conducted general reconnaissance surveys in this region in the 1960s and 1970s (Narimanov 1987). He reported many prehistoric tepe (mound) sites spread across all regions of Azerbaijan and provided us with detailed information on the Neolithic and Chalcolithic periods (referred to as "Aeneolithic" by Narimanov). It is of particular note that he sufficiently described several mound sites in the Tovuz region, along with the excavation results at important sites like Shomutepe and Toiretepe.

After Narimanov's work, the northwestern region of Azerbaijan extending from Agstafa to Shamkir and further south to the Gedebey region was extensively surveyed by Bertille Lyonnet and Farhad Guliyev from 2006 to 2007. Although their research emphasized prehistoric mining activities, they identified a number of hitherto unknown archaeological sites distributed in western Azerbaijan (Lyonnet and Guliyev 2009).

On the basis of their work, our survey projects were planned for more restricted areas in the vicinity of Göytepe. While we proceeded to excavate the Neolithic settlement of Göytepe, our goal was to find contemporaneous or earlier settlements in order to understand the evolution of the Shomutepe cultural entity and later developments in the region. The surveyed area encompasses approximately $200 \mathrm{~km}^{2}$ within a radius of ca. $15 \mathrm{~km}$ from Göytepe (Fig. 8.1).
The site is situated near the modern town of Qovlar, Tovuz district, an area bordered by the Zayam River to the east and the Tovuz River to the west. Located between the two deeply cut rivers, the target area is more or less a flat plain formed by wide alluvial fans. A small tributary called Esrik River flows west of Qovlar to the north before joining the Tovuz River. As shown in the Fig. 8.1 map, south and north are demarcated by the northern flanks of the Lesser Caucasus mountains and the Shamkir reservoir dam of the Middle Kura River.

\subsection{Research objectives}

The research objectives of the Tovuz-Qovlar survey are twofold. First, field surveys attempted to find any unidentified Neolithic sites that may predate the occupations of Göytepe so that the historical backgrounds of Göytepe occupations are properly understood. As Narimanov (1987) already reported, there are several Neolithic and Chalcolithic settlements such as Huseingulu Tepesi and Cheltiktepe, but no aceramic site has ever been identified. Detection of such a new site might be valuable for understanding the Neolithisation process in the region. Another objective is to register as many archaeological settlements as possible in the Tovuz district, especially tepe sites. Based on these collected data, we can reconstruct the settlement and land use patterns over time, from the Neolithic to subsequent periods. To understand the microregional occupational history and relationships between human and landscape developments, we initiated our survey in the immediate neighboring area of Göytepe. 


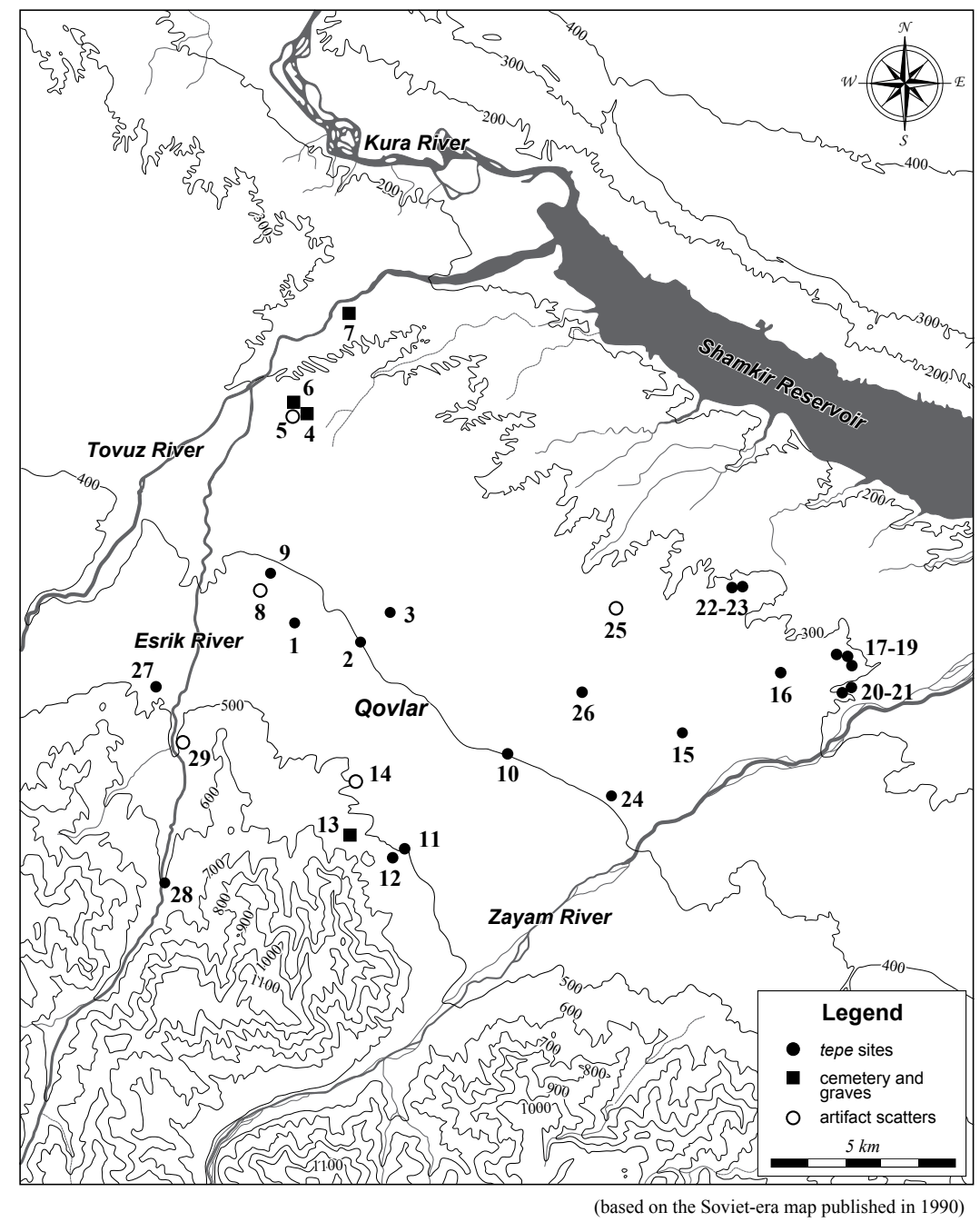

Fig. 8.1 Distribution of archaeological sites in the surveyed area.

\subsection{Survey methodology}

In addition to the site information from Narimanov's studies, we applied remote sensing techniques before the fieldwork. In this case, Google Earth images are extremely useful for simple field surveys like ours. Any possible artificial mounds are pinpointed in advance based on satellite images in Google Earth (accessed in early July 2011). With the help of high resolution images, every field site was located and visited by motor vehicle. However, this method has some limitations with respect to detecting archaeological sites. First and foremost, since sites without a visible mound can hardly be identified, we focused primarily on elevated mounds such as tepe sites that represent any substantial dimensions in size and/or height. It may mean that we overlooked low or small mound sites as well as buried cemeteries and other past activity areas. Identifying some of those less prominent sites could be complemented by careful surveys, so some parts of the areas in question were subject to pedestrian field survey without establishing transects (for instance, around the lower Tovuz river). Only a few sites were found on highly exploited or cultivated lands. It is likely that a fair number of ancient sites must have been destroyed in the course of both modern agricultural activities and Holocene geophysical changes. Other limitations in site detectability are due to the area's landforms. The surveyed area comprises not only flat plains, piedmont zones, and intermontane valleys, but also high mountains greater than $600 \mathrm{~m}$ a.s.l. to the south, an arid steppe area heavily dissected by numerous gorges. As such, some parts of the region were too difficult to be accessed by vehicle or were otherwise inaccessible.

When an archaeological site was recognized, a range of geographical information (e.g., exact locations in latitude/longitude, altitude, and the extent 
of a dense artifact-scattered area) was documented using a mobile GPS device (GPSMAP60CSx, Garmin Ltd.) and a range finder (TruePulse 200, Laser Technology Inc.). Surface finds were randomly collected by several surveyors. Specifically diagnostic sherds that could potentially be dated were collected. The discovered sites were then dated by the artifacts, primarily based on ceramic sherds.

Nevertheless, it should be noted that the reconnaissance survey reported here is neither completely systematic nor comprehensive at this preliminary stage of research.

\subsection{Results of the Tovuz-Qovlar survey}

During a total of three days of field survey in the 2011 season, 29 archaeological sites were documented within the Tovuz-Qovlar survey area (Fig. 8.1; Table 8.1). Twenty-one of them are tepe sites with more or less elevated mounds. We also registered constructed features identified as cemeteries or in some cases kurgans (ancient tumuli) at four locales as well as sparse artifact distributions. The periods confirmed by associated surface finds (mainly pottery sherds) range from the Neolithic to the Late Medieval periods (Figs. 8.2 and 8.3). However, chronological dating of the pottery collections is not yet fully complete. This is partly because well-dated excavated materials are not available in most of the periods, making exact dating of collected sherds difficult. Thus, we use a rather rough chronological frame with a broad time range here.

\subsubsection{Neolithic period}

At least four sites including Göytepe (TQ001) are currently assigned to the Neolithic period (Fig. 8.2: a).

Huseingulu Tepesi (TQ003) and Cheltiktepe (TQ010) are possible Neolithic settlement sites, both of which were first discovered by Narimanov. The former Huseingulu Tepesi is a small site of 0.5 ha, located ca. $2.5 \mathrm{~km}$ east of Göytepe. At this site, Narimanov reported collecting stone implements including obsidian sickle blades, burins, scrapers, and blades and chaff-tempered coarse burnished pottery or sand-tempered sherds, thus designating the site as "completely Aeneolithic" (Narimanov 1987:
31, fig. 33: 1-4). At Huseingulu our survey collected a chaff-tempered sherd of a large bowl with a knoblike ledge and a burnished base fragment, which show some affinity in ware type with the Göytepe assemblage (Fig. 8.4: 5 and 6). Most of the stone implements collected on site are chipped obsidian artifacts, including burins on blades, retouched blades, and a single splintered piece (Fig. 8.5: 5-7). We also collected a few flakes of andesite and green tuff. The latter rock type was also found as a core for flakes (Fig. 8.5: 7). In addition, a piece of core pounder was also recovered.

Cheltiktepe (TQ010) was also reported by Narimanov. This mound site was assigned to the "Aeneolithic," yielding two distinct pottery wares and sickle blades of both flint and obsidian at the time of his visit (Narimanov 1987: 31). Similar to TQ024, this site (ca. $0.58 \mathrm{ha}$ ) is located beside the modern railway and visible from the highway. At the time of our fieldwork, thick vegetation covered the mound entirely, which made it difficult to find artifacts on the surface. Three ceramic sherds, not illustrated here due to extremely fragmented pieces, show a rather homogeneous surface color of light gray or browngray. They contain small amounts of vegetal temper and fine sand, with slight polishing. We also found a few examples of chipped stone artifacts, including a bladelet, splintered piece of obsidian, and an andesite flake. Although these finds are broadly assignable to the Neolithic, it remains difficult to determine the precise date of occupations at this site.

One of the most interesting sites discovered in 2011 is a small mound locally known as Hac1 Elamxanlı (TQ009), which was not mentioned in Narimanov's report. Situated ca. $1.1 \mathrm{~km}$ northwest of Göytepe, Hacı Elamxanlı is a low oval mound measuring $60 \times 80 \mathrm{~m}$ (ca. $0.4 \mathrm{ha}$ ) in plan and 1.5 $\mathrm{m}$ in height (Fig. 8.6). The mound is surrounded by a modern field and covered with a large quantity of metal wire and recent vinicultural refuse. However, large-scale destruction of the original mound cannot be observed except animal burrows or artificial pits for soil collection on the edge. It thus seems that the cultural deposits may not be significantly disturbed. A handful of artifacts were collected in the surrounding field beyond the mound, but it is premature to suggest whether or not the original occupied area extended 


\begin{tabular}{|c|c|c|c|c|c|c|c|c|c|c|c|c|c|c|c|c|}
\hline 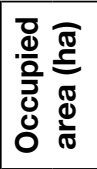 & $\stackrel{\leftrightarrow}{\leftrightarrow}$ & : & $\stackrel{\infty}{\stackrel{\infty}{0}}$ & . & ' & I & I & 1 & 文 & 占 & $\stackrel{m}{r}$ & 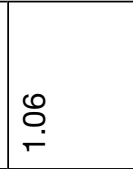 & . & I & ' & $\begin{array}{c}\infty \\
\stackrel{\infty}{0} \\
0\end{array}$ \\
\hline 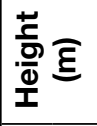 & $\infty$ & $\stackrel{\sim}{r}$ & $\begin{array}{l}L \\
\oplus\end{array}$ & 1 & $\stackrel{i}{\rho}$ & $\stackrel{\infty}{+}$ & , & ' & $\stackrel{2}{\longrightarrow}$ & \begin{tabular}{|l}
10 \\
10 \\
\end{tabular} & $\begin{array}{l}\stackrel{\ln }{+} \\
+\end{array}$ & 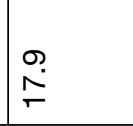 & $\begin{array}{l}m \\
\dot{\sim}\end{array}$ & $\begin{array}{l}10 \\
\infty \\
\infty\end{array}$ & , & $\begin{array}{l}10 \\
10 \\
\end{array}$ \\
\hline 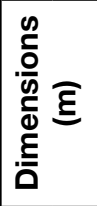 & $\underset{L}{\stackrel{L}{f}}$ & $\approx$ & $\begin{array}{l}\text { Nִ } \\
\stackrel{0}{0}\end{array}$ & $\begin{array}{l}\infty \\
\stackrel{N}{N} \\
\text { Non }\end{array}$ & $\begin{array}{l}\infty \\
\stackrel{\sim}{\infty} \\
\end{array}$ & $\begin{array}{l}\infty \\
\stackrel{i}{ } \\
\text {. }\end{array}$ & , & 1 & $\begin{array}{l}8 \\
\varnothing \\
\times \\
\varnothing \\
\infty\end{array}$ & $\begin{array}{l}\forall \\
\infty \\
\infty\end{array}$ & 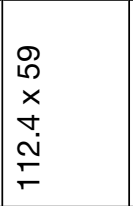 & 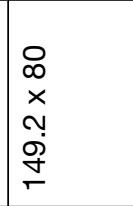 & 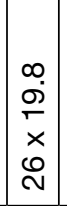 & $\begin{array}{l} \\
m \\
\dot{q} \\
\dot{q}\end{array}$ & , & $\bar{\infty}^{-}$ \\
\hline 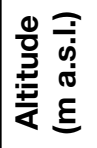 & $\begin{array}{l}\hat{N} \\
\stackrel{N}{N} \\
\stackrel{y}{*}\end{array}$ & 㐫 & న్ల & \& & $\begin{array}{l}\infty \\
\stackrel{0}{\infty}\end{array}$ & $\begin{array}{l}0 \\
0 \\
0\end{array}$ & 1 & $\frac{\sigma}{\gamma}$ & $\frac{10}{\gamma}$ & 承 & 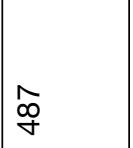 & 孪 & 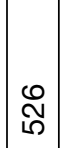 & $\stackrel{\mathscr{P}}{\stackrel{\sim}{\sim}}$ & 只 & ల్ల \\
\hline$=$ & $\begin{array}{l}\infty \\
\stackrel{\infty}{\sim} \\
\Gamma\end{array}$ & \begin{tabular}{|l}
$\mid \infty$ \\
$\stackrel{\infty}{+}$ \\
f
\end{tabular} & $\begin{array}{l}\underset{N}{N} \\
\infty \\
\sim\end{array}$ & 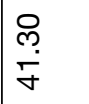 & 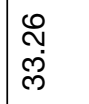 & 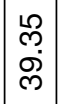 & & $\begin{array}{l}\bar{n} \\
\dot{\omega} \\
i\end{array}$ & $\begin{array}{l}50 \\
\varnothing 0 \\
\varnothing 0\end{array}$ & 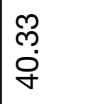 & 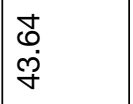 & $\begin{array}{l}\bar{\delta} \\
\ddot{~}\end{array}$ & $\underset{\dot{m}}{\ddot{m}}$ & 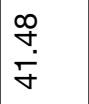 & $\begin{array}{l}\infty \\
\infty \\
\infty \\
0 \\
0\end{array}$ & $\frac{N}{\overline{5}}$ \\
\hline ש & テ & $\stackrel{\mathscr{P}}{\stackrel{9}{*}}$ & $\underset{\forall}{\forall}$ & テ & テ & ฯ & \& & ₹ & ๆ & $\stackrel{\circ}{q}$ & 尹 & \& & ஜ & भ̊ & g & 5 \\
\hline$\circ$ & $\stackrel{8}{\&}$ & L & $\stackrel{\text { L }}{q}$ & L & L & L & \& & L & $\stackrel{2}{f}$ & L & L & L & 过 & L & L & \& \\
\hline \multirow{3}{*}{$\mathbf{z}$} & 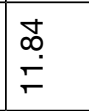 & \begin{tabular}{|l|}
8 \\
$\circ$ \\
$\circ$ \\
\end{tabular} & 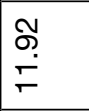 & $\begin{array}{l}m \\
\dot{0} \\
\end{array}$ & \begin{tabular}{|l|} 
\\
\\
0 \\
\multirow{2}{*}{}
\end{tabular} & $\begin{array}{l}\bar{\sim} \\
\infty \\
\sim \\
\end{array}$ & & \begin{tabular}{|l|}
$\infty$ \\
$\infty$ \\
$\infty$ \\
$\stackrel{\infty}{ }$ \\
\end{tabular} & $\begin{array}{l} \\
\dot{0} \\
\dot{\sigma}\end{array}$ & \begin{tabular}{l}
\multirow{0}{*}{} \\
$\stackrel{8}{\circ}$ \\
\end{tabular} & $\begin{array}{l}\overline{0} \\
\dot{g}\end{array}$ & 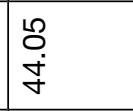 & 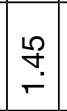 & 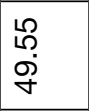 & 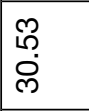 & $\frac{m}{\grave{N}}$ \\
\hline & $\begin{array}{l}\infty \\
i \\
\end{array}$ & in & $\stackrel{\infty}{\infty}$ & 5 & 5 & 5 & $\widetilde{0}$ & $\stackrel{\infty}{\infty}$ & $\stackrel{\infty}{\infty}$ & $\begin{array}{l}0 \\
\end{array}$ & L & 它 & $\begin{array}{ll}10 \\
10\end{array}$ & in & $\begin{array}{l}0 \\
\end{array}$ & $\hat{\omega}$ \\
\hline & 우 & 우 & 우 & テ & $\bar{\nabla}$ & 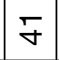 & $\bar{\gamma}$ & 웅 & 우 & 우 & 우 & 우 & q & ㅇ & 우 & 우 \\
\hline 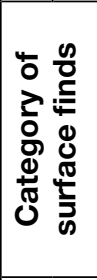 & 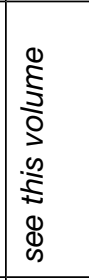 & 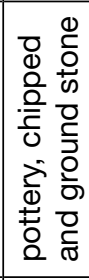 & 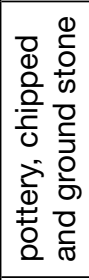 & 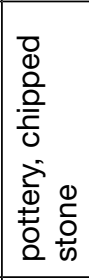 & 更 & 1 & 1 & $\begin{array}{l}\frac{Z}{0} \\
\stackrel{0}{*} \\
\stackrel{0}{0} \\
\end{array}$ & 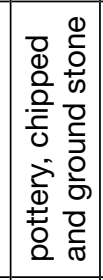 & 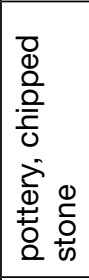 & 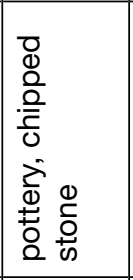 & 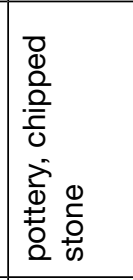 & 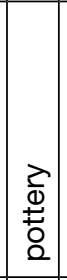 & 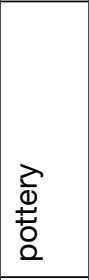 & 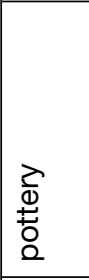 & 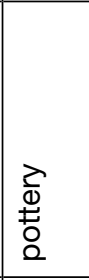 \\
\hline 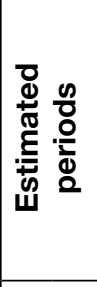 & 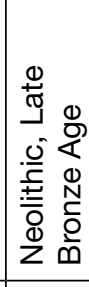 & 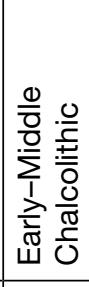 & 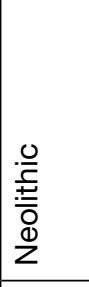 & $\begin{array}{l}0 \\
\frac{0}{3} \\
\frac{0}{\underline{T}} \\
\frac{\pi}{<}\end{array}$ & 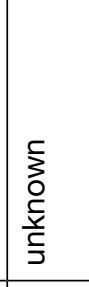 & \begin{tabular}{|l|} 
\\
$\vdots$ \\
0 \\
$\frac{5}{5}$ \\
J \\
\\
\end{tabular} & $\left|\begin{array}{l}\frac{5}{3} \\
0 \\
\frac{5}{5} \\
\frac{5}{J}\end{array}\right|$ & 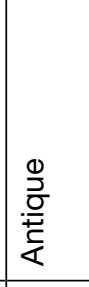 & 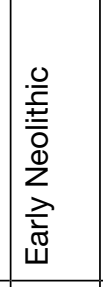 & 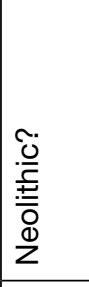 & 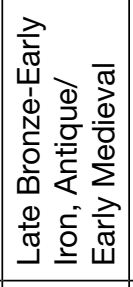 & 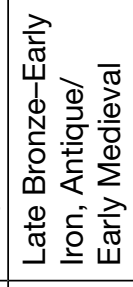 & 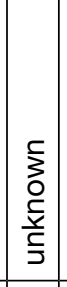 & 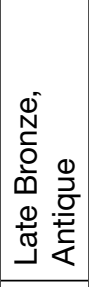 & 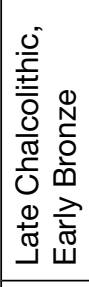 & 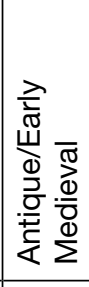 \\
\hline 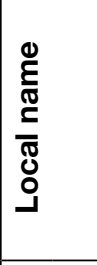 & 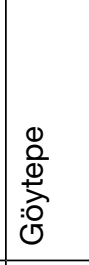 & 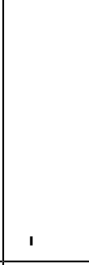 & 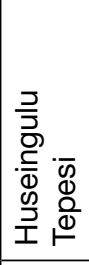 & . & 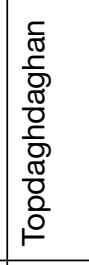 & \begin{tabular}{|l|}
$\frac{1}{\pi}$ \\
$\frac{0}{0}$ \\
$\frac{\pi}{0}$ \\
$\frac{0}{0}$ \\
$\frac{5}{0}$ \\
$\frac{\pi}{0}$ \\
0 \\
$\circ$ \\
0 \\
\end{tabular} & , & . & 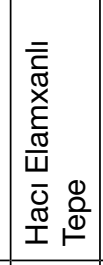 & 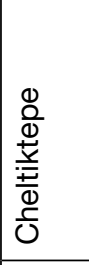 & 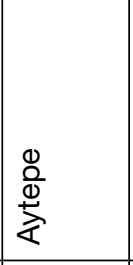 & 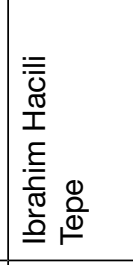 & 1 & & 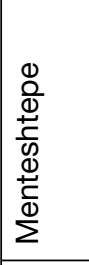 & 1 \\
\hline 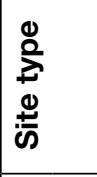 & $\frac{\mathbb{0}}{\varrho}$ & $\stackrel{\otimes}{\otimes}$ & $\stackrel{\otimes}{\infty}$ & 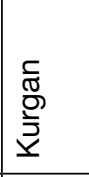 & 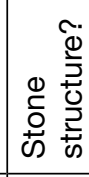 & 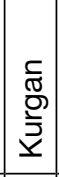 & 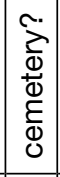 & 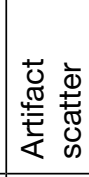 & 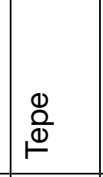 & $\stackrel{\otimes}{\otimes}$ & $\frac{\otimes}{0}$ & $\stackrel{\mathbb{Q}}{\mathbb{0}}$ & 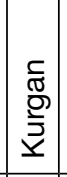 & 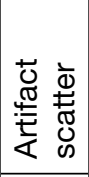 & $\stackrel{\otimes}{\stackrel{0}{\omega}}$ & $\stackrel{\otimes}{\stackrel{0}{\Theta}}$ \\
\hline 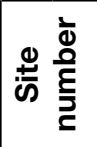 & - & N & $\infty$ & $\nabla$ & in & 0 & $\sim$ & $\infty$ & 0 & 우 & $\mp$ & $\stackrel{\Upsilon}{ }$ & 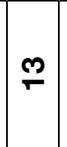 & $\stackrel{\nabla}{ }$ & 10 & $\stackrel{\odot}{\circ}$ \\
\hline
\end{tabular}




\begin{tabular}{|c|c|c|c|c|c|c|c|c|c|c|c|c|c|}
\hline 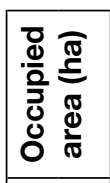 & $\begin{array}{l} \\
0 \\
2 \\
0 \\
0\end{array}$ & $\begin{array}{l}- \\
0 \\
0\end{array}$ & $\begin{array}{l}\ell \\
0 \\
0\end{array}$ & 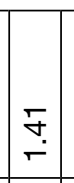 & 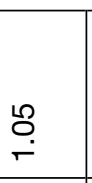 & $\bar{\sigma}_{0}$ & $\begin{array}{l}0 \\
0 \\
0\end{array}$ & $\begin{array}{l}\widehat{\infty} \\
\infty \\
0 \\
0\end{array}$ & , & 怘 & 苫 & $\stackrel{\text { fo }}{\stackrel{r}{r}}$ & \\
\hline 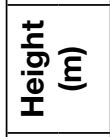 & $\begin{array}{l}0 \\
0 \\
0\end{array}$ & 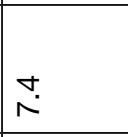 & 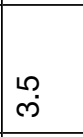 & $\hat{\infty}$ & 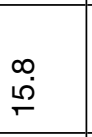 & $\begin{array}{l}\omega_{0} \\
\stackrel{\alpha}{*}\end{array}$ & $\begin{array}{l}1 \\
0 \\
0\end{array}$ & 0 & م & $\infty$ & 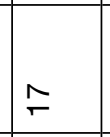 & $\wedge$ & 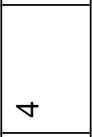 \\
\hline 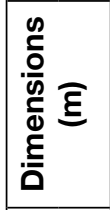 & $\bar{\sigma}$ & $\stackrel{م}{\rightleftharpoons}$ & $\hat{\imath}$ & $\begin{array}{l}\stackrel{o}{\mathrm{i}} \\
\stackrel{\mathrm{O}}{\sim}\end{array}$ & $\stackrel{\infty}{\stackrel{\infty}{\sim}}$ & $\begin{array}{c}0 \\
\dot{j} \\
\dot{1}\end{array}$ & \ & $\infty$ & 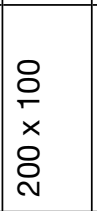 & 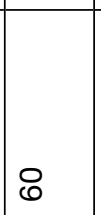 & 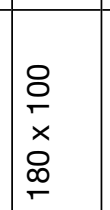 & $\stackrel{\stackrel{p}{r}}{r}$ & \begin{tabular}{|l}
$\dot{0}$ \\
$\times$ \\
0 \\
0 \\
\end{tabular} \\
\hline 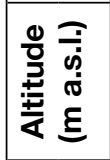 & $\frac{10}{m}$ & $\stackrel{\vec{m}}{\mathrm{~m}}$ & 悉 & $\frac{0}{m}$ & ले & $\frac{0}{m}$ & $\frac{N}{m}$ & 움 & 黛 & : & 음 & 安 & 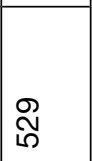 \\
\hline$=$ & 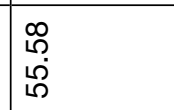 & 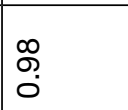 & 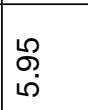 & \begin{tabular}{|l}
$\stackrel{0}{0}$ \\
$\stackrel{\sim}{\sim}$
\end{tabular} & $\stackrel{\stackrel{\infty}{\infty}}{\sim}$ & 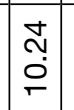 & $\begin{array}{c}\bar{f} \\
i\end{array}$ & 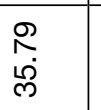 & $\begin{array}{l}0 \\
0 \\
0 \\
\infty\end{array}$ & $\stackrel{m}{i}$ & $\frac{m}{i}$ & \begin{tabular}{|l|}
$g$ \\
0 \\
0 \\
0
\end{tabular} & 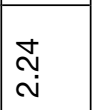 \\
\hline ш- & ஸิ & 吕 & 足 & $\begin{array}{l}0 \\
0 \\
0\end{array}$ & 艿 & in & in & $\stackrel{\infty}{+}$ & 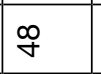 & $\stackrel{\infty}{+\infty}$ & q & g & q \\
\hline$\circ$ & 过 & $\stackrel{\text { \& }}{q}$ & \& & \begin{tabular}{|l|}
$g$ \\
\end{tabular} & \& & \begin{tabular}{|l|}
$g$ \\
\end{tabular} & 守 & \& & L & \& & L & \& & 多 \\
\hline$=$ & \begin{tabular}{|l|} 
\\
$\infty$ \\
$\infty$ \\
$\infty$ \\
$\infty$
\end{tabular} & $\begin{array}{l}\tilde{m} \\
\dot{\tilde{m}} \\
\end{array}$ & 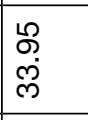 & \begin{tabular}{|l|l|} 
& \\
$\infty$ \\
$\infty$ \\
\end{tabular} & $\stackrel{N}{N}$ & \begin{tabular}{|l|}
9 \\
$\dot{0}$ \\
$\dot{\omega}$
\end{tabular} & \begin{tabular}{|c|}
$\bar{m}$ \\
$\omega$ \\
$\omega$
\end{tabular} & $\begin{array}{l}\infty \\
\stackrel{\infty}{\infty} \\
\infty \\
\infty \\
\infty\end{array}$ & \begin{tabular}{|l|l|}
\multirow{2}{*}{} \\
$\stackrel{\Gamma}{\circ}$ \\
\end{tabular} & 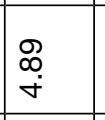 & 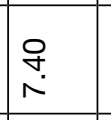 & \begin{tabular}{|l|}
$\infty$ \\
$\stackrel{\leftrightarrow}{N}$ \\
$\dot{\dot{N}}$ \\
\end{tabular} & \begin{tabular}{|l|}
\multirow{N}{N}{} \\
\\
\\
\end{tabular} \\
\hline $\mathbf{z}$ & in & in & in & in & in & 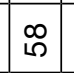 & \begin{tabular}{|c|} 
\\
0 \\
0
\end{tabular} & 足 & $\overbrace{i \infty}^{\infty}$ & in & in & in & i \\
\hline$\circ$ & fo & o & g & g & g & g & $q$ & g & q & q & q & q & q \\
\hline 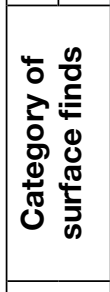 & 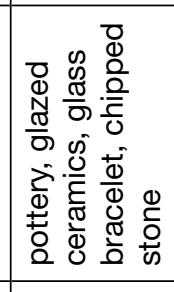 & 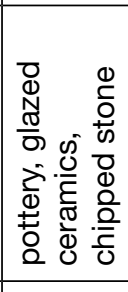 & 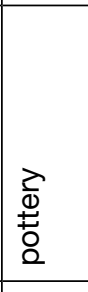 & 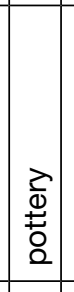 & 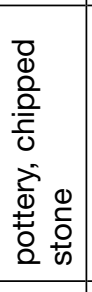 & 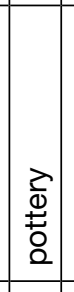 & 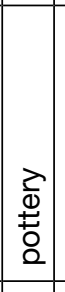 & 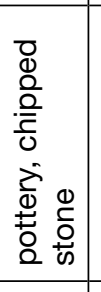 & 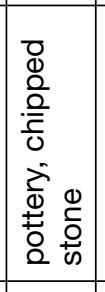 & 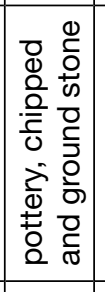 & 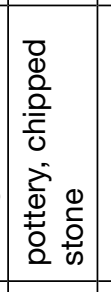 & 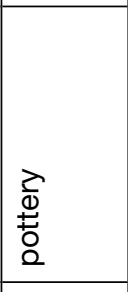 & 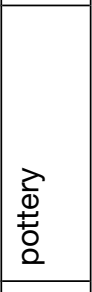 \\
\hline 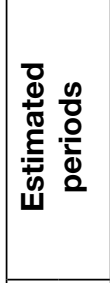 & 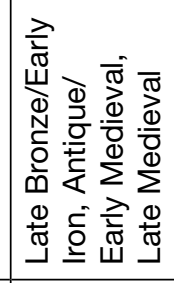 & 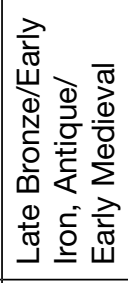 & 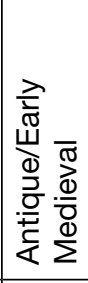 & 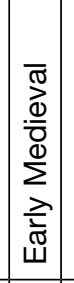 & 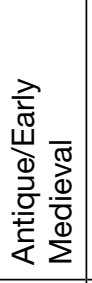 & 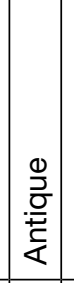 & 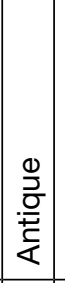 & 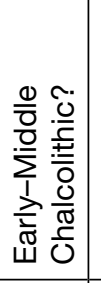 & 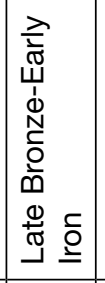 & 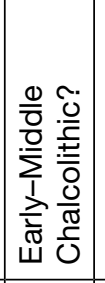 & 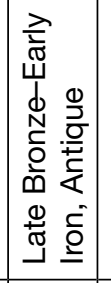 & 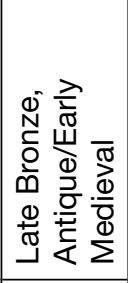 & 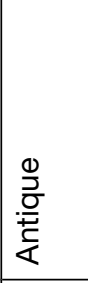 \\
\hline لَّ & 1 & 1 & 5 &. & . &. &. & 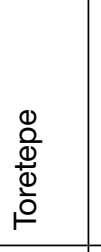 & & 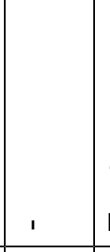 & 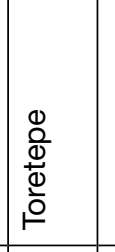 & & \\
\hline 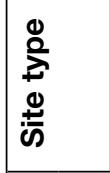 & $\frac{\Phi}{\sigma}$ & $\begin{array}{l}\stackrel{\otimes}{\sigma} \\
\stackrel{\alpha}{\Theta}\end{array}$ & 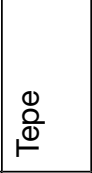 & \begin{tabular}{|l|}
$\mathbf{0}$ \\
$\stackrel{0}{\varrho}$ \\
\end{tabular} & $\stackrel{0}{\stackrel{o}{\varrho}}$ & 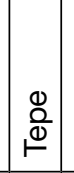 & 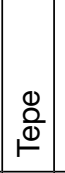 & 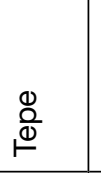 & 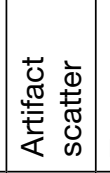 & 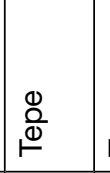 & 递 & 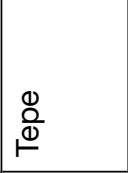 & 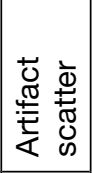 \\
\hline 离 & 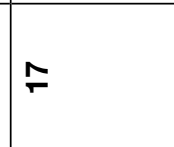 & $\stackrel{\infty}{-}$ & $\stackrel{P}{P}$ & 尺े & $\bar{\Lambda}$ & สิ & $\mathscr{N}$ & A & ผ & 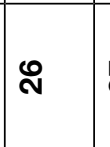 & $\hat{N}$ & $\stackrel{\infty}{\infty}$ & న \\
\hline
\end{tabular}




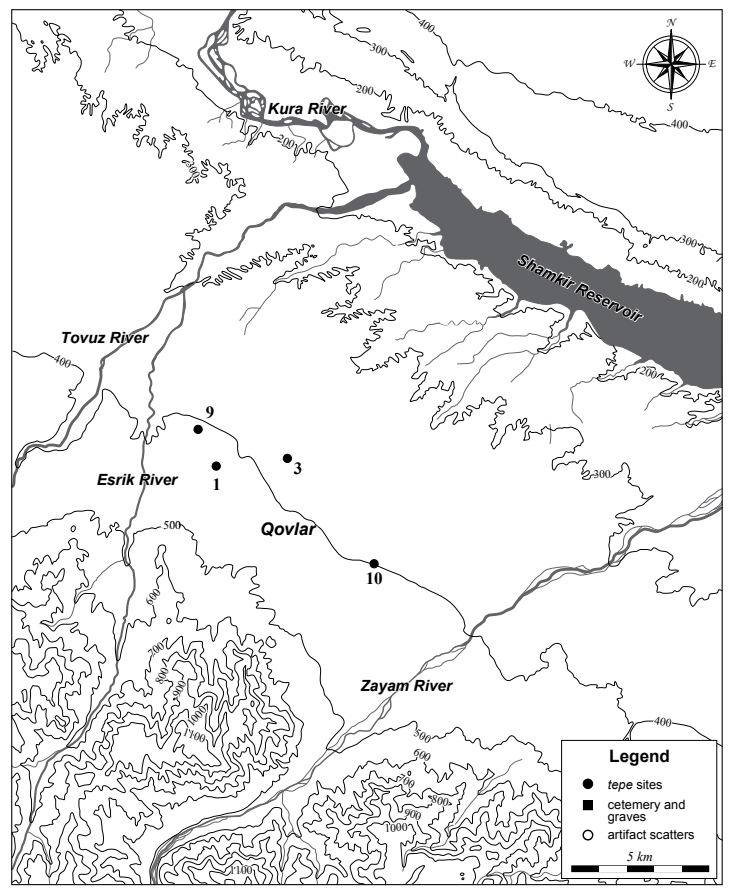

a. Neolithic

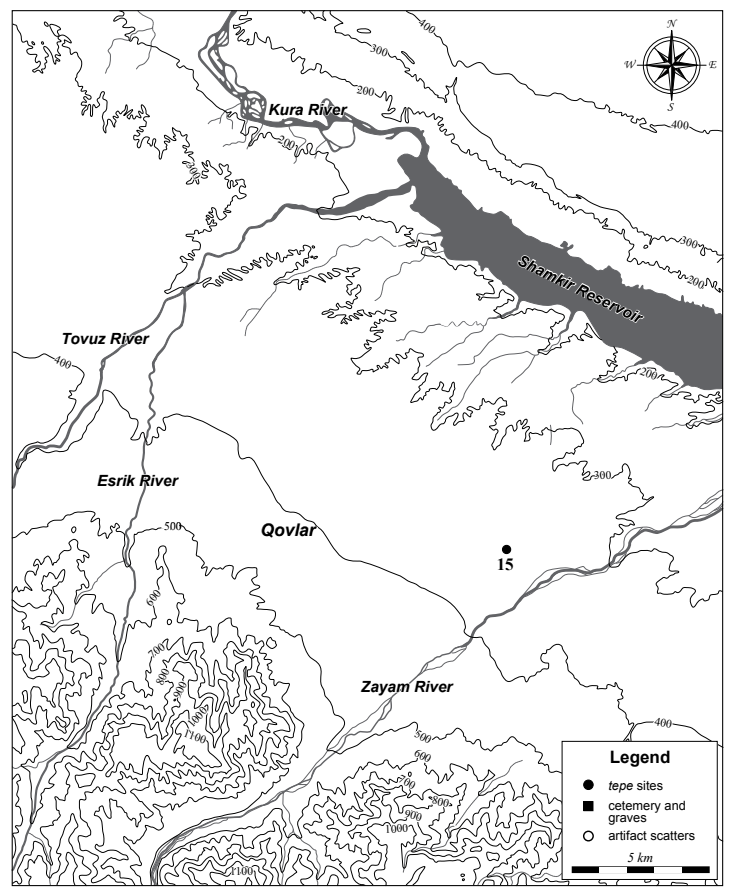

c. Late Chalcolithic-Early Bronze Age

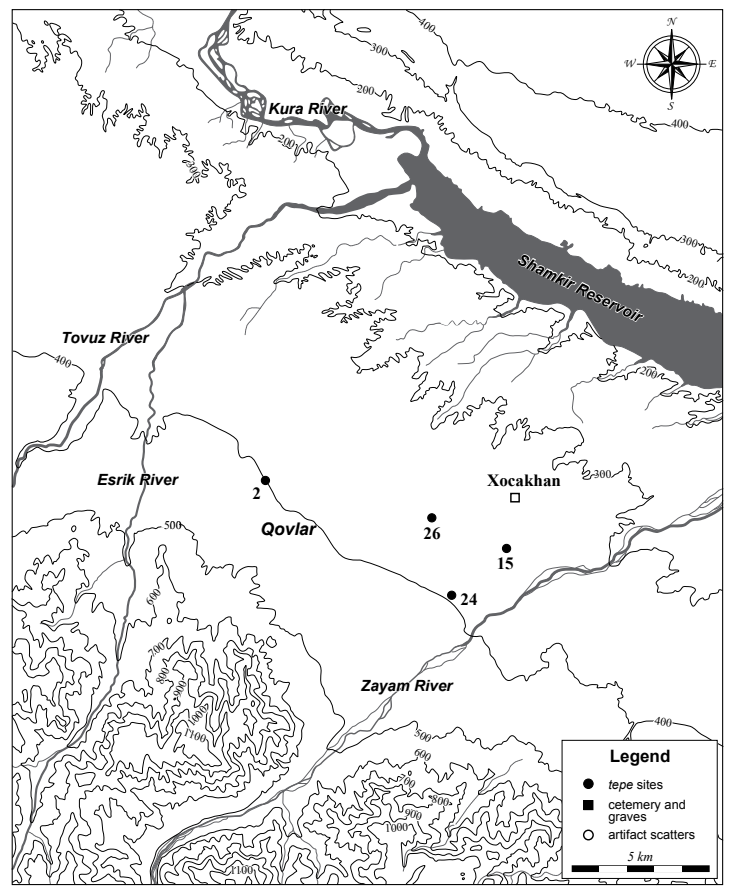

b. Early-Middle Chalcolithic

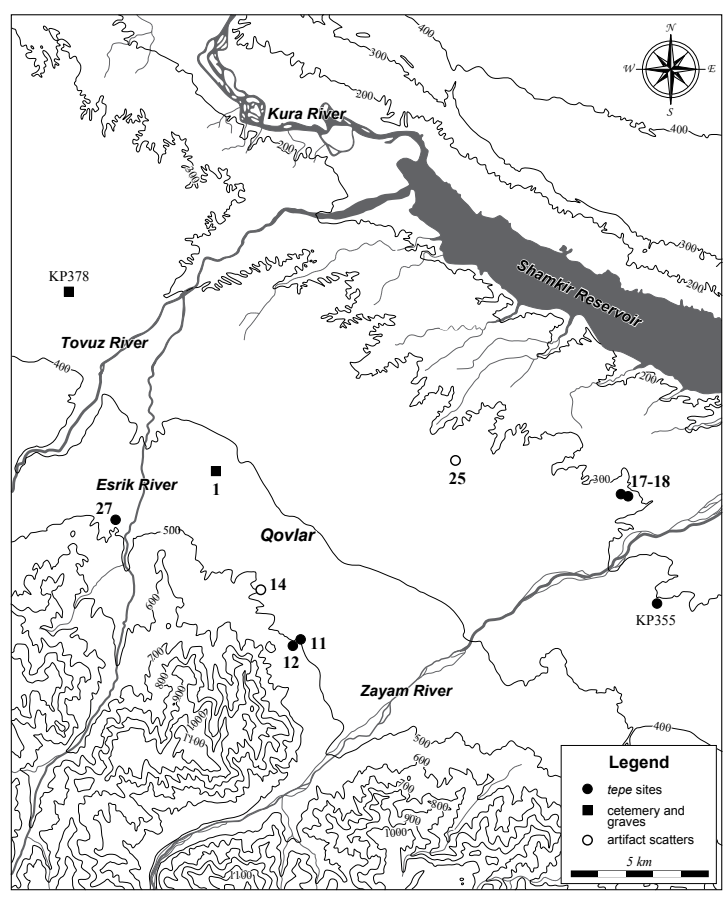

d. Late Bronze-Early Iron Age

Fig. 8.2 Distribution of archaeological sites over time.

much beyond the extant mound.

The surface collection from the site contains more than 100 stone artifacts. The main raw material type is obsidian, which frequently occurs in blade forms (Fig. 8.7). Obsidian blade tools include burins, retouched blades, and nibbled blades. We also found a single-platform blade core and a few platform tablets, which indicate blade production on site. A few flake tools were also recovered, such as splintered pieces and retouched/nibbled flakes. Besides obsidian, a 


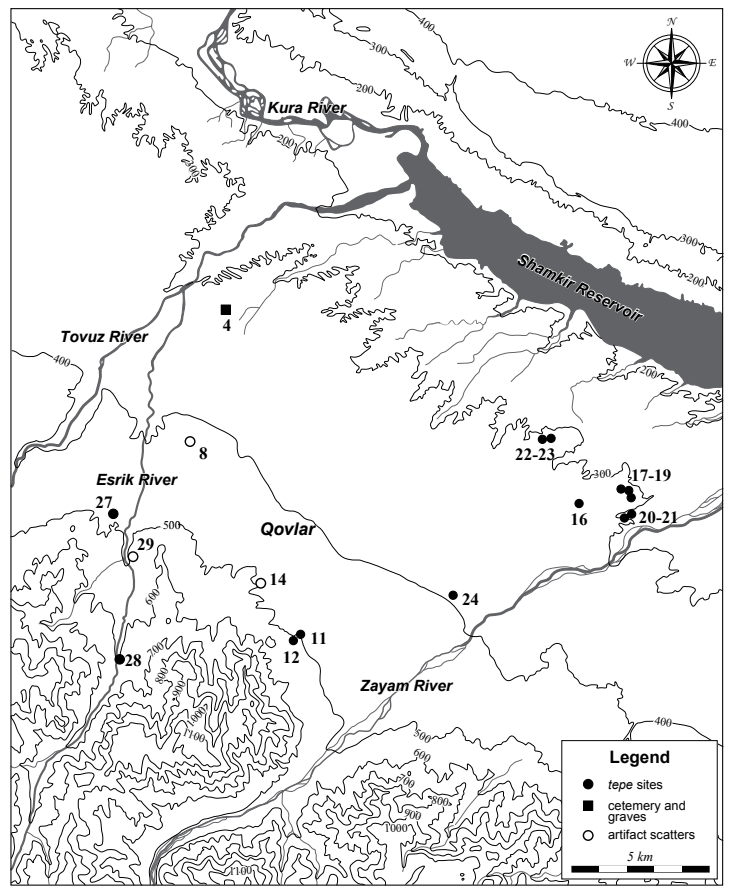

e. Antique-Early Medieval

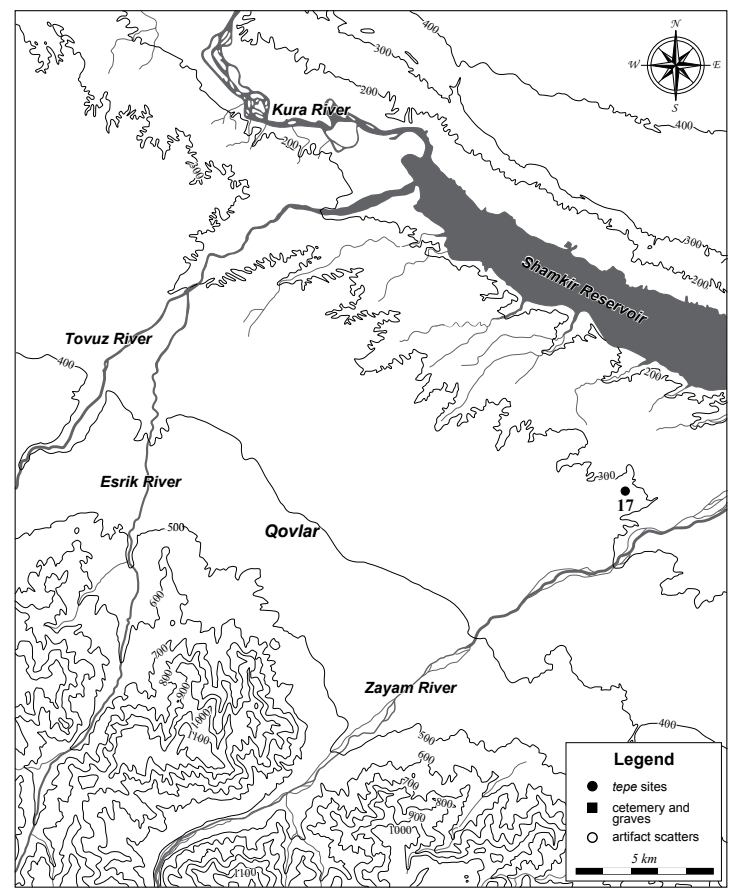

f. Late Medieval

Fig. 8.2 Continued.

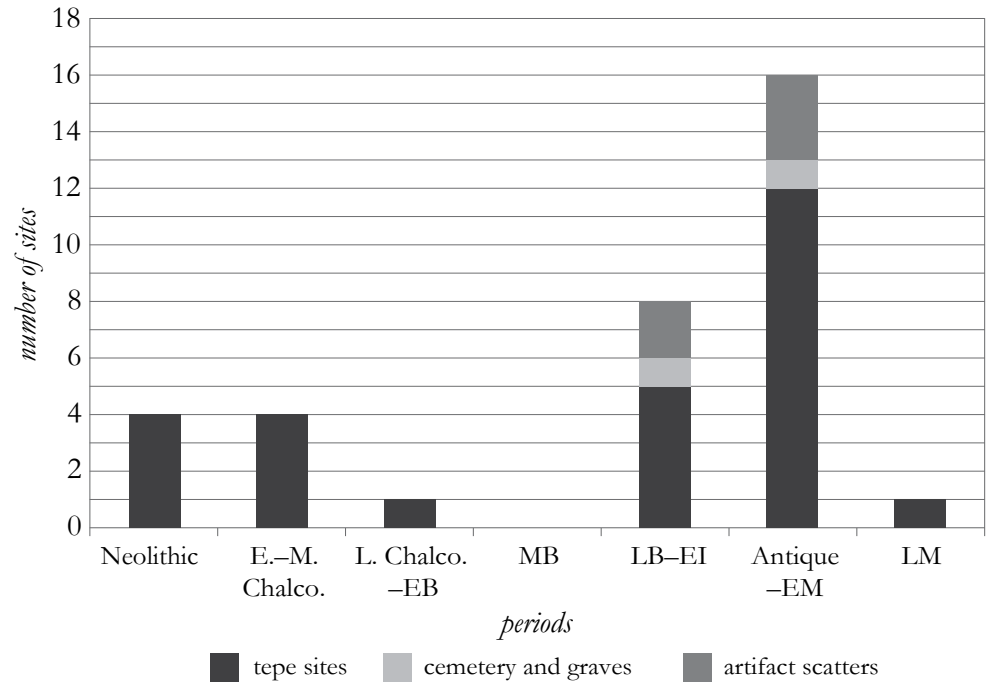

Fig. 8.3 Diagram of the numbers of sites from each time period. Abbreviations: E.-M. Chalco. = Early-Middle Chalcolithic; L. Chalco. = Late Chalcolithic; EB = Early Bronze Age; $\mathrm{MB}=$ Middle Bronze Age; LB = Late Bronze Age; $\mathrm{El}=$ Early Iron Age; $\mathrm{EM}=$ Early Medieval; LM = Late Medieval. variety of other materials were used, including red dacite/rhyolite, red brown flint, and green tuff. These types of rocks occur in flake forms and some pieces are modified into tools (Fig. 8.8). In addition, a single piece of small perforated stone (limestone?) was collected. In contrast to the abundance of lithics, we found very few potsherds. Among some later sherds that are clearly intrusive, one single sherd with grit and fine sand temper seems at first comparable in paste with Neolithic pottery (Fig. 8.4: 8). This dark brown sherd is possibly a part of bowl or jar with a ridge on which fine mat impressions are visible. To date, however, similar decorations have never been found in any Neolithic context, making its dating less plausible. The extreme paucity of pottery fragments at TQ009 implies that in all likelihood it was occupied earlier than Göytepe.

The survey results led us to examine the 

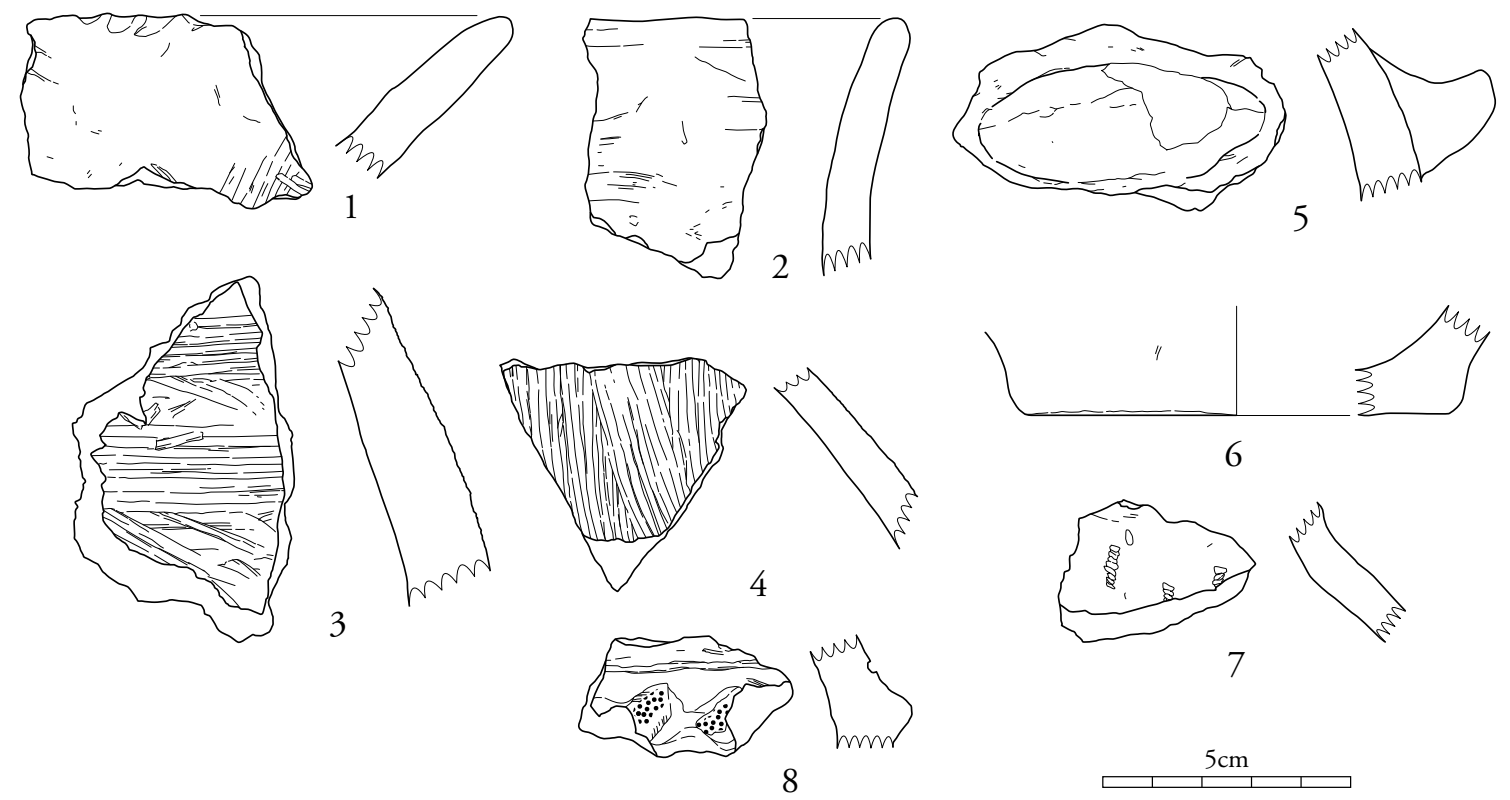

6
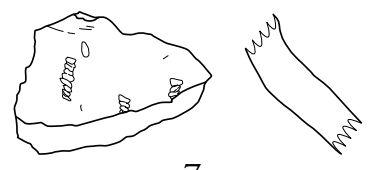

7

$5 \mathrm{~cm}$

Fig. 8.4 Pottery from the Tovuz-Qovlar Survey in 2011. 1-4: TQ002; 5-6: TQ003; 7: TQ004; 8: TQ009. Legends apply to all the pottery figures of this chapter: obliquely hatched = slipped; light gray = burnished/ polished; blurred light gray = sooty area; dark gray = glazed; black = painted decorations; dotted area = impressions.

stratigraphy of TQ009, now called Hac1 Elamxanl1 Tepe, and excavations began since the 2012 season (for more details on the fieldworks, see Nishiaki et al. 2013; Nishiaki et al. 2015a).

There is currently no evidence of Mesolithic or earlier prehistoric sites in this region. Since some open-air sites or rockshelters are located on the piedmont area near Agstafa and Kazakh (Jeferov 2008), they may also be found to the south in the high altitude montane or piedmont areas.

For the Neolithic period, radiocarbon dates obtained from Göytepe and other related sites strongly suggest that these localities must have been inhabited during the 6th millennium BC (Nishiaki et al. 2015a; Nishiaki et al. 2018; Hansen et al. 2008; Lyonnet et al. 2012). As shown in Fig. 8.2: a, the distribution of all four mound sites (including Göytepe) is confined to the intermediate flat area between foothills in the south and arid steppes in the north. Interestingly, they appear to be situated on a line at $400 \mathrm{~m}$ a.s.l. Another notable fact is that these Neolithic sites are not found near large rivers like Zayam or Tovuz River, but rather on rolling alluvial fans, which may have facilitated the underground water supply. With regard to settlement size, each site generally tends to be small, no more than 0.6 ha, with the clear exception of Göytepe (ca. 1.65 ha). We suggest that the occupation periods of all sites were not necessarily contemporaneous. For example, at least one site (TQ009) may have earlier Neolithic or possibly aceramic deposits, while others were occupied somewhat later than Göytepe. This suggestion was proved later. TQ009 (Hac1 Elamxanl1) was securely dated to the early centuries of the 6 th millennium $\mathrm{BC}$, predating the occupations at Göytepe (Nishiaki et al. 2013; Nishiaki et al. 2015a; Nishiaki et al. 2015b).

\subsubsection{Early-Middle Chalcolithic period}

For the Early-Middle Chalcolithic (Fig. 8.2: b) in the surveyed area, useful information comes from the French-Azerbaijani excavations at Mentesh (Lyonnet and Guliyev 2012; Lyonnet and Guliyev 2017) and the Azerbaijani excavations at Xocakhan (Huseynov and Jalilov 2007). The Early and Middle Chalcolithic of Mentesh, albeit provisional, roughly correspond with Period II (first half of the 5th millennium BC) and Period III (second half of the 5th millennium $\mathrm{BC}$ ), respectively. Three new sites identified by our survey are added to the published data of these time periods.

A small site, registered as TQ002, lies in the vicinity of Göytepe to the southeast. Due to heavy destruction from recent agricultural activities such 

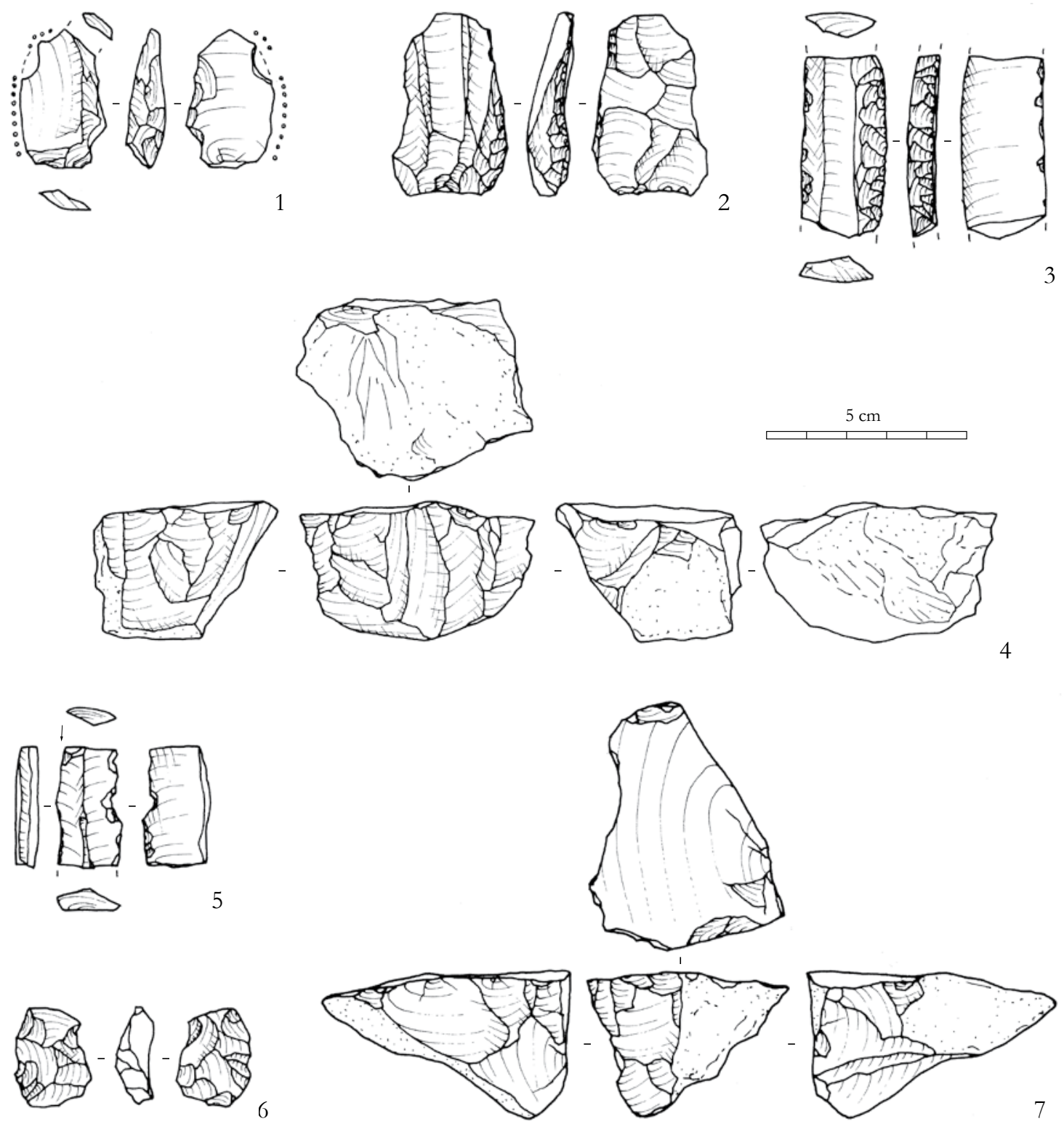

Fig. 8.5 Chipped stone artifacts from Site TQ002 (1-4) and TQ003 (5-7). 1: Sickle element; 2: Splintered piece; 3: Blade with invasive retouch; 4: Single-platform core; 5: Burin; 6: Splintered piece; 7: Change-oforientation core (1, 4, and 7: Green tuff; 2-3 and 5-6: Obsidian).

as soil removal, its surface was completely altered into an irregularly shaped mound that eroded away (at least ca. 0.5 ha of the original mound is missing). Nevertheless, a large number of pottery fragments and both chipped and ground stone tools were collected from the surface. More than 50 potsherds are classified into two groups. The first group consists of gray ware with fine sand temper that was fired under medium temperatures. The much larger second group is chaff-tempered coarse ware, usually with thick walls and an unoxidized gray/black core. This coarse ware group has comb-scraped exterior surfaces in some cases (Fig. 8.4: 3 and 4), which is analogous to the Period III pottery from Mentesh (Lyonnet et al. 2012: 98, figs.143-146) and Xocakhan (Huseynov and Jalilov 2007: 43). However, other type of decorations such as applied pellets, incised rim, or bitumen paint that were attested at Mentesh, were not seen in this group. Complete vessel shapes could rarely be reconstructed because most sherds represent undiagnostic parts. Chipped stone artifacts are mostly made of obsidian and found in blade forms (Fig. 8.5: 1-4). Some obsidian blades have marginal or occasionally invasive retouch. We also collected a 


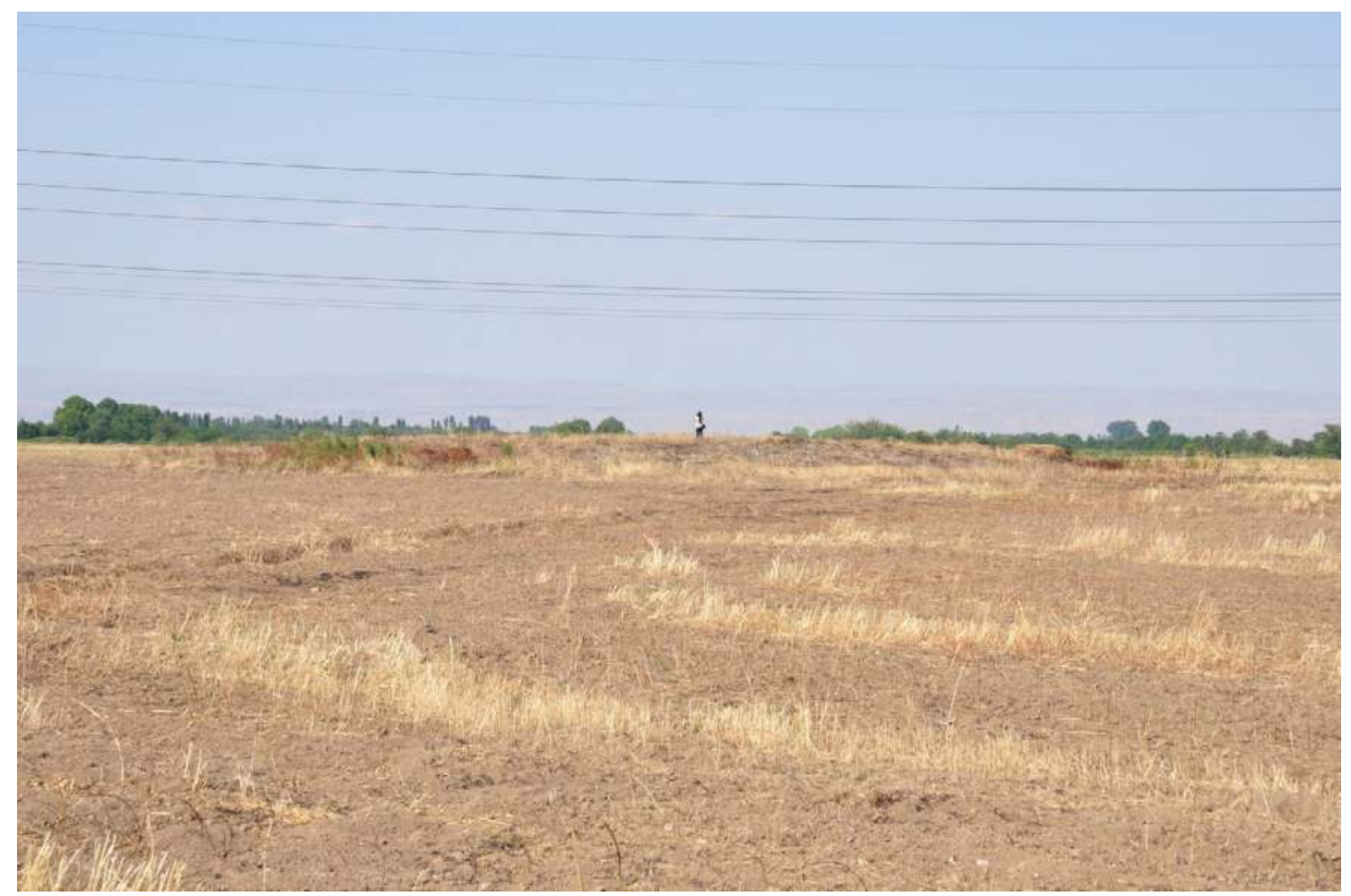

Fig. 8.6 Site TQ009 (Hacı Elamxanlı), seen from the southwest.

few splintered obsidian pieces. Another raw material type is green tuff, represented by a flake, core, and sickle element. In addition, a fragment of a basalt handstone was collected. Excavations of this site, later called Kichik Tepe, has begun on behalf of an Azerbaijan-French team since 2017 (Palumbi et al. 2018).

Toretepe (TQ024) was reported as Toretepe II by Narimanov (1987: 31). Situated close to the BakuTbilisi railway, this site is extensively covered with modern graves of the nearby town of Ashagi Ayibli (Fig. 8.9). It is also a small mound covering ca. 0.87 ha, but easily recognizable from a distance. Planttempered pottery, sometimes with slipped, reddish fabric, and ridges on the rim, was designated as "new elements" in the Aeneolithic, according to Narimanov. However, our surface finds contain not only examples of red-slipped chaff-tempered ware, but also coarse thick-walled pottery (Fig. 8.16: 8, 9) without slip and sand-tempered red-brown ware. One bowl fragment bears a fine incision on its exterior surface (Fig. 8.16: 10), comparable with the excavated sherds of Mentesh Period IV (Lyonnet et al. 2012: fig. 146: 4, 5, fig. 147), which can be dated between the end of the Middle Chalcolithic and transition to Late Chalcolithic. We also collected a small number of chipped stone artifacts, most of which are obsidian (Fig. 8.10: 1-3). Although blade forms occur, some of which were made into burins, flakes are also abundant. Basalt represents another rock type that is represented by some flakes and a single-platform core.

Another new site known as TQ026 is located slightly northwest of Mentesh in the middle of a modern cemetery. This is another low, humble mound of about $60 \mathrm{~m}$ in diameter that covers 0.3 ha. Only four sherds were collected (not illustrated) but these fragments are too small for us to reconstruct the entire vessel. Fine sand-tempered examples have red slip and burnishing on their exterior sides, while two coarser fragments show chaff or grit-temper similar to the Mentesh assemblage. This fine ware may be associated with the Neolithic pottery from Göytepe, suggesting a date earlier than the Chalcolithic. We also collected a small number of chipped stone artifacts, most of which are obsidian, including one borer on a blade with alternate retouch, as well as a burin, splintered piece, and a platform tablet (Fig. 8.10: 4 and 5). In addition, basalt core pounders were also recovered.

The French-Azerbaijani survey in western Azerbaijan also recorded several archaeological 

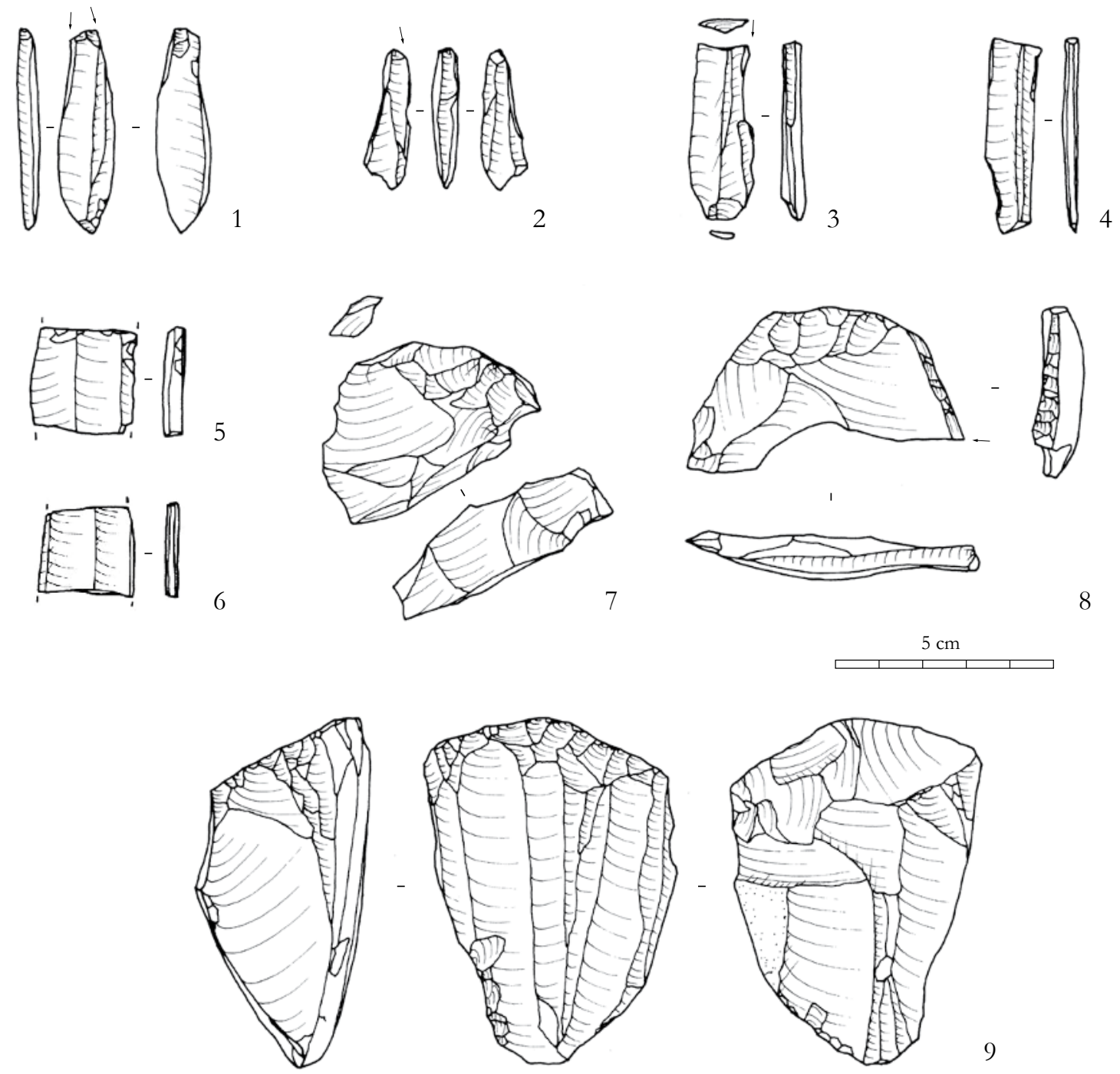

Fig. 8.7 Chipped stone artifacts (obsidian) from TQ009. 1-3: Burins; 4: Retouched blade; 5 and 6: Blades; 7 and 8: Platform tablets; 9: Single-platform core for blades.

sites in this area that yielded Chalcolithic ceramic sherds (Lyonnet and Guliyev 2009). All of the sites are small mounds situated at the northern edge of modern cultivated fields around Xocakhan and are known as Madat Yeri, Aghili Dara, and Gambar Tepe (not shown in Fig. 8.2: b).

In comparison with the preceding period, there seems to be an occupational shift in this period for most settlements to the southeast belonging to the Zayam River catchment (Fig. 8.2: b). Estimated sizes of each settlement vary but are basically less than 1 ha, similar to the Neolithic sites. Although there remain problems in pinpointing the precise dates of each site, the above-mentioned sites will nonetheless be quite promising for future excavations aimed at better understanding the aftermath of Neolithic occupations at Göytepe. However, the distribution patterns of Neolithic and Chalcolithic sites demonstrate that they are similar to each other in their settings between the foothills and modern agricultural fields.

\subsubsection{Late Chalcolithic, Early Bronze Age, and Middle Bronze Age}

The Late Chalcolithic period in this region is exemplified by the local Leylatepe culture and related material cultures. The nature of their cultural interactions with Northern Mesopotamia or the Uruk expansion is much debated (Marro 2007; Akhundov 2007; Narimanov et al. 2007; Munchaev and Amirov 2012). This period is eventually followed by the Early Bronze Age Kura-Araxes cultural complex, an archaeological entity of the Transcaucasus that 

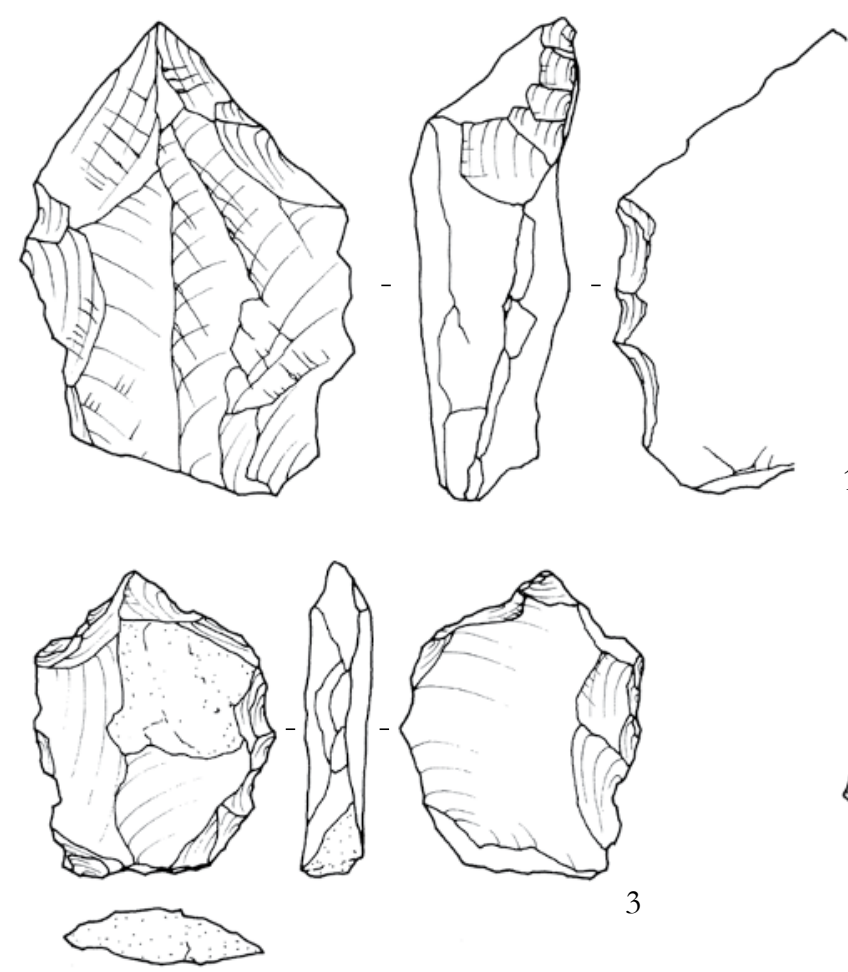

3

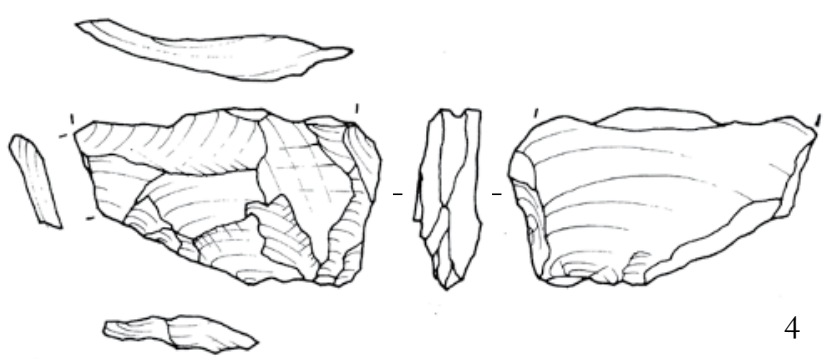

Fig. 8.8 Chipped stone artifacts (non-obsidian) from TQ009. 1: Denticulate; 2: Round scraper; 3: Retouched flake; 4: Flake; 5: Single-platform core (1 and 2: Andesite; 3 and 4: Red dacite/rhyolite; 5: Green tuff).

later expanded into much broader geographical extents. Despite its historical significance in the southern Caucasus, the origin and development of the Kura-Araxes phenomenon are still controversial (e.g., Kiguradze and Sagona 2003; Palumbi and Chataigner 2014). In relation to this period, kurgan burials dating to the early 4 th millennium $\mathrm{BC}$ were recently investigated at Soyuq Bulaq in northwestern Azerbaijan (Lyonnet et al. 2008). This study provided some clues allowing us to elucidate the socio-political process during this important Late Prehistoric period.

The only site dated to the Late Chalcolithic and Early Bronze Age (Fig. 8.2: c) in this area is Mentesh (TQ015) (Lyonnet et al. 2012; Lyonnet and Guliyev 2017). In Period IV defined at this site (from late 4th to early 3 rd millennium $\mathrm{BC}$ ), a collective burial was
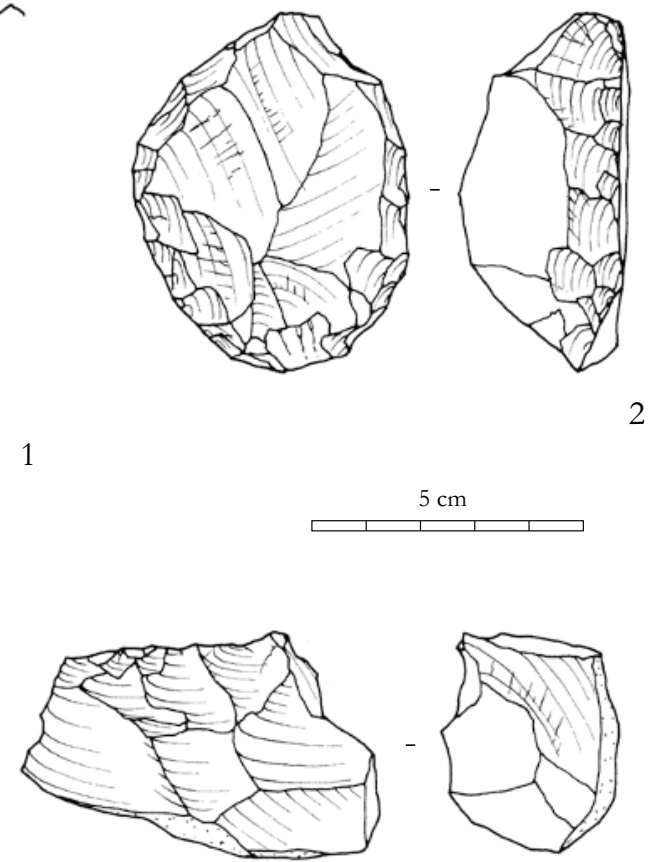

constructed from which two groups of pottery, an early red-brown group and a later black-burnished ware, were found (Lyonnet et al. 2012: 103; Lyonnet and Guliyev 2017; Lyonnet 2017). However, no comparable surface finds from the surveyed area have yet been collected.

Likewise, no Middle Bronze Age sites were found in our survey area. The limited Early Bronze Age settlements and complete absence of Middle Bronze Age sites in this region are somewhat curious. This may be explained either by a possible occupational hiatus or by distinctive subsistence patterns differing from those in other periods, for instance, pastoral nomadism. It is also possible that relevant mound sites had been largely destroyed or are too small to be detected. Because of the lack of available data or 


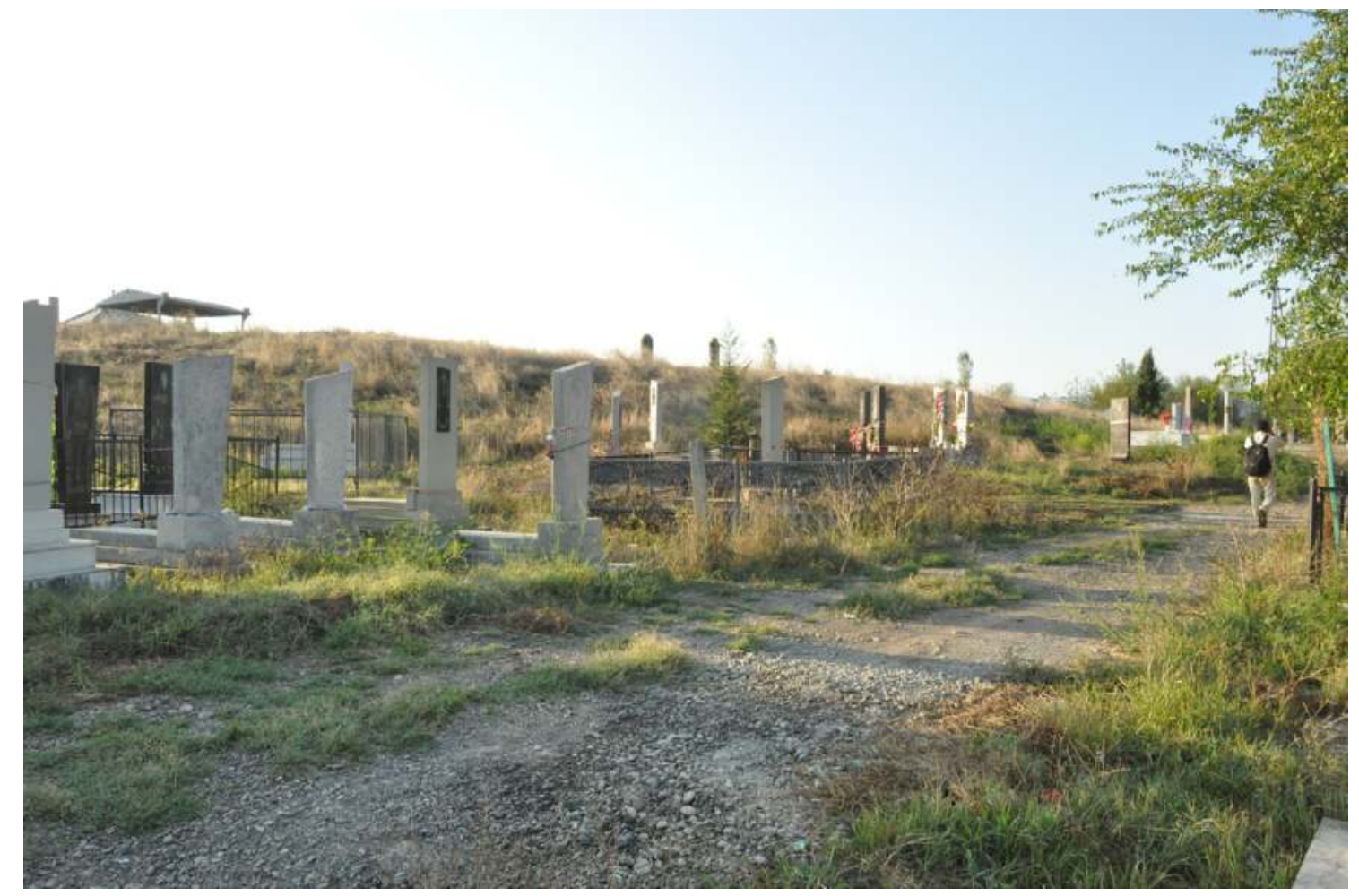

Fig. 8.9 Site TQ024 (Toretepe), seen from the southeast.
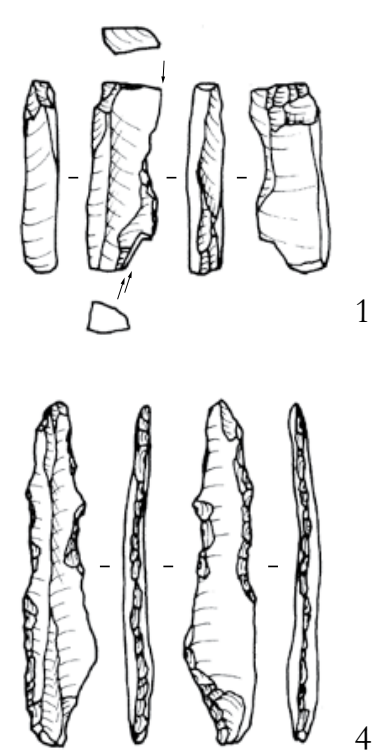
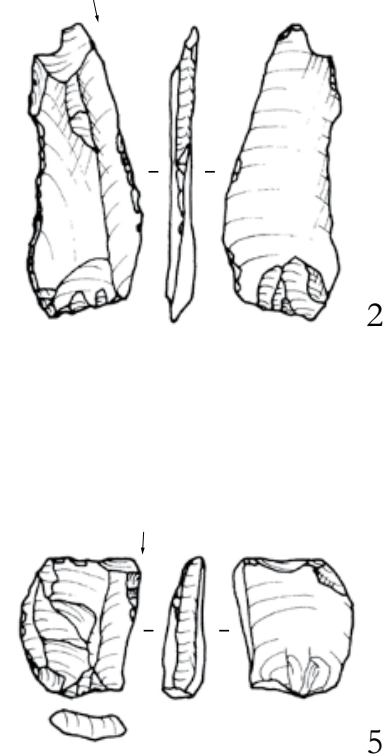

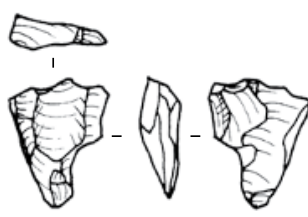

3

Fig. 8.10 Chipped stone artifacts (obsidian) from TQ024 (1-3), TQ026 (4 and 5), and TQ027 (6). 1 and 2: Burins; 3: Truncated-faceted flake; 4: Borer; 5: Burin; 6: Splintered piece.

cultural evidence from these periods, more intensive surveys must be necessary.

\subsubsection{Late Bronze Age and Early Iron Age}

In contrast to the scarce Early-Middle Bronze Age sites, a number of settlements were established around Göytepe in the Late Bronze to Early Iron Ages (Fig. 8.2: d). In total, five tepe sites, two artifact scatters, and one cemetery site were registered. Not only are the settlements more numerous than in the 
preceding period (Fig. 8.3), but hitherto unexploited areas were also used in these periods. Particularly remarkable is that some large settlements forming high mounds began to be occupied in some areas.

TQ011 (Aytepe) on the left bank of the Zayam River lies immediately adjacent to the similar mound of TQ012 (Ibrahim Hacili Tepe), south of Qovlar (Fig. 8.11). In terms of dimensions, both of these sites exceed $100 \mathrm{~m}$ in length and are more than 14 $\mathrm{m}$ high, resulting in a substantial mound over 1 ha. Located south of the Ashagi Qushchu village, Toretepe (TQ027) on the left bank of the Esrik River is also one of the largest settlements of this period, having almost the same dimensions as the other two sites (Fig. 8.12). Chronologically, the majority of surface finds from all the sites consist of gray burnished ware, which is characteristic for the Late Bronze Age and Early Iron Age (Figs. 8.13, 8.14, and 8.15 ). The gray burnished ware usually consists of pottery made on a wheel with sand temper, easily recognized by burnishing or polishing treatment on a gray surface (Figs. 8.13: 5, 8, 9, 8.14: 4, 6, 8.15: 4). In most cases, such treatment represents a patterned burnish resembling multiple vertical lines (Figs. 8.13: 11, 8.15: 1), combined bands and lines (Fig. 8.13: 12), a fine wavy line (Fig. 8.15: 2), coupled strokes (Fig. 8.15: 9), or sometimes vertical excisions (Fig. 8.15: 10). Oblique impressed decorations (Fig. 8.13: 7), incised patterns (Fig. 8.15: 6, 8), and low horizontal ribs on the jar body (Figs. 8.13: 12, 8.14: 6, 8.15: 9) are also included. For this ware group, the predominant forms are carinated bowls with thickened beaded rims (Figs. 8.13: 5, 6, 8, 8.15: 1 and 2) and jars with similar rims (Figs. 8.13: 10, 8.15:4), but beakers with small ring bases (Fig. 8.14:8) are also found. In addition to gray burnished ware, grittempered coarse ware likely dates to this time period as well (Fig. 8.13: 13). Although the paired sites of TQ011 and TQ012 are situated side by side, we could not determine whether they were contemporaneous or were occupied in temporal succession. In addition, a few obsidian flakes were collected at TQ011, TQ012, and TQ027 (Fig. 8.10: 6).

Göytepe (TQ001) is also unique because in the course of our excavations it revealed many stone graves dug into the Neolithic deposits (Guliyev et al. 2017: 69-74). Since the graves mostly concentrate on the mound's flat summit, it is likely that it served as a cemetery for the Late Bronze Age and Early Iron Age inhabitants. Those buried in the Göytepe cemetery possibly came from Toretepe (TQ027), as they lie in closer proximity than other sites (the distance between them is approximately $3 \mathrm{~km}$ ). With the exception of the Göytepe case, no further evidence of cemeteries exists during this period. According to the Baku-Tbilisi-Ceyhan (BTC) pipeline salvage excavations, however, numerous underground graves or "necropoleis" have been incidentally discovered on hills at KP378 west of the Tovuz River (Museibli et al. 2008). At KP355 on the right bank of the Zayam River (Ashurov 2007), a settlement site called Sari Reme or Yataq Remesi is also located near a catacomb of unknown age. Thus, it is very likely that more undiscovered graveyards or ancient settlements than we expected actually exist underground on the flat alluvial plain. However, this kind of sites would be extremely hard to identify through survey methods such as ours.

Site TQ025 is recognized as an area scattered with artifacts on the low steppe plain. A modern cemetery lies over more than half of the site. It is not certain whether this flat and large area of $200 \times$ $100 \mathrm{~m}$ belongs to an artificial mound or if it is only a natural hill cut by seasonal streams on all sides. Sparse distributions of archaeological materials are recorded across the extensive area and comprised potsherds and obsidian flakes. Pottery fragments collected here consist primarily of gray burnished ware and some coarse examples of brown ware, possibly of the Late Bronze Age or Early Iron Age (Fig. 8.16: 12-16). Although we cannot confirm an exact date of the site from small pottery fragments, it is clear that a well-polished bowl rim with an incised wavy design has a different age (Fig. 8.16: 11).

No prominent mound is identified at site TQ014, which lies on piedmont grassland. Some sherds were encountered on the surface (Fig. 8.14: 11 and 12), most of which belong to gray coarse ware with sand temper, together with fine ware of the Antique period. Although their surfaces were unburnished and one body fragment is decorated with uncommon diagonal grooves (Fig. 8.14: 12), these fragments are provisionally assigned to this period.

Sites TQ017 and TQ018 are tentatively dated to 


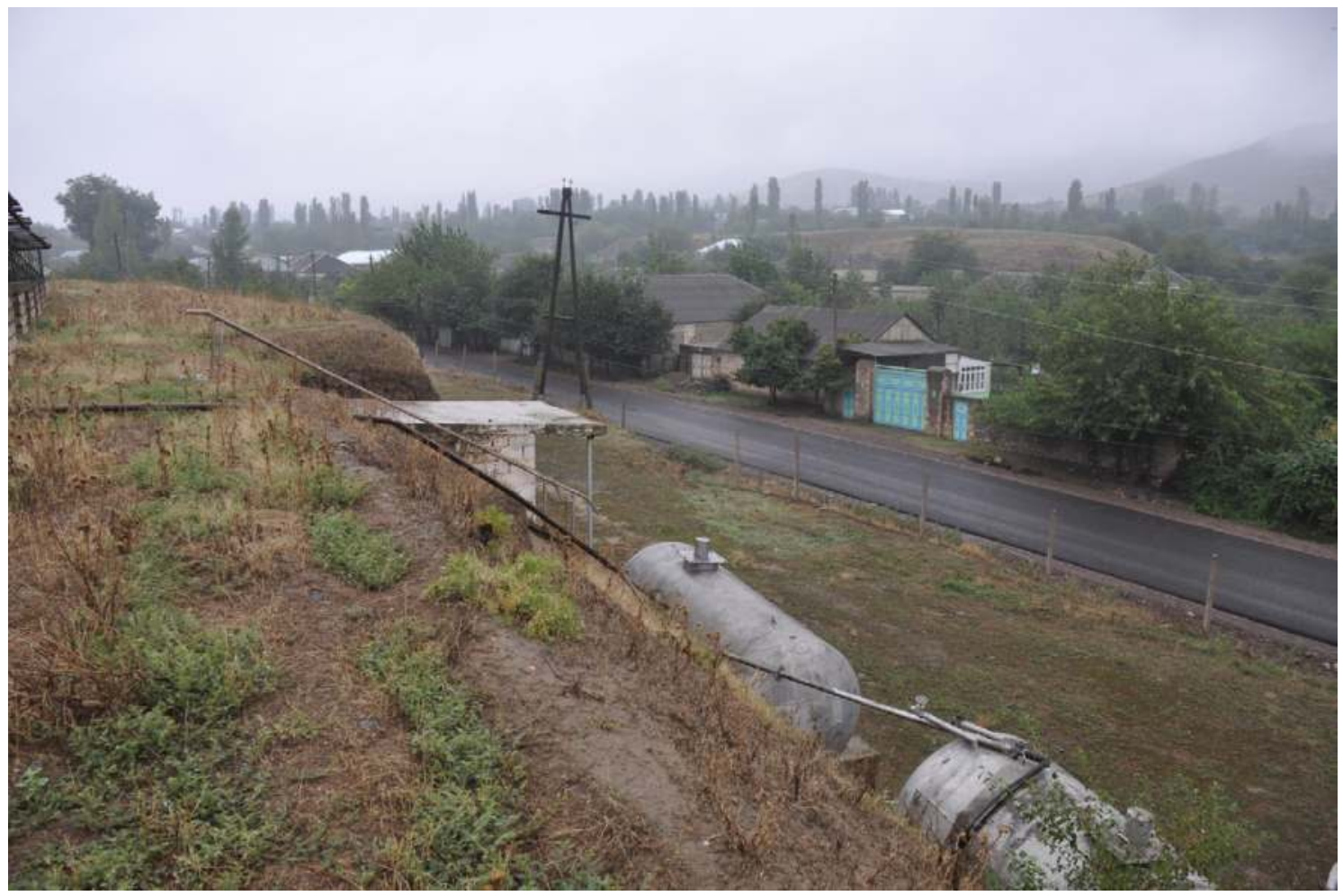

Fig. 8.11 Site TQ011 (Aytepe) cut by modern road in the foreground and TQ012 (Ibrahim Hacili Tepe) in the background behind village houses, seen from the north.

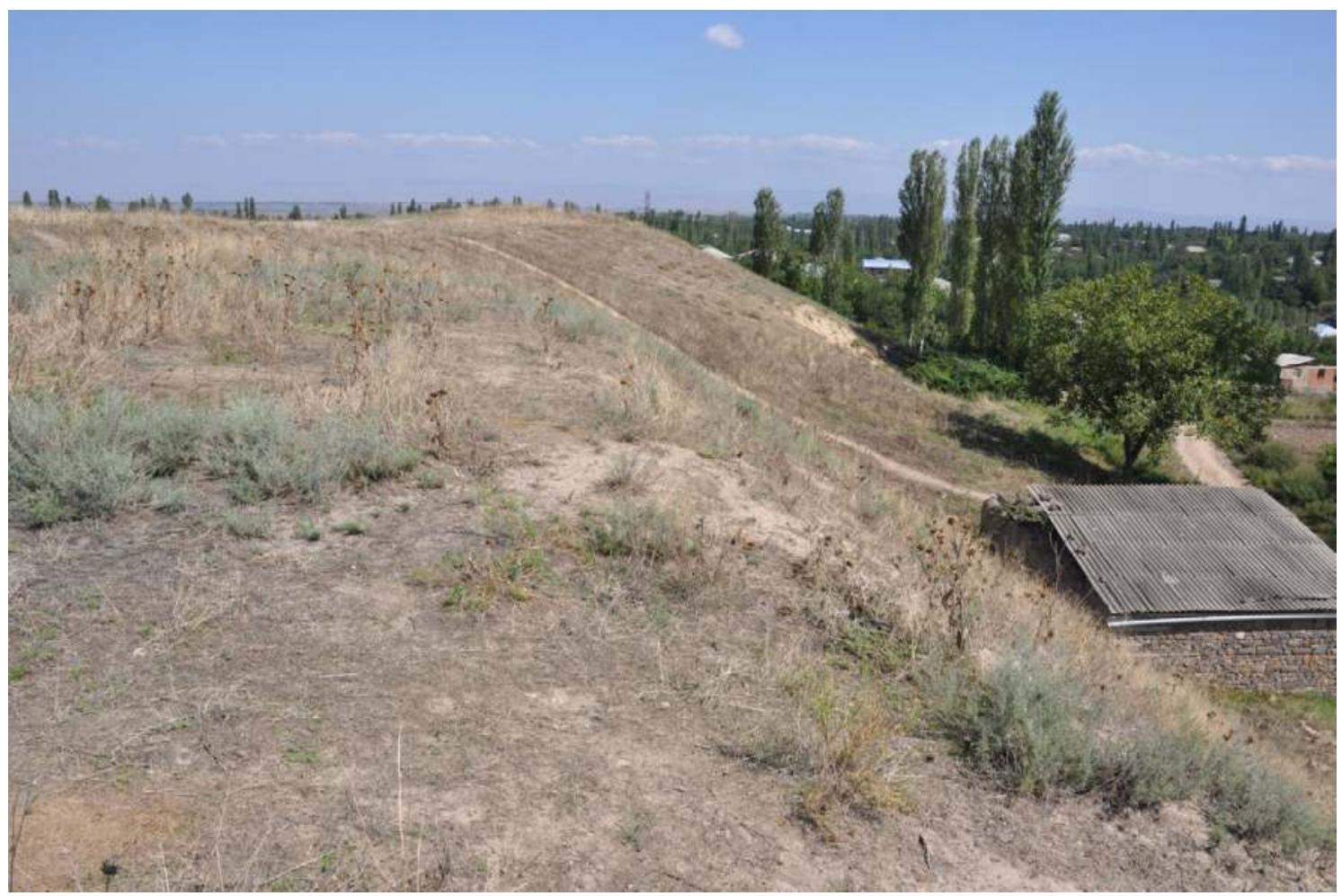

Fig. 8.12 Site TQ027 (Toretepe), seen from the south. 


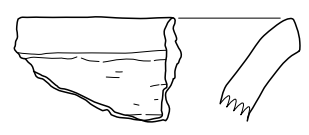

1

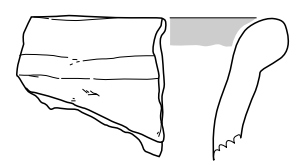

4

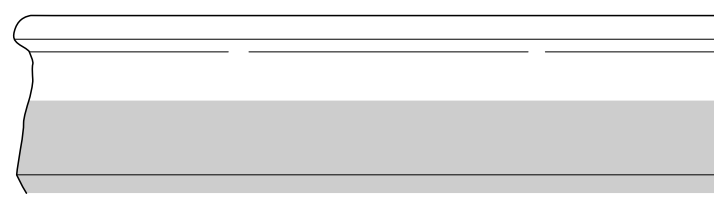

8

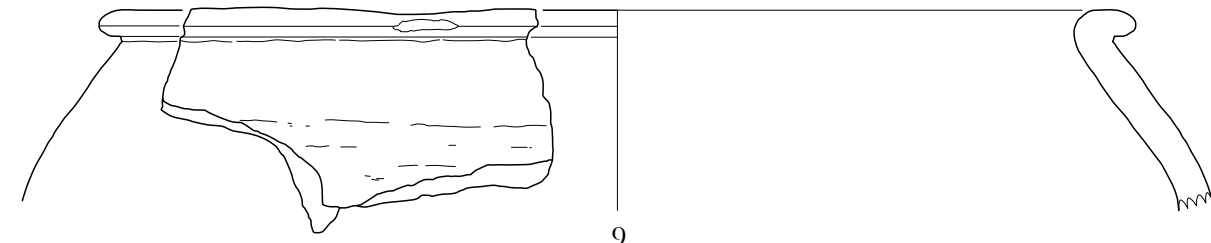

9

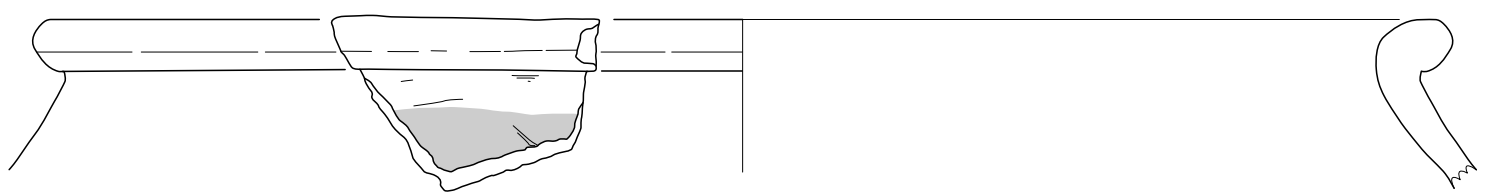

10

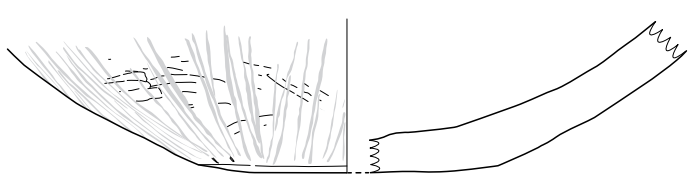

11

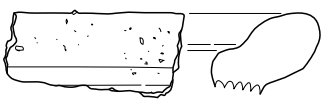

13

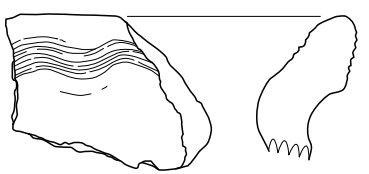

14

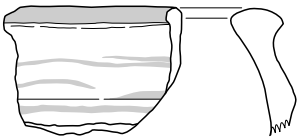

6
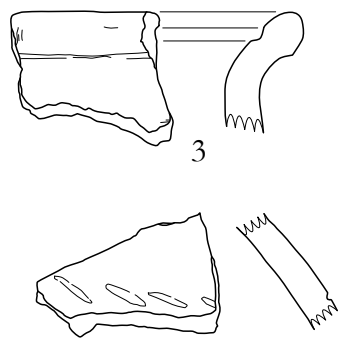

7

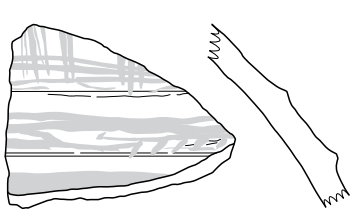

12
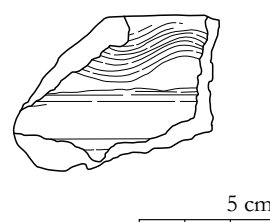

$5 \mathrm{~cm}$
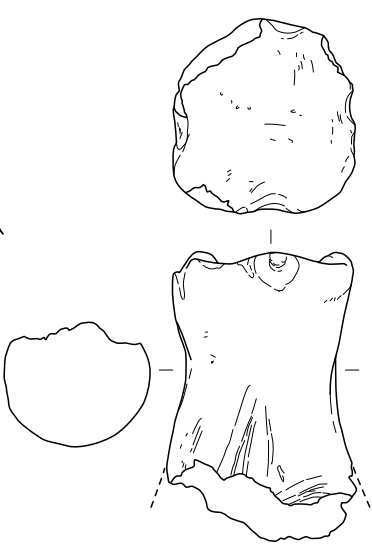

15

Fig. 8.13 Pottery from the Tovuz-Qovlar Survey in 2011. 1-15: TQ011.

later periods, but both yielded a few possible Late Bronze or Early Iron Age ceramic collections (not illustrated here because of their small fragmented size) and some obsidian flakes. This implies that these post-Antique period settlements were originally occupied as early as the Late Bronze or Early Iron Ages.

The Late Bronze and Early Iron Ages in this region, roughly dating to the late 2 nd millennium to early 1st millennium BC (Badalyan et al. 2003), are less known archaeologically because few settlement sites have been excavated. It is reasonable to posit from our survey results that after some hiatus in occupation during the Early and Middle Bronze Ages, the Tovuz-Qovlar region was both intensively and extensively settled during the Late Bronze and Early Iron Ages. The presence of several sites larger than 1 ha (TQ011, TQ012, TQ027) discovered in this survey is especially remarkable. Since those sites have substantial dimensions, they must have served 


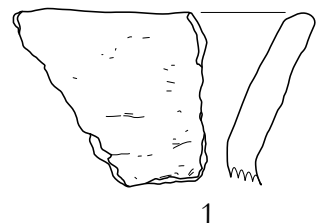

1

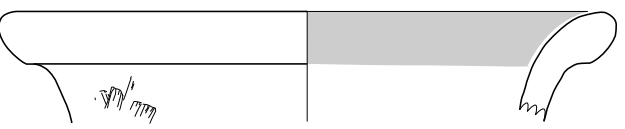

4

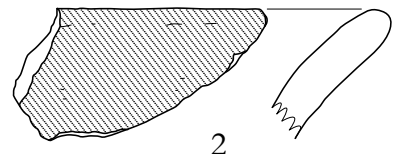

2
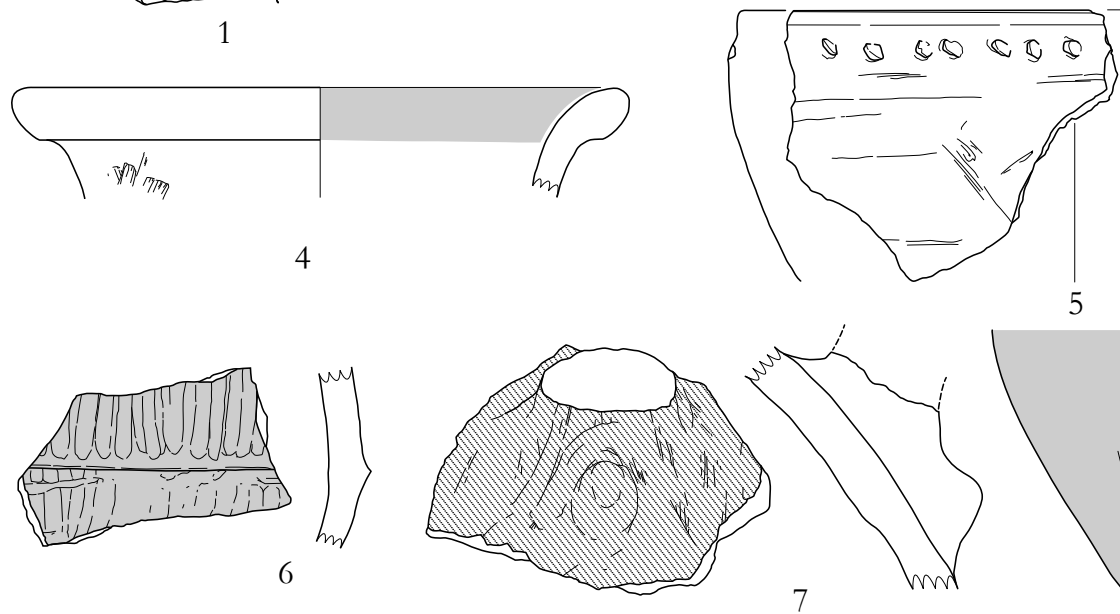

7

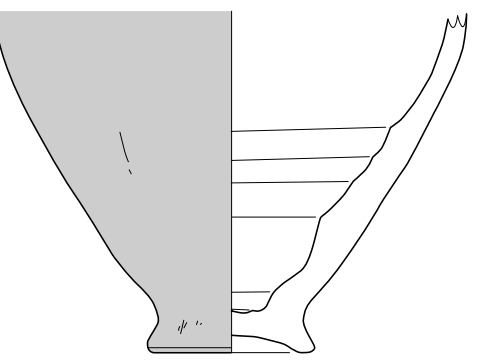

8

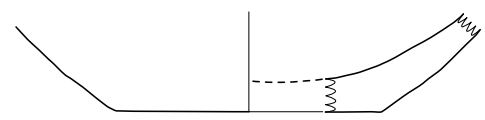

10

9

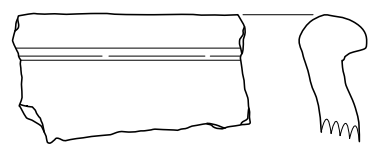

11

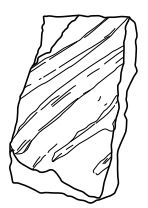

12

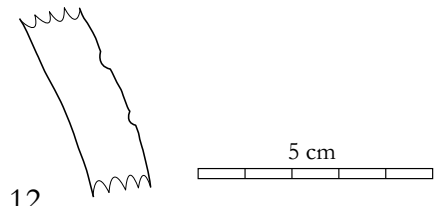

Fig. 8.14 Pottery from the Tovuz-Qovlar Survey in 2011. 1-10: TQ012; 11-12: TQ014.

as microregional centers playing an important role in the local community. It is also noteworthy that they are all situated in close proximity to the northern foothills of the Lesser Caucasus mountains (Fig. 8.2: d). This location was probably related to resource abundance and accessibility in the local environment and its defensive factors. First, one could easily access riverine resources including abundant fresh water and cobbles for stone tool production or construction materials for settlements situated high up on river terraces. Second, these large tepe sites concentrate along a line at ca. $500 \mathrm{~m}$ a.s.l., reflecting a significant settlement pattern. It also suggests that occupation sites were preferentially chosen in piedmont locations having long distance views of the low alluvial lands. The occupants presumably had fortification systems for the main habitation areas on the mounds, though this has not yet been confirmed by our surveys. As we often see in the Bronze Age sites of the Near East, the local communities in this region apparently achieved some level of sociopolitical complexity by this time.

Excluding artifact scatters, no hierarchy in settlement size can be observed among the identified sites. As mentioned above, however, smaller village settlements may exist underneath the surface of the flat alluvial plain.

Survey and excavations on the Tsaghkahovit plain of Armenia also demonstrate that fortified settlements flourished during the Late Bronze Age, which showed a similar tendency in settlement pattern to ours (Badalyan et al. 2003). Recent surveys in the Nakhchivan area revealed an increase in large settlement sites in the slightly earlier Middle 


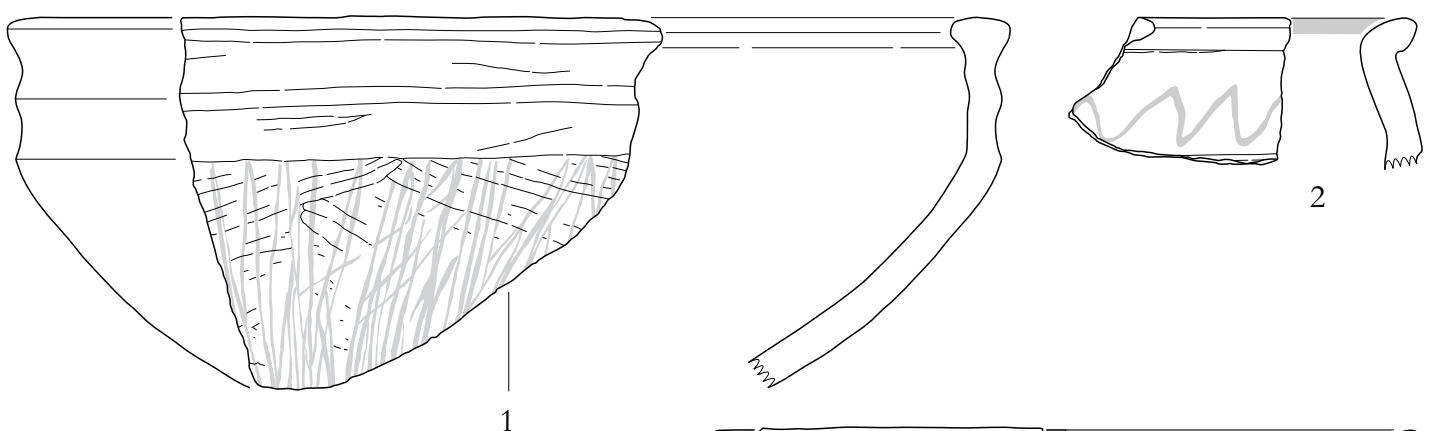

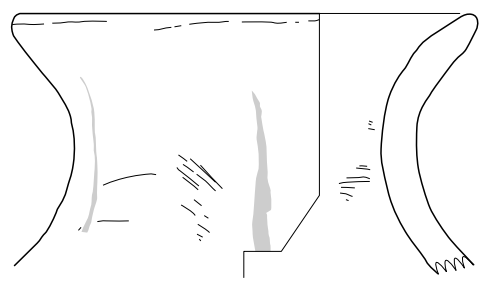

3

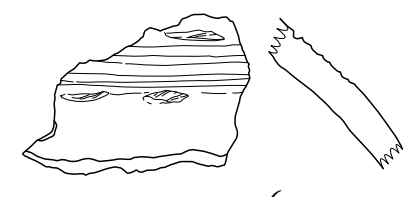

6

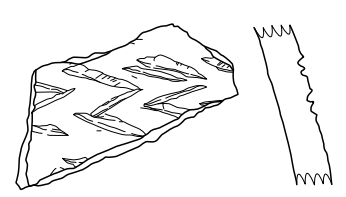

8

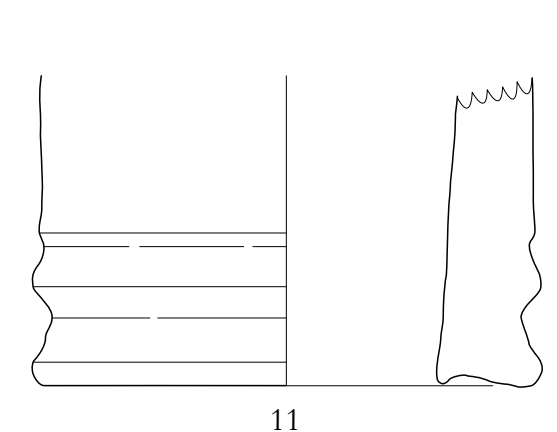

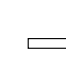

Fig. 8.15 Pottery from the Tovuz-Qovlar Survey in 2011. 1-12: TQ027.

$5 \mathrm{~cm}$

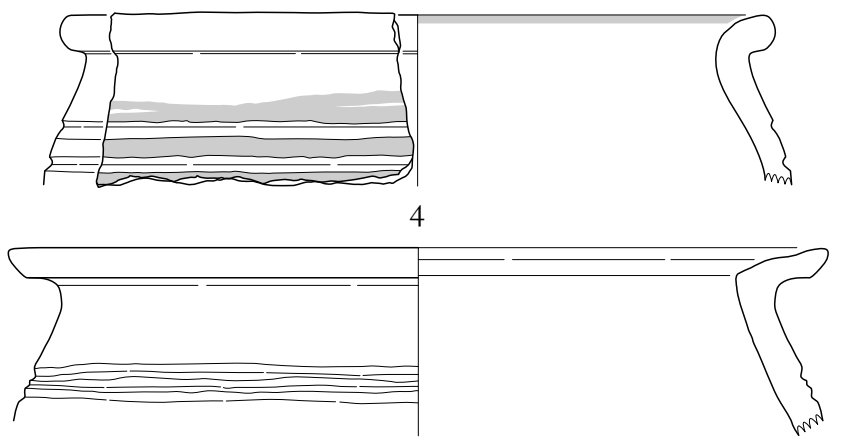

5
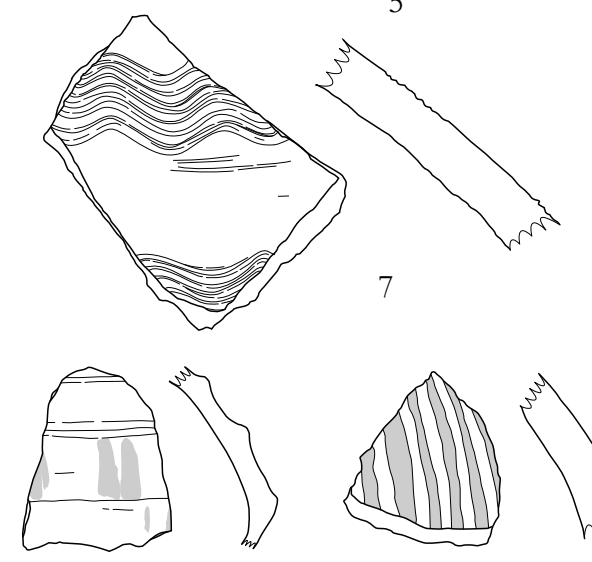

9

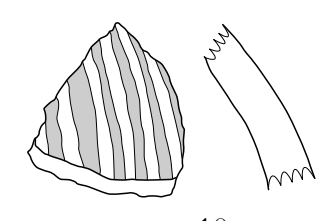

10

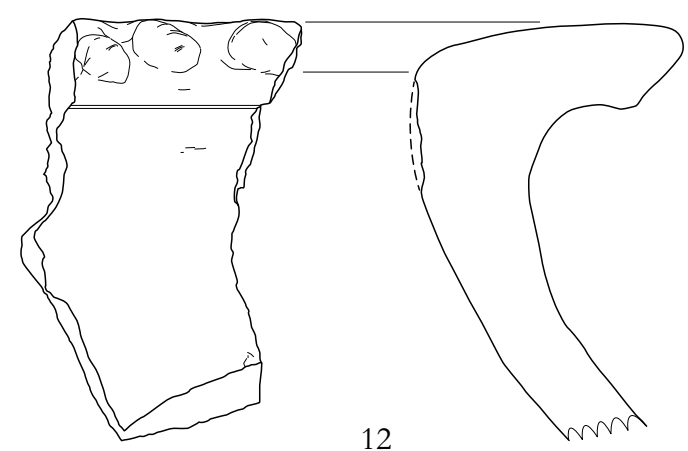

12 

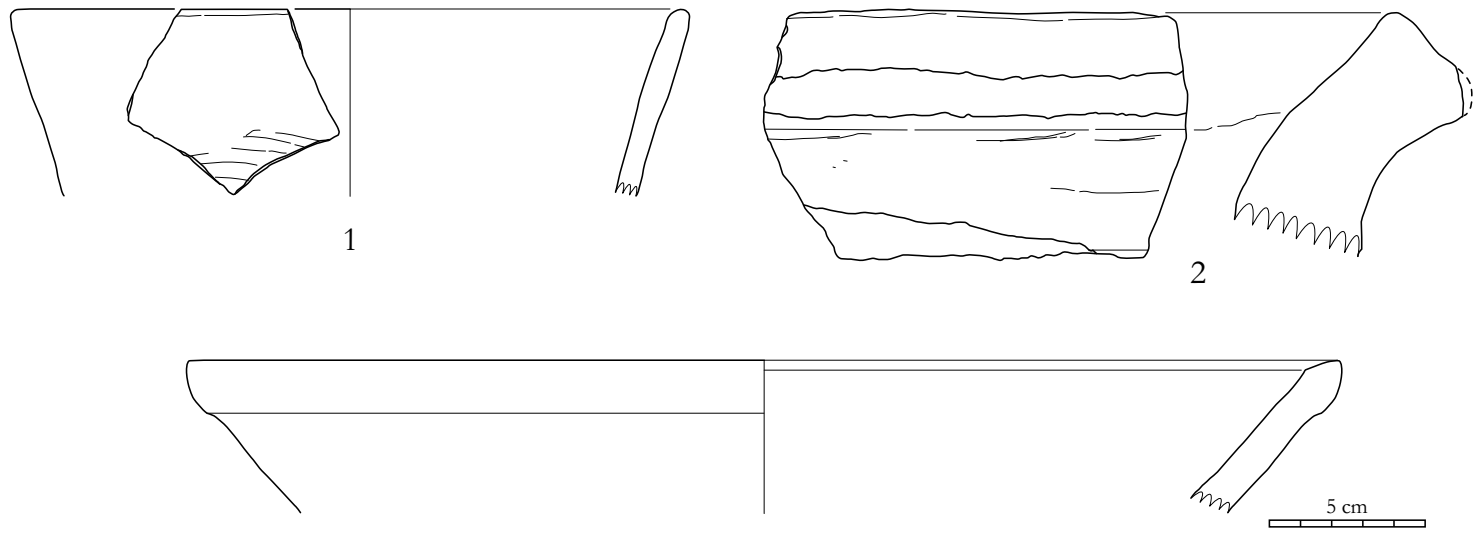

3

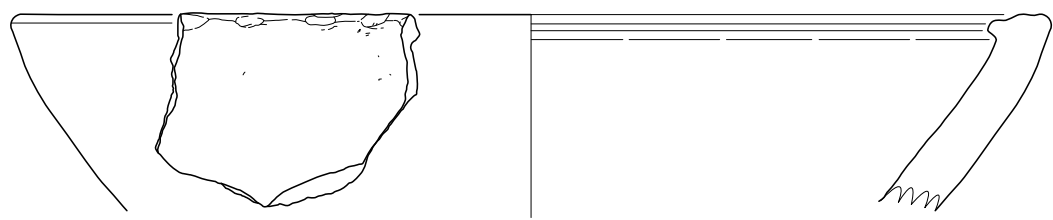

4

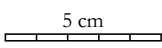

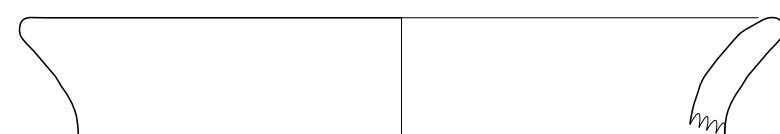

5

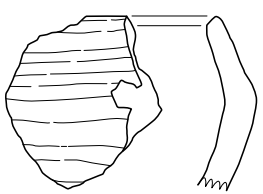

7

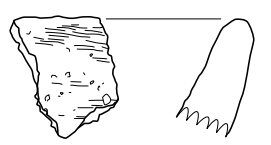

8

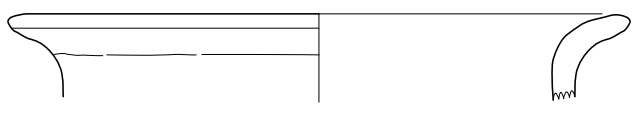

6
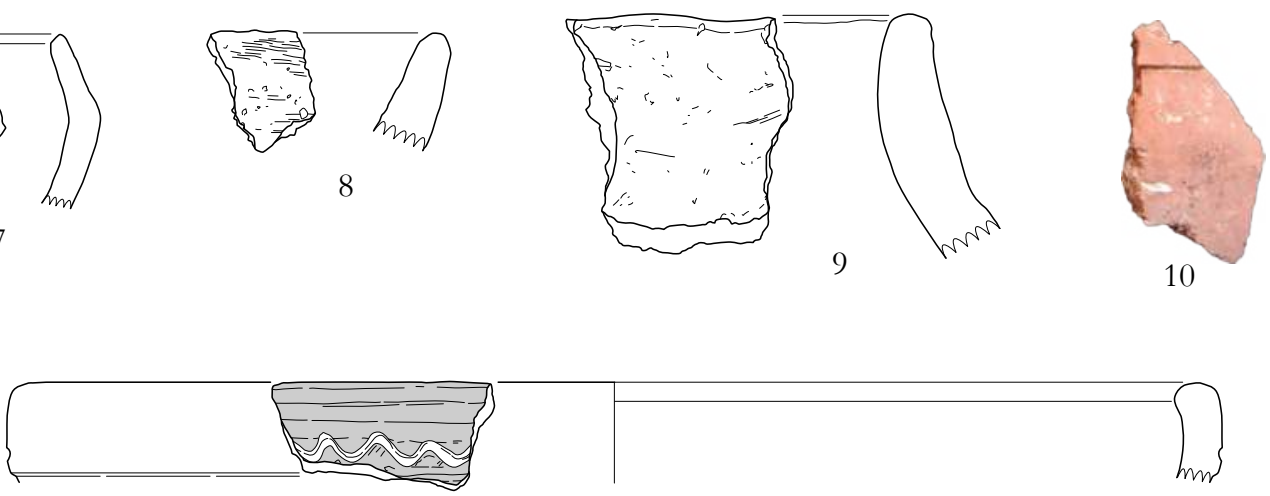

11

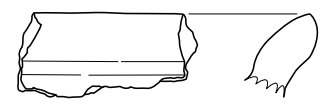

12

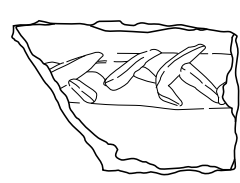

15

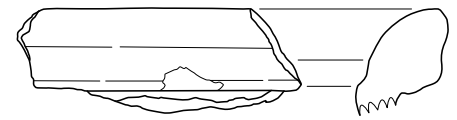

13

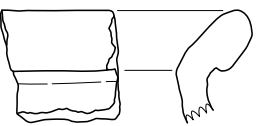

14

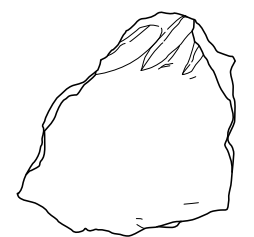

16

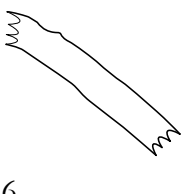

$$
\frac{5 \mathrm{~cm}}{\frac{1}{\text { (scale except nos. } 3 \text { and 4) }}}
$$

Fig. 8.16 Pottery from the Tovuz-Qovlar Survey in 2011. 1-4: TQ021; 5: TQ022; 6-7: TQ023; 8-10: TQ024; 11-16: TQ025. 
Bronze Age and more fortresses in the Middle Iron Age (Ristvet et al. 2011). In order to understand what motivated this change in settlement pattern in the later Bronze Age in a much wider context, more evidence and data from excavations are needed.

\subsubsection{Antique and Early Medieval periods}

A significant change in settlement pattern occurred during the following Antique and Early Medieval periods (Fig. 8.2: e) when the region witnessed a marked increase in occupation. Thirteen tepe sites, one possible burial mound, and three artifact scatters were identified for these periods, increasing almost twofold from the preceding period (Fig. 8.3). Not only did the number and density of occupied sites increase and spread extensively across the region, but many large settlements of the Late Bronze and Early Iron Ages appear to have been continuously occupied or resettled in the subsequent Antique period. Together with gray burnished pottery, there are high numbers of coarser red-brown ware at some large sites including TQ011, TQ012, and TQ027; for instance, a jar rim with a wavy comb-incision on both sides (Fig. 8.13: 14). However, since some groups of ceramics dated to the Early Medieval period occur only rarely among the surface finds, including fine painted or glazed wares, we believe that most or all of the settlements no longer persisted after that time. In comparison with the previous period, we cannot specify how large the occupied area in each site was during the Antique and Early Medieval periods. Nevertheless, some tiers of settlement can be distinguished for a series of sites more than 1 ha, such as TQ020, TQ021 and TQ028, while others (TQ022, TQ023) represent extremely small sites less than 0.3 ha and intermediate sizes of about $0.4-1.0$ ha (TQ016-TQ018). Still uncertain are how and when such diversity in settlement size became common.

A wide variety of ceramic categories should be assigned to the Antique and Early Medieval periods: lid handle fragments (Fig. 8.13: 15); jar with an appliqué band with impressions (Fig. 8.14: 9); saucer lid with bands of comb-impressed crosshatches (Fig. 8.19: 3); red-brown ware with wavy combed lines (Fig. 8.15: 7) and a pattern-burnished jar with everted rim (Fig. 8.15: 3); pedestal or terracotta tube (?) (Fig. 8.15: 11); large pithoi (Fig. 8.15: 12); and a bowl with semi-circular handle (Fig. 8.21: 8).

In addition, red-slipped pottery typical of this period was frequently collected at several sites, represented by simple or carinated bowls (Fig. 8.18: 2, 8.21: 1) and handled jars (Fig. 8.14: 7). Similar pottery fragments are also attested at TQ011 and TQ029. In addition, we see elaborately formed spouted vessels with dark red paste and red slip or burnishing at TQ017 (Fig. 8.19: 4, 5). Another interesting ware group involves very fine painted pottery from TQ018 and TQ019. It has highly fine texture of yellow or reddish buff and frequently displays wet-smoothed and red-painted surfaces without any inclusions (Fig. 8.20: 4, 5). This ware is undoubtedly dated to the Early Medieval period in this region. As far as decorations are concerned, various punctated impressions exclusively on handmade crude pottery were also encountered at many sites (Figs. 8.14: 5, 8.17: 3, 8.21: 3-5, 10), characteristic of the Early Medieval period (possibly prior to the 9th century AD).

Among the glazed ceramics, dark green monochrome wares are common. These rarely have any definite decorative motifs like paint or incision and are often glazed on the interior surface and around the rim (Figs. 8.18: 1, 7, 8.20:2). An exception to this rule involves a single example with a glazed outer surface (Fig. 8.21: 7). This kind of monochrome glazed ware probably dates to around the 9th to 10th centuries AD. A base fragment of polychrome glazed ceramic with green bands on buff yellow glaze (Fig. 8.18: 4) and a severely eroded sherd of grey-black on dark yellow glazed ware (Fig. 8.18: 5) are different types obtained from TQ017. In particular, the former type belongs to the so-called "early glazed ware" or tri-colored glazed pottery (Dostiyev 2008: table 65. 4).

Among the surface finds from TQ017, a single blue glass bracelet with a triangular section is included (Fig. 8.18: 11). Similar glass ornaments have been unearthed from many Early Medieval sites in Azerbaijan (Dostiyev 2008: table 96.18). Given that the great majority of finds constitutes relatively later Early Medieval ceramics, it is likely that most settlements were established and densely occupied in the late 1st millennium AD. The distribution of sites across the entire region shows that since the previous 

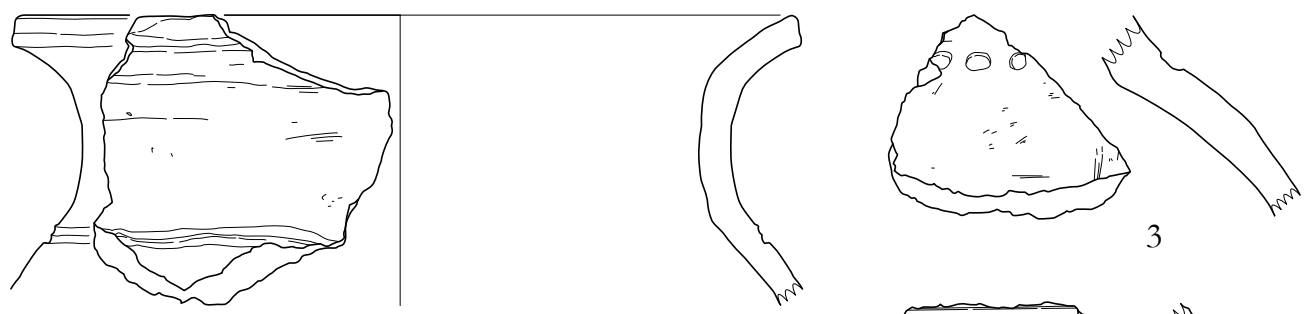

3
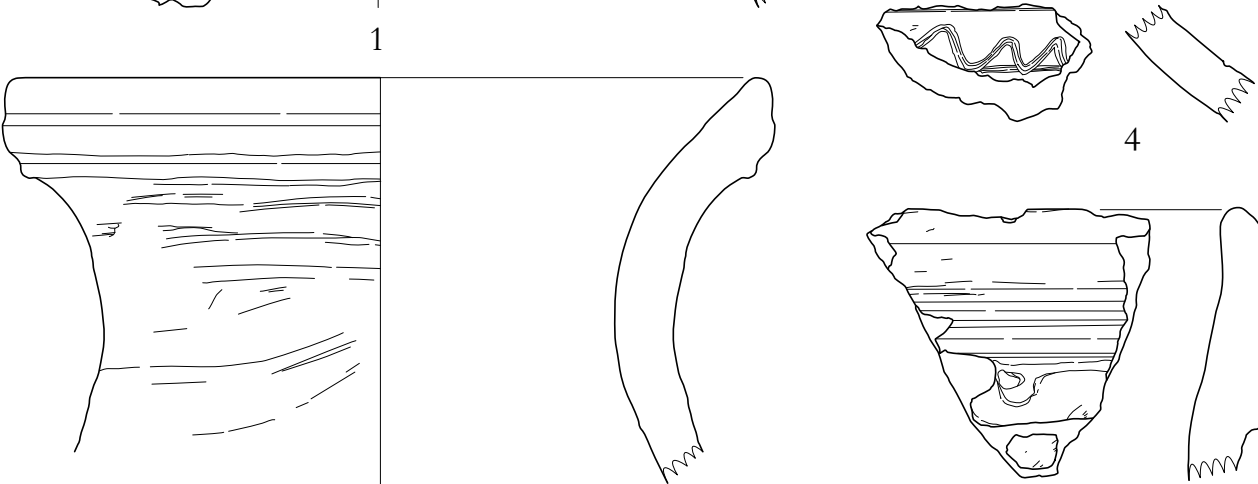

4

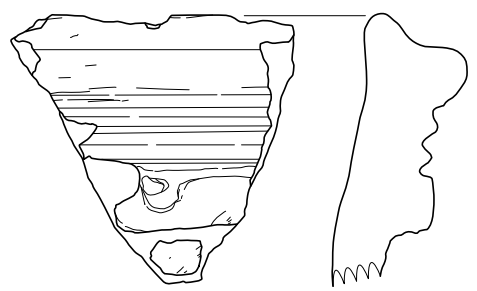

5

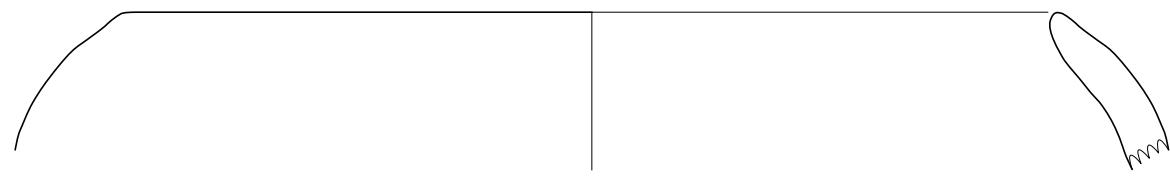

6

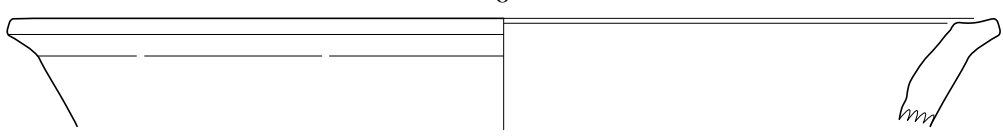

7
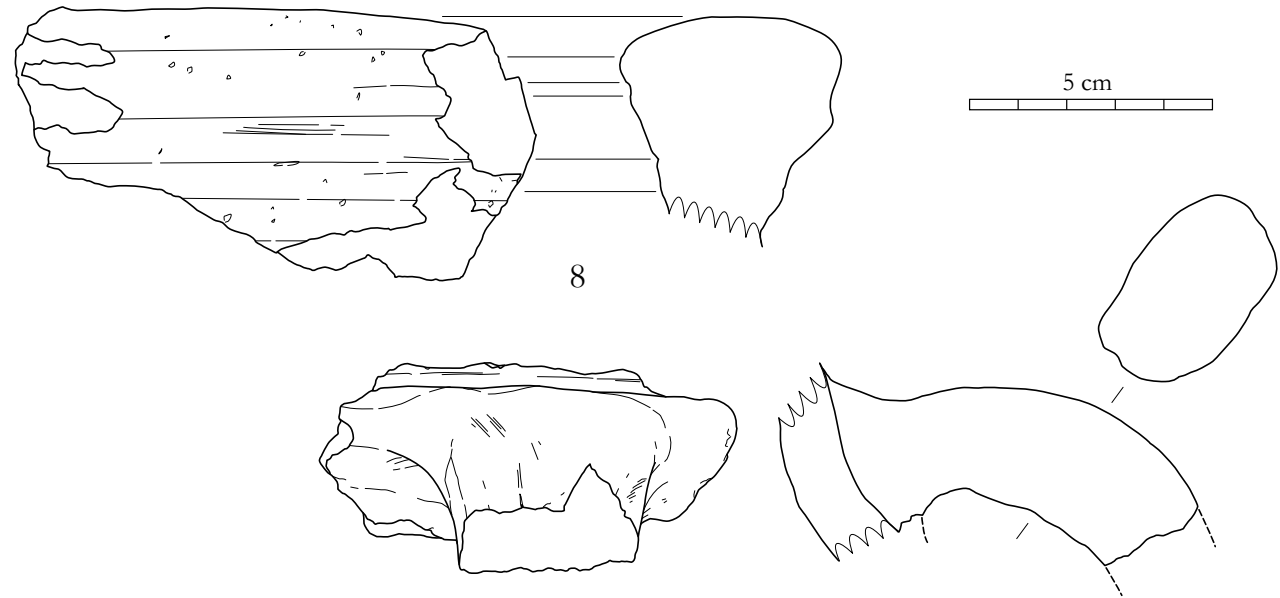

9

Fig. 8.17 Pottery from the Tovuz-Qovlar Survey in 2011. 1-9: TQ016.

time period, one group of settlements appears to form a cluster on the left bank of the lower Zayam River in the north, while others are more interspersed and persist in the piedmont zone to the south. This somewhat bimodal distribution may suggest different land use patterns between each group at that time.
Evidence of funerary practices of this period have so far been shown only in the lowland area. At KP356, a Muslim cemetery consisting of more than seventy graves was unearthed, which was attributed to the 9th and 10th centuries AD by the excavators (Museibli and Kvachidze 2006). Site TQ004 in our 

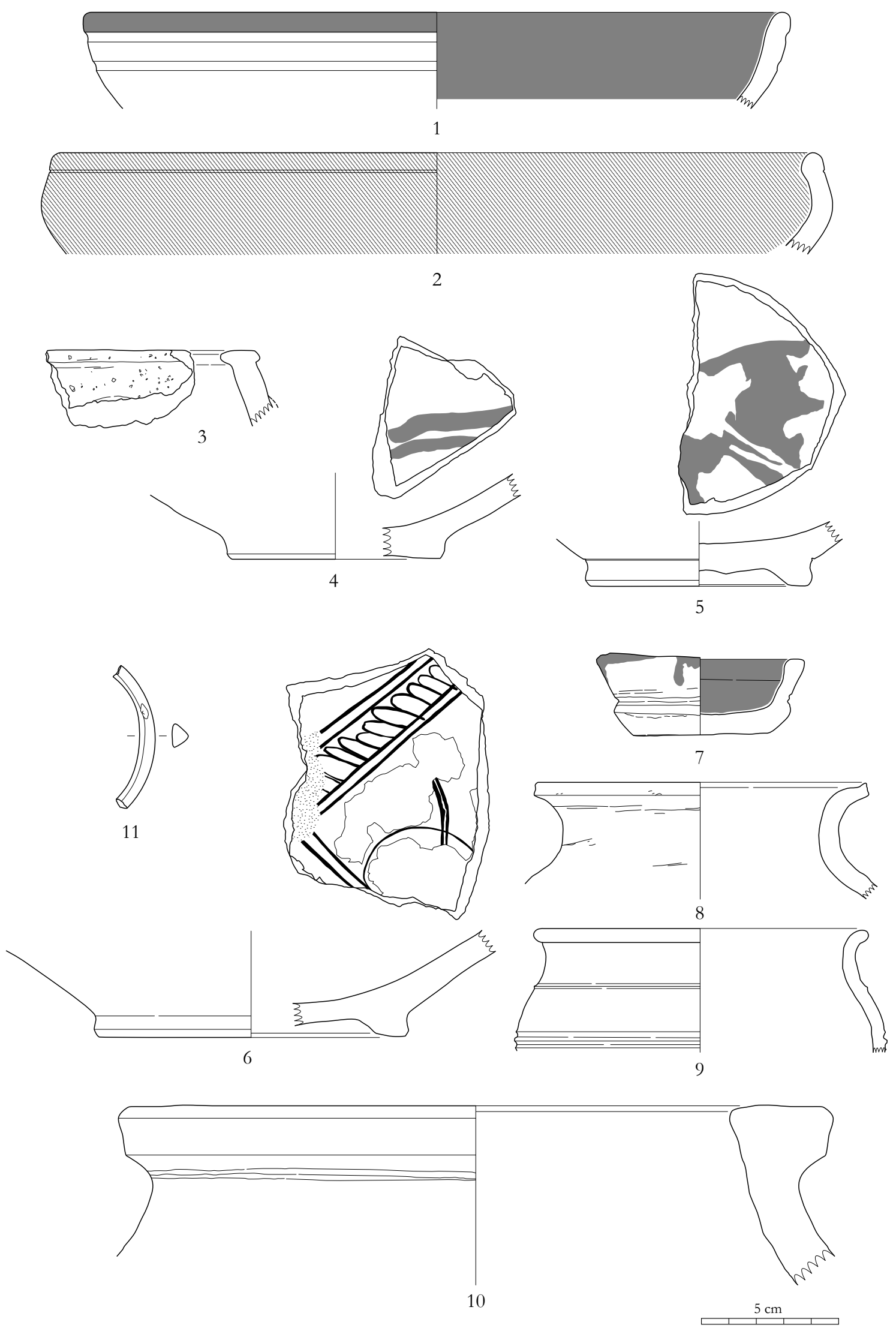

Fig. 8.18 Pottery and small finds from the Tovuz-Qovlar Survey in 2011. 1-10: TQ017. 

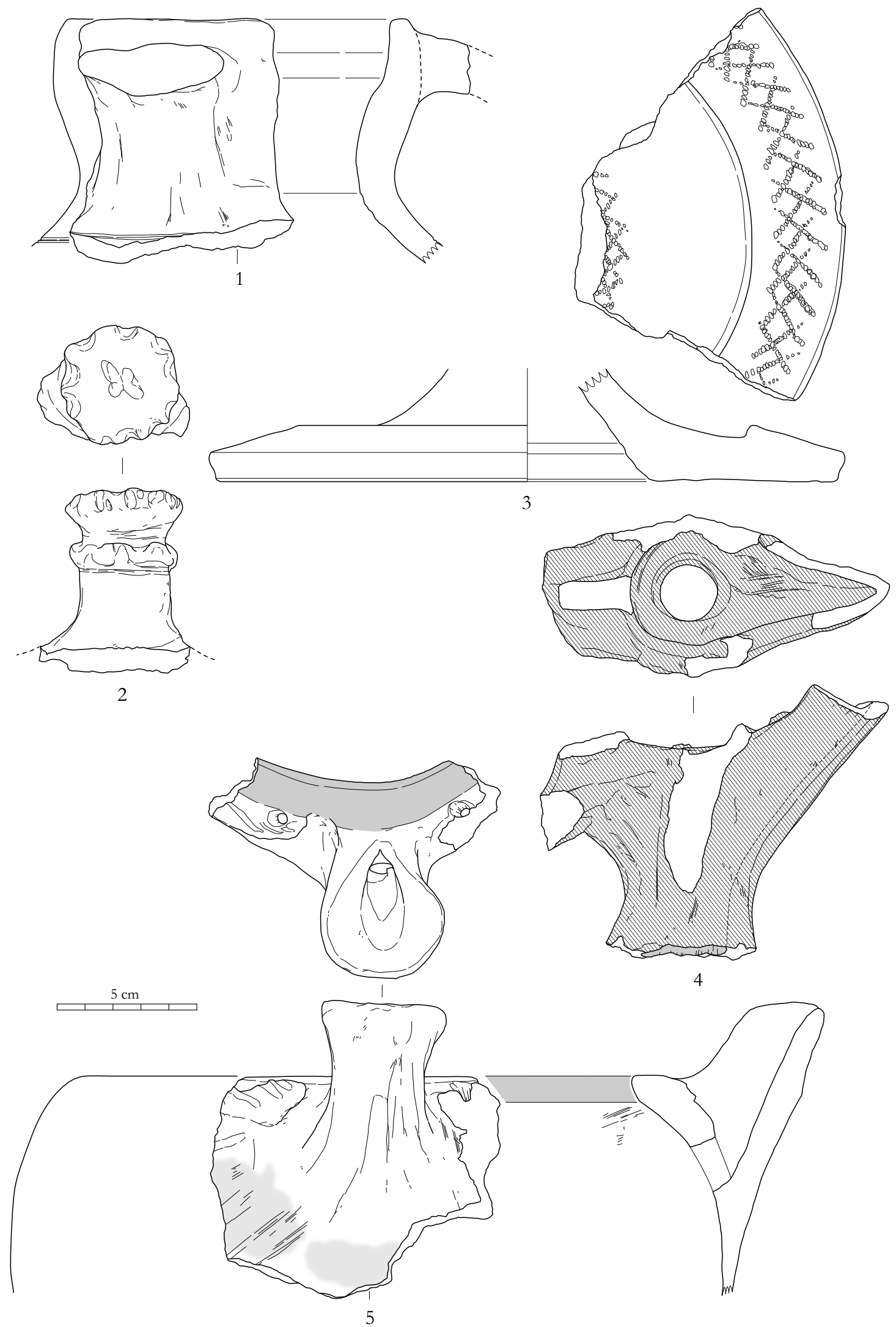

Fig. 8.19 Pottery from the Tovuz-Qovlar Survey in 2011. 1-5: TQ017 (continued). 

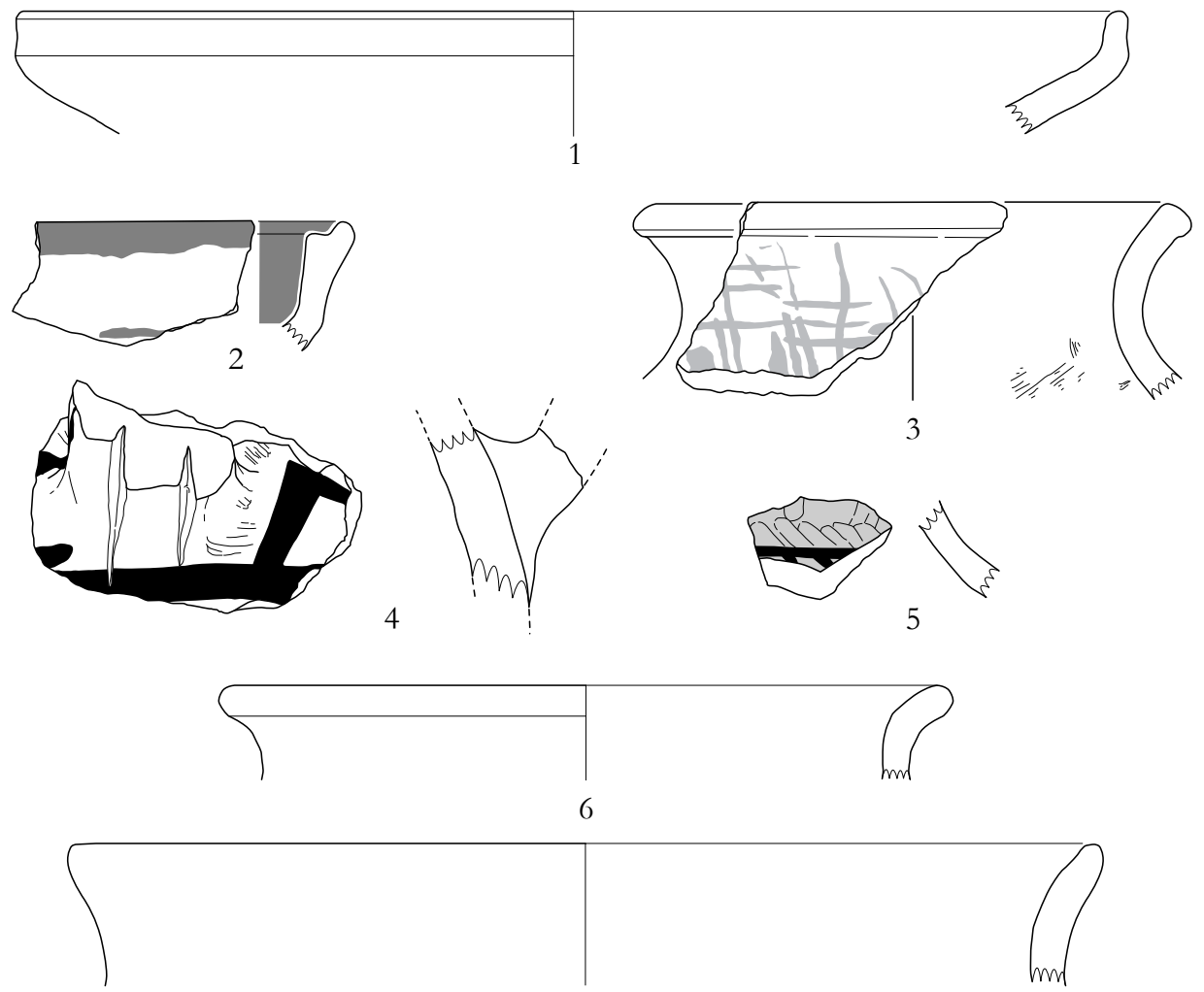

7

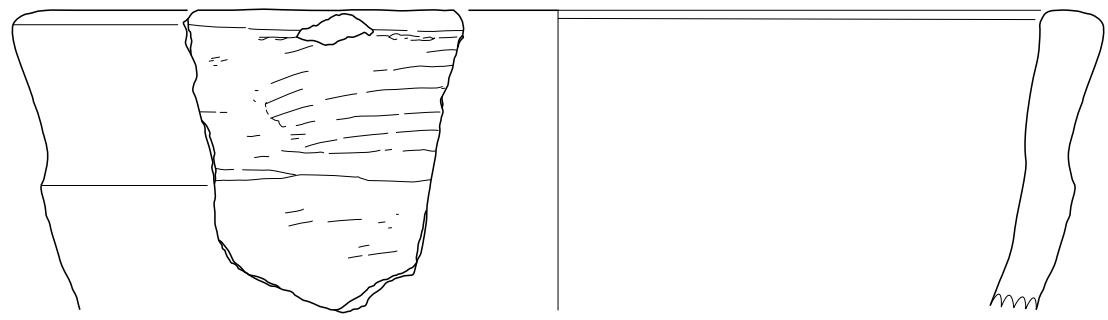

8

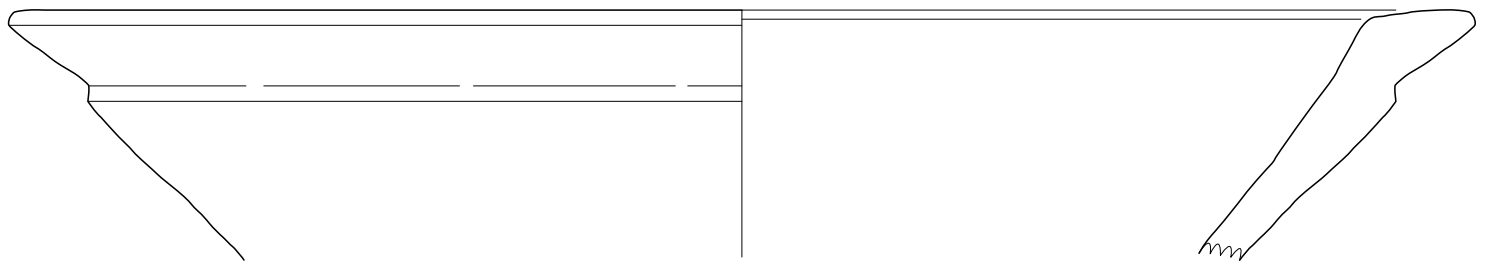

9
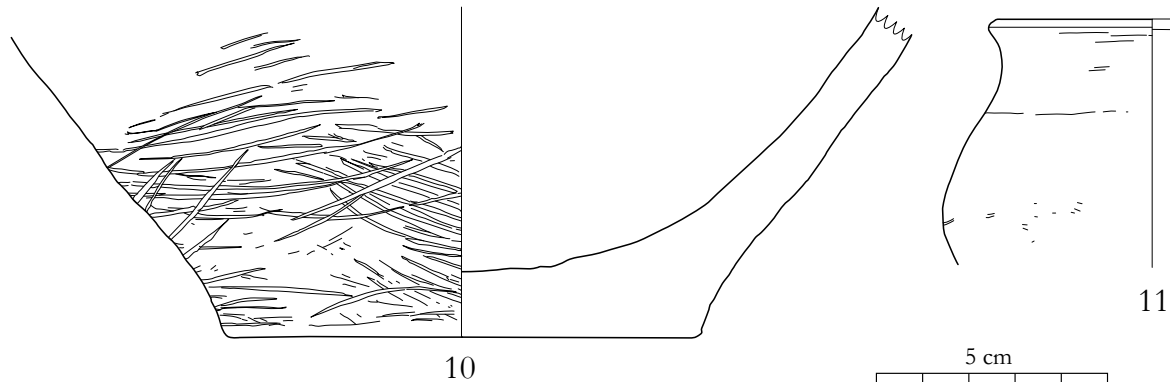

10

$5 \mathrm{~cm}$

Fig. 8.20 Pottery from the Tovuz-Qovlar Survey in 2011. 1-4: TQ018; 5: TQ019; 6-11: TQ020. 

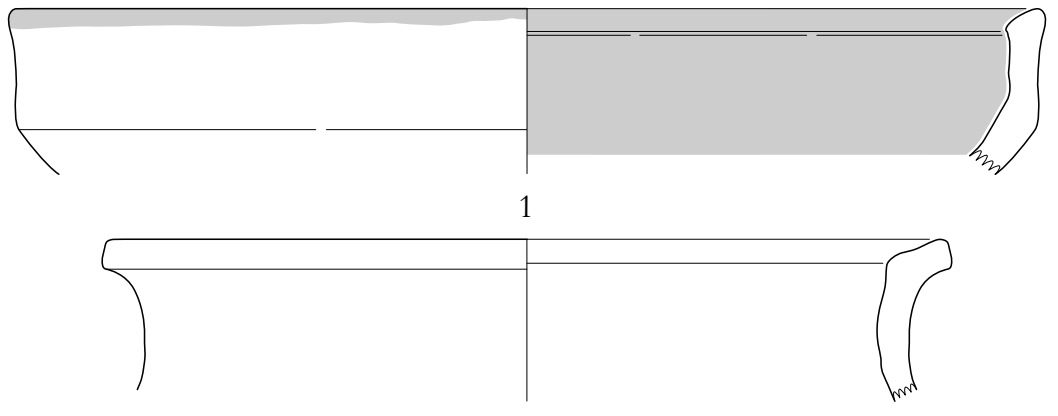

2

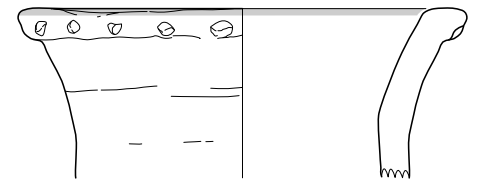

3

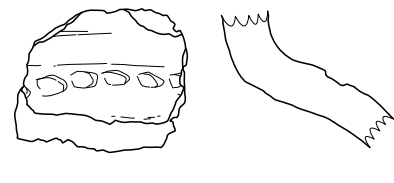

4

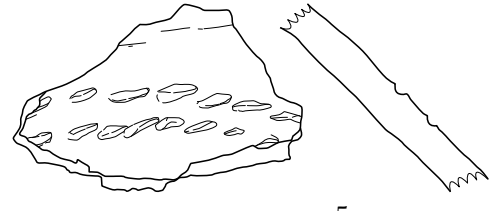

5

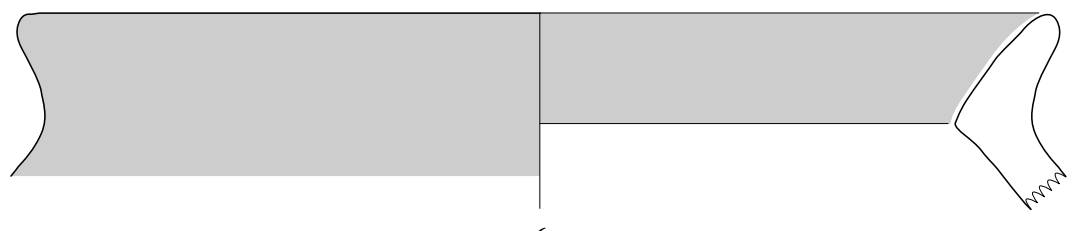

6
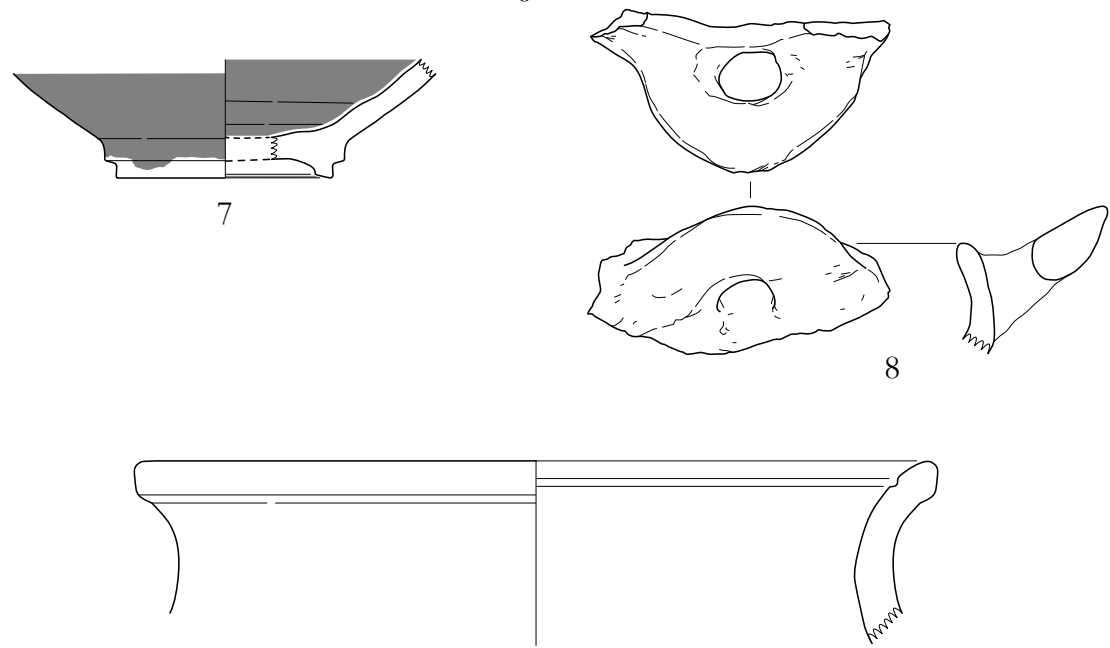

9

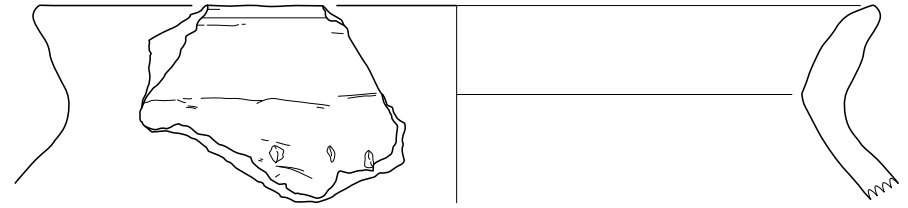

10

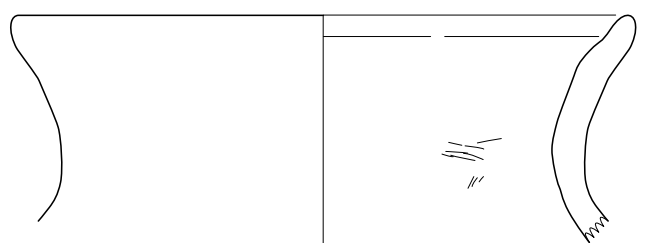

11

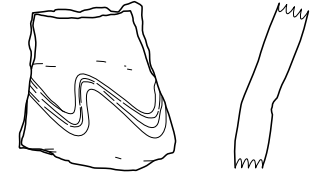

12

Fig. 8.21 Pottery from the Tovuz-Qovlar Survey in 2011. 1-8: TQ028; 9-12: TQ029. 
survey may also be part of a cemetery; round cobbles between 20 and $30 \mathrm{~cm}$ in size were exposed on the river terrace, probably showing the existence of grave stones. Only a few isolated sherds collected near the stones show some affinity with the Antique period pottery. It is possible that settlement sites were located near this place in the lower Tovuz River, considering that KP356 cemetery had a nearby site cluster in the lower Zayam River region.

\subsubsection{Late Medieval period}

In the Late Medieval period (Fig. 8.2: f), whose onset was marked by Mongol invasions, numerous settlements were abandoned and their overall number dwindled dramatically to only one site (TQ017) in this region. This site is represented by a green glazed sherd with black geometric decorations (Fig. 8.18: 6 ), showing the approximate date of the 12th-13th centuries that should correspond to the very beginning of the Late Medieval period (a similar glazed bowl is illustrated in Dostiyev [2008: table 147.3], though the geometric designs on this item consist of incised lines). Another possible addition to this inventory is a rim fragment of fine orange ware from TQ021 (Fig. 8.16: 1), so rare a find that no analogous examples were found at other sites.

\subsubsection{Archaeological sites of unidentified age}

Other than the archaeological sites with partially or well-established radiocarbon chronologies, some sites of unknown age are mentioned in this section.

Site TQ005 lies on a hill in the lower Tovuz River region, an area locally known as Topdaghdaghan (Figs. 8.1 and 8.22). This mound-like feature is composed of a large quantity of rounded cobbles, but whether this is a human-made or natural hill remains unknown at the time of our visit, since no artifacts were detected nearby. However, site TQ006 was identified in the vicinity of this stone accumulation (Figs. 8.1 and 8.23). The mound is circular in shape, ca. $20 \mathrm{~m}$ in diameter and $1.8 \mathrm{~m}$ high, forming a true kurgan with some stones exposed on the surface. The absence of surface finds prohibits us from specifying the mound's date. But some of the mounds in the kurgan group of Topdaghdaghan were recently excavated by the Azerbaijani team in 2013, and they

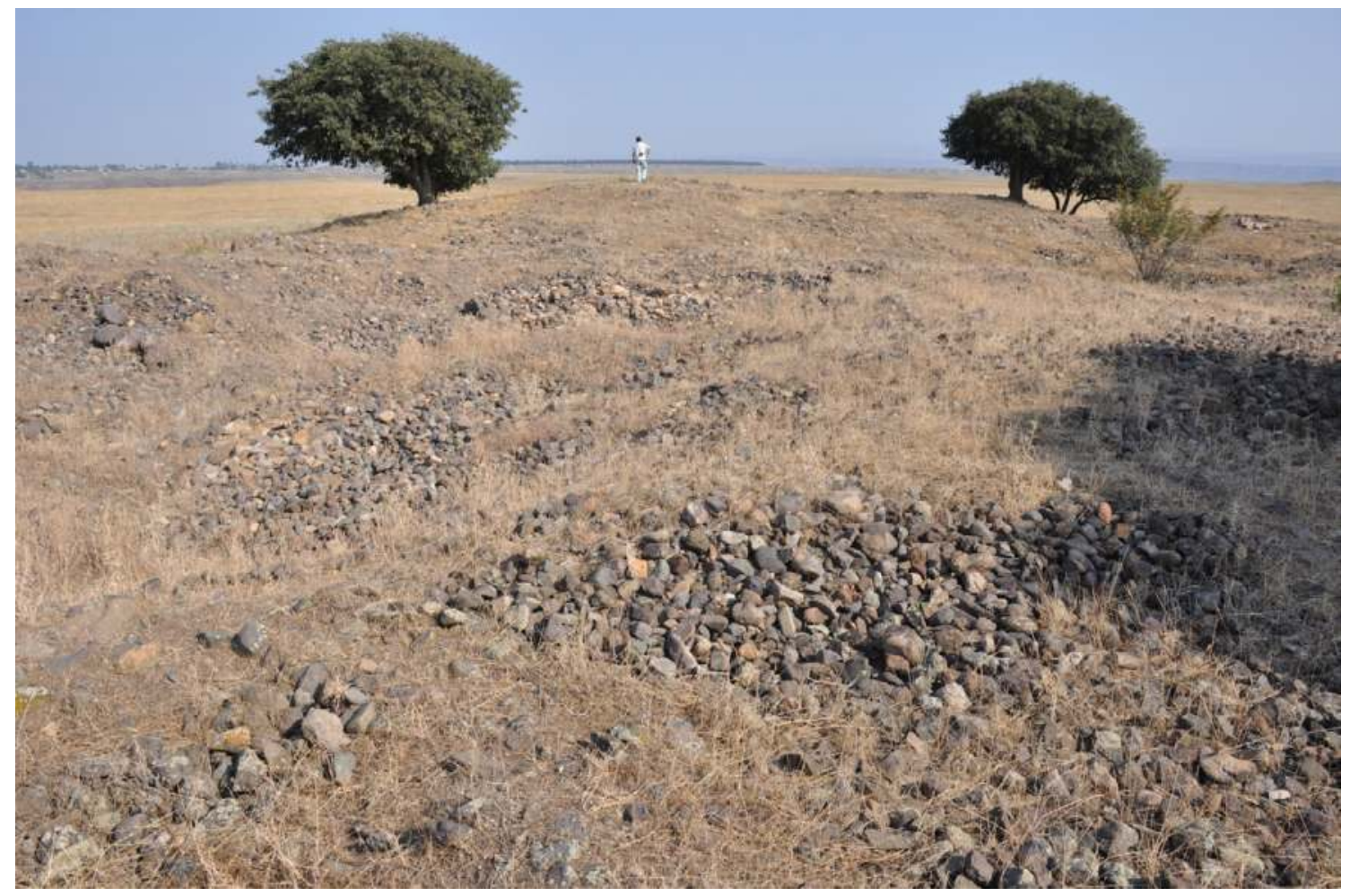

Fig. 8.22 Site TQ005, seen from the south. 


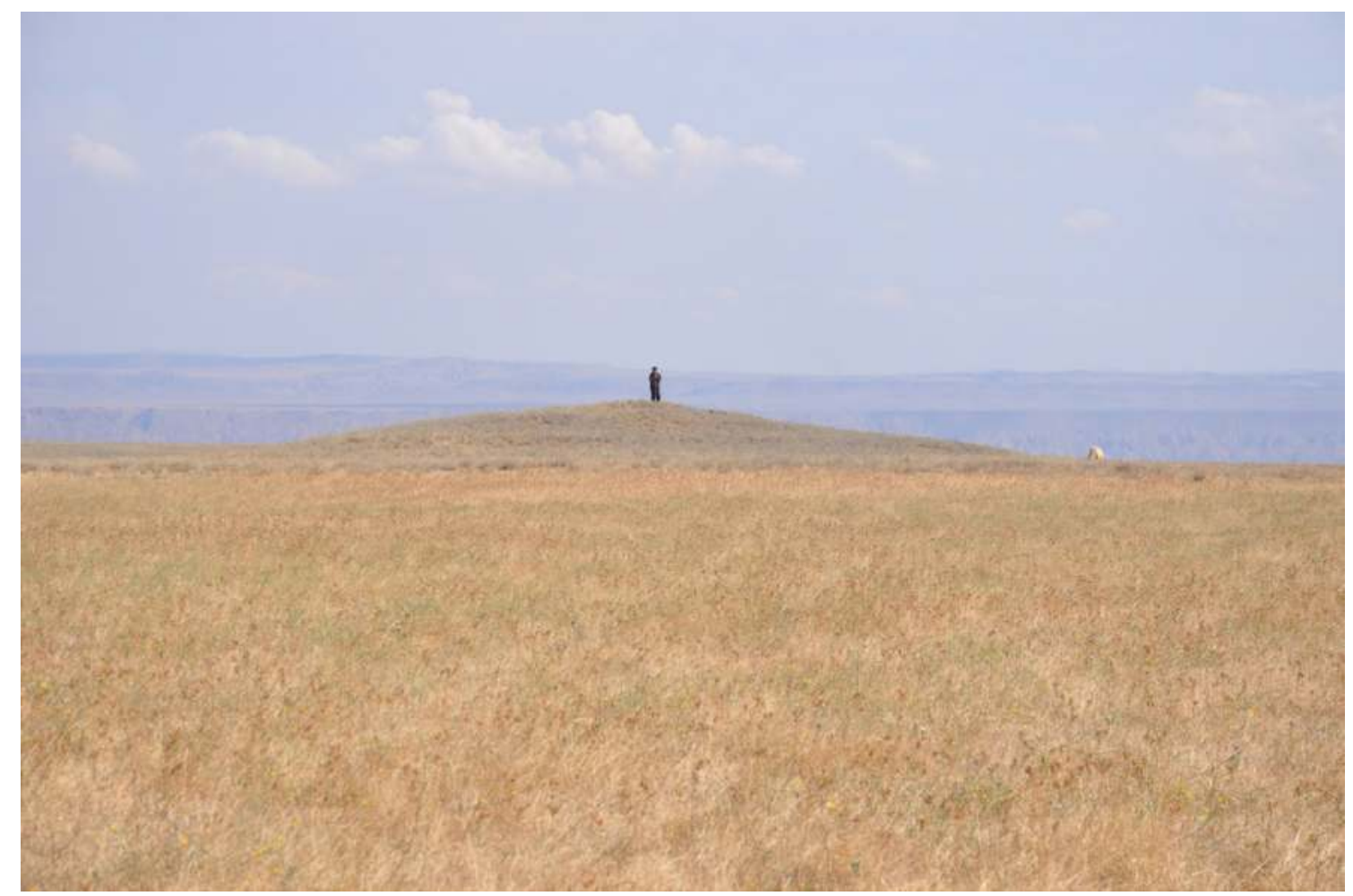

Fig. 8.23 Site TQ006, seen from the southwest.

revealed that on the basis of the associated finds and radiocarbon dates the burial mounds were dated to the Late Bronze/Early Iron Age (Guliyev et al. 2015).

Downstream in the floodplain of the Kura River, a possible cemetery site (TQ007) was found (Fig. 8.1). Round gravels and cobbles are sparsely distributed on hill-like terrace, but no diagnostic and datable archaeological finds were collected.

Finally, TQ013 is another possible kurgan located in the piedmont area near the large settlement sites of TQ011 and TQ012 (Fig. 8.1). Several small mounds, with both circular and irregular, elongated shapes with various diameters from $5-10 \mathrm{~m}$, were identified within an area covering a few hundred meters.

\subsection{Settlement and land use patterns over time}

The results of our survey in the Tovuz area between the Zayam and Tovuz Rivers revealed nearly 30 various ancient sites and settlements. The spatial distributions of these sites by time periods demonstrate that the topographical locations of each site reflect different historical and environmental factors such as climate, resources, subsistence strategy, and socio-political or economic conditions at the time of occupations.
In the Neolithic and Chalcolithic, most of the settlements are sporadically found on the flat alluvial fans within the confines of $350-450 \mathrm{~m}$ a.s.l. This suggests that subsistence activities such as agriculture and animal husbandry were primarily centered around this zone during these periods. After a hiatus in settlement in the Early and Middle Bronze Ages, sizeable settlements were established over a much wider area by the Late Bronze and Early Iron Ages. The lower parts of alluvial fans that were settled in the preceding periods were now supposedly utilized as funerary areas. More importantly, a series of large mounded sites provisionally interpreted as microregional centers appeared for the first time in this region, all of which tend to be positioned along a line at $500 \mathrm{~m}$ a.s.1. In the subsequent Antique and Early Medieval periods, the surveyed area was more extensively inhabited. Some of the settlements of these periods may have been resettled on the large mounds of the Late Bronze and Early Iron Ages, and some settlement clusters of various sizes that can be identified to the northeast also appeared. Yet most areas in the flat alluvial lands were occupied less intensively than in the preceding period and many sites were finally abandoned in the Late Medieval period. 
In terms of geomorphology, settlement sites of any period do not lie in the immediate vicinity of major rivers like the Tovuz, Esrik, and Zayam. The floodplains in river valleys could have been difficult places to live due to the flooding or riverbed fluctuations that occurred in the Holocene. This fact probably influenced humans to build their settlements on alluvial fans during all cultural periods. This general tendency suggests that the regional settlement pattern might have changed in connection with water table fluctuations (Chapter 2). At the same time, it should be noted that cultural factors such as major communication routes or defensive requirements greatly influenced the location of settlements. However, the current stage of research does not allow us to address this issue in more depth. Large-scale excavations planned for the future will hopefully provide the relevant data.

Another important fact is that very few sites were continuously settled or resettled after abandonment in a given period, excluding periods later than the Late Bronze and Early Iron Ages. This settlement pattern is in strong contrast with evidence from the Near East, where elevated mounds with considerable cultural deposits were often found, particularly in the historical periods. Examining the interrelation and long-term transformation of subsistence strategy, resource management, and occupation pattern in more detail will shed new light on many issues involving the developing settlement pattern in the Tovuz-Qovlar region.

\subsection{Concluding remarks}

It should be emphasized that this chapter presents us our preliminary results of the 2011 survey season, which were supplemented by the subsequent seasons. Nevertheless, distinctive diachronic patterns are apparent in terms of the distribution of settlements and tepe sites. In order to study the settlement history of the Tovuz-Qovlar region more extensively, some research problems must be solved. First, a refined chronology is urgently needed for material remains, especially pottery assemblages from excavated sites. This would allow more accurate dating of the surface finds and help us to consider the broader historical implications of the survey data. Second, further investigations will rectify some potential biases that exist as the result of methodological limitations, like the detection of non-tepe sites and the rather restricted area covered in this survey research.

\section{Bibliography}

Akhundov, T. (2007) Sites de migrants venus du Proche-Orient en Transcaucasie. In: Les Cultures du Caucase (Vle-Ille Millénaires avant Notre Ere): Leur Relations avec le Proche-Orient, edited by B. Lyonnet, pp. 95-121. Paris: Éditions Recherche sur les Civilisations.

Ashurov, S. (2007) Zayamchai Report on Excavations of a Catacomb Burial at Kilometre Point 355 of BakuTbilisi-Ceyhan and South Caucasus Pipelines Right of Way. Baku: Azerbaijan National Academy of Sciences, Institute of Archaeology and Ethnography.

Badalyan, R. S., A. T. Smith, and P. S. Avetisyan (2003) The emergence of sociopolitical complexity in Southern Caucasia: An interim report on the research of project ArAGATS. In: Archaeology in the Borderlands: Investigations in Caucasia and Beyond, edited by A. T. Smith and K. S. Rubinson, pp. 144-166. Los Angeles: Cotsen Institute of Archaeology, University of California.

Dostiyev, T. (ed.) (2008) Azerbaijan Archaeology. Vol. VI: Middle Ages. Institute of Archaeology and Ethnography, National Academy of Sciences of Azerbaijan. Baku: Sherg-Garb (in Azerbaijani).

Guliyev, F. E., P. P. Gasimov, F. R. Huseynov, O. Brileva, S. A. Salimbeyov, and V. A. Alakbarov (2015) The Necropolis of Topdghdaghan (2013). In: Archaeological Researches in Azerbaijan 2013 -2014, edited by M. N. Ragimova, pp. 384-393. Baku: Khazar University Publishing (in Azerbaijani).

Guliyev, F. E., P. P. Gasimov, V. A. Alakbarov, S. A. Salimbeyov, and Y. V. Mammadov (2017) Archaeological excavations at Göytepe ancient settlement. In: Archaeological Researches in Azerbaijan 2015-2016, edited by M. N. Ragimova, pp. 62-75. Baku: Institute of Archaeology and Ethnography, National Academy of Sciences of Azerbaijan (in Azerbaijani).

Hansen, S., G. Mirtskhulava, K. Bastert-Lamprichs, J. Görsdorf, D. Neumann, M. Ullrich, I. Gatsov, and P. Nedelcheva (2008) Aruchlo 2007. Bericht über die Ausgrabungen im Neolithischen Siedlungshügel. Archäologische Mitteilungen aus Iran und Turan, 39: 1-30.

Huseynov, M. M. and B. M. Jalilov (2007) Xocakhan Settlement. Azerbaijan Archaeology and Ethnography 2007(2): 42-56 (in Azerbaijani).

Jeferov, E. (2008) Azerbaijan Archaeology. Vol. I: Stone Age. Institute of Archaeology and Ethnography, 
National Academy of Sciences of Azerbaijan. Baku: Sherg-Garb (in Azerbaijani).

Kiguradze, T. and A. Sagona (2003) On the origins of the Kura-Araxes cultural complex. In: Archaeology in the Borderlands: Investigations in Caucasia and Beyond, edited by A. T. Smith and K. S. Rubinson, pp. 38-94. Los Angeles: Cotsen Institute of Archaeology, University of California.

Lyonnet, B. (2017) Mentesh Tepe 2012-2014. The Pottery. In: The Kura Projects: New Researches on the Later Prehistory of the Southern Caucasus, edited by B. Helwing, T. Aliyev, B. Lyonnet, F. Guliyev, S. Hansen, and G. Mirtskkhulava, pp. 141151. Berlin: Dietrich Reimer Verlag.

Lyonnet, B., T. Akhundov, K. Almamedov, L. Bouquet, A. Courcier, B. Jellilov, F. Huseynov, S. Loute, Z. Makharadze, and S. Raynard (2008) Late Chalcolithic kurgans in Transcaucasis: The cemetery of Soyuq Bulaq (Azerbaijan). Archäologische Mitteilungen aus Iran und Turan, 40: 27-44.

Lyonnet, B. and F. Guliyev (2009) Mission «Boyuk Kesik», Azerbaïdjan. Rapport 2009. Preliminary Report of the Franco-Azeri Mission. Unpublished report.

Lyonnet, B. and F. Guliyev (2012) Mentesh Tepe. In: Ancient Kura 2010-2011: The First Two Seasons of Joint Field Work in the Southern Caucasus, edited by B. Lyonnet, F. Guliyev, B. Helwing, T. Aliyev, S. Hansen, and G. Mirtskhulava, pp. 86-21.

Lyonnet, B. and F. Guliyev (2017) Mentesh Tepe (Azerbaijan), a preliminary report on the 2012-2014 excavations. In: The Kura Projects: New Researches on the Later Prehistory of the Southern Caucasus, edited by B. Helwing, T. Aliyev, B. Lyonnet, F. Guliyev, S. Hansen, and G. Mirtskkhulava, pp. 125140. Berlin: Dietrich Reimer Verlag.

Lyonnet, B., F. Guliyev, B. Helwing, T. Aliyev, S. Hansen, and G. Mirtskhulava (eds.) (2012) Ancient Kura 2010-2011: The First Two Seasons of Joint Field Work in the Southern Caucasus. Archäologische Mitteilungen aus Iran und Turan, 44: 1-190.

Marro, C. (2007) Upper-Mesopotamia and Transcaucasia in the Late Chalcolithic period (4000-3500 BC). In: Les Cultures du Caucase (Vle-llle Millénaires avant Notre Ere): Leur Relations avec le Proche-Orient, edited by B. Lyonnet, pp. 77-94. Paris: Éditions Recherche sur les Civilisations.

Munchaev, R. M. and S. O. Amirov (2012) Еще раз о Месопотамско-Кавказских связах в IV -III тыс. до н.э. Российская Археология 2012(4): 37-46.

Museibli, N. and V. Kvachidze (2006) Zayamchai Cemetery Report on Excavations of a Muslim Cemetery at Kilometre Point 356 of Baku-TbilisiCeyhan and South Caucasus Pipelines Right of Way. Baku: Azerbaijan National Academy of Sciences, Institute of Archaeology and Ethnography.

Museibli, N., G. Agayev, S. Ashurov, I. Aliyev, M.
Huseynov, Sh. Najarov, and F. Guliyev (2008) Tovuzchai Necropolis Report on Excavations of Tovuzchai Necropolis at Kilometre Point 378 of Baku-Tbilisi-Ceyhan and South Caucasus Pipelines Right of Way. Baku: Azerbaijan National Academy of Sciences, Institute of Archaeology and Ethnography.

Narimanov, I. (1987) The Culture of the Most Ancient Farming and Stock-Breeding Population of Azerbaijan. Baku: National Academy of Sciences (in Russian with an English summary).

Narimanov, I. G., T. I. Akhundov, and N. G. Aliyev (2007) Лейлатепе: Поселение, Традиция, Этап в Этно-культурной Истории Южного Кавказа. Baku: Издательство Элм.

Nishiaki, Y., F. Guliyev, S. Kadowaki, Y. Arimatsu, Y. Hayakawa, K. Shimogama, T. Miki, C. Akashi, S. Arai, and S. Salimbeyov (2013) Hacı Elamxanlı Tepe: Excavations of the earliest Pottery Neolithic occupations on the Middle Kura, Azerbaijan, 2012. Archäologische Mitteilungen aus Iran und Turan, 45: 1-25.

Nishiaki, Y., F. Guliyev, and S. Kadowaki (2015a) Chronological contexts of the earliest Pottery Neolithic in the South Caucasus: Radiocarbon dates for Göytepe and Hacı Elamxanlı Tepe, Azerbaijan. American Journal of Archaeology, 119(3): 279-294.

Nishiaki, Y., F. Guliyev, S. Kadowaki, V. Alakbarov, T. Miki, S. Salimbeyov, C. Akashi, and S. Arai (2015b) Investigating cultural and socioeconomic change at the beginning of the Pottery Neolithic in the Southern Caucasus: The 2013 excavations at $\mathrm{Hacl}$ Elamxanlı Tepe, Azerbaijan. Bulletin of the American Schools of Oriental Research, 374: 1-28.

Nishiaki, Y., F. Guliyev, S. Kadowaki, and T. Omori (2018) Neolithic residential patterns in the southern Caucasus: Radiocarbon analysis of rebuilding cycles of mudbrick architecture at Göytepe, west Azerbaijan. Quaternary International, 474: 119-130.

Palumbi, G. and C. Chataigner (2014) The Kura-Araxes culture from the Caucasus to Iran, Anatolia and the Levant: between unity and diversity. A synthesis. Paléorient, 40(2): 247-260.

Palumbi, G., F. Guliyev, M. Poulmarc'h, E. Muradova, L. Cinque, J. Faßbinder, M. Scheiblecker, F. Becker, S. Gondet, and B. Lyonnet (2018) The 2017 investigations at Kiçik Tepe (Tovuz, Azerbaidjan), new data on the neolithic and chalcolithic periods in the Kura river valley. In: Abstract Book of the 11th International Congress on the Archaeology of the Ancient Near East, edited by A. Otto, p. 147. Ludwig-Maximilians-Universität Munich.

Ricci, A. (2012) Archaeological landscape studies: the Mil-Qarabağ plain and the Kvemo Kartli survey projects - a preliminary account of the first two field seasons (2010-2011). In: Ancient Kura 2010- 
2011: The First Two Seasons of Joint Field Work in the Southern Caucasus, edited by B. Lyonnet, F. Guliyev, B. Helwing, T. Aliyev, S. Hansen, and G. Mirtskhulava, pp. 127-145.

Ristvet, L., V. Bakhsheliyev, and S. Ashrov (2011) Settlement and society in Naxçıvan: 2006 excavations and survey of the Naxçivan Archaeological Project. Iranica Antiqua, 46: 1-53. 
Part II: Technology and Subsistence 



\title{
Chapter 9
}

\section{Neolithic flaked stone assemblages from Göytepe}

\author{
Yoshihiro Nishiaki
}

\subsection{Introduction}

The lithic collection described in this chapter is derived from the excavations conducted at Göytepe between 2008 and 2013. The collection consists of assemblages from the sounding (Squares $3 \mathrm{~B} / 4 \mathrm{~B}$ ) and upper (Squares $99 \mathrm{~A} / \mathrm{B}$ to $3 \mathrm{~A} / 4 \mathrm{~A}$ ) areas (see Chapter 3). Thus far, we have examined all material from the sounding area but have only partially analyzed those finds from the upper area because their stratigraphic assignment to proper architectural levels has not yet been completed. Accordingly, the material presented in this study as from the "Upper Area" indicates the part of the lithic artifacts securely assigned to Neolithic levels (Levels 1-5) of the upper squares (Table 9.1). The following accounts are based on our latest studies of the collection, which may differ in breakdowns presented in the previous publication (Nishiaki and Guliyev 2019).

\subsection{Raw material}

Obsidian is the most commonly used lithic raw material at Göytepe and comprises about $80 \%$ to $90 \%$ throughout the occupation levels $(3,911 / 4,447$; Table 9.1). In order to determine the provenance of the obsidian sources, we conducted a trace-element analysis using energy-dispersive $\mathrm{X}$-ray fluorescence (XRF) methods as described elsewhere (Nishiaki et al. 2019a). The analysis was unique in that it analyzed a complete, non-selected obsidian assemblage from one particular area of the sounding squares. The analysis yielded remarkable results as follows.

First, the use of obsidian from diversified sources is remarkable. The nearest source is Guntansar, an outcrop to the south of Lake Sevan in Armenia, about $100 \mathrm{~km}$ southwest of Göytepe across the Lesser
Caucasus Mountains. On the other hand, the farthest source area we identified was in northeast Anatolia of modern Turkey, almost $300 \mathrm{~km}$ southwest of Göytepe. Among the regions between these two sources, we identified more than 20 outcrops as potential sources that could have provided obsidian to the Göytepe communities. This result is rather unexpected as traditional views believed the Neolithic communities in the Middle Kura Valley primarily utilized the sources in Chikiani, Georgia, which is located in the province where the Shomutepe culture originated (Akhundov 2004).

Second, the analyses revealed a diachronic change in obsidian use between the earlier (Levels 14-8) and later phases (Levels 7-1), the boundary being around $5530 \mathrm{cal}$. BC (Nishiaki et al. 2018). The combined use of obsidian from the Anatolian and Lesser Caucasus mountain regions was replaced in the later phase by the almost exclusive use of obsidian from the Lesser Caucasus mountains (Fig. 9.1). However, the use of obsidian from Chikiani in Georgia, some $150 \mathrm{~km}$ west of Göytepe, remained uncommon throughout the sequence of this site. The change in location from which the Göytepe communities sourced their obsidian should be examined from multiple viewpoints. The technotypological analyses of the lithic artifacts presented in this chapter will help develop their holistic interpretation.

Lithic raw materials other than obsidian include flint (chert), quartzite, tuff, mud stone, slate, and andesite. Most of these were likely procured from secondary sources within a range of $10 \mathrm{~km}$ from Göytepe (Chapter 1). However, our field surveys suggest that sources of reddish-brown flint were situated more remotely, in the upstream area of the 


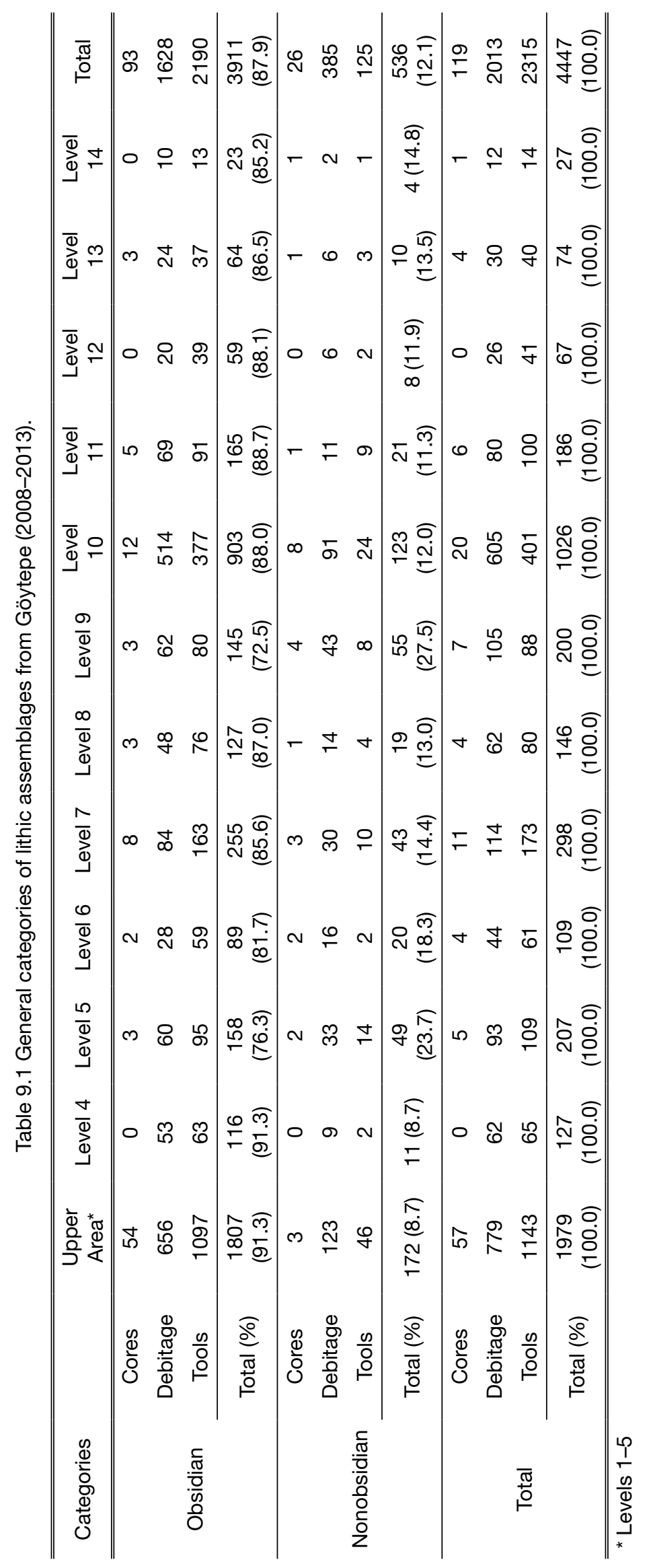




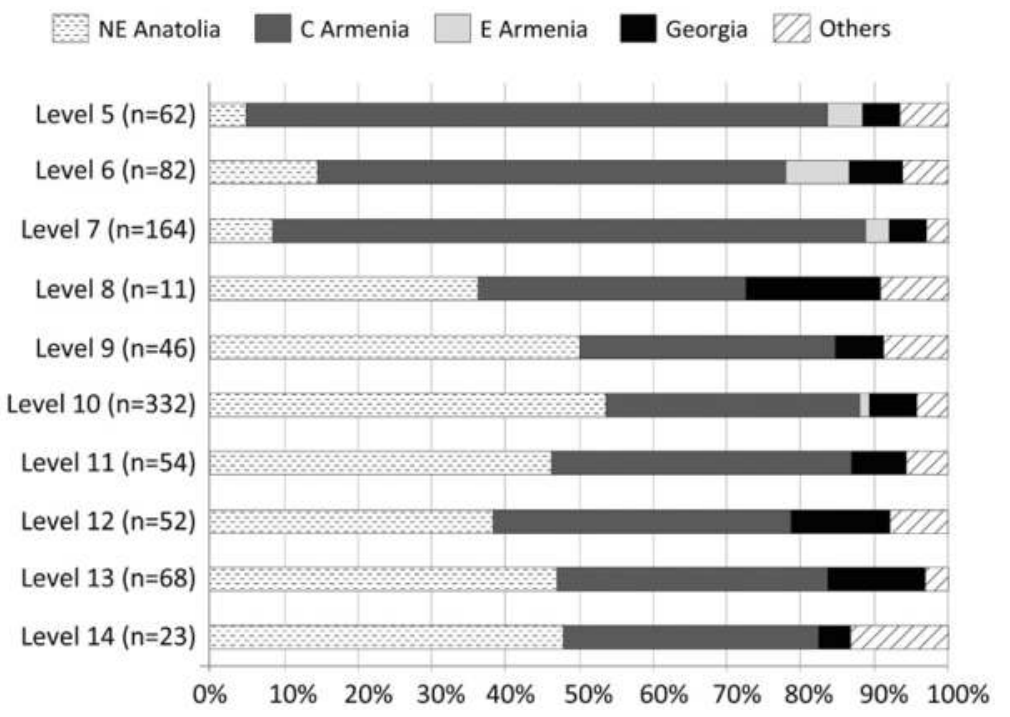

Fig. 9.1 Stratigraphic changes in the use of obsidian at Göytepe. Northeast Anatolia (Cildir Golu, Sarikamis); Georgia (Chikiani); Central Anatolia (Geghasar, Spitakasar, Hatis, Gutansar, Ttvakar, Damlik, Arteni); East Anatolia (Satanakar, Sevkar) (after Nishiaki et al. 2019a).
Agstafa River, some $40 \mathrm{~km}$ or more from Göytepe (Kadowaki et al. 2016). As they exhibit marked differences in techno-typology as well as use patterns, we describe obsidian and non-obsidian artifacts separately below.

\subsection{Lithic assemblages}

\subsubsection{Obsidian assemblage}

\section{(1) Cores}

The samples of this study include 93 obsidian cores. As Table 9.2 shows, the largest portion is exhausted cores $(40.9 \%)$, which no longer show traces of original blank removal technology. They usually take the form of small chunks covered with flake removal scars that originate from multiple directions. The abundance of these exhausted cores is best interpreted as a result of the intensive reduction of obsidian due to its precious nature. The relatively higher occurrence of simple on-flake cores (16.1\%), which includes truncated-faceted pieces (see Nishiaki 1985), may also be interpreted in this manner. Although many cores, even well-prepared blade cores, could have been made on flake blanks, the ones in this category are distinguished by their diminutive size and the small number of blank removal scars.

Flake cores: The formal cores are divided into flake (33.3\%) and blade cores (6.5\%). Flake cores predominantly exhibit flake removal scars (Fig. 9.2). They may include cores that were originally used for blade production and later transformed into those for flake removals. We can classify the flake cores into several types according to the number and configuration of striking platforms: single- (Fig. 9.2: 1), opposed- (Fig. 9.2: 3), multiple-platform cores with two crossed-working surfaces, and multipleplatform cores with more than two working surfaces. The most common category is opposed-platform cores (Fig. 9.2: 3). One should note that the profile of these cores is more or less wedge-shaped. When the cores become small enough, they can be similar to the splintered pieces described later in (3) retouched tools.

Blade cores: All blade cores in the present collection belong to the single-platform type (Figs. 9.2: 2, 9.3 and 9.4). The blade removal scars with regular parallel ridges suggest the use of pressure debitage (Fig. 9.3). It is important to note that the working surfaces are located on one side only, which leaves the backside unexploited. The presence of tiny scars and scratches along the edges and ridges of the back may be an indication that these cores were held in a device that kept them immobile during pressuring (see Clark 2012). The relatively large size of the detached blades implied by the analysis of those removal scars and debitage composition suggests the use of a lever pressure technique (see below).

Two of the blade cores exhibit not only pressure but also percussion removal scars, which cut through the preceding pressure removals (Fig. 9.4: 1, 2). The remaining blade cores show traces of percussion debitage alone (Fig. 9.2: 2). Overall, the scarcity of blade cores in the study sample is remarkable when we consider the much higher percentage of blades in the debitage assemblages (Table 9.2). This disparity 


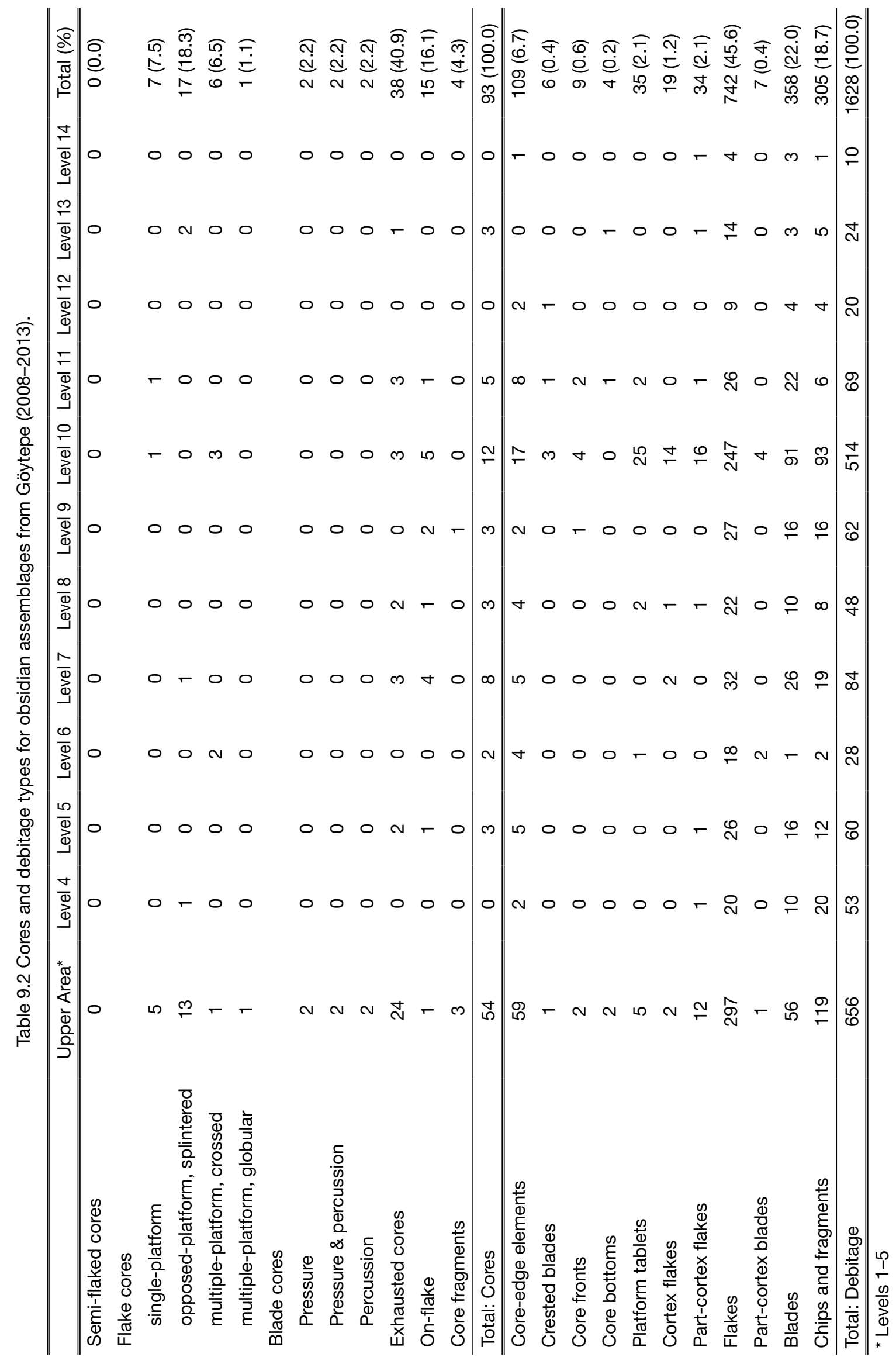



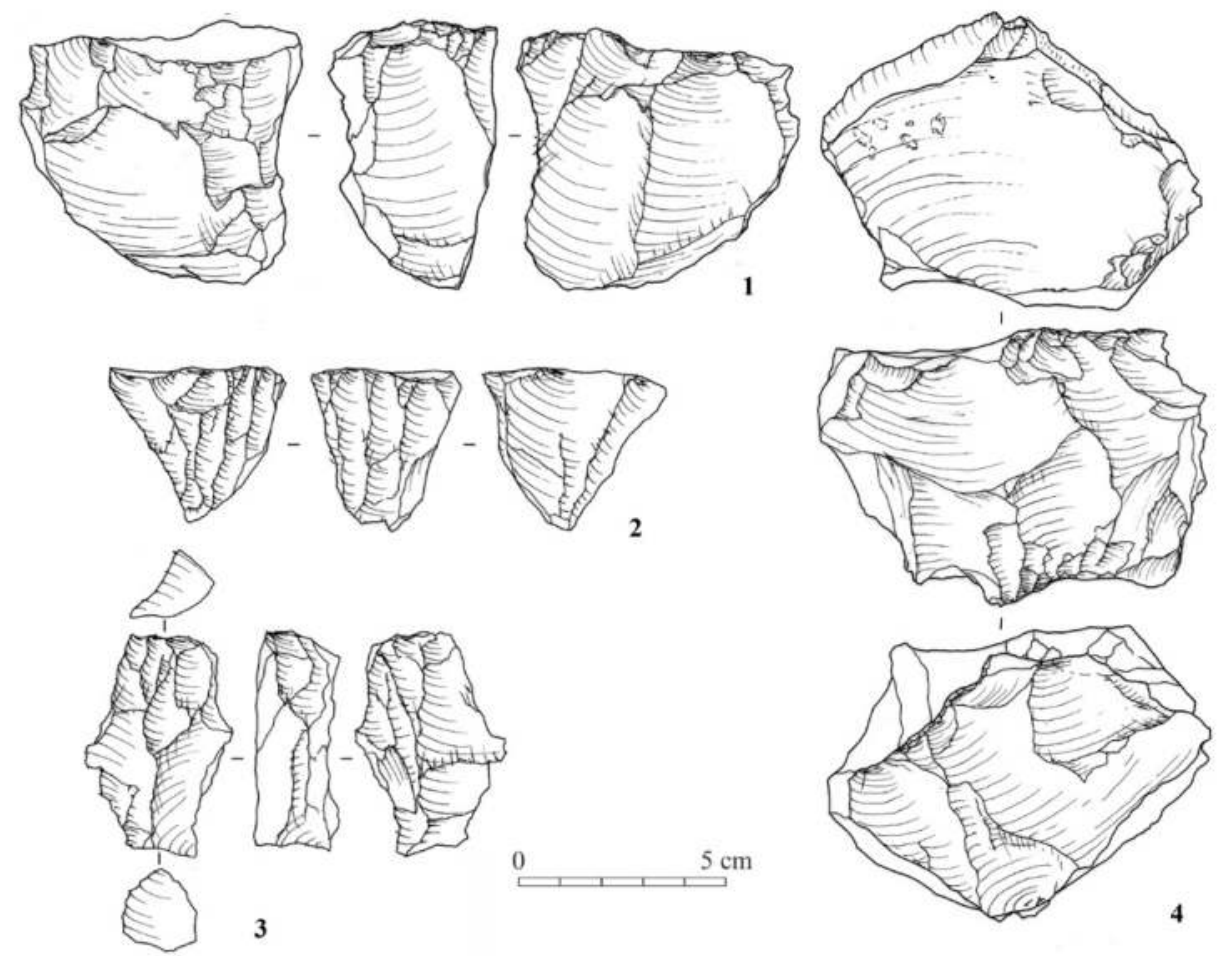

Fig. 9.2 Obsidian percussion-flaked cores from Göytepe. 1: Single-platform flake core (2B-corridor, Upper Area); 2: Single-platform blade core (3A, Upper Area); 3: Opposed-platform flake core (4BIIX-59, Level 10); 4: Multiple-platform flake core (2B, Upper Area).
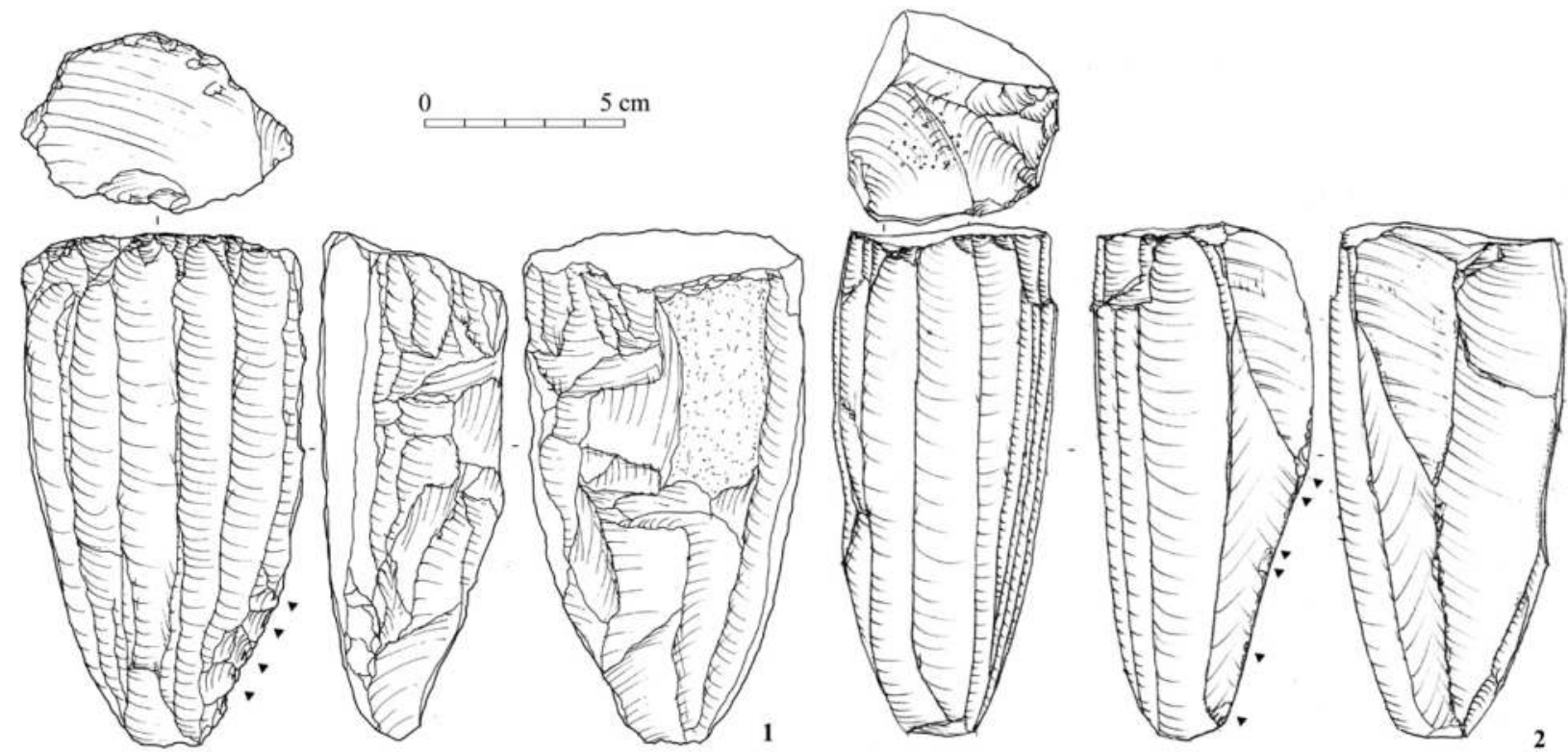

Fig. 9.3 Obsidian pressure-flaked cores from Göytepe. 1: Single-platform pressure blade core (2B-Loc. 3, Upper Area); 2: Single-platform pressure blade core (3All, Upper Area). Note that both retain edge damages (black triangles) caused by core-holding devices. 

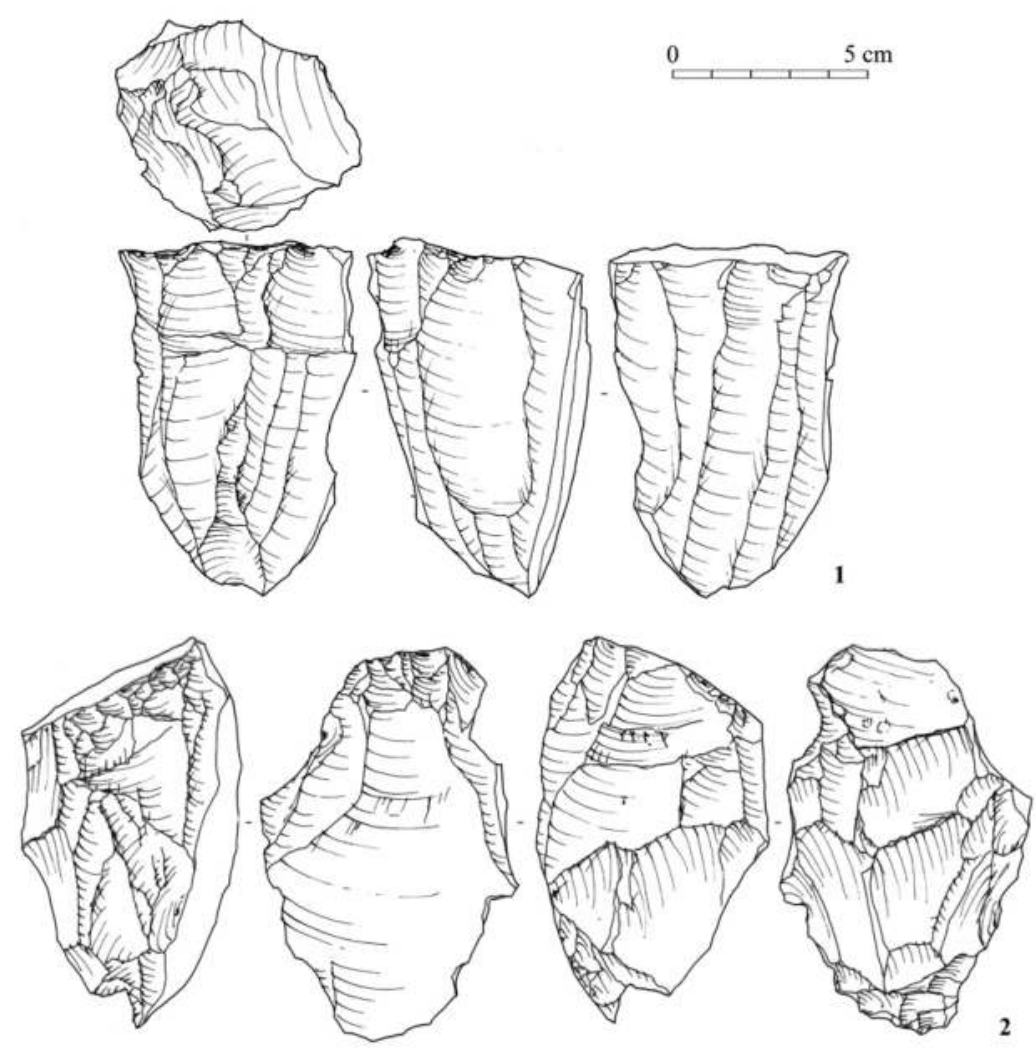

Fig. 9.4 Obsidian pressure/percussion-flaked cores from Göytepe. 1: Single-platform pressure/percussionflaked blade core (2B-Loc. 3, Upper Area); 2: Single-platform pressure blade core (1B2, Upper Area). On number 2, pressure-flaked scars running perpendicular to the core axis are visible on the back side only (far right drawing).

suggests that at least some of the flake cores in this collection could in fact represent the final forms of blade cores, whose blade removal scars were obliterated by later, nonsystematic flake detachment.

\section{(2) Debitage}

Debitage denotes two things (Inizan et al. 1999): the action of stone knapping itself, and the products that result from knapping. In the paper, this term is used in both senses. The debitage assemblage includes core management pieces that were detached in order to adjust cores for the proper production of tool blanks, as well as flakes and blades intentionally produced to manufacture tools.

Compared to the number of cores, core management pieces are rare at Göytepe, fewer than two pieces per core (159/93). Moreover, the majority of them are simple core-edge elements (Table 9.2), followed by core tablets (Fig. 9.5: 2), core fronts (Fig. 9.5: 4), crested pieces (Fig. 9.5: 1), and core bottoms (Fig. 9.5: 3). The uncommon occurrence of crested pieces, which many scholars believe were produced from the earlier stages of core reduction, may imply the practice of initial core preparation at locations near the obsidian outcrops. We can support this inference through the rarity of cortical pieces in the assemblages (cortex flakes, part-cortex flakes, and part-cortex blades; 3.7\%; Table 9.2).

The core reduction of obsidian was oriented toward blade production (Fig. 9.5: 5-9). About onethird of the blanks (i.e., flakes and blades) are blades (Table 9.2). The blade widths are shown in Fig. 9.6 by phases, which appear to follow a normal distribution with a focus on the production of blades 17 to 18 $\mathrm{mm}$ wide. The average width may have slightly decreased in the later phase (Table 9.3; $\mathrm{t}=-2.57693$; $\mathrm{df}=1230 ; \mathrm{p}=0.010084)$. Many of these blades show parallel sides and ridges, symmetrical horizontal shape, regular profile, and small butt with a diffused bulb, which all indicate the use of pressure debitage (see Inizan et al. 1999). A fracture-wing analysis confirms this interpretation, which can differentiate 

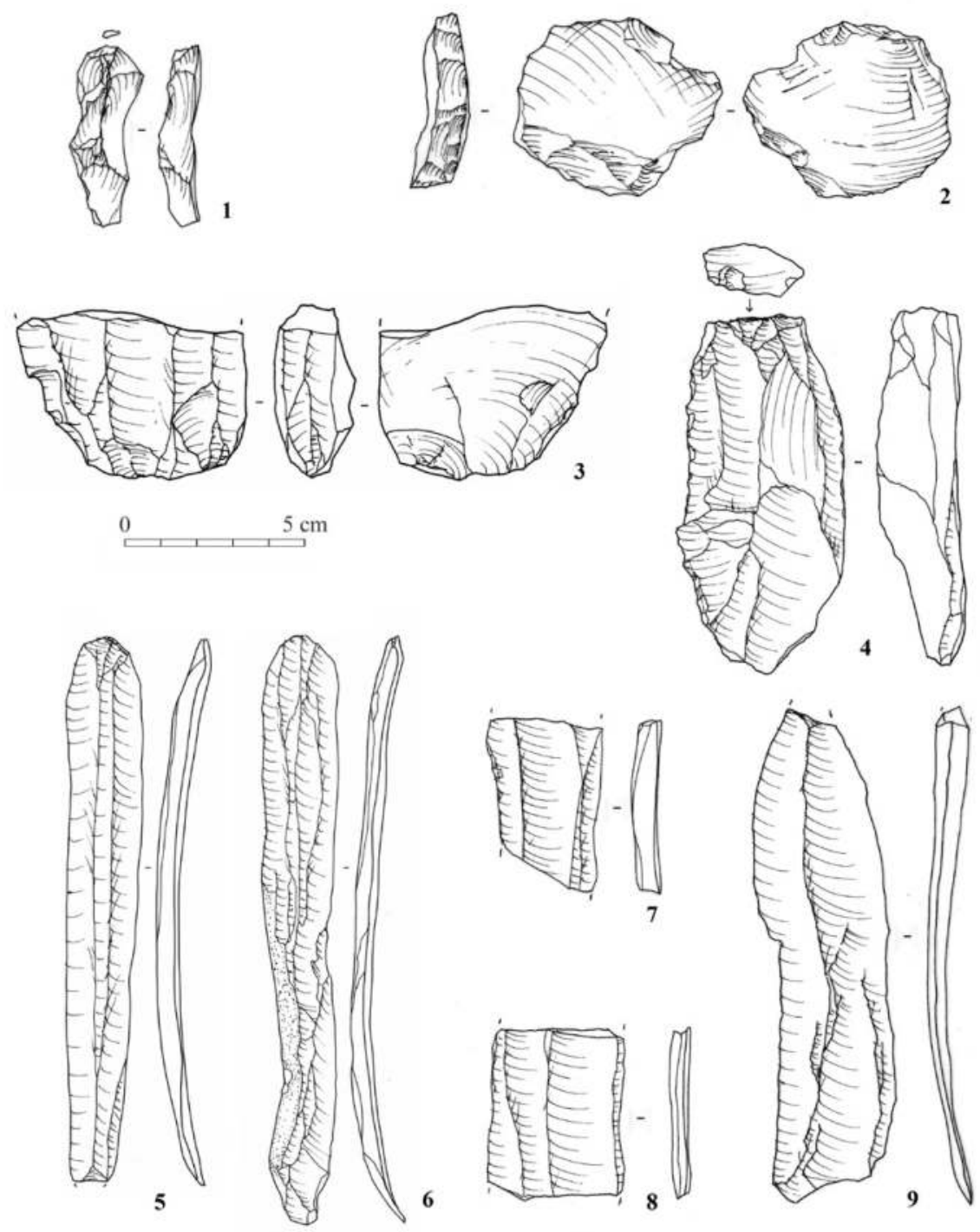

Fig. 9.5 Obsidian core management pieces from Göytepe. 1: Crested blade (99B2, Upper Area); 2: Platform tablet (3All, Upper Area); 3: Core-bottom flake (2B-Loc. 3, Upper Area); 4: Core-front flake (4BI-111S, Level 10); 5: Large blade (4A, Upper Area); 6: Large blade (Cache, Upper Area); 7: Large blade (99A1, Levels 1-5); 8: Large blade (99A2, Upper Area); 9: Large blade (Cache, Upper Area).

the pressure debitage from other sorts of debitage techniques (Chapter 11). Particularly interesting is the fact that large blades wider than $25 \mathrm{~mm}$ also show traces diagnostic to the pressure debitage. The widest blade with parallel ridges and edges measures $37 \mathrm{~mm}$ (Fig. 9.5: 8). The obsidian knappers at Göytepe may have employed lever pressuring as well as a range of other pressuring techniques (Pelegrin 2012; Chabot and Pelegrin 2012).

(3) Retouched tools

Tools made from retouched blanks, together with spalls from burin/splinter blows and unidentified tool fragments, constitute more than half of the obsidian assemblages (2190/3911; Tables 9.1 and 9.3). More than two-thirds of the tools, excluding heavily retouched pieces, are made on blade blanks (778/1093 or $71.1 \%$ ). Given that we have found blades in only about one-third of the debitage assemblages, the much higher occurrence of blade tools indicates a preference for this blank form for tool production.

Trapezes: The tools include trapeze arrowheads that were manufactured on snapped blade blanks by steep direct retouch on both lateral edges (Fig. 9.7: $1-4)$. The working edges, which remain unretouched, occasionally show "impact fractures" (Fig. 9.7: 4) 


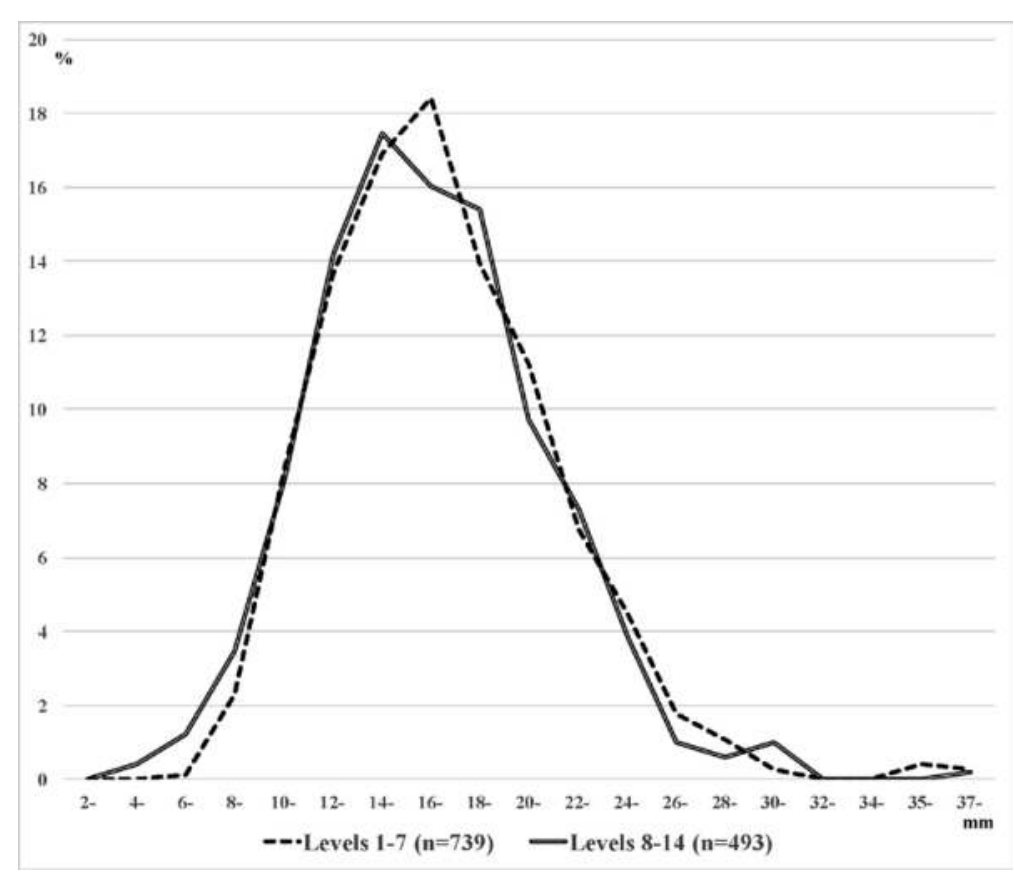

Fig. 9.6 Widths of obsidian blades from Göytepe by phases. The samples from Levels 1-7 include blades from the Upper Area.

Table 9.3 Mean widths of obsidian blades from Göytepe (2008-2013).

\begin{tabular}{lccccc}
\hline \hline & No. & Mean* & S.D. & Min & Max \\
\hline \hline Levels 1-7 & 739 & 16.82 & 4.648 & 4.5 & 39 \\
Levels 8-14 & 493 & 17.52 & 4.706 & 7.9 & 37 \\
\hline \hline Total & 1232 & 17.27 & 4.717 & 4.5 & 39 \\
\hline \hline * millimeters & & & &
\end{tabular}

* millimeters

that likely derived from their use as projectiles. These trapeze arrowheads measure 12 to $23 \mathrm{~mm}$ long and 10.3 to $24.5 \mathrm{~mm}$ wide, but their length is generally greater than their width (Nishiaki et al. 2019b: fig. 11). It is also noteworthy that some of the arrowheads exhibit invasive pressure retouching from the snapped bilateral ends on the dorsal surface (Fig. 9.7: 2 and 3). One can also find this type of retouching on the trapeze arrowheads from contemporaneous sites in the South Caucasus, including Mentesh and Kamiltepe, Azerbaijan (Gilbeau et al. 2017), and Aruchlo, Georgia (Gatsov and Nedelcheva 2017). In addition, excavations in northern Syria and the Levant have yielded almost identical pieces from the early 6th millennium BC (Copeland 1996), which signifies a cultural link between the South Caucasus and the Levant and Southwest Asia (Guliyev and Nishiaki 2014: 7).

Sickle elements: A tool falls into this category when "sickle gloss" is visible on the cutting edges through the naked eye examination. However, in the case of glossy obsidian, it is often difficult to identify sickle gloss. Instead, the use-wear indicators for this material usually take the form of a matte surface or visible scratches (Astruc et al. 2012). Indeed, the present collection consists of flakes and blades with matte surfaces along the edge. Our microscopic analyses also indicate that all four of the "sickle elements" we have examined to date show wear from "cutting/sawing grass plants" (Chapter 10).

As we have defined sickle elements in this study functionally, their techno-morphological traits can be heterogeneous. However, these elements generally retain a rectangular to oblong shape of 17 to $58 \mathrm{~mm}$ long and 12 to $28 \mathrm{~mm}$ wide. The working edges are either retouched (Fig. 9.7: 6, 7, 9), denticulated (Fig. 9.7: 8), or unmodified (Fig. 9.7: 5). Technologically, we have identified three major types (Table 9.4): blades or flakes with snapped ends (Fig. 9.7: 5 and 6), burinated edges (Fig. 9.7: 7 and 8), and backed edges (Fig. 9.7: 9). The most unique examples are those with burinated edges. The burination is 

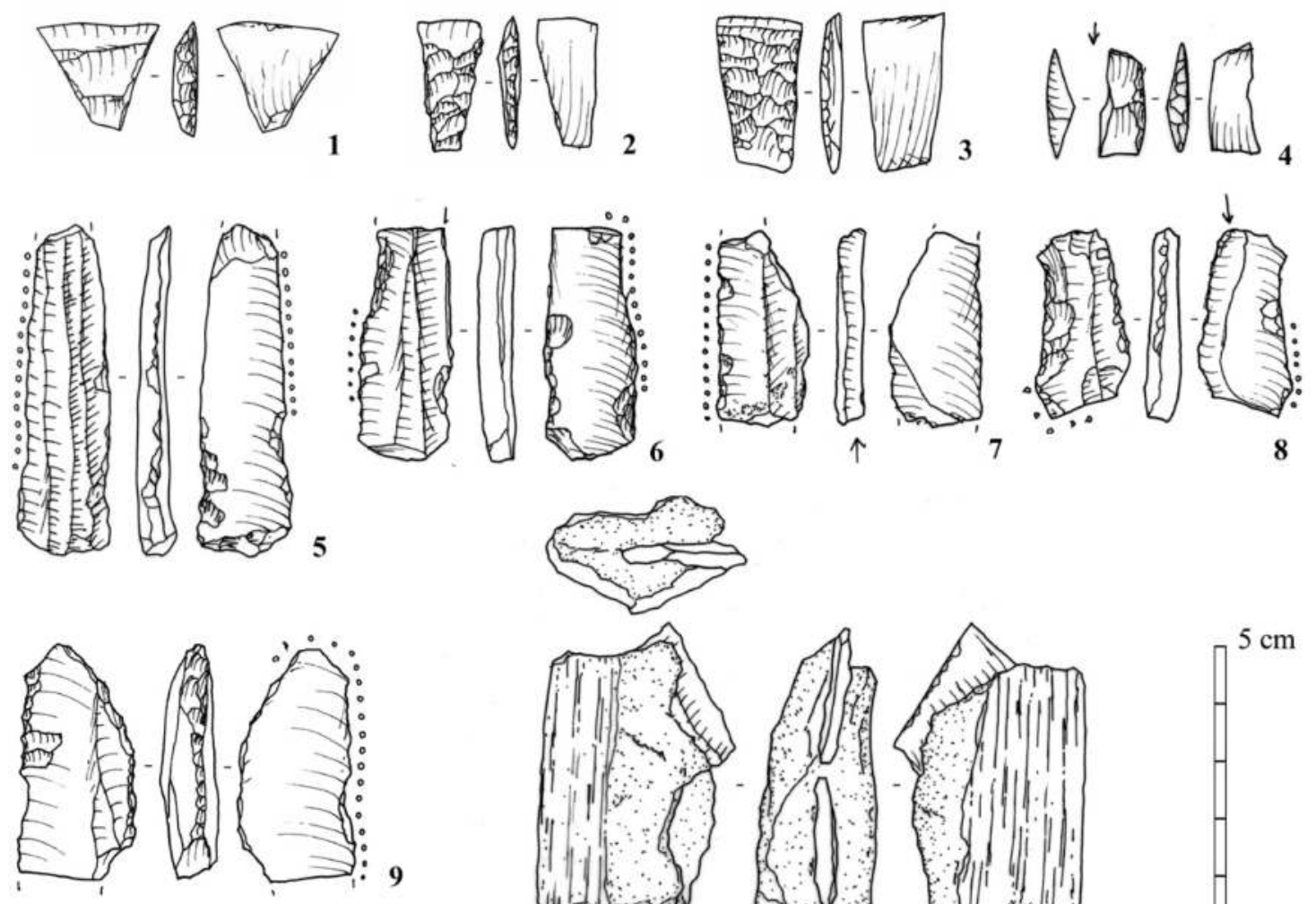

9
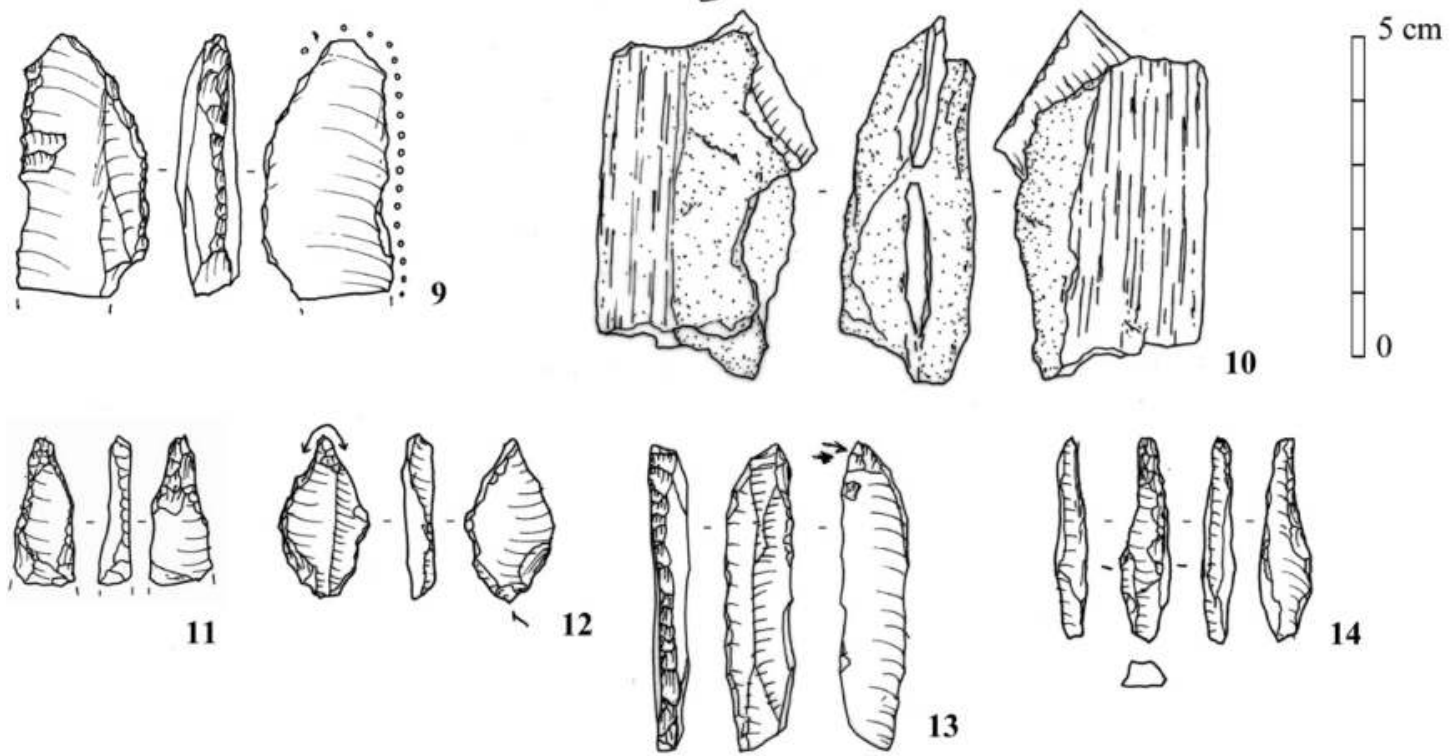

Fig. 9.7 Obsidian retouched tools from Göytepe (I). 1: Trapeze, bilaterally retouched (4A, Upper Area); 2: Trapeze, bidirectionally pressure retouched (2B-Loc. 2, Upper Area); 3: Trapeze, bidirectionally pressure retouched (3AI, Upper Area); 4: Trapeze, bilaterally retouched, with an impact fracture (3All, Upper Area); 5: Sickle element, snapped (4BII-23, Level 7); 6: Sickle element, snapped (3B-corridor, Upper Area); 7: Sickle element, burinated, bitumen traces near the proximal end (2B-Loc. 3, Upper Area); 8: Sickle element, burinated (3B-Loc. 1, Upper Area); 9: Sickle element, backed (3B corridor, Upper Area); 10: Sickle fragment retaining an obsidian blade coated with bitumen, which shows imprints of a handle (3Al, Upper Area); 11: Piercer (4BI-14, 6); 12: Piercer (3B-corridor, Upper Area); 13: Reamer with torsion fractures at the tip (2A2, Upper Area); 14: Reamer made on burin spall (4BI-113D, Level 10).

introduced by one or more overshot angle burin blows along the edge opposite to the working edge in order to produce a back for the tool (Fig. 9.7: 7 and 8). Varoutsikos (2015) first identified this technique and called the tools that utilized it "Aknashen tools." The discovery of such tools used as sickle elements at Göytepe supports the identification of this type of burination as a distinct retouch technique. Regardless of the retouch technique, the distribution of use- wear of sickle elements often slants to the cutting edge (also see Chapter 10), which indicates that the elements were hafted to the handle in a jagged way. The recovered sickles support this interpretation (Figs. 9.7: 10, 9.8).

Backed pieces: There is a small number of backed blades and flakes (Table 9.3). Although, without matting on the edge that is visible to the naked eye, they may include sickle elements. 
Table 9.4 Obsidian tools from Göytepe (2008-2013).

\begin{tabular}{|c|c|c|c|c|c|c|c|c|c|c|c|c|c|}
\hline & $\begin{array}{l}\text { Upper } \\
\text { Area* }^{*}\end{array}$ & $\begin{array}{c}\text { Level } \\
4\end{array}$ & $\begin{array}{c}\text { Level } \\
5\end{array}$ & $\begin{array}{c}\text { Level } \\
6\end{array}$ & $\begin{array}{c}\text { Level } \\
7\end{array}$ & $\begin{array}{c}\text { Level } \\
8\end{array}$ & $\begin{array}{c}\text { Level } \\
9\end{array}$ & $\begin{array}{c}\text { Level } \\
10\end{array}$ & $\begin{array}{c}\text { Level } \\
11\end{array}$ & $\begin{array}{c}\text { Level } \\
12\end{array}$ & $\begin{array}{c}\text { Level } \\
13\end{array}$ & $\begin{array}{c}\text { Level } \\
14\end{array}$ & Total \\
\hline \multicolumn{14}{|l|}{ Trapezes } \\
\hline Bilaterally backed & 4 & 0 & 2 & 1 & 0 & 0 & 0 & 1 & 0 & 0 & 0 & 0 & 8 \\
\hline Flat faced & 2 & 0 & 0 & 0 & 0 & 0 & 0 & 0 & 0 & 0 & 0 & 0 & 2 \\
\hline Unfinished or broken & 1 & 0 & 0 & 0 & 0 & 0 & 0 & 0 & 1 & 0 & 0 & 0 & 2 \\
\hline \multicolumn{14}{|l|}{ Sickle elements } \\
\hline Snapped end & 7 & 0 & 0 & 0 & 1 & 0 & 1 & 3 & 0 & 0 & 2 & 0 & 14 \\
\hline Burinated & 7 & 0 & 0 & 0 & 0 & 1 & 0 & 1 & 0 & 0 & 0 & 0 & 9 \\
\hline Backed & 5 & 0 & 1 & 0 & 0 & 0 & 0 & 1 & 0 & 0 & 0 & 0 & 7 \\
\hline \multicolumn{14}{|l|}{ Backed pieces } \\
\hline on blade & 2 & 0 & 0 & 1 & 0 & 0 & 1 & 0 & 0 & 0 & 1 & 0 & 5 \\
\hline on flake & 2 & 0 & 0 & 0 & 0 & 0 & 0 & 0 & 0 & 0 & 0 & 0 & 2 \\
\hline \multicolumn{14}{|l|}{ Perforators } \\
\hline Piercers & 6 & 0 & 1 & 0 & 1 & 0 & 0 & 1 & 0 & 0 & 0 & 0 & 9 \\
\hline Reamers & 7 & 1 & 2 & 1 & 2 & 0 & 1 & 4 & 2 & 0 & 0 & 0 & 20 \\
\hline \multicolumn{14}{|l|}{ Side scrapers } \\
\hline on blade & 1 & 0 & 0 & 0 & 0 & 0 & 0 & 1 & 0 & 0 & 0 & 0 & 2 \\
\hline on flake & 11 & 0 & 0 & 0 & 1 & 1 & 0 & 0 & 0 & 0 & 0 & 0 & 13 \\
\hline \multicolumn{14}{|l|}{ End scrapers } \\
\hline on blade & 3 & 1 & 0 & 0 & 0 & 0 & 0 & 1 & 0 & 0 & 0 & 0 & 5 \\
\hline on flake & 5 & 0 & 0 & 0 & 0 & 1 & 0 & 1 & 0 & 0 & 0 & 0 & 7 \\
\hline \multicolumn{14}{|l|}{ Burinated pieces } \\
\hline Angle & 144 & 6 & 10 & 12 & 21 & 15 & 7 & 67 & 15 & 6 & 2 & 0 & 305 \\
\hline Dihedral & 32 & 1 & 0 & 0 & 5 & 1 & 2 & 13 & 4 & 1 & 1 & 1 & 61 \\
\hline Transverse & 24 & 0 & 0 & 0 & 1 & 0 & 1 & 5 & 3 & 0 & 3 & 0 & 37 \\
\hline Splintered pieces & 168 & 23 & 23 & 11 & 32 & 11 & 15 & 52 & 6 & 5 & 7 & 3 & 356 \\
\hline \multicolumn{14}{|l|}{ Truncated pieces } \\
\hline on blade & 9 & 0 & 0 & 0 & 3 & 0 & 0 & 2 & 1 & 0 & 0 & 1 & 16 \\
\hline on flake & 2 & 0 & 0 & 0 & 0 & 0 & 0 & 0 & 0 & 0 & 0 & 0 & 2 \\
\hline \multicolumn{14}{|l|}{ Denticulated pieces } \\
\hline on blade & 39 & 3 & 1 & 1 & 8 & 9 & 6 & 9 & 5 & 6 & 0 & 0 & 87 \\
\hline on flake & 15 & 0 & 0 & 2 & 1 & 0 & 0 & 3 & 1 & 0 & 0 & 0 & 22 \\
\hline \multicolumn{14}{|l|}{ Notched pieces } \\
\hline on blade & 44 & 7 & 4 & 1 & 7 & 4 & 2 & 14 & 1 & 1 & 0 & 0 & 85 \\
\hline on flake & 24 & 0 & 1 & 1 & 3 & 3 & 5 & 8 & 1 & 0 & 1 & 0 & 47 \\
\hline \multicolumn{14}{|l|}{ Retouched pieces } \\
\hline on blade & 150 & 5 & 10 & 7 & 19 & 10 & 3 & 46 & 17 & 6 & 12 & 1 & 286 \\
\hline on flake & 87 & 1 & 14 & 7 & 12 & 3 & 9 & 31 & 11 & 3 & 1 & 0 & 179 \\
\hline \multicolumn{14}{|l|}{ Edge-damaged pieces } \\
\hline on blade & 126 & 7 & 8 & 4 & 23 & 4 & 11 & 39 & 15 & 5 & 1 & 3 & 246 \\
\hline on flake & 27 & 0 & 1 & 1 & 1 & 0 & 1 & 5 & 1 & 0 & 0 & 0 & 37 \\
\hline Tool fragments & 15 & 0 & 0 & 0 & 3 & 0 & 2 & 8 & 1 & 0 & 0 & 0 & 29 \\
\hline \multicolumn{14}{|l|}{ Tool spalls } \\
\hline Burin spalls & 86 & 4 & 14 & 9 & 15 & 12 & 13 & 50 & 4 & 6 & 4 & 4 & 221 \\
\hline Splintered spalls & 42 & 4 & 3 & 0 & 4 & 1 & 0 & 11 & 2 & 0 & 2 & 0 & 69 \\
\hline Total & 1097 & 63 & 95 & 59 & 163 & 76 & 80 & 377 & 91 & 39 & 37 & 13 & 2190 \\
\hline
\end{tabular}




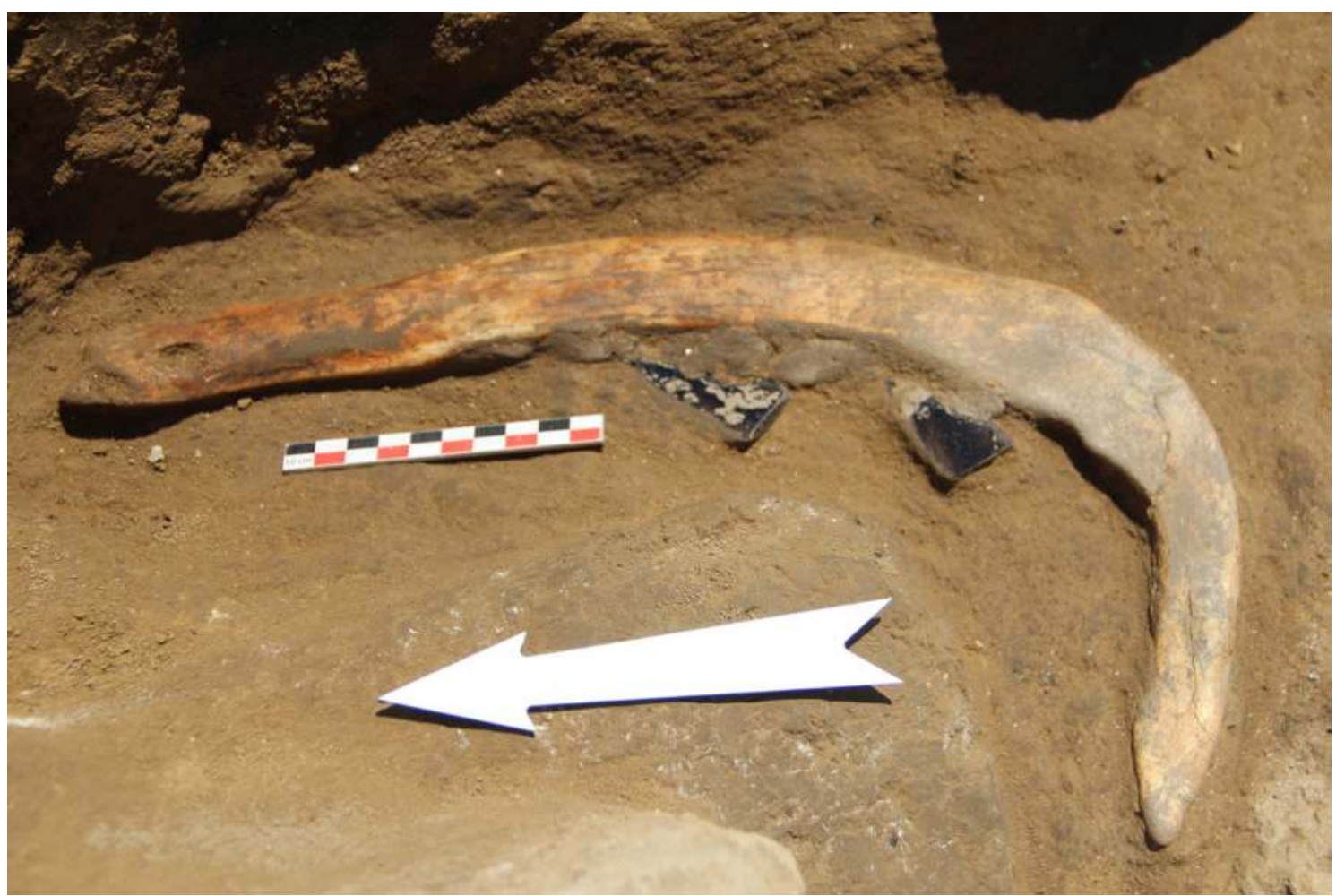

Fig. 9.8 Complete sickle with obsidian blades inserted in a jagged way (Upper Area).

Perforators: This tool group consists of at least two morphological types. One has a pointed end that is separated from the body by intentional retouch to the blank. The body is also retouched but less intensively (Fig. 9.7: 11 and 12). In this study, we call this type piercers. We refer to the other type as reamers, and these show a rod shape whose pointed end is not delineated from the body (Fig. 9.7: 13 and 14). Rather, it exhibits steep retouches along both edges, often made in an alternate fashion. The piercers were more commonly manufactured on flakes, while the reamers were on blades. The latter even used burin spalls as blanks (Fig. 9.7: 14).

Burinated pieces: These represent the numerous tools in the obsidian assemblages from Göytepe. Although they have at least one burin facet, they are not called "burins" in this study. This is because burin faceting was applied at this site not only to produce burins but also to shape the blanks to make other types of tools. For example, we discussed earlier the employment of this technique to make sickle elements. When the faceting is overshot, the resultant tool can be considered an Aknashen tool (Varoutiskos 2015).

The burin faceting was executed in varied ways. The most common among these is faceting along the blank axis, with the angle type made on an unretouched edge or snapped end of the blank (Fig. 9.9: 1 and 2). Faceting on a retouched truncation is uncommon. Burinated pieces with dihedral (Fig. 9.9:3) and transversal faceting (Fig. 9.9:4) are less common. The dihedral facet is often made at a corner of one end of the blank and is thereby regarded as demonstrating a combination of angle and transversal faceting.

Splintered pieces: These are the second most frequently found pieces (Table 9.3). In this study, the term "splintered pieces" denotes flakes or blades with a series of flat faceting scars on the blank face (Fig. 9.9: 5 and 6). The scars can run from one or both ends or the side edges. In either case, the profile of these pieces shows a wedge-shape, similar to that of opposed-platform cores. Those with facets inclined to an edge of the blank show similarities to the burinated pieces as well.

Scrapers: The two major types of scrapers are sidescrapers (Fig. 9.9: 8) and endscrapers (Fig. 9.9: 9 and 10). These are distinguished from each other by differences in the location of the scraping edges relative to the blank axis. Both types are more often made from flake blanks, which include core management pieces (Fig. 9.9: 8). 

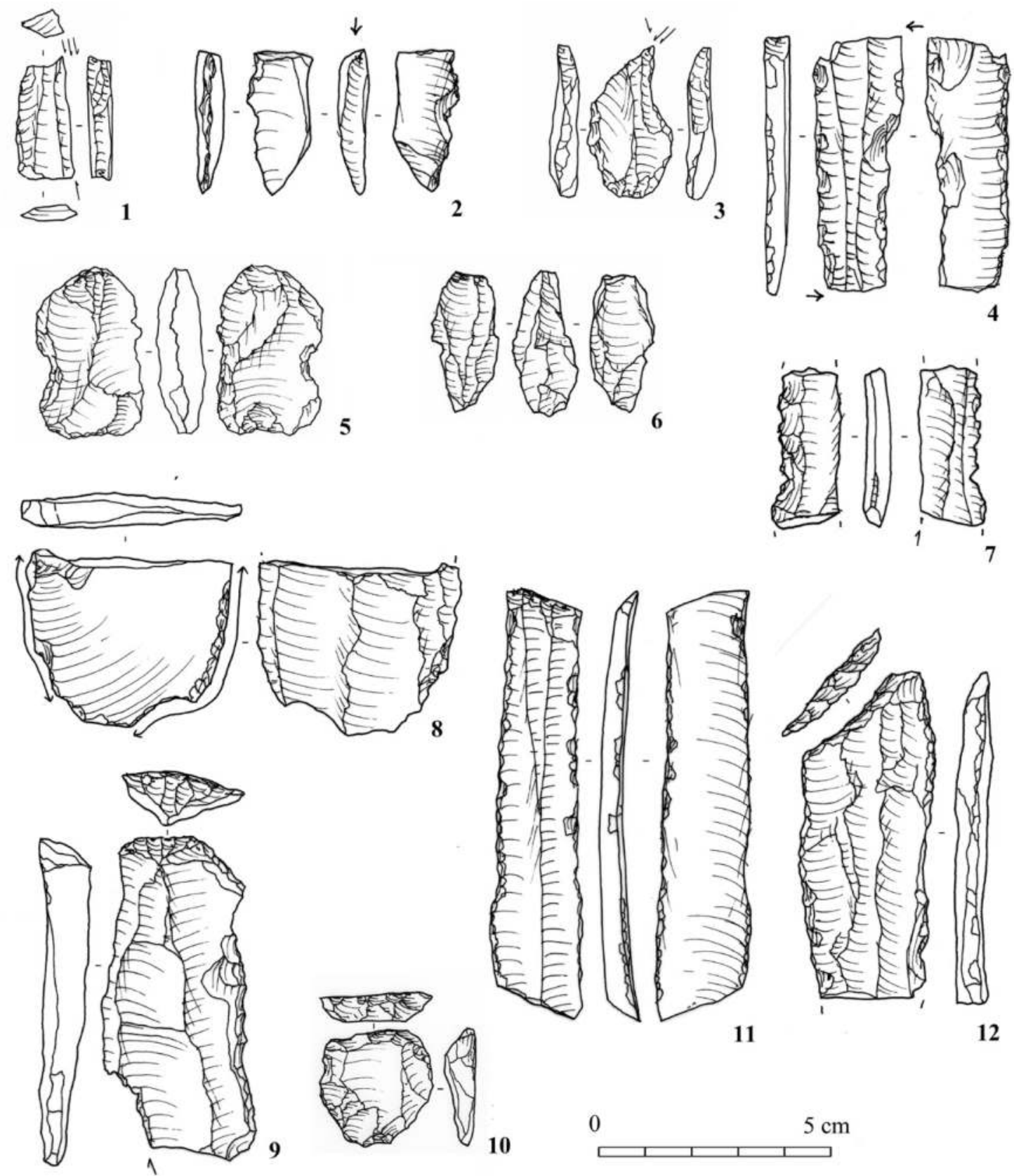

Fig. 9.9 Obsidian retouched tools from Göytepe (II). 1: Burinated piece, angle type (4BIIX-33, Levels 10); 2: Burinated piece, angle type, overshot (2B-Loc. 8, Upper Area); 3: Burinated piece, dihedral type (3All, Upper Area); 4: Burinated piece, transversal type (3All, Upper Area); 5: Splintered piece (4BIIX-8, Level 10); 6: Splintered piece (3B-Loc. 1, Upper Area); 7: Denticulate (4BI-67, Level 9); 8: Side-scraper on flake (4BI62, Level 8); 9: End-scraper on blade (4BI-111N, Level 10); 10: End-scraper on flake (4BI-111, Level 10); 11: Truncation (GT09, Upper Area); 12: Truncation, oblique type (4BI-62, Level 8).

Truncated pieces: Blades or flakes with steep straight retouches at one or both ends fall into this category. The truncation can be perpendicular (Fig. 9.9: 11) or oblique to the blank axis (Fig. 9.9: 12). These were often made from blade blanks.

Miscellaneous tools: In addition to the above categories, the site yielded retouched tools that do not show standardized techno-morphological features. According to the form of the retouched edge and retouching intensity, they are classified as denticulated (Fig. 9.9: 7), notched, retouched, and edge-damaged pieces. It is uncertain whether all of 
them were intentionally modified to produce a certain type of working edge. They may include accidentally shaped or use-retouched pieces.

Tool spalls: These are not tools themselves but byproducts from retouching. The high number of burin spalls is in proportion to the occurrence of burinated pieces. The splintered spalls are less common in relation to the high number of splintered pieces. However, this is primarily because it is difficult to identify them; there is little distinction between splintered spalls and waste from obsidian knapping. The pieces we have identified as splintered spalls in this study are small and flat elongated pieces with a shattered butt and include Kombewa flakes.

\subsubsection{Non-obsidian artifacts}

(1) Cores

The non-obsidian core assemblages differ from the obsidian assemblages. The relatively common occurrence of semi-flaked cores, which are absent from the obsidian assemblages, is remarkable.
Conversely, exhausted cores are quite rare in the non-obsidian core assemblages (Table 9.5). This undoubtedly reflects the local availability of the non-obsidian rocks in the Göytepe communities. The cores include a few specimens with traces of blade removals, which were made by hard-hammer percussion but not by pressuring. The dominant specimens are hard-hammer struck flake cores with few traces of core preparation, among which globular cores with multi-directional scars are most common (Fig. 9.10: 2 and 3), followed by singleplatform cores (Fig. 9.10: 1). Opposed-platform cores are almost absent in this site. The reduction of cores might have started from single-platforms and proceeded to further reduction, which ultimately resulted in globular cores.

All of the cores in the present sample, with the exception of a few pieces of tuff and quartzite, are made on flint. The absence of andesite and basalt cores is noteworthy because debitage and tools made from these rocks constitute a significant portion of

Table 9.5 Flint cores, core management pieces, and debitage from Göytepe (2008-2013).

\begin{tabular}{|c|c|c|c|c|c|c|c|c|c|c|c|c|c|}
\hline & $\begin{array}{l}\text { Upper } \\
\text { Area* }^{*}\end{array}$ & $\begin{array}{c}\text { Level } \\
4\end{array}$ & $\begin{array}{c}\text { Level } \\
5\end{array}$ & $\begin{array}{l}\text { Level } \\
6\end{array}$ & $\begin{array}{c}\text { Level } \\
7\end{array}$ & $\begin{array}{c}\text { Level } \\
8\end{array}$ & $\begin{array}{c}\text { Level } \\
9\end{array}$ & $\begin{array}{c}\text { Level } \\
10\end{array}$ & $\begin{array}{c}\text { Level } \\
11\end{array}$ & $\begin{array}{c}\text { Level } \\
12\end{array}$ & $\begin{array}{c}\text { Level } \\
13\end{array}$ & $\begin{array}{c}\text { Level } \\
14\end{array}$ & Total \\
\hline Semi-flaked cores & 0 & 0 & 1 & 0 & 0 & 1 & 1 & 1 & 0 & 0 & 1 & 0 & 5 \\
\hline \multicolumn{14}{|l|}{ Flake cores } \\
\hline single-platform & 2 & 0 & 1 & 1 & 0 & 0 & 0 & 0 & 0 & 0 & 0 & 0 & 4 \\
\hline opposed-platform & 1 & 0 & 0 & 0 & 0 & 0 & 0 & 0 & 0 & 0 & 0 & 0 & 1 \\
\hline multiple-platform, crossed & 0 & 0 & 0 & 0 & 1 & 0 & 0 & 1 & 0 & 0 & 0 & 0 & 2 \\
\hline multiple-platform, globular & 0 & 0 & 0 & 1 & 2 & 0 & 1 & 3 & 1 & 0 & 0 & 0 & 8 \\
\hline Exhausted cores & 0 & 0 & 0 & 0 & 0 & 0 & 0 & 0 & 0 & 0 & 0 & 1 & 1 \\
\hline On-flake cores & 0 & 0 & 0 & 0 & 0 & 0 & 1 & 3 & 0 & 0 & 0 & 0 & 4 \\
\hline Core fragment & 0 & 0 & 0 & 0 & 0 & 0 & 1 & 0 & 0 & 0 & 0 & 0 & 1 \\
\hline Total: Cores & 3 & 0 & 2 & 2 & 3 & 1 & 4 & 8 & 1 & 0 & 1 & 1 & 26 \\
\hline Core-edge elements & 6 & 3 & 1 & 1 & 1 & 0 & 3 & 1 & 2 & 0 & 1 & 0 & 19 \\
\hline Cortex flakes & 9 & 0 & 2 & 1 & 2 & 0 & 1 & 9 & 3 & 2 & 0 & 0 & 29 \\
\hline Part-cortical flakes & 36 & 2 & 3 & 8 & 9 & 6 & 14 & 27 & 2 & 2 & 0 & 0 & 109 \\
\hline Flakes & 55 & 3 & 23 & 4 & 9 & 6 & 14 & 45 & 4 & 2 & 3 & 2 & 170 \\
\hline Part-cortex blades & 2 & 0 & 0 & 0 & 0 & 0 & 0 & 0 & 0 & 0 & 0 & 0 & 2 \\
\hline Blades & 1 & 0 & 1 & 0 & 0 & 0 & 1 & 0 & 0 & 0 & 0 & 0 & 3 \\
\hline Chips and fragments & 15 & 1 & 3 & 2 & 9 & 2 & 10 & 10 & 0 & 0 & 2 & 0 & 54 \\
\hline Total: Debitage & 123 & 9 & 33 & 16 & 30 & 14 & 43 & 92 & 11 & 6 & 6 & 2 & 385 \\
\hline
\end{tabular}




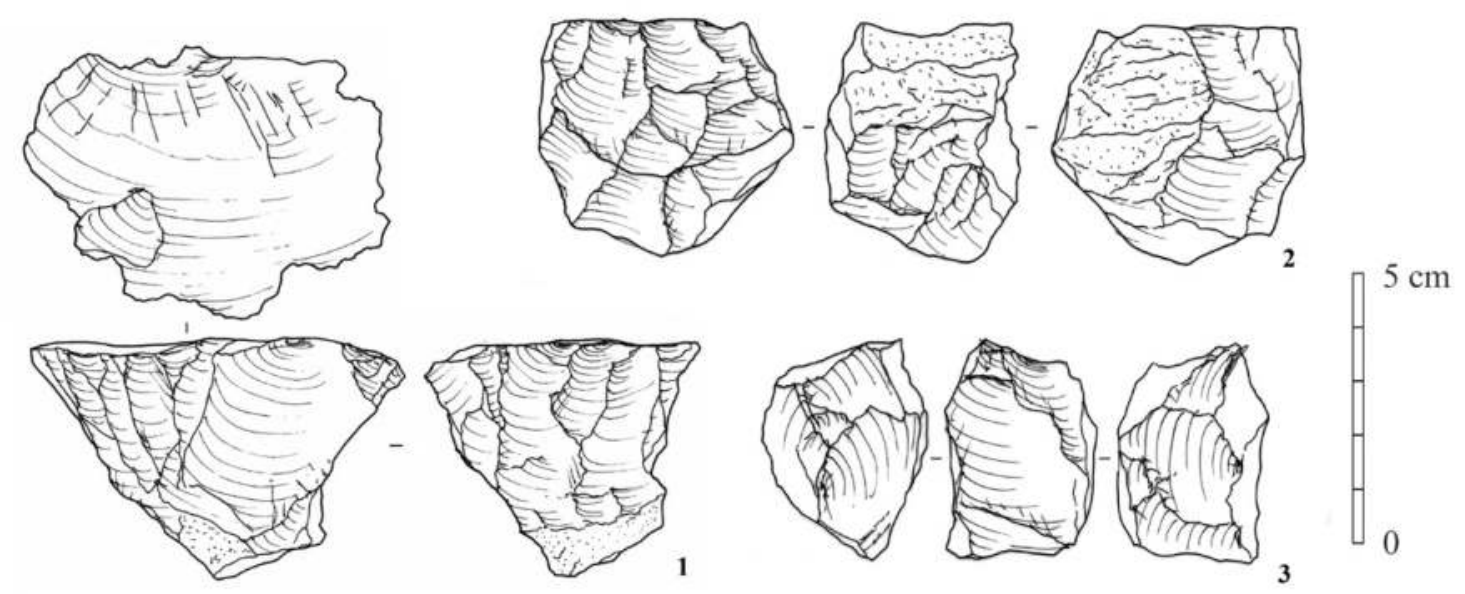

Fig. 9.10 Non-obsidian flake cores from Göytepe. 1: Single-platform flake core, flint (2B-Loc. 1, Upper Area); 2: Multiple-platform flake core, globular type, flint (4BIIX-59, Level 10); 3: Multiple-platform flake core, globular type, quartzite (4BI-106, Level 10).

the non-obsidian assemblages. The andesite and basalt pieces are likely products from the fashioning of heavy-duty tools.

\section{(2) Debitage}

Debitage also displays distinct differences between the non-obsidian and obsidian assemblages. The non-obsidian debitage includes a larger amount of cortex and part-cortex flakes, which points to onsite knapping from the early stages of core reduction (Table 9.5). Cortical nodules were likely reduced within the settlement. The near absence of coremanagement pieces reflects a simple core reduction technology. There is no evidence of pressure debitage in the non-obsidian assemblages.

\section{(3) Tools}

The non-obsidian retouched tool assemblages demonstrate two key characteristics. Most of them are made on flake blanks. The range of tool types is smaller than for obsidian tools (Table 9.5), although this may at least partly be a reflection of the smaller number of non-obsidian tools, which include heavyduty tools such as picks and choppers (see below).

Sickle elements: The most abundant non-obsidian tools are sickle elements (Table 9.6). Interestingly, despite the marked typological contrast between obsidian and non-obsidian assemblages in general, this tool type demonstrates common features across both categories of raw materials (Fig. 9.11). We can categorize the non-obsidian sickle elements into snapped/unmodified (Fig. 9.11:2-4), burinated (Fig.
9.11: 7), and backed pieces (Fig. 9.11: 5 and 6). The snapped/unmodified pieces are the most common of these sickle elements. As with the obsidian sickle elements, the use-wear and traces of bitumen typically have an oblique distribution in a portion of the working edge. The discovery of several complete sickles illustrates how these elements were hafted into a handle (Fig. 9.11: 1).

Perforators: As in the obsidian assemblages, we can classify non-obsidian perforators as piercers and reamers. However, the overall look of the non-obsidian perforators differs greatly from the obsidian examples because of the distinct blank use. Non-obsidian pieces are made on much larger blanks, which are mostly flakes (Fig. 9.12: 1 and 2) and include core management pieces (Fig. 9.12: 3). Bitumen remains at the base indicate their use as hafted (Fig. 9.12: 1).

Scrapers: Non-obsidian scrapers differ from obsidian samples in the same way as the perforators. Both sidescrapers and endscrapers are made on larger flake blanks. While the sidescrapers tend to be made by inverse retouch (Fig. 9.12: 4 and 5), the endscrapers are almost always manufactured by obverse retouch at Göytepe (Fig. 9.13: 1).

Burinated and splintered pieces: These are commonly manufactured from obsidian but rarely from other rocks, which indicates a raw material preference. However, it is important to note that while non-obsidian burinated and splintered pieces may be much rarer, we still find evidence for the use of burin faceting to shape tool forms in the non- 
Table 9.6 Flint tools from Göytepe (2008-2013).

\begin{tabular}{|c|c|c|c|c|c|c|c|c|c|c|c|c|c|}
\hline & $\begin{array}{l}\text { Upper } \\
\text { Area* }\end{array}$ & $\begin{array}{c}\text { Level } \\
4\end{array}$ & $\begin{array}{l}\text { Level } \\
5\end{array}$ & $\begin{array}{l}\text { Level } \\
6\end{array}$ & $\begin{array}{l}\text { Level } \\
7\end{array}$ & $\begin{array}{c}\text { Level } \\
8 \\
\end{array}$ & $\begin{array}{c}\text { Level } \\
9\end{array}$ & $\begin{array}{c}\text { Level } \\
10\end{array}$ & $\begin{array}{c}\text { Level } \\
11\end{array}$ & $\begin{array}{c}\text { Level } \\
12\end{array}$ & $\begin{array}{c}\text { Level } \\
13\end{array}$ & $\begin{array}{c}\text { Level } \\
14\end{array}$ & Total \\
\hline \multicolumn{14}{|l|}{ Sickle elements } \\
\hline Snapped/plain & 10 & 1 & 4 & 0 & 1 & 0 & 2 & 4 & 4 & 0 & 1 & 0 & 27 \\
\hline Burinated (siret) & 2 & 0 & 0 & 0 & 1 & 0 & 0 & 0 & 0 & 0 & 0 & 0 & 3 \\
\hline Backed & 8 & 0 & 0 & 0 & 0 & 0 & 0 & 2 & 0 & 0 & 0 & 0 & 10 \\
\hline \multicolumn{14}{|l|}{ Perforators } \\
\hline Piercers & 1 & 0 & 0 & 0 & 0 & 0 & 0 & 0 & 0 & 0 & 1 & 0 & 2 \\
\hline Reamers & 1 & 0 & 1 & 0 & 0 & 0 & 0 & 1 & 1 & 0 & 0 & 0 & 4 \\
\hline Side scrapers on flake & 0 & 0 & 1 & 0 & 2 & 1 & 1 & 2 & 0 & 0 & 0 & 0 & 7 \\
\hline End scrapers on flake & 2 & 0 & 0 & 0 & 0 & 0 & 0 & 0 & 0 & 1 & 1 & 0 & 4 \\
\hline \multicolumn{14}{|l|}{ Burinated pieces } \\
\hline Angle & 1 & 0 & 0 & 0 & 0 & 0 & 0 & 1 & 0 & 0 & 0 & 0 & 2 \\
\hline Dihedral & 2 & 0 & 0 & 0 & 0 & 0 & 0 & 0 & 0 & 0 & 0 & 0 & 2 \\
\hline Truncation & 1 & 0 & 0 & 0 & 0 & 0 & 0 & 0 & 0 & 0 & 0 & 0 & 1 \\
\hline Splintered piece & 0 & 0 & 0 & 0 & 0 & 0 & 0 & 1 & 0 & 0 & 0 & 0 & 1 \\
\hline Denticulated flakes & 2 & 0 & 0 & 0 & 0 & 0 & 0 & 2 & 0 & 0 & 0 & 0 & 4 \\
\hline Notched flakes & 1 & 0 & 2 & 0 & 0 & 0 & 1 & 0 & 0 & 0 & 0 & 0 & 4 \\
\hline Retouched blades & 2 & 0 & 0 & 0 & 0 & 0 & 1 & 0 & 0 & 0 & 0 & 0 & 3 \\
\hline Retouched flakes & 8 & 1 & 4 & 1 & 1 & 3 & 1 & 6 & 0 & 1 & 0 & 1 & 27 \\
\hline Edge-damaged flakes & 3 & 0 & 0 & 0 & 0 & 0 & 1 & 0 & 0 & 0 & 0 & 0 & 4 \\
\hline Picks & 0 & 0 & 0 & 0 & 2 & 0 & 0 & 2 & 3 & 0 & 0 & 0 & 7 \\
\hline Rods & 0 & 0 & 0 & 0 & 0 & 0 & 0 & 3 & 0 & 0 & 0 & 0 & 3 \\
\hline Choppers & 0 & 0 & 1 & 0 & 2 & 0 & 0 & 0 & 0 & 0 & 0 & 0 & 3 \\
\hline Flaked axe & 0 & 0 & 0 & 0 & 0 & 0 & 1 & 0 & 0 & 0 & 0 & 0 & 1 \\
\hline Tabular scraper & 1 & 0 & 0 & 0 & 0 & 0 & 0 & 0 & 1 & 0 & 0 & 0 & 2 \\
\hline Hammerstones & 1 & 0 & 0 & 1 & 1 & 0 & 0 & 0 & 0 & 0 & 0 & 0 & 3 \\
\hline Tool fragment & 0 & 0 & 1 & 0 & 0 & 0 & 0 & 0 & 0 & 0 & 0 & 0 & 1 \\
\hline Total & 46 & 2 & 14 & 2 & 10 & 4 & 8 & 24 & 9 & 2 & 3 & 1 & 125 \\
\hline
\end{tabular}

* Levels 1-5

obsidian pieces (Fig. 9.11: 7).

Miscellaneous tools: Denticulated (Fig. 9.13: 5), notched, retouched (Fig. 9.13: 6), and edgedamaged pieces are also commonly found in the non-obsidian assemblages. However, unlike their obsidian counterparts, most non-obsidian examples in this category are made on flake blanks.

Heavy-duty tools: This tool group is unique to the non-obsidian assemblages. Many can be classified as picks, which have a pointed end created by a series of steep retouches separate from the body (Fig. 9.14: 3-5). Rods, or thick bilaterally retouched blades reminiscent of Palaeolithic limaces and narrow convergent scrapers (Debenath and Dibble 1994), are also common (Fig. 9.14: 1 and 2). Choppers and chopping tools are another important part of this group (Fig. 9.13: 8), followed by a smaller number of other tools such as flaked axes (Fig. 9.13: 7), bifacial knives, and tabular scrapers (Fig. 9.13: 2). The blanks used for tabular scrapers are slate slabs. Further, hammerstones are present.

\subsection{Discussion}

The above accounts demonstrate that the Neolithic knappers of Göytepe handled obsidian and nonobsidian raw materials differently. They reduced exotic obsidian with a more controlled technology 

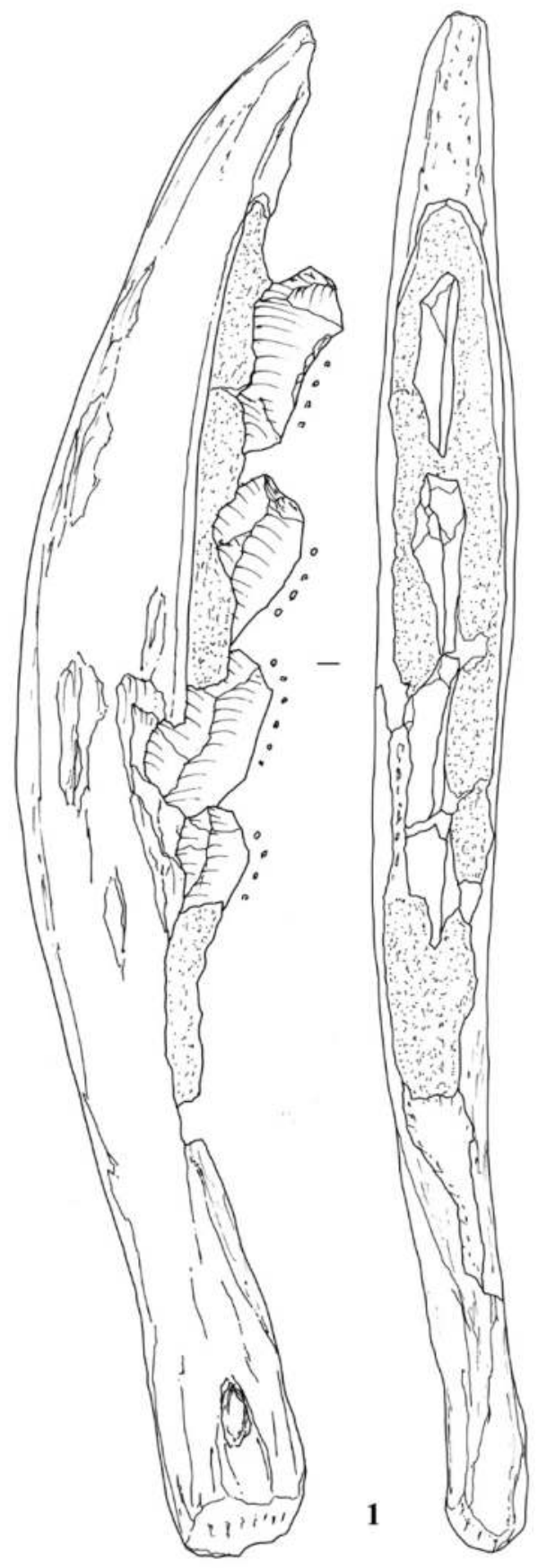
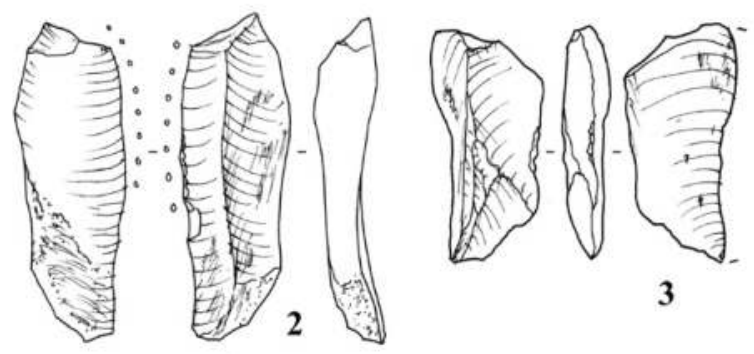

3
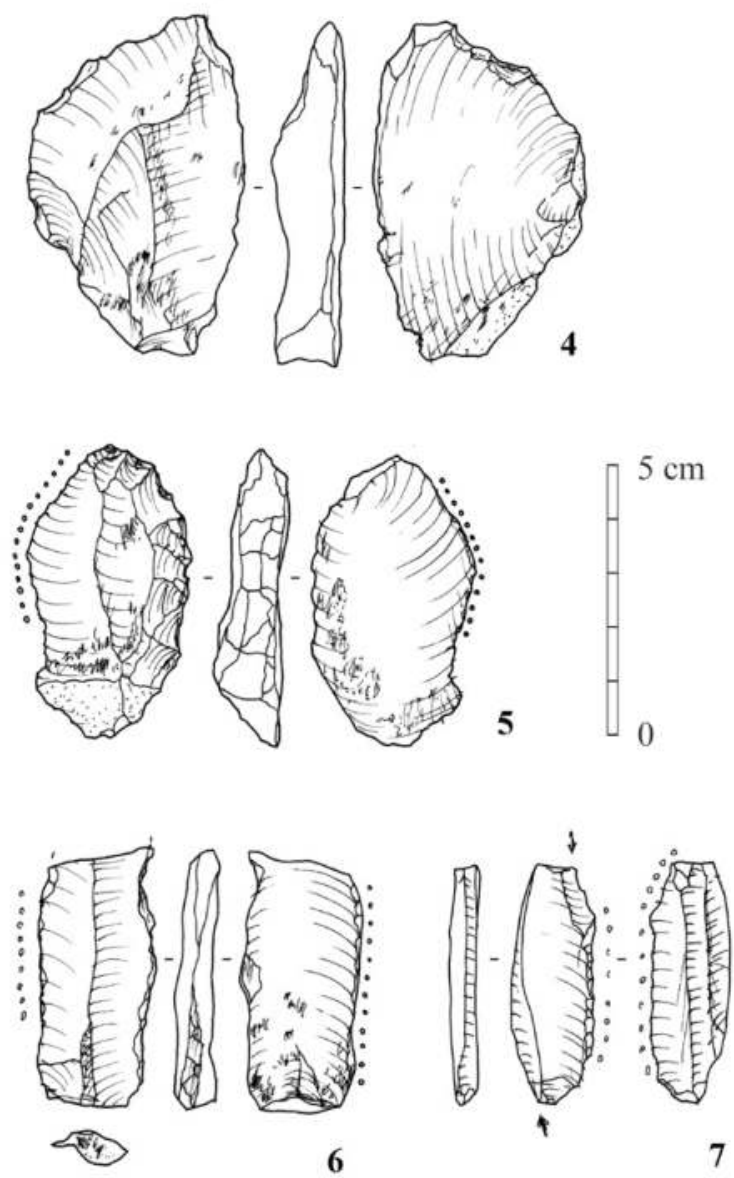

Fig. 9.11 Non-obsidian sickle and sickle elements from Göytepe. 1: Complete sickle made of four flint elements inserted to a bone handle with bitumen (3Al, Upper Area); 2: Sickle element, snapped type, flint (3BII-7, Level 5); 3: Sickle element, unretouched type on a Siret-fractured flake, flint (3B-Loc. 7, Upper Area); 4: Sickle element, unretouched type using the butt as the back, flint (2B-Loc. 2, Upper Area); 5: Sickle element, backed type, flint (3A-Loc. 2, Upper Area); 6: Sickle element, backed type, flint (4BI-58. Level 7); 7 : Sickle element, burinated type, flint (2All, Upper Area). 2-6: Note that bitumen remains are visible.

in order to produce a certain group of tools, notably arrowheads and cutting tools. The communities brought the obsidian from numerous sources situated in regions between $100 \mathrm{~km}$ and nearly $400 \mathrm{~km}$ from Göytepe. Interestingly, the reduction and use patterns do not differ by sources (Nishiaki et al. 2019a). On the other hand, the Göytepe communities brought in non-obsidian rocks, which were largely available near the settlement, with little prior preparation and reduced them by a rather $a d$ hoc percussion in order 

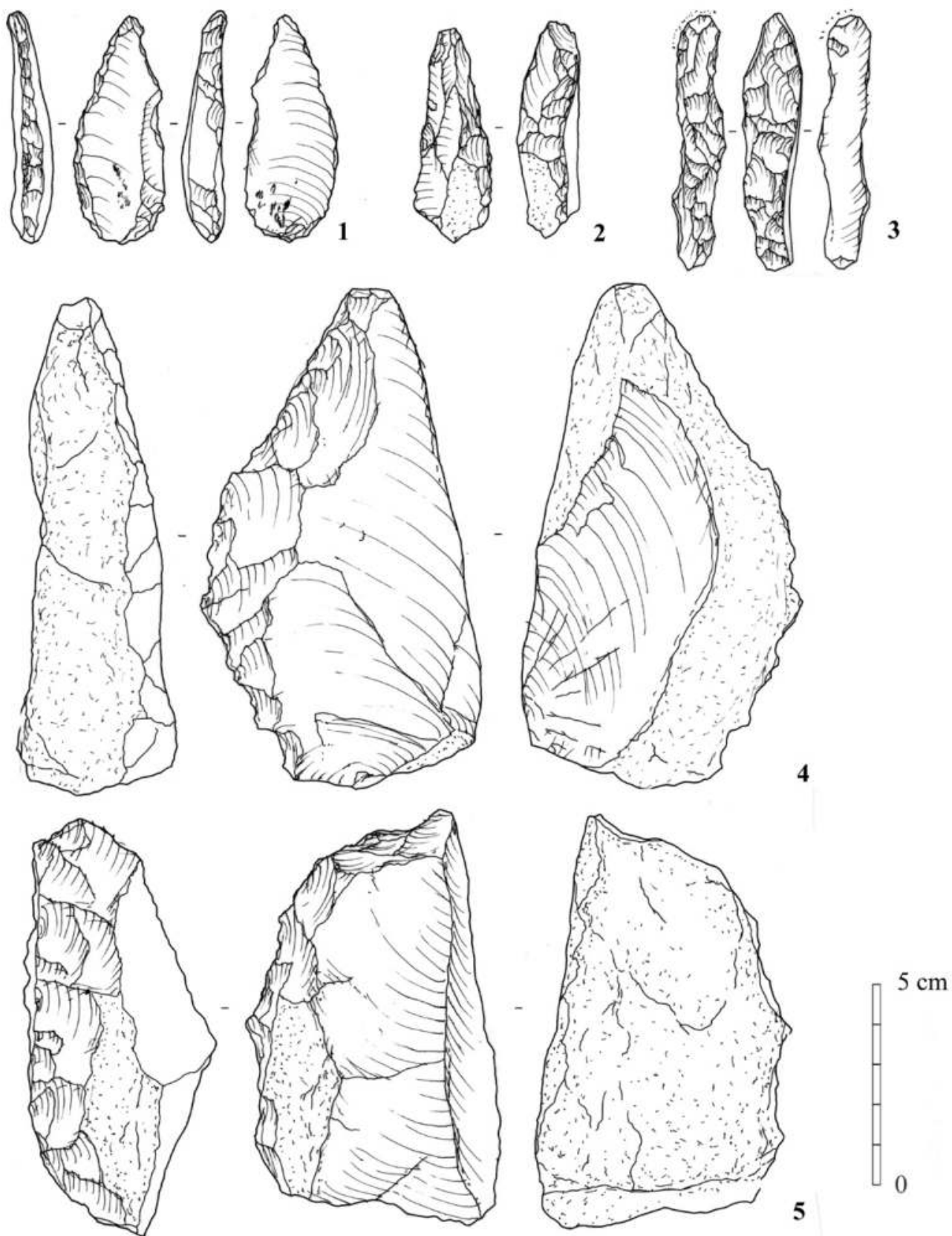

Fig. 9.12 Non-obsidian retouched tools from Göytepe (I). 1: Piercer, with bitumen traces at the proximal end, flint (2B-corridor, Upper Area); 2: Reamer, flint (3A, Upper Area); 3: Reamer, flint (4BI-93, Level 10); 4: Side-scraper, andesite (4BII-14, Level 7); 5: Side-scraper, andesite (4BI-9, Level 5).

to produce amorphous flakes for more robust tools. The knappers also produced heavy-duty tools such as picks and choppers with non-obsidian materials. As is the case of Neolithic Southwest Asia (Nishiaki $2000)$, this sort of dichotomy is likely related to the differences in the procurement costs and physical properties of these two groups of raw materials. One of the rare tool types that we can find in both types of raw materials is sickle elements.
Accordingly, the lithic assemblages from Göytepe can provide an important insight into the raw material economy during the early stages of the development of Neolithic societies in the Southern Caucasus. Simultaneously, the Göytepe data has a strong advantage in the determination of chronological changes in the lithic industry. We obtained this data from strictly controlled stratigraphic excavations with reliable radiocarbon dates (Nishiaki et al. 

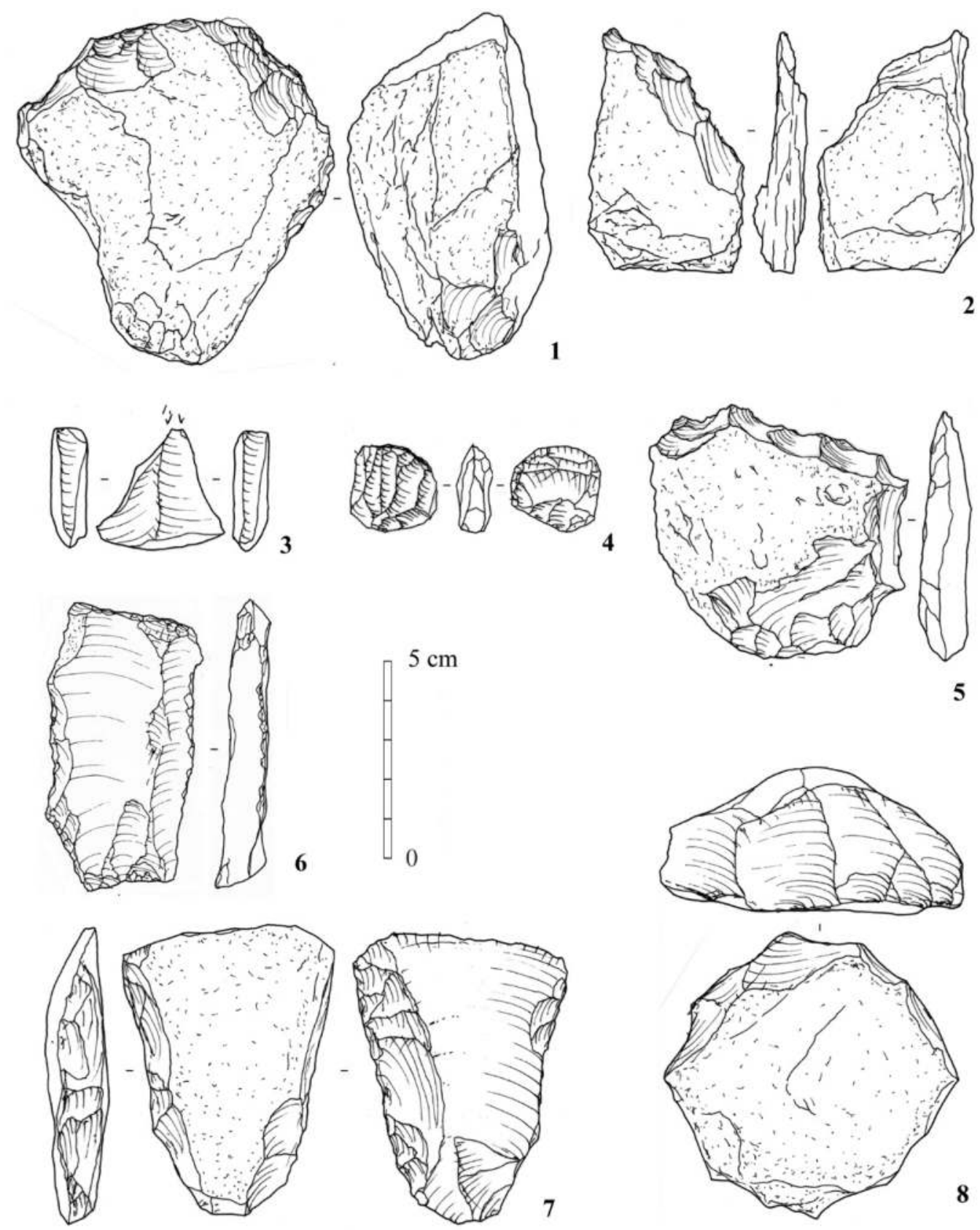

Fig. 9.13 Non-obsidian retouched tools from Göytepe (II). 1: End-scraper, andesite (4BI-54, Level 7); 2: Tabular scraper, slate (2B/3B baulk, Upper Area); 3: Burin, tuff (3B-corridor, Upper Area); 4: Splintered piece, flint (1B1, Upper Area); 5: Denticulate, andesite (4BI-111N, Level 10); 6: Retouched blade (4BIIX-33, Level 10); 7: Flaked axe, andesite (4BIIX-65, Level 9); 8: Chopper, andesite (4BII-9, Level 7).

2018). In the subsequent paragraphs, we discuss the chronological changes in the lithic industry within the Göytepe sequence and the Shomutepe culture in general.

This study has divided the occupational sequence of Göytepe into two phases (Levels 14-8 and Levels $7-1$ ) based on differences in the archaeological record, such as the changes in pottery manufacture and obsidian sources. Therefore, it is interesting to examine how the lithic industry changed over the sequence of the levels. However, as Tables 9.1-9.5 show, we are unable to identify significant changes between the two phases. The particular pattern for the obsidian/non-obsidian use, blank production technology, and tool composition seems to have remained virtually the same from one phase to the 


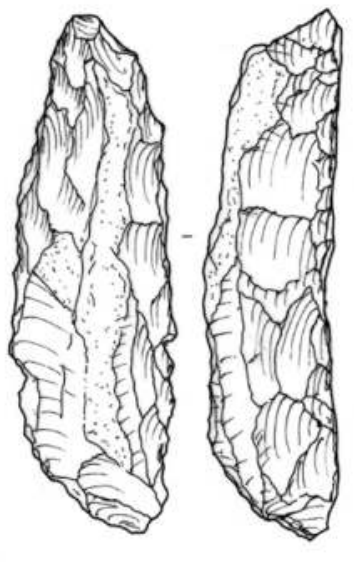

1
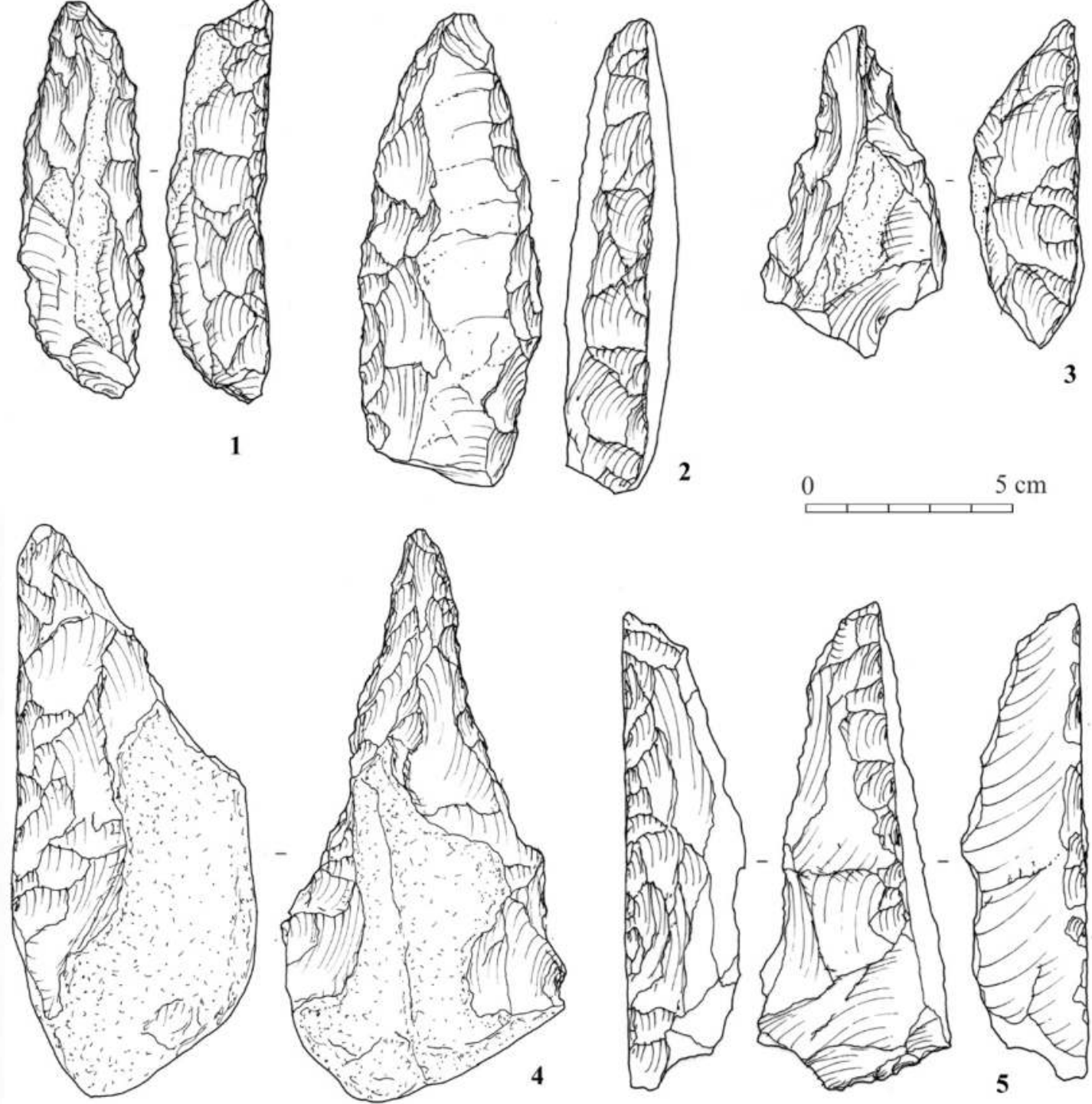

Fig. 9.14 Non-obsidian retouched tools from Göytepe (III). 1: Rod, andesite (4BI-113D, Level 10); 2: Rod, andesite (4BI-114CC, Level 10); 3: Pick, quartzite (4BI-117FF, Level 11); 4: Pick, andesite (4BI-56, Level 7); 5: Pick, andesite (4BI-131DD, Level 10).

next. One identifiable difference is the increase of splintered pieces over burinated pieces in the later phase (Table 9.5; Nishiaki and Guliyev 2019). As these two tool categories exhibit interchangeable technological strategies, there may have been some fluctuation in retouching technology. Overall, however, we can describe the lithic assemblages of the two phases of Göytepe as a representation of a single industry and of the same tradition.

The Göytepe sequence dates from $5650-5460$ $\mathrm{BC}$, which was the late stage of the Shomutepe culture. Our comparison of the general features of the Göytepe lithic industry with the lithic data from the earlier site Hac1 Elamxanlı Tepe has yielded the following observations (Nishiaki and Guliyev 2019):

(1) The use of obsidian at Göytepe seems more common than at Hac1 Elamxanlı (Kadowaki et al. 2016). However, the difference is rather small and may reflect different excavation strategies. The employment of sediment sieving at Hac1 Elamxanl1 may have facilitated a greater recovery of nonobsidian artifacts that are otherwise less visible than obsidian in the field. At this stage of research, it is not reasonable to emphasize the change in proportion of obsidian between the two sites. This issue, as well as the potential changes in the obsidian sources, should 
await future studies.

(2) The preferential use of pressure debitage for obsidian blade production and percussion for nonobsidian flake production characterizes the lithic assemblages of both sites. However, the size of blade products may have increased from Hac1 Elamxanl (average $=14.6 \mathrm{~mm} ; \mathrm{n}=469$; standard deviation $=4.5$ $\mathrm{mm}$; Nishiaki and Guliyev 2019) to the Göytepe stages, where the average blade widths are 16 to 17 $\mathrm{mm}$. If we adopt the current assumption that large blades were detached through lever pressuring (Pelegrin 2012; Chabot and Pelegrin 2012), this novel technique may have become more popular in the later stage of the Shomutepe culture.

(3) In terms of typological aspects, we notice diachronic changes. Trapeze arrowheads were important hunting tools at both settlements. However, they became less common over time. Moreover, their size increased from the Hac1 Elamxanlı (around 1 $\mathrm{cm}$ in both length and width) to the Göytepe periods (often longer than $2 \mathrm{~cm}$ ) (Nishiaki et al. 2019b: fig. 11). The increase in trapeze size is likely related to the increased production of wider blades, as trapezes were manufactured on blade blanks with truncations at both ends.

(4) Scrapers are common at both sites, but a potential typological change may exist. The artifacts most often found at Hacı Elamxanlı Tepe are flake scrapers made from thick non-obsidian materials (Kadowaki et al. 2016: fig. 5: 13 and 14). This tool type is rarely seen at Göytepe, where scrapers are more often made from small obsidian flat flakes.

(5) The relative frequencies of burinated pieces and splintered pieces demonstrated chronological patterns. Burinated pieces were most numerous at Hac1 Elamxanli and sharply decreased over the two phases of the Göytepe sequence. Conversely, splintered pieces became progressively more common in the late Neolithic stages.

\subsection{Conclusions}

This chapter describes the lithic assemblages we excavated in the seasons of 2008-2013. While our description of the Upper Area (the time period included in Levels 1-5) was based on a particular selection of material from thousands of recovered artifacts, the features we have identified in this chapter would remain consistent across the overall examination of the Göytepe lithic industry. This study provides the first detailed dataset in this craft for the late stage of the Shomutepe-Shulaveri culture in the Middle Kura Valley.

The industry shows both similarities and differences from the lithic assemblages that belong to the early stage of the Shomutepe culture (Hac1 Elamxanl1). Diachronic changes do exist in blank size and burinated/splintered piece frequency; however, a continuity over time is more evident in the first half of the early 6th millennium BC. This preliminary conclusion may seem at odds with major changes in cultural elements over this period. We find changes in the uses of obsidian and pottery during this period, and the architectural plan further shows significant changes (Nishiaki et al. 2015). The curious combination of continuities and discontinuities is worthy of future research. We can better understand these characteristics with reference to data from other related Neolithic sites in the Southern Caucasus. Shomutepe lithic assemblages can be found at many sites, including Mentesh (Gilbeau et al. 2017) and Damjili Cave (Nishiaki et al. 2019b), Azerbaijan; Archulo (Gatsov and Nedelcheva 2017) and Gadachrili Gora (Hamon et al. 2016), Georgia; and Aknashen, Aratashen (Varoutsikos 2015), and Masis Blur (Martirosyan-Olshansky et al. 2013), Armenia. These data suggest potential variabilities in technotypological details. In order to define the meaning of such variability, a comparison of the reliable datasets from controlled excavations will be indispensable. We hope that this study can provide a basis for future comparative studies of the lithic assemblages from these sites.

\section{References}

Akhundov, T. (2004) South Caucasus in the Neolithic to Early Bronze Age: The question of epochs and periods. In: A View from the Highlands: Archaeological Studies in Honour of Charles Burney, edited by A. Sagona, pp. 421-436. Leuven: Peeters.

Astruc, L., M. Ben Tkaya, L. Torchy, C. Altinbilek, S. Balcı, C. Bontemps, B. Gassin, S. Ducret, N. Kayacan, K. Kayan, N. Kurt, O. Oral, M. Özbaşaran, 
J. Pelegrin, A. Rodriguez Rodriguez, and Ö. Toprak (2012) De l'efficacité des faucilles néolithiques au Proche-Orient: Approche expérimentale. Bulletin de la Société Préhistorique Française, 109(4): 671-687.

Chabot, J. and J. Pelegrin (2012). Two examples of pressure blade production with a lever: Recent research from the southern Caucasus (Armenia) and Northern Mesopotamia (Syria, Iraq). In: The Emergence of Pressure Blade Making, edited by P. M. Desrosiers, pp. 181-198. New York: Springer.

Clark, J. E. (2012) Stoneworkers' approaches to replicating prismatic blades. In: The Emergence of Pressure Blade Making, edited by P. M. Desrosiers, pp. 43-135. New York: Springer.

Copeland, L. (1996) The flint and obsidian industries. In: Tell Sabi Abyad: The Late Neolithic Settlement. Report on the Excavations of the University of Amsterdam (1988) and the National Museum of Antiquities Leiden (1991-1993) in Syria, edited by P. M. M. G. Akkermans, pp. 285-339. Istanbul: Nederlands Historisch-Archaeologisch Instituut te Istanbul.

Debenath, A. and H. L. Dibble (1994) Handbook of Paleolithic Typology. Volume One: Lower and Middle Paleolithic of Europe. Philadelphia: The University Museum, University of Pennsylvania.

Gatsov, I and P. Nedelcheva (2017) Latest results on the obsidian assemblages from the Neolithic period of Aruchlo I, Georgia. In: The Kura Projects: New Research on the Later Prehistory of the Southern Caucasus, edited by B. Helwing, T. Aliyev, B. Lyonnet, F. Guliyev, S. Hansen, and G. Mirtskhulava, pp. 247-253. Berlin: German Institute of Archaeology, Eurasian Department.

Gilbeau, D., L. Astruc, and A. Samzun (2017) Chipped stone industries from Mil Plain (Kamiltepe) and the Middle Kura Valley (Menteshtepe), Azerbaijan. In: The Kura Projects: New Research on the Later Prehistory of the Southern Caucasus, edited by B. Helwing, T. Aliyev, B. Lyonnet, F. Guliyev, S. Hansen, and G. Mirtskhulava, pp. 385-398. Berlin: German Institute of Archaeology, Eurasian Department.

Guliyev, F. and Y. Nishiaki (2014) Excavations at the Neolithic settlement of Göytepe, West Azerbaijan, 2010-2011. In: Proceedings of the 8th International Congress of the Archaeology of the Ancient Near East, Vol. 2, edited by P. Bieliński, M. Gawlikowski, R. Koliński, D. Ławecka, A. Sołtysiak, and Z. Wygnańska, pp. 3-16. Wiesbaden: Harrassowitz Verlag.

Hamon, C., M. Jalabadze, T. Agapishvili, E. Baudouin, I. Koridze, and E. Messager (2016) Gadachrili Gora: Architecture and organisation of a Neolithic settlement in the middle Kura Valley (6th millennium BC, Georgia). Quaternary International, 395: 154169.
Inizan, M. L., M. Reduron-Ballinger, H. Roche, and J. Tixier (1999) Technology and Terminology of Knapped Stone. Nanterre: CREP.

Kadowaki, S., F. Guliyev, and Y. Nishiaki (2016) Chipped stone technology of the earliest agricultural village in the Southern Caucasus: Hacı Elamxanlı Tepe (the beginning of the 6th millennium BC). In: Proceedings of the 9th International Congress on the Archaeology of the Ancient Near East, Vol. 3, edited by O. Kaelin and H. -P. Mathys, pp. 709-723. Wiesbaden: Harrassowitz Verlag.

Martirosyan-Olshansky, K., G. E. Areshian, P. S. Avestiyan, and A. Hayrapetyan (2013) Masis Blur: A Late Neolithic settlement in the plain of Ararat, Armenia. Backdirt, 2013: 142-146.

Nishiaki, Y. (1985) Truncated-faceted flakes from Levantine Mousterian assemblages. Bulletin of Department of Archaeology, The University of Tokyo, 4: 215-226.

Nishiaki, Y. (2000) Lithic Technology of Neolithic Syria. Oxford: Archaeopress.

Nishiaki, Y., F. Guliyev, S. Kadowaki, V. Alakbarov, T. Miki, S. Salimbeyov, C. Akashi, and S. Arai (2015) Investigating cultural and socioeconomic change at the beginning of the Pottery Neolithic in the Southern Caucasus - The 2013 excavations at Hacl Elamxanlı Tepe, Azerbaijan. Bulletin of the American School of Oriental Research, 374: 1-28.

Nishiaki, Y. and F. Guliyev (2019) Neolithic lithic industries of the Southern Caucasus: Göytepe and Hacı Elamxanlı Tepe, West Azerbaijan (early 6th millennium BC). In: Near Eastern Lithics on the Move: Interaction and Contexts in Neolithic Traditions, edited by L. Astruc, F. Briois, C. McCartney, and L. Kassianidou, pp. 471-483. Nicosia: Astrom Editions.

Nishiaki, Y., F. Guliyev, S. Kadowaki, and T. Omori (2018) Neolithic residential patterns in the southern Caucasus: Radiocarbon analysis of rebuilding cycles of mudbrick architecture at Göytepe, west Azerbaijan. Quaternary International, 474: 119-130.

Nishiaki, Y., O. Maeda, T. Kannari, M. Nagai, E. Healey, F. Guliyev, and S. Campbell (2019a) Obsidian provenance analyses at Göytepe, Azerbaijan: Implications for understanding Neolithic socioeconomies in the Southern Caucasus. Archaeometry, 61(4): 765-782.

Nishiaki, Y., A. Zeynalov, M. Mansrov, C. Akashi, S. Arai, K. Shimogama, and F. Guliyev (2019b) The Mesolithic-Neolithic interface in the Southern Caucasus: 2016-2017 excavations at Damjili Cave, West Azerbaijan. Archaeological Research in Asia, 19: 100140.

Pelegrin, J. (2012). New experimental observations for the characterization of pressure blade production techniques. In: The Emergence of Pressure Blade 
Making, edited by P. M. Desrosiers, pp. 465-500. New York: Springer.

Varoutsikos, B. (2015) The Mesolithic-Neolithic Transition in the South Caucasus: Cultural Transmission and Technological Transfer. Cambridge: Harvard University (Ph.D. Dissertation). 
Chapter 10

\title{
Use-wear analysis of chipped stone artifacts from Göytepe
}

\author{
Katsunori Takase
}

\subsection{Objective}

Stone tools have played an important role in our understanding of Neolithic subsistence economies. In particular, along the paleoethnobotanical approach, examinations of tool function are indispensable for studying the use of major cereals. Neolithic case studies of lithic use-wear analysis have been, thus far, mainly applied to major types of flint tools related to agriculture. At Göytepe, however, the discovery of a number of obsidian artifacts with bone implements may suggest that the stone tools had a close relationship not only with plant use but also with crafting bone tools. The purpose of this study is to reveal the functions of Göytepe stone tools and to collect information about the use of plant and animal resources by the Neolithic society in the southern Caucasus.

\subsection{Specimens and method}

The specimens analyzed in this study are chipped stone artifacts excavated at Göytepe in 2009 and 2010. Artifacts with glossed/smoked surfaces, microflakings, or distinct striations from each level of the site were identified by naked-eye observation. The total number of selected specimens was 70 (Table 10.1). Sixty-seven (96\%) are made of obsidian (Figs. 10.1-10.6) and the remaining three are made of flint (Fig. 10.7: 1 and 2). The preservation condition of these stone tools is generally good. Under the microscope, slight weathering can be seen, such as cracks, pits, and irregular striations, likely formed in the post-depositional process. However, the influence of weathering has only a limited effect on the analysis.

The present study employed the high-powered technique of lithic use-wear analysis (Keeley 1977, 1980). At $100 \times, 200 \times$, and $500 \times$ magnifications, the specimens were observed using a metallographic microscope with incident light (Olympus BX-FM) and photomicrographs were taken with a digital camera (Olympus Camedia C-4040 Zoom) mounted on the microscope. Before microscopic observation, fat on the surface of the stone artifacts was removed using scientific-grade paper wipers laced with ethanol. Worked materials were mainly assessed by referring to the classification scheme of use-wear polish established by Kajiwara and Akoshima (1981), Akoshima (1989), Akoshima and Hong (2017), and Midoshima (1986).

\subsection{Results and brief discussion}

Among the 70 artifacts (87 edges), striations or use-wear polish can be seen on 40 specimens (57 edges). This means that - although micro-flaking can be seen by naked-eye observation - more than $40 \%$ of the stone artifacts have no traces of use at the microscopic level (Table 10.1). Use-wear polish was detected on three retouched blades (four edges), a denticulate (two edges), a retouched flake (one edge), 11 burinated pieces (12 edges), two splintered pieces (two edges), an exhausted core (one edge), and four sickle elements (four edges). No distinct use traces could be observed on blades, a scraper, a borer, a flake, notched flakes, and spalls. Results of the analysis are outlined below. For the typological description of these tools, see Chapter 9.

10.3.1 Edge-damaged blades, denticulates, and exhausted cores

Worked materials could not be specified due to the 


\begin{tabular}{|c|c|c|c|c|c|c|c|c|c|c|c|c|c|}
\hline 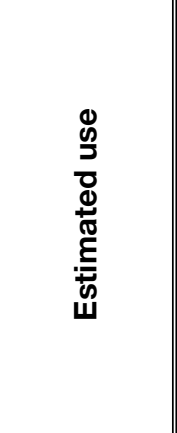 & 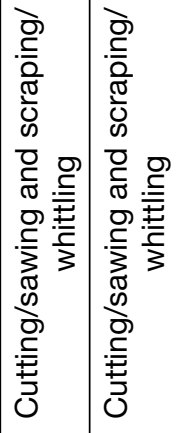 & 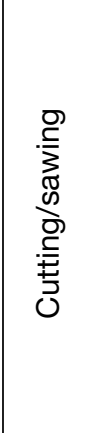 & 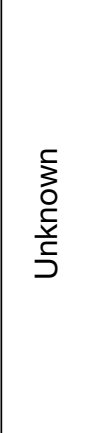 & 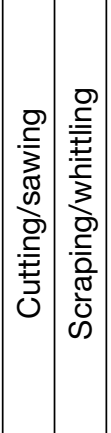 & 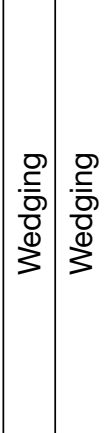 & $\begin{array}{l}\frac{5}{3} \\
0 \\
\frac{5}{5} \\
\frac{5}{5}\end{array}$ & $\begin{array}{l}\frac{5}{3} \\
0 \\
\frac{5}{5} \\
\text { J̦ }\end{array}$ & 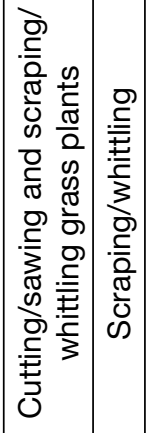 & 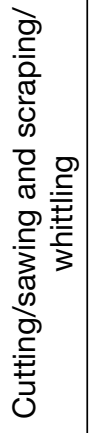 & 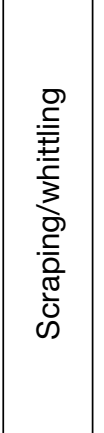 & 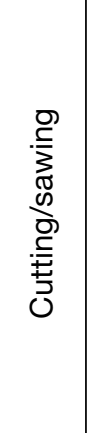 & 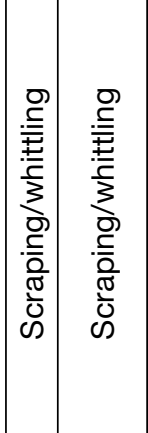 & $\begin{array}{l}\frac{1}{3} \\
0 \\
\frac{5}{5} \\
\text { c } \\
\end{array}$ \\
\hline 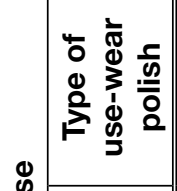 & & & & & & & & $\begin{array}{l}\stackrel{0}{2} \\
\stackrel{2}{0} \\
0 \\
0 \\
0\end{array}$ & & & & & \\
\hline 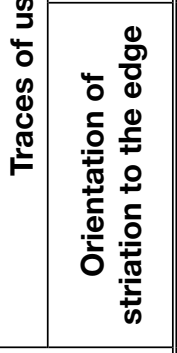 & 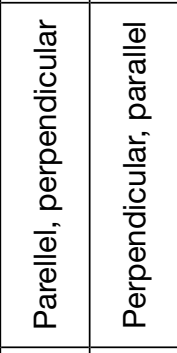 & $\begin{array}{l}\frac{\bar{\Phi}}{\overline{\widetilde{\sigma}}} \\
\overline{\widetilde{\sigma}} \\
\alpha\end{array}$ & & 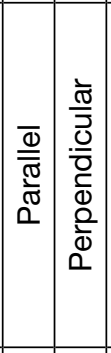 & 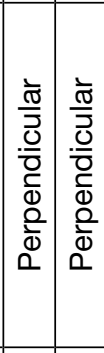 & & & 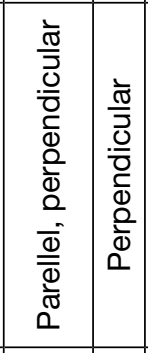 & 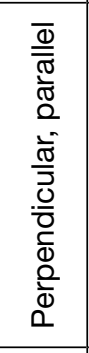 & 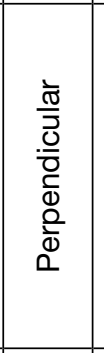 & $\begin{array}{l}\frac{\bar{\Phi}}{\overline{\widetilde{N}}} \\
\frac{\bar{\sigma}}{\alpha} \\
\frac{0}{0}\end{array}$ & 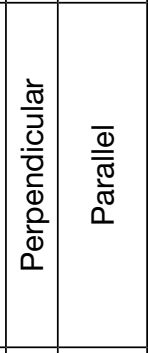 & \\
\hline 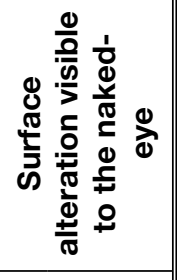 & & & & & & & & 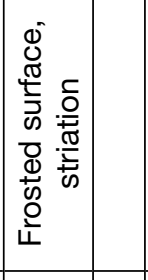 & & 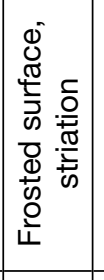 & 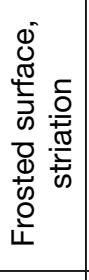 & & \\
\hline 壱 & 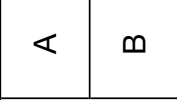 & & & $\ll \infty$ & $\ll$ & & & $\varangle \quad \infty$ & $\varangle$ & $\varpi$ & 0 & 0 & \\
\hline 竞 & & & & & & & & $\begin{array}{l}\check{\check{C}} \\
\dot{\circ} \\
\dot{0} \\
\dot{\square}\end{array}$ & & & & & \\
\hline 竞 & $\begin{array}{l}\frac{1}{0} \\
\frac{0}{0} \\
\frac{0}{0} \\
\frac{0}{0}\end{array}$ & $\begin{array}{l}\frac{.}{.0} \\
\frac{0}{0} \\
\frac{0}{00} \\
\frac{0}{0}\end{array}$ & $\begin{array}{l}\frac{1}{.0} \\
\frac{0}{0} \\
\frac{0}{00} \\
0 \\
0\end{array}$ & $\begin{array}{l}\frac{.}{0} \\
\frac{.0}{00} \\
\frac{0}{0}\end{array}$ & $\begin{array}{l}\frac{.0}{0} \\
\frac{.0}{00} \\
\frac{0}{0}\end{array}$ & $\begin{array}{l}\frac{. \pi}{0} \\
\frac{.0}{0} \\
\frac{0}{0}\end{array}$ & $\begin{array}{l}\frac{.0}{0} \\
\frac{.0}{0} \\
\frac{0}{0}\end{array}$ & 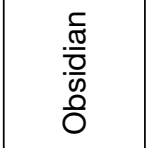 & & 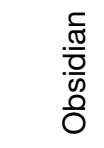 & & $\begin{array}{l}\frac{.0}{\frac{\pi}{0}} \\
\frac{.0}{00} \\
\frac{0}{0}\end{array}$ & $\begin{array}{l}\frac{.}{0} \\
\frac{.0}{0} \\
\frac{0}{0}\end{array}$ \\
\hline 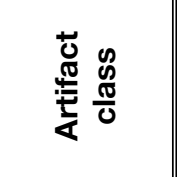 & 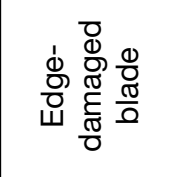 & 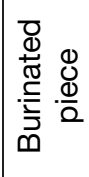 & 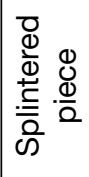 & 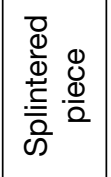 & 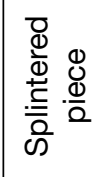 & 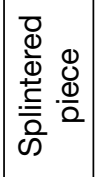 & 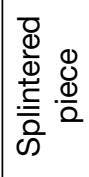 & 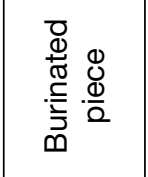 & & 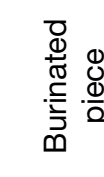 & & 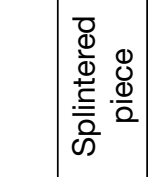 & 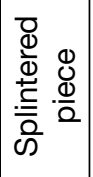 \\
\hline ग्త & $\begin{array}{l}\overline{\bar{o}} \\
\stackrel{0}{0} \\
\end{array}$ & $\infty$ & 10 & 10 & 10 & ما & ما & 10 & & 10 & & ما & 10 \\
\hline 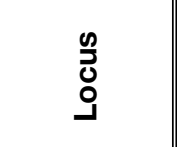 & $\frac{\bar{T}}{\overline{\bar{g}}}$ & $\frac{p}{\frac{p}{q}}$ & $\frac{\varphi}{\frac{1}{q}}$ & $\frac{\infty}{\frac{1}{1}}$ & $\frac{\frac{9}{1}}{\frac{1}{q}}$ & $\frac{\hat{\frac{1}{n}}}{\frac{1}{q}}$ & $\frac{\stackrel{t}{I}}{\stackrel{g}{q}}$ & 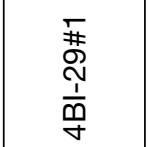 & & 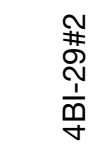 & & 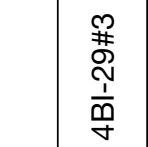 & 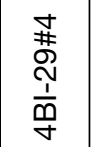 \\
\hline í & $r$ & $\sim$ & $m$ & $\nabla$ & م & 0 & $\wedge$ & $\infty$ & & $\sigma$ & & 으 & $\mp$ \\
\hline
\end{tabular}




\begin{tabular}{|c|c|c|c|c|c|c|c|c|c|c|c|c|c|c|}
\hline 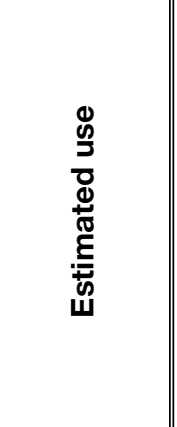 & 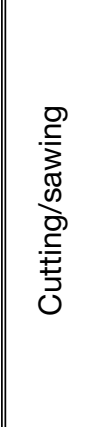 & 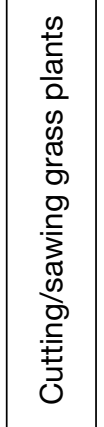 & 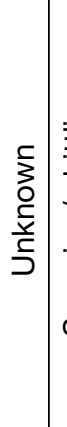 & 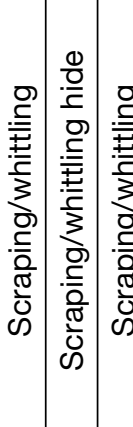 & 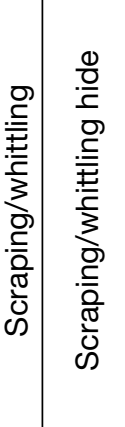 & 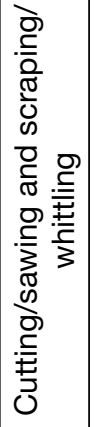 & 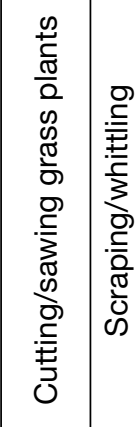 & 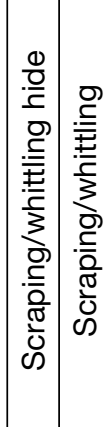 & 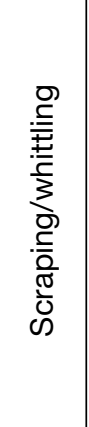 & $\begin{array}{l}\frac{1}{3} \\
0 \\
\frac{5}{2} \\
\frac{5}{5}\end{array}$ & $\begin{array}{l}\frac{5}{3} \\
0 \\
\frac{1}{2} \\
\text { c } \\
\text { J }\end{array}$ & 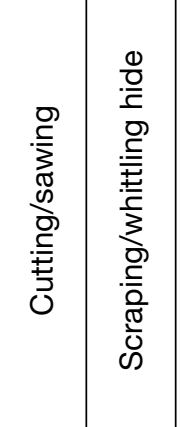 & 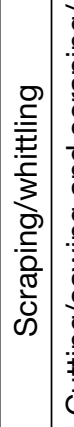 & 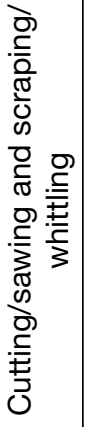 \\
\hline 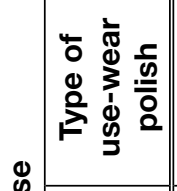 & & $\begin{array}{l}\stackrel{0}{2} \\
\stackrel{2}{+} \\
1 \\
0 \\
0\end{array}$ & & $\mid$\begin{tabular}{l}
0 \\
$\stackrel{2}{2}$ \\
\multirow{2}{1}{} \\
$\vdots$ \\
0 \\
0
\end{tabular} & 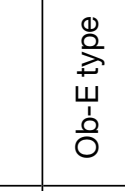 & & \begin{tabular}{l}
0 \\
$\stackrel{2}{2}$ \\
\multirow{1}{1}{} \\
0 \\
0
\end{tabular} & 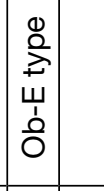 & & & & 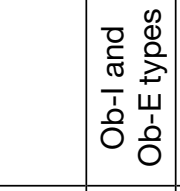 & & \\
\hline 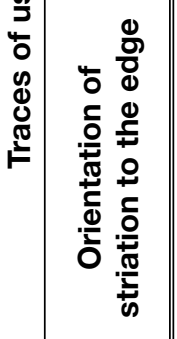 & $\begin{array}{l}\frac{\bar{\Phi}}{\overline{\widetilde{\sigma}}} \\
\frac{\widetilde{\sigma}}{\alpha}\end{array}$ & $\begin{array}{l}\bar{\Phi} \\
\frac{\bar{\sigma}}{\bar{\sigma}} \\
\frac{\bar{\sigma}}{0} \\
0\end{array}$ & & 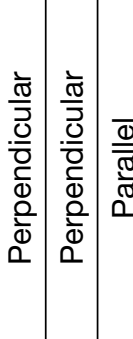 & 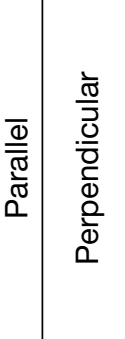 & 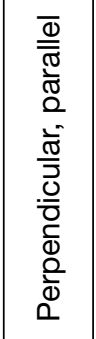 & 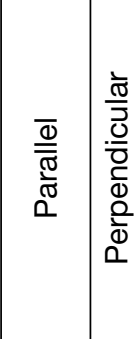 & 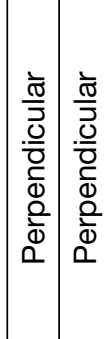 & 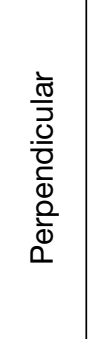 & & & 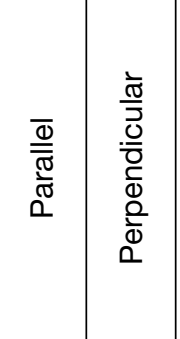 & 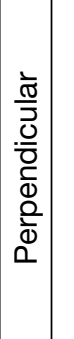 & 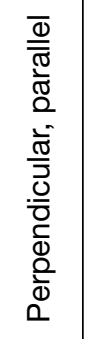 \\
\hline 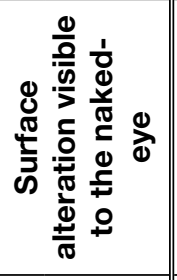 & & 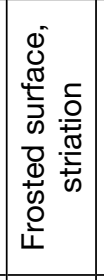 & & & 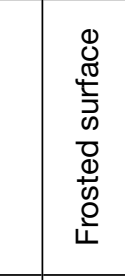 & & 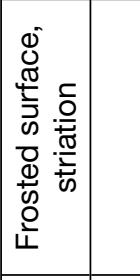 & 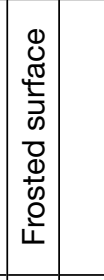 & & & & 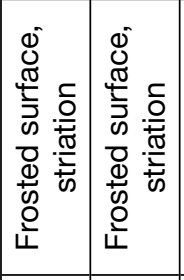 & & 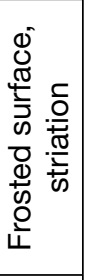 \\
\hline 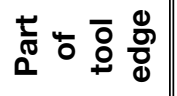 & & & & $\ll \mid \infty \quad 0$ & 0 & & $\varangle \quad \infty$ & $<\mid$ & & & & $\varangle$ & $\varangle$ & $\varpi$ \\
\hline 高 & & $\begin{array}{l}\stackrel{\sim}{\check{0}} \\
\stackrel{\circ}{\circ} \\
\dot{0}\end{array}$ & & 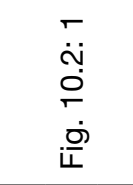 & \begin{tabular}{l}
$m$ \\
$\ddot{0}$ \\
$\dot{0}$ \\
\hdashline \\
$\dot{0}$ \\
$\ddot{0}$
\end{tabular} & & 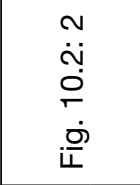 & $\begin{array}{l}\bar{r} \\
\ddot{g} \\
\stackrel{0}{0} \\
\dot{\rho} \dot{i}\end{array}$ & & & & & & \\
\hline 竞 & $\begin{array}{l}\frac{1}{0} \\
\frac{0}{0} \\
\frac{0}{0} \\
0\end{array}$ & $\begin{array}{l}\frac{c}{0} \\
\frac{.0}{0} \\
\frac{0}{0}\end{array}$ & 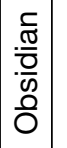 & $\begin{array}{l}\frac{1}{0} \\
\frac{.0}{00} \\
\frac{0}{0}\end{array}$ & $\begin{array}{l}\frac{.}{0} \\
\frac{00}{00} \\
\frac{0}{0}\end{array}$ & $\begin{array}{l}\frac{.}{0} \\
\frac{.0}{0} \\
\frac{0}{0}\end{array}$ & $\begin{array}{l}\frac{.}{0} \\
\frac{.0}{0} \\
\frac{0}{0}\end{array}$ & $\begin{array}{l}\frac{1}{.0} \\
\frac{.0}{00} \\
\frac{0}{0} \\
0\end{array}$ & $\begin{array}{l}\frac{.0}{0} \\
\frac{0}{0} \\
\frac{0}{0}\end{array}$ & $\begin{array}{l}\frac{.0}{0} \\
\frac{0}{0} \\
\frac{0}{0}\end{array}$ & $\begin{array}{l}\frac{.}{0} \\
\frac{.0}{0} \\
\frac{0}{0}\end{array}$ & $\begin{array}{l}\frac{1}{0} \\
\frac{.0}{0} \\
\frac{0}{0} \\
0\end{array}$ & & $\begin{array}{l}\frac{.}{0} \\
\frac{.0}{0} \\
\frac{0}{0} \\
0\end{array}$ \\
\hline 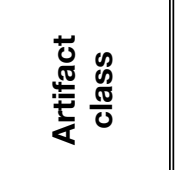 & 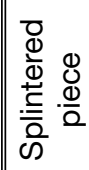 & 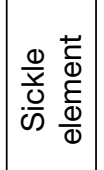 & $\begin{array}{l}\overline{\bar{\sigma}} \\
\frac{0}{0} \\
\end{array}$ & 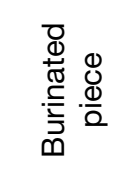 & 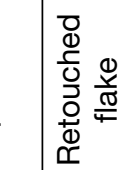 & 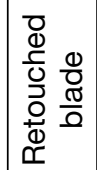 & 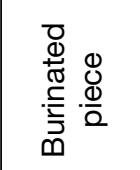 & 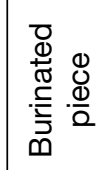 & 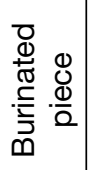 & 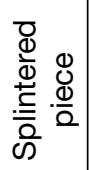 & 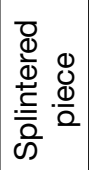 & 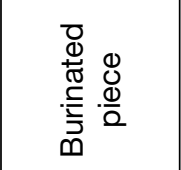 & & : \\
\hline \্త & 10 & 10 & 10 & 10 & 10 & 0 & 0 & 0 & 0 & 0 & 0 & 0 & & 0 \\
\hline 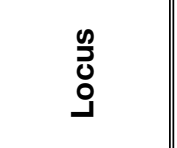 & 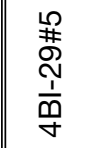 & $\begin{array}{l}0 \\
\stackrel{0}{7} \\
\stackrel{N}{1} \\
\frac{1}{9}\end{array}$ & 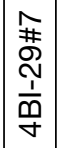 & $\begin{array}{l}\mathscr{Q} \\
\stackrel{1}{1} \\
\frac{1}{q}\end{array}$ & $\frac{\underline{p}}{\frac{p}{q}}$ & $\begin{array}{l}\text { 䓪 } \\
\frac{1}{\frac{1}{9}} \\
\frac{9}{q}\end{array}$ & 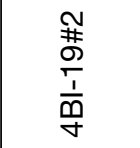 & 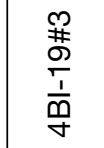 & $\begin{array}{l}\underset{+}{+} \\
\stackrel{\frac{1}{4}}{\frac{1}{9}}\end{array}$ & $\begin{array}{l}\text { N } \\
\stackrel{\#}{\sim} \\
\frac{1}{1} \\
\stackrel{q}{q}\end{array}$ & 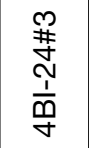 & $\frac{\stackrel{\infty}{N}}{\frac{1}{\dddot{q}}}$ & & 等 \\
\hline í & $\stackrel{\simeq}{\leftarrow}$ & $\stackrel{m}{\square}$ & $\stackrel{\nabla}{\sim}$ & $\stackrel{n}{\sim}$ & $\stackrel{\varphi}{\leftarrow}$ & 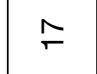 & $\stackrel{\infty}{\leftarrow}$ & $\stackrel{\circ}{\square}$ & 尺 & $\bar{N}$ & N & $\stackrel{\mathscr{N}}{ }$ & & $\stackrel{d}{\sim}$ \\
\hline
\end{tabular}




\begin{tabular}{|c|c|c|c|c|c|c|c|c|c|c|c|c|c|c|c|c|}
\hline 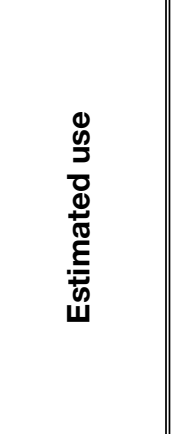 & 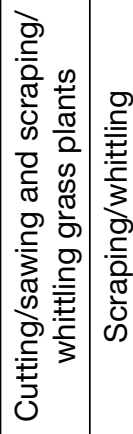 & 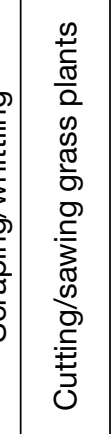 & 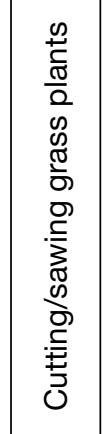 & 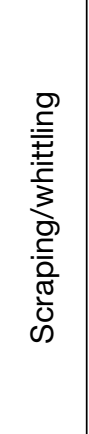 & 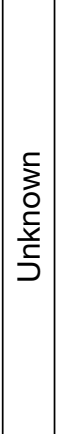 & 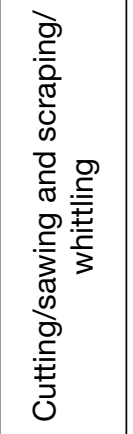 & 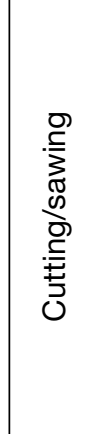 & 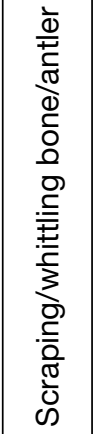 & & & $\begin{array}{l}\frac{5}{3} \\
\frac{5}{5} \\
\frac{5}{5} \\
\text { د }\end{array}$ & $\begin{array}{l}5 \\
\vdots \\
0 \\
\frac{5}{5} \\
5\end{array}$ & 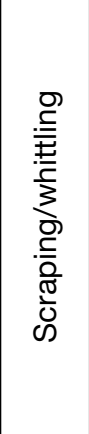 & $\begin{array}{l}\frac{5}{3} \\
0 \\
\frac{5}{5} \\
\text { د }\end{array}$ & 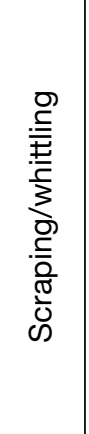 & $\begin{array}{l}\frac{5}{3} \\
\frac{5}{5} \\
\frac{5}{5}\end{array}$ \\
\hline 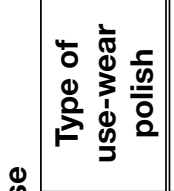 & 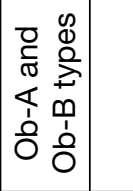 & $\begin{array}{ll}0 & 0 \\
0 & 0 \\
\tau & 0 \\
\pi & 2 \\
1 & 0 \\
1 & 1 \\
0 & 0 \\
0 & 0 \\
0\end{array}$ & $\left|\begin{array}{ll}0 & 0 \\
0 & 0 \\
0 & 0 \\
0 & 2 \\
1 & 0 \\
0 & 1 \\
0 & 0 \\
0 & 0\end{array}\right|$ & & & & & $\begin{array}{l}\stackrel{0}{2} \\
\stackrel{2}{2} \\
0 \\
0 \\
0\end{array}$ & & & & & & & & \\
\hline 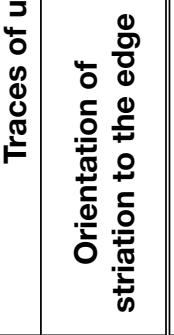 & 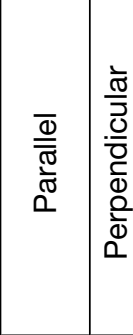 & $\begin{array}{l}\frac{\bar{\Phi}}{\overline{\bar{\sigma}}} \\
\overline{\widetilde{\sigma}} \\
\Omega\end{array}$ & $\begin{array}{l}\frac{\bar{\Phi}}{\overline{\widetilde{\pi}}} \\
\frac{\bar{\sigma}}{\alpha}\end{array}$ & 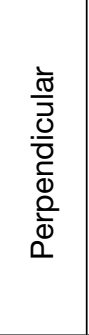 & & 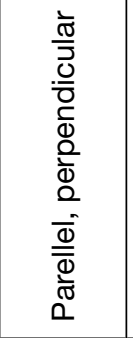 & $\begin{array}{l}\frac{\bar{\Phi}}{\overline{\widetilde{\sigma}}} \\
\overline{\widetilde{\sigma}} \\
\alpha\end{array}$ & 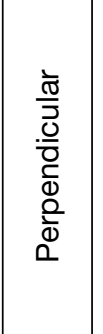 & & & & & 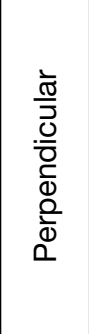 & & $\begin{array}{l}\frac{\bar{\sigma}}{\overline{0}} \\
\frac{0}{\overline{0}} \\
\frac{0}{0} \\
\frac{0}{0} \\
\frac{0}{0} \\
0\end{array}$ & \\
\hline 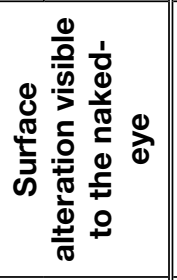 & 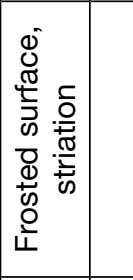 & 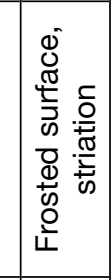 & & & & & & & & & & & & & & \\
\hline 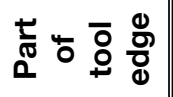 & $\varangle \quad \infty$ & $\varangle$ & $\infty$ & & & & & & & & & & & & & \\
\hline 高 & 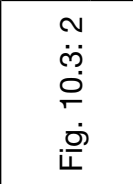 & & $\begin{array}{l}\check{r} \\
\ddot{8} \\
\dot{0} \\
\dot{0} \\
\dot{i}\end{array}$ & & & & & $\begin{array}{l}N \\
\ddot{\forall} \\
\dot{0} \\
\dot{0} \\
\dot{\square}\end{array}$ & & & & & & & & \\
\hline 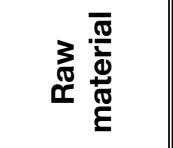 & $\begin{array}{l}\frac{7}{.0} \\
\frac{.0}{00} \\
\frac{0}{0} \\
0\end{array}$ & & $\begin{array}{l}\frac{.}{.0} \\
\frac{0}{6} \\
\frac{0}{00} \\
0\end{array}$ & $\begin{array}{l}\frac{\sqrt{\pi}}{\frac{0}{0}} \\
\frac{0}{0} \\
0 \\
0\end{array}$ & 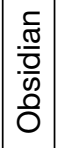 & 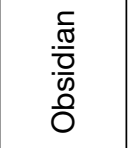 & $\begin{array}{l}\frac{7}{\frac{\pi}{0}} \\
\frac{.0}{0} \\
\frac{0}{0}\end{array}$ & $\begin{array}{l}\frac{.}{\frac{\pi}{0}} \\
\frac{.0}{0} \\
\frac{0}{0}\end{array}$ & $\begin{array}{ll}\frac{.}{0} & \\
\frac{.0}{0} & : \\
0 & 0 \\
0 & 0\end{array}$ & $\begin{array}{l}\frac{c}{0} \\
\frac{0}{0} \\
\frac{0}{0} \\
0\end{array}$ & $\begin{array}{l}\frac{.}{0} \\
\frac{0}{0} \\
\frac{0}{0}\end{array}$ & $\begin{array}{l}\frac{.}{\frac{\pi}{0}} \\
\frac{.0}{00} \\
\frac{0}{0}\end{array}$ & $\begin{array}{l}\frac{.}{0} \\
\frac{.0}{00} \\
\frac{0}{0}\end{array}$ & $\mid$\begin{tabular}{l|}
$\frac{c}{.0}$ \\
$\frac{.0}{0}$ \\
$\frac{0}{00}$ \\
$\frac{0}{0}$
\end{tabular} & $\begin{array}{l}\frac{.}{\frac{\pi}{0}} \\
\frac{0}{0} \\
\frac{0}{0}\end{array}$ & $\begin{array}{l}\frac{.}{\frac{\pi}{0}} \\
\frac{.0}{0} \\
\frac{0}{0}\end{array}$ \\
\hline 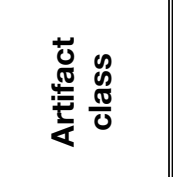 & 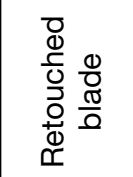 & 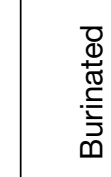 & 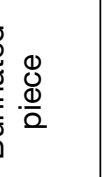 & 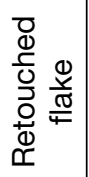 & $\frac{\frac{0}{0}}{\frac{\pi}{0}}$ & 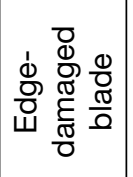 & 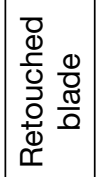 & 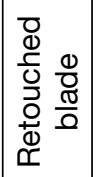 & $\frac{\frac{0}{0}}{\frac{\pi}{\infty}}$ & 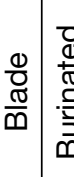 & 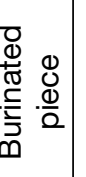 & $\begin{array}{l}\overline{\overline{\widetilde{J}}} \\
\text { के }\end{array}$ & 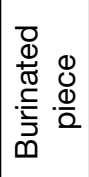 & $\begin{array}{l}\overline{\bar{\sigma}} \\
\text { की }\end{array}$ & $\begin{array}{l}\frac{\delta}{d} \\
\frac{d}{0} \\
\frac{0}{0} \\
\frac{0}{0} \\
\frac{0}{0} \\
\widetilde{0}\end{array}$ & 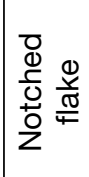 \\
\hline ब্ & $\wedge$ & & N & $\wedge$ & $\wedge$ & $\wedge$ & $\wedge$ & $\Lambda$ & $\wedge$ & $\wedge$ & $\infty$ & $\infty$ & $a$ & a & $\sigma$ & $a$ \\
\hline בִ & $\frac{\stackrel{\mathscr{N}}{\stackrel{I}{\prime}}}{\overline{\bar{M}}}$ & & $\begin{array}{l}\text { 导 } \\
\frac{1}{9} \\
\end{array}$ & $\frac{\stackrel{P}{I}}{\frac{1}{q}}$ & $\begin{array}{l}\text { 움 } \\
\frac{\dot{x}}{\bar{g}} \\
\frac{\bar{g}}{y}\end{array}$ & $\frac{\check{\check{I}}}{\overline{\bar{q}}}$ & $\frac{\bar{N}}{\stackrel{\grave{1}}{\bar{M}}}$ & $\frac{\bar{q}}{\frac{1}{q}}$ & 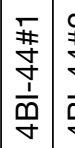 & 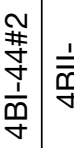 & 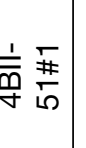 & $\frac{1}{\overline{\underline{m}}} \frac{\tilde{\#}}{5}$ & 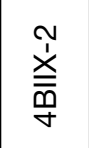 & 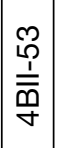 & 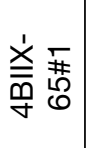 & 蒿䓀 \\
\hline í & $\stackrel{\llcorner}{\sim}$ & & $\stackrel{\sim}{\sim}$ & $\hat{N}$ & $\stackrel{\infty}{N}$ & $\stackrel{\sim}{N}$ & ᄋ్ల & $\bar{m}$ & $\approx$ & m & ले & $\stackrel{\infty}{m}$ & @ & $\hat{m}$ & $\stackrel{\infty}{\infty}$ & ஜ \\
\hline
\end{tabular}




\begin{tabular}{|c|c|c|c|c|c|c|c|c|c|c|c|c|c|c|c|c|c|}
\hline 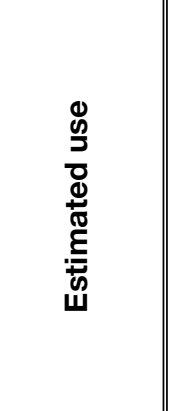 & $\begin{array}{l}\frac{5}{3} \\
\frac{5}{5} \\
\frac{5}{5}\end{array}$ & $\begin{array}{l}\frac{5}{3} \\
0 \\
\frac{5}{5} \\
\text { c }\end{array}$ & 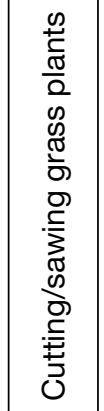 & 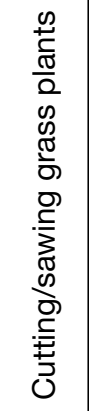 & 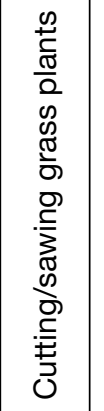 & 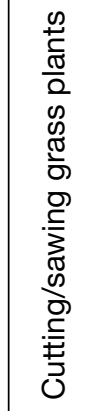 & 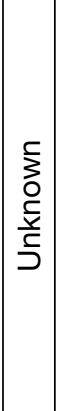 & $\begin{array}{l}\frac{5}{3} \\
0 \\
\frac{5}{5} \\
\frac{5}{5}\end{array}$ & $\begin{array}{l}\frac{c}{2} \\
0 \\
\frac{\Sigma}{2} \\
\text { c }\end{array}$ & 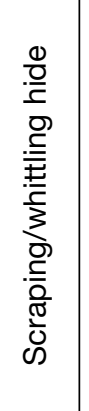 & 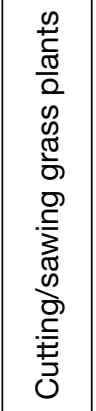 & 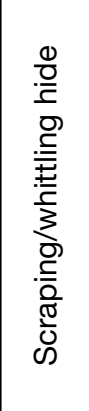 & 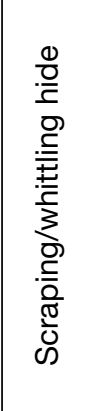 & $\begin{array}{l}\frac{5}{3} \\
0 \\
\frac{5}{5} \\
\frac{5}{5}\end{array}$ & 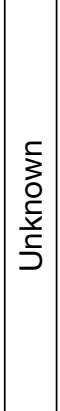 & $\begin{array}{l}\frac{5}{3} \\
0 \\
\frac{1}{5} \\
\frac{5}{5}\end{array}$ & 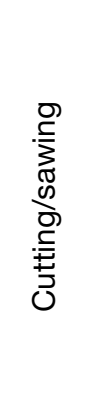 \\
\hline 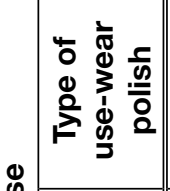 & & & 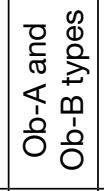 & 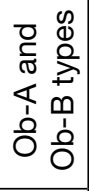 & 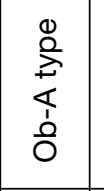 & \begin{tabular}{l}
0 \\
\multirow{2}{2}{} \\
\multirow{1}{1}{} \\
0 \\
0
\end{tabular} & & & & 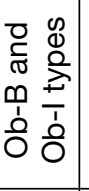 & $\left|\begin{array}{ll}0 & 0 \\
0 & 0 \\
\sigma & 0 \\
\frac{0}{2} & 0 \\
0 & 0 \\
0 & 1 \\
0 & 0 \\
0\end{array}\right|$ & $\begin{array}{l}\frac{0}{2} \\
\frac{0}{1} \\
\frac{0}{0}\end{array}$ & $\begin{array}{l}\frac{0}{2} \\
\frac{0}{1} \\
\frac{1}{0}\end{array}$ & & & & \\
\hline 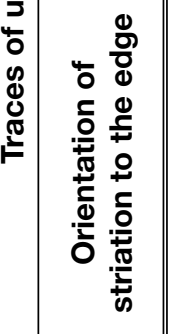 & & & $\begin{array}{l}\frac{\bar{\Phi}}{\overline{\bar{\sigma}}} \\
\overline{\bar{\sigma}} \\
\bar{\alpha}\end{array}$ & $\begin{array}{l}\overline{\bar{\Phi}} \\
\overline{\overline{\widetilde{\sigma}}} \\
\overline{\widetilde{\sigma}} \\
\bar{\alpha}\end{array}$ & 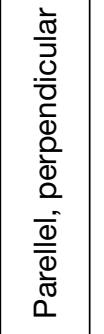 & 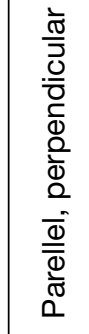 & & & & 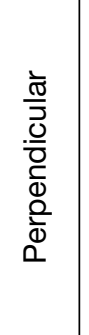 & $\begin{array}{l}\frac{\bar{\Phi}}{\overline{\bar{\sigma}}} \\
\frac{\bar{\sigma}}{\alpha} \\
\frac{\alpha}{\alpha}\end{array}$ & 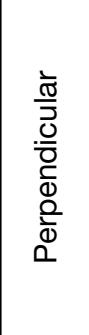 & 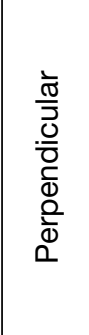 & & & & $\begin{array}{l}\overline{\bar{\Phi}} \\
\overline{\bar{\sigma}} \\
\overline{\widetilde{\sigma}} \\
\alpha\end{array}$ \\
\hline 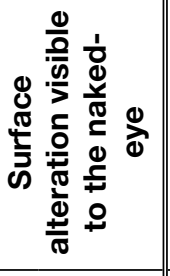 & & & & 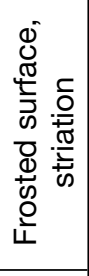 & 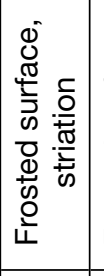 & 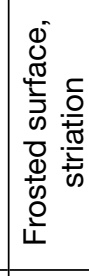 & & & & & 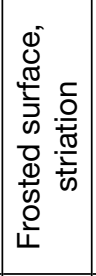 & & & & & & \\
\hline$\frac{t}{\pi} \div \overline{0} \frac{0}{8}$ & & & & & $\varangle$ & $\infty$ & & & & & & & & & & & \\
\hline 高 & & & & 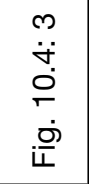 & 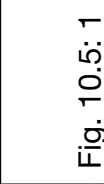 & 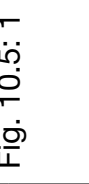 & & & & & 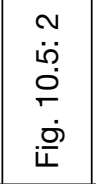 & & $\begin{array}{l}\stackrel{N}{0} \\
\ddot{\dot{\theta}} \\
+ \\
\dot{0}\end{array}$ & & & & \\
\hline 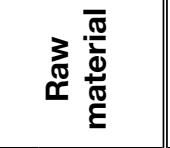 & $\begin{array}{l}\frac{c}{\frac{\pi}{0}} \\
\frac{0}{0} \\
\frac{0}{0}\end{array}$ & $\begin{array}{l}\frac{7}{.0} \\
\frac{0}{0} \\
\frac{0}{0} \\
0 \\
0\end{array}$ & 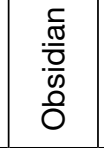 & $\begin{array}{l}\frac{c}{\frac{\pi}{0}} \\
\frac{0}{0} \overline{0} \\
\frac{0}{0}\end{array}$ & & 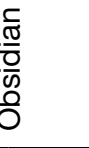 & 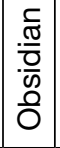 & $\begin{array}{l}\frac{.}{\bar{w}} \\
\frac{.0}{0} \\
\frac{0}{0} \\
\end{array}$ & $\begin{array}{l}\frac{.}{0} \\
\frac{0}{0} \\
\frac{0}{0}\end{array}$ & $\begin{array}{l}\frac{.0}{0} \\
\frac{.0}{0} \\
\frac{0}{0} \\
0\end{array}$ & 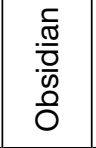 & 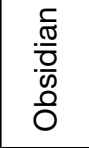 & $\begin{array}{l}\frac{7}{\frac{0}{0}} \\
\frac{0}{0} \\
\frac{0}{0}\end{array}$ & $\begin{array}{l}\frac{7}{\frac{0}{0}} \\
\frac{0}{0} \\
\frac{0}{0}\end{array}$ & \begin{tabular}{|l|}
$\frac{c}{-\frac{0}{0}}$ \\
$\frac{0}{0}$ \\
$\frac{0}{0}$ \\
\end{tabular} & $\begin{array}{l}\frac{.}{0} \\
\frac{0}{0} \\
\frac{0}{0} \\
0 \\
0\end{array}$ & $\begin{array}{l}\frac{.0}{0.0} \\
\frac{.0}{0} \\
\frac{0}{0}\end{array}$ \\
\hline 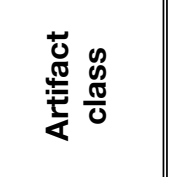 & 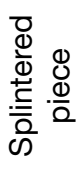 & 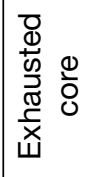 & 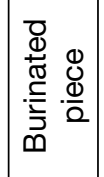 & 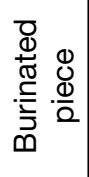 & $\begin{array}{l}0 \\
d \\
\frac{1}{0} \\
0 \\
0 \\
0 \\
\square\end{array}$ & $\frac{\pi}{0}$ & 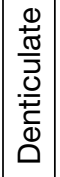 & $\begin{array}{l}\frac{D}{d} \\
\frac{d}{0} \\
\frac{0}{0} \\
\frac{0}{4} \\
\frac{0}{4}\end{array}$ & $\begin{array}{l}\overline{\bar{\sigma}} \\
\text { के }\end{array}$ & 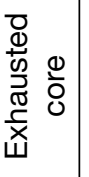 & 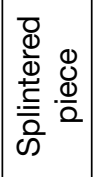 & 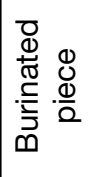 & 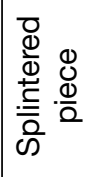 & 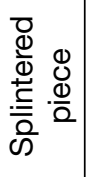 & 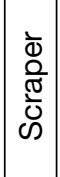 & $\begin{array}{l}\bar{\Phi} \\
\stackrel{\bar{\nu}}{\varnothing}\end{array}$ & 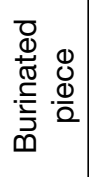 \\
\hline ब্ & 으 & 으 & 으 & 으 & & 우 & 으 & 으 & $\mp$ & $\mp$ & $\mp$ & $\mp$ & $\mp$ & $\mp$ & $F$ & $\mp$ & $\mp$ \\
\hline بٌ & 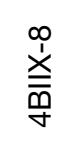 & 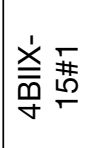 & 紊辛 & 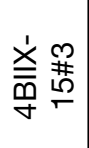 & & $\begin{array}{l}\frac{P}{\dot{y}} \\
\frac{\dot{x}}{\bar{y}} \\
\frac{y}{y}\end{array}$ & $\begin{array}{l}0 \\
\hat{1} \\
\stackrel{x}{\bar{y}} \\
\bar{q}\end{array}$ & 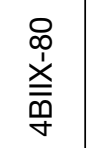 & $\begin{array}{l}\infty \\
0 \\
\dot{p} \\
\overline{\underline{y}} \\
\bar{q}\end{array}$ & 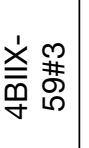 & $\begin{array}{l}\stackrel{0}{\hat{x}} \\
\stackrel{x}{\bar{g}} \\
\text { 学 }\end{array}$ & 吝荞 & $\begin{array}{l}\bar{r} \\
\stackrel{x}{\bar{x}} \\
\underline{\bar{g}}\end{array}$ & $\begin{array}{l}\frac{\infty}{\hat{1}} \\
\underline{x} \\
\underline{\bar{y}}\end{array}$ & $\frac{\infty}{\frac{\infty}{\frac{1}{m}}}$ & 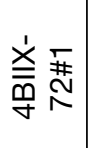 & 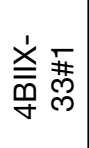 \\
\hline$\dot{z}$ & 우 & $\bar{\forall}$ & $\stackrel{\sim}{\sim}$ & $\stackrel{\mathscr{q}}{\rightarrow}$ & & $\forall$ & \& & $\mathscr{f}$ & $\hat{f}$ & $\stackrel{\infty}{+}$ & g & 오 & 5 & ธิ & న్ & نే & 员 \\
\hline
\end{tabular}




\begin{tabular}{|c|c|c|c|c|c|c|c|c|c|c|c|c|c|c|c|c|}
\hline 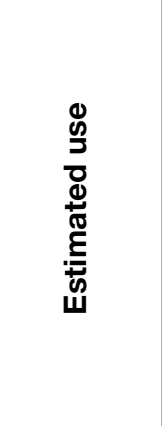 & 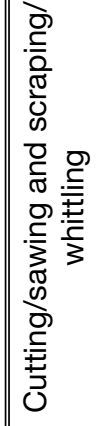 & $\begin{array}{l}\frac{5}{3} \\
\frac{5}{5} \\
\frac{5}{5} \\
\text { J }\end{array}$ & $\begin{array}{l}\frac{1}{3} \\
0 \\
\frac{5}{5} \\
\frac{5}{5}\end{array}$ & 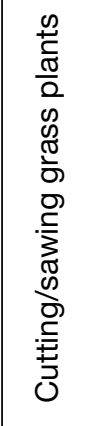 & $\begin{array}{l}\frac{5}{3} \\
0 \\
\frac{1}{2} \\
\text { c }\end{array}$ & 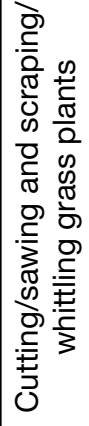 & 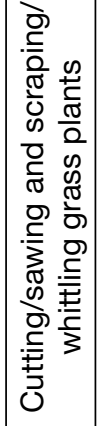 & 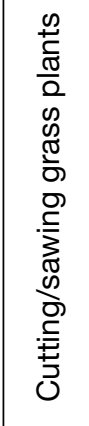 & 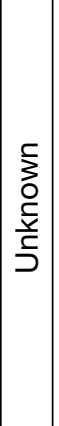 & $\begin{array}{l}\frac{5}{3} \\
0 \\
\frac{5}{5} \\
\frac{5}{5}\end{array}$ & 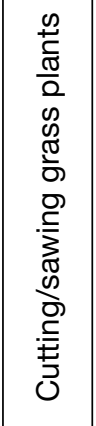 & $\begin{array}{l}5 \\
\vdots \\
0 \\
\frac{5}{5} \\
5\end{array}$ & 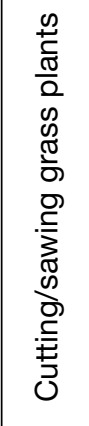 & $\begin{array}{l}\frac{1}{3} \\
0 \\
\frac{5}{5} \\
\text { c } \\
\text { J }\end{array}$ & 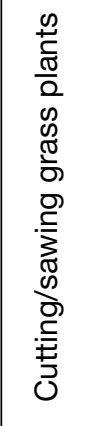 & $\begin{array}{l}\frac{5}{3} \\
\frac{5}{5} \\
\frac{5}{5}\end{array}$ \\
\hline 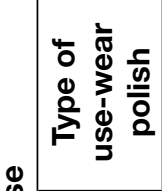 & & & & $\begin{array}{l}\infty \\
0 \\
0 \\
0 \\
\frac{0}{\pi} \\
\frac{0}{2}\end{array}$ & & $\begin{array}{l}0 \\
\stackrel{0}{2} \\
\substack{1 \\
1 \\
0} \\
0\end{array}$ & \begin{tabular}{l}
0 \\
$\stackrel{2}{2}$ \\
\multirow{1}{1}{} \\
0
\end{tabular} & 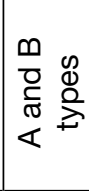 & & & 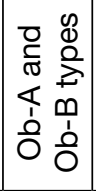 & & $\begin{array}{ll}0 & 0 \\
0 & 0 \\
\pi & 0 \\
\pi & 0 \\
1 & 0 \\
0 & 0 \\
0 & 0 \\
0\end{array}$ & & $\begin{array}{ll}\infty & \\
0 & 0 \\
0 & 0 \\
\frac{1}{\sigma} & 0 \\
< & 7\end{array}$ & \\
\hline 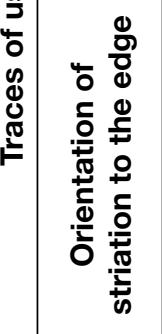 & 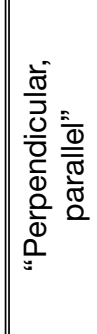 & & & $\begin{array}{l}\frac{\bar{\Phi}}{\overline{\widetilde{\sigma}}} \\
\frac{\tilde{\sigma}}{\alpha} \\
\end{array}$ & & 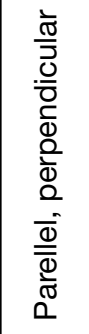 & 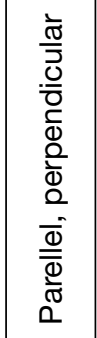 & 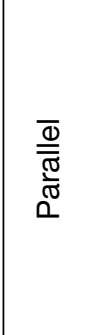 & & & $\frac{\bar{\Phi}}{\frac{\bar{\sigma}}{\bar{\sigma}}}$ & & $\begin{array}{l}\frac{\bar{\Phi}}{\overline{\bar{\sigma}}} \\
\frac{\bar{\sigma}}{\alpha}\end{array}$ & & $\frac{\overline{\bar{d}}}{\overline{\bar{\sigma}}}$ & \\
\hline 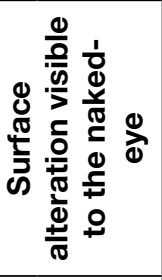 & & & & $\begin{array}{l}\infty \\
\text { OD } \\
\text { OO }\end{array}$ & & 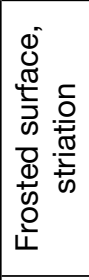 & 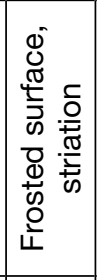 & $\begin{array}{l}0 \\
\text { OD } \\
\text { 은 }\end{array}$ & & & 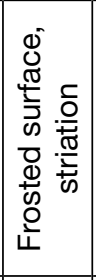 & & 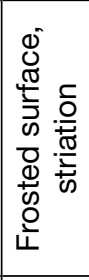 & & 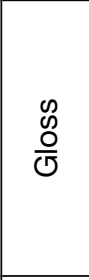 & \\
\hline ড় ๘ & & & & & & $\varangle$ & 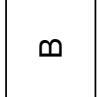 & & & & & & & & & \\
\hline 产 & & & & $\begin{array}{l}\check{r} \\
\hat{\sim} \\
+ \\
\dot{0} \\
\dot{0}\end{array}$ & & $\begin{array}{l}c \\
\dot{c} \\
c \\
\dot{c} \\
\dot{c}\end{array}$ & 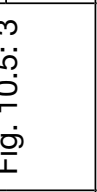 & $\begin{array}{l}\check{1} \\
\ddot{0} \\
\dot{0} \\
\dot{0}\end{array}$ & & & $\begin{array}{l}m \\
\stackrel{m}{\hat{0}} \\
\dot{0} \\
\dot{0}\end{array}$ & & & & $\begin{array}{l}\stackrel{N}{N} \\
\stackrel{\hat{O}}{\circ} \\
\dot{0} \\
\dot{0}\end{array}$ & \\
\hline 离 & $\begin{array}{l}\frac{5}{6} \\
\frac{0}{00} \\
\frac{0}{00} \\
0\end{array}$ & $\begin{array}{l}\frac{.}{\frac{\pi}{0}} \\
\frac{0}{0} \\
0 \\
0\end{array}$ & $\begin{array}{l}\frac{.5}{\frac{.0}{0}} \\
\frac{.0}{0} \\
00 \\
0\end{array}$ & $\stackrel{\overrightarrow{\underline{\underline{L}}}}{\underline{\underline{u}}}$ & $\begin{array}{l}\frac{.}{0} \\
\frac{.0}{0} \\
\frac{0}{0} \\
0\end{array}$ & & $\begin{array}{l}\frac{1}{0} \\
\frac{0}{0} \\
0 \\
0 \\
0\end{array}$ & $\stackrel{\overrightarrow{\underline{\underline{I}}}}{\underline{4}}$ & 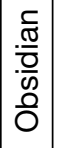 & $\begin{array}{l}\frac{.}{\frac{\pi}{0}} \\
\frac{.0}{\overline{0}} \\
0 \\
0\end{array}$ & $\begin{array}{l}\frac{.}{0} \\
\frac{.0}{0} \\
\frac{0}{0} \\
\frac{0}{0}\end{array}$ & $\begin{array}{l}\frac{.}{\frac{\pi}{0}} \\
\frac{.0}{\overline{0}} \\
0 \\
0\end{array}$ & $\begin{array}{l}\frac{1}{.0} \\
\frac{.0}{0} \\
\frac{0}{0} \\
0\end{array}$ & $\begin{array}{l}\frac{.0}{\frac{\pi}{0}} \\
\frac{0}{0} \\
\frac{0}{0}\end{array}$ & $\overrightarrow{\underline{\underline{\underline{E}}}}$ & $\begin{array}{l}\frac{c}{.0} \\
\frac{.0}{0} \\
\frac{0}{00} \\
0\end{array}$ \\
\hline 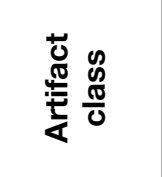 & 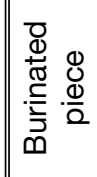 & 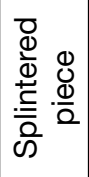 & 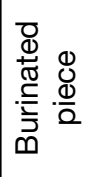 & 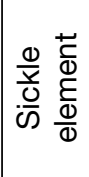 & 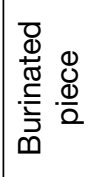 & 定 & 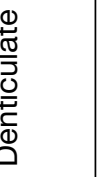 & 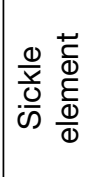 & $\frac{\frac{0}{0}}{\frac{\pi}{0}}$ & 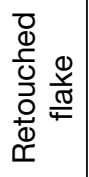 & 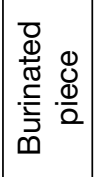 & $\begin{array}{l}\overline{\overline{0}} \\
\text { की }\end{array}$ & 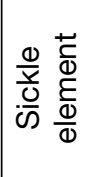 & 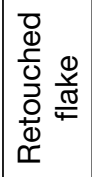 & 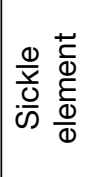 & $\begin{array}{l}\frac{\mathscr{N}}{\pi} \\
\frac{\pi}{L}\end{array}$ \\
\hline Ð্త & $\mp$ & $\mp$ & $\mp$ & $\mp$ & $\mp$ & $T$ & $F$ & $\mp$ & $\stackrel{\sim}{\sim}$ & $\stackrel{\sim}{\leftarrow}$ & $\stackrel{\sim}{\sim}$ & $\stackrel{N}{\sim}$ & $\stackrel{\sim}{\sim}$ & $\stackrel{N}{\sim}$ & $\stackrel{\sim}{\leftarrow}$ & $\stackrel{\sim}{\leftarrow}$ \\
\hline 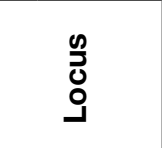 & 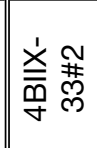 & $\begin{array}{l}\hat{y} \\
\dot{x} \\
\underline{\overline{\bar{y}}}\end{array}$ & $\begin{array}{l}\stackrel{g}{+} \\
\stackrel{x}{\underline{\underline{x}}}\end{array}$ & 蒿葍 & 蒿吾 & i & $\begin{array}{l}\frac{x}{x} \\
\frac{x}{y} \\
\frac{x}{y}\end{array}$ & $\begin{array}{l}\hat{o} \\
\dot{x} \\
\underline{\underline{x}} \\
\underline{q}\end{array}$ & $\mid \begin{array}{l}\tilde{y} \\
\dot{x} \\
\underline{\underline{x}} \\
\bar{q} \\
\end{array}$ & 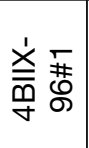 & 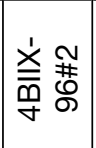 & 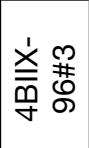 & 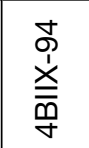 & 﨎菀 & 﨎葛 & 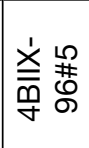 \\
\hline í & $\mathscr{L}$ & $\hat{\omega}$ & 曲 & ஜூ & 8 & ¿ & 5 & $\widetilde{~ ช ్ ~}$ & 8 & ఠ & ஜ̊ & $\mathscr{8}$ & $\hat{\vartheta}$ & $\mathscr{\varnothing}$ & 8 & R \\
\hline
\end{tabular}




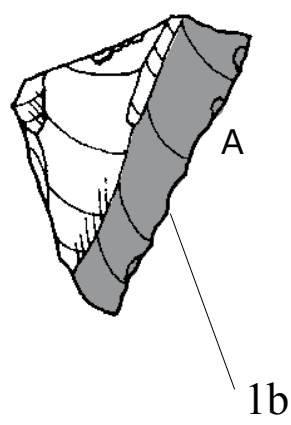

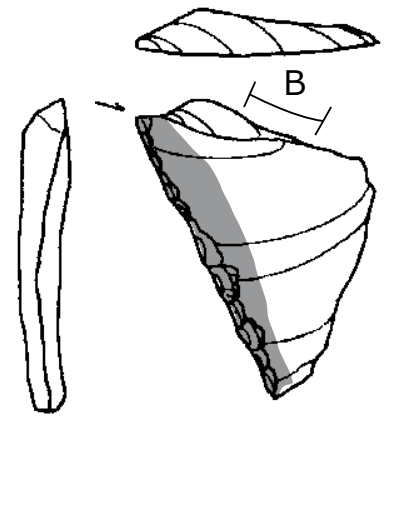

$1 \mathbf{a}$

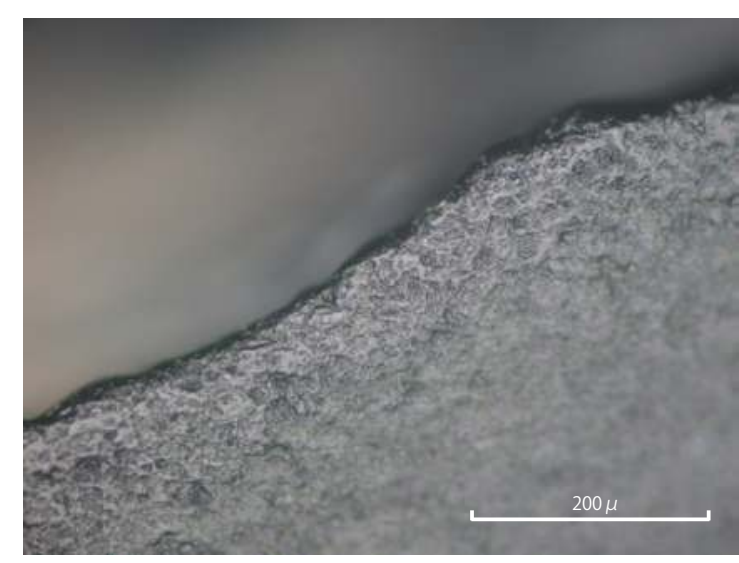

$1 \mathrm{~b}$
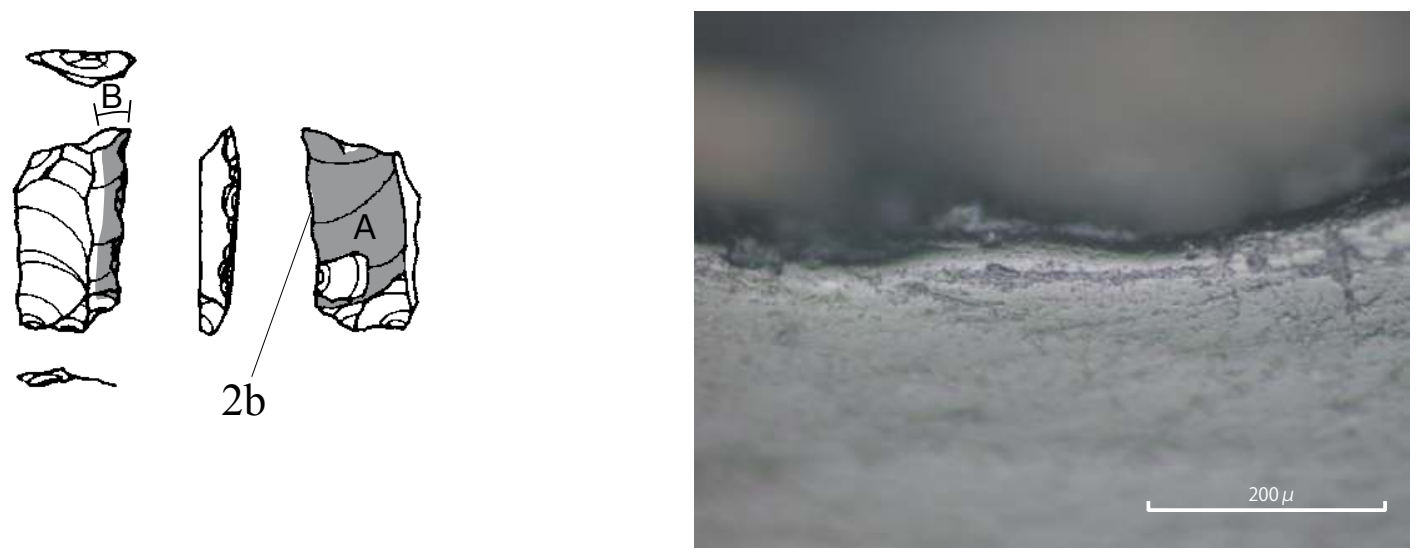

2a

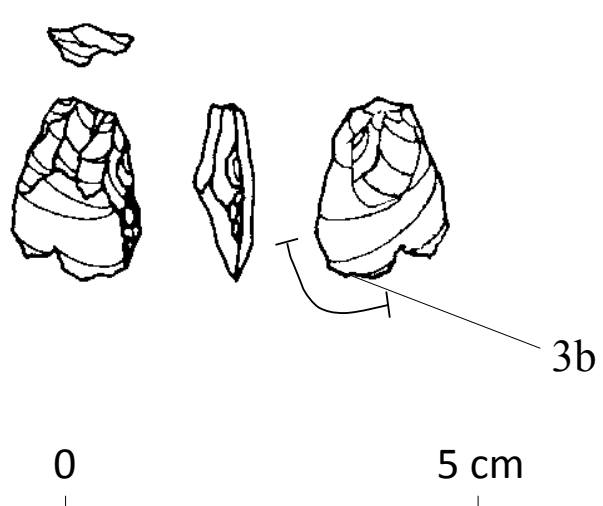

$3 \mathbf{a}$

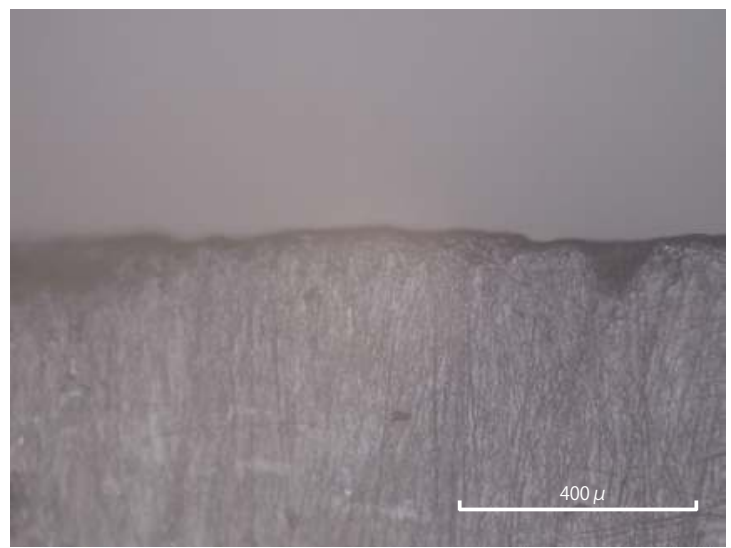

$3 \mathbf{b}$

Fig. 10.1 Polish distribution on obsidian artifacts (shaded areas and lines along the edge) from Göytepe (I). 1: Burinated piece (4BI-29\#1, Level 5); 2: Sickle element (4BI-29\#6, Level 5); 3: Retouched flake (4BI-5, Level 5). 


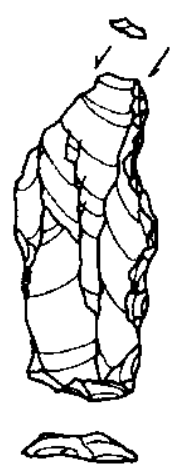

0

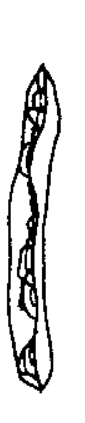

$1 \mathrm{~b}$

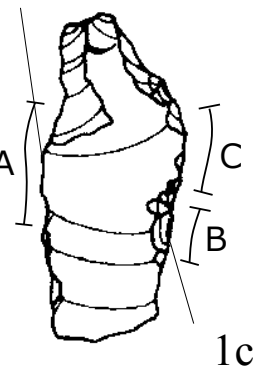

$5 \mathrm{~cm}$

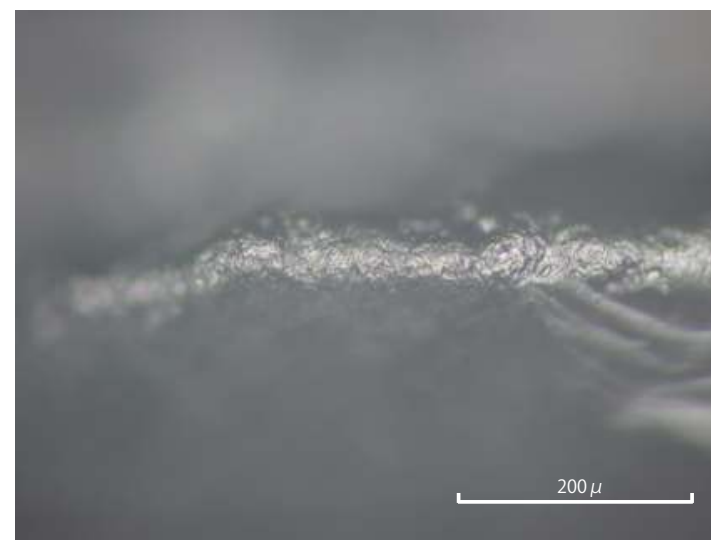

$1 \mathbf{b}$

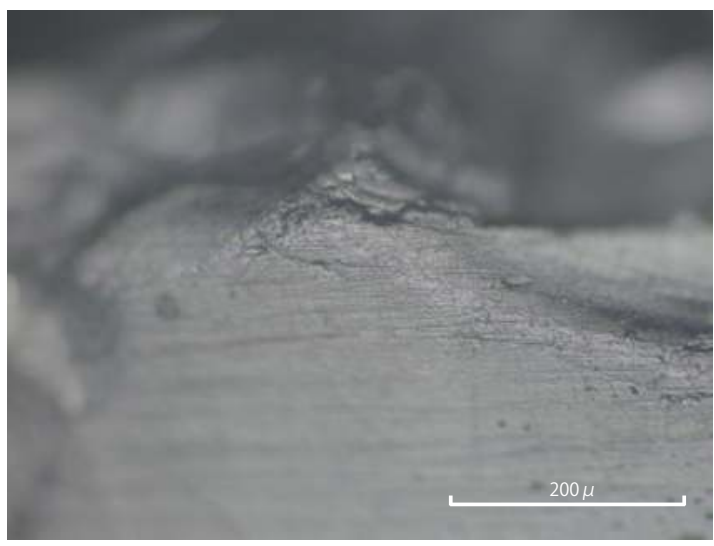

$1 \mathrm{c}$
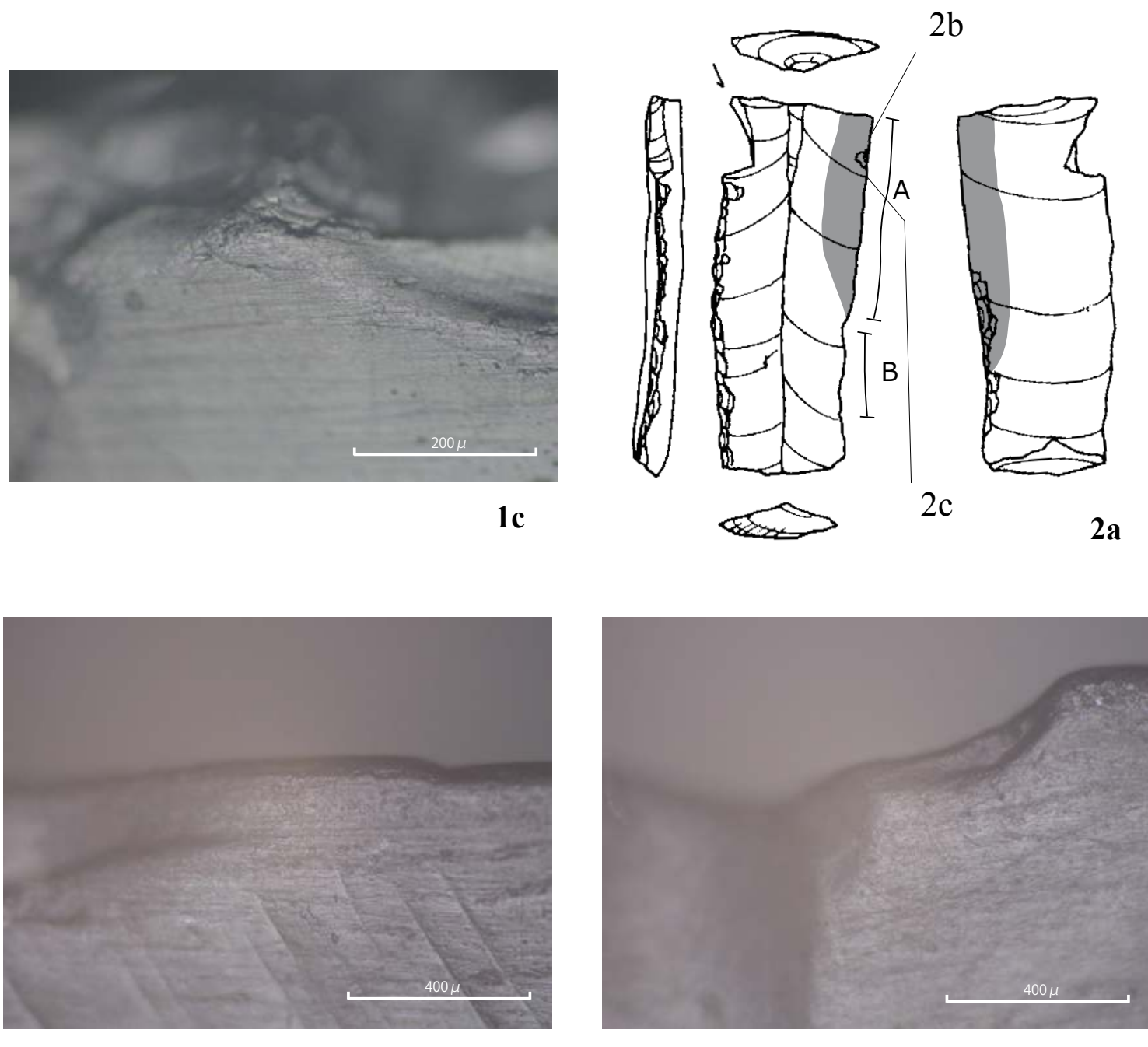

$2 \mathbf{b}$

2c

Fig. 10.2 Polish distribution on obsidian artifacts (shaded areas and lines along the edge) from Göytepe (II). 1: Burinated piece (4BI-36, Level 5); 2: Burinated piece (4BI-19\#2, Level 6). 

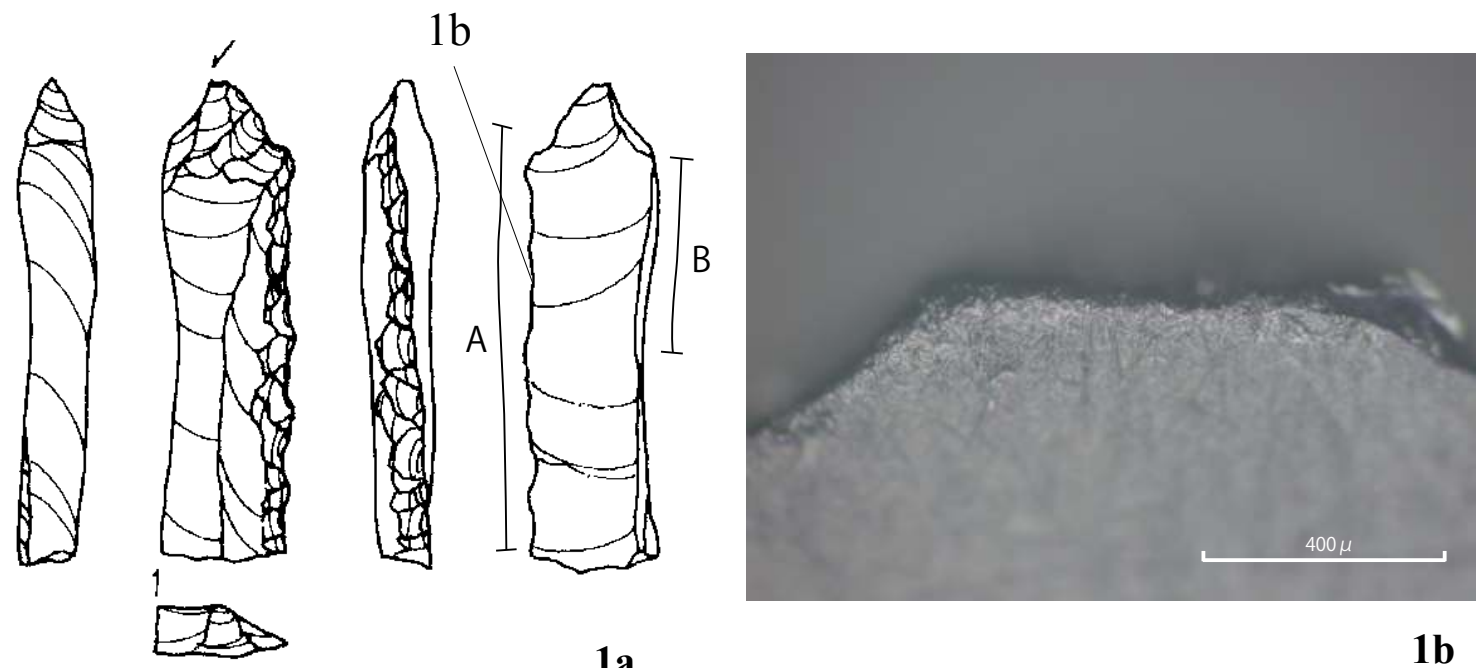

$1 \mathrm{a}$

$1 \mathbf{b}$
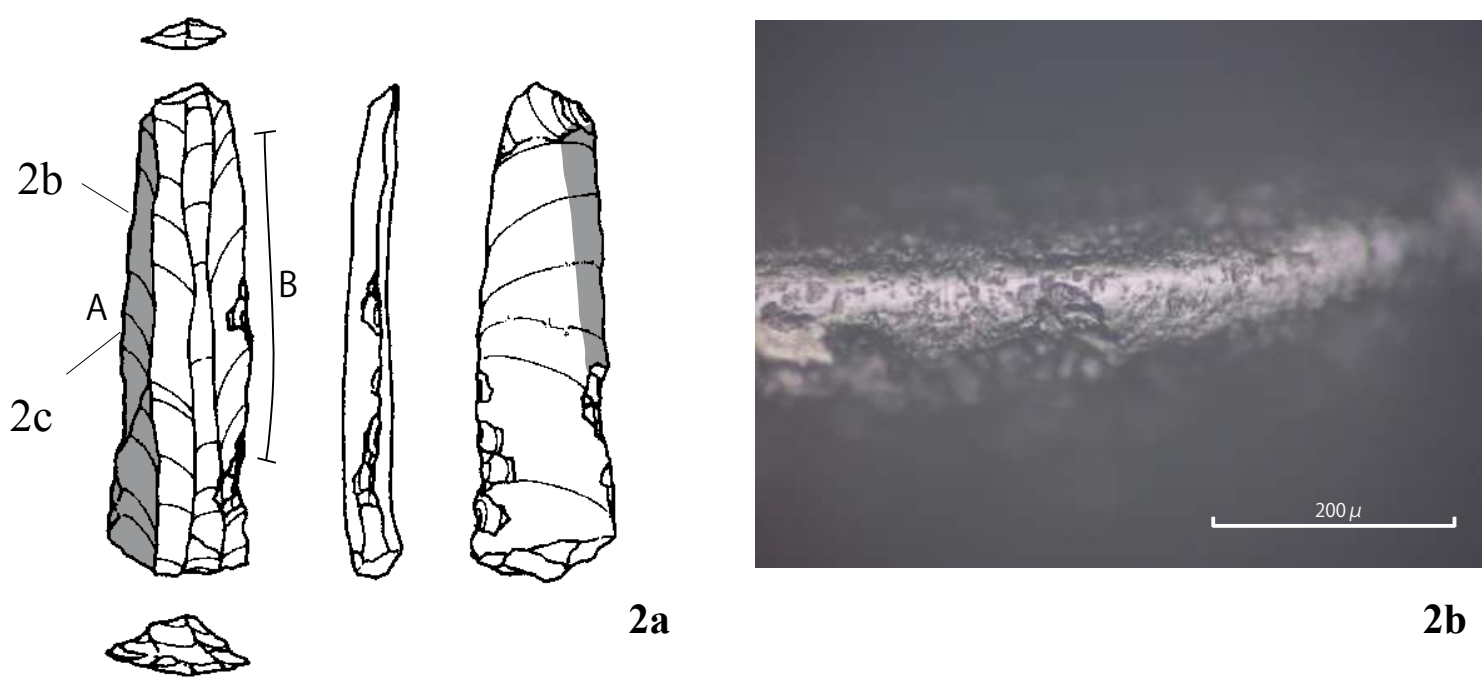

$\mathbf{2 a}$

2b
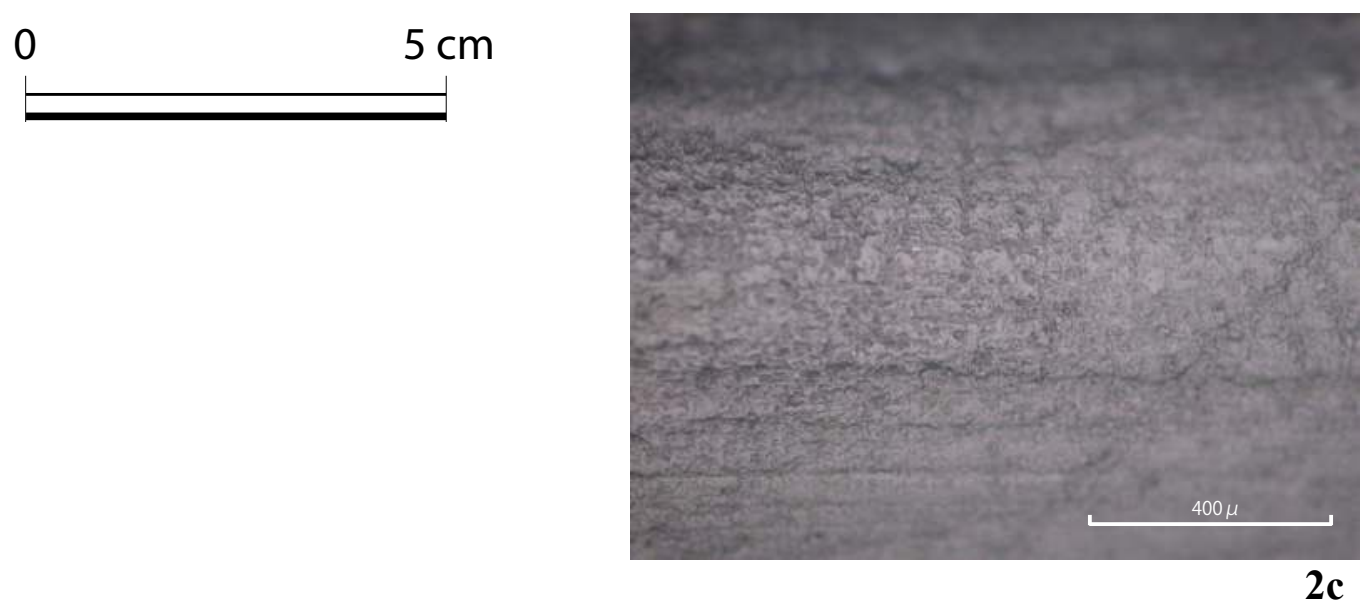

Fig. 10.3 Polish distribution on obsidian artifacts (shaded areas and lines along the edge) from Göytepe (III). 1: Burinated piece (4BI-19\#3, Level 6); 2: Retouched blade (4BII-23, Level 7). 

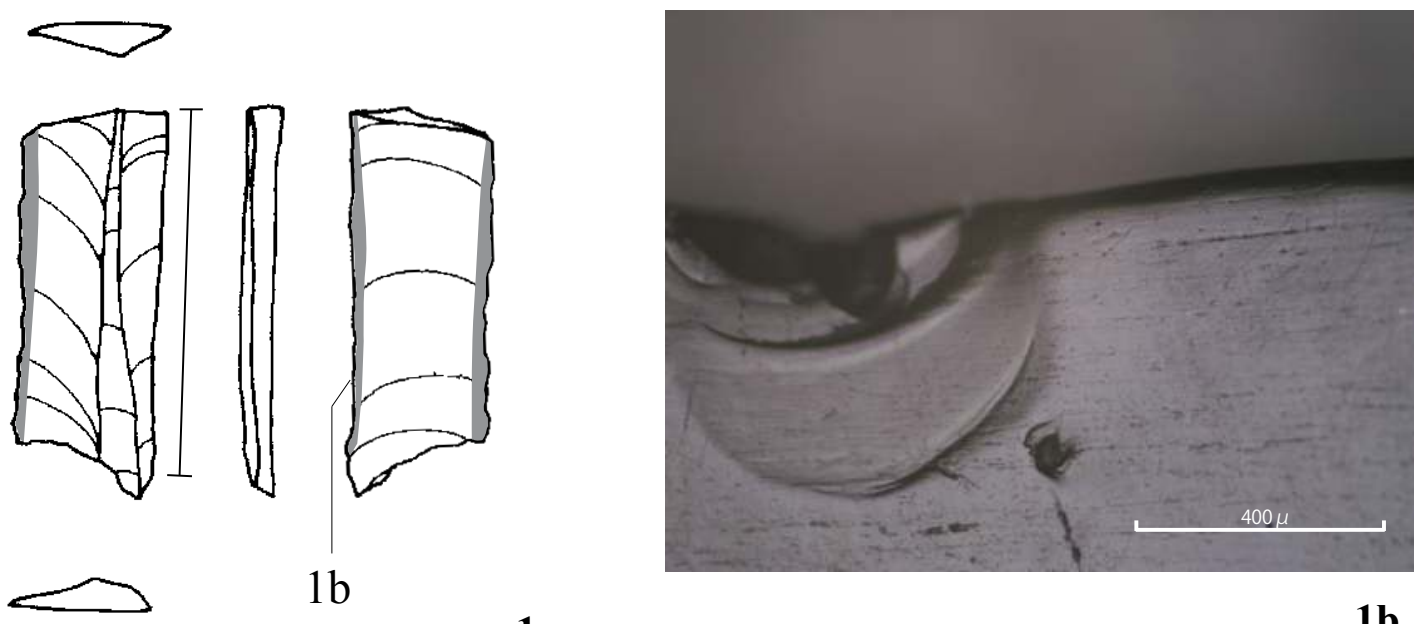

$1 \mathbf{a}$

$1 \mathbf{b}$
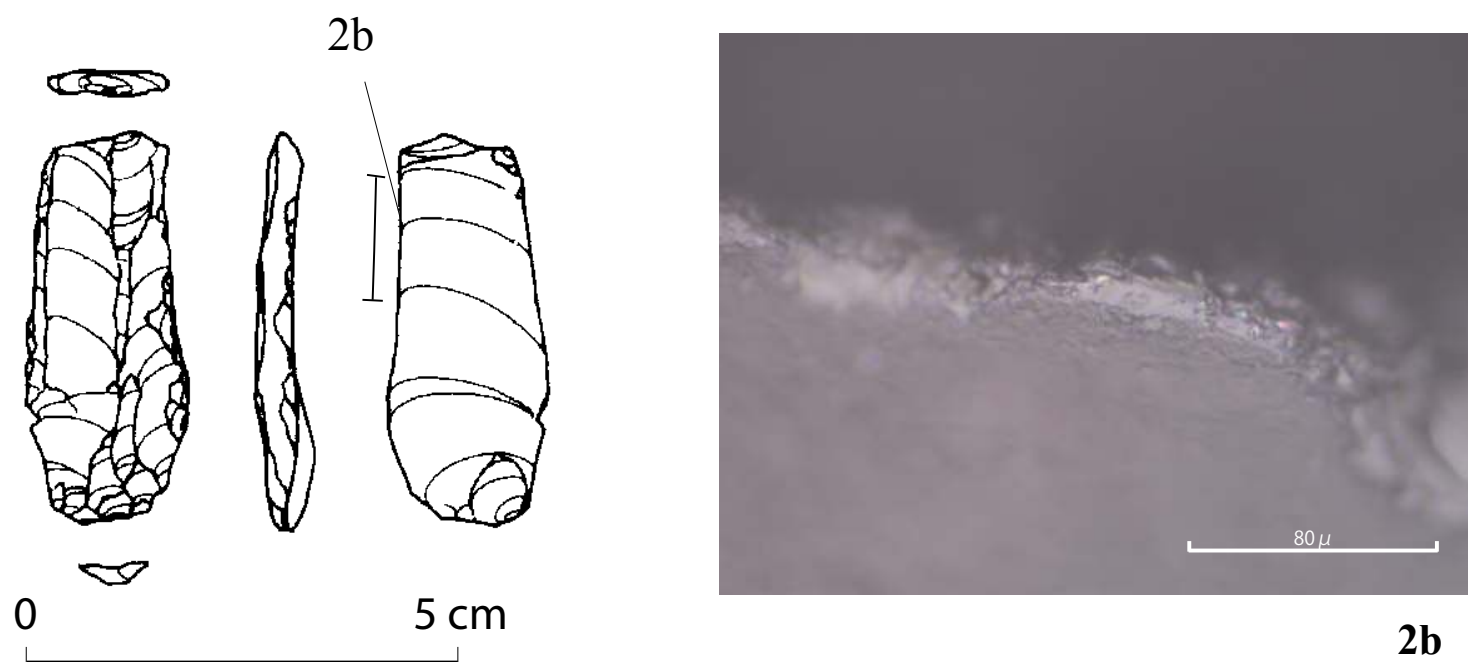

2b

2a
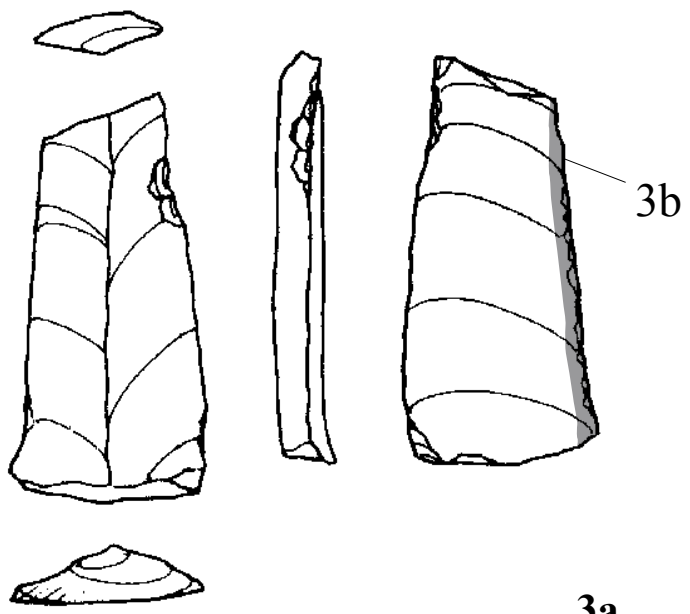

3a

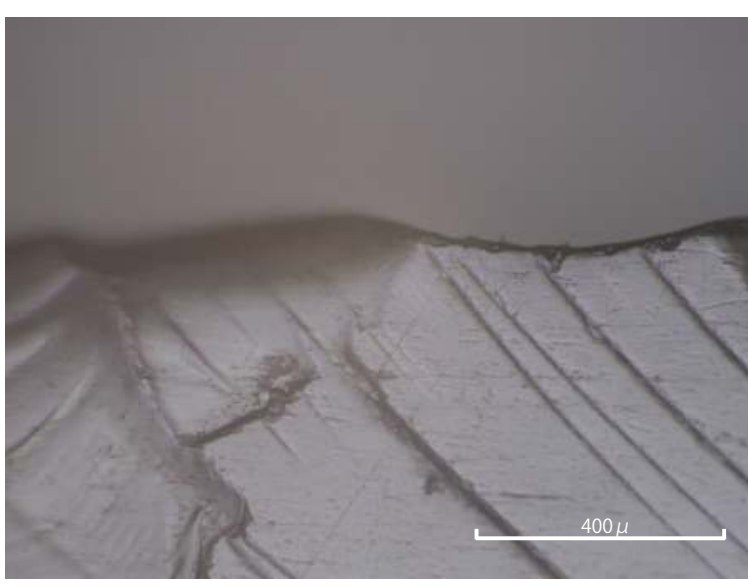

$\mathbf{3 b}$

Fig. 10.4 Polish distribution on obsidian artifacts (shaded areas and a line along the edge) from Göytepe (IV). 1: Burinated piece (4BI-44, Level 7); 2: Retouched blade (4BI-41, Level 7); 3: Burinated piece (4BIIX15\#3, Level 10). 


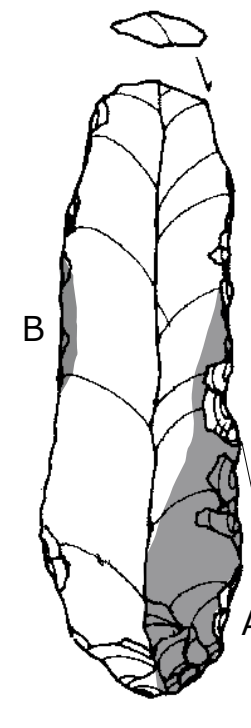

$\varnothing$

$2 b$

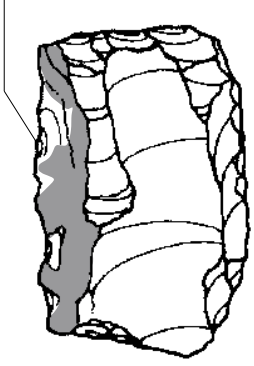

0

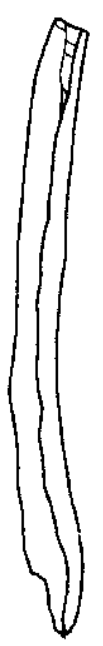

$1 b$
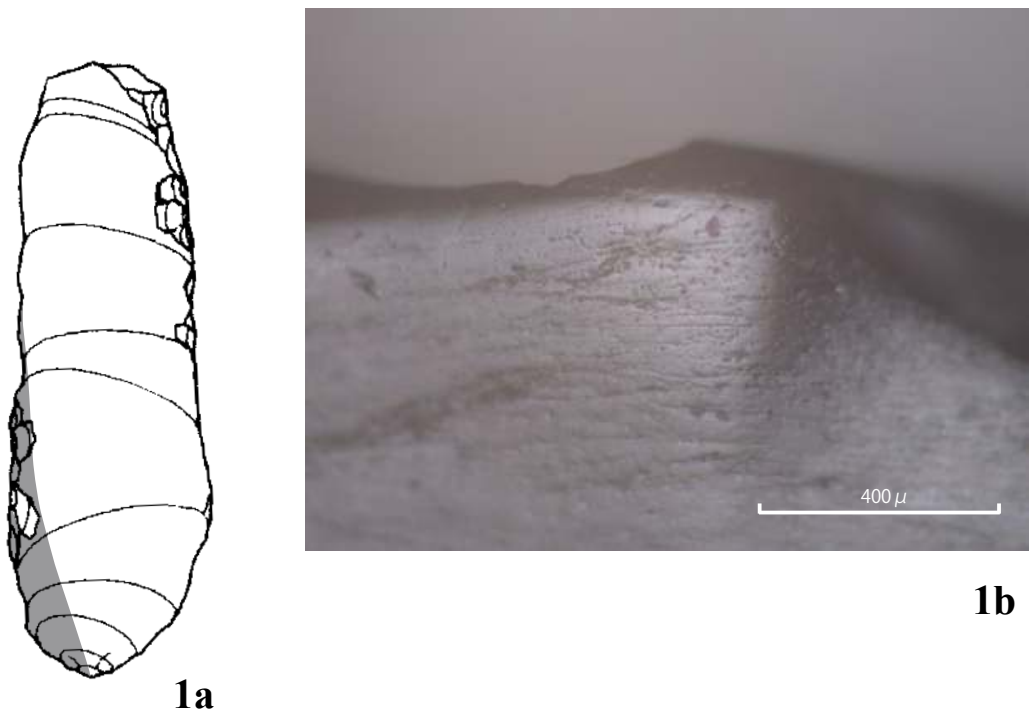

1b

1a

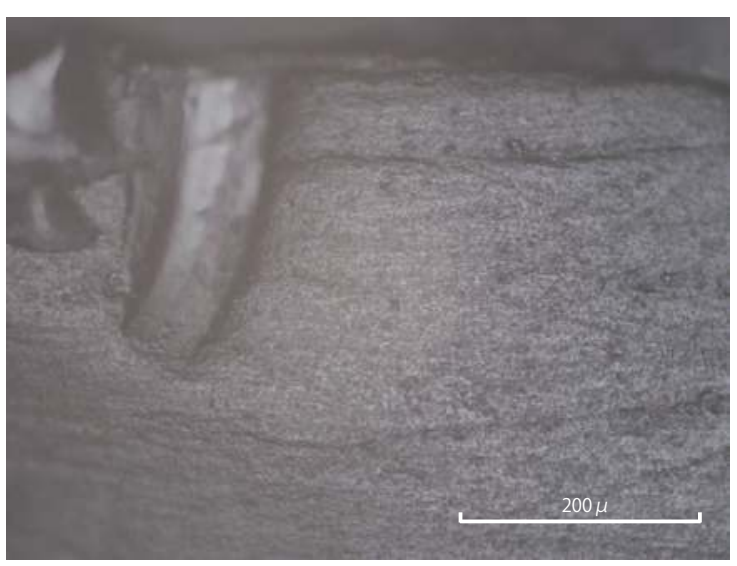

2b
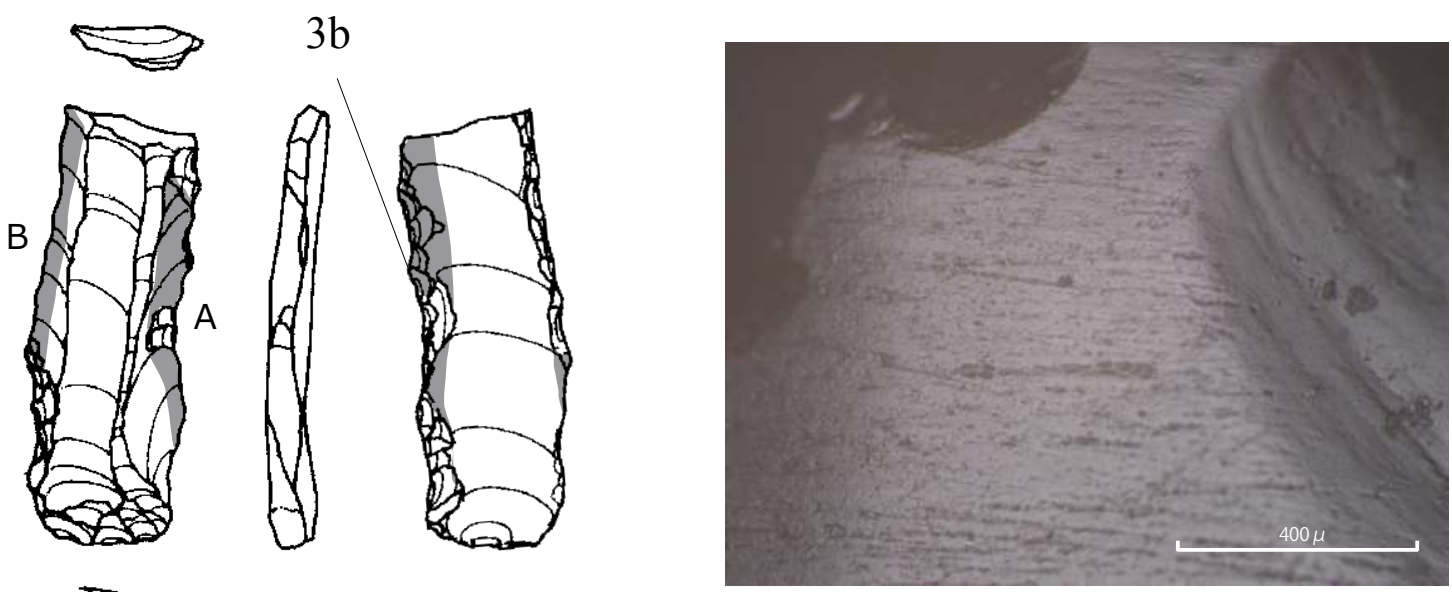

$\mathbf{3 b}$

Fig. 10.5 Polish distribution on obsidian artifacts (shaded areas) from Göytepe (V). 1: Retouched blade (4BIIX-40, Level 10); 2: Splintered piece (4BIIX-76, Level 11); 3: Denticulate (4BIIX-73, Level 11). 

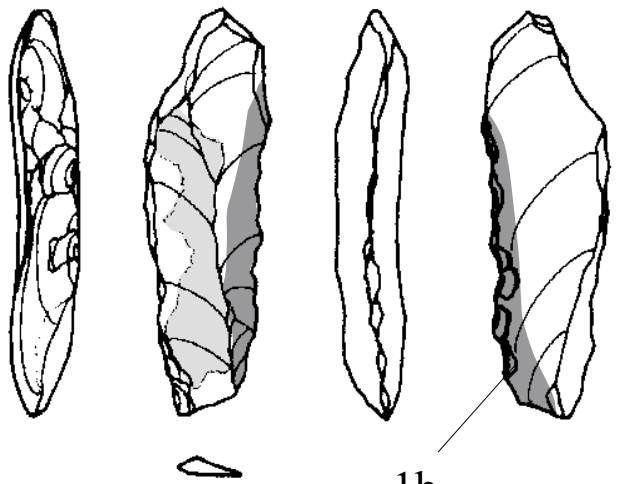

$1 \mathrm{~b}$

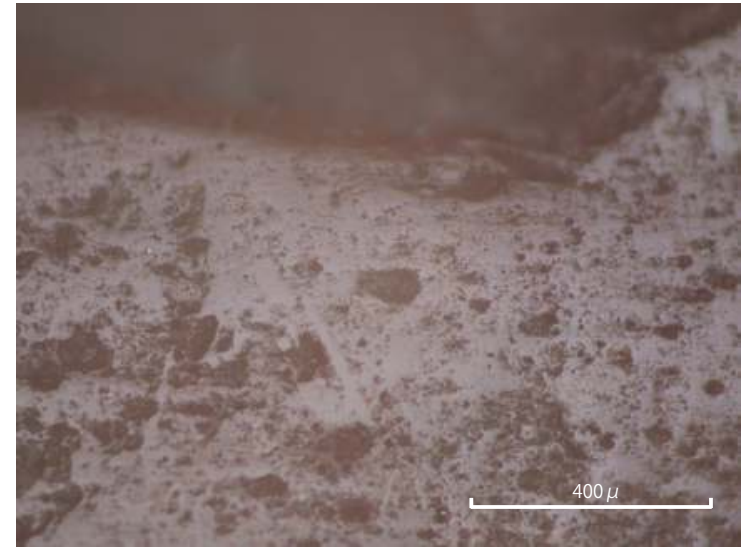

1b

$1 \mathbf{a}$

$5 \mathrm{~cm}$
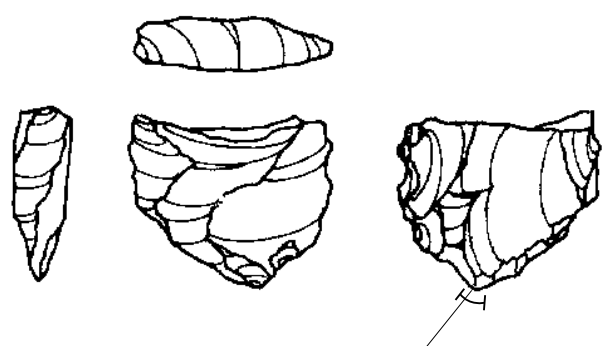

$2 b$

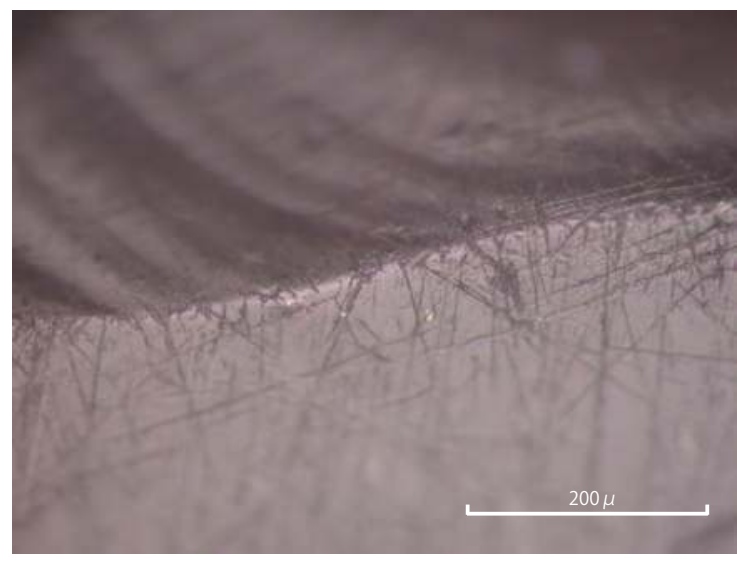

2b

2a

Fig. 10.6 Polish distribution on obsidian artifacts (shaded areas and a line along the edge) from Göytepe (VI). 1: Sickle element (4BIIX-87, Level 11); 2: Splintered piece (4BIIX-71, Level 11).

lack of use-wear polish. Yet, based on striations perpendicular and parallel to the edge, it could be determined that edge-damaged blades were used in both cutting/sawing and scraping/whittling motions (Table 10.2). Obsidian denticulates are also believed to have been used in the same tool movement. Ob-A and $\mathrm{Ob}-\mathrm{B}$ types of use-wear polish suggest that the grass plant was a strong candidate for a worked material (Table 10.2, Fig. 10.5: 3). Striations perpendicular to the edge and types of use-wear polish (Ob-B and ObI) indicate that one of the exhausted cores was used for scraping/whittling relatively soft materials, such as plants and hides (Table 10.2). Because Ob-I type has a close relationship with animal skin, this artifact is estimated to have been used for hide-working.
10.3.2 Burinated pieces, splintered pieces, retouched flakes, and retouched blades

Results of the analysis indicate that the major tool types at Göytepe, burinated and splintered pieces, were used for broader purposes (Table 10.2). At least one splintered piece is considered to have been a wedge because of the perpendicular striations on its opposed edges. As for other specimens, a wide variety of striations and use-wear polish (Ob-A, Ob$\mathrm{B}, \mathrm{Ob}-\mathrm{E}$ and $\mathrm{Ob}-\mathrm{I}$ types) suggest that they were used for processing plants and soft animals in cutting/ sawing and scraping/whittling motions (Figs. 10.1: 1, 10.2: 1 and 2, 10.3: 1, 10.4: 1 and 3, 10.5: 2, 10.6: 2, and 10.7: 3). Retouched blades and retouched flakes were also likely to be used for similar purposes (Table 10.2, Figs. 10.1: 3, 10.3: 2, and 10.5: 1), although a retouched blade exhibited traces showing 

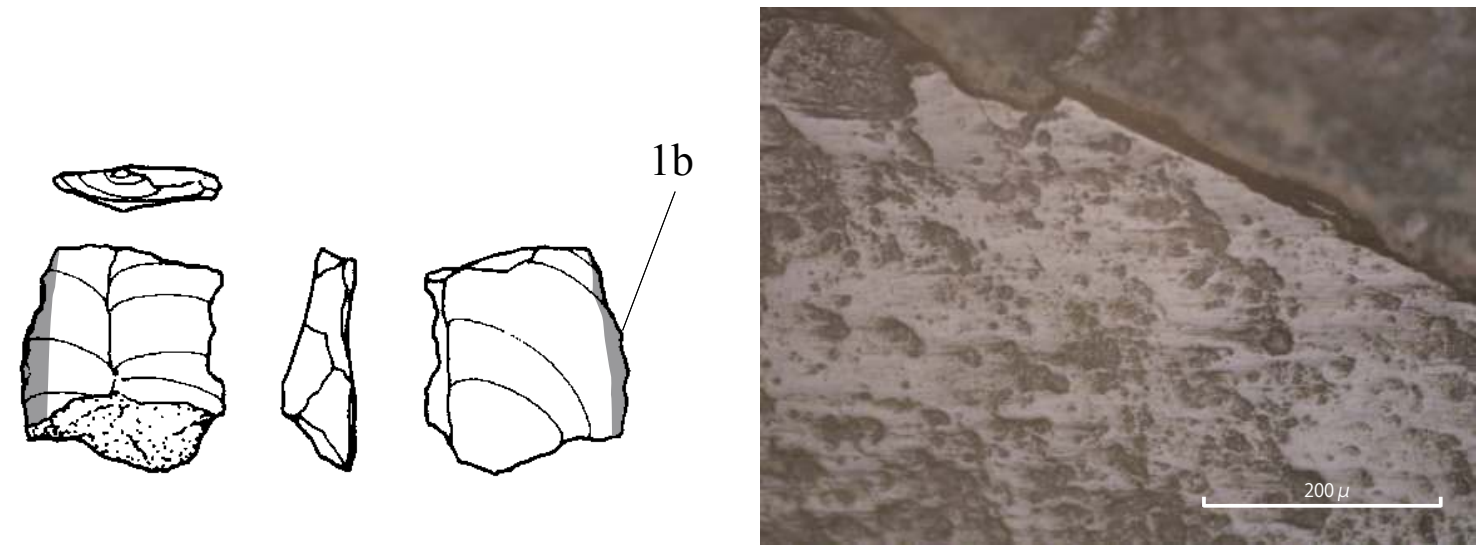

$1 \mathbf{a}$

$\mathbf{1 b}$
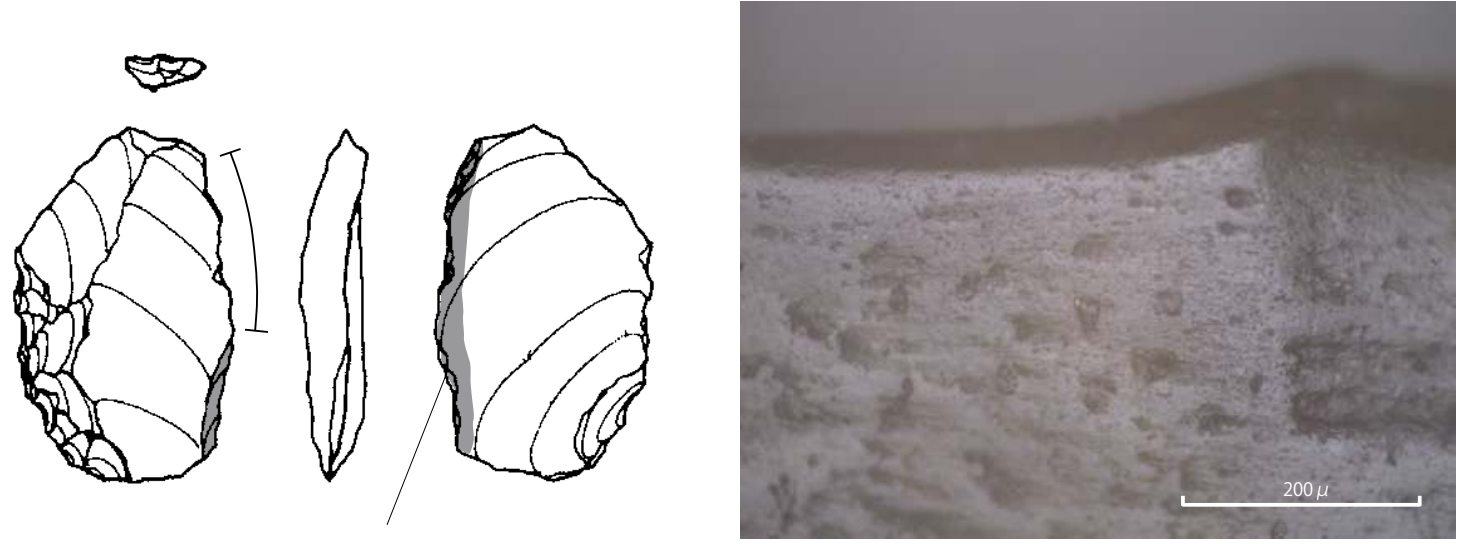

$2 b$

2a

2b
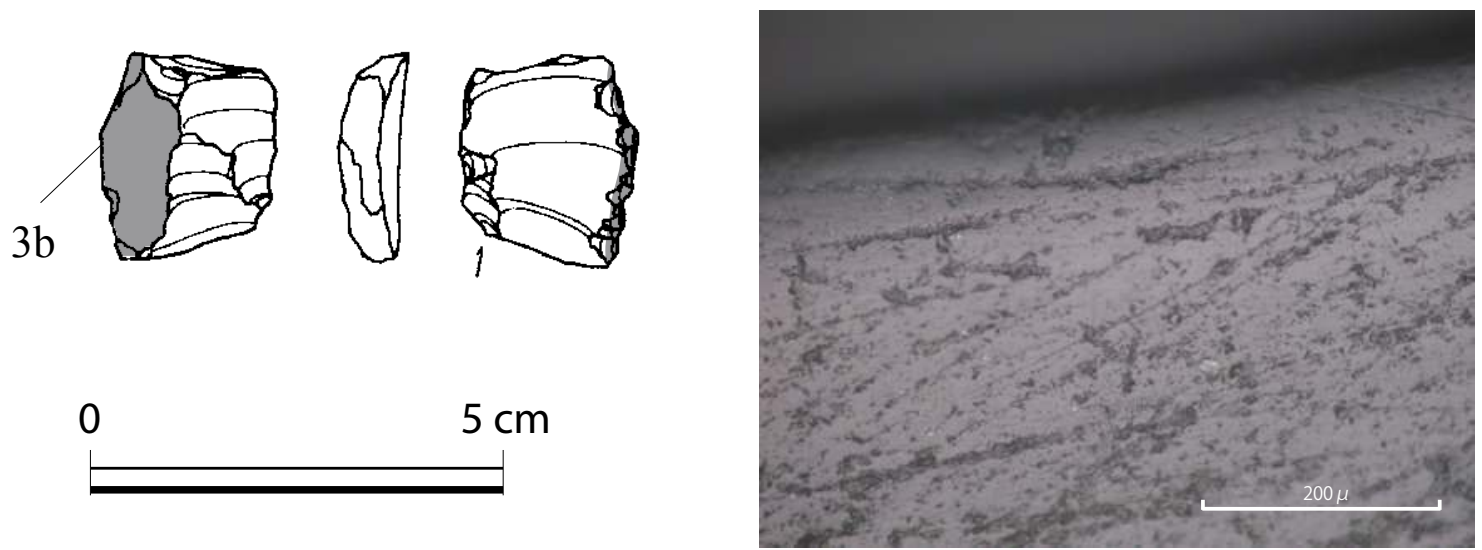

3a

3b

Fig. 10.7 Polish distribution on obsidian and flint artifacts (shaded areas and a line along the edge) from Göytepe. 1: Sickle element (4BIIX-59\#1, Level 11); 2: Sickle element (4BIIX-59\#2, Level 12); 3: Burinated piece (4BIIX-96\#2, Level 12). 
Chapter 10

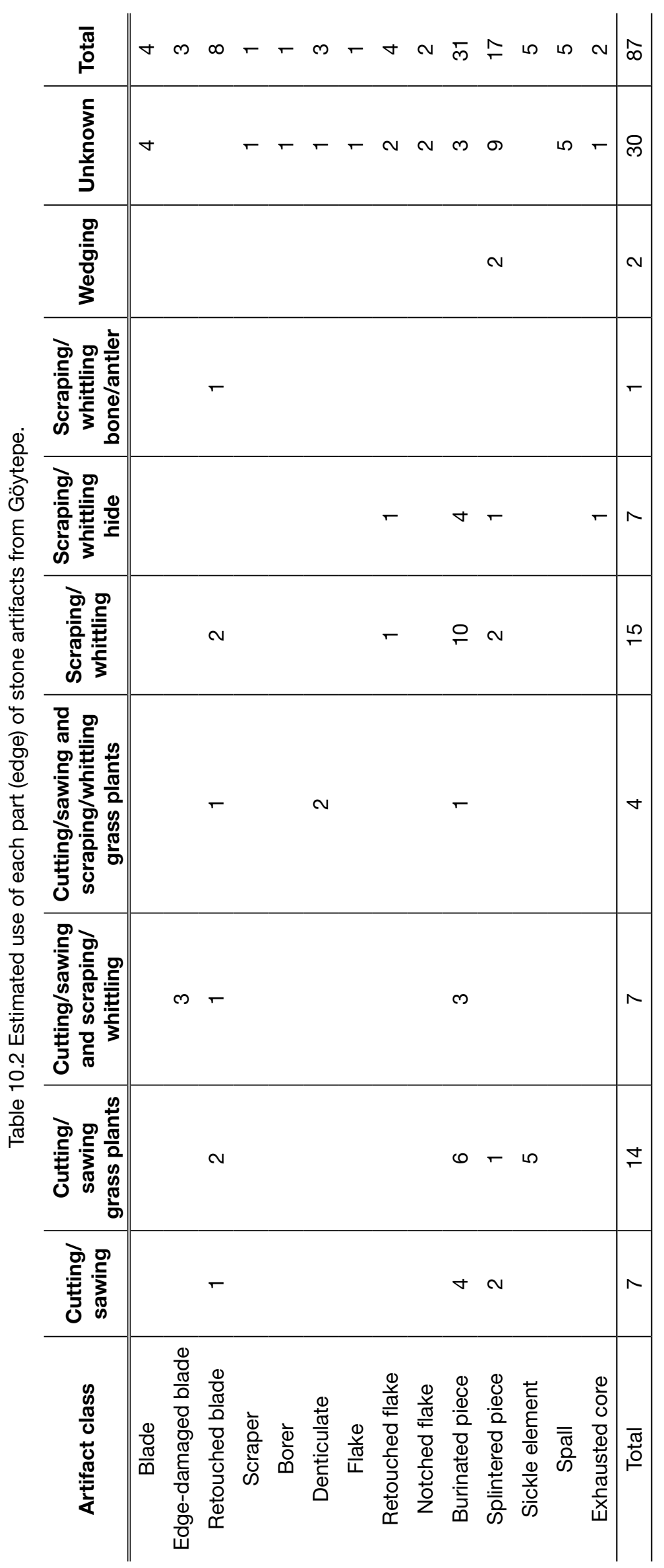


bone or antler scraping/whittling (Fig. 10.4: 2). Overall, major types of obsidian tools from this site were used for plant- and hide-working. Nishiaki and Guliyev (2019) speculate that there is a functional relationship between burinated pieces (burins) and splintered pieces because of the unchanged total frequency of these tools throughout this site and similarities in the retouch technology used to make them. This hypothesis is supported by results from this lithic use-wear analysis.

The lithic assemblage of this site is characterized by the high frequency of burinated and splintered pieces (Chapter 9), and a lot of bone tools have been discovered as well (Chapter 15). Before analysis, we hypothesized that these tools could have been the main implements for the production of bone tools. However, only one stone tool, which was a retouched blade instead of a burinated/splintered piece, was estimated to be used for processing bone or antler. This indicates that chipped stone tools, including burinated and splintered pieces, were not intensively used for manufacturing bone tools (Table 10.1). Remarkably, ridges formed by burin-like facets and other surfaces on burinated pieces were infrequently utilized at this site; these parts were primary working edges for Paleolithic burins in the Eurasian continent. In contrast, the functions of Neolithic burins have not yet been thoroughly revealed because they have a tendency to show indistinct use-wear patterns (Yamada 2012). Moreover, experiments using stone tools for mammal butchering demonstrate that burinlike facets can occasionally be produced on obsidian tools due to unintended contact with hard materials, such as bones and floors (Ono et al. 2005). This indicates that burin-like facets may not be generated intentionally. The cause of the high proportion of burinated pieces in the lithic assemblage at this site still remains a mystery.

A close examination of technology for making bone tools indicates scarce use of the groove-andsplinter technique at this site (Chapter 15; see also Clark and Thompson 1953). Rough knapping was frequently used in the initial stage of bone tool production. Abrasion and polishing with ground stones were more significant methods for finishing bone implements, rather than processing techniques that used chipped stone tools. Thus, it is not necessarily surprising that there are little traces of bone-working on chipped stone tools. However, bone tools from this site occasionally have holes. Stone perforators must have been used to make them. Unfortunately, no use-wear polish was detected on an obsidian borer in this study, but future studies should explore stone perforators.

\subsubsection{Sickle elements}

On sickle elements, well-developed microwear polish related to plant-working with smooth surface textures and domed topography was distributed widely on the dorsal and ventral surfaces of their edges (Figs. 10.1: 2, 10.6: 1, 10.7: 1 and 2). Obviously, the dominant orientation of striation is parallel to the edge on all specimens. There are some studies that distinguish "plant/cereal polish" from "reed polish," based on polish morphology and demarcations between polished and unaltered areas (Unger-Hamilton 1991; Vaughan 1985; Juel-Jensen 1994; Anderson 1994; Anderson et al. 1998). Certainly, sickle elements from Göytepe have use-wear polish similar to "plant/ cereal polish." However, further analysis based on larger samples is necessary because the different patterns of gray level distribution expected between the two types of polish cannot be seen in an image analysis (Yamada 2000). Thus, we do not identify these types of polish in this study. Nevertheless, it is safe to say that use-wear polish on sickle elements was generated by cutting and/or sawing grass plants (Table 10.2).

Through traceological examinations of ethnographic and experimental threshing sledges, Skakun (1992: 203-204) points out that tribulum inserts have 1) blunted edges, 2) edge deformation and deep linear traces with pitted appearance, 3) indistinct demarcation between the worn and the unworn surfaces, and 4) dull mirror polish. Kardulias and Yerkes (1996) also confirm that threshing sledge inserts exhibit heavier abrasion than sickle elements and that use-wear polish is equally distributed over both faces, while use-wear polish is often heavier on one surface of sickle blades. In specimens from Göytepe, heavily rounded edges and large-scale edge deformation cannot be seen. Also, the demarcation between the worn and the unworn surfaces tends to be distinct. Furthermore, use-wear polish has an 
asymmetric distribution on both surfaces. As such, there is no clear evidence showing the existence of tribulum inserts in the specimens examined in this study.

Anderson (1992) reports that visible striations occur on experimental tools when the harvest is carried out near ground level (20 to $30 \mathrm{~cm}$ from the soil), but visible striations rarely occur on tools used to harvest cereals high on the stems. Using this idea, important issues about prehistoric agriculture, such as types of dry fields (tilled or untilled) and harvesting methods (ear cutting or stem cutting) have been discussed (e.g., Unger-Hamilton 1992; Clemente and Gibaja 1998). In Göytepe, a couple of non-obsidian sickle elements show relatively distinct striations (Figs. 10.1, 10.7: 1 and 2), indicating that they were used in an environment where soil mineral particles were prevalent. Other sickle elements without distinct striations may have been used in different places. Yet, a careful consideration is also necessary because striation development patterns can differ depending on the location of stone elements (e.g., distal and proximal parts) in a sickle (Clemente and Gibaja 1998).

Arazova and Skakun (2017) reveled the morphological variety of Neolithic sickles from Azerbaijan. They also recognized two methods of inserting stone elements into a sickle shaft. The first method is to put stone elements parallel to the shaft. As a result, use-wear polish can be seen along the edge of stone tools. This is highly relevant for sickle elements from Göytepe because polish is distributed along the edges (Figs. 10.1: 2, 10.6: 1, 10.7: 1 and 2 ). In the second method, stone tools are embedded diagonally in a groove of a shaft so that a sickle has a large denticulate edge. In this case, use-wear polish is distributed on the corner of stone inserts. There might be artifacts used in this method. For instance, use-wear polish from grass plants is distributed on the corner of a burinated piece (Fig. 10.2: 2) and a retouched blade (Fig. 10.5: 1). In fact, a complete sickle with blades embedded diagonally in a cattle mandible with bitumen was discovered at Göytepe (Nishiaki and Guliyev 2019). Possibly, sickle blades were involved not only in sickle elements but also in parts of other tool classes.

Focusing on comet-shaped pits that indicate unidirectional movement of the tool (Semenov 1964; Witthoft 1967), Yamada (2012) argues the hafting method of sickle blades. According to an examination of materials from the Nahal Zehora I site in Israel, most sickle blades were hafted with the ventral side facing the ground (if the sickles were operated by right-handed workers). At Göytepe, distinct cometshaped pits can be seen on two flint specimens. One of them is estimated to be used in the same way as the blades found at Nahal Zehora I (Fig. 10.7: 1). Conversely, the other specimen is considered to be hafted with the ventral side facing up (Fig. 10.7: 2). Interestingly, this understanding is not consistent with our interpretation of an asymmetrical distribution of use-wear polish on both sides because the polish should have a wider distribution on the groundfacing surface. For example, based on comet-shapedpits, a specimen shown in Fig. 10.7: 2 was estimated to be hafted with the dorsal side facing the ground. However, use-wear polish is distributed in a wider area on the ventral face. One possible explanation is that this was attached to a sickle used by lefthanded workers. This estimation can be supported by an excavated sickle from Göytepe because stone elements are fixed with the ventral side facing the ground if the sickle is used by left-handed individuals (Nishiaki and Guliyev 2019).

It is also notable that an archaeological sickle from Shomutepe (Narimanov 1987) suggests that stone inserts were fixed in diverse manners. We should take into account the possibility that there was no strict rule for fixing stone elements in a sickle shaft. Thus, the polish distribution on stone elements, even a sickle, would show diverse patterns. In this study, a typical method for hafting sickle elements is not identified due to the small sample size. However, such a functional analysis will provide effective clues for revealing the spacio-temporal change in the use of farming implements. Future research would contribute to a better understanding of the Neolithization processes of the southern Caucasus.

\section{Acknowledgement}

I thank Yoshihiro Nishiaki for giving me the opportunity to analyze materials from Göytepe. My thanks also go to Shoh Yamada, Hirohiko Saino, 
Tadashi Midoshima, Atsushi Sawada, and Motoki Harada for providing useful advice and literature regarding this study.

\section{References}

Akoshima, K. (1989) Use-wear of Stone Tools. Tokyo: Nyusaiensusha (in Japanese).

Akoshima, K. and H. Hong (2017) Standard use-wear chart of TUMRT (3): Polish (1). Bulletin of the Tohoku University Museum, 16: 69-86.

Anderson, P. C. (1992) Experimental cultivation, harvest, and threshing of wild cereals: Their relevance for interpreting the use of Epipaleolithic and Neolithic artifacts. In: Prehistoric Agriculture: New Experimental and Ethnographic Approaches, edited by P. C. Anderson, pp. 118-144. Los Angels: Institute of Archaeology University of California.

Anderson, P. C. (1994) Insights into plant harvesting and other activities at Hatoula as revealed by microscopic functional analysis of selected chipped stone tools. In: Le Site de Hatoula en Judée Occidentale, Israel, edited by M. Lechevallier and A. Rosen, pp. 277-315. Mémoires et Travaux du Centre de Recherche Fraçais de Jérusalem 8. Paris: Association Paléorient.

Anderson, P. C., L. Astruc, R. Vargiolu, and H. Zahouani (1998) Contribution of quantitative analysis of surface states to a multi-method approach for characterizing plant-processing traces on flint tools with gloss. Proceedings of the XIII U.I.S.P.P. Congress, Forli-Italia, 8-14 September 1996, 6(2): 1151-1160.

Arazova, R. and N. Skakun (2017) The oldest harvesting tools of Azerbaijan (according to experimentaltraceological research). Cuadernos de Prehistoria y Arqueología de la Universidad de Granada, 27: 121-132.

Clark, J. G. D. and M. W. Thompson (1953) The groove and splinter technique of working antler in Upper Palaeolithic and Mesolithic Europe. Proceedings of the Prehistoric Society, 19: 148-160.

Clemente, I. and J. F. Gibaja (1998) Working processes on cereals: An approach through microwear analysis. Journal of Archaeological Science, 25: 546-464.

Juel-Jensen, H. (1994) Flint Tools and Plant Working: Hidden Traces of Stone Age Technology. Aarhus: Aarhus University Press.

Kardulias, P. N. and R. W. Yerkes (1996) Microwear and metric analysis of threshing sledge flints from Greece and Cyprus. Journal of Archaeological Science, 23: 657-666.

Kajiwara, H. and K. Akoshima (1981) An experimental study of microwear polish on shale artifacts. Journal of Archaeological Society of Nippon (Kokogaku Zasshi), 67(1): 1-36 (in Japanese with English summary).

Keeley, L. H. (1977) The functions of paleolithic flint tools. Scientific American, 237(5): 108-126.

Keeley, L. H. (1980) Experimental Determination of Stone Tool Uses: A Microwear Analysis. Chicago: University of Chicago Press.

Midoshima, T. (1986) Use-wear of obsidian stone tools: An experimental study on use-wear polish. Kanagawakouko, 22: 51-78 (in Japanese).

Narimanov, I. (1987) The Culture of the Most Ancient Faming and Stock-Breeding Population of Azerbaijan. Baku: National Academy of Sciences (in Russian with an English summary).

Nishiaki, Y. and F. Guliyev (2019) Neolithic lithic industries of the Southern Caucasus: Göytepe and $\mathrm{Hacl}$ Elamxanlı Tepe, west Azerbaijan (early 6th millennium cal. BC). In: Near Eastern Lithics on the Move: Interaction and Contexts in Neolithic Traditions, edited by L. Astruc, F. Briois, C. McCartney, and L. Kassianidou, pp. 471-483. Nicosia: Astrom Editions.

Semenov. S. A. (translated by M. W. Thompson) (1964) Prehistoric Technology. London: Cory, Adams, and Mackay.

Skakun, N. N. (1992) Evolution of agricultural techniques in Eneolithic (Chalcolithic) Bulgaria: Data from use-wear analysis. In: Prehistoric Agriculture: New Experimental and Ethnographic Approaches, edited by P. C. Anderson, pp. 199-222. Los Angels: Institute of Archaeology University of California.

Ono, A., K. Takase, R. Ishii, G. Shiotani, and K. Segawa (2005) Butchering experimentation of a Japanese sika Cervus Nippon in Kamogawa and Chikura, Chiba Prefecture, Japan. Reports of Human Action and Society, 2004: 46-67. Tokyo: Archaeology Laboratory, Tokyo Metropolitan University (in Japanese).

Unger-Hamilton, R. (1991) Natufian plant husbandry in the southern Levant and comparison with that of the Neolithic periods: The lithic perspective. In: The Natufian Culture in the Levant, edited by $O$. Bar-Yosef and F. R. Valla, pp. 483-520. Ann Arbor: International Monographs in Prehistory.

Unger-Hamilton, R. (1992) Harvesting wild cereals and other plants: Experimental observations. In: Prehistoric Agriculture: New Experimental and Ethnographic Approaches, edited by P. C. Anderson, pp. 145-155. Los Angels: Institute of Archaeology University of California.

Vaughan, P. (1985) Use-wear Analysis of Flaked Stone Tools. Tucson: The University of Arizona Press.

Witthoft, J. (1967) Glazed polish on flint tools, American Antiquity, 32: 383-388.

Yamada, S. (2000) Development of the Neolithic: 
Lithic Use-Wear Analysis of Major Tool Types in the Southern Levant. Harvard University (Ph.D. dissertation).

Yamada, S. (2012) Use-wear Analysis of tools from Nahal Zehora I. In: Village Communities of the Pottery Neolithic Period in the Menashe Hills, Israel: Archaeological Investigations at the Site of Nahal Zehora, Volume II, edited by A. Gopher, pp. 9781010. Tel Aviv: Institute of Archaeology, Tel Aviv University. 


\title{
Fracture wing analysis for identification of obsidian blank production techniques at Göytepe
}

\author{
Jun Takakura and Yoshihiro Nishiaki
}

\subsection{Introduction}

There has been consensus among researchers of the South Caucasian Neolithic that pressure debitage was the main core reduction technology in the Middle Kura Valley region, where the site of Göytepe is situated (see Chapter 9). This understanding is based on techno-morphological observations of recovered obsidian products, which display features often recognized in replicative experiments of pressure debitage (Inizan et al. 1999: 79):

- parallel edges and arrises, which tend to be rectilinear;

- constant thickness, mesial section included;

- no obvious ripples on the lower face;

- a butt always narrower than the maximum width of the blades, which is very rapidly reached.

Identification of pressure debitage using such morphological criteria has been widely employed, often taking into consideration the comparable features of the cores and other technological traits of the striking platform and distal end of the products (see Desrosiers 2012). However, the reliability of such empirical identification has not been verified quantitatively. In this chapter, we present results of our attempt to examine whether pressure debitage was employed for blade and flake production at Göytepe (Fig. 11.1). The method we employ is "fracture wing" analysis.

\subsection{Method and Material}

Fracture wings imply microscopic markings visible on fracture surfaces of glassy materials that contain sufficient amounts of vitreous substance. The markings usually take a V-shape feature with its apex pointing toward the direction of fracture propagation and typically originate from an impurity in the material (Figs. 11.2-11.4). Since the formation of fracture wings, triggered by the passing of a fracture wave, is largely determined by the crack velocity and the material substance, its identification directly contributes to a better understanding of chipped stone technology. Particularly relevant to our concern is the crack velocity, which may vary according to different ways of flaking, that is, pressure, indirect, and direct flakings. Guided by exploratory work of the 1970s to 1980s (e.g., Faulkner 1972; Cotterell et al. 1985; Cotterell and Kamminga 1979, 1992), Tomenchuk (1988) and Hutchings (1999) developed a method to determine the crack velocity of obsidian flake scars based on the analysis of fracture wings. Hutchings' (1999) analysis revealed that channel flaking retouch on obsidian projectile Clovis points shows a significantly slower velocity than experimental scars made by direct percussion, suggesting the use of pressure flaking for retouch.

One of the authors of this chapter was on a team that substantiated this analytical method for identifying debitage techniques (Takakura and Izuho 2004). We conducted a series of obsidian knapping experiments using a combination of different techniques (pressure, indirect, and direct percussions) and hammers (soft and hard hammers for percussion). The results demonstrated a strong correlation between crack velocity and flaking technologies. At least three groups of flaking techniques were defined: I) pressure, II) indirect percussion/direct percussion using soft hammers, and III) direct percussion using hard hammers. 

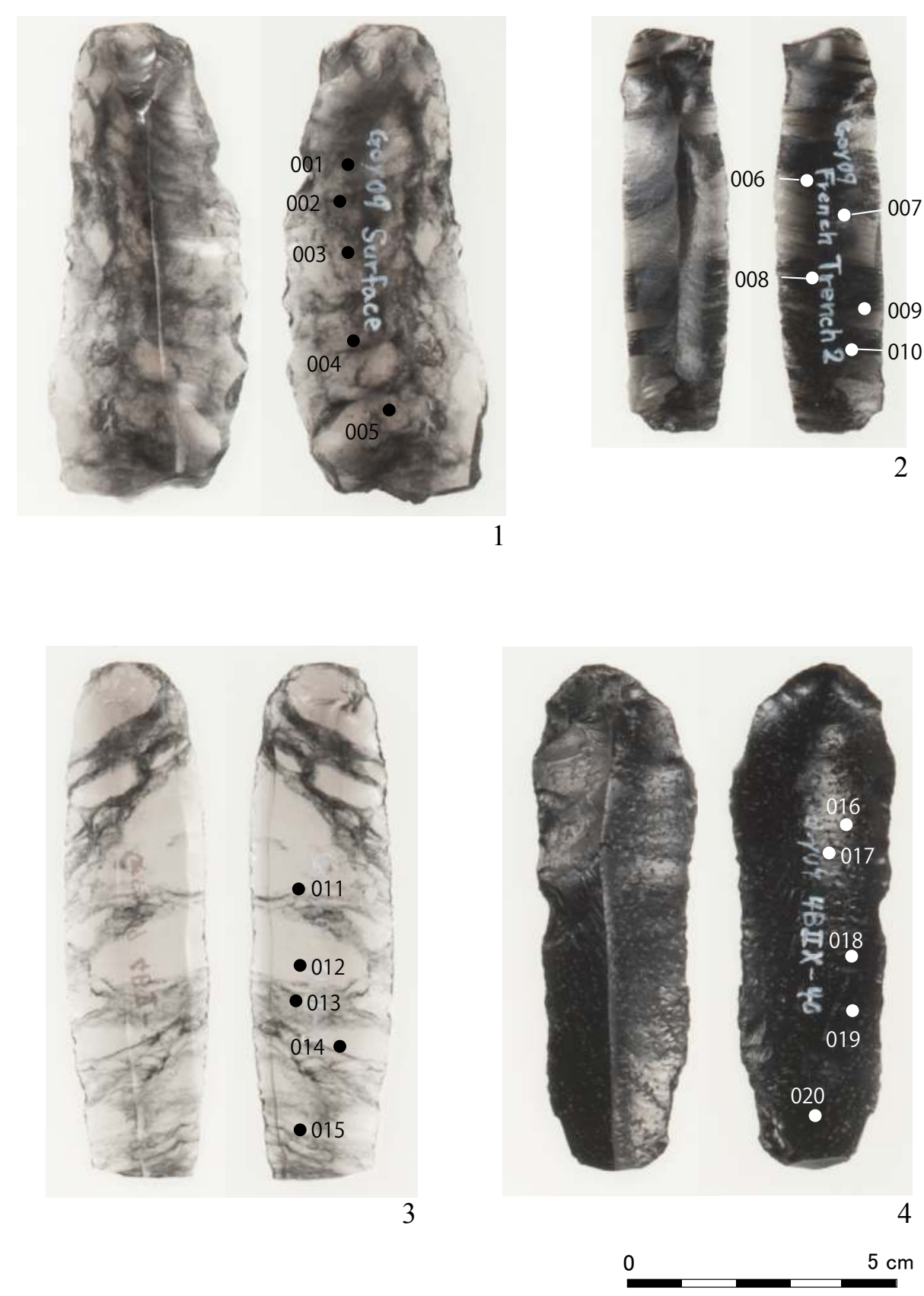

Fig. 11.1 Obsidian artifacts from Göytepe showing the location of fracture wing measurement points. 1: Unretouched blade (Surface); 2: Unretouched blade (FT 2); 3: Unretouched blade (4BII-1); 4: Unretouched blade (4BIIX-40). The numbers on each specimen indicate the measurement points (AZGY).

Although this method does not distinguish indirect percussion from soft hammer direct percussion, it is appropriate for the present Göytepe study, which asks if pressure debitage was employed for obsidian blade production. The material for analysis consists of selected obsidian flakes and blades from the 2009-2010 excavations of this site. Regardless of their excavation contexts, complete (or nearly complete) specimens with a butt were selected (Fig. 11.1; Table 11.1). The samples were in good condition for observation of fracture wings because of the absence of heavily weathered and heat-altered surfaces. Considering the possibility that crack velocity may differ according to the distance from the percussion point, the measurement of fracture wings was conducted at several points (four to seven) of the ventral surface of each specimen, avoiding the bulb of percussion. A high- 

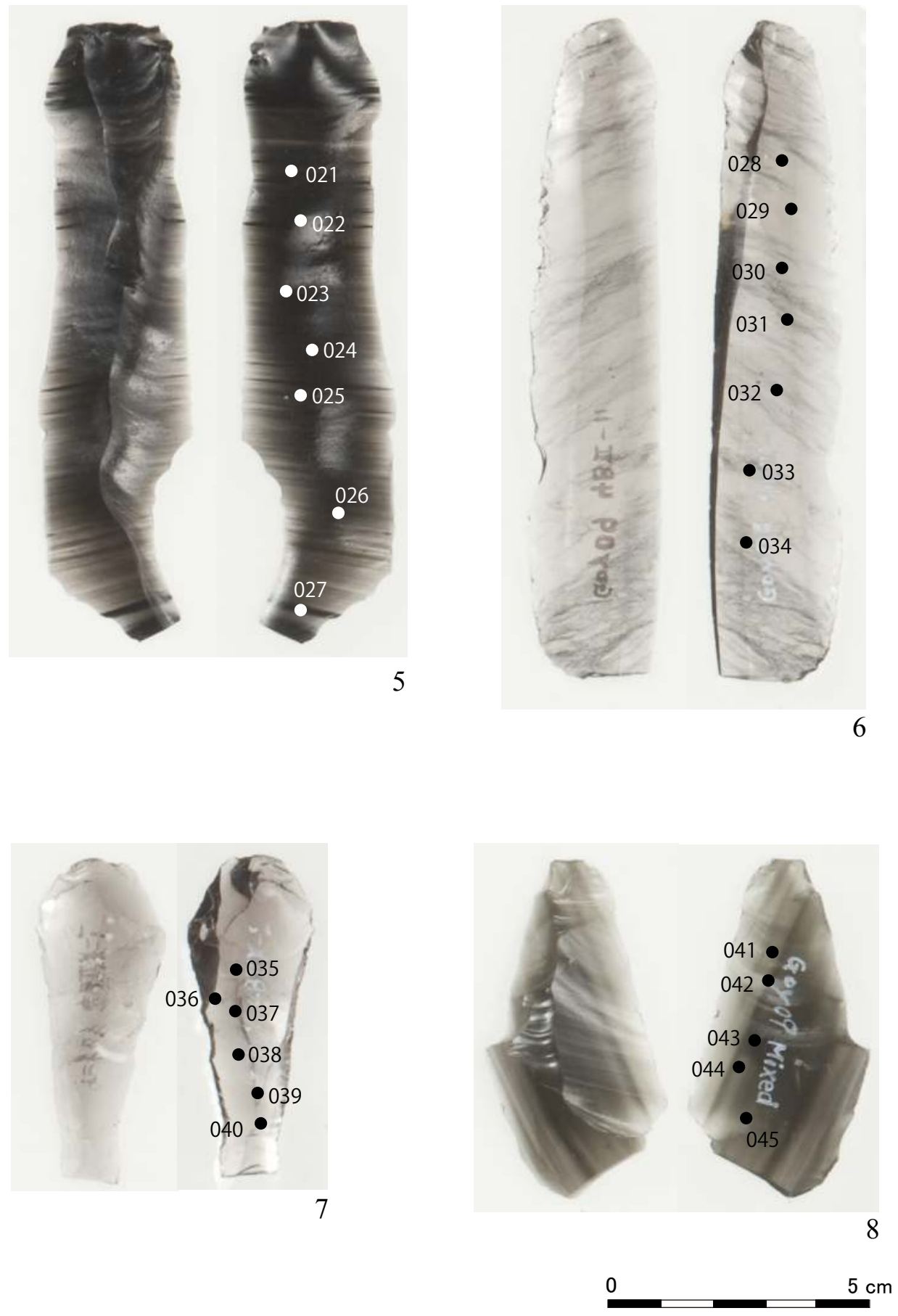

Fig. 11.1 Continued. 5: Unretouched blade (4BIIX-10); 6: Unretouched blade (4BII-11); 7: Unretouched blade (4BIIX-21); 8: Unretouched blade (4BII-mixed). The numbers on each specimen indicate the measurement points (AZGY).

powered microscope with magnification of $200 \times$ was employed to measure the angles of divergence of fracture wings in this research. The crack velocity (C) was calculated by the following formula, where $\Psi$ represents the angle of divergence of fracture wing and $\mathrm{C}^{2}$ indicates the elastic wave velocity
(Tomenchuk 1988): $\mathrm{C} / \mathrm{C}^{2}=\cos \Psi / 2$. The elastic wave velocity of obsidian is considered to be $3,507 \mathrm{~m} / \mathrm{s}$, which is an average of the $\mathrm{C}^{2}$ values of multiple obsidian specimens measured by Tomenchuk (1985). The angle of divergence of each fracture wing on the plane primary crack front was measured to determine 

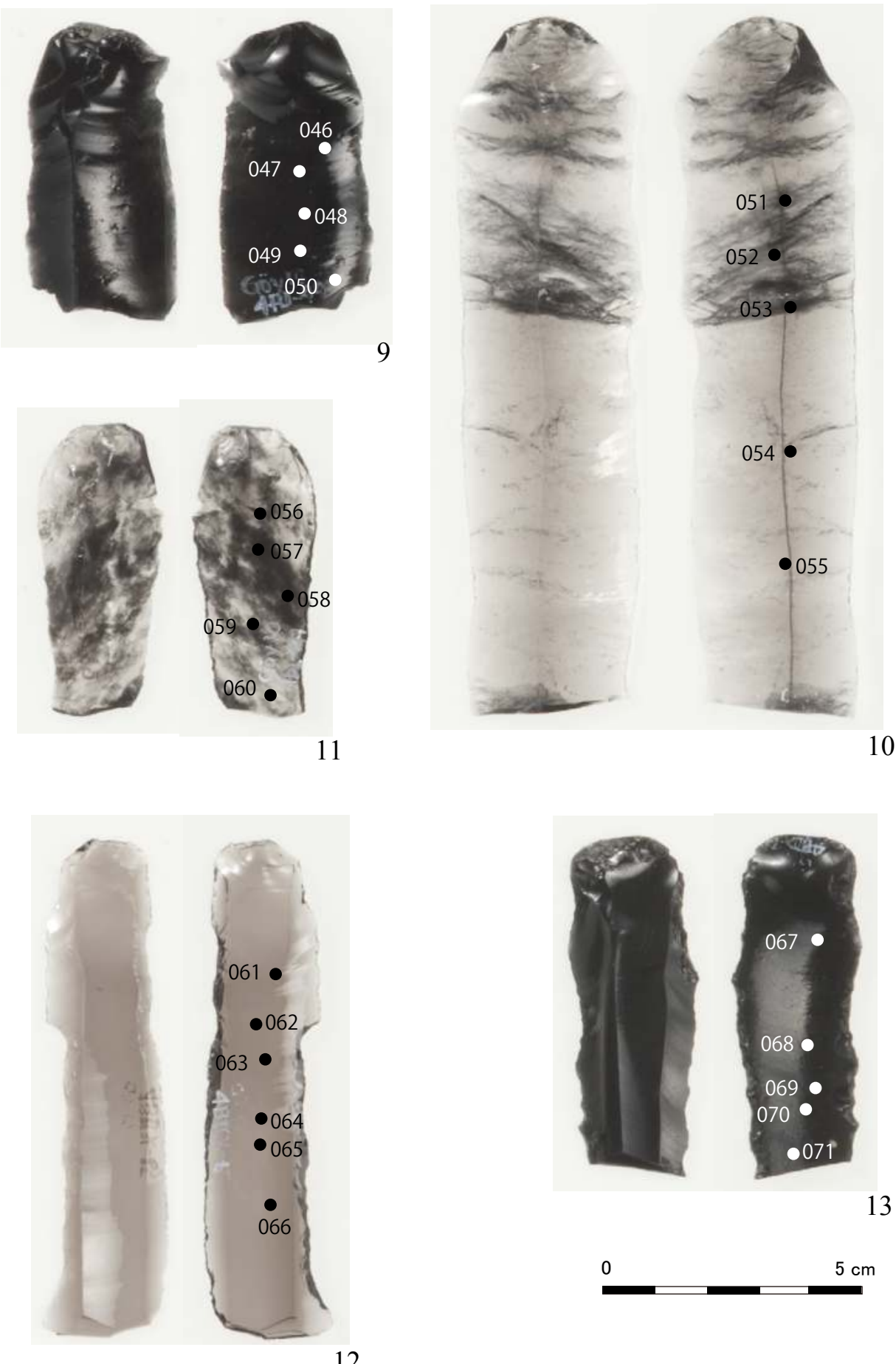

12

Fig. 11.1 Continued. 9: Unretouched blade (4BI-44); 10: Unretouched blade (4BI-44); 11: Unretouched blade (4BI-41); 12. Retouched blade (4BIIX-65); 13: Retouched blade (4BIIX-73). The numbers on each specimen indicate the measurement points (AZGY).

the crack velocity. This method of calculation was also applied in the experimental study by Takakura and Izuho (2004).

\subsection{Results}

The results are shown in Table 11.1 and Figs. 11.211.4. The average crack velocity for each specimen clearly points to the existence of three groups among 

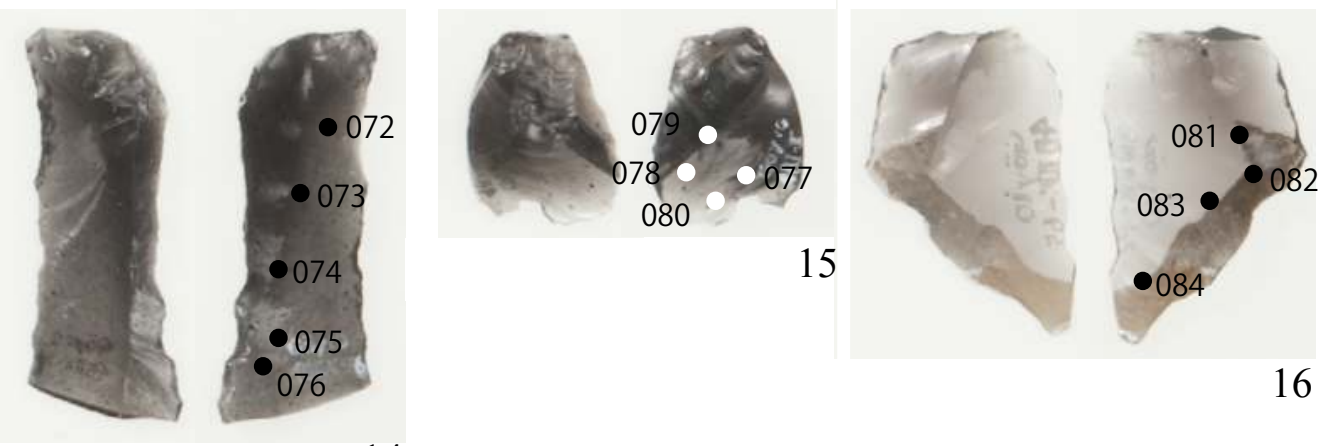

14
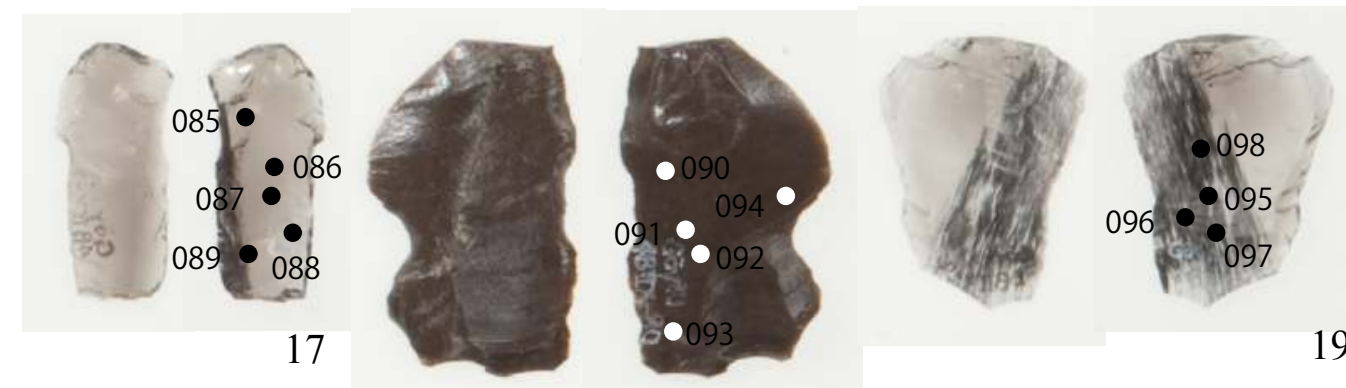

18

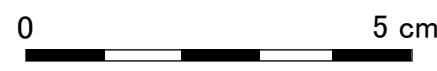

Fig. 11.1 Continued. 14: Retouched blade (4BIIX-76); 15: Retouched blade (4BI-5); 16: Unretouched blade (4BIIX-65); 17: Unretouched blade (4BIIX-72); 18: Retouched blade (4BIIX-80); 19: Unretouched flake (4BIIX-96). The numbers on each specimen indicate the measurement points (AZGY).

the study samples (Fig. 11.5). The first group shows the slowest velocity, 300-500 m/s (Table 11.1: 1-6, 9-13, and 17), while the second shows $600-800 \mathrm{~m} / \mathrm{s}$ (Table 11.1: 7, 8, 14, 18, and 19), and the third group shows significantly faster velocities, $1,000-1,400 \mathrm{~m} / \mathrm{s}$ (Table 11.1: 15 and 16). The velocities of these three groups almost perfectly match with those defined by the replicative experiments of Takakura and Izuho (2004: 43, fig. 4): crack velocities of I) pressure ranging $178-620 \mathrm{~m} / \mathrm{s}$, II) indirect percussion/direct percussion using soft hammers ranging 459-1,055 $\mathrm{m} / \mathrm{s}$, and III) direct percussion using hard hammers ranging $764-1,353 \mathrm{~m} / \mathrm{s}$. As a matter of fact, Group I specimens exhibit symmetrical shapes, along with parallel edges and ridges, and other morphological features that are associated with pressure debitage (Fig. 11.1: 1-6, 9-13, and 17). On the other hand, Group II (Fig. 11.1: 7, 8, 14, 18, and 19) and Group III (Fig. 11.1: 15 and 16) show more varied morphological traits.

The findings above provide compelling evidence to argue that obsidian assemblages from Göytepe include products made by pressure debitage. This is the first time that fracture mechanics, rather than empirical morphological traits, were used to assert the use of pressure technology during the Neolithic in the South Caucasus. At the same time, the results indicate that the blank production technology at Göytepe was performed by a combination of different techniques: not only pressure (I), but also indirect percussion/soft hammer percussion (II), and hard hammer percussion (III). This combination has been suggested only from morphological observations in the current literature. The obsidian cores of Göytepe are considered to have been knapped by hard hammer percussion in the early reduction stage, followed by indirect percussion/soft hammer, and pressure flaking in the later stages (Nishiaki and Guliyev 2019; Chapter 9). The present study provides evidence to verify the widely acknowledged, but unverified, interpretation of these technological strategies.

Our primary concern is pressure debitage, which is believed to constitute the major blank production technology of the Neolithic South Caucasus. The seminal replicative studies by Pelegrin (2012) model pressure techniques potentially employed 

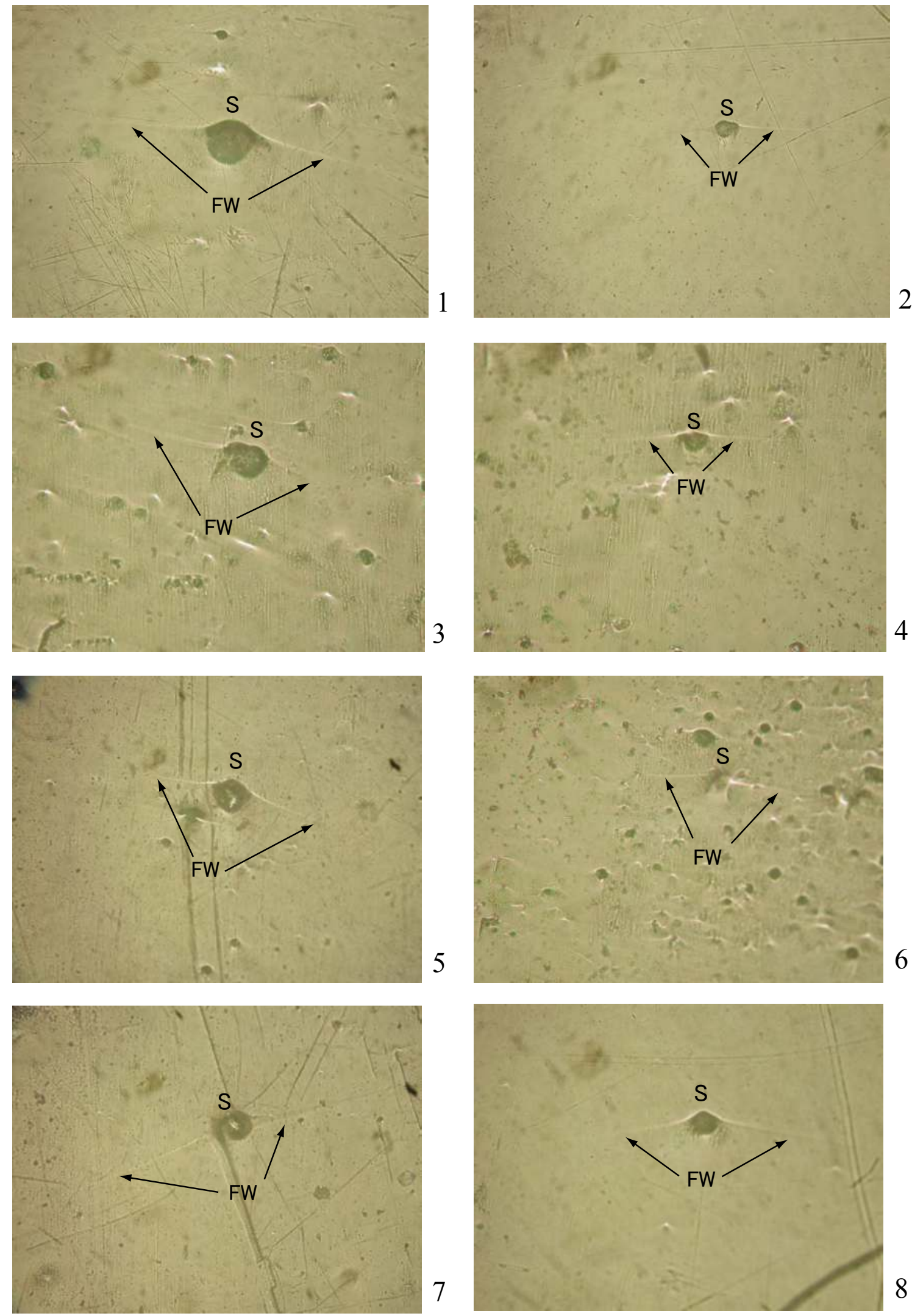

Fig. 11.2 Microphotographs of fracture wings from Göytepe (I). Width of field of view is $1 \mathrm{~mm}$. 1: AZGY-001 (Fig. 11.1: 1); 2: AZGY-005 (Fig. 11.1: 1); 3: AZGY-008 (Fig. 11.1: 2); 4: AZGY-010 (Fig. 11.1: 2); 5: AZGY-013 (Fig. 11.1: 3); 6: AZGY-023 (Fig. 11.1: 5); 7: AZGY-031 (Fig. 11.1: 6); 8: AZGY-039 (Fig. 11.1: 7). S: point of origin of the elastic wave; FW: fracture wing. 

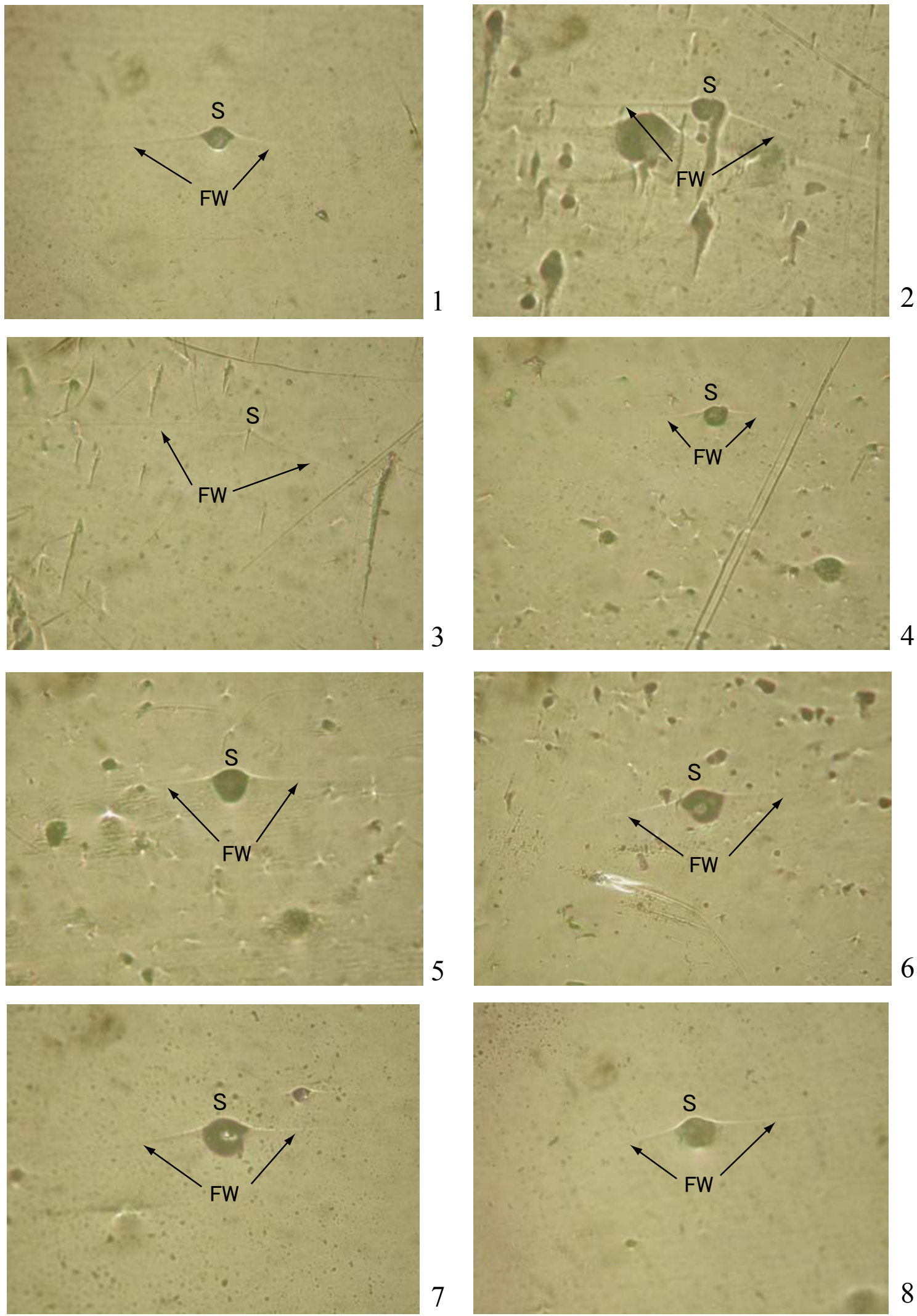

Fig. 11.3 Microphotographs of fracture wings from Göytepe (II). Width of field of view is $1 \mathrm{~mm} .1$ : AZGY-040 (Fig. 11.1: 7); 2: AZGY-042 (Fig. 11.1: 8); 3: AZGY-043 (Fig. 11.1: 8); 4: AZGY-048 (Fig. 11.1: 9); 5: AZGY-049 (Fig. 11.1: 9); 6: AZGY-050 (Fig. 11.1: 9); 7: AZGY-053 (Fig. 11.1: 10); 8: AZGY-054 (Fig. 11.1: 10). S: point of origin of the elastic wave; FW: fracture wing. 

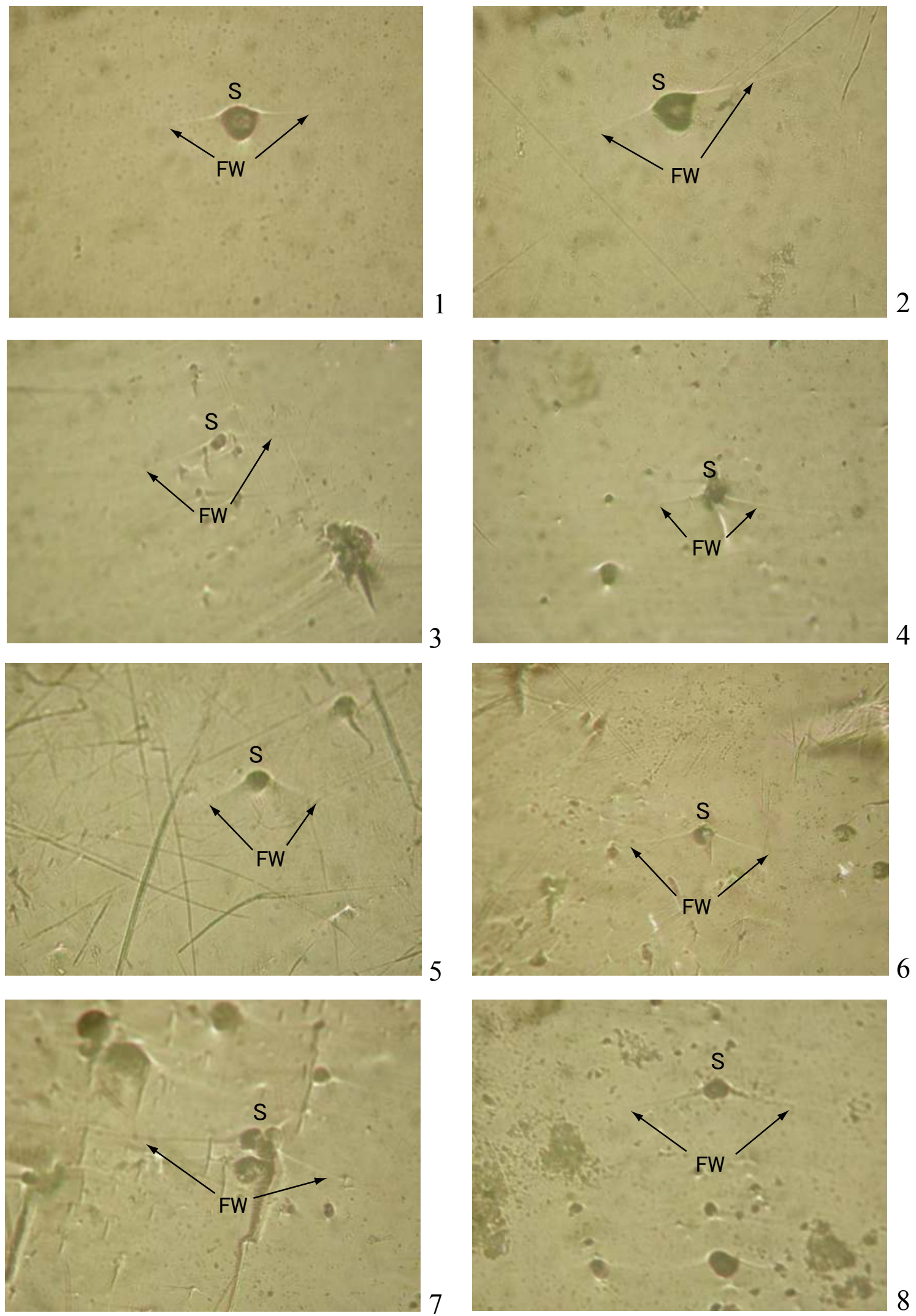

Fig. 11.4 Microphotographs of fracture wings from Göytepe (III). Width of field of view is $1 \mathrm{~mm}$. 1: AZGY064 (Fig. 11.1: 12); 2: AZGY-066 (Fig. 11.1: 12); 3: AZGY-073 (Fig. 11.1: 14); 4: AZGY-076 (Fig. 11.1: 14); 5: AZGY-080 (Fig. 11.1: 15); 6: AZGY-091 (Fig. 11.1: 18); 7: AZGY-093 (Fig. 11.1: 18); 8: AZGY-098 (Fig. 11.1: 19). S: point of origin of the elastic wave; FW: fracture wing. 
Table 11.1 The study samples and their measurement results. The distance denotes between the percussion points and the locations of fracture wing measurement points.

\begin{tabular}{|c|c|c|c|c|c|c|}
\hline Fig. 11.1: & Context & Description & $\begin{array}{c}\text { Measurement } \\
\text { point }\end{array}$ & $\begin{array}{l}\text { Distance } \\
(\mathbf{m m})\end{array}$ & $\begin{array}{l}\text { Velocity } \\
(\mathrm{m} / \mathrm{s})\end{array}$ & $\begin{array}{l}\text { Debitage } \\
\text { technique }\end{array}$ \\
\hline \multirow{5}{*}{1} & \multirow{5}{*}{ Surface (topsoil) } & \multirow{5}{*}{ Unretouched blade } & AZGY-001 & 20 & 426.3 & \multirow{5}{*}{1} \\
\hline & & & AZGY-002 & 26 & 383.8 & \\
\hline & & & AZGY-003 & 34 & 406.1 & \\
\hline & & & AZGY-004 & 46 & 329.0 & \\
\hline & & & AZGY-005 & 56 & 317.8 & \\
\hline \multirow{5}{*}{2} & \multirow{5}{*}{ FT 2 (Level 7) } & \multirow{5}{*}{ Unretouched blade } & AZGY-006 & 22 & 345.2 & \multirow{5}{*}{ I } \\
\hline & & & AZGY-007 & 26 & 283.2 & \\
\hline & & & AZGY-008 & 38 & 381.7 & \\
\hline & & & AZGY-009 & 40 & 363.5 & \\
\hline & & & AZGY-010 & 48 & 308.7 & \\
\hline \multirow{5}{*}{3} & \multirow{5}{*}{ 4BII-1 (topsoil) } & \multirow{5}{*}{ Unretouched blade } & AZGY-011 & 35 & 426.3 & \multirow{5}{*}{1} \\
\hline & & & AZGY-012 & 43 & 527.4 & \\
\hline & & & AZGY-013 & 49 & 403.0 & \\
\hline & & & AZGY-014 & 57 & 299.5 & \\
\hline & & & AZGY-015 & 70 & 366.5 & \\
\hline \multirow{5}{*}{4} & \multirow{5}{*}{ 4BIIX-40 (Level 11) } & \multirow{5}{*}{ Unretouched blade } & AZGY-016 & 23 & 446.6 & \multirow{5}{*}{ I } \\
\hline & & & AZGY-017 & 27 & 428.4 & \\
\hline & & & AZGY-018 & 43 & 411.1 & \\
\hline & & & AZGY-019 & 50 & 541.5 & \\
\hline & & & AZGY-020 & 68 & 463.8 & \\
\hline \multirow{7}{*}{5} & \multirow{7}{*}{ 4BIIX-10 (Level 10) } & \multirow{7}{*}{ Unretouched blade } & AZGY-021 & 24 & 524.4 & \multirow{7}{*}{ I } \\
\hline & & & AZGY-022 & 31 & 488.0 & \\
\hline & & & AZGY-023 & 43 & 371.6 & \\
\hline & & & AZGY-024 & 49 & 384.8 & \\
\hline & & & AZGY-025 & 56 & 411.1 & \\
\hline & & & AZGY-026 & 72 & 358.4 & \\
\hline & & & AZGY-027 & 88 & 410.1 & \\
\hline \multirow{7}{*}{6} & \multirow{7}{*}{ 4BII-11 (Level 8) } & \multirow{7}{*}{ Unretouched blade } & AZGY-028 & 19 & 299.5 & \multirow{7}{*}{1} \\
\hline & & & AZGY-029 & 28 & - & \\
\hline & & & AZGY-030 & 37 & 397.0 & \\
\hline & & & AZGY-031 & 47 & 379.7 & \\
\hline & & & AZGY-032 & 58 & 299.5 & \\
\hline & & & AZGY-033 & 68 & 332.0 & \\
\hline & & & AZGY-034 & 81 & 278.2 & \\
\hline \multirow{6}{*}{7} & & & AZGY-035 & 16 & 655.1 & \\
\hline & & & AZGY-036 & 21 & 755.0 & \\
\hline & 4RIIX-21 (l evel 11) & & AZGY-037 & 23 & 593.9 & $\|$ \\
\hline & 4BIIX-21 (Level 11) & Unretouched blade & AZGY-038 & 29 & 742.1 & II \\
\hline & & & AZGY-039 & 36 & 633.0 & \\
\hline & & & AZGY-040 & 41 & 600.9 & \\
\hline
\end{tabular}


Table 11.1 Continued.

\begin{tabular}{|c|c|c|c|c|c|c|}
\hline Fig. 11.1: & Context & Description & $\begin{array}{c}\text { Measurement } \\
\text { point }\end{array}$ & $\begin{array}{c}\text { Distance } \\
(\mathrm{mm})\end{array}$ & $\begin{array}{c}\text { Velocity } \\
(\mathrm{m} / \mathrm{s})\end{array}$ & $\begin{array}{c}\text { Debitage } \\
\text { technique }\end{array}$ \\
\hline \multirow{5}{*}{8} & \multirow{5}{*}{ 4BII (mixed) } & \multirow{5}{*}{ Unretouched blade } & AZGY-041 & 13 & 734.1 & \multirow{5}{*}{ II } \\
\hline & & & AZGY-042 & 18 & 654.1 & \\
\hline & & & AZGY-043 & 26 & 611.9 & \\
\hline & & & AZGY-044 & 32 & 791.8 & \\
\hline & & & AZGY-045 & 40 & 576.8 & \\
\hline \multirow{5}{*}{9} & \multirow{5}{*}{ 4BI-44 (Level 8) } & \multirow{5}{*}{ Unretouched blade } & AZGY-046 & 23 & 278.2 & \multirow{5}{*}{1} \\
\hline & & & AZGY-047 & 25 & 348.3 & \\
\hline & & & AZGY-048 & 30 & 342.2 & \\
\hline & & & AZGY-049 & 35 & 249.7 & \\
\hline & & & AZGY-050 & 40 & 237.5 & \\
\hline \multirow{5}{*}{10} & \multirow{5}{*}{ 4BI-44 (Level 8) } & \multirow{5}{*}{ Unretouched blade } & AZGY-051 & 32 & 299.5 & \multirow{5}{*}{1} \\
\hline & & & AZGY-052 & 41 & 275.1 & \\
\hline & & & AZGY-053 & 47 & 296.5 & \\
\hline & & & AZGY-054 & 65 & 314.8 & \\
\hline & & & AZGY-055 & 81 & 275.1 & \\
\hline \multirow{5}{*}{11} & \multirow{5}{*}{ 4BI-41 (Level 8) } & \multirow{5}{*}{ Unretouched blade } & AZGY-056 & 14 & 301.5 & \multirow{5}{*}{ I } \\
\hline & & & AZGY-057 & 19 & 272.1 & \\
\hline & & & AZGY-058 & 27 & 406.1 & \\
\hline & & & AZGY-059 & 31 & 314.8 & \\
\hline & & & AZGY-060 & 41 & 453.7 & \\
\hline \multirow{6}{*}{12} & \multirow{6}{*}{ 4BIIX-65 (Level 10) } & \multirow{6}{*}{ Retouched blade } & AZGY-061 & 22 & 266.0 & \multirow{6}{*}{1} \\
\hline & & & AZGY-062 & 29 & 356.4 & \\
\hline & & & AZGY-063 & 34 & 278.2 & \\
\hline & & & AZGY-064 & 43 & 334.1 & \\
\hline & & & AZGY-065 & 48 & 267.0 & \\
\hline & & & AZGY-066 & 61 & 250.7 & \\
\hline \multirow{5}{*}{13} & \multirow{5}{*}{ 4BIIX-73 (Level 12) } & \multirow{5}{*}{ Retouched blade } & AZGY-067 & 16 & 229.3 & \multirow{5}{*}{ I } \\
\hline & & & AZGY-068 & 31 & 290.4 & \\
\hline & & & AZGY-069 & 37 & 366.5 & \\
\hline & & & AZGY-070 & 43 & 326.9 & \\
\hline & & & AZGY-071 & 49 & 287.3 & \\
\hline \multirow{5}{*}{14} & & & AZGY-072 & 11 & 769.0 & \\
\hline & & & AZGY-073 & 14 & 632.0 & \\
\hline & 4BIIX-76 (Level 11) & Retouched blade & AZGY-074 & 23 & 648.1 & II \\
\hline & & & AZGY-075 & 31 & 592.9 & \\
\hline & & & AZGY-076 & 35 & 634.0 & \\
\hline & & & AZGY-077 & 15 & 1309.0 & \\
\hline 15 & API-5 (1) & Retourhed hlado & AZGY-078 & 14 & 1305.2 & $\mu$ \\
\hline 10 & 4DT-U (LEVETO) & hervuctied Niace & AZGY-079 & 12 & 1436.6 & III \\
\hline & & & AZGY-080 & 17 & 1443.1 & \\
\hline
\end{tabular}


Table 11.1 Continued.

\begin{tabular}{|c|c|c|c|c|c|c|}
\hline Fig. 11.1: & Context & Description & $\begin{array}{c}\text { Measurement } \\
\text { point }\end{array}$ & $\begin{array}{c}\text { Distance } \\
(\mathrm{mm})\end{array}$ & $\begin{array}{c}\text { Velocity } \\
(\mathrm{m} / \mathrm{s})\end{array}$ & $\begin{array}{c}\text { Debitage } \\
\text { technique }\end{array}$ \\
\hline \multirow{4}{*}{16} & \multirow{4}{*}{ 4BIIX-65 (Level 10) } & \multirow{4}{*}{ Unretouched flake } & AZGY-081 & 13 & 1321.3 & \multirow{4}{*}{ III } \\
\hline & & & AZGY-082 & 16 & 1086.6 & \\
\hline & & & AZGY-083 & 20 & 1015.5 & \\
\hline & & & AZGY-084 & 26 & 972.5 & \\
\hline \multirow{5}{*}{17} & \multirow{5}{*}{ 4BIIX-72 (Level 12) } & \multirow{5}{*}{ Unretouched blade } & AZGY-085 & 7 & 296.5 & \multirow{5}{*}{1} \\
\hline & & & AZGY-086 & 12 & 244.6 & \\
\hline & & & AZGY-087 & 17 & 345.2 & \\
\hline & & & AZGY-088 & 19 & 349.3 & \\
\hline & & & AZGY-089 & 22 & 375.7 & \\
\hline \multirow{5}{*}{18} & \multirow{5}{*}{ 4BIIX-80 (Level 11) } & \multirow{5}{*}{ Retouched blade } & AZGY-090 & 14 & 884.0 & \multirow{5}{*}{ II } \\
\hline & & & AZGY-091 & 19 & 943.1 & \\
\hline & & & AZGY-092 & 22 & 735.1 & \\
\hline & & & AZGY-093 & 30 & 708.1 & \\
\hline & & & AZGY-094 & 18 & 678.1 & \\
\hline \multirow{4}{*}{19} & \multirow{4}{*}{ 4BIIX-96 (Level 13) } & \multirow{4}{*}{ Unretouched flake } & AZGY-095 & 16 & 562.7 & \multirow{4}{*}{ II } \\
\hline & & & AZGY-096 & 20 & 642.1 & \\
\hline & & & AZGY-097 & 22 & 535.5 & \\
\hline & & & AZGY-098 & 12 & 722.1 & \\
\hline
\end{tabular}

in prehistoric times: Mode 1: hand-held, Mode 2: shoulder crutch, Mode 3: short crutch in a standing position, Mode 4: long crutch in a standing position, and Mode 5: lever use, corresponding to the use of different knapping devices respectively. Pelegrin suggested the width of pressure products (blades) as a useful indicator to distinguish which modes/ techniques were employed in the prehistoric lithic assemblages being studied. According to Pelegrin (2012: 479, fig. 18: 12), the upper limit of the width of producible blades increases from Modes 1 to 5 . Apparently, the threshold exists between Modes 4 and 5. Mechanical devices used for Mode 5, that is, lever and other tools, enable production of much wider blades than the other modes that principally used human body power alone. The border seems to be drawn about 2 to $2.5 \mathrm{~cm}$ in width. Considering that Mode 4 can occasionally produce blades of 2 to $2.5 \mathrm{~cm}$ in width (Pelegrin 2012: 479, fig. 18: 12), those wider than $2.5 \mathrm{~cm}$ may be safely assumed to be produced by lever pressure. The 13 blades assigned to Group 1 include large blades up to $3.7 \mathrm{~cm}$ in width. Even if excluding the largest one because of its uncertain stratigraphic context (Fig. 11.1: 1, Göy09Surface), the stratified study samples include large blades as wide as $2.8 \mathrm{~cm}$ (Fig. 11.1: 10), suggesting the use of a lever pressure technique. This data is in accordance with the current interpretation of lever use for obsidian knapping in the Neolithic period of the South Caucasus (see Chabot and Pelegrin 2012).

\subsection{Conclusions}

The present study opens a promising avenue to understand the details of the Neolithic lithic technology in the Southern Caucasus. The results support the general consensus that pressure debitage was the predominant technique for obsidian blade blank production. The distribution of the samples by different occupational levels shows that the use of this technology was common throughout the occupational sequence of this large Neolithic settlement. At the same time, the study indicates that other debitage techniques were also used for core reduction. As the specimens with fracture wings inconsistent with pressure debitage contain irregular flakes, we believe that (among our selected study samples) techniques other than pressuring were likely employed for different purposes, including core preparation and management. 


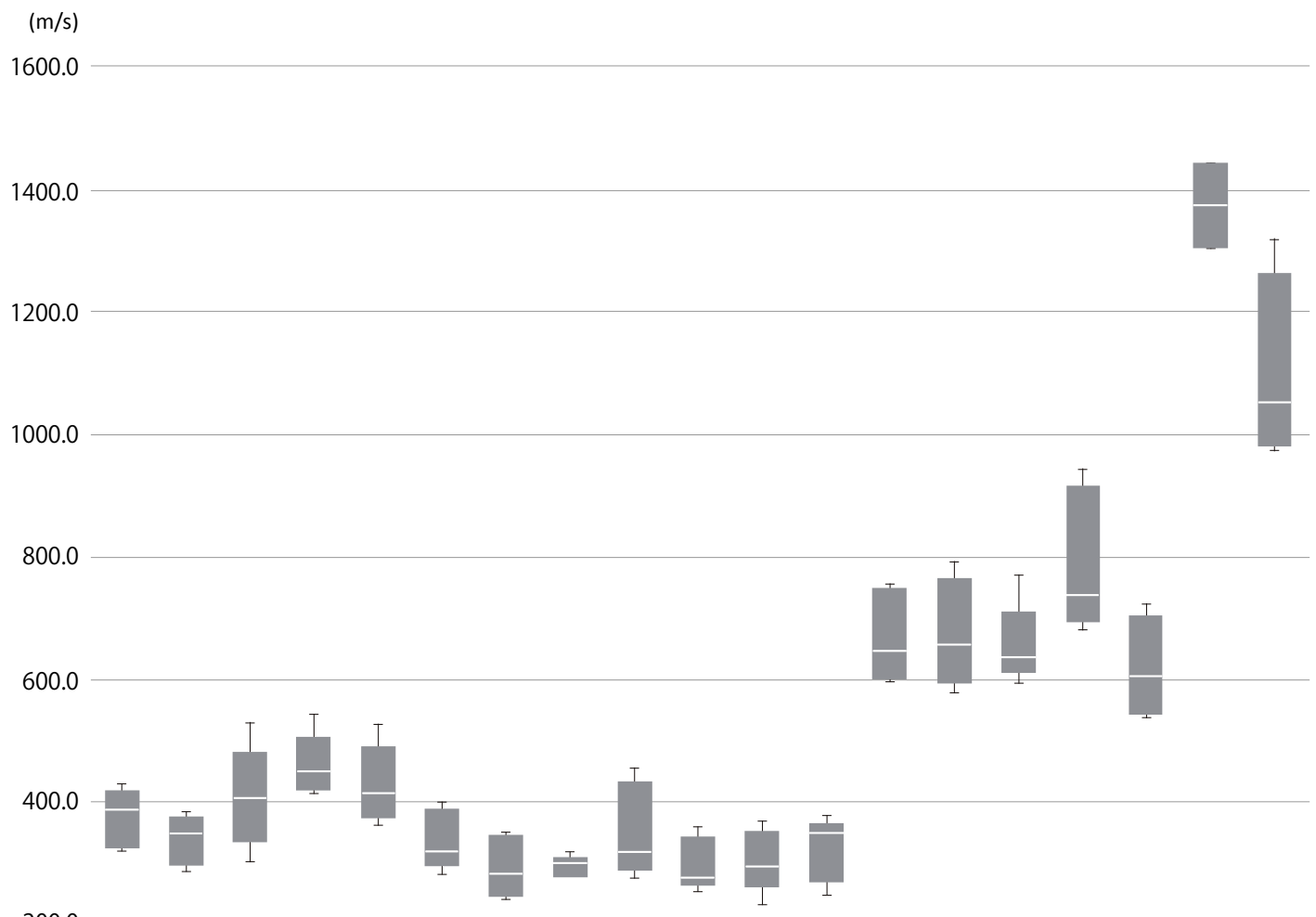

200.0

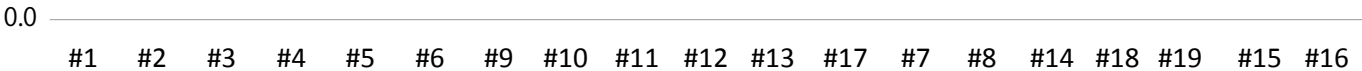

Fig. 11.5 Measurement values of the crack velocity of 19 samples from Göytepe. Each box plot shows minimum value, $25 \%$ value, median, $75 \%$ value and maximum value.

Fracture wing analysis is not a perfect research tool for identification of knapping techniques at least in the current stage. A notable limitation is the difficulty of distinguishing the use of indirect percussion from soft hammer direct percussion. Nevertheless, the present study shows great potential for this analytical approach when the identification of pressure debitage and retouch is the focus of a research, such as in our case. Although the present paper dealt with only a small portion of the huge obsidian artifact collection of Göytepe, more systematic studies based on nonselective collections will produce fresh insights into the Neolithic technology of the South Caucasus.

\section{References}

Chabot, J. and J. Pelegrin (2012) Two examples of pressure blade production with a lever: Recent research from the Southern Caucasus (Armenia) and Northern Mesopotamia (Syria, Iraq). In: The Emergence of Pressure Blade Making: From
Origin to Modern Experimentation, edited by P. M. Desrosiers, pp. 181-198. New York: Springer.

Cotterell, B. and J. Kamminga (1979) The mechanics of flaking. In: Lithic Use-wear Analysis, edited by B. Hayden, pp. 97-112. New York: Academic Press.

Cotterell, B. and J. Kamminga (1992) Mechanics of Pre-industrial Technology: An Introduction to the Mechanics of Ancient and Traditional Material Culture. Cambridge: Cambridge University Press.

Cotterell, B., J. Kamminga, and F. P. Dickson (1985) The essential mechanics of conchoidal flaking. International Journal of Fracture, 29(4): 205-221.

Desrosiers, P. M. (2012) The Emergence of Pressure Blade Making: From Origin to Modern Experimentation. New York: Springer.

Faulkner, A. (1972) Mechanical Principles of Flintworking. Washington State University. Ann Arbor: University Microfilms (Ph.D. Dissertation).

Hutchings, W. K. (1999) Quantification of fracture propagation velocity employing a sample of Clovis channel flakes. Journal of Archaeological Science, 26(12): 1437-1447.

Inizan, M. -L., M. Reduron-Ballinger, H. Roche, and J. Tixier (1999) Technology and Terminology of 
Knapped Stone. Nanterre: C.R.E.P.

Nishiaki, Y. and F. Guliyev (2019) Neolithic lithic industries of the Southern Caucasus: Göytepe and Hacı Elamxanlı Tepe, West Azerbaijan (early 6th millennium BC). In: Near Eastern Lithics on the Move: Interaction and Contexts in Neolithic Traditions, edited by L. Astruc, F. Briois, C. McCartney, and L. Kassianidou, pp. 471-483. Nicosia: Astrom Editions.

Pelegrin, J. (2012) New experimental observations for the characterization of pressure blade production techniques. In: The Emergence of Pressure Blade Making: From Origin to Modern Experimentation, edited by P. M. Desrosiers, pp. 465-500. New York: Springer.

Takakura, J. and M. Izuho (2004) Identification of flaking techniques: From the analysis of fracture wings. The Quaternary Research, 43(1): 37-48 (in Japanese with English summary).

Tomenchuk, J. (1985) The Development of a Wholly Parametric Use-wear Methodology and its Application to Two Selected Samples of Epipaleolithic Chipped Stone Tools from Hayonim Cave, Israel. University of Toronto (Ph.D. Dissertation).

Tomenchuk, J. (1988) Effects of loading rate on the reliability of the engineering use-wear models. In: Industries Lithiques: Tracéologie et Technologie, edited by S. S. Beyries, pp. 115132. BAR International Series 411. Oxford: British Archaeological Reports. 



\title{
Neolithic ground stone typology and technology at Göytepe
}

\author{
Seiji Kadowaki
}

\subsection{Introduction}

This chapter reports ground stones from Göytepe by describing their typology and examining technological behaviors in the production and use of ground stones. The chapter then presents stratigraphic changes in ground stone assemblages of Göytepe, followed by comparisons with other Neolithic sites in the southern Caucasus and in wider regions.

Four seasons of excavations at Göytepe from 2008 to 2011 recovered a large number of ground stone artifacts, of which 1,762 pieces are reported in this chapter. These specimens consist of four sets of collections differing from each other in recovery methods or contexts. The first and main group $(n=1,506)$ was obtained from Neolithic Levels 4-11 that were excavated by the Japanese team at Squares 3BI, 4BI, and 4BII. The excavation kept all the ground stone items, including unmodified ad hoc tools, fragments, and debitage. Another set of ground stones $(\mathrm{n}=58)$ were collected by the same excavation methods from small pits opened by the Japanese mission at the periphery of the site (Squares 5BI, 96F, $97 \mathrm{~F}$, and $97 \mathrm{G}$ ). Because the stratigraphic connection between the pits and main excavation areas (Squares $1 \mathrm{~A}, 1 \mathrm{~B}, 2 \mathrm{~A}, 2 \mathrm{~B}, 3 \mathrm{~A}, 3 \mathrm{~B}, 4 \mathrm{~A}$, and 4B) is still unclear, the artifacts from the pits have not been assigned to Neolithic Levels. The third collection $(\mathrm{n}=195)$ is from the excavations by the Azerbaijani mission at Squares 1A, 1B, 2A, 2B, 3A, 3BII, and 4A (see Chapter 3 ), where the recovery of ground stones focused on formal tool types, such as grinding slabs, mortars, grinders, handstones, and axes. The limited contextual data of these artifacts allow us to make broad assignment of the artifacts to Neolithic levels among Levels $1-6$. The last small set $(n=3)$ includes selective finds from the eroded surface of the site.
Because the ground stones from these four collections share similar characteristics inmorphology and production technology, all the specimens are used for setting the ground stone typology at Göytepe. The same samples are also used for proposing chaînes opératoires involved in the production, use, and maintenance of ground stones. On the other hand, the stratigraphic examination of ground stones uses the main collection by the Japanese mission from Neolithic Levels 4-11 with some references to the Azerbaijani selective collections from Levels 1-6. This chapter also compares the ground stones from Göytepe with those from other sites affiliated to the Shomutepe culture. As a part of this purpose, tool compositions are examined by using quantitative data on some Neolithic ground stone assemblages from the Kwemo-Kartli region (Hamon 2008). Lastly, the chapter briefly discusses what implications can be drawn from the Göytepe ground stones in light of its temporal and geographic contexts in the Neolithization processes in the southern Caucasus.

\subsection{Typology}

The classification of the ground stone artifacts from Göytepe is based on the morphology of tools and working surfaces as well as the kind and extent of modification traces, such as flaking, pecking, and grinding, which occurred in the course of production, use, and maintenance of the tools. The following describes the definition of the tool types presented in Table 12.1 with some references to other ground stone typologies in west Asia, such as those by Wright (1992) and Hamon (2008), for the facilitation of inter-site comparisons. The relationship between raw material types and tool types is presented in Table 12.2. 
Table 12.1 Inventory of ground stone artifacts from Göytepe (2008-2011 seasons).

\begin{tabular}{|c|c|c|c|c|}
\hline Type group & Type name & $\#$ & $\%$ & $\begin{array}{l}\text { Without pebbles } \\
\text { and flakes }\end{array}$ \\
\hline \multirow[t]{3}{*}{ Slabs and Querns } & Grinding slabs & 38 & $2.2 \%$ & $9.3 \%$ \\
\hline & Grinding querns & 5 & $0.3 \%$ & $1.2 \%$ \\
\hline & Working slab & 1 & $0.1 \%$ & $0.2 \%$ \\
\hline Mortars & Mortars & 5 & $0.3 \%$ & $1.2 \%$ \\
\hline \multirow[t]{5}{*}{ Grinders and Handstones } & Grinders & 26 & $1.5 \%$ & $6.3 \%$ \\
\hline & Handstones & 58 & $3.3 \%$ & $14.1 \%$ \\
\hline & Handstone/pestles & 10 & $0.6 \%$ & $2.4 \%$ \\
\hline & Handstone/crushing cobbles & 2 & $0.1 \%$ & $0.5 \%$ \\
\hline & Handstone/pestle/crushing cobbles & 2 & $0.1 \%$ & $0.5 \%$ \\
\hline \multirow[t]{2}{*}{ Pestles } & Pestles & 6 & $0.3 \%$ & $1.5 \%$ \\
\hline & Pestle/crushing cobbles & 2 & $0.1 \%$ & $0.5 \%$ \\
\hline \multirow[t]{2}{*}{ Pounders } & Core pounders & 111 & $6.3 \%$ & $27.1 \%$ \\
\hline & Pounders & 25 & $1.4 \%$ & $6.1 \%$ \\
\hline \multirow[t]{7}{*}{ Cobble/pebble tools } & Crushing cobbles & 4 & $0.2 \%$ & $1.0 \%$ \\
\hline & Pecked cobbles & 14 & $0.8 \%$ & $3.4 \%$ \\
\hline & Flaked cobbles & 18 & $1.0 \%$ & $4.4 \%$ \\
\hline & Ground cobbles & 10 & $0.6 \%$ & $2.4 \%$ \\
\hline & Flat cobbles & 14 & $0.8 \%$ & $3.4 \%$ \\
\hline & Elongated cobbles & 8 & $0.5 \%$ & $2.0 \%$ \\
\hline & Pebbles & 989 & $56.1 \%$ & NA \\
\hline \multirow[t]{2}{*}{ Axes/Chisels } & Axes & 15 & $0.9 \%$ & $3.7 \%$ \\
\hline & Chisel & 1 & $0.1 \%$ & $0.2 \%$ \\
\hline \multirow[t]{2}{*}{ Abraders/Polishers } & Abraders & 14 & $0.8 \%$ & $3.4 \%$ \\
\hline & Floor polishers & 2 & $0.1 \%$ & $0.5 \%$ \\
\hline \multicolumn{2}{|l|}{ Perforated stone } & 1 & $0.1 \%$ & $0.2 \%$ \\
\hline Debitage & Flakes & 363 & $20.6 \%$ & NA \\
\hline \multirow[t]{2}{*}{ Fragments } & Fragments of handstones or grinding slabs & 17 & $1.0 \%$ & $4.1 \%$ \\
\hline & Unidentifiable fragment & 1 & $0.1 \%$ & $0.2 \%$ \\
\hline \multicolumn{2}{|l|}{ Total } & 1762 & $100.0 \%$ & NA \\
\hline \multicolumn{2}{|l|}{ Without pebbles and flakes } & 410 & NA & $100.0 \%$ \\
\hline
\end{tabular}

\subsubsection{Grinding slabs (Fig. 12.1)}

Raw material: Mainly andesite with some basalt boulders.

Morphology: Oval to rectangular in plan. The longitudinal cross-section of working surface is concave in a varying degree, showing a saddleshaped form.

Modification traces: The working surface is pecked and ground, showing longitudinal striations (Fig. 12.2). The sides and the back of the slabs are usually ground with some pecking and flaking scars.

Notes: This type corresponds to saddle-shaped grinding slabs in Wright's type 6 (Wright 1992:

63 ) and saddle-shaped slabs in Hamon (2008: 93).

\subsubsection{Grinding querns}

Raw material: Mainly andesite boulders.

Morphology: Oval to quadrangular in plan. The working surface forms a shallow depression.

Modification traces: The working surface is pecked 


\begin{tabular}{|c|c|c|c|c|c|c|c|c|c|c|c|c|c|c|c|c|c|c|c|c|c|c|c|c|}
\hline & 必 & & -1 & ऽ) & & $\stackrel{5}{\circ}$ & 20 & $\sim \sim$ & 10 & & $\Xi$ & $\stackrel{\sim}{2}$ & & $\pm \$$ & 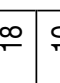 & 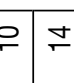 & $\infty$ & $\stackrel{2}{\circ}$ & $-F$ & $\sim$ & $-\mathbb{m}_{\bar{c}}$ & 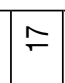 & & א \\
\hline 志 & ○े̀ & ఫे & ڤें & 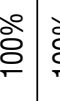 & se & : & 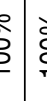 & 3 & ò & oे & 잉 & ذిڤ & ڤ⿳亠口冋口 & ڤें & ڤें & ô & ठे & 容 & & ○े̀े & ڤి̊ & oे & & 产 \\
\hline 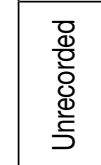 & $\AA^{\circ}$ & ৪े & 今े & ڤें & \&े & $\stackrel{e}{e}$ & 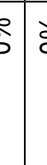 & $b$ & 8 & ठั & $\therefore$ & 8̊ & ঃे & $8^{\circ}$ & ১े & $\therefore$ & $\therefore$ & ১े & $\stackrel{\circ}{\circ}$ & ठे & ১̊ & ò & s̊ & 究 \\
\hline 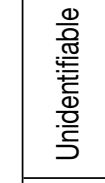 & $\AA^{\circ}$ & ১े & 今े & ஃे & \&े & ڤे & 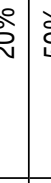 & : वీ & ळे & i̊ & 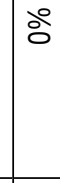 & ठ̊ & ஃें & ஓें & ڤั) & : & 怘 & 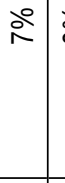 & 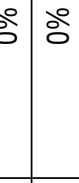 & 总 & ठे ले & $\stackrel{\text { ڤે }}{\stackrel{0}{0}}$ & & वें \\
\hline 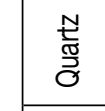 & ذ̊ & ठे & ஃे & ঃें & 今े & bi & co & $\stackrel{\circ}{\vdots}$ & $\mathrm{a}^{\circ}$ & ठั & $\stackrel{8}{\circ}$ & ১̊ & ঃे & ঃ̊ & ¿े & ¿̊ & $0^{\circ}$ & ठे & 今 & 今े & ১̊ & o̊ & ঃ̊ & $\frac{\circ}{0}$ \\
\hline 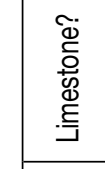 & $\stackrel{\circ}{\circ}$ & ஃे & ঃे & ঃे a & ○े & â & ¿ & : á & 8 & वे & 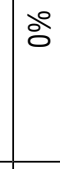 & ठ̊ & ঃे & ถें & ¿ें & ১̊ & ò & ذें & $\stackrel{8}{\circ}$ & $\AA^{\circ}$ & ถిㅇ & 今̊ & & هें \\
\hline 壱 & $\stackrel{\circ}{\circ}$ & ஃे & \&े & ঃे & ○े & bे & ¿ & : á & 3 & ¿ & $\stackrel{\circ}{\circ}$ & ळ & ঃे & ฉ̊ & ১े & ১̊ & $\therefore$ & ठे & 今 & 今े & ஃे & 今े & & 总 \\
\hline 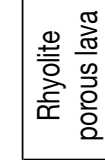 & 8 & ১े & ১ें & ঃे & ஓे & s̀ & co & $\vdots$ & 8 & ठั & $\stackrel{8}{\circ}$ & ठ̊ & ঃे & ঃे & ১े & ১̊ & $\therefore$ & ฉ̊ & ஃ̊ & ठे & $\therefore$ & ○े & & 今े \\
\hline 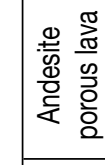 & 80 & ठे & ৪े & ১े| & $\therefore$ & $\dot{b}$ & $\dot{s}$ & $\vdots a$ & 8 & 8 & $\therefore$ & 今े & ঃे & 今े & ১ें & sั & ০ั & ذें & : & 今े & वें & $8^{\circ}$ & & @̊̀ \\
\hline 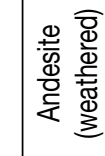 & $\stackrel{\circ}{\stackrel{\circ}{\circ}}$ & ১̊̀ & ஃे & ১̊ำ & ণे & ¿े & ¿ & : á & 8 & वे & 8 & ठ̊ & 产 & ถें & ¿ें & ১े & ठ̊ & ذें & ஃ̊ & ذे & ১े & ठ̊ & ঃे & @̊ \\
\hline 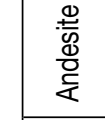 & 吕 & ڤें & $8^{\circ}$ & ১ें & 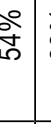 & : & colo & $\stackrel{\circ}{\vdots}$ & 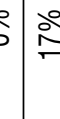 & ¿े & ৯े & ذ̊ & $\mathrm{s}^{\circ}$ & ১̊ & ১े? & 仓े & $\therefore$ & ذे & 今ं & 今े & ठें & $\mid$\begin{tabular}{c}
2 \\
2 \\
\multirow{2}{*}{}
\end{tabular} & & $\begin{array}{l}\text { 今े } \\
\text { 。이 }\end{array}$ \\
\hline 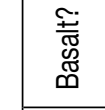 & $\AA^{\circ}$ & वें & $\stackrel{8}{\circ}$ & ১̊ا & ஃे & 今̀ & $s$ & $g_{0}^{\circ}$ & 8 & ذి & $\therefore$ & ठ̊ & ঃे & ঃे & ১̊ & ১̊ & 灾 & ठे & ஃ̊ & ஓे & ¿े & 8 & ঃे & $\begin{array}{l}\circ \\
\text { ळo } \\
0\end{array}$ \\
\hline 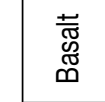 & ঐे & ১े & ڤั̀े & ১े & ڤे & 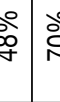 & 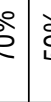 & : & ì & ذे & $\frac{8}{2}$ & ๙े & 产 & ટે & ڤे̀े & ১ें & ì & 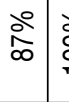 & : & $\AA^{\circ}$ & ठั & $\frac{2}{\dot{\gamma}}$ & & 总 \\
\hline 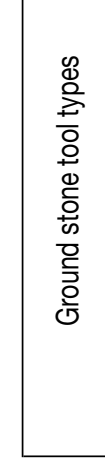 & 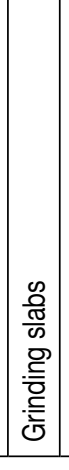 & & & & & & & 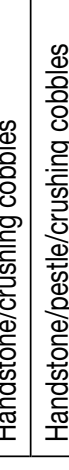 & & 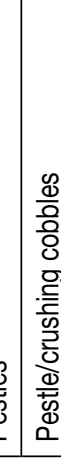 & 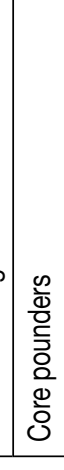 & & 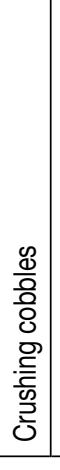 & 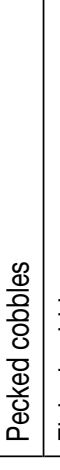 & & & 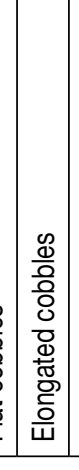 & & 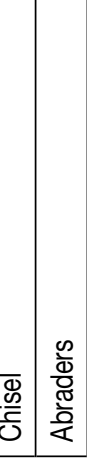 & 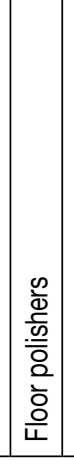 & 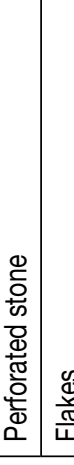 & 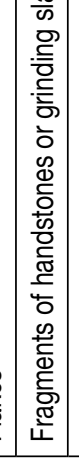 & 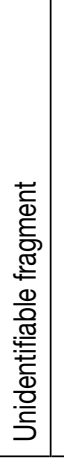 & 袬 \\
\hline
\end{tabular}



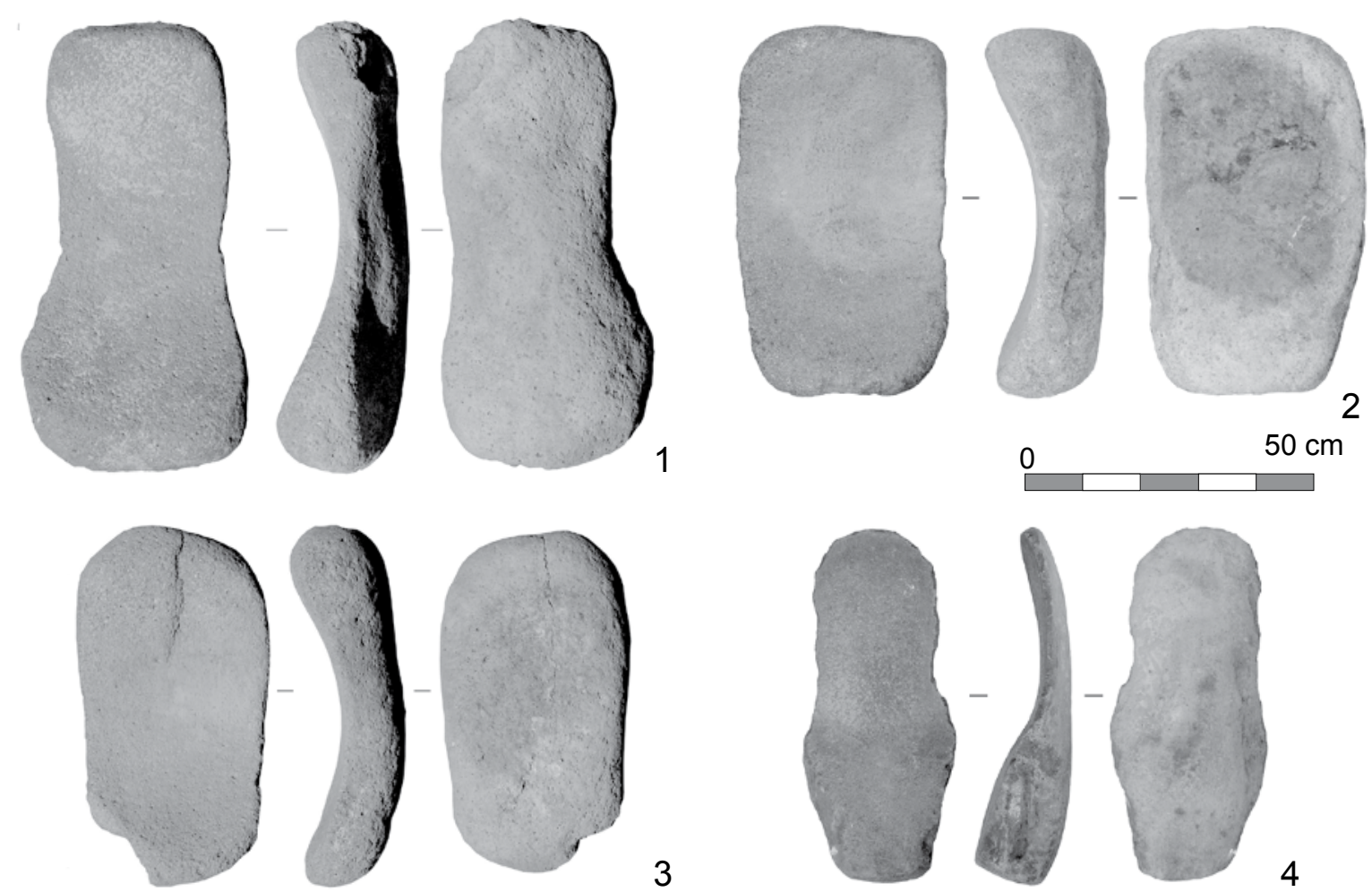

3
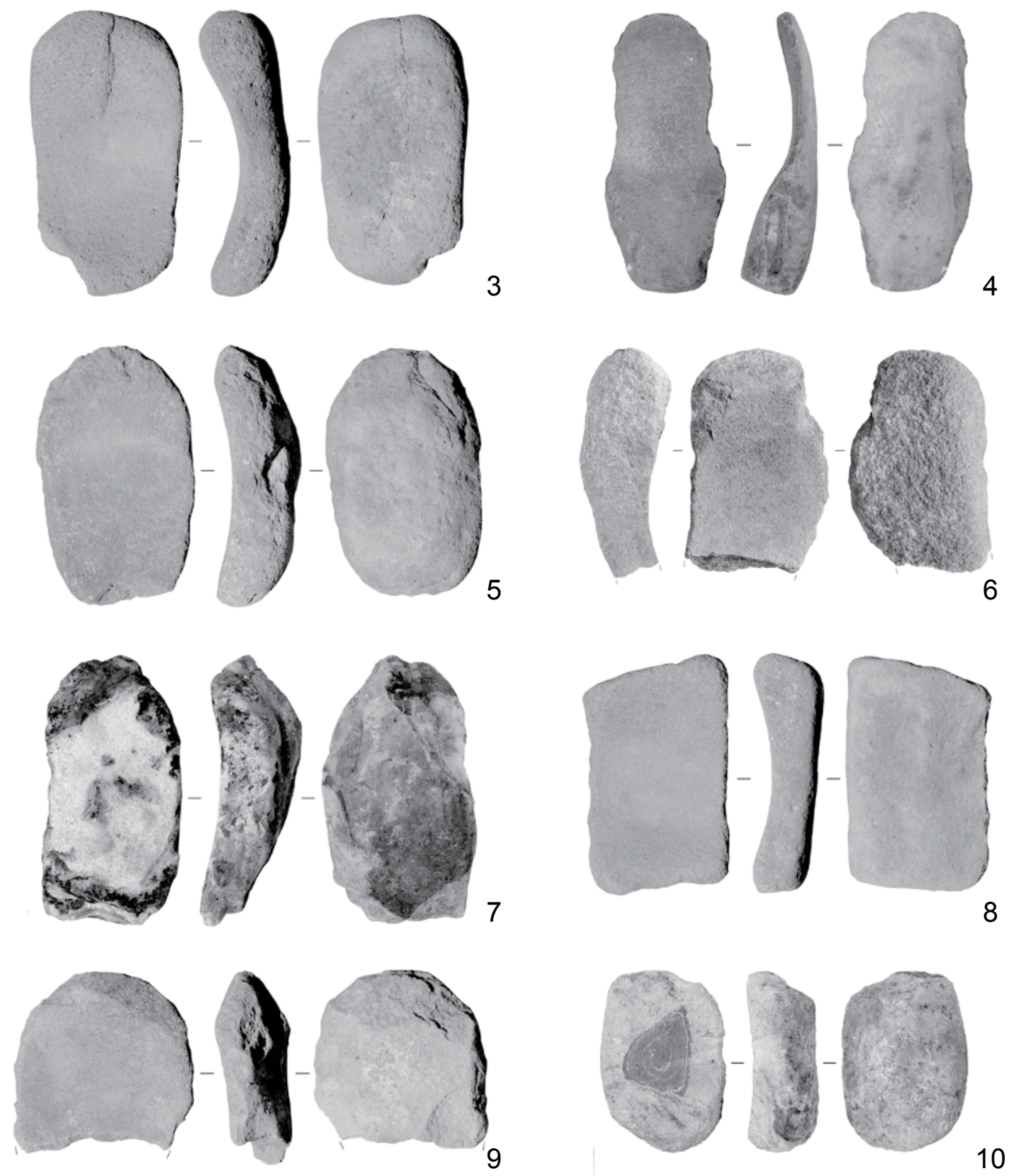

Fig. 12.1 Grinding slabs from Göytepe. 


\begin{tabular}{|c|c|c|c|c|c|c|c|}
\hline & Area & Context & Level & Type & Subtype & $\begin{array}{c}\text { Raw } \\
\text { material }\end{array}$ & Notes \\
\hline 1 & $\begin{array}{l}1 \mathrm{~A}, 1 \mathrm{~B}, \\
2 \mathrm{~A}, 2 \mathrm{~B}\end{array}$ & & $1-4$ & $\begin{array}{l}\text { Grinding } \\
\text { slab }\end{array}$ & $\begin{array}{l}\text { Saddle- } \\
\text { shaped }\end{array}$ & Andesite & \\
\hline 2 & $4 \mathrm{~A}$ & & $5-6$ & $\begin{array}{l}\text { Grinding } \\
\text { slab }\end{array}$ & $\begin{array}{l}\text { Saddle- } \\
\text { shaped }\end{array}$ & Andesite & Longitudinal striations on the working surface \\
\hline 3 & $\begin{array}{l}1 \mathrm{~A}, 1 \mathrm{~B} \\
2 \mathrm{~A}, 2 \mathrm{~B}\end{array}$ & & $1-4$ & $\begin{array}{l}\text { Grinding } \\
\text { slab }\end{array}$ & $\begin{array}{l}\text { Saddle- } \\
\text { shaped }\end{array}$ & Andesite & Longitudinal striations on the working surface \\
\hline 4 & $4 \mathrm{~A}$ & & $5-6$ & $\begin{array}{l}\text { Grinding } \\
\text { slab }\end{array}$ & $\begin{array}{l}\text { Saddle- } \\
\text { shaped }\end{array}$ & Basalt & Longitudinal striations on the working surface \\
\hline 5 & $\begin{array}{l}1 \mathrm{~A}, 1 \mathrm{~B} \\
2 \mathrm{~A}, 2 \mathrm{~B}\end{array}$ & & $1-4$ & $\begin{array}{l}\text { Grinding } \\
\text { slab }\end{array}$ & $\begin{array}{l}\text { Saddle- } \\
\text { shaped }\end{array}$ & Andesite & \\
\hline 6 & 4BIIX & 92 & 10 & $\begin{array}{l}\text { Grinding } \\
\text { slab }\end{array}$ & $\begin{array}{l}\text { Saddle- } \\
\text { shaped }\end{array}$ & Andesite & \\
\hline 7 & $1 \mathrm{Al}$ & & 3 & $\begin{array}{l}\text { Grinding } \\
\text { slab }\end{array}$ & $\begin{array}{l}\text { Saddle- } \\
\text { shaped }\end{array}$ & $\begin{array}{l}\text { Weathered } \\
\text { andesite }\end{array}$ & $\begin{array}{l}\text { Longitudinal striations on the working surface. Dark areas are } \\
\text { natural reddish, probably oxidized, portions of the rock. }\end{array}$ \\
\hline 8 & $\begin{array}{l}1 \mathrm{~A}, 1 \mathrm{~B}, \\
2 \mathrm{~A}, 2 \mathrm{~B}\end{array}$ & & $1-4$ & $\begin{array}{l}\text { Grinding } \\
\text { slab }\end{array}$ & $\begin{array}{l}\text { Saddle- } \\
\text { shaped }\end{array}$ & Andesite & Longitudinal striations on the working surface \\
\hline 9 & $\begin{array}{l}1 \mathrm{~A}, 1 \mathrm{~B}, \\
2 \mathrm{~A}, 2 \mathrm{~B}\end{array}$ & & $1-4$ & $\begin{array}{l}\text { Grinding } \\
\text { slab }\end{array}$ & $\begin{array}{l}\text { Saddle- } \\
\text { shaped }\end{array}$ & Andesite & Pigment residues on the working surface \\
\hline 10 & $1 \mathrm{Al}$ & & 3 & $\begin{array}{l}\text { Grinding } \\
\text { slab }\end{array}$ & $\begin{array}{l}\text { Saddle- } \\
\text { shaped }\end{array}$ & $\begin{array}{l}\text { Weathered } \\
\text { andesite }\end{array}$ & $\begin{array}{l}\text { Longitudinal striations on the working surface. Dark areas are } \\
\text { natural reddish, probably oxidized, portions of the rock. }\end{array}$ \\
\hline
\end{tabular}

Fig. 12.1 Descriptions.

and ground, showing longitudinal striations in two cases (4BI-51 and 97G-4). The piece from 4BI-51 was shaped by flaking on the sides.

Notes: Grinding querns are characterized by the shallowly dished working surfaces. The samples are too few and fragmentary for further classification. This type may correspond to saddle-shaped querns and/or ovoid querns in Hamon's type list (Hamon 2008: 93-94).

\subsubsection{Working slab (Fig. 12.3)}

Raw material: Basalt slab.

Morphology: Discoidal.

Modification traces: The discoidal form is created by unifacial flaking around the periphery of the slab. The un-flaked surface is flat and smooth, showing no clear traces of grinding.

Notes: Although this type corresponds to grinding slabs in Hamon's type list (Hamon 2008: 97), it is called working slab here due to the absence of traces of grinding.

\subsubsection{Mortars (Fig. 12.4)}

Raw material: Mainly andesite boulders.

Morphology: Quadrangular in plan. Near the center of the top surface is located a round hole with its diameter of $6-17 \mathrm{~cm}$.

Modification traces: The hollow working surfaces often show pecking scars at the bottom and are ground near the opening. The side and the back of the mortars are ground with occasional flaking scars.

Note: This type corresponds to boulder mortars (No. 17) in Wright's type list (Wright 1992: 66) and mortars in Hamon's (Hamon 2008: 96).

\subsubsection{Grinders (Figs. 12.5 and 12.6)}

Raw material: Mainly andesite with some basalt boulders.

Morphology: Plan forms are most frequently loafshaped $(n=20)$ and rarely quadrangular $(n=2)$. Both forms are elongated with a length/width ratio ranging from 1.83 to 2.55 for the loaf form and that of 1.82 to 2.03 for the quadrangular shape. The plano-convex cross-sectional form often occurs in association with the unifacial working surface $(n=18)$, which are more numerous than the bifacial working surface $(n=8)$. The distinction between short and flat type (Hamon 2008: 94) could not be made in the Göytepe specimens.

Modification traces: Macroscopic striations on the working surface are transversally oriented. The side and the back of the tools are smooth, showing pecking scars.

Note: Grinders can be distinguished from handstones 

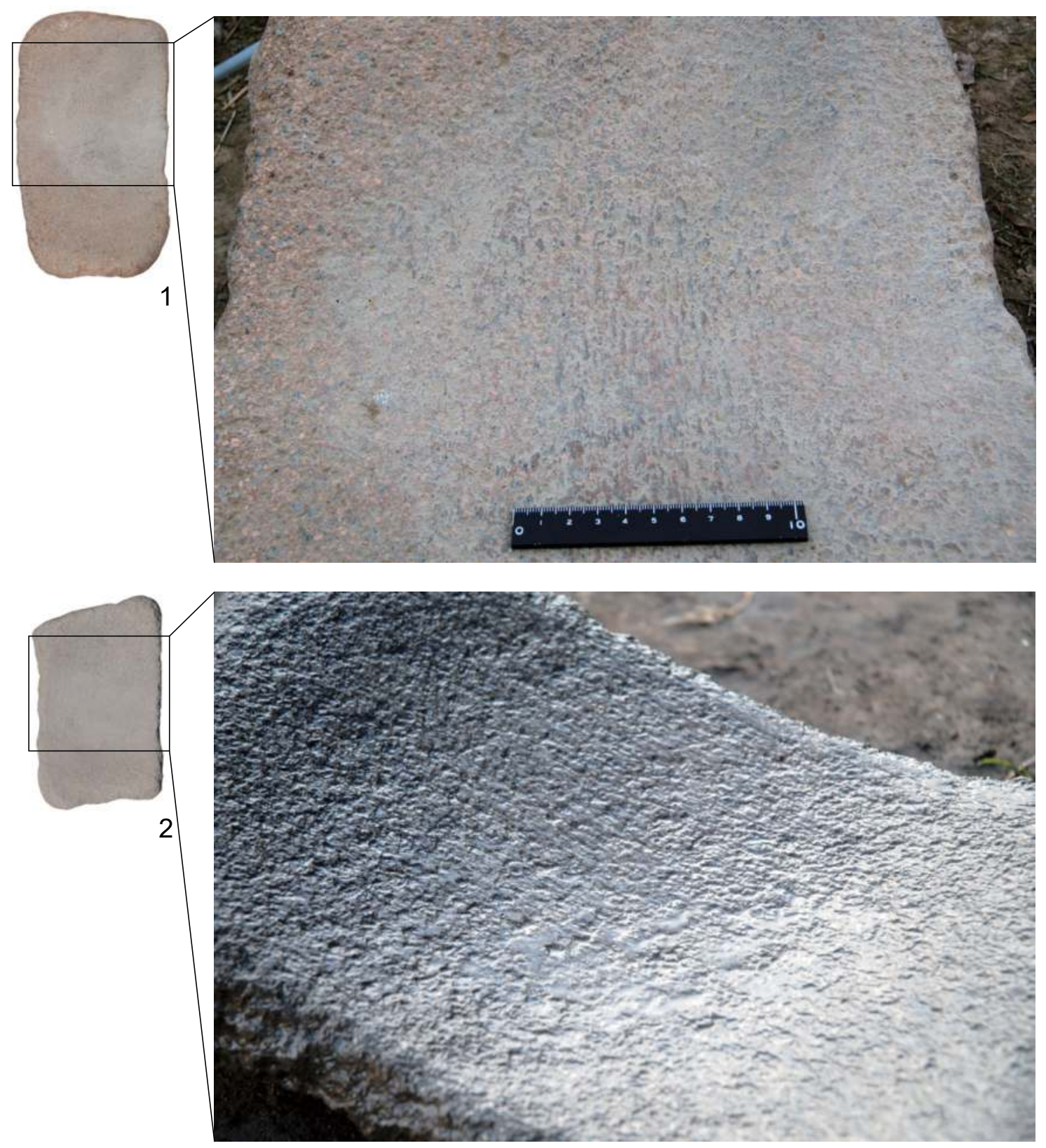

Fig. 12.2 Striations on the working surfaces of grinding slabs. 1: Fig. 12.1: 2; 2: Fig. 12.1: 8.

by their larger size (Fig. 12.7), the dominance of loaf-shaped plan form (Table 12.3), and the greater preference of andesite as raw material for grinders (Table 12.2). This type corresponds to grinders in Hamon's type list (Hamon 2008: 94). The size-frequency distribution of the length of complete grinders overlaps with that of the width of grinding slabs (Fig. 12.7), indicating that grinders were set across the working surface of the grinding slabs to be used in the back-and-forth grinding motion. This idea is also supported by the fact that observable striations on the working surfaces of grinders are oriented transversal to their long axis (Figs. 12.5 and 12.6), while those of grinding slabs are in parallel to their long axes (Fig. 12.2).

\subsubsection{Handstones (Fig. 12.8)}

Raw material: Basalt or andesite cobbles.

Morphology: In contrast to grinders, ovate forms with the length/width ratio between 1.19 and 1.68 are more numerous $(n=20)$ than loaf forms $(n=10)$, which is defined by the length/width ratio between 1.87 and 2.50 (Table 12.3). There 


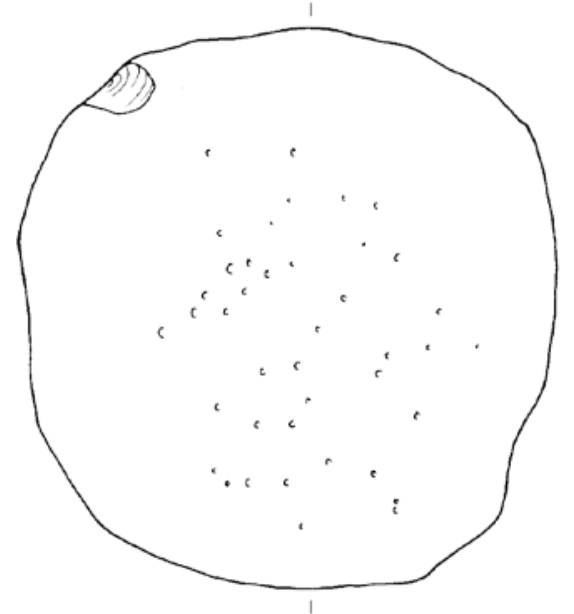

$\therefore$ Pecking or battering scars
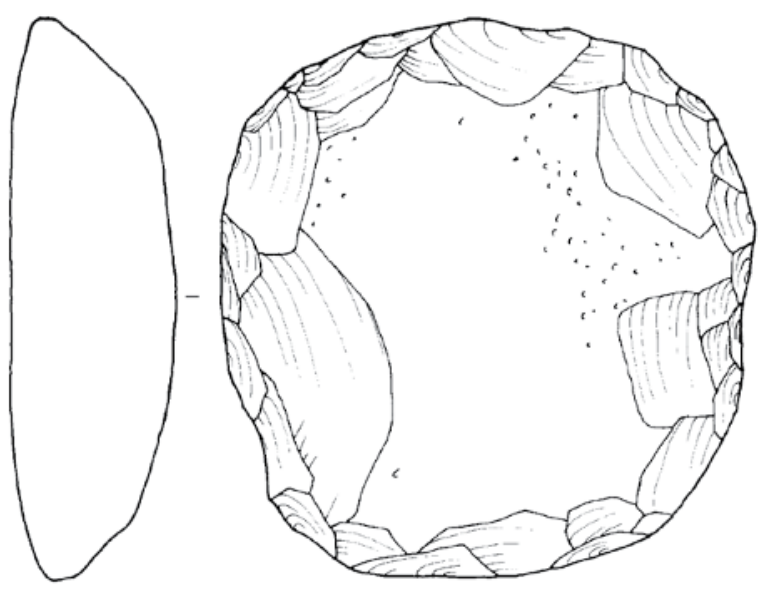

0

$10 \mathrm{~cm}$

\begin{tabular}{|c|c|c|c|c|c|}
\hline & Area & Context & Level & Type & Raw material \\
\hline 1 & $3 \mathrm{BI}$ & 1 & topsoil & Working slab & Basalt \\
\hline
\end{tabular}

Fig. 12.3 Working slab from Göytepe.
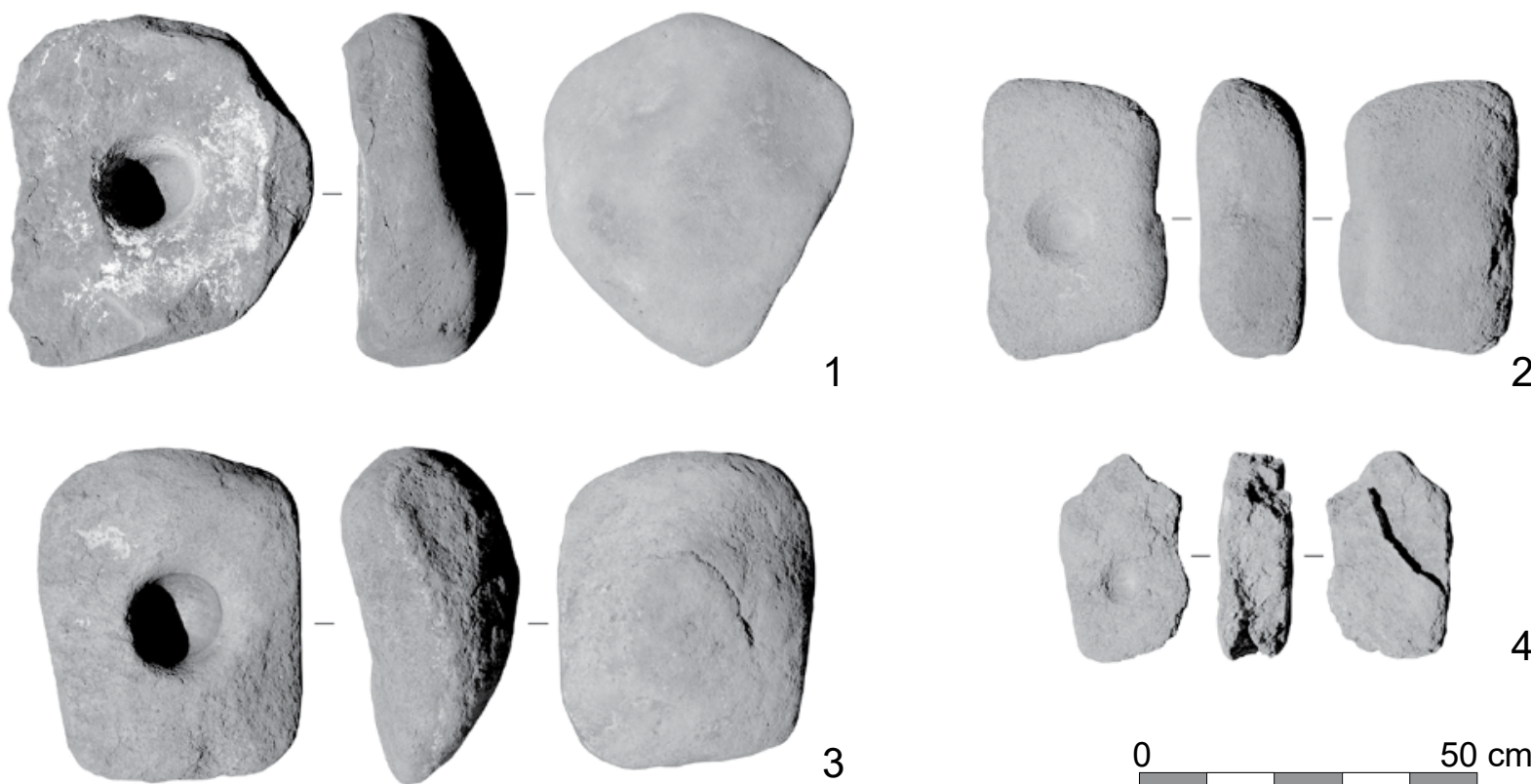

3

0

$50 \mathrm{~cm}$

\begin{tabular}{|c|c|c|c|c|c|c|c|l|}
\hline & Area & Context & Level & Type & Subtype & $\begin{array}{c}\text { Raw } \\
\text { material }\end{array}$ & $\begin{array}{c}\text { Depression size } \\
\text { (diameter } \times \text { depth) }\end{array}$ & $\begin{array}{c}\text { Use and/or maintenance } \\
\text { traces in the depression }\end{array}$ \\
\hline 1 & $1 \mathrm{~A}, 1 \mathrm{~B}, 2 \mathrm{~A}, 2 \mathrm{~B}$ & & $1-4$ & Mortar & Boulder & Andesite & $14.5-15 \times 11.3 \mathrm{~cm}$ & Pecked and ground surfaces \\
\hline 2 & $1 \mathrm{~A}, 1 \mathrm{~B}, 2 \mathrm{~A}, 2 \mathrm{~B}$ & & $1-4$ & Mortar & Boulder & Andesite & $10.5 \times 3.5 \mathrm{~cm}$ & Pecking scars \\
\hline 3 & $1 \mathrm{~A}, 1 \mathrm{~B}, 2 \mathrm{~A}, 2 \mathrm{~B}$ & & $1-4$ & Mortar & Boulder & Andesite & $16-17 \times 11 \mathrm{~cm}$ & Pecked and ground surfaces \\
\hline 4 & $1 \mathrm{~A}, 1 \mathrm{~B}, 2 \mathrm{~A}, 2 \mathrm{~B}$ & & $1-4$ & Mortar & Boulder & Andesite & $6 \times 2.1 \mathrm{~cm}$ & Pecking scars \\
\hline
\end{tabular}

Fig. 12.4 Mortars from Göytepe. 


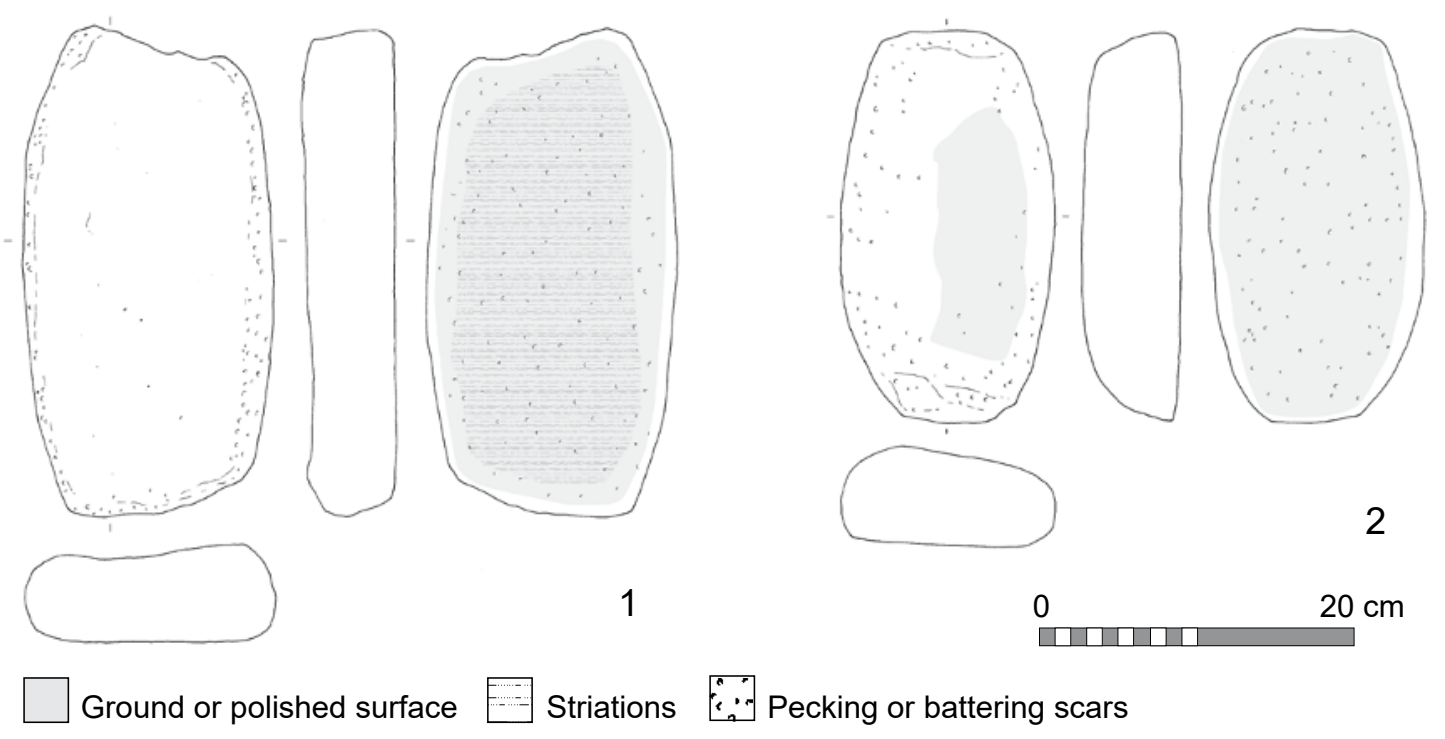

\begin{tabular}{|c|c|c|c|c|c|c|}
\hline & Area & Context & Level & Type & Subtype & Raw material \\
\hline 1 & $4 \mathrm{BIIX}$ & 13 & 9 & Grinder & Quadrangular, unifacial & Andesite \\
\hline 2 & $2 \mathrm{AI}$ & 1 & $4 ?$ & Grinder & Quadrangular, unifacial & Andesite \\
\hline
\end{tabular}

Fig. 12.5 Grinders from Göytepe. 1 and 2: Quadrangular unifacial.

are two pieces of quadrangular forms that are also elongated with the length/width ratio around 2 . Bifacial working surfaces $(n=34)$ occurs more frequently than unifacial ones $(n=15)$.

Modification traces: Macroscopic striations on the working surface are transversally oriented. The sides and the back of the tools are smooth, showing pecking scars.

Notes: This type corresponds to handstones in Wright's type list (Wright 1992: 67) and hand stones in Hamon's (Hamon 2008: 102). Like grinders, handstones of Göytepe are also likely to have been used as upper grinding tools on grinding slabs given their use-wears, i.e., ground surfaces and transversal striations. On the other hand, handstones are smaller than grinders (Fig. 12.7) and show traces of other uses, such as pounding. The handstones with traces of several different uses are classed as multiple tools, including handstone/pestle, handstone/crushing cobble, and handstone/pestle/crushing cobble (Table 12.1).

\subsubsection{Pestles (Fig. 12.9)}

Raw material: Basalt or andesite cobbles.

Morphology: Plan forms are cylindrical $(n=3)$, conical $(n=3)$, or rectangular $(n=1)$ with one unidentifiable piece. The plan forms are not correlated with the number of used ends as five pestles are bipolar and two pieces are unipolar.

Modification traces: Pecking and occasional flaking scars indicate the used ends, while the lateral surfaces are often ground.

Notes: This type corresponds to pestles in Wright's (Wright 1992: 69) and Hamon's type list (Hamon 2008: 97). Two pieces from Göytepe have pecked depressions on the side, indicating their re-use as crushing cobbles (Fig. 12.9: 3; cf. Hamon 2008: 97). One basalt pestle (4BI-56) may be a re-use of a broken axe (Fig. 12.9: 2).

\subsubsection{Crushing cobbles (Fig. 12.10)}

Raw material: Basalt or weathered andesite cobbles. Morphology: Discoidal or ovate in the plan form. Two parallel flat surfaces create oval or quadrangular cross-sectional forms.

Modification traces: A pecked depression on the flat surface forms a working area. Three pieces have a pecked depression on each of the flat surfaces (bifacial), while one piece is unifacially worked.

Notes: This type corresponds to crushing cobble in Hamon's type list (Hamon 2008: 100). 
12.2.9 Core pounders (Fig. 12.11 and 12.19)

Raw material: Mostly basalt cobbles $(\mathrm{n}=108)$ with some andesite $(\mathrm{n}=2)$ or flint $(\mathrm{n}=1)$ cobbles.

Morphology: A wide range of forms, including spherical, polyhedral, triangular, and tabular, depending on the natural shapes of cobbles.

Modification traces: Angular edges, created by unifacial or bifacial flaking, are battered and also often ground.

Notes: This type is included in hammer stones in Hamon's type list (Hamon 2008: 99) and corresponds to irregular core pounders (No. 75) and spherical/irregular pounders (No. 76) in Wright's type list (Wright 1992: 70) although there are differences in raw material selections.

12.2.10 Pounders (Fig. 12.11 and 12.19)

Raw material: Mostly basalt cobbles $(n=23)$ with two pieces of flint cobbles.

Morphology: A variety of forms, depending on the natural shapes of cobbles, are mostly unstandardized polyhedrons and rarely spherical, discoidal, or triangular.

Modification traces: Pounders show clear hammering or pecking scars covering more than a quarter of the surface. The absence of flaking scars makes this type distinct from core pounders.

Notes: This type is included in hammer stones in Hamon's type list (Hamon 2008: 99) and spherical/irregular pounders (No. 76) in Wright's type list (Wright 1992: 70) although there are differences in raw material selections.

\subsubsection{Pecked cobbles}

Raw material: Mostly basalt cobbles $(\mathrm{n}=8)$ with a few pieces of other rocks.

Morphology: Ovoid.

Modification traces: Hammering and pecking scars are restricted to less than a quarter of the surface.

Notes: This type is included in hammer stones in Hamon's type list (Hamon 2008: 99) and corresponds to pecked cobbles/pebbles (No. 85) in Wright's type list (Wright 1992: 70).

12.2.12 Flaked cobbles (Fig. 12.11)

Raw material: Mostly basalt cobbles $(n=17)$.
Morphology and modification traces: Irregular, depending on the natural forms of cobbles. Unifacial and occasionally bifacial flaking creates the overall shapes like choppers or chopperchopping tools although no macroscopic wears are visible on the edges.

Notes: This type may correspond to miscellaneous flaked choppers (No. 98), flaked cobbles/pebbles (No. 86), or flake cores (No. 142) in Wright's type list (Wright 1992: 71).

\subsubsection{Ground cobbles}

Raw material: Mostly basalt cobbles ( $\mathrm{n}=8)$.

Morphology: Irregular or spherical.

Modification traces: The surface is smooth, showing the traces of grinding to a varying degree.

Notes: This type corresponds to ground cobbles/ pebbles (No. 83) and ground spheres (No. 84) in Wright's type list (Wright 1992: 71).

\subsubsection{Flat cobbles (Fig. 12.12)}

Raw material: Raw material types are unidentifiable for more than half $(n=10)$ due to weathered surfaces, while identified pieces are all made of basalt ( $\mathrm{n}=4)$.

Morphology: All the pieces are discoidal with two flat surfaces in parallel, except for two pieces (4BI114BB and 3BI-1) showing slightly concave surfaces (Fig. 12.12: 5 and 7).

Modification traces: The flat surfaces are smooth, but there are no clear traces of modification or ochre.

Notes: The form is similar to palettes in Hamon's type list (Hamon 2008: 103), but the flat cobbles from Göytepe show no macroscopic signs of use, such as striations or ochre.

\subsubsection{Elongated cobbles (Fig. 12.12)}

Raw material: Identifiable pieces are all made of basalt $(n=4)$.

Morphology: Cobbles of cylindrical or long ovoid forms.

Modification traces: Two pieces (4BII-43 and 4BIIX88) show pecking and flaking scars at opposed ends, probably corresponding to splintered tools in Hamon's type list (Hamon 2008: 105). A single piece (4BIIX-95) shows pecking and 

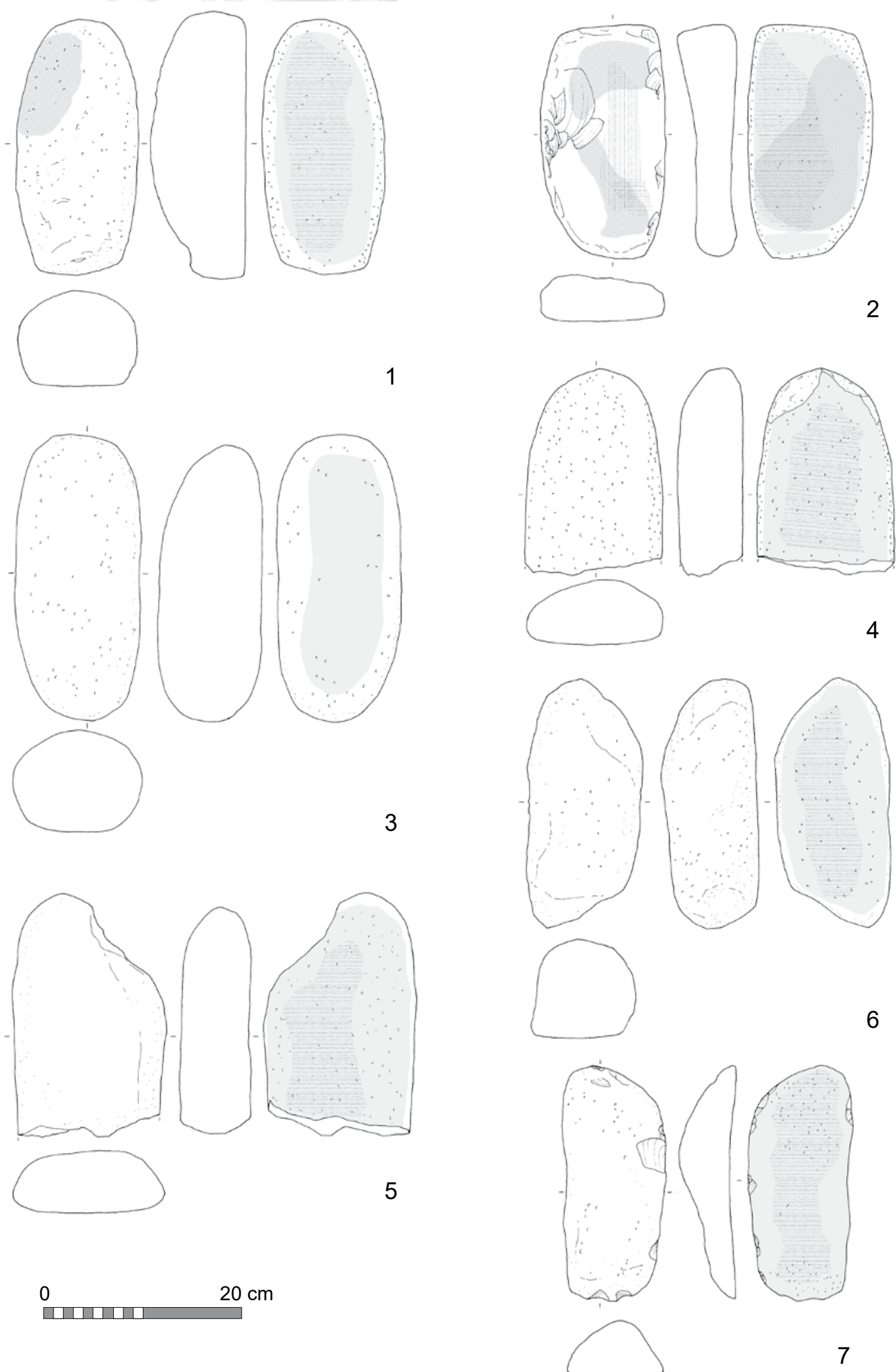

Ground or polished surface E Striations $\because$ Pecking or battering scars

Fig. 12.6 Grinders from Göytepe. 1-7: Loaf unifacial. 


\begin{tabular}{|c|c|c|c|c|c|c|l|}
\hline & Area & Context & Level & Type & Subtype & Raw material & Notes \\
\hline 1 & $4 \mathrm{BIIX}$ & $94 \mathrm{~b}$ & 10 & Grinder & Loaf, unifacial & Rhyolite porous lava & $\begin{array}{l}\text { Stored in the clay bin (4BIIX-94). Sediments on the } \\
\text { working surface were analyzed for phytoliths (See } \\
\text { Chapter 7 and Kadowaki et al. 2015). A photo of this } \\
\text { piece in shown in Fig. 12.18: 1. }\end{array}$ \\
\hline 2 & $4 \mathrm{BIIX}$ & $92 \mathrm{a}$ & 10 & Grinder & Loaf, bifacial & Weathered andesite & $\begin{array}{l}\text { Re-used as a lower grinding stone. A photo of this } \\
\text { piece is shown in Fig. 12.18: 2. }\end{array}$ \\
\hline 3 & $4 \mathrm{BI}$ & 34 & 5 & Grinder & Loaf, unifacial & Andesite & \\
\hline 4 & $4 \mathrm{BI}$ & 37 & 7 & Grinder & Loaf, unifacial & Andesite & \\
\hline 5 & $4 \mathrm{BI}$ & $128 \mathrm{FF}$ & 11 & Grinder & Loaf, unifacial & Andesite & \\
\hline 6 & $4 \mathrm{BII}$ & 45 & 7 & Grinder & Loaf, unifacial & Andesite & \\
\hline 7 & $3 \mathrm{~B} 1$ & 1 & mixed & Grinder & Loaf, unifacial & Weathered andesite & \\
\hline
\end{tabular}

Fig. 12.6 Descriptions.

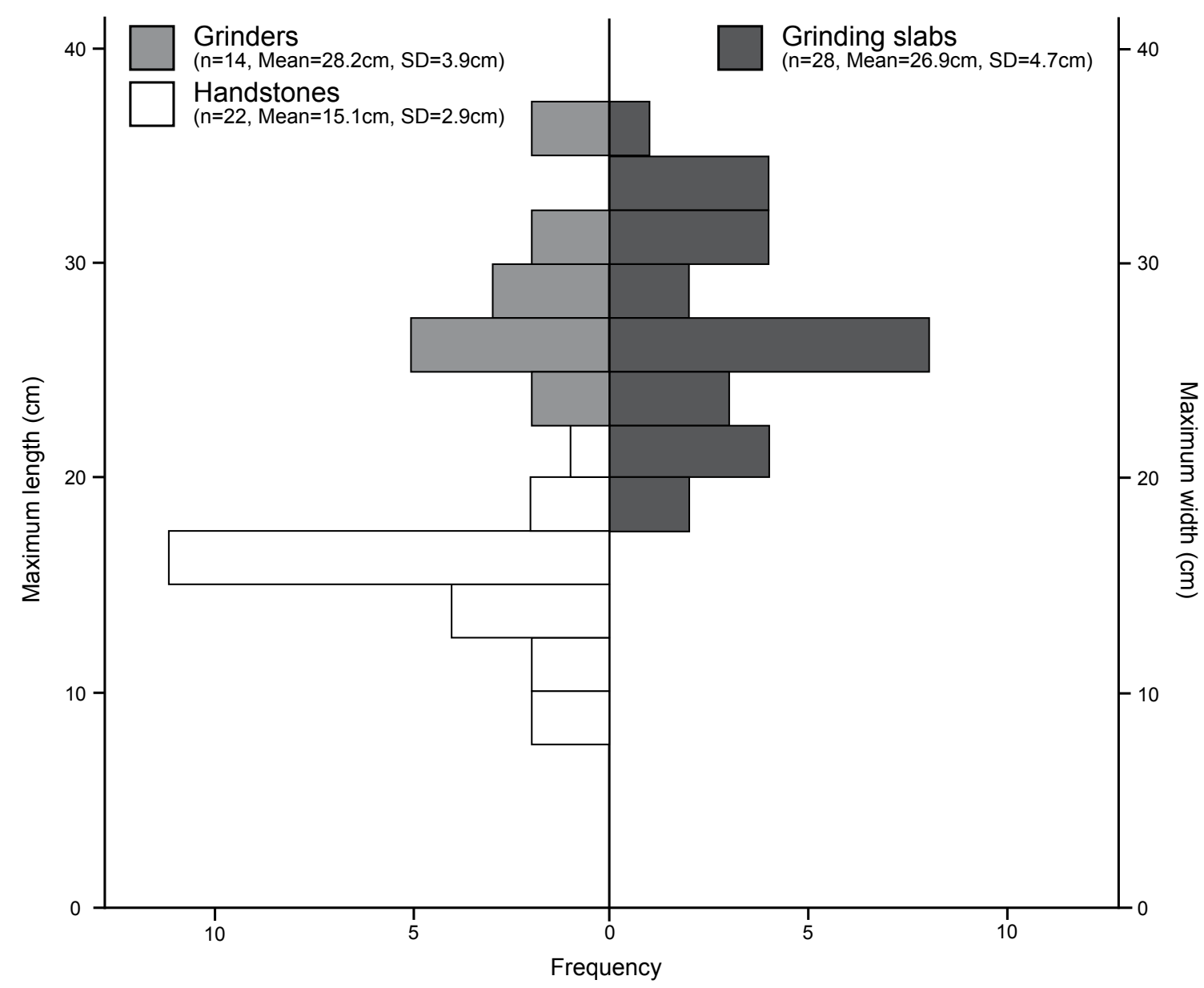

Fig. 12.7 Size-frequency distributions of complete grinders and handstones in comparison with grinding slabs.

flaking scars only at one end. Other pieces have no macroscopic traces of production or use.

\subsubsection{Pebbles (Fig. 12.13)}

Raw material: An assemblage from a cache found at $4 \mathrm{BI}-40(\mathrm{n}=38)$ were examined for raw materials.
More than half $(n=21)$ are andesite, while the rest consist of various raw materials, including rhyolite, tuff breccia, sandstone, and mudstone.

Morphology: Unstandardized ovoid forms of unmodified pebbles.

Modification traces: No clear traces of production or 
Table 12.3 Frequencies of plan forms of grinders and handstones.

\begin{tabular}{|c|c|c|c|c|c|c|}
\hline \multirow{3}{*}{ Plan forms } & \multicolumn{3}{|c|}{ Grinders } & \multicolumn{3}{|c|}{ Handstones } \\
\hline & \multirow[t]{2}{*}{$\mathbf{n}$} & \multicolumn{2}{|c|}{$\begin{array}{l}\text { Length/width ratio of } \\
\text { complete pieces }\end{array}$} & \multirow[t]{2}{*}{$\mathbf{n}$} & \multicolumn{2}{|c|}{$\begin{array}{l}\text { Length/width ratio of } \\
\text { complete pieces }\end{array}$} \\
\hline & & Mean & $1 \sigma$ & & Mean & $1 \sigma$ \\
\hline Loaf & 20 & 2.17 & 0.25 & 10 & 2.15 & 0.30 \\
\hline Ovate & 0 & NA & NA & 20 & 1.45 & 0.16 \\
\hline Quadrangular & 2 & 1.93 & 0.15 & 2 & 2.00 & NA \\
\hline Irregular & 0 & NA & NA & 3 & 1.36 & 0.35 \\
\hline Unidentifiable & 4 & NA & NA & 23 & NA & NA \\
\hline TOTAL & 26 & & & 58 & & \\
\hline
\end{tabular}

use are visible.

Notes: Despite the absence of production- or usewears, pieces of this type are included in ground stones because of their frequent occurrences as a cache located near domestic buildings at the site. This type corresponds to sling-stones in Hamon's type list (Hamon 2008: 105).

\subsubsection{Axes (Fig. 12.14)}

Raw material: Mostly basalt $(n=13)$ cobbles with a single piece made of rhyolite porous lava. Two wide axes (2AII-locus 14 and 4BII-25) and one unfinished axe (4BI-1) show positive flaking scars, indicating flakes were used as tool blanks (Fig. 12.14: 9, 10, 13).

Morphology: Three groups, Long, Short, and Wide, are recognizable according to the size and the plan form (Fig. 12.15). Long axes are the largest and most elongated, while Short axes are smaller and have the reduced length/width ratio in comparison with Long axes. Long and Short axes are ovate in plan, being widest at a mid-point between the cutting edge and the butt. Wide axes are broader than the former two types and have a point of maximum width near the cutting edge, assuming trapezoidal plan forms.

Modification traces: All the axes have polished convex cutting-edges that are more or less symmetrical in the profile. The body is covered with pecking scars and occasionally polished facets. Some pieces, particularly Wide axes, show flaking scars from lateral sides. Three pieces are identified as unfinished axes. They are shaped by flaking and pecking to create elongated plan forms, which fall within a range of Long and Short axes. A thinned edge is observable at one end although they are asymmetric in plan and not completely sharpened.

Notes: This type corresponds to azes in Hamon's terminology (Hamon 2008: 102-103). Long and short axes are similar to ovate celts (No. 91) in Wright's type list (Wright 1992: 73), while Wide axes are comparable to trapezoidal celts (No. 90; Wright 1992: 72).

\subsubsection{Chisel (Fig. 12.14)}

Raw material: Basalt.

Morphology: Elongated with parallel lateral sides. The cutting edge is narrower than those of axes.

Modification traces: The cutting edge and the body are all polished.

Notes: This type corresponds to chisels in Hamon's (Hamon 2008: 103) and Wright's type list (No. 92; Wright 1992: 73).

\subsubsection{Abraders (Fig. 12.16)}

Raw material: Andesite porous lava.

Morphology: Four groups of abraders are distinguishable according to their sizes, overall shapes, and the form of grinding surfaces. Large prismatic abraders $(n=2)$ are distinct from the rest by their exceeding size $(>24 \mathrm{~cm})$, and their cross-section is more or less quadrangular. Small prismatic abraders $(n=9)$ is a hand-held size, smaller than $15 \mathrm{~cm}$. Flat concave abraders are characterized by flat cross-sections and concave working surfaces. A single broken piece has an irregular globular form.

Modification traces: Ground facets are observable on both large and small prismatic abraders, some of which have concave ground surfaces and/or shallow incisions, which could be related to their 
use as abraders (Fig. 12.16: 1, 2, 4, and 6). Such a function is also indicated by the ground working surface with striations of flat concave abraders (Fig. 12.16: 7 and 8).

Notes: Small prismatic abraders may correspond to prismatic polishers in Hamon's type list (Hamon 2008: 100), and flat concave abraders may compare to some of handheld polishers (Hamon 2008: 100).

\subsubsection{Floor polishers (Fig. 12.18: 16)}

Raw material: Unidentifiable.

Morphology: Oval to discoidal in plan, and quadrangular in cross-section. One face is shallowly dished with a narrow rim $(3-4 \mathrm{~cm})$, and the other face is either flat or convex.

Modification traces: The concave working surface is ground with no clear striations. The peripheral side and the other face are smoothly ground on one piece (1AII, M, d=1.9 m, locus 14 ), while the other specimen $(1 \mathrm{~A}, 1 \mathrm{~B}, 2 \mathrm{~A}, 2 \mathrm{~B})$ shows extensive pecking scars.

Notes: No tools in Hamon 2008 or Wright 1992 are comparable to this type. A similar type is floor polishers in Adams' type list (Adams 2002: 9496).

12.2.21 Perforated stone (Fig. 12.17: 1)

Raw material: Unidentifiable.

Morphology: A disc $(5.4 \mathrm{~cm}$ in diameter and 3.8 $\mathrm{cm}$ in thickness) with a perforation $(1.5 \mathrm{~cm}$ in diameter)

Modification traces: The disc is worked from two opposed surfaces to create a perforation with a bi-conical section.

Notes: This piece is morphologically similar to loomweights (No. 109) in Wright's type list (Wright 1992: 75) and should be included in perforated objects in Hamon's (Hamon 2008: 105).

12.2.22 Flakes (Fig. 12.17: 2-5)

Raw material: Mostly basalt $(\mathrm{n}=341)$ and rarely andesite $(\mathrm{n}=10)$.

Morphology: Unstandardized.

Modification traces: Unretouched flakes are included in this category, while retouched flakes are classed as chipped stone tools. Three flakes have battered edges.

Notes: This type corresponds to flakes (No. 143) in Wright's type list (Wright 1992: 78).

\subsubsection{Fragments of grinders/handstones or grinding slabs}

Raw material: Identifiable pieces are either andesite or basalt.

Morphology and modification traces: Although the overall shape is unidentifiable, the presence of flat ground surface and pecked side walls indicates that the fragments are from either grinders/handstones or grinding slabs.

Notes: This type corresponds to possible handstones/ grinding slabs (No. 145) in Wright's type list (Wright 1992: 78).

\subsubsection{Unidentifiable fragment}

Raw material: Unidentifiable.

Morphology and modification traces: Despite the presence of ground surface, the piece is too fragmented for the identification of the tool types.

Notes: This type corresponds to unidentifiable ground stone fragments (No. 147) in Wright's type list (Wright 1992: 78).

\subsection{Chaîne opératoir of ground stone technology at Göytepe}

This section presents chaînes opératoires involved in the production, use, and maintenance of ground stones at Göytepe on the basis of the above typology and the observations of production technology as well as inferred functions of the tools. Fig. 12.20 shows a schematic chart of activities related to ground stones, including raw material selection, food processing, craft production, tool maintenance, pigment processing, and other unknown activities. These activities are marked by thick lined rectangles, which contain tool types and raw material stones involved in each of the activities. Some tool types are grouped within thin lined rectangles that indicate inferred roles in the activities, such as worked materials, tools, debitage, lower stones, upper stones, tools to be maintained, and maintaining tools. Arrows 

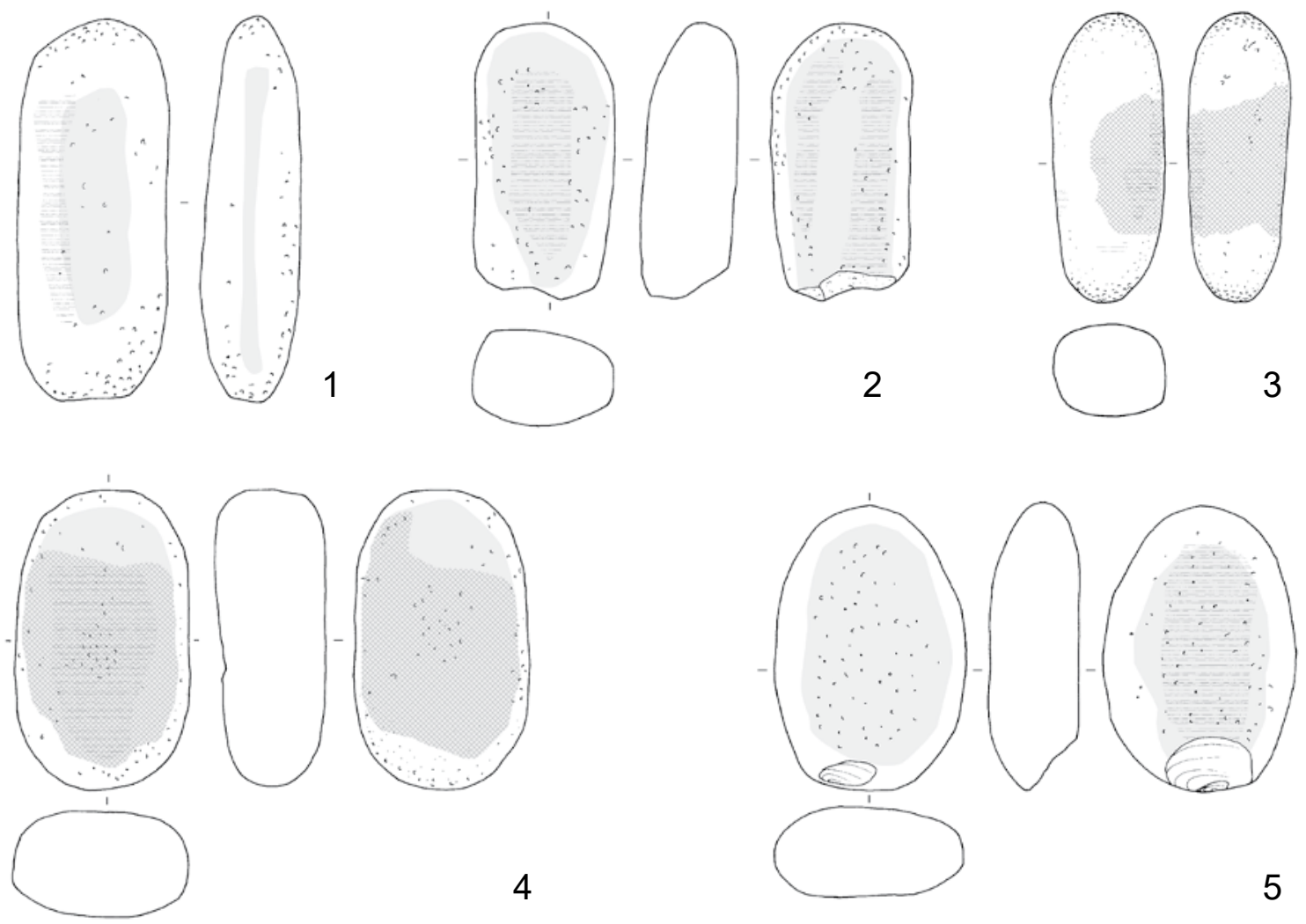

4

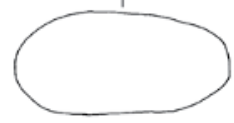

5
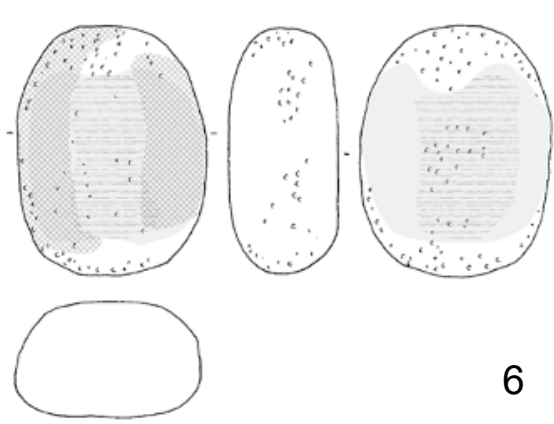

6
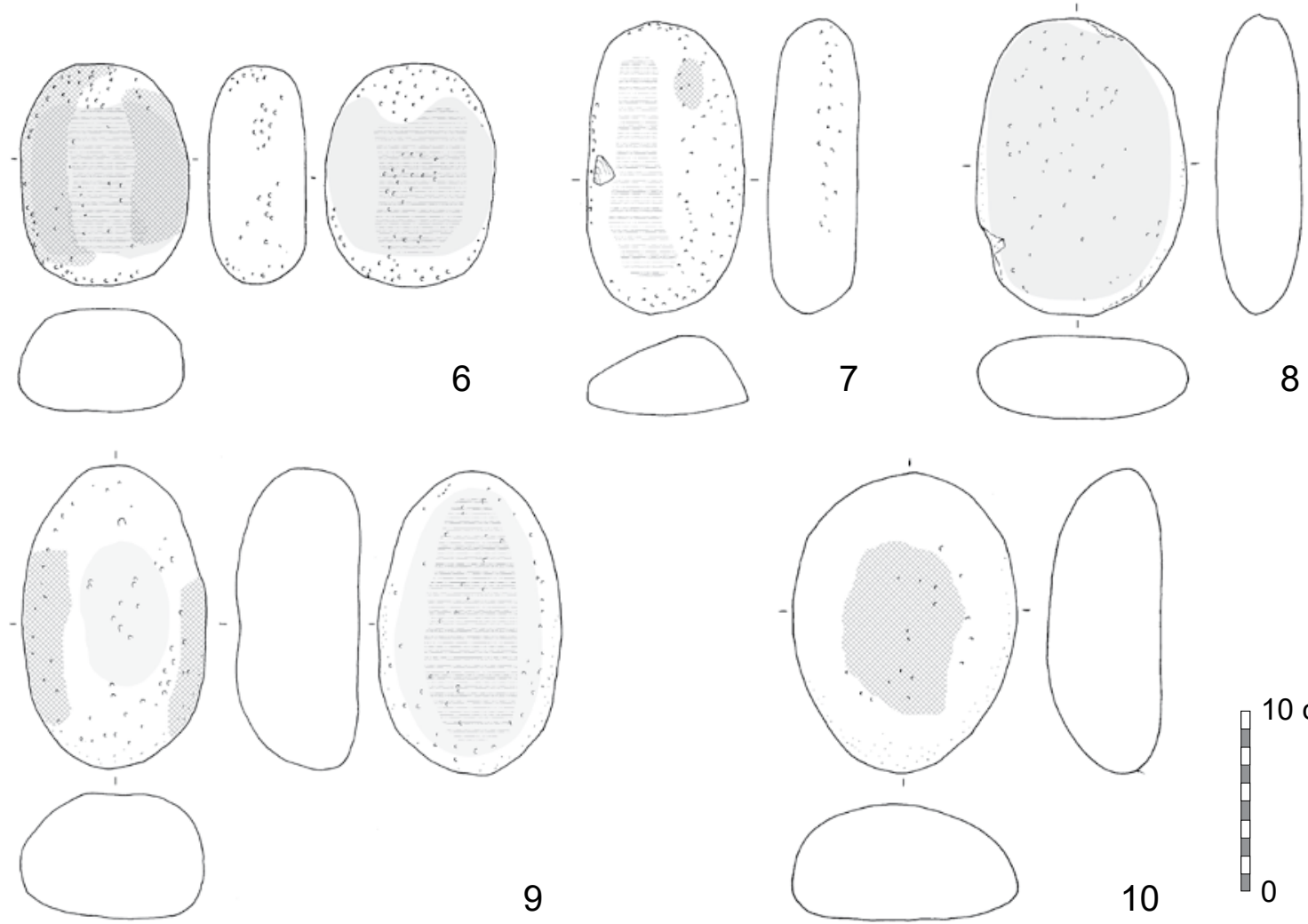

9

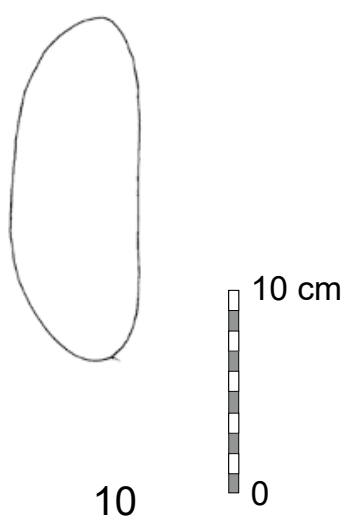

Ground or polished surface $\boxminus$ Striations $\because$ Pecking or battering scars

Fig. 12.8 Handstones from Göytepe. 1 and 2: Loaf bifacial; 3: Loaf unifacial; 4-8: Ovate bifacial; 9 and 10 : Ovate unifacial. 


\begin{tabular}{|c|c|c|c|c|c|c|c|}
\hline & Area & Context & Level & Type & Subtype & Raw material & Notes \\
\hline 1 & 1 All & $\begin{array}{c}\mathrm{N}, \mathrm{d}=1.9 \mathrm{~m}, \\
\text { locus } 14\end{array}$ & $1-4$ & Handstone & Loaf, bifacial & Andesite & \\
\hline 2 & $3 \mathrm{BI}$ & 1 & mixed & Handstone & Loaf, bifacial & Basalt & \\
\hline 3 & $3 \mathrm{All}$ & & $4-5$ & Handstone & Loaf, unifacial & Basalt? & \\
\hline 4 & $4 \mathrm{BIIX}$ & $94 \mathrm{a}$ & 10 & Handstone & Ovate, bifacial & Basalt & $\begin{array}{l}\text { Stored in the clay bin (4BIIX-94). Sediments on the } \\
\text { working surface were analyzed for phytoliths (See } \\
\text { Chapter 7 and Kadowaki et al. 2015). A photo of } \\
\text { this piece is shown in Fig. 12.18: 3. }\end{array}$ \\
\hline 5 & $3 \mathrm{BI}$ & 1 & mixed & Handstone & Ovate, bifacial & Basalt & \\
\hline 6 & $4 \mathrm{BI}$ & 44 & 7 & Handstone & Ovate, bifacial & Andesite & \\
\hline 7 & $1 \mathrm{All}$ & locus 14 & $1-4$ & Handstone & Loaf, bifacial & Unrecorded & \\
\hline 8 & $3 \mathrm{BI}$ & 2 & topsoil & Handstone & Ovate, bifacial & Andesite & \\
\hline 9 & $4 \mathrm{BI}$ & 1 & topsoil & Handstone & Ovate, unifacial & Andesite & \\
\hline 10 & $4 \mathrm{BI}$ & 33 & 5 & Handstone & Ovate, unifacial & Basalt & \\
\hline
\end{tabular}

Fig. 12.8 Descriptions.

in the chart show trajectories of ground stones from raw material selection, through modification and use, to re-use.

A notable structure in the chaînes opératoires of ground stones at Göytepe is three empirical size categories of ground stones. The three size classes, marked by different colours in the chart (Fig. 12.20), differ from each other in the occurrences of raw material types and tool types. The ground stones in the large-size class, ranging from 35 to $71 \mathrm{~cm}$, are mainly made of andesite boulders, and the tool types are all lower stones of food processing tools, such as grinding slabs, grinding querns, and mortars. Andesite is still dominant in the medium-size class, measuring from 23 to $32 \mathrm{~cm}$, but other raw materials occur more frequently than the large-size class. A main tool type of the medium-size class is grinders that were probably used as upper stones in food processing. Another characteristic tool type in the medium class is large prismatic abraders that are exclusively made of andesite porous lava. In contrast to the dominance of andesite in the large and medium classes, basalt is the main raw material type in the small-size group that ranges from 4 to $21 \mathrm{~cm}$ in maximum length, excluding pebbles and flakes. This class includes various tool types that are described below in terms of their roles in the ground stone related activities.

\subsubsection{Raw material selection}

Table 12.2 shows the frequency of raw materials by ground stone tool types at Göytepe. Basalt is dominant for many tool types, including axes, core pounders, flakes, flaked cobbles, ground cobbles, various types of handstones and pestles, pecked cobbles, and pounders. Andesite tends to be used for food processing tools, including grinders, grinding slabs, handstones, handstone/grinding slab fragments, and mortars. Some andesite cobbles and boulders are weathered in white colour with red bands that probably resulted from oxidization (Fig. 12.1: 7 and 10). This type of weathered andesite is also used for food processing tools, such as grinders, grinding querns, and grinding slabs. Weathered andesite is also used for two crushing cobbles. Another type of andesite is porous lava, which is exclusively used for abraders.

Size is an important factor in the raw material selection for ground stone artifacts at Göytepe. Fig. 12.21 shows the distribution of maximum length of complete ground stones, excluding flakes and pebbles, by raw material types. A notable difference is observable in the size-frequency distribution between andesite and basalt tools. Complete tools of andesite are significantly larger than those of basalt. Size is also related to tool types as Fig. 12.22 shows that grinding slabs are the largest, followed by mortars and grinders, and the rest of the tool types are smaller than the former types. Such dimensional patterns in the raw material and tool types can be summarized as the three size categories, i.e., large, medium, and small, of ground stones (Fig. 12.20). Andesite is dominant in the large and medium classes, while basalt is the major raw material in the small group. It is unclear how this pattern in the raw material selection is influenced by natural occurrences of 

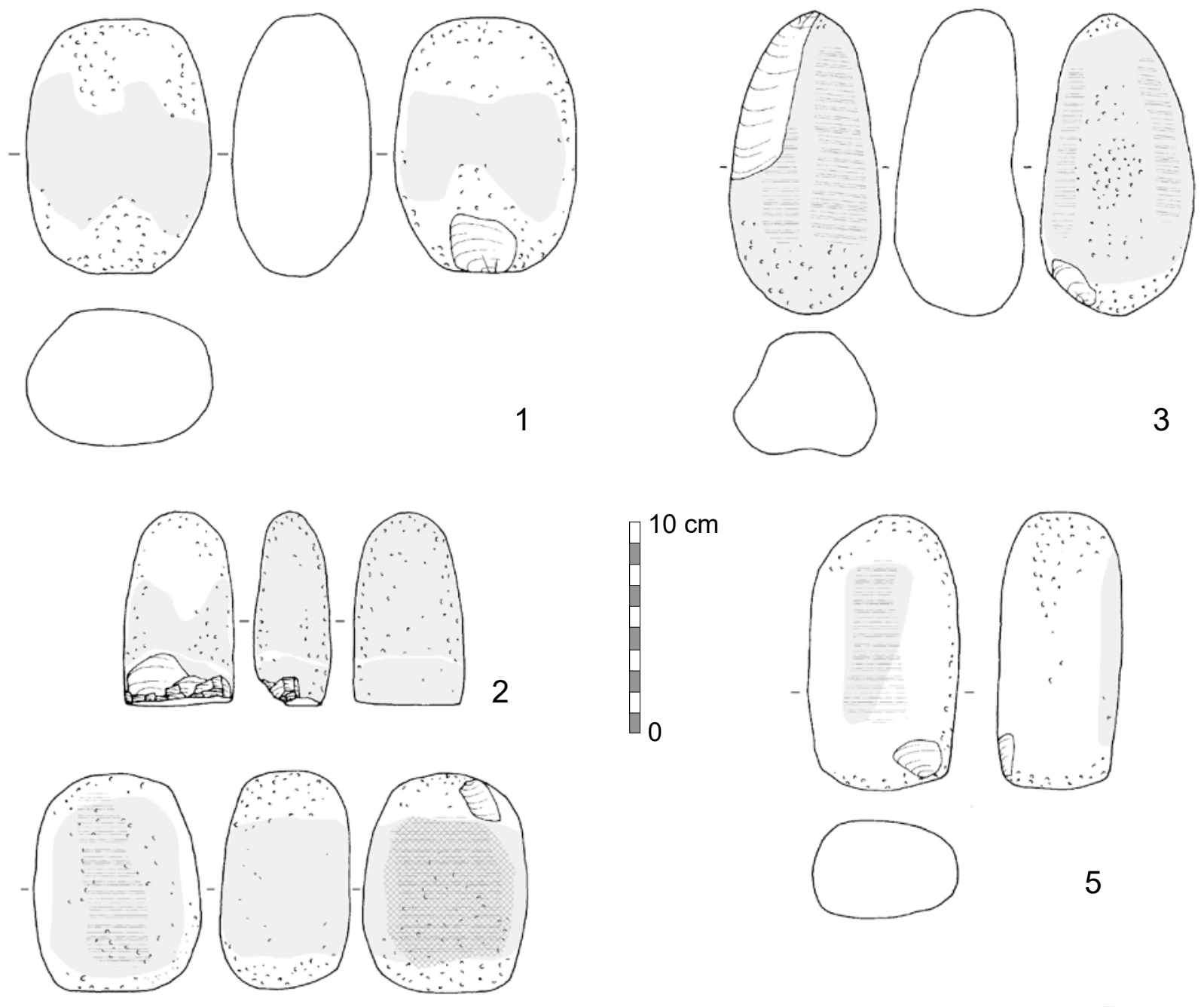

5
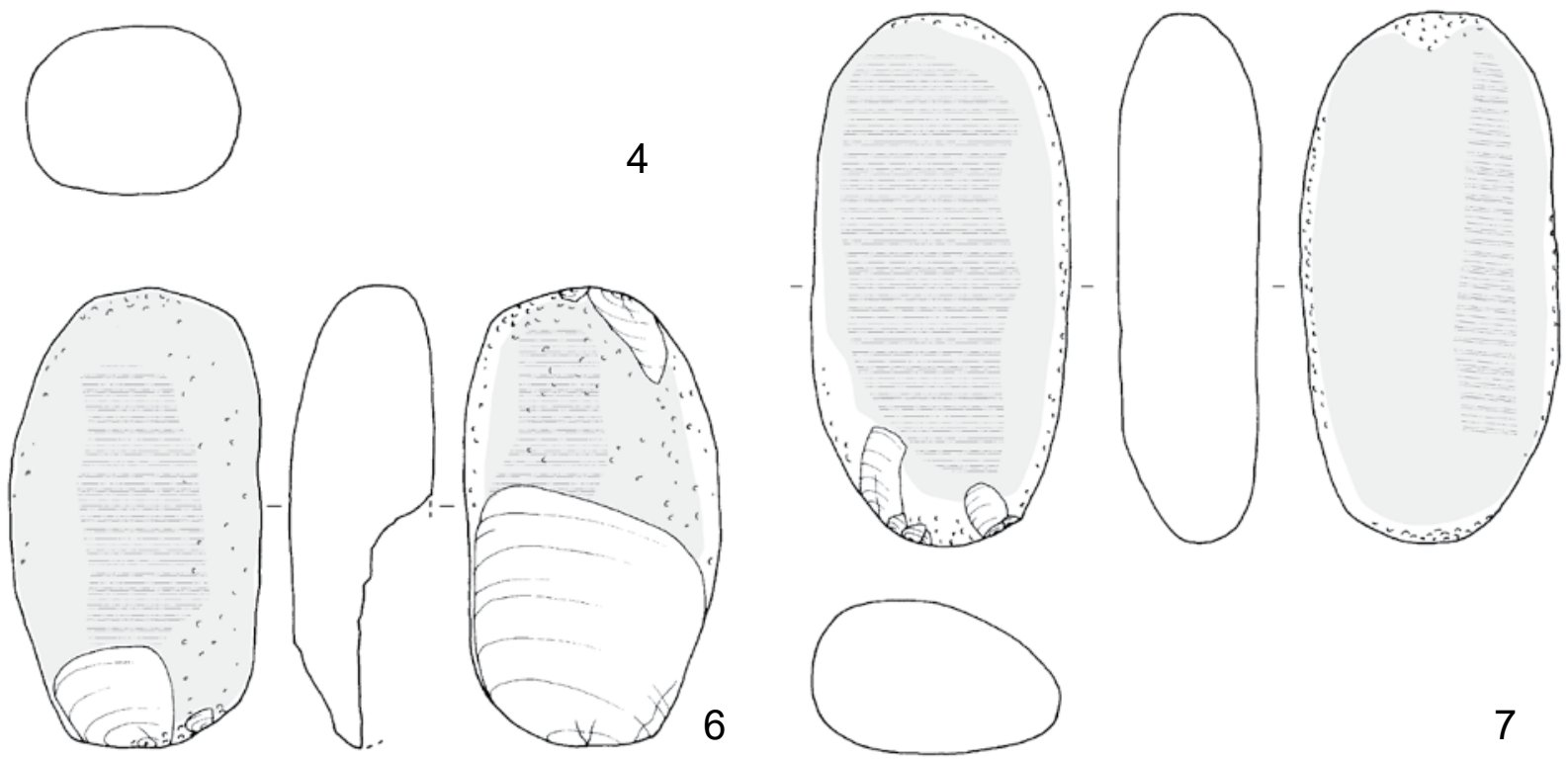

7

Ground or polished surface $\equiv$ Striations

\section{$\therefore$ Pecking or battering scars Ochre stains}

Fig. 12.9 Pestles from Göytepe. 1: Bipolar cylindrical; 2: Unipolar cylindrical, re-use of an broken axe?; 3: Pestle/ crushing cobble; 4-7: Handstone/pestles. 


\begin{tabular}{|c|c|c|c|c|c|c|c|}
\hline & Area & Context & Level & Type & Subtype & Raw material & Notes \\
\hline 1 & 4BI & $131 \mathrm{EE}$ & 11 & Pestle & Bipolar, cylindrical & Basalt & \\
\hline 2 & 4BI & 56 & 7 & Pestle & Unipolar, cylindrical & Basalt & Re-use of an broken axe? \\
\hline 3 & $4 \mathrm{BI}$ & 6 & 5 & $\begin{array}{l}\text { Pestle/crushing } \\
\text { cobble }\end{array}$ & Bipolar, conical & Basalt & $\begin{array}{l}\text { This piece may also have been } \\
\text { used as a handstone. A photo is } \\
\text { shown in Fig. 12.18: } 4 \text {. }\end{array}$ \\
\hline 4 & $4 \mathrm{BI}$ & 1 & topsoil & Handstone/pestle & Rectangular, bifacial, bipolar & Andesite & \\
\hline 5 & 2All & $\mathrm{d}=1.839 \mathrm{~m}$ & $2-4$ & Handstone/pestle & Loaf, bifacial, unipolar & Basalt & \\
\hline 6 & 4BI & 104 & 10 & Handstone/pestle & Ovate, bifacial, bipolar & Basalt & \\
\hline 7 & 4BI & 11 & mixed & Handstone/pestle & Loaf, bifacial, bipolar & Basalt & \\
\hline
\end{tabular}

Fig. 12.9 Descriptions.
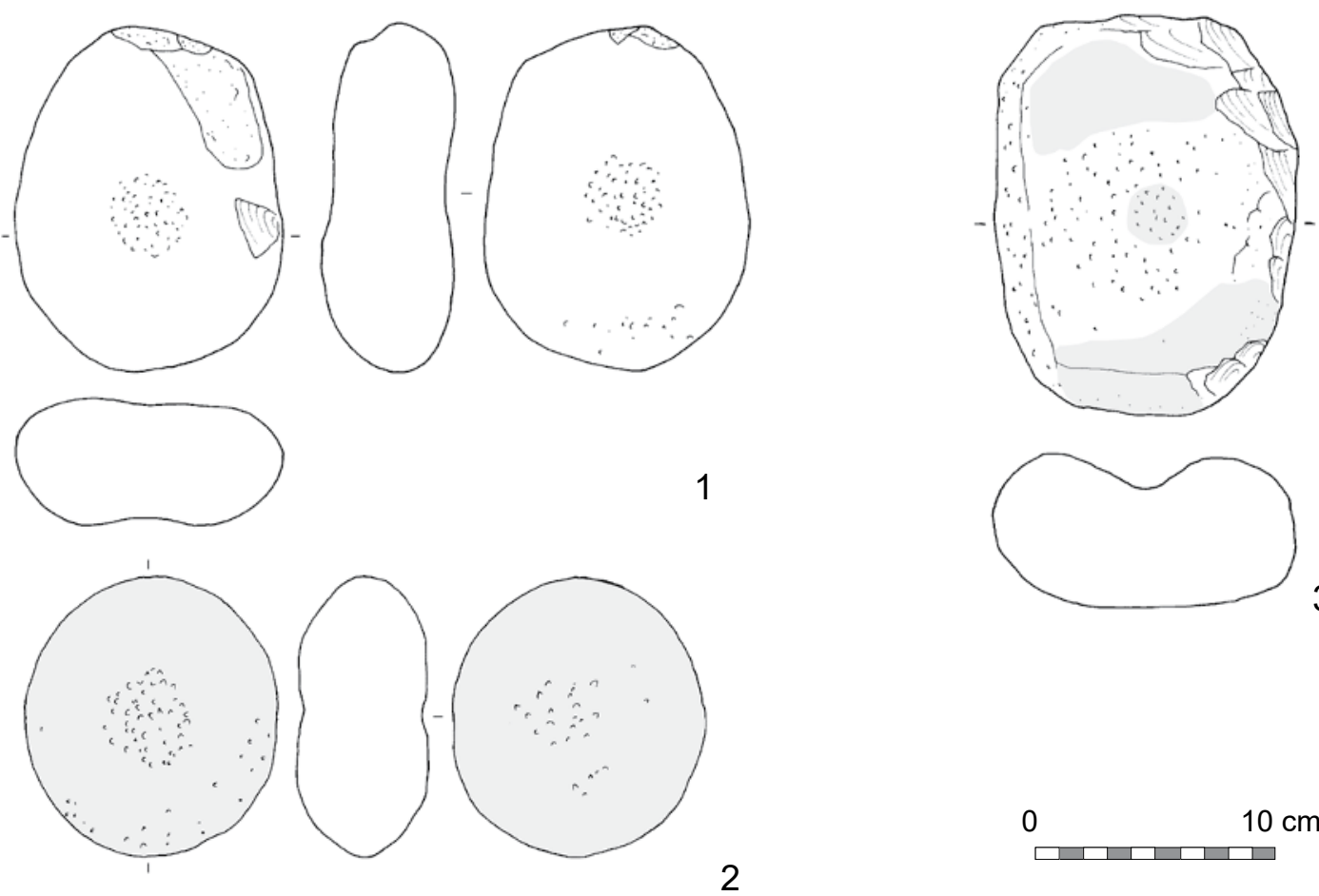

1

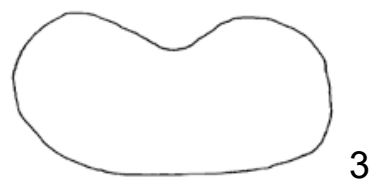

3

2

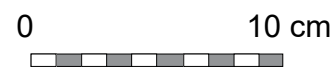

Ground or polished surface

\begin{tabular}{|c|c|c|c|c|c|c|c|}
\hline & Area & Context & Level & Type & Subtype & Raw material & Notes \\
\hline 1 & $3 \mathrm{~B}$ & 1 & mixed & Crushing cobble & Bifacial & Basalt & \\
\hline 2 & $3 \mathrm{BI}$ & 10 & topsoil & Crushing cobble & Bifacial & Weathered andesite & \\
\hline 3 & $\begin{array}{c}1 \mathrm{~A}, 1 \mathrm{~B}, \\
2 \mathrm{~A}, 2 \mathrm{~B}\end{array}$ & & $1-4$ & Crushing cobble & Unifacial & Weathered andesite & A photo of this piece is shown in Fig. 12.18: 17. \\
\hline
\end{tabular}

Fig. 12.10 Crushing cobbles from Göytepe. 1 and 2: Bifacial; 3: Unifacial.

rocks around the site. The exact raw material sources have yet to be identified although most pieces show rolled smooth surfaces that indicate their origins from nearby river beds.

\subsubsection{Ground stone production}

Pieces in this activity consist of three groups. The first group includes raw material stones to be modified, such as rocks of various sizes from boulders to coarse pebbles. These pieces are modified and left on site as 

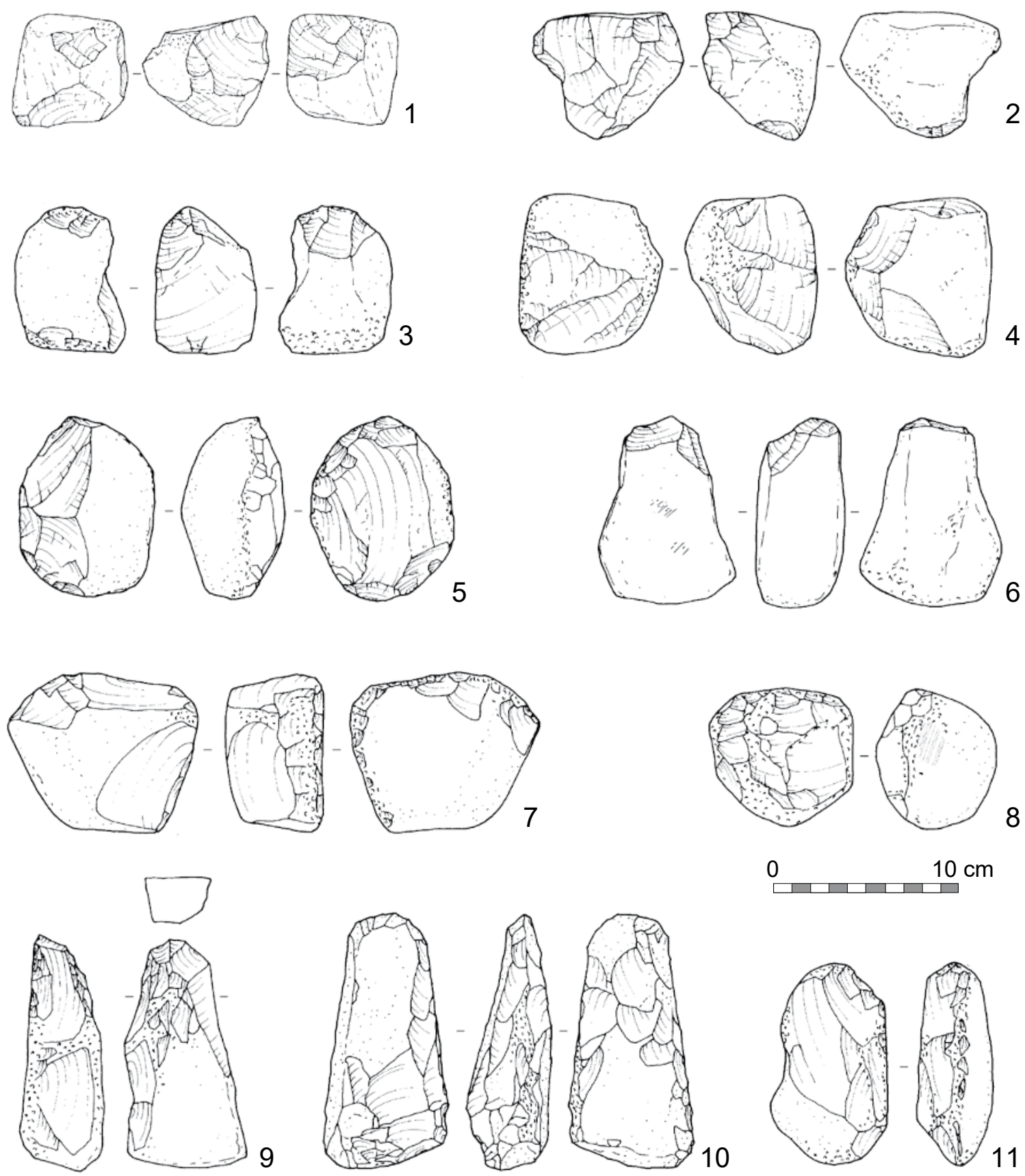

0

$10 \mathrm{~cm}$
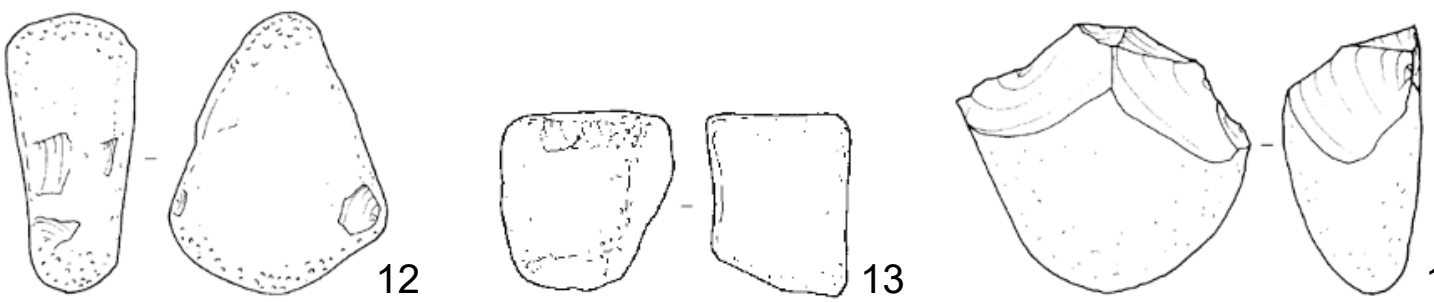

$\therefore$ Pecking or battering scars

Fig. 12.11 Pounders and flaked cobble from Goytepe. 1-11: Core pounders; 12 and 13: Pounders; 14: Flaked cobble. 


\begin{tabular}{|c|c|c|c|c|c|c|}
\hline & Area & Context & Level & Type & Raw material & Notes \\
\hline 1 & $3 \mathrm{BI}$ & 13.6 & 4 & Core pounder & Basalt & A photo of this piece is shown in Fig. 12.19: 4. \\
\hline 2 & $3 \mathrm{BI}$ & 13.2 & 4 & Core pounder & Basalt & A photo of this piece is shown in Fig. 12.19: 1. \\
\hline 3 & $3 \mathrm{BI}$ & 13.3 & 4 & Core pounder & Basalt & A photo of this piece is shown in Fig. 12.19: 3. \\
\hline 4 & $3 \mathrm{BI}$ & 13.4 & 4 & Core pounder & Basalt & A photo of this piece is shown in Fig. 12.19: 2. \\
\hline 5 & $3 \mathrm{BI}$ & 13.5 & 4 & Core pounder & Basalt & \\
\hline 6 & $3 \mathrm{BI}$ & 13.1 & 4 & Core pounder & Basalt & \\
\hline 7 & $4 \mathrm{BI}$ & 56 & 7 & Core pounder & Basalt & \\
\hline 8 & $4 \mathrm{BI}$ & 60 & 8 & Core pounder & Basalt & \\
\hline 9 & $1 \mathrm{All}$ & locus 14 & $1-4$ & Core pounder & Basalt & \\
\hline 10 & $3 \mathrm{All}$ & & $4-5$ & Core pounder & Basalt & \\
\hline 11 & $4 \mathrm{BI}$ & 60 & 8 & Core pounder & Basalt & \\
\hline 12 & $4 \mathrm{BI}$ & 60 & 8 & Pounder & Basalt & \\
\hline 13 & $3 \mathrm{BI}$ & 13.8 & 4 & Pounder & Basalt & A photo of this piece is shown in Fig. 12.19: 5. \\
\hline 14 & $4 \mathrm{BI}$ & 60 & 8 & Flaked cobble & Basalt & \\
\hline
\end{tabular}

Fig. 12.11 Descriptions.
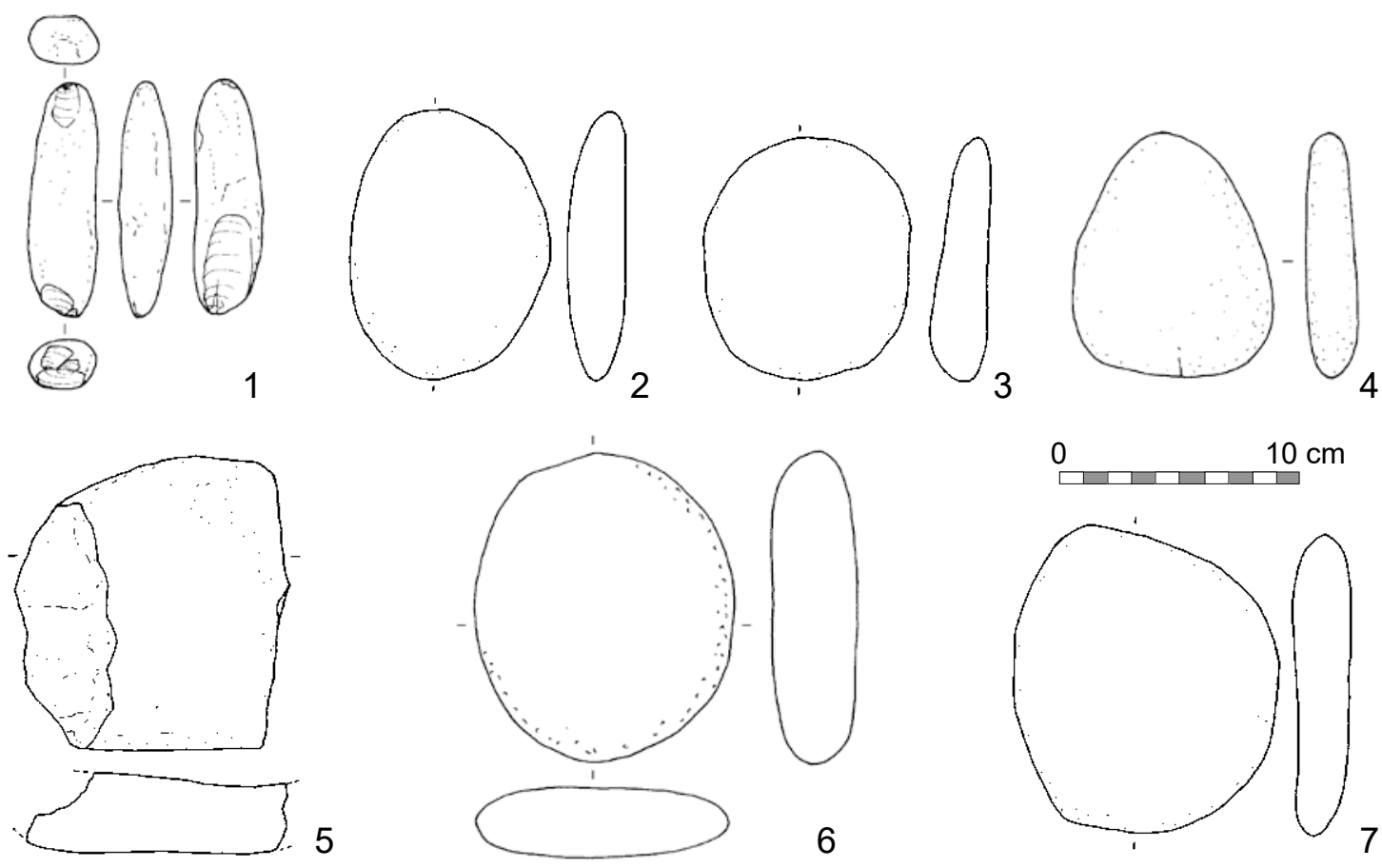

6

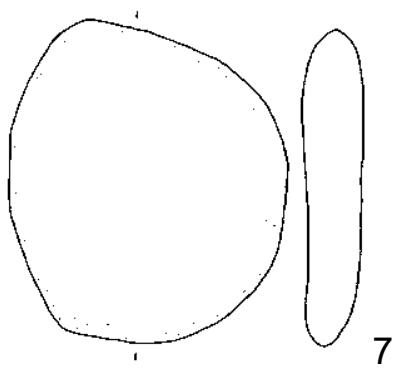

\begin{tabular}{|c|c|c|c|c|c|}
\hline & Area & Context & Level & Type & Raw material \\
\hline 1 & $4 \mathrm{BIIX}$ & 88 & modern & Elongated cobble & Basalt? \\
\hline 2 & $4 \mathrm{BI}$ & $109(\mathrm{~N})$ & 10 & Flat cobble & Unidentifiable \\
\hline 3 & $3 \mathrm{BI}$ & 12 & 4 & Flat cobble & Basalt \\
\hline 4 & $4 \mathrm{BI}$ & 60 & 8 & Flat cobble & Unidentifiable \\
\hline 5 & $4 \mathrm{BI}$ & $114 \mathrm{BB}$ & 11 & Flat cobble & Unidentifiable \\
\hline 6 & $2 \mathrm{All}$ & $\mathrm{d}=1.839 \mathrm{~m}$ & $2-4$ & Flat cobble & Unrecorded \\
\hline 7 & $3 \mathrm{BI}$ & 1 & topsoil & Flat cobble & Basalt \\
\hline
\end{tabular}

Fig. 12.12. Unmodified cobbles from Göytepe. 1: Elongated cobble; 2-7: Flat cobbles. 

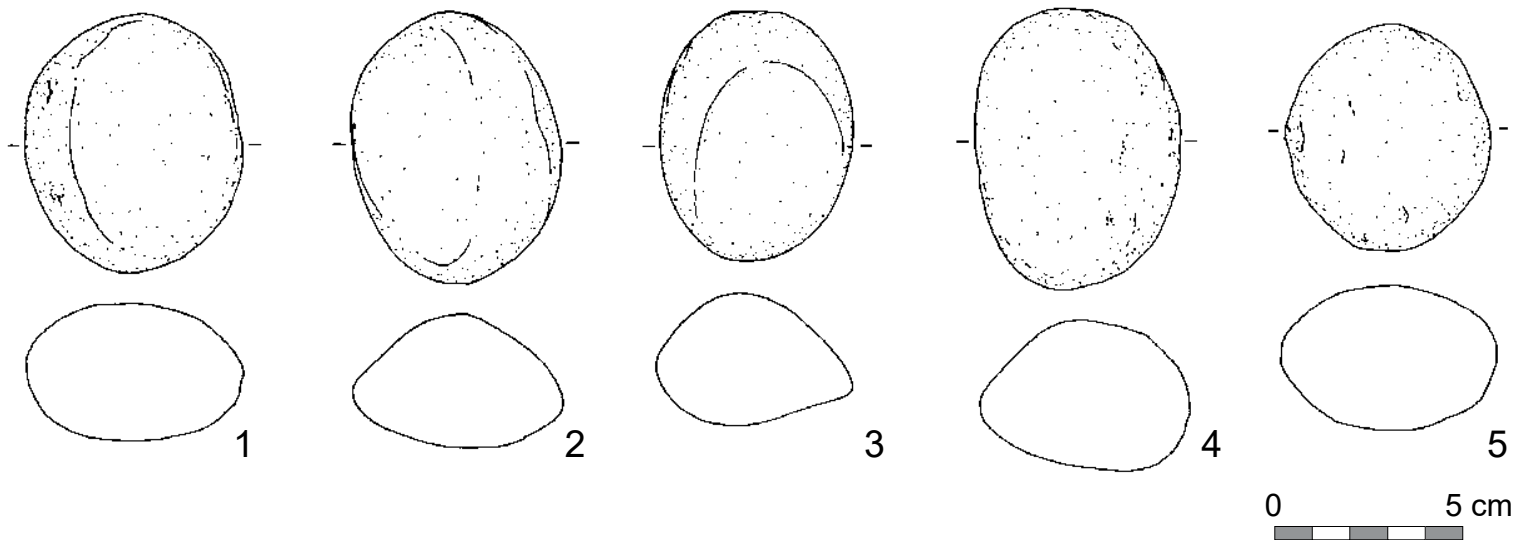

\begin{tabular}{|c|c|c|c|c|c|c|}
\hline & Area & Context & Level & Type & Raw material & Notes \\
\hline 1 & $4 \mathrm{BI}$ & 40 & 7 & Pebble & Sandstone? & From a cache of 38 pieces \\
\hline 2 & $4 \mathrm{BI}$ & 40 & 7 & Pebble & Limestone? & From a cache of 38 pieces \\
\hline 3 & $4 \mathrm{BI}$ & 40 & 7 & Pebble & Rhyolite & From a cache of 38 pieces \\
\hline 4 & $4 \mathrm{BI}$ & 40 & 7 & Pebble & Andesite & From a cache of 38 pieces \\
\hline 5 & $4 \mathrm{BI}$ & 40 & 7 & Pebble & Andesite & From a cache of 38 pieces \\
\hline
\end{tabular}

Fig. 12.13 Pebbles from Göytepe.

finished products, such as grinding slabs, grinding querns, mortars, handstones, pestles, crushing cobbles, axes, chisels, working slabs, floor polishers, showing flaking and pecking scars. See descriptions of each tool type for the details of modification traces.

The second group includes production tools, such as core pounders, pounders, and pecked cobbles, which are mostly made of fine-grained dense basalt. Core pounders can be effective tools for producing ground stones by flaking and pecking according to ethnographic observations (e.g., Hayden 1987), experimental production (e.g., Wilke and Quintero 1996), and studies of archaeological workshops of ground stones (e.g., Roubet 1989). Flaked cobbles do not show battered edges but included here because they are probably preforms of core pounders given their forms and dimensions similar to core pounders. However, they could also be cores for basalt flakes that are sometimes retouched. In this case, flaked cobbles should be included in chipped stone assemblages.

As the third group, numerous flakes mostly of basalt are probably debitage in the production of ground stones. The few occurrences of andesite flakes, despite the presence of flaking scars on andesite tools, indicate three possibilities. First, flaking of andesite boulders and coarse cobbles were performed outside the site. Second, flaking was not a major method in the modification of andesite tools. Lastly, it is possible that andesite flakes were not collected in the excavations because flakes of andesite with coarse phenocrysts do not show clear ventral or dorsal surfaces. The second scenario is likely for two reasons. First, andeside boulders have naturally flat surfaces that are suitable either for grinding surfaces or for the bottom of lower grinding stones without extensive modification. Second, andesite with large phenocrysts can be readily modified by removing large phenocrysts by pecking rather than flaking. Thus, the modification of andesite rocks by flaking may not have been as frequent as basalt because of the natural forms suitable for grinding tasks and the lithological properties of andesite.

\subsubsection{Food processing}

Food processing tools comprise lower stones and upper stones. Lower stones include grinding slabs, grinding querns, and mortars, which are mostly made of andesite boulders. Upper stones are grinders, handstones, and pestles. Grinders are distinctively larger than handstones (Fig. 12.7) and made of small boulder and coarse cobbles of andesite and other raw materials. Handstones and pestles are mostly made of basalt (Table 12.2). Mortar and pestles are few in number and grinding slabs/querns and grinders/handstones are major food processing 
tools at Göytepe. One grinder and one handstone retained grass phytoliths that are likely to represent infloresence part of wheat. In addition, the phytoliths attached on the grinding surfaces include higher percentage of fragments than those from other contexts, which is consistent with the experimental results of grain processing (see Chapter 7 and Kadowaki et al. 2015).

\subsubsection{Craft production}

There are two kinds of tools, probably related to craft production, distinguished by raw materials. The first is abraders that are exclusively made of andesite porous lava. Small size is more numerous and various in type, including prismatic, flat concave, and irregular, while there are a few large prismatic pieces. Brittle vesicular surfaces of these tools often show shallow grooves, indicating that they were used for abrading relatively soft narrow materials, possibly bones.

The second group includes, crushing cobbles, axes, chisels, working slabs, and floor polishers, which are made of basalt and other raw materials. Their exact functions have not been examined. In addition, core pounders, pounders, and pecked cobbles could have been used for craft production in addition to ground stone production.

\subsubsection{Tool maintenance}

Traces of tool maintenance is observable on grinding tools, such as grinding slabs, grinding querns, grinders, and handstones. Grinding surfaces of these tools show pecking marks, indicating that the surfaces were roughened periodically to keep efficiency of pulverizing food stuff as observed ethnographically and confirmed by grinding experiments (Wright, $\mathrm{M}$. 1990). Hammers for pecking the grinding surfaces are probably core pounders, pounders, and pecked cobbles.

\subsubsection{Pigment processing}

Tools stained with red pigment $(\mathrm{n}=23)$ indicate this activity. They include core pounders $(\mathrm{n}=2)$, grinders $(\mathrm{n}=2)$, broken grinding slabs $(\mathrm{n}=4)$, handstones $(\mathrm{n}=14)$, and a pecked cobble $(\mathrm{n}=1)$. These tools are likely to represent the re-use of food processing tools or craft production tools rather than specialized tools for pigment processing.

\subsubsection{Unknown activities}

Unmodified cobbles or pebbles with various forms (i.e., elongated, flat, ground cobbles and pebbles) could have been used for unknown activities. A perforated stone (Fig. 12.17: 1) received intentional modifications, but its function is unclear.

\subsubsection{Re-use}

The re-use of tools is observable for handstones and pestles as multi-purpose tools (Table 12.1). Some grinders, handstones, and broken grinding slabs were re-used for pigment processing as described above.

\subsubsection{Storage}

Table 12.4 shows the list of ground stone artifacts recovered in the clay bins. All such cases do not necessarily have resulted from the storage of tools because abandoned bins could have been used as receptacles of garbage, such as broken objects and byproducts of stone tool production, i.e., flakes.

However, two cases are notable. One of them is the clay bin at 4BIIX-58 in Level 10 (Fig. 12.23: left), which contained a complete handstone and a complete pounder in addition to bone artifacts, including a hoe-like tool. Another case is the clay bin at 4BIIX-94 (Fig. 12.23: right) also in Level 10, where a complete grinder and a complete handstone were lying on top of the $4 \mathrm{~cm}$-thick bottom fill consisting of high concentration of grass, probably wheat, phytoliths. As suggested by micromorphological observations (see Chapter 7 and Kadowaki et al. 2015 in detail), the bottom fill, rich in phytoliths, is most likely primary deposits of grains, which were stored on mats to control moisture in the clay bin. This find provides contextual evidence for the use of the complete grinder and handstone in relation to cereal grains stored together in the same bin.

\subsection{Stratigraphic examination of ground stones at Göytepe}

For the stratigraphic examination of ground stones, the main collection by the Japanese mission from 

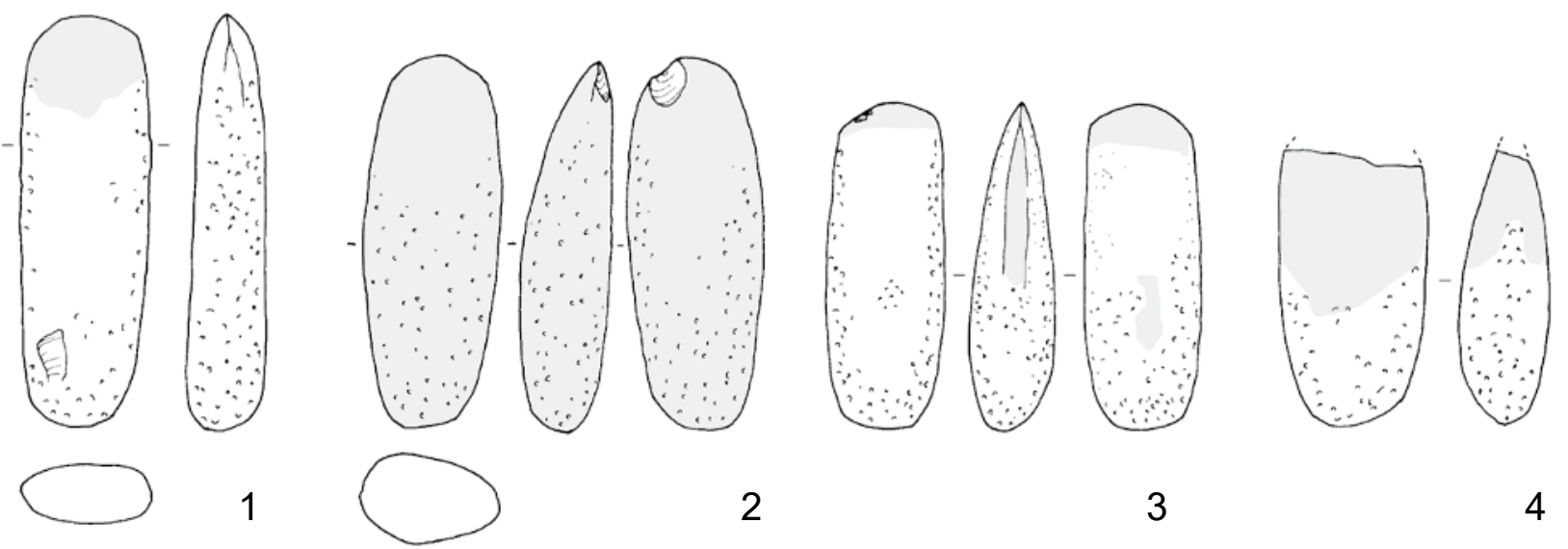

2

3

4
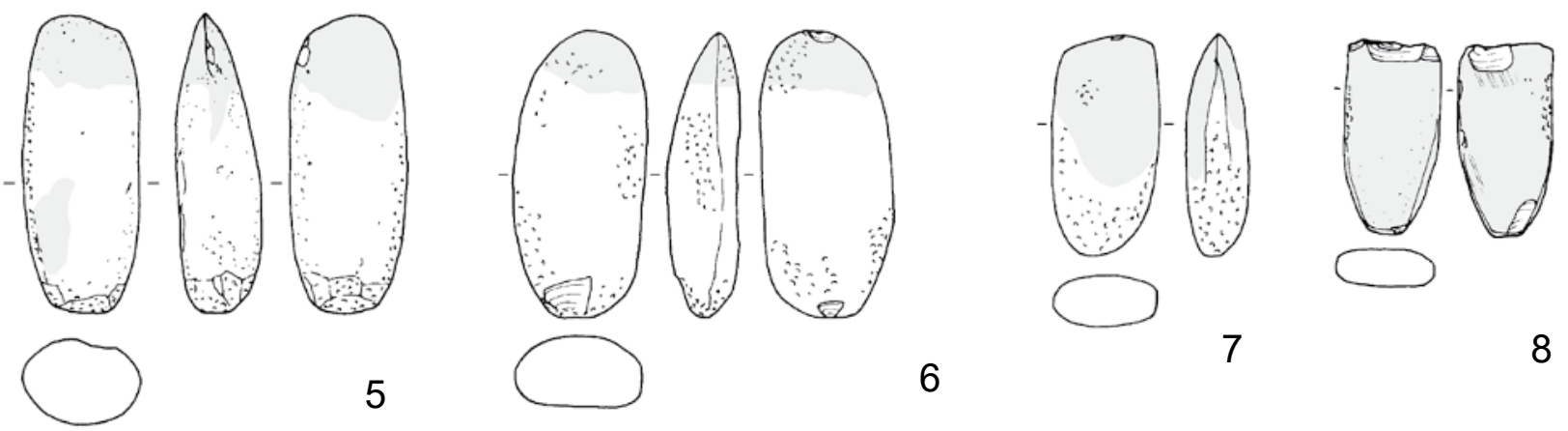

6

8
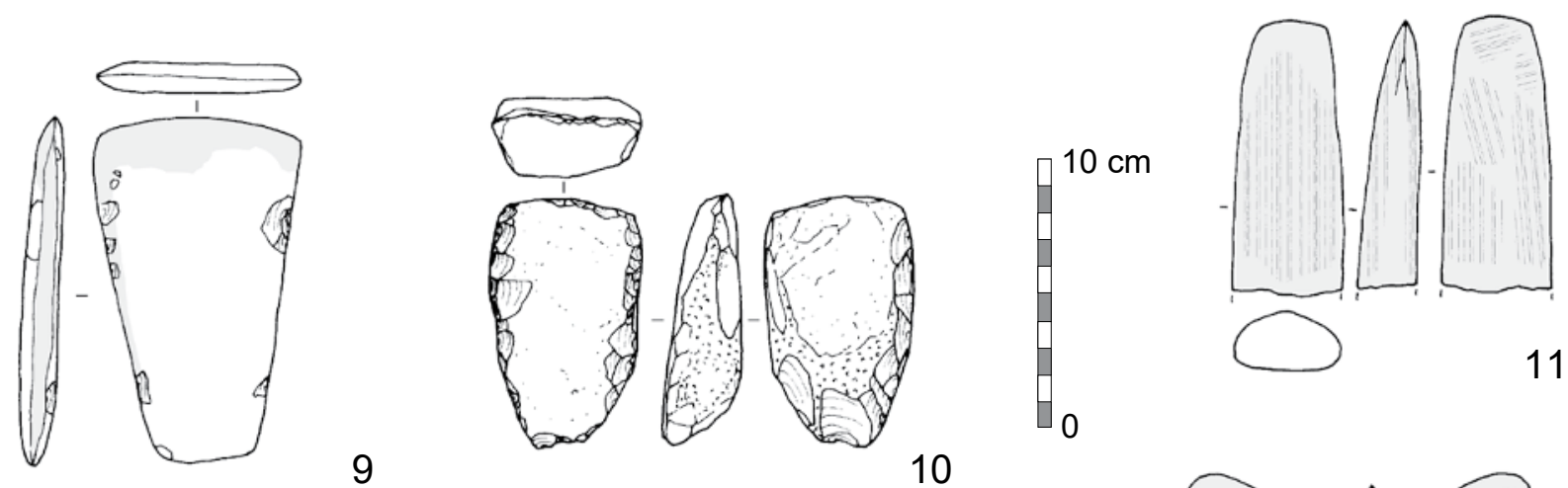

9

10
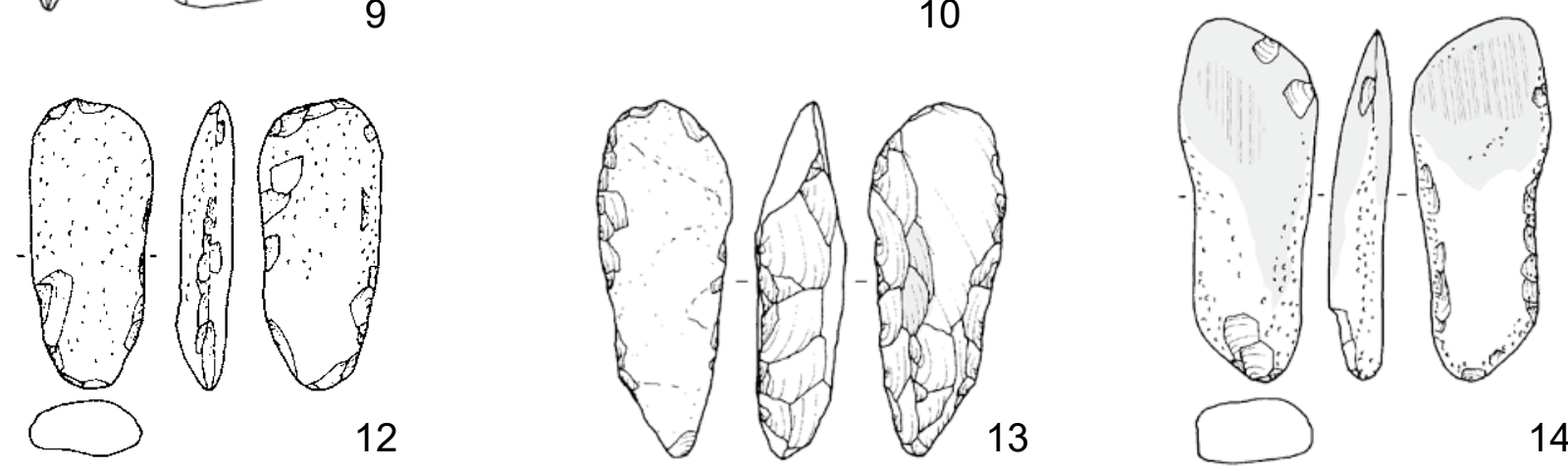

$\square$ Ground or polished surface $\boxminus$ Striations

Fig. 12.14 Axes and chisel from Göytepe. 1-3: Long type; 4-8: Short type; 9-10: Wide type; 11: Chisel; 12-14: Unfinished axes. 


\begin{tabular}{|c|c|c|c|c|c|l|l|}
\hline & Area & Context & Level & Type & Subtype & Raw material & Notes \\
\hline 1 & $1 \mathrm{Al}$ & $\mathrm{d}=40 \mathrm{~cm}$ & 3 & Axe & Long & Basalt & \\
\hline 2 & $3 \mathrm{BI}$ & 12 & 4 & Axe & Long & Basalt & A photo of this piece is shown in Fig. 12.18: 9. \\
\hline 3 & $4 \mathrm{BI}$ & 60 & 8 & Axe & Long & Basalt & \\
\hline 4 & $1 \mathrm{Al}$ & $\mathrm{d}=36.5 \mathrm{~cm}$ & 3 & Axe & Short & Unidentifiable & \\
\hline 5 & $3 \mathrm{All}$ & & $4-5$ & Axe & Short & Basalt & \\
\hline 6 & $3 \mathrm{~B}$ & 1 & mixed & Axe & Short & Basalt & \\
\hline 7 & $2 \mathrm{All}$ & & $2-4$ & Axe & Short & Basalt & \\
\hline 8 & $\mathrm{~N} / \mathrm{A}$ & & Surface & Axe & Short & Basalt & \\
\hline 9 & $2 \mathrm{All}$ & locus 14 & $2-4$ & Axe & Wide & Basalt & $\begin{array}{l}\text { Retaining a part of ventral surface of a flake, } \\
\text { probably used as a blank }\end{array}$ \\
\hline 10 & $4 \mathrm{BIl}$ & 25 & 6 & Axe & Wide & Basalt & $\begin{array}{l}\text { Retaining a part of ventral surface of a flake, } \\
\text { probably used as a blank. A photo of this piece is } \\
\text { shown in Fig. 12.18: 5. }\end{array}$ \\
\hline 11 & $4 \mathrm{BI}$ & $114 \mathrm{BB}$ & 11 & Chisel & & Basalt & A photo of this piece is shown in Fig. 12.18: 6. \\
\hline 12 & $1 \mathrm{~A}, 1 \mathrm{~B}, 2 \mathrm{~A}, 2 \mathrm{~B}$ & & $1-4$ & Axe & Unfinished & Basalt & \\
\hline 13 & $4 \mathrm{BI}$ & 1 & topsoil & Axe & Unfinished & Basalt & $\begin{array}{l}\text { Retaining a part of ventral surface of a flake, } \\
\text { probably used as a blank. A photo of this piece is } \\
\text { shown in Fig. 12.18: 7. }\end{array}$ \\
\hline 14 & 2B & & $1-4$ & Axe & Unfinished & Basalt & A photo of this piece is shown in Fig. 12.18: 8. \\
\hline
\end{tabular}

Fig. 12.14 Descriptions.

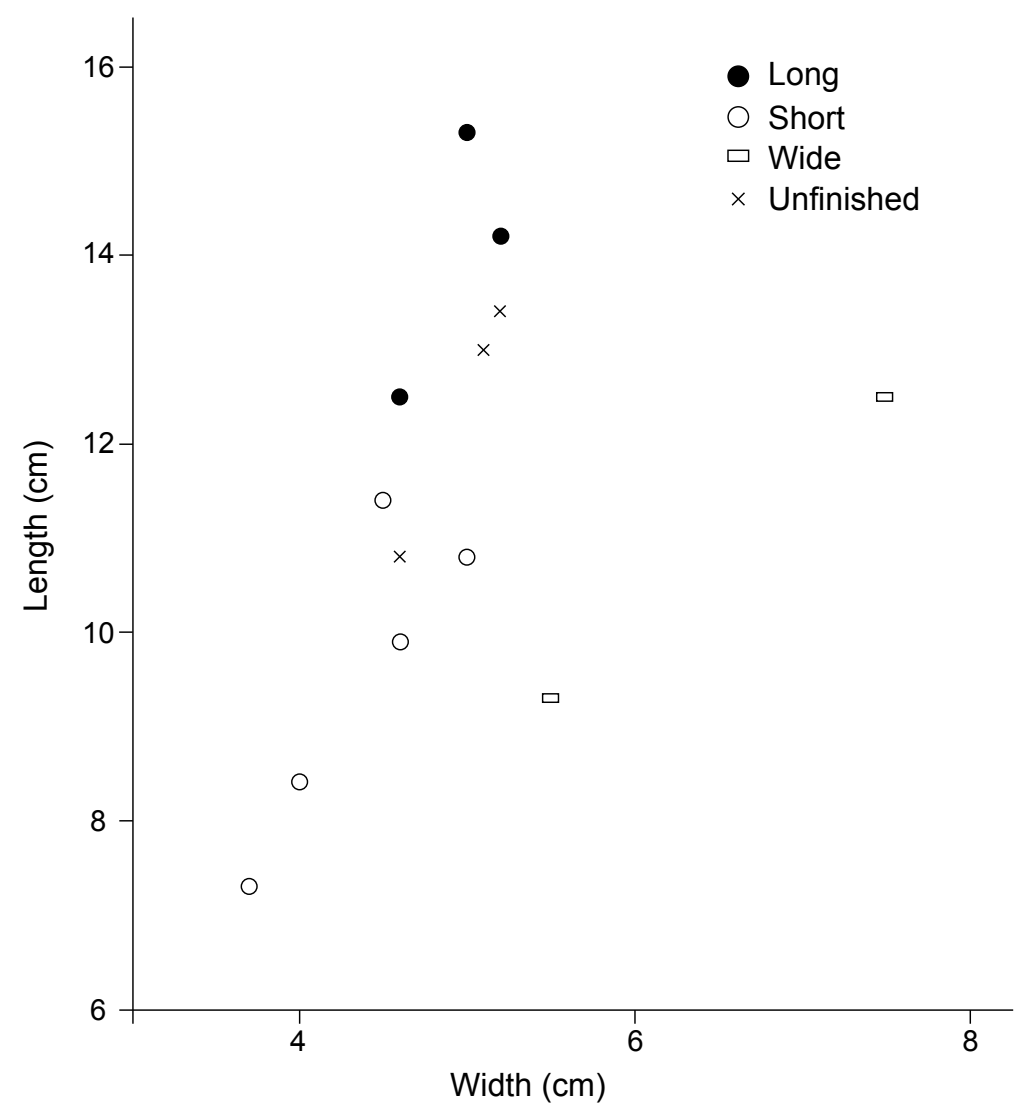

Fig. 12.15 Size of complete axes by subtypes. 

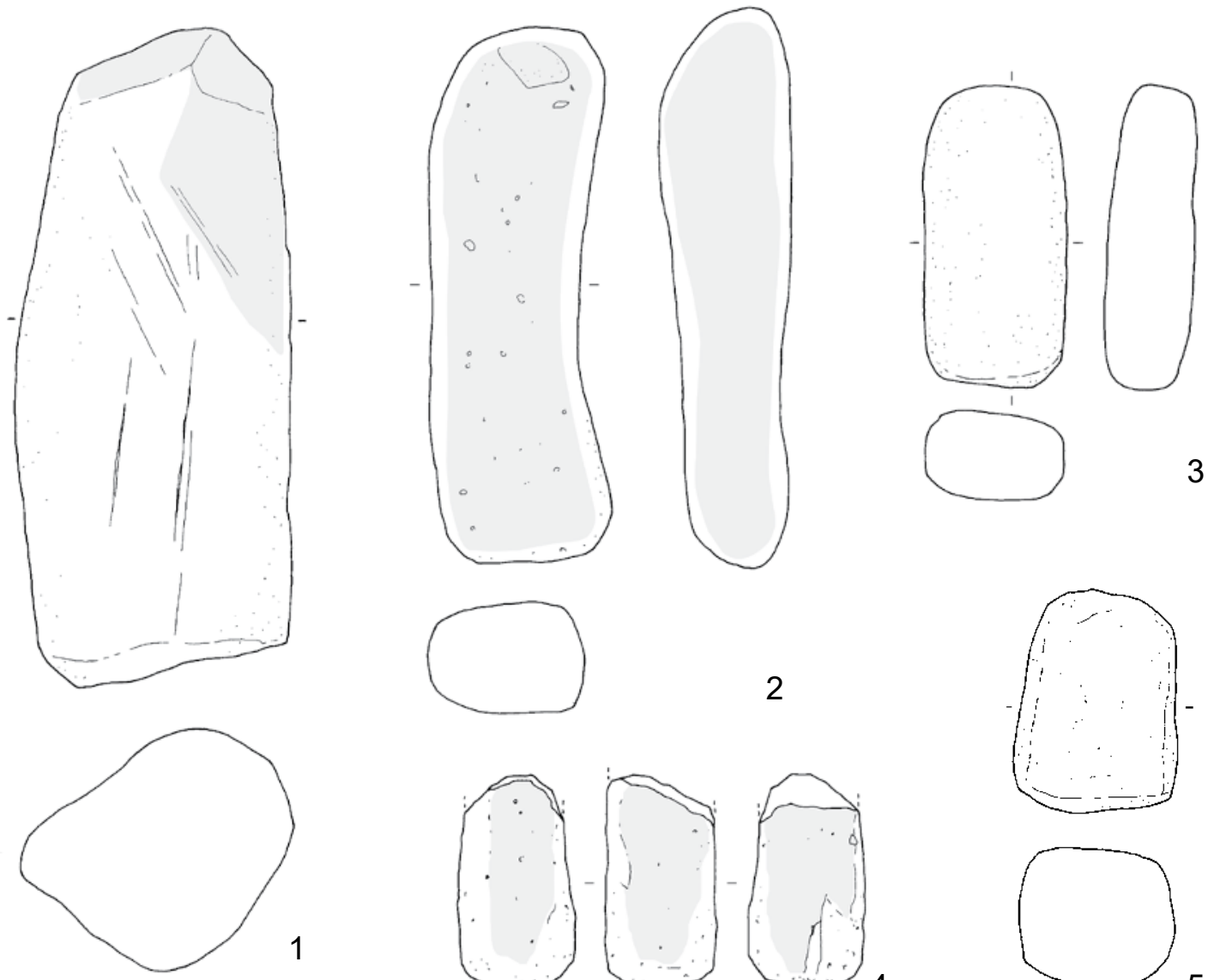

2
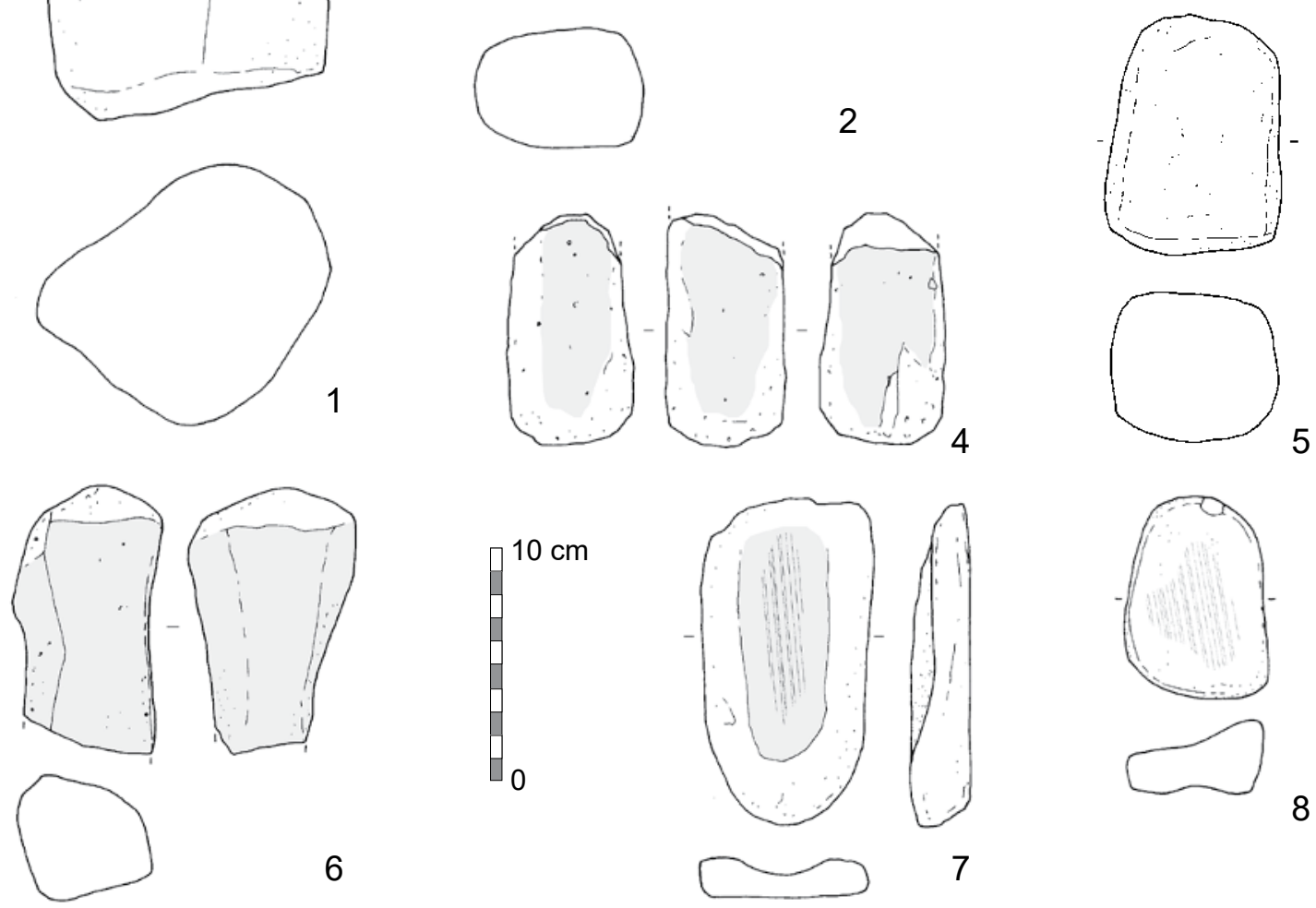

6
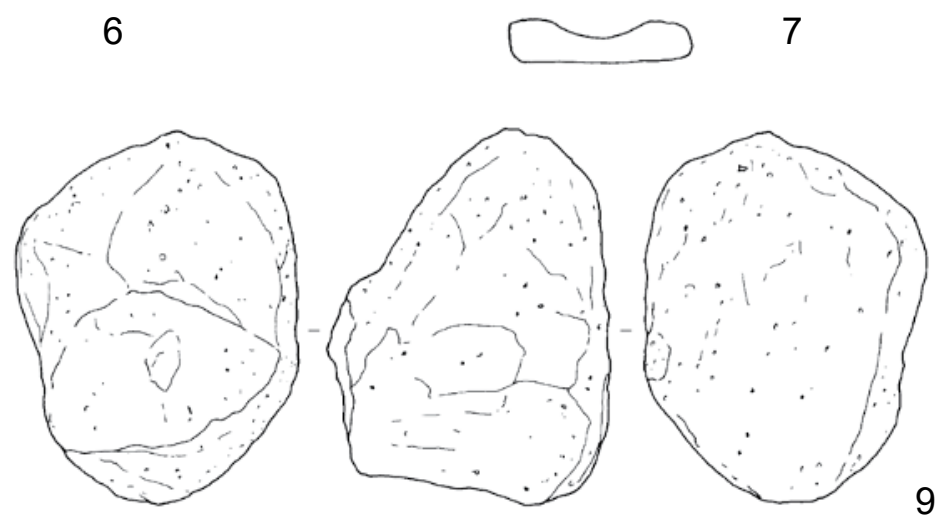

Ground or polished surface $E$ Striations $\therefore$ Pecking or battering scars

Fig. 12.16 Abraders from Göytepe. 1 and 2: Large prismatic; 3-6: Small prismatic; 7 and 8: Flat with concave surfaces; 9: Irregular form. 


\begin{tabular}{|c|c|c|c|c|c|c|c|}
\hline & Area & Context & Level & Type & Subtype & Raw material & Notes \\
\hline 1 & $4 \mathrm{BIIX}$ & 95 & 10 & Abrader & $\begin{array}{c}\text { Large } \\
\text { prismatic }\end{array}$ & $\begin{array}{c}\text { Andesite } \\
\text { porous lava }\end{array}$ & A photo of this piece is shown in Fig. 12.18: 10. \\
\hline 2 & $1 \mathrm{Al}$ & 3 & 3 & Abrader & $\begin{array}{c}\text { Large } \\
\text { prismatic }\end{array}$ & $\begin{array}{c}\text { Andesite } \\
\text { porous lava }\end{array}$ & A photo of this piece is shown in Fig. 12.18: 11. \\
\hline 3 & $\begin{array}{c}1 \mathrm{~A}, 1 \mathrm{~B}, \\
2 \mathrm{~A}, 2 \mathrm{~B}\end{array}$ & $1-4$ & Abrader & $\begin{array}{c}\text { Small } \\
\text { prismatic }\end{array}$ & $\begin{array}{c}\text { Andesite } \\
\text { porous lava }\end{array}$ & A photo of this piece is shown in Fig. 12.18: 12. \\
\hline 4 & $4 \mathrm{BI}$ & 60 & 8 & Abrader & $\begin{array}{c}\text { Small } \\
\text { prismatic }\end{array}$ & $\begin{array}{c}\text { Andesite } \\
\text { porous lava }\end{array}$ & \\
\hline 5 & $\begin{array}{c}1 \mathrm{~A}, 1 \mathrm{~B}, \\
2 \mathrm{~A}, 2 \mathrm{~B}\end{array}$ & 3 & 3 & Abrader & $\begin{array}{c}\text { Small } \\
\text { prismatic }\end{array}$ & $\begin{array}{c}\text { Andesite } \\
\text { porous lava }\end{array}$ & A photo of this piece is shown in Fig. 12.18: 13. \\
\hline 6 & $1 \mathrm{Al}$ & $3-4$ & Abrader & $\begin{array}{c}\text { Small } \\
\text { prismatic }\end{array}$ & $\begin{array}{c}\text { Andesite } \\
\text { porous lava }\end{array}$ & And \\
\hline 7 & $1 \mathrm{Al}$ & $\mathrm{d}=36 \mathrm{~cm}$ & 3 & Abrader & $\begin{array}{c}\text { Flat } \\
\text { concave }\end{array}$ & $\begin{array}{c}\text { Andesite } \\
\text { porous lava }\end{array}$ & A photo of this piece is shown in Fig. 12.18: 14. \\
\hline 8 & $2 \mathrm{All}$ & $\mathrm{d}=1.839 \mathrm{~m}$ & $2-4$ & Abrader & $\begin{array}{c}\text { Flat } \\
\text { concave }\end{array}$ & $\begin{array}{c}\text { Andesite } \\
\text { porous lava }\end{array}$ & \\
\hline 9 & $4 \mathrm{BI}$ & 43 & 7 & Abrader & $\begin{array}{c}\text { Irregular } \\
\text { Andesite } \\
\text { porous lava }\end{array}$ & \\
\hline
\end{tabular}

Fig. 12.16 Descriptions.
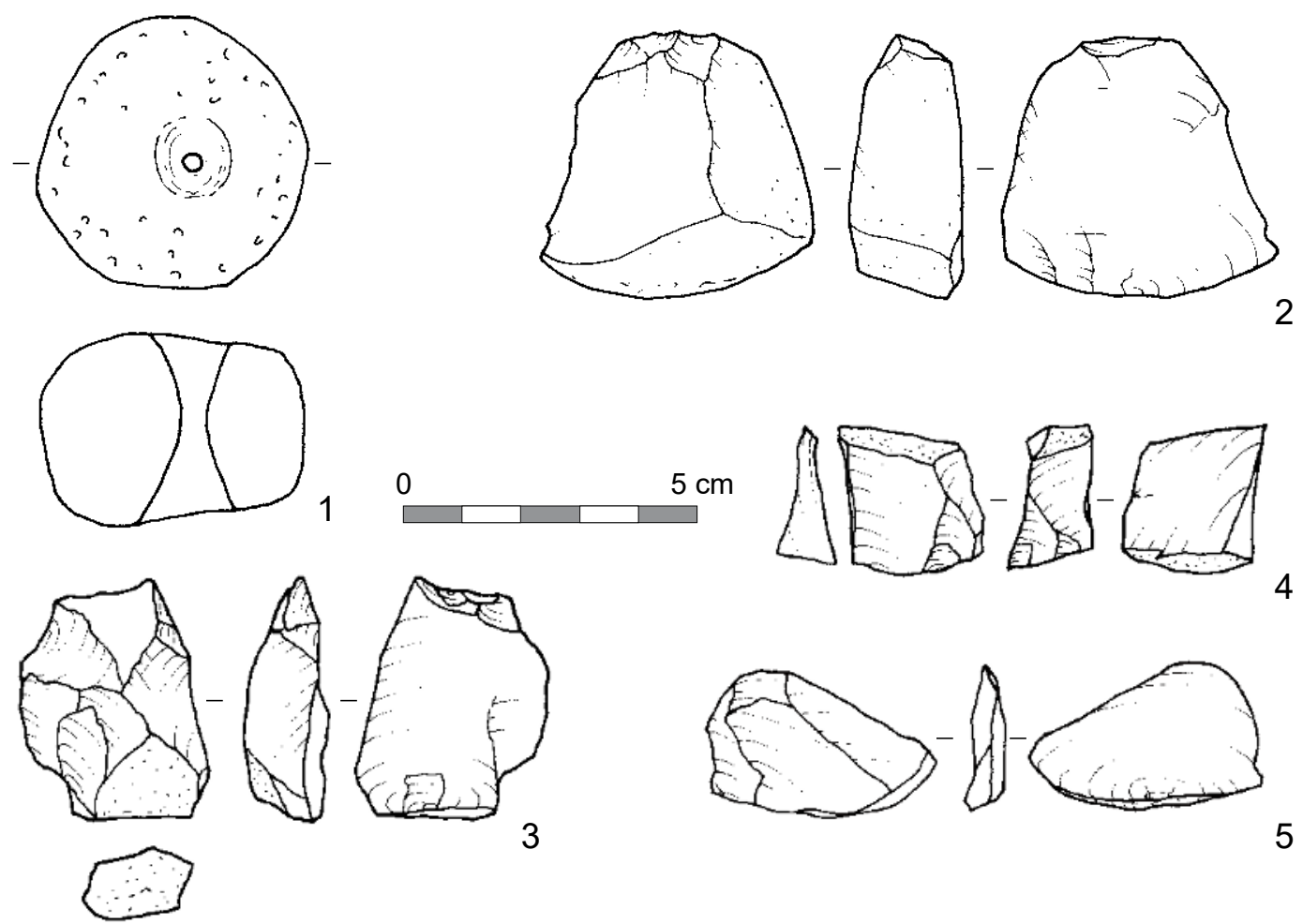

$\therefore$ Pecking or battering scars

\begin{tabular}{|c|c|c|c|c|c|}
\hline & Area & Context & Level & Type & Raw material \\
\hline 1 & 2 All & locus 6 & $2-4$ & Perforated stone & Unidentifiable \\
\hline 2 & $3 \mathrm{BI}$ & 13.7 & 4 & Flake & Basalt \\
\hline 3 & $4 \mathrm{BII}$ & 25 & 6 & Flake & Basalt \\
\hline 4 & $4 \mathrm{BII}$ & 25 & 6 & Flake & Basalt \\
\hline 5 & $4 \mathrm{BII}$ & 25 & 6 & Flake & Basalt \\
\hline
\end{tabular}

Fig. 12.17 Perforated stone and flakes from Göytepe. 1: Perforated stone, 2-5: Flakes. 

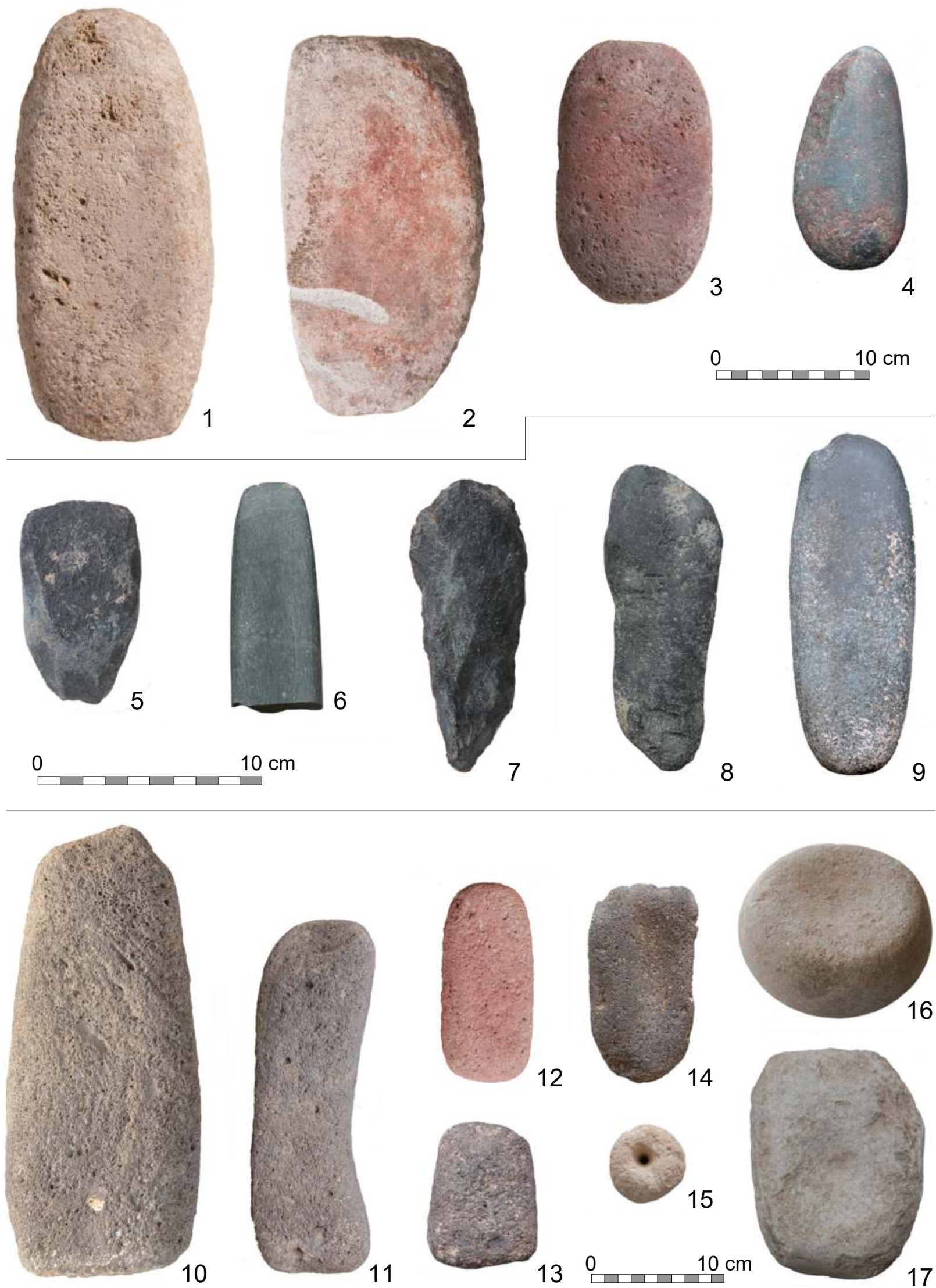

12
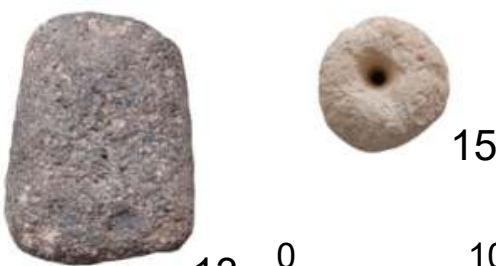

13
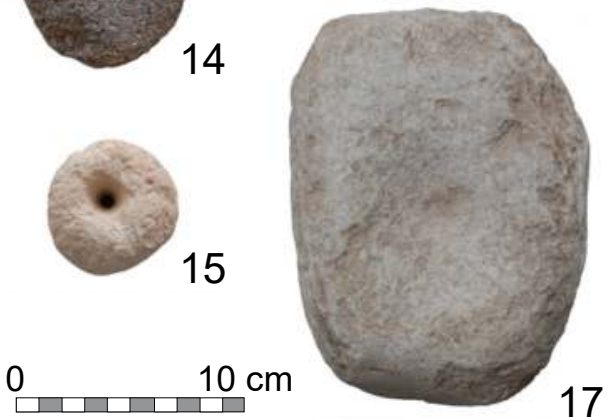

Fig. 12.18 Various ground stones from Göytepe. 1-2: Grinders; 3: Handstone; 4: Pestle/crushing cobble; 5-9: Axes and chisel; 10-14: Abraders, 15: Perforated stone; 16: Floor polisher; 17: Crushing cobble. 


\begin{tabular}{|c|c|l|}
\hline & Type & \multicolumn{1}{|c|}{ Notes } \\
\hline 1 & Grinder & Fig. 12.6: 1 shows the drawing. \\
\hline 2 & Grinder & Fig. 12.6: 2 shows the drawing. \\
\hline 3 & Handstone & Fig. 12.8: 4 shows the drawing. \\
\hline 4 & Pestle/Crushing cobble & Fig. 12.9: 3 shows the drawing. \\
\hline 5 & Axe & Fig. 12.14: 10 shows the drawing. \\
\hline 6 & Chisel & Fig. 12.14: 11 shows the drawing. \\
\hline 7 & Axe & Fig. 12.14: 13 shows the drawing. \\
\hline 8 & Axe & Fig. 12.14: 14 shows the drawing. \\
\hline 9 & Axe & Fig. 12.14: 2 shows the drawing. \\
\hline 10 & Abrader & Fig. 12.16: 1 shows the drawing. \\
\hline 11 & Abrader & Fig. 12.16: 2 shows the drawing. \\
\hline 12 & Abrader & Fig. 12.16: 3 shows the drawing. \\
\hline 13 & Abrader & Fig. 12.16: 5 shows the drawing. \\
\hline 14 & Abrader & Fig. 12.16: 7 shows the drawing. \\
\hline 15 & Perforated stone & Fig. 12.17: 1 shows the drawing. \\
\hline 16 & Floor polisher & 1All (M) d=1.9, locus 14, Level 1-4 \\
\hline 17 & Crushing cobble & Fig. 12.10: 3 shows the drawing. \\
\hline
\end{tabular}

Fig. 12.18 Descriptions.

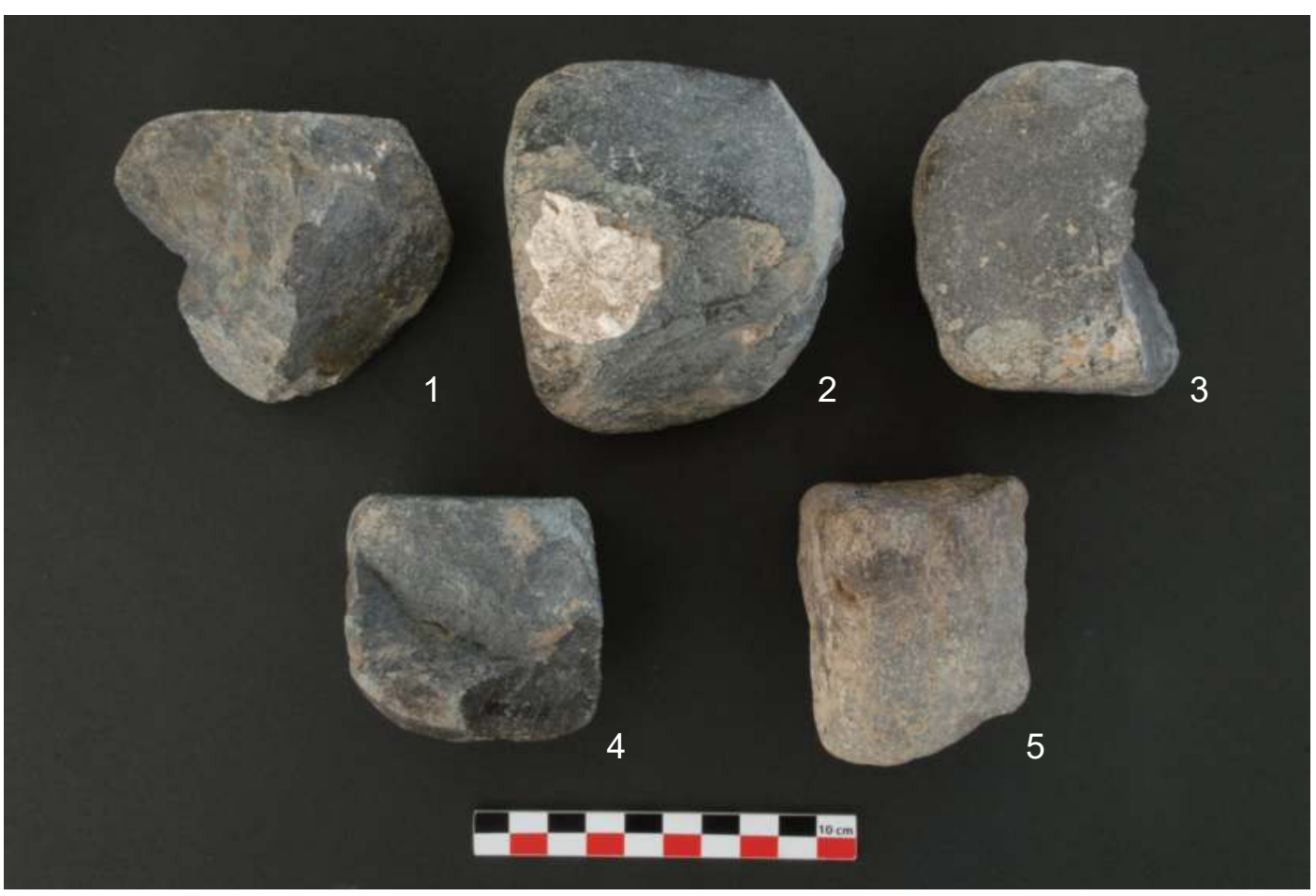

\begin{tabular}{|l|l|l|}
\hline & \multicolumn{1}{|c|}{ Type } & \multicolumn{1}{c|}{ Notes } \\
\hline 1 & Core pounder & Fig. 12.11: 2 shows the drawing. \\
\hline 2 & Core pounder & Fig. 12.11: 4 shows the drawing. \\
\hline 3 & Core pounder & Fig. 12.11: 3 shows the drawing. \\
\hline 4 & Core pounder & Fig. 12.11: 1 shows the drawing. \\
\hline 5 & Pounder & Fig. 12.11: 13 shows the drawing. \\
\hline
\end{tabular}

Fig. 12.19 Pounders from Göytepe. 1-4: Core pounders; 5: Pounder. 


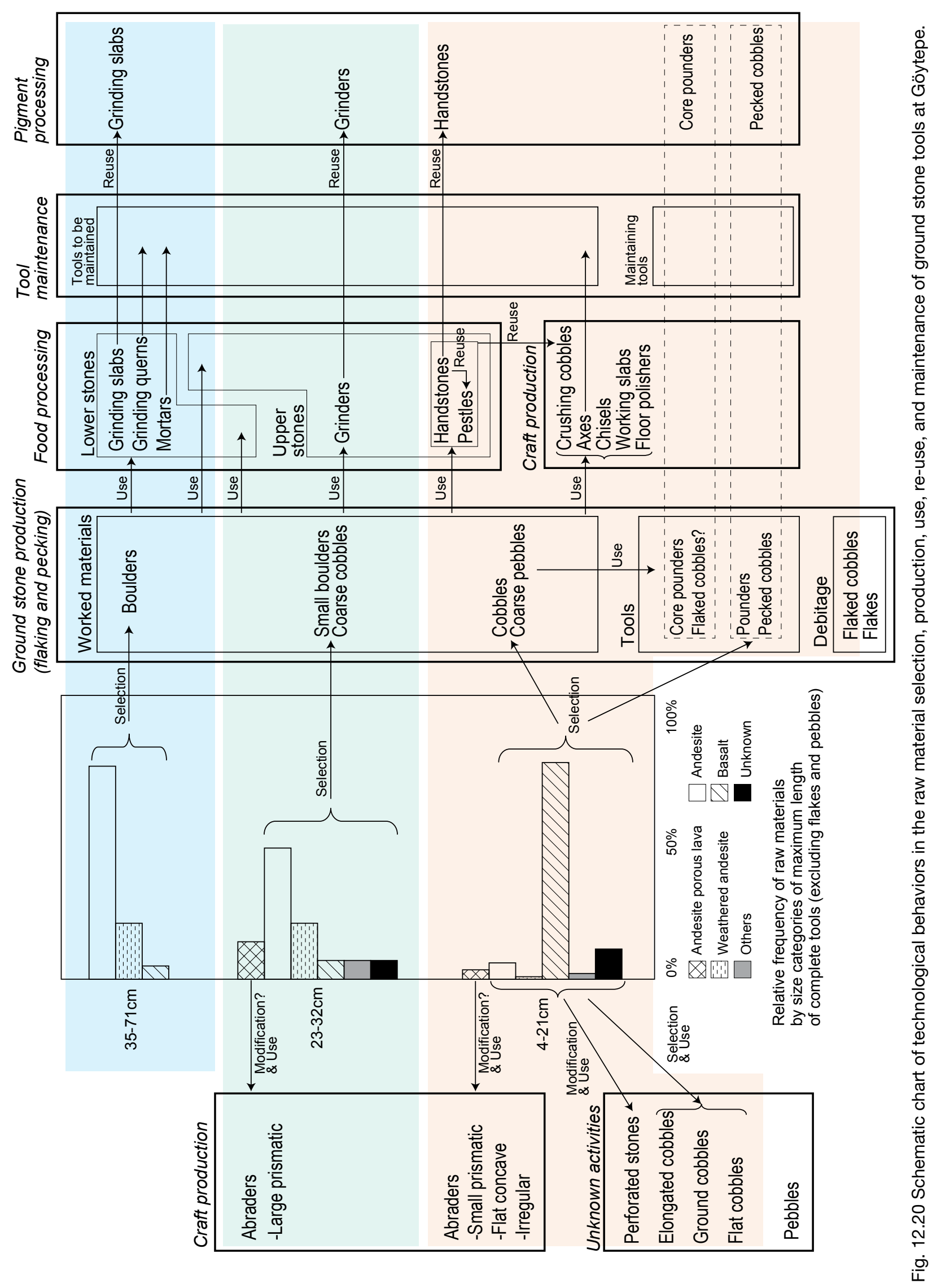




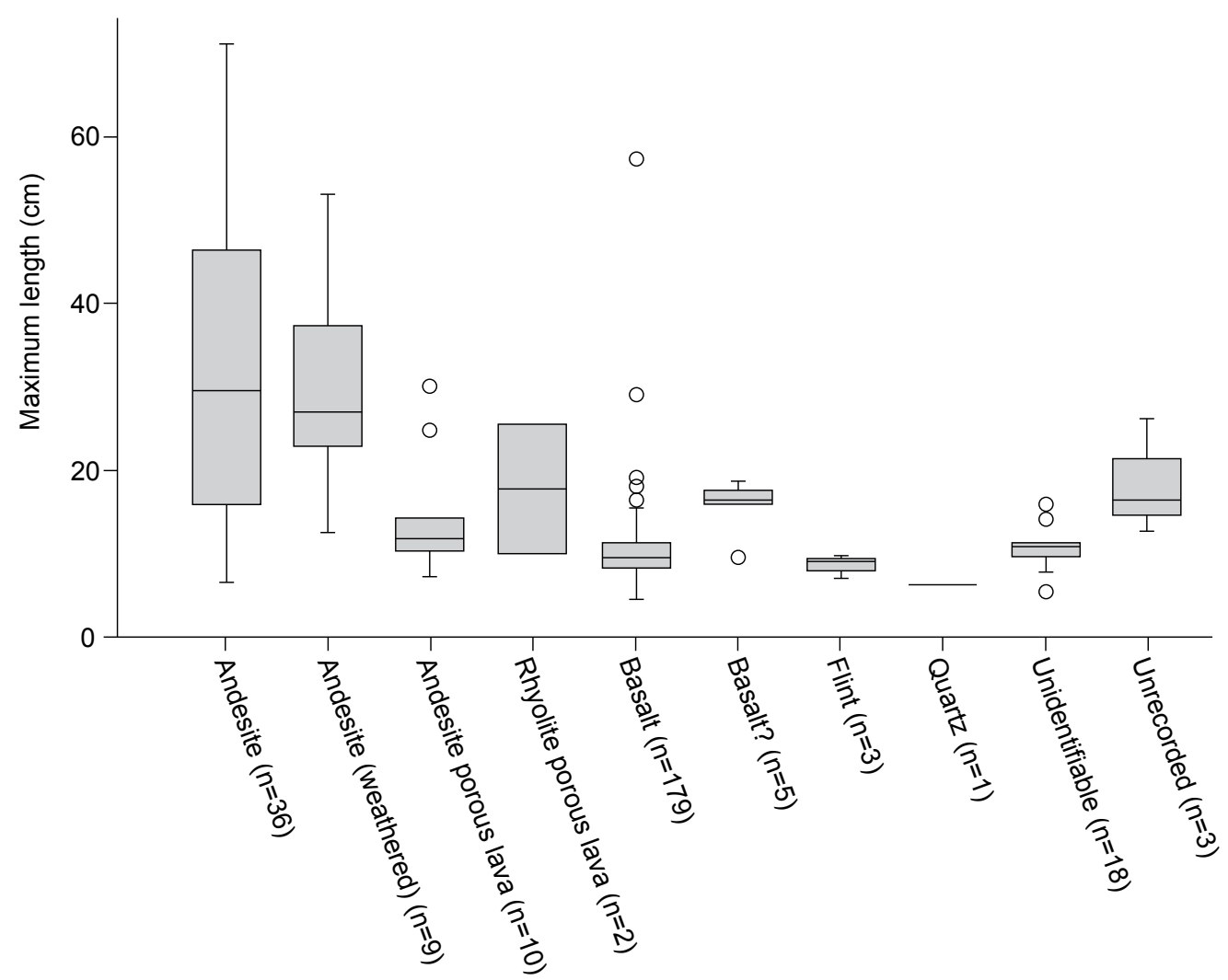

Fig. 12.21 Maximum length of complete ground stones, excluding flakes and pebbles, by raw material types.

Neolithic Levels 4-11 are used with some references to the Azerbaijani selective collections from Levels $1-6$.

Table 12.5 shows the occurrences of food processing and other tools by Neolithic Levels 4-11. This functional division is simply meant to show overall stratigraphic patterns in the occurrences of tool types and does not negate the possible uses of individual tools for multiple purposes, as suggested by ethnographic observations and use-wear observations (Adams 1988; Schneider 1993; Hamon 2008).

Despite the variation of sample size by levels, there is a generally decreasing trend in the proportion of food processing tools from Level 11 to Level 4. One of the factors for this trend is apparently decreasing number of grinders and handstones in upper levels. Another factor is the appearance of some craft production tools in upper levels. For example axes have been recovered only from Levels 4 to 8 , and a crushing cobble occurs only in Level 4 . The latter tool type $(n=3)$ has been also recovered from topsoil or Levels 1-4 in the Azerbaijani excavation areas. The increase in the variety of craft production tools in upper levels is also indicated by the recovery of a perforated stone, a working slab, and floor polishers only from Levels 1-4 or topsoil in the Azerbaijani excavation areas. These tool types do not occur in Levels 4-11 in the Japanese excavation areas.

These observations on the decreasing proportions of food processing tools need to be tested with larger sample size in future. On the other hand, it is notable that food processing tool kits were already developed in early stages of occupations at Göytepe, suggesting that the development of milling technology predates Level 11 of Göytepe.

\subsection{Comparisons with other Neolithic ground stone assemblages in the southern Caucasus}

\subsubsection{Tool composition}

The description of finds from Shomutepe by Narimanov (1992: 14) mentions stone axes, grinding stones, pestles, mortars, mace heads, and sling stones. The last tool type corresponds to pebbles in this report, and mace heads may correspond to a perforated stone (Fig. 12.17: 1) at Göytepe. A similar 


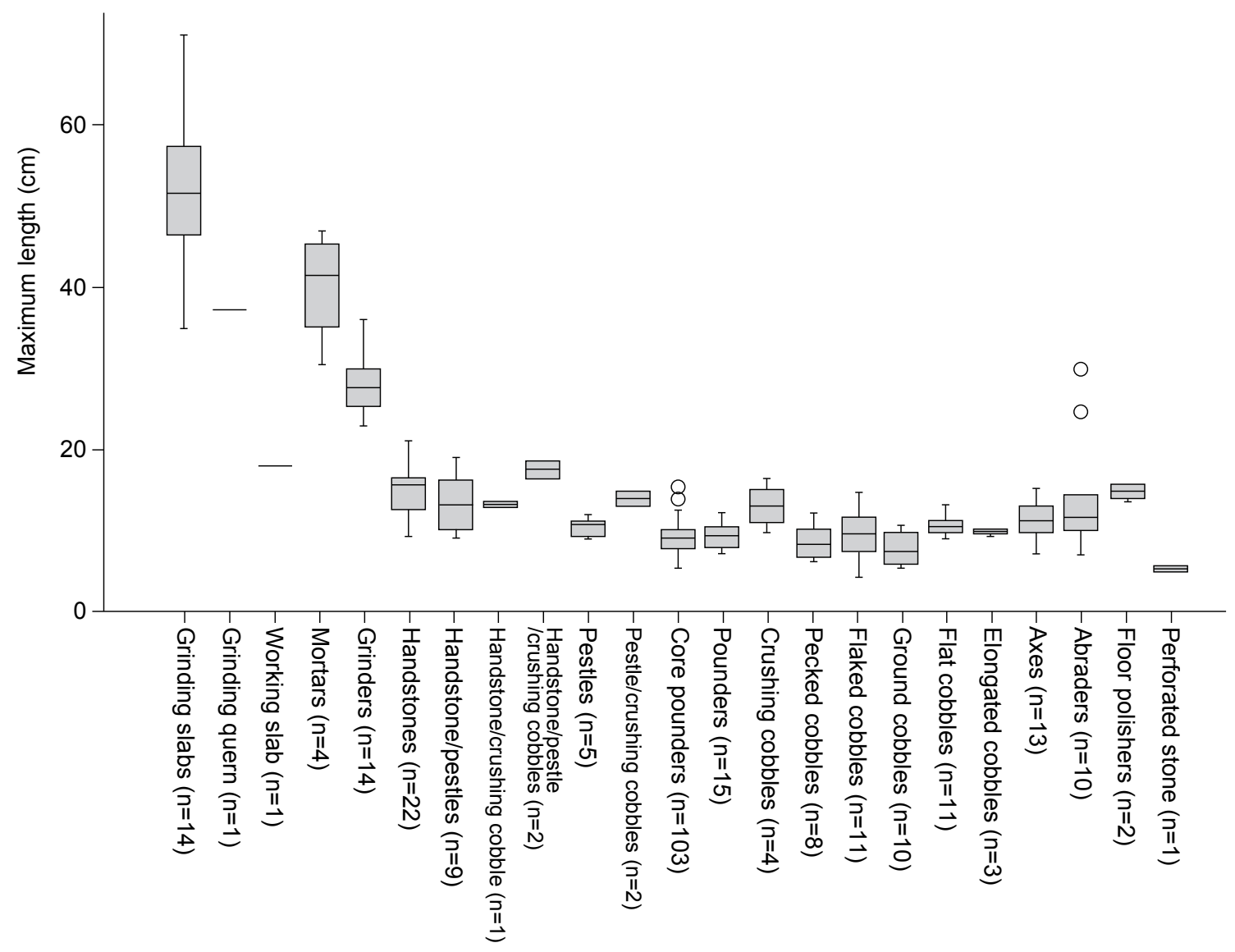

Fig. 12.22 Maximum length of complete ground stones, excluding flakes and pebbles, by tool types.

Table 12.4 List of ground stone artifacts recovered in clay bins.

\begin{tabular}{|l|r|l|l|l|l|l|}
\hline \multicolumn{1}{|c|}{ Context } & Level & \multicolumn{1}{|c|}{ Type } & $\#$ & \multicolumn{1}{|c|}{ Raw material } & Completeness & Notes \\
\hline 4BIIX-58 & 10 & Handstone & 1 & Basalt & Complete & \\
\hline & & Pounder & 1 & Basalt & Complete & \\
\hline 4BIIX-68 & 10 & Flake & 1 & Basalt & Complete & \\
\hline 4BIIX-69 & 10 & Flake & 2 & Basalt & Unrecorded & \\
\hline 4BIIX-74 & 10 & Core pounder & 1 & Basalt & Complete & \\
\hline $\begin{array}{l}\text { 4BIIX-94 (see } \\
\text { Chapter 7 and } \\
\text { Kadowaki et al. } \\
\text { 2015) }\end{array}$ & 10 & Handstone & 1 & Basalt & Fragment & \\
\cline { 2 - 7 } & & Handstone & 1 & Basalt & Complete & Fig. 12.8: 4, Fig. 12.18: 3. \\
\hline
\end{tabular}

range of ground stone tools is reported by Chataigner (1995: 147-170) in her compilation of Neolithic and Chalcolithic archaeological records of the Caucasus.

More recently, Hamon (2008) describes ground stone assemblages in the Kwemo-Kartli regions with quantitative data and proposes diachronic trends, in which activities with ground stones (macrolithic implements) become diversified. Using her data, Table 12.6 compares the frequencies of ground stone tool types at Göytepe with those at some Neolithic sites in the Kwemo-Kartli region. Comparisons exclude pebbles, which correspond to sling-stones in Hamon (2008) because the latter does not report the number of specimens. Also excluded are flakes, unidentifiable pieces, and some ad hoc tools, such as ground cobbles, flaked cobbles, and flat cobbles, which are not included in the type list of Hamon (2008). 


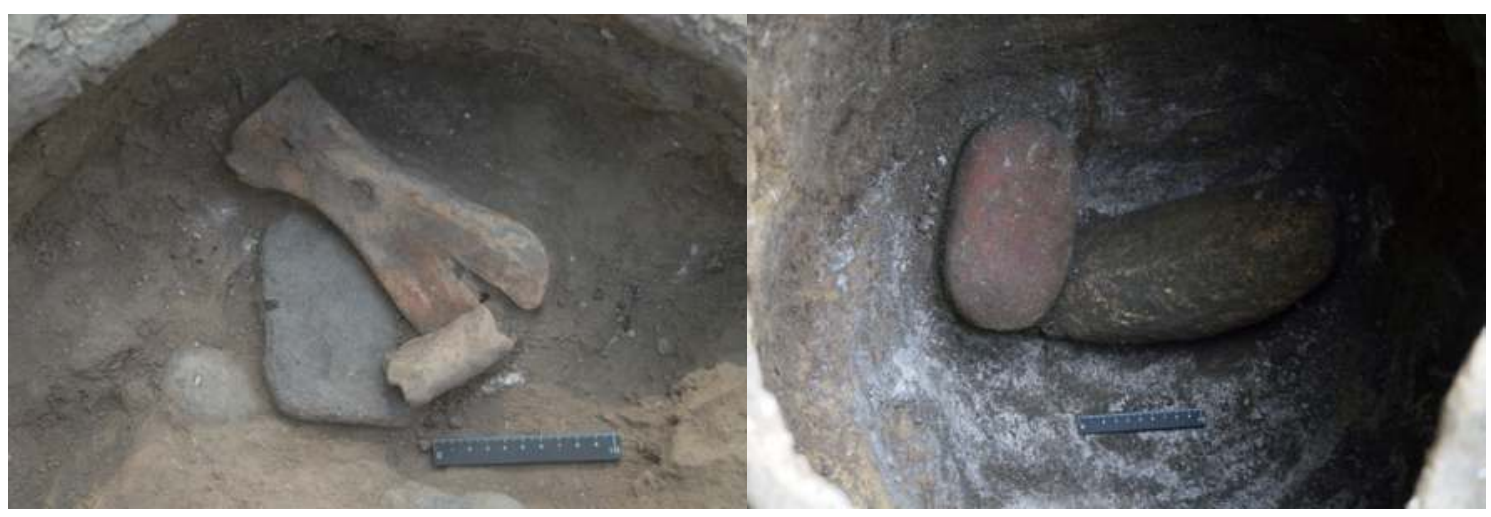

Fig. 12.23 Ground stones and other tools recovered in the clay bins. Left: In the middle fill (4BIIX-58, Level 10). Right: On top of the bottom fill with high concentration of grass, probably wheat, phytoliths (4BIIX-94, Level 10).

Fig. 12.24 shows the result of the Correspondence Analysis that examines the compositions of ground stone tool types at Göytepe and other sites in Table 12.6. The result indicates that the tool kit of Göytepe is most similar to that of Shulaveri Gora. The same result occurs whether the Göytepe samples consist of only those of the Japanese excavations (Levels 4-11) or include also the Azerbaijani selective collections.

A seriation of the Kwemo-Kartlian sites in Fig. 12.24 apparently fits their chronological order suggested by Hamon (2008: 108), who suggests a trend from the dominance of food procurement at Shulaveri Gora to the diversification of activities involving a wider range of macrolithic implements at Khramis Digi Gora. According to this scheme, the ground stone types at Göytepe are characterized by strong association with food processing tools, which in turn means the smaller variability of tool types, indicating its early chronological position. However, radiocarbon dates of Levels 1-14 at Göytepe range between 5650 and $5450 \mathrm{cal}$. BC (Nishiaki et al. 2015a) and slightly younger than the date (5770-5660 cal. BC) in the recent report of Aruchlo I (Hansen et al. 2007), which are apparently associated with nonfood processing tools more strongly. Thus, further analyses may be necessary to clarify potentially varied timings and scales of increasing variability of ground stones in the middle Kura region.

\subsubsection{Size range}

Fig. 12.25 shows that complete querns/slabs and mortars from Göytepe are generally larger than those from the Kwemo-Kartlian sites. Despite the limited sample size, such dimensional differences may be related to a number of factors, e.g., the distance to raw material sources, the kinds and sizes of available raw materials, the intensity of tool use, and potentially methodological differences in collecting and classifying artifacts.

Fig. 12.26 compares the length of complete grinders and handstones from Göytepe with those of grinders from the Kwemo-Kartlian sites. The dimensional range of grinders from Göytepe is similar to that of Aruchlo and overlaps with the upper range of Imiris and Khramis Digi gora. If handstones are added in the Göytepe samples, their distributions become more similar to that of the Imiris grinders. This indicates that criteria for classifying grinders and handstones differ between this report and Hamon (2008). In any case, it is notable that grinders of Göytepe are apparently larger than those of Shulaveri gora despite their resemblance in the tool compositions that are characterized by the dominance of food processing tools.

\subsubsection{Raw material use}

In contrast to the frequent use of basalt and sandstone for grinding querns, mortars, and grinders in the Kwemo-Kartli region (Hamon 2008), andesite is the dominant raw material type for such tools at Göytepe. However, as for the distinction between andesite and basalt, both of which are volcanic rocks, it is possible that similar raw materials are classified into different categories depending on researchers.

As described in the chaines opératoires of ground stone production at Göytepe (Fig. 12.20), large $(35-71 \mathrm{~cm})$ and medium $(23-32 \mathrm{~cm})$ size tools, such as grinding slabs, mortars, and grinders, are mostly made of andesite, while basalt is dominant in the small tools $(4-21 \mathrm{~cm})$, including handstones, 
Table 12.5 Occurrences of ground stone artifact types, excluding flakes, pebbles, and unidentifiable pieces, by levels.

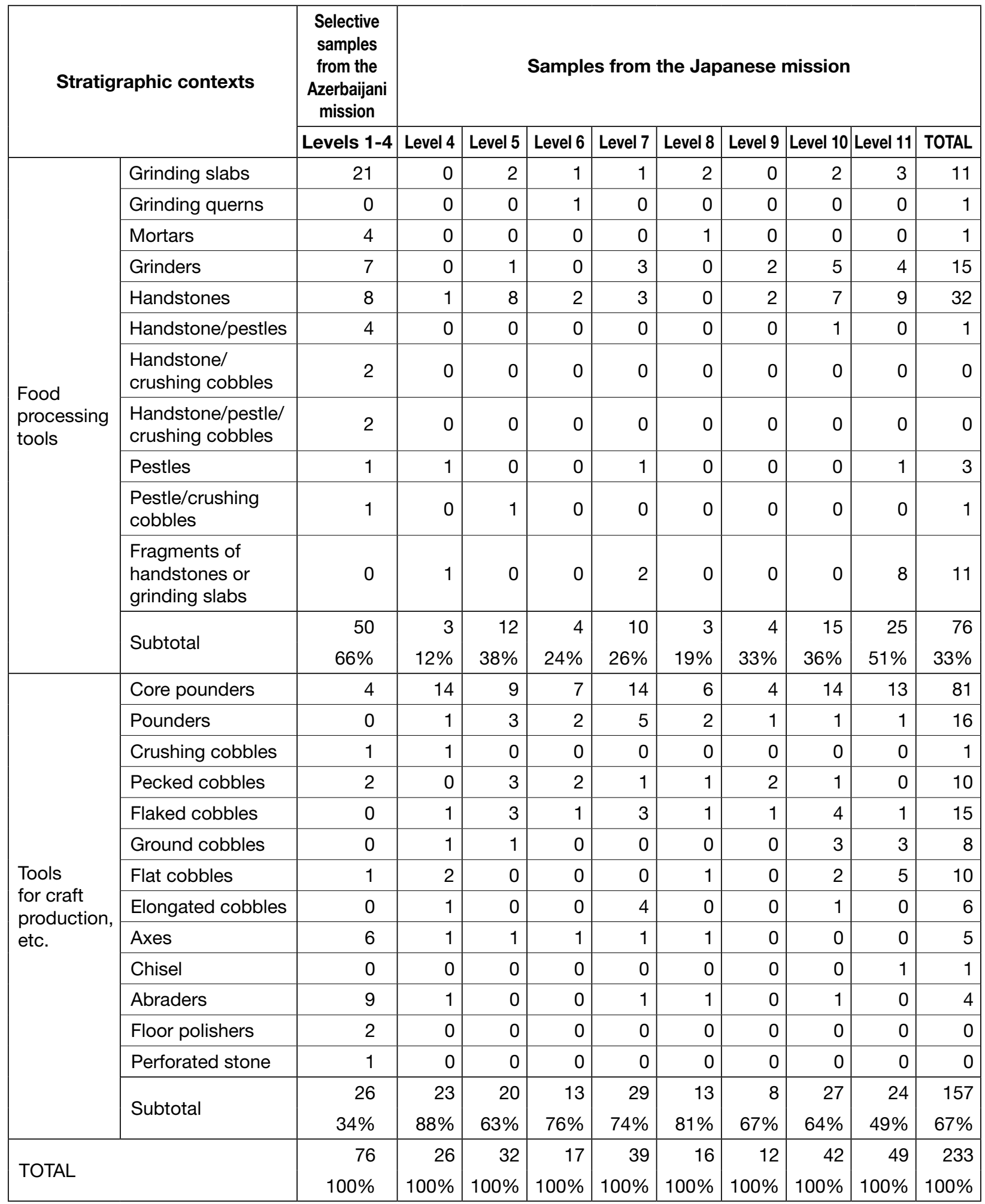

pestles, axes, pounders, and various cobble tools. This pattern in the raw material selection is probably influenced by the natural occurrences of rocks around the site. Andesite outcrops are widely distributed in the foothills south to Göytepe and large andesite clasts are abundantly available near the outcrops. Because andesite and basalt tools from Göytepe often retain naturally rolled surfaces, nearby riverbeds are 
Table 12.6 Frequencies of ground stone tool types at Göytepe and select Neolithic sites in the Kwemo-Kartli region.

\begin{tabular}{|c|c|c|c|c|c|c|c|c|}
\hline & \multicolumn{2}{|c|}{ Correspondence of tool types } & \multicolumn{2}{|c|}{ Göytepe } & \multirow[b]{2}{*}{ Shulaveri } & \multirow[b]{2}{*}{ Imiris } & \multirow[b]{2}{*}{ Aruchlo I } & \multirow[b]{2}{*}{$\begin{array}{c}\text { Khramis } \\
\text { Didi }\end{array}$} \\
\hline & Göytepe & Hamon 2008 & Total & $\begin{array}{c}\text { Samples } \\
\text { from Levels } \\
4-11 \text { by the } \\
\text { Japanese } \\
\text { excavations }\end{array}$ & & & & \\
\hline \multirow{19}{*}{$\begin{array}{l}\text { Types } \\
\text { used for } \\
\text { comparison }\end{array}$} & $\begin{array}{l}\text { Grinding slabs and } \\
\text { grinding querns }\end{array}$ & $\begin{array}{l}\text { Quadrangular querns, flat } \\
\text { basaltic querns, ovoid } \\
\text { querns, and narrow querns }\end{array}$ & 43 & 12 & 6 & 13 & 5 & 1 \\
\hline & Working slabs & Grinding slabs & 1 & 0 & 1 & 7 & 2 & 1 \\
\hline & Mortars & Mortars & 5 & 1 & 0 & 3 & 2 & 0 \\
\hline & Grinders & Grinders (flat and short) & 26 & 15 & 14 & 23 & 5 & 8 \\
\hline & $\begin{array}{l}\text { Handstones including } \\
\text { multiple tools }\end{array}$ & Hand stones & 72 & 44 & 7 & 11 & 1 & 14 \\
\hline & $\begin{array}{l}\text { Pestles including } \\
\text { multiple tools }\end{array}$ & Pestles & 8 & 4 & 5 & 21 & 12 & 30 \\
\hline & $\begin{array}{l}\text { Core pounders, } \\
\text { pounders, and } \\
\text { pecked cobbles }\end{array}$ & Hammer stones & 150 & 107 & 27 & 37 & 2 & 82 \\
\hline & Crushing cobbles & Crushing cobbles & 4 & 1 & 0 & 13 & 3 & 9 \\
\hline & $\begin{array}{l}\text { Elongated cobbles } \\
\text { with flaking scars }\end{array}$ & Splintered tools & 3 & 2 & 0 & 3 & 1 & 4 \\
\hline & Axes and chisels & Azes and axes & 16 & 6 & 2 & 4 & 3 & 87 \\
\hline & $\begin{array}{l}\text { Small prismatic } \\
\text { abraders }\end{array}$ & Prismatic polishers & 9 & 2 & 0 & 1 & 0 & 2 \\
\hline & $\begin{array}{l}\text { Flat concave } \\
\text { abraders }\end{array}$ & Handheld abraders & 2 & 0 & 1 & 3 & 1 & 2 \\
\hline & Perforated stones & Perforated objects & 1 & 0 & 1 & 7 & 3 & 7 \\
\hline & NA & Anvils & 0 & 0 & 2 & 4 & 0 & 0 \\
\hline & NA & Grooved abraders & 0 & 0 & 0 & 4 & 0 & 3 \\
\hline & NA & Sharpeners & 0 & 0 & 0 & 5 & 1 & 4 \\
\hline & NA & Limestone items & 0 & 0 & 0 & 3 & 0 & 25 \\
\hline & NA & Palettes & 0 & 0 & 0 & 4 & 1 & 0 \\
\hline & TOTAL & & 340 & 194 & 66 & 166 & 42 & 279 \\
\hline \multirow{10}{*}{$\begin{array}{l}\text { Types } \\
\text { excluded } \\
\text { from } \\
\text { comparison }\end{array}$} & Flaked cobbles & $?$ & 18 & 15 & $?$ & $?$ & $?$ & $?$ \\
\hline & Ground cobbles & $?$ & 10 & 8 & $?$ & $?$ & $?$ & $?$ \\
\hline & Flat cobbles & $?$ & 14 & 10 & $?$ & $?$ & $?$ & $?$ \\
\hline & Elongated cobbles & $?$ & 5 & 4 & $?$ & $?$ & $?$ & $?$ \\
\hline & Pebbles & Sling-stones & 989 & 935 & unreported & unreported & unreported & unreported \\
\hline & $\begin{array}{l}\text { Large prismatic } \\
\text { abraders }\end{array}$ & $?$ & 2 & 1 & $?$ & $?$ & $?$ & $?$ \\
\hline & Irregular abraders & $?$ & 1 & 1 & $?$ & $?$ & $?$ & $?$ \\
\hline & Floor polishers & $?$ & 2 & 0 & $?$ & $?$ & $?$ & $?$ \\
\hline & Flakes & $?$ & 363 & 277 & $?$ & $?$ & $?$ & $?$ \\
\hline & Unidentifiable objects & Undetermined & 1 & 1 & 4 & 3 & 1 & 6 \\
\hline
\end{tabular}

Data of Göytepe are from this paper, and those of other sites are from Hamon 2008. 


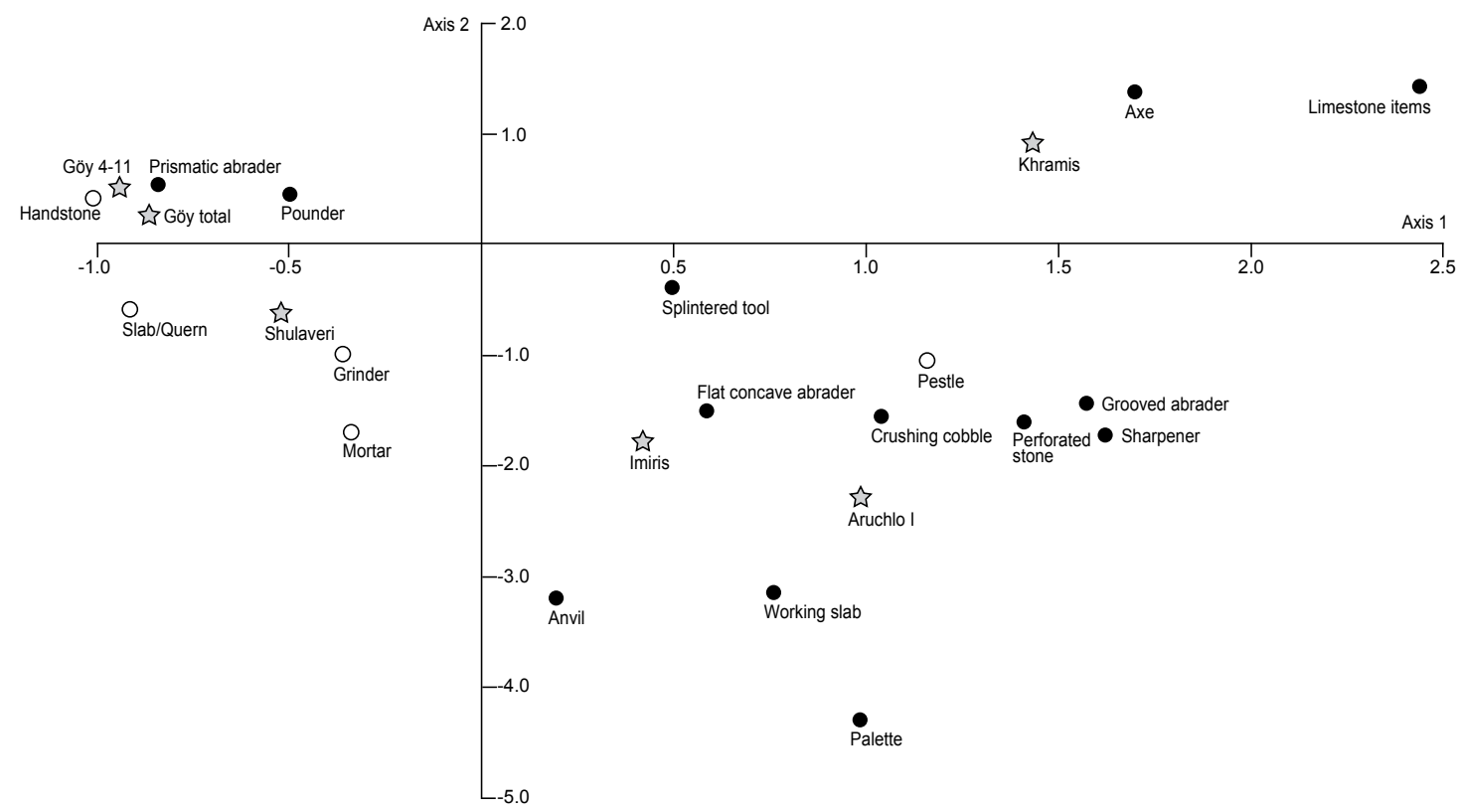

Fig. 12.24 Symmetric display of sites (stars) and their ground stone tool types (circles, in which open ones indicate food processing tools) in the space defined by Axes 1 and 2 that account for $54.0 \%$ and $35.4 \%$ of inertia of tool types respectively. See Table 12.6 for the data used in the analysis.

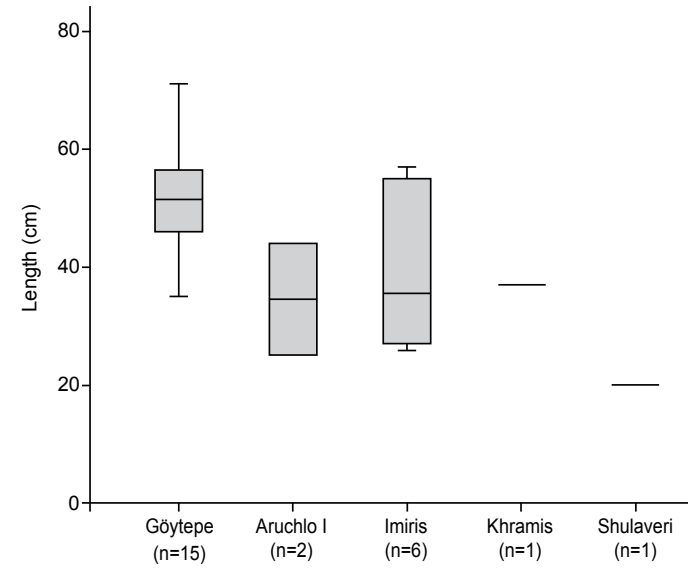

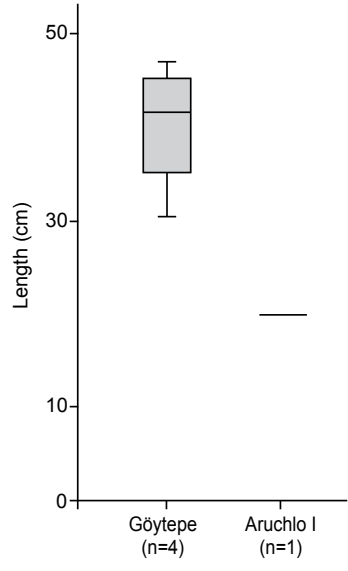

Fig. 12.25 Length of complete querns/ slabs (left) and mortars (right) at Göytepe and select Neolithic sites in the KwemoKartli region (Data of Göytepe are from this report, and those of other sites are from Hamon 2008). likely sources of boulders, cobbles, and pebbles of these rocks.

As noted earlier, andesite porous lava is used for abraders at Göytepe, which may or may not have parallels in other Neolithic sites in the southern Caucasus. In contrast to Khramis Digi gora, the use of limestone is not frequent at Göytepe like Shulaveri, Imiris, and Aruchlo.

\subsection{Summary}

To summarize the above examinations of ground stone assemblages from Göytepe, two technotypological characteristics are noteworthy for their culture-historical and behavioral implications.

The first is the preponderance of food-processing tools, such as grinding slabs, grinders, handstones, mortars, and pestles, in the tool composition at Göytepe. This is indicated by the comparison of tooltype frequencies among some Neolithic sites in the middle Kura region (Fig. 12.24). In comparison with 


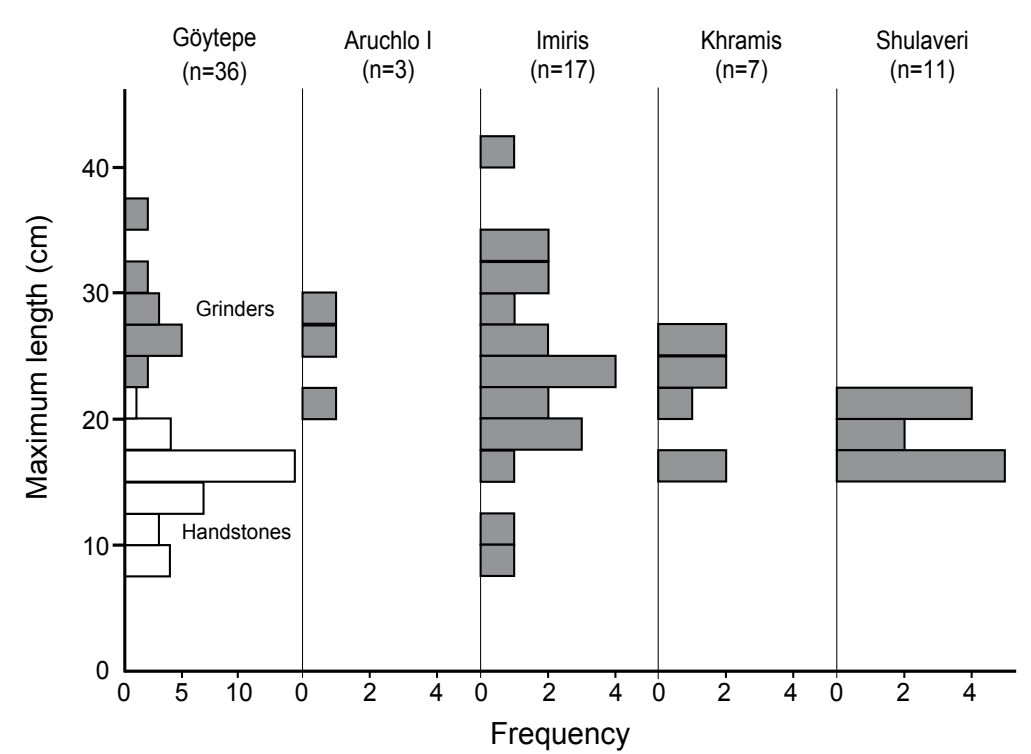

Fig. 12.26 Size-frequency distributions of the maximum length of complete grinders (filled bars) and handstones (open bars) at Göytepe and select Neolithic sites in the Kwemo-Kartli region (Data of Göytepe are from this report, and those of other sites are from Hamon 2008).
Göytepe, ground stones of other sites include higher percentages of non-milling implements, such as axes, grooved abraders, perforated stones, sharpeners, and various limestone items.

Such typological variability may represent a diachronic trend, in which the early dominance of food-processing tools shifts to later, more diverse tool-kits, as suggested by Hamon (2008). In fact, the ground stones from the Japanese excavations at Göytepe show stratigraphic changes towards higher percentages of non-milling tools (Table 12.5). Moreover, the samples from Levels 1-4, excavated by the Azerbaijani mission, include new tool types, such as crushing cobbles, perforated stones, and floor polishers. However, the latter case can merely be the result of larger excavation areas by the Azerbaijani mission. Thus, further chronological studies are necessary to clarify the timing and the regional variability in the process of diversification of ground stones in the middle Kura region.

At a wider regional scale, greater typological ranges, including elaborate stone vessels and various perforated stones, are known from some Late Neolithic sites in southern Turkey, northern Mesopotamia, and the Levant (e.g., Carter et al. 2003; Collet and Spoor 1996; Gopher and Orelle 1995; Kadowaki 2007), where the Halaf and the Wadi Rabah chrono-cultural entities are roughly contemporary to the ShomutepeShulaveri culture. Future studies can address the question of whether similar craft production was performed in the southern Caucasus, and if it was, when and how it developed.

Another notable characteristic is the appearance of developed grinding technology since earlier occupations at Göytepe. Grinding tools in the early levels (e.g., Levels 10 and 11) mainly consist of grinding slabs and grinders with some handstones and a few pestles. The form of pestles is rather short and actually includes handstones re-used for pounding at their distal ends. The grinding slabs have more or less concave grinding surfaces, assuming a saddle shape (Fig. 12.1: 6), and the large size of grinders would have required both hands to manipulate them (Figs. 12.5 and 12.6). The production of these large and medium grinding tools was systematically achieved by the selection of andesite boulders and coarse cobbles, which were modified by pounding tools made of basalt cobbles (Fig. 12.20).

In the Levant, saddle-shaped grinding slabs and large handstones developed in the late Pre-Pottery Neolithic B period (late eighth millennium cal. BC) in tandem with the growth of settlement size and demands for greater intensity of cereal processing (Wright 1993; Kadowaki 2014). Although it is hardly surprising that similar grinding technology was employed by early agriculturalists in the middle 6th millennium cal. BC in the southern Caucasus, the issue is when and how this grinding technology developed in the latter region. The use of grinding slabs and grinders for cereal processing at Göytepe is suggested by their morphologies, macroscopic usewears, i.e., patterned striations, and the recovery of two upper grinding stones in the storage bin for cereal grains (Fig. 12.23; also see Chapter 7 and Kadowaki et al. 2015 for phytoliths analyses). Given this close relationship between grinding tools and cereal 
processing, clarifying when and how this grinding technology emerged should provide significant insights into the development of agricultural practices in this region.

Regarding this problem, recent studies in West Azerbaijan suggest a sudden development of grinding tools in association with the appearance of domesticated cereals at the transition from a final Mesolithic campsite at Damjili Cave (Nishiaki et al. 2019) to a Neolithic settlement at Hac1 Elamxanlı Tepe that predates Göytepe (Nishiaki et al. 2015a and 2015b). These new records suggest introductions of cereal production and processing technology from southern regions, such as Anatolia and northern Mesopotamia, where such technologies developed earlier. This cultural influx that triggered the Neolithization in the southern Caucasus may have been associated with migrations of farmers and/or achieved by local Mesolithic foragers through their contacts with the southern farmers. Further studies are necessary to clarify more concrete pictures about the Neolithization in the southern Caucasus.

\section{References}

Adams, J. L. (1988) Use-wear analyses on manos and hide-processing stones. Journal of Field Archaeology, 15: 307-315.

Adams, J. L. (2002) Ground Stone Analysis: A Technological Approach. Utah: University of Utah Press.

Carter, E., S. Campbell, and S. Gauld (2003) Elusive complexity: New data from late Halaf Domuztepe in south central Turkey. Paléorient, 29(2): 117-133.

Chataigner, C. (1995) La Transcaucasie au Néolithique et au Chalcolithique. BAR Internatinal Series 624. Oxford: Tempus Reparatum.

Collet, P. and R. H. Spoor (1996) The ground-stone industry. In: Tell Sabi Abyad: The Late Neolithic Settlement, edited by P. M. M. G. Akkermans, pp. 415-438. Leiden and Istanbul: Nederlands Historisch-Archaeologisch Instituut.

Gopher, A. and E. Orelle (1995) The Ground Stone Assemblages of Munhata, A Neolithic Site in the Jordan Valley - Israel. A Report. Les Cahiers des Missions Archéologiques Françaises en Israel. Paris: Association Paleorient.

Hamon, C. (2008) From Neolithic to Chalcolithic in the southern Caucasus: Economy and macrolithic implements from Shulaveri-Shomu sites of KwemoKartli (Georgia). Paléorient, 34(2): 85-135.
Hansen, S., G. Mirtskhulava, and K. Bastert-Lamprichs (2007) Aruchlo: A Neolithic settlement mound in the Caucasus. Neo-Lithics, 1/07: 13-19.

Hayden, B. (1987) Lithic Studies Among the Contemporary Highland Maya. Tucson: The University of Arizona Press.

Kadowaki, S. (2007) Changing Community Life at a Late Neolithic Farmstead: Built Environments and the Use of Space at Tabaqat al-Bûma in Wadi Ziqlab, Northern Jordan. Tronto: Department of Anthropology, University of Toronto (Ph.D. Dissertation).

Kadowaki, S. (2014) Groundstones and grinding technology. In: The Sands of Time: The Desert Neolithic Settlement at Ayn Abū Nukayla, edited by D. O. Henry and J. E. Beaver, pp. 259-285. Berlin: ex oriente.

Kadowaki, S., L. Maher, M. Portillo, R. M Albert, C. Akashi, F. Guliyev, and Y. Nishiaki (2015) Geoarchaeological and palaeobotanical evidence for prehistoric cereal storage at the Neolithic settlement of Göytepe (mid 8th millennium BP) in the southern Caucasus. Journal of Archaeological Science, 53: 408-425.

Narimanov, I. G. (1992) The earliest agricultural settlements in the territory of Azerbaidzhan. In: Recent Discoveries in Transcaucasia, edited by P. L. Kohl, pp. 9-66. Soviet Anthropology and Archeology 30(4). M. E. Sharpe, N.Y. (English translation of Narimanov 1987: pp. 14-69).

Nishiaki, Y., F. Guliyev, and S. Kadowaki (2015a) Chronological contexts of the earliest Pottery Neolithic in the Southern Caucasus: Radiocarbon dates for Göytepe and Hacı Elamxanlı Tepe, West Azerbaijan. American Journal of Archaeology, 119(3): 279-294.

Nishiaki, Y., F. Guliyev, S. Kadowaki, V. Alakbarov, T. Miki, S. Salimbeyov, C. Akashi, and S. Arai (2015b) Investigating cultural and socioeconomic change at the beginning of the Pottery Neolithic in the Southern Caucasus - The 2013 Excavations at Hacl Elamxanlı Tepe, Azerbaijan. Bulletin of the American School of Oriental Research, 374: 1-28.

Nishiaki, Y., A. Zeynalov, M. Mansrov, C. Akashi, S. Arai, K. Shimogama, and F. Guliyev (2019) The Mesolithic-Neolithic interface in the Southern Caucasus: 2016-2017 excavations at Damjili Cave, West Azerbaijan. Archaeological Research in Asia, 19: 100140.

Roubet, C. (1989) Report on Site E-82-1: A workshop for the manufacture of grinding stones at Wadi Kubbaniya. In: The Prehistory of Wadi Kubbaniya, Volume 3, edited by A. E. Close, pp. 588-608. Dallas: Southern Methodist University Press.

Schneider, J. S. (1993) Milling implements: biases and problems in their use as indicators of prehistoric 
behavior and paleoenvironment. Pacific Coast Archaeological Society Quarterly, 29(4): 5-21.

Wilke, P. J. and L. A. Quintero (1996) Near Eastern Neolithic millstone production: Insights from research in the arid southwestern United States. In: Neolithic Chipped Stone Industries of the Fertile Crescent, and their Contemporaries in Adjacent Regions, edited by S. K. Kozlowski and H. G. K. Gebel, pp. 243-260. Berlin: ex oriente.

Wright, K. I. (1992) A classification system for ground stone tools from the prehistoric Levant. Paléorient, 18(2): 53-81.

Wright, K. I. (1993) Early Holocene ground stone assemblages in the Levant. Levant, 25: 93-111.

Wright, M. K. (1990) Sandstone and Cornmeal: Experimental Use of Early Pueblo Maize Grinding Tools from Southwestern Colorado. Washington: Department of Anthropology, Washington State University (MA Thesis). 

Chapter 13

\title{
Neolithic pottery from Göytepe
}

\author{
Yui Arimatsu
}

\subsection{Introduction}

A large amount of pottery was recovered from the excavations of Göytepe. In order to define its technostylistic characteristics and diachronic changes, this chapter examines pottery assemblages by their stratigraphic context. The study samples consist of all pottery specimens excavated from Square 4B (Sectors 4BI and 4BII) and part of the materials from Square 3B (3BI and 3BII), both of which were recovered under strict stratigraphic control (Chapters 3 and 4). The sample size numbers 2,098 finds. The distribution of pottery specimens by squares and levels is shown in Table 13.1. Since the two squares are situated close to the edge of the mound, the samples belong to the earlier levels of the Neolithic sequence, Levels 4-13. They include not only sherds of body, rim, and base, but also spindle whorls made on reused pottery. Some of these are decorated. We have not yet found a complete pottery vessel in these levels.
All of the pottery assemblages from Göytepe are dated to the Pottery Neolithic period, representing typical Shomutepe assemblages. They can be divided into several types based on their technological and morphological attributes. Several stages can be identified on the basis of the stratigraphic changes of pottery. The changes quite likely reflect important aspects relating to the appearance and acceptance processes of pottery production in the Southern Caucasus. Quantitative data on these features are presented below (Figs. 13.1-13.6) and illustrations and photos of the pottery are found at the end of this chapter (Figs. 13.7-13.21).

\subsection{Classification of Göytepe pottery}

We can discern some variety in both pottery production techniques and vessel form. It is likely that all pottery was handmade with coils or small slabs. However, other techniques (e.g., preparation

Table 13.1 The pottery assemblages described in this chapter by squares and levels.

\begin{tabular}{|c|c|c|c|c|c|}
\hline Squares & 3BI & 3BII & 4BI & 4BII & Total \\
\hline Level 4 & 473 & 5 & & & 478 \\
\hline Level 5 & 496 & & 258 & & 754 \\
\hline Level 6 & & 8 & 81 & 98 & 187 \\
\hline Level 7 & & & 161 & 101 & 262 \\
\hline Level 8 & & & 43 & & 43 \\
\hline Level 9 & & & 61 & 20 & 81 \\
\hline Level 10 & & & 76 & 150 & 226 \\
\hline Level 11 & & & 26 & 15 & 41 \\
\hline Level 12 & & & & 19 & 19 \\
\hline Level 13 & & & & 7 & 7 \\
\hline Total & 969 & 13 & 706 & 410 & 2098 \\
\hline
\end{tabular}


of paste, surface treatment, decoration techniques, and firing method) show a great deal of variability. The variation of technical attributes corresponds to the differences in form and decoration, and probably their function as well. Although we have found some restorable sherds that were once shallow bowls (Figs. 13.7: 12-13 and 13.19: 5) and neckless jars (Figs. 13.12: 1 and 13.16), it was difficult to determine the complete shape of a vessel from most of the excavated sherds. The rim shape does not vary much: it is either simple or round. We cannot find sufficient characteristics for estimating their precise shape. Only a few of fragments of handle (Fig. 13.8: 3), leg, and spout were found.

Although there are some chronological/regional variations in pottery of the Shomutepe culture, we can say that its forms are limited: they mainly comprise truncated cone-shaped bowls, shortnecked jars, long-necked jars, cylindrical shallow bowls, deep bowls, barrel-shaped vessels, and eggshaped jars (Chataigner 1995: 87-119). Generally, the truncated cone-shaped bowl has a convex wall. The cylindrical shallow bowl has straight walls, whereas the all of a deep bowl is not sloped around the rim. The barrel-shaped vessel and egg-shaped jar both have concave rims. The convex and straight rim could be part of the jar's neck. Compared with examples of other sites, we could roughly estimate the following correspondence: a concave rim to eggshaped jar or barrel-shaped vessel, a straight rim to deep bowl, a cylindrical shallow bowl or neck part of jar, a convex rim to truncated cone-shaped bowl or neck of jar. On the other hand, all identifiable base parts are flat (Figs. 13.14 and 13.15). A part of the convex-shaped or round base could not be precisely identified. Basically, it is difficult to distinguish between two forms of base. However, considering that the flat base predominates at other representative sites of the Shomutepe culture (Chataigner 1995: 100), we propose a similar situation for Göytepe.

After integrating these technological and morphological variations, excavated pottery sherds of Göytepe can be classified into seven categories (Table 13.2). Division between the first four vessel categories and the others depends on inclusions in the clay. Types I to IV contain organic inclusions, while the others contain mainly mineral inclusions.
The proportion of these two categories is similar (Fig. 13.1). Each group is subdivided by the amount/ combination of inclusion and surface treatment.

\subsubsection{Organic-tempered wares: Types I to IV}

Type $I$ is the finest and the rarest ware in this site (Fig. 13.1). It rarely contains small grains of organic inclusions in its paste. Other inclusions are small, brown minerals with $0.1-0.5 \mathrm{~mm}$ diameters. Interior and exterior surfaces are carefully wet-smoothed or lightly burnished. Their surface is invariably wellfinished, and slip is also applied on some of them. In this case, surfaces are dull brown, dull orange, or reddish brown. The cross-section shows these same colors, often with a gray core. This type is relatively well-fired without any traces of a secondary firing. The wall is relatively thin, measuring less than 10 $\mathrm{mm}$. This type probably corresponds to open shaped vessels (Fig. 13.7: 1-3).

Type II is an organic- and mineral-tempered common ware. Proportions of these two kinds of inclusion are approximately the same. The brown or reddish-brown mineral inclusions are about $0.5-1.0$ $\mathrm{mm}$ in diameter. Vessel surfaces were treated by light burnishing or careful wet-smoothing. After that, a buff slip was applied on the surfaces in some cases. They are generally a dull brown or dull orange color. These sherds were relatively well-fired but sometimes maintain a gray core. This ware type is represented by both open and closed vessels (Figs. 13.7: 4-5 and 7, and 13.10: 1). In some cases, a reddish horizontal line was painted on the rim.

Type III is also a common ware. This type represents the majority of organic-tempered wares (Fig. 13.1). It contains many 3.0-6.0 mm-sized organic inclusions and black and dark-reddish mineral inclusions with $1.0-5.0 \mathrm{~mm}$ in diameters. Traces of organic inclusions are visible on the surface. The surfaces were treated by wet-smoothing or, sometimes light-burnishing or application of slip. They are dull orange or bright reddish brown in color (Figs. 13.17: 1, 13.18: 5, and 13.19: 2-6). This type was relatively poorly-fired and a gray core remains visible in cross-section. There are some examples decorated with paint or relief(Figs. 13.13: 15-18 and 13.19: 5 and 6). The painted decoration is simple, consisting of only horizontal lines on the rim. The 
Table 13.2 Classification of pottery types for Göytepe.

\begin{tabular}{|c|c|c|c|c|c|}
\hline \multirow{2}{*}{ TYPE } & \multicolumn{5}{|c|}{ ATTRIBUTES OF POTTERY } \\
\hline & Paste/Inclusion & Surface treatment & Decoration & Form & Firing \\
\hline I & $\begin{array}{l}\text { Organic inclusions } \\
\text { and a small } \\
\text { mineral inclusions. } \\
\text { Inclusion itself is } \\
\text { rare. }\end{array}$ & $\begin{array}{l}\text { Careful wet smooth or light burnish. } \\
\text { Sometimes slipped. Surface presents } \\
\text { dull orange or bright reddish brown. } \\
\text { Sections is dull orange or dull brown. }\end{array}$ & Nothing. & $\begin{array}{l}\text { Out-curving } \\
\text { rim. Wall is } \\
\text { thin: } 5-10 \\
\mathrm{~mm} \text {. }\end{array}$ & $\begin{array}{l}\text { Relatively } \\
\text { well fired. } \\
\text { Gray core } \\
\text { remains in } \\
\text { some cases. }\end{array}$ \\
\hline II & $\begin{array}{l}\text { A lot of organic and } \\
\text { mineral inclusions: } \\
\text { same amount or } \\
\text { mineral ones are } \\
\text { more. }\end{array}$ & $\begin{array}{l}\text { Careful wet smooth or light burnish. } \\
\text { Sometimes with slip. Surfaces } \\
\text { present dull orange or bright reddish } \\
\text { brown. Section is dull orange or dull } \\
\text { brown. }\end{array}$ & $\begin{array}{l}\text { Sometimes } \\
\text { painted } \\
\text { decoration. }\end{array}$ & $\begin{array}{l}\text { Out-sloping } \\
\text { rim, and } \\
\text { sometimes } \\
\text { in-sloping. } \\
\text { Wall is thin: } \\
5-10 \mathrm{~mm} \text {. }\end{array}$ & $\begin{array}{l}\text { Relatively } \\
\text { well fired. } \\
\text { Sometimes } \\
\text { gray core } \\
\text { remains. }\end{array}$ \\
\hline III & $\begin{array}{l}\text { Organic inclusions } \\
\text { with } 3-6 \text { mm in } \\
\text { diameter and a little } \\
\text { mineral inclusions. } \\
\text { Dark brown mineral } \\
\text { inclusions with 0.5- } \\
1 \mathrm{~mm} \text { in diameter } \\
\text { are the majority. }\end{array}$ & $\begin{array}{l}\text { Often horizontal wet smooth. } \\
\text { Sometimes light burnish or red } \\
\text { slip. Traces of vegetable tempers } \\
\text { are visible on the surfaces. Surface } \\
\text { presents dull orange, bright reddish } \\
\text { brown, or sometimes more dark } \\
\text { color. Section is dull orange or dull } \\
\text { brown. }\end{array}$ & $\begin{array}{l}\text { Painted or } \\
\text { slipped. } \\
\text { Sometimes } \\
\text { reliefed. }\end{array}$ & $\begin{array}{l}\text { Out-sloping } \\
\text { and in- } \\
\text { sloping } \\
\text { rim. Wall is } \\
\text { reratively } \\
\text { thick: } 10-15 \\
\text { mm. }\end{array}$ & $\begin{array}{l}\text { Relatively } \\
\text { poorly fired } \\
\text { and crumbly. } \\
\text { Gray or black } \\
\text { core often } \\
\text { remains. }\end{array}$ \\
\hline IV & $\begin{array}{l}\text { A lot of organic } \\
\text { inclusions. Mainly } \\
1-3 \mathrm{~mm} \text { mineral } \\
\text { inclusions are } \\
\text { included. }\end{array}$ & $\begin{array}{l}\text { Light was smooth is major. } \\
\text { Sometimes with light burnish. } \\
\text { Surfaces present dull orange or } \\
\text { bright reddish brown. section is dull } \\
\text { orange or dull brown. }\end{array}$ & Nothing. & $\begin{array}{l}\text { Straight rim } \\
\text { is major and } \\
\text { in-sloping rim } \\
\text { also exists. } \\
\text { Wall is thick: } \\
10-25 \mathrm{~mm} \text {. }\end{array}$ & $\begin{array}{l}\text { Poorly fired } \\
\text { and generally } \\
\text { crumbly. } \\
\text { Sometimes } \\
\text { secondarily } \\
\text { fired. }\end{array}$ \\
\hline V & $\begin{array}{l}\text { Mineral inclusions: } \\
0.5-1 \mathrm{~mm} \text { in } \\
\text { diameter. }\end{array}$ & $\begin{array}{l}\text { Careful wet smooth or light burnish. } \\
\text { Sometimes with slip. Surface are } \\
\text { dull orange or bright reddish brown. } \\
\text { Section presents dull orange or } \\
\text { dull brown. Rarely inner and outer } \\
\text { surfaces are dark colored. }\end{array}$ & Nothing. & $\begin{array}{l}\text { Closed form. } \\
\text { Rim is in- } \\
\text { curving or } \\
\text { straight. Wall } \\
\text { is often thin: } \\
5-10 \mathrm{~mm} \text {. }\end{array}$ & Well fired. \\
\hline $\mathrm{VI}$ & $\begin{array}{l}\text { A lot of mineral } \\
\text { inclusions: } 3-5 \mathrm{~mm} \\
\text { in diameter. }\end{array}$ & $\begin{array}{l}\text { Mainly wet smooth: rough and } \\
\text { irregular. Sometimes mat impression } \\
\text { remains on the base. Surface } \\
\text { presents dull orange or bright } \\
\text { reddish brown. Section is dull } \\
\text { orange, dull brown, orange or bright } \\
\text { reddish brown. }\end{array}$ & $\begin{array}{l}\text { Relief } \\
\text { decorations: } \\
\text { knobs or } \\
\text { wavy line. }\end{array}$ & $\begin{array}{l}\text { Rim is in- } \\
\text { sloping and } \\
\text { exceptionally } \\
\text { out-sloping. } \\
\text { Wall is thin } \\
\text { or relatively } \\
\text { thick: } 5-15 \\
\text { mm. }\end{array}$ & $\begin{array}{l}\text { Well fired } \\
\text { and often } \\
\text { secodarily } \\
\text { fired. }\end{array}$ \\
\hline VII & $\begin{array}{l}\text { A lot of diverse } \\
\text { mineral inclusions. }\end{array}$ & $\begin{array}{l}\text { Light wet smooth. Surfaces present } \\
\text { dark browm or brownish black. } \\
\text { Section is grayish brown or brownish } \\
\text { black. Surfaces are often secodarily } \\
\text { fired and dark. }\end{array}$ & Nothing. & $\begin{array}{l}\text { Rim is in- } \\
\text { sloping or } \\
\text { straight. } \\
\text { Wall is thick: } \\
10-25 \mathrm{~mm} \text {. }\end{array}$ & $\begin{array}{l}\text { Generally } \\
\text { crumbly. } \\
\text { Often } \\
\text { secodarily } \\
\text { fired. }\end{array}$ \\
\hline
\end{tabular}

relief decoration is varied and commonly present in Type VI (see below). This type shows some variety in regard to paste and surface treatment. The walls are relatively thick, about $10-15 \mathrm{~mm}$. Diverse vessel forms probably correspond to this type (Figs. 13.7: 8-13, 13.8: 1-5, 8-9, 11-12, 13.9: 10-13, and 13.10: $2-4)$.
Type $I V$ is the coarsest of the organic-tempered wares. This type contains many $1.0-10.0 \mathrm{~mm}$ chaff inclusions, sometimes including grains. In addition, it has 1-3 $\mathrm{mm}$ mineral inclusions. The walls are thick (approximately 10-25 $\mathrm{mm}$ ). Surfaces were wet-smoothed or sometimes lightly burnished. In any case, the finishing treatment was not careful. 


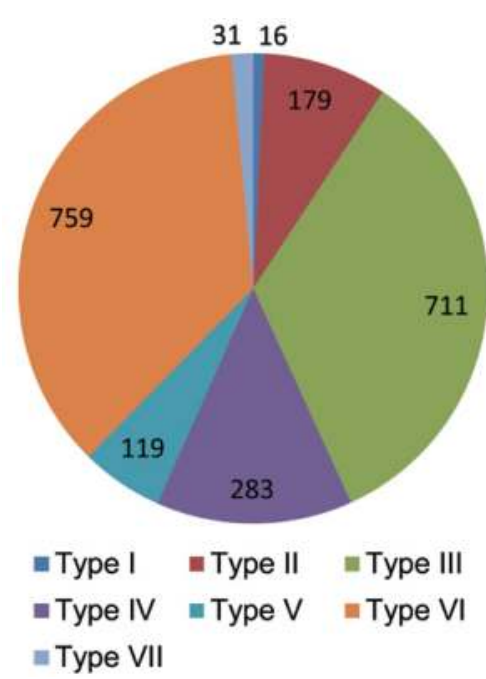

Fig. 13.1 Proportion of pottery types in the study collection of Göytepe.
Surfaces are dull brown or dull orange in color. This type was poorly-fired and crumbly in texture. A gray core was identified in the sherd's cross sections. This type corresponds to deep bowls or closed vessels.

\subsubsection{Mineral tempered wares: Types V to VII}

Type $V$ contains only small mineral inclusions (less than $1 \mathrm{~mm}$ ) in the paste. Surface treatments were made with careful wet-smoothing or light burnishing. Some vessels were then slipped, generally showing careful finishing treatment. They are dull brown or brownish gray in color. They were well-fired without any traces of secondary firing. The walls are relatively thin, measuring less than $10 \mathrm{~mm}$. Generally, this type would correspond to bowl type vessels or closed vessels (Figs. 13.7: 6, 13.8: 6-7, 13.9: 1-2, and 13.10: 5-6).

Type $V I$ is a mineral-tempered common ware. This type is the most frequent among mineraltempered wares overall (Fig. 13.1). At the same time, this type is characteristic of Shomutepe pottery (Chataigner 1995: 91-96, 112-117). It has brown or reddish-brown mineral inclusions about $3.0-5.0 \mathrm{~mm}$ in diameter. Though infrequent, obsidian was also used as an inclusion. Surfaces are treated by simple wet-smoothing at the early stage of drying. These vessels were well-fired and a gray core remains visible in cross-section. Interior and exterior surfaces were often secondarily fired (Figs. 13.16, 13.17: 4, and 13.20: 5). This type is limited to closed vessels or deep bowl-shaped vessels (Figs. 13.7: 14-15, 13.9: 3-9, 13.10: 7-12, 13.11: 3-10, and 13.16). On the base, a mat impression sometimes remains (Figs. 13.18: 6 and 13.21: 2). Relief decoration is also characteristic of this ware type (Figs. 13.12 and 13.13: 1-14).

Type VII is a very coarse ware. It has various mineral inclusions with diameters of $1.0-5.0 \mathrm{~mm}$. The walls are thick, approximately $10.0-25.0 \mathrm{~mm}$. The surfaces were treated by simple wet-smoothing and are grayish-brown or brownish-black in color. Interior and exterior surfaces were often secondarily fired. This type is generally very crumbly in texture. Its form is either a closed vessel or deep bowl (Fig. 13.11: 1).

\subsection{Stratigraphic changes of pottery}

Based on the above classification, we will survey the stratigraphic changes of pottery at Göytepe (Fig. 13.2). The analyzed data come from the Japanese trenches, since all excavated sherds have been recorded with contextual information. The earlier two-thirds of Göytepe, from Level 4 to Level 13, are the focus of this analysis. Three evolutionary stages could be identified in these levels.

\subsubsection{Development of mineral-tempered wares: Level 13 to Level 10}

In this stage, Type VI always predominates and Type III is simultaneously adopted. In Level $13(\mathrm{n}=7)$, which is the lowest level so far studied by the present author, the assemblage consists of common wares: Type III and Type VI. Pottery is limited overall. We found no rim parts and it is impossible to reconstruct any vessel form from the recovered sherds. At present, no decorative elements exist. In Level 12 ( $\mathrm{n}=19)$, we can confirm almost the same situation as in Level 13. 


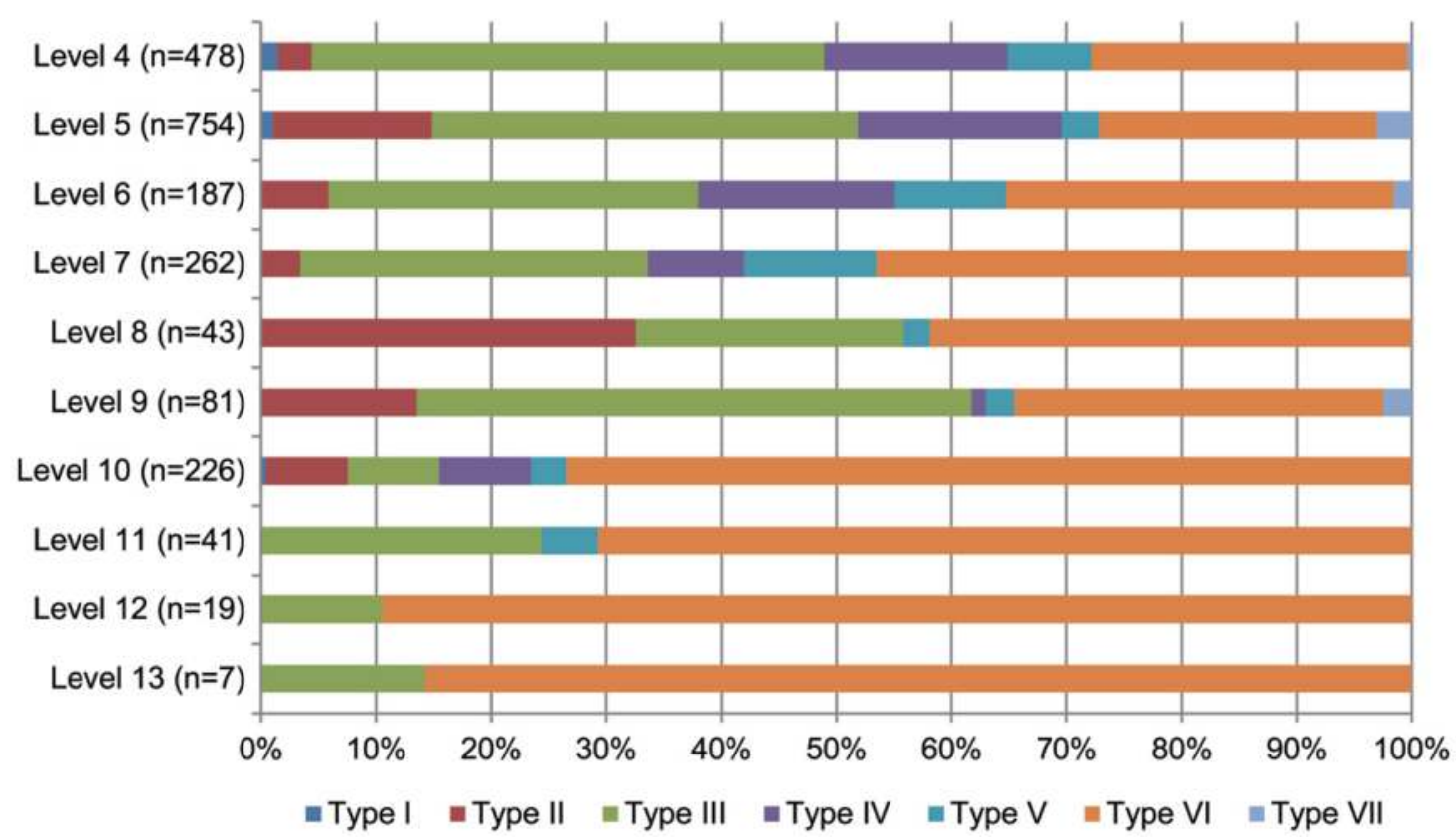

Fig. 13.2 Diachronic changes in the pottery types by level at Göytepe.

As far as we can detect, form is limited to concavewalled vessels in both types (Fig. 13.3). On the other hand, pottery itself increased relatively little. Level $11(\mathrm{n}=41)$ is characterized by an increasing diversity of the mineral-tempered ware. Although scarce $(n=2)$, Type V appears in this level. At Göytepe, this could represent the first clear example of a fine ware. However, we only identified concave shape (Fig.

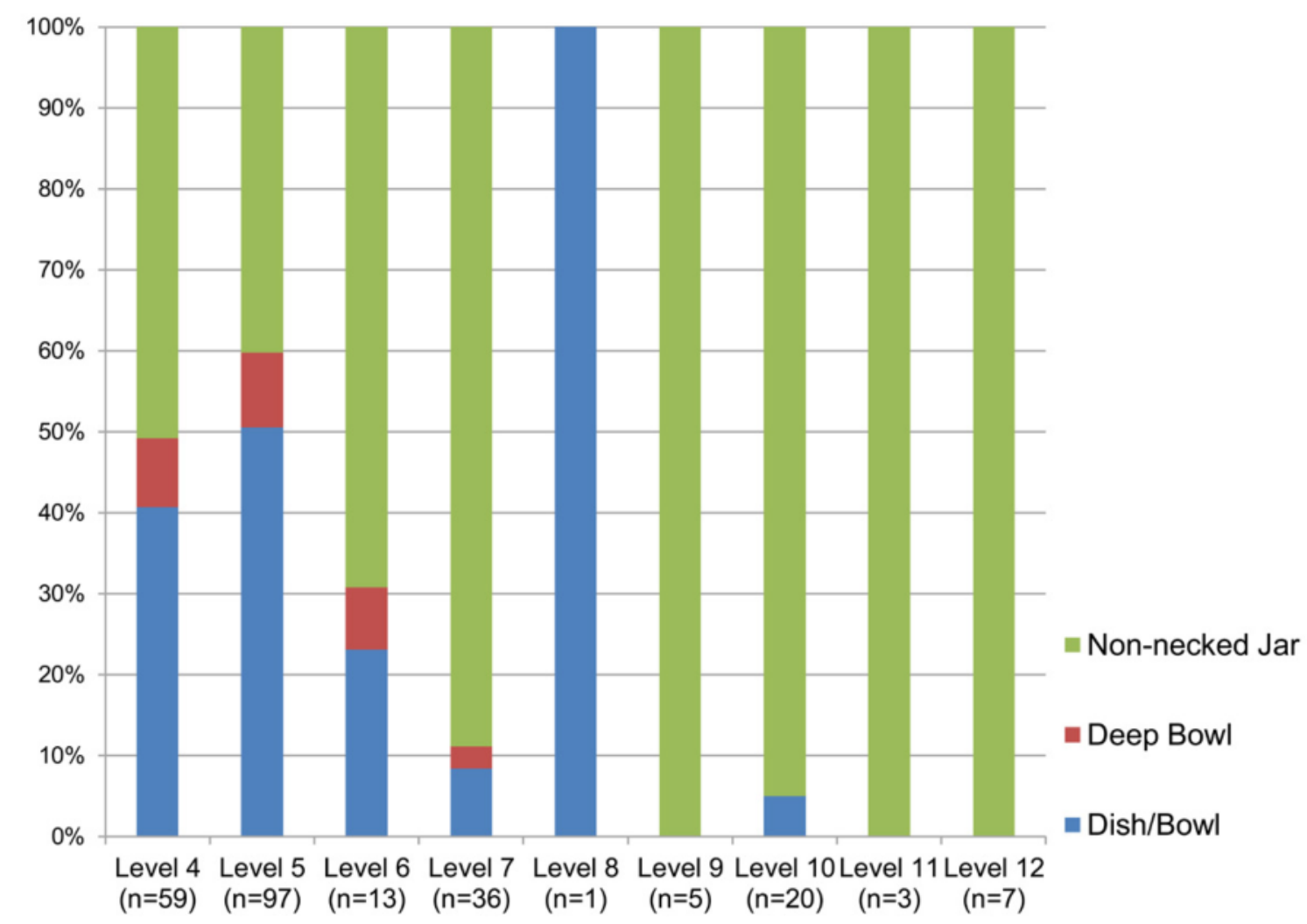

Fig. 13.3 Diachronic changes in the pottery shapes by level at Göytepe. 
13.3) and always lacking decoration (Fig. 13.6).

In Level 10, we found more abundant pottery sherds $(n=226)$. Although it might be a result of the abundance of finds, it appears that the pottery is diverse. Fine wares appeared along with organictempered wares: Type I and Type II. The coarsest type of organic-tempered ware, Type IV, is also confirmed in this level. It could be said that organictempered wares diversified most remarkably. On the other hand, mineral-tempered ware, especially Type VI, is always predominates (Fig. 13.2). Furthermore, open-shaped vessels appear in this level but are only represented in Type V (Fig. 13.4). The other types always correspond to closed shaped forms.

\subsubsection{Increase of organic tempered wares: Level 9 to Level 6}

A comparison of mineral- and organic-tempered wares shows that the proportions of these two categories reversed in Level $9(\mathrm{n}=81)$. The mineraltempered ware of Type VI accounted for more than
$70 \%$ up until Level 10. In Level 9, however, organictempered wares markedly increased $(63.0 \%)$. Considering the stability of ware type in the previous stage, the qualitative change in this stage cannot be considered part of a gradual evolution. However, Type III continuously predominates among the organic-tempered wares in this level and we cannot confirm any morphological diversification. It is in Level $8(\mathrm{n}=43)$ that technological and morphological diversifications of organic-tempered wares occurred. In this level, organic-tempered wares are most common $(55.8 \%)$. The finer organic-tempered ware, Type II, increased $(32.6 \%)$. Furthermore, openshaped forms appear in Type III (Figs. 13.4 and 13.5).

In Level 7 (total 262 sherds), organic-tempered wares decreased slightly (42.0\%). An increase of coarser organic-tempered ware, Type IV (8.4\%), is characteristic of this level. Relief decoration appeared among the mineral-tempered wares (Figs. 13.12: $2-7$ and 13.17: 4 and 6) and was common in the following levels. In regard to these mineral-

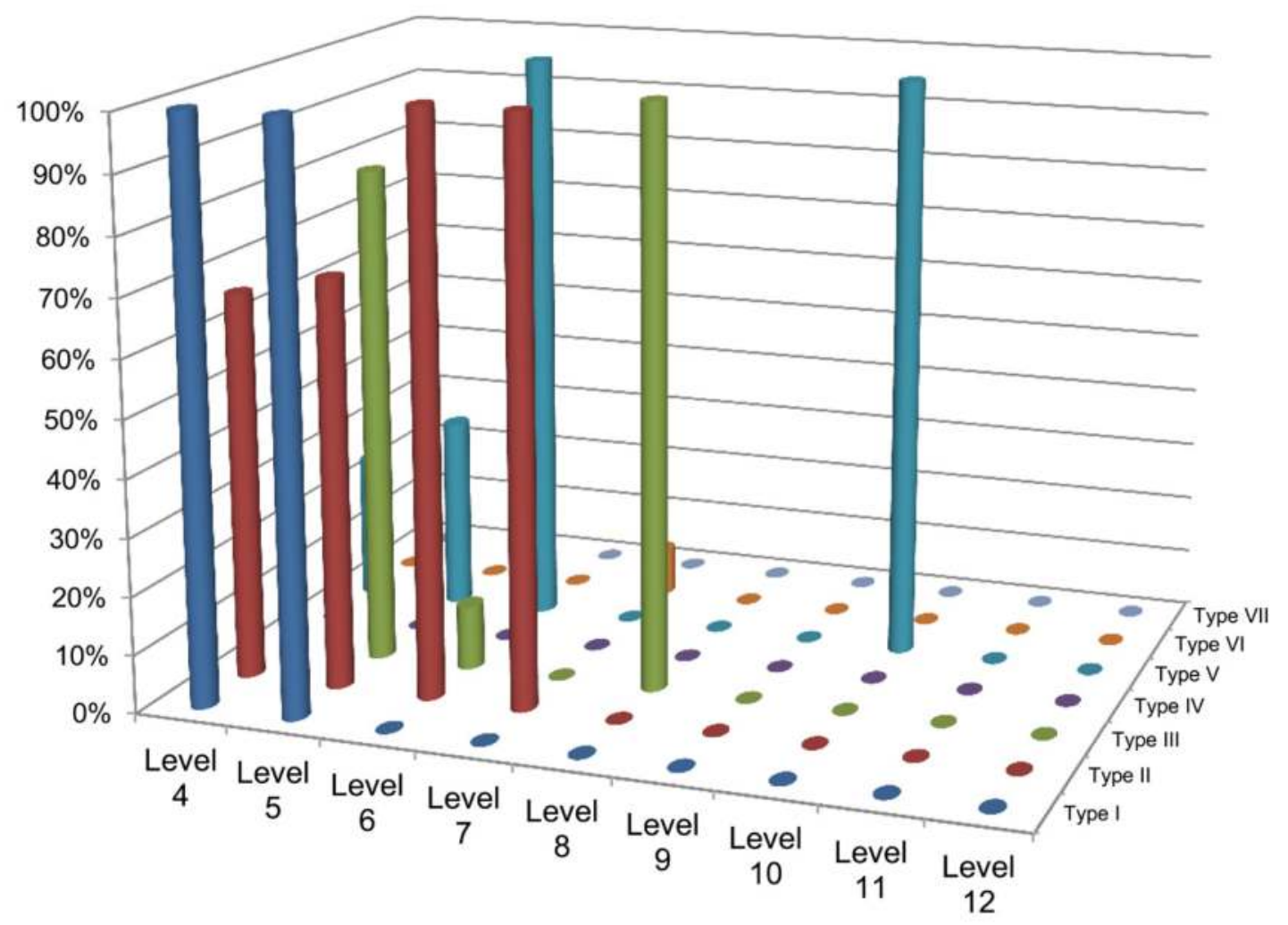

Fig. 13.4 Proportion of pottery types in open-shape pottery assemblages by level at Göytepe. 
tempered wares, we can detect an increase of finer Type V (11.5\%). In this way, Level 7 marks the beginning of the diversification of pottery. This trend became conspicuous in the following level. Level 6 $(n=187)$ is characterized by the full-scale introduction of fine and coarse types in both of organic- and mineral-tempered wares (Type II, Type IV, Type V, and Type VII) (Fig. 13.2). Furthermore, open-shaped and probably deep bowl forms increased, especially in Types II and V (Fig. 13.4). Relief decoration continued to be applied (Figs. 13.12: 8-10 and 13.18: $1-2)$.

\subsubsection{Functional diversification of pottery: Level 5 to Level 4}

After Level $5(\mathrm{n}=754 ; \mathrm{n}=478$ in Level 4$)$, more functional changes in pottery developed. First, pottery generally increased and morphological diversification occurred. Nearly half of the rims were open-shaped $(50.5 \%$ in Level $5,40.7 \%$ in Level 4). The finest (Type I) and the coarsest (Type
IV) organic-tempered wares increased. Use of characteristic inclusions began in Type VI mineraltempered wares. This dark reddish sand was utilized more clearly in the upper levels.

Decorative elements emerged more frequently (5.1\% in Level 5, 10.3\% in Level 4) (Fig. 13.6). The motifs of relief decoration diversified: we detected wavy lines (Fig. 13.13: 5-7), large circles (Fig. 13.13: 10), large ovals (Figs. 13.13: 2-4, 9, and 11-18 and 13.18: 3 and 6), and double-lined circles (Fig. 13.13: 8). Furthermore, an important development in this level is that relief decoration moved from mineraltempered ware (Type VI) into organic-tempered ware (Type III) (Figs. 13.13: 15-17 and 13.19: 5). This kind of decoration had been limited to Type VI prior to Level 6.

\subsection{Position of Göytepe pottery}

\subsubsection{Chronological position}

Göytepe, situated in the central region of the

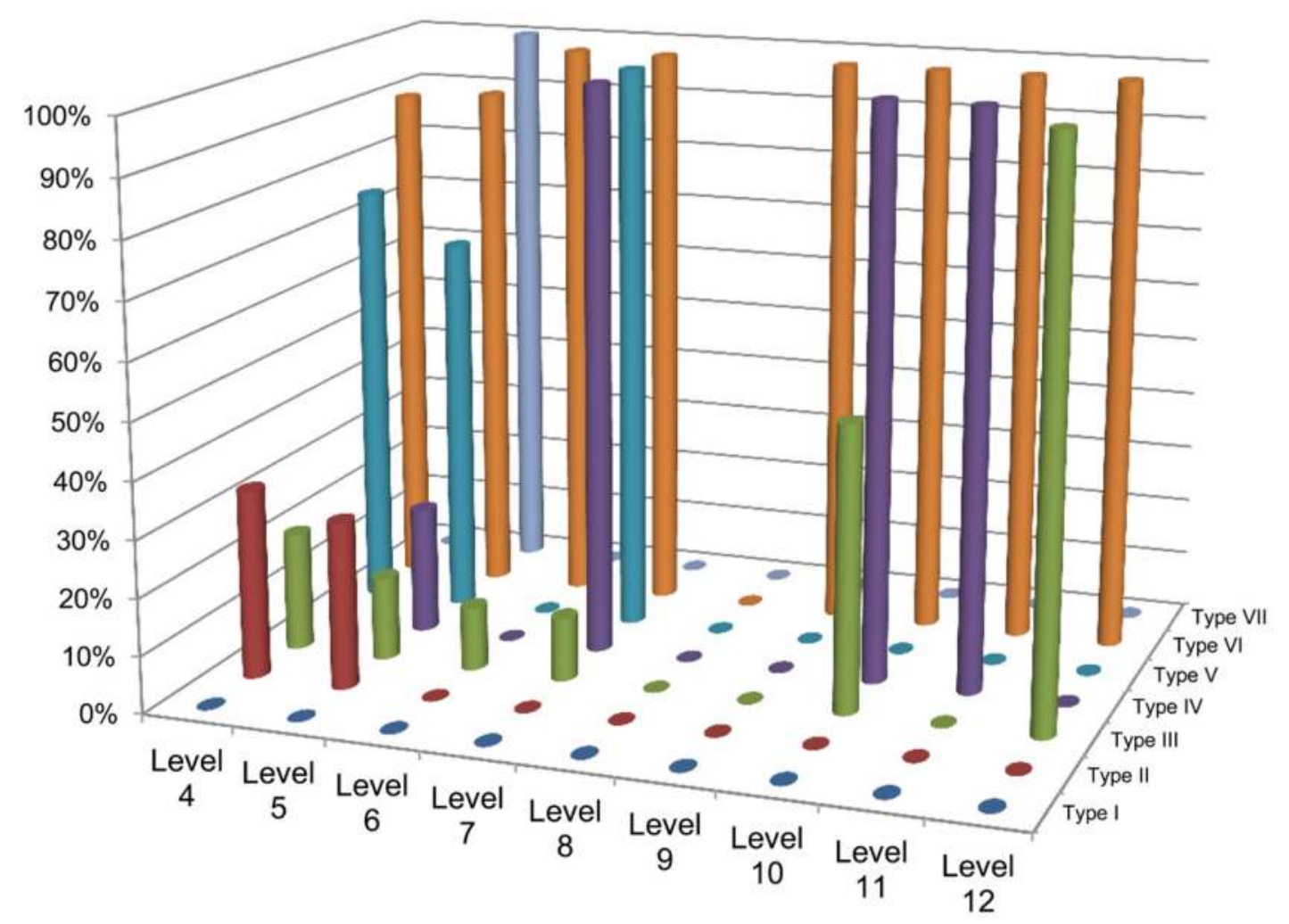

Fig. 13.5 Proportion of pottery types in closed-shape pottery assemblages by level at Göytepe. 


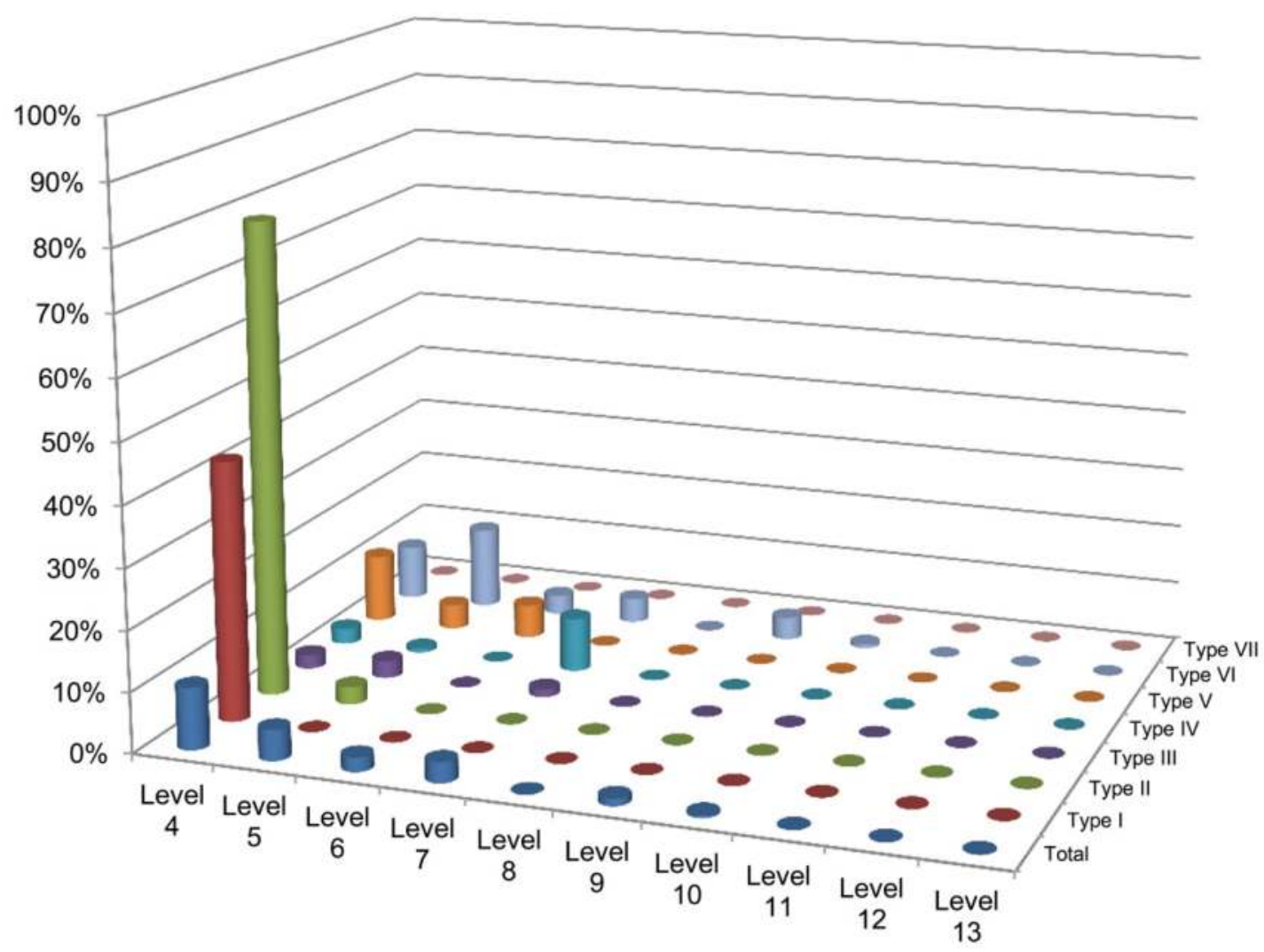

Fig. 13.6 Proportion of pottery types in decorated pottery assemblages by level at Göytepe.

Shomutepe culture, is surrounded by many representative sites of this Pottery Neolithic culture of the Southern Caucasus. The pottery from Göytepe shares several common elements with these sites. The most characteristic attribute is relief decoration. In addition, the main vessel shape has a concave rim, probably representing a neckless jar. A mat impression on the base is also characteristic of this culture's pottery.

It is important to note that these features of the Shomutepe culture are limited to only part of the occupation sequence at Göytepe. Relief decoration is principally unique to Type VI, but it was also adopted in Type III. Although these two types always predominate in the assemblage, their percentage varies. Furthermore the use of relief decoration started later in this site, in Types VI and III. Type VI represents other typical features of the Shomutepe culture: closed shape, mat impression on the base, and solely mineral inclusions. On the other hand, Type III is an organic-tempered ware in the first place. This kind of inclusion is not common in Shomutepe pottery (Chataigner 1995: pls. 29 and 35, 101-102) and Type III would become predominant only in the upper layers. Relief decoration also emerged on this type even in the latter levels.

The Shomutepe culture is principally dated to the 6th millennium BC (Connor and Sagona 2007: 29; Lyonnet 2007: 12) and divided in to five phases: Phase I to Phase V (Chataigner 1995: 91-92, pls. 2934; Kushnareva 1997: 23-25). Excavated pottery of Göytepe is dated to the relatively early stage (Stages I and II, possibly a part of III) of this culture. The motifs of relief decoration are limited to abstract forms: a dot, oval, or exceptionally circle (Fig. 13.6: 6, 13-19). There are the Göytepe assemblages include no complicated motifs typical of the Phase IV such as the human-like relief (Chataigner 1995: pls. 32: 11, 33: 13 and 14) and small dots on a circle (Chataigner 1995: pl. 33: 11 and 12). Combined motifs of circle and dots that are characteristic of Phases III and IV (Chataigner 1995: pls. 31: 13, 32: 7 and 12) are also rare. On the other hand, the earlier levels of Göytepe, where the relief decoration does not exist, might be dated to the previous stage of Phase I (Chataigner 1995: 113). 


\subsubsection{Regional position}

As mentioned above, Göytepe lies in the central region of the Shomutepe culture. Among the regional divisions of this culture (Chataigner 1995: 94, pl. 35), it is situated in "the left bank of the Akstafa River." Excavated pottery from Göytepe demonstrates the similarities with the sites in the same region: the coexistence of mineral-tempered and organic-tempered wares, round, wavy-lined, or oval-shaped relief decorations, and mat impressions. It would be said that Göytepe represents one part of the Shomutepe material culture.

A comparison of the recent investigations shows that the pottery of Göytepe and Aruchlo (Hansen et al. 2006, 2007; Lyonnet et al. 2012) have several similarities. These include a predominance of concave-walled forms (Hansen et al. 2006: 17-18, tab. 5), prosperity of oval-shaped relief decoration (Hansen et al. 2006: 20, tab. 8), and mat impression on the base (Hansen et al. 2006: 18-19, tab. 5, fig. 38). Mineral-tempered ware with similar relief decoration has also been found at Kkhzjak Blur and Shengavit on the Ararat plain (Chataigner 1995: 99-100, pl. 42). It is clear that Göytepe shares some common features with the region south of Shomutepe and Shulaveri Gora.

Some different types of pottery sherds have also been excavated. Organic-tempered ware decorated by nail-marked incisions is one typical type (Fig. 13.6: 1). Similar examples have been excavated from Ilanly Tepe (Chataigner 1995: 119), a site located on the southern region of the Shomutepe culture's core area. However, such sherds are few in number and thus exceptional.

In this way, the pottery of Göytepe belongs to the same regional unit as the neighboring sites of Shomutepe and Aruchlo upstream, sharing form, decoration, and production techniques (especially the mat impression on the base). At the same time, it can be said that this site composed part of the cultural network that included the Kura River valley and reached to the Ararat plain.

\subsection{Conclusion: New insight on the Pottery Neolithic period in the Southern Caucasus}

At first, the excavated pottery of Göytepe gives the impression of uniformity without significant change over time. In this analysis, however, we have distinguished three stages for which the boundary lines can be drawn between Levels 10 and 9 and Levels 6 and 5. It is certain that some functional/ technological changes occurred step by step regarding clay vessels used as ordinary objects. As mentioned above, the amount of pottery increased after Level 5 and all types of pottery were manufactured in later levels. It is probable that pottery types correspond to vessel shapes to some extent. An increase in types could reflect diversification in the shape and use of vessels. Ways of using clay vessels expanded at this time and evidence of this functional diversification occurred after the increase of organic-tempered wares in Level 9. Differences between organic-tempered and mineral-tempered wares could have resulted from the expanding demand for pottery vessels.

Based on the radiocarbon data from Göytepe (Nishiaki et al. 2018), it is apparent that each chronological change in pottery occurred over one or two generations. We can say that the development of pyrotechnology was important to the daily life and overall lifestyle, and this craft advanced relatively rapidly. That could be one of the most important characteristics of the Pottery Neolithic in the Southern Caucasus.

In this region, only the Shomutepe culture has representative the Neolithic period. However, absolute dates and the chronological division of this culture have remained unclear. Discerning the stratigraphic evolution of pottery in Göytepe would be useful for defining the periodization. Some of the phenomena discussed above represent new aspects about this culture's pottery. Furthermore, the early levels (before Level 10) of Göytepe are especially valuable for defining the beginnings of the Shomutepe culture. The origin of this culture is also debatable and we need to investigate pottery in this site more closely. Such efforts would lead to a better understanding of the prosperity of the Pottery Neolithic period in the Southern Caucasus. They could also reveal any epochdefining phases in Neolithic society in this region. 


\section{References}

Chataigner, C. (1995) La Transcaucasie au Néolithique et au Chalcolithique. BAR Internatinal Series 624. Oxford: Tempus Reparatum.

Connor, S. and A. Sagona (2007) Environment and society in the late prehistory of southern Georgia and Caucasus. In: Les Cultures du Caucase (VleIIle Millénaires avant notre ère): Leur Relations avec le Proche-Orient, edited by B. Lyonnet, pp. 21-36. Paris: Éditions Recherche sur les Civilisations.

Hansen, S., G. Mirtskhulava, and K. BastertLamprichs (2006) Aruchlo 2005-2006. Bericht über die Ausgrabungen in einem neolithischen Siedlungshügel. Archäologische Mitteilungen aus Iran und Turan, 38: 1-34.

Hansen, S., G. Mirtskhulava, and K. Bastert-Lamprichs (2007) Aruchlo: A Neolithic settlement mound in the Caucasus. Neo-Lithics, 1/07: 13-19.

Kushnareva, K. K. (1997) The Southern Caucasus in Prehistory: Stages of Cultural and Socioeconomic Development from the Eighth to the Second Millennium B.C. Philadelphia: The University of Pennsylvania Museum.

Lyonnet, B. (2007) Introduction. In: Les Cultures du Caucase (Vle-Ille Millénaires avant notre ère): Leur Relations avec le Proche-Orient, edited by $\mathrm{B}$. Lyonnet, pp. 11-19. Paris: Éditions Recherche sur les Civilisations.

Lyonnet, B., F. Guliyev, B. Helwing, T. Aliyev, S. Hansen, and G. Mirtskhulava (eds.) (2012) Ancient Kura 2010-2011: The First Two Seasons of Joint Field Work in the Southern Caucasus. Archäologische Mitteilungen aus Iran und Turan, 44: 1-190.

Nishiaki, Y., F. Guliyev, S. Kadowaki, and T. Omori (2018) Neolithic residential patterns in the southern Caucasus: Radiocarbon analysis of rebuilding cycles of mudbrick architecture at Göytepe, west Azerbaijan. Quaternary International, 474: 119-130. 


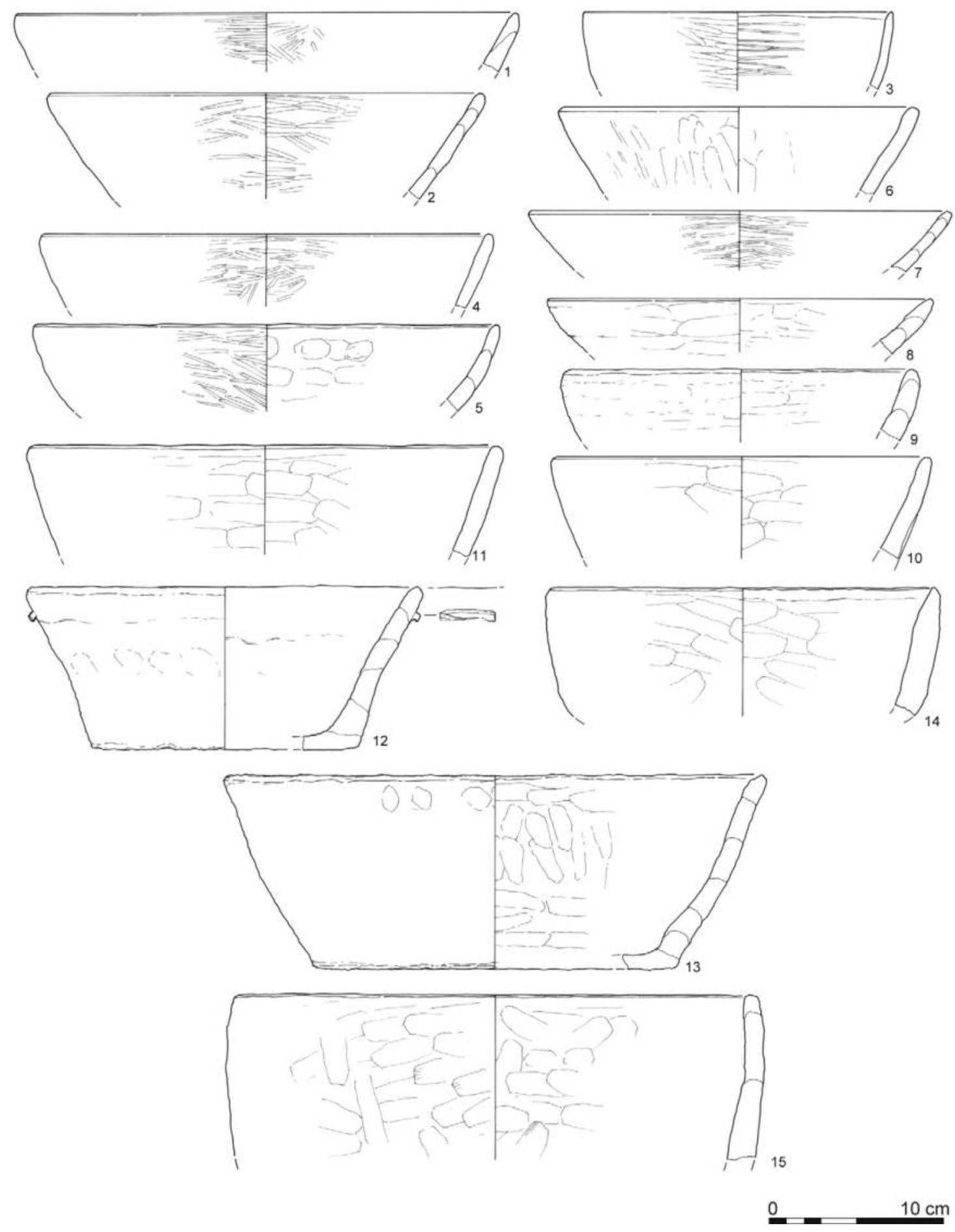

Fig. 13.7 Pottery from Göytepe (I). 1-2: Type I from Level 4; 3: Type I from Level 5; 4-5: Type II from Level 5; 6: Type V from Level 5; 7: Type II from Level 7; 8: Type III from Level 6; 9-13: Type III from Level 5; 14: Type VI from Level 4; 15: Type VI from Level 5. 

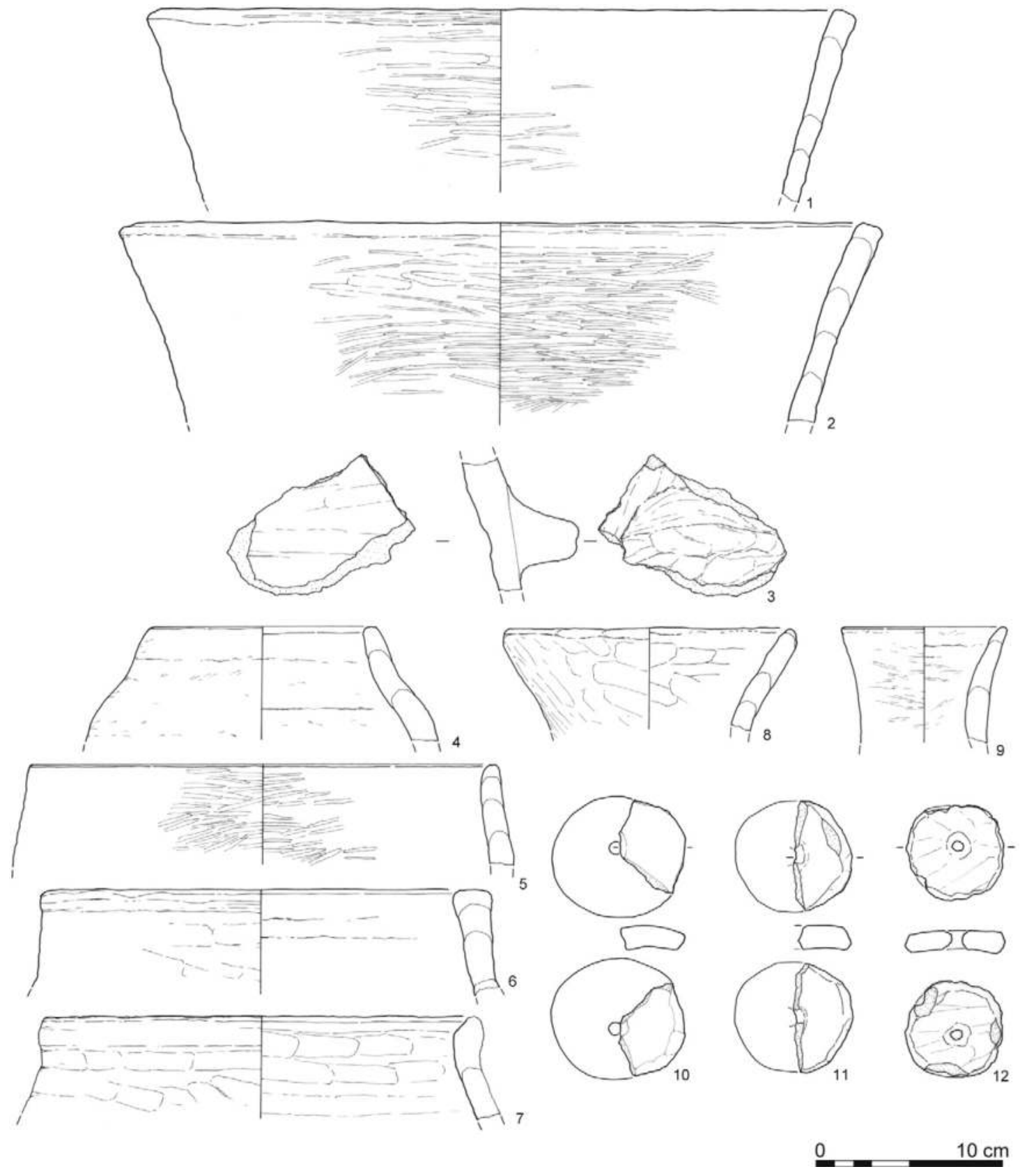

Fig. 13.8 Pottery from Göytepe (II). 1-4: Type III from Level 5; 5: Type III from Level 7; 6: Type V from Level 4; 7: Type V from Level 5; 8-9: Type III from Level 5; 10: Type II from Level 5; 11: Type III from Level 5; 12 : Type III from Topsoil. 


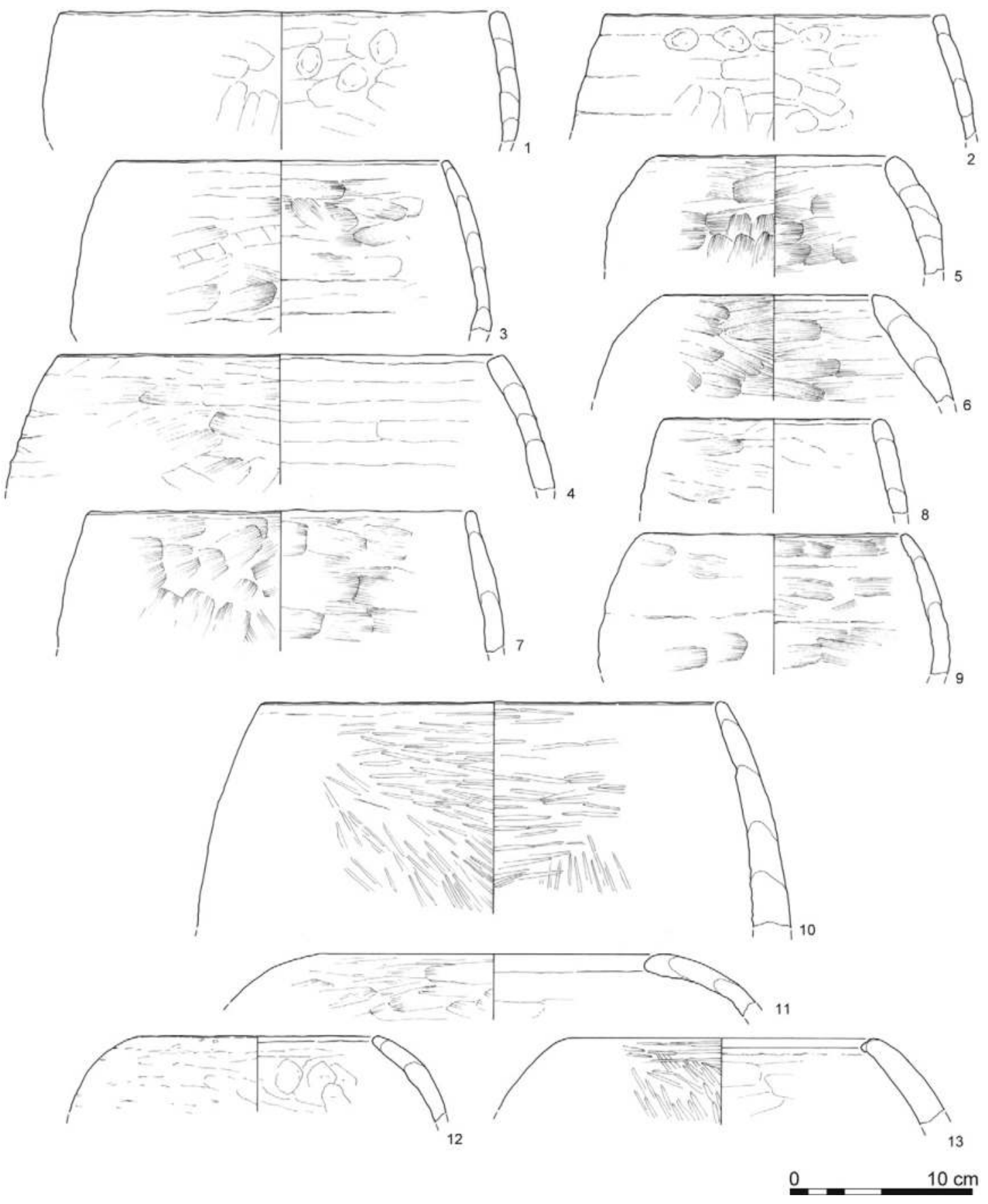

Fig. 13.9 Pottery from Göytepe (III). 1-2: Type V from Level 5; 3-5: Type VI from Level 5; 6: Type VI from Level 6; 7: Type VI from Level 7; 8-9: Type VI from Level 11; 10: Type III from topsoil; 11-12: Type III from Level 6; 13: Type III from Level 11. 

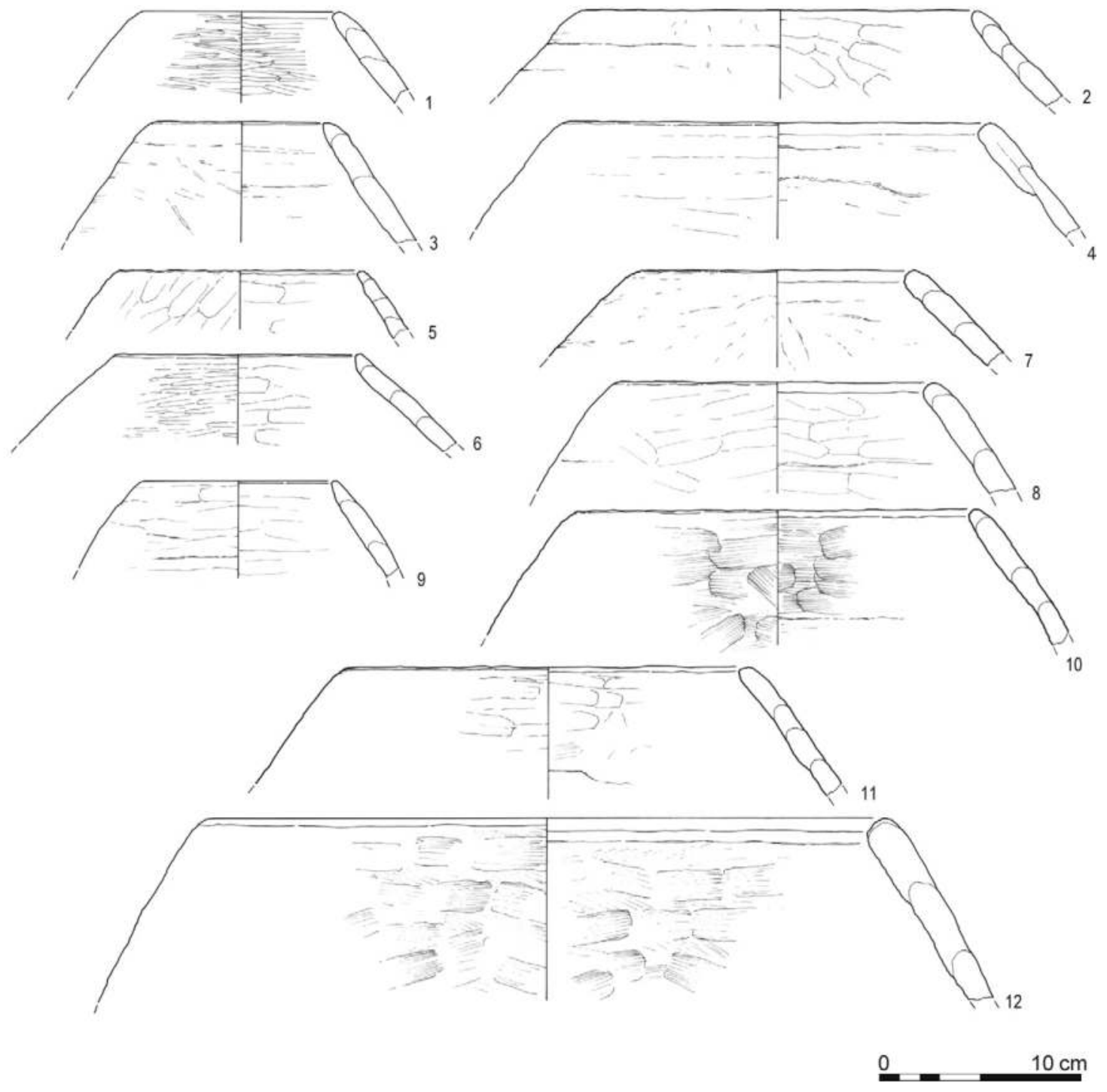

Fig. 13.10 Pottery from Göytepe (IV). 1: Type II from Level 7; 2: Type III from Level 4; 3-4: Type III from Level 5; 5-6: Type V from Level 5; 7-8: Type VI from Level 4; 9-12: Type VI from Level 5. 


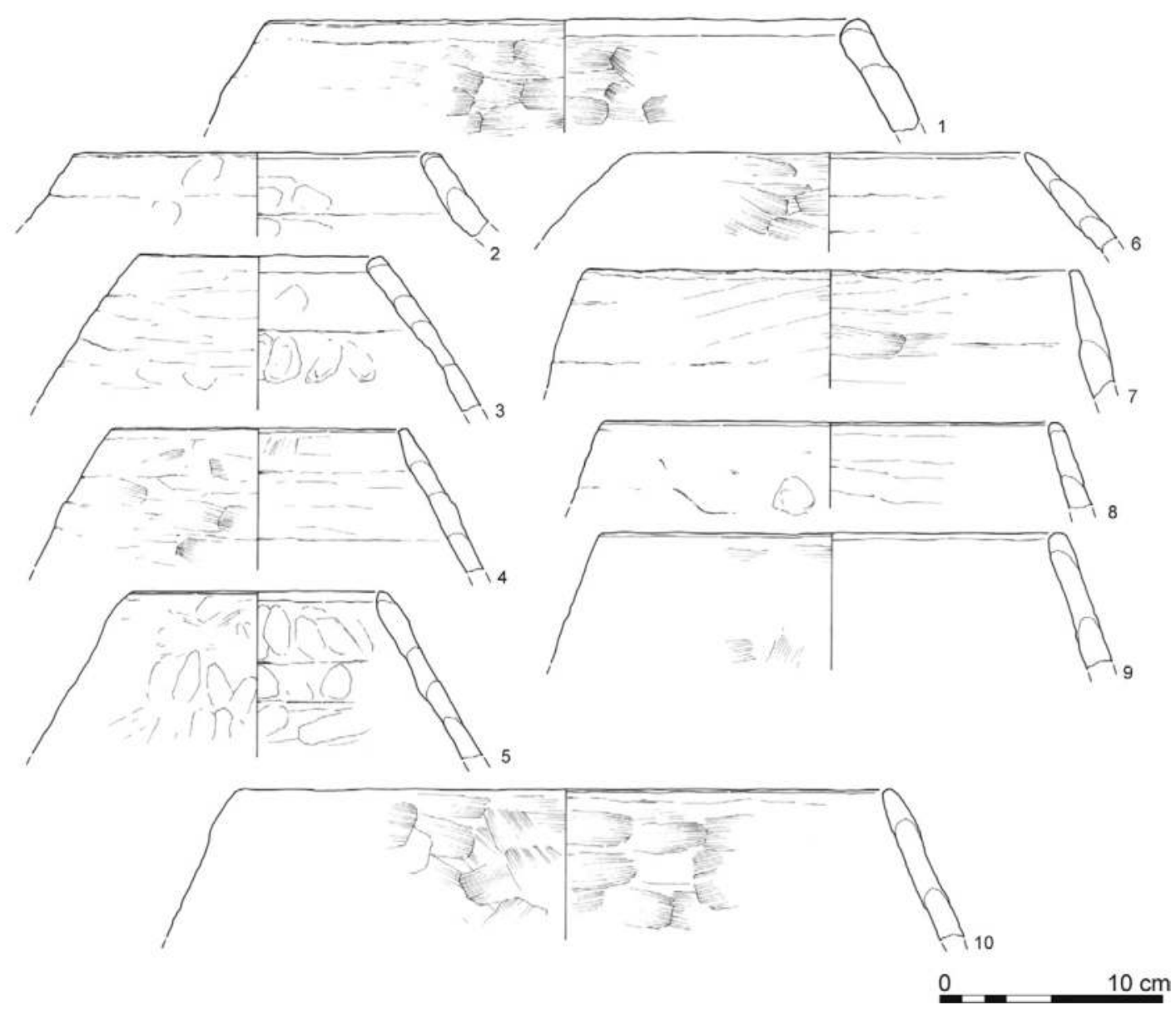

Fig. 13.11 Pottery from Göytepe (V). 1: Type VII from Level 5; 2: Type VI from Topsoil; 3-10: Type VI from Level 7. 

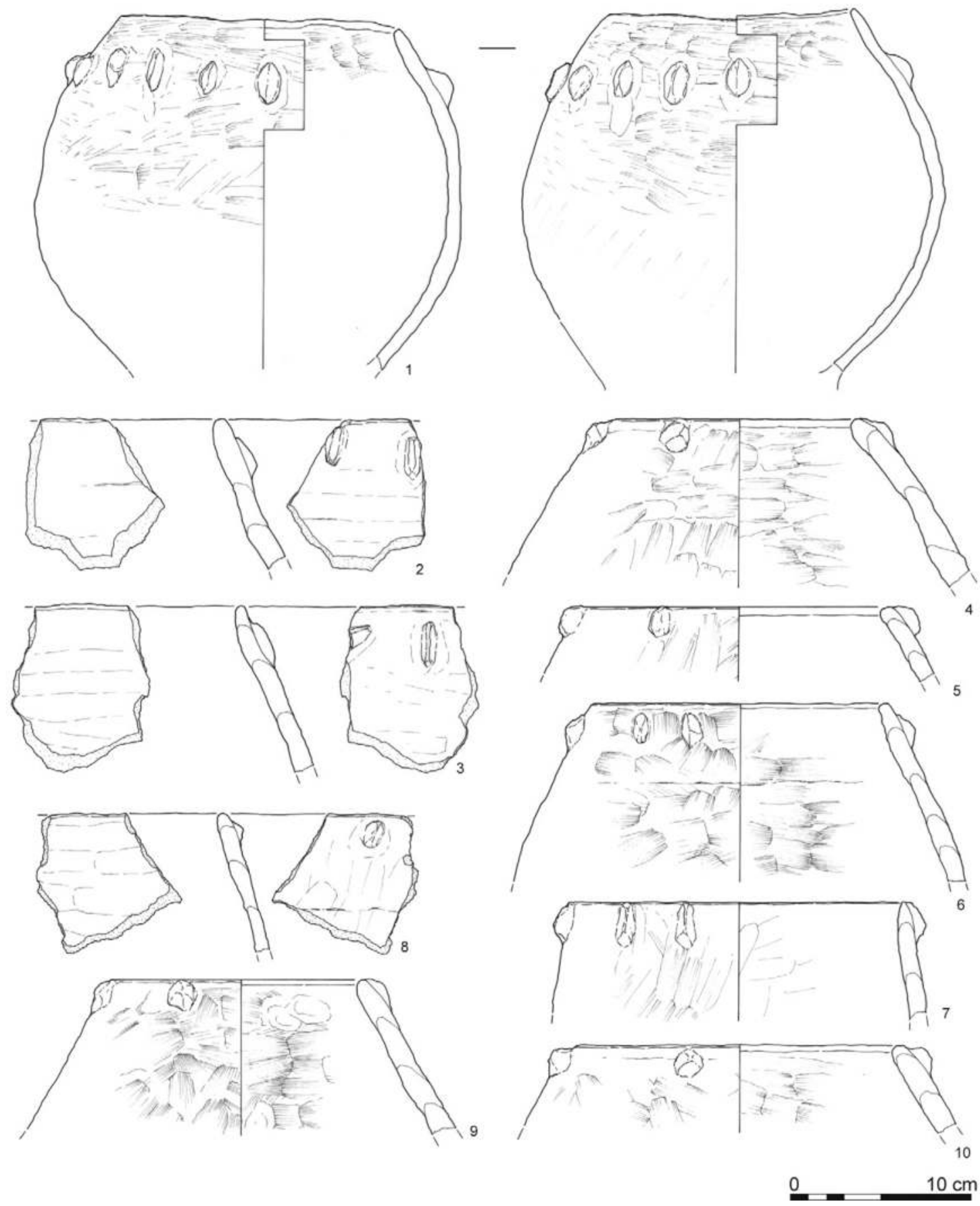

Fig. 13.12 Pottery from Göytepe (VI). 1: Type VI from Level 11; 2-7: Type VI from Level 7; 8-10: Type VI from Level 6. 


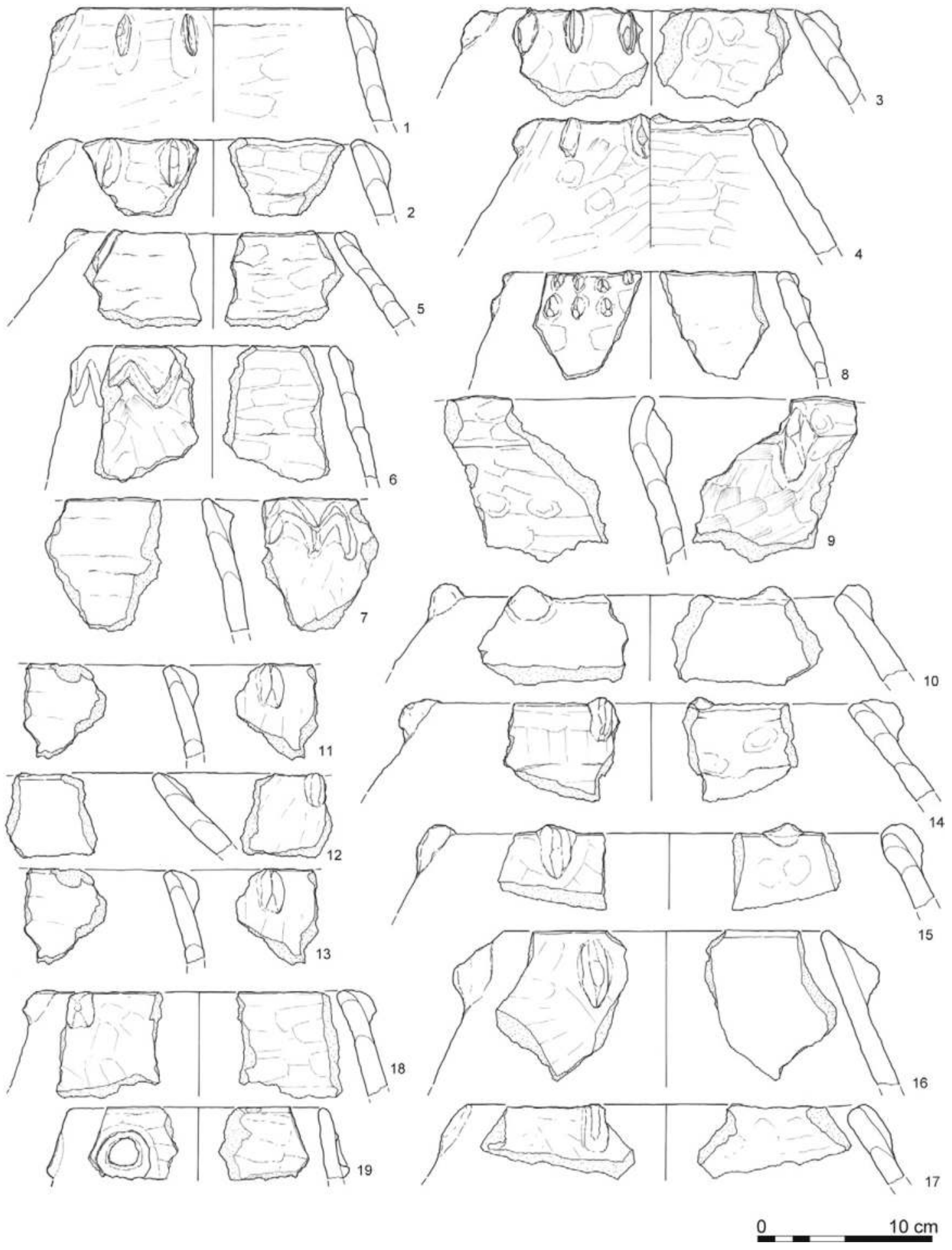

Fig. 13.13 Pottery from Göytepe (VII). 1-10: Type VI from Level 5; 11-14: Type VI from Level 4; 15-16: Type III from Level 5; 17: Type III from Level 4; 18: Type III from topsoil; 19: Type II from topsoil. 


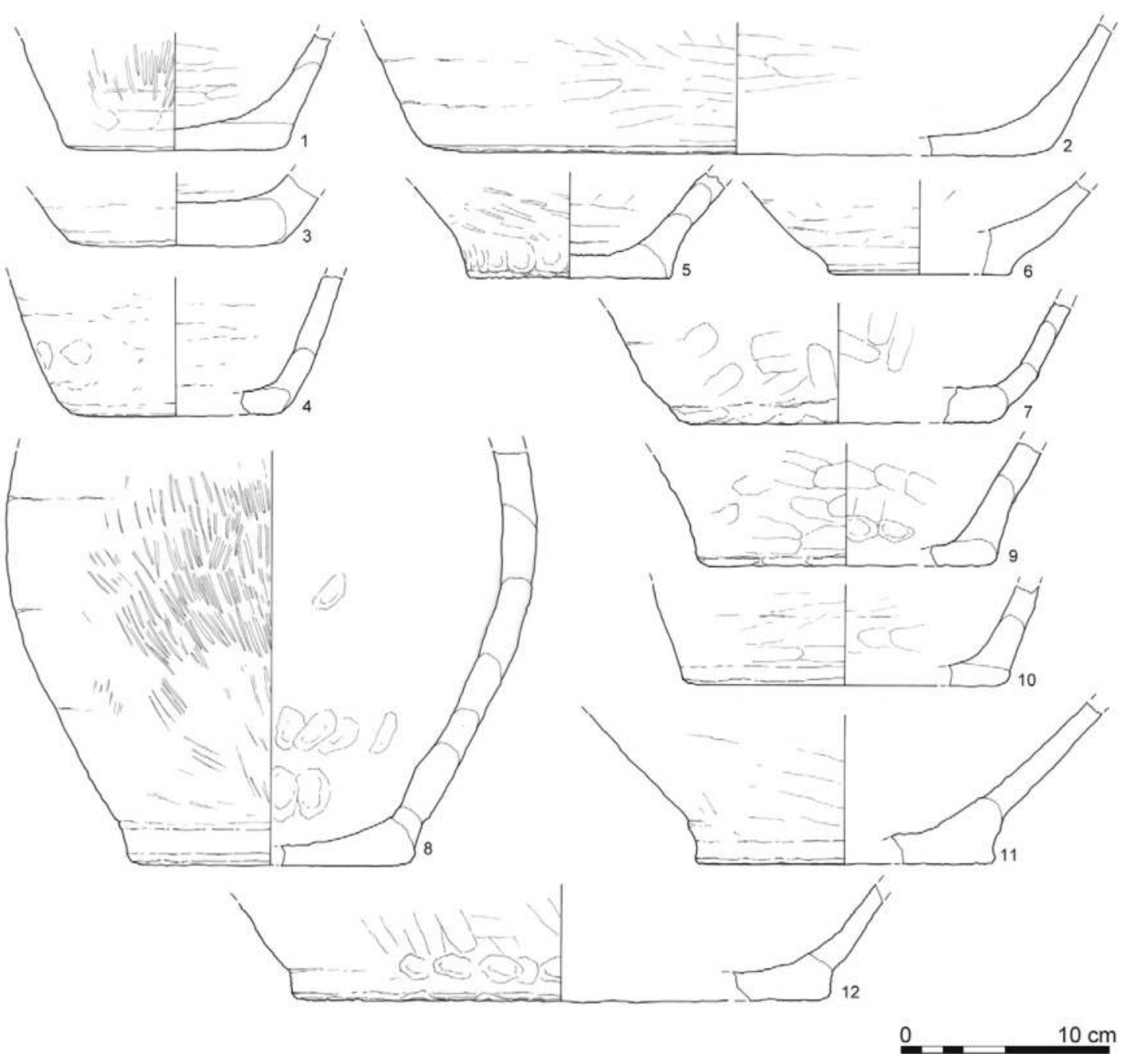

Fig. 13.14 Pottery from Göytepe (VIII). 1-2: Type III from Level 5; 3: Type III from Level 6; 4: Type VI from Level 4; 5: Type IV from Level 4; 6: Type III from Level 7; 7: Type VI from Level 5; 8: Type VI from Level 10; 9: Type VI from Level 5; 10-12: Type III from Level 5. 


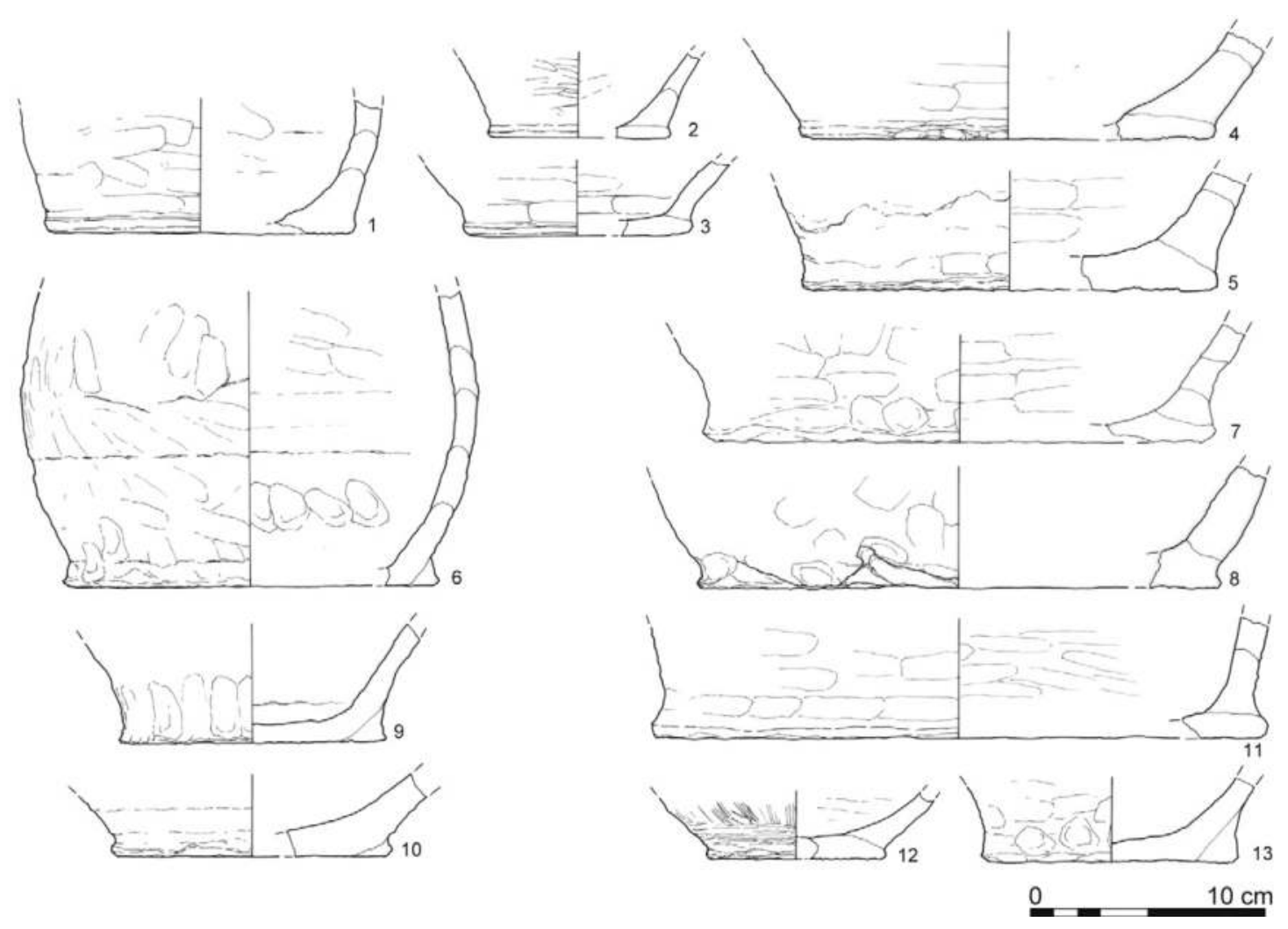

Fig. 13.15 Pottery from Göytepe (IX). 1-2: Type III from Level 5; 3: Type VI from Level 5; 4: Type IV from Level 5; 5: Type III from Level 5; 6-9: Type VI from Level 5; 10: Type VI from Level 7; 11: Type III from Level 5; 12: Type III from Level 10; 13: Type V from Level 4. 


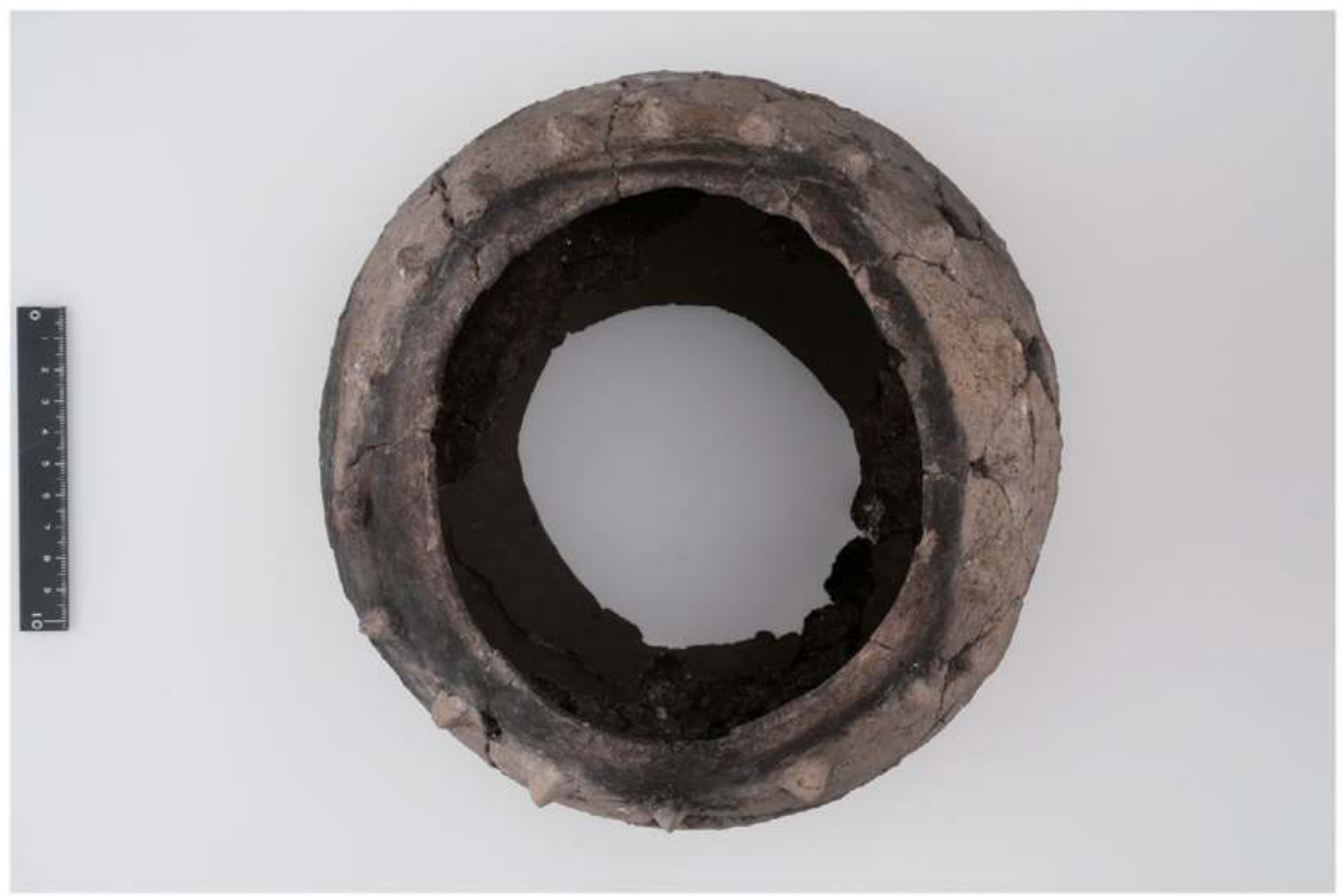

(1) Level 11

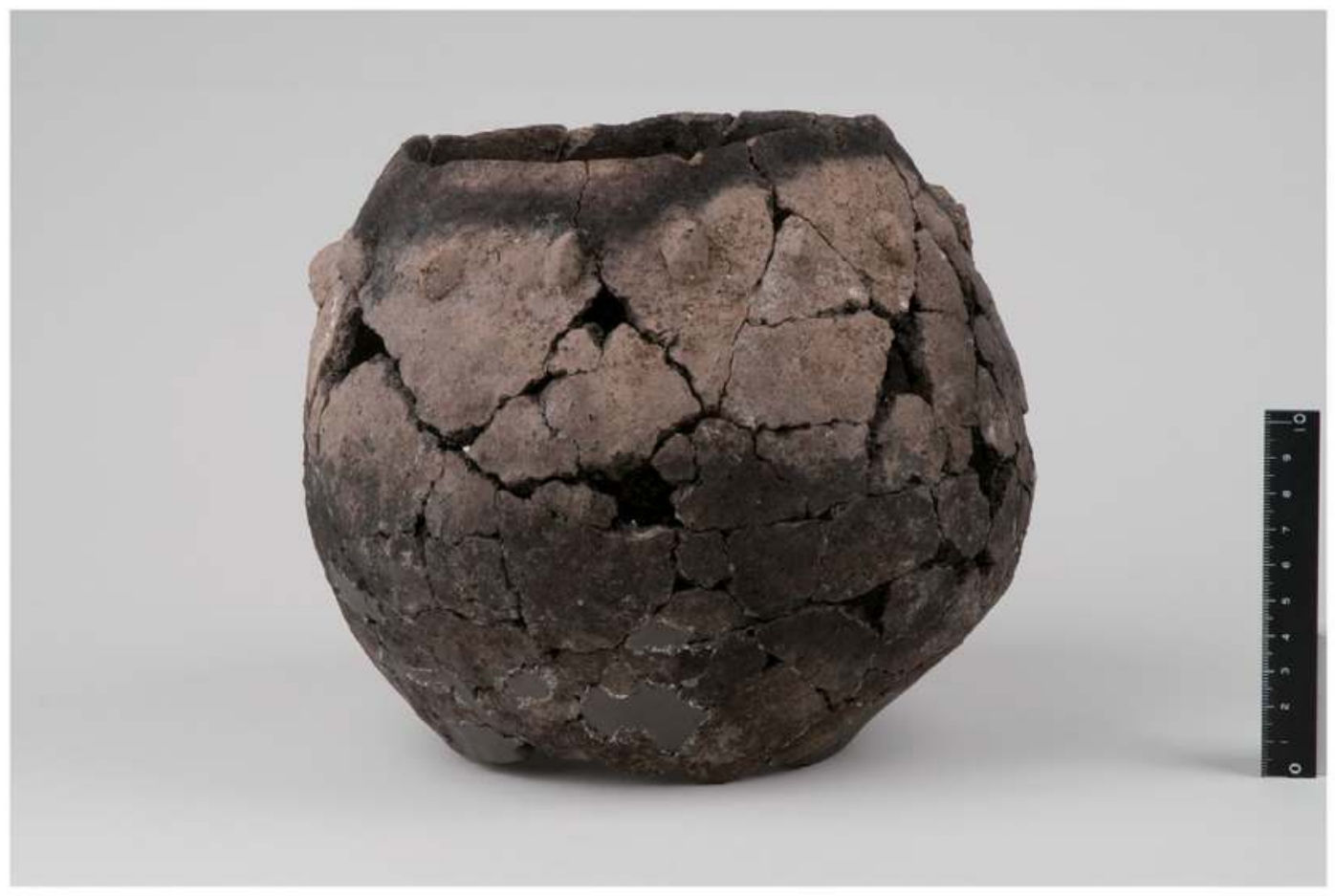

(2) Level 11

Fig. 13.16 Pottery from Göytepe (X). Nearly complete pottery from Level 11. 


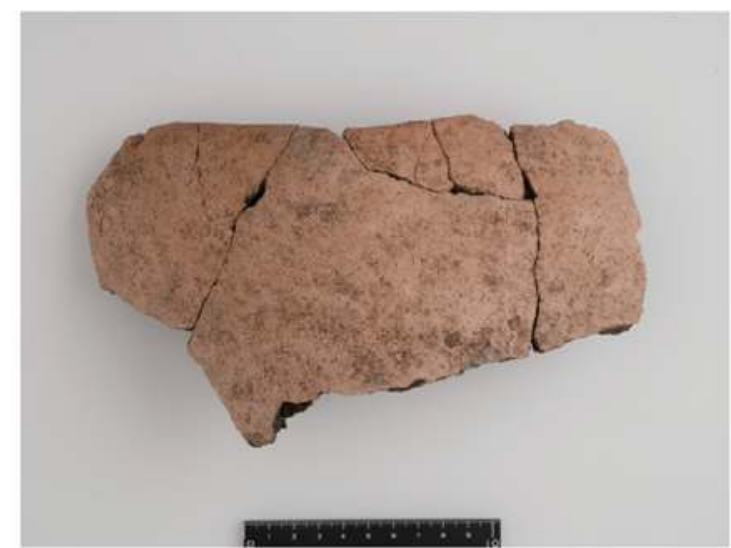

(1) Topsoil

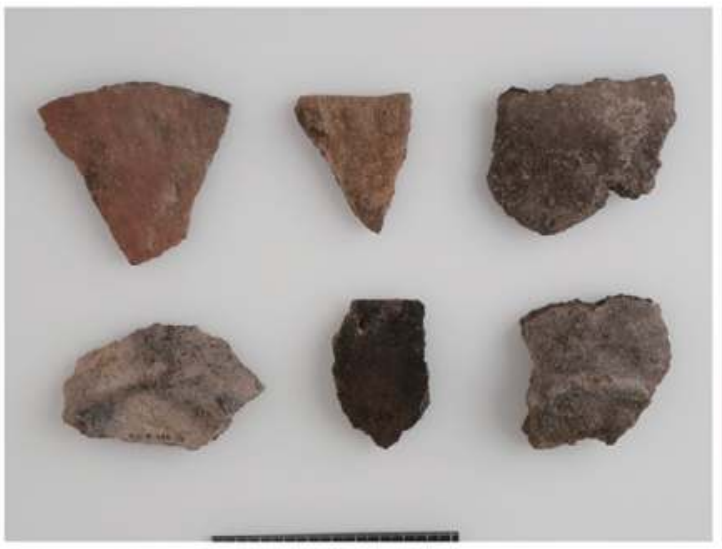

(3) Level 7

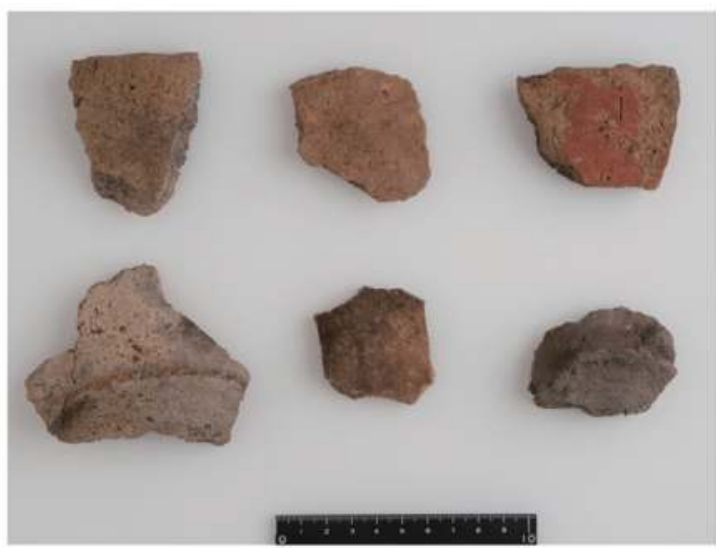

(5) Level 7

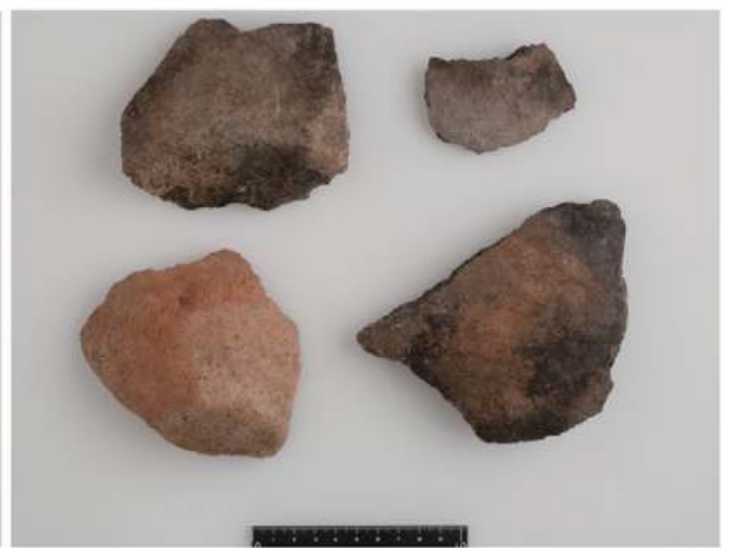

(2) Level 9

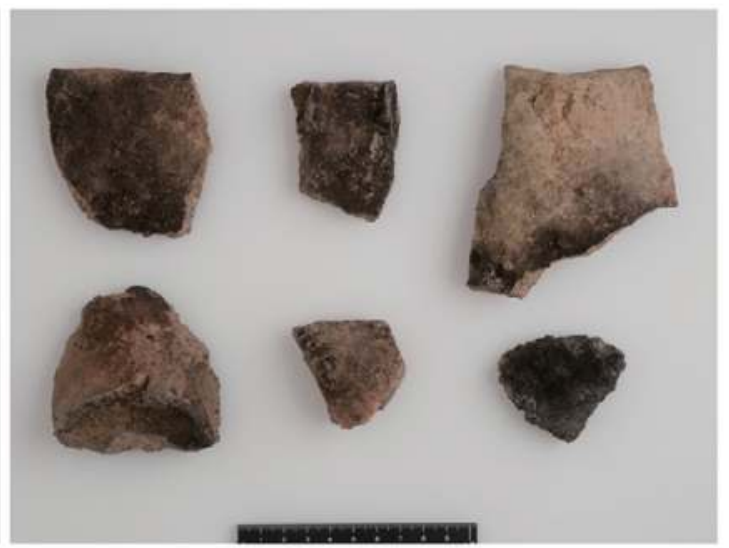

(4) Level 7

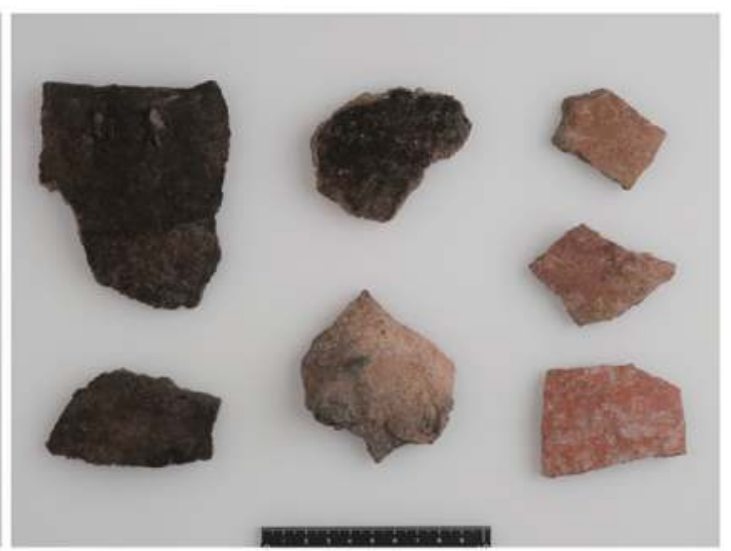

(6) Level 7

Fig. 13.17 Pottery from Göytepe (XI). 1: Topsoil; 2: Level 9; 3-6: Level 7. 


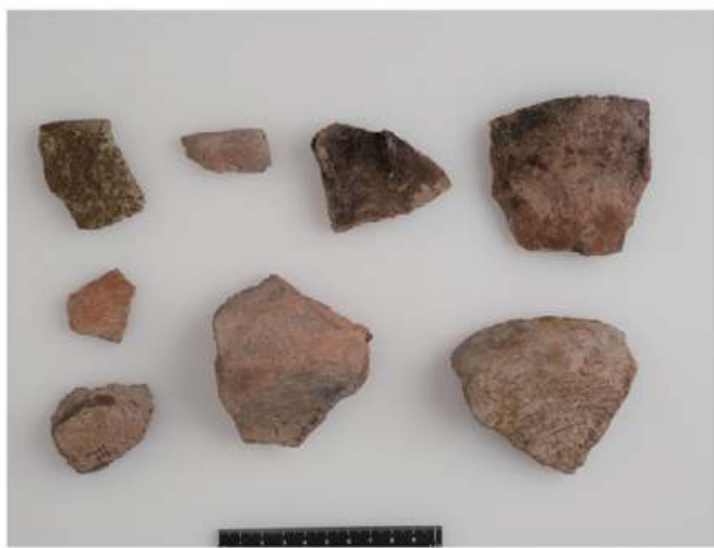

(1) Level 6

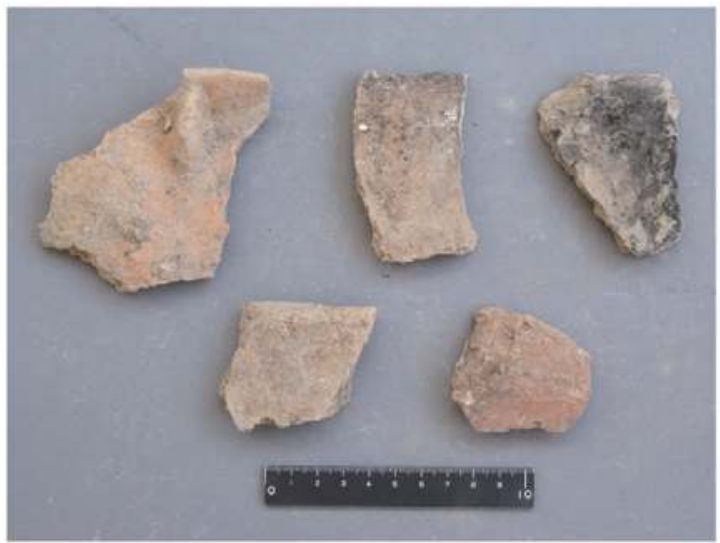

(3) Level 5

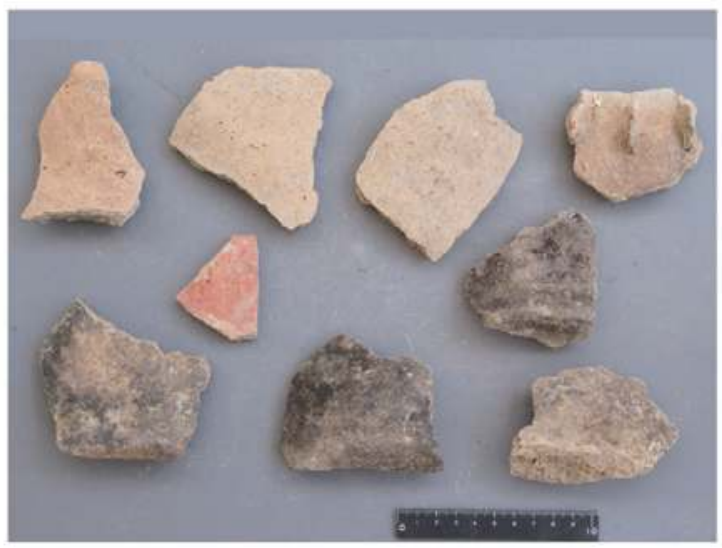

(5) Level 5

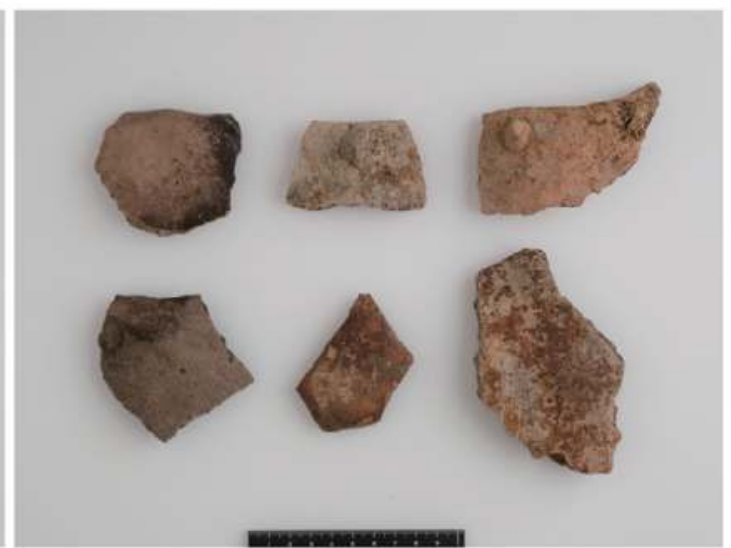

(2) Level 6

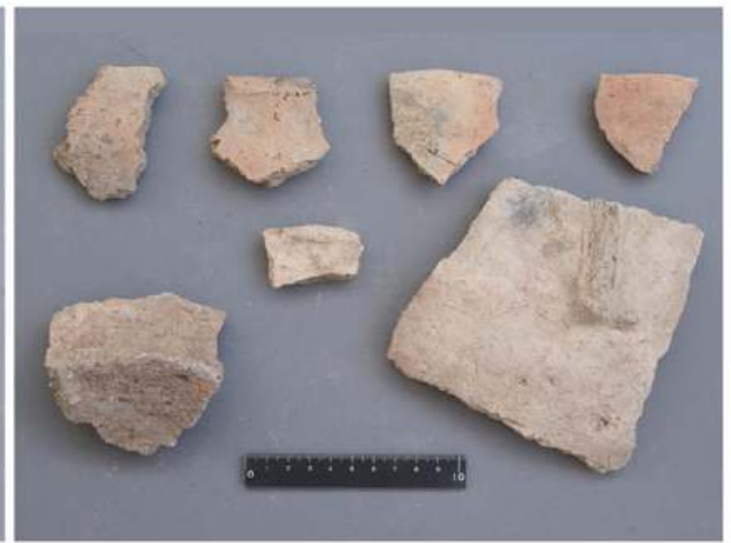

(4) Level 5

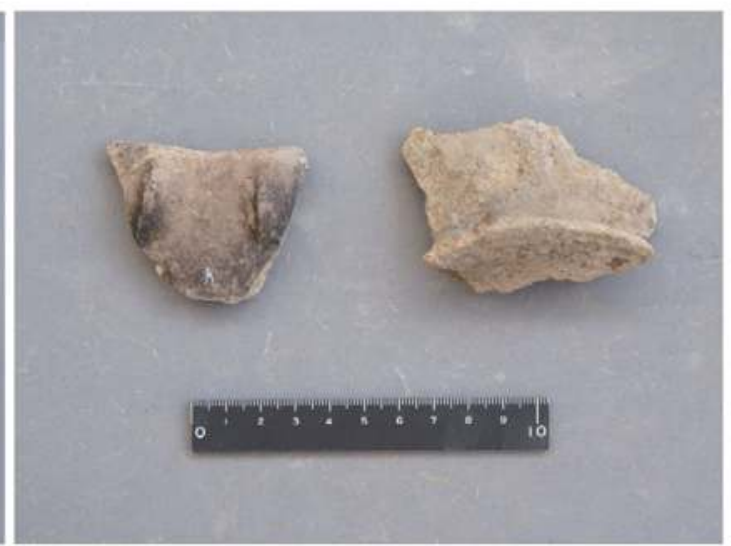

(6) Level 5

Fig. 13.18 Pottery from Göytepe (XII). 1-2: Level 6; 3-6: Level 5. 


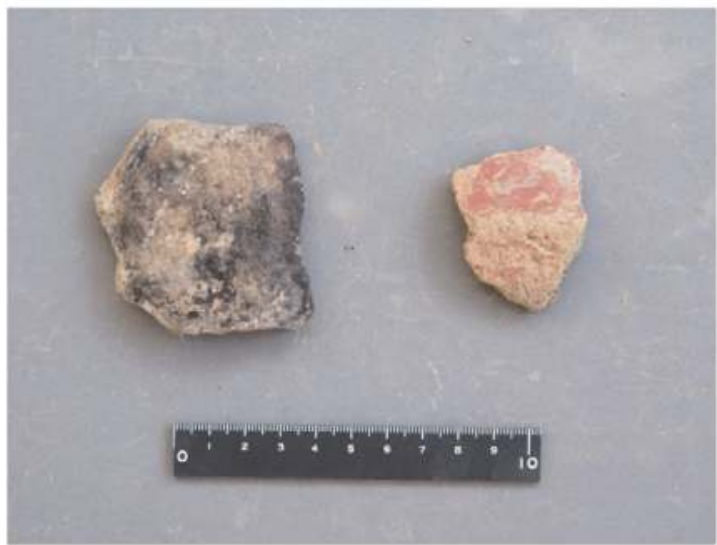

(1) Level 5

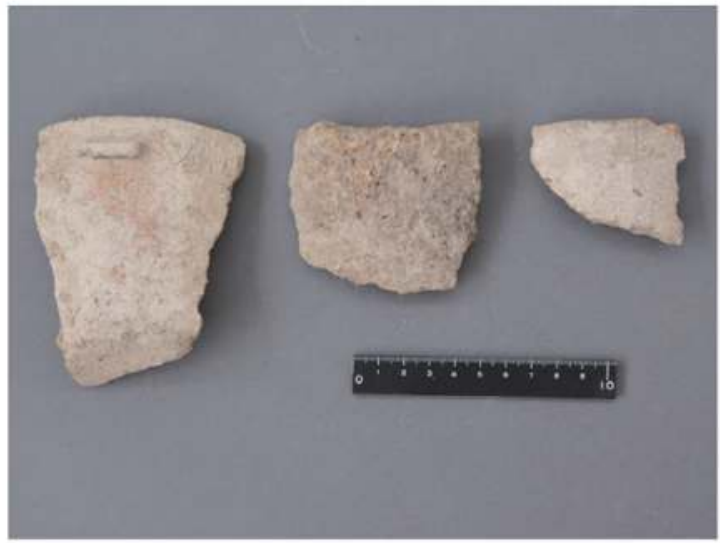

(3) Level 5

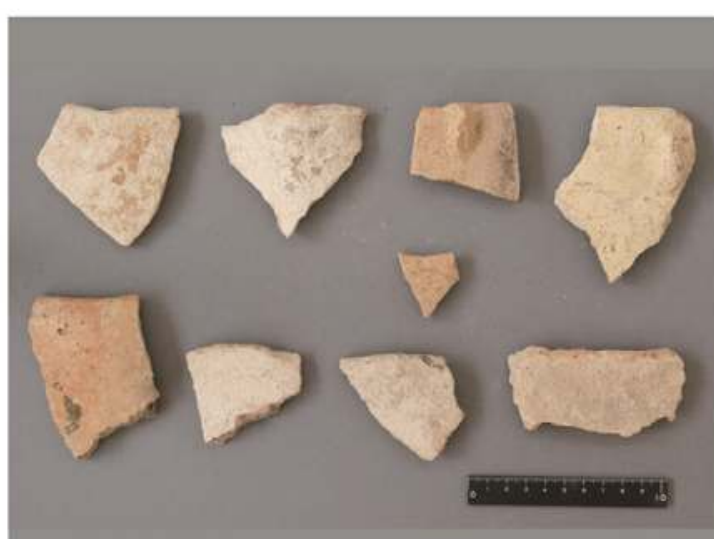

(5) Level 5

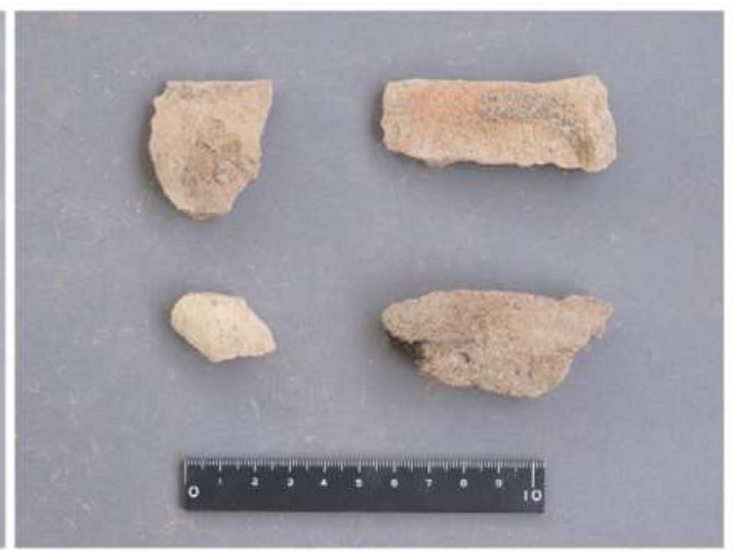

(2) Level 5

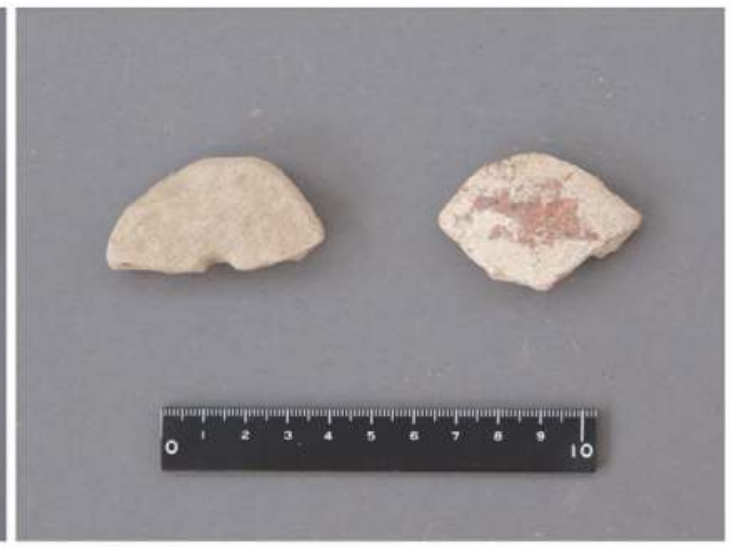

(4) Level 5

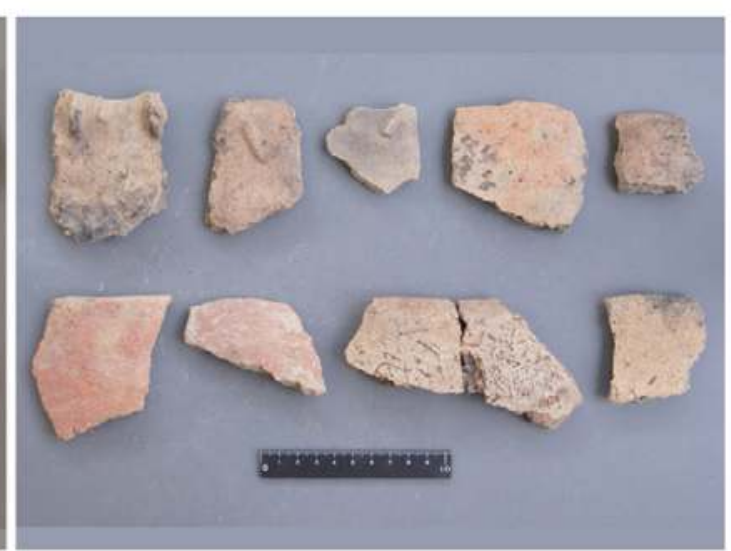

(6) Level 5

Fig. 13.19 Pottery from Göytepe (XIII). 1-6: Level 5. 


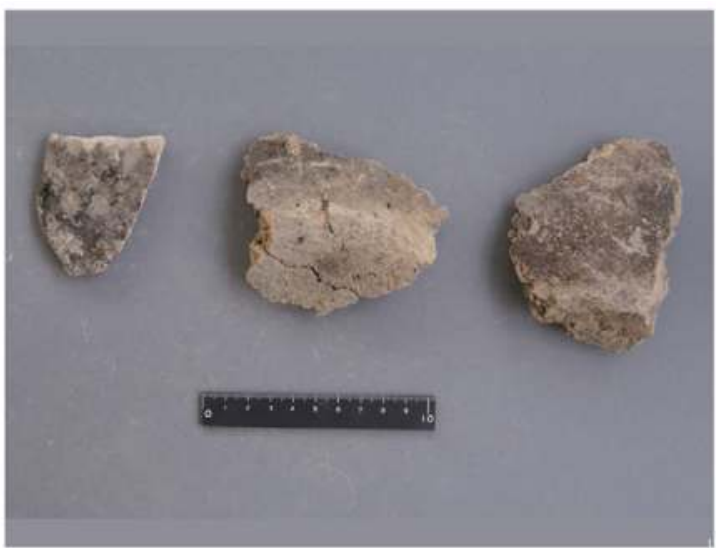

(1) Level 5

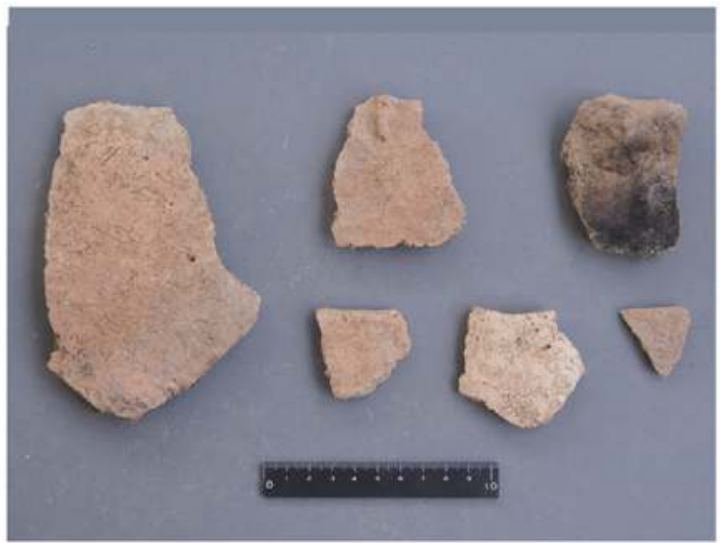

(3) Level 5

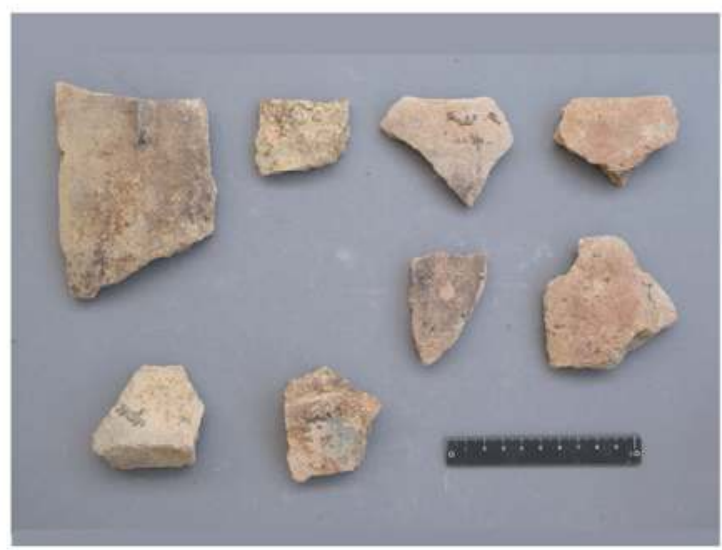

(5) Level 4

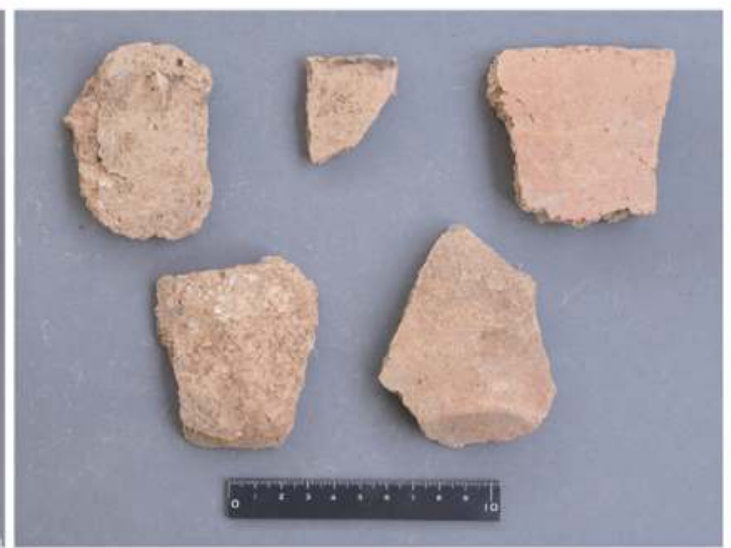

(2) Level 5

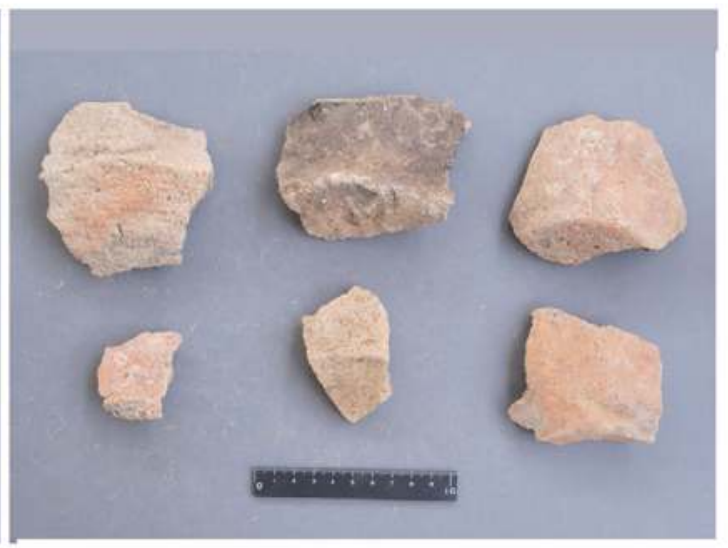

(4) Level 5

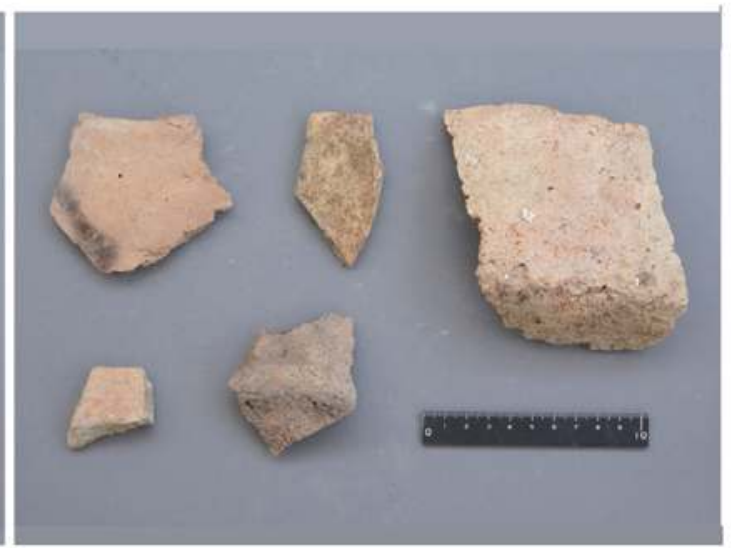

(6) Level 4

Fig. 13.20 Pottery from Göytepe (XIV). 1-4: Level 5; 5-6: Level 4. 


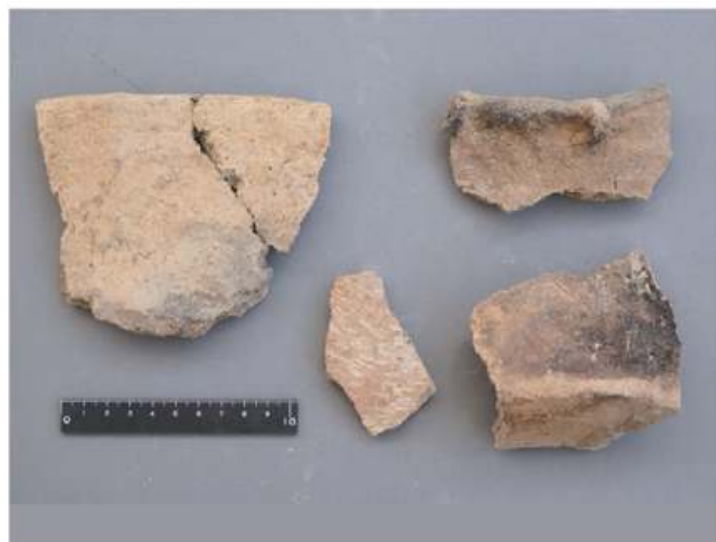

(1) Level 4

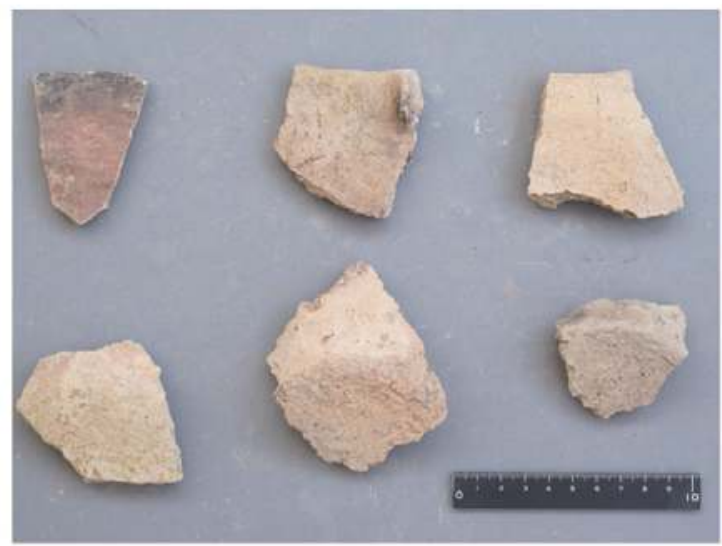

(3) Level 4

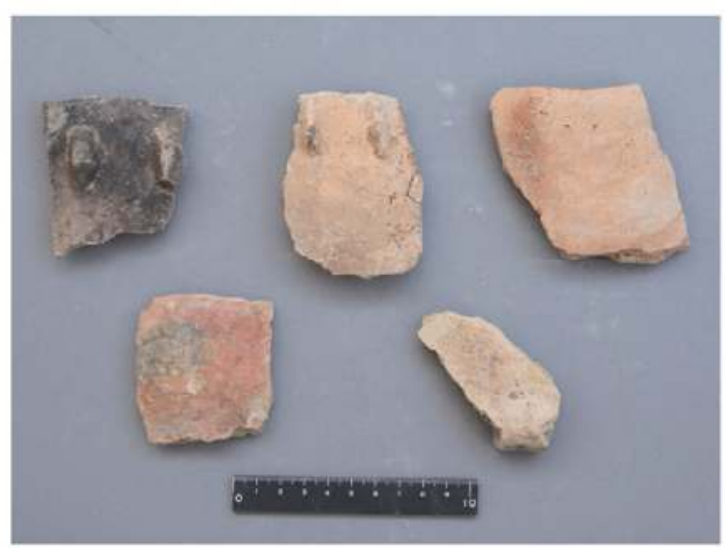

(5) Level 4

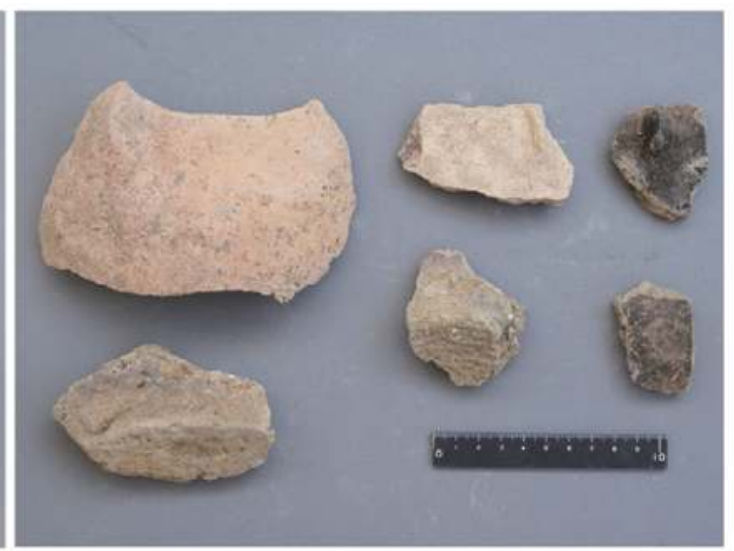

(2) Level 4

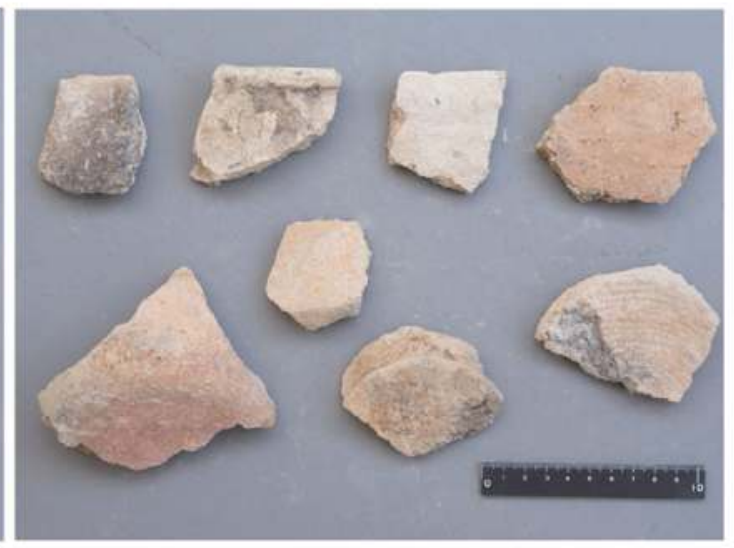

(4) Level 4

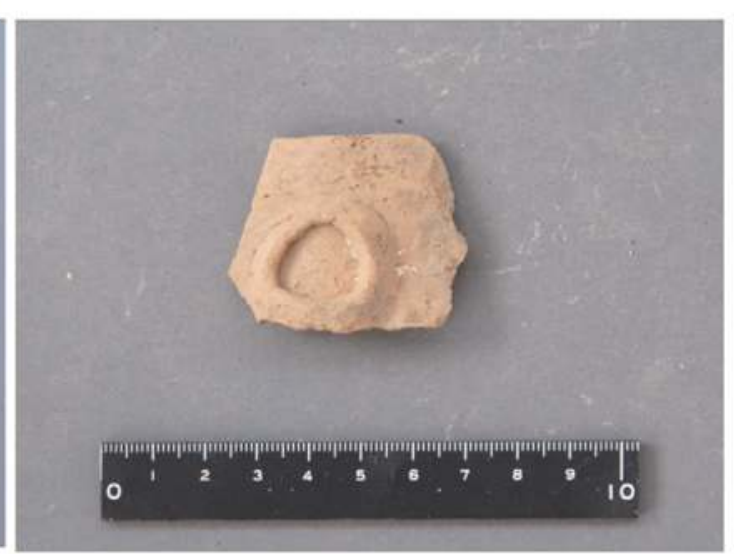

(6) Topsoil

Fig. 13.21 Pottery from Göytepe (XV). 1-5: Level 4; 6: Topsoil. 
Chapter 13: Appendix

\title{
Pottery from Level 14 of Göytepe
}

\author{
Takahiro Odaka
}

In addition to the Neolithic pottery reported in the main text of this chapter, three plain body sherds were recovered from Level 14 at Square 4BII in the 2013 season.

One of them (4BIIX-129\#1) is organic-tempered ware with mineral inclusions and a wall thickness measuring $12 \mathrm{~mm}$. Its roughly smoothed surface presents a dull orange color and its section reveals a dull brown color. This specimen can be assigned to our Type III. The other two sherds include minerals in paste without organic temper. The first is lightly burnished ware including fine sand (4BIIX$129 \# 2)$. Its surface color is orange, and the section measuring $10 \mathrm{~mm}$ in thickness shows a dark core. These attributes demonstrate that this sherd can be classified under Type V. The second sherd includes a large amount of minerals (4BIIX-128). Both its exterior and interior surfaces are wet-smoothed and seem stained with soot. The section of the thin wall measuring $8 \mathrm{~mm}$ in thickness is also black-colored. This specimen can be classified under Type VI.

Although the number is limited and the original vessel shapes can hardly be reconstructed, these three sherds possibly represent the oldest ceramic assemblage at Göytepe. The presence of a sherd belonging to Type $\mathrm{V}$ apparently suggests that mineral-tempered ware had already become diversified by then, and the so-called fine ware appeared even at the lowest level. Besides this point, no other contradictions were observed in view of the stratigraphic change mentioned in the main text. 


\title{
Neolithic figurines from Göytepe
}

\author{
Kazuya Shimogama
}

Among clay figurines discovered from the Neolithic levels at Göytepe, two examples clearly belonging to anthropomorphic figurines are described in this chapter.

\subsection{Figurine 1}

One of the clay figurines was retrieved alongside a wall at the southwestern corner of the Square 1BII (Fig. 14.1). This location constitutes just outside one of Level 2 building complexes (south of building 207; see Fig. 3.2 and Fig. 3.6 in Chapter 3) which was composed of four round structures with its center in the neighboring Square 1BI. The figurine was found within soft ash deposits along with other artifacts and animal bones, showing no significant context but refuse deposits. The stratigraphic and depositional context indicates that it derives without doubt from Level 2.

The Figurine 1, $4.5 \mathrm{~cm}$ tall, is made of fine clay with sand inclusion of less than $1 \mathrm{~mm}$. The fabric is also extremely fine and unfired. Surface color is grayish yellow brown (10YR5/2 of Munsell Chart) while the color of its matrix is relatively lighter (10YR6/2). The overall shape of this hand-modelled figurine looks like more or less elongated cone, with a tapered head drawing somewhat backward and a facetted face as observed in profile and section. Its lower body or leg part is broken, making it difficult to reconstruct the complete shape. Perhaps it does not have arms, showing a very schematic shape with a rounded section. As to face representations, eyes and a possible mouth deeply stubbed into the matrix are recognizable. The orbital depressions are also depicted probably by a sharp pointed tool. Its nose is remarkably pronounced, clearly modelled as shown in curved profile. In addition, an ear is represented on the left side, also impressed just below the end of an eyebrow.

Special attention is paid to the fact that it has some sets of impressed dots. They are ornamented by impressing each dot separately on the surface using tube-like pointed tool (possibly a sort of thorn or fine reed) as they make continuous dotted lines. Irregular rendering for these punctures support that they are not impressed by comb-shaped tool. At the same time, such lines are positioned as at least six horizontal rows on both sides of a central groove down the mouth. Two lines of similar impressed dots are also depicted as if they radiate from each eye. It is argued that the two dotted lines under eyes may represent flowing tears or a sort of tattooing (comparable depiction can be found on a Samarran painted jar decoration from Tell Hassuna, Lloyd and Safar 1945: fig. 1, 2 and pl. XVII, 2). Impressed decorations similar to this figurine have many parallels from Neolithic sites like such as Khramis Didi Gora (Kiguradze 1986: Abb. 83.2-3, Abb. 85.11), Gargalartepesi (Narimanov 1987: fig. 28), and Hajji Firuz Tepe in northwestern Iran (Voigt 1983: fig. 101b, pl. 27a).

Traces of breasts or sexual organ cannot be identified clearly, since the figurine lacks the lower part. Thus, the extant morphological feature prevents us from determining whether this figurine represents male or female.

\subsection{Figurine 2}

The provenience of the second clay figurine was attributed to the Square 2AII (Fig. 14.2). Exactly where or how this object was found is regrettably unknown. But the figurine can be related to the Level 

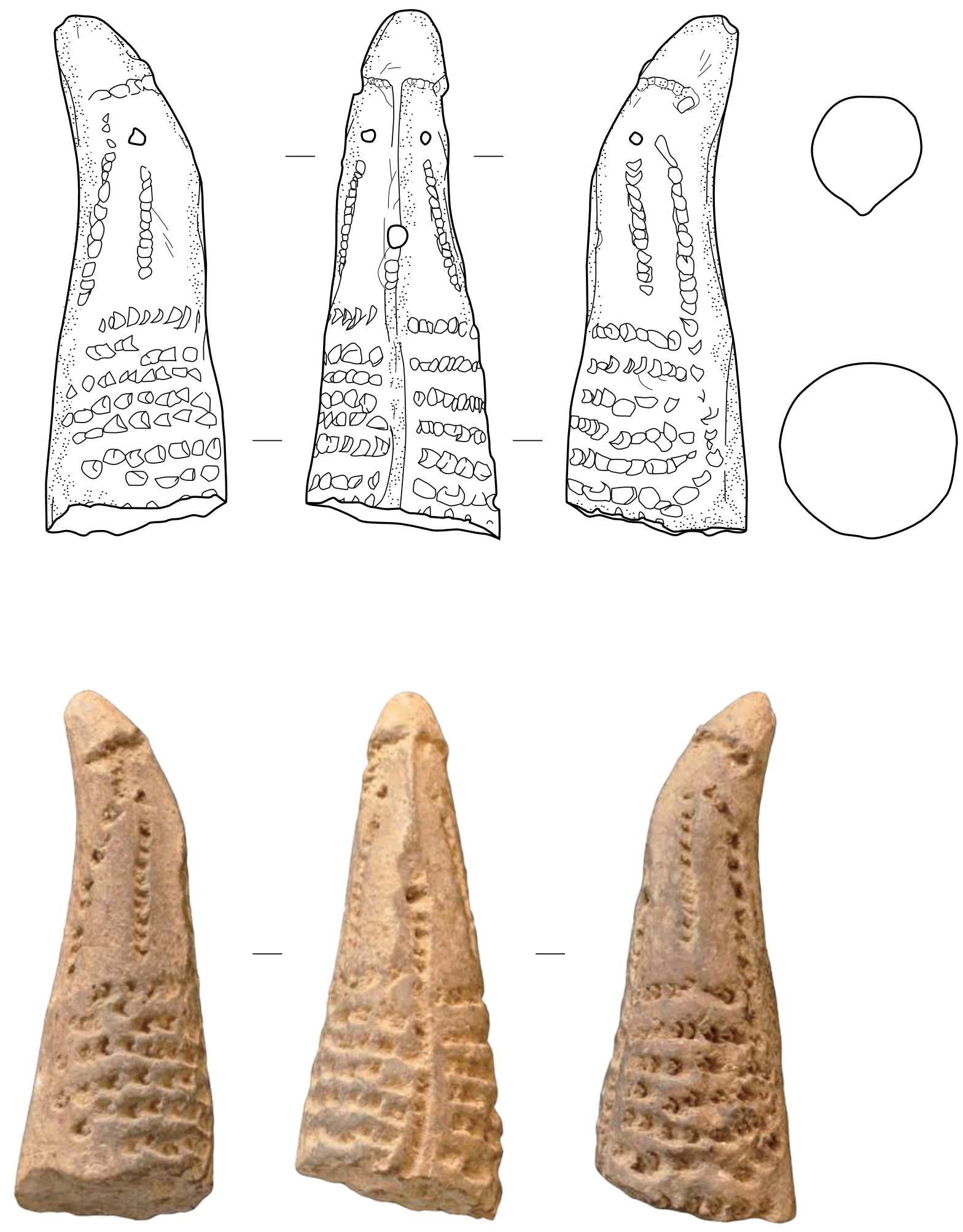

\section{$5 \mathrm{~cm}$}

Fig. 14.1 Göytepe Figurine 1 (Square 1BII). 

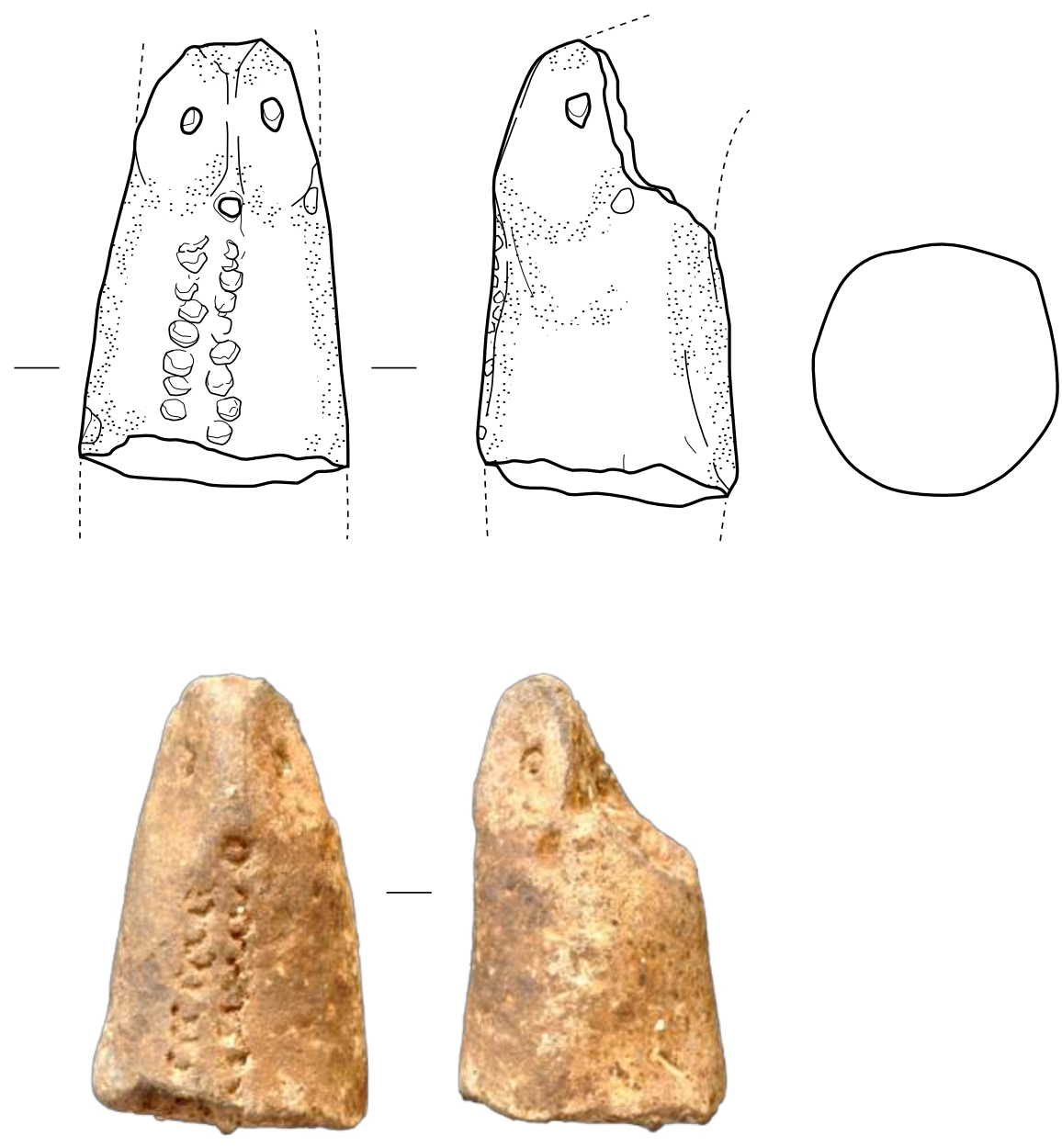

\section{$5 \mathrm{~cm}$}

Fig. 14.2 Göytepe Figurine 2 (Square 2All).

4 building complex, and presumably coming from the northeastern corner of the square. This area is considered to be outside the complex, where at least two ovens are installed. Such situations indicate that the figurine was discarded in ashy refuse deposits mixed with other artifacts.

The Figurine 2 has many common features with the Figurine 1 in terms of producing technique. It is also made of unfired fine clay with a small amount of sand temper (less than $1 \mathrm{~mm}$ ). The matrix color is grayish brown (7.5YR5/2), and surface color ranges from grayish brown $(7.5 \mathrm{YR} 4 / 2)$ to brownish black (7.5YR3/2) possibly due to depositional condition. The extant height measures approximately $2.5 \mathrm{~cm}$, lacking the tip of the upper part (head) and larger part of the body. Its head also seems to be somewhat protruded backward as the forehead shows an inclinated surface. Again its face is facetted, but the nose line is pinched out but less pronounced than the Figurine 1 described above. Eyes are impressed using a tube-like tool with the size of $1.0-1.5 \mathrm{~mm}$ in diameter, while a small punctuation under the nose may represent a mouth. A shallow depression on its left may depict a single ear. Also remarkable are two vertical lines of impressed dots. The rendering method is similar to that of the Figurine 1, but the punctated pattern differs.

\subsection{Discussion}

Anthropomorphic clay figurines have been attested from some Shomutepe sites in the adjacent region. 
Most extraordinary and near-complete seated figurine ever discovered is a female figurine from Gargalartepesi (Narimanov 1987: fig. 28). This figurine has a pillar-like torso, bent at the buttock with tapered head and legs, and equipped with a featureless protrusion in the abdominal part. On the leg part of this figurine triangular impressed dots cover its surface, and on the upper body two vertical lines of dots are flanked on both sides by zigzag-like dotted lines (Munchaev 1982: table XL, 3). Although the head and face are broken, this example can be comparable well with the Göytepe Figurine 1 with regard to both proportions and decorative patterns.

A lower part of similar clay statuette has been reported from Aruchlo (Hansen et al. 2006: Abb. 56). According to the preliminary report, found from a round building AR05A108, this is a seated figurine with punctated decorations on its back and abdomen and a deep groove dividing its tapered legs. Diverse types of clay figurine including seated ones were found at Khramis Didi-Gora as well (Kiguradze 1986). Kiguradze classified various figurines into two groups, naturalistic and schematic, with the former subdivided into two types: figurines with bent legs may usually have upright stature and facetted face (Kiguradze 1986: Abb. 83.4, Abb. 86.1), while another seated type with extended legs (ibid., Abb. 82.8) show a more abstract form to which Gargalartepesi and Aruchlo figurines belong. The latter group includes conical clay objects and geometric figurines, but both of our examples cannot be attributed to this group due to clear morphological differences.

The Göytepe figurines presented here are, on the whole, well manufactured and highly stylized but with meticulous facial features. Both examples, Figurines 1 and 2, appear to share many features (cone-shaped proportion, elongated head, facetted face, and punctated ornaments) with each other, so that both may belong to the same type of extended legs. However, compared with complete examples such as Khramis Didi Gora figurines which have bent legs and facetted faces in some cases (Kiguradze 1986: Abb. 83.4, Abb. 86.1), it is also possible to suggest that our Figurine 2 in particular would also be part of a figurine with bent legs. As with sex determination of each figurine, they are possibly recognized as female in comparison with other excavated materials in the region, although the fragmented figurines from our site do not show any definite distinguishing traits.

Other than anthropomorphic figurines, different categories of clay figurines are widely known. At Aruchlo, a ram figurine and stone figurines representing human heads have been obtained (Chelidze and Gogelia 2004; Hansen et al. 2006; Kushnareva and Chubinishvili 1970: 28). In fact, animal figurines, which are abundantly encountered in the Near Eastern Neolithic sites, seem to occur very sporadically in the Transcaucasian Neolithic as a whole. 'Token'-like small clay objects have been found from Khramis Didi Gora (Kiguradze 1986: Abb. 82.12-15), and similar ones recently excavated from Aruchlo (Hansen et al. 2006: Abb.57) as well as from a site called MPS 4 near Kamiltepe in the Mil steppe (Helwing and Aliyev 2012: fig. 13). But to date, there are no evidence of animal figurines nor other clay objects comparable to these at Göytepe.

Functional interpretations of clay figurines in prehistoric periods are still poorly studied and debated in different ways across various regions (cultic depot, vehicle of magic, initiation cult, dolls of teaching aids, or simply toys for children) (Voigt 1983: 186-202). Yet, as many scholars agree (Lesure 2011; McAdam 1997), spacial and contextual analysis for excavated objects would provide some clues to understand them. For our examples, both figurines were exclusively found not inside any constructions but in the refuse deposits outside buildings in both cases. It suggests that they must have been discarded as rubbish after their uses. In contrast to Göytepe, figurines of Khramis Didi Gora were found from different context, where 17 figurines were discovered side by side near a hearth in the middle of structure (Bauhorizont II) (Kiguradze 1986). Not all figurines from this site seem to be associated with hearth, for a large number of figurines were retrieved from other contexts as well. Also of any significance is the fact that most figurines are found in fragmented or damaged pieces (head, body, legs are often recovered separately). Bearing in mind that most figurines were found unfired and often broken off, it seems more likely that whatever their function be, they were utilized or treated for a considerably brief period before discarded in refuse deposits. 
The figurines of Shomutepe cultural region remain to be fully analyzed not only because we do not have enough excavated specimens at our disposal, but because there are very few information available for analyses. A combination of more quantitative data and contextual analysis will shed more light on the interpretation of Neolithic clay figurines in this region.

\section{Reference}

Chataigner, C. (1995) La Transcaucasie au Néolithique et au Chalcolithique. BAR Internatinal Series 624. Oxford: Tempus Reparatum.

Chelidze, L. and D. Gogelia (2004) Arukhlo I: An earlyfarming site. Journal of Georgian Archaeology, 1: 46-92.

Kiguradze, T. (1986) Neolithische Siedlungen von Kvemo-Kartli, Georgien. Materialien zur Allgemeinen und Vergleichenden Archäologie, Bd 29. München: Verlag C. H. Beck.

Hansen, S., G. Mirtskhulava, K. Bastern-Lamprichs, N. Benecke, I. Gatsov, and P. Nedelcheva (2006) Aruchlo 2005-2006: Bericht über die Ausgrabungen in einem neolithischen Siedlungshügel. Archäologische Mitteilungen aus Iran und Turan, 38: 1-34.

Helwing, B. and T. Aliyev (2012) Field work in the Mil Plain: The 2010-2011 Expedition. Archäologische Mitteilungen aus Iran und Turan, 44: 4-17.

Kushnareva, K. Kh. and T. N. Chubinishvili (1970) Древние Культуры Южного Кавказа (V -III тыс. до H. Э.). Leningrad: Nauka.

Lesure, R. G. (2011) Interpreting Ancient Figurines: Context, Comparison, and Prehistoric Art. Cambridge: Cambridge University Press.

Lloyd, S. and F. Safar (1945) Tell Hassuna. Excavations by the Iraq Government Directorate General of Antiquities in 1943 and 1944. Journal of Near Eastern Studies, 4: 255-289.

McAdam, E. (1997) The figurines from the 1982-5 seasons of excavations at Ain Ghazal. Levant, 29: 115-145.

Munchaev, R. М. (1982) Энеолит Кавказа. In: Энеолит CCCP, edited by V. M. Masson and N. Merpert, pp. 100-164. Moscow: Nauka.

Narimanov, I. (1987) The Culture of the Most Ancient Farming and Stock-Breeding Population of Azerbaijan. Baku: National Academy of Sciences (in Russian with an English summary).

Voigt, M. M. (1983) Hajji Firuz Tepe, Iran: The Neolithic Settlement. Hasanlu Excavation Reports, Vol. I. Philadelphia: The University Museum, University of Pennsylvania. 

Chapter 15

\section{The Neolithic bone and antler industry from Göytepe}

Saiji Arai

\subsection{Introduction}

The rich worked bone industry is one of the most important characteristics of Neolithic sites in the Southern Caucasus. Conforming to this trend, many bone and antler artifacts have been recovered from Göytepe. This chapter presents the general typology and technology of the worked bone industry from the site. The study sheds light on several significant aspects of the Neolithic culture in the region.

\subsection{Worked bone assemblage from Göytepe}

During the 2008 to 2011 seasons, a total of 357 bone and antler artifacts have been collected by the Japanese expedition from the primary contexts of Square 4B (Sectors 4BI and 4BII) and 32 pieces out of context (Table 15.1). The total number accounts for $6.3 \%$ of the faunal remains overall. The artifacts were classified based on their shape and raw material. Their general typology is described below. Quantitative data, manufacturing traces and schemes

Table 15.1 Worked bone materials from Square 4B at Göytepe.

\begin{tabular}{|l|c|r|r|r|r|r|r|r|r|}
\hline \multicolumn{1}{|c|}{ Type } & Level 12 & Level 11 & Level 10 & Level 9 & Level 8 & Level 7 & Level 6 & Level 5 & Total \\
\hline Awl & 3 & 23 & 21 & 8 & 3 & 30 & 8 & 50 & 146 \\
\hline Spatula & 0 & 9 & 3 & 0 & 0 & 5 & 1 & 2 & 20 \\
\hline Palette & 0 & 1 & 0 & 0 & 0 & 1 & 0 & 3 & 5 \\
\hline Hoe & 0 & 9 & 5 & 5 & 1 & 0 & 2 & 7 & 29 \\
\hline Bone dibble & 0 & 6 & 1 & 2 & 2 & 3 & 0 & 4 & 18 \\
\hline Antler dibble & 1 & 1 & 4 & 0 & 0 & 2 & 1 & 2 & 11 \\
\hline Hammer & 0 & 2 & 1 & 0 & 1 & 1 & 0 & 2 & 7 \\
\hline Dibble/hammer & 0 & 0 & 0 & 0 & 1 & 0 & 0 & 0 & 1 \\
\hline Flesher & 2 & 2 & 0 & 0 & 0 & 2 & 1 & 2 & 9 \\
\hline Arrowhead & 0 & 0 & 1 & 1 & 0 & 0 & 0 & 0 & 2 \\
\hline Buttonette & 1 & 0 & 0 & 0 & 0 & 0 & 0 & 0 & 1 \\
\hline Knife & 1 & 2 & 3 & 0 & 0 & 0 & 0 & 0 & 6 \\
\hline Counter & 0 & 1 & 0 & 1 & 0 & 0 & 0 & 0 & 2 \\
\hline Sickle handle & 0 & 0 & 1 & 0 & 0 & 0 & 0 & 0 & 1 \\
\hline Debitage & 0 & 1 & 4 & 0 & 0 & 2 & 0 & 1 & 8 \\
\hline Unclassifiable & 0 & 26 & 16 & 4 & 13 & 21 & 4 & 7 & 91 \\
\hline Total & 8 & 83 & 60 & 21 & 21 & 67 & 17 & 80 & 357 \\
\hline
\end{tabular}


of worked bones are presented in the text (Figs. 15.115.9), while illustrations and photos of the worked bones are found at the end of this chapter (Figs. 15.10-15.30).

\subsubsection{Awl (Figs. 15.10 and 15. 11)}

The "awl" is the predominant bone tool type at Göytepe, accounting for about half of the assemblage $(n=146)$. They are mainly made on long bones of medium-sized ungulates. The distal metapodial is the most common, but the proximal radius and distal tibia were also used as raw material. Since most specimens have only been processed around their distal tips, the proximal areas show no traces of processing. Interestingly, red ochre is often present on the surface of awls. Since the same situation has been attested at Mentesh Tepe (Le Dosseur 2012: 121), it would be useful to discuss these tools' function and the relationship between the two different industries. Lengths of the tools vary, whereas the tip's diameter is quite uniform (ca. $2.8 \mathrm{~mm}$ on average). This fact may be related to their manufacturing technology (see below).

\subsubsection{Spatula (Fig. 15.12)}

The "spatula" is a common tool type at Göytepe $(n=20)$ similar to other Neolithic sites in the regions of Eurasia.

We could identify two different types according to raw materials: long bones (mainly tibiae of sheep/ goat) and scapulae (spine) of large ungulates. Spatulas made on long bones show traces of processing only around the edges. This type is quite common in the Shomutepe culture. Specimens made on scapulae are larger than those on long bones and show traces of abrasion around their edges. Raw material of the tools on scapulae may be debitage flaked during the production of other tools (see below).

\subsubsection{Palette (Figs. 15.12 and 15.13)}

Five implements made on the flat parts of scapula were classified as "palettes." Although their actual functions are unclear, similar objects are reported from other prehistoric sites around the region. They are generally semi-circular in shape and show traces of polishing on their entire surfaces. Although specimens incised around part of the edge have been reported from other sites (Badalyan et al. 2007: fig. $6 \mathrm{~g}$ ), no such specimens were found at Göytepe.

15.2.4 Hoe (Figs. 15.14-15.16)

"Hoes" made on scapulae of large-sized ungulates are the most characteristic implement among Neolithic sites in the Kura valley. Many complete or fragmented hoes have also been found at Göytepe $(n=29)$. Their edges are of different shapes (e.g., linear, oblique, forked), suggesting their frequent use. The lateral or medial surfaces and articular ends are perforated for hafting.

\subsubsection{Dibble (Bone) (Fig. 15.17)}

Bone "dibbles" made of various skeletal elements of large-sized ungulates are also characteristic with a total of 18 specimens found at Göytepe. We could identify implements made on the proximal radius, distal tibia, proximal or distal metapodial. In order to insert a handle, specimens made from the distal part are perforated on the diaphysis and others on the proximal articular surface. Despite of the difference in raw materials, all implements have a similar shape and are made using similar technologies. These implements are well-known in the Kura valley, whereas comparable implements are reported from Neolithic sites in other regions such as Beidha in southern Jordan (Stordeur 1999: fig. 1) and Tell Hassuna in northern Iraq (Lloyd et al. 1945: pl. X-2).

\subsubsection{Dibble (Antler) (Figs. 15.18-15.20)}

Besides bone dibbles, several antler "dibbles" (or picks?) have been found at Göytepe ( $\mathrm{n}=11)$. Although they are classified as the same type, it is remarkable that the antler dibbles are made on blanks of three different types. The first type is the largest, using the antler base. These have a relatively flat edge and are perforated where the tine was removed. The second type uses the beam portion including the branch. Similar to the first type, these have flat edges and are perforated for hafting. The third type uses the tine part and has a pointed tip unlike the other types. It is possible that a functional difference exists between the groups. For example, some tine specimens without perforation may have been a "punch" for 
flaking lithics (Fig. 15.12: 5).

\subsubsection{Hammer (Fig. 15.21 and 15.22)}

The "hammer" is another tool type made on antler. Seven specimens have been found at Göytepe. In contrast to dibbles, they are completely transformed, shaped like an ovoid or handle. Some specimens with decorations on their surfaces might have served for symbolic uses, and do not show use wear on the functional portion. Both the base and beam of antler appear to have been used equally as raw materials. All of the specimens have been perforated for hafting.

15.2.8 Flesher (or Burnisher) (Figs. 15.23 and 15.24)

Nine implements with flat edges were classified as a "flesher" or "burnisher." Unlike spatulas, fleshers have narrower edges and were made from relatively robust skeletal elements as raw material. To date, specimens made on scapulae or metapodials of large ungulate have been found.

\subsubsection{Arrowhead (Fig. 15.25: 1)}

Two "arrowheads" have been recovered from Levels 10 and 9 . Since the degree of processing was extensive, we could not identify the raw material. However, considering their length and thickness, the raw material appears to be bone (metapodials) of large-sized ungulates. Christidou (2009) notes that they may be made on metapodials of large cervids. Similar specimens have been found at Khramis Didi Gora (Munchaev 1982: tab. XXXVI-15), Imiris Gora, and Aruchlo I in Georgia (Kiguradze 1986: figs. 38-12 and 55-5), Aratashen in Armenia (Badalyan et al. 2007: fig. 6c), and Tilkitepe near Lake Van (Korfmann 1982).

15.2.10 Buttonette (Figs. 15.25: 2 and 15.26: right)

One small button-shaped implement was found in Level 12. Although the shape and length resemble to the arrowheads, this "butonette" lacks a pointed tip. Instead, one extremity has a rounded tip and the other is flat in cross-section. The raw material could not be determined. Traces of heavy abrasion are observed on the entire surface.
15.2.11 Knife (Figs. 15.25: $3-4$ and 15.26: left, center)

Four knife-shaped implements have been found in the lower levels (Levels 12-9). These consist of two parts: edge and handle. These "knives" are probably made on a flat bone such as the scapula. Some are perforated on the proximal end of the handle.

\subsubsection{Counter (Fig. 15.25: 5)}

Implements with parallel incisions were classified as a "counter" (Redman 1973: 258). Two specimens were found at Göytepe. Both of them were made on scapulas of medium- or large-sized ungulates. Although these tools are classified as one independent type, it was undecided whether they actually functioned as a counter, because one specimen from another sector of the site has both parallel incisions and a pointed tip.

\subsubsection{Ornament (Fig. 15.25: 6-7)}

A few ornaments made on bones, antlers, or teeth have been found at Göytepe. These include incised antlers, beads made on bird bone, and perforated incisors. Only one unfinished bead made on a bird radius has been recovered during the Japanese excavations. In general, the evidence for artistic representation is poor in the Middle Kura during the Neolithic period.

\subsubsection{Sickle handle (Figs. 15.27 and 15.28)}

Sickle handles made on antler or mandible are well known from Neolithic sites in the Kura valley (Narimanov 1987). At Göytepe, however, only the mandibles of cattle were used as raw material. This tool type used almost the entire mandible. After removing the cheek teeth, sickle elements made on stone were attached with bitumen to the alveolar part of the mandible.

\subsubsection{Debitage}

There are some bone fragments classified as debitage, consisting mainly of long bone fragments with percussion marks or the isolated scapula spine. In addition to these, one large-sized ungulate tibia splintered by grooving was found in Level 8 (Fig. 15.29). This is an interesting find because most tools made on long bones were splintered by percussion, 
as described below.

\subsubsection{Unclassifiable worked bone/antler}

Many bone or antler fragments with traces of processing have been recovered from each level. Most specimens are "unclassifiable" to tool type because it was difficult to determine the function from their shapes, or they were fragmented and do not retain their original shapes. Among these, the most interesting finds are two worked dog mandibles from Levels 7 and 10 (Figs. 15.25: 8 and 15.30). Similar objects have been reported as possible "sickles" from Aratashen in Armenia (Badalyan et al. 2007: fig. 6h).

\subsection{Raw material selection}

An archaeozoological study of worked bone shows how raw materials were selected and procured by the site's inhabitants. Table 15.2 presents the number of identified specimens used as raw material. It is clear that bones of domestic livestock, that is, wastes from daily activities, were mainly selected. On the other hand, antlers of red deer were also frequently used. Implements made on deer antlers are ubiquitous among archaeological sites worldwide, since they are a particularly suitable raw material. In addition, people can easily procure shed antlers during foraging in the forest. The high frequency of antlers in the assemblage might indicate that inhabitants of Göytepe had often exploited forests resources.

With respect to each tool type, various skeletal elements were selected as raw material. For example, bone awls are made on radii, tibiae, and metapodials of medium-sized ungulates. Materials with unfused epiphysis were also used. Difference in raw material possibly reflects the preferences of individual manufacturers or low availability of proper materials at the time of manufacturing. Distal metapodials are always the most frequent. However, the status in raw material selection changes slightly in the upper levels: the frequency of tools made on distal metapodials and proximal radii increase, whereas proximal metapodials decrease (Fig. 15.1).

The diversity of implements made from scapulae is the most notable. Hoes, palettes, spatulas, counters, fleshers were all similarly made from scapulae of large-sized ungulates. Of these, the blanks for spatulas, counters, and fleshers probably represent broken hoes based on traces of perforation visible on the distal articular surface (Fig. 15.2). The same strategy may be applicable to the antler industry (Fig. 15.3). The choices of broken tools as raw material

Table 15.2 Animal species used as raw material for bone tools.

\begin{tabular}{|l|r|r|r|r|r|r|r|r|r|}
\hline \multicolumn{1}{|c|}{ Species } & Level 12 & Level 11 & Level 10 & Level 9 & Level 8 & Level 7 & Level 6 & Level 5 & Total \\
\hline Ovis & 1 & 7 & 8 & 1 & 0 & 15 & 3 & 11 & 46 \\
\hline Capra & 0 & 1 & 1 & 0 & 0 & 5 & 1 & 8 & 16 \\
\hline O/C & 1 & 14 & 11 & 6 & 2 & 9 & 1 & 10 & 54 \\
\hline Sus & 0 & 0 & 0 & 0 & 0 & 1 & 0 & 0 & 1 \\
\hline Bos & 0 & 11 & 2 & 2 & 0 & 2 & 0 & 2 & 19 \\
\hline Gazella & 0 & 0 & 0 & 0 & 0 & 0 & 0 & 2 & 2 \\
\hline Cervus & 1 & 4 & 5 & 0 & 3 & 4 & 1 & 5 & 23 \\
\hline Canis & 0 & 0 & 1 & 0 & 0 & 1 & 0 & 0 & 2 \\
\hline Ave & 0 & 1 & 0 & 0 & 0 & 0 & 0 & 0 & 1 \\
\hline Medium-sized & 0 & 24 & 15 & 3 & 9 & 16 & 8 & 22 & 97 \\
\hline Large-sized & 5 & 16 & 10 & 7 & 6 & 12 & 3 & 15 & 74 \\
\hline M/L. sized & 0 & 5 & 7 & 2 & 1 & 2 & 0 & 5 & 22 \\
\hline Total & 8 & 83 & 60 & 21 & 21 & 67 & 17 & 80 & 357 \\
\hline
\end{tabular}


may reflect the expedient nature of the industry at Göytepe (see below).

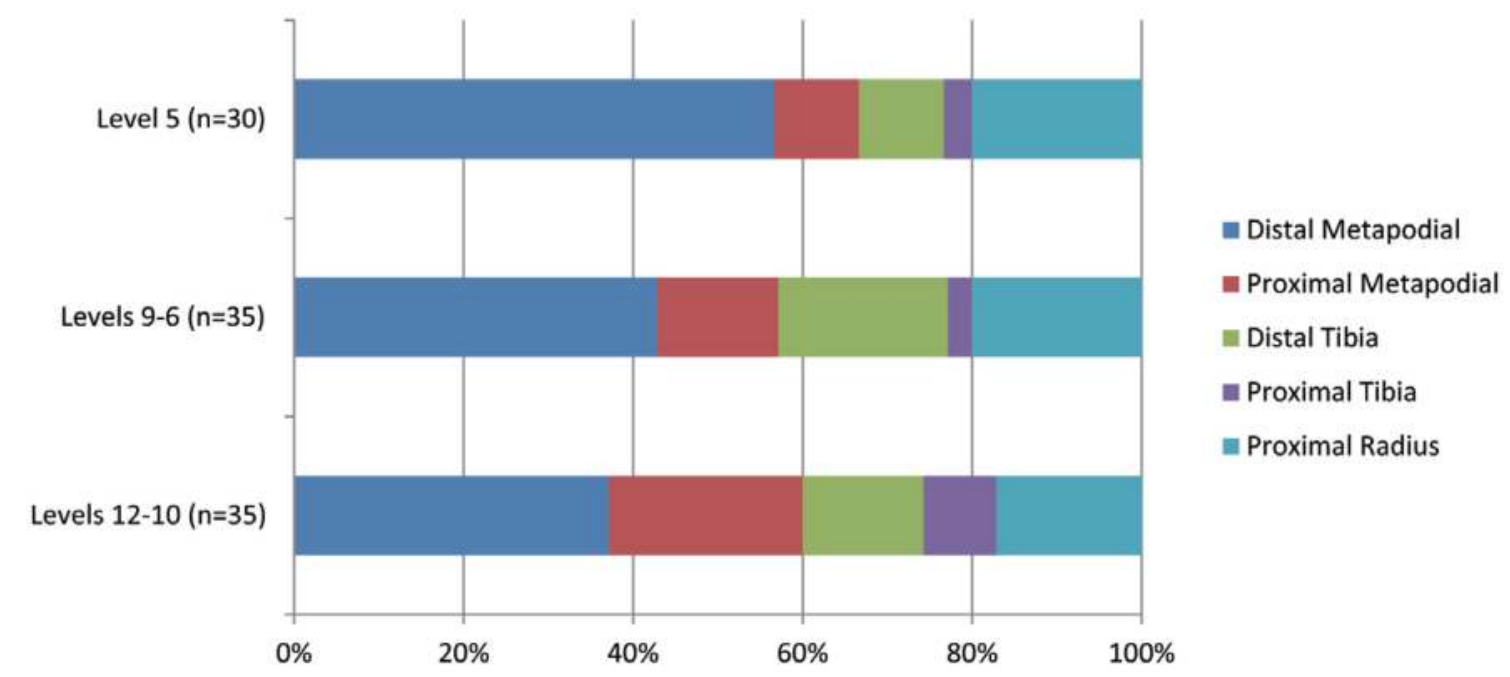

Fig. 15.1 Chronological changes in the use of skeletal parts used to manufacture awls at Göytepe. 

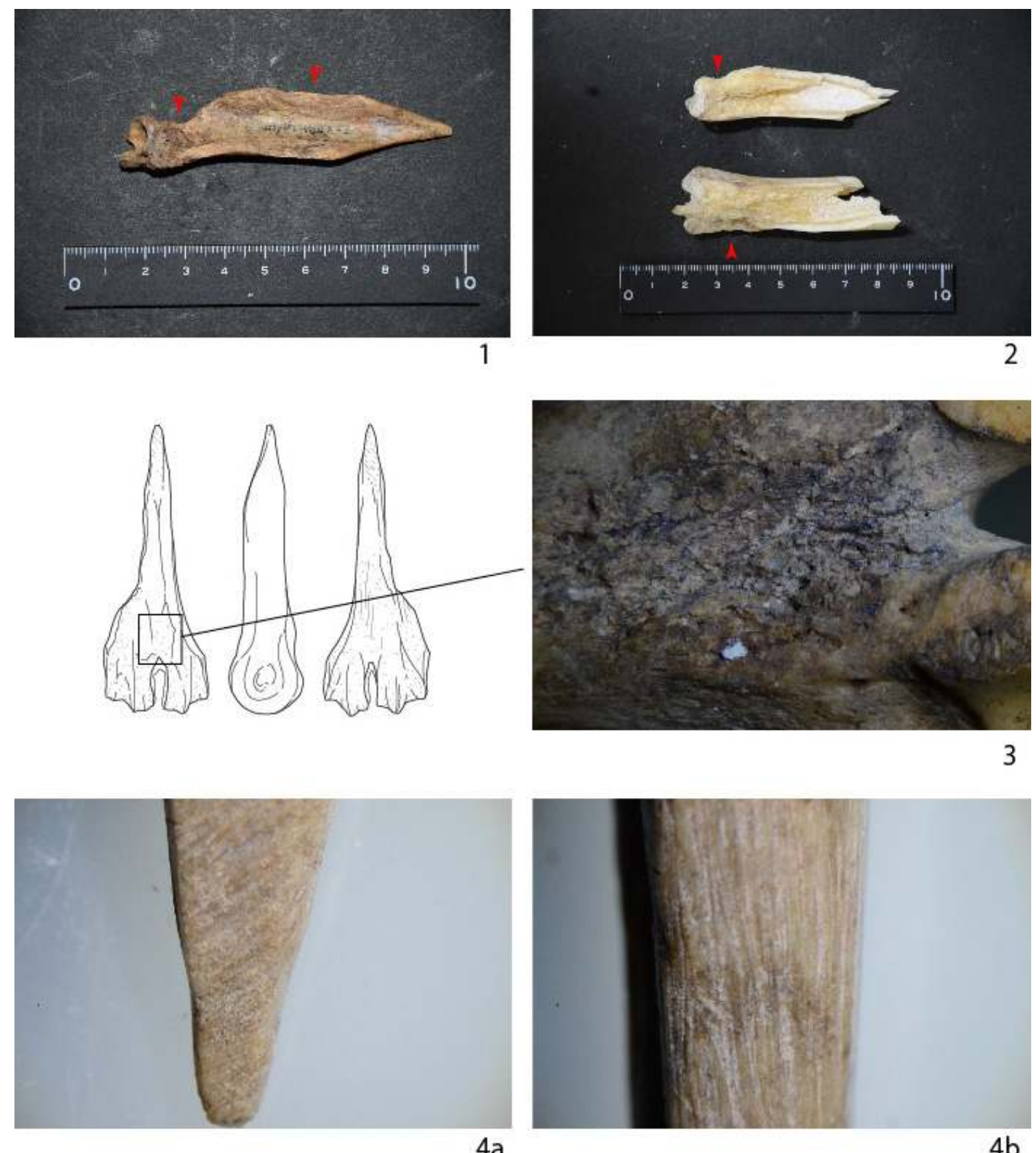

Fig. 15.2 Micrographs of bone awls from Göytepe. 1: Negative flake scars; 2: Replicated blank; 3: Percussion marks on the surface; $4 a-b$ : Traces of abrasion around the tip. Red arrow: impact point. 


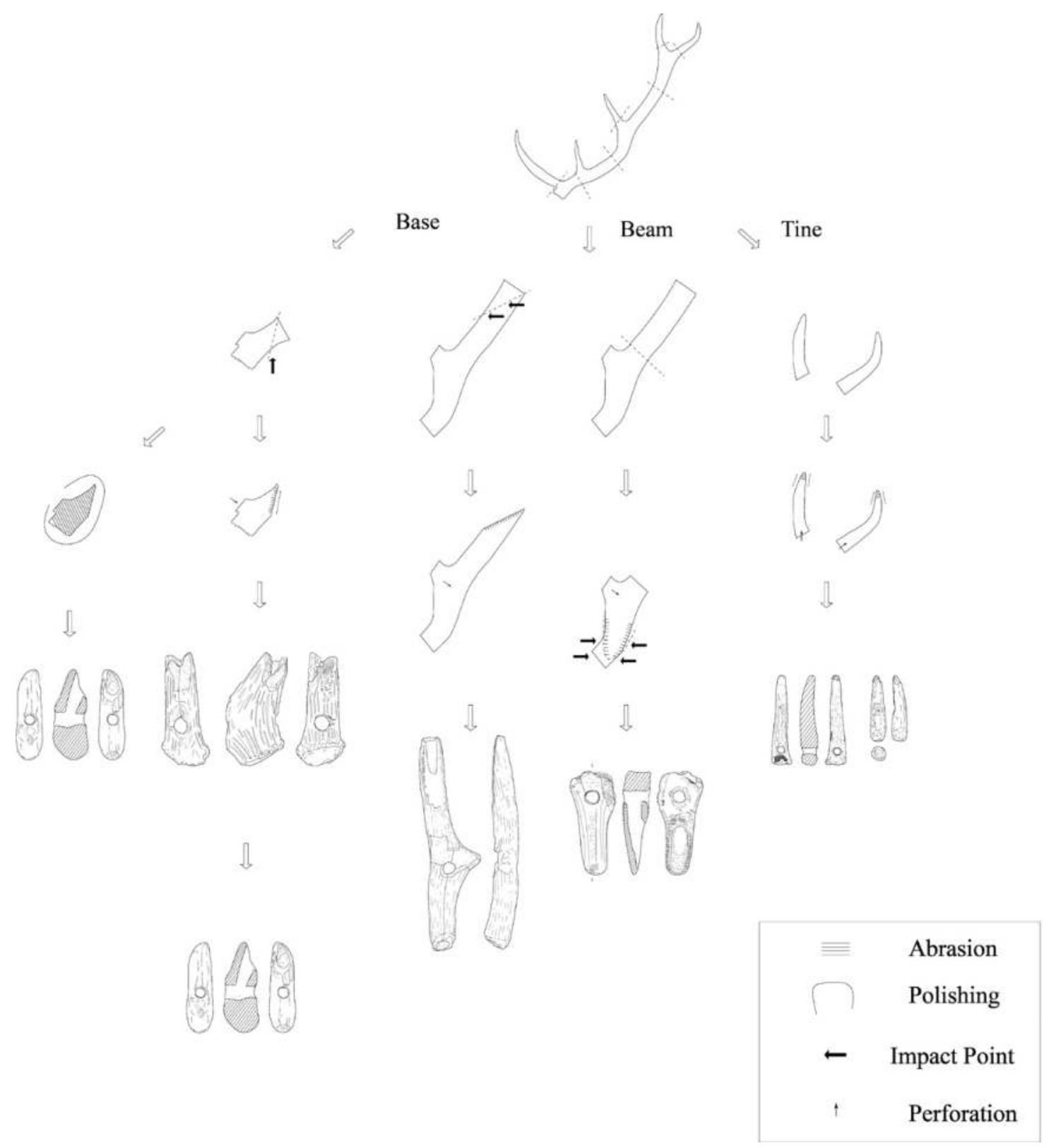

Fig. 15.3 Manufacturing scheme of worked antler implements at Göytepe. 


\subsection{Manufacturing process and its technology}

Here we present the technology used for working bone and antler at Göytepe. Generally speaking, most tools were manufactured on "less time-consuming" way as reported from the nearby site of Mentesh (Le Dosseur 2012: 119). The results of technological studies are described below by raw material type.

Implements using long bones of medium-sized ungulates (awls, spatulas) are made on blanks that were flaked by percussion. For example, most awls that were split longitudinally have negative flake scars and were spirally flaked (Fig. 15.2: 1-2). Even awls retaining intact epiphyses show percussion marks on the anterior or posterior surface, indicating the intention to split the blank (Fig. 15.2: 3). For spatula blanks, most specimens were made form tibiae that were fractured around the proximal epiphysis to the middle of the diaphysis. The finds from 4BI-131KK demonstrate that these skeletal parts were actually selected for blanks. In this excavation context, plenty of tools, blanks, and raw materials were found in a pile (Fig. 15.4). These blanks were also spirally fractured. It is possible that some blanks selected for these tools were produced through butchering activities (e.g., marrow-cracking). After the blanks were prepared, they were shaped into their final forms. This was mainly achieved by abrasion on a hard material including coarse-grained ground stones. Traces of horizontal or longitudinal abrasions are seen on many tools (Fig. 15.2: 4a-b). Although a shaving technique was also used, it was applied to a much smaller number of tools.

Heavy-duty tools such as bone or antler dibbles and hammers were made through a similar process. Some blanks found at Göytepe show traces of direct or indirect percussion. It is important to note that the groove-and-splinter technique (Clark and Thompson 1953) has been rarely attested here

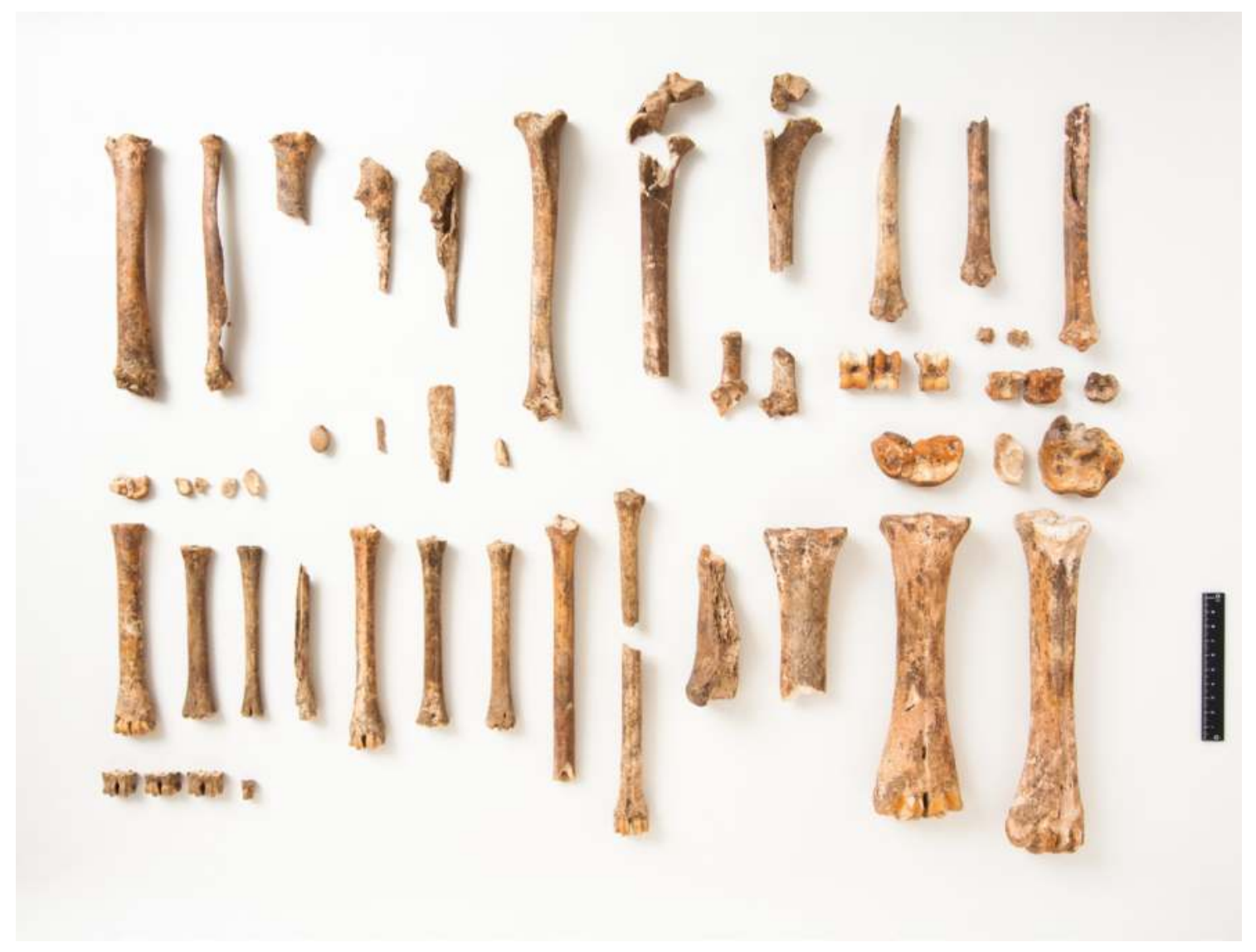

Fig. 15.4 Bone tools and non-worked boned from the locus 4BI-131KK at Göytepe. 

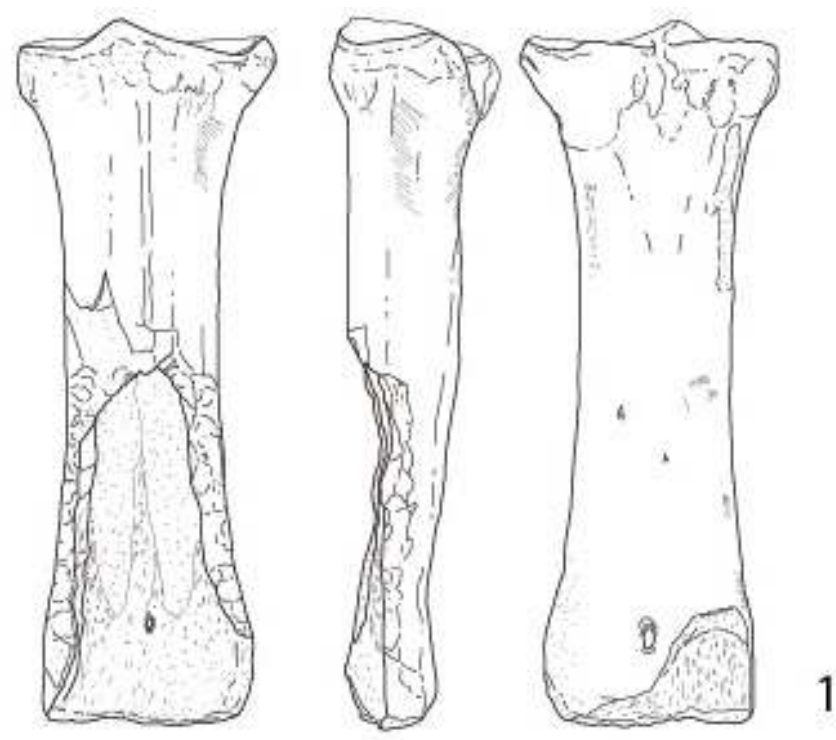

0 $5 \mathrm{~cm}$
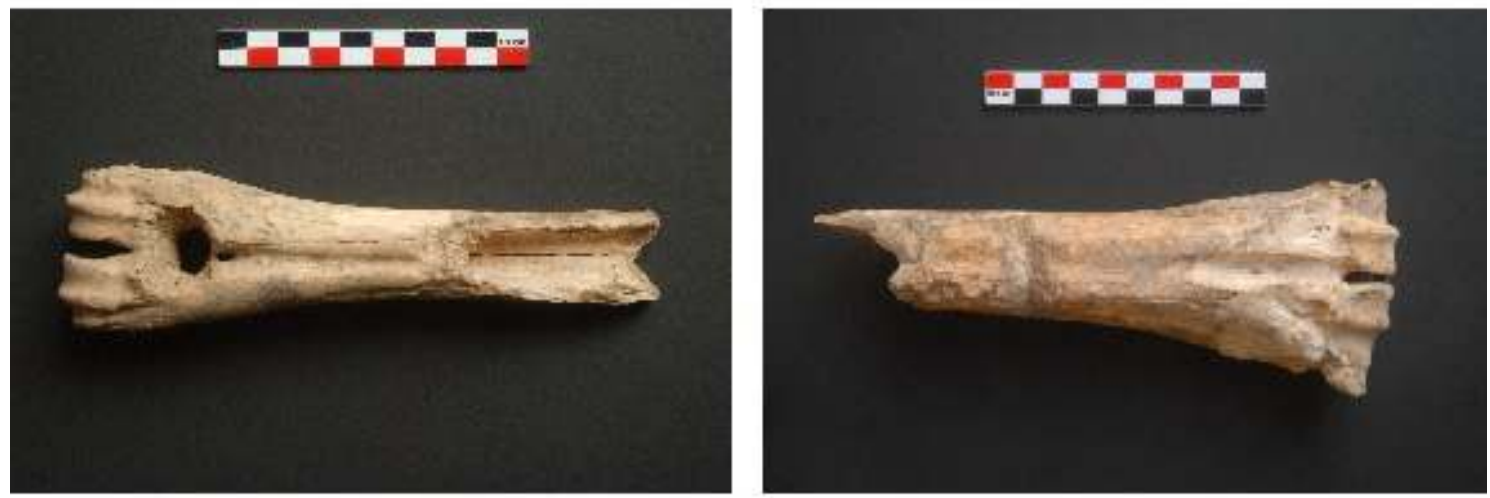

$2 a$

$2 b$
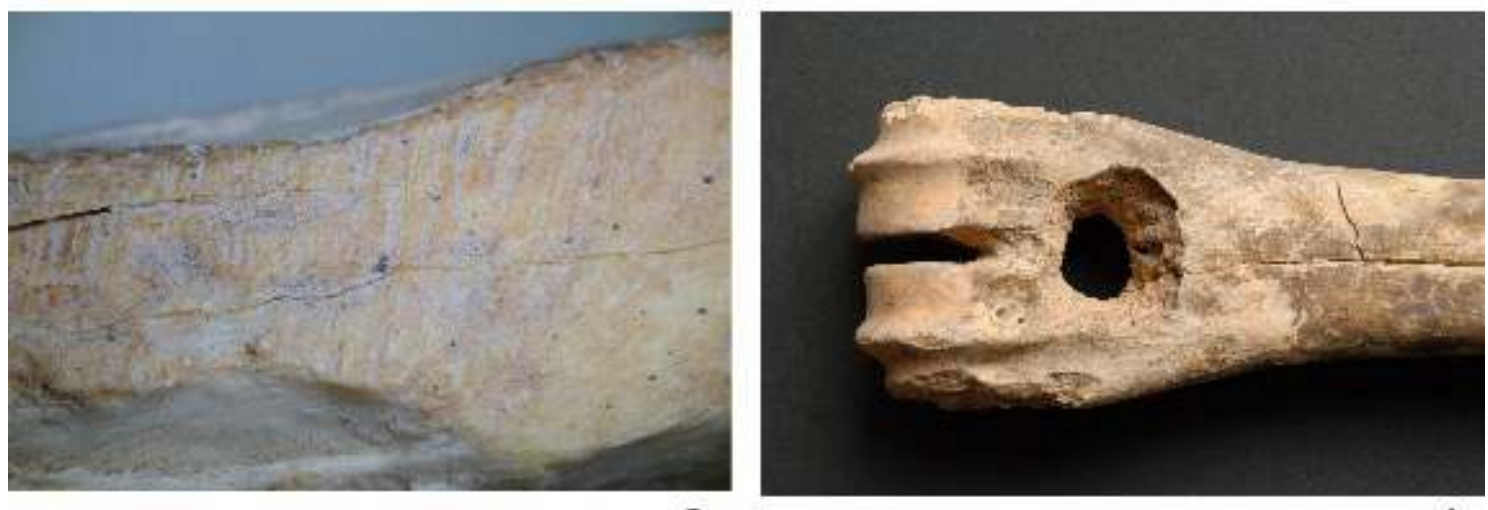

3

4

Fig. 15.5 Micrographs of bone dibbles from Göytepe. 1, 2a-b: Unfinished tool; 3: Traces of abrasion on the edge; 4: Perforation.

(Fig. 15.5: 1, 2a-b), even on antlers (but see Fig. 15.6: 2). The perforation technique for hafting is still unclear. However, it was probably finished by pecking directly or indirectly, since the perforations are irregularly shaped and many pecking marks were observed around them (Figs. 15.5: 4 and 15.6: $3)$. In this way, the perforations were enlarged. The edges were finished by abrasion on coarse-grained 

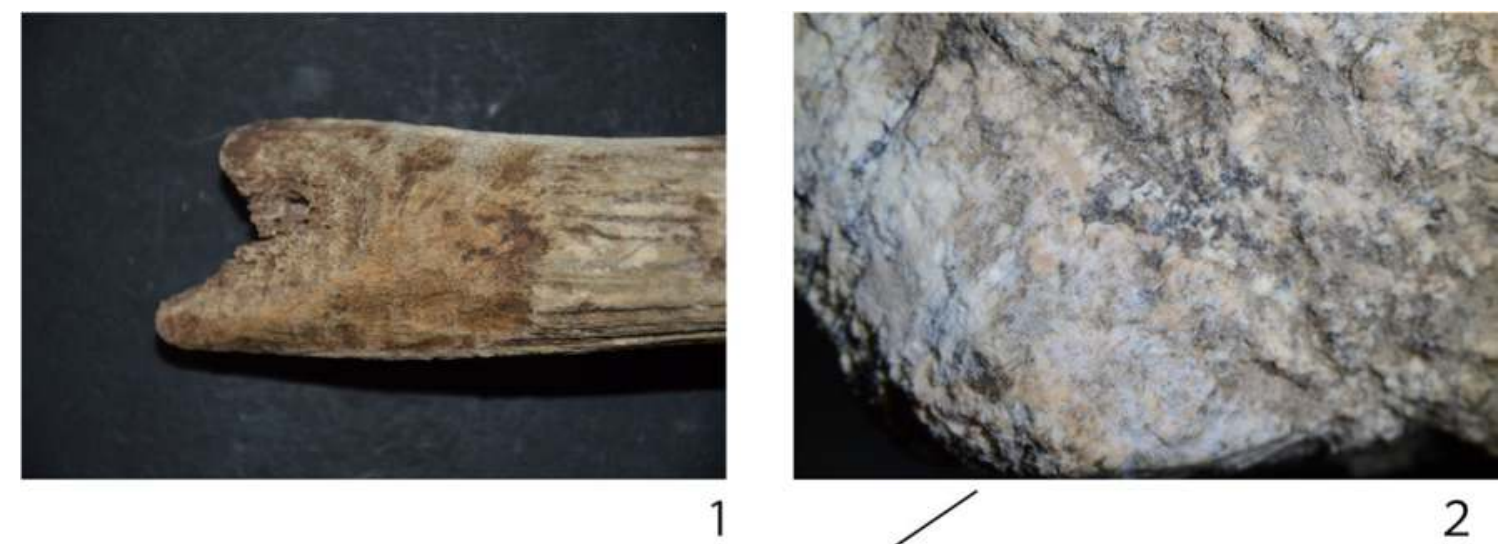

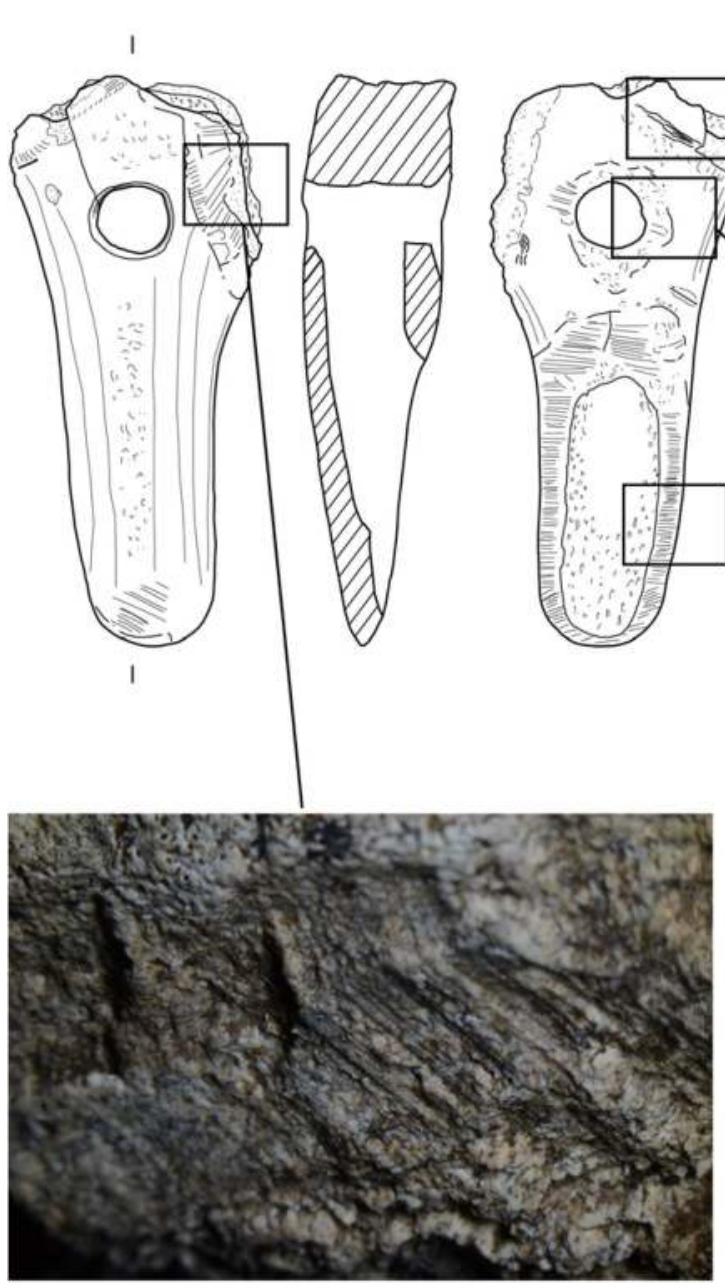

4

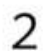

\section{政}

(

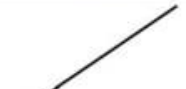

2 

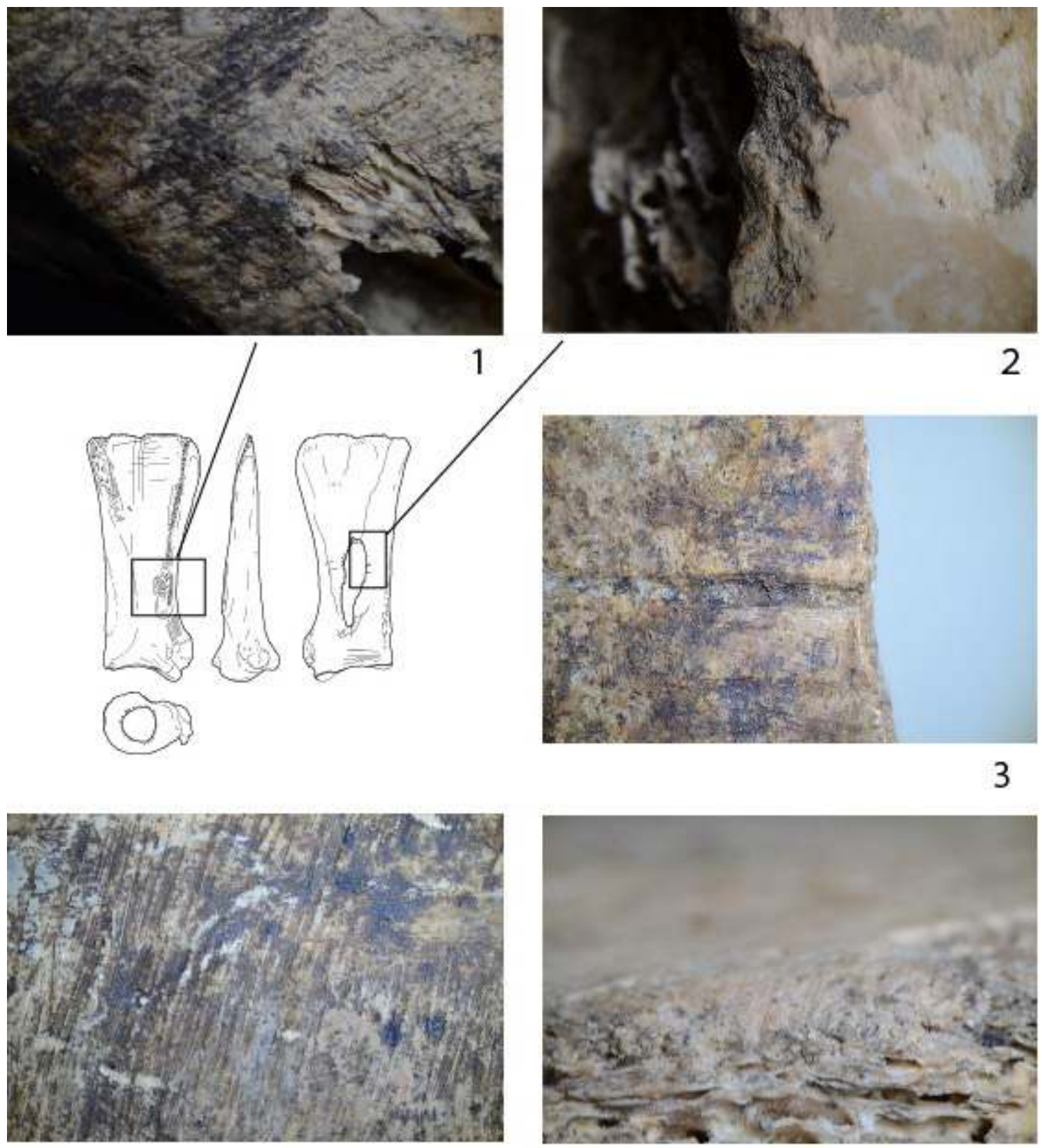

Fig. 15.7 Micrographs of scapulae from Göytepe. 1: Traces of abrasion on hoe; 2: Edge of perforation; 3: Traces of grooving on palette; $4-5$ : Traces of abrasion on counter and spatula.

separated by grooving. This piece may have been used as a blank for a palette. In the third stage, the other (distal) half of the scapula was perforated on its medial, lateral surface, or distal articular surface. This appears to have been achieved by pecking motions similar to the manufacture of bone dibbles. After its final shape was created, each implement was finished by abrasion probably on coarse- or finegrained ground stone.

Other tools that were completely transformed and no longer retain the blank's original form could not be identified in regard to either raw material or flaking technique. It is possible that some specimens were flaked by grooving because of their long length. In any case, they were finished by abrasion, as seen in clear parallel traces on their surfaces (Fig. 15.9). 

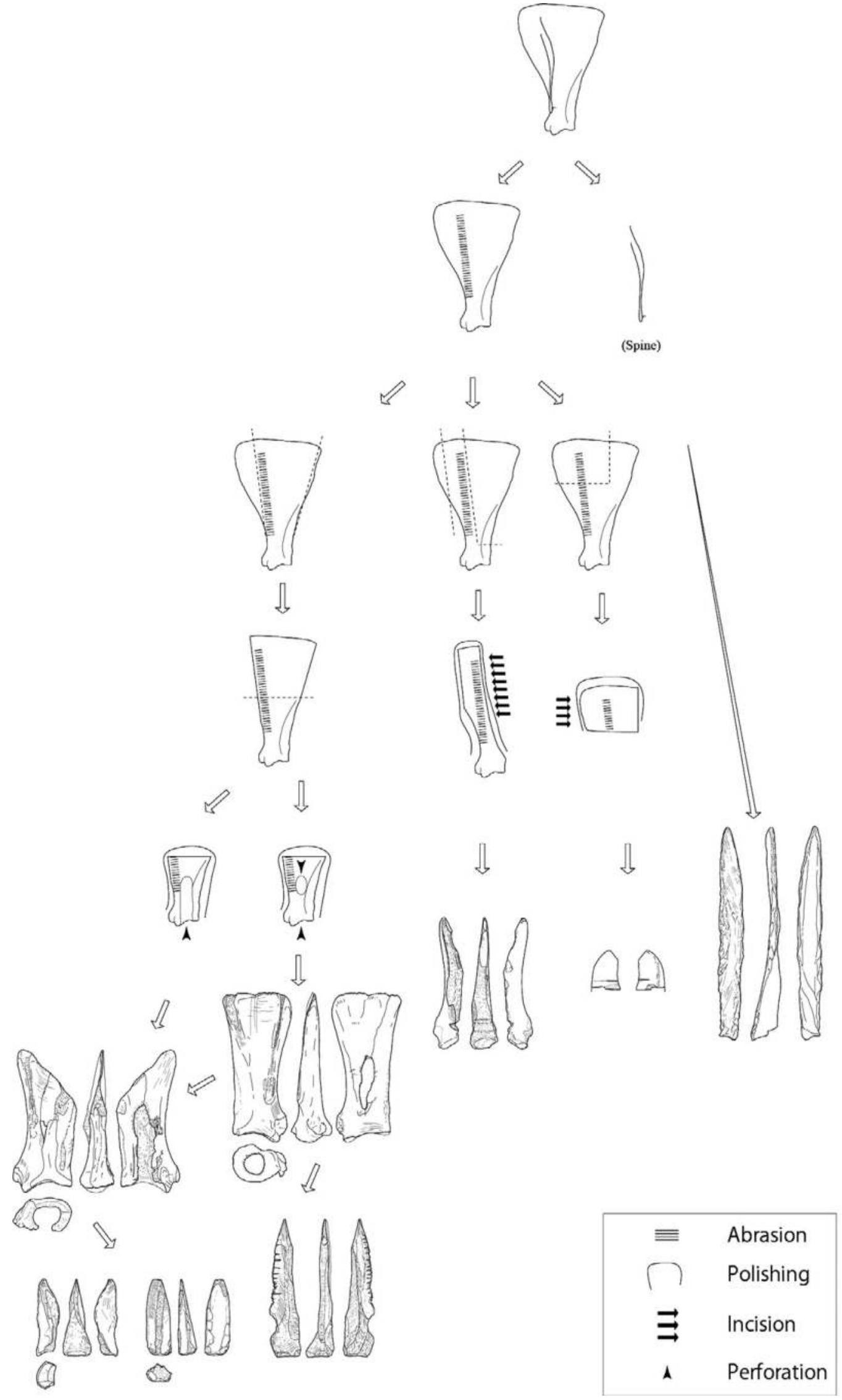

\& $\mathbb{V}$

Fig. 15.8 Manufacturing scheme of worked scapula implements. 

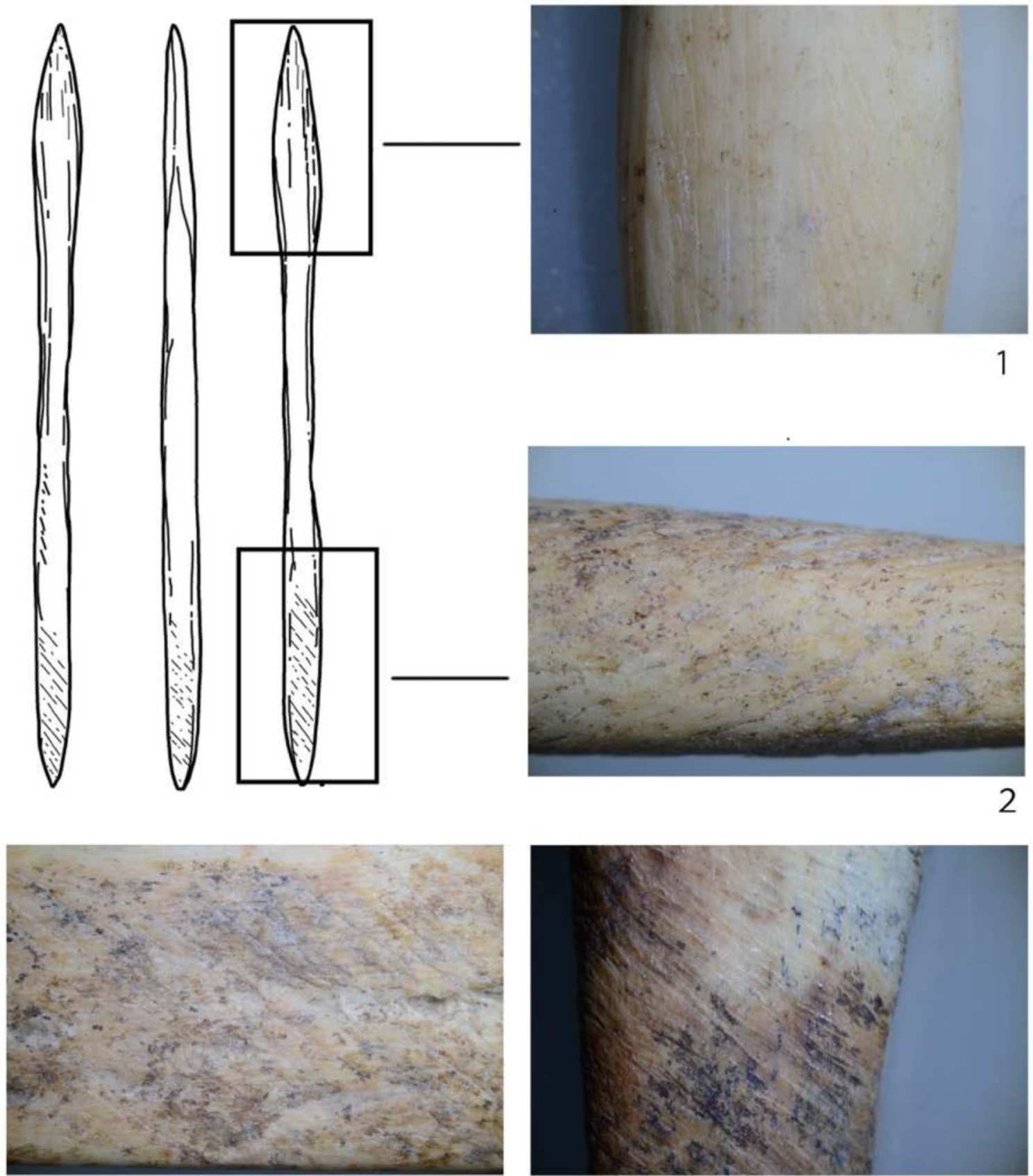

3

Fig. 15.9 Micrographs of various tools from Göytepe. 1-2: Arrowhead; 3: Knife; 4: Buttonette.

\subsection{Göytepe worked bone in a wider context}

Once Kiguradze established a chronology of Shomutepe culture based on his reanalysis of materials from sites in the Kvemo-Kartli region, Georgia (Kiguradze 1986), he claimed that some changes in the worked bone assemblage occurred during the Neolithic period. In the earliest Phase I of his chronology, the quantity of worked bone is limited but includes awls, arrowheads, spatulas made on long bone, and antler dibbles without perforation. In the following Phase II, spatulas made from rib or flat bone, knives, pendants made from boar tusk, and perforated antler adzes are added. During Phase III, antler or bone dibbles, bone sickle handles are present. In Phase IV, he notes hoes made from scapulae, perforated needles, spoons, and incised 
spatulas. In the final Phase V, the quantity of scapula hoes increases, while decorated antler hammers, beads with one or several perforations are evident. However, it appears that this chronological scheme is not applicable to the sequence from Göytepe, because all tool types simultaneously exist from the earliest level onward. Yet, arrowheads, knives, and buttonettes are present only in the lower levels (129 ). These types, which are relatively time-consuming to manufacture, may serve as chronological markers of that period.

While the worked bone industry of Göytepe is comparable to other sites from the Shomutepe culture in the Middle Kura, some differences exist among the assemblages from each site. For example, "spoon" tools have been reported from Shulaveris Gora and Imiris Gora in Georgia (Munchaev 1982: tab. XXX37; Kiguradze 1986: fig. 38-15), while such tools are completely absent at Göytepe. These tools have been also found at Aratashen and Aknashen in Armenia (Badalyan et al. 2007: fig. 6f; Badalyan et al. 2010: fig. 15-12), and Tilkitepe near Lake Van (Korfmann 1982: Abb. 18-8). On the other hand, bone arrowhead and hammer also have been found at Tilkitepe (Phase III) (Korfmann 1982: Abb. 18-7 and 8). At present, it remains unclear whether the differences among assemblages reflect chronological or regional variation. However, some tool types might be useful markers for discussing the chronological context of sites and the relationship between geographical regions.

\section{References}

Badalyan, R., P. Lombard, P. Avetisyan, C. Chataigner, J. Chabot, E. Vila, R. Hovsepyan, G. Willcox, and H. Pessin (2007) New data on the late prehistory of the southern Caucasus: The excavations at Aratashen (Armenia) -Preliminary report. In: Les Cultures du Caucase (Vle-Ille Millénaires avant notre ère): Leur Relations avec le Proche-Orient, edited by B. Lyonnet, pp. 37-61. Paris: Éditions Recherche sur les Civilisations.

Badalyan, R. S., A. A. Harutyunyan, C. Chataigner, F. Le Mort, J. Chabot, J. -E. Brochier, A. Balalescu, V. Radu, and R. Hovsepyan (2010) The settlement of Aknashen-Khatunarkh. A Neolithic site in the Ararat plain (Armenia): Excavation results (2004-2009). Turkiye Bilimler Akademisi Arkeoloji Dergisi (TÜBA-
$A R), 13: 187-220$.

Chataigner, C. (1995) La Transcaucasie au Néolithique et au Chalcolithique. BAR Internatinal Series 624. Oxford: Tempus Reparatum.

Christidou, R. (2009) La industrie osseus de l'etablissement Néolithique d'Aratashen. In: Mission Caucase. Rapport Scientifique 2009, edited by C. Chataigner, pp. 71-78. Lyon: Maison de l'Orient et de la Méditerranée.

Clark, J. G. D. and M. W. Thompson (1953) The groove and splinter technique of working antler in Upper Palaeolithic and Mesolithic Europe. Proceedings of the Prehistoric Society, 19: 148-160.

Kiguradze, T. (1986) Neolithische Siedlungen von Kvemo-Kartli, Georgien. Materialien zur Allgemeinen und Vergleichenden Archäologie Band 29. Munchen: Verlag C. H. Beck.

Korfmann, M. (1982) Tilkitepe. Die ersten Ansätze prähistorischer Forschung in der Östlichen Türkei. Istanbuler Mitteilungen Beiheft 26. Tübingen: Verlag Ernst Wasmuth.

Le Dosseur, G. (2012) The bone industry from Mentesh Tepe. In: Ancient Kura 2010-2011: The First Two Seasons of Joint Field Work in the Southern Caucasus, edited by B. Lyonnet, F. Guliev, B. Helwing, T. Aliyev, S. Hansen, and G. Mirtskhulava, pp. 119-121.

Lloyd S., F. Safar, and R. J. Braidwood (1945) Tell Hassuna. Excavations by the Iraq Government Directorate General of Antiquities in 1943 and 1944. Journal of Near Eastern Studies, 4(4): 255-289.

Munchaev, R. М. (1982) Энеолит Кавказа. In: Энеолит CCCP, edited by V. M. Masson and N. Merpert, pp. 100-164. Moscow: Nauka.

Narimanov, I. G. 1987 The Culture of the Most Ancient Farming and Stock-Breeding Population of Azerbaijan. Baku: National Academy of Sciences (in Russian with an English summary).

Redman, C. L. (1973) Early village technology: A view through the microscope. Paléorient, 1(2): 249-261.

Stordeur, D. (1999) Néolithisation et outillage osseux. La revolution a-t-elle eu lieu? In: Préhistoire D'Os. Recuil d'Etudes sur I'Industrie Osseuse Préhistorique, edited by H. Camps-Fabrer, pp. 261272. Aix-en-Provence: Publications de l'Université de Provence. 


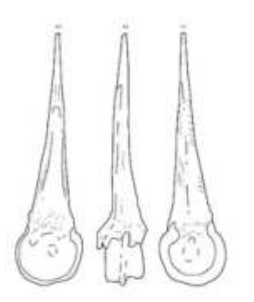

1

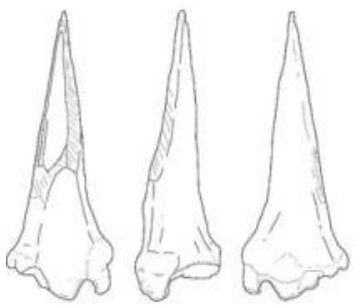

6

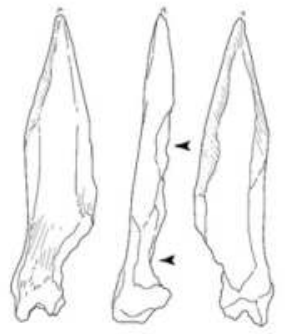

10

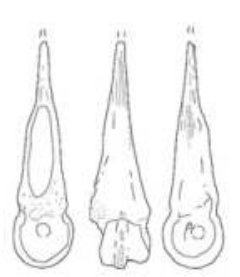

14

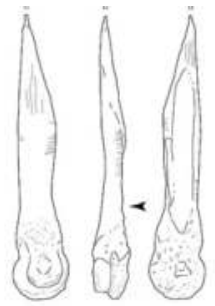

2

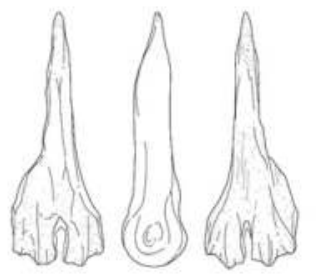

7

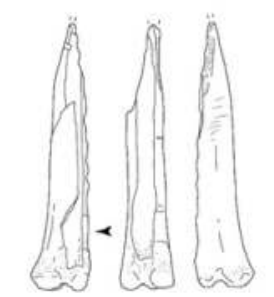

11

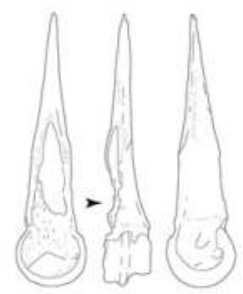

15
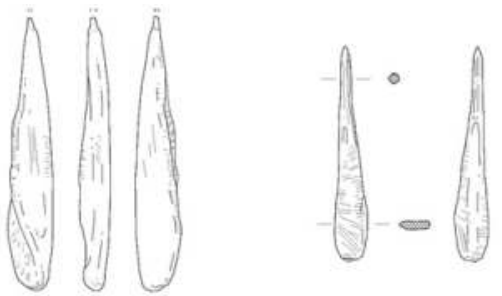

3
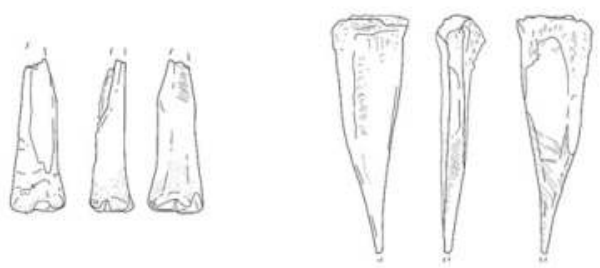

8
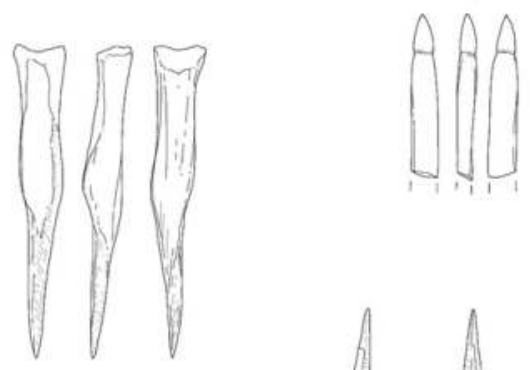

13
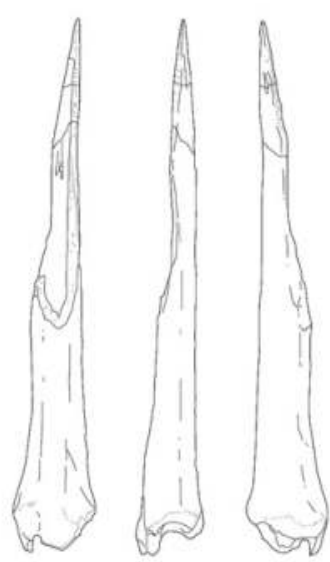

17

$5 \mathrm{~cm}$

Fig. 15.10 Bone awls from Göytepe*. 1-3, 7-9, 11-12, 14-15: Metapodial; 4: Rib; 6, 10, 16: Tibia; 15: Radius; 5, 13: Indeterminate long bone. *The drawings inserted in this chapter reflect the anatomical orientation of the skeletal element used as raw material. 

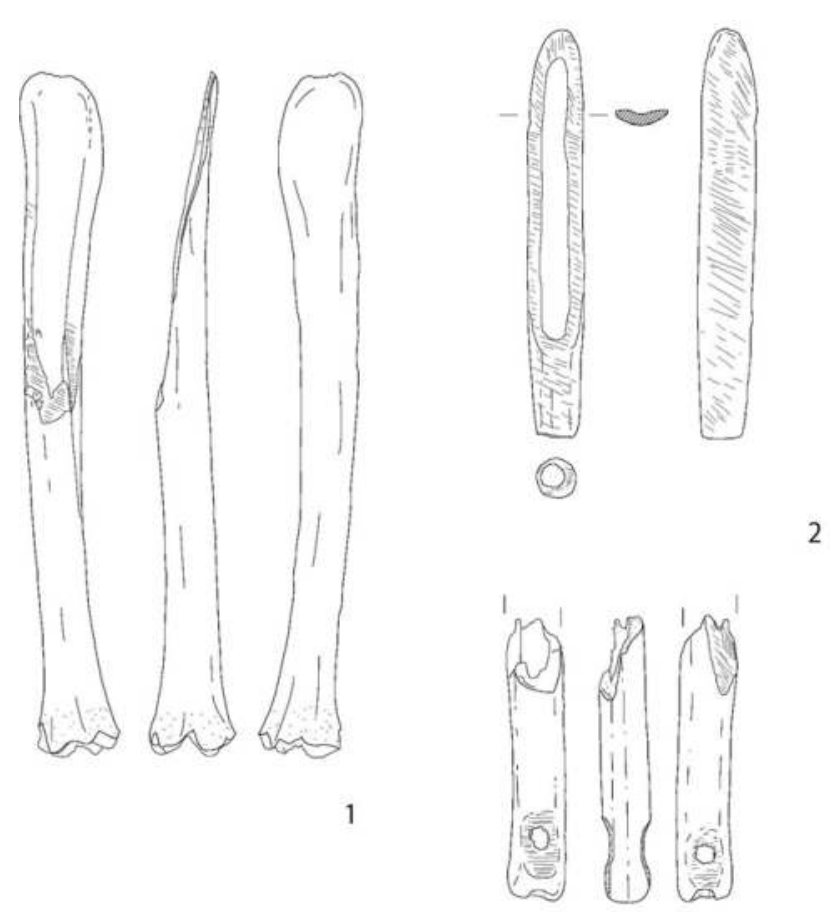

(6)

3
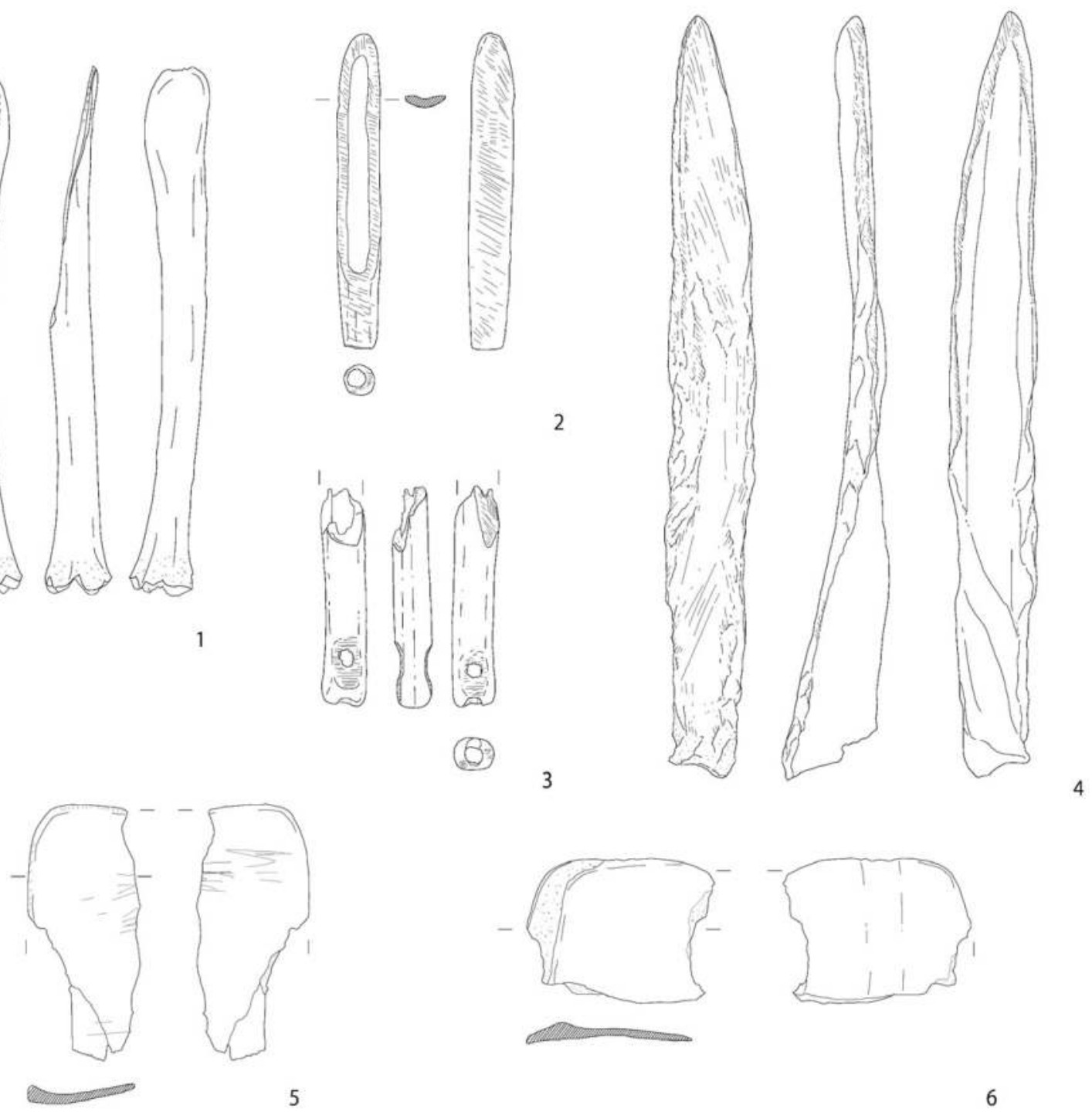

6
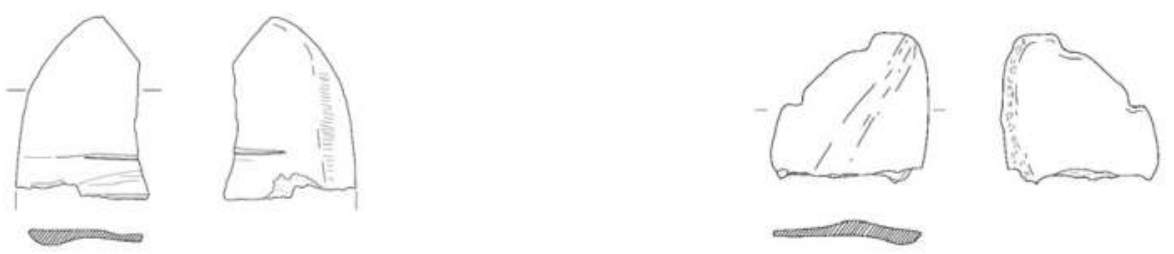

8

0

$5 \mathrm{~cm}$

Fig. 15.11 Bone spatulae and palette from Göytepe. 1-3: Spatula made on tibia; 4: Spatula made on scapula; 5-8: Palettes. 

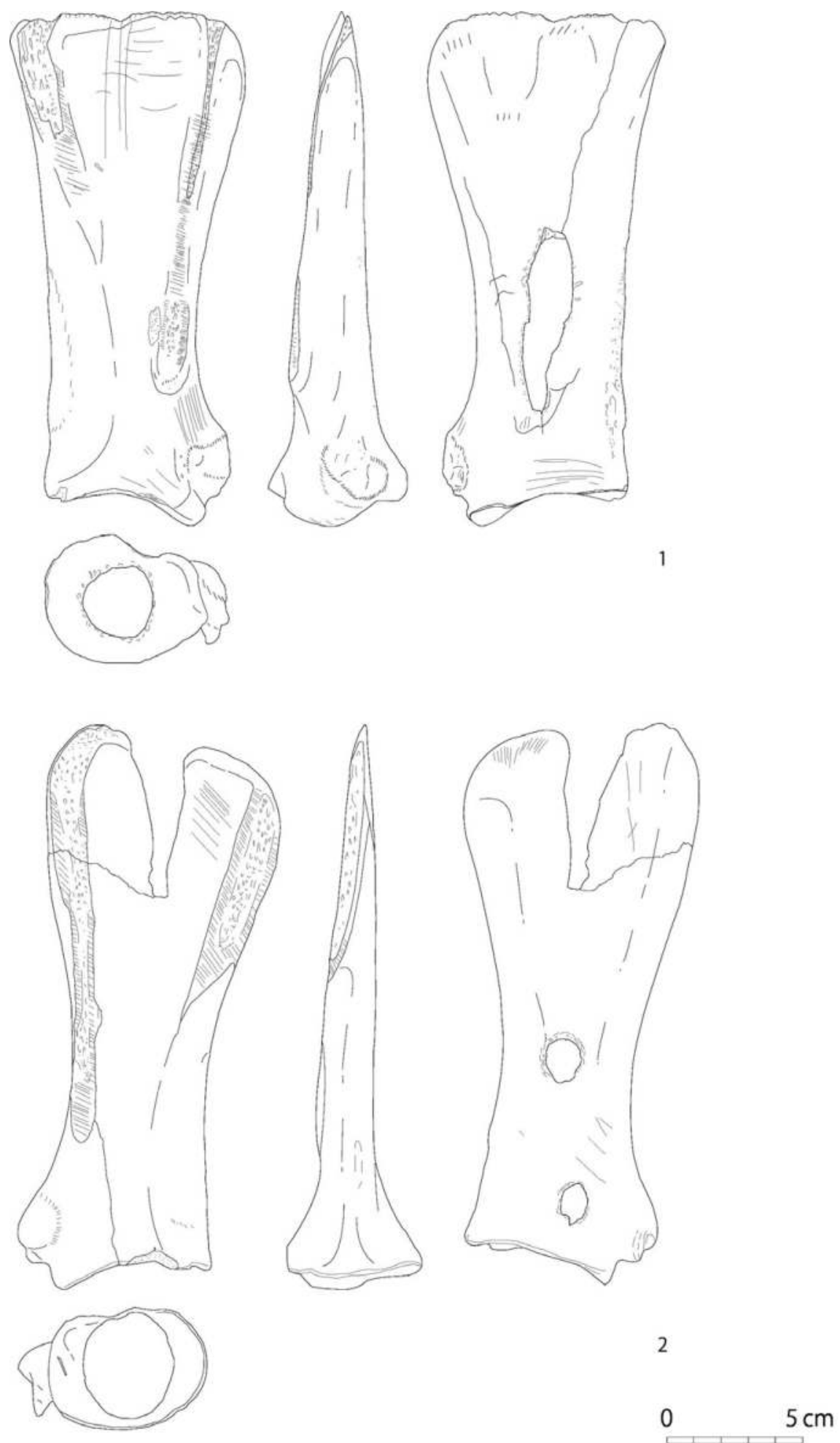

Fig. 15.12 Bone hoes from Göytepe. 

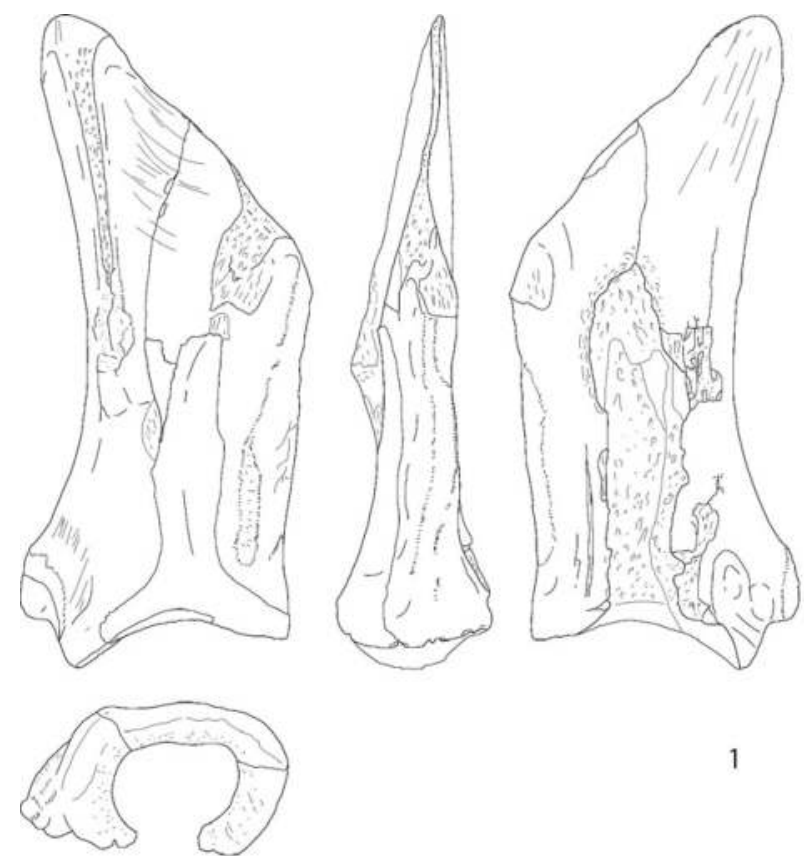

1
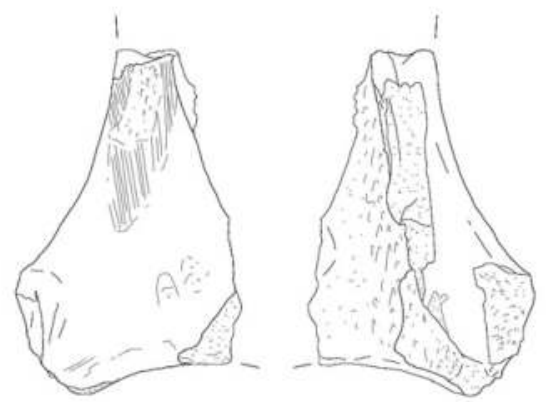

2

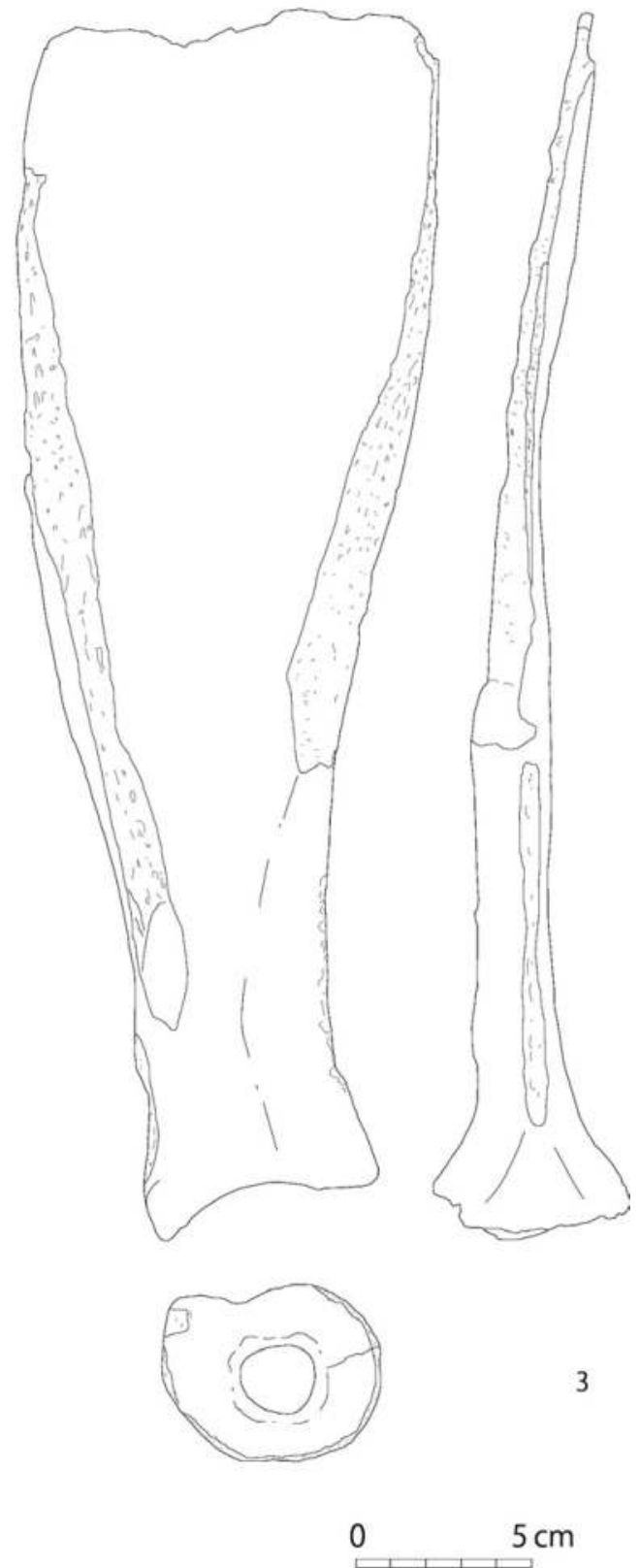

Fig. 15.13 Bone hoes $(1,2)$ and an unfinished bone hoe (3) from Göytepe. 


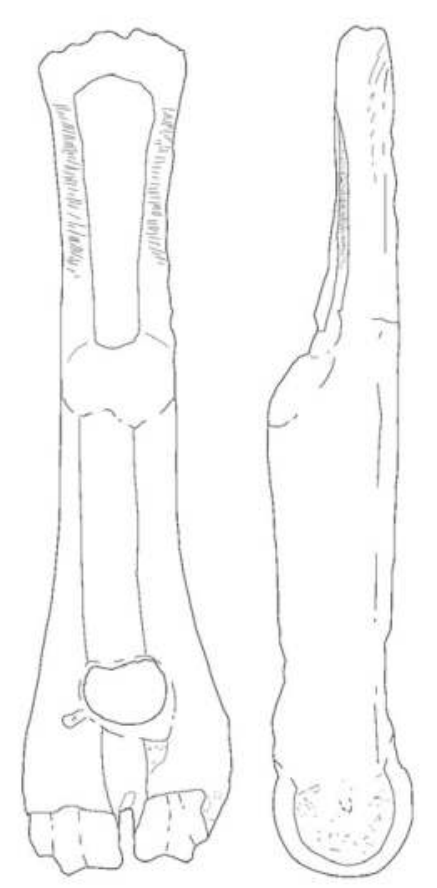

1
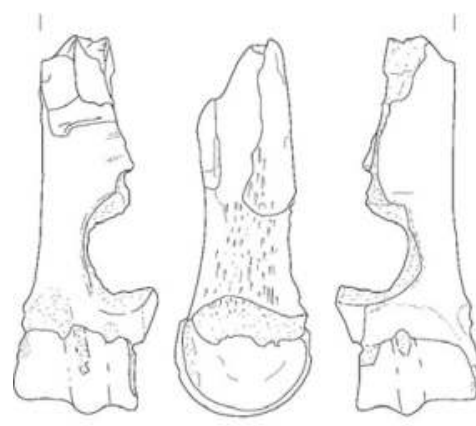

4

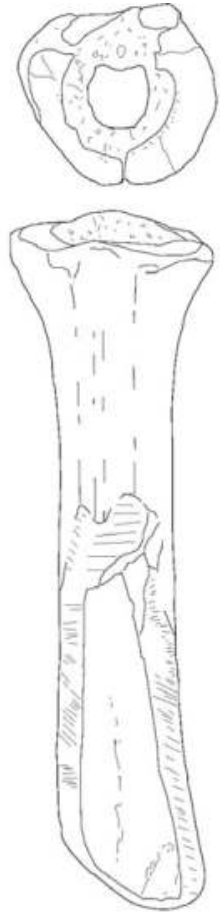

2
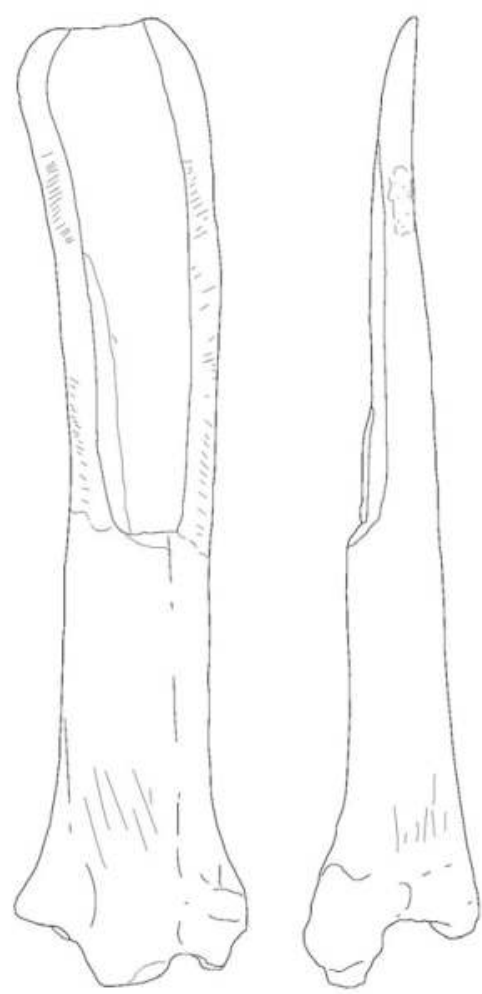

3

Fig. 15.14 Bone dibbles from Göytepe. 1, 4: Metacarpal; 3: Tibia; 2, 5: Metatarsal. 

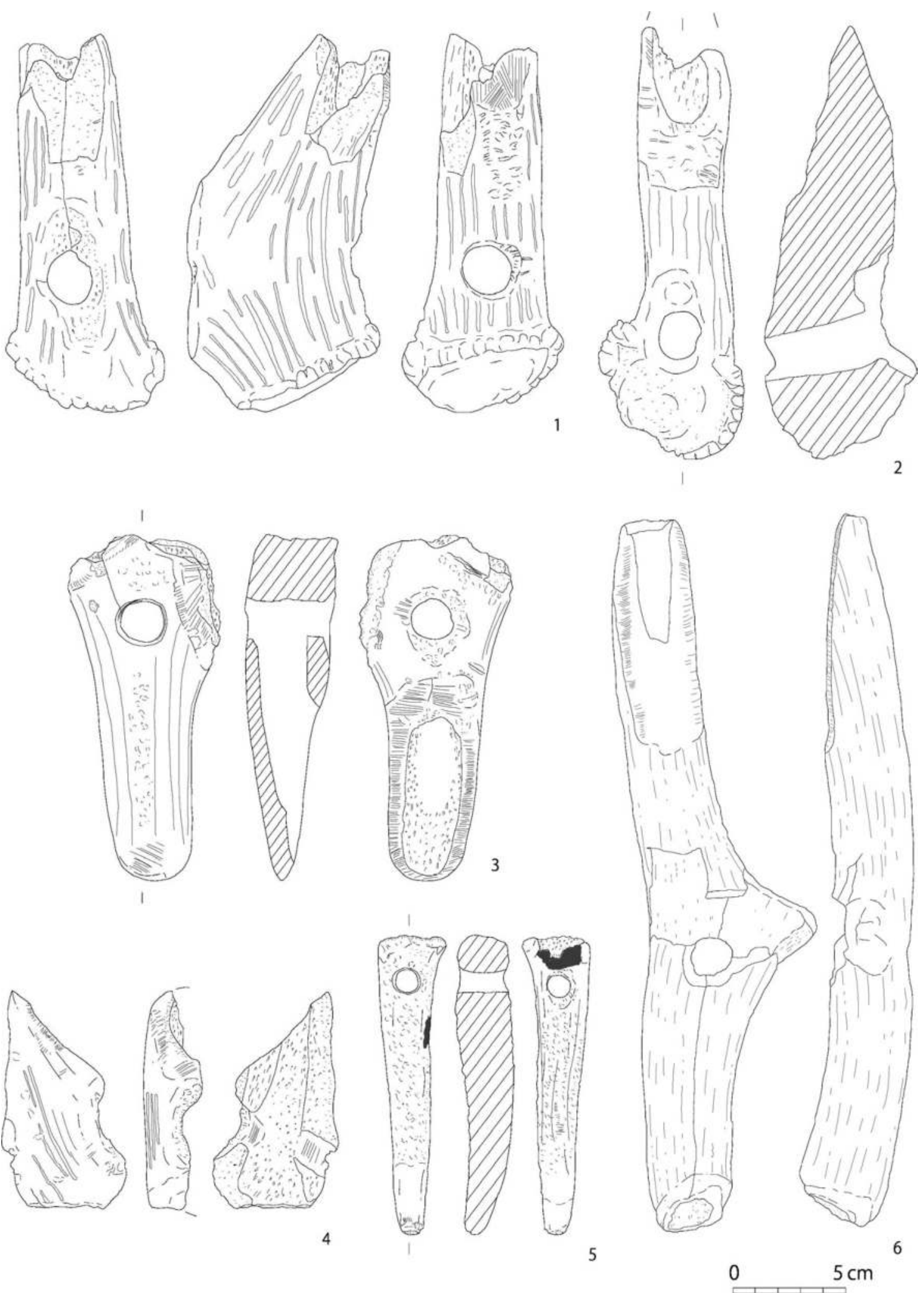

Fig. 15.15 Antler dibbles from Göytepe. 

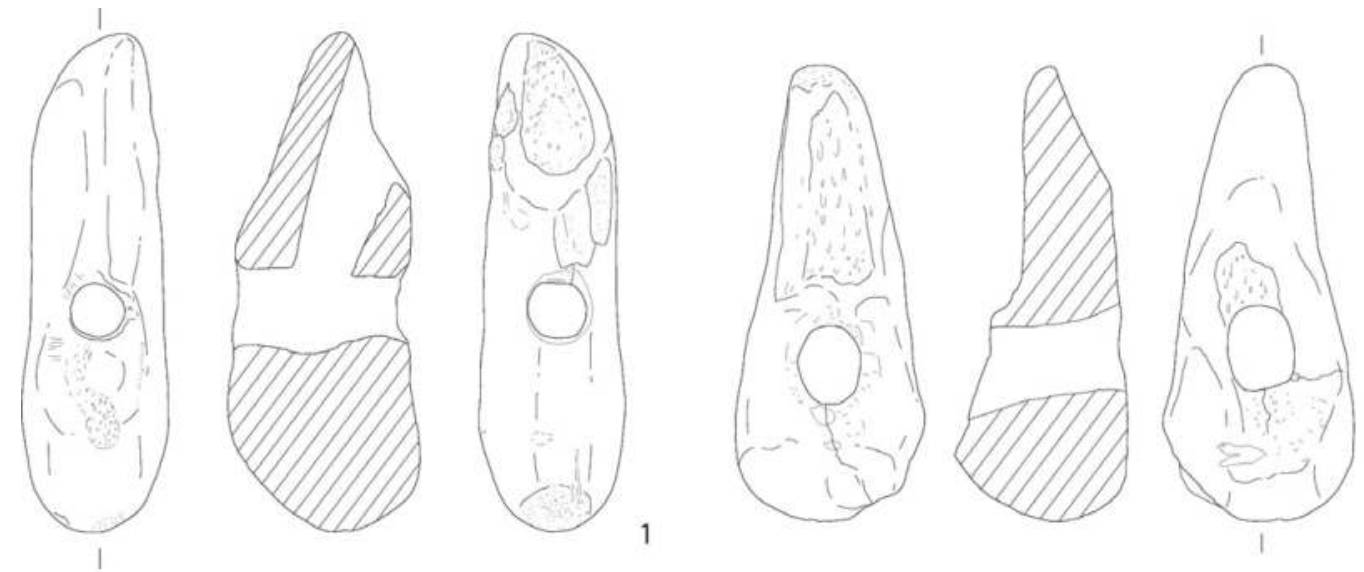

2
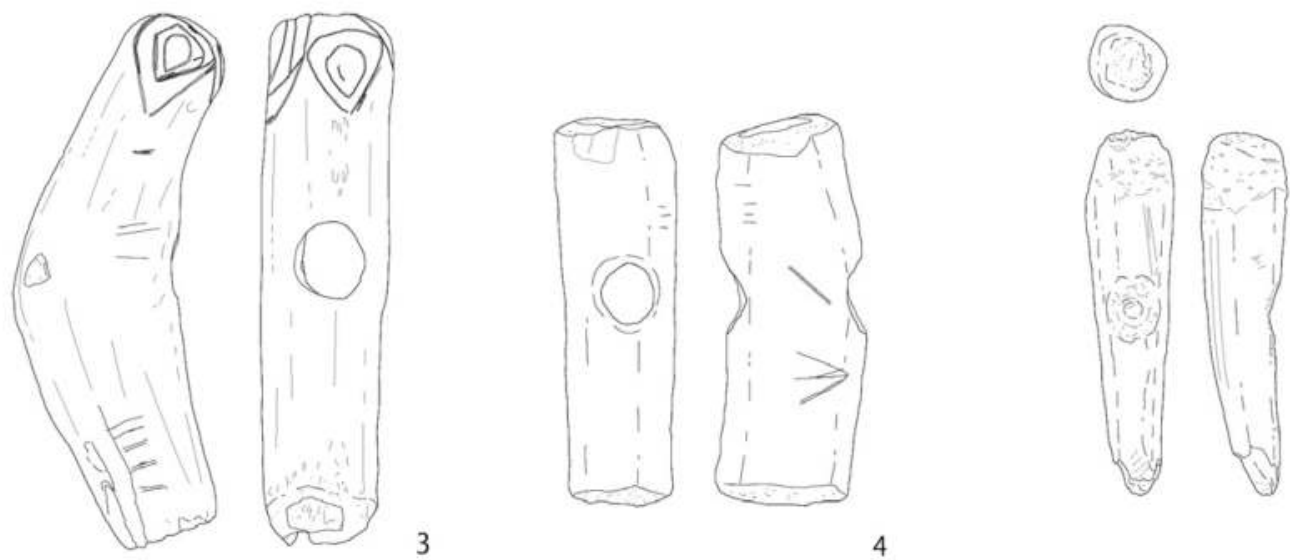

4 $5 \mathrm{~cm}$

Fig. 15.16 Antler hammers (1-4) and a possible punch (5) from Göytepe. 

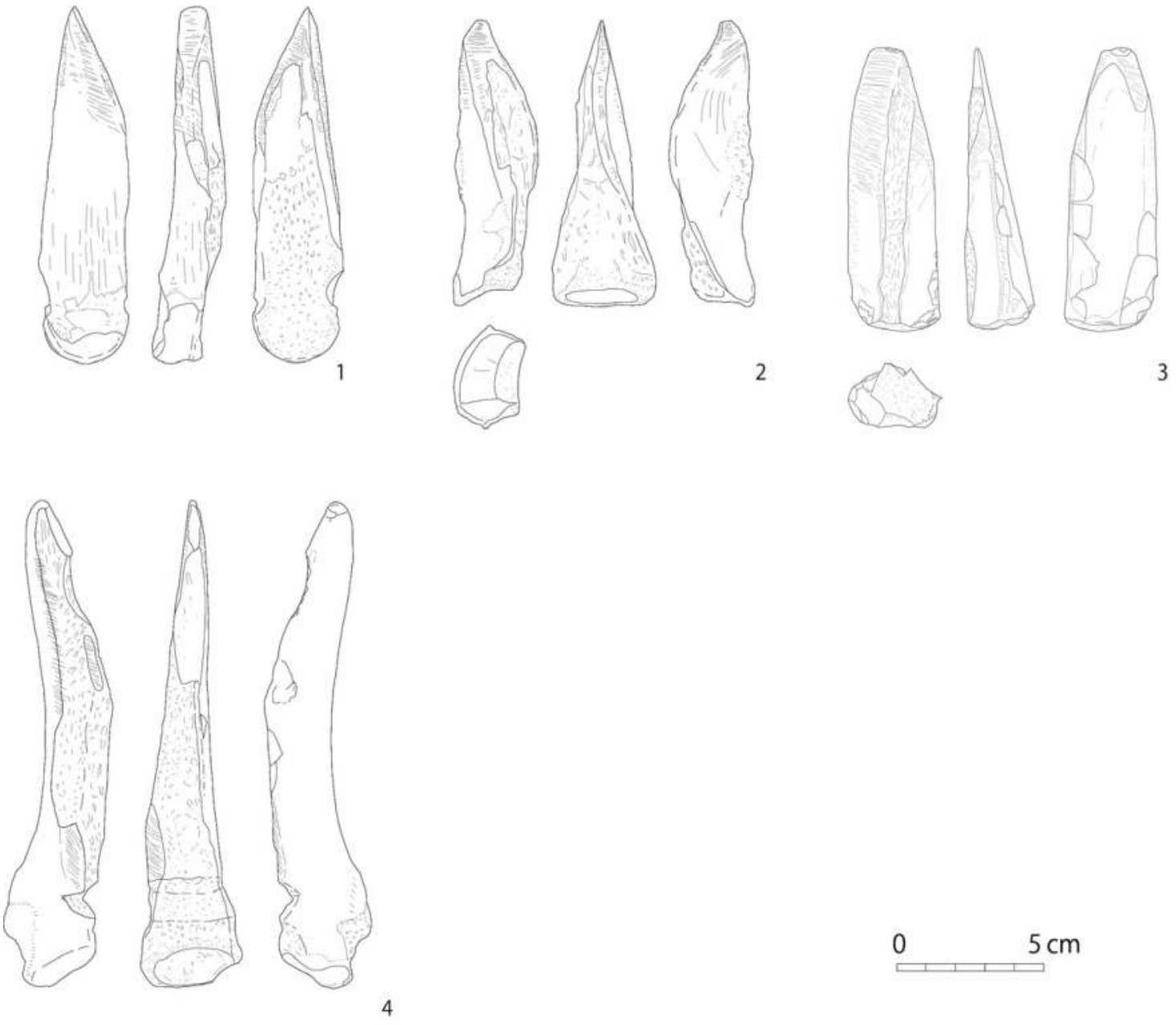

Fig. 15.17 Bone fleshers from Göytepe. 1: metacarpal; 2-4: scapula. 

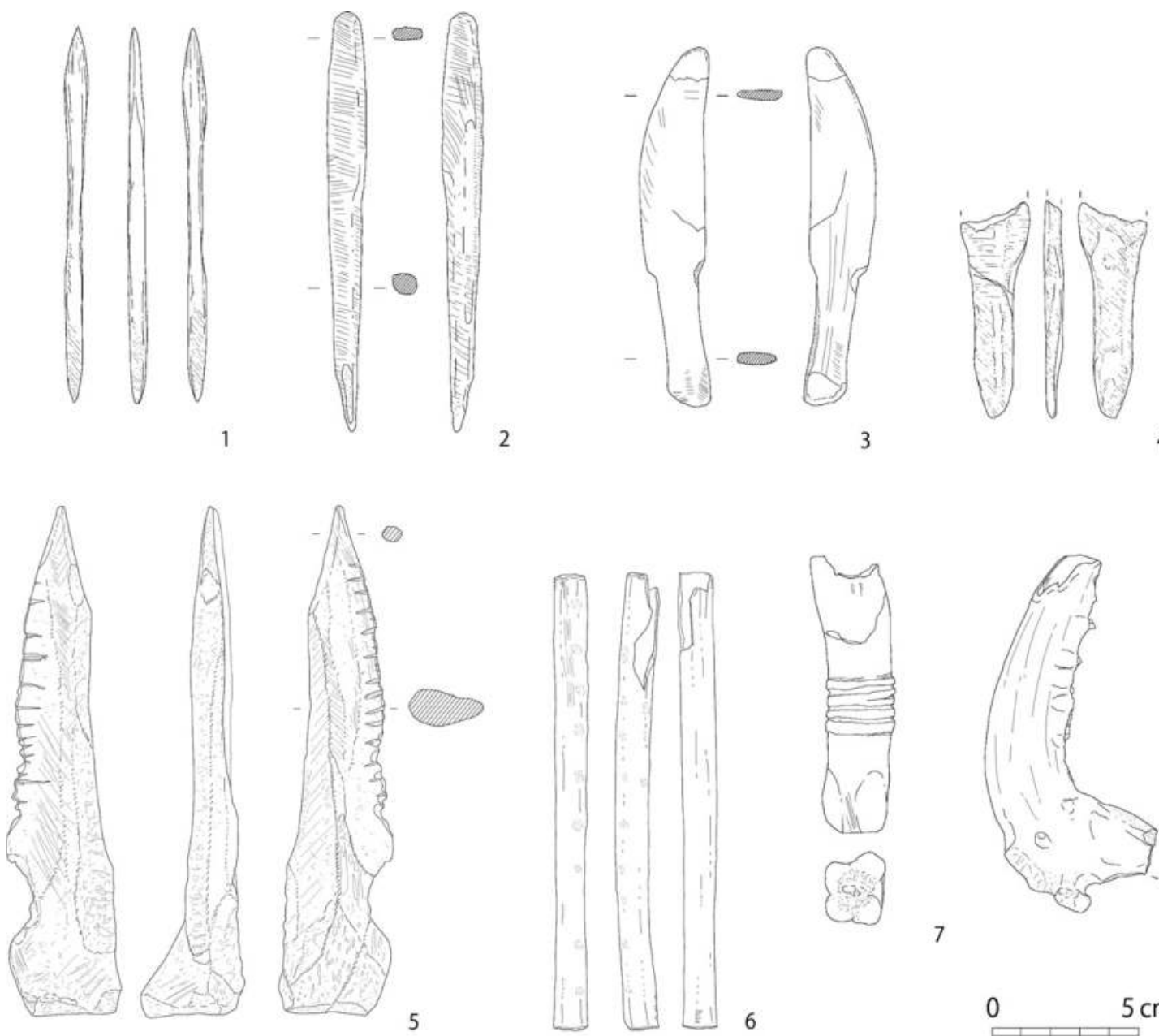

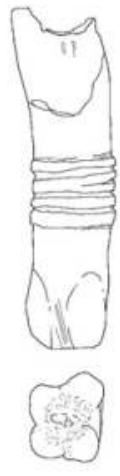

0

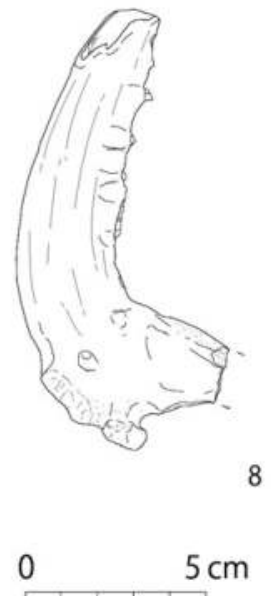

Fig. 15.18 Arrowhead (1), buttonette (2), knife (3-4), counter (5), ornament (6-7), and sickle? (8) from Göytepe. 

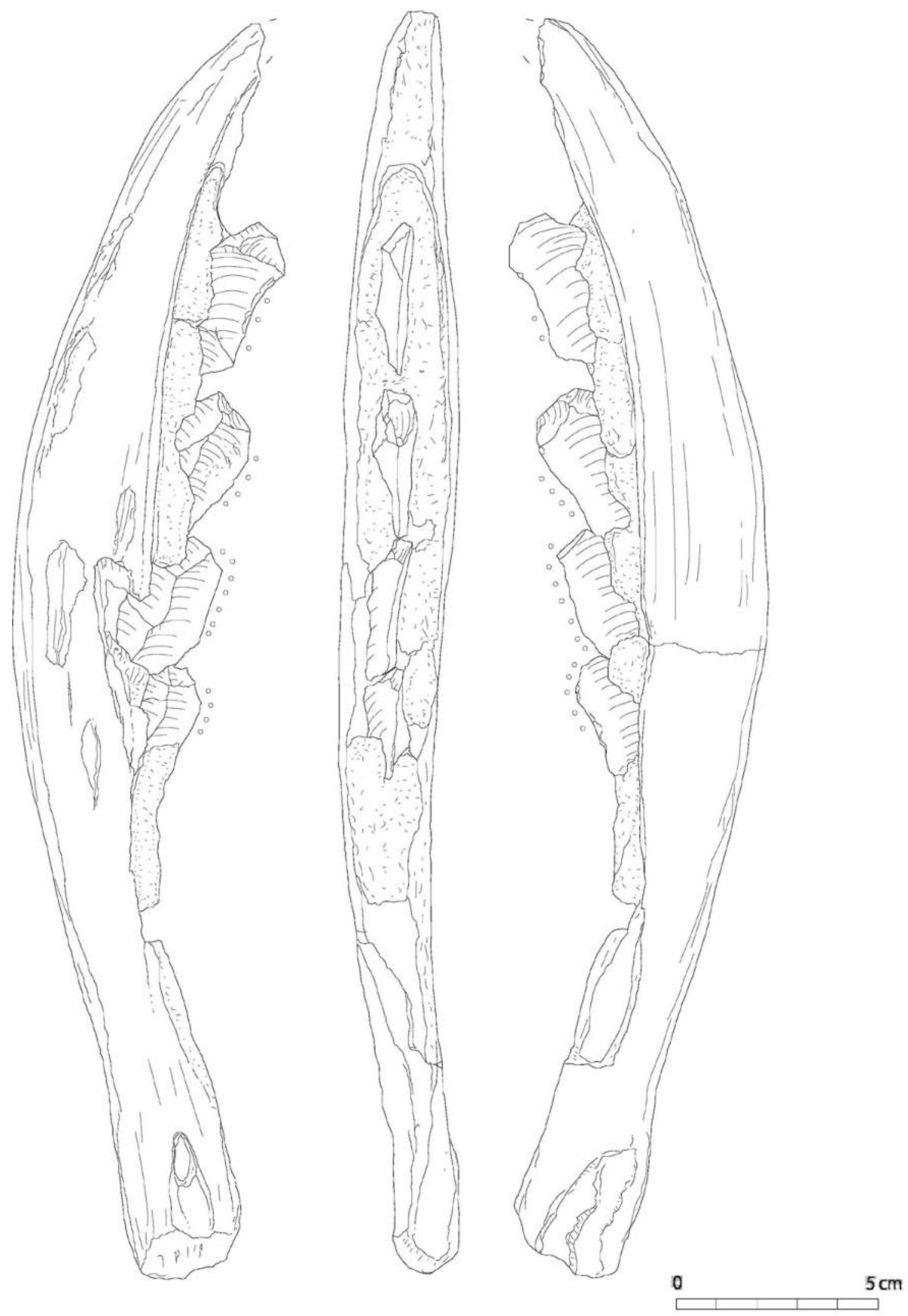

Fig. 15.19 Sickle made on mandible from Göytepe. 

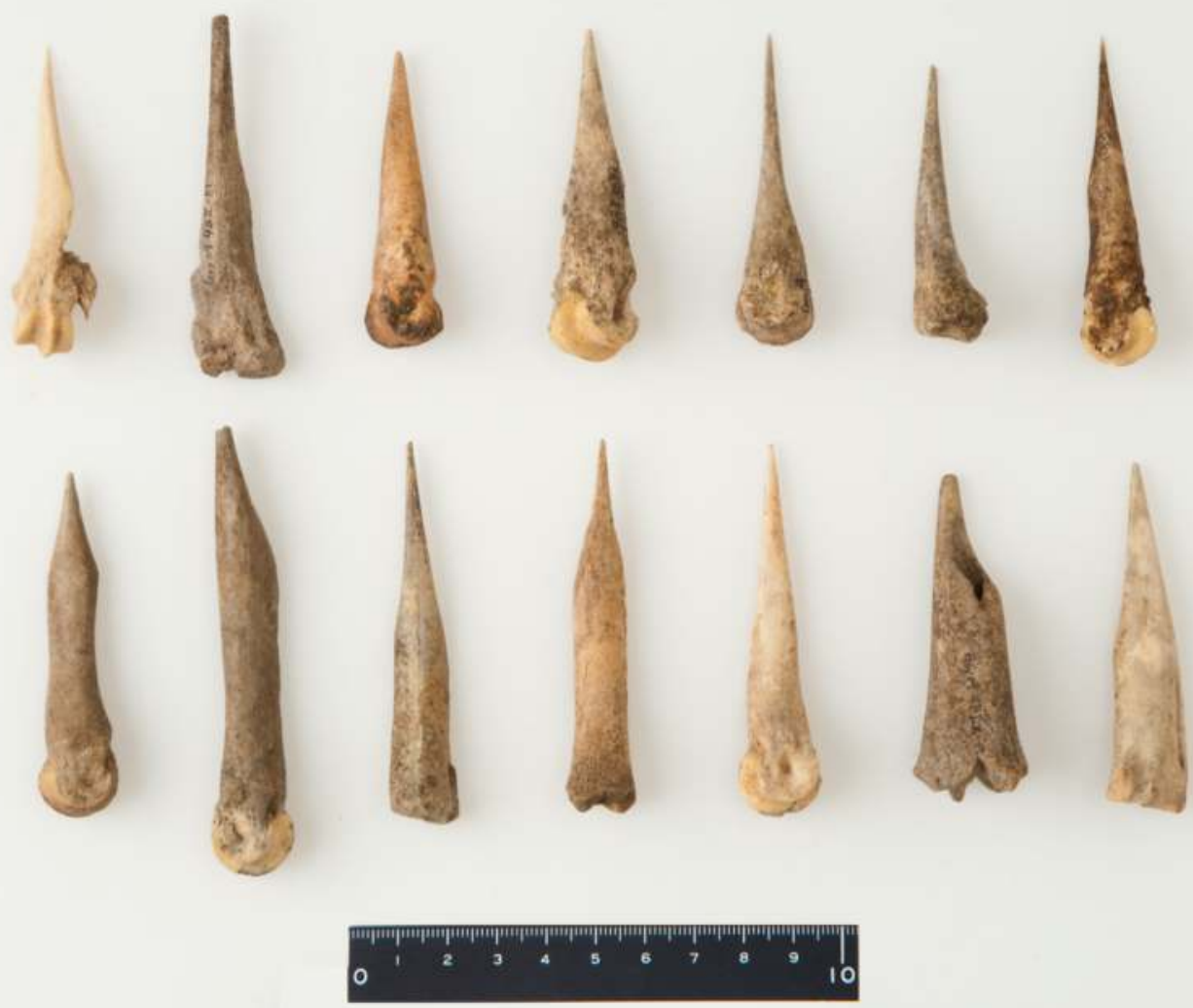

Fig. 15.20 Representative bone awls from Göytepe.
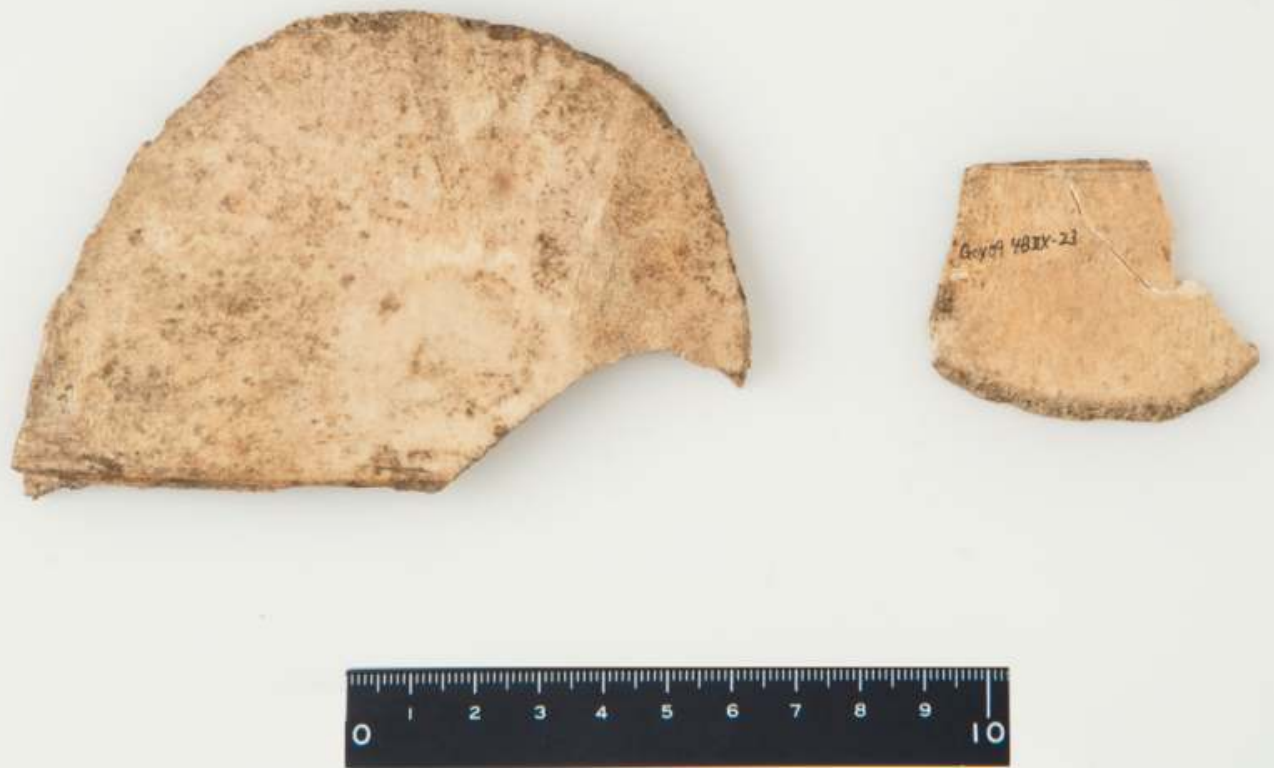

Fig. 15.21 Representative bone palettes from Göytepe. 

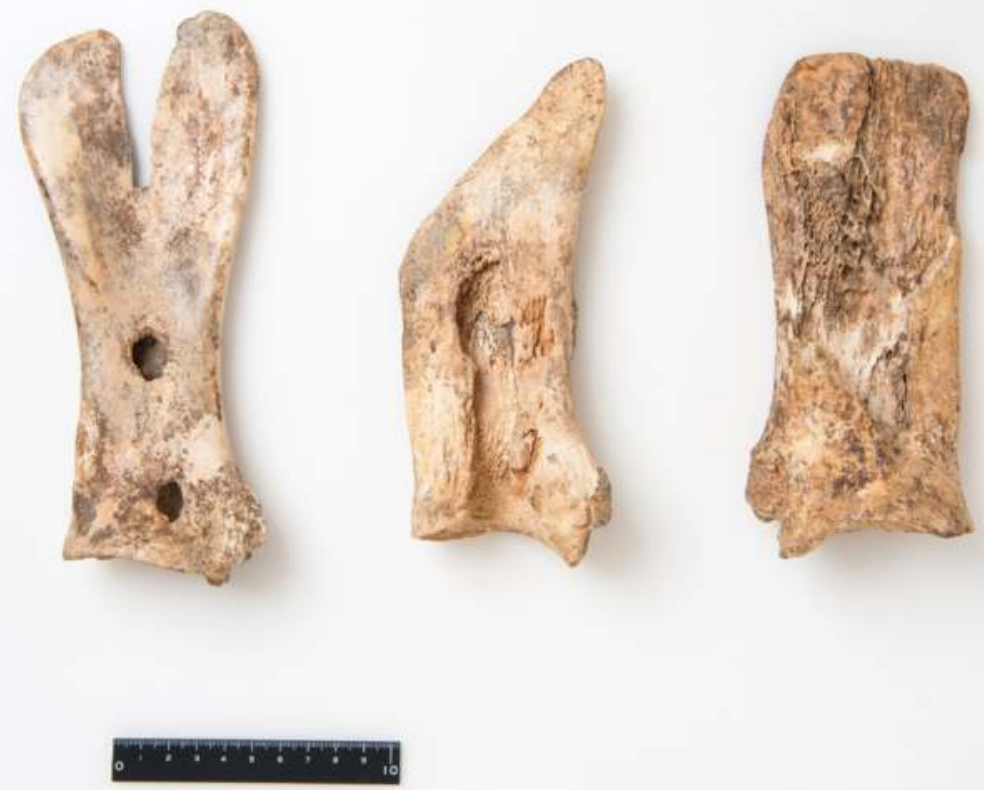

Fig. 15.22 Representative bone hoes from Göytepe.
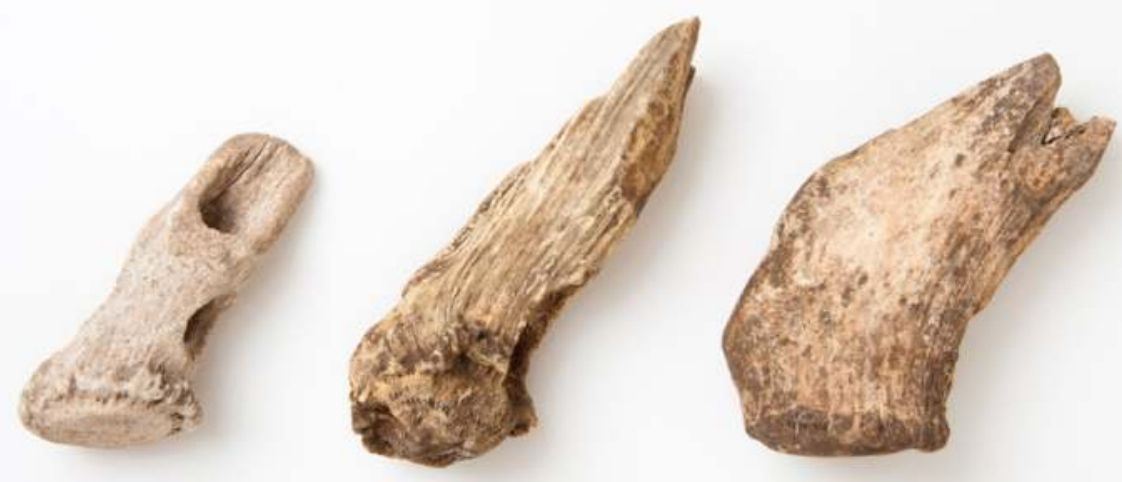

Fig. 15.23 Representative antler dibbles from Göytepe. 

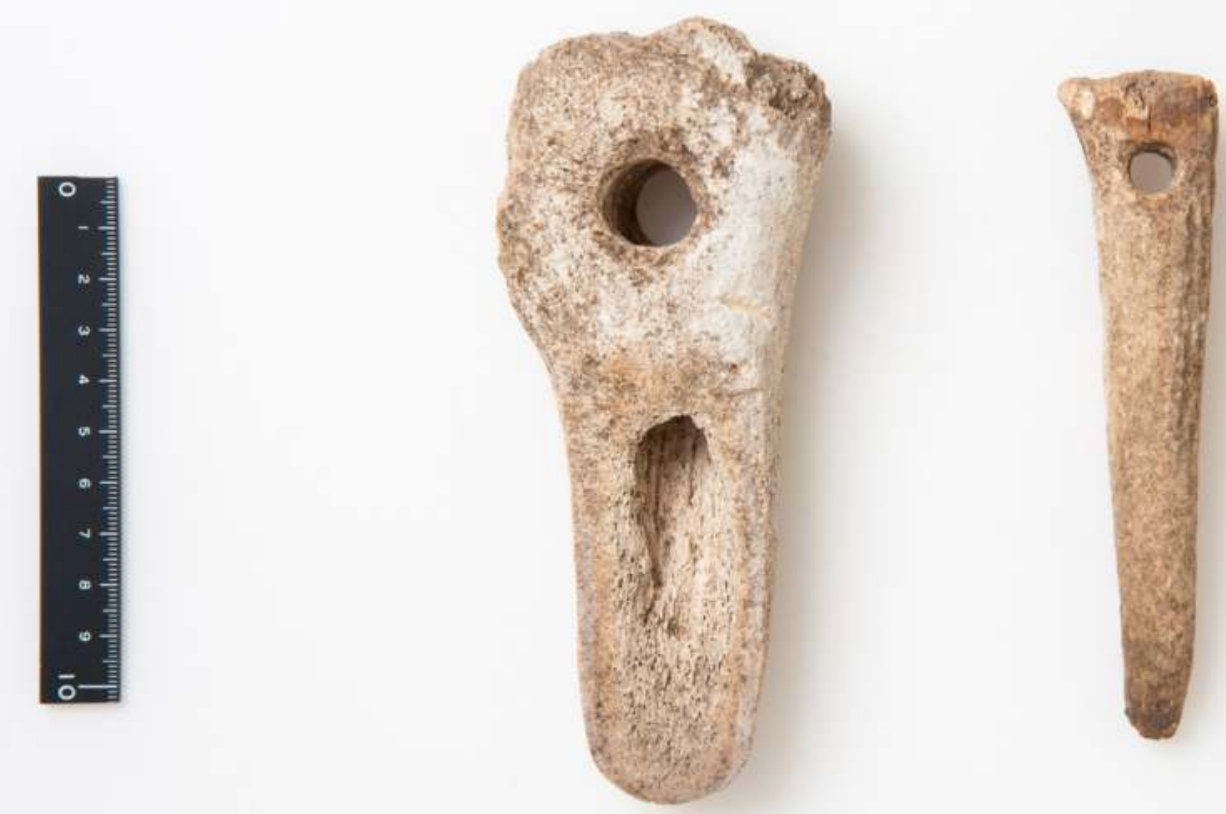

Fig. 15.24 Antler dibble or pick from Göytepe.

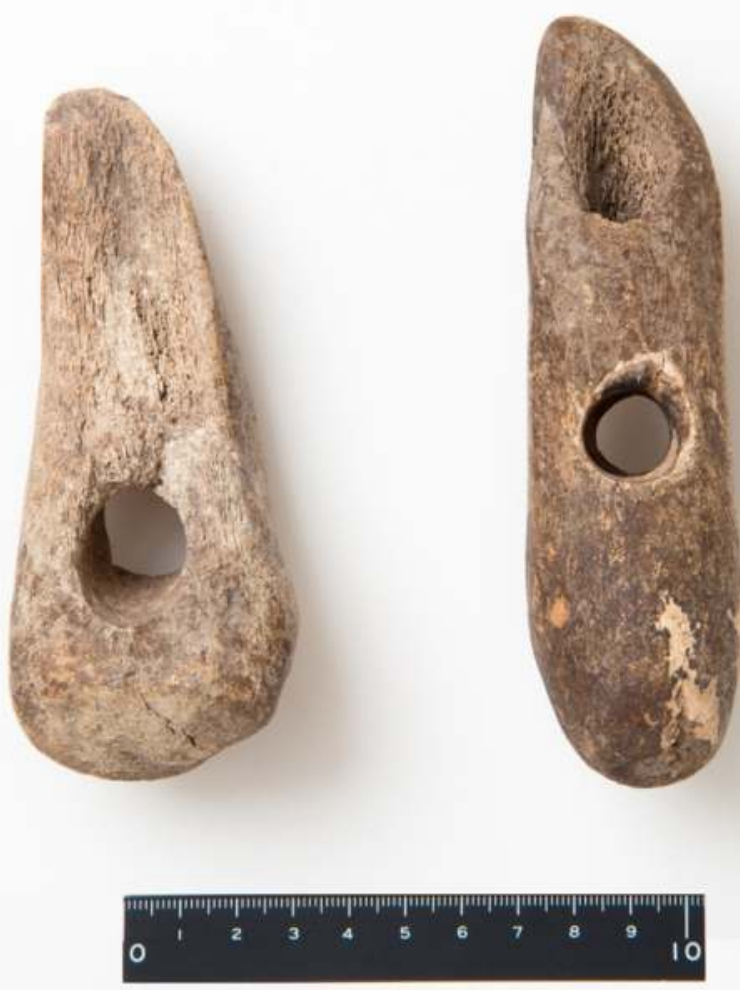

Fig. 15.25 Representative antler hammers from Göytepe. 

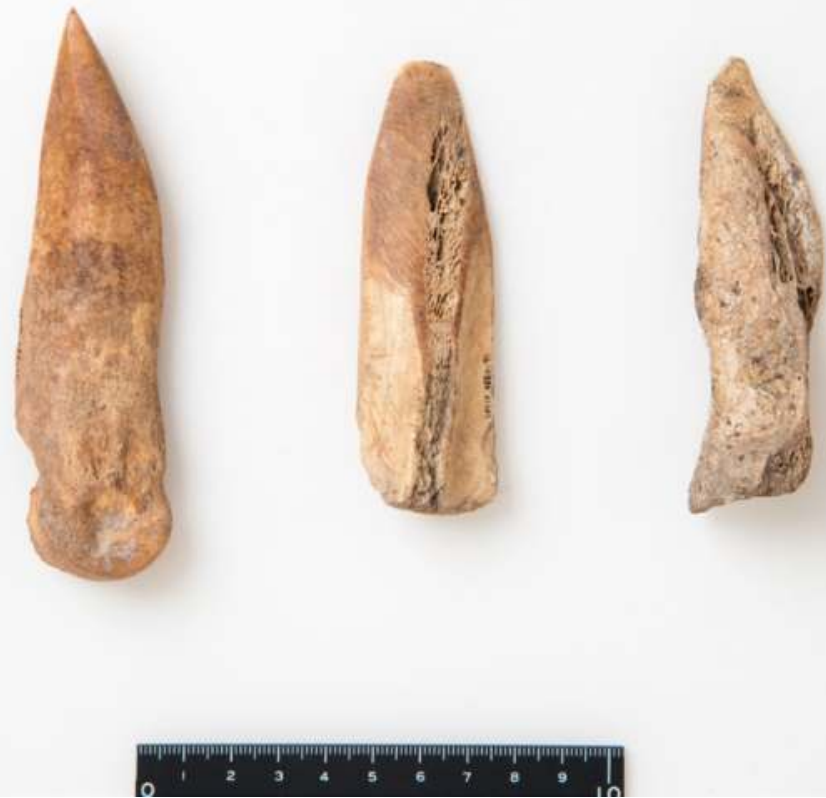

Fig. 15.26 Fleshers made on various elements from Göytepe.
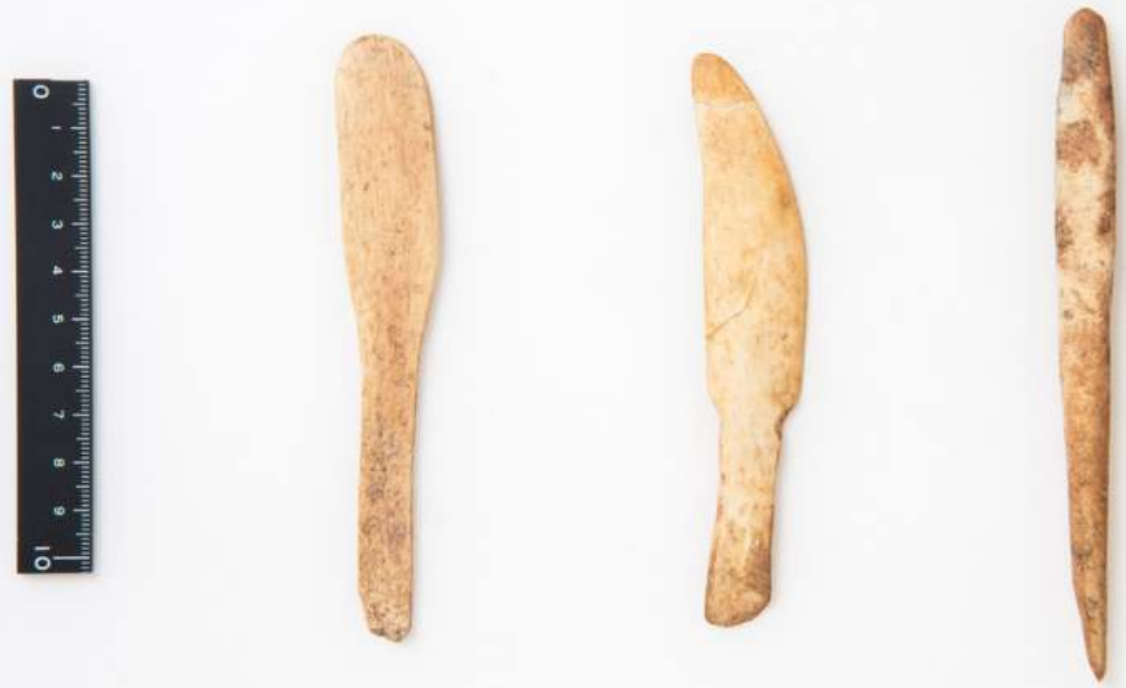

Fig. 15.27 Knives (left, center) and butonette (right) from Göytepe. 


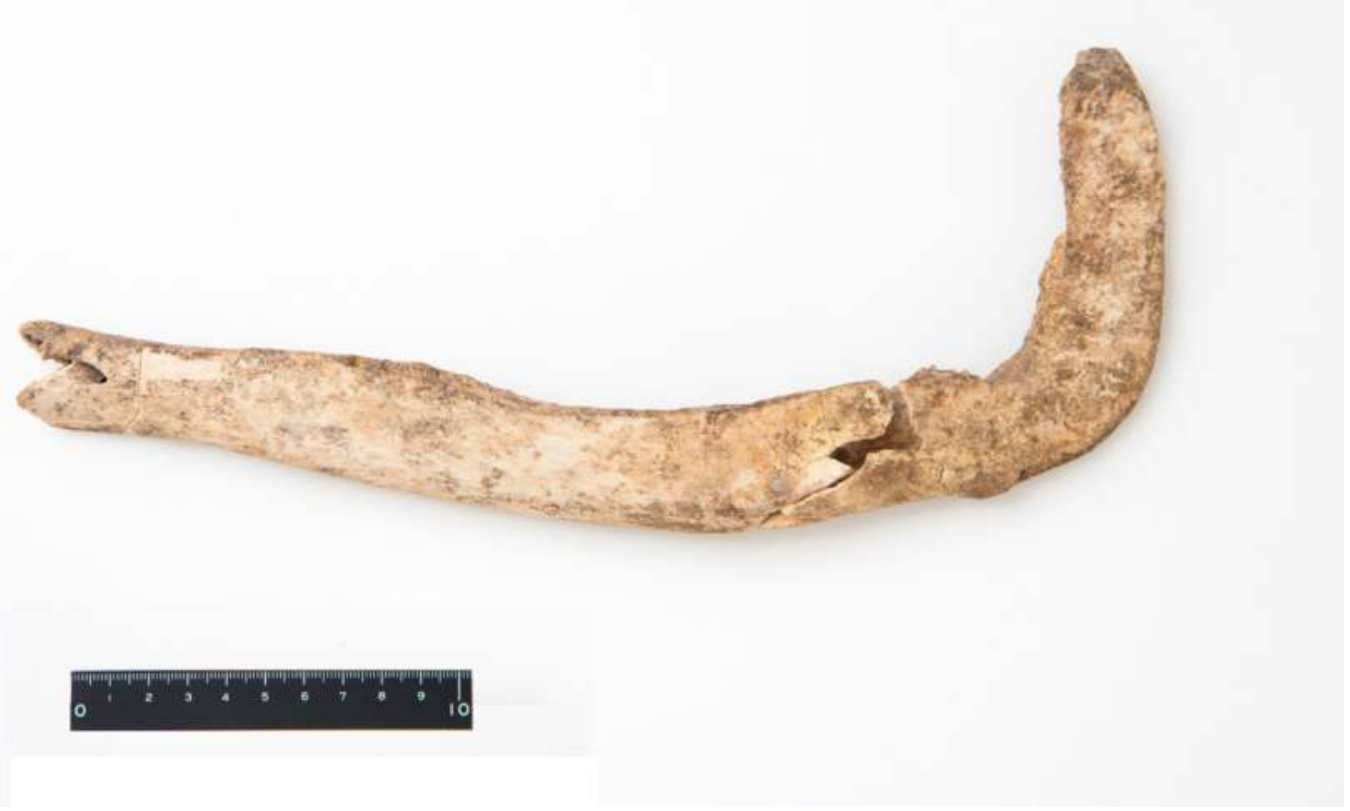

Fig. 15.28 Unfinished sickle handle made on cattle mandible from Göytepe.

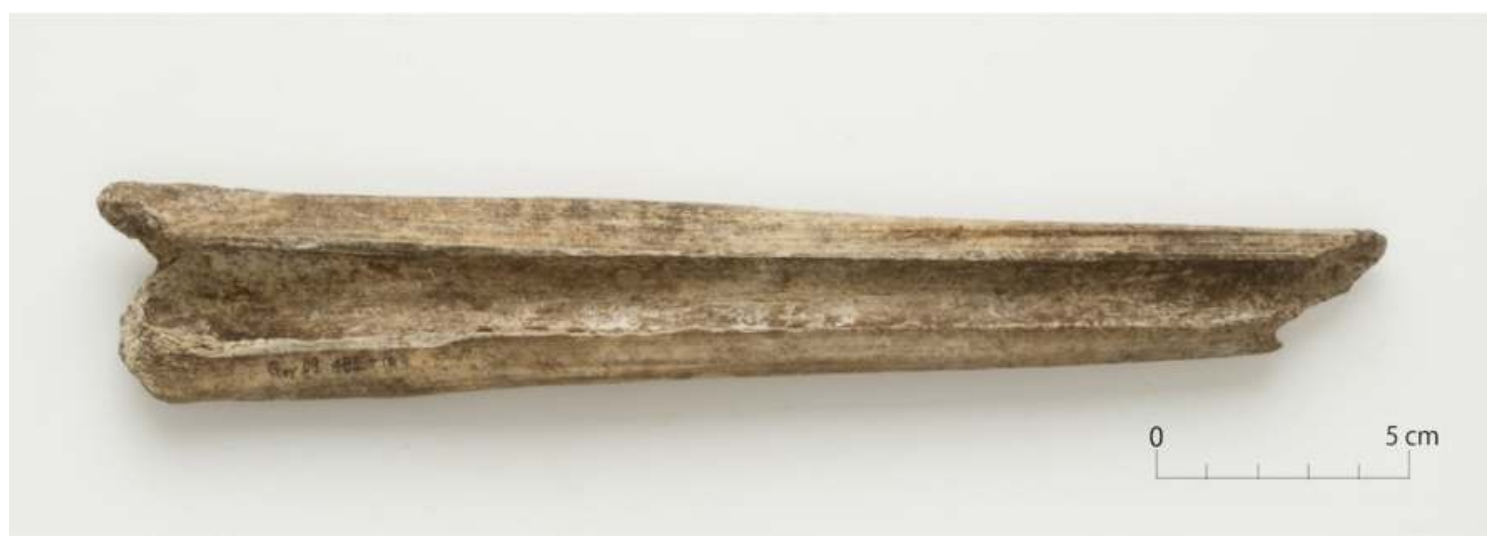

Fig. 15.29 Debitage splintered by grooving technique from Göytepe. 


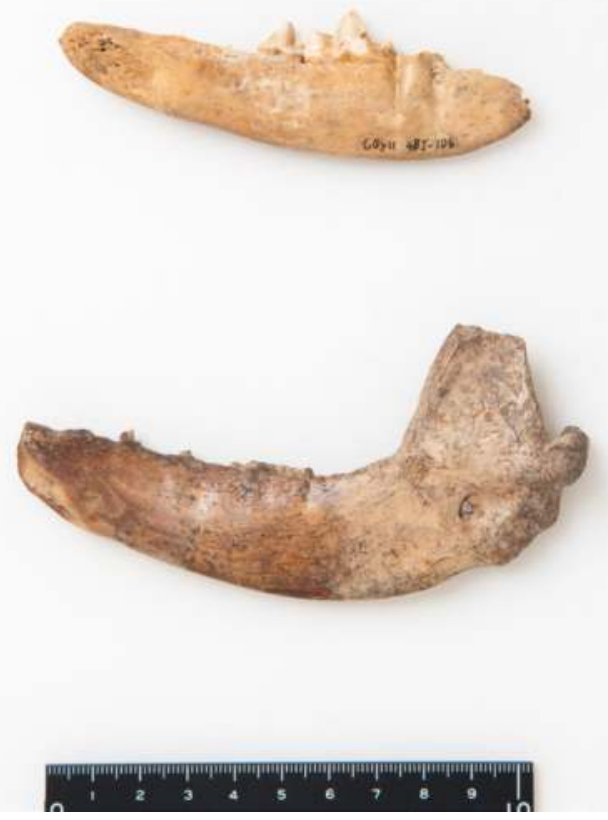

Fig. 15.30 Worked dog mandibles (sickle?) from Göytepe. 


\title{
Plant remains from Göytepe
}

\author{
Chie Akashi and Ken-ichi Tanno
}

\subsection{Introduction}

It is certain that the introduction of agriculture in the South Caucasus occurred as a result of influence from West Asia. However, there is very little evidence about when, how and why the new subsistence strategy was accepted in this area. The Shomutepe culture flourished in the Kura region around the 6th millennium $\mathrm{BC}$ represents the earliest food-producing society known to date, but archaeobotanical data obtained during the Soviet period prohibit us from connecting the plant identifications with stratigraphic or contextual information. This complicates any discussion beyond the presence or absence of cultivated plants.

However, archaeobotanical data on the first farmers of South Caucasus is increasing rapidly with recent excavations of early Neolithic sites on the Middle Kura and Araxes rivers and the Mil plain (Akashi et al. 2018; Decaix et al. 2016; Neef et al. 2017; Hovsepyan and Willcox 2008; Lyonnet et al. 2012). Among those, Göytepe is especially rich in organic remains and can be a key site to understand the subsistence and the plant use of this period. This chapter presents the results of the macrobotanical analysis on 19 samples from Göytepe, with references to data from Hac1 Elamxanlı when appropriate (Akashi et al. 2018). The two sites are only about $1 \mathrm{~km}$ apart and were occupied continuously with a short hiatus (Nishiaki et al. 2013). The archaeobotanical data of Göytepe provide us with the basis for discussion about the process of establishing agriculture in the region and a comparison between other areas in the South Caucasus.

\subsection{Sampling}

During the three excavation seasons from 2009 to 2011, the authors took more than 200 soil samples for water flotation. In the first season in 2009, the main purpose of sampling was to obtain as many charred remains as possible to assess the plant assemblage from Göytepe. Therefore, we focused on contexts where charred remains were abundantly visible with the naked eye, such as hearths and ashy layers. In total, 27 flotation samples were obtained: two from Square 2AI, ten from 4BIIX, and 15 from 4BII.

In the second and the third seasons, we aimed to collect samples based more on context. In the second season, a cluster of bins was recovered in Level 10 of 4BIIX. Similar structures were also found in the upper layers excavated by the Azerbaijani team. We attempted to reconstruct the bins' function using several scientific methods including macro-botanical analysis (see Chapter 7 and Kadowaki et al. 2015). Sediments from inside and outside the bins were collected for later comparison. We took 27 samples in 4BIIX, 19 of which are from inside the bins. The other eight were from outside or from fireplaces. Two bins were sampled in 3AII with one sample collected outside the bins. Furthermore, four samples in 3BI and nine in 4BI were taken from the other contexts. This report is dealing with 19 samples related to the bins collected during the second season.

In the third season, excavation of the large round structure (Wall 16) in 4BI enabled us to reconstruct its construction, use, abandonment, and the postabandonment use (see Chapter 4). We divided the floor into 36 sub-squares (A to Y, AA to KK) and took sediment from each one. We conducted this grid sampling four times (in the room fill, the floor, and below the floor). Besides the grid sampling, a total of 
27 samples were collected from 16 contexts in 4BI. Four samples were also taken in the Pit 97F.

We also conducted sampling in the section wall of 4BII, which is equivalent to part of the open area just outside Wall 16. We took 15 macrobotanical samples from top to bottom in order to clarify the change of the plant assemblage through time.

Abundant charcoal fragments were also found. They were either hand-picked during the excavation or obtained by water flotation. Many fragments are large and solid enough for species identification, the results of which will come out in future reports.

\subsection{Water flotation, sorting and identification}

Water flotation of the sediment samples was conducted in the excavation house. The charred remains of Göytepe were extremely well preserved so we tried to collect even the tiniest and most fragile ones. The flotation device is composed of a large tank and a smaller basin with two sieves (Fig. 16.1). The tank and basins were connected with a hose and pump to create circulation and let the water overflow from the large tank. A fine sieve $(0.3 \mathrm{~mm}$-mesh $)$ was set below the tank's spout to retrieve the light fractions. The light fractions were dried and sent to Japan for microscopic observation.

Heavy fractions were collected with a $0.5 \mathrm{~mm}$ mesh sieve set inside the large tank just below the water level. They included pottery sherds, stone chips, bone fragments, and other small objects. These heavy fractions were also dried and sorted, but few charred remains were present among them and not included in this paper.

The charred remains were examined with a microscope (NIKON SEM1500) under magnification of $\times 8-\times 50$. A combination of published pictures and modern plant samples collected in Azerbaijan and other West Asian countries helped in their identification. Due to the insufficiency of the comparative samples gathered from the Southern Caucasus, most wild taxa were identified only to genus or family level.

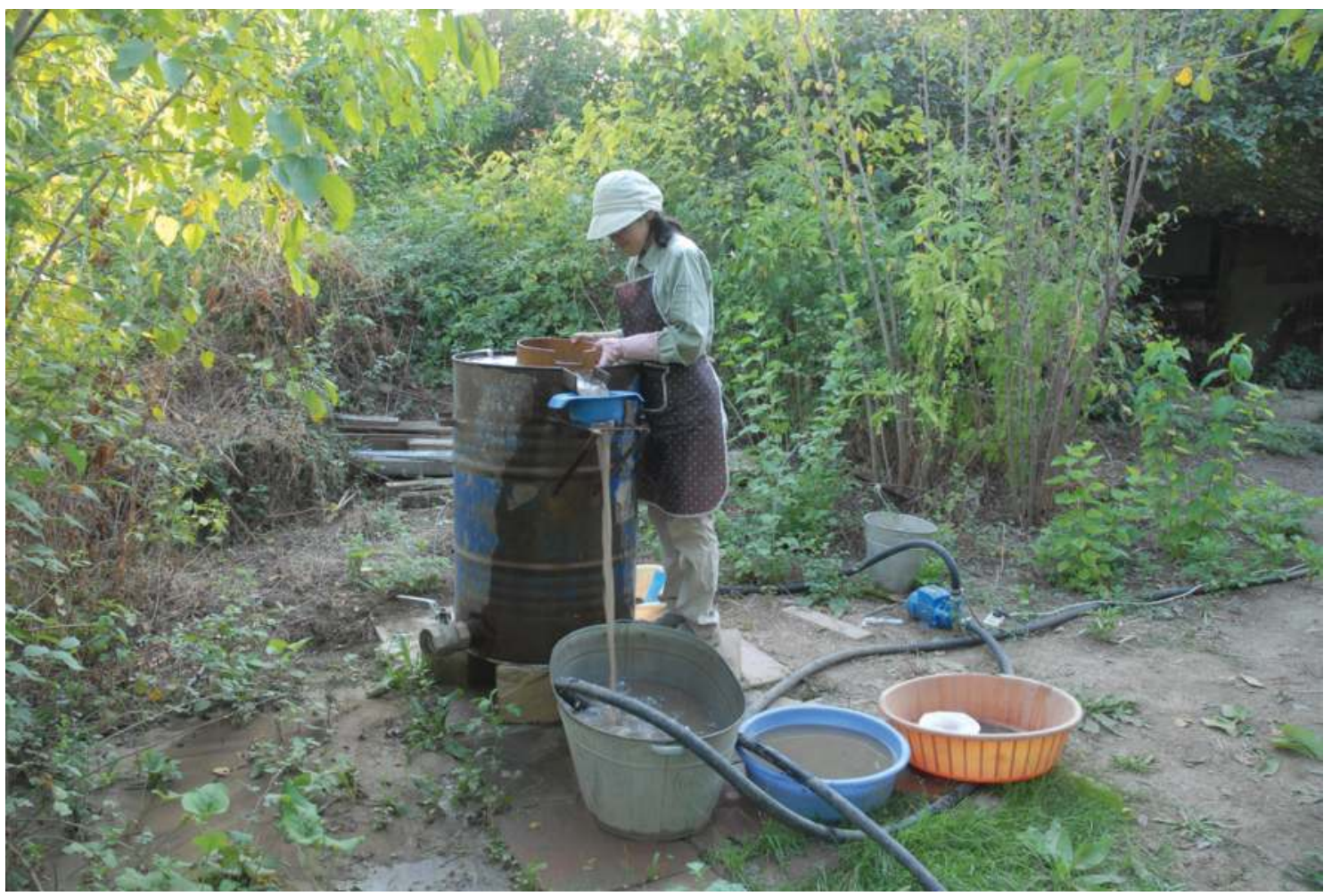

Fig. 16.1 Water flotation device for soil samples. 


\subsection{Samples}

This report represents 19 samples from 4BIIX (Levels 10-11) and two from 3AII (Table 16.1). The amount of soil samples varied between $0.2-6.0$ liters (average 3.4 liters) with the overall total being 64.7 liters. They produced approximately $420 \mathrm{ml}$ of charred remains. Fourteen of these were taken from the clay bins, four from near these bins, and one from a hearth. Each bin was divided into two or four layers (from bottom to top) and each fill was sampled to investigate if the original contents of the bins still remain.

Table 16.2 shows the list of plant macro-remains from the 19 samples (the seven samples already published in Kadowaki et al. 2015 are included). Many of the samples presented in this report came from the storage bins, but there were no burned parts of these bins and most of their macro-botanical remains produced little evidence of the original contents stored in them. They all seem to be from secondary deposits of residues of daily activities except one: one of the bins (4BIIX-94) retained possible traces of its use. A layer of white silicified cereal chaff was found on its bottom, along with some charred grains and rachises and wild seeds like Artemisia-type, which was interpreted as storage of cereal by-products (see Chapter 7 and Kadowaki et al. 2015 for the detail). No grain deposits were recognized from Göytepe.

\subsection{Preservation of seeds and chaff}

The seed density was 180 items per liter, which is remarkably high compared to the other contemporary sites. For example, the seed density was a maximum of 5.0 per liter in Aratashen and Aknashen (Hovsepyan and Wilcox 2008), 12.15 per liter in Mil Plain sites, and 23.63 in Mentesh (Lyonnet et al. 2012).

Most seeds were charred, but some Boraginaceae stones were found mineralized. A part of chaffs was recovered in silicified form, especially in abundance in the lower fills of the bin (4BIIX-94), preserving its shape of glume tip or awn. Impressions of grains and chaff also appeared often on the surface of pottery sherds and mud bricks, which will be discussed elsewhere.

\subsection{Results (Fig. 16.2, Table 16.2)}

Large amount of cereal grains, rachises and glume fragments were found. The most predominant cereal was barley, followed by free-threshing wheat and hulled wheat. Lentil was a part of crop assemblage. The other legumes and tree fruits were represented in a limited number.

\subsubsection{Food plants}

Barley

Barley is the most predominant species in the cultivated plant assemblage of Göytepe, possibly indicating that it was the most important food crop for the inhabitants. Both grains and rachis of barley were abundant. Only five grains were securely identified as hulled type and the many grains had characters of naked barley, like round and plump shape with only a vague ventral groove.

More than 2,000 barley rachis fragments were recovered. This number is twice as many as total wheat spikelet bases. The barley rachis is clearly domesticated type (Tanno and Wilcox 2012) and found occasionally with more than two segments attached together. Some of the well-preserved rachis represented six-row type.

Table 16.1 Summary of the samples.

\begin{tabular}{lr}
\hline number of samples & 19 \\
soil amount (litre) & 64.7 \\
charred remains $(\mathrm{ml})$ & 420 \\
charcoal amount $(\mathrm{ml})$ & 371 \\
number of items identified & \\
number of items / 1 litre soil $^{*}$ & 11,652 \\
\hline
\end{tabular}

* light chaff excluded. 


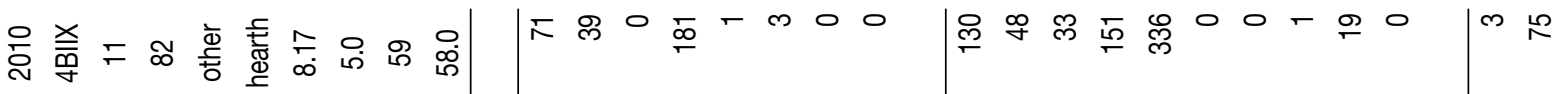

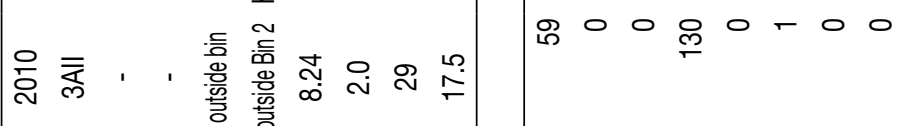
응

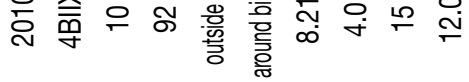

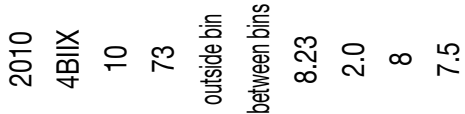

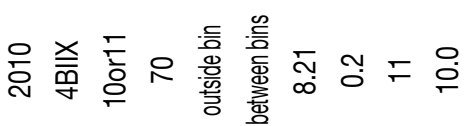

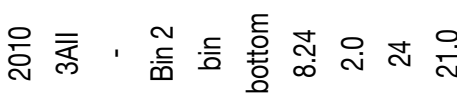

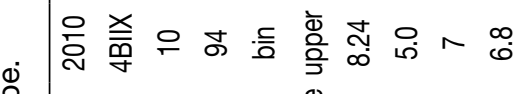

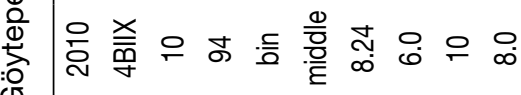

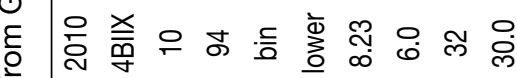

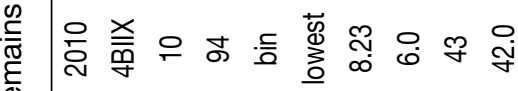

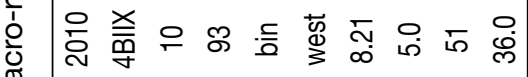

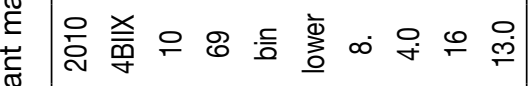
을 䒽 으 顿 을 즘 으

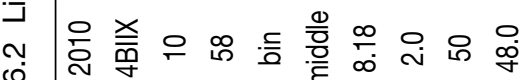

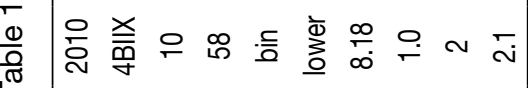
을 즘 으 ⿱口

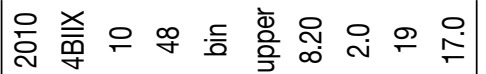

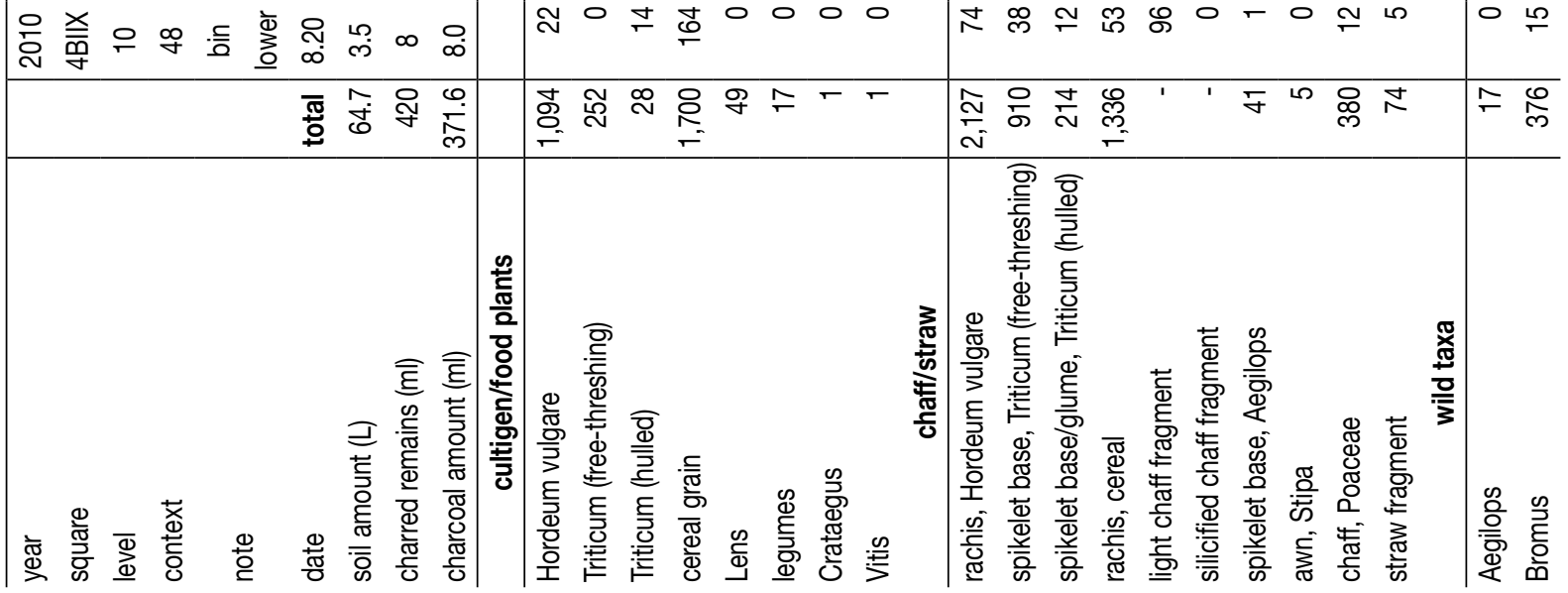

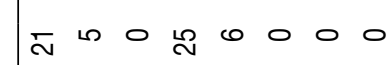
$n \rightarrow 0$ n 0000 무으 œ N 000000

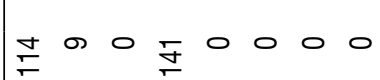
E ล A c m s n n g $\approx \sim 00000$ R A 0 告 -000 $\sim \sim 0$ n $\sim$ \% ह1-8

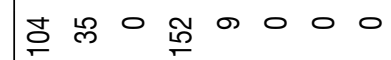
$0+0 \underline{\underline{0}}-000$ m n N 象

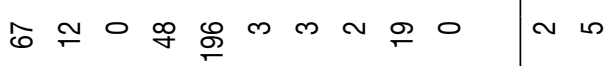
б d

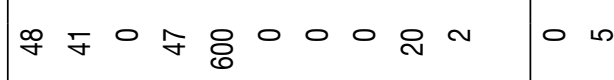

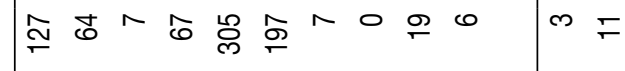
뜬 조일 흐

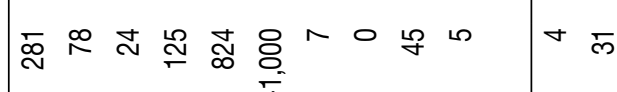

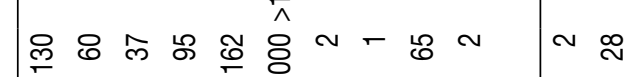

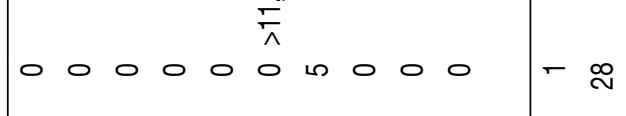

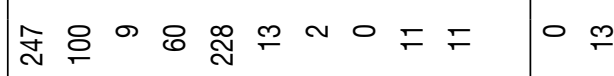
m m 卢

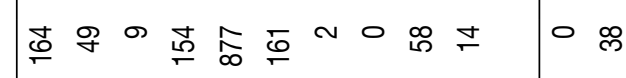
i a

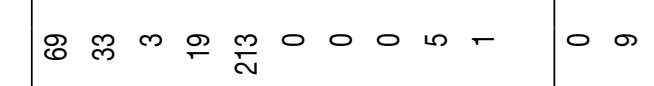

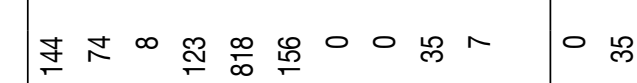


గ $\operatorname{n}$ h $m-000-N 0-00+000000+00000000000000$ $0000 F-0000 m 000000000000000000-000$

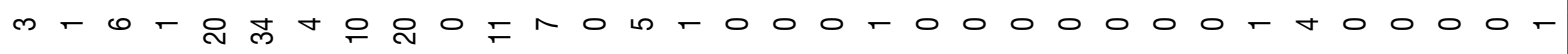

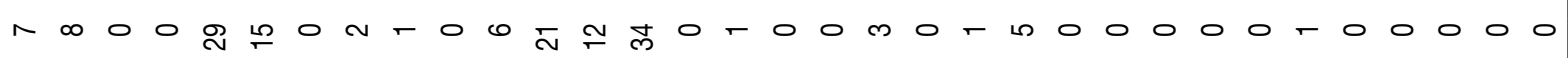

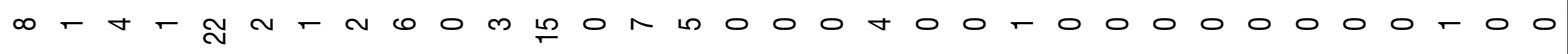
ヘ O O M

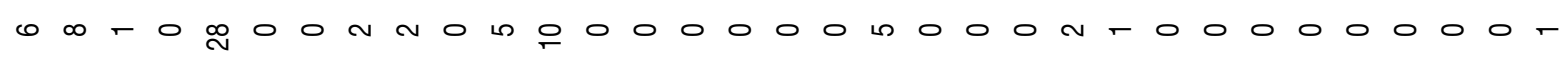
$0000 N 000000+000000000000000000000$ otonfNo $\rightarrow t \rightarrow 0$ L -000 N $000 N 000000000000000000000000$ $0+-0$ T

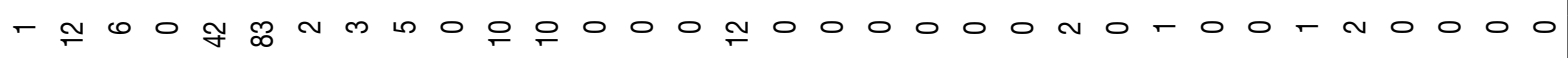
of 8 융

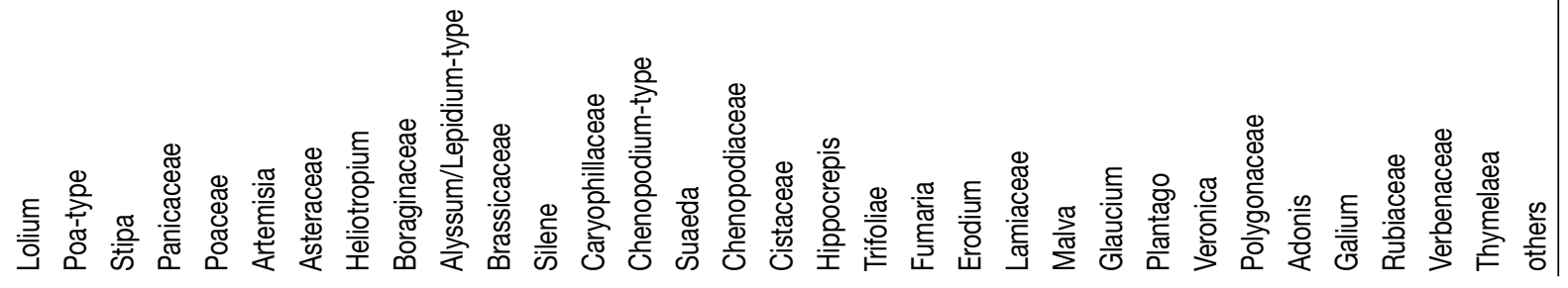



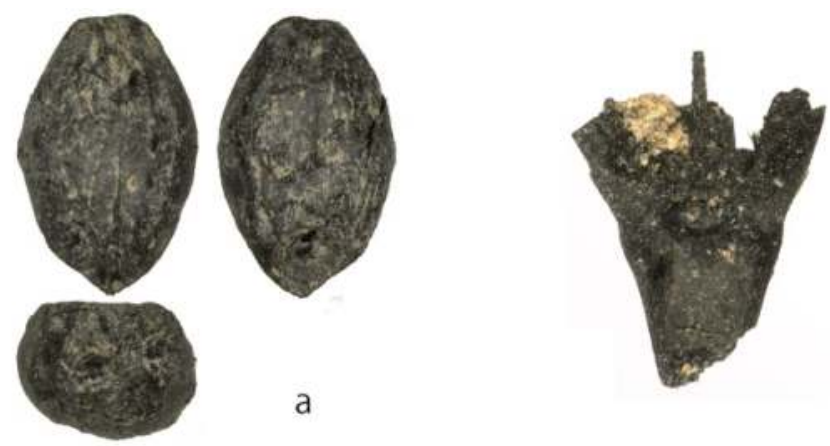

b

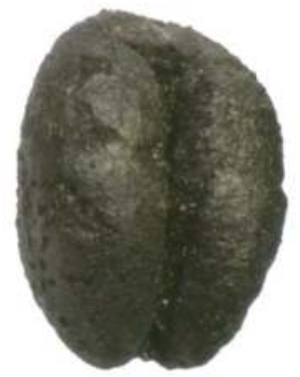

C
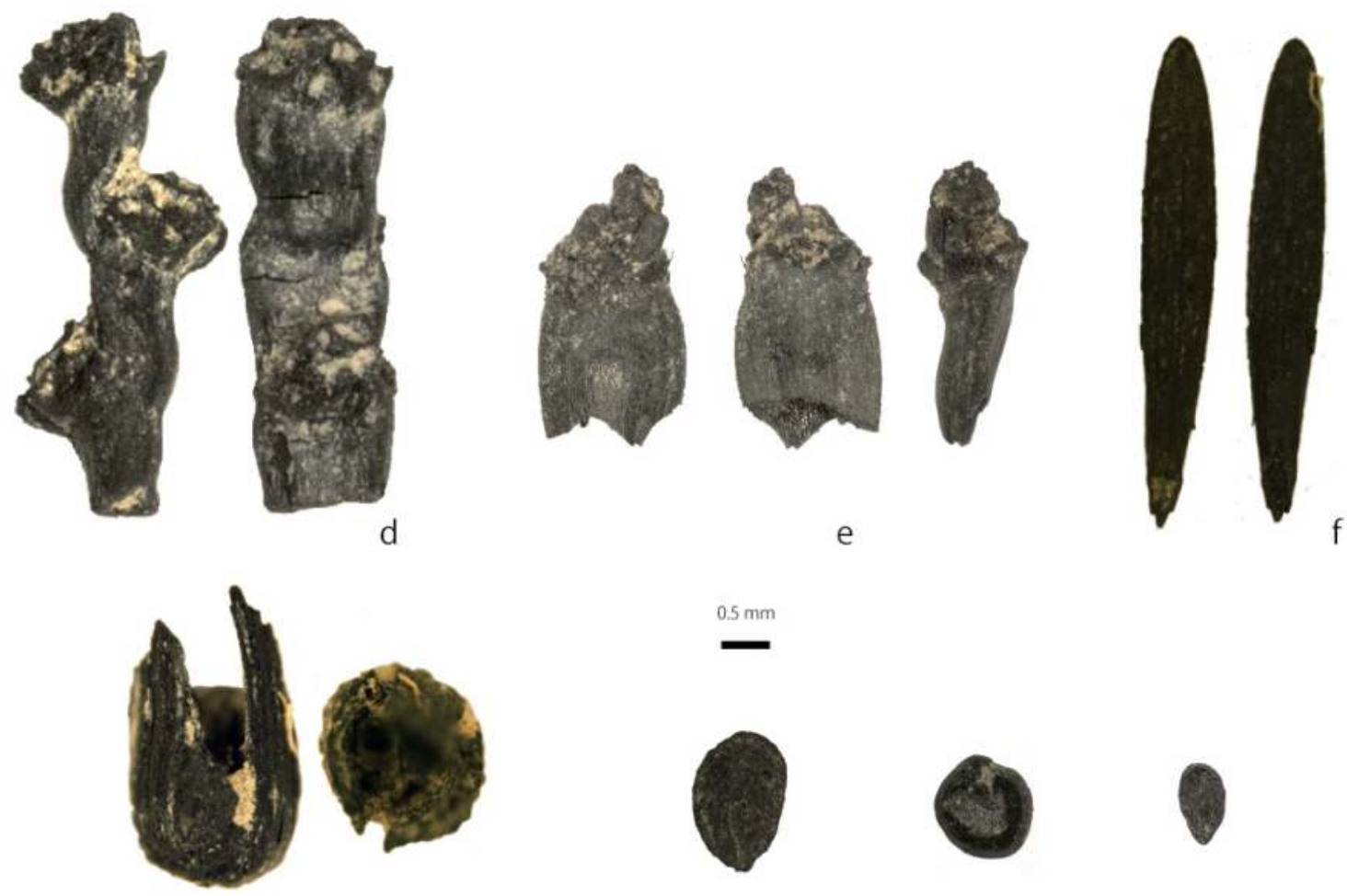

g

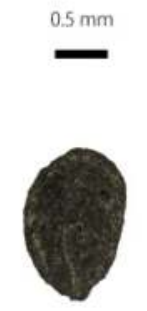

h

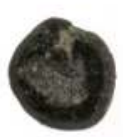

i

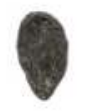

j

Fig. 16.2 Major plant remains from Göytepe. a: Barley grain; b: Barley rachis; c: Free-threshing wheat grain; $\mathrm{d}$ : Free-threshing wheat spikelet bases (tetraploid-type); e: Free-threshing wheat spikelet base (hexaploidtype); f: Bromus sp.; g: Aegilops spikelet base; h: Brassicaceae; i: Chenopodium-type; j: Artemisia-type.

\section{Wheat}

Free-threshing type is the most common wheat in Göytepe. Rotund, round seeds of free-threshing or naked wheat counted about 250. Spikelet bases of free-threshing wheat were also abundant. About 100 spikelet bases were found with more than two segments attached. So obviously the people of Göytepe cultivated the free-threshing wheat as one of their main crops.

Some of these spikelet bases of free-threshing wheat had features of hexaploid (Triticum aestivum) characters (Fig. 16.2: e). Their shield-shaped outline and thinner section are different from the tetraploidtype of spikelet bases (Fig. 16.2: d), and many colleagues agreed that this type is more likely to be hexaploid in the laboratory sessions in the 16th International Work Group for Palaeoethnobotany (IWGP). Some of the naked wheat seeds have almost globular shape, which occurs in hexaploid wheat rather than tetraploid naked wheats like Triticum durum.

The hulled wheat was represented mainly by spikelet bases and glume fragments, and the emmer type was included. The presence of hulled wheat 
seems to be minor compared to barley and naked wheat, and this is interesting because only about 150 years before, in Hac1 Elamxanl, hulled wheat was the most common wheat and naked type was very few (Akashi et al. 2018; Nishiaki et al. 2013).

The grain fragments that could not be identified as either barley or wheat are indicated as "cereal fragments" in Table 16.2.

\section{Other cereal parts}

As mentioned above, the organic materials from Göytepe are very well preserved. Fragile parts like the palea, lemma, awn, or tip of glume, which rarely survive the charring process, were also retrieved in abundance. Fragments of such fragile parts counted more than 7,000 in number and are indicated as "light chaff" in Table 16.2.

In the field excavators frequently encountered a whitish powdery substance that accumulated on the house floor or was mixed in the fill. This was found to be silicified chaff that still retained the shape of glume parts (Chapter 7; Kadowaki et al. 2015). Such chaff was present in eleven of the samples, in exceptionally large number in the bottom and lower fills of the bin (4BIIX-94). Most of them were fragmented glume/palea/lemma, and glume tips of naked wheat and awn fragments of wheat and barley were also included.

\section{Legumes}

Forty-nine seeds of lentil (Lens sp.) were found. The other large legumes could not be identified to genus level due to the fragmented condition. Such scarcity of legumes compared to cereal remains is commonly seen all over West Asian Pottery Neolithic sites. Alternatively, this may partly due to the sampling contexts.

\section{Fruits}

Fruit remains are very scarce. Only one grape (Vitis sp.) pip and one hawthorn (Crataegus sp., 3-stone type) stone were recovered. The South Caucasus is considered as a candidate for the origin of grape domestication (McGovern et al. 2017), but our result shows that grape consumption was limited in Göytepe. Similar to legumes, this scarcity may also be due to the sampling context. It is also noted that the absence of mineralized hackberry (Celtis sp.) is clearly different from Hac1 Elamxanl, where a number of Celtis stones were observed with the naked eye during excavation (Nishiaki et al. 2013).

\subsubsection{Wild or weed plants}

At least 36 taxa belonging to 21 families were represented. Bromus (Poaceae) seeds appeared both in high number $(\mathrm{n}=376)$ and frequency in the overall samples $(100 \%)$, but Artemisia-type (Asteraceae) was the most predominant wild species $(\mathrm{n}=910,74 \%)$. More than 200 remains of Silene sp. (Caryophyllaceae) and Chenopodiumtype (Chenopodiaceae) were collected from various samples. Seeds of at least four kinds of Brassicaceae were also frequently seen.

Minor species that were indentified include grasses such as Poa-type, Lolium sp., Stipa sp., grains and spikelet bases of Aegilops sp. (Poaceae), Heliotropium sp. (Boraginaceae), Alyssum/ Lepidium-type (Brassicaceae), Medicago sp. (Fabaceae), Plantago (Plantaginaceae), Verbena sp. (Verbenaceae), Galium sp. (Rubiaceae), Lamiaceae and Cistaceae, were attested.

Among the major wild species, Bromus sp. and Silene sp. seeds were consistently found from both inside and outside the bins, but the Artemisia-type showed a different trend. This is the most predominant wild species in Göytepe, but they were concentrated in a few samples. A sample from the lower layer of the bin (4BIIX-94), which we reconstructed as storage of chaff, contained more than 500 remains while the upper layers of this bin had only 17 Artemisia-type seeds.

Its concentration in the lower fills indicates that this plant was original content of the bin, and the different usage of this plant compared to other wild plants. Artemisia sp. seed is rarely reported found charred in macro-botanical record, despite it is very common in pollen diagrams of West and Central Asia. But in prehistoric sites of West Azerbaijan, Göytepe and Hacı Elamxanl1, its seeds were found in quantity. It is likely that Artemisia was gathered by Neolithic people of this region for some use.

Artemisia sp. is distributed all over Eurasia, and its ethnopharmacological exploitation is also widely spread. Some Artemisia sp. contain useful medicinal 
ingredients for treating various anthelmintic (parasites) and digestive problems, diabetes, high blood pressure, rheumatism, dysmenorrhea, and various dermatological and respiratory ailments. Artemisine contained in Artemisia annua is used in modern medicine to cure malaria (Mueller et al. 2000).

However, the large number and high ubiquity of charred Artemisia-type of Göytepe, and its concentration in the storage of chaffs suggests more daily use of the plant, rather than occasional oral administration. Artemisia sp. has strong aroma and many of them are known to have chemical components effective as pest control and fungicide (e.g., Dib et al. 2017; Kordali et al. 2006; Abiri et al. 2018), and often used to keep insects and snakes away in ethnography (e.g., Cas et al. 2015; Mohammad Al Sayed 2010), so this plant may have been put in the bin for a sort of preservative.

\subsection{Conclusion}

Barley, free-threshing wheat, hulled wheat, and lentil were cultivated during the Neolithic at Göytepe. This domesticated plant assemblage is quite similar to many other Neolithic sites in the Southern Caucasus. A strong interest of naked cereals in prehistoric Caucasus has been stated by many researchers (Lisitsina and Prishchepenko 1976), and our results from Göytepe also confirmed the preference of naked cereals in this period. The existence of hexaploid free-threshing wheat rachis is of particular interest, for the origin of hexaploid wheat is still in debate.

However, the beginning and the motive of such preference of free-threshing cereals are still unclear. Cereal assemblage of Hac1 Elamxanll, dated in the first quarter of the 6th millennium $\mathrm{BC}$, showed scarcity of naked cereals and much higher proportion of hulled wheat (Akashi et al. 2018). The change in crop choice may indicate the local process of establishment of agricultural society in this region.

Wild plant assemblages seem to have more diversity from site to site and the abundance of Artemisia-type, Bromus sp., Chenopodium sp., and Silene sp. characterize the wild taxa of Göytepe. Especially Artemisia sp. may have been intentionally gathered for insecticidal or fungicidal purposes.
Göytepe and Hacı Elamixanlı represent the two oldest sites with cultivated plants in the South Caucasus. Further analysis of more samples will not only enable us to reconstruct plant distribution and the diachronic changes of the plant use through time and it will also provide crucial information on the introduction and establishment of agriculture in this region.

\section{References}

Abiri, R, A. L. M. Silva, L. S. S. de Mesquita, J. W. C. de Mesquita, N. Atabaki, E. B. de Almeida Jr., N. A. Shaharuddin, and S. Malik (2018) Towards a better understanding of Artemisia vulgaris: Botany, phytochemistry, pharmacological and biotechnological potential. Food Research International, 109: 403-415.

Akashi, C., K. Tanno, F. Guliyev, and Y. Nishiaki (2018) Neolithisation processes of the South Caucasus: As viewed from macro-botanical analyses at $\mathrm{Hacl}$ Elamxanlı Tepe, west Azerbaijan. Paléorient, 44(2): 75-89.

Decaix, A., E. Messager, M. Tengberg, R. Neef, B. Lyonnet, and F. Guliyev (2016) Vegetation and plant exploitation at Mentesh Tepe (Azerbaijan), 6th-3rd millennium BC initial results of the archaeobotanical study. Quaternary International, 395: 19-30.

Cas, L. D., F. Pugni, and G. Fico (2015) Tradition of use on medicinal species in Valfurva (Sondrio, Italy). Journal of Ethnopharmacology, 163: 113-134.

Dib, I., L. Angenot, A. Mihamou, A. Ziyyat, and M. Tits (2017) Artemisia campestris I.: Ethnomedicinal, phytochemical and pharmacological review. Journal of Herbal Medicine, 7: 1-10.

Hovsepyan, R. and G. Willcox (2008) The earliest finds of cultivated plants in Armenia: Evidence from charred remains and crop processing residues in pise from the Neolithic settlements of Aratashen and Aknashen. Vegetation History and Archaeobotany, 17(Supplement 1): S63-71.

Kadowaki, S., L. Maher, M. Portillo, R. M Albert, C. Akashi, F. Guliyev, and Y. Nishiaki (2015) Geoarchaeological and palaeobotanical evidence for prehistoric cereal storage at the Neolithic settlement of Göytepe (mid 8th millennium BP) in the southern Caucasus. Journal of Archaeological Science, 53: 408.

Kordali, S., I. Aslan, O. Çalmaşur, and A. Cakir (2006) Toxicity of essential oils isolated from three Artemisia species and some of their major components to granary weevil, Sitophilus granarius (I.) (coleoptera: Curculionidae). Industrial Crops and Products, 23: 162-170. 
Lisitsina, G. N. and L. V. Prishchepenko (1976) The significance of paleoethnobotanical remains for the reconstruction of early farming in the arid regions of the USSR. Folia Quaternaria, 47: 83-88.

Lyonnet, B., F. Guliyev, B. Helwing, T. Aliyev, S. Hansen, and G. Mirtskhulava (eds.) (2012) Ancient Kura 2010-2011: The First Two Seasons of Joint Field Work in the Southern Caucasus. Archäologische Mitteilungen aus Iran und Turan, 44: 1-190.

McGovern, P. E., M. Jalabadze, S. Batiuk, M. P. Callahan, K. E. Smith, G. R. Hall, E. Kvavadze, D. Maghradze, N. Rusishvili, L. Bouby, O. Failla, G. Cola, L. Mariani, E. Boaretto, R. Bacilieri, P. This, N. Wales, and D. Lordkipanidze (2017) Early Neolithic wine of Georgia in the South Caucasus. Proceedings of the National Academy of Sciences, 114(48): E10309-E10318.

Mohammad Al Sayed, A. (2010) Medicinal Herbal Guide. Alfa Publishing (in Arabic).

Mueller, M. S., I. B. Karhagomba, H. M. Hirt, and E. Wemakor (2000) The potential of Artemisia annua I. As a locally produced remedy for malaria in the tropics: Agricultural, chemical and clinical aspects. Journal of Ethnopharmacology, 73: 487-493.

Neef, R., A. Decaix, and M. Tengberg (2017) Agricultural Practices and Paleoenvironment of the Southern Caucasus during the Neolithic: A Transect along the Kura River. In: The Kura Projects: New Research on the Later Prehistory of the Southern Caucasus, edited by B. Helwing, T. Aliyev, B. Lyonnet, F. Guliyev, S. Hansen, and G. Mirtskhulava, pp. 371-377. Berlin: German Institute of Archaeology, Eurasian Department.

Nishiaki, Y., F. Guliyev, S. Kadowaki, Y. Arimatsu, Y. Hayakawa, K. Shimogama, T. Miki, C. Akashi, S. Arai, and S. Salimbeyov (2013) Hacı Elamxanlı Tepe: Excavations of the earliest Pottery Neolithic occupations on the Middle Kura, Azerbaijan, 2012. Archäologische Mitteilungen aus Iran und Turan, 45: $1-25$.

Tanno, K. and G. Willcox (2012) Distinguishing wild and domestic wheat and barley spikelets from early holocene sites in the near east. Vegetation History and Archaeobotany, 21: 107-115. 



\title{
Faunal remains from Göytepe
}

\author{
Saiji Arai
}

\subsection{Introduction}

Over the past 50 years, archaeological research in the Old World has increased our knowledge of the origins and spread of food production economies. For the matter of the domestication of animals, archaeozoological studies in Turkey, Syria, Jordan, Israel, Iraq, and Iran have provided much information on its origin and development. Many scholars now agree that the domestication process advanced in Southeastern Anatolia and North Syria (Peters et al. 1999; Helmer et al. 2007).

Information about Neolithic subsistence economies in the Southern Caucasus, just north of the area where primary domestication advanced, however, is still limited. Only a few studies were conducted during the second half of the 20th century under the regime of the Soviet Union. However, recent research in Azerbaijan, Georgia, and Armenia has shed light on early animal husbandry in the region (Badalyan et al. 2007, 2010; Balasescu et al. 2010; Benecke 2012). Thus, a comprehensive study between different sites has become available now. It is important to note that a recent aDNA analysis at Aruchlo in Georgia, which is a contemporary Neolithic site to Göytepe, shows that sheep and cattle from the site have relatively diverse mitochondrial haplotypes, in contrast to Western Anatolia and Southeast Europa. Thus, this might be indicative of the fact that the Southern Caucasus was close to the areas where primary domestication of these species happened, or that they were domesticated in this region independently (Benecke 2012: 155). Therefore, the study of early animal husbandry in the Southern Caucasus will be helpful to understand the spread process of the Neolithic package.

This chapter presents the preliminary result of the analysis of faunal remains from Göytepe. The identified species, their size distribution, and culling profile are described, and the results were compared with those from other Neolithic sites in the Southern Caucasus region.

\subsection{The site and its environmental setting}

Göytepe is the largest Neolithic mound in the region (approximately $2 \mathrm{ha}$ ), located $8 \mathrm{~km}$ east of Tovuz city in western Azerbaijan. It is situated on the edge of the alluvial fan formed by the rivers draining from the Lesser Caucasus to the Kura River, and approximately $425 \mathrm{~m}$ above sea level. Although the site is located roughly $13 \mathrm{~km}$ south of the main course of the Kura at present, geomorphological study around the site suggests that there was once a river eastward of the site (Chapter 2).

The modern climate in the region is warm and humid. Annual precipitation is reported to be about $400 \mathrm{~mm}$ (Chataigner 1995). The vegetation around the site today is characterized by steppe, while it is thought that there was also an open forest environment during the Neolithic period.

\subsection{Materials analyzed}

During the 2009-2011 seasons, a total of 6,105 (including worked bone; see Chapter 15) faunal remains were yielded from Japanese expeditions in Square 4B, consisting of 4BI and 4BII (Table 17.1). The dimension of each sector is $5 \mathrm{~m} \times 10 \mathrm{~m}$. Since the Japanese expeditions were limited to these small trenches on the edge of the mound, the sequence of materials do not cover the upper occupation levels of the site. Although the materials were handpicked 


\begin{tabular}{|c|c|c|c|c|c|c|c|c|c|c|c|c|c|c|c|c|c|c|c|c|}
\hline \multirow[t]{2}{*}{ s } & 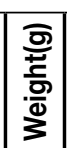 & $\begin{array}{l}0 \\
\tilde{\rho} \\
\tilde{o}\end{array}$ & 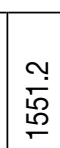 & $\begin{array}{l}\bar{p} \\
\infty \\
\infty \\
\infty\end{array}$ & $\begin{array}{l}\text { Ñ } \\
\text { ర్ } \\
\text { ֶু }\end{array}$ & 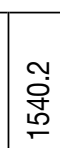 & $\begin{array}{l}\infty \\
\infty \\
\stackrel{\infty}{N}\end{array}$ & 站 & 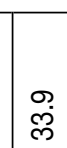 & $\begin{array}{l}\infty \\
\stackrel{\infty}{q}\end{array}$ & $\stackrel{m}{\sim}$ & 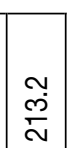 & $\stackrel{m}{\circ}$ & 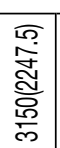 & $\begin{array}{l}\stackrel{\leftrightarrow}{\sim} \\
\stackrel{\sim}{n}\end{array}$ & o & 옹 & $\stackrel{m}{\Gamma}$ & & 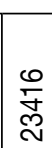 \\
\hline & $\frac{\text { os }}{z}$ & న్ & 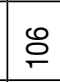 & : & $\bar{\Sigma}$ & 음 & ㄱ & ని & $\simeq$ & $\infty$ & - & $\stackrel{d}{\sim}$ & $\nabla$ & $\overline{\bar{m}}$ & $m$ & - & 0 & $\mp$ & $\sim$ & \begin{tabular}{|l} 
\\
$g$ \\
$g$
\end{tabular} \\
\hline \multirow[b]{2}{*}{ 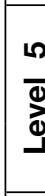 } & 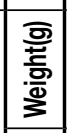 & 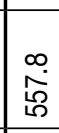 & 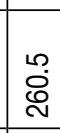 & $\begin{array}{l}\text { 官 } \\
\dot{\alpha}+\end{array}$ & $\begin{array}{l}\check{\tilde{O}} \\
\mathbb{N}\end{array}$ & $\begin{array}{l}\text { ó } \\
\stackrel{\leftrightarrow}{N}\end{array}$ & $\begin{array}{l}\text { مٌ } \\
\stackrel{\sim}{N}\end{array}$ & $\stackrel{\text { Po }}{\longrightarrow}$ & $\stackrel{L}{+}$ & 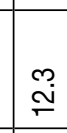 & 0 & $\overline{\mathrm{D}}$ & 0 & $\begin{array}{l}\vec{g} \\
\bar{o} \\
o \\
o \\
\sim\end{array}$ & 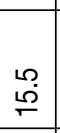 & 0 & 0 & $\overline{\dot{v}}$ & & \begin{tabular}{|l} 
\\
$\stackrel{0}{0}$ \\
$\frac{\rho}{m}$ \\
\end{tabular} \\
\hline & $\frac{\text { os }}{2}$ & 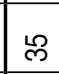 & $\stackrel{2}{\sim}$ & $\bar{\epsilon}$ & $\stackrel{\infty}{\sim}$ & $F$ & $m$ & $\sim$ & $\sim$ & - & 0 & $\sim$ & 0 & $\underset{N}{\mathbb{N}}$ & - & 0 & 0 & - & - & 守 \\
\hline \multirow{2}{*}{$\frac{0}{0}$} & 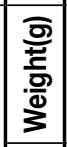 & 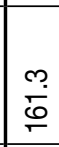 & 형 & $\frac{m}{m}$ & $\begin{array}{l}\hat{\sim} \\
\stackrel{6}{0} \\
\end{array}$ & 苂 & $\begin{array}{l}\text { 定 } \\
\stackrel{\rho}{=}\end{array}$ & $\stackrel{\sim}{\sim}$ & 0 & $\stackrel{0}{\stackrel{d}{d}}$ & 0 & 0 & 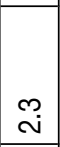 & 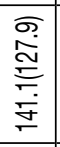 & 0 & 0 & $\stackrel{9}{\circ}$ & ח. & 0 & 항 \\
\hline & $\frac{5}{\frac{5}{2}}$ & 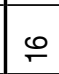 & $\stackrel{m}{\leftarrow}$ & ₹ & க & $\simeq$ & $\sim$ & $\theta$ & 0 & $\sim$ & 0 & 0 & - & 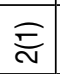 & 0 & 0 & $m$ & $\sim$ & 0 & 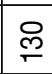 \\
\hline \multirow{2}{*}{ 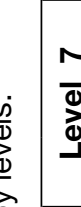 } & 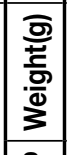 & ్ㅠ & 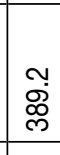 & 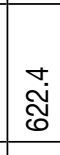 & 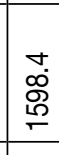 & 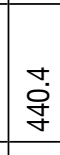 & 0 & $\begin{array}{l}\infty \\
\infty \\
\infty\end{array}$ & 0 & $\check{f}^{\prime}$ & $\stackrel{?}{\sim}$ & 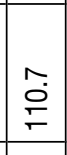 & 0 & 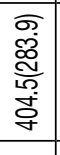 & ஸี & 0 & 0 & 0 & 0 & $\begin{array}{l}\check{\dot{d}} \\
\stackrel{+}{+}\end{array}$ \\
\hline & $\frac{\text { os }}{2}$ & กิ & $\stackrel{i}{2}$ & ळ & 将 & $\stackrel{\sim}{N}$ & 0 & m & 0 & $\sim$ & - & $\infty$ & 0 & $\underset{N}{\mathbb{N}}$ & - & 0 & 0 & 0 & 0 & $\underset{\sim}{\stackrel{g}{d}}$ \\
\hline \multirow[t]{2}{*}{$\frac{\infty}{\bar{d}}$} & 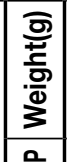 & \begin{tabular}{|l} 
\\
g. \\
$\stackrel{\sigma}{\sigma}$ \\
\end{tabular} & $\stackrel{d}{a}$ & $\begin{array}{l}10 \\
\infty \\
\infty \\
\infty\end{array}$ & $\begin{array}{l}0 \\
\dot{\sigma}\end{array}$ & $\stackrel{-}{\infty}$ & $\stackrel{\sim}{\sim}$ & $\stackrel{m}{i}$ & $\stackrel{\leftrightarrow}{\leftarrow}$ & 0 & 0 & 0 & $\stackrel{\sim}{-}$ & 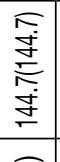 & 0 & 0 & 0 & 0 & 0 & \begin{tabular}{|l} 
\\
0 \\
0 \\
\end{tabular} \\
\hline & $\frac{20}{2}$ & $\approx$ & $\sim$ & $\stackrel{\mathscr{N}}{2}$ & $\stackrel{m}{\longrightarrow}$ & - & $\tau$ & - & - & 0 & 0 & 0 & - & $\frac{\widehat{D}}{\omega n}$ & 0 & 0 & 0 & 0 & 0 & 0 \\
\hline \multirow[t]{2}{*}{ a } & 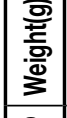 & $\stackrel{\circ}{\circ}$ & \begin{tabular}{l}
$\infty$ \\
்ָ \\
\cline { 1 - 1 }
\end{tabular} & 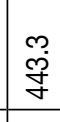 & 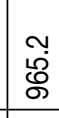 & $\hat{\grave{\rho}}$ & $\begin{array}{l}\infty \\
\text { Li } \\
\text { م. }\end{array}$ & $\begin{array}{l}\infty \\
\stackrel{0}{\circ} \\
\end{array}$ & $\stackrel{⿱}{\leftarrow}$ & $\stackrel{m}{i}$ & 0 & $\tilde{\sim}$ & 0 & $\begin{array}{l}\text { 응 } \\
\infty \\
\overline{0} \\
\end{array}$ & $\stackrel{\sim}{+}$ & o & 0 & 0 & 0 & \begin{tabular}{l}
0 \\
\multirow{2}{\alpha}{} \\
\end{tabular} \\
\hline & $\frac{\mathrm{og}}{2}$ & $\approx$ & 0 & $\overline{6}$ & $\stackrel{\infty}{\sim}$ & $\stackrel{m}{-}$ & 0 & $\nabla$ & $\sim$ & - & 0 & - & 0 & $\frac{o}{y}$ & - & - & 0 & 0 & 0 & $\stackrel{8}{10}$ \\
\hline \multirow{2}{*}{$\frac{5}{9}$} & 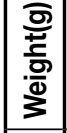 & 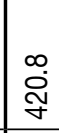 & $\begin{array}{l}10 \\
8 \\
\circ \\
\end{array}$ & \begin{tabular}{|c}
$\infty$ \\
$\dot{\infty}$ \\
$\infty$ \\
$\infty$
\end{tabular} & $\begin{array}{l}\text { 동 } \\
\text { 吕 }\end{array}$ & $\begin{array}{l}\infty \\
\dot{f} \\
\stackrel{0}{0}\end{array}$ & $\begin{array}{l}10 \\
\text { ద్ల }\end{array}$ & $\stackrel{\sim}{\sim}$ & o & $\stackrel{\sim}{\sim}$ & 0 & $\overline{\dot{m}}$ & 0 & 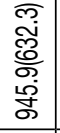 & 0 & 0 & $\overline{0}$ & ஸ̃ & & 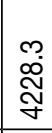 \\
\hline & $\frac{\mathrm{e}}{2}$ & 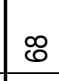 & 응 & 号 & $\mp$ & $\stackrel{m}{\rightleftarrows}$ & $\theta$ & $m$ & $\theta$ & $\sim$ & 0 & $\infty$ & 0 & 응 & 0 & 0 & - & $\sim$ & - & F \\
\hline & 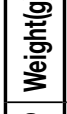 & No & 0 & 0 & প্ল & 0 & 0 & 0 & 0 & 0 & 0 & $\underset{+}{+}$ & 0 & 0 & 0 & 0 & 0 & 0 & 0 & $\bar{ळ}$ \\
\hline & $\frac{\rho}{\frac{0}{z}}$ & $\tau$ & 0 & 0 & $\sim$ & 0 & 0 & 0 & 0 & 0 & 0 & - & 0 & 0 & 0 & 0 & 0 & 0 & 0 & $\nabla$ \\
\hline \multirow{2}{*}{$\frac{\mp}{\bar{d}}$} & 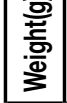 & \begin{tabular}{|l}
0 \\
$\dot{\infty}$ \\
$\dot{\infty}$
\end{tabular} & ন্ল্] & 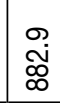 & 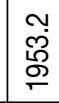 & হ্লల & $\begin{array}{l}m \\
\stackrel{f}{f}\end{array}$ & $\stackrel{?}{\sim}$ & $\stackrel{\vec{F}}{\rightleftharpoons}$ & 0 & 0 & $\hat{i}$ & $\begin{array}{l}3 \\
0 \\
6\end{array}$ & $\begin{array}{l}\overline{\tilde{d}} \\
\bar{N} \\
\bar{\alpha}\end{array}$ & 0 & 0 & 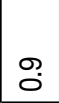 & $\stackrel{\infty}{\infty}$ & 0 & 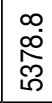 \\
\hline & $\frac{\rho}{2}$ & 交 & 오 & $\underset{\mathbb{N}}{\stackrel{\mathbb{N}}{ }}$ & க & প্ল & 8 & m & m & 0 & 0 & $\theta$ & $\sim$ & $\frac{\widehat{Q}}{\infty}$ & 0 & 0 & - & 10 & 0 & $\frac{m}{m}$ \\
\hline \multirow{2}{*}{$\begin{array}{l}\text { N } \\
\bar{d} \\
\text { d }\end{array}$} & 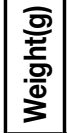 & 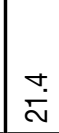 & $\stackrel{\mathbb{N}}{\mathrm{N}}$ & 8 & $\begin{array}{l}0 \\
\infty \\
\infty\end{array}$ & 0 & 0 & 0 & 0 & 0 & 0 & 0 & 0 & 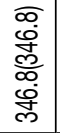 & 0 & 0 & 0 & $\dot{v}$ & 0 & \begin{tabular}{|l|}
$\infty$ \\
\\
0
\end{tabular} \\
\hline & $\frac{\text { Dos }}{z}$ & $\sim$ & $\sim$ & 0 & $m$ & 0 & 0 & 0 & 0 & 0 & 0 & 0 & 0 & $\equiv$ & 0 & 0 & 0 & - & 0 & $\stackrel{20}{2}$ \\
\hline 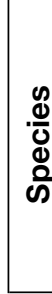 & 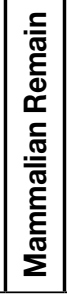 & $\begin{array}{l}\dot{0} \\
\text { की } \\
\stackrel{5}{0} \\
0\end{array}$ & 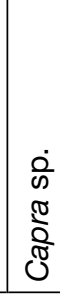 & 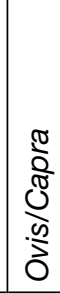 & $\begin{array}{l}\text { के } \\
\text { कू } \\
\text { कि }\end{array}$ & $\begin{array}{l}0 \\
\text { क } \\
\text { s } \\
\text { क }\end{array}$ & 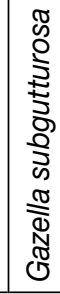 & $\begin{array}{l}0 \\
0 \\
0 \\
0\end{array}$ & $\begin{array}{l}0 \\
0 \\
0 \\
0 \\
0\end{array}$ & 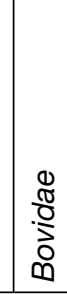 & 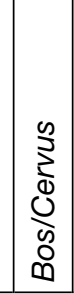 & 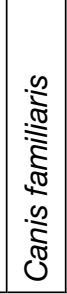 & 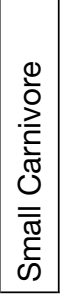 & 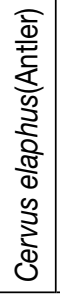 & 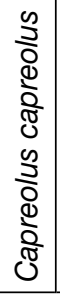 & $\begin{array}{l}.0 \\
\frac{2}{D} \\
0 \\
0\end{array}$ & \begin{tabular}{|l}
0 \\
0 \\
0 \\
0 \\
0 \\
0 \\
\\
\end{tabular} & 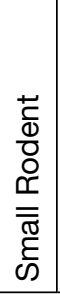 & 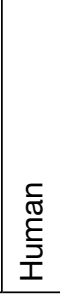 & 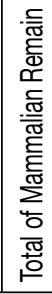 \\
\hline
\end{tabular}




\begin{tabular}{|c|c|c|c|c|c|c|c|c|c|c|c|c|c|c|}
\hline $\begin{array}{l}\bar{\pi} \\
\text { 。̊ }\end{array}$ & 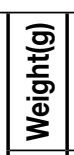 & 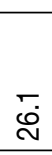 & ্ֻণ் & $\stackrel{\infty}{i}$ & $\begin{array}{l}0 \\
\stackrel{8}{2} \\
\stackrel{2}{2}\end{array}$ & $\theta$ & 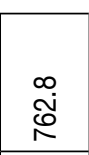 & & ల్ల & \begin{tabular}{|l}
0 \\
$\stackrel{\dot{J}}{\mathbf{N}}$ \\
\end{tabular} & $\begin{array}{l}\infty \\
0 \\
0 \\
0 \\
0\end{array}$ & $\begin{array}{l}L \rho \\
0 \\
0 \\
0 \\
\end{array}$ & $\begin{array}{l}\tilde{J} \\
\text { ثें }\end{array}$ & $\begin{array}{l}\bar{c} \\
\stackrel{6}{\circ} \\
\stackrel{0}{-} \\
\end{array}$ \\
\hline & $\frac{\text { os }}{2}$ & $\underset{\nabla}{ }$ & $\mp$ & $\simeq$ & న & 10 & $\stackrel{10}{\leftarrow}$ & & 过 & $\bar{\Sigma}$ & $\stackrel{\mathbb{O}}{\stackrel{0}{\sim}}$ & ஜ뭉 & 怘 & 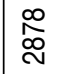 \\
\hline$\frac{\omega}{\frac{\sigma}{d}}$ & 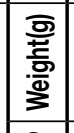 & $\underset{+}{\check{\sigma}}$ & $\stackrel{\leftrightarrow}{\leftarrow}$ & 0 & $\bar{N}$ & $\stackrel{m}{\leftarrow}$ & $\stackrel{\infty}{\sim}$ & & $m$ & $\begin{array}{l}\stackrel{\infty}{\sim} \\
\stackrel{\sim}{N}\end{array}$ & $\begin{array}{l}\text { L } \\
\stackrel{N}{N} \\
\end{array}$ & 总 & $\begin{array}{l}\underset{\tilde{N}}{\mathrm{~N}} \\
\text { }\end{array}$ & $\begin{array}{l}\dot{0} \\
\stackrel{0}{0} \\
\stackrel{0}{\sim} \\
\end{array}$ \\
\hline$\stackrel{\Xi}{\unlhd}$ & \begin{tabular}{|l|}
$\frac{2}{2}$ \\
\end{tabular} & $m$ & $\tau$ & 0 & $m$ & $r$ & $\infty$ & & 10 & 으 & 穴 & $\stackrel{m}{\div}$ & $\overline{6}$ & 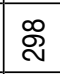 \\
\hline $\begin{array}{l}0 \\
\bar{\Phi} \\
\text { త్ }\end{array}$ & 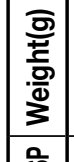 & $\stackrel{\leftrightarrow}{i}$ & 0 & $\overline{0}$ & 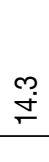 & 0 & $\begin{array}{l}\stackrel{乃}{0} \\
\stackrel{6}{\circ}\end{array}$ & & $\begin{array}{l}\infty \\
0 \\
0\end{array}$ & 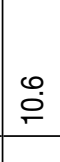 & $\begin{array}{l}\infty \\
\stackrel{0}{j} \\
0 \\
0\end{array}$ & $\begin{array}{c}\infty \\
\stackrel{\infty}{+} \\
\stackrel{\alpha}{q}\end{array}$ & $\begin{array}{l}1 \\
0 \\
0 \\
0 \\
0 \\
\end{array}$ & $\frac{\infty}{\stackrel{\infty}{-}}$ \\
\hline & $\frac{25}{2}$ & $m$ & 0 & 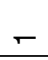 & $\nabla$ & 0 & $\infty$ & & 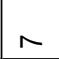 & 0 & $\stackrel{?}{\gtrless}$ & 0 & $\stackrel{\sim}{\sim}$ & $\stackrel{\infty}{i}$ \\
\hline ก & 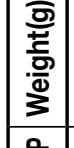 & 0 & ָூ & $\stackrel{m}{\square}$ & $\stackrel{?}{10}$ & ๙ָ & 品 & & $\stackrel{n}{\infty}$ & 水 & $\begin{array}{l}0 \\
\infty \\
\infty \\
\stackrel{0}{\infty}\end{array}$ & $\mathfrak{N}$ & $\begin{array}{l}\hat{\rho} \\
\tilde{\infty}\end{array}$ & 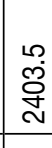 \\
\hline & $\frac{\frac{5}{2}}{2}$ & 0 & $\sim$ & $r$ & N & - & $\mp$ & & 10 & $\stackrel{\infty}{\infty}$ & 足 & $\mp$ & 守 & $\mathbb{N}$ \\
\hline 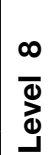 & 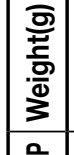 & 0 & 0 & 0 & $\sim$ & 0 & $\sim$ & & 0 & 0 & ì & ז্ & $\begin{array}{l}\infty \\
\stackrel{\infty}{0} \\
\stackrel{్}{.}\end{array}$ & $\widetilde{\tilde{ర}}$ \\
\hline & $\frac{5}{\frac{5}{2}}$ & 0 & 0 & 0 & - & 0 & $\tau$ & & 0 & 0 & $\stackrel{\Re}{\stackrel{N}{\sim}}$ & $\mp$ & 임 & 神 \\
\hline$\frac{\sigma}{\rho}$ & 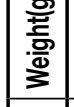 & $\stackrel{m}{\risingdotseq}$ & 0 & 0 & $\begin{array}{l}\infty \\
\stackrel{\infty}{\sigma} \\
\stackrel{\sigma}{\sigma}\end{array}$ & 0 & ¿্d & & $\stackrel{\infty}{\circ}$ & 요 & 㝘 & $\stackrel{\stackrel{\circ}{\leftarrow}}{\leftarrow}$ & 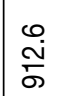 & 형 \\
\hline 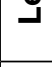 & $\frac{0}{2}$ & $\sim$ & 0 & 0 & $\stackrel{\nabla}{\nabla}$ & 0 & $\stackrel{\circ}{\leftarrow}$ & & m & $\stackrel{\sim}{\sim}$ & ৪) & m & గొ & $\underset{\Xi}{\mp}$ \\
\hline$\frac{0}{\bar{\rho}}$ & 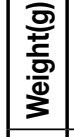 & ח) & ช్ & $\stackrel{\text { مִ }}{\leftarrow}$ & $\begin{array}{l}\frac{m}{\rho} \\
\stackrel{\rho}{\rho}\end{array}$ & 5 & 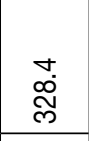 & & in & $\begin{array}{l}m \\
0 \\
0\end{array}$ & $\begin{array}{l}\stackrel{\infty}{\sim} \\
\stackrel{\sim}{\sim}\end{array}$ & ָ̦ & $\begin{array}{l}\tilde{N} \\
\stackrel{N}{\infty} \\
\infty\end{array}$ & $\begin{array}{l}0 \\
\dot{0} \\
\stackrel{N}{N} \\
\stackrel{N}{2}\end{array}$ \\
\hline & $\frac{\mathrm{o}}{\mathbf{2}}$ & m & $m$ & 으 & $\hat{\sim}$ & - & 寸 & & $\mp$ & $R$ & ₹ & g్ల & $\stackrel{R}{\circ}$ & $\widehat{\mathscr{\infty}}$ \\
\hline $\begin{array}{l}\text { 운 } \\
\text { 훋 }\end{array}$ & 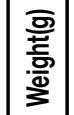 & 0 & 0 & 0 & $\stackrel{\llcorner}{\tilde{N}}$ & 0 & $\stackrel{\llcorner}{\text { ஸ̊ }}$ & & 0 & 0 & $\stackrel{10}{\square}$ & 0 & [ై & ని \\
\hline 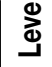 & \begin{tabular}{|l|}
$\frac{D}{2}$ \\
\end{tabular} & 0 & 0 & 0 & - & 0 & - & & 0 & 0 & 10 & 0 & - & 0 \\
\hline$\frac{\Xi}{\Phi}$ & 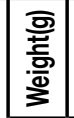 & $\stackrel{\infty}{\sim}$ & గ్రి & 0 & $\frac{+}{\infty}$ & $\stackrel{\sim}{\sim}$ & ه્ఠ & & $\stackrel{\sim}{\text { ָे }}$ & ڤ̊. & $\begin{array}{l}\hat{\alpha} \\
\infty \\
\stackrel{\infty}{\sim} \\
\stackrel{\sim}{\sim}\end{array}$ & S̆ & 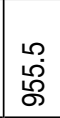 & $\stackrel{\text { P. }}{\stackrel{\Sigma}{+}}$ \\
\hline د & $\frac{\mathrm{o}_{2}}{\mathrm{~m}}$ & m & مـ & 0 & $\stackrel{\text { م }}{\complement}$ & $\sim$ & $\stackrel{\llcorner}{\sim}$ & & $\underset{\leftarrow}{ }$ & is & 会 & $\stackrel{\infty}{\stackrel{\infty}{2}}$ & $\mathscr{C}$ & $\stackrel{m}{\Gamma}$ \\
\hline$\frac{\text { T }}{\Phi}$ & 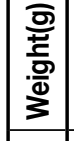 & 0 & 0 & 0 & $\bar{\infty}_{\infty}^{-}$ & 0 & $\check{\infty}^{-}$ & & 0 & 0 & 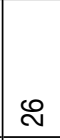 & $\stackrel{\Re}{\stackrel{2}{r}}$ & $\begin{array}{l}\text { N } \\
\text { ஸ్ }\end{array}$ & $\begin{array}{l}\stackrel{\circ}{0} \\
\stackrel{\sigma}{\sigma} \\
\stackrel{2}{\circ}\end{array}$ \\
\hline & $\frac{\mathrm{o}}{\mathrm{m}}$ & 0 & 0 & 0 & $\tau$ & 0 & $\tau$ & & 0 & 0 & 0 & $\sim$ & 0 & $\approx$ \\
\hline 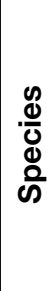 & 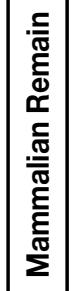 & $\frac{\overline{0}}{\overline{0}}$ & $\frac{\frac{c}{\omega}}{i \frac{1}{L}}$ & $\frac{\text { ox }}{\stackrel{\text { ph }}{\overline{\bar{O}}}}$ & $\begin{array}{l}0 \\
. \frac{N}{0} \\
\bar{O} \\
\stackrel{t}{0} \\
\end{array}$ & $\frac{O}{\frac{O}{L}}$ & 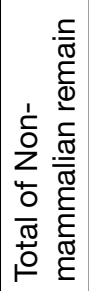 & 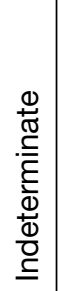 & 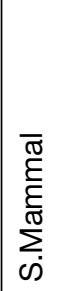 & 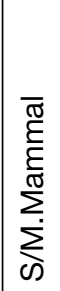 & 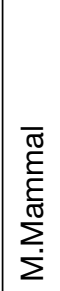 & 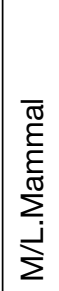 & $\begin{array}{l}\overline{\widetilde{\sigma}} \\
\sum_{j}^{\underline{\xi}} \\
\sum_{j}^{\tilde{\sigma}}\end{array}$ & 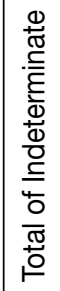 \\
\hline
\end{tabular}


by the excavators, small specimens, such as rodents' bones, are also included in the assemblage. The state of preservation of materials is favorable. The rapid deposition during the site formation and the humidity may have contributed to the extremely good condition of these materials.

\subsection{The faunal assemblage}

\subsubsection{Domestic species}

\section{Sheep and goat}

Caprines are the most common taxa at Göytepe, accounting for more than $60 \%$ of the faunal remains based on the number of identified specimens (NISP). Almost all of the specimens are identified as domestic. At the same time, some specimens appear to be their wild ancestors (see below). Given their natural habitats and the location of the site, wild sheep and goats appear to have been hunted around the northern foothills of the Lesser Caucasus.

Sheep remains outnumber goat remains in all phases. However, the frequency of sheep to goats changed from the lower levels onward. The sheep to goat ratio is 4:1 in the lower levels (Levels 12-9), whereas it is 2:1 in the upper level (Level
5) (Fig. 17.1). This suggests that some changes in animal exploitation strategies occurred during the occupation of this site.

The size distributions of sheep and goats from Göytepe were analyzed using the logarithmic size index (LSI) method (Meadow 1981, 1999). In this method, each measurement from the different elements is converted into logarithms and compared with those from a standard animal. The standards used here are a modern female Ovis orientalis from Iran and the averaged measurements of the modern male and female Capra aegagurus from the Taurus (Uerpmann and Uerpmann 1994). Then, the LSI values are calculated as per the formula: $d=\log x-$ $\log m=\log (x / m)$, where $x$ equals the measurement from the archaeological specimen, and $m$ equals the corresponding measurement from the standard animal. Since no measurement data from other sites in the region have been published, this result could not be compared at an inter-site level.

Because the sample size is small, specimens from Levels 12-9 were integrated as "lower levels" and from $8-5$ as "upper levels." Fig. 17.2 shows the distributions of the LSI values of sheep and goats from Göytepe. It is clear that most Göytepe

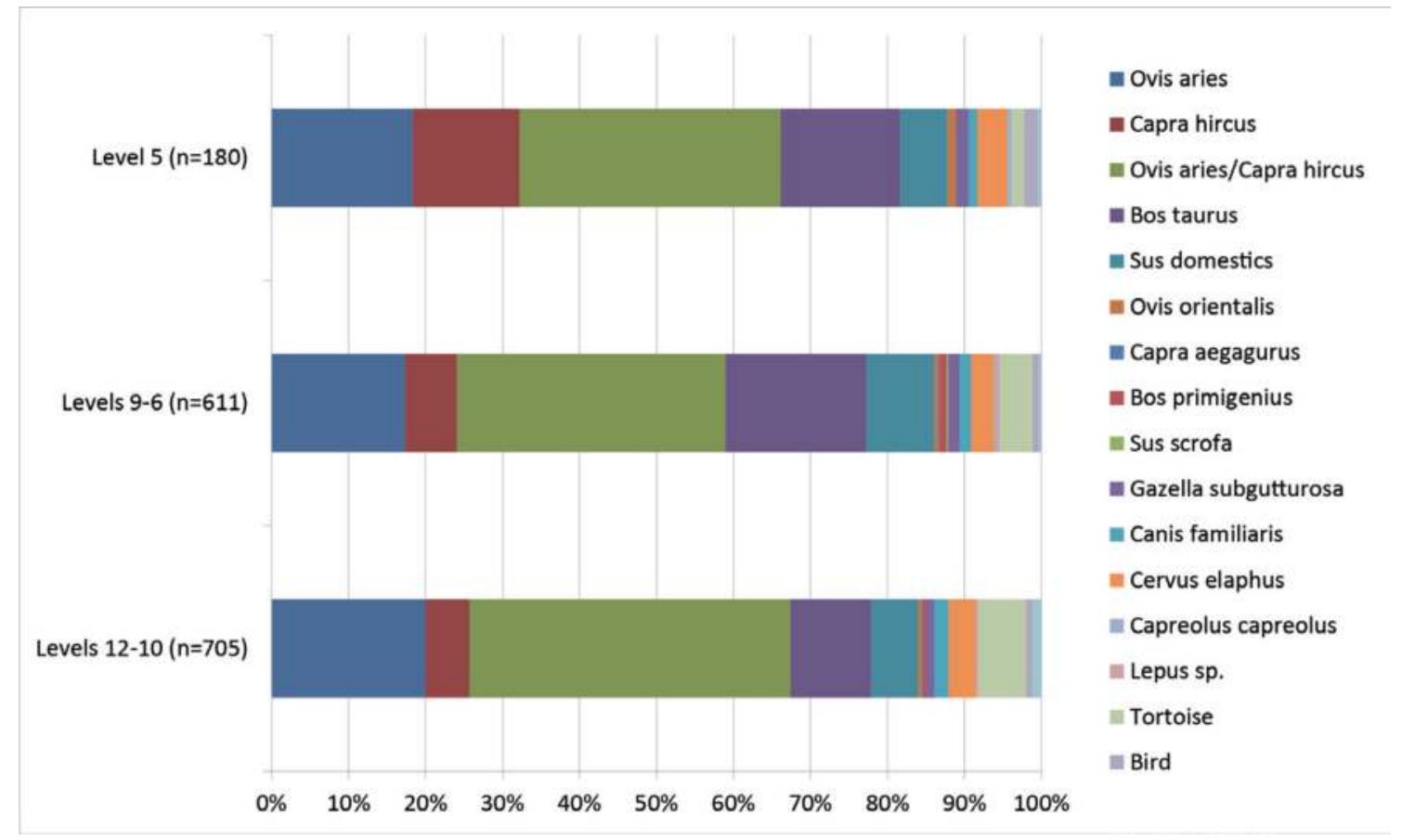

Fig. 17.1 Frequency of faunal remains from Göytepe. 


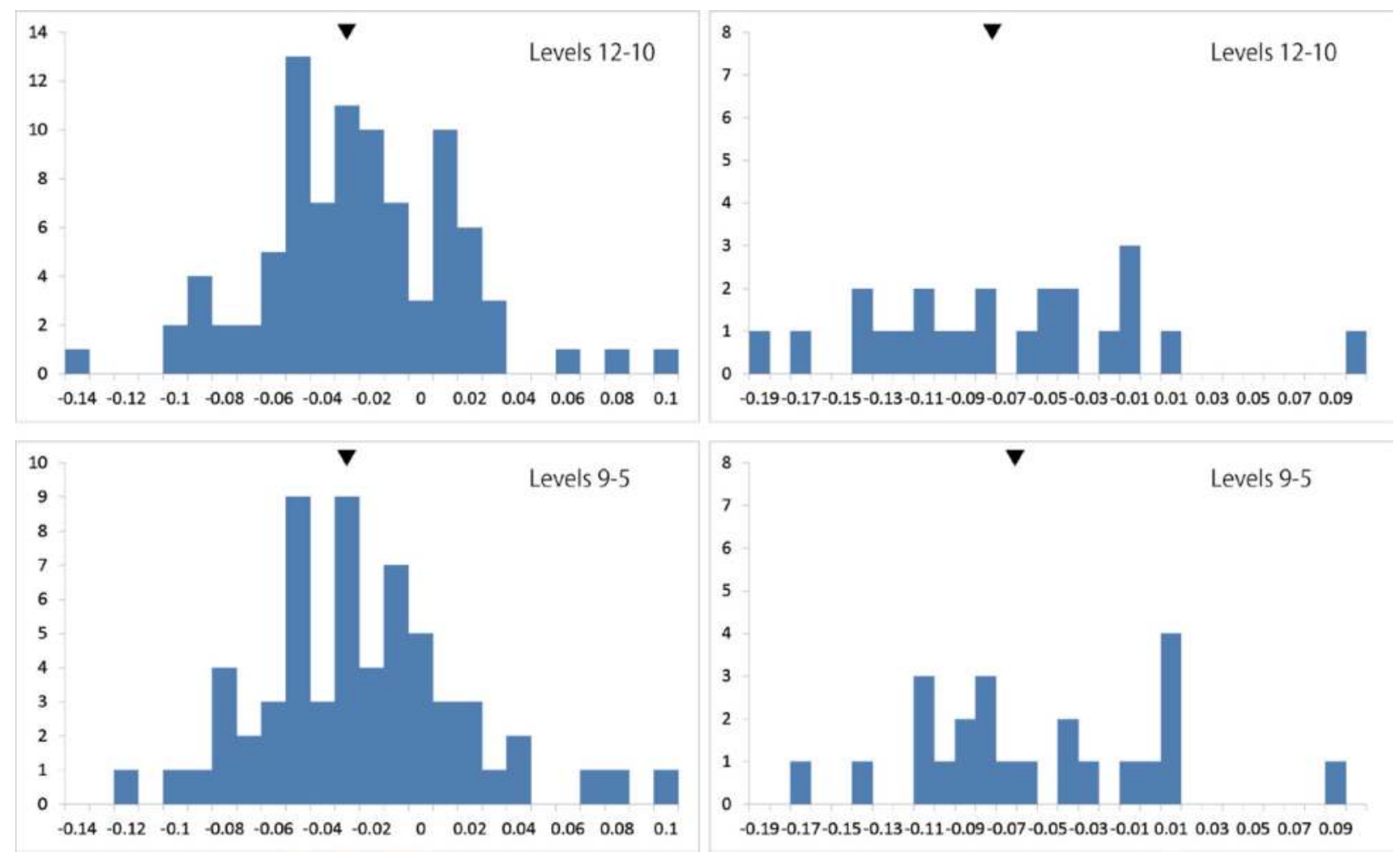

Fig. 17.2 Histogram showing the distribution of LSI values of sheep and goat (left: sheep; right: goat).

specimens of both species are lower in value than the standard in both levels. In the case of sheep, the male/ female border is not obvious. This is partly due to the lower sexual dimorphism of sheep than of goats. Given that two peaks are observed within the ranges from -0.05 to -0.03 and -0.01 to 0.01 respectively, the boundary between the size ranges of both sexes appears to have existed at around -0.02. At the same time, specimens with a value larger than 0.06 appear to have been from a wild population. With respect to the LSI distribution of goats, most specimens are distributed within the range -0.1 (or smaller than the value) to 0.01 . Since the sample size is small, no clear peaks were evident. However, a gap exists around -0.05 in both levels. In any case, extremely large specimens from both levels can be identified as wild. Interestingly, both specimens with large values $(0.09,0.1)$ were worked, possibly from raw materials to be used for implements.

The culling profile of sheep and goats was established for reconstructing past management strategies (Fig. 17.3). The method used here was proposed by Helmer (1995). It combines methods adopted by Ducos (1968) and Payne (1973). In this method, the age at death of each individual is estimated according to indices of each cheek tooth, which are calculated by dividing the dental crown heights by the vestibulo-lingual breadth taken at the collar. Then the specimens are sorted into age classes. Because of the scarcity of samples, specimens from Levels 12-9 were integrated as "lower," and from 8-5 were as "upper." The culling profile established here suggests that sheep and goats had been exploited in different strategies between the two phases. In the lower levels, two peaks were observed at age Classes $\mathrm{A}$ and $\mathrm{C}$, and some individuals survived for over six years. This pattern indicates that they were exploited for their tender meat, and possibly for their milk. However, in upper levels, most individuals were slaughtered at the ages of juvenile and immature classes (Classes C and D), indicating a strategy toward meat production. In any case, it is clear that caprines had been kept primarily for their meat during the occupation of the site. However, the use of fleece is not obvious, although some adult individuals appear to have been kept for their wool. Considering the lack of spindle whorls, and a large number of bone spatulas and obsidian tools used for skinning (see Chapter 15), the main fabric used at Göytepe seems to have been animal skin. 


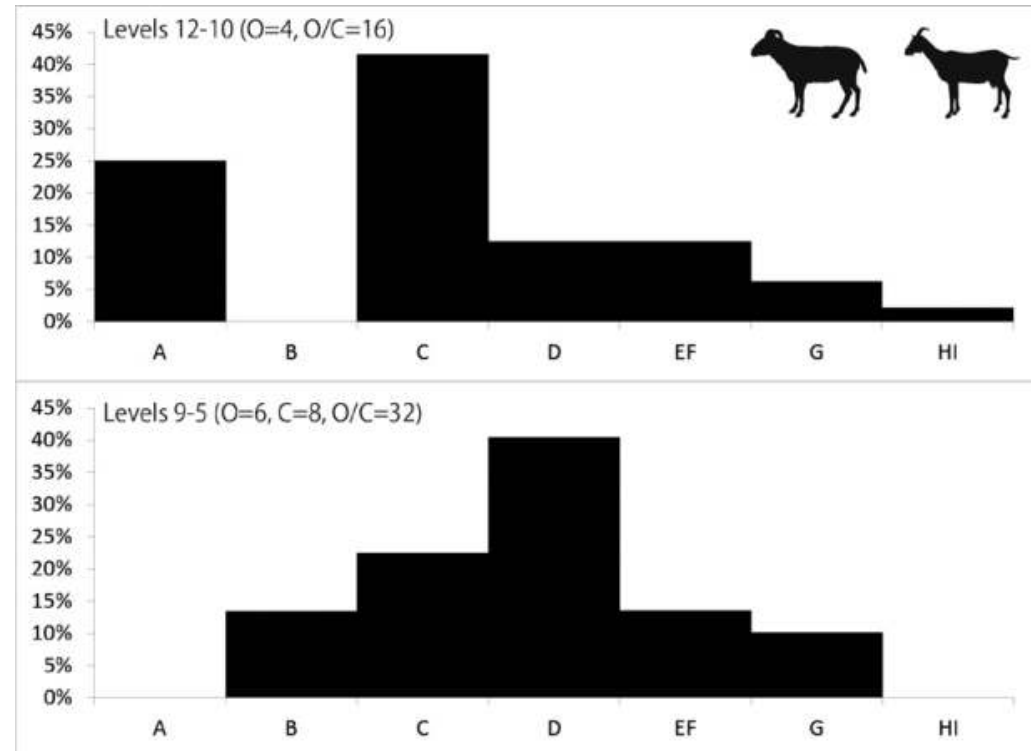

Fig. 17.3 Culling profile of sheep and goat (A: 0-0.2 years; B: 0.2-0.5 years; C: $0.5-1$ years; D: 1-2 years; EF: 2-4 years; G: 5-6 years; $\mathrm{HI}:>6$ years. Age data based on Helmer et al. 2007).

\section{Cattle}

Cattle were the second abundant species based on NISP (ca. 15-20\%), and the most significant based on their weight of identified specimens (WISP) (Table 17.1). This means that cattle were most important for meat provision. At the same time, they were important for their skeletons, which served as the raw materials for various bone implements (see Chapter 15).

Most specimens were identified as domestic. Fig. 17.4 presents the distribution of the LSI values of cattle from Göytepe. Measurements of a female aurochs found at Ullerslev (Denmark) were used here as the standard (for detailed data, see Steppen 2001: Appendix). In both levels, the distributions are clearly biased to the left of the graph (smaller than the standard). Although some possible remains of their wild ancestors (aurochs: Bos primigenius) also appear to be present in the assemblage, they are not significant in number. It is possible that there were also some remains of bison (see Benecke 2012; Uerpmann 1987), although, no such specimens have been identified from their morphological character. It is interesting to note that larger specimens, possibly male, increase in the upper levels. Considering that the entire range of distribution is almost consistent at both phases, it appears to reflect a certain change in the management strategy. The reason for this cannot be determined at present. An increase in males or older populations and the misidentification of different sub-species are both possible explanations.

Unfortunately, survivorship data for cattle is not available. This is because most skeletal elements of cattle were used as raw material for bone tools. Even mandibles were used for manufacturing sickle handles. Thus, there are too small a number of isolated teeth or postcranial elements to establish the culling profile.

Pigs

Pig remains were frequently encountered in the assemblage. Although the frequency varies by level, pigs constantly account for ca. $10 \%$ of mammal remains based on NISP, which is indicating their importance in the subsistence economy. Although pigs can provide only meat, they have the highest reproduction and growth rate among livestock, and their meat has the highest values of fats and calories (Flannery 1969).

Since most specimens had not reached maturity (see below) and only a limited number could be used for measurement, size distribution data for Sus is not available. However one possible set of remains of a wild boar has been identified at Level 7 .

Demographic profiles of domestic pigs in the prehistoric Near East tend to comprise predominantly (ca. 85-100\%) of individuals under two years old (see Price and Arbuckle 2015). The age data from Göytepe shows that they were also significantly biased for young ages (up to ca. 12 months old) (Fig. 17.5). Thus it is clear that they were consumed when they reached a reproductive age. One almost complete skeleton of a fetal pig was found at 4BI, suggesting a winter-early spring occupation of the site (Fig. 17.6). 


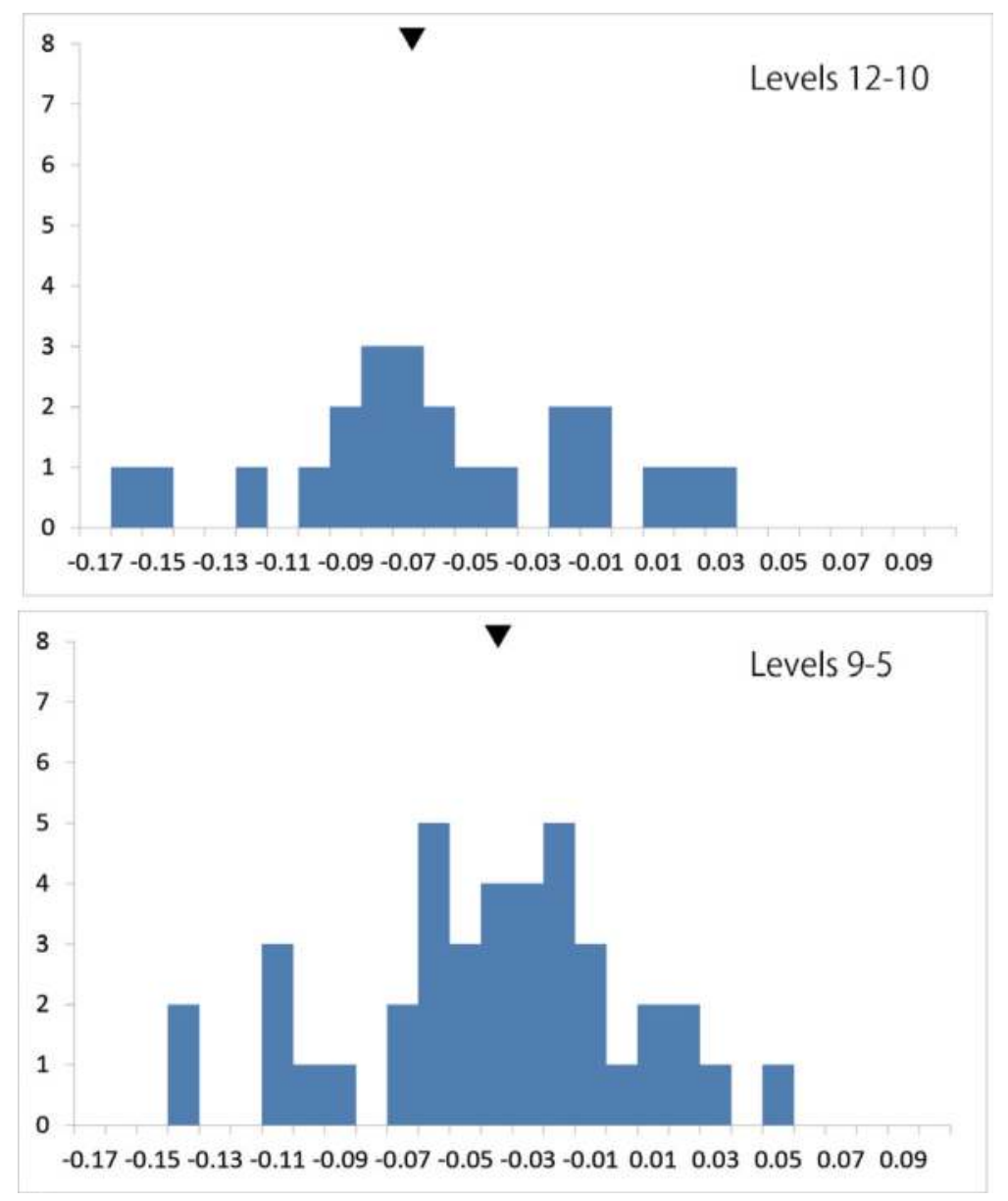

Fig. 17.4 Histogram showing the distribution of LSI values of cattle.

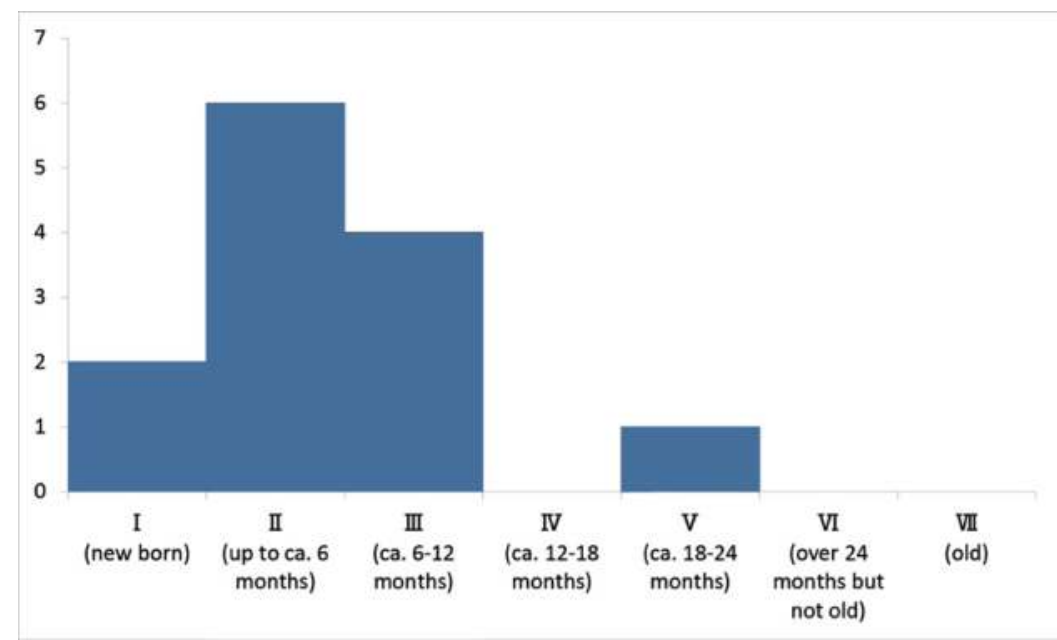

Fig. 17.5 Histogram showing the age composition of pig (Age stage data from Hongo and Meadow 2000).

\section{Dog}

Although the frequency is not abundant, dog remains appear consistently at each level. At the same time, some fragments of bones with bite marks are represented in the assemblage. Thus, it seems that dogs had a close relationship with the inhabitants of the site. At Aratashen, some fragments of dog remains with cut-marks indicating consumption by humans were found (Badalyan et al. 2007: 56). However, there is no evidence of killing-off in the present data. Therefore, it is not clear whether dogs used to be consumed as a meat resource at Göytepe. 


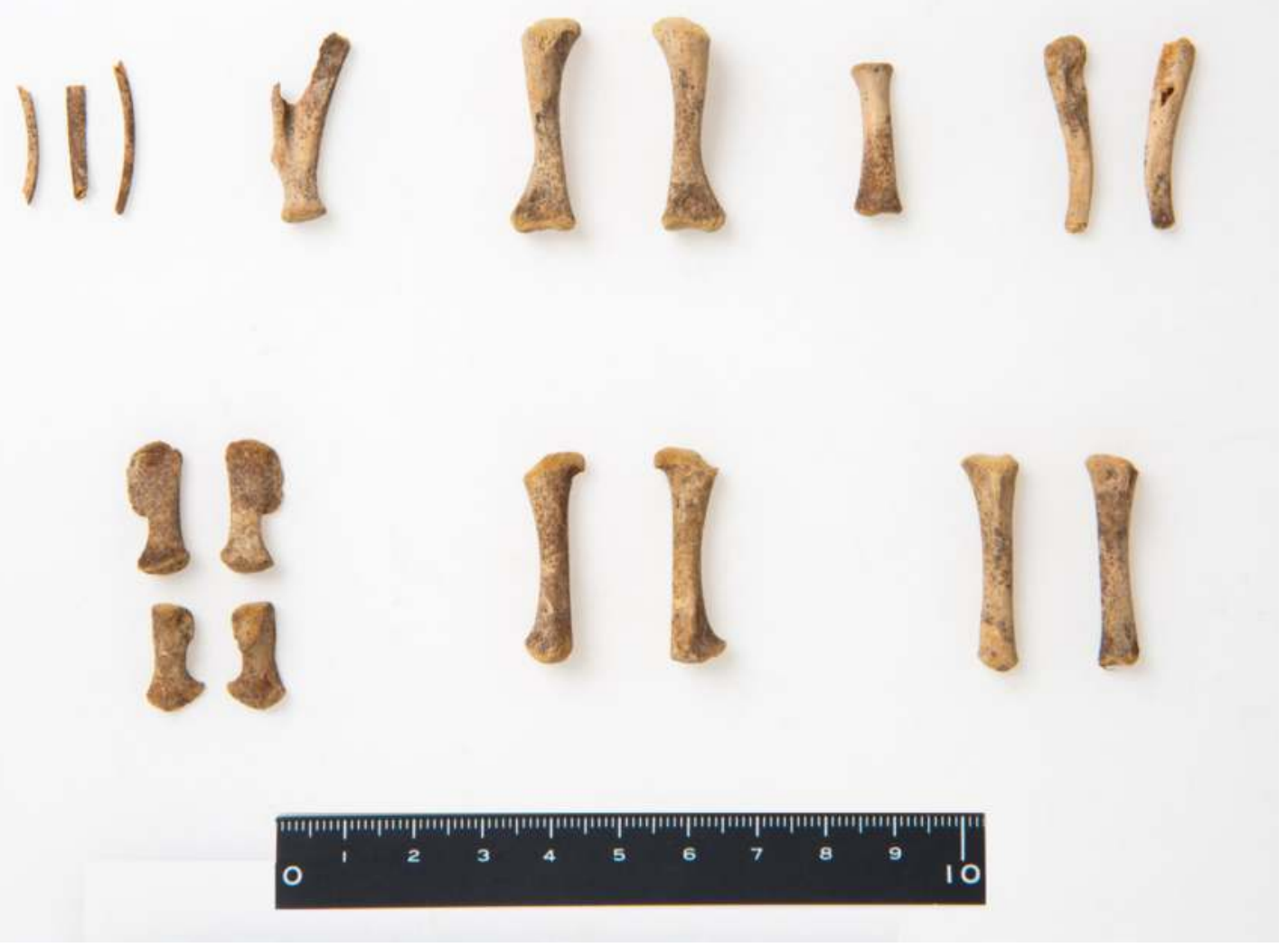

Fig. 17.6 Fetal bones of pig.

\subsubsection{Wild animals}

\section{Game species}

The assemblage of game animals consists mainly of species that inhabit either forest or steppe environments. During the 2009-2011 excavations, the remains of red deer (Cervus elaphus), gazelle (Gazella subgutturosa), roe deer (Capreolus capreolus), aurochs, wild sheep and goats, boar, hare (Lepus sp.), tortoise (Testudo gracea), various birds and fishes have been identified. Besides these, some freshwater mollusks, rodents and frog have been recovered from the site. Mollusks and rodents are particular to the lower levels.

Among them, red deer is known as the most important game through the Neolithic period in the Southern Caucasus. This is probably because the people had exploited the surrounding forest. Their antlers were preferred as raw material for equipment. Various implements made of the antlers have been reported from Neolithic sites in the Middle Kura. Considering that some postcranial elements of red deer have also been found at Göytepe (Fig. 17.7), the inhabitants of the site appear to have not only collected shed antlers, but also hunted the deer. Yet the vertebra and ribs of large-sized ungulates are almost absent in the assemblage. Thus, only selected parts (head and limbs) of the body appear to have been brought into the site. Because of the scarcity of the elements with measurable elements, further information about red deer is not available at present.

Gazelle is the second important hunted species at the site. Both cranial and postcranial elements appear in the assemblage. One grooved horn core from Level 6 and some worked metapodia indicate that gazelle was important not only as a meat resource but also as raw material for implements.

Some postcranial remains of roe deer appear in the assemblage in small numbers. Since roe deer antlers have not been found-as opposed to antlers of red deer-roe deer appears to have been mostly a source of meat. It appears that their antlers were too small to make tools. It is possible that the bias reflects hunting season. In other words, it is possible that the main hunting season for this species was spring. Male deer are known to shed their antlers annually in spring. Thus, if they were hunted in 


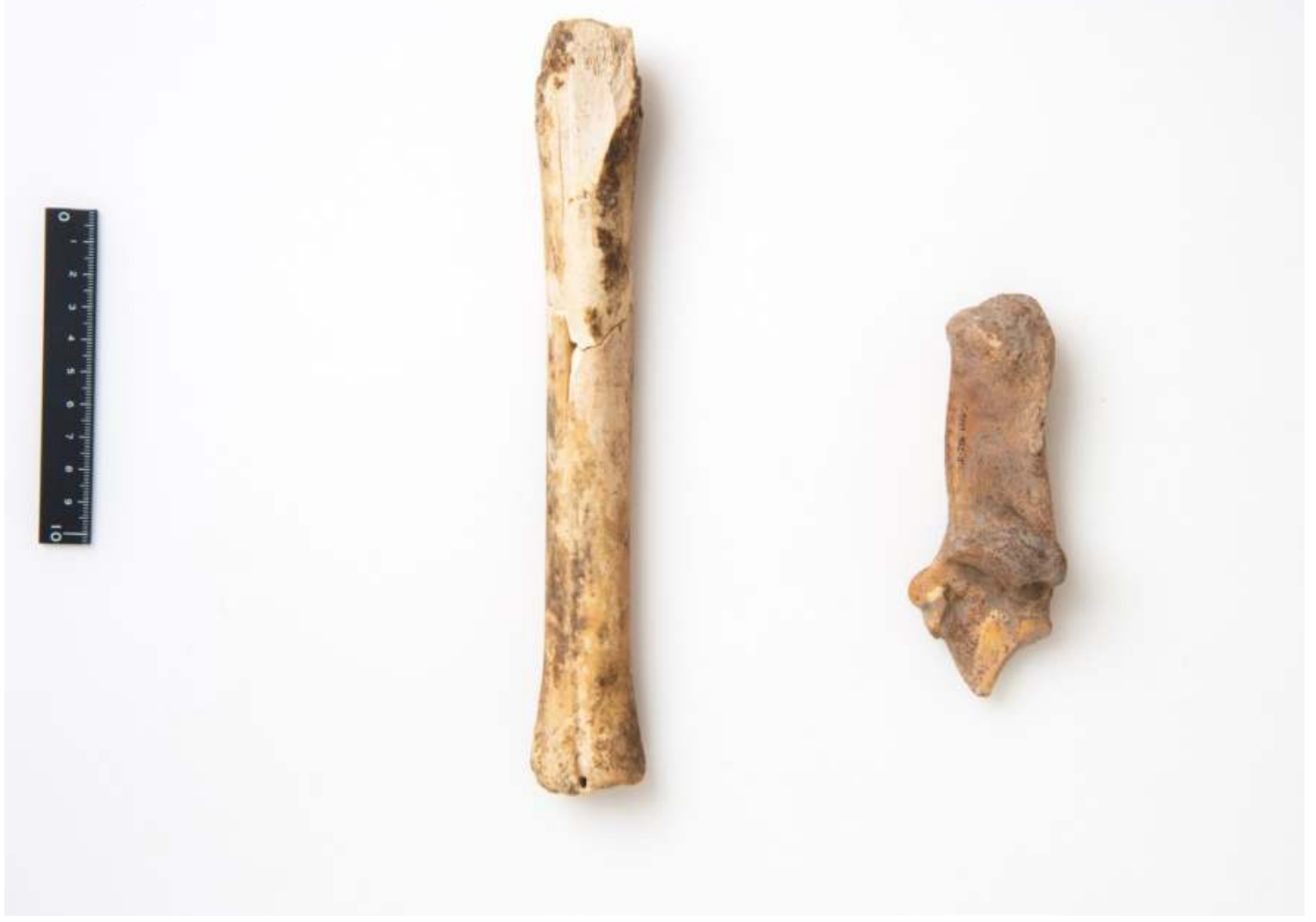

Fig. 17.7 Postcranial remains of red deer (Cervus elaphus).

the summer, antler growth would have been little. Selected hunting of the female of the species would have caused a similar result. An analysis of the male to female roe deer ratio is required, however, as this was not available because of the small sample size.

The remains of small game, such as hare and tortoise, are quite common at the site, especially tortoise. This is partially because of the high frequency of the carapace fragments (although the fragments from the same context were counted as a single specimen). However, the frequency of small game clearly decreased in the upper levels (Fig. 17.8). This may reflect the exhaustion of resources around the site.

\section{Birds}

Thirteen specimens were identified as remains of birds (Fig. 17.9). Although none of them has been identified at a species level, at least three genera (Corvus, Columba, and Tetrax) are represented in the assemblage. These remains include both fore- and hind-limb elements. One ulna of a medium-sized bird is sawn at both proximal and distal epiphysis, demonstrating that it was used as raw material for a bone artifact. Therefore, birds were clearly hunted for various purposes at the site.

Fish

Some fish remains were identified at Göytepe $(n=11)$. They represent the skeletal remains of mandible, crania, fin and vertebra (Fig. 17.10). One vertebra shows a transversal cut mark indicating a technique of butchering. At least two different species of fish have been identified - the Siluriformes and Cyprinidae. The techniques of fishing have not been identified, however, given the estimated size of the fish, they appear to have been hunted with tools like harpoons and spears. Some arrowheads made of bone may be connected to this activity.

\subsection{Neolithic animal exploitation in the Middle Kura valley and the Ararat Plain}

There is only limited information on animal 


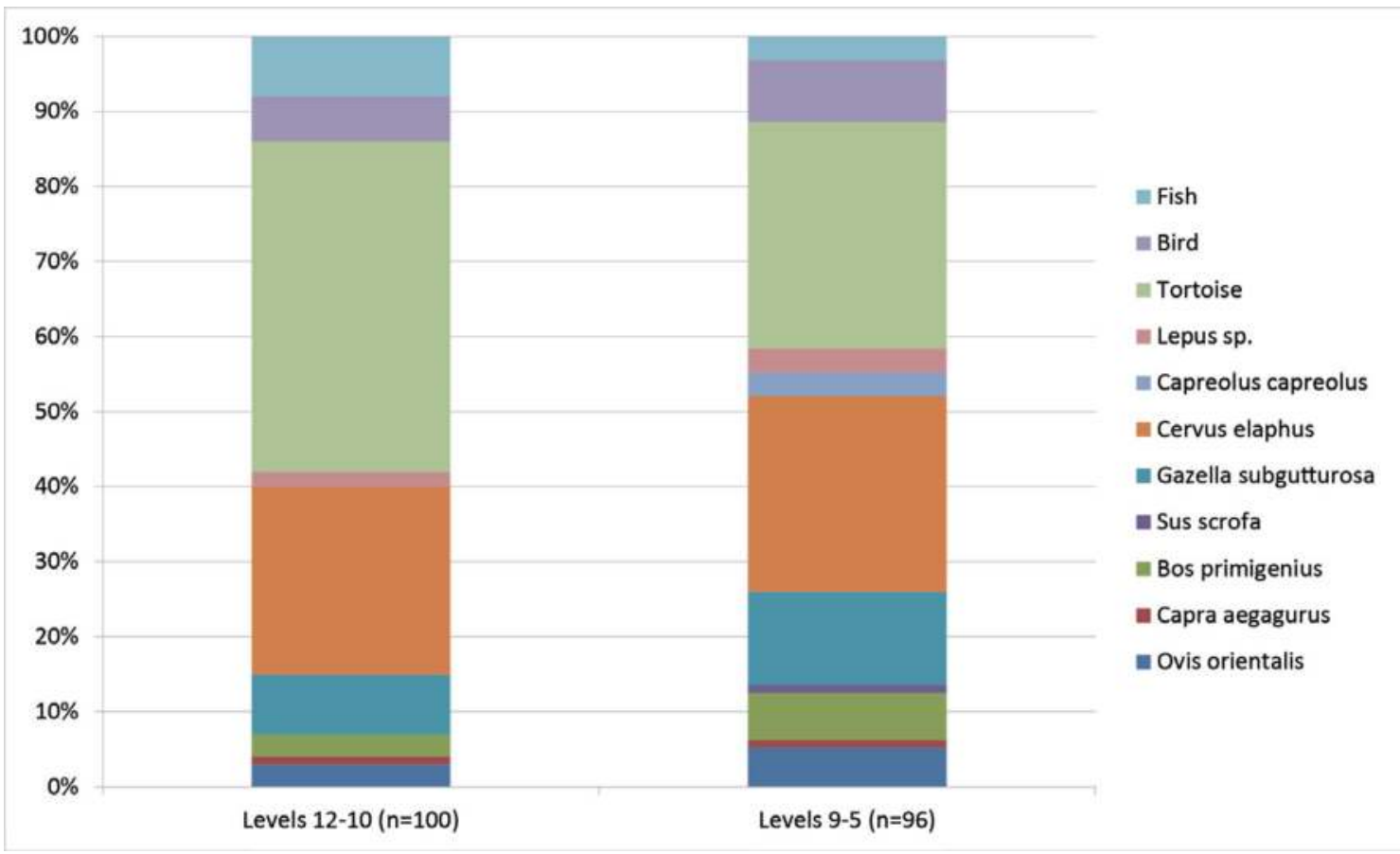

Fig. 17.8 Sequential change in the frequency of game species.
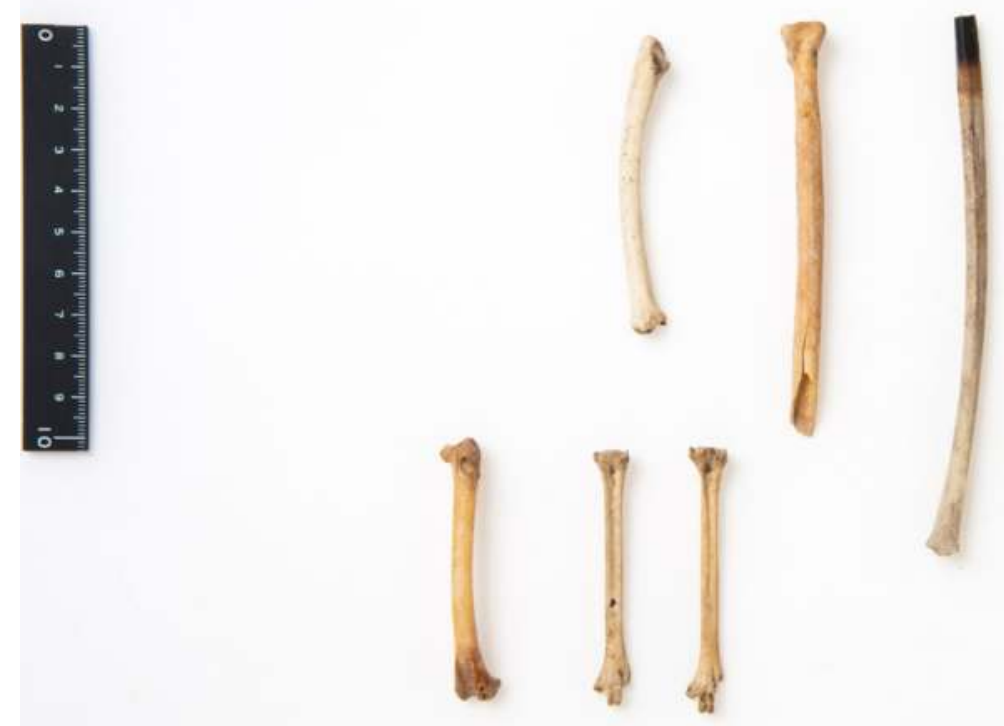

Fig. 17.9 Fore- and hindlimb elements of various birds. 


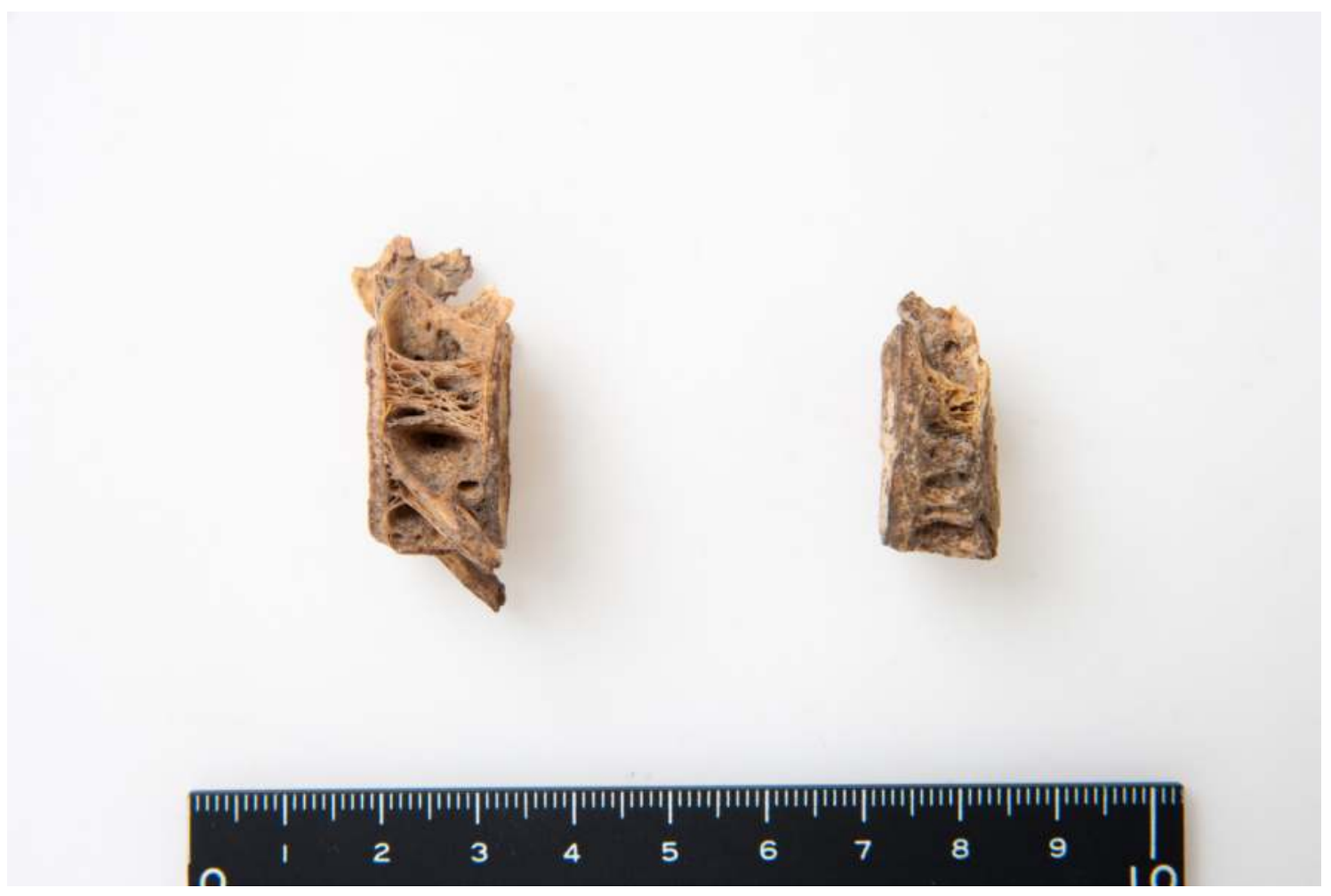

Fig. 17.10 Vertebrae of freshwater fish.

exploitation strategies in the Southern Caucasus during the Neolithic period. However, we can now compare the results of analyses from Göytepe with other contemporary sites, and summarize animal exploitation strategies during the period.

To begin with, the frequencies of animal species from each site in the Middle Kura are very consistent. Therefore, it is suggested that similar strategies had been adopted at Göytepe, Aruchlo (Benecke 2012), and other contemporary sites summarized by Chataigner (1995). At these sites, sheep and goats are predominant, accounting for ca. 50-60\% of the total faunal remains. They were kept primarily for their meats, and possibly for their milk, but not intensively. Sheep are often more frequent than goats, although the frequency of goats increased in the upper levels at Göytepe. Similar trends have been observed at contemporary sites in the Ararat Plain. At Aratashen, the ratio of sheep to goats is 4:1 in the lowest level (IId). However, the ratio changed to 2:1 in the upper level (IIa) (Badalyan et al. 2007). Balasescu et al. (2010) note that sheep and goats were kept primarily for meat based on their mortality pattern. It is therefore suggested that these sites had shared a common subsistence strategy in spite of their geographical difference. Cattle are the other important animal, accounting for ca. 30\% of faunal remains at each site. At Aratashen, the frequency of cattle had increased from the beginning of the occupation, and reached ca. $20 \%$ of the assemblage in the latest level (Badalyan et al. 2007). The most notable difference between the two regions is the frequency of pig remains. In the Ararat Plain, pigs are rarely found, whilst in the Middle Kura pig remains comprise $5-15 \%$ of the total fauna. The difference may be related to the environments and subsistence economies in both regions.

The inventories of wild fauna are diverse, suggesting their significance in the subsistence economies. It is also suggested that diverse environments were exploited by the occupants. In all the occupation levels, the main hunted species is red deer. They were hunted not only for their meat but also for their antlers. At present, the seasons for the hunting of each species are not clear. Further research is required to reconstruct a definite seasonality of the site.

\section{References}

Badalyan, R., P. Lombard, P. Avetisyan, C. Chataigner, J. Chabot, E. Vila, R. Hovsepyan, G. Willcox, and H. 
Pessin (2007) New data on the late prehistory of the southern Caucasus: the excavations at Aratashen (Armenia) - Preliminary report. In: Les Cultures du Caucase (Vle-Ille Millénaires avant notre ère): Leur Relations avec le Proche-Orient, edited by B. Lyonnet, pp. 37-61. Paris: Éditions Recherche sur les Civilisations.

Badalyan, R. S., A. A. Harutyunyan, C. Chataigner, F. Le Mort, J. Chabot, J. -E. Brochier, A. Balalescu, V. Radu, and R. Hovsepyan (2010) The settlement of Aknashen-Khatunarkh. A Neolithic site in the Ararat plain (Armenia): Excavation results (2004-2009). Turkiye Bilimler Akademisi Arkeoloji Dergisi (TÜBAAR), 13: 185-218.

Balasescu, A., E. Vila, V. Radu, R. Badalyan, and C. Chataigner (2010) Production animale et économie de subsistence au néolithique dans la plaine de l'Ararat (Arménie). Annales d'Université "Valahia" Tâgovişte. Section d'Archéologie et d'Histoire Tome, 12(1): 25-38.

Benecke, R. (2012) Archaeozoological studies. In: Ancient Kura 2010-2011: The First Two Seasons of Joint Field Work in the Southern Caucasus, Archäologische Mitteilungen aus Iran und Turan 44, edited by B. Lyonnet, F. Guliev, B. Helwing, T. Aliyev, S. Hansen, and G. Mirtskhulava, pp. 154-155.

Chataigner, C. (1995) La Transcaucasie au Néolithique et au Chalcolithique. BAR Internatinal Series 624. Oxford: Tempus Reparatum.

Ducos, P. (1968) L'Origine des Animaux Domestiques en Palestine. Bordeaux: Institut de Préhistoire de l'Université.

Flannery, K. (1969) Origins and ecological effects of early domestication in Iran and the Near East. In: The Domestication and Exploitation of Plants and Animal, edited by P. J. Ucko and G. W. Dimbleby, pp. 73-100. Chicago: Aldine.

Helmer, D., L. Gourichon, and E. Vila (2007) The development of the exploitation of products from Capra and Ovis (meat, milk and fleece) from the PPNB to the Early Bronze in the northern Near East (8700 to 2000 BC Cal.). Anthropozoologica, 42(2): 41-69.

Meadow, R. H. (1981) Early animal domestication in South Asia: a first report of the faunal remains from Mehrgarh, Pakistan. In: South Asian Archaeology 1979, edited by H. Hartel, pp. 143-179. Berlin: Dietrich Reimer.

Meadow, R. H. (1999) The use of size index scaling techniques for research on archaeozoological collection from the Middle East. In: Historia Animalium ex Ossibus: Beiträge auf Paläoanatomie, Archäologie, Ägyptologie, Ethnologie und Geschichte der Tiermedizin, edited by C. Becker, $\mathrm{H}$. Manhart, J. Peters, and J. Schibler, pp. 285-300. Rahden/Westf: Verlag Marie Leidorf.
Payne, S. (1973) Kill-off patterns in sheep and goats: the mandibles from Aşvan kale. Anatolian Studies, 23: 281-303.

Peters, J., A. Von den Driesch, and M. Saña Segui (1999) Early animal husbandry in the northern Levant. Paléorient, 25(2): 27-47.

Peters, J., A. Von den Driesch, and D. Helmer (2005) The Upper Tigris-Euphrates basin: Cradle of agro-pastoralism? In: The First Steps of Animal Domestication. Proceedings of the $9^{\text {th }}$ International Council of Archaeozoology (ICAZ, Durham, 2002), edited by J. -D. Vigne, J. Peters, and D. Helmer, pp. 96-123. Oxford: Oxbowbooks.

Price, M. D. and B. S. Arbuckle (2015) Early pig management in the Zagros flanks: Reanalysis of the fauna from Neolithic Jarmo, northern Iraq. International Journal of Osteoarchaeology, 25(4): 441-453.

Steppen, K. (2001) Ur oder Hausrind? Die variablität der wildtieranteile in Linearbandkeramischen tierknochenkomplexen. In: Rôlle et statut de la chasse dans le Néolithique ancien danubien (5500-4900 av. J.-C.), edited by R.-M. Arbogast, C. Jeunesse, and H. Schibler, pp. 171-186. Rahden/ Westf: Verlag Marie Leidorf $\mathrm{GmbH}$.

Uerpmann, H.-P. (1987) The Ancient Distribution of Ungulate Mammals in the Middle East: Fauna and Archaeological Sites in Southwester Asia and Northeast Africa. Beihefie zum Tübinger Atlas des Vorderen Orients Reiche A (Naturwissenschaften 27). Wiesbaden Dr: Ludwig Reichert Verlag.

Uerpmann, H.-P. and M. Uerpmann 1994 Animal bone finds from excavation 520 at Qala'at al-Bahrain. In: Qala'at al-Bahrain I. The Northern City Wall and the Isramic Fortress, edited by F. Höljund and $\mathrm{H}$. H. Andersen, pp. 417-444. Aahus: Jutland Archaeological Society. 


\section{Appendix}

Table A17.1 Measurements of Ovis sp.

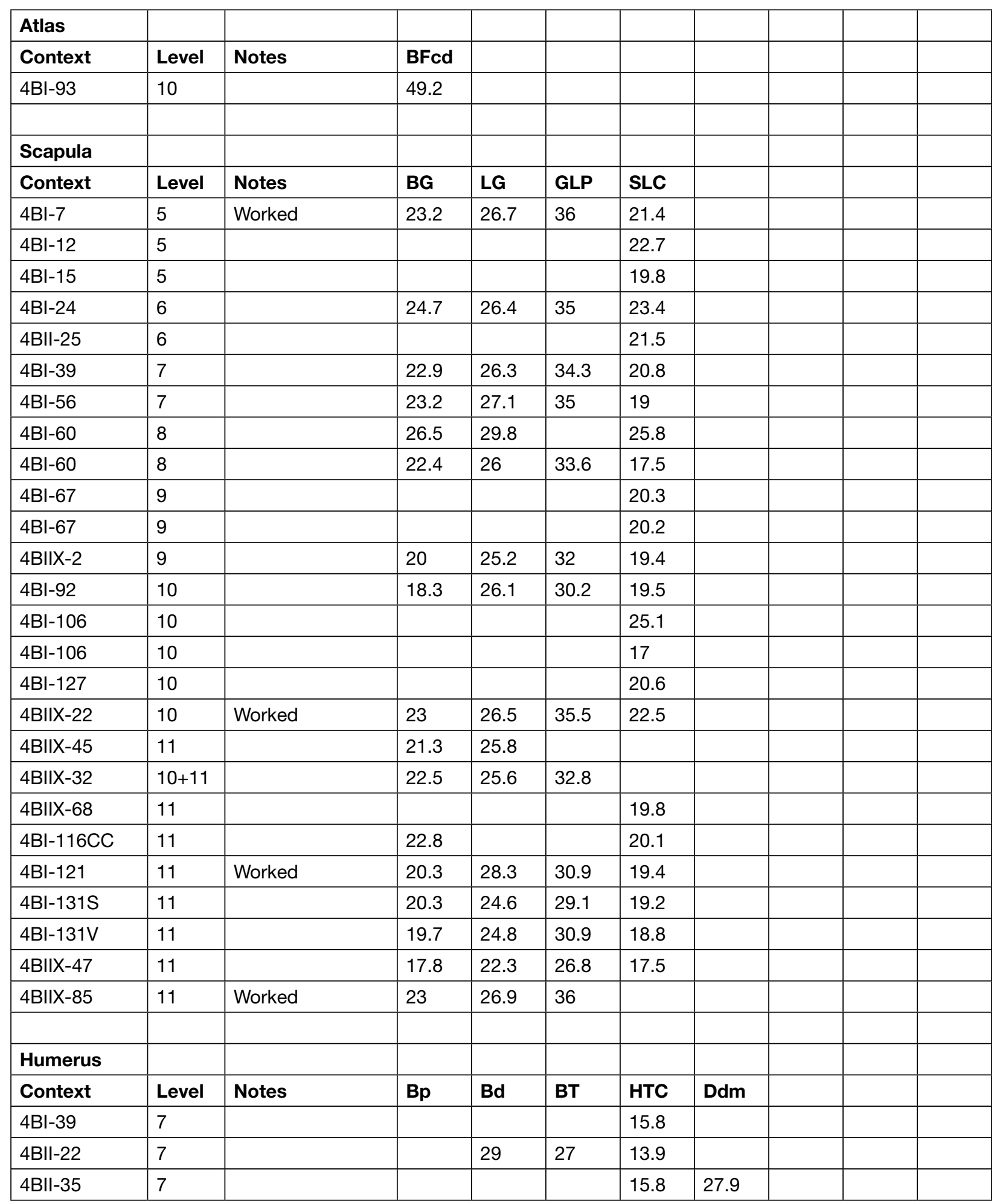


Table A17.1 Continued.

\begin{tabular}{|c|c|c|c|c|c|c|c|c|c|c|}
\hline 4BI-60 & 8 & & & 25.9 & 25.2 & 11.7 & 21.4 & & & \\
\hline 4BI-71 & 9 & & & 29.2 & 28 & 13.8 & 26.8 & & & \\
\hline 4BI-93 & 10 & Burnt & & & 26.9 & 14.2 & & & & \\
\hline 4BI-93 & 10 & & & 31.2 & 30.3 & 15.5 & 28.2 & & & \\
\hline $4 \mathrm{BI}-93$ & 10 & & & 29.5 & 29 & 14.8 & 26.9 & & & \\
\hline 4BI-97 & 10 & & & & & 15 & & & & \\
\hline $4 \mathrm{BI}-97$ & 10 & & & & & 14 & & & & \\
\hline 4BI-106 & 10 & & & & 31.1 & 15.2 & & & & \\
\hline $4 \mathrm{BI}-120$ & 10 & & & 30.5 & 30 & 15.2 & 28.7 & & & \\
\hline $4 \mathrm{BI}-113 \mathrm{~N}$ & 11 & & & 28.6 & 27.4 & 12.9 & 26 & & & \\
\hline $4 \mathrm{BI}-115 \mathrm{H}$ & 11 & Burnt & & 27.1 & 25.6 & 13.2 & & & & \\
\hline 4BI-128U & 11 & & & 29.4 & 29 & 14 & 27.5 & & & \\
\hline 4BIIX-35 & 11 & & 47.1 & & & & & & & \\
\hline 4BIIX-64 & 11 & & & 30.5 & 27.4 & 15.7 & 27.3 & & & \\
\hline \multicolumn{11}{|l|}{ Radius } \\
\hline Context & Level & Notes & $\mathrm{Bp}$ & BFp & $\mathrm{Dp}$ & SD & Bd & BFd & Dd & GL \\
\hline $4 \mathrm{BI}-60$ & 8 & Unfused & & & & & 30.1 & 24.6 & 19.2 & \\
\hline 4BIIX-44 & 10 & Unfused & & & & & 27.9 & & 18.1 & \\
\hline $4 \mathrm{BI}-93$ & 10 & & & & 15.6 & & & & & \\
\hline $4 \mathrm{BI}-106$ & 10 & & 32.7 & 30.3 & 16.2 & & & & & \\
\hline $4 \mathrm{BI}-127$ & 10 & Fusing & 30.3 & 27.6 & 16.2 & & & & & \\
\hline $4 \mathrm{BI}-113 \mathrm{G}$ & 11 & & 27.4 & 26.5 & & 17.9 & & & & \\
\hline 4BI-114BB & 11 & Dist. Unfused & 33.1 & 30.9 & 18.2 & 17.3 & 31.8 & 27 & 21 & 171 \\
\hline 4BI-128KK & 11 & Unfused & & & & & 26.8 & & 17.3 & \\
\hline 4BI-131FF & 11 & Dist. Unfused & & & & & 29.5 & 22.5 & 19.7 & 15.3 \\
\hline 4BI-131FF & 11 & Unfused & & & & 19.4 & 29 & 24.4 & 18.7 & \\
\hline 4BI-131FF & 11 & Dist. Unfused & 34.7 & 31.8 & 17.9 & 17.6 & 32.2 & 26.6 & 22.6 & 160 \\
\hline 4BI-131KK & 11 & Dist. Unfused & 34.4 & 31.4 & 18.3 & 18.8 & 30.5 & 24.9 & 20.2 & 176 \\
\hline 4BIIX-57 & 11 & Burnt & & & & & 30.2 & & & \\
\hline \multicolumn{11}{|l|}{ Ulna } \\
\hline Context & Level & Notes & DPA & SDO & LO & BPC & & & & \\
\hline $4 \mathrm{BI}-6$ & 5 & & 23.5 & 19.6 & 40.1 & & & & & \\
\hline 4BI-39 & 7 & Unfused & 25.7 & 21.2 & & 19.6 & & & & \\
\hline 4BII-22 & 7 & Unfused & 26.5 & 22.2 & & 17.6 & & & & \\
\hline $4 \mathrm{BI}-9$ & 9 & & 22.5 & 19.4 & & & & & & \\
\hline 4BI-114BB & 11 & Unfused & 26.1 & 22.1 & 43.7 & 20 & & & & \\
\hline 4BI-117X & 11 & & 25.3 & 21.1 & & 17.3 & & & & \\
\hline 4BI-131FF & 11 & Unfused & 28.3 & 24.7 & & 19.5 & & & & \\
\hline 4BI-131KK & 11 & Unfused & 29.5 & 25.5 & & & & & & \\
\hline 4BIIX-24 & 11 & Unfused & 27 & 22.9 & & & & & & \\
\hline 4BIIX-64 & 11 & & 19.6 & 23.6 & & 14.7 & & & & \\
\hline & & & & & & & & & & \\
\hline
\end{tabular}


Table A17.1 Continued.

\begin{tabular}{|c|c|c|c|c|c|c|c|c|c|c|}
\hline Metacarpal & & & & & & & & & & \\
\hline Context & Level & Notes & $\mathrm{Bp}$ & Dp & SD & Bd & Dd & GL & & \\
\hline $4 \mathrm{BI}-53$ & 6 & & & & & & 17 & & & \\
\hline 4BII-2 & 6 & & 21.9 & 15.3 & 13.3 & & & & & \\
\hline $4 \mathrm{BI}-44$ & 7 & & 22.1 & 15.9 & & & & & & \\
\hline 4BII-16 & 7 & Worked & & & & & 15.6 & & & \\
\hline $4 \mathrm{BI}-60$ & 8 & & 20.8 & 14.3 & & & & & & \\
\hline $4 \mathrm{BI}-73$ & 9 & & 20.9 & 15.1 & & & & & & \\
\hline $4 \mathrm{BI}-88$ & 10 & & & & & 23.7 & 15.9 & & & \\
\hline 4BI-106 & 10 & & & & & 24.7 & & & & \\
\hline $4 \mathrm{BI}-114 \mathrm{CC}$ & 11 & Unfused & 20.7 & 14.7 & 11.6 & 22.8 & 15.1 & 111.5 & & \\
\hline 4BI-131KK & 11 & & 24 & 17.7 & 16 & 28.1 & 17.1 & 146.3 & & \\
\hline 4BI-131KK & 11 & Unfused & 21.4 & 15.1 & 13.7 & 24.8 & 14.6 & 133.1 & & \\
\hline 4BI-131KK & 11 & Unfused & & & & 24.5 & 16.1 & & & \\
\hline 4BI-131KK & 11 & Unfused & & & & 23.4 & 15.2 & & & \\
\hline 4BI-133EE & 11 & Unfused & & & & 26.3 & 16.3 & & & \\
\hline 4BIIX-64 & 11 & & 23.9 & 18 & 16.3 & & & 146 & & \\
\hline \multicolumn{11}{|l|}{ Pelvis } \\
\hline Context & Level & Notes & LA & & & & & & & \\
\hline $4 \mathrm{BI}-17$ & 5 & & 32.5 & & & & & & & \\
\hline 4BII-27 & 7 & & 30.9 & & & & & & & \\
\hline \multicolumn{11}{|l|}{ Femur } \\
\hline Context & Level & Notes & $\mathrm{Bp}$ & DC & & & & & & \\
\hline $4 \mathrm{BI}-50$ & 7 & & & 19.4 & & & & & & \\
\hline $4 \mathrm{BI}-60$ & 8 & & 38 & 18.9 & & & & & & \\
\hline $4 \mathrm{BI}-133 \mathrm{~S}$ & 11 & & & 21 & & & & & & \\
\hline 4BIIX-91 & 11 & & & 20.3 & & & & & & \\
\hline \multicolumn{11}{|l|}{ Tibia } \\
\hline Context & Level & Notes & SD & Bd & & & & & & \\
\hline $4 \mathrm{BI}-8$ & 5 & & 15.4 & 25.4 & & & & & & \\
\hline $4 \mathrm{BI}-17$ & 5 & & & 26.5 & & & & & & \\
\hline $4 \mathrm{BI}-44$ & 7 & Unfused & & 26.5 & & & & & & \\
\hline 4BII-27 & 7 & Worked & 13.8 & 25.9 & & & & & & \\
\hline $4 \mathrm{BI}-60$ & 8 & & & 23.7 & & & & & & \\
\hline 4BIIX-10 & 9 & & & 25 & & & & & & \\
\hline $4 \mathrm{BI}-97$ & 10 & & & 28.2 & & & & & & \\
\hline $4 \mathrm{BI}-131 \mathrm{KK}$ & 11 & Fusing & 14.2 & 26.8 & & & & & & \\
\hline 4BI-131KK & 11 & & 15.6 & 27.2 & & & & & & \\
\hline 4BI-131KK & 11 & Fusing, Worked & 15.4 & 27.1 & & & & & & \\
\hline 4BI-131P & 11 & Unfused & & 25.5 & & & & & & \\
\hline & & & & & & & & & & \\
\hline
\end{tabular}


Table A17.1 Continued.

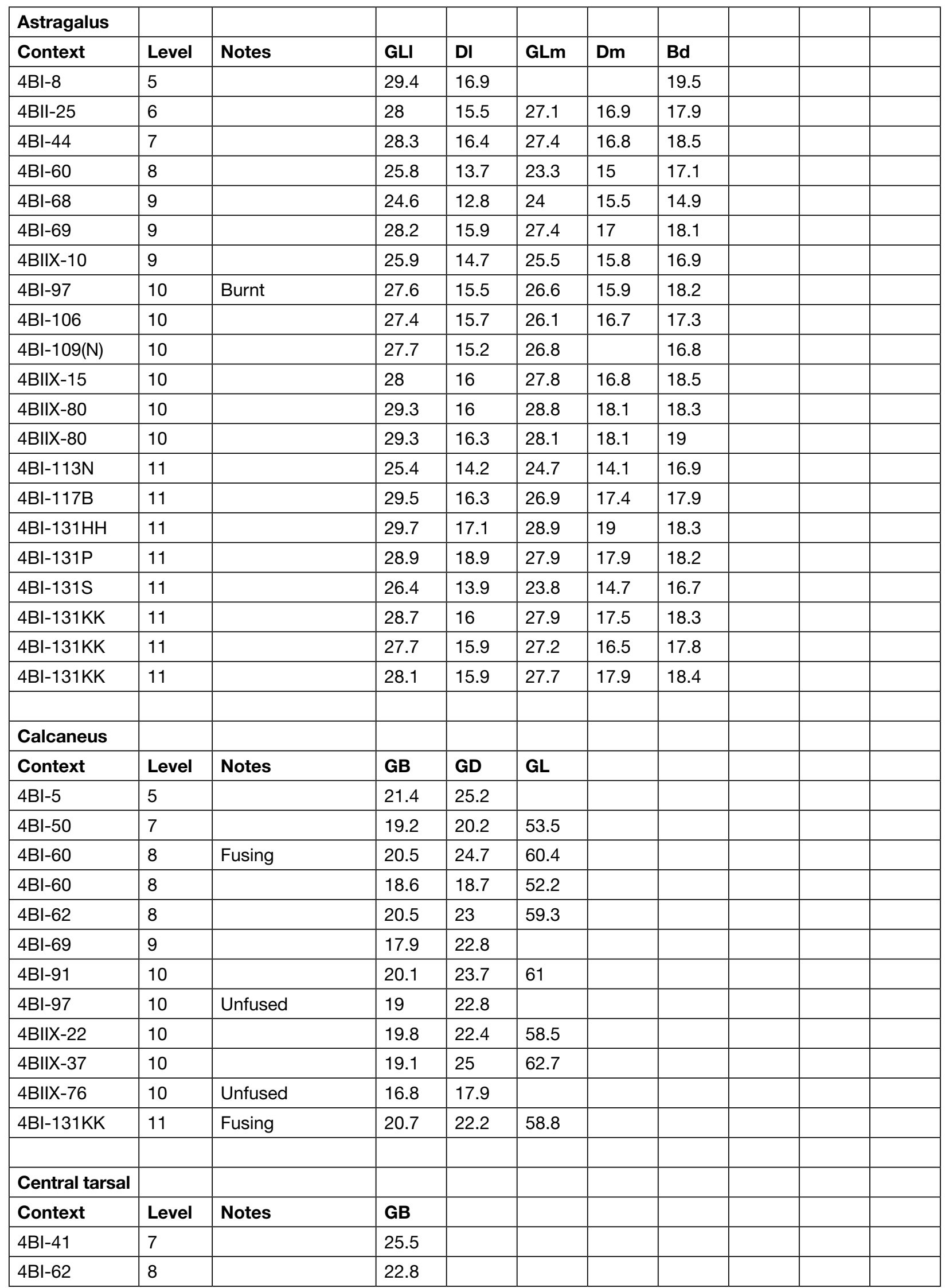


Table A17.1 Continued.

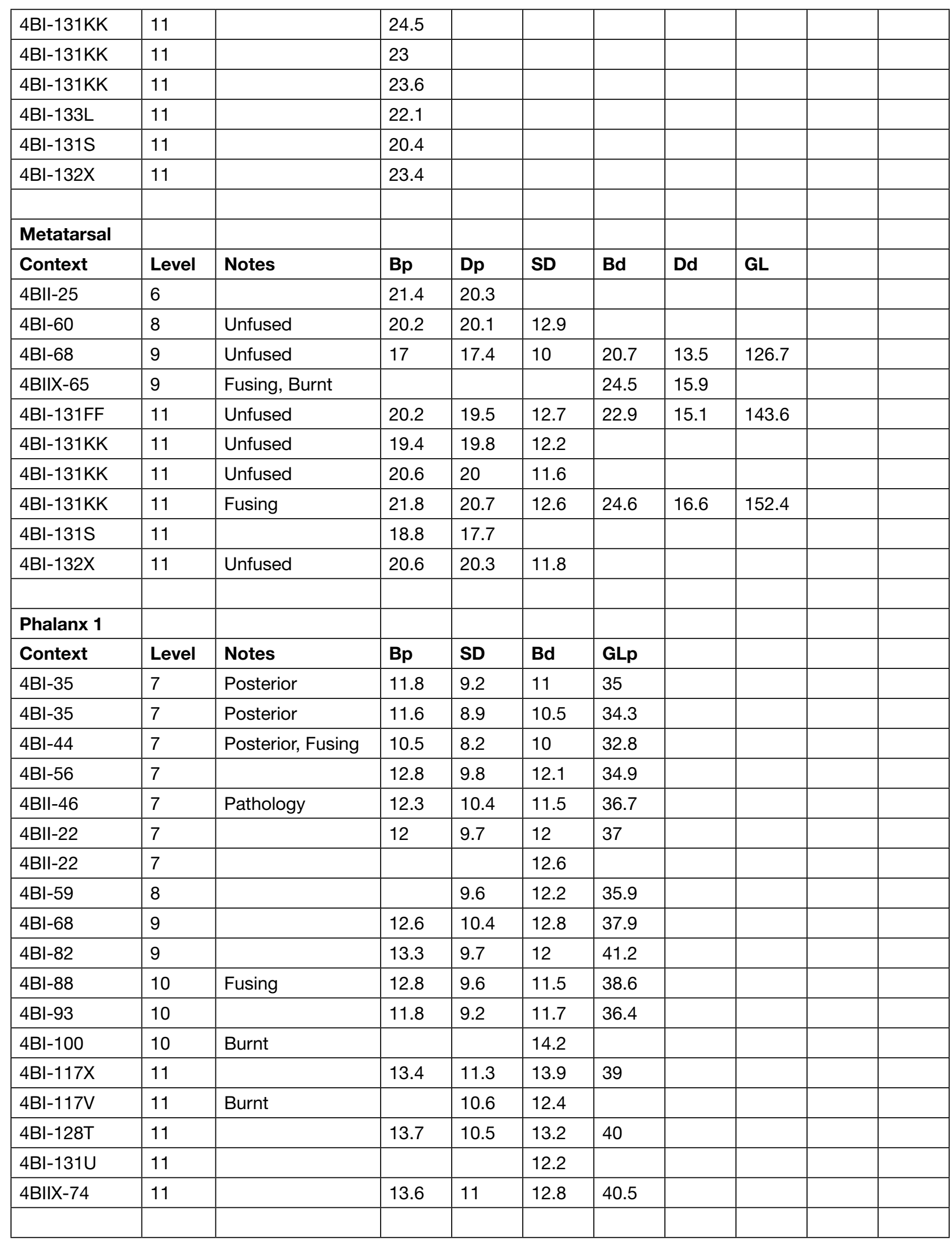


Table A17.1 Continued.

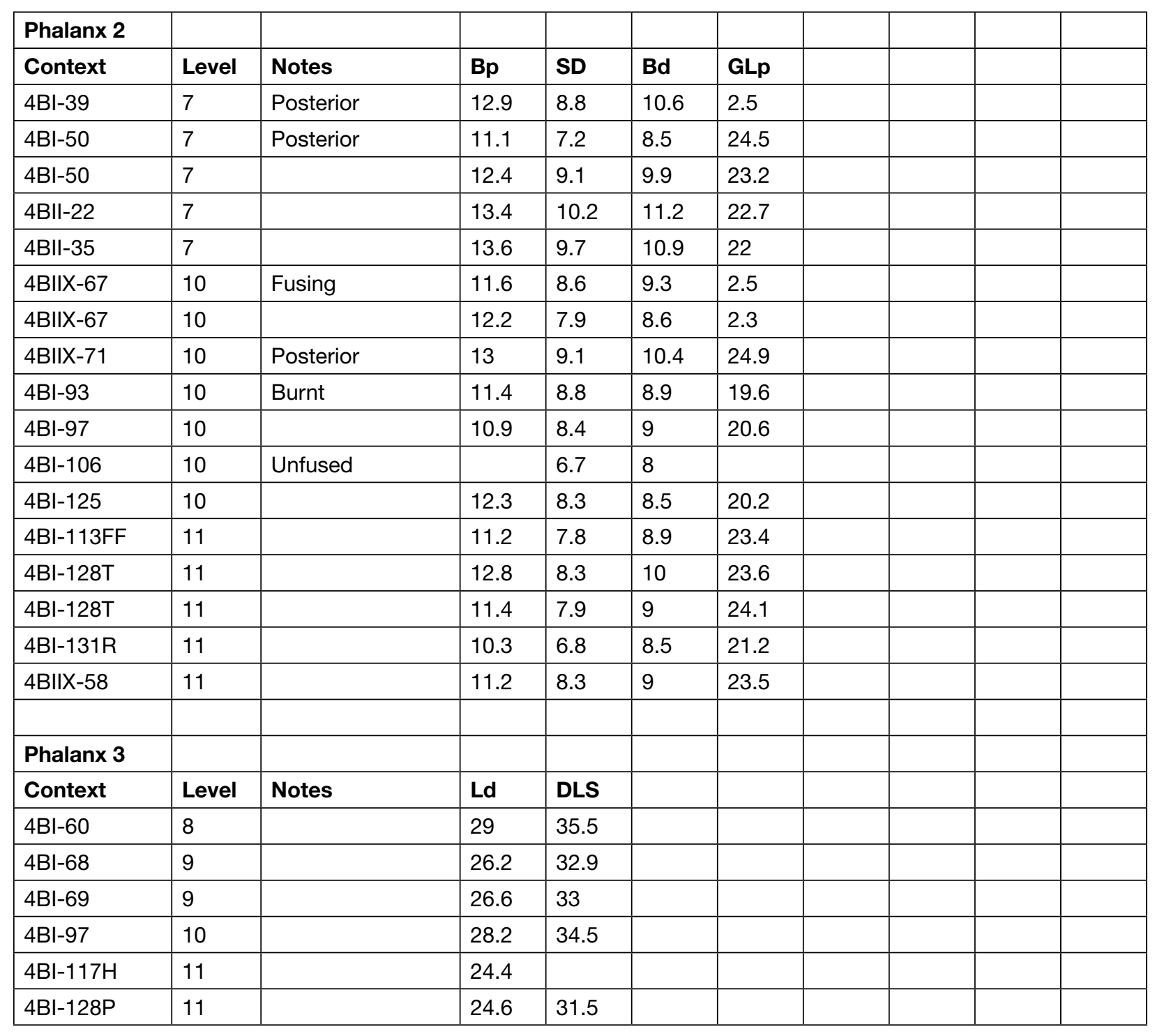

Table A17.2 Measurements of Capra sp.

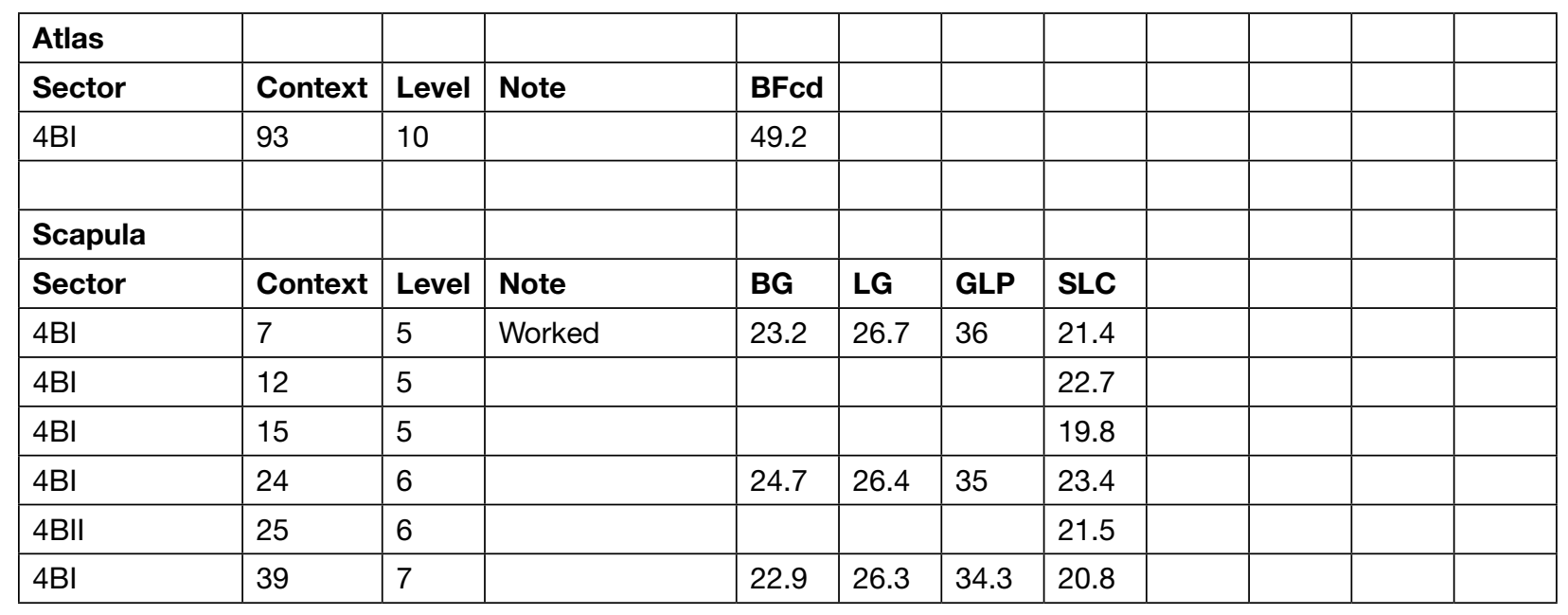


Table A17.2 Continued.

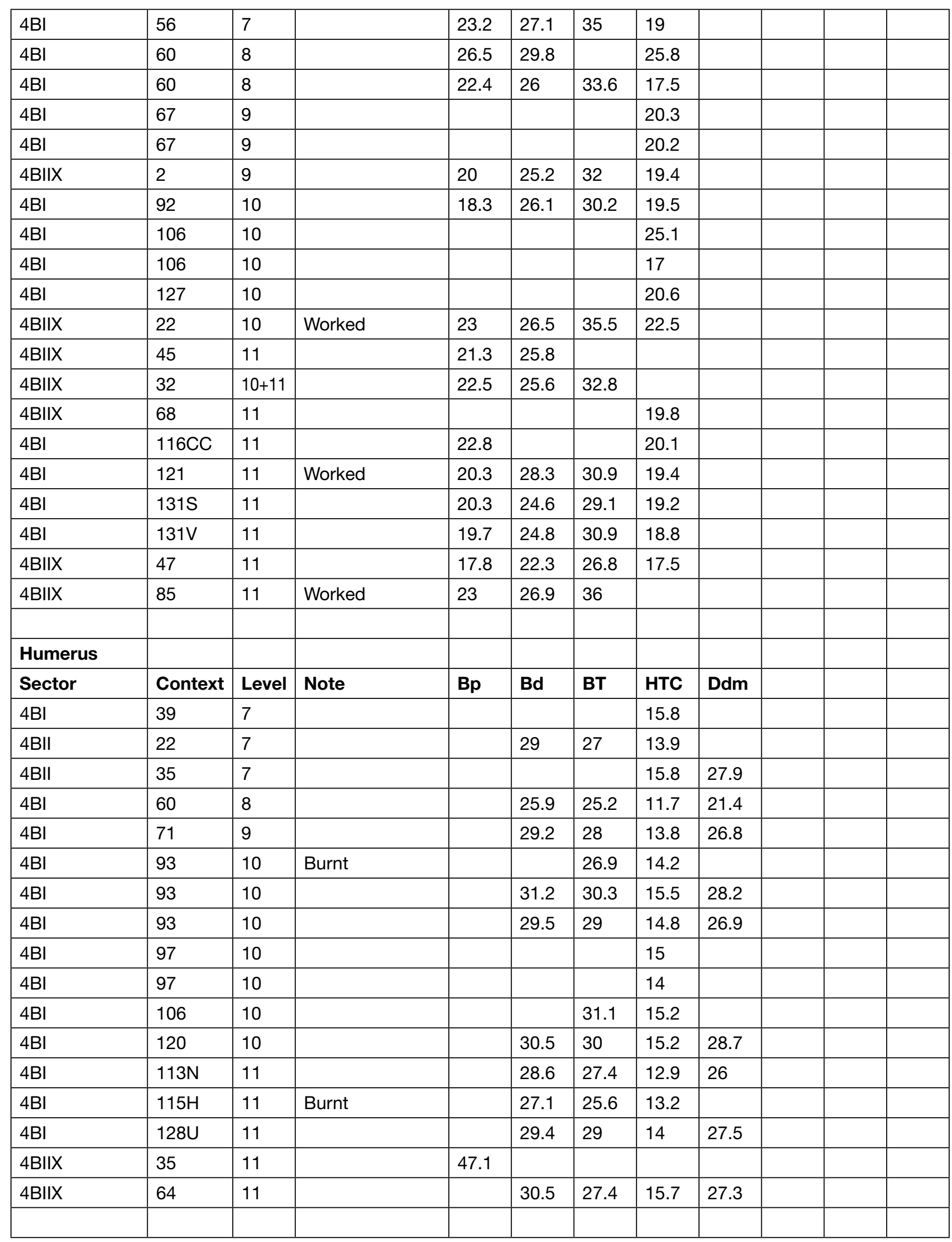


Table A17.2 Continued.

\begin{tabular}{|c|c|c|c|c|c|c|c|c|c|c|c|}
\hline \multicolumn{12}{|l|}{ Radius } \\
\hline Sector & Context & Level & Note & Bp & BFp & $\mathrm{Dp}$ & SD & Bd & BFd & Dd & GL \\
\hline 4BI & 60 & 8 & Unfused & & & & & 30.1 & 24.6 & 19.2 & \\
\hline 4BIIX & 44 & 10 & Unfused & & & & & 27.9 & & 18.1 & \\
\hline 4BI & 93 & 10 & & & & 15.6 & & & & & \\
\hline 4BI & 106 & 10 & & 32.7 & 30.3 & 16.2 & & & & & \\
\hline 4BI & 127 & 10 & Fusing & 30.3 & 27.6 & 16.2 & & & & & \\
\hline 4BI & $113 G$ & 11 & & 27.4 & 26.5 & & 17.9 & & & & \\
\hline 4BI & 114BB & 11 & Dist. Unfused & 33.1 & 30.9 & 18.2 & 17.3 & 31.8 & 27 & 21 & 171 \\
\hline 4BI & $128 \mathrm{KK}$ & 11 & Unfused & & & & & 26.8 & & 17.3 & \\
\hline 4BI & $131 \mathrm{FF}$ & 11 & Dist. Unfused & & & & & 29.5 & 22.5 & 19.7 & 15.3 \\
\hline 4BI & $131 \mathrm{FF}$ & 11 & Unfused & & & & 19.4 & 29 & 24.4 & 18.7 & \\
\hline 4BI & $131 \mathrm{FF}$ & 11 & Dist. Unfused & 34.7 & 31.8 & 17.9 & 17.6 & 32.2 & 26.6 & 22.6 & 160 \\
\hline 4BI & $131 \mathrm{KK}$ & 11 & Dist. Unfused & 34.4 & 31.4 & 18.3 & 18.8 & 30.5 & 24.9 & 20.2 & 176 \\
\hline 4BIIX & 57 & 11 & Burnt & & & & & 30.2 & & & \\
\hline \multicolumn{12}{|l|}{ Ulna } \\
\hline Sector & Context & Level & Note & DPA & SDO & LO & BPC & & & & \\
\hline 4BI & 6 & 5 & & 23.5 & 19.6 & 40.1 & & & & & \\
\hline 4BI & 39 & 7 & Unfused & 25.7 & 21.2 & & 19.6 & & & & \\
\hline 4BII & 22 & 7 & Unfused & 26.5 & 22.2 & & 17.6 & & & & \\
\hline 4BI & 79 & 9 & & 22.5 & 19.4 & & & & & & \\
\hline 4BI & 114BB & 11 & Unfused & 26.1 & 22.1 & 43.7 & 20 & & & & \\
\hline 4BI & $117 X$ & 11 & & 25.3 & 21.1 & & 17.3 & & & & \\
\hline 4BI & $131 \mathrm{FF}$ & 11 & Unfused & 28.3 & 24.7 & & 19.5 & & & & \\
\hline 4BI & $131 \mathrm{KK}$ & 11 & Unfused & 29.5 & 25.5 & & & & & & \\
\hline 4BIIX & 24 & 11 & Unfused & 27 & 22.9 & & & & & & \\
\hline 4BIIX & 64 & 11 & & 19.6 & 23.6 & & 14.7 & & & & \\
\hline \multicolumn{12}{|c|}{ Metacarpal } \\
\hline Sector & Context & Level & Note & $\mathrm{Bp}$ & $\mathrm{Dp}$ & SD & Bd & Dd & GL & & \\
\hline 4BI & 53 & 6 & & & & & & 17 & & & \\
\hline 4BII & 2 & 6 & & 21.9 & 15.3 & 13.3 & & & & & \\
\hline 4BI & 44 & 7 & & 22.1 & 15.9 & & & & & & \\
\hline 4BII & 16 & 7 & Worked & & & & & 15.6 & & & \\
\hline 4BI & 60 & 8 & & 20.8 & 14.3 & & & & & & \\
\hline 4BI & 73 & 9 & & 20.9 & 15.1 & & & & & & \\
\hline 4BI & 88 & 10 & & & & & 23.7 & 15.9 & & & \\
\hline 4BI & 106 & 10 & & & & & 24.7 & & & & \\
\hline 4BI & 114CC & 11 & Unfused & 20.7 & 14.7 & 11.6 & 22.8 & 15.1 & 111.5 & & \\
\hline $4 \mathrm{BI}$ & $131 \mathrm{KK}$ & 11 & & 24 & 17.7 & 16 & 28.1 & 17.1 & 146.3 & & \\
\hline 4BI & $131 \mathrm{KK}$ & 11 & Unfused & 21.4 & 15.1 & 13.7 & 24.8 & 14.6 & 133.1 & & \\
\hline 4BI & $131 \mathrm{KK}$ & 11 & Unfused & & & & 24.5 & 16.1 & & & \\
\hline
\end{tabular}


Table A17.2 Continued.

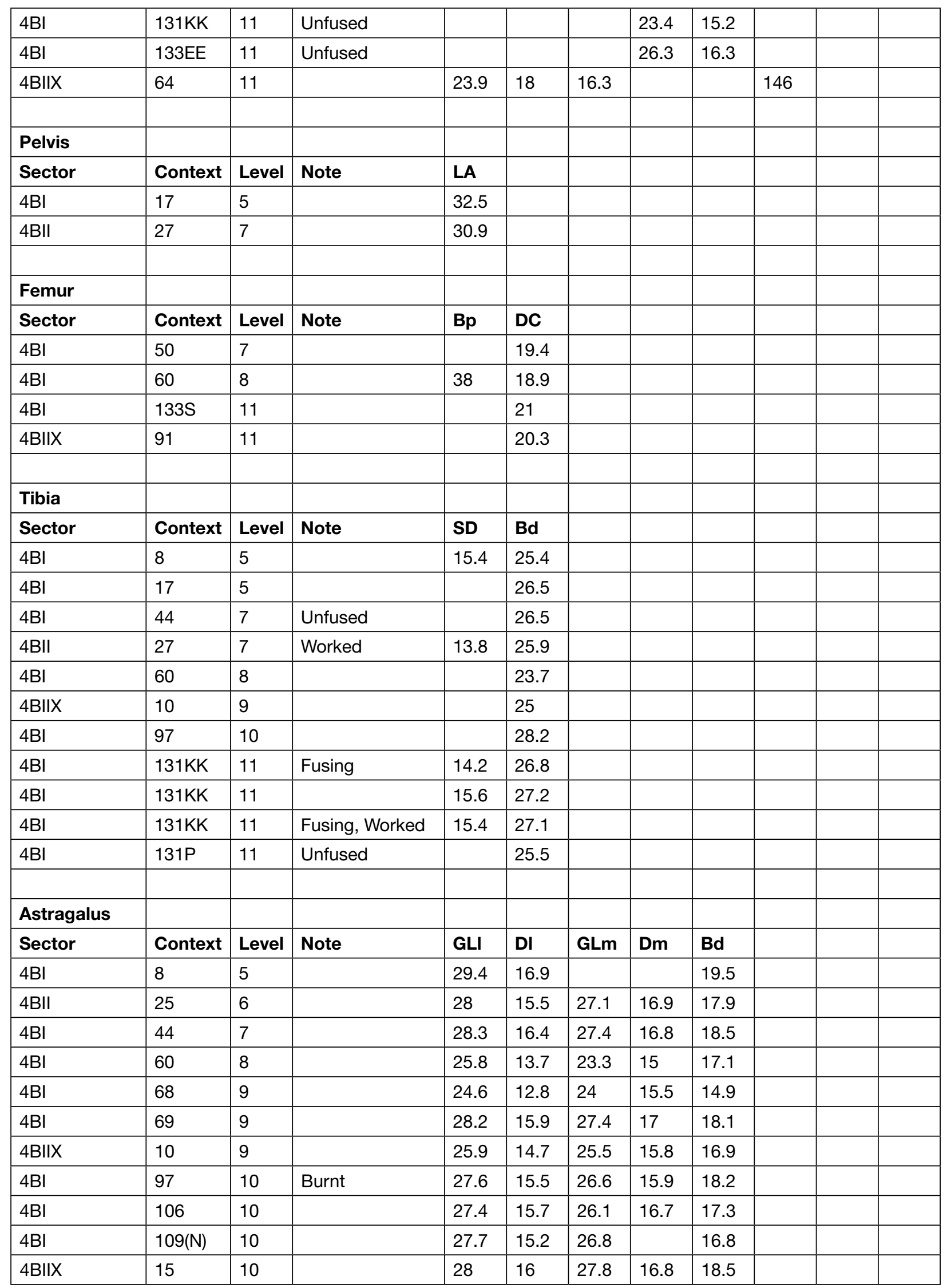


Table A17.2 Continued.

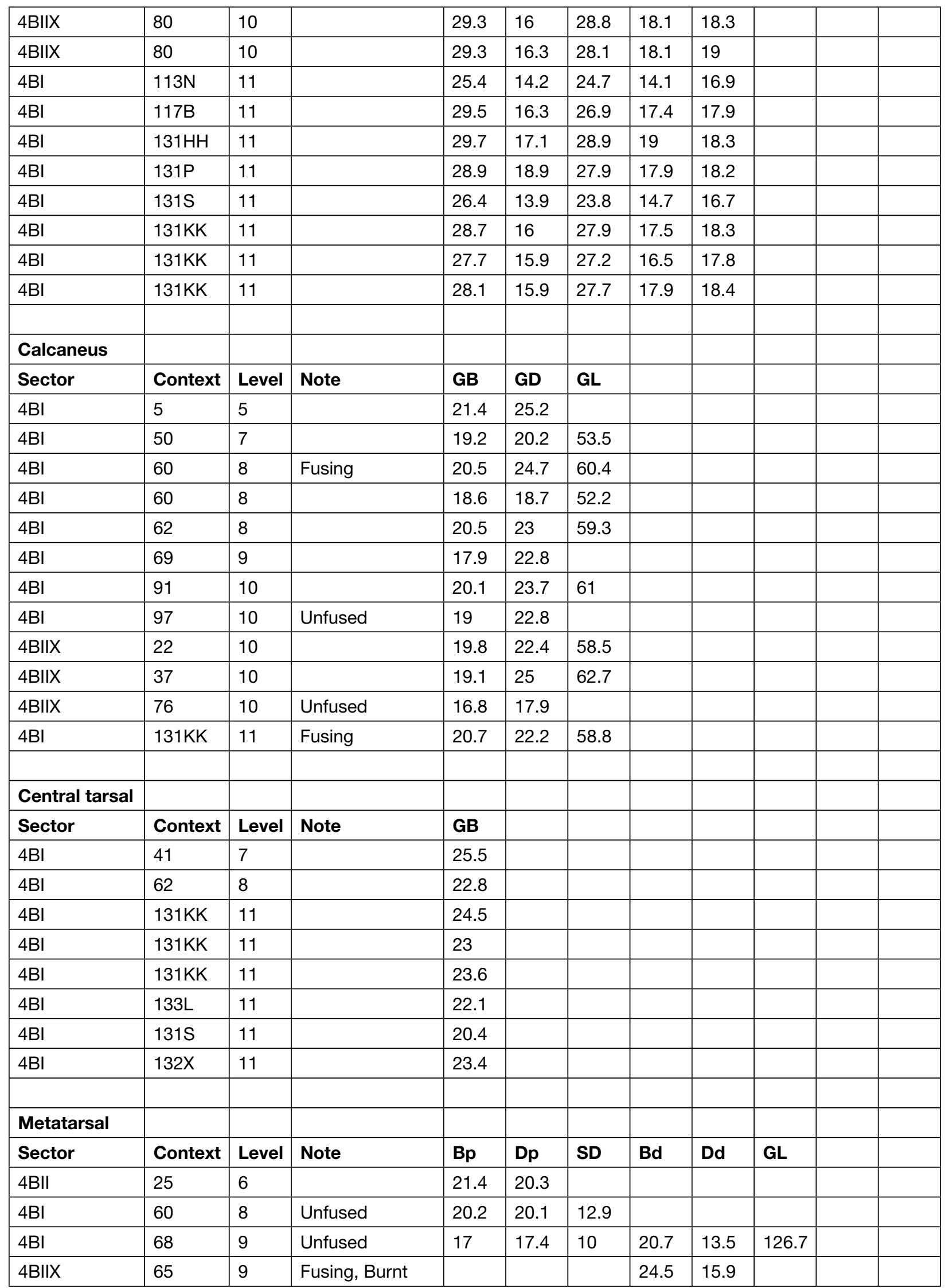


Table A17.2 Continued.

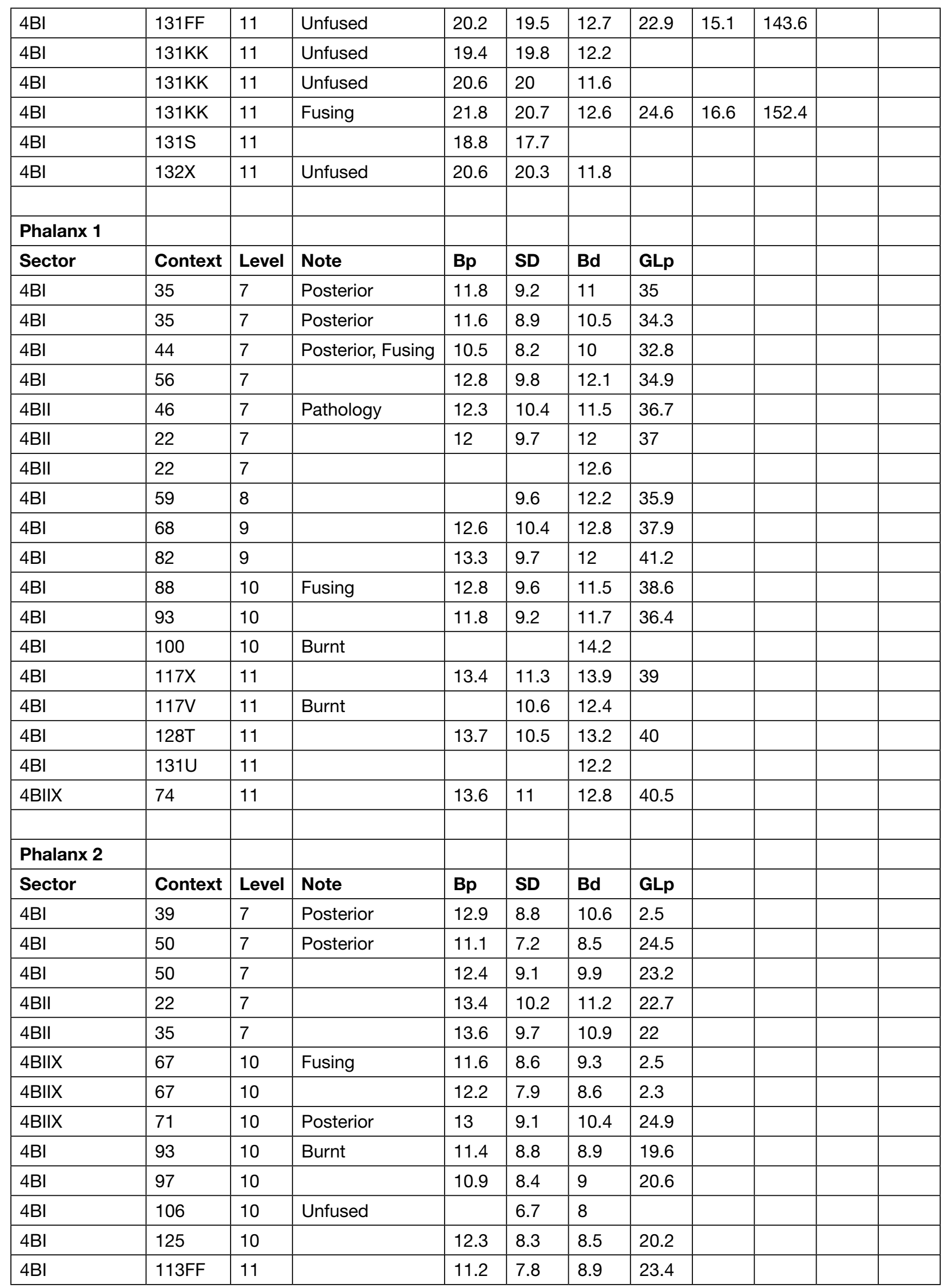


Table A17.2 Continued.

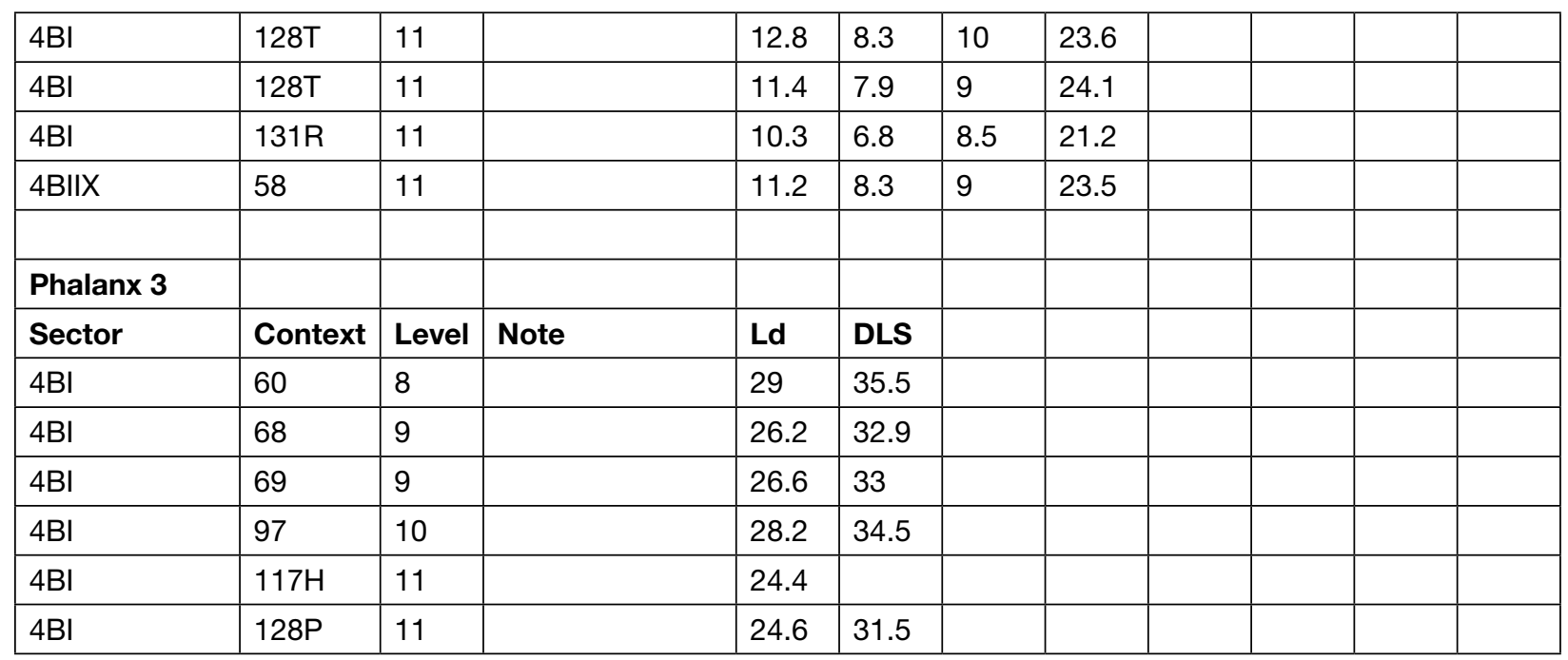

Table A17.3 Measurements of Bos sp.

\begin{tabular}{|c|c|c|c|c|c|c|c|}
\hline \multicolumn{8}{|l|}{ Atlas } \\
\hline Sector & Context & Level & Note & BFcd & & & \\
\hline 4BII & 22 & 7 & & 48.7 & & & \\
\hline \multicolumn{8}{|c|}{ Scapula } \\
\hline Sector & Context & Level & Note & BG & LG & GLP & SLC \\
\hline 4BI & 8 & 5 & & & & & 19.9 \\
\hline 4BI & 44 & 7 & & 20.6 & 22.6 & 27.6 & 17.3 \\
\hline 4BI & 37 & 7 & & & & & 16.1 \\
\hline 4BI & 39 & 7 & Worked & 20.5 & 22.5 & 29.5 & 16.8 \\
\hline 4BII & 21 & 7 & Worked & & & & 24.8 \\
\hline 4BII & 43 & 7 & Worked & 23 & 28 & 31.4 & 20.3 \\
\hline 4BII & 49 & 8 & & & & & 18.1 \\
\hline 4BIIX & 80 & 10 & Fusing & 18.8 & 24 & 29.4 & 14.6 \\
\hline 4BI & $117 \mathrm{CC}$ & 11 & & 19.5 & 22.8 & & 15.6 \\
\hline 4BIIX & 90 & 11 & Worked & 27.5 & 28.5 & 37.2 & 25.3 \\
\hline \multicolumn{8}{|c|}{ Humerus } \\
\hline Sector & Context & Level & Note & Bd & BT & HTC & Ddm \\
\hline 4BI & 97 & 10 & & 28.1 & 26.7 & 12 & \\
\hline 4BIIX & 15 & 10 & & 28.9 & 27.6 & 12.2 & \\
\hline 4BIIX & 64 & 11 & Fusing & 26.8 & 24.9 & 12.6 & 23.1 \\
\hline 4BIIX & 96 & 12 & Fusing & & 26.8 & 13.6 & \\
\hline \multicolumn{8}{|l|}{ Radius } \\
\hline Sector & Context & Level & Note & $\mathrm{Bp}$ & BFp & $\mathrm{Dp}$ & SD \\
\hline $4 \mathrm{BI}$ & 12 & 5 & & 36.5 & & 18.4 & \\
\hline
\end{tabular}


Table A17.3 Continued.

\begin{tabular}{|c|c|c|c|c|c|c|c|c|}
\hline 4BI & $111(N)$ & 10 & & 27.3 & 26.7 & 14.1 & 15.1 & \\
\hline 4BI & $117 \mathrm{~T}$ & 11 & & 34 & 32 & 18.3 & & \\
\hline 4BI & $131 \mathrm{KK}$ & 11 & & 32.1 & 29.3 & 16.2 & 19.5 & \\
\hline 4BIIX & 64 & 11 & & 28.5 & 26.7 & 15.3 & 16.9 & \\
\hline \multicolumn{9}{|l|}{ Ulna } \\
\hline Sector & Context & Level & Note & DPA & SDO & LO & BPC & \\
\hline 4BII & 22 & 7 & & 27 & 22.2 & 41 & 18.6 & \\
\hline 4BI & $111(\mathrm{~N})$ & 10 & Unfused & 21.5 & 19.1 & 36.1 & 21.2 & \\
\hline 4BI & $131 \mathrm{KK}$ & 11 & Unfused & 25.9 & 21.6 & & 22.8 & \\
\hline \multicolumn{9}{|c|}{ Metacarpal } \\
\hline Sector & Context & Level & Note & Dd & & & & \\
\hline 4BI & 48 & 7 & Worked & 15.2 & & & & \\
\hline \multicolumn{9}{|l|}{ Pelvis } \\
\hline Sector & Context & Level & Note & LA & & & & \\
\hline 4BI & 15 & 5 & & 27.4 & & & & \\
\hline \multicolumn{9}{|l|}{ Tibia } \\
\hline Sector & Context & Level & Note & Bp & Dp & SD & Bd & GL \\
\hline 4BI & 5 & 5 & Fusing, Worked & & & & 28.5 & \\
\hline $4 \mathrm{BI}$ & 15 & 5 & & & & & 23.7 & \\
\hline 4BI & 21 & 6 & Worked & & & & 21.5 & \\
\hline $4 \mathrm{BI}$ & 38 & 7 & & & & & 23.5 & \\
\hline $4 \mathrm{BI}$ & 106 & 10 & & & & & 25.8 & \\
\hline 4BI & $110(\mathrm{~S})$ & 10 & Unfused & & & & 23.2 & \\
\hline 4BI & 115EE & 11 & & & & & 27.1 & \\
\hline $4 \mathrm{BI}$ & $131 \mathrm{FF}$ & 11 & & 37.1 & 37.5 & 13.9 & 22.9 & 186 \\
\hline 4BI & $131 \mathrm{KK}$ & 11 & Unfused & & & 14.4 & 25 & \\
\hline \multicolumn{9}{|c|}{ Astragalus } \\
\hline Sector & Context & Level & Note & GLI & DI & GLm & Dm & Bd \\
\hline 4BII & 10 & 7 & & 31 & 17.1 & 27.8 & 17.9 & 18.3 \\
\hline 4BIIX & 37 & 10 & & & 16.4 & 28.8 & 18.1 & 18.3 \\
\hline $4 \mathrm{BI}$ & 115EE & 11 & & 32.7 & 17.3 & 29.9 & 18.5 & 17.9 \\
\hline \multicolumn{9}{|c|}{ Calcaneus } \\
\hline Sector & Context & Level & Note & GB & GD & GL & & \\
\hline $4 \mathrm{BII}$ & 22 & 7 & & 21.3 & 24.7 & 62 & & \\
\hline $4 \mathrm{BI}$ & $110(S)$ & 10 & Unfused & 21.1 & 21.6 & 63.3 & & \\
\hline
\end{tabular}


Table A17.3 Continued.

\begin{tabular}{|c|c|c|c|c|c|c|c|c|c|}
\hline \multicolumn{10}{|c|}{ Central Tarsal } \\
\hline Sector & Context & Level & Note & GB & & & & & \\
\hline 4BI & 32 & 5 & & 24.9 & & & & & \\
\hline 4BI & $110(S)$ & 10 & & 25.4 & & & & & \\
\hline 4BI & $131 \mathrm{KK}$ & 11 & & 54.9 & & & & & \\
\hline \multicolumn{10}{|c|}{ Metatarsal } \\
\hline Sector & Context & Level & Note & $\mathrm{Bp}$ & Dp & SD & Bd & Dd & GL \\
\hline 4BI & 32 & 5 & & & & & 22.1 & 15 & \\
\hline 4BI & 38 & 7 & Worked & & & & 22.5 & 15 & \\
\hline 4BI & 71 & 9 & & 19.5 & 18.2 & 12.4 & 23.7 & & 108.5 \\
\hline \multicolumn{10}{|c|}{ Phalanx 1} \\
\hline Sector & Context & Level & Note & $\mathrm{Bp}$ & SD & Bd & GLp & & \\
\hline 4BI & 32 & 5 & & 11.9 & & & & & \\
\hline 4BI & 24 & 6 & & 10.6 & 9.3 & 10.5 & 33.2 & & \\
\hline 4BII & 22 & 7 & & & & 10.8 & 35.5 & & \\
\hline 4BII & 45 & 7 & & & & 13.1 & & & \\
\hline 4BI & 68 & 9 & & & & 9.7 & & & \\
\hline 4BI & 106 & 10 & & & & 9.3 & & & \\
\hline \multicolumn{10}{|c|}{ Phalanx 2} \\
\hline Sector & Context & Level & Note & $B p$ & SD & Bd & GLp & & \\
\hline 4BI & 71 & 9 & & 12.9 & 8.5 & 10.6 & 25.1 & & \\
\hline 4BI & $133 Q$ & 11 & & 12.3 & 8.7 & 9.9 & 21.1 & & \\
\hline
\end{tabular}

Table A17.4 Measurements of Sus sp.

\begin{tabular}{|l|l|l|l|l|l|l|l|l|l|}
\hline Scapula & & & & & & & & \\
\hline Sector & Context & Level & Note & BG & LG & GLP & SLC & \\
\hline 4BI & $131 \mathrm{M}$ & 11 & Worked & 57.8 & 69.4 & & & \\
\hline 4BI & $132 \mathrm{X}$ & 11 & Worked & 51.6 & 62.3 & & 66.1 & \\
\hline 4BIIX & 47 & 11 & Worked & 48 & 60.5 & 68.2 & & & \\
\hline 4BIIX & 47 & 11 & Worked & 57 & 67 & 78 & 67.2 & & \\
\hline 4BIIX & 58 & 11 & Worked & 44.1 & 60 & 72 & & & \\
\hline 4BIIX & 64 & 11 & Worked & 37.2 & 47.2 & 58.7 & & & \\
\hline 4BIIX & 69 & 11 & Worked & 43.3 & 62.5 & 74.8 & & & \\
\hline & & & & & & & & & \\
\hline Radius & & & & & & & & & \\
\hline Sector & Context & Level & Note & Bd & BFd & & & & \\
\hline 4BI & 50 & 7 & Unfused & 63.2 & & & & & \\
\hline 4BI & 66 & 9 & Unfused & 76.1 & 61.5 & & & & \\
\hline 4BI & 67 & 9 & Unfused & 80 & 63 & & & & \\
\hline & & & & & & & & & \\
\hline
\end{tabular}


Table A17.4 Continued.

\begin{tabular}{|c|c|c|c|c|c|c|c|c|c|}
\hline \multicolumn{10}{|c|}{ Metacarpal } \\
\hline Sector & Context & Level & Note & Bp & Dp & SD & Bd & Dd & GL \\
\hline 4BI & 58 & 7 & Worked & & & 32.3 & 59.1 & 36.4 & \\
\hline 4BIIX & 14 & 10 & & & & & 59 & 31.8 & \\
\hline 4BI & $131 \mathrm{KK}$ & 11 & Worked & 60.9 & 38.7 & 32.8 & 60 & 32.8 & 210 \\
\hline 4BI & $131 \mathrm{KK}$ & 11 & Worked & 56.1 & 34.7 & & & & \\
\hline \multicolumn{10}{|l|}{ Femur } \\
\hline Sector & Context & Level & Note & DC & Bd & & & & \\
\hline 4BI & 35 & 7 & & 56.8 & & & & & \\
\hline \multicolumn{10}{|c|}{ Astragalus } \\
\hline Sector & Context & Level & Note & GLI & DI & GLm & Dm & Bd & \\
\hline 4BI & 6 & 5 & & 79.2 & 44.8 & 72 & 45 & 50.1 & \\
\hline 4BI & 44 & 7 & & 73.4 & 42 & 66.5 & 41.9 & 48.7 & \\
\hline 4BII & 11 & 7 & & & & & & 48 & \\
\hline 4BI & 60 & 8 & & 73.2 & 41.2 & 66.8 & 42.5 & 49.5 & \\
\hline \multicolumn{10}{|c|}{ Calcaneus } \\
\hline Sector & Context & Level & Note & GB & GD & GL & & & \\
\hline 4BII & 25 & 6 & Gnawed & 51 & 57.4 & & & & \\
\hline 4BI & 104 & 10 & & 55 & 55.4 & 141.3 & & & \\
\hline 4BIIX & 36 & 11 & Unfused & 46.8 & 52.9 & & & & \\
\hline \multicolumn{10}{|c|}{ Central tarsal } \\
\hline Sector & Context & Level & Note & GB & & & & & \\
\hline 4BII & 25 & 6 & & 64 & & & & & \\
\hline 4BII & 25 & 6 & & 52 & & & & & \\
\hline 4BII & 22 & 7 & & 52.1 & & & & & \\
\hline 4BII & 43 & 7 & & 57.5 & & & & & \\
\hline \multicolumn{10}{|c|}{ Metatarsal } \\
\hline Sector & Context & Level & Note & $\mathrm{Bp}$ & Dp & SD & Bd & Dd & GL \\
\hline 4BI & 128DD & 11 & & 49.2 & 49.7 & 27.4 & 57 & 30.9 & 230.5 \\
\hline 4BI & $131 \mathrm{KK}$ & 11 & & 48.8 & 44.7 & 26.9 & 55.3 & 32.1 & 236 \\
\hline \multicolumn{10}{|c|}{ Phalanx 1} \\
\hline Sector & Context & Level & Note & $\mathrm{Bp}$ & SD & Bd & GLp & & \\
\hline 4BI & 6 & 5 & Anterior, Pathology & 34.8 & 29.5 & 33.7 & 60.5 & & \\
\hline 4BI & 12 & 5 & Posterior & 30.6 & 25.9 & 29.4 & 70.9 & & \\
\hline 4BI & 12 & 5 & Posterior & & & 27.9 & & & \\
\hline 4BI & 13 & 5 & Posterior & 33.1 & & & & & \\
\hline 4BI & 15 & 5 & Posterior & 25.5 & 21.2 & 25.3 & 55.6 & & \\
\hline
\end{tabular}


Table A17.4 Continued.

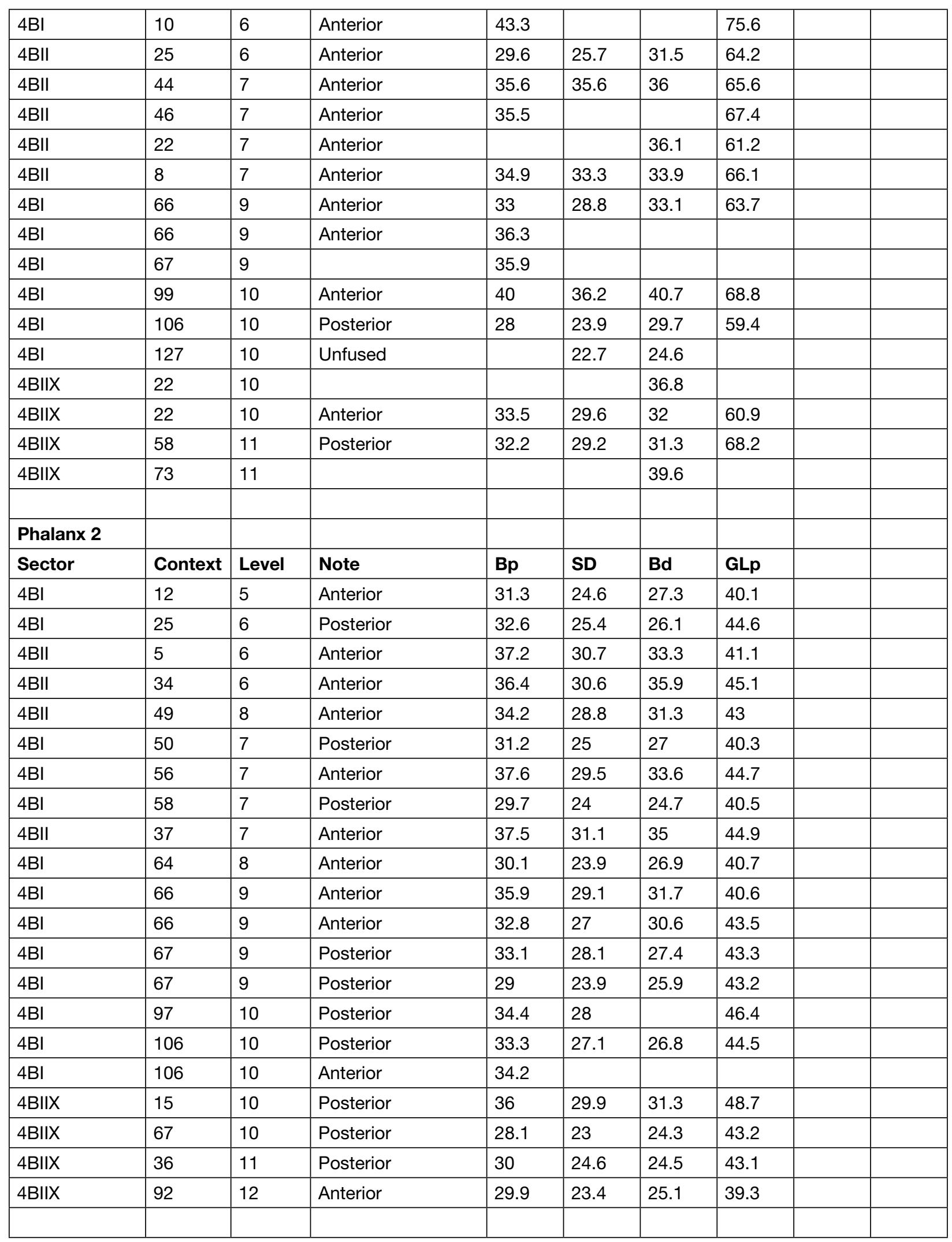


Table A17.4 Continued.

\begin{tabular}{|l|l|l|l|l|l|l|l|l|l|}
\hline Phalanx 3 & & & & & & & & \\
\hline Sector & Context & Level & Note & Ld & DLS & & & & \\
\hline 4BI & 15 & 5 & & 63.9 & & & & & \\
\hline 4BII & 5 & 6 & & 64.7 & 91.2 & & & & \\
\hline 4BII & 25 & 6 & & 76.9 & 10.2 & & & & \\
\hline 4BII & 25 & 6 & & 72.8 & 97.2 & & & & \\
\hline 4BI & 46 & 7 & & 62.5 & 76.7 & & & & \\
\hline 4BI & 67 & 9 & & 72.3 & 93.4 & & & & \\
\hline 4BI & 100 & 10 & & 62.4 & 80.5 & & & & \\
\hline 4BIIX & 15 & 10 & & 65.5 & 84.7 & & & & \\
\hline 4BIIX & 44 & 10 & & 54.8 & 50 & & & & \\
\hline 4BIIX & 71 & 10 & & 60.9 & 81.2 & & & & \\
\hline 4BIIX & 32 & $10+11$ & & 60.5 & 78.7 & & & & \\
\hline 4BIIX & 36 & 11 & & 65.7 & 80.3 & & & & \\
\hline 4BIIX & 64 & 11 & & 70.8 & 85.7 & & & & \\
\hline
\end{tabular}

Table A17.5 Measurements of Canis familiaris.

\begin{tabular}{|c|c|c|c|c|c|c|c|}
\hline \multicolumn{8}{|l|}{ Atlas } \\
\hline Sector & Context & Level & Note & BFcr & H & GB & GL \\
\hline 4BII & 5 & 6 & & 49 & 47 & 71 & 43.5 \\
\hline \multicolumn{8}{|c|}{ Scapula } \\
\hline Sector & Context & Level & Note & $\mathbf{B G}$ & LG & GLP & SLC \\
\hline 4BI & 4 & 5 & & & & & 21.6 \\
\hline 4BII & 25 & 6 & Unfused & & & & 19.3 \\
\hline 4BI & 35 & 7 & Unfused & 20.6 & & & 17.7 \\
\hline 4BI & 54 & 7 & & & & & 19.9 \\
\hline 4BII & 28 & 7 & & 26.1 & 29.1 & 36.2 & 22.3 \\
\hline 4BII & 14 & 7 & & 22 & & & 19.6 \\
\hline \multicolumn{8}{|c|}{ Humerus } \\
\hline Sector & Context & Level & Note & Bd & BT & HTC & Ddm \\
\hline 4BI & 41 & 7 & & 40.7 & 31.9 & 18.7 & 41.8 \\
\hline $4 \mathrm{BII}$ & 43 & 7 & & 39.3 & 31.7 & 18.2 & \\
\hline \multicolumn{8}{|l|}{ Ulna } \\
\hline Sector & Context & Level & Note & DPA & SDO & LO & BPC \\
\hline 4BII & 14 & 7 & & 36.4 & & & 22.9 \\
\hline \multicolumn{8}{|l|}{ Pelvis } \\
\hline Sector & Context & Level & Note & LA & & & \\
\hline 4BII & 20 & 7 & & 35 & & & \\
\hline
\end{tabular}


Table A17.5 Continued.

\begin{tabular}{|c|c|c|c|c|c|c|c|}
\hline \multicolumn{8}{|c|}{ Astragalus } \\
\hline Sector & Context & Level & Note & GLI & DI & GLm & Bd \\
\hline 4BI & 44 & 7 & & 41.4 & 20 & 37.5 & 22.7 \\
\hline \multicolumn{8}{|c|}{ Phalanx 1} \\
\hline Sector & Context & Level & Note & Bp & SD & Bd & GLp \\
\hline 4BI & 6 & 5 & & 19.8 & 15.2 & 16.9 & 35 \\
\hline 4BI & 24 & 6 & Unfused & & 12.3 & 14.5 & \\
\hline 4BI & 44 & 7 & Unfused & 14.4 & & & \\
\hline 4BI & 58 & 7 & & 17.2 & 13.4 & 15.6 & 35.5 \\
\hline 4BII & 20 & 7 & Unfused & 18.7 & 10 & 18 & 26.2 \\
\hline 4BII & 22 & 7 & & 17.2 & & & \\
\hline 4BI & 67 & 9 & Unfused & 17 & 14.2 & 15.5 & 37 \\
\hline 4BI & 69 & 9 & Unfused & & 13.1 & 15.1 & \\
\hline 4BII & 53 & 9 & Unfused & 11.8 & 9.2 & 11.4 & 24.4 \\
\hline 4BII & 53 & 9 & Unfused & 11.8 & 9.7 & 10.7 & 24.5 \\
\hline 4BI & $131 \mathrm{M}$ & 11 & Fusing & 15.1 & 11.6 & 14.5 & 32.5 \\
\hline 4BIIX & 59 & 11 & Unfused & & 11.4 & 13.9 & \\
\hline \multicolumn{8}{|c|}{ Phalanx 2} \\
\hline Sector & Context & Level & Note & Bp & SD & Bd & GLp \\
\hline 4BI & 69 & 9 & & 15.9 & 13.8 & 13.5 & 23.2 \\
\hline 4BI & 71 & 9 & & 17 & 14.4 & 15 & 23.8 \\
\hline \multicolumn{8}{|c|}{ Phalanx 3} \\
\hline Sector & Context & Level & Note & Ld & DLS & & \\
\hline 4BII & 5 & 6 & & 30.6 & 32.7 & & \\
\hline
\end{tabular}

Table A17.6 Measurements of Cervus elaphus.

\begin{tabular}{|l|l|l|l|l|l|l|l|}
\hline UIna & & & & & & & \\
\hline Sector & Context & Level & Note & DPA & SDO & LO & BPC \\
\hline 4BIIX & 36 & 11 & & 14.7 & 12 & 18.9 & 9.2 \\
\hline & & & & & & & \\
\hline Femur & & & & & & & \\
\hline Sector & Context & Level & Note & Bp & DC & SD & Bd \\
\hline 4BI & 97 & 10 & & & & & 19.8 \\
\hline 4BIIX & 69 & 11 & & 32.5 & 16.2 & & \\
\hline 4BIIX & 85 & 11 & Unfused & & & 82 & 21.4 \\
\hline
\end{tabular}


Table A17.7 Measurements of Gazella subgutturosa.

\begin{tabular}{|c|c|c|c|c|c|c|c|}
\hline \multicolumn{8}{|c|}{ Scapula } \\
\hline Sector & Context & Level & Note & BG & LG & GLP & SLC \\
\hline 4BI & $111(\mathrm{~N})$ & 10 & Unfused & 42.3 & 47 & 58.9 & 32.3 \\
\hline \multicolumn{8}{|c|}{ Humerus } \\
\hline Sector & Context & Level & Note & Bd & BT & HTC & Ddm \\
\hline 4BII & 48 & 8 & & 54.6 & 54 & 29.4 & 57.6 \\
\hline \multicolumn{8}{|c|}{ Metacarpal } \\
\hline Sector & Context & Level & Note & Bd & & & \\
\hline 4BI & 41 & 7 & & 48.2 & & & \\
\hline \multicolumn{8}{|l|}{ Femur } \\
\hline Sector & Context & Level & Note & Bd & & & \\
\hline 4BI & 93 & 10 & & 72 & & & \\
\hline \multicolumn{8}{|c|}{ Calcaneus } \\
\hline Sector & Context & Level & Note & GB & GD & GL & \\
\hline 4BI & 15 & 5 & & 39 & 45.2 & 125 & \\
\hline 4BI & 67 & 9 & & 39 & 47.6 & & \\
\hline \multicolumn{8}{|c|}{ Phalanx 1} \\
\hline Sector & Context & Level & Note & $\mathrm{Bp}$ & SD & Bd & GLp \\
\hline 4BIIX & 5 & 9 & & 20.3 & 16.7 & 20.2 & 52.8 \\
\hline 4BI & 106 & 10 & & 21.7 & 16.6 & 20.1 & 58.4 \\
\hline 4BI & $117 \mathrm{H}$ & 11 & & 22.1 & 19.5 & 22.5 & 55.7 \\
\hline \multicolumn{8}{|c|}{ Phalanx 2} \\
\hline Sector & Context & Level & Note & Bp & SD & Bd & GLp \\
\hline 4BI & 38 & 7 & & 25.8 & 18 & 22.1 & 48.8 \\
\hline 4BI & 127 & 10 & & 19.5 & 13.5 & 17.8 & 42 \\
\hline
\end{tabular}

Table A17.8 Measurements of Capreolus capreolus.

\begin{tabular}{|l|l|l|l|l|l|l|l|l|l|}
\hline Scapula & & & & & & & & & \\
\hline Sector & Context & Level & Note & BG & LG & GLP & SLC & & \\
\hline 4BI & 66 & 9 & & 23 & 25.3 & 33.4 & 16.7 & & \\
\hline & & & & & & & & & \\
\hline Radius & & & & & & & & & \\
\hline Sector & Context & Level & Note & Bp & BFp & Dp & SD & & \\
\hline 4BIIX & 65 & 9 & & 30.9 & 29.5 & 15.7 & & & \\
\hline 4BI & $111(\mathrm{~N})$ & 10 & & 27.9 & 25.6 & 15.6 & 16.3 & & \\
\hline & & & & & & & & & \\
\hline
\end{tabular}


Table A17.8 Continued.

\begin{tabular}{|c|c|c|c|c|c|c|c|c|c|}
\hline \multicolumn{10}{|l|}{ Ulna } \\
\hline Sector & Context & Level & Note & DPA & SDO & LO & BPC & & \\
\hline 4BI & $111(\mathrm{~N})$ & 10 & & 21.8 & 18.7 & 37.2 & 14.8 & & \\
\hline \multicolumn{10}{|c|}{ Metacarpal } \\
\hline Sector & Context & Level & Note & Bp & Dp & SD & Bd & Dd & GL \\
\hline 4BI & 6 & 5 & Worked Bone & & & & & 18 & \\
\hline 4BI & 32 & 5 & Worked Bone & & & & 22.8 & 18.1 & \\
\hline $4 \mathrm{BI}$ & 123 & 11 & & & & & 22.4 & 16.6 & \\
\hline $4 \mathrm{BI}$ & $131 \mathrm{KK}$ & 11 & & & & 12.8 & 24 & 18.8 & \\
\hline \multicolumn{10}{|l|}{ Tibia } \\
\hline Sector & Context & Level & Note & Bd & & & & & \\
\hline 4BII & 5 & 6 & & 21.6 & & & & & \\
\hline \multicolumn{10}{|c|}{ Metatarsal } \\
\hline Sector & Context & Level & Note & Bp & Dp & SD & Bd & Dd & GL \\
\hline 4BI & $131 \mathrm{KK}$ & 11 & & 21.1 & 23.9 & 11.2 & & & \\
\hline 4BI & $131 \mathrm{KK}$ & 11 & & 23.2 & 26.9 & 13.6 & & & \\
\hline \multicolumn{10}{|c|}{ Phalanx 1} \\
\hline Sector & Context & Level & Note & Bp & SD & Bd & GLp & & \\
\hline 4BII & 51 & 8 & & 10.4 & 8.2 & 9.9 & 34.2 & & \\
\hline 4BIIX & 65 & 9 & & 11.2 & 8.1 & 10.2 & 49.3 & & \\
\hline 4BIIX & 65 & 9 & & 10.9 & 8.1 & 10.4 & 50.5 & & \\
\hline 4BIIX & 47 & 11 & & 11.7 & 8.3 & 10.2 & 44.7 & & \\
\hline \multicolumn{10}{|c|}{ Phalanx 2} \\
\hline Sector & Context & Level & Note & $\mathrm{Bp}$ & SD & Bd & GLp & & \\
\hline 4BI & $128 \mathrm{CC}$ & 11 & & 10.3 & 6.7 & 7.2 & 23.4 & & \\
\hline
\end{tabular}

Table A17.9 Measurements of Lepus sp.

\begin{tabular}{|l|l|l|l|l|l|l|l|l|}
\hline Astragalus & & & & & & & & \\
\hline Sector & Context & Level & Note & GLI & DI & GLm & Dm & Bd \\
\hline 4BI & 41 & 7 & & 25.1 & 15.2 & 24.6 & 15.8 & 16.5 \\
\hline & & & & & & & & \\
\hline Calcaneus & & & & & & & & \\
\hline Sector & Context & Level & Note & GB & GD & GL & & \\
\hline 4BIIX & 5 & 9 & & 17.5 & 19.5 & 51.7 & & \\
\hline
\end{tabular}




\section{REZUME}

2008-ci ildə Azərbaycanda ilkin sivilizasiyaların başlanğıc ərəfəsini əks etdirən erkən əkinçi maldar tayfaların qədim yaşayış yerlərinin öyrənilməsi məqsədilə, Tovuz bölgəsində yerləşən Göytəpə qədim yaşayış yerində arxeoloji qazıntı işləri təşkil edildi.

Bundan əvvəl isə, Azərbaycan Milli Elmlər Akademiyasının, Arxeologiya və Etnoqrafiya İnstitutunun ekspedisiyası 2007-ci ildə, Neolit və Xalkolit dövrünə aid qədim yaşayış yerlərinin monitorinqi ilə bağl1, Azərbaycan (Fərhad Quliyev) və Fransa (Bertil Lyonnet) arxeoloqları ilə birgə çöl tədqiqatlarını həyata keçirmiş oldu. Ekspedisiyanın məqsədi bölgənin qədim yaşayış yerlərinə nəzər keçirmək və yeni araşdırmalar üçün strateji proqram hazırlamaq idi.

Ümumiyyətlə, Azərbaycan alimləri tərəfindən oturaq həyat tərzli erkən əkinçi tayfaların yaşayış yerlərinin aşkar edilməsi faktı (60 il bundan əvvəl) tədqiqatçı Osman Həbibullayevin adı ilə bağlıdır. Bu baxımdan Naxçıvan bölgəsində I Kültəpə qədim yaşayış yerinin arxeoloji qazıntıları, Azərbaycanın Ön Asiyanın qədim mədəniyyətləri sistemində öyrənilməsi üçün yeni səhifə açmışdır. Azərbaycan arxeoloqu İdeal Nərimanov, Azərbaycan ərazisində erkən əkinçi tayfalara aid arxeoloji kompleksləri əsaslı şəkildə araşdırmış və Kür çayının orta axarında yayılmış olan abidələr kompleksini dünya elmi ictimaiyyətinə "Şomutəpə" (ilk araşdırılan qədim yaşayış yeri 1964-cü ildə "Şomutəpə" idi) arxeoloji mədəniyyəti kimi tanıtmışdır. İri və kiçik süni təpə formasında olan qədim yaşayış yerləri, erkən əkinçilik və maldarlıq təsərrüfatını, ilkin saxsı məmulatının geniş̧ istifadəsini və çiy kərpicdən tikilən dairəvi formalı evlərin özündə əks etdirir. İdeal Nərimanov bölgədə apardığı araşdırmaları zamanı (60-80 illərdə) Tovuz rayonunda yerləşən Göytəpə qədim yaşayış yerini aşkar edərək abidə haqqında ilkin informasiyanı öz nəşrində çap etmişdir.

2008-ci ildə, İdeal Nərimanovun yetirməsi tərəfindən (Fərhad Quliyev) Göytəpə qədim yaşayış yerində əsaslı arxeoloji qazıntı işləri başlanılmışdır.

2009-cu ildə, tədqiqatçı Fərhad Quliyev Yaponiyanın Tokio Universitetinin Professoru, Orta Şərqin neolit dövrü üzrə mütəxəssisi Yoşihiro Nişiyaki ilə Göytəpə qədim yaşayış yerində və ətrafında olan (Tovuz rayonunda yerləşən) digər arxeoloji abidələrdə müş̧ərək araşdırmalar aparılması ilə bağlı ilkin razılığa gəldikdən sonra tərəflər arasında (Azərbaycan Milli Elmlər Akademiyasının, Arxeologiya və Etnoqrafiya İnstitutu və Yaponiyanın Tokio Universiteti) rəsmi olaraq əməkdaşlıq memorandumu imzalanmışdır. Azərbaycan və Yaponiya beynəlxalq arxeoloji ekspedisiyasının əsas məqsədi: qədim dövrün oturaq həyat tərzinin xüsusiyyətlərini, qida istehsalının yüksəliş mərhələsini, qazıntılar zamanı aşkar olunmuş maddi mədəniyyət nümunələrinin mədəni mənşəyinin müəyyən edilməsi və region üzrə Neolit dövrü yaşayış yerlərinin dəqiq xronoloji çərçivəsini təyin etməkdən ibarət olmuşdur.

Bu baxımdan Azərbaycan və Yaponiya mütəxəssisləri tərəfindən təşkil olunmuş yaradıcı kollektivin, bizim eramızdan əvvəl VI minilliyə aid ilk kəndlərdən olan Göytəpə arxeoloji kompleksində aparılmış tədqiqat işlərinin nəticələrinə həsr olunmuş birinci kitabı oxucuların müzakirəsinə təqdim edirik.

Göytəpədə arxeoloji qazıntı işləri abidənin üst hissəsindən başlayaraq, iki əsas strategiyadan ibarət olmuşdur. Geniş ölçülü arxeoloji qazıntılar qədim yaşayış yerinin ümumi tərtibatını və quruluşunu təyin etməyə yönəlmişdir. Təpənin şimal yamacında $10 \times 10 \mathrm{~m}$ ölçüsündə ümumilikdə, 1000 kvadrat metr sahədə 10 kvadratda qazıntılar aparılmışdır. Qazıntı kvadratları arasında yalnız təpənin şimal-şərq hissəsində yerləşən 4B kvadratı materikə qədər öyrənilmişdir. Nəticədə qədim yaşayış yerinin 14 memarlıq tikili horizontundan ibarət 11 metr Neolit təbəqəsi üzə çıxarılmışdır. Yüksək yaşam tərzini əks etdirən qalın mədəni qat yatımında on dörd (14) tikili horizontların hər birinin quruluşu haqqında dəqiq informasiyalar əldə edilmişdir. Heç bir regional Neolit abidəsində belə bir mükəmməl stratiqrafik quruluşa, mədəni təbəqə ardıcıllığına rast gəlinməmişdir.

Göytəpədə memarlıq tikililəri 2-6 m diametrində dairəvi formalı tikililərdən ibarətdir. Tikililər bir-birinə çiy kərpicdən ibarət əyri formalı divarlar vasitəsilə birləşir və həyətə aç1lan dairəvi kompleks əmələ gətirir. Kompleksə eyni zamanda ocaq yerləri, təsərrüfat anbarları və digər məişət tikililəri də daxildir. Mövcud materiallar klassik cəhətdən Neolit dövrü ilə birbaşa uzlaşır: saxsı məmulatı, iri və xırda daş alətlər və cilalanmış daş artefakt nümunələr, sümük alətlər, həmçinin əhliləşdirilmiş heyvan sümükləri və qida məqsədilə istifadə olunan bitki qalıqları. Saxsı məmulatları arasında kobud formalı, yapma dekorlu keramika nümunələri üstünlük təşkil edir. Mədəniyyətin erkən çağında əsasən mineral tərkibli keramika nümunələrinə, daha sonrakı mərhələlərdə isə bitki 
tərkibli keramika nümunələrinə rast gəlinir. Daş məmulatına gəldikdə, əsasən Kiçik Qafqaz mənbələrindən əldə edilən obsidiandan hazırlanmış ülgüclü bıçaqlar daha xarakterik alətlər hesab edilir. Sümük alətlər isə başlıca olaraq heyvan sümüyündən hazırlanmış bizlər, çəkiclər, baltalar və oraqların dəstəklərindən ibarətdir.

Göytəpə qədim yaşayış yerinin aid olduğu məlum "Şomutəpə" arxeoloji mədəniyyəti abidələri bəzi ədəbiyyatlarda bizim eradan əvvəl V-ci minilliyə aid edilir. Lakin son illərdə aparılmış daha etibarlı və dəqiq radiokarbon analizlər bu mədəniyyətin bizim eradan əvvəl VI-c1 minilliyə aid olduğunu deməyə əsas verir. Keramikalı Neolit dövrünü (b.e.ə. VI minillik) özündə əks etdirən Göytəpə arxeoloji abidəsinin öyrənilməsi məqsədilə, 46 ədəd nümunə üzərində radiokarbon (C14) analizlər aparılmışdır. Abidə üzərində aparılmış müqayisəli stratiqrafik analizlər erkən Keramikalı Neolit dövründə sabit bir yaşayışın mövcud olduğunu göstərmiş̧ir.

Göytəpə son Neolit dövrünün bütün xüsusiyyətlərini özündə əks etdirən Azərbaycan ərazisində ilk kənd tipli yaşayış yerlərindən biri olmuşdur. Mükəmməl şəkildə Neolit dövrünün sakinləri tərəfindən inşa olunan qədim yaşayış yeri dövrün inkişaf mərhələsini özündə cəmləşdirmiş̧ir. Qədim yaşayış yerinin sakinlərinin zəngin maddi mədəni irsi, bizim eradan əvvəl VI-cı minillikdə Orta Kür hövzəsində məskunlaşmış erkən əkinçi maldar tayfaların yüksək mədəniyyətə malik olduqlarını sübuta yetirir. Đldə olunmuş elmi nəticələr bir neçə mərhələdən ibarət olmuş beynəlxalq və innovasiya xarakterli müxtəlif elmlərin qovşağında aparılan Azərbaycan və Yaponiya arxeoloqlarının müştərək tədqiqatlarını əks etdirir. Arxeoloji abidədən aşkar olunmuş maddi-mədəniyyət nümunələrinin hər biri öz təyinatı üzrə xarici ölkələrin reytinqli elmi tədqiqat laboratoriyalarında analizlərə göndərilmiş və bununla arxeologiya elm sahəsində multidissiplinar xarakterli araşdırmaların aparılmasına şərait yaratmışdır.

Göytəpədə bu illər ərzində böyük bir komanda çalışmış̧ır. Hər il qazıntı mövsümündə müxtəlif ölkələrdən olan tələbə və mütəxəssislərə rast gəlmək mümkün idi. Yaponiyanın müxtəlif Universitetlərini və elmi ocaqlarını təmsil edən tanınmış arxeoloqlar Seydji Kodavaki, Kazuya Şimoqama, Çiya Akaşi, Saydji Aray, Azərbaycan tərəfindən çalışan arxeoloqlarımız Valeh Oləkbərov, Fuad Hüseynov, Xəqani Alməmmədov, Yaqub Məmmədov, Şahin Səlimbəyov, Pərviz Qasımov, Zəki Cəfərov və digərlərinin əməyini xüsusi qeyd etmək istərdik.

Tədqiqatlarda iştirak etmiş mütəxəssislər çöl-tədqiqat işlərinin aparılmasına görə Azərbaycan Respublikasının Prezidenti yanında Elmin İnkişafı Fonduna, Azərbaycan Milli Elmlər Akademiyasının, Arxeologiya və Etnoqrafiya İnstitutuna o cümlədən Yaponiyanın Təhsil, Mədəniyyət, Elm, İdman Texnologiya nazirliyinə, Yaponiyanın Elmin İnkişafı Cəmiyyətinə, Heiva Nakajima Fonduna maliyyə dəstəklərinə görə öz dərin təşəkkürünü bildirirlər.

Forhad Quliyev

AMEA Arxeologiya və Etnoqrafiya İnstitutunun şöbə rəhbəri, tarix üzrə fəlsəfə doktoru

Yoşihiro Nişiyaki

Tokio Universiteti, Universitet Muzeyinin direktoru, Professor 